UNITED STATES

DEPARTMENT OF THE INTERIOR

GEOLOGICAL SURVEY

FIELD DATA DESCRIBING THE MOVEMENT AND STORAGE OF SEDIMENT

IN THE EAST FORK RIVER, WYOMING

PART V. Bed-Material Tracers, 1979 and 1980

By William W. Emmett and Robert M. Myrick

Open-File Report 85-169

Denver, Colorado

1985 


\author{
UNITED STATES DEPARTMENT OF THE INTERIOR \\ DONALD PAUL HODEL, Secretary \\ GEOLOGICAL SURVEY \\ Dallas L. Peck, Director
}

For additional information write to:

U.S. Geological Survey

Water Resources Division

Mail Stop 413, Box 25046

Denver Federal Center

Denver, Colorado 80225

(303) 236-4998

For purchase, write to:

Open File Services Section

U.S. Geological Survey

Box 25425

Denver Federal Center

Denver, Colorado 80225

(303) 236-7476 


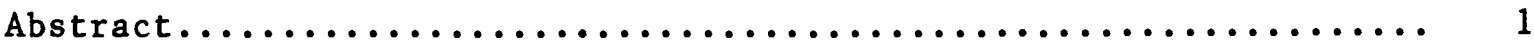

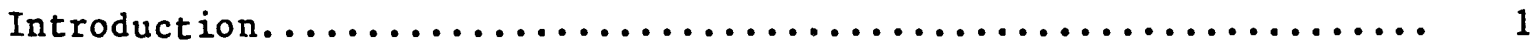

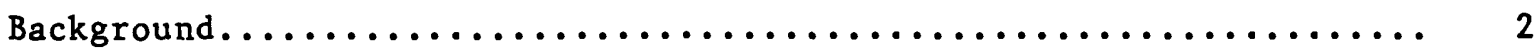

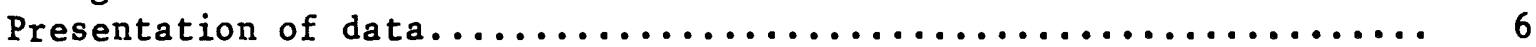

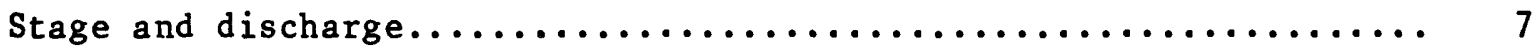

Bed material...................................... 8

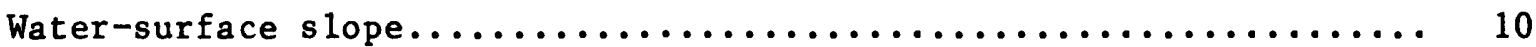

Stream power, bedload, and scour and fill...................... 13

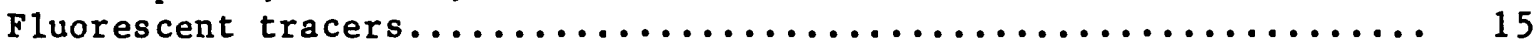

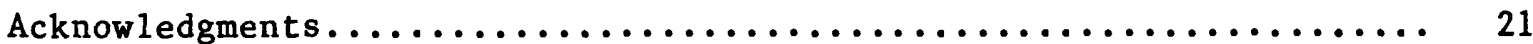

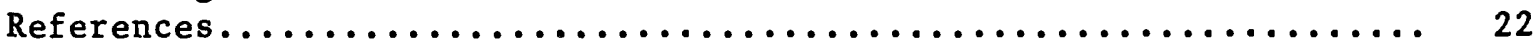

\section{ILLUSTRATIONS}

Figure 1. Map of the East Fork River, Wyoming, drainage area...... 3

2. Map showing location of cross sections along the $3.3-\mathrm{km}$ study reach, 1979 , East Fork River, Wyoming......... 4

3. Map showing location of cross sections along the $1.83-\mathrm{km}$ study reach, 1980 , East Fork River, Wyoming...........

4. Graph showing discharge hydrographs for 1979 and 1980 snowmelt runoff, section 0000 , East Fork River,

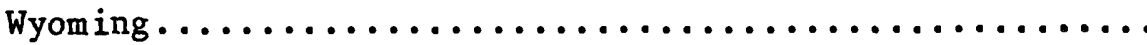

5. Graph showing downstream distribution of bed-material particle size, 1979, East Fork River, Wyoming........

6. Graph showing downstream distribution of volume of bed material, 1979, East Fork River, Wyoming...........

7. Graph showing longitudinal profiles of the water surface for low to high flow, 1979, East Fork River,

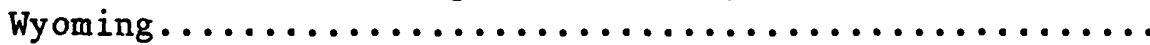

8. Graphs showing discharge, mean water-surface slope, and examples of local water-surface slope for a pool and a riffle, May-June 1980, East Fork River, Wyoming.. 0

9. Graphs showing discharge, and examples of stream power and bedload-transport rate for a pool and a riffle, May-June 1980, East Fork River, Wyoming.............

10. Graphs showing discharge, and examples of scour and fill for a pool and a riffle, May-June 1980, East Fork

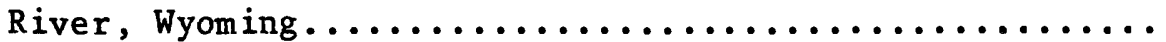

11. Graph showing downstream distribution of bed-material tracers, June 3,1979, the 16 th day after injection,

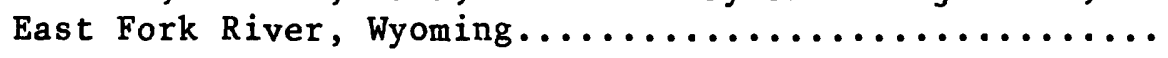




\section{ILLUSTRATIONS--Continued}

Page

Figure 12. Graph showing downstream daily displacement of the peak concentration of $0.5-$ to 1.0 -mm bed-material tracers, 1979, East Fork River, Wyoming............

13. Graph showing downstream particle speeds and travel distances for 0.5 - to 1.0 -mm bed-material tracers,

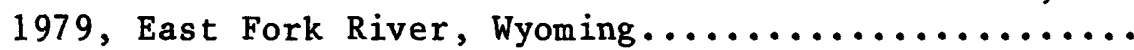

\section{TABLES}

Table 1. Bihourly discharge, in cubic meters per second, at section 0000 , East Fork River, Wyoming, 1979.........

2. Bihourly discharge, in cubic meters per second, at section 3295, East Fork River, Wyoming, 1979......... 30

3. Bihourly discharge, in cubic meters per second, at section 0000 , East Fork River, Wyoming, $1980 \ldots \ldots \ldots \ldots . . .34$

4. Bihourly discharge, in cubic meters per second, at section 2505, East Fork River, Wyoming, $1980 \ldots \ldots \ldots \ldots$....

5. Number of pink tracer particles between 0.25 and 8.00 millimeters, per 100 grams of bed material, as a function of date and section, East Fork River, Wyoming,

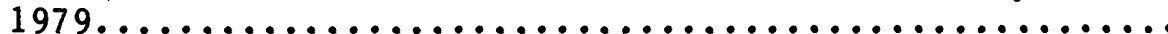

6. Number of blue tracer particles between 0.25 and 8.00 millimeters, per 100 grams of bed material, as a function of date and section, East Fork River, Wyoming,

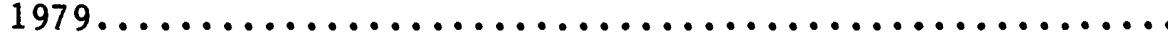

7. Number of orange tracer particles between 0.25 and 8.00 millimeters, per 100 grams of bed material, as a function of date and section, East Fork River, Wyoming,

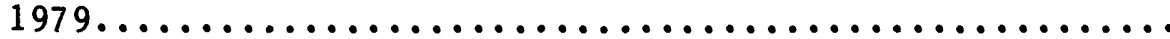

8. Number of pink tracer particles between 0.25 and 8.00 millimeters, per 100 grams of bedload, as a function of date and section, East Fork River, Wyoming,

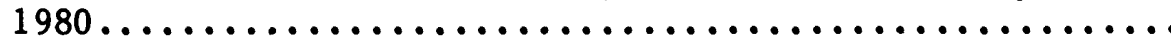

9. Number of blue tracer particles between 0.25 and 8.00 millimeters, per 100 grams of bedload, as a function of date and section, East Fork River, Wyoming, 1980....

10. Number of orange tracer particles between 0.25 and 8.00 millimeters, per 100 grams of bedload, as a function of date and section, East Fork River, Wyoming, 1980....

11. Number of green tracer particles between 0.25 and 8.00 millimeters, per 100 grams of bedload, as a function of date and section, East Fork River, Wyoming, 1980....

12. Number of pink tracer particles, per 100 grams of bed material, East Fork River, Wyoming, 1979............ 
TABLES--Continued

Page

Table 13. Number of blue tracer particles, per $100 \mathrm{grams}$ of bed material, East Fork River, Wyoming, 1979........... 91

14. Number of orange tracer particles, per 100 grams of bed material, East Fork River, Wyoming, 1979........... 104

15. Number of pink tracer particles, per 100 grams of bedload sample, composite from cross-channel sampling positions spaced at 1 -meter intervals across the channel width, East Fork River, Wyoming, $1980 \ldots \ldots \ldots \ldots$

16. Number of blue tracer particles, per 100 grams of bedload sample, composite from cross-channel sampling positions spaced at 1 -meter intervals across the channel width, East Fork River, Wyoming, $1980 \ldots \ldots \ldots \ldots$

17. Number of orange tracer particles, per 100 grams of bedload sample, composite from cross-channel sampling positions spaced at 1 -meter intervals across the channel width, East Fork River, Wyoming, 1980........

18. Number of green tracer particles, per 100 grams of bedload sample, composite from cross-channel sampling positions spaced at 1 -meter intervals across the channel width, East Fork River, Wyoming, $1980 \ldots \ldots \ldots \ldots$

19. Number of pink tracer particles, per 100 grams of bedload sample, collected at one or more cross-channel sampling positions spaced at 1 -meter intervals across the channel width, East Fork River, Wyoming, $1980 \ldots \ldots$.

20. Number of blue tracer particles, per 100 grams of bedload sample, collected at one or more cross-channel sampling positions spaced at 1-meter intervals across the channel width, East Fork River, Wyoming, $1980 \ldots \ldots$.

21. Number of green tracer particles, per 100 grams of bedload sample, collected at one or more crosschannel sampling positions spaced at 1-meter intervals across the channel width, East Fork River, Wyoming,

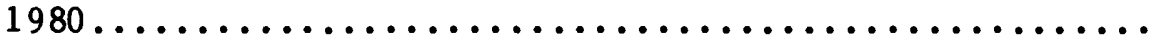

22. Number of pink tracer particles, per 100 grams of bed material sample, East Fork River, Wyoming, 1982...... 333

23. Number of blue tracer particles, per 100 grams of bed material sample, East Fork River, Wyoming, $1982 . \ldots \ldots$.

24. Number of orange tracer particles, per 100 grams of bed material sample, East Fork River, Wyoming, 1982...... 337

25. Number of green tracer particles, per 100 grams of bed material sample, East Fork River, Wyoming, 1982....... 


\title{
FIELD DATA DESCRIBING THE MOVEMENT AND STORAGE OF SEDIMENT IN THE EAST FORK RIVER, WYOMING \\ Part V. Bed-Material Tracers, 1979 and 1980
}

By William W. Emmett and Robert M. Myrick

\begin{abstract}
Bed-material particles, mostly coarse sand and fine gravel, were extracted from the streambed, dyed a fluorescent color, and reinjected into the streambed. During 1979, by bed-material sampling, and during 1980, by bedload sampling, the downstream dispersed distribution of tracer particles was measured. Complete data of the tracer studies are presented in a series of tables. The text contains supplementary explanation of the data, and examples of data are illustrated in graphical format. The text also contains some background information, complementary hydraulic information, and complete reference to other research on the East Fork River, Wyoming.
\end{abstract}

\section{INTRODUCTION}

Since 1967, the East Fork River in western Wyoming has been a field laboratory for the study of fluvial processes. Studies on the East Fork River and its principal tributary, Muddy Creek, have been reported by Andrews (1977,1979a,1979b,1981,1982a,1982b), Bagnold (1977, 1980), Bennett and Nordin (1977), Dietrich (1982a, 1982b), Dietrich, Smith, and Dunne (1979), Dunne and Leopold (1978), Emmett (1980a, 1980b, 1981, 1982a, 1982b), Emmett and Leopold (1977), Emmett, Leopold, and Myrick (1983), Emmett, Myrick, and Meade (1980, 1982), Klingeman and Emmett (1982), Leopold (1982a, 1982b), Leopold and Emmett (1976, 1977, 1982), Lisle (1976, 1979, 1982), Mahoney and others (1976), Meade, Emmett, and Myrick (1981a, $1981 \mathrm{~b})$, Meade, Myrick, and Emmett (1980,1982), and Prestegaard (1982a, $1982 \mathrm{~b})$.

Beginning in 1979 and continuing through 1980, the program was intensified to provide more definitive data on bedload transport along a reach of stream channel. Two principal differences distinguish the 1979 and 1980 data collections. First, during 1979, 41 cross sections were spaced approximately at equal distances along a 3.3-km (kilometer) reach; during 1980,44 cross sections were spaced approximately at equal distances along the downstream most $1.83 \mathrm{~km}$ of the 1979 reach. Second, during 1979, bed-material sampling at each cross section was the dominant sediment measurement; during 1980 , bedload measurements were the dominant sediment data collected at each cross section. 
Data for the 1979 field season have been published as parts I and II of this series of reports (Emmett, Myrick and Meade, 1980; Meade, Myrick, and Emmett, 1980). Data for the 1980 field season have been published as parts III and IV (Emmett, Myrick, and Meade, 1982; Meade, Myrick, and Emmett, 1982). Parts I and III contain data on river hydraulics, channel geometry, and rate and gradation of transported sediment. Parts II and IV tabulate the river-bed elevations that were measured and the types of bed material that were observed during several months at cross sections along the channel.

Prior to the 1979 field season, bed-material particles were dyed with a fluorescent resin coating, and separate-colored particles were injected as bed-material tracers at three sections within the study reach. This report (Part V) primarily tabulates observations related to tracking of fluorescent particles and is the last in the series of data compilations for the intensified studies begun during 1979 on the East Fork River.

\section{BACKGROUND}

The East Fork River originates in the Wind River Range of Wyoming west of the Continental Divide and east and south of Mt. Bonneville (fig. 1). From a series of small alpine lakes and an altitude of approximately 3,400 $m$ (meters), the East Fork River descends about 1,250 m in 50 river km to the project reach described in this report. Downstream from the study reach, it continues another $50 \mathrm{~km}$ to its confluence with the New Fork River, tributary to the Green River.

During 1979, the study reach was $3.3 \mathrm{~km}$ long and terminated downstream at a bedload trap constructed across the river (Leopold and Emmett, 1976; Emmett, 1980a). The general configuration of this study reach is shown in figure 2; the number shown at each section is the centerline distance in meters upstream from the bedload trap. During 1980 , only the downstream most $1.83 \mathrm{~km}$ of the 1979 reach were extensively measured. Exceptions were data on river stage, water discharge, and suspended sediment that were collected at a distance of $2505 \mathrm{~m}, 5 \mathrm{~m}$ downstream from section 2510 . (Given distances, for example $2505 \mathrm{~m}$, are hereafter referred to as sections, for example section 2505, if the distance is being used as a reference location.) Additional cross sections and the general configuration of the 1980 study reach are shown in figure 3 . 


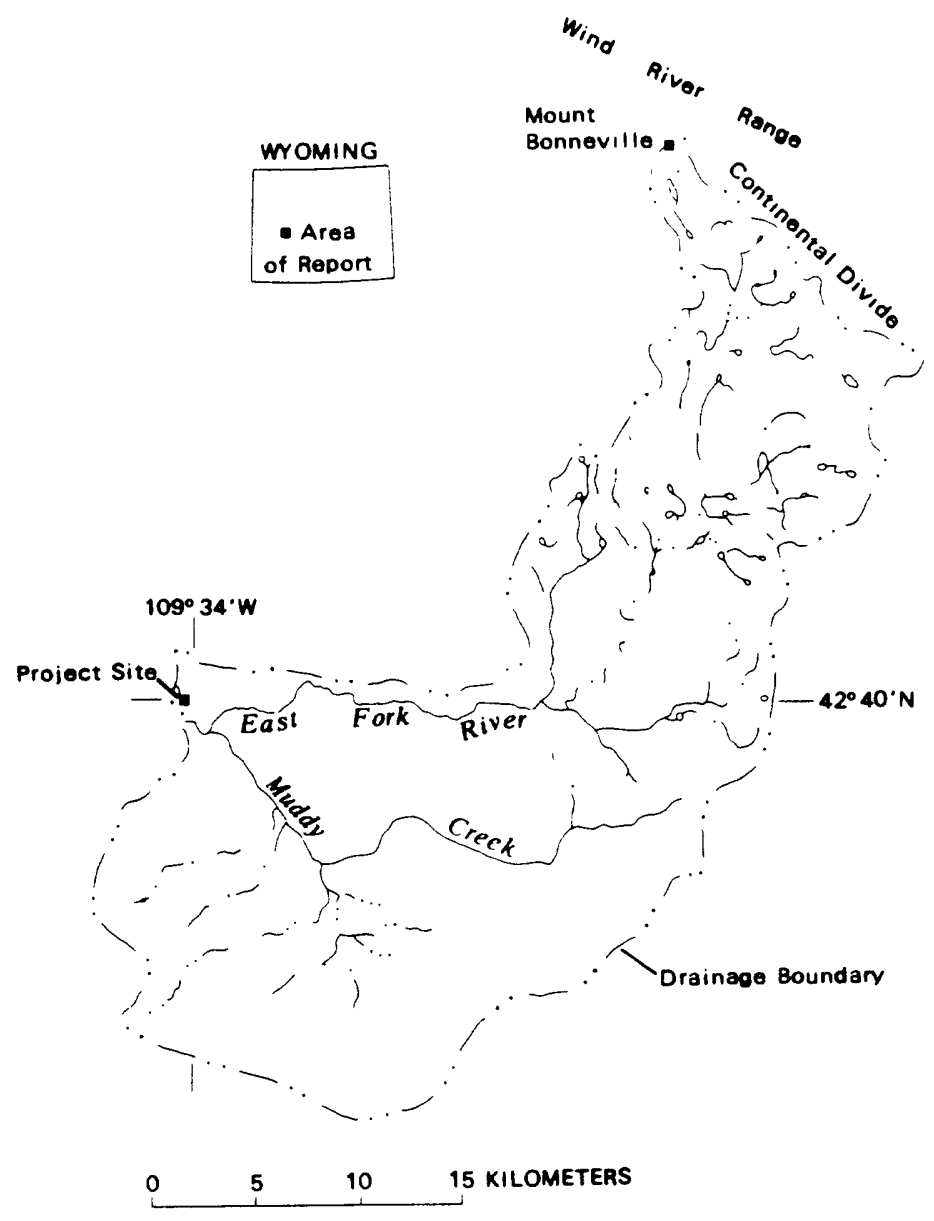

Figure 1. East Fork River, Wyoming, drainage area. 


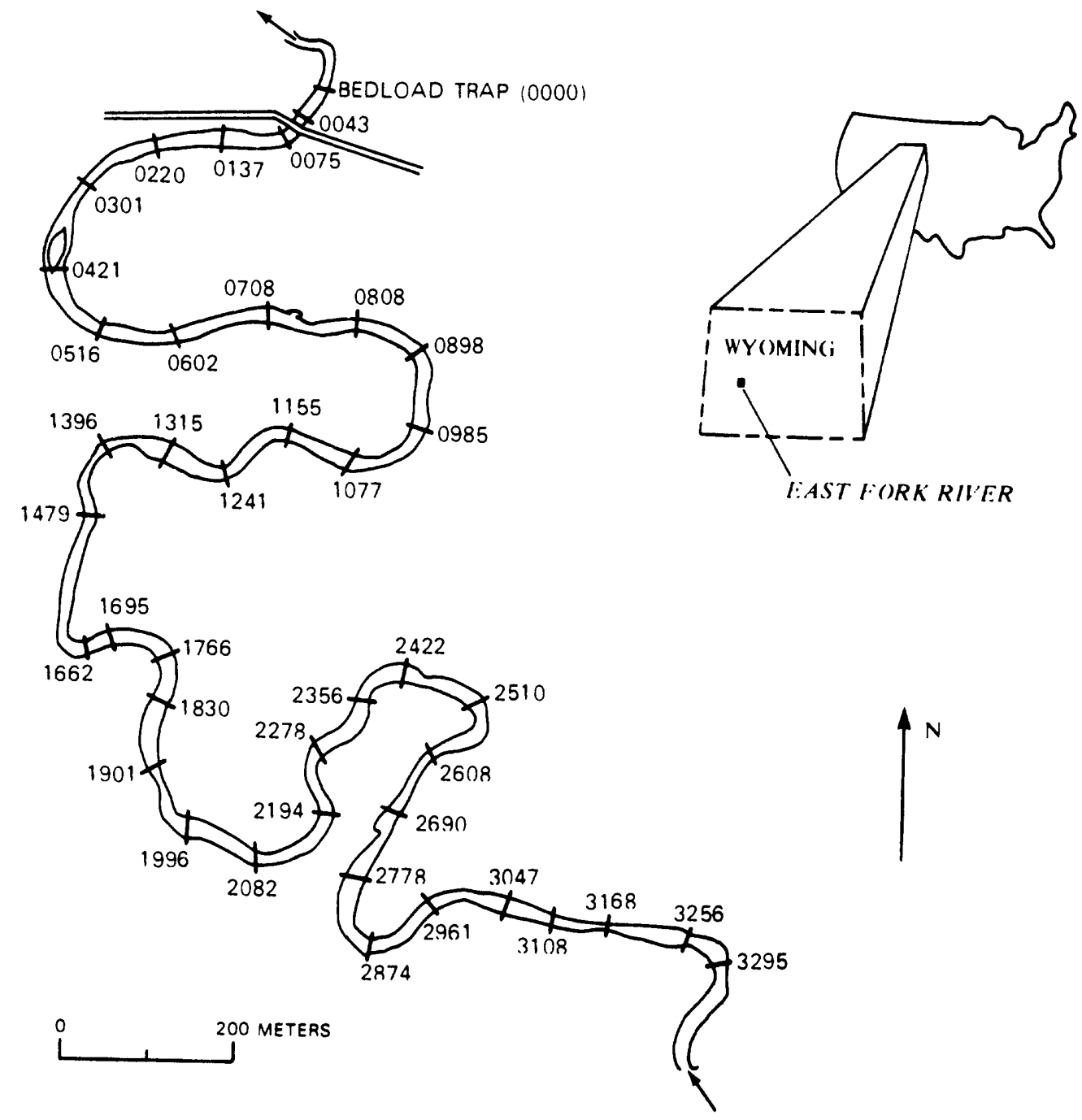

Figure 2. Location of cross sections along the 3.3-km study reach, 1979, East Fork River, Wyoming. 


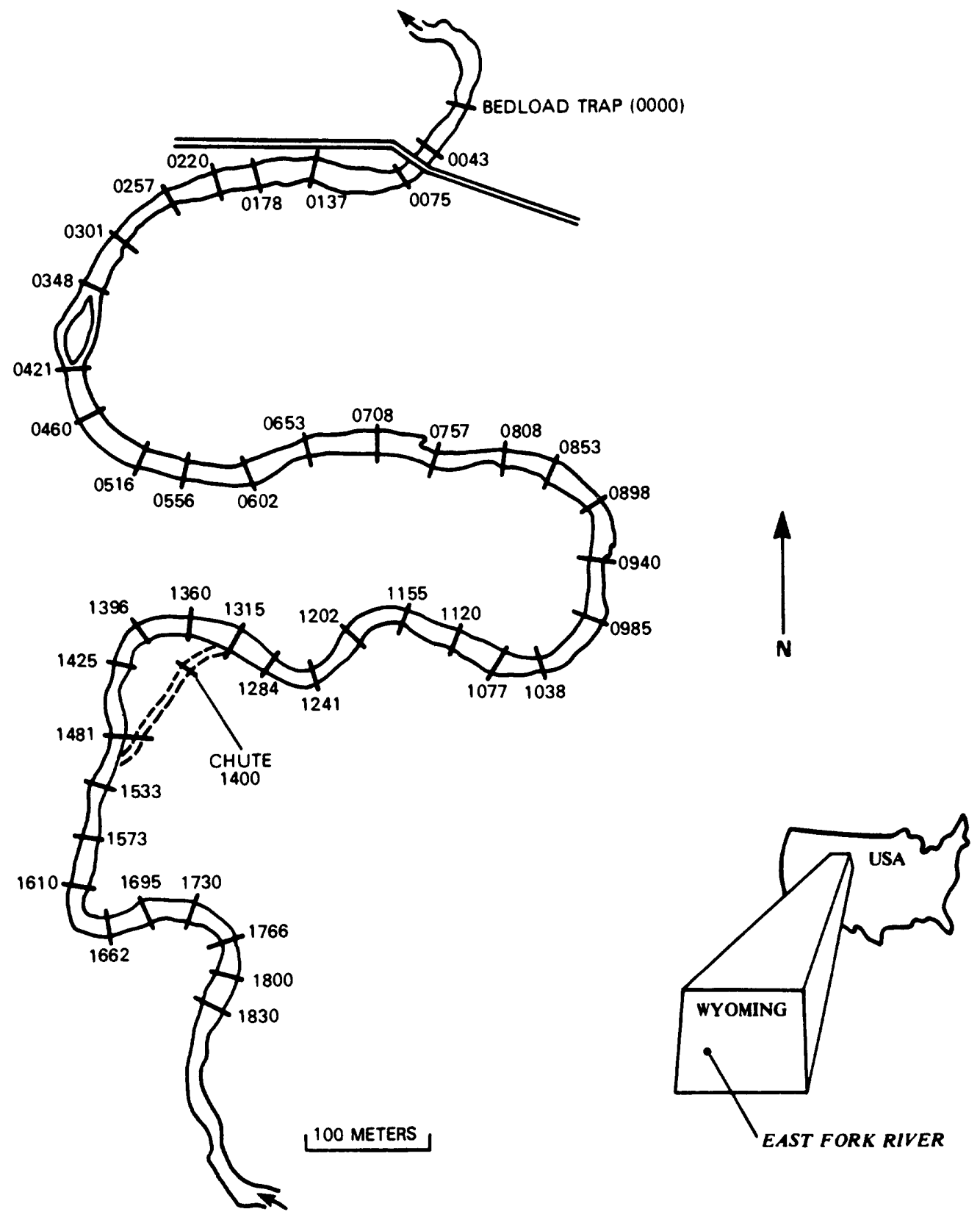

Figure 3. Location of cross sections along the $1.83-\mathrm{km}$ study reach, 1980 , East Fork River, Wyoming. 
The bedload trap was installed at latitude $42^{\circ} 40^{\prime} 23^{\prime \prime}$ N., longitude $109^{\circ} 34^{\prime} 16^{\prime \prime}$ W. The drainage area of the East Fork River at this point is about $500 \mathrm{~km}^{2}$ (square kilometer). About one-half of this basin area lies within the Wind River Mountains; the other one-half of the basin area is drained by a major tributary, Muddy Creek, that enters the East Fork River about $4 \mathrm{~km}$ upstream from the bedload trap and drains an upland of rolling hills underlain by lower Tertiary sandstone and shale of the Wasatch Formation. The mountainous part of the basin is underlain by granitic and metamorphic rocks, mostly of Precambrian age. Much of the sand part of the sediment load for the East Fork River comes from the Muddy Creek basin, but most of the water during high flow comes from melting snow in the mountain area.

Along the study reach, the East Fork River meanders in a flood plain averaging $100 \mathrm{~m}$ in width, which, in turn, is confined by the Wasatch Formation or by glacial outwash terraces of sand and gravel. The tread or surface of the most prominent terrace is about $5 \mathrm{~m}$ above the flood plain. The terraces and outcrops of the Wasatch Formation are sources of fresh sand and gravel debris wherever the river impinges laterally against them.

All elevations reported herein are referenced to the National Geodetic Vertical Datum (NGVD) of 1929 by three complete leveling surveys, conducted during May and October 1979, and during September 1980. Partial leveling surveys were conducted to re-establish NGVD elevations to reference markers when they were repositioned after the river had damaged or destroyed them.

\section{PRESENTATION OF DATA}

Each type of data is presented in a table or series of tables; all tables are included at the end of this report. In most instances, footnotes make the tables self-explanatory. As tables are presented, additional explanation is provided in the text. Some data also are illustrated in graphical form.

This report is intended to be supplemented by concurrent use of parts I-IV of the report series. However, some discussion from the earlier report is included here to make information on the bed-material tracers more readily understandable. 


\section{STAGE AND DISCHARGE}

Mean annual precipitation in the drainage basin ranges from about 300 $\mathrm{mm}$ (millimeters) in the vicinity of the study reach to an estimated 1,200 $\mathrm{mm}$ in the headwater areas. This can be compared to a mean annual runoff of about $450 \mathrm{~mm}$ at gaging station 09203000 , East Fork River near Big Sandy, which is located about 22 river $\mathrm{km}$ upstream from the study reach. This value of runoff corresponds to a mean annual discharge of about $3 \mathrm{~m}^{3} / \mathrm{s}$ (cubic meters per second) which is approximately valid for the study reach as well. Mean annual discharge is equaled or exceeded about 25 percent of the time. The level of the flood plain corresponds with the bankfull stage of the river, at which the water has an average depth of about $1.2 \mathrm{~m}$. The bankfull discharge is about $20 \mathrm{~m}^{3} / \mathrm{s}$.

Most precipitation occurs as snow. The high-flow season caused by spring snowmelt in the mountains extends from mid-May to early July and accounts for about 75 percent of the total annual flow. During spring runoff, diurnal fluctuations through the study reach are characterized by a rising stage during the morning, a peak stage at midday, and a declining stage during the afternoon. This fluctuation at the study reach reflects snowmelt in the mountains from the previous day.

Bihourly values of discharge, in cubic meters per second, for 1979 are listed in tables 1 and 2 (sections 0000 and 3295 , respectively) and for 1980 are 1 isted in tables 3 and 4 (sections 0000 and 2505 , respectively). Discharge hydrographs for spring runoff during 1979 and 1980 are shown in figure 4; discharges shown occurred at noon at the bedload trap (section 0000 ). 


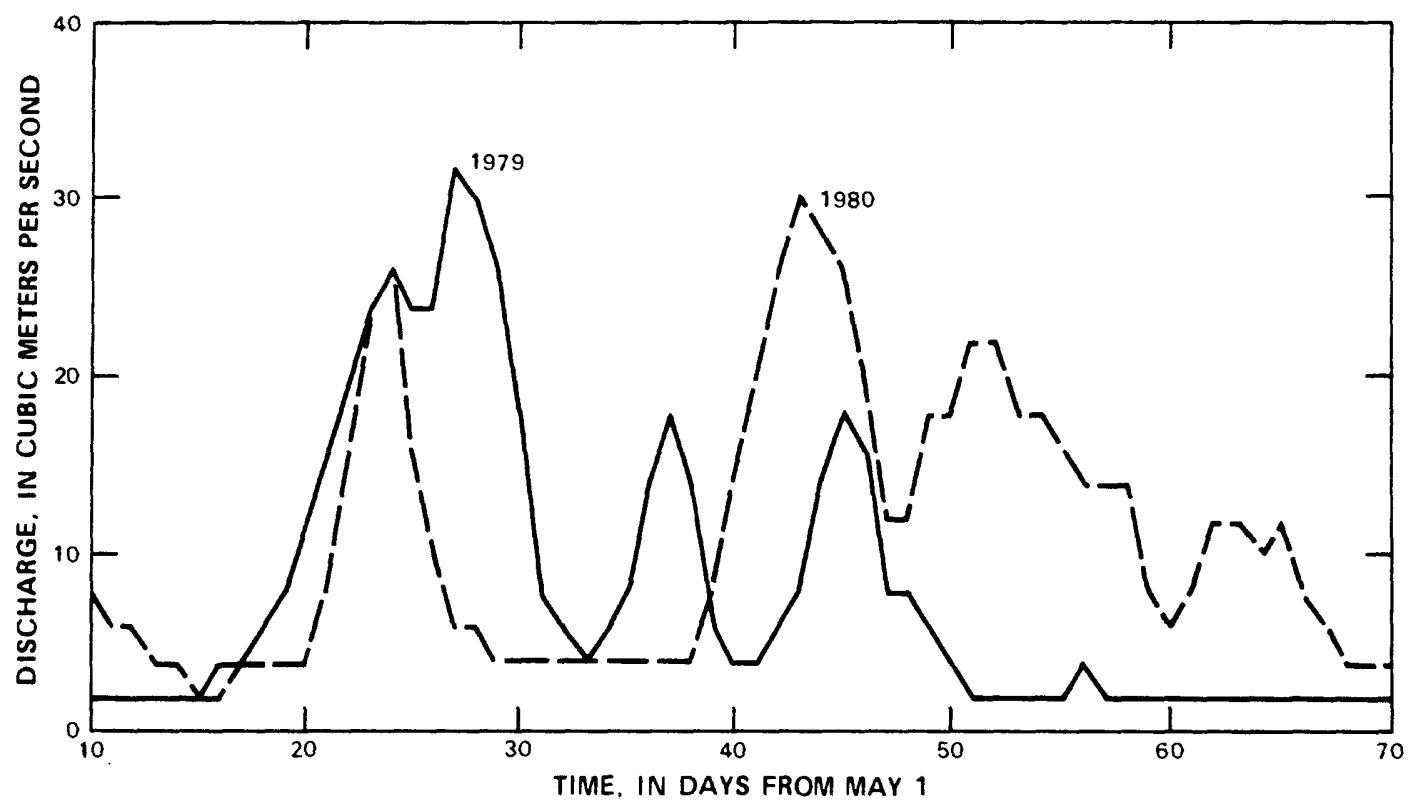

Figure 4. Discharge hydrographs for 1979 and 1980 snowmelt runoff, section 0000 , East Fork River, Wyoming.

\section{BED MATERIAL}

Composition of the streambed of the East Fork River in the study reach is predominant 1 y sand, but gravel bars are spaced at regular intervals. The downstream distribution of particle size as measured in 1979 is shown in figure 5. Within the study reach, six gravelly areas, spaced at about $600-m$ increments, are each followed by a sandy area. Composition of bed material varies greatly between the gravelly riffles and the sandy pools, but overall, the median grain size (particle diameter at the 50th percentile), $D_{50}$, is $1.28 \mathrm{~mm}$, or coarse sand. However, as can be seen in figure 5, particle sizes from fine sand to medium gravel generally are available at all sections. Bed material larger than about 8 to $16 \mathrm{~mm}$ is common in some central gravel bars, but so seldom do these sizes move that they represent only a small part of bedload caught in a sampler. 


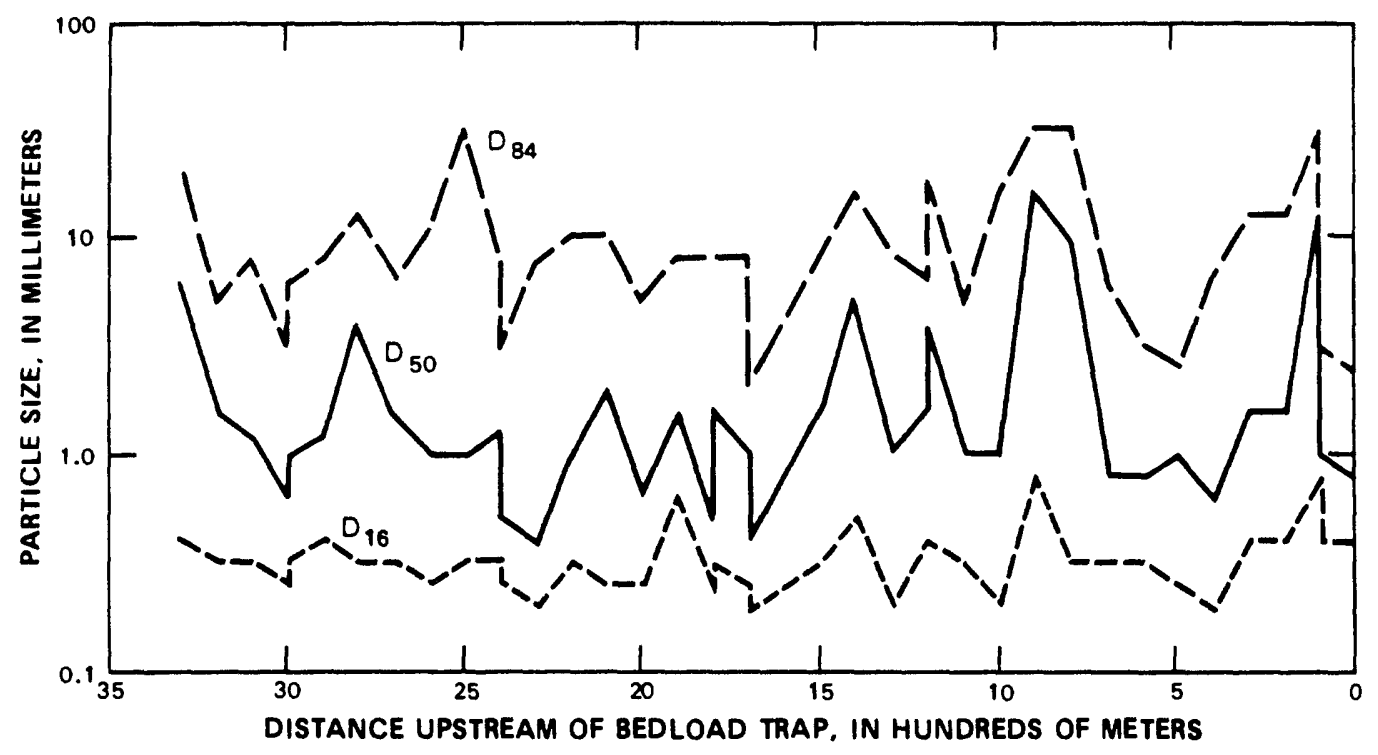

Figure 5. Downstream distribution of bed-material particle size, May 1819, 1979, East Fork River, Wyoming (data from Emmett, Myrick, and Meade, 1980).

Underlying the bed material is a stratum of imbricated coarse gravel and, sporadically, bedrock. The position of this stratum was determined at each section by probing the bed material with a steel rod. Although scour seldom extends to the full depth of bed material, this underlying stratum, or gravel base, represents the maximum extent of possible scour. The volume of bed material above this gravel base is shown in figure 6 for the day of highest flow in 1979 (May 27) and for a day during low flow in 1979 (June 28). Although the largest quantities of bed material occur at the sandy-pool sections, some bed material is in storage at all sections, during both high- and low-flow periods. The combined data in figures 5 and 6 indicate that some sediment of all sizes is everywhere available for transport. 


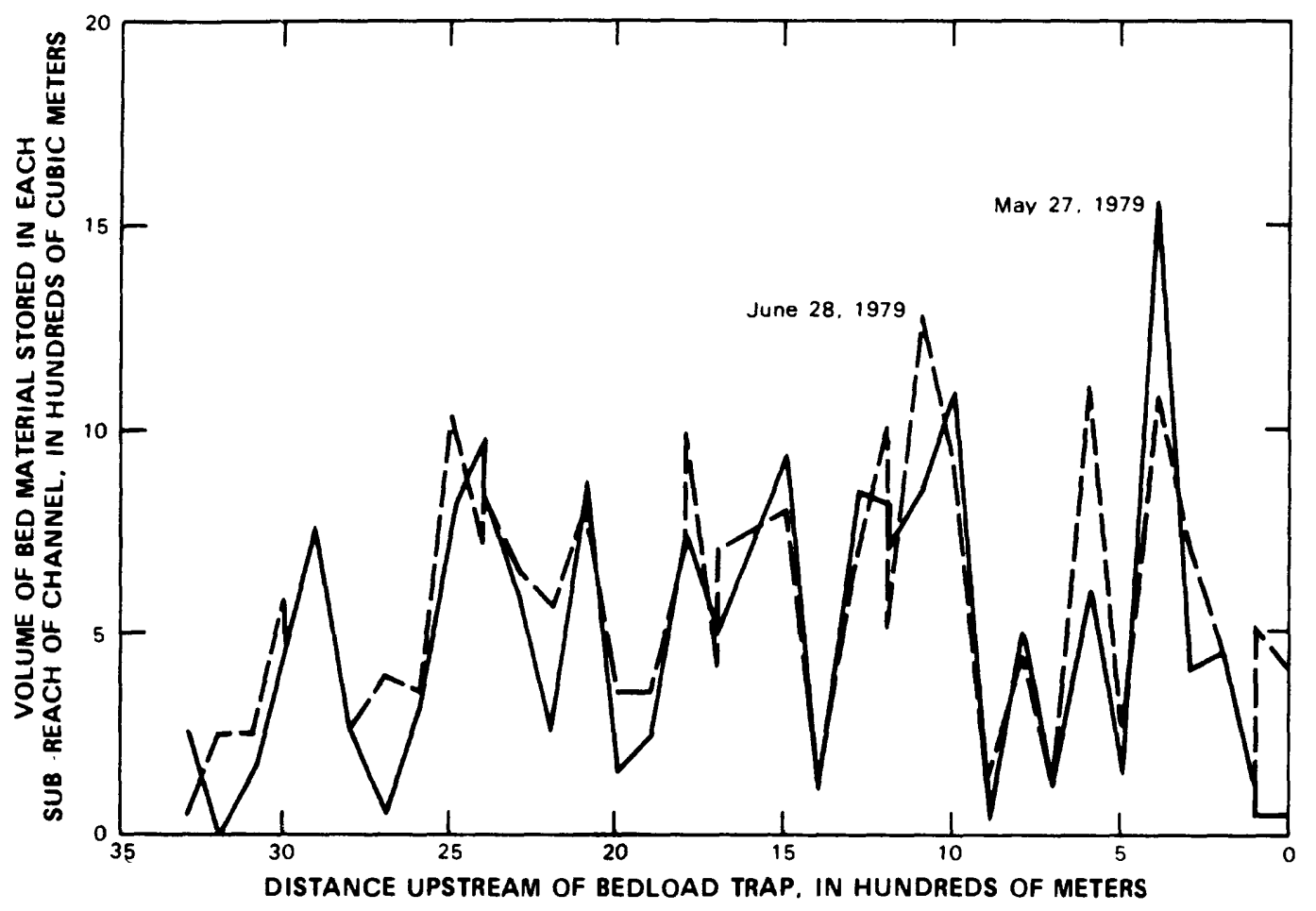

Figure 6. Downstream distribution of volume of bed material, May 27 and June 28, 1979, East Fork River, Wyoming (data from Meade, Myrick, and Emmett, 1980).

\section{WATER-SURFACE SLOPE}

Water-surface slopes are dependent on river stage at the time of measurement and the reach length along which the measurements were made. For the 3.3-km reach length, water-surface slope corresponds closely to riverbed slope; water-surface profiles are shown in figure 7 for five stages of flow. However, along the downstream one-half of this reach, slope flattens with stage and ranges from 0.0083 at low flow to 0.0066 at high flow. This is shown graphically in the middle part of figure 8 for values of water-surface slope computed along the downstream $1.8 \mathrm{~km}$ of the reach (reach length equivalent to 100 channel widths). 


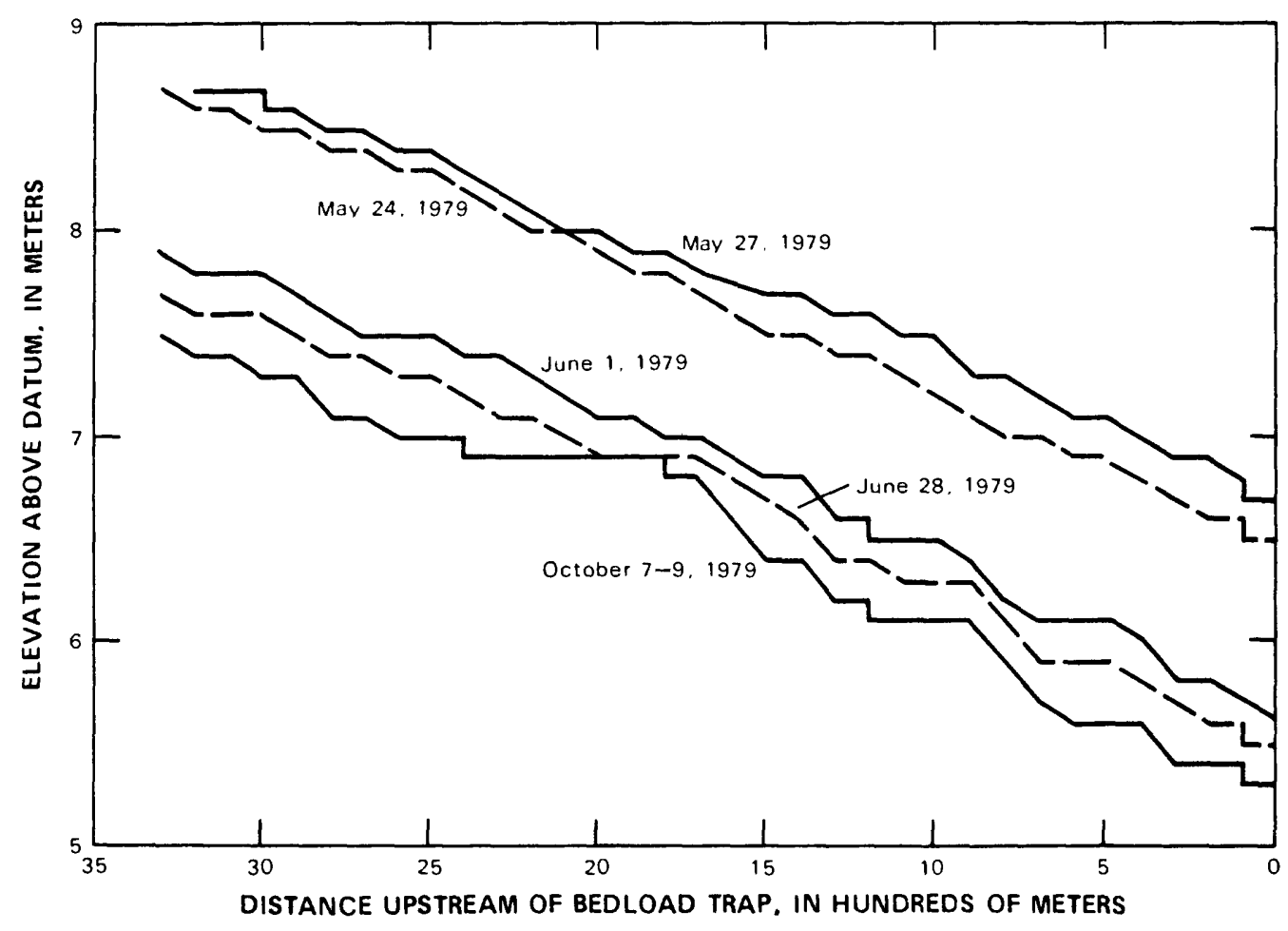

Figure 7. Longitudinal profiles of the water surface for low to high flow, 1979, East Fork River, Wyoming (data from Emmett, Myrick, and Meade, 1980).

Locally, water-surface slope varies greatly with stage. In figure 7, the water-surface slope at the pool section near $1100 \mathrm{~m}$ changes from nearly flat to steep as stage increases, whereas at the riffle section near 1300 $m$, the water-surface slope changes from steep to nearly flat. These changes are more evident in the bottom graph of figure 8 for values of slope computed along a reach length equivalent to 15 channel widths, or about $275 \mathrm{~m}$. At the riffle (section 1315), slope decreased from about 0.0011 at 10 f flow to about 0.0004 at high flow. Conversely, at the poo 1 (section 1077), the slope increased from about 0.0004 at low flow to a little more than 0.0008 at high flow. There is clearly a reversal in magnitude of slope; at high flow, the water-surface slope in the pool became steeper than that in the riffle. 

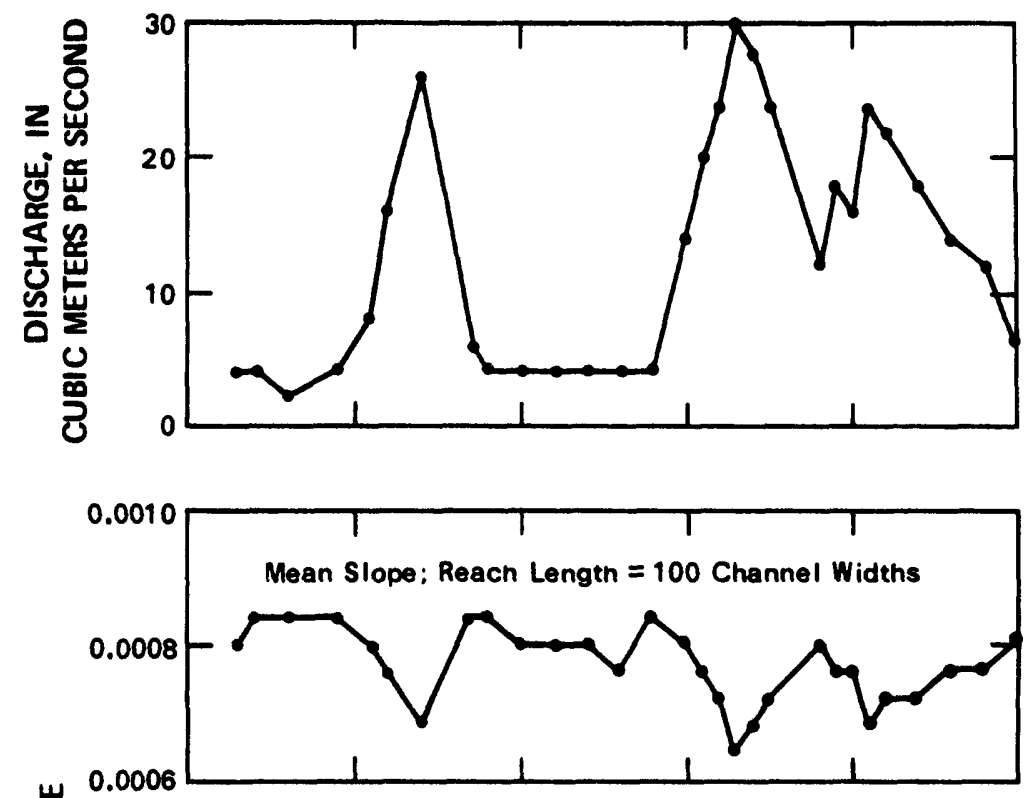

岁

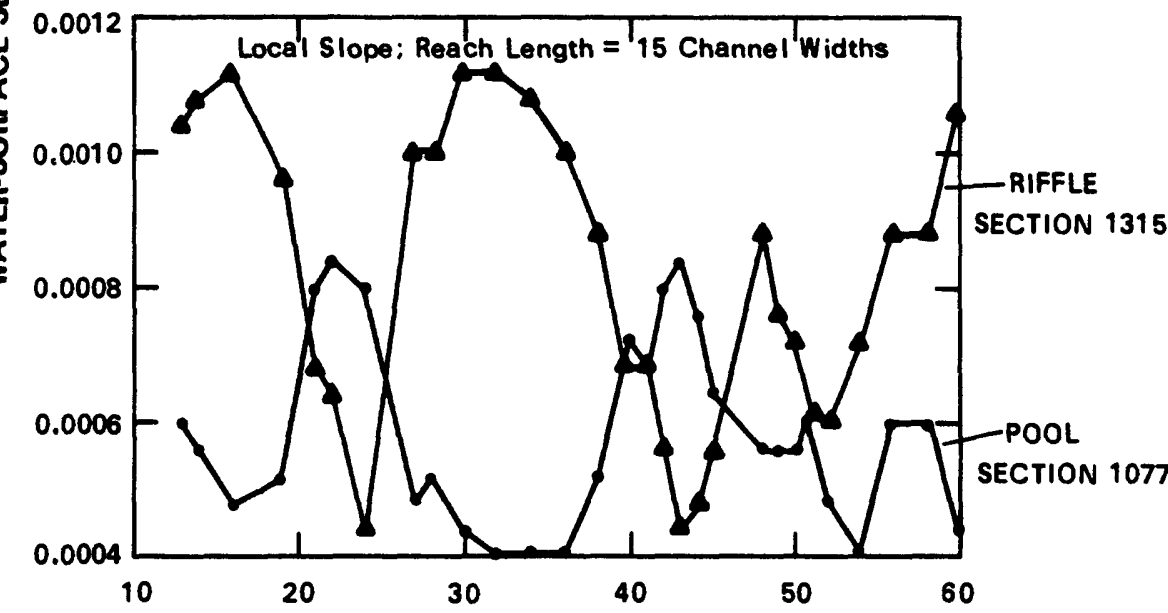

TIME, IN DAYS FROM MAY 1, 1980

Figure 8. Discharge, mean water-surface slope, and examples of local water-surface slope for a riffle and a pool, May-June 1980, East Fork River, Wyoming (from Emmett, Myrick, and Meade, 1982). 
STREAM POWER, BEDLOAD, AND SCOUR AND FILL

Stream power is a measure of the work rate of a stream; it is computed as the product of gravitational acceleration, mass density of the fluid flow, discharge, and water-surface slope. Dimensions of stream power are watts per meter ( $W / m$ ) of channel length, numerically and physically equivalent to newtons per second (N/s), and represent the power available per unit length of channel, including that power dissipated in the transport of sediment. For the same pool and riffle sections as shown in figure 8 , the middle part of figure 9 shows the range in values of stream power during spring runoff. At most sections, stream power is greatest at high flow; at low flow, pools are likely to have negligible sediment transporting capability. However, because of the reversal in maximum values of water-surface slope, at high flow, pools are likely to have greater stream power than riffles.

Measured bedload-transport rates, expressed in watts per meter (newtons per second) of power dissipation (immersed weight per unit time), are shown in the bottom part of figure 9 for the pool and riffle sections. As may be expected from the stream-power relations, bedload-transport rates are greatest in the pools during high flow, are negligible in the pools during low flow, and are of intermediate values in the riffles during all flows. In terms of efficiency, about 10 percent of available stream power is dissipated in the transport of bedload.

The spatially-varied transport rates shown in figure 9 indicate that bed material is being stored and being removed from storage at various sections along the channel. That is, continuity in the total quantity of sediment requires that with spatially variable bedload-transport rates, some sections must be filling and other sections must be scouring. This is illustrated in the bottom part of figure 10 for the pool and riffle sections. Whereas some sections show little change in mean bed elevation as the sediment supplied to them is transported past, other sections show alternating scour and fill as the sections have fluctuating availability of stream power to transport sediment. At the end of the spring runoff, most sections have about the same value of mean bed elevation as they had prior to spring runoff. 

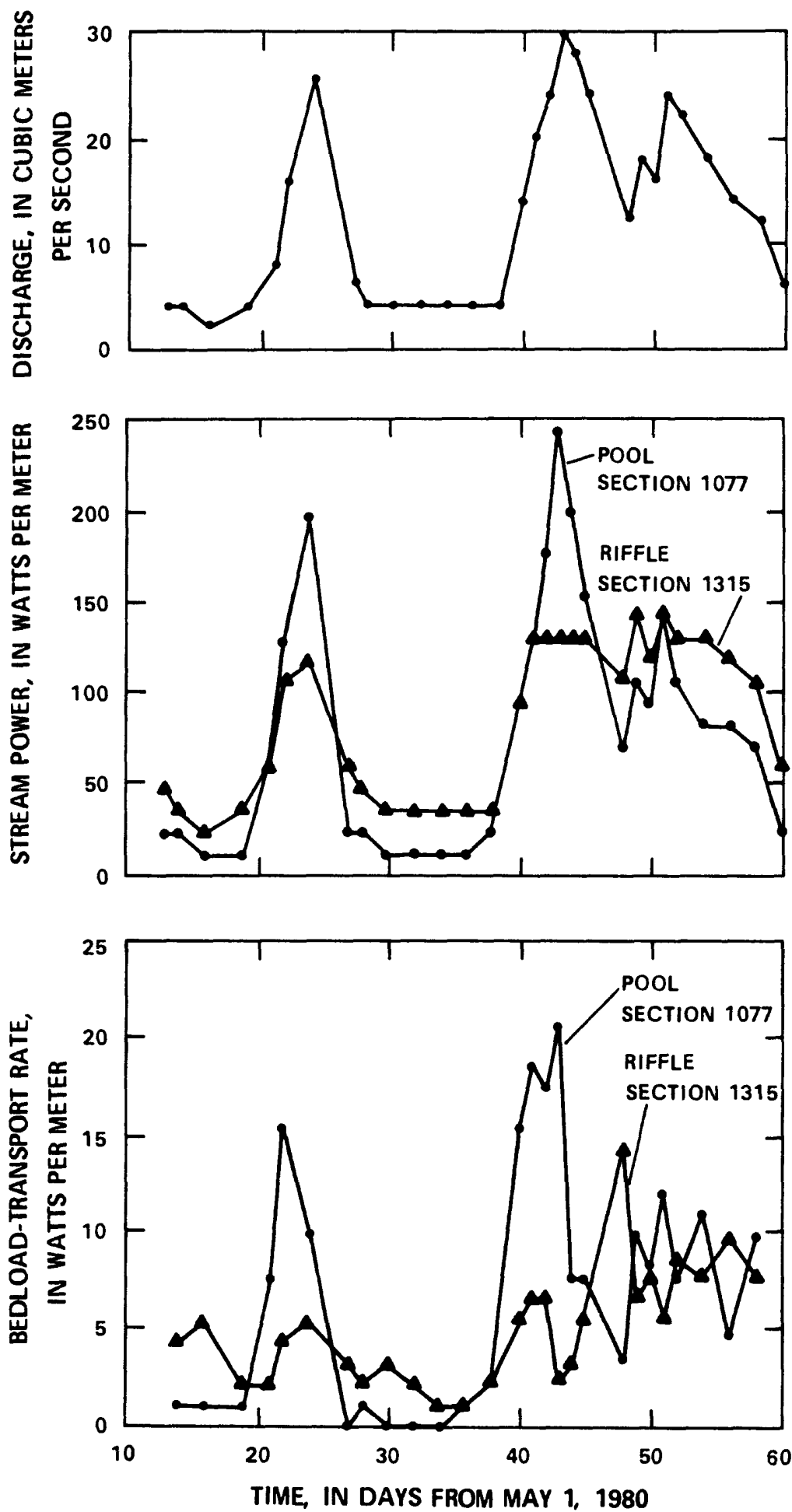

Figure 9. Discharge, and examples of stream power and bedload-transport rate for a riffle and a pool, May-June 1980, East Fork River, Wyoming (from Emmett, Myrick, and Meade, 1982). 

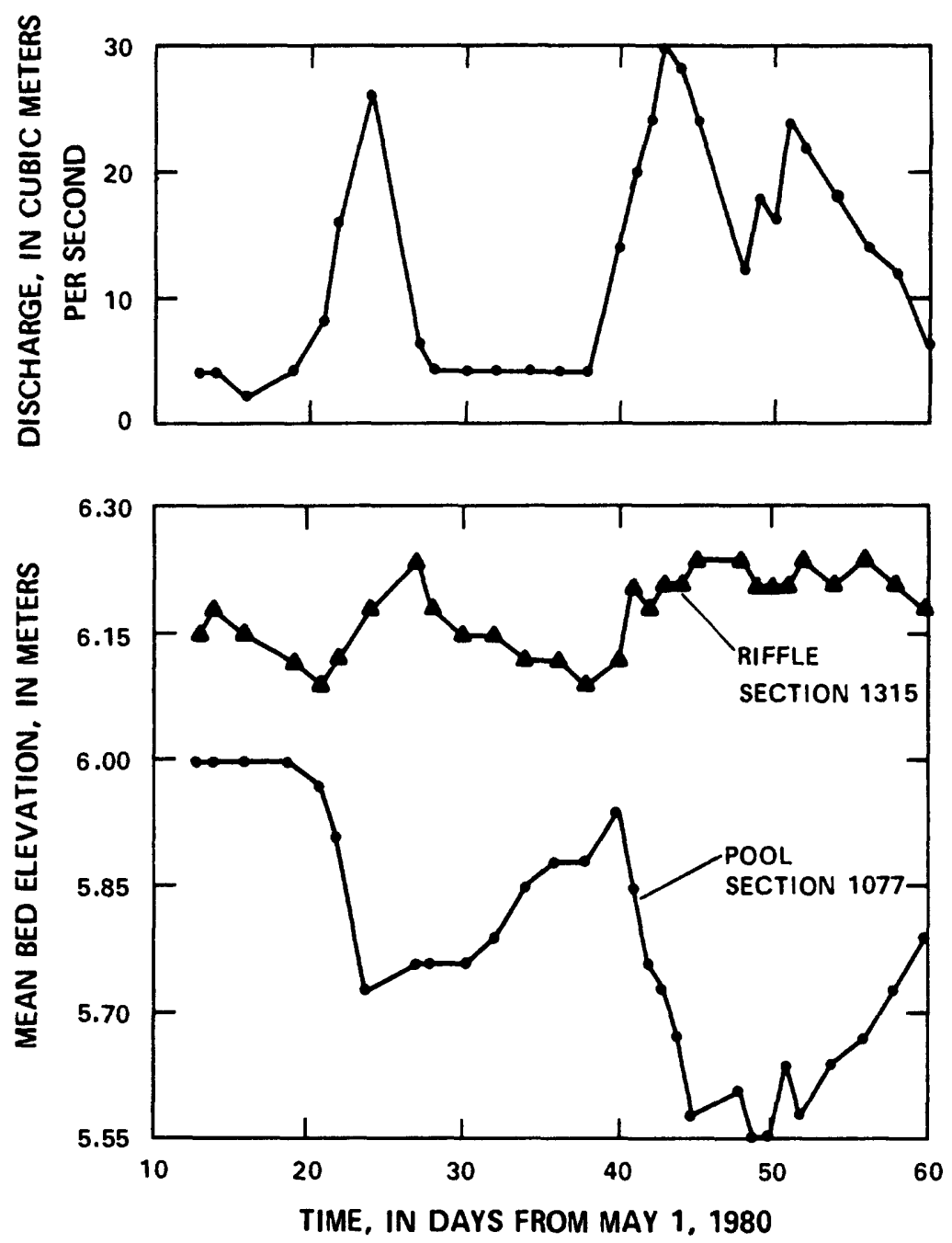

Figure 10. Discharge, and examples of scour and fill for a pool and a riffle, May-June 1980 , East Fork River, Wyoming (from Emmett, Myrick, and Meade, 1982).

\section{FLUORESCENT TRACERS}

Prior to the 1979 runoff season (May 1, 1979), about $6,000 \mathrm{~kg}$ (kilograms) of bed material was excavated from the streambed near the right bank at section 0570 . The $6,000 \mathrm{~kg}$ represented only a small part of bed material available at that section (see fig. 6; approximately less than 0.5 percent) and, accordingly, created little or no sink for subsequent storage of material. The excavated material was dried and individual particles were coated with a fluorescent-dyed acrylic. A summary of particle size for the dyed particles is given below. 


\begin{tabular}{lccc}
$\begin{array}{c}\text { Particle } \\
\text { size } \\
\text { range }\end{array}$ & $\begin{array}{c}\text { Percent } \\
\text { retained } \\
\text { in size range }\end{array}$ & $\begin{array}{c}\text { Percent } \\
\text { finer than } \\
\text { upper 1imit of } \\
\text { size range } \\
\text { (by weight) }\end{array}$ & $\begin{array}{c}\text { Percent } \\
\text { finer than } \\
\text { upper limit of } \\
\text { size range } \\
\text { (by number) }\end{array}$ \\
\hline $\begin{array}{l}\text { (billimeters) } \\
\text { (by weight) }\end{array}$ & 5 & 5 & 90.8 \\
$.25-.50$ & 14 & 19 & 98.1 \\
$.50-1.0$ & 9 & 28 & 99.6 \\
$1.0-2.0$ & 15 & 43 & 99.97 \\
$2.0-4.0$ & 33 & 97 & 99.99 \\
$4.0-8.0$ & 21 & 100 & $>99.99$ \\
$>8.0$ & 3 & & 100.00 \\
\hline
\end{tabular}

A small percentage of particles, by weight, were smaller than $0.25 \mathrm{~mm}$ or larger than $8 \mathrm{~mm}$. Most particles were between 2.0 and $4.0 \mathrm{~mm}$; the median diameter was a little larger than $2 \mathrm{~mm}$. Because particle weight is proportional to about the cube of particle size, the most individual dyed particles were in the smallest size categories and, by computation, 99.97 percent of the dyed particles were smaller than $2 \mathrm{~mm}$.

Extreme values of the actual size distribution of dyed material are unimportant; particles smaller than $0.25 \mathrm{~mm}$ or larger than $8 \mathrm{~mm}$ were rejected from the analysis. In retained samples, an electronic image scanner and microcomputer counted and measured the tracer particles and results are expressed as total number of fluorescent particles or as number of fluorescent particles in a particle-size category. In the analytical procedure, it was determined that each kilogram of tracer material contained about $1 \times 10^{6}$ particles. The total number of fluorescent particles available for transport was thus very large.

The fluorescent coating was sufficiently thin that it negligibly changed the size and shape of particles. About 2,000 kg of sediment was dyed each of 3 colors. This dyed material subsequently was reinjected into the streambed as 1 ine sources of tracer particles. Injection was accomplished by pouring dyed particles down a $0.4-\mathrm{m}$ diameter pipe placed into the streambed at verticals along the 1 ine of the section. Each 1 ine was located near the upstream end of every other naturally occurring accumulation of sediment within the study reach. Specifically, pinkcolored particles were injected at section 3037 on May 18, 1979; blue particles were injected at section 1685 on May 19 , and orange particles were injected in a line at section 0506 on May 19, 1979. In a separate experiment, green-colored particles were placed as a point source on the right bank of section 1620 on May 31,1980 . 
As an indication of the approximate concentration of fluorescent particles of the three principal colors, from figure 6 it is computed that about $2 \times 10^{4} \mathrm{~m}^{3}$ of sediment are within the study reach, or about $3 \times 10^{7} \mathrm{~kg}$. As stated, about $2 \times 10^{3} \mathrm{~kg}$ of sediment were dyed each principal color. Assuming complete mixing of dyed particles with all particles, the minimum concentration of fluorescent particles was projected at 50 to $100 \mathrm{ppm}$ (parts per million). Based on yearly operation of the bedload trap, all particles within the study reach represent about a 6 - to 10-year supply of bedload and complete mixing of dyed particles with all particles was not expected. Accordingly, actual measured concentrations ranged from zero at no mixing, to very large concentrations near the locations of injection.

During 1979 , the movement of fluorescent bed-material tracers was measured daily by bed material sampling at downstream sections. The total number of tracer particles as measured per 100 grams of bed material is tabulated in tables 5-7 and the same data tabulated by particle size category are listed in tables 12-14.

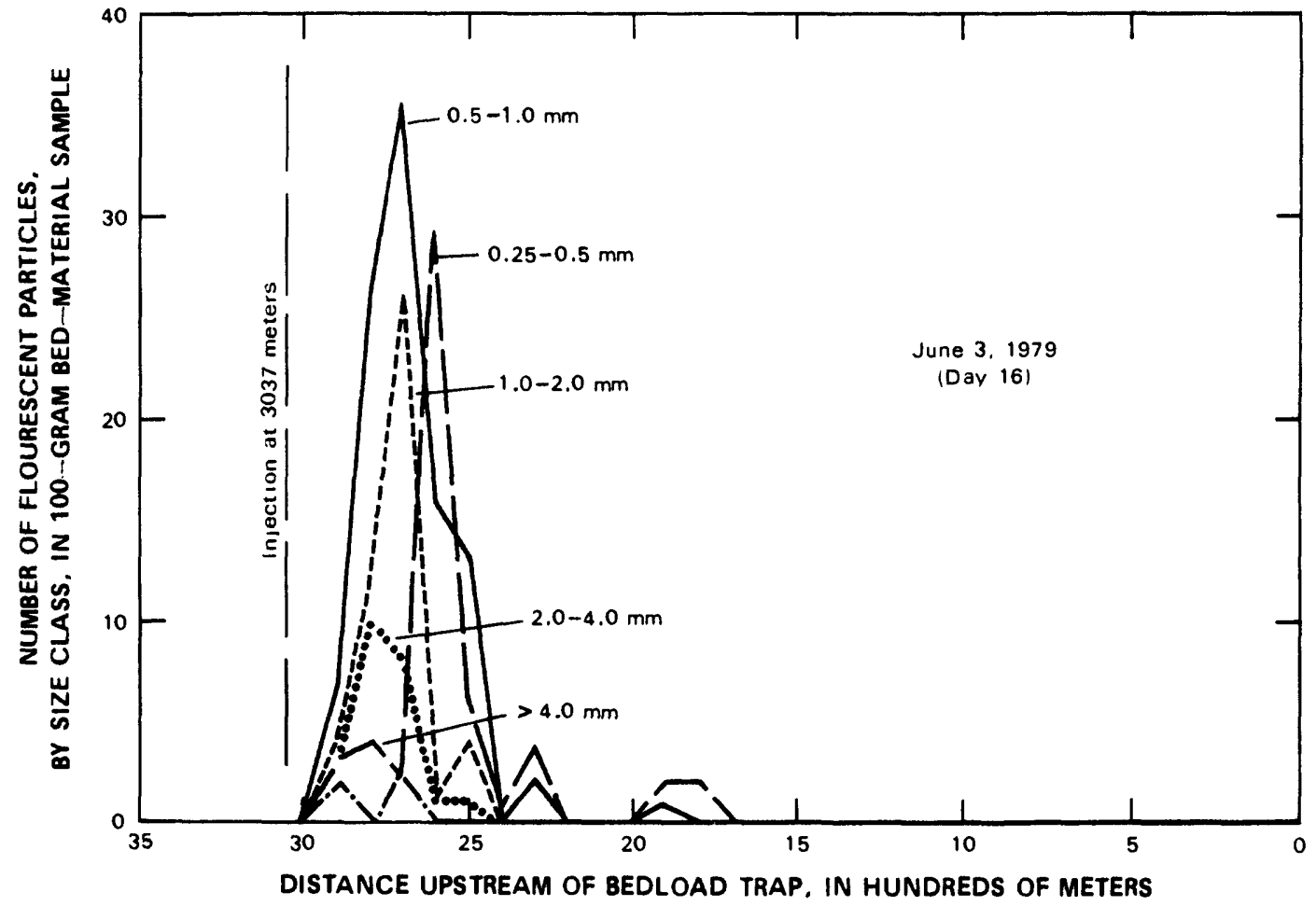

Figure 11. Downstream distribution of bed-material tracers, June 3, 1979, the 16th day after injection, East Fork River, Wyoming. 
Data from the tables are exemplified by a sample from table 12 for the pink material injected as a line source at section 3037 . Typical results, separated into five particle-size categories, are shown in figure 11 . The data shown are for June 3,1979 , the 16 th day from the time of injection (May 18, 1979). As can be inferred from figure 11, it appears that the smaller particles move faster and further than the larger particles. The daily downstream displacement of the peak concentration (approximate centroid), for tracer particles of size between 0.5 to $1.0 \mathrm{~mm}$, is shown in figure 12. Because downstream displacement of the tracer was recorded only at measurement sections, the relation that describes the displacement shown in figure 12 is a curve that envelops the data rather than a best-fit to the data.

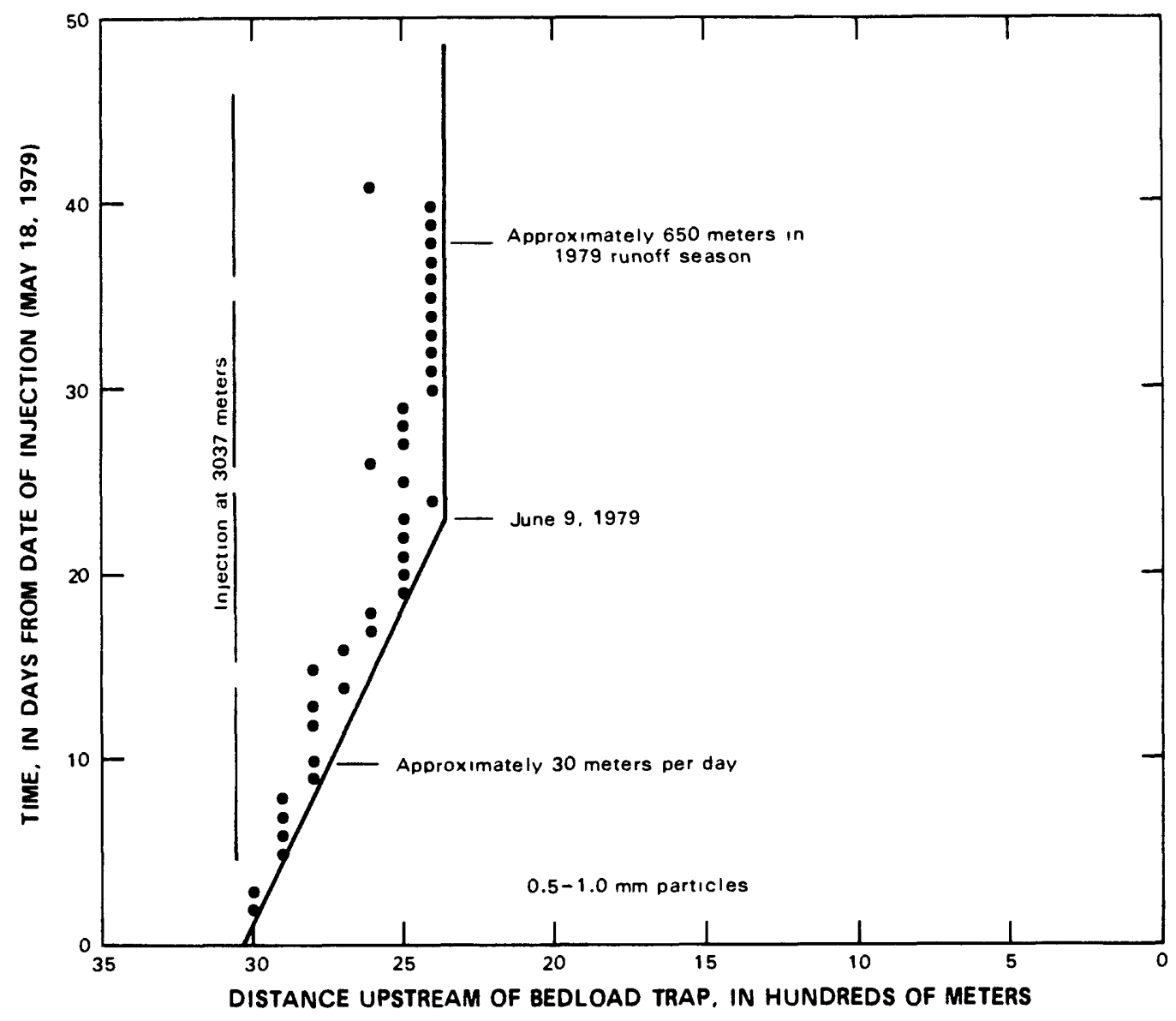

Figure 12. Downstream daily displacement of the peak concentration of 0.5to 1.0-mm bed-material tracers, 1979, East Fork River, Wyoming. 
Graphs similar to figure 12 could be prepared for other particle-size classes or for other characteristics of the dispersed tracer. For example, the leading edge of the tracer defines first arrival time, or maximum particle speed, whereas the centroid of the distribution defines mean particle speed. For particles of size between 0.5 to $1.0 \mathrm{~mm}$, figure 13 shows downstream particle speed and travel distance for the leading edge (maximum values), for significant quantities of tracer, and for the peak concentration (approximate mean values). For 0.5- to 1.0-mm particles, mean particle speed is about $30 \mathrm{~m} / \mathrm{d}$ (meters per day); transport occurred during about 22 days, giving an annual downstream travel distance of about

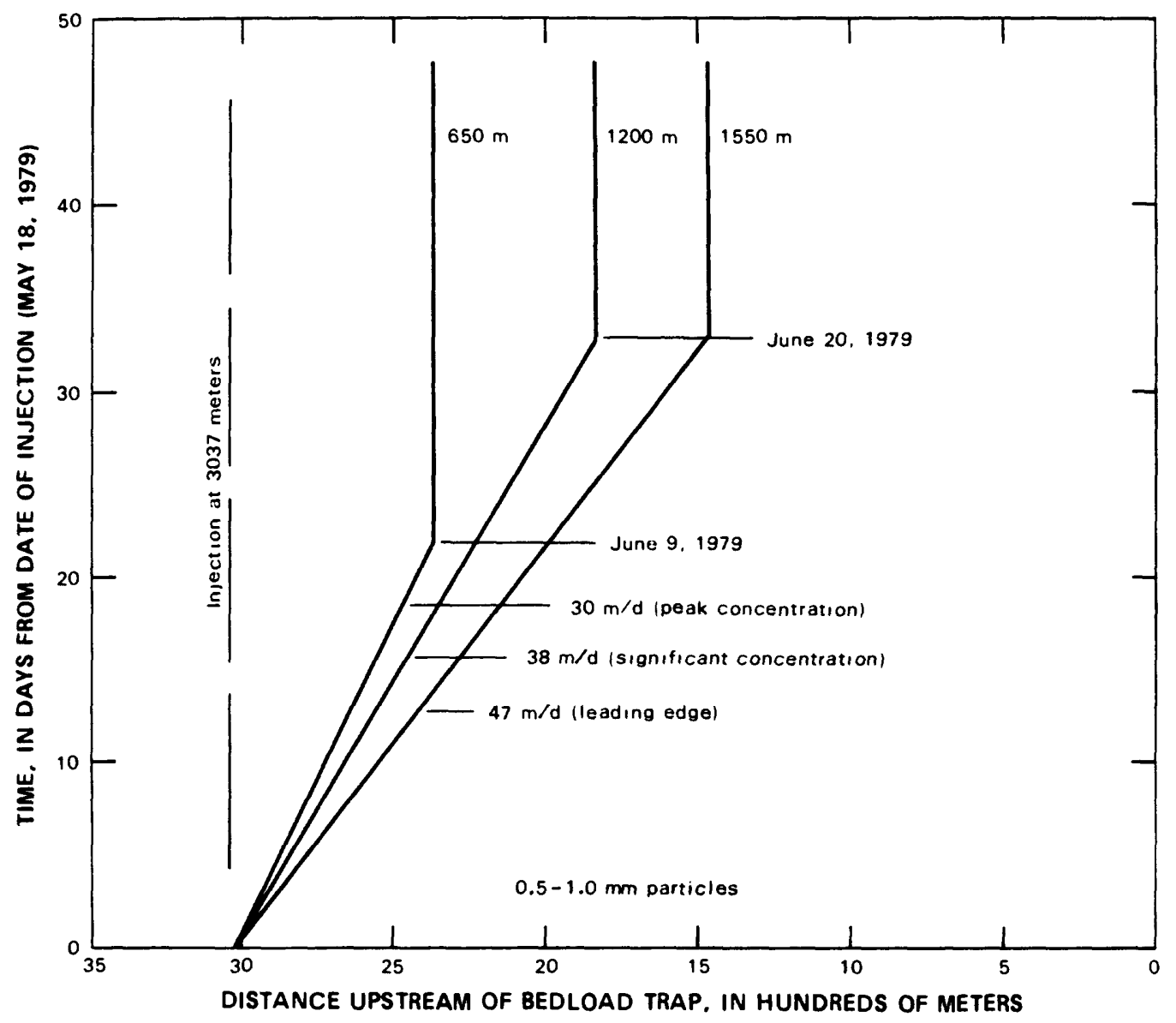

Figure 13. Downstream particle speeds and travel distances for 0.5 - to 1.0-mm bed-material tracers, 1979, East Fork River, Wyoming. 
$650 \mathrm{~m}$. A significant (more than the single or several grains defining the leading edge) but minority part of the tracer travelled at $38 \mathrm{~m} / \mathrm{d}$ to a total distance of $1,200 \mathrm{~m}$, whereas the leading edge of the tracer moved at $47 \mathrm{~m} / \mathrm{d}$ and went a distance of $1,550 \mathrm{~m}$. Particles that travelled farthest moved for about 33 days after injection, with little movement recorded after June 20.

There is a modest day-to-day decrease in the total number of observed tracer particles as the sediment moves downstream. As shown in figure 11, some particles move faster than the majority and some lag behind. While some particles may move a great distance in a short time, others are consumptively used in river-building processes (for example, floodplain building); however, they may be replaced by particles supplied from other processes (for example, bank cutting). Downstream continuity in transport may be maintained, but there is replacement of particles comprising the transport.

During 1980 , the second season of runoff, movement of fluorescent particles was measured by bedload sampling using a Helley-Smith sampler. Details of the sampling are provided in Emmett, Myrick and Meade (1982). Summary data for the four fluorescent colors during 1980 are tabulated in tables 8-11, are listed as a function of particle-size category in tables 15-18, and are shown by cross-channel location in tables 19-21 for those sections and colors for which cross-channel position was measured.

During 1982 there was an attempt to describe the distribution of bedmaterial tracers after four seasons of runoff. Core samples of bed material were collected and analyzed for concentration of fluorescent particles. Although the number of fluorescent-tracer particles found and counted was small, the data are presented in tables 22-25.

For all data contained in the tables, careful attention needs to be given to information 1 isted in the titles and footnotes of the tables. 


\section{ACKNOWLEDGMENTS}

Many people have contributed greatly to the collection and analysis of data presented herein. Luna B. Leopold helped design the study and collect the data as well as providing stimulating discussion and companionship in the field. Robert H. Meade served as project co-chief during both years of field study and had principal responsibility for bed-elevation data presented in Parts II and IV of the basic-data reports. Bruce Parks' early participation in 1979 was invaluable in the fluorescent dying and injection of bed-material tracers.

Others who assisted in either, or both years, of the data-collecting effort include R. C. Averett, K. D. Cartier, K. D. Crowley, W. F. Curtis, T. R. Eschner, D. J. Evans, J. Florsheim, E. M. Freyer, G. J. Freyer, D. C. Geiger, J. L. Glenn, E. M. Held, T. A. Herrett, J. E. Kircher, P. C. Klingeman, L. B. Laird, B. B. Leopold, J. Lewin, J. S. Luhr, H. A. Martinson, R. C. Mengis, J. A. Moody, M. P. Muir, S. M. Myrick, R. J. $0^{\prime}$ Brien, C. T. Nelson, T. L. Nelson, L. R. Newmyer, C. F. Nordin, J. J. Patry, K. L. Prestegaard, S. A. Stewart, A. L. Strong, S. Takayama, A. V. Vecchia, K. R. Vincent, A. Werritty, G. P. Williams, and K. M. Williams. The assistance of all these individuals is gratefully acknowledged. 


\section{REFERENCES}

Andrews, E. D., 1977, Hydraulic adjustment of an alluvial stream channel to the supply of sediment, western Wyoming: Berkeley, University of California, unpublished Ph.D. Dissertation, 152 p.

- $1979 \mathrm{a}$, Scour and fill in a stream channel, East Fork River, western Wyoming: U.S. Geological Survey Professional Paper 1117, 49 p.

- $1979 \mathrm{~b}$, Hydraulic adjustment of the East Fork River, Wyoming to the supply of sediment, in Rhodes, D. D., and Williams, G. P., eds., Adjustments of the fluvial system: Dubuque, Iowa, Kendall/Hunt Publishing Co., p. 69-94.

- 1981, Measurement and computation of bed-material discharge in a shallow sand-bed stream, Muddy Creek, Wyoming: Water Resources Research, v. 17, no. 1, p. 131-141.

-- 1982a, Adjustment of the East Fork River to bedload sediment contributed by Muddy Creek, in Leopold, L. B., ed., Field Trip Guidebook: American Geomorphological Field Group 1982 Conference, p. $57-68$.

- 1982b, Bank stability and channel width adjustment, East Fork River, Wyoming: Water Resources Research, v. 18, no. 4, p. 1184-1192.

Bagnold, R. A., 1977, Bedload transport by natural rivers: Water Resources Research, v. 13, no. 2, p. 303-312.

- 1980, An empirical correlation of bedload transport rates in flumes and natural rivers: Royal Society of London Proceedings, v. 372A, 0ct. 31, p. 453-473.

Bennett, J. P., and Nordin, C. F., 1977 , Simulation of sediment transport and armouring: Hydrological Sciences Bulletin, v. 22, no. 4, p. 555569.

Dietrich, W. E., $1982 \mathrm{a}$, Mechanics of a river meander, in Leopold, L. B., ed., Field Trip Guidebook: American Geomorphological Field Group 1982 Conference, p. 18-29.

- 1982b, Flow, boundary shear stress, and sediment transport in a river meander: Seattle, University of Washington, unpublished Ph.D. Dissertation, $261 \mathrm{p}$.

Dietrich, W. E., Smith, J. D., and Dunne, Thomas, 1979 , Flow and sediment transport in a sand bedded meander: Journal of Geology, v. 87, no. 3, p. 305-315.

Dunne, Thomas, and Leopold, L. B., 1978, Water in environmental planning: San Francisco, W. H. Freeman and Co., 818 p.

Emmett, W. W., 1980 a, A field calibration of the sediment-trapping characteristics of the Helley-Smith bedload sampler: U.S. Geological Survey Professional Paper 1139, 44 p.

- $1980 \mathrm{~b}$, Bedload sampling in rivers: Proceedings, International Symposium on River Sedimentation, Beijing, China, March 24-29, 1980 , Guanghua Press, p. 991-1017.

--- 1981, Measurement of bedload in rivers, in Erosion and sediment transport measurement: International Association of Hydrological Sciences Publication 133, p. 3-15. 
1982a, Helley-Smith bedload sampler, in Leopold, L. B., ed., Field Trip Guidebook: American Geomorphological Field Group 1982 Conference, p. 41-48.

- $1982 b$, Variability of bedload and some hydraulic characteristics along a reach of East Fork River, in Leopold, L. B., ed., Field Trip Guidebook: American Geomorphological Field Group 1982 Conference, p. 49-56.

Emmett, W. W., and Leopold, L. B., 1977, A comparison of observed sedimenttransport rates with rates computed using existing formulas, in Doehring, D. D., ed., Geomorphology in Arid Regions: Proceedings, Eighth Annual Geomorphology Symposium, Publications in Geomorphology, p. $187-188$.

Emmett, W. W., Leopold, L. B., and Myrick R. M., 1983, Some characteristics of fluvial processes in rivers: Proceedings, Second International Symposium on River Sedimentation, Nanjing, China, October 11-16, 1983, Water Resources and Electric Power Press, p. 730754.

Emmett, W. W., Myrick, R. M., and Meade, R. H., 1980, Field data describing the movement and storage of sediment in the East Fork River, Wyoming: Part I. River hydraulics and sediment transport, 1979: U.S. Geological Survey Open-File Report 80-1189, 43 p.

- 1982, Field data describing the movement and storage of sediment in the East Fork River, Wyoming: Part III. River hydraulics and sediment transport, 1980: U.S. Geological Survey Open-File Report 82$359,289 \mathrm{p}$.

Kl ingeman, P. C., and Emmett, W. W., 1982, Gravel bedload transport processes, in Hey, R. D., Bathurst, J. C., and Thorne, C. R., eds., Grave1-bed rivers: New York, John Wiley and Sons, p. 141-179.

Leopold, L. B., 1982a, Geologic setting (of East Fork River, Wyoming), in Leopold, L. B., ed., Field Trip Guidebook: American Geomorphological Field Group 1982 Conference, p. 4-13.

-- 1982b, Water surface topography in river channels and implications for meander development, in Hey, R. D., Bathurst, J. C., and Thorne, C. R., eds., Gravel-bed rivers: New York, John Wiley and Sons, p. 359-388.

Leopold, L. B., and Emmett, W. W., 1976, Bedload measurements, East Fork River, Wyoming: [U.S.] National Academy of Sciences Proceedings, v. 74 , no. 4, p. 1000-1004.

-- 1977, 1976 bedload measurements, East Fork River, Wyoming: [U.S.] National Academy of Sciences Proceedings, v. 74, no. 7, p. 2644-2648.

---- 1982, Bedload trap, East Fork River, in Leopold, L. B., ed., Field Trip Guidebook: American Geomorphological Field Group 1982 Conference, p. 30-40.

Lisle, T. E., 1976, Components of flow resistance in a natural channel: Berkeley, University of California, unpublished Ph.D. Dissertation, 66 p.

- 1979, A sorting mechanism for a riffle-pool sequence: Geological Society of America Bulletin, v. 90, pt. II, p. 1142-1157. 
1982, Variations of hydraulic friction and roughness in a pool, riffle, and two bends of the East Fork River during a period of $h i g h$ runoff, in Leopold, L. B., ed., Field Trip Guidebook: American Geomorphological Field Group 1982 Conference, p. 69-76.

Mahoney, H. A., Andrews, E. D., Emmett, W. W., Leopold, L. B., Meade, R. H., Myrick, R. M., and Nordin, C. F., 1976, Data for calibrating unsteady-flow sediment-transport models, East Fork River, Wyoming, 1975: U.S. Geological Survey Open-File Report 76-22, 293 p.

Meade, R. H., Emmett, W. W., and Myrick, R. M., 1981a, Movement and storage of bed material during 1979 in East Fork River, Wyoming, USA, in Erosion and sediment transport in Pacific rim steep lands: International Association of Hydrological Sciences Publication 132, p. 225-235.

$1981 \mathrm{~b}$, Wavelike movement of bedload, East Fork River, Wyoming USA, in Abstracts: Symposium on Modern and Ancient Fluvial Systems, University of Keele, United Kingdom, p. 81 .

Meade, R H., Myrick, R. M., and Emmett, W. W., 1980, Field data describing the movement and storage of sediment in the East Fork River, Wyoming: Part II. Bed elevations, 1979: U.S. Geological Survey Open-File Report 80-1190, $172 \mathrm{p}$.

- - 1982, Field data describing the movement and storage of sediment in the East Fork River, Wyoming: Part IV. Bed elevations, 1980: U.S. Geological Survey Open-File Report 82-360, 197 p.

Prestegaard, K. L., 1982a, Variables influencing water surface slope in gravel and coarse sand streams: Berkeley, University of California, unpublished $\mathrm{Ph} . \mathrm{D}$. Dissertation, $111 \mathrm{p}$. + appendices.

-.- 1982b, Components of flow resistance in the East Fork River, Wyoming, in Leopold, L. B., ed., Field Trip Guidebook: American Geomorphological Field Group 1982 Conference, p. 77-85. 


\section{TABLES}


TABLE 1.- BIHOURLY DISCHARGE (1), IN CUBIC METERS PER SECOND, AT SECTION 0000, EAST FORK RIVER, WYOMING, 1979

\begin{tabular}{|c|c|c|c|c|c|c|}
\hline DATE & $\begin{array}{r}2 \\
14 \\
\end{array}$ & $\begin{array}{r}4 \\
16 \\
\end{array}$ & $\begin{array}{c}\text { TIME IN } \\
6 \\
18\end{array}$ & $\begin{array}{r}\text { HOURS } \\
8 \\
20\end{array}$ & $\begin{array}{r}10 \\
22 \\
\end{array}$ & $\begin{array}{l}12 \\
24 \\
\end{array}$ \\
\hline $5-10$ & $\begin{array}{l}2.11 \\
1.73\end{array}$ & $\begin{array}{l}2.02 \\
1.73\end{array}$ & $\begin{array}{l}2.02 \\
1.73\end{array}$ & $\begin{array}{l}1.94 \\
1.73\end{array}$ & $\begin{array}{l}1.85 \\
1.73\end{array}$ & $\begin{array}{l}1.77 \\
1.81\end{array}$ \\
\hline $5-11$ & $\begin{array}{l}1.89 \\
1.69\end{array}$ & $\begin{array}{l}1.85 \\
1.65\end{array}$ & $\begin{array}{l}1.85 \\
1.61\end{array}$ & $\begin{array}{l}1.81 \\
1.61\end{array}$ & $\begin{array}{l}1.81 \\
1.65\end{array}$ & $\begin{array}{l}1.77 \\
1.73\end{array}$ \\
\hline $5-12$ & $\begin{array}{l}1.85 \\
1.77\end{array}$ & $\begin{array}{l}1.81 \\
1.73\end{array}$ & $\begin{array}{l}1.81 \\
1.73\end{array}$ & $\begin{array}{l}1.81 \\
1.73\end{array}$ & $\begin{array}{l}1.77 \\
1.69\end{array}$ & $\begin{array}{l}1.77 \\
1.69\end{array}$ \\
\hline $5-13$ & $\begin{array}{l}1.73 \\
1.61\end{array}$ & $\begin{array}{l}1.73 \\
1.54\end{array}$ & $\begin{array}{l}1.73 \\
1.46\end{array}$ & $\begin{array}{l}1.69 \\
1.39\end{array}$ & $\begin{array}{l}1.69 \\
1.35\end{array}$ & $\begin{array}{l}1.65 \\
1.35\end{array}$ \\
\hline $5-14$ & $\begin{array}{l}1.43 \\
1.61\end{array}$ & $\begin{array}{l}1.46 \\
1.61\end{array}$ & $\begin{array}{l}1.50 \\
1.54\end{array}$ & $\begin{array}{l}1.54 \\
1.50\end{array}$ & $\begin{array}{l}1.61 \\
1.46\end{array}$ & $\begin{array}{l}1.61 \\
1.54\end{array}$ \\
\hline $5-15$ & $\begin{array}{l}1.61 \\
2.25\end{array}$ & $\begin{array}{l}1.73 \\
2.20\end{array}$ & $\begin{array}{l}1.89 \\
2.11\end{array}$ & $\begin{array}{l}2.02 \\
2.11\end{array}$ & $\begin{array}{l}2.11 \\
2.20\end{array}$ & $\begin{array}{l}2.20 \\
2.34\end{array}$ \\
\hline $5-16$ & $\begin{array}{l}2.90 \\
3.40\end{array}$ & $\begin{array}{l}2.90 \\
3.28\end{array}$ & $\begin{array}{l}3.17 \\
3.12\end{array}$ & $\begin{array}{l}3.40 \\
3.12\end{array}$ & $\begin{array}{l}3.52 \\
3.17\end{array}$ & $\begin{array}{l}3.40 \\
3.28\end{array}$ \\
\hline $5-17$ & $\begin{array}{l}3.34 \\
4.08\end{array}$ & $\begin{array}{l}3.64 \\
4.01\end{array}$ & $\begin{array}{l}3.95 \\
3.82\end{array}$ & $\begin{array}{l}4.21 \\
3.58\end{array}$ & $\begin{array}{l}4.21 \\
3.64\end{array}$ & $\begin{array}{l}4.14 \\
3.89\end{array}$ \\
\hline $5-18$ & $\begin{array}{l}4.75 \\
6.04\end{array}$ & $\begin{array}{l}5.64 \\
5.88\end{array}$ & $\begin{array}{l}6.37 \\
5.88\end{array}$ & $\begin{array}{l}6.79 \\
6.04\end{array}$ & $\begin{array}{l}6.79 \\
6.12\end{array}$ & $\begin{array}{l}6.62 \\
6.62\end{array}$ \\
\hline $5-19$ & $\begin{array}{l}7.32 \\
7.68\end{array}$ & $\begin{array}{l}8.15 \\
7.32\end{array}$ & $\begin{array}{l}8.54 \\
7.32\end{array}$ & $\begin{array}{l}8.64 \\
7.05\end{array}$ & $\begin{array}{l}8.64 \\
7.23\end{array}$ & $\begin{array}{l}8.15 \\
8.06\end{array}$ \\
\hline $5-20$ & 19.14 & $\begin{array}{l}10.5 \\
11.3\end{array}$ & $\begin{array}{l}11.5 \\
11.5\end{array}$ & $\begin{array}{l}12.0 \\
11.5\end{array}$ & $\begin{array}{l}12.2 \\
11.3\end{array}$ & $\begin{array}{l}12.0 \\
12.0\end{array}$ \\
\hline $5-21$ & $\begin{array}{l}13.0 \\
14.5\end{array}$ & $\begin{array}{l}13.7 \\
13.7\end{array}$ & $\begin{array}{l}14.6 \\
13.2\end{array}$ & $\begin{array}{l}15.2 \\
12.8\end{array}$ & $\begin{array}{l}15.3 \\
13.1\end{array}$ & $\begin{array}{l}15.0 \\
14.2\end{array}$ \\
\hline $5-22$ & $\begin{array}{l}15.4 \\
19.8\end{array}$ & $\begin{array}{l}16.7 \\
18.9\end{array}$ & $\begin{array}{l}17.9 \\
17.7\end{array}$ & $\begin{array}{l}19.1 \\
16.7\end{array}$ & $\begin{array}{l}20.0 \\
16.4\end{array}$ & $\begin{array}{l}20.3 \\
16.7\end{array}$ \\
\hline $5-23$ & $\begin{array}{l}17.7 \\
22.8\end{array}$ & $\begin{array}{l}18.9 \\
21.6\end{array}$ & $\begin{array}{l}20.3 \\
20.3\end{array}$ & $\begin{array}{l}21.8 \\
19.1\end{array}$ & $\begin{array}{l}22.8 \\
18.4\end{array}$ & $\begin{array}{l}23.3 \\
18.4\end{array}$ \\
\hline $5-24$ & $\begin{array}{l}19.2 \\
25.2\end{array}$ & $\begin{array}{l}20.6 \\
24.5\end{array}$ & $\begin{array}{l}22.1 \\
23.6\end{array}$ & $\begin{array}{l}23.5 \\
22.8\end{array}$ & $\begin{array}{l}24.5 \\
21.9\end{array}$ & $\begin{array}{l}25.2 \\
21.3\end{array}$ \\
\hline $5-25$ & $\begin{array}{l}20.9 \\
24.0\end{array}$ & $\begin{array}{l}21.3 \\
23.6\end{array}$ & $\begin{array}{l}21.8 \\
22.9\end{array}$ & $\begin{array}{l}23.1 \\
22.1\end{array}$ & $\begin{array}{l}24.0 \\
21.6\end{array}$ & $\begin{array}{l}24.1 \\
21.3\end{array}$ \\
\hline $5-26$ & $\begin{array}{l}21.6 \\
24.7\end{array}$ & $\begin{array}{l}21.9 \\
24.7\end{array}$ & $\begin{array}{l}22.8 \\
24.3\end{array}$ & $\begin{array}{l}23.5 \\
23.8\end{array}$ & $\begin{array}{l}24.1 \\
23.3\end{array}$ & $\begin{array}{l}24.5 \\
23.1\end{array}$ \\
\hline
\end{tabular}


TABLE 1.- BIHOURLY DISCHARGE (1), IN CUBIC METERS PER SECOND, AT SECTION 0000, EAST FORK RIVER, WYOMING, 1979--CONTINUED

\begin{tabular}{|c|c|c|c|c|c|c|}
\hline DATE & $\begin{array}{r}2 \\
14\end{array}$ & $\begin{array}{r}4 \\
15\end{array}$ & $\begin{array}{c}\text { TIME IN } \\
6 \\
18\end{array}$ & $\begin{array}{r}\text { HOURS } \\
8 \\
20\end{array}$ & $\begin{array}{l}10 \\
22\end{array}$ & $\begin{array}{l}12 \\
24\end{array}$ \\
\hline $5-27$ & $\begin{array}{l}23.6 \\
32.3\end{array}$ & $\begin{array}{l}25.2 \\
32.1\end{array}$ & $\begin{array}{l}27.2 \\
31.1\end{array}$ & $\begin{array}{l}29.5 \\
29.9\end{array}$ & $\begin{array}{l}31.5 \\
28.6\end{array}$ & $\begin{array}{l}32.3 \\
27.8\end{array}$ \\
\hline $5-28$ & $\begin{array}{l}27.0 \\
28.9\end{array}$ & $\begin{array}{l}27.0 \\
28.4\end{array}$ & $\begin{array}{l}27.6 \\
27.6\end{array}$ & $\begin{array}{l}28.6 \\
26.7\end{array}$ & $\begin{array}{l}29.1 \\
25.6\end{array}$ & $\begin{array}{l}29 \cdot 3 \\
24.5\end{array}$ \\
\hline $5-29$ & $\begin{array}{l}23.6 \\
27.2\end{array}$ & $\begin{array}{l}23.3 \\
28.0\end{array}$ & $\begin{array}{l}23.6 \\
28.6\end{array}$ & $\begin{array}{l}24.3 \\
28.2\end{array}$ & $\begin{array}{l}25.2 \\
27.4\end{array}$ & $\begin{array}{l}26.1 \\
26.7\end{array}$ \\
\hline $5-30$ & $\begin{array}{l}25.8 \\
16.3\end{array}$ & $\begin{array}{l}24.7 \\
15.0\end{array}$ & $\begin{array}{l}23.6 \\
13.7\end{array}$ & $\begin{array}{l}22.3 \\
13.6\end{array}$ & $\begin{array}{l}20.6 \\
13.3\end{array}$ & $\begin{array}{l}18.4 \\
13.0\end{array}$ \\
\hline $5-31$ & $\begin{array}{r}12.1 \\
8.44\end{array}$ & 11.3 & $\begin{array}{c}10.5 \\
7.59\end{array}$ & $\begin{array}{l}9.76 \\
7.41\end{array}$ & $9 \cdot 14$ & $\begin{array}{l}8.64 \\
6.96\end{array}$ \\
\hline $6-1$ & $\begin{array}{l}6.79 \\
5.04\end{array}$ & $\begin{array}{l}6.53 \\
4.61\end{array}$ & $\begin{array}{l}6.37 \\
4.41\end{array}$ & $\begin{array}{l}6.12 \\
4.48\end{array}$ & $\begin{array}{l}5.88 \\
4.83\end{array}$ & $\begin{array}{l}5.49 \\
4.68\end{array}$ \\
\hline $6-2$ & $\begin{array}{l}4.48 \\
4.08\end{array}$ & $\begin{array}{l}4.48 \\
3.89\end{array}$ & $\begin{array}{l}4.48 \\
3.64\end{array}$ & $\begin{array}{l}4.48 \\
3.52\end{array}$ & $\begin{array}{l}4.41 \\
3.76\end{array}$ & $\begin{array}{l}4.28 \\
3.82\end{array}$ \\
\hline $6-3$ & $\begin{array}{l}3.89 \\
5.41\end{array}$ & $\begin{array}{l}4.41 \\
5.26\end{array}$ & $\begin{array}{l}5.04 \\
5.04\end{array}$ & $\begin{array}{l}5.41 \\
5.26\end{array}$ & $\begin{array}{l}5.57 \\
5.41\end{array}$ & $\begin{array}{l}5.57 \\
5.49\end{array}$ \\
\hline $6-4$ & $\begin{array}{l}5.88 \\
8.83\end{array}$ & $\begin{array}{l}6.79 \\
8.54\end{array}$ & $\begin{array}{l}7.68 \\
8.34\end{array}$ & $\begin{array}{l}8.44 \\
8.15\end{array}$ & $\begin{array}{l}8.83 \\
8.15\end{array}$ & $\begin{array}{l}8.93 \\
8.64\end{array}$ \\
\hline $6-5$ & $\begin{array}{r}9.24 \\
14.2\end{array}$ & $\begin{array}{l}10.3 \\
13.8\end{array}$ & $\begin{array}{l}11.3 \\
13.3\end{array}$ & $\begin{array}{l}12.4 \\
12.7\end{array}$ & $\begin{array}{l}13.2 \\
12.1\end{array}$ & $\begin{array}{l}14.0 \\
11.6\end{array}$ \\
\hline $6-6$ & $\begin{array}{l}12.1 \\
17.4\end{array}$ & $\begin{array}{l}13.1 \\
17.3\end{array}$ & $\begin{array}{l}14.0 \\
16.8\end{array}$ & $\begin{array}{l}15.2 \\
16.3\end{array}$ & $\begin{array}{l}16.1 \\
15.7\end{array}$ & $\begin{array}{l}17.0 \\
15.0\end{array}$ \\
\hline $6-7$ & $\begin{array}{l}14.4 \\
12.4\end{array}$ & $\begin{array}{l}14.4 \\
11.2\end{array}$ & $\begin{array}{l}14.4 \\
10.7\end{array}$ & $\begin{array}{l}14.6 \\
10.7\end{array}$ & $\begin{array}{l}14.2 \\
10.7\end{array}$ & $\begin{array}{l}13.6 \\
10.1\end{array}$ \\
\hline $6-8$ & $\begin{array}{l}9.14 \\
6.53\end{array}$ & $\begin{array}{l}8.64 \\
6.12\end{array}$ & $\begin{array}{l}8.15 \\
5.72\end{array}$ & $\begin{array}{l}7.77 \\
5.41\end{array}$ & $\begin{array}{l}7.32 \\
5.26\end{array}$ & $\begin{array}{l}6.96 \\
5.41\end{array}$ \\
\hline $6-9$ & $\begin{array}{l}5.26 \\
3.82\end{array}$ & $\begin{array}{l}5.04 \\
3.64\end{array}$ & $\begin{array}{l}4.83 \\
3.46\end{array}$ & $\begin{array}{l}4.55 \\
3.46\end{array}$ & $\begin{array}{l}4.28 \\
3.76\end{array}$ & $\begin{array}{l}3.95 \\
3.70\end{array}$ \\
\hline $6-10$ & $\begin{array}{l}3.64 \\
3.64\end{array}$ & $\begin{array}{l}3.58 \\
3.52\end{array}$ & $\begin{array}{l}3.58 \\
3.46\end{array}$ & $\begin{array}{l}3.70 \\
3.40\end{array}$ & $\begin{array}{l}3.76 \\
3.46\end{array}$ & $\begin{array}{l}3.70 \\
3.52\end{array}$ \\
\hline $6-11$ & $\begin{array}{l}3.58 \\
6.28\end{array}$ & $\begin{array}{l}3.89 \\
6.12\end{array}$ & $\begin{array}{l}4.75 \\
5.96\end{array}$ & $\begin{array}{l}5.57 \\
5.80\end{array}$ & $\begin{array}{l}5.96 \\
5.64\end{array}$ & $\begin{array}{l}6.20 \\
5.72\end{array}$ \\
\hline $6-12$ & $\begin{array}{l}6.04 \\
8.74\end{array}$ & $\begin{array}{l}6.70 \\
8.54\end{array}$ & $\begin{array}{l}7.50 \\
8.06\end{array}$ & $\begin{array}{l}8.15 \\
7.77\end{array}$ & $\begin{array}{l}8.64 \\
7.32\end{array}$ & $\begin{array}{l}8.83 \\
7.50\end{array}$ \\
\hline
\end{tabular}




\begin{tabular}{|c|c|c|c|c|c|c|}
\hline DATE & $\begin{array}{r}2 \\
14\end{array}$ & 16 & $\begin{array}{c}\text { TIME IN } \\
6 \\
18\end{array}$ & $\begin{array}{r}\text { HOURS }_{8} \\
20\end{array}$ & $\begin{array}{l}10 \\
22\end{array}$ & $\frac{12}{24}$ \\
\hline $6-13$ & 14.25 & 19.34 & $\begin{array}{l}10.6 \\
13.2\end{array}$ & $\begin{array}{l}12.0 \\
12.1\end{array}$ & $\begin{array}{l}13.0 \\
11.1\end{array}$ & $\begin{array}{l}13.7 \\
10.6\end{array}$ \\
\hline $6-14$ & $\begin{array}{l}10.6 \\
17.7\end{array}$ & $\frac{11}{17.9}$ & $\begin{array}{l}13.3 \\
16.3\end{array}$ & $\begin{array}{l}15.2 \\
15.0\end{array}$ & $\begin{array}{l}16.3 \\
13.7\end{array}$ & $\frac{17}{12}: \frac{1}{7}$ \\
\hline $6-15$ & $\begin{array}{l}12.2 \\
15.2\end{array}$ & $\begin{array}{l}12.8 \\
14.2\end{array}$ & $\begin{array}{l}13.7 \\
12.8\end{array}$ & $\begin{array}{l}14.8 \\
11.4\end{array}$ & $\frac{15.4}{10.3}$ & $\begin{array}{c}15.6 \\
9.55\end{array}$ \\
\hline $6-16$ & $\begin{array}{l}8.93 \\
8.64\end{array}$ & $\begin{array}{l}8.83 \\
8.15\end{array}$ & $\begin{array}{l}8.83 \\
7.77\end{array}$ & $\begin{array}{l}9.04 \\
7.23\end{array}$ & $\begin{array}{l}9.04 \\
6.79\end{array}$ & $\begin{array}{l}8.83 \\
6.45\end{array}$ \\
\hline $6-17$ & $\begin{array}{l}6.28 \\
6.88\end{array}$ & $\begin{array}{l}6.37 \\
6.45\end{array}$ & $\begin{array}{l}6.79 \\
6.12\end{array}$ & $\begin{array}{l}7.05 \\
5.64\end{array}$ & $\begin{array}{l}7.32 \\
5.26\end{array}$ & $\begin{array}{l}7.32 \\
4.97\end{array}$ \\
\hline $6-18$ & $\begin{array}{l}4.83 \\
6.12\end{array}$ & $\begin{array}{l}5.04 \\
6.04\end{array}$ & $\begin{array}{l}5.49 \\
5.64\end{array}$ & $\begin{array}{l}5.88 \\
5.41\end{array}$ & $\begin{array}{l}6.12 \\
5.12\end{array}$ & $\begin{array}{l}6.28 \\
4.90\end{array}$ \\
\hline $6-19$ & $\begin{array}{l}4.75 \\
3.76\end{array}$ & $\begin{array}{l}4.55 \\
3.64\end{array}$ & $\begin{array}{l}4.41 \\
3.46\end{array}$ & $\begin{array}{l}4.21 \\
3.34\end{array}$ & $\begin{array}{l}4.08 \\
3.23\end{array}$ & $\begin{array}{l}3.95 \\
3.12\end{array}$ \\
\hline $6-20$ & $\begin{array}{l}3.06 \\
2.59\end{array}$ & $\begin{array}{l}3.00 \\
2.54\end{array}$ & $\begin{array}{l}2.95 \\
2.44\end{array}$ & $\begin{array}{l}2.90 \\
2.39\end{array}$ & $\begin{array}{l}2.84 \\
2.34\end{array}$ & $\begin{array}{l}2.79 \\
2.29\end{array}$ \\
\hline $6-21$ & $\begin{array}{l}2.25 \\
2.25\end{array}$ & $\begin{array}{l}2.25 \\
2.20\end{array}$ & $\begin{array}{l}2.20 \\
2.20\end{array}$ & $\begin{array}{l}2.20 \\
2.16\end{array}$ & $\begin{array}{l}2.25 \\
2.11\end{array}$ & $\begin{array}{l}2.29 \\
2.07\end{array}$ \\
\hline $6-22$ & $\begin{array}{l}2.07 \\
2.79\end{array}$ & $\begin{array}{l}2.02 \\
2.74\end{array}$ & $\begin{array}{l}2.02 \\
2.64\end{array}$ & $\begin{array}{l}2.11 \\
2.54\end{array}$ & $\begin{array}{l}2.44 \\
2.49\end{array}$ & $\begin{array}{l}2.74 \\
2.39\end{array}$ \\
\hline $6-23$ & $\begin{array}{l}2.29 \\
2.84\end{array}$ & $\begin{array}{l}2.20 \\
2.79\end{array}$ & $\begin{array}{l}2.20 \\
2.74\end{array}$ & $\begin{array}{l}2.44 \\
2.59\end{array}$ & $\begin{array}{l}2.74 \\
2.49\end{array}$ & $\begin{array}{l}2.84 \\
2.39\end{array}$ \\
\hline $6-24$ & $\begin{array}{l}2.34 \\
2.95\end{array}$ & $\begin{array}{l}2.29 \\
2.90\end{array}$ & $\begin{array}{l}2.25 \\
2.79\end{array}$ & $\begin{array}{l}2.34 \\
2.69\end{array}$ & $\begin{array}{l}2.69 \\
2.59\end{array}$ & $\begin{array}{l}2.90 \\
2.49\end{array}$ \\
\hline $6-25$ & $\begin{array}{l}2.39 \\
3.17\end{array}$ & $\begin{array}{l}2.29 \\
3.06\end{array}$ & $\begin{array}{l}2.25 \\
2.95\end{array}$ & $\begin{array}{l}2.39 \\
2.84\end{array}$ & $\begin{array}{l}2.79 \\
2.74\end{array}$ & $\begin{array}{l}3.00 \\
2.64\end{array}$ \\
\hline $6-26$ & $\begin{array}{l}2.49 \\
2.79\end{array}$ & $\begin{array}{l}2.39 \\
2.84\end{array}$ & $\begin{array}{l}2.34 \\
2.79\end{array}$ & $\begin{array}{l}2.44 \\
2.69\end{array}$ & $\begin{array}{l}2.64 \\
2.59\end{array}$ & $\begin{array}{l}2.79 \\
2.49\end{array}$ \\
\hline $6-27$ & $\begin{array}{l}2.44 \\
2.95\end{array}$ & $\begin{array}{l}2.34 \\
3.58\end{array}$ & $\begin{array}{l}2.29 \\
3.34\end{array}$ & $\begin{array}{l}2.39 \\
2.84\end{array}$ & $\begin{array}{l}2.69 \\
2.64\end{array}$ & $\begin{array}{l}2.90 \\
2.54\end{array}$ \\
\hline $6-28$ & $\begin{array}{l}2.49 \\
2.69\end{array}$ & $\begin{array}{l}2.39 \\
2.64\end{array}$ & $\begin{array}{l}2.34 \\
2.54\end{array}$ & $\begin{array}{l}2.44 \\
2.44\end{array}$ & $\begin{array}{l}2.54 \\
2.39\end{array}$ & $\begin{array}{l}2.64 \\
2.34\end{array}$ \\
\hline $6-29$ & $\begin{array}{l}2.29 \\
2.64\end{array}$ & $\begin{array}{l}2.25 \\
2.64\end{array}$ & $\begin{array}{l}2.20 \\
2.54\end{array}$ & $\begin{array}{l}2.20 \\
2.44\end{array}$ & $\begin{array}{l}2.34 \\
2.34\end{array}$ & $\begin{array}{l}2.54 \\
2.25\end{array}$ \\
\hline
\end{tabular}


TABLE 1.- BIHOURLY DISCHARGE (1), IN CUBIC METERS PER SECOND, AT SECTION 0000, EAST FORK RIVER, WYOMING, 1979--CONTINUED

\begin{tabular}{|c|c|c|c|c|c|c|}
\hline DATE & $\begin{array}{r}2 \\
14\end{array}$ & $\begin{array}{r}46 \\
\end{array}$ & $\begin{array}{c}\text { TIME IN } \\
68 \\
18\end{array}$ & $\begin{array}{r}\text { HOURS } \\
80 \\
20\end{array}$ & $\begin{array}{l}10 \\
22\end{array}$ & $\begin{array}{l}12 \\
24\end{array}$ \\
\hline $6-30$ & $\begin{array}{l}2.25 \\
2.49\end{array}$ & $\begin{array}{l}2.20 \\
2.54\end{array}$ & $\begin{array}{l}2.16 \\
2.49\end{array}$ & $\begin{array}{l}2.16 \\
2.39\end{array}$ & $\begin{array}{l}2.20 \\
2.34\end{array}$ & $\begin{array}{l}2.39 \\
2.25\end{array}$ \\
\hline $7-1$ & $\begin{array}{l}2.20 \\
2.02\end{array}$ & $\begin{array}{l}2.16 \\
2.02\end{array}$ & $\begin{array}{l}2.16 \\
1.98\end{array}$ & $\begin{array}{l}2.11 \\
1.94\end{array}$ & $\begin{array}{l}2.11 \\
1.94\end{array}$ & $\begin{array}{l}2.07 \\
1.89\end{array}$ \\
\hline $7-2$ & $\begin{array}{l}1.85 \\
1.89\end{array}$ & $\begin{array}{l}1.85 \\
1.89\end{array}$ & $\begin{array}{l}1.81 \\
1.85\end{array}$ & $\begin{array}{l}1.77 \\
1.81\end{array}$ & $\begin{array}{l}1.81 \\
1.81\end{array}$ & $\begin{array}{l}1.85 \\
1.77\end{array}$ \\
\hline $7-3$ & $\begin{array}{l}1.77 \\
1.73\end{array}$ & $\begin{array}{l}1.73 \\
1.73\end{array}$ & $\begin{array}{l}1.73 \\
1.69\end{array}$ & $\begin{array}{l}1.69 \\
1.69\end{array}$ & $\begin{array}{l}1.69 \\
1.65\end{array}$ & $\begin{array}{l}1.69 \\
1.65\end{array}$ \\
\hline $7-4$ & $\begin{array}{l}1.65 \\
1.61\end{array}$ & $\begin{array}{l}1.61 \\
1.61\end{array}$ & $\begin{array}{l}1.61 \\
1.61\end{array}$ & $\begin{array}{l}1.61 \\
1.57\end{array}$ & $\begin{array}{l}1.61 \\
1.57\end{array}$ & $\begin{array}{l}1.61 \\
1.57\end{array}$ \\
\hline $7-5$ & $\begin{array}{l}1.57 \\
1.54\end{array}$ & $\begin{array}{l}1.57 \\
1.54\end{array}$ & $\begin{array}{l}1.57 \\
1.54\end{array}$ & $\begin{array}{l}1.57 \\
1.54\end{array}$ & $\begin{array}{l}1.54 \\
1.54\end{array}$ & $\begin{array}{l}1.54 \\
1.54\end{array}$ \\
\hline $7-6$ & $\begin{array}{l}1.54 \\
1.46\end{array}$ & $\begin{array}{l}1.54 \\
1.46\end{array}$ & $\begin{array}{l}1.54 \\
1.46\end{array}$ & $\begin{array}{l}1.50 \\
1.46\end{array}$ & $\begin{array}{l}1.50 \\
1.46\end{array}$ & $\begin{array}{l}1.46 \\
1.46\end{array}$ \\
\hline $7-7$ & $\begin{array}{l}1.46 \\
1.46\end{array}$ & $\begin{array}{l}1.46 \\
1.46\end{array}$ & $\begin{array}{l}1.46 \\
1.46\end{array}$ & $\begin{array}{l}1.46 \\
1.43\end{array}$ & $\begin{array}{l}1.46 \\
1.43\end{array}$ & $\begin{array}{l}1.46 \\
1.43\end{array}$ \\
\hline $7-8$ & $\begin{array}{l}1.43 \\
1.39\end{array}$ & $\begin{array}{l}1.43 \\
1.39\end{array}$ & $\begin{array}{l}1.43 \\
1.39\end{array}$ & $\begin{array}{l}1.43 \\
1.35\end{array}$ & $\begin{array}{l}1.43 \\
1.35\end{array}$ & $\begin{array}{l}1.43 \\
1.35\end{array}$ \\
\hline $7-9$ & $\begin{array}{l}1.35 \\
1.35\end{array}$ & $\begin{array}{l}1.35 \\
1.32\end{array}$ & $\begin{array}{l}1.35 \\
1.32\end{array}$ & $\begin{array}{l}1.35 \\
1.32\end{array}$ & $\begin{array}{l}1.35 \\
1.29\end{array}$ & $\begin{array}{l}1.35 \\
1.29\end{array}$ \\
\hline $7-10$ & $\begin{array}{l}1.29 \\
1.29\end{array}$ & $\begin{array}{l}1.29 \\
1.29\end{array}$ & $\begin{array}{l}1.29 \\
1.25\end{array}$ & $\begin{array}{l}1.29 \\
1.25\end{array}$ & $\begin{array}{l}1.29 \\
1.25\end{array}$ & $\begin{array}{l}1.29 \\
1.22\end{array}$ \\
\hline $7-11$ & $\begin{array}{l}1.22 \\
1.25\end{array}$ & $\begin{array}{l}1.22 \\
1.25\end{array}$ & $\begin{array}{l}1.22 \\
1.22\end{array}$ & $\begin{array}{l}1.25 \\
1.22\end{array}$ & $\begin{array}{l}1.25 \\
1.19\end{array}$ & $\begin{array}{l}1.25 \\
1.19\end{array}$ \\
\hline $7-12$ & $\begin{array}{l}1.19 \\
1.22\end{array}$ & $\begin{array}{l}\frac{1}{1} .15 \\
.22\end{array}$ & $\begin{array}{l}1.15 \\
1.22\end{array}$ & $\begin{array}{l}1.19 \\
1.19\end{array}$ & $\begin{array}{l}1.19 \\
1.19\end{array}$ & $\begin{array}{l}1.22 \\
1.15\end{array}$ \\
\hline $7-13$ & $\begin{array}{l}1.15 \\
1.19\end{array}$ & $\begin{array}{l}1.15 \\
1.19\end{array}$ & $\begin{array}{l}1.15 \\
1.15\end{array}$ & $\begin{array}{l}1.19 \\
1.15\end{array}$ & $\frac{1.22}{1.15}$ & $\frac{1.22}{1.12}$ \\
\hline $7-14$ & $\frac{1}{1.12}$ & $\begin{array}{l}1.12 \\
1.15\end{array}$ & $\frac{1}{1}: 12$ & $\begin{array}{l}1.15 \\
1.09\end{array}$ & $\begin{array}{l}1.15 \\
1.09\end{array}$ & $\begin{array}{l}1.15 \\
1.06\end{array}$ \\
\hline $7-15$ & $\begin{array}{l}1.06 \\
1.09\end{array}$ & $\begin{array}{l}1.06 \\
1.06\end{array}$ & $\begin{array}{l}1.06 \\
1.06\end{array}$ & $\begin{array}{l}1.06 \\
1.06\end{array}$ & $\begin{array}{l}1.09 \\
1.06\end{array}$ & $\begin{array}{l}1.09 \\
1.06\end{array}$ \\
\hline $7-16$ & $\begin{array}{l}1.06 \\
1.12\end{array}$ & $\begin{array}{l}1.06 \\
1.12\end{array}$ & $\begin{array}{l}1.06 \\
1.09\end{array}$ & $\begin{array}{l}1.09 \\
1.09\end{array}$ & $\begin{array}{l}1.09 \\
1.09\end{array}$ & $\begin{array}{l}1.12 \\
1.06\end{array}$ \\
\hline
\end{tabular}

(1) DATA FROM EMMETT, MYRICK, AND MEADE, 1980, TABLE 6. 
TABLE 2.- BIHOURLY DISCHARGE (1), IN CUBIC METERS PER SECOND, AT SECTION 3295, EAST FORK RIVER, WYOMING, 1979

\begin{tabular}{|c|c|c|c|c|c|c|}
\hline DATE & $\begin{array}{r}2 \\
14 \\
-\end{array}$ & $\begin{array}{r}4 \\
16 \\
\end{array}$ & $\begin{array}{c}\text { TIME IN } \\
6 \\
18\end{array}$ & $\begin{array}{r}\text { HOURS } \\
8 \\
20\end{array}$ & $\begin{array}{l}10 \\
22 \\
\end{array}$ & $\begin{array}{r}12 \\
24 \\
\end{array}$ \\
\hline $5-10$ & $=-$ & $=-$ & $=-$ & $=-$ & $=-$ & $=-$ \\
\hline $5-11$ & --- & $=--$ & $=-$ & $=-$ & $=-$ & $=-$ \\
\hline $5-12$ & --- & --- & $=-$ & $=-$ & $=-$ & $=-$ \\
\hline $5-13$ & $\overline{--}$ & $=-$ & $=-$ & $=-$ & $\ddot{--}$ & $=-$ \\
\hline $5-14$ & $=--$ & $=--$ & $=--$ & $=-$ & --- & $=-$ \\
\hline $5-15$ & $=-$ & $=-$ & $\overline{---}$ & $=-$ & $=-$ & $=-$ \\
\hline $5-16$ & --- & --- & --- & $\overline{--}$ & $\overline{--}$ & --- \\
\hline $5-17$ & $\begin{array}{l}3.90 \\
3.90\end{array}$ & $\begin{array}{l}3.90 \\
3.69\end{array}$ & $\begin{array}{l}3.90 \\
3.49\end{array}$ & $\begin{array}{l}3.90 \\
3.49\end{array}$ & $\begin{array}{l}3.90 \\
3.76\end{array}$ & $\begin{array}{l}3.90 \\
4.61\end{array}$ \\
\hline $5-18$ & $\begin{array}{l}5.43 \\
5.66\end{array}$ & $\begin{array}{l}6.21 \\
5.43\end{array}$ & $\begin{array}{l}6.29 \\
5.43\end{array}$ & $\begin{array}{l}6.29 \\
5.74\end{array}$ & $\begin{array}{l}6.13 \\
5.98\end{array}$ & $\begin{array}{l}5.90 \\
6.78\end{array}$ \\
\hline $5-19$ & $\begin{array}{l}7.62 \\
6.62\end{array}$ & $\begin{array}{l}7.88 \\
6.62\end{array}$ & $\begin{array}{l}8.14 \\
6.29\end{array}$ & $\begin{array}{l}7.88 \\
6.29\end{array}$ & $\begin{array}{l}7.62 \\
6.95\end{array}$ & $\begin{array}{l}7.03 \\
8.14\end{array}$ \\
\hline $5-20$ & $\begin{array}{r}9.40 \\
10.4\end{array}$ & $\begin{array}{l}10.5 \\
10.7\end{array}$ & $\begin{array}{l}11.2 \\
10.4\end{array}$ & $\begin{array}{l}11.4 \\
10.3\end{array}$ & $\begin{array}{l}11.3 \\
10.9\end{array}$ & $\begin{array}{l}11.0 \\
11.8\end{array}$ \\
\hline $5-21$ & $\begin{array}{l}12.9 \\
12.7\end{array}$ & $\begin{array}{l}13.5 \\
12.1\end{array}$ & $\begin{array}{l}13.9 \\
11.5\end{array}$ & $\begin{array}{l}13.9 \\
11.8\end{array}$ & $\begin{array}{l}13.7 \\
12.9\end{array}$ & $\begin{array}{l}13.3 \\
14.0\end{array}$ \\
\hline $5-22$ & $\begin{array}{l}15.1 \\
17.3\end{array}$ & $\begin{array}{l}16.1 \\
16.5\end{array}$ & $\begin{array}{l}17.1 \\
15.9\end{array}$ & $\begin{array}{l}17.9 \\
15.4\end{array}$ & $\begin{array}{l}18.2 \\
15.4\end{array}$ & $\begin{array}{l}17.7 \\
15.9\end{array}$ \\
\hline $5-23$ & $\begin{array}{l}17.5 \\
20.2\end{array}$ & $\begin{array}{l}19.3 \\
18.8\end{array}$ & $\begin{array}{l}20.9 \\
17.5\end{array}$ & $\begin{array}{l}21.6 \\
16.5\end{array}$ & $\begin{array}{l}21.9 \\
16.5\end{array}$ & $\begin{array}{l}21.6 \\
17.7\end{array}$ \\
\hline $5-24$ & $\begin{array}{l}19.3 \\
24.9\end{array}$ & $\begin{array}{l}21.6 \\
23.4\end{array}$ & $\begin{array}{l}23.4 \\
22.3\end{array}$ & $\begin{array}{l}24.1 \\
21.6\end{array}$ & $\begin{array}{l}24.9 \\
20.9\end{array}$ & $\begin{array}{l}25.2 \\
20.6\end{array}$ \\
\hline $5-25$ & $\begin{array}{l}21.1 \\
24.4\end{array}$ & $\begin{array}{l}22.1 \\
23.9\end{array}$ & $\begin{array}{l}23.4 \\
23.4\end{array}$ & $\begin{array}{l}24.4 \\
22.1\end{array}$ & $\begin{array}{l}24.7 \\
21.6\end{array}$ & $\begin{array}{l}24.9 \\
22.1\end{array}$ \\
\hline $5-26$ & $\begin{array}{l}22.6 \\
25.5\end{array}$ & $\begin{array}{l}23.6 \\
25.2\end{array}$ & $\begin{array}{l}24.4 \\
24.7\end{array}$ & $\begin{array}{l}24.9 \\
24.4\end{array}$ & $\begin{array}{l}25.5 \\
24.1\end{array}$ & $\begin{array}{l}25.7 \\
24.4\end{array}$ \\
\hline
\end{tabular}


TABLE 2.- BIHOURLY DISCHARGE (1), IN CUBIC METERS PER SECOND, AT SECTION 3295, EAST FORK RIVER, WYOMING, 1979--CONTINUED

\begin{tabular}{|c|c|c|c|c|c|c|}
\hline DATE & $\begin{array}{r}2 \\
14 \\
\end{array}$ & 16 & $\begin{array}{c}\text { TIME IN } \\
6 \\
18\end{array}$ & $\begin{array}{r}\text { HOURS } \\
80 \\
20\end{array}$ & $\begin{array}{r}10 \\
22 \\
\end{array}$ & $\begin{array}{l}12 \\
24\end{array}$ \\
\hline $5-27$ & $\begin{array}{l}25.7 \\
32.5\end{array}$ & $\begin{array}{l}27.7 \\
31.5\end{array}$ & $\begin{array}{l}29.7 \\
30.6\end{array}$ & $\begin{array}{l}31.8 \\
29.4\end{array}$ & $\begin{array}{l}33.4 \\
28.8\end{array}$ & $\begin{array}{l}33.4 \\
28.2\end{array}$ \\
\hline $5-28$ & $\begin{array}{l}28.2 \\
29.4\end{array}$ & $\begin{array}{l}28.8 \\
28.8\end{array}$ & $\begin{array}{l}29.4 \\
28.0\end{array}$ & $\begin{array}{l}30.0 \\
27.1\end{array}$ & $\begin{array}{l}30.3 \\
26.3\end{array}$ & $\begin{array}{l}30.3 \\
25.7\end{array}$ \\
\hline $5-29$ & $\begin{array}{l}25.2 \\
29.4\end{array}$ & $\begin{array}{l}25.2 \\
30.3\end{array}$ & $\begin{array}{l}25.7 \\
30.3\end{array}$ & $\begin{array}{l}26.8 \\
29.7\end{array}$ & $\begin{array}{l}27.7 \\
28.8\end{array}$ & $\begin{array}{l}28.8 \\
28.0\end{array}$ \\
\hline $5-30$ & 27.1 & $\begin{array}{l}25.7 \\
14.4\end{array}$ & $\begin{array}{l}24.1 \\
14.0\end{array}$ & $\begin{array}{l}22.1 \\
14.0\end{array}$ & $\begin{array}{l}19.7 \\
13.6\end{array}$ & $\begin{array}{l}17.5 \\
13.1\end{array}$ \\
\hline $5-31$ & $\begin{array}{r}12.4 \\
9.22\end{array}$ & $1 \frac{11.8}{8.76}$ & 11.10 & $\begin{array}{c}10.5 \\
8.32\end{array}$ & $\begin{array}{c}10.0 \\
7.88\end{array}$ & $\begin{array}{l}9.68 \\
7.71\end{array}$ \\
\hline $6-1$ & $\begin{array}{l}7.54 \\
5.13\end{array}$ & $\begin{array}{l}7.28 \\
4.83\end{array}$ & $\begin{array}{l}6.95 \\
4.90\end{array}$ & $\begin{array}{l}6.70 \\
5.28\end{array}$ & $\begin{array}{l}6.29 \\
5.20\end{array}$ & $\begin{array}{l}5.74 \\
5.05\end{array}$ \\
\hline $6-2$ & $\begin{array}{l}4.90 \\
3.97\end{array}$ & $\begin{array}{l}4.75 \\
3.69\end{array}$ & $\begin{array}{l}4.75 \\
3.49\end{array}$ & $\begin{array}{l}4.75 \\
3.90\end{array}$ & $\begin{array}{l}4.68 \\
3.90\end{array}$ & $\begin{array}{l}4.32 \\
3.90\end{array}$ \\
\hline $6-3$ & $\begin{array}{l}4.39 \\
5.43\end{array}$ & $\begin{array}{l}4.90 \\
5.20\end{array}$ & $\begin{array}{l}5.35 \\
5.43\end{array}$ & $\begin{array}{l}5.74 \\
5.58\end{array}$ & $\begin{array}{l}5.90 \\
5.58\end{array}$ & $\begin{array}{l}5.66 \\
6.05\end{array}$ \\
\hline $6-4$ & $\begin{array}{l}7.12 \\
9.49\end{array}$ & $\begin{array}{l}8.23 \\
9.31\end{array}$ & $\begin{array}{l}9.13 \\
9.13\end{array}$ & $\begin{array}{l}9.68 \\
9.13\end{array}$ & $\begin{array}{l}9.86 \\
9.31\end{array}$ & $\begin{array}{l}9.77 \\
10.0\end{array}$ \\
\hline $6-5$ & $\begin{array}{l}11.0 \\
14.5\end{array}$ & $\begin{array}{l}12.0 \\
14.0\end{array}$ & $\begin{array}{l}12.9 \\
13.4\end{array}$ & $\begin{array}{l}13.8 \\
12.9\end{array}$ & $\begin{array}{l}14.6 \\
12.8\end{array}$ & $\begin{array}{l}14.8 \\
13.0\end{array}$ \\
\hline $6-6$ & $\begin{array}{l}13.8 \\
19.0\end{array}$ & $\begin{array}{l}14.8 \\
18.4\end{array}$ & $\begin{array}{l}15.6 \\
17.5\end{array}$ & $\begin{array}{l}17.1 \\
16.5\end{array}$ & $\begin{array}{l}18.2 \\
15.6\end{array}$ & $\begin{array}{l}18.8 \\
15.2\end{array}$ \\
\hline $6-7$ & $\begin{array}{l}14.9 \\
13.1\end{array}$ & $\begin{array}{l}14.9 \\
12.1\end{array}$ & $\begin{array}{l}15.5 \\
11.7\end{array}$ & $\begin{array}{l}15.5 \\
12.0\end{array}$ & $\begin{array}{l}14.9 \\
11.6\end{array}$ & $\begin{array}{l}14.0 \\
10.9\end{array}$ \\
\hline $6-8$ & $\begin{array}{c}10.3 \\
7.37\end{array}$ & $\begin{array}{l}9.68 \\
6.87\end{array}$ & $\begin{array}{l}9.13 \\
6.46\end{array}$ & $\begin{array}{l}8.76 \\
6.21\end{array}$ & $\begin{array}{l}8.32 \\
6.21\end{array}$ & $\begin{array}{l}7.88 \\
6.13\end{array}$ \\
\hline $6-9$ & $\begin{array}{l}5.82 \\
3.97\end{array}$ & $\begin{array}{l}5.43 \\
3.76\end{array}$ & $\begin{array}{l}5.20 \\
3.63\end{array}$ & $\begin{array}{l}5.05 \\
3.97\end{array}$ & $\begin{array}{l}4.68 \\
3.97\end{array}$ & $\begin{array}{l}4.25 \\
3.83\end{array}$ \\
\hline $6-10$ & $\begin{array}{l}3.76 \\
3.30\end{array}$ & $\begin{array}{l}3.69 \\
3.43\end{array}$ & $\begin{array}{l}3.63 \\
3.43\end{array}$ & $\begin{array}{l}3.56 \\
3.63\end{array}$ & $\begin{array}{l}3.43 \\
3.76\end{array}$ & $\begin{array}{l}3.30 \\
3.76\end{array}$ \\
\hline $6-11$ & $\begin{array}{l}3.63 \\
6.62\end{array}$ & $\begin{array}{l}4.11 \\
6.38\end{array}$ & $\begin{array}{l}5.28 \\
6.21\end{array}$ & $\begin{array}{l}6.29 \\
6.05\end{array}$ & $\begin{array}{l}6.70 \\
5.90\end{array}$ & $\begin{array}{l}6.70 \\
6.13\end{array}$ \\
\hline $6-12$ & $\begin{array}{l}6.87 \\
9.31\end{array}$ & $\begin{array}{l}7.62 \\
8.85\end{array}$ & $\begin{array}{l}8.59 \\
8.41\end{array}$ & $\begin{array}{l}9.22 \\
7.97\end{array}$ & $\begin{array}{l}9.58 \\
7.88\end{array}$ & $\begin{array}{l}9.49 \\
8.32\end{array}$ \\
\hline
\end{tabular}


TABLE 2.- BIHOURLY DISCHARGE (1), IN CUBIC METERS PER SECOND, AT SECTION 3295, EAST FORK RIVER, WYOMING, 1979--CONTINUED

\begin{tabular}{|c|c|c|c|c|c|c|}
\hline DATE & $\begin{array}{r}2 \\
14\end{array}$ & $\begin{array}{r}4 \\
16\end{array}$ & $\begin{array}{c}\text { TIME IN } \\
18\end{array}$ & $\begin{array}{r}\text { HOURS } \\
8 \\
20\end{array}$ & $\begin{array}{l}10 \\
22\end{array}$ & $\begin{array}{l}12 \\
24\end{array}$ \\
\hline $6-13$ & $\begin{array}{r}9.40 \\
15.1\end{array}$ & $\begin{array}{l}10.7 \\
14.5\end{array}$ & $\begin{array}{l}12.1 \\
13.4\end{array}$ & $\begin{array}{l}13.4 \\
12.6\end{array}$ & $\begin{array}{l}14.5 \\
11.6\end{array}$ & $\begin{array}{l}15.0 \\
11.5\end{array}$ \\
\hline $6-14$ & $\begin{array}{l}12.4 \\
18.8\end{array}$ & $\begin{array}{l}13.8 \\
17.7\end{array}$ & $\begin{array}{l}15.2 \\
15.9\end{array}$ & $\begin{array}{l}16.7 \\
14.4\end{array}$ & $\begin{array}{l}18.2 \\
13.4\end{array}$ & $\begin{array}{l}18.8 \\
12.9\end{array}$ \\
\hline $6-15$ & $\begin{array}{l}12.8 \\
15.2\end{array}$ & $\begin{array}{l}13.4 \\
13.9\end{array}$ & $\begin{array}{l}14.3 \\
12.8\end{array}$ & $\begin{array}{l}15.4 \\
11.4\end{array}$ & $\begin{array}{l}16.5 \\
10.4\end{array}$ & $\begin{array}{l}15.9 \\
10.0\end{array}$ \\
\hline $6-16$ & $\begin{array}{l}9.86 \\
9.22\end{array}$ & $\begin{array}{l}9.86 \\
8.76\end{array}$ & $\begin{array}{l}9.95 \\
8.14\end{array}$ & $\begin{array}{c}10.0 \\
7.80\end{array}$ & $\begin{array}{c}10.0 \\
7.45\end{array}$ & $\begin{array}{l}9.68 \\
7.20\end{array}$ \\
\hline $6-17$ & $\begin{array}{l}7.20 \\
7.28\end{array}$ & $\begin{array}{l}7.45 \\
6.78\end{array}$ & $\begin{array}{l}7.71 \\
6.21\end{array}$ & $\begin{array}{l}8.06 \\
5.74\end{array}$ & $\begin{array}{l}8.06 \\
5.43\end{array}$ & $\begin{array}{l}7.62 \\
5.20\end{array}$ \\
\hline $6-18$ & $\begin{array}{l}5.28 \\
6.21\end{array}$ & $\begin{array}{l}5.74 \\
5.98\end{array}$ & $\begin{array}{l}6.13 \\
5.58\end{array}$ & $\begin{array}{l}6.62 \\
5.35\end{array}$ & $\begin{array}{l}6.62 \\
5.05\end{array}$ & $\begin{array}{l}6.46 \\
4.90\end{array}$ \\
\hline $6-19$ & $\begin{array}{l}4.75 \\
3.63\end{array}$ & $\begin{array}{l}4.54 \\
3.49\end{array}$ & $\begin{array}{l}4 \cdot 32 \\
3.36\end{array}$ & $\begin{array}{l}4.18 \\
3.23\end{array}$ & $\begin{array}{l}3.97 \\
3.17\end{array}$ & $\begin{array}{l}3.76 \\
3.04\end{array}$ \\
\hline $6-20$ & $\begin{array}{l}2.98 \\
2.49\end{array}$ & $\begin{array}{l}2.91 \\
2.43\end{array}$ & $\begin{array}{l}2.85 \\
2.31\end{array}$ & $\begin{array}{l}2.73 \\
2.25\end{array}$ & $\begin{array}{l}2.67 \\
2.14\end{array}$ & $\begin{array}{l}2.54 \\
2.02\end{array}$ \\
\hline $6-21$ & $\begin{array}{l}2.02 \\
1.91\end{array}$ & $\begin{array}{l}1.97 \\
1.91\end{array}$ & $\begin{array}{l}1.97 \\
1.86\end{array}$ & $\begin{array}{l}2.02 \\
1.81\end{array}$ & $\begin{array}{l}2.14 \\
1.75\end{array}$ & $\begin{array}{l}1.97 \\
1.70\end{array}$ \\
\hline $6-22$ & $\begin{array}{l}1.65 \\
2.60\end{array}$ & $\begin{array}{l}1.70 \\
2.49\end{array}$ & $\begin{array}{l}1.81 \\
2.37\end{array}$ & $\begin{array}{l}2.31 \\
2.25\end{array}$ & $\begin{array}{l}2.67 \\
2.14\end{array}$ & $\begin{array}{l}2.67 \\
2.02\end{array}$ \\
\hline $6-23$ & $\begin{array}{l}1.97 \\
2.67\end{array}$ & $\begin{array}{l}1.86 \\
2.54\end{array}$ & $\begin{array}{l}1.86 \\
2.43\end{array}$ & $\begin{array}{l}2.31 \\
2.31\end{array}$ & $\begin{array}{l}2.67 \\
2.19\end{array}$ & $\begin{array}{l}2.73 \\
2.14\end{array}$ \\
\hline $6-24$ & $\begin{array}{l}2.02 \\
2.73\end{array}$ & $\begin{array}{l}1.91 \\
2.67\end{array}$ & $\begin{array}{l}1.97 \\
2.54\end{array}$ & $\begin{array}{l}2.43 \\
2.37\end{array}$ & $\begin{array}{l}2.73 \\
2.25\end{array}$ & $\begin{array}{l}2.85 \\
2.14\end{array}$ \\
\hline $6-25$ & $\begin{array}{l}2.02 \\
2.91\end{array}$ & $\begin{array}{l}2.02 \\
2.79\end{array}$ & $\begin{array}{l}2.31 \\
2.67\end{array}$ & $\begin{array}{l}2.67 \\
2.54\end{array}$ & $\begin{array}{l}2.98 \\
2.43\end{array}$ & $\begin{array}{l}3.04 \\
2.31\end{array}$ \\
\hline $6-26$ & $\begin{array}{l}2.19 \\
2.67\end{array}$ & $\begin{array}{l}2.02 \\
2.60\end{array}$ & $\begin{array}{l}2.14 \\
2.49\end{array}$ & $\begin{array}{l}2.54 \\
2.43\end{array}$ & $\begin{array}{l}2.67 \\
2.31\end{array}$ & $\begin{array}{l}2.67 \\
2.25\end{array}$ \\
\hline $6-27$ & $\begin{array}{l}2.14 \\
3.43\end{array}$ & $\begin{array}{l}2.08 \\
3.04\end{array}$ & $\begin{array}{l}2.31 \\
2.60\end{array}$ & $\begin{array}{l}2.54 \\
2.49\end{array}$ & $\begin{array}{l}2.73 \\
2.37\end{array}$ & $\begin{array}{l}2.73 \\
2.25\end{array}$ \\
\hline $6-28$ & $\begin{array}{l}2.14 \\
2.37\end{array}$ & $\begin{array}{l}2.08 \\
2.31\end{array}$ & $\begin{array}{l}2.25 \\
2.19\end{array}$ & $\begin{array}{l}2.31 \\
2.14\end{array}$ & $\begin{array}{l}2.37 \\
2.02\end{array}$ & $\begin{array}{l}2.43 \\
1.97\end{array}$ \\
\hline $6-29$ & $\begin{array}{l}1.91 \\
2.37\end{array}$ & $\begin{array}{l}1.86 \\
2.31\end{array}$ & $\begin{array}{l}1.91 \\
2.19\end{array}$ & $\begin{array}{l}2.08 \\
2.02\end{array}$ & $\begin{array}{l}2.31 \\
1.91\end{array}$ & $\begin{array}{l}2.37 \\
1.86\end{array}$ \\
\hline
\end{tabular}


TABLE 2.- BIHOURLY DISCHARGE(1), IN CUBIC METERS PER SECOND AT SECTION 3295, EAST FORK RIVER, WYOMING, 1979--CONTINUED

\begin{tabular}{|c|c|c|c|c|c|c|}
\hline DATE & $\begin{array}{r}2 \\
14 \\
\end{array}$ & 16 & $\begin{array}{c}\text { TIME } \text { IN } \\
18 \\
18\end{array}$ & $\begin{array}{r}80 U R S \\
20 \\
\end{array}$ & $\begin{array}{r}10 \\
22 \\
\end{array}$ & $\frac{12}{24}$ \\
\hline $6-30$ & $\frac{1}{2} \cdot 81$ & $\begin{array}{l}1.75 \\
2.19\end{array}$ & $\begin{array}{l}1.70 \\
2.02\end{array}$ & $\frac{1}{1.91}$ & $\begin{array}{l}2.14 \\
1.97\end{array}$ & $\begin{array}{l}2.31 \\
1.91\end{array}$ \\
\hline $7-1$ & $\begin{array}{l}1.86 \\
1.70\end{array}$ & $\frac{1}{1.86}$ & $\frac{1}{1.81}$ & $\frac{1.75}{1.70}$ & $\begin{array}{l}1.75 \\
1.70\end{array}$ & $\begin{array}{l}1.70 \\
1.65\end{array}$ \\
\hline $7-2$ & $\begin{array}{l}1.60 \\
1.44\end{array}$ & $\begin{array}{l}1.60 \\
1.44\end{array}$ & $\begin{array}{l}1.54 \\
1.44\end{array}$ & $\begin{array}{l}1.49 \\
1.44\end{array}$ & $\begin{array}{l}1.44 \\
1.44\end{array}$ & $\begin{array}{l}1.39 \\
1.39\end{array}$ \\
\hline
\end{tabular}

(1) DATA FROM EMMETT, MYRICK, AND MEADE, 1980, TABLE 7. 
TABLE 3.- BIHOURLY DISCHARGE (1), IN CUBIC METERS PER SECOND, AT SECTION 0000, EAST FORK RIVER, WYOMING, 1980

\begin{tabular}{|c|c|c|c|c|c|c|}
\hline DATE & $\begin{array}{r}2 \\
14 \\
\end{array}$ & $\begin{array}{r}4 \\
16\end{array}$ & $\begin{array}{c}\text { TIME IN } \\
6 \\
18\end{array}$ & $\begin{array}{r}8 \\
20 \\
\end{array}$ & $\begin{array}{r}10 \\
22 \\
\end{array}$ & $\begin{array}{r}12 \\
24\end{array}$ \\
\hline $5-10$ & $\begin{array}{l}9.38 \\
8.21\end{array}$ & $\begin{array}{l}9.38 \\
7.93\end{array}$ & $\begin{array}{l}9.28 \\
7.84\end{array}$ & $\begin{array}{l}9.08 \\
7.84\end{array}$ & $\begin{array}{l}8.88 \\
8.12\end{array}$ & $\begin{array}{l}8.59 \\
8.31\end{array}$ \\
\hline $5-11$ & $\begin{array}{l}8.31 \\
6.67\end{array}$ & $\begin{array}{l}8.02 \\
6.50\end{array}$ & $\begin{array}{l}7.65 \\
6.41\end{array}$ & $\begin{array}{l}7.29 \\
6.41\end{array}$ & $\begin{array}{l}7.02 \\
6.33\end{array}$ & $\begin{array}{l}6.85 \\
6.50\end{array}$ \\
\hline $5-12$ & $\begin{array}{l}6.85 \\
5.83\end{array}$ & $\begin{array}{l}6.85 \\
5.67\end{array}$ & $\begin{array}{l}6.76 \\
5.67\end{array}$ & $\begin{array}{l}6.50 \\
5.67\end{array}$ & $\begin{array}{l}6.16 \\
5.67\end{array}$ & $\begin{array}{l}5.99 \\
5.67\end{array}$ \\
\hline $5-13$ & $\begin{array}{l}5.59 \\
4.59\end{array}$ & $\begin{array}{l}5.51 \\
4.51\end{array}$ & $\begin{array}{l}5.35 \\
4.51\end{array}$ & $\begin{array}{l}5.19 \\
4.51\end{array}$ & $\begin{array}{l}4.96 \\
4.51\end{array}$ & $\begin{array}{l}4.74 \\
4.59\end{array}$ \\
\hline $5-14$ & $\begin{array}{l}4.66 \\
3.81\end{array}$ & $\begin{array}{l}4.74 \\
3.67\end{array}$ & $\begin{array}{l}4.66 \\
3.74\end{array}$ & $\begin{array}{l}4.59 \\
3.41\end{array}$ & $\begin{array}{l}4.37 \\
3.47\end{array}$ & $\begin{array}{l}4.08 \\
3.47\end{array}$ \\
\hline $5-15$ & $\begin{array}{l}3.41 \\
2.79\end{array}$ & $\begin{array}{l}3.34 \\
2.67\end{array}$ & $\begin{array}{l}3.28 \\
2.73\end{array}$ & $\begin{array}{l}3.22 \\
2.73\end{array}$ & $\begin{array}{l}3.09 \\
2.85\end{array}$ & $\begin{array}{l}2.91 \\
2.73\end{array}$ \\
\hline $5-16$ & $\begin{array}{l}2.73 \\
2.67\end{array}$ & $\begin{array}{l}2.73 \\
2.61\end{array}$ & $\begin{array}{l}2.79 \\
2.67\end{array}$ & $\begin{array}{l}2.85 \\
2.85\end{array}$ & $\begin{array}{l}2.79 \\
2.85\end{array}$ & $\begin{array}{l}2.73 \\
3.09\end{array}$ \\
\hline $5-17$ & $\begin{array}{l}3.41 \\
3.28\end{array}$ & $\begin{array}{l}3.61 \\
3.28\end{array}$ & $\begin{array}{l}3.61 \\
3.34\end{array}$ & $\begin{array}{l}3.54 \\
3.41\end{array}$ & $\begin{array}{l}3.47 \\
3.54\end{array}$ & $\begin{array}{l}3.41 \\
3.74\end{array}$ \\
\hline $5-18$ & $\begin{array}{l}3.81 \\
3.61\end{array}$ & $\begin{array}{l}3.88 \\
3.28\end{array}$ & $\begin{array}{l}3.94 \\
3.03\end{array}$ & $\begin{array}{l}3.88 \\
3.09\end{array}$ & $\begin{array}{l}3.81 \\
3.22\end{array}$ & $\begin{array}{l}3.74 \\
3.22\end{array}$ \\
\hline $5-19$ & $\begin{array}{l}3.34 \\
3.28\end{array}$ & $\begin{array}{l}3.54 \\
3.15\end{array}$ & $\begin{array}{l}3.61 \\
3.03\end{array}$ & $\begin{array}{l}3.67 \\
3.09\end{array}$ & $\begin{array}{l}3.61 \\
3.09\end{array}$ & $\begin{array}{l}3.47 \\
3.15\end{array}$ \\
\hline $5-20$ & $\begin{array}{l}3.28 \\
4.15\end{array}$ & $\begin{array}{l}3.61 \\
4.08\end{array}$ & $\begin{array}{l}3.94 \\
4.01\end{array}$ & $\begin{array}{l}4.22 \\
4.08\end{array}$ & $\begin{array}{l}4 \cdot 30 \\
4.30\end{array}$ & $\begin{array}{l}4.30 \\
4.74\end{array}$ \\
\hline $5-21$ & $\begin{array}{l}5.99 \\
8.40\end{array}$ & $\begin{array}{l}7.47 \\
8.21\end{array}$ & $\begin{array}{l}8.50 \\
7.93\end{array}$ & $\begin{array}{l}8.88 \\
8.12\end{array}$ & $\begin{array}{l}8.88 \\
8.88\end{array}$ & $\begin{array}{c}8.69 \\
10.4\end{array}$ \\
\hline $5-22$ & $\begin{array}{l}12.2 \\
16.4\end{array}$ & $\begin{array}{l}13.9 \\
15.7\end{array}$ & $\begin{array}{l}15.2 \\
15.2\end{array}$ & $\begin{array}{l}16.2 \\
15.2\end{array}$ & $\begin{array}{l}16.7 \\
15.9\end{array}$ & $\begin{array}{l}16.7 \\
16.8\end{array}$ \\
\hline $5-23$ & $\begin{array}{l}17.9 \\
25.8\end{array}$ & $\begin{array}{l}19.2 \\
26.4\end{array}$ & $\begin{array}{l}20.7 \\
26.8\end{array}$ & $\begin{array}{l}22.3 \\
26.7\end{array}$ & $\begin{array}{l}23.8 \\
26.5\end{array}$ & $\begin{array}{l}24.8 \\
26.1\end{array}$ \\
\hline $5-24$ & $\begin{array}{l}26.1 \\
25.4\end{array}$ & $\begin{array}{l}26.1 \\
24.5\end{array}$ & $\begin{array}{l}26.1 \\
23.5\end{array}$ & $\begin{array}{l}26.2 \\
22.3\end{array}$ & $\begin{array}{l}26.2 \\
21.4\end{array}$ & $\begin{array}{l}25.9 \\
20.6\end{array}$ \\
\hline $5-25$ & $\begin{array}{l}19.8 \\
15.1\end{array}$ & $\begin{array}{l}19.2 \\
14.0\end{array}$ & $\begin{array}{l}18.4 \\
12.9\end{array}$ & $\begin{array}{l}17.5 \\
12.1\end{array}$ & $\begin{array}{l}16.6 \\
11.7\end{array}$ & $\begin{array}{l}15.8 \\
11.4\end{array}$ \\
\hline $5-26$ & 11.18 & $\begin{array}{c}10.8 \\
8.88\end{array}$ & $\begin{array}{r}10.5 \\
8.59\end{array}$ & $\begin{array}{r}10.2 \\
8.40\end{array}$ & $\begin{array}{l}9.78 \\
7.84\end{array}$ & $\begin{array}{l}9.48 \\
7.84\end{array}$ \\
\hline
\end{tabular}


TABLE 3.- BIHOURLY DISCHARGE (1), IN CUBIC METERS PER SECOND, AT SECTION 0000, EAST FORK RIVER, WYOMING, 1980--CONTINUED

\begin{tabular}{|c|c|c|c|c|c|c|}
\hline DATE & $\begin{array}{r}2 \\
14 \\
\end{array}$ & $\begin{array}{r}4 \\
16 \\
\end{array}$ & $\begin{array}{c}\text { TIME IN } \\
6 \\
18\end{array}$ & $\begin{array}{r}\text { HOURS } \\
8 \\
20\end{array}$ & $\begin{array}{r}10 \\
22 \\
\end{array}$ & $\frac{12}{2}$ \\
\hline $5-27$ & $\begin{array}{l}7.74 \\
5.91\end{array}$ & $\begin{array}{l}7.56 \\
5.59\end{array}$ & $\begin{array}{l}7.38 \\
6.24\end{array}$ & $\begin{array}{l}7.02 \\
6.24\end{array}$ & $\begin{array}{l}6.67 \\
6.08\end{array}$ & $\begin{array}{l}6.33 \\
5.99\end{array}$ \\
\hline $5-28$ & $\begin{array}{l}6.08 \\
5.04\end{array}$ & $\begin{array}{l}6.08 \\
4.74\end{array}$ & $\begin{array}{l}5.99 \\
4.66\end{array}$ & $\begin{array}{l}5.83 \\
4.51\end{array}$ & $\begin{array}{l}5.67 \\
4.51\end{array}$ & $\begin{array}{l}5.43 \\
4.51\end{array}$ \\
\hline $5-29$ & $\begin{array}{l}4.59 \\
4.30\end{array}$ & $\begin{array}{l}4.74 \\
4.15\end{array}$ & $\begin{array}{l}4.66 \\
4.08\end{array}$ & $\begin{array}{l}4.66 \\
4.08\end{array}$ & $\begin{array}{l}4.59 \\
4.01\end{array}$ & $\begin{array}{l}4.51 \\
3.88\end{array}$ \\
\hline $5-30$ & $\begin{array}{l}3.81 \\
3.28\end{array}$ & $\begin{array}{l}3.81 \\
3.28\end{array}$ & $\begin{array}{l}3.74 \\
3.15\end{array}$ & $\begin{array}{l}3.67 \\
3.28\end{array}$ & $\begin{array}{l}3.61 \\
3.54\end{array}$ & $\begin{array}{l}3.47 \\
3.41\end{array}$ \\
\hline $5-31$ & $\begin{array}{l}3.41 \\
3.47\end{array}$ & $\begin{array}{l}3.47 \\
3.28\end{array}$ & $\begin{array}{l}3.54 \\
3.28\end{array}$ & $\begin{array}{l}3.61 \\
3.41\end{array}$ & $\begin{array}{l}3.61 \\
3.61\end{array}$ & $\begin{array}{l}3.54 \\
3.54\end{array}$ \\
\hline $6-1$ & $\begin{array}{l}3.47 \\
3.22\end{array}$ & $\begin{array}{l}3.47 \\
3.15\end{array}$ & $\begin{array}{l}3.47 \\
3.15\end{array}$ & $\begin{array}{l}3.41 \\
3.22\end{array}$ & $\begin{array}{l}3.28 \\
3.28\end{array}$ & $\begin{array}{l}3.28 \\
3.28\end{array}$ \\
\hline $6-2$ & $\begin{array}{l}3.22 \\
3.34\end{array}$ & $\begin{array}{l}3.28 \\
3.34\end{array}$ & $\begin{array}{l}3.34 \\
3.28\end{array}$ & $\begin{array}{l}3.41 \\
3.34\end{array}$ & $\begin{array}{l}3.54 \\
3.41\end{array}$ & $\begin{array}{l}3.41 \\
3.47\end{array}$ \\
\hline $6-3$ & $\begin{array}{l}3.54 \\
3.34\end{array}$ & $\begin{array}{l}3.61 \\
3.34\end{array}$ & $\begin{array}{l}3.61 \\
3.28\end{array}$ & $\begin{array}{l}3.54 \\
3.22\end{array}$ & $\begin{array}{l}3.47 \\
3.09\end{array}$ & $\begin{array}{l}3.41 \\
3.03\end{array}$ \\
\hline $6-4$ & $\begin{array}{l}3.03 \\
3.15\end{array}$ & $\begin{array}{l}3.03 \\
3.09\end{array}$ & $\begin{array}{l}3.09 \\
3.03\end{array}$ & $\begin{array}{l}3.15 \\
2.97\end{array}$ & $\begin{array}{l}3.15 \\
2.85\end{array}$ & $\begin{array}{l}3.15 \\
2.85\end{array}$ \\
\hline $6-5$ & $\begin{array}{l}3.03 \\
3.81\end{array}$ & $\begin{array}{l}3.22 \\
3.81\end{array}$ & $\begin{array}{l}3.41 \\
3.74\end{array}$ & $\begin{array}{l}3.61 \\
3.81\end{array}$ & $\begin{array}{l}3.74 \\
3.81\end{array}$ & $\begin{array}{l}3.81 \\
3.94\end{array}$ \\
\hline $6-6$ & $\begin{array}{l}4.30 \\
4.89\end{array}$ & $\begin{array}{l}4.51 \\
4.81\end{array}$ & $\begin{array}{l}4.74 \\
4.89\end{array}$ & $\begin{array}{l}4.81 \\
4.74\end{array}$ & $\begin{array}{l}4.96 \\
4.74\end{array}$ & $\begin{array}{l}4.96 \\
4.81\end{array}$ \\
\hline $6-7$ & $\begin{array}{l}4.96 \\
4.66\end{array}$ & $\begin{array}{l}5.19 \\
4.51\end{array}$ & $\begin{array}{l}5.27 \\
4.59\end{array}$ & $\begin{array}{l}5.35 \\
5.12\end{array}$ & $\begin{array}{l}5.19 \\
5.04\end{array}$ & $\begin{array}{l}4.96 \\
5.04\end{array}$ \\
\hline $6-8$ & $\begin{array}{l}5.59 \\
7.11\end{array}$ & $\begin{array}{l}6.33 \\
6.93\end{array}$ & $\begin{array}{l}6.76 \\
6.85\end{array}$ & $\begin{array}{l}7.02 \\
7.02\end{array}$ & $\begin{array}{l}7.02 \\
7.11\end{array}$ & $\begin{array}{l}7.11 \\
7.74\end{array}$ \\
\hline $6-9$ & $\begin{array}{l}9.08 \\
13.7\end{array}$ & $\begin{array}{l}10.8 \\
13.7\end{array}$ & $\begin{array}{l}11.8 \\
13.5\end{array}$ & $\begin{array}{l}12.6 \\
13.2\end{array}$ & $\begin{array}{l}13.2 \\
12.9\end{array}$ & $\begin{array}{l}13.5 \\
13.2\end{array}$ \\
\hline $6-10$ & $\begin{array}{l}13.7 \\
19.8\end{array}$ & $\begin{array}{l}14.8 \\
20.0\end{array}$ & $\begin{array}{l}15.9 \\
19.8\end{array}$ & $\begin{array}{l}17.0 \\
19.3\end{array}$ & $\begin{array}{l}18.1 \\
18.8\end{array}$ & $\begin{array}{l}19.2 \\
18.5\end{array}$ \\
\hline $6-11$ & $\begin{array}{l}18.7 \\
26.1\end{array}$ & $\begin{array}{l}19.3 \\
26.7\end{array}$ & $\begin{array}{l}20.7 \\
26.2\end{array}$ & $\begin{array}{l}22.3 \\
25.0\end{array}$ & $\begin{array}{l}23.8 \\
24.1\end{array}$ & $\begin{array}{l}25.3 \\
23.2\end{array}$ \\
\hline $6-12$ & $\begin{array}{l}22.5 \\
30.7\end{array}$ & $\begin{array}{l}22.7 \\
30.6\end{array}$ & $\begin{array}{l}24.7 \\
30.1\end{array}$ & $\begin{array}{l}26.5 \\
29.4\end{array}$ & $\begin{array}{l}29.1 \\
28.4\end{array}$ & $\begin{array}{l}30.4 \\
27.3\end{array}$ \\
\hline
\end{tabular}


TABLE 3.- BIHOURLY DISCHARGE (1), IN CUBIC METERS PER SECOND AT SECTION O000, EAST FORK RIVER, WYOMING, 1980--CONTINUED

\begin{tabular}{|c|c|c|c|c|c|c|}
\hline DATE & 12 & 16 & $\begin{array}{c}\text { TIME IN } \\
6 \\
18\end{array}$ & $\begin{array}{r}\text { HOURS } \\
20 \\
\end{array}$ & $\begin{array}{r}10 \\
22 \\
\end{array}$ & $\frac{12}{24}$ \\
\hline $6-13$ & $\begin{array}{l}26.2 \\
28.4\end{array}$ & $\begin{array}{l}25.8 \\
28.1\end{array}$ & $\begin{array}{l}25.8 \\
27.8\end{array}$ & $\begin{array}{l}26.4 \\
25.8\end{array}$ & $\begin{array}{l}27.6 \\
24.5\end{array}$ & $\begin{array}{l}28.4 \\
23.2\end{array}$ \\
\hline $6-14$ & $\begin{array}{l}22.0 \\
25: 3\end{array}$ & $\begin{array}{l}21.7 \\
25.0\end{array}$ & $\begin{array}{l}22.2 \\
24.4\end{array}$ & $\begin{array}{l}23.2 \\
23.2\end{array}$ & $\begin{array}{l}24.1 \\
21.6\end{array}$ & $\begin{array}{l}25.0 \\
20.3\end{array}$ \\
\hline $6-15$ & $\begin{array}{l}19.5 \\
20.5\end{array}$ & $\begin{array}{l}19.2 \\
19.6\end{array}$ & $\begin{array}{l}19.6 \\
18.9\end{array}$ & 19.9 & $\begin{array}{l}20.5 \\
16.7\end{array}$ & $\begin{array}{l}20.7 \\
15.7\end{array}$ \\
\hline $6-16$ & $\begin{array}{l}14.8 \\
10.7\end{array}$ & $\begin{array}{r}14.1 \\
9.99\end{array}$ & 13.4 & $\begin{array}{c}12.7 \\
9.28\end{array}$ & $\begin{array}{r}12.1 \\
9.08\end{array}$ & $\begin{array}{c}11.5 \\
8.98\end{array}$ \\
\hline $6-17$ & $\begin{array}{r}9.38 \\
12.4\end{array}$ & $\begin{array}{l}10.2 \\
12.1\end{array}$ & $\begin{array}{l}10.9 \\
11.8\end{array}$ & $\begin{array}{l}11.5 \\
11.5\end{array}$ & $\begin{array}{l}12.0 \\
11.3\end{array}$ & 12.2 \\
\hline $6-18$ & $\begin{array}{l}11.7 \\
19.2\end{array}$ & $\begin{array}{l}12.7 \\
19.9\end{array}$ & 20.1 & $\begin{array}{l}15.4 \\
19.5\end{array}$ & $\begin{array}{l}16.8 \\
18.4\end{array}$ & $\frac{18.1}{17.0}$ \\
\hline $6-19$ & $\frac{16}{17} \cdot \frac{1}{3}$ & $\begin{array}{l}15.7 \\
16.8\end{array}$ & $\begin{array}{l}16.2 \\
15.9\end{array}$ & $\begin{array}{l}16.7 \\
15.1\end{array}$ & $\begin{array}{l}17: \frac{1}{14: 6}\end{array}$ & $\begin{array}{l}17.3 \\
14.3\end{array}$ \\
\hline $6-20$ & $\begin{array}{l}14.7 \\
24.5\end{array}$ & $\begin{array}{l}15.7 \\
25.4\end{array}$ & $\begin{array}{l}17.1 \\
25.4\end{array}$ & $\begin{array}{l}18.7 \\
24.4\end{array}$ & $\begin{array}{l}20.7 \\
22.6\end{array}$ & $\begin{array}{l}22.9 \\
20.5\end{array}$ \\
\hline $6-21$ & $\begin{array}{l}18.8 \\
21.7\end{array}$ & $\frac{18.1}{21.6}$ & $\begin{array}{l}18.5 \\
20.7\end{array}$ & $\begin{array}{l}19.2 \\
19.2\end{array}$ & $\begin{array}{l}20.2 \\
17.6\end{array}$ & $\begin{array}{l}21.2 \\
16.1\end{array}$ \\
\hline $6-22$ & $\begin{array}{l}15.3 \\
18.3\end{array}$ & $\begin{array}{l}15.3 \\
17.7\end{array}$ & $\begin{array}{l}15.9 \\
16.6\end{array}$ & $\begin{array}{l}16.7 \\
15.3\end{array}$ & $\begin{array}{l}17.5 \\
14: 1\end{array}$ & $\begin{array}{l}18.1 \\
13.0\end{array}$ \\
\hline $6-23$ & $\begin{array}{l}12.9 \\
18.7\end{array}$ & $\begin{array}{l}13.4 \\
18.9\end{array}$ & $\begin{array}{l}14.5 \\
18.3\end{array}$ & $\begin{array}{l}15.8 \\
16.8\end{array}$ & $\begin{array}{l}17.0 \\
14: 8\end{array}$ & $\begin{array}{l}17.9 \\
13.3\end{array}$ \\
\hline $6-24$ & $\begin{array}{l}12.7 \\
16.4\end{array}$ & $\begin{array}{l}12.9 \\
15.9\end{array}$ & $\begin{array}{l}13.6 \\
14.7\end{array}$ & $\begin{array}{l}14.6 \\
13.4\end{array}$ & $\begin{array}{l}15.4 \\
12.0\end{array}$ & $\begin{array}{l}16.2 \\
11.3\end{array}$ \\
\hline $6-25$ & $\begin{array}{l}10.8 \\
15.2\end{array}$ & $\frac{11.3}{14: 7}$ & $\begin{array}{l}12.2 \\
13.7\end{array}$ & $\begin{array}{l}13.3 \\
12.6\end{array}$ & $\begin{array}{l}14.2 \\
11.5\end{array}$ & $\begin{array}{l}14.9 \\
10.4\end{array}$ \\
\hline $6-26$ & $\begin{array}{r}9.99 \\
14.6\end{array}$ & $\begin{array}{l}10.3 \\
14.2\end{array}$ & $\frac{11}{13}: 5$ & $\begin{array}{l}12.6 \\
11: 8\end{array}$ & $\begin{array}{l}13.6 \\
10: 7\end{array}$ & 14.3 \\
\hline $6-27$ & 19.48 & $\begin{array}{r}9.68 \\
13.3\end{array}$ & $\begin{array}{l}10.7 \\
12.2\end{array}$ & $\begin{array}{l}12.0 \\
11.0\end{array}$ & $\begin{array}{l}13.0 \\
10.1\end{array}$ & $\begin{array}{c}13.6 \\
9.28\end{array}$ \\
\hline $6-28$ & $\begin{array}{l}8.59 \\
8.31\end{array}$ & $\begin{array}{l}8.12 \\
7.38\end{array}$ & $\begin{array}{l}8.02 \\
6.76\end{array}$ & $\begin{array}{l}8.12 \\
6.33\end{array}$ & $\begin{array}{l}8.12 \\
6.08\end{array}$ & $\begin{array}{l}7.93 \\
5.75\end{array}$ \\
\hline $6-29$ & $\begin{array}{l}5.43 \\
5.99\end{array}$ & $\begin{array}{l}5.35 \\
5.75\end{array}$ & $\begin{array}{l}5.59 \\
5.51\end{array}$ & $\begin{array}{l}5.91 \\
5.27\end{array}$ & $\begin{array}{l}6.16 \\
5.04\end{array}$ & $\begin{array}{l}6.24 \\
4.89\end{array}$ \\
\hline
\end{tabular}




\begin{tabular}{|c|c|c|c|c|c|c|}
\hline DATE & $\begin{array}{r}2 \\
14\end{array}$ & $\begin{array}{r}4 \\
16\end{array}$ & $\begin{array}{c}\text { TIME IN } \\
6 \\
18\end{array}$ & $\begin{array}{r}\text { HOURS } \\
8 \\
20\end{array}$ & $\begin{array}{l}10 \\
22\end{array}$ & $\begin{array}{l}12 \\
24\end{array}$ \\
\hline $6-30$ & $\begin{array}{l}4.74 \\
7.29\end{array}$ & $\begin{array}{l}4.81 \\
7.29\end{array}$ & $\begin{array}{l}5.35 \\
7.02\end{array}$ & $\begin{array}{l}6.08 \\
6.85\end{array}$ & $\begin{array}{l}6.76 \\
6.76\end{array}$ & $\begin{array}{l}7.11 \\
6.76\end{array}$ \\
\hline $7-1$ & $\begin{array}{c}7.11 \\
13.0\end{array}$ & 13.02 & $\begin{array}{r}9.38 \\
13.0\end{array}$ & $\begin{array}{l}10.7 \\
12.4\end{array}$ & $\begin{array}{l}11.7 \\
11.6\end{array}$ & $\begin{array}{l}12.6 \\
11.0\end{array}$ \\
\hline $7-2$ & $\begin{array}{l}10.7 \\
13.0\end{array}$ & $\begin{array}{l}10.4 \\
12.9\end{array}$ & $\begin{array}{l}10.6 \\
12.1\end{array}$ & $\begin{array}{l}11.4 \\
11.4\end{array}$ & $\begin{array}{l}12.1 \\
10.5\end{array}$ & $\begin{array}{r}12.9 \\
9.89\end{array}$ \\
\hline $7-3$ & $\begin{array}{c}9.38 \\
10.9\end{array}$ & 10.28 & $\begin{array}{r}9.58 \\
10.8\end{array}$ & $\begin{array}{c}9.99 \\
11.0\end{array}$ & $\begin{array}{l}10.4 \\
11.4\end{array}$ & $\begin{array}{l}10.8 \\
11.5\end{array}$ \\
\hline $7-4$ & $\begin{array}{l}11 \\
12.1\end{array}$ & $\begin{array}{l}11.8 \\
11.4\end{array}$ & $\begin{array}{l}12.2 \\
10.6\end{array}$ & $\begin{array}{c}12.6 \\
9.89\end{array}$ & $\begin{array}{r}12.8 \\
9.18\end{array}$ & $\begin{array}{r}12.7 \\
8.69\end{array}$ \\
\hline $7-5$ & $\begin{array}{l}8.21 \\
7.38\end{array}$ & $\begin{array}{l}7.84 \\
7.11\end{array}$ & $\begin{array}{l}7.56 \\
6.76\end{array}$ & $\begin{array}{l}7.56 \\
6.41\end{array}$ & $\begin{array}{l}7.56 \\
5.99\end{array}$ & $\begin{array}{l}7.47 \\
5.75\end{array}$ \\
\hline $7-6$ & $\begin{array}{l}5.51 \\
5.91\end{array}$ & $\begin{array}{l}5.35 \\
5.75\end{array}$ & $\begin{array}{l}5.51 \\
5.51\end{array}$ & $\begin{array}{l}5.75 \\
5.19\end{array}$ & $\begin{array}{l}5.91 \\
4.96\end{array}$ & $\begin{array}{l}6.08 \\
4.66\end{array}$ \\
\hline $7-7$ & $\begin{array}{l}4.44 \\
4.51\end{array}$ & $\begin{array}{l}4.22 \\
4.37\end{array}$ & $\begin{array}{l}4.08 \\
4.08\end{array}$ & $\begin{array}{l}4.22 \\
3.81\end{array}$ & $\begin{array}{l}4.44 \\
3.74\end{array}$ & $\begin{array}{l}4.59 \\
3.61\end{array}$ \\
\hline $7-8$ & $\begin{array}{l}3.54 \\
3.22\end{array}$ & $\begin{array}{l}3.41 \\
.3 .09\end{array}$ & $\begin{array}{l}3.41 \\
3.15\end{array}$ & $\begin{array}{l}3.34 \\
3.22\end{array}$ & $\begin{array}{l}3.28 \\
3.15\end{array}$ & $\begin{array}{l}3.28 \\
3.15\end{array}$ \\
\hline $7-9$ & $\begin{array}{l}3.15 \\
3.34\end{array}$ & $\begin{array}{l}3.09 \\
3.28\end{array}$ & $\begin{array}{l}3.09 \\
3.15\end{array}$ & $\begin{array}{l}3.15 \\
3.03\end{array}$ & $\begin{array}{l}3.34 \\
2.79\end{array}$ & $\begin{array}{l}3.41 \\
2.73\end{array}$ \\
\hline $7-10$ & $\begin{array}{l}2.61 \\
2.73\end{array}$ & $\begin{array}{l}2.55 \\
2.61\end{array}$ & $\begin{array}{l}2.55 \\
2.50\end{array}$ & $\begin{array}{l}2.55 \\
2.38\end{array}$ & $\begin{array}{l}2.67 \\
2.27\end{array}$ & $\begin{array}{l}2.79 \\
2.22\end{array}$ \\
\hline $7-11$ & $\begin{array}{l}2.22 \\
2.44\end{array}$ & $\begin{array}{l}2.11 \\
2.44\end{array}$ & $\begin{array}{l}2.06 \\
2.33\end{array}$ & $\frac{2.11}{2.27}$ & $\begin{array}{l}2.22 \\
2.22\end{array}$ & $\begin{array}{l}2.38 \\
2.11\end{array}$ \\
\hline $7-12$ & $\begin{array}{l}2.06 \\
1.96\end{array}$ & $\begin{array}{l}2.01 \\
1.96\end{array}$ & $\begin{array}{l}1.96 \\
1.96\end{array}$ & $\frac{1}{1.91}$ & $\begin{array}{l}1.86 \\
1.91\end{array}$ & $\begin{array}{l}1.91 \\
1.86\end{array}$ \\
\hline $7-13$ & $\frac{1}{1.81}$ & $\begin{array}{l}1.81 \\
1.66\end{array}$ & $\begin{array}{l}1.76 \\
1.66\end{array}$ & $\begin{array}{l}1.76 \\
1.66\end{array}$ & $\begin{array}{l}1.76 \\
1.61\end{array}$ & $\begin{array}{l}1.71 \\
1.61\end{array}$ \\
\hline $7-14$ & $\begin{array}{l}1.61 \\
1.52\end{array}$ & $\frac{1}{1.61}$ & $\frac{1}{1.61}$ & $\begin{array}{l}1.57 \\
1.52\end{array}$ & $\begin{array}{l}1.57 \\
1.52\end{array}$ & $\begin{array}{l}1.57 \\
1.57\end{array}$ \\
\hline $7-15$ & $\frac{1}{1.81}$ & $\frac{1}{1.71}$ & $\frac{1}{1.81}$ & $\frac{1}{1.81}$ & $\begin{array}{l}1.76 \\
1.61\end{array}$ & $\begin{array}{l}1.76 \\
1.61\end{array}$ \\
\hline $7-16$ & $\begin{array}{l}1.61 \\
1.38\end{array}$ & $\begin{array}{l}1.57 \\
1.43\end{array}$ & $\begin{array}{l}1.52 \\
1.43\end{array}$ & $\begin{array}{l}1.43 \\
1.43\end{array}$ & $\begin{array}{l}1.38 \\
1.43\end{array}$ & $\begin{array}{l}1.38 \\
1.38\end{array}$ \\
\hline
\end{tabular}

(1) DATA FROM EMMETT, MYRICK, AND MEADE, 1982, TABLE 5. 
TABLE 4.- BIHOURLY DISCHARGE ( 1 ), IN CUBIC METERS PER SECOND, AT SECTION 2505, EAST FORK RIVER, WYOMING, 1980

\begin{tabular}{|c|c|c|c|c|c|c|}
\hline DATE & $\begin{array}{r}2 \\
14\end{array}$ & $\begin{array}{r}4 \\
16\end{array}$ & $\begin{array}{c}\text { TIME IN } \\
6 \\
18\end{array}$ & $\begin{array}{r}\text { HOURS } \\
8 \\
20\end{array}$ & $\begin{array}{l}10 \\
22\end{array}$ & $\begin{array}{l}12 \\
24\end{array}$ \\
\hline $5-10$ & $=-$ & -- & --- & --- & $=-$ & $=$ \\
\hline $5-11$ & $=-$ & $=-$ & $\overline{---}$ & -- & $\overline{--}$ & $=-$ \\
\hline $5-12$ & $\overline{--}$ & $=-$ & $=-$ & $\overline{--}$ & $\overline{---}$ & $=-$ \\
\hline $5-13$ & $=-$ & $=-$ & $=-$ & $=$ & $=-$ & $=-$ \\
\hline $5-14$ & $=-$ & $=-$ & --- & $=-$ & -- & $=-$ \\
\hline $5-15$ & -- & -- & --- & $=-$ & -- & $=-$ \\
\hline $5-16$ & $\begin{array}{l}2.60 \\
2.50\end{array}$ & $\begin{array}{l}2.60 \\
2.45\end{array}$ & $\begin{array}{l}2.60 \\
2.60\end{array}$ & $\begin{array}{l}2.60 \\
2.70\end{array}$ & $\begin{array}{l}2.60 \\
2.81\end{array}$ & $\begin{array}{l}2.55 \\
2.91\end{array}$ \\
\hline $5-17$ & $\begin{array}{l}3.19 \\
3.25\end{array}$ & $\begin{array}{l}3.36 \\
3.25\end{array}$ & $\begin{array}{l}3.54 \\
3.25\end{array}$ & $\begin{array}{l}3.54 \\
3.36\end{array}$ & $\begin{array}{l}3.48 \\
3.54\end{array}$ & $\begin{array}{l}3.42 \\
3.78\end{array}$ \\
\hline $5-18$ & $\begin{array}{l}3.91 \\
3.60\end{array}$ & $\begin{array}{l}3.97 \\
3.25\end{array}$ & $\begin{array}{l}4.03 \\
3.08\end{array}$ & $\begin{array}{l}4.03 \\
3.08\end{array}$ & $\begin{array}{l}3.84 \\
3.25\end{array}$ & $\begin{array}{l}3.72 \\
3.25\end{array}$ \\
\hline $5-19$ & $\begin{array}{l}3.31 \\
3.42\end{array}$ & $\begin{array}{l}3.42 \\
3.19\end{array}$ & $\begin{array}{l}3.60 \\
3.08\end{array}$ & $\begin{array}{l}3.66 \\
3.08\end{array}$ & $\begin{array}{l}3.66 \\
3.14\end{array}$ & $\begin{array}{l}3.54 \\
3.19\end{array}$ \\
\hline $5-20$ & $\begin{array}{l}3.31 \\
4.16\end{array}$ & $\begin{array}{l}3.54 \\
4.16\end{array}$ & $\begin{array}{l}3.84 \\
4.10\end{array}$ & $\begin{array}{l}4.16 \\
4.10\end{array}$ & $\begin{array}{l}4.22 \\
4.16\end{array}$ & $\begin{array}{l}4.22 \\
4.42\end{array}$ \\
\hline $5-21$ & $\begin{array}{l}6.61 \\
8.31\end{array}$ & $\begin{array}{l}7.87 \\
8.04\end{array}$ & $\begin{array}{l}8.58 \\
7.95\end{array}$ & $\begin{array}{l}9.03 \\
8.22\end{array}$ & $\begin{array}{c}8.94 \\
10.3\end{array}$ & $\begin{array}{r}8.67 \\
12.6\end{array}$ \\
\hline $5-22$ & $\begin{array}{l}14.6 \\
16.0\end{array}$ & $\begin{array}{l}15.6 \\
15.5\end{array}$ & $\begin{array}{l}16.5 \\
15.5\end{array}$ & $\begin{array}{l}16.9 \\
16.0\end{array}$ & $\begin{array}{l}17.0 \\
17.1\end{array}$ & $\begin{array}{l}16.7 \\
18.3\end{array}$ \\
\hline $5-23$ & $\begin{array}{l}19.6 \\
23.4\end{array}$ & $\begin{array}{l}20.5 \\
23.5\end{array}$ & $\begin{array}{l}21.4 \\
23.6\end{array}$ & $\begin{array}{l}22.2 \\
23.8\end{array}$ & $\begin{array}{l}22.6 \\
23.6\end{array}$ & $\begin{array}{l}23.1 \\
23.6\end{array}$ \\
\hline $5-24$ & $\begin{array}{l}23.5 \\
22.9\end{array}$ & $\begin{array}{l}23.5 \\
22.3\end{array}$ & $\begin{array}{l}23.5 \\
21.6\end{array}$ & $\begin{array}{l}23.4 \\
21.1\end{array}$ & $\begin{array}{l}23.4 \\
20.4\end{array}$ & $\begin{array}{l}23.2 \\
19.7\end{array}$ \\
\hline $5-25$ & $\begin{array}{l}19.2 \\
14.5\end{array}$ & $\begin{array}{l}18.5 \\
13.3\end{array}$ & $\begin{array}{l}17.8 \\
12.4\end{array}$ & $\begin{array}{l}17.0 \\
12.0\end{array}$ & $\begin{array}{l}16.3 \\
11.7\end{array}$ & $\begin{array}{l}15.4 \\
11.5\end{array}$ \\
\hline $5-26$ & 11.3 & $\begin{aligned} 11.0 \\
8.85\end{aligned}$ & $\begin{array}{c}10.6 \\
8.58\end{array}$ & $\begin{array}{c}10.2 \\
7.87\end{array}$ & $\begin{array}{l}9.79 \\
7.87\end{array}$ & $\begin{array}{l}9.41 \\
7.87\end{array}$ \\
\hline
\end{tabular}


TABLE 4.- BIHOURLY DISCHARGE(1), IN CUBIC METERS PER SECOND, AT SECTION 2505, EAST FORK RIVER, WYOMING, 1980--CONTINUED

\begin{tabular}{|c|c|c|c|c|c|c|}
\hline DATE & $\begin{array}{r}2 \\
14\end{array}$ & $\begin{array}{r}4 \\
16\end{array}$ & $\begin{array}{c}\text { TIME IN } \\
6 \\
18\end{array}$ & $\begin{array}{r}\text { HOURS } \\
8 \\
20\end{array}$ & $\begin{array}{l}10 \\
22\end{array}$ & $\begin{array}{l}12 \\
24\end{array}$ \\
\hline $5-27$ & $\begin{array}{l}7.78 \\
5.54\end{array}$ & $\begin{array}{l}7.61 \\
5.76\end{array}$ & $\begin{array}{l}7.44 \\
6.38\end{array}$ & $\begin{array}{l}7.10 \\
6.22\end{array}$ & $\begin{array}{l}6.53 \\
6.06\end{array}$ & $\begin{array}{l}6.14 \\
6.06\end{array}$ \\
\hline $5-28$ & $\begin{array}{l}6.14 \\
4.96\end{array}$ & $\begin{array}{l}6.14 \\
4.83\end{array}$ & $\begin{array}{l}5.99 \\
4.76\end{array}$ & $\begin{array}{l}5.84 \\
4.76\end{array}$ & $\begin{array}{l}5.61 \\
4.69\end{array}$ & $\begin{array}{l}5.39 \\
4.62\end{array}$ \\
\hline $5-29$ & $\begin{array}{l}4.69 \\
4.29\end{array}$ & $\begin{array}{l}4.76 \\
4.22\end{array}$ & $\begin{array}{l}4.76 \\
4.22\end{array}$ & $\begin{array}{l}4.69 \\
4.22\end{array}$ & $\begin{array}{l}4.55 \\
4.03\end{array}$ & $\begin{array}{l}4.35 \\
3.97\end{array}$ \\
\hline $5-30$ & $\begin{array}{l}3.91 \\
3.31\end{array}$ & $\begin{array}{l}3.91 \\
3.31\end{array}$ & $\begin{array}{l}3.91 \\
3.31\end{array}$ & $\begin{array}{l}3.84 \\
3.36\end{array}$ & $\begin{array}{l}3.72 \\
3.54\end{array}$ & $\begin{array}{l}3.54 \\
3.54\end{array}$ \\
\hline $5-31$ & $\begin{array}{l}3.54 \\
3.60\end{array}$ & $\begin{array}{l}3.60 \\
3.48\end{array}$ & $\begin{array}{l}3.66 \\
3.42\end{array}$ & $\begin{array}{l}3.72 \\
3.54\end{array}$ & $\begin{array}{l}3.72 \\
3.66\end{array}$ & $\begin{array}{l}3.66 \\
3.66\end{array}$ \\
\hline $6-1$ & $\begin{array}{l}3.66 \\
3.36\end{array}$ & $\begin{array}{l}3.66 \\
3.36\end{array}$ & $\begin{array}{l}3.66 \\
3.36\end{array}$ & $\begin{array}{l}3.66 \\
3.36\end{array}$ & $\begin{array}{l}3.54 \\
3.36\end{array}$ & $\begin{array}{l}3.42 \\
3.42\end{array}$ \\
\hline $6-2$ & $\begin{array}{l}3.42 \\
3.54\end{array}$ & $\begin{array}{l}3.42 \\
3.54\end{array}$ & $\begin{array}{l}3.48 \\
3.48\end{array}$ & $\begin{array}{l}3.54 \\
3.42\end{array}$ & $\begin{array}{l}3.54 \\
3.48\end{array}$ & $\begin{array}{l}3.54 \\
3.54\end{array}$ \\
\hline $6-3$ & $\begin{array}{l}3.60 \\
3.42\end{array}$ & $\begin{array}{l}3.60 \\
3.36\end{array}$ & $\begin{array}{l}3.66 \\
3.31\end{array}$ & $\begin{array}{l}3.66 \\
3.25\end{array}$ & $\begin{array}{l}3.60 \\
3.19\end{array}$ & $\begin{array}{l}3.54 \\
3.14\end{array}$ \\
\hline $6-4$ & $\begin{array}{l}3.08 \\
3.19\end{array}$ & $\begin{array}{l}3.08 \\
3.14\end{array}$ & $\begin{array}{l}3.14 \\
3.08\end{array}$ & $\begin{array}{l}3.19 \\
3.02\end{array}$ & $\begin{array}{l}3.19 \\
2.97\end{array}$ & $\begin{array}{l}3.19 \\
2.91\end{array}$ \\
\hline $6-5$ & $\begin{array}{l}2.97 \\
3.72\end{array}$ & $\begin{array}{l}3.08 \\
3.78\end{array}$ & $\begin{array}{l}3.25 \\
3.78\end{array}$ & $\begin{array}{l}3.42 \\
3.78\end{array}$ & $\begin{array}{l}3.60 \\
3.84\end{array}$ & $\begin{array}{l}3.66 \\
3.91\end{array}$ \\
\hline $6-6$ & $\begin{array}{l}4.10 \\
4.89\end{array}$ & $\begin{array}{l}4.29 \\
4.89\end{array}$ & $\begin{array}{l}4.55 \\
4.89\end{array}$ & $\begin{array}{l}4.76 \\
4.83\end{array}$ & $\begin{array}{l}4.83 \\
4.83\end{array}$ & $\begin{array}{l}4.89 \\
4.83\end{array}$ \\
\hline $6-7$ & $\begin{array}{l}4.96 \\
4.83\end{array}$ & $\begin{array}{l}5.11 \\
4.69\end{array}$ & $\begin{array}{l}5.32 \\
4.62\end{array}$ & $\begin{array}{l}5.32 \\
4.96\end{array}$ & $\begin{array}{l}5.25 \\
5.03\end{array}$ & $\begin{array}{l}5.03 \\
5.11\end{array}$ \\
\hline $6-8$ & $\begin{array}{l}5.69 \\
7.27\end{array}$ & $\begin{array}{l}6.45 \\
6.94\end{array}$ & $\begin{array}{l}6.86 \\
7.02\end{array}$ & $\begin{array}{l}7.19 \\
7.10\end{array}$ & $\begin{array}{l}7.35 \\
7.52\end{array}$ & $\begin{array}{l}7.27 \\
8.49\end{array}$ \\
\hline $6-9$ & $\begin{array}{l}10.4 \\
14.8\end{array}$ & $\begin{array}{l}11.9 \\
14.6\end{array}$ & $\frac{13}{14.1}$ & $\begin{array}{l}14.0 \\
14.0\end{array}$ & $\begin{array}{l}14.6 \\
14.0\end{array}$ & $\begin{array}{l}14.8 \\
14.6\end{array}$ \\
\hline $6-10$ & $\begin{array}{l}15.5 \\
20.3\end{array}$ & $\begin{array}{l}16.5 \\
20.3\end{array}$ & $\begin{array}{l}17.5 \\
20.1\end{array}$ & $\begin{array}{l}18.5 \\
19.7\end{array}$ & $\begin{array}{l}19.3 \\
19.5\end{array}$ & $\begin{array}{l}19.9 \\
19.2\end{array}$ \\
\hline $6-11$ & $\begin{array}{l}19.7 \\
23.8\end{array}$ & $\begin{array}{l}20.4 \\
23.6\end{array}$ & $\begin{array}{l}21.2 \\
23.5\end{array}$ & $\begin{array}{l}22.2 \\
22.9\end{array}$ & $\begin{array}{l}22.9 \\
22.3\end{array}$ & $\begin{array}{l}23.4 \\
21.9\end{array}$ \\
\hline $6-12$ & $\begin{array}{l}21.8 \\
25.7\end{array}$ & $\begin{array}{l}22.2 \\
25.7\end{array}$ & $\begin{array}{l}23.2 \\
25.3\end{array}$ & $\begin{array}{l}24.2 \\
24.5\end{array}$ & $\begin{array}{l}25.1 \\
23.9\end{array}$ & $\begin{array}{l}25.7 \\
23.5\end{array}$ \\
\hline
\end{tabular}


TABLE 4.- BIHOURLY DISCHARGE(1), IN CUBIC METERS PER SECOND, AT SECTION 2505, EAST FORK RIVER, WYOMING, 1980--CONTINUED

\begin{tabular}{|c|c|c|c|c|c|c|}
\hline DATE & $\begin{array}{r}2 \\
14\end{array}$ & $\begin{array}{r}4 \\
16\end{array}$ & $\begin{array}{c}\text { TIME IN } \\
6 \\
18\end{array}$ & $\begin{array}{r}\text { HOURS } \\
8 \\
20\end{array}$ & $\begin{array}{l}10 \\
22\end{array}$ & $\begin{array}{l}12 \\
24\end{array}$ \\
\hline $6-13$ & $\begin{array}{l}23.1 \\
23.9\end{array}$ & $\begin{array}{l}23.1 \\
23.6\end{array}$ & $\begin{array}{l}23.2 \\
23.2\end{array}$ & $\begin{array}{l}23.5 \\
22.5\end{array}$ & $\begin{array}{l}23.8 \\
21.8\end{array}$ & $\begin{array}{l}24.1 \\
21.1\end{array}$ \\
\hline $6-14$ & $\begin{array}{l}20.7 \\
22.8\end{array}$ & $\begin{array}{l}21.1 \\
22\end{array}$ & $\begin{array}{l}21.8 \\
21.9\end{array}$ & $\begin{array}{l}22.1 \\
20.9\end{array}$ & $\begin{array}{l}22.6 \\
20.1\end{array}$ & $\begin{array}{l}22.8 \\
19.5\end{array}$ \\
\hline $6-15$ & $\begin{array}{l}19.2 \\
19.7\end{array}$ & $\begin{array}{l}19.5 \\
19.1\end{array}$ & $\begin{array}{l}19.9 \\
18.3\end{array}$ & $\begin{array}{l}20.1 \\
17.4\end{array}$ & $\begin{array}{l}20.3 \\
16.5\end{array}$ & $\begin{array}{l}20.3 \\
15.8\end{array}$ \\
\hline $6-16$ & $\begin{array}{l}14.9 \\
10.8\end{array}$ & $\begin{array}{l}14.2 \\
10.2\end{array}$ & $\begin{array}{r}13.5 \\
9.79\end{array}$ & $\begin{array}{r}13.0 \\
9.41\end{array}$ & 12.3 & $\begin{array}{r}11.6 \\
9.50\end{array}$ \\
\hline $6-17$ & $\begin{array}{l}10.4 \\
13.2\end{array}$ & $\begin{array}{l}11.4 \\
12.9\end{array}$ & $\begin{array}{l}12.2 \\
12.4\end{array}$ & $\begin{array}{l}12.9 \\
12.1\end{array}$ & $\begin{array}{l}13.3 \\
12.0\end{array}$ & $\begin{array}{l}13.4 \\
12.3\end{array}$ \\
\hline $6-18$ & $\begin{array}{l}13.3 \\
19.7\end{array}$ & $\begin{array}{l}14.7 \\
19.7\end{array}$ & $\begin{array}{l}16.0 \\
19.5\end{array}$ & $\begin{array}{l}17.4 \\
18.7\end{array}$ & $\begin{array}{l}18.5 \\
17.6\end{array}$ & $\begin{array}{l}19.2 \\
16.7\end{array}$ \\
\hline $6-19$ & $\begin{array}{l}16.3 \\
17.8\end{array}$ & $\begin{array}{l}16.5 \\
17.0\end{array}$ & $\begin{array}{l}17.0 \\
16.3\end{array}$ & $\begin{array}{l}17.4 \\
15.6\end{array}$ & $\begin{array}{l}17.8 \\
15.0\end{array}$ & $\begin{array}{l}17.9 \\
14.9\end{array}$ \\
\hline $6-20$ & $\begin{array}{l}15.6 \\
22.9\end{array}$ & $\begin{array}{l}17.0 \\
22.9\end{array}$ & $\begin{array}{l}18.3 \\
22.6\end{array}$ & $\begin{array}{l}19.6 \\
21.8\end{array}$ & $\begin{array}{l}20.9 \\
20.5\end{array}$ & $\begin{array}{l}22.1 \\
19.2\end{array}$ \\
\hline $6-21$ & $\begin{array}{l}18.4 \\
21.1\end{array}$ & $\begin{array}{l}18.4 \\
20.8\end{array}$ & $\begin{array}{l}19.3 \\
20.1\end{array}$ & $\begin{array}{l}19.9 \\
18.8\end{array}$ & $\begin{array}{l}20.4 \\
17.4\end{array}$ & $\begin{array}{l}20.9 \\
16.1\end{array}$ \\
\hline $6-22$ & $\begin{array}{l}15.6 \\
18.4\end{array}$ & $\begin{array}{l}16.0 \\
17.8\end{array}$ & $\begin{array}{l}16: 9 \\
16.7\end{array}$ & $\begin{array}{l}17.6 \\
15.3\end{array}$ & $\begin{array}{l}18.3 \\
14.1\end{array}$ & $\begin{array}{l}18.5 \\
13.3\end{array}$ \\
\hline $6-23$ & $\begin{array}{l}13.5 \\
19.7\end{array}$ & $\begin{array}{l}14.6 \\
19.5\end{array}$ & $\begin{array}{l}15.9 \\
18.4\end{array}$ & $\begin{array}{l}17.2 \\
16.7\end{array}$ & $\begin{array}{l}18.3 \\
14.8\end{array}$ & $\begin{array}{l}19.2 \\
13.5\end{array}$ \\
\hline $6-24$ & $\begin{array}{l}13.2 \\
17.0\end{array}$ & $\begin{array}{l}13.7 \\
16.3\end{array}$ & $\begin{array}{l}14.7 \\
14.9\end{array}$ & $\begin{array}{l}15.8 \\
13.5\end{array}$ & $\begin{array}{l}16.6 \\
12.3\end{array}$ & 17.1 \\
\hline $6-25$ & $\begin{array}{l}11.2 \\
16.0\end{array}$ & $\begin{array}{l}11.8 \\
15.3\end{array}$ & $\begin{array}{l}13.1 \\
14.0\end{array}$ & $\begin{array}{l}14.3 \\
12.7\end{array}$ & $\begin{array}{l}15.4 \\
11.4\end{array}$ & $\begin{array}{l}16.0 \\
10.6\end{array}$ \\
\hline $6-26$ & $\begin{array}{l}10.5 \\
15.2\end{array}$ & $\begin{array}{l}11.5 \\
14.0\end{array}$ & $\begin{array}{l}12.9 \\
12.7\end{array}$ & $\begin{array}{l}14.2 \\
11.4\end{array}$ & $\begin{array}{l}15.2 \\
10.3\end{array}$ & $\begin{array}{c}15.5 \\
9.60\end{array}$ \\
\hline $6-27$ & $\begin{array}{r}9.60 \\
14.3\end{array}$ & $\begin{array}{l}10.8 \\
13.1\end{array}$ & $\begin{array}{l}12.2 \\
11.7\end{array}$ & $\begin{array}{l}13.5 \\
10.6\end{array}$ & $\begin{array}{c}14.6 \\
9.69\end{array}$ & $\begin{array}{c}14.8 \\
8.85\end{array}$ \\
\hline $6-28$ & $\begin{array}{l}8.13 \\
8.31\end{array}$ & $\begin{array}{l}8.04 \\
7.44\end{array}$ & $\begin{array}{l}8.04 \\
6.61\end{array}$ & $\begin{array}{l}8.04 \\
6.14\end{array}$ & $\begin{array}{l}8.04 \\
5.76\end{array}$ & $\begin{array}{l}7.95 \\
5.54\end{array}$ \\
\hline $6-29$ & $\begin{array}{l}5.46 \\
5.84\end{array}$ & $\begin{array}{l}5.39 \\
5.69\end{array}$ & $\begin{array}{l}5.39 \\
5.54\end{array}$ & $\begin{array}{l}5.61 \\
5.69\end{array}$ & $\begin{array}{l}6.14 \\
5.54\end{array}$ & $\begin{array}{l}6.06 \\
5.18\end{array}$ \\
\hline
\end{tabular}


TABLE 4.- BIHOURLY DISCHARGE (1), IN CUBIC METERS PER SECOND, AT SECTION 2505, EAST FORK RIVER, WYOMING, 1980--CONTINUED

\begin{tabular}{|c|c|c|c|c|c|c|}
\hline DATE & $\begin{array}{r}2 \\
14\end{array}$ & $\begin{array}{r}4 \\
16\end{array}$ & $\begin{array}{c}\text { TIME IN } \\
6 \\
18\end{array}$ & $\begin{array}{r}\text { HOURS } \\
8 \\
20\end{array}$ & $\begin{array}{l}10 \\
22\end{array}$ & $\frac{12}{24}$ \\
\hline $6-30$ & $\begin{array}{l}4.89 \\
7.35\end{array}$ & $\begin{array}{l}4.76 \\
7.27\end{array}$ & $\begin{array}{l}4.89 \\
6.94\end{array}$ & $\begin{array}{l}5.91 \\
6.69\end{array}$ & $\begin{array}{l}6.69 \\
6.69\end{array}$ & $\begin{array}{l}7.19 \\
6.61\end{array}$ \\
\hline $7-1$ & $\begin{array}{r}7.02 \\
14.0\end{array}$ & $\begin{array}{r}8.31 \\
14.0\end{array}$ & $\begin{array}{r}9.98 \\
13.7\end{array}$ & $\frac{1}{12} .5$ & $\begin{array}{l}12.8 \\
11.8\end{array}$ & $\begin{array}{l}13.7 \\
11.4\end{array}$ \\
\hline $7-2$ & $\frac{11}{13.0}$ & $\begin{array}{l}10.7 \\
13.5\end{array}$ & $\begin{array}{l}10.9 \\
12.6\end{array}$ & $\frac{11}{11.6}$ & $\begin{array}{l}12.7 \\
10.8\end{array}$ & $\begin{array}{c}13.5 \\
9.89\end{array}$ \\
\hline $7-3$ & 11.41 & 19.31 & $\begin{array}{l}9.69 \\
11.2\end{array}$ & $\begin{array}{l}10.2 \\
11.4\end{array}$ & $\begin{array}{l}10.7 \\
11.7\end{array}$ & $\begin{array}{l}11.0 \\
11.9\end{array}$ \\
\hline $7-4$ & $\begin{array}{l}12.0 \\
12.2\end{array}$ & $\begin{array}{l}12.3 \\
11.4\end{array}$ & $\begin{array}{l}12.8 \\
10.5\end{array}$ & $\begin{array}{r}13.2 \\
9.60\end{array}$ & $\begin{array}{c}13.2 \\
8.94\end{array}$ & $\begin{array}{c}13.0 \\
8.31\end{array}$ \\
\hline $7-5$ & $\begin{array}{l}7.87 \\
7.10\end{array}$ & $\begin{array}{l}7.61 \\
6.86\end{array}$ & $\begin{array}{l}7.52 \\
6.53\end{array}$ & $\begin{array}{l}7.52 \\
6.14\end{array}$ & $\begin{array}{l}7.52 \\
5.84\end{array}$ & $\begin{array}{l}7.35 \\
5.61\end{array}$ \\
\hline $7-6$ & $\begin{array}{l}5.54 \\
5.76\end{array}$ & $\begin{array}{l}5.39 \\
5.61\end{array}$ & $\begin{array}{l}5.32 \\
5.46\end{array}$ & $\begin{array}{l}5.46 \\
5.25\end{array}$ & $\begin{array}{l}5.69 \\
5.03\end{array}$ & $\begin{array}{l}5.76 \\
4.83\end{array}$ \\
\hline $7-7$ & $\begin{array}{l}4.62 \\
4.29\end{array}$ & $\begin{array}{l}4.42 \\
4.29\end{array}$ & $\begin{array}{l}4.29 \\
4.16\end{array}$ & $\begin{array}{l}4.22 \\
3.97\end{array}$ & $\begin{array}{l}4.22 \\
3.84\end{array}$ & $\begin{array}{l}4.29 \\
3.72\end{array}$ \\
\hline $7-8$ & $\begin{array}{l}3.60 \\
3.14\end{array}$ & $\begin{array}{l}3.48 \\
3.02\end{array}$ & $\begin{array}{l}3.42 \\
3.02\end{array}$ & $\begin{array}{l}3.36 \\
3.02\end{array}$ & $\begin{array}{l}3.25 \\
3.02\end{array}$ & $\begin{array}{l}3.19 \\
3.02\end{array}$ \\
\hline $7-9$ & $\begin{array}{l}2.97 \\
3.02\end{array}$ & $\begin{array}{l}2.97 \\
3.02\end{array}$ & $\begin{array}{l}2.97 \\
2.97\end{array}$ & $\begin{array}{l}2.97 \\
2.86\end{array}$ & $\begin{array}{l}2.97 \\
2.75\end{array}$ & $\begin{array}{l}3.02 \\
2.65\end{array}$ \\
\hline $7-10$ & $\begin{array}{l}2.60 \\
2.45\end{array}$ & $\begin{array}{l}2.50 \\
2.45\end{array}$ & $\begin{array}{l}2.45 \\
2.45\end{array}$ & $\begin{array}{l}2.40 \\
2.30\end{array}$ & $\begin{array}{l}2.40 \\
2.20\end{array}$ & $\begin{array}{l}2: 35 \\
2.20\end{array}$ \\
\hline
\end{tabular}

(1) DATA FROM EMMETT, MYRICK, AND MEADE, 1982, TABLE 6 . 
TABLE 5.- NUMBER OF PINK TRACER PARTICLES(1) BETWEEN 0.25 AND 8.00 MILLIMETERS, PER 100 GRAMS OF BED MATERIAL, AS A FUNCTION OF DATE AND SECTION, EAST FORK RIVER, WYOMING, 1979

\begin{tabular}{|c|c|c|c|c|c|c|c|c|c|}
\hline \multirow{2}{*}{$\begin{array}{l}\text { SECTION } \\
\text { NUMBER }\end{array}$} & \multicolumn{9}{|c|}{ DATE } \\
\hline & $5-20$ & $5-21$ & $5-23$ & $5-24$ & $5-25$ & $5-26$ & $5-27$ & $5-28$ & $5-30$ \\
\hline $\begin{array}{l}0043 \\
0075 \\
0137 \\
0220 \\
0301\end{array}$ & $\begin{array}{l}-- \\
-\overline{0} \\
0\end{array}$ & $\begin{array}{r}0 \\
-0 \\
0 \\
0\end{array}$ & $\begin{array}{l}-- \\
0 \\
0 \\
0 \\
0\end{array}$ & $\begin{array}{l}0 \\
0 \\
0 \\
0 \\
0\end{array}$ & $\begin{array}{l}0 \\
0 \\
0 \\
0 \\
0\end{array}$ & $\begin{array}{l}0 \\
0 \\
0 \\
0 \\
0\end{array}$ & $\begin{array}{l}-- \\
0 \\
0 \\
0 \\
0\end{array}$ & $\begin{array}{l}0 \\
0 \\
0 \\
0 \\
0\end{array}$ & $\begin{array}{l}0 \\
0 \\
0 \\
0 \\
0\end{array}$ \\
\hline $\begin{array}{l}0301(2) \\
0421 \\
0516 \\
0602 \\
0708\end{array}$ & $\begin{array}{l}0 \\
0 \\
-- \\
--\end{array}$ & $\begin{array}{r}0 \\
0 \\
-- \\
-- \\
--\end{array}$ & $\begin{array}{r}0 \\
0 \\
-- \\
--\end{array}$ & $\begin{array}{l}0 \\
0 \\
-- \\
--\end{array}$ & $\begin{array}{r}0 \\
0 \\
-- \\
--\end{array}$ & $\begin{array}{r}0 \\
0 \\
-- \\
--\end{array}$ & $\begin{array}{r}0 \\
0 \\
-- \\
--\end{array}$ & $\begin{array}{l}-- \\
-- \\
--\end{array}$ & $\begin{array}{l}-- \\
0 \\
0 \\
0 \\
0\end{array}$ \\
\hline $\begin{array}{l}0808 \\
0808(2) \\
0898 \\
0985 \\
1077\end{array}$ & $\begin{array}{l}-- \\
-- \\
--\end{array}$ & $\begin{array}{l}-z \\
z- \\
--\end{array}$ & $\begin{array}{l}-- \\
-- \\
--\end{array}$ & $\begin{array}{l}-- \\
=- \\
--\end{array}$ & $\begin{array}{l}-- \\
=- \\
-- \\
--\end{array}$ & $\begin{array}{l}-- \\
-\overline{0} \\
-\overline{0}\end{array}$ & $\begin{array}{l}-- \\
-\overline{0} \\
0\end{array}$ & $\begin{array}{r}-0 \\
-0 \\
0 \\
0\end{array}$ & $\begin{array}{r}0 \\
-0 \\
0 \\
0\end{array}$ \\
\hline $\begin{array}{l}1155 \\
1241 \\
1315 \\
1396 \\
1400(3)\end{array}$ & $\begin{array}{r}-- \\
-\overline{0} \\
0 \\
--\end{array}$ & $\begin{array}{l}-- \\
-\overline{0} \\
--\end{array}$ & $\begin{array}{c}-- \\
0 \\
0\end{array}$ & $\begin{array}{r}-- \\
0 \\
0 \\
0 \\
--\end{array}$ & $\begin{array}{l}0 \\
0 \\
0 \\
0 \\
0\end{array}$ & $\begin{array}{l}0 \\
0 \\
0 \\
0 \\
0\end{array}$ & $\begin{array}{l}0 \\
0 \\
0 \\
0 \\
0\end{array}$ & $\begin{array}{r}0 \\
0 \\
0 \\
0 \\
--\end{array}$ & $\begin{array}{r}3 \\
0 \\
0 \\
0 \\
--\end{array}$ \\
\hline $\begin{array}{l}1481 \\
1481(2) \\
1573 \\
1662 \\
1695\end{array}$ & $\begin{array}{r}0 \\
0 \\
0 \\
0 \\
--\end{array}$ & $\begin{array}{r}0 \\
0 \\
0 \\
0 \\
--\end{array}$ & $\begin{array}{r}0 \\
1 \\
1 \\
0 \\
--\end{array}$ & $\begin{array}{r}0 \\
0 \\
0 \\
0 \\
--\end{array}$ & $\begin{array}{r}1 \\
0 \\
0 \\
5 \\
-\end{array}$ & $\begin{array}{r}0 \\
0 \\
0 \\
0 \\
--\end{array}$ & $\begin{array}{r}0 \\
0 \\
0 \\
0 \\
--\end{array}$ & $\begin{array}{r}0 \\
-0 \\
0 \\
-\end{array}$ & $\begin{array}{l}0 \\
0 \\
0 \\
0 \\
0\end{array}$ \\
\hline $\begin{array}{l}1766 \\
1830 \\
1901 \\
1996 \\
2082\end{array}$ & $\begin{array}{l}=- \\
=- \\
=-\end{array}$ & $\begin{array}{l}-- \\
-- \\
--\end{array}$ & $\begin{array}{l}=- \\
=- \\
=-\end{array}$ & $\begin{array}{l}=- \\
=- \\
=-\end{array}$ & $\begin{array}{l}=- \\
=- \\
=-\end{array}$ & $\begin{array}{l}-- \\
-- \\
--\end{array}$ & $\begin{array}{l}-- \\
-- \\
--\end{array}$ & $\begin{array}{l}=- \\
=- \\
--\end{array}$ & $\begin{array}{l}0 \\
0 \\
0 \\
0 \\
0\end{array}$ \\
\hline $\begin{array}{l}2194 \\
2278 \\
2356 \\
2422 \\
2510\end{array}$ & $\begin{array}{l}-- \\
-- \\
--\end{array}$ & $\begin{array}{l}-- \\
-- \\
--\end{array}$ & $\begin{array}{l}-- \\
-- \\
--\end{array}$ & $\begin{array}{l}-- \\
-\overline{0} \\
-\overline{0}\end{array}$ & $\begin{array}{r}-- \\
-- \\
0 \\
1\end{array}$ & $\begin{array}{c}-- \\
-0 \\
0 \\
1\end{array}$ & $\begin{array}{c}-- \\
0 \\
1 \\
0 \\
1\end{array}$ & $\begin{array}{l}0 \\
0 \\
0 \\
0 \\
4\end{array}$ & $\begin{array}{l}1 \\
0 \\
0 \\
2 \\
6\end{array}$ \\
\hline $\begin{array}{l}2608 \\
2690 \\
2778 \\
2874 \\
2961\end{array}$ & $\begin{array}{r}-- \\
-\overline{0} \\
133\end{array}$ & $\begin{array}{r}-- \\
0 \\
0 \\
10 \\
148\end{array}$ & $\begin{array}{r}0 \\
1 \\
0 \\
131 \\
57\end{array}$ & $\begin{array}{r}0 \\
2 \\
7 \\
42 \\
46\end{array}$ & $\begin{array}{r}1 \\
0 \\
1 \\
59 \\
26\end{array}$ & $\begin{array}{r}2 \\
0 \\
7 \\
59 \\
12\end{array}$ & $\begin{array}{r}2 \\
2 \\
43 \\
22 \\
10\end{array}$ & $\begin{array}{r}0 \\
1 \\
78 \\
25 \\
10\end{array}$ & $\begin{array}{r}5 \\
0 \\
49 \\
8 \\
9\end{array}$ \\
\hline
\end{tabular}


TABLE 5.- NUMBER OF PINK TRACER PARTICLES(1) BETWEEN 0.25 AND 8.00 MILLIMETERS, PER 100 GRAMS OF BED MATERIAL, AS A FUNCTION OF DATE AND SECTION, EAST FORK RIVER, WYOMING， 1979--CONTINUED

\begin{tabular}{|c|c|c|c|c|c|c|c|c|c|}
\hline \multirow{2}{*}{$\begin{array}{r}\text { SECTION } \\
\text { NUMBER }\end{array}$} & \multicolumn{9}{|c|}{ DATE } \\
\hline & $5-31$ & $6-01$ & $6-02$ & $6-03$ & $6-04$ & $6-05$ & $6-0 \overline{6}$ & $6-07$ & $6-0$ \\
\hline $\begin{array}{l}0043 \\
0075 \\
0137 \\
0220 \\
0301\end{array}$ & $\begin{array}{l}0 \\
0 \\
0 \\
0 \\
0\end{array}$ & $\begin{array}{l}0 \\
0 \\
0 \\
0 \\
0\end{array}$ & $\begin{array}{l}0 \\
0 \\
0 \\
0 \\
0\end{array}$ & $\begin{array}{l}0 \\
0 \\
0 \\
0 \\
0\end{array}$ & $\begin{array}{l}1 \\
0 \\
1 \\
0 \\
0\end{array}$ & $\begin{array}{l}0 \\
0 \\
0 \\
0 \\
0\end{array}$ & $\begin{array}{l}0 \\
2 \\
1 \\
0 \\
0\end{array}$ & $\begin{array}{l}0 \\
2 \\
0 \\
0 \\
0\end{array}$ & $\begin{array}{l}0 \\
0 \\
0 \\
0 \\
0\end{array}$ \\
\hline $\begin{array}{l}0301(2) \\
0421 \\
0516 \\
0602 \\
0708\end{array}$ & $\begin{array}{l}-\overline{0} \\
0 \\
0 \\
0\end{array}$ & $\begin{array}{l}-\overline{0} \\
0 \\
0 \\
0\end{array}$ & $\begin{array}{c}-- \\
0 \\
0 \\
0 \\
0\end{array}$ & $\begin{array}{c}-\overline{0} \\
0 \\
0 \\
0\end{array}$ & $\begin{array}{c}-- \\
0 \\
0 \\
0 \\
0\end{array}$ & $\begin{array}{l}0 \\
0 \\
0 \\
0 \\
0\end{array}$ & $\begin{array}{l}0 \\
0 \\
0 \\
0 \\
0\end{array}$ & $\begin{array}{l}0 \\
0 \\
0 \\
0 \\
0\end{array}$ & $\begin{array}{l}-\overline{0} \\
0 \\
0 \\
0\end{array}$ \\
\hline $\begin{array}{l}0808 \\
0808(2) \\
0898 \\
0985 \\
1077\end{array}$ & $\begin{array}{r}-0 \\
-0 \\
0 \\
0\end{array}$ & $\begin{array}{r}0 \\
-0 \\
0 \\
0 \\
0\end{array}$ & $\begin{array}{r}0 \\
-0 \\
0 \\
0\end{array}$ & $\begin{array}{r}0 \\
-0 \\
0 \\
0\end{array}$ & $\begin{array}{l}0 \\
0 \\
0 \\
0 \\
0\end{array}$ & $\begin{array}{l}0 \\
0 \\
0 \\
0 \\
0\end{array}$ & $\begin{array}{l}0 \\
0 \\
0 \\
0 \\
0\end{array}$ & $\begin{array}{l}0 \\
0 \\
0 \\
0 \\
0\end{array}$ & $\begin{array}{l}0 \\
0 \\
0 \\
0 \\
0\end{array}$ \\
\hline $\begin{array}{l}1155 \\
1241 \\
1315 \\
1396 \\
1400(3)\end{array}$ & $\begin{array}{l}0 \\
0 \\
0 \\
0 \\
0\end{array}$ & $\begin{array}{l}0 \\
0 \\
0 \\
0 \\
0\end{array}$ & $\begin{array}{l}0 \\
0 \\
0 \\
0 \\
0\end{array}$ & $\begin{array}{l}0 \\
0 \\
0 \\
0 \\
0\end{array}$ & $\begin{array}{l}0 \\
0 \\
0 \\
0 \\
0\end{array}$ & $\begin{array}{l}0 \\
0 \\
0 \\
0 \\
0\end{array}$ & $\begin{array}{l}0 \\
0 \\
0 \\
0 \\
0\end{array}$ & $\begin{array}{l}0 \\
0 \\
0 \\
0 \\
0\end{array}$ & $\begin{array}{l}0 \\
0 \\
0 \\
0 \\
0\end{array}$ \\
\hline $\begin{array}{l}1481 \\
1481(2) \\
1573 \\
1662 \\
1695\end{array}$ & $\begin{array}{l}0 \\
0 \\
0 \\
0 \\
0\end{array}$ & $\begin{array}{l}0 \\
0 \\
0 \\
0 \\
0\end{array}$ & $\begin{array}{l}0 \\
0 \\
0 \\
0 \\
0\end{array}$ & $\begin{array}{l}0 \\
0 \\
0 \\
0 \\
0\end{array}$ & $\begin{array}{l}0 \\
0 \\
0 \\
0 \\
0\end{array}$ & $\begin{array}{l}0 \\
0 \\
0 \\
0 \\
0\end{array}$ & $\begin{array}{l}0 \\
0 \\
0 \\
0 \\
1\end{array}$ & $\begin{array}{l}0 \\
0 \\
0 \\
0 \\
1\end{array}$ & $\begin{array}{r}-0 \\
-0 \\
0 \\
0\end{array}$ \\
\hline $\begin{array}{l}1766 \\
1830 \\
1901 \\
1996 \\
2082\end{array}$ & $\begin{array}{l}0 \\
0 \\
0 \\
0 \\
0\end{array}$ & $\begin{array}{l}0 \\
0 \\
0 \\
0 \\
0\end{array}$ & $\begin{array}{l}3 \\
0 \\
0 \\
0 \\
0\end{array}$ & $\begin{array}{l}2 \\
2 \\
3 \\
0 \\
0\end{array}$ & $\begin{array}{l}0 \\
2 \\
0 \\
1 \\
1\end{array}$ & $\begin{array}{l}1 \\
4 \\
0 \\
0 \\
1\end{array}$ & $\begin{array}{l}0 \\
3 \\
1 \\
0 \\
3\end{array}$ & $\begin{array}{l}0 \\
2 \\
0 \\
0 \\
5\end{array}$ & $\begin{array}{l}1 \\
0 \\
0 \\
0 \\
1\end{array}$ \\
\hline $\begin{array}{l}2194 \\
2278 \\
2356 \\
2422 \\
2510\end{array}$ & $\begin{array}{r}0 \\
0 \\
2 \\
1 \\
17\end{array}$ & $\begin{array}{r}0 \\
1 \\
\frac{1}{3} \\
31\end{array}$ & $\begin{array}{r}0 \\
1 \\
2 \\
5 \\
21\end{array}$ & $\begin{array}{r}0 \\
6 \\
0 \\
1 \\
24\end{array}$ & $\begin{array}{r}0 \\
6 \\
2 \\
0 \\
28\end{array}$ & $\begin{array}{r}\frac{1}{3} \\
2 \\
0 \\
53\end{array}$ & $\begin{array}{r}2 \\
4 \\
8 \\
32 \\
109\end{array}$ & $\begin{array}{l}4 \\
13 \\
13 \\
31 \\
58\end{array}$ & $\begin{array}{r}9 \\
3 \\
15 \\
29 \\
72\end{array}$ \\
\hline $\begin{array}{l}2608 \\
2690 \\
2778 \\
2874 \\
2961\end{array}$ & $\begin{array}{r}8 \\
15 \\
55 \\
7 \\
6\end{array}$ & $\begin{array}{r}11 \\
87 \\
95 \\
12 \\
4\end{array}$ & $\begin{array}{r}22 \\
86 \\
104 \\
16 \\
3\end{array}$ & $\begin{array}{r}47 \\
75 \\
52 \\
19 \\
2\end{array}$ & $\begin{array}{r}65 \\
78 \\
30 \\
7 \\
4\end{array}$ & $\begin{array}{r}85 \\
81 \\
21 \\
18 \\
6\end{array}$ & $\begin{array}{l}50 \\
73 \\
20 \\
33 \\
65\end{array}$ & $\begin{array}{r}42 \\
43 \\
11 \\
50 \\
3\end{array}$ & $\begin{array}{r}59 \\
32 \\
12 \\
17 \\
4\end{array}$ \\
\hline
\end{tabular}


TABLE 5.- NUMBER OF PINK TRACER PARTICLES(1) BETWEEN 0.25 AND 8.00 MILIIMETERS, PER 100 GRAMS OF BED MATERIAL, AS A FUNCTION OF DATE AND SECTION, EAST FORK RIVER, WYOMING, 1979--CONTINUED

\begin{tabular}{|c|c|c|c|c|c|c|c|c|c|}
\hline \multirow{2}{*}{$\begin{array}{l}\text { SECTION } \\
\text { NUMBER }\end{array}$} & \multicolumn{9}{|c|}{ DATE } \\
\hline & $6-09$ & $6-10$ & $6-11$ & $6-12$ & $6-13$ & $6-14$ & $6-15$ & $6-16$ & $6-17$ \\
\hline $\begin{array}{l}0043 \\
0075 \\
0137 \\
0220 \\
0301\end{array}$ & $\begin{array}{l}0 \\
0 \\
0 \\
0 \\
0\end{array}$ & $\begin{array}{l}0 \\
0 \\
0 \\
0 \\
0\end{array}$ & $\begin{array}{l}0 \\
0 \\
0 \\
0 \\
0\end{array}$ & $\begin{array}{l}0 \\
0 \\
0 \\
0 \\
0\end{array}$ & $\begin{array}{l}0 \\
0 \\
0 \\
0 \\
0\end{array}$ & $\begin{array}{l}0 \\
0 \\
0 \\
0 \\
0\end{array}$ & $\begin{array}{l}0 \\
0 \\
0 \\
1 \\
0\end{array}$ & $\begin{array}{l}0 \\
0 \\
0 \\
0 \\
0\end{array}$ & $\begin{array}{l}0 \\
0 \\
0 \\
0 \\
0\end{array}$ \\
\hline $\begin{array}{l}0301(2) \\
0421 \\
0516 \\
0602 \\
0708\end{array}$ & $\begin{array}{l}-\overline{0} \\
0 \\
0 \\
0\end{array}$ & $\begin{array}{l}-- \\
0 \\
0 \\
0 \\
0\end{array}$ & $\begin{array}{r}-\overline{0} \\
0 \\
0 \\
0\end{array}$ & $\begin{array}{l}0 \\
0 \\
0 \\
0 \\
0\end{array}$ & $\begin{array}{l}0 \\
0 \\
0 \\
0 \\
0\end{array}$ & $\begin{array}{l}0 \\
0 \\
0 \\
0 \\
0\end{array}$ & $\begin{array}{l}0 \\
0 \\
0 \\
0 \\
0\end{array}$ & $\begin{array}{l}0 \\
1 \\
0 \\
1 \\
0\end{array}$ & $\begin{array}{l}0 \\
0 \\
1 \\
0 \\
0\end{array}$ \\
\hline $\begin{array}{l}0808 \\
0808(2) \\
0898 \\
0985 \\
1077\end{array}$ & $\begin{array}{r}-0 \\
0 \\
0 \\
0\end{array}$ & $\begin{array}{r}0 \\
-\frac{0}{0} \\
0 \\
0\end{array}$ & $\begin{array}{r}-0 \\
-0 \\
0 \\
0\end{array}$ & $\begin{array}{l}0 \\
0 \\
0 \\
0 \\
0\end{array}$ & $\begin{array}{l}0 \\
0 \\
0 \\
0 \\
0\end{array}$ & $\begin{array}{l}0 \\
0 \\
0 \\
0 \\
0\end{array}$ & $\begin{array}{l}0 \\
0 \\
0 \\
0 \\
0\end{array}$ & $\begin{array}{l}4 \\
0 \\
0 \\
0 \\
1\end{array}$ & $\begin{array}{l}2 \\
0 \\
0 \\
0 \\
0\end{array}$ \\
\hline $\begin{array}{l}1155 \\
1241 \\
1315 \\
1396 \\
1400(3)\end{array}$ & $\begin{array}{l}0 \\
0 \\
0 \\
0 \\
0\end{array}$ & $\begin{array}{l}0 \\
0 \\
0 \\
0 \\
0\end{array}$ & $\begin{array}{l}0 \\
0 \\
0 \\
0 \\
0\end{array}$ & $\begin{array}{l}0 \\
0 \\
0 \\
0 \\
0\end{array}$ & $\begin{array}{l}0 \\
0 \\
0 \\
0 \\
0\end{array}$ & $\begin{array}{l}0 \\
0 \\
0 \\
0 \\
0\end{array}$ & $\begin{array}{l}0 \\
0 \\
0 \\
0 \\
0\end{array}$ & $\begin{array}{l}0 \\
0 \\
0 \\
0 \\
0\end{array}$ & $\begin{array}{l}1 \\
5 \\
0 \\
0 \\
1\end{array}$ \\
\hline $\begin{array}{l}1481 \\
1481(2) \\
1573 \\
1662 \\
1695\end{array}$ & $\begin{array}{l}0 \\
0 \\
0 \\
0 \\
2\end{array}$ & $\begin{array}{l}0 \\
0 \\
0 \\
0 \\
0\end{array}$ & $\begin{array}{l}0 \\
0 \\
0 \\
0 \\
1\end{array}$ & $\begin{array}{l}0 \\
0 \\
0 \\
0 \\
4\end{array}$ & $\begin{array}{l}0 \\
0 \\
0 \\
0 \\
7\end{array}$ & $\begin{array}{l}0 \\
1 \\
2 \\
4 \\
3\end{array}$ & $\begin{array}{l}0 \\
0 \\
0 \\
0 \\
3\end{array}$ & $\begin{array}{l}4 \\
4 \\
0 \\
2 \\
3\end{array}$ & $\begin{array}{l}0 \\
1 \\
0 \\
3 \\
4\end{array}$ \\
\hline $\begin{array}{l}1766 \\
1830 \\
1901 \\
1996 \\
2082\end{array}$ & $\begin{array}{l}1 \\
1 \\
2 \\
0 \\
0\end{array}$ & $\begin{array}{l}2 \\
0 \\
0 \\
1 \\
0\end{array}$ & $\begin{array}{l}0 \\
7 \\
6 \\
1 \\
4\end{array}$ & $\begin{array}{l}1 \\
0 \\
0 \\
1 \\
0\end{array}$ & $\begin{array}{l}0 \\
4 \\
0 \\
0 \\
0\end{array}$ & $\begin{array}{r}1 \\
5 \\
1 \\
1 \\
18\end{array}$ & $\begin{array}{l}0 \\
2 \\
9 \\
0 \\
5\end{array}$ & $\begin{array}{r}1 \\
2 \\
4 \\
4 \\
11\end{array}$ & $\begin{array}{r}4 \\
6 \\
11 \\
11 \\
11\end{array}$ \\
\hline $\begin{array}{l}2194 \\
2278 \\
2356 \\
2422 \\
2510\end{array}$ & $\begin{array}{r}10 \\
3 \\
24 \\
39 \\
67\end{array}$ & $\begin{array}{l}10 \\
13 \\
62 \\
56 \\
94\end{array}$ & $\begin{array}{r}2 \\
7 \\
38 \\
71 \\
69\end{array}$ & $\begin{array}{r}1 \\
16 \\
16 \\
32 \\
68\end{array}$ & $\begin{array}{l}12 \\
24 \\
35 \\
13 \\
92\end{array}$ & $\begin{array}{l}11 \\
15 \\
29 \\
11 \\
63\end{array}$ & $\begin{array}{l}21 \\
12 \\
17 \\
38 \\
42\end{array}$ & $\begin{array}{l}23 \\
31 \\
43 \\
43 \\
66\end{array}$ & $\begin{array}{l}25 \\
22 \\
43 \\
41 \\
36\end{array}$ \\
\hline $\begin{array}{l}2608 \\
2690 \\
2778 \\
2874 \\
2961\end{array}$ & $\begin{array}{r}79 \\
47 \\
33 \\
15 \\
5\end{array}$ & $\begin{array}{r}67 \\
77 \\
62 \\
13 \\
9\end{array}$ & $\begin{array}{l}84 \\
44 \\
12 \\
17 \\
11\end{array}$ & $\begin{array}{r}52 \\
53 \\
26 \\
12 \\
5\end{array}$ & $\begin{array}{l}86 \\
45 \\
20 \\
27 \\
13\end{array}$ & $\begin{array}{r}21 \\
36 \\
40 \\
67 \\
6\end{array}$ & $\begin{array}{l}33 \\
40 \\
32 \\
23 \\
5\end{array}$ & $\begin{array}{r}11 \\
33 \\
28 \\
14 \\
2\end{array}$ & $\begin{array}{r}30 \\
44 \\
28 \\
13 \\
2\end{array}$ \\
\hline
\end{tabular}


TABLE 5.- NUMBER OF PINK TRACER PARTICLES(1) BETWEEN 0.25 AND 8.00 MILLIMETERS, PER 100 GRAMS OF BED MATERIAL, AS A FUNCTION OF DATE AND SECTION, EAST FORK RIVER, WYOMING, 1979--CONTINUED

\begin{tabular}{|c|c|c|c|c|c|c|c|c|c|}
\hline \multirow{2}{*}{$\begin{array}{l}\text { SECTION } \\
\text { NUMBER }\end{array}$} & \multicolumn{9}{|c|}{ DATE } \\
\hline & $6-18$ & $6-19$ & $6-20$ & $6-21$ & $6-22$ & $6-23$ & $6-24$ & $6-25$ & $6-26$ \\
\hline $\begin{array}{l}0043 \\
0075 \\
0137 \\
0220 \\
0301\end{array}$ & $\begin{array}{l}0 \\
0 \\
0 \\
0 \\
0\end{array}$ & $\begin{array}{l}0 \\
0 \\
0 \\
0 \\
0\end{array}$ & $\begin{array}{l}0 \\
0 \\
0 \\
0 \\
0\end{array}$ & $\begin{array}{l}0 \\
0 \\
2 \\
1 \\
0\end{array}$ & $\begin{array}{l}0 \\
0 \\
0 \\
0 \\
2\end{array}$ & $\begin{array}{l}0 \\
0 \\
0 \\
0 \\
0\end{array}$ & $\begin{array}{l}0 \\
0 \\
0 \\
0 \\
0\end{array}$ & $\begin{array}{l}0 \\
0 \\
0 \\
0 \\
0\end{array}$ & $\begin{array}{l}0 \\
0 \\
0 \\
0 \\
0\end{array}$ \\
\hline $\begin{array}{l}0301(2) \\
0421 \\
0516 \\
0602 \\
0708\end{array}$ & $\begin{array}{r}-\overline{0} \\
0 \\
0 \\
0\end{array}$ & $\begin{array}{c}-\overline{0} \\
0 \\
0 \\
0\end{array}$ & $\begin{array}{r}-- \\
0 \\
0 \\
0 \\
0\end{array}$ & $\begin{array}{l}-\overline{0} \\
0 \\
0 \\
0\end{array}$ & $\begin{array}{c}-\overline{0} \\
0 \\
0 \\
0\end{array}$ & $\begin{array}{c}-- \\
0 \\
0 \\
0 \\
0\end{array}$ & $\begin{array}{c}-- \\
0 \\
0 \\
0 \\
0\end{array}$ & $\begin{array}{c}-\overline{0} \\
--\overline{0} \\
-\overline{0}\end{array}$ & $\begin{array}{r}-\overline{0} \\
-\overline{0} \\
0\end{array}$ \\
\hline $\begin{array}{l}0808 \\
0808(2) \\
0898 \\
0985 \\
1077\end{array}$ & $\begin{array}{l}0 \\
0 \\
1 \\
0 \\
0\end{array}$ & $\begin{array}{r}-0 \\
-\frac{0}{0} \\
0 \\
0\end{array}$ & $\begin{array}{r}-0 \\
-0 \\
0 \\
0\end{array}$ & $\begin{array}{r}0 \\
-1 \\
0 \\
3 \\
0\end{array}$ & $\begin{array}{r}-0 \\
-0 \\
0 \\
0\end{array}$ & $\begin{array}{r}-0 \\
-0 \\
0 \\
0\end{array}$ & $\begin{array}{r}-0 \\
-0 \\
0 \\
0\end{array}$ & $\begin{array}{l}-- \\
-\overline{-} \\
-\overline{0}\end{array}$ & $\begin{array}{l}=- \\
=- \\
-\overline{0}\end{array}$ \\
\hline $\begin{array}{l}1155 \\
1241 \\
1315 \\
1396 \\
1400(3)\end{array}$ & $\begin{array}{l}4 \\
0 \\
0 \\
0 \\
0\end{array}$ & $\begin{array}{l}0 \\
3 \\
0 \\
0 \\
0\end{array}$ & $\begin{array}{r}0 \\
0 \\
0 \\
0 \\
--\end{array}$ & $\begin{array}{r}1 \\
3 \\
0 \\
0 \\
--\end{array}$ & $\begin{array}{r}0 \\
0 \\
0 \\
0 \\
--\end{array}$ & $\begin{array}{r}0 \\
0 \\
0 \\
0 \\
--\end{array}$ & $\begin{array}{r}0 \\
0 \\
0 \\
0 \\
--\end{array}$ & $\begin{array}{l}0 \\
0 \\
0 \\
0 \\
--\end{array}$ & $\begin{array}{r}0 \\
0 \\
0 \\
0 \\
--\end{array}$ \\
\hline $\begin{array}{l}1481 \\
1481(2) \\
1573 \\
1662 \\
1695\end{array}$ & $\begin{array}{l}0 \\
0 \\
0 \\
6 \\
3\end{array}$ & $\begin{array}{l}0 \\
0 \\
2 \\
0 \\
2\end{array}$ & $\begin{array}{r}0 \\
-\frac{0}{0} \\
3 \\
2\end{array}$ & $\begin{array}{r}0 \\
-\frac{1}{0} \\
10 \\
2\end{array}$ & $\begin{array}{r}2 \\
-\frac{1}{0} \\
5 \\
0\end{array}$ & $\begin{array}{r}-0 \\
-0 \\
0 \\
4\end{array}$ & $\begin{array}{r}-\frac{0}{0} \\
0 \\
0 \\
3\end{array}$ & $-\frac{0}{0}$ & $\begin{array}{l}-- \\
-0 \\
0 \\
0\end{array}$ \\
\hline $\begin{array}{l}1766 \\
1830 \\
1901 \\
1996 \\
2082\end{array}$ & $\begin{array}{r}1 \\
10 \\
3 \\
5 \\
16\end{array}$ & $\begin{array}{r}7 \\
6 \\
22 \\
\frac{1}{3}\end{array}$ & $\begin{array}{r}4 \\
5 \\
18 \\
2 \\
8\end{array}$ & $\begin{array}{r}3 \\
4 \\
29 \\
2 \\
3\end{array}$ & $\begin{array}{r}4 \\
4 \\
21 \\
7 \\
1\end{array}$ & $\begin{array}{r}0 \\
8 \\
13 \\
4 \\
2\end{array}$ & $\begin{array}{r}0 \\
2 \\
10 \\
9 \\
5\end{array}$ & $\begin{array}{r}2 \\
-4 \\
-2 \\
5\end{array}$ & $-\frac{4}{5}$ \\
\hline $\begin{array}{l}2194 \\
2278 \\
2356 \\
2422 \\
2510\end{array}$ & $\begin{array}{r}7 \\
22 \\
60 \\
32 \\
49\end{array}$ & $\begin{array}{l}11 \\
28 \\
60 \\
49 \\
37\end{array}$ & $\begin{array}{r}4 \\
28 \\
76 \\
47 \\
34\end{array}$ & $\begin{array}{r}6 \\
8 \\
65 \\
42 \\
36\end{array}$ & $\begin{array}{r}6 \\
15 \\
78 \\
63 \\
20\end{array}$ & $\begin{array}{l}10 \\
18 \\
83 \\
58 \\
32\end{array}$ & $\begin{array}{l}12 \\
32 \\
64 \\
37 \\
35\end{array}$ & $\begin{array}{l}-- \\
\overline{61} \\
42 \\
33\end{array}$ & $\begin{array}{l}-- \\
\overline{64} \\
65 \\
--\end{array}$ \\
\hline $\begin{array}{l}2608 \\
2690 \\
2778 \\
2874 \\
2961\end{array}$ & $\begin{array}{l}37 \\
18 \\
10 \\
15 \\
1\end{array}$ & $\begin{array}{r}24 \\
53 \\
29 \\
7 \\
3\end{array}$ & $\begin{array}{r}28 \\
36 \\
40 \\
9 \\
3\end{array}$ & $\begin{array}{r}18 \\
21 \\
62 \\
7 \\
0\end{array}$ & $\begin{array}{r}25 \\
34 \\
86 \\
2 \\
2\end{array}$ & $\begin{array}{r}33 \\
79 \\
69 \\
4 \\
1\end{array}$ & $\begin{array}{r}35 \\
76 \\
63 \\
7 \\
2\end{array}$ & $\begin{array}{l}69 \\
60 \\
54 \\
-- \\
--\end{array}$ & $\begin{array}{l}64 \\
78 \\
-- \\
--\end{array}$ \\
\hline
\end{tabular}


TABLE 5.- NUMBER OF PINK TRACER PARTICLES(1) BETWEEN 0.25 AND 8.00 MILLIMETERS, PER 100 GRAMS OF BED MATERIAL, AS A FUNCTION OF DATE AND SECTION, EAST FORK RIVER, WYOMING, 1979--CONTINUED

\begin{tabular}{|c|c|c|c|c|}
\hline \multirow{2}{*}{$\begin{array}{r}\text { SECTION } \\
\text { NUMBER }\end{array}$} & \multirow{2}{*}{$6-27$} & \multirow[b]{2}{*}{$6-28$} & \multirow[b]{2}{*}{$7-11$} & \multirow[b]{2}{*}{$10-07$} \\
\hline & & & & \\
\hline $\begin{array}{l}0043 \\
0075 \\
0137 \\
0220 \\
0301\end{array}$ & $\begin{array}{l}0 \\
0 \\
0 \\
0 \\
0\end{array}$ & $\begin{array}{l}0 \\
0 \\
0 \\
0 \\
0\end{array}$ & $\begin{array}{l}0 \\
0 \\
0 \\
0 \\
0\end{array}$ & $\begin{array}{l}2 \\
1 \\
0 \\
0 \\
0\end{array}$ \\
\hline $\begin{array}{l}0301(2) \\
0421 \\
0516 \\
0602 \\
0708\end{array}$ & $\frac{-\overline{0}}{--}$ & $\begin{array}{r}-- \\
0 \\
2 \\
0 \\
0\end{array}$ & $\begin{array}{r}-- \\
0 \\
2 \\
2 \\
1\end{array}$ & $\begin{array}{c}-- \\
1 \\
0 \\
0 \\
3\end{array}$ \\
\hline $\begin{array}{l}0808 \\
0808(2) \\
0898 \\
0985 \\
1077\end{array}$ & $\begin{array}{l}= \\
= \\
= \\
=\end{array}$ & $\begin{array}{c}1 \\
-\frac{1}{0} \\
0 \\
0\end{array}$ & $\begin{array}{r}0 \\
-0 \\
0 \\
0 \\
1\end{array}$ & $\begin{array}{r}-2 \\
0 \\
0 \\
0\end{array}$ \\
\hline $\begin{array}{l}1155 \\
1241 \\
1315 \\
1396 \\
1400(3)\end{array}$ & $\begin{array}{r}0 \\
0 \\
0 \\
0 \\
--\end{array}$ & $\begin{array}{r}0 \\
2 \\
0 \\
0 \\
--\end{array}$ & $\begin{array}{r}2 \\
0 \\
0 \\
3 \\
--\end{array}$ & $\begin{array}{r}1 \\
3 \\
3 \\
1 \\
--\end{array}$ \\
\hline $\begin{array}{l}1481 \\
1481(2) \\
1573 \\
1662 \\
1695\end{array}$ & $\begin{array}{r}-\frac{0}{2} \\
2 \\
4\end{array}$ & $\begin{array}{r}-0 \\
-0 \\
0 \\
4\end{array}$ & $\begin{array}{r}0 \\
-1 \\
6 \\
1\end{array}$ & $\begin{array}{r}0 \\
-0 \\
3 \\
7\end{array}$ \\
\hline $\begin{array}{l}1766 \\
1830 \\
1901 \\
1996 \\
2082\end{array}$ & $\begin{array}{l}3 \\
-\overline{12} \\
--\end{array}$ & $\begin{array}{r}3 \\
1 \\
18 \\
7 \\
4\end{array}$ & $\begin{array}{l}0 \\
0 \\
5 \\
1 \\
3\end{array}$ & $\begin{array}{r}3 \\
0 \\
10 \\
6 \\
7\end{array}$ \\
\hline $\begin{array}{l}2194 \\
2278 \\
2356 \\
2422 \\
2510\end{array}$ & $\begin{array}{l}-- \\
\overline{76} \\
45 \\
--\end{array}$ & $\begin{array}{r}6 \\
26 \\
64 \\
52 \\
19\end{array}$ & $\begin{array}{r}9 \\
7 \\
29 \\
33 \\
29\end{array}$ & $\begin{array}{l}17 \\
22 \\
70 \\
49 \\
47\end{array}$ \\
\hline $\begin{array}{l}2608 \\
2690 \\
2778 \\
2874 \\
2961\end{array}$ & $\begin{array}{l}87 \\
67 \\
-2 \\
--\end{array}$ & $\begin{array}{r}122 \\
76 \\
50 \\
10 \\
2\end{array}$ & $\begin{array}{r}74 \\
42 \\
24 \\
2 \\
3\end{array}$ & $\begin{array}{r}29 \\
19 \\
24 \\
6 \\
2\end{array}$ \\
\hline
\end{tabular}
DATE

$$
\begin{aligned}
& 2 \\
& 1 \\
& 0 \\
& 0 \\
& 0
\end{aligned}
$$

$$
\begin{aligned}
& -1 \\
& 0 \\
& 0 \\
& 3
\end{aligned}
$$


TABLE 6.- NUMBER OF BLUE TRACER PARTICLES(1) BETWEEN 0.25 AND 8.00 MILLIMETERS, PER 100 GRAMS OF BED MATERIAL, AS A FUNCTION OF DATE AND SECTION, EAST FORK RIVER, WYOMING, 1979

\begin{tabular}{|c|c|c|c|c|c|c|c|c|c|}
\hline \multirow{2}{*}{$\begin{array}{l}\text { SECTION } \\
\text { NUMBER }\end{array}$} & \multicolumn{9}{|c|}{ DATE } \\
\hline & $5-20$ & $5-21$ & $5-23$ & $5-24$ & $5-25$ & $5-26$ & $5-27$ & $5-28$ & $5-30$ \\
\hline $\begin{array}{l}0043 \\
0075 \\
0137 \\
0220 \\
0301\end{array}$ & $\begin{array}{c}-- \\
-\overline{0} \\
0\end{array}$ & $\begin{array}{r}0 \\
-0 \\
0 \\
1\end{array}$ & $\begin{array}{l}-\overline{0} \\
0 \\
0 \\
0\end{array}$ & $\begin{array}{l}0 \\
0 \\
0 \\
0 \\
0\end{array}$ & $\begin{array}{l}0 \\
0 \\
0 \\
0 \\
0\end{array}$ & $\begin{array}{l}0 \\
0 \\
0 \\
0 \\
0\end{array}$ & $\begin{array}{r}-\overline{0} \\
0 \\
1 \\
0\end{array}$ & $\begin{array}{l}0 \\
0 \\
0 \\
0 \\
0\end{array}$ & $\begin{array}{l}2 \\
4 \\
1 \\
0 \\
0\end{array}$ \\
\hline $\begin{array}{l}0301(2) \\
0421 \\
0516 \\
0602 \\
0708\end{array}$ & $\begin{array}{l}0 \\
0 \\
-- \\
--\end{array}$ & $\begin{array}{c}0 \\
0 \\
-- \\
--\end{array}$ & $\begin{array}{r}0 \\
0 \\
-- \\
--\end{array}$ & $\begin{array}{l}0 \\
0 \\
-- \\
--\end{array}$ & $\begin{array}{c}0 \\
0 \\
-- \\
--\end{array}$ & $\begin{array}{r}0 \\
1 \\
-- \\
--\end{array}$ & $\begin{array}{r}0 \\
0 \\
-- \\
--\end{array}$ & $\begin{array}{l}-\overline{0} \\
-- \\
--\end{array}$ & $\begin{array}{r}-\overline{0} \\
0 \\
0 \\
0\end{array}$ \\
\hline $\begin{array}{l}0808 \\
0808(2) \\
0898 \\
0985 \\
1077\end{array}$ & $\begin{array}{l}-- \\
=- \\
=-\end{array}$ & $\begin{array}{l}=- \\
=- \\
=-\end{array}$ & $\begin{array}{l}=- \\
=- \\
=-\end{array}$ & $\begin{array}{l}-- \\
=- \\
-- \\
--\end{array}$ & $\begin{array}{l}-- \\
=- \\
=- \\
--\end{array}$ & $\begin{array}{l}=- \\
\overline{--} \\
-\overline{0}\end{array}$ & $\begin{array}{c}-- \\
-\overline{0} \\
0\end{array}$ & $\begin{array}{r}0 \\
-0 \\
0 \\
1\end{array}$ & $\begin{array}{r}-0 \\
-0 \\
0 \\
7\end{array}$ \\
\hline $\begin{array}{l}1155 \\
1241 \\
1315 \\
1396 \\
1400(3)\end{array}$ & $\begin{array}{l}-- \\
312 \\
28 \\
--\end{array}$ & $\begin{array}{l}-- \\
\overline{42} \\
--\end{array}$ & $\begin{array}{l}-- \\
\overline{12} \\
10 \\
--\end{array}$ & $\begin{array}{r}-5 \overline{2} \\
35 \\
125 \\
--\end{array}$ & $\begin{array}{r}4 \\
15 \\
49 \\
78 \\
187\end{array}$ & $\begin{array}{r}2 \\
27 \\
112 \\
33 \\
83\end{array}$ & $\begin{array}{r}8 \\
20 \\
68 \\
30 \\
123\end{array}$ & $\begin{array}{r}3 \\
1 \\
20 \\
25 \\
--\end{array}$ & $\begin{array}{r}11 \\
9 \\
72 \\
22 \\
--\end{array}$ \\
\hline $\begin{array}{l}1481 \\
1481(2) \\
1573 \\
1662\end{array}$ & $\begin{array}{r}19 \\
7 \\
75 \\
222\end{array}$ & $\begin{array}{r}93 \\
52 \\
288 \\
260\end{array}$ & $\begin{array}{r}159 \\
249 \\
122 \\
23\end{array}$ & $\begin{array}{r}109 \\
232 \\
65 \\
2\end{array}$ & $\begin{array}{r}68 \\
121 \\
38 \\
3\end{array}$ & $\begin{array}{r}98 \\
59 \\
15 \\
8\end{array}$ & $\begin{array}{r}27 \\
33 \\
6 \\
1\end{array}$ & $-\frac{8}{5}$ & $\begin{array}{r}12 \\
15 \\
5 \\
2\end{array}$ \\
\hline
\end{tabular}


TABLE 6.- NUMBER OF BLUE TRACER PARTICLES(1) BETWEEN 0.25 AND 8.00

MILL IMETERS, PER 100 GRAMS OF BED MATERIAL, AS A FUNCTION OF

DATE AND SECTION, EAST FORK RIVER, WYOMING, 1979--CONTINUED

\begin{tabular}{|c|c|c|c|c|c|c|c|c|c|}
\hline \multirow{2}{*}{$\begin{array}{l}\text { SECTION } \\
\text { NUMBER }\end{array}$} & \multicolumn{9}{|c|}{ DATE } \\
\hline & $5-31$ & $6-01$ & $6-02$ & $6-03$ & $6-04$ & $6-05$ & $6-06$ & $6-07$ & $6-08$ \\
\hline $\begin{array}{l}0043 \\
0075 \\
0137 \\
0220 \\
0301\end{array}$ & $\begin{array}{l}0 \\
0 \\
0 \\
1 \\
0\end{array}$ & $\begin{array}{l}0 \\
0 \\
0 \\
0 \\
0\end{array}$ & $\begin{array}{l}0 \\
0 \\
0 \\
0 \\
0\end{array}$ & $\begin{array}{l}0 \\
0 \\
0 \\
0 \\
0\end{array}$ & $\begin{array}{l}1 \\
1 \\
0 \\
1 \\
0\end{array}$ & $\begin{array}{l}1 \\
0 \\
0 \\
0 \\
0\end{array}$ & $\begin{array}{l}0 \\
2 \\
1 \\
1 \\
1\end{array}$ & $\begin{array}{l}1 \\
0 \\
0 \\
0 \\
0\end{array}$ & $\begin{array}{l}1 \\
3 \\
0 \\
0 \\
1\end{array}$ \\
\hline $\begin{array}{l}0301(2) \\
0421 \\
0516 \\
0602 \\
0708\end{array}$ & $\begin{array}{r}-\overline{0} \\
0 \\
0 \\
0\end{array}$ & $\begin{array}{r}-\overline{0} \\
0 \\
0 \\
2\end{array}$ & $\begin{array}{c}-- \\
0 \\
1 \\
2 \\
6\end{array}$ & $\begin{array}{c}-- \\
0 \\
0 \\
0 \\
0\end{array}$ & $\begin{array}{r}-- \\
0 \\
0 \\
12 \\
0\end{array}$ & $\begin{array}{l}\frac{1}{1} \\
\frac{1}{2} \\
8 \\
0\end{array}$ & $\begin{array}{r}2 \\
2 \\
12 \\
6 \\
0\end{array}$ & $\begin{array}{l}4 \\
6 \\
4 \\
0 \\
3\end{array}$ & $\begin{array}{r}-- \\
2 \\
5 \\
3 \\
21\end{array}$ \\
\hline $\begin{array}{l}0808 \\
0808(2) \\
0898 \\
0985 \\
1077\end{array}$ & $\begin{array}{r}-0 \\
-\frac{1}{0} \\
0 \\
16\end{array}$ & $\begin{array}{r}0 \\
-\frac{1}{3} \\
0 \\
10\end{array}$ & $\begin{array}{r}-\frac{2}{0} \\
0 \\
13\end{array}$ & $\begin{array}{r}-\frac{0}{0} \\
0 \\
0 \\
0\end{array}$ & $\begin{array}{r}2 \\
4 \\
0 \\
1 \\
16\end{array}$ & $\begin{array}{r}5 \\
5 \\
3 \\
0 \\
40\end{array}$ & $\begin{array}{r}3 \\
0 \\
6 \\
2 \\
169\end{array}$ & $\begin{array}{r}12 \\
25 \\
6 \\
54 \\
24\end{array}$ & $\begin{array}{r}6 \\
52 \\
1 \\
13 \\
58\end{array}$ \\
\hline $\begin{array}{l}1155 \\
1241 \\
1315 \\
1396 \\
1400(3)\end{array}$ & $\begin{array}{l}23 \\
61 \\
77 \\
31 \\
92\end{array}$ & $\begin{array}{r}3 \\
66 \\
50 \\
9 \\
87\end{array}$ & $\begin{array}{r}74 \\
72 \\
74 \\
109 \\
13\end{array}$ & $\begin{array}{l}2 \\
32 \\
25 \\
28 \\
86\end{array}$ & $\begin{array}{r}148 \\
72 \\
60 \\
6 \\
68\end{array}$ & $\begin{array}{r}28 \\
40 \\
68 \\
3 \\
138\end{array}$ & $\begin{array}{r}95 \\
69 \\
76 \\
19 \\
199\end{array}$ & $\begin{array}{l}54 \\
69 \\
61 \\
13 \\
56\end{array}$ & $\begin{array}{l}52 \\
66 \\
20 \\
82 \\
19\end{array}$ \\
\hline $\begin{array}{l}1481 \\
1481(2) \\
1573 \\
1662\end{array}$ & $\begin{array}{r}11 \\
13 \\
1 \\
0\end{array}$ & $\begin{array}{r}14 \\
16 \\
7 \\
0\end{array}$ & $\begin{array}{r}15 \\
10 \\
5 \\
0\end{array}$ & $\begin{array}{r}13 \\
19 \\
10 \\
0\end{array}$ & $\begin{array}{r}8 \\
11 \\
27 \\
0\end{array}$ & $\begin{array}{r}32 \\
17 \\
73 \\
0\end{array}$ & $\begin{array}{r}35 \\
21 \\
34 \\
1\end{array}$ & $\begin{array}{r}26 \\
64 \\
7 \\
2\end{array}$ & $\frac{33}{11}$ \\
\hline
\end{tabular}


TABLE 6.- NUMBER OF BLUE TRACER PARTICLES(1) BETWEEN 0.25 AND 8.00 MILI IMETERS, PER 100 GRAMS OF BED MATERIAL, AS A FUNCTION OF DATE AND SECTION, EAST FORK RIVER, WYOMING, 1979--CONTINUED

\begin{tabular}{|c|c|c|c|c|c|c|c|c|c|}
\hline \multirow{2}{*}{$\begin{array}{l}\text { SECTION } \\
\text { NUMBER }\end{array}$} & \multicolumn{9}{|c|}{ DATE } \\
\hline & $6-09$ & $6-10$ & $6-11$ & $6-12$ & $6-13$ & $6-14$ & $6-15$ & $6-16$ & $6-17$ \\
\hline $\begin{array}{l}0043 \\
0075 \\
0137 \\
0220 \\
0301\end{array}$ & $\begin{array}{l}2 \\
7 \\
0 \\
0 \\
0\end{array}$ & $\begin{array}{l}0 \\
3 \\
2 \\
0 \\
1\end{array}$ & $\begin{array}{l}5 \\
0 \\
0 \\
1 \\
1\end{array}$ & $\begin{array}{l}1 \\
4 \\
6 \\
2 \\
2\end{array}$ & $\begin{array}{l}0 \\
0 \\
0 \\
0 \\
0\end{array}$ & $\begin{array}{l}1 \\
1 \\
1 \\
3 \\
0\end{array}$ & $\begin{array}{l}\frac{1}{2} \\
\frac{1}{1} \\
\frac{1}{1}\end{array}$ & $\begin{array}{l}0 \\
3 \\
1 \\
0 \\
0\end{array}$ & $\begin{array}{l}1 \\
4 \\
0 \\
2 \\
0\end{array}$ \\
\hline $\begin{array}{l}0301(2) \\
0421 \\
0516 \\
0602 \\
0708\end{array}$ & $\begin{array}{r}-\overline{0} \\
4 \\
1 \\
4 I\end{array}$ & $\begin{array}{r}-\overline{3} \\
4 \\
3 \\
4\end{array}$ & $\begin{array}{r}- \\
3 \\
9 \\
10\end{array}$ & $\begin{array}{r}0 \\
1 \\
12 \\
5 \\
5\end{array}$ & $\begin{array}{l}6 \\
1 \\
0 \\
0 \\
0\end{array}$ & $\begin{array}{l}2 \\
7 \\
0 \\
2 \\
5\end{array}$ & $\begin{array}{l}5 \\
3 \\
3 \\
4 \\
5\end{array}$ & $\begin{array}{r}0 \\
3 \\
6 \\
12 \\
5\end{array}$ & $\begin{array}{l}3 \\
1 \\
3 \\
0 \\
8\end{array}$ \\
\hline $\begin{array}{l}0808 \\
0808(2) \\
0898 \\
0985 \\
1077\end{array}$ & $\begin{array}{r}-9 \\
10 \\
3 \\
79\end{array}$ & $\begin{array}{l}-3 \\
24 \\
13 \\
33\end{array}$ & $\begin{array}{l}17 \\
16 \\
23 \\
29\end{array}$ & $\begin{array}{r}7 \\
18 \\
5 \\
5 \\
33\end{array}$ & $\begin{array}{r}4 \\
11 \\
9 \\
16 \\
44\end{array}$ & $\begin{array}{r}8 \\
20 \\
18 \\
5 \\
60\end{array}$ & $\begin{array}{r}16 \\
8 \\
7 \\
21 \\
70\end{array}$ & $\begin{array}{l}22 \\
11 \\
25 \\
48 \\
62\end{array}$ & $\begin{array}{r}10 \\
9 \\
9 \\
21 \\
62\end{array}$ \\
\hline $\begin{array}{l}1155 \\
1241 \\
1315 \\
1396 \\
1400(3)\end{array}$ & $\begin{array}{l}73 \\
37 \\
22 \\
30 \\
47\end{array}$ & $\begin{array}{r}70 \\
54 \\
37 \\
82 \\
9\end{array}$ & $\begin{array}{r}124 \\
20 \\
31 \\
14 \\
69\end{array}$ & $\begin{array}{l}56 \\
28 \\
24 \\
13 \\
54\end{array}$ & $\begin{array}{l}54 \\
47 \\
18 \\
28 \\
74\end{array}$ & $\begin{array}{l}45 \\
38 \\
41 \\
16 \\
67\end{array}$ & $\begin{array}{l}44 \\
37 \\
57 \\
16 \\
40\end{array}$ & $\begin{array}{r}42 \\
40 \\
26 \\
42 \\
0\end{array}$ & $\begin{array}{r}50 \\
43 \\
31 \\
9 \\
32\end{array}$ \\
\hline $\begin{array}{l}1481 \\
1481(2) \\
1573 \\
1662\end{array}$ & $\begin{array}{r}15 \\
48 \\
24 \\
4\end{array}$ & $\begin{array}{r}51 \\
12 \\
8 \\
1\end{array}$ & $\begin{array}{r}22 \\
18 \\
8 \\
0\end{array}$ & $\begin{array}{r}14 \\
69 \\
6 \\
2\end{array}$ & $\begin{array}{r}38 \\
48 \\
0 \\
0\end{array}$ & $\begin{array}{r}17 \\
54 \\
3 \\
2\end{array}$ & $\begin{array}{r}7 \\
10 \\
4 \\
0\end{array}$ & $\begin{array}{r}16 \\
22 \\
3 \\
0\end{array}$ & $\begin{array}{r}12 \\
8 \\
1 \\
0\end{array}$ \\
\hline
\end{tabular}


TABLE 6.- NUMBER OF BLUE TRACER PARTICLES(1) BETWEEN 0.25 AND 8.00 MILLIMETERS, PER 100 GRAMS OF BED MATERIAL, AS A FUNCTION OF DATE AND SECTION, EAST FORK RIVER, WYOMING, 1979--CONTINUED

\begin{tabular}{|c|c|c|c|c|c|c|c|c|c|}
\hline \multirow{2}{*}{$\begin{array}{l}\text { SECTION } \\
\text { NUMBER }\end{array}$} & \multicolumn{9}{|c|}{ DATE } \\
\hline & $6-18$ & $6-19$ & $6-20$ & $6-21$ & $6-22$ & $6-23$ & $6-24$ & $6-25$ & $6-26$ \\
\hline $\begin{array}{l}0043 \\
0075 \\
0137 \\
0220 \\
0301\end{array}$ & $\begin{array}{l}0 \\
3 \\
2 \\
2 \\
1\end{array}$ & $\begin{array}{l}1 \\
4 \\
1 \\
1 \\
6\end{array}$ & $\begin{array}{r}6 \\
0 \\
0 \\
6 \\
12\end{array}$ & $\begin{array}{l}1 \\
3 \\
0 \\
2 \\
8\end{array}$ & $\begin{array}{l}6 \\
0 \\
0 \\
3 \\
3\end{array}$ & $\begin{array}{l}5 \\
2 \\
2 \\
3 \\
6\end{array}$ & $\begin{array}{l}3 \\
1 \\
2 \\
2 \\
4\end{array}$ & $\begin{array}{l}1 \\
1 \\
0 \\
0 \\
3\end{array}$ & $\begin{array}{r}2 \\
2 \\
1 \\
11 \\
0\end{array}$ \\
\hline $\begin{array}{l}0301(2) \\
0421 \\
0516 \\
0602 \\
0708\end{array}$ & $\begin{array}{r}-- \\
4 \\
0 \\
10 \\
7\end{array}$ & $\begin{array}{r}-- \\
1 \\
9 \\
8 \\
14\end{array}$ & $\begin{array}{r}-\overline{0} \\
\frac{1}{2} \\
24\end{array}$ & $\begin{array}{r}-- \\
0 \\
2 \\
20 \\
32\end{array}$ & $\begin{array}{r}-\overline{0} \\
2 \\
6 \\
10\end{array}$ & $\begin{array}{r}-- \\
8 \\
8 \\
16 \\
14\end{array}$ & $\begin{array}{r}-\overline{1} \\
9 \\
14 \\
16\end{array}$ & $\begin{array}{l}-\overline{0} \\
-\overline{-} \\
\overline{24}\end{array}$ & $\begin{array}{r}-\frac{2}{2} \\
\overline{20} \\
21\end{array}$ \\
\hline $\begin{array}{l}0808 \\
0808(2) \\
0898 \\
0985 \\
1077\end{array}$ & $\begin{array}{r}12 \\
17 \\
18 \\
4 \\
48\end{array}$ & $\begin{array}{l}28 \\
\frac{47}{7} \\
22\end{array}$ & $\begin{array}{l}14 \\
-\frac{1}{35} \\
\frac{1}{33}\end{array}$ & $\begin{array}{l}38 \\
66 \\
29 \\
53\end{array}$ & $\begin{array}{l}-9 \\
10 \\
24 \\
35\end{array}$ & $\begin{array}{l}18 \\
33 \\
15 \\
49\end{array}$ & $\begin{array}{l}43 \\
-\frac{1}{60} \\
18 \\
62\end{array}$ & $\begin{array}{l}=- \\
=- \\
-- \\
5 \overline{6}\end{array}$ & $\begin{array}{l}-- \\
=- \\
\overline{--} \\
21\end{array}$ \\
\hline $\begin{array}{l}1155 \\
1241 \\
1315 \\
1396 \\
1400(3)\end{array}$ & $\begin{array}{r}51 \\
29 \\
39 \\
4 \\
32\end{array}$ & $\begin{array}{l}26 \\
53 \\
26 \\
10 \\
25\end{array}$ & $\begin{array}{l}38 \\
38 \\
15 \\
14 \\
--\end{array}$ & $\begin{array}{r}31 \\
39 \\
7 \\
7 \\
--\end{array}$ & $\begin{array}{r}38 \\
25 \\
12 \\
6 \\
--\end{array}$ & $\begin{array}{r}33 \\
24 \\
10 \\
8 \\
--\end{array}$ & $\begin{array}{l}42 \\
13 \\
23 \\
11 \\
-\end{array}$ & $\begin{array}{l}70 \\
24 \\
14 \\
15 \\
\end{array}$ & $\begin{array}{l}47 \\
26 \\
19 \\
10 \\
-\end{array}$ \\
\hline $\begin{array}{l}1481 \\
1481(2) \\
1573 \\
1662\end{array}$ & $\begin{array}{r}20 \\
20 \\
2 \\
3\end{array}$ & $\begin{array}{r}19 \\
8 \\
2 \\
1\end{array}$ & $\frac{11}{4}$ & $-\frac{9}{8}$ & $-\frac{9}{7}$ & $\frac{22}{1}$ & $\frac{12}{-\frac{1}{6}}$ & $\frac{14 .}{3}$ & $\begin{array}{c}-- \\
-3 \\
0\end{array}$ \\
\hline
\end{tabular}


TABLE 6.- NUMBER OF BLUE TRACER PARTICLES(1) BETWEEN 0.25 AND 8.00 MILL IMETERS, PER 100 GRAMS OF BED MATERIAL, AS A FUNCTION OF DATE AND SECTION, EAST FORK RIVER, WYOMING, 1979--CONTINUED

\begin{tabular}{|c|c|c|c|c|}
\hline \multirow{2}{*}{$\begin{array}{l}\text { SECTION } \\
\text { NUMBER }\end{array}$} & \multirow{2}{*}{$6-27$} & \multirow[b]{2}{*}{$6-28$} & \multirow[b]{2}{*}{$7-11$} & \multirow[b]{2}{*}{$10-0$} \\
\hline & & & & \\
\hline $\begin{array}{l}0043 \\
0075 \\
0137 \\
0220 \\
0301\end{array}$ & $\begin{array}{l}1 \\
1 \\
1 \\
2 \\
0\end{array}$ & $\begin{array}{l}3 \\
4 \\
1 \\
1 \\
0\end{array}$ & $\begin{array}{l}2 \\
5 \\
1 \\
0 \\
3\end{array}$ & \\
\hline $\begin{array}{l}0301(2) \\
0421 \\
0516 \\
0602 \\
0708\end{array}$ & $\frac{--}{1}$ & $\begin{array}{r}-- \\
5 \\
2 \\
11 \\
16\end{array}$ & $\begin{array}{r}-5 \\
5 \\
17 \\
9\end{array}$ & \\
\hline $\begin{array}{l}0808 \\
0808(2) \\
0898 \\
0985 \\
1077\end{array}$ & $\begin{array}{l}=- \\
=- \\
=-\end{array}$ & $\begin{array}{l}18 \\
29 \\
29 \\
33\end{array}$ & $\begin{array}{r}-\frac{4}{31} \\
20\end{array}$ & \\
\hline $\begin{array}{l}1155 \\
1241 \\
1315 \\
1396 \\
1400(3)\end{array}$ & $\begin{array}{r}25 \\
22 \\
3 \\
14 \\
\end{array}$ & $\begin{array}{l}35 \\
38 \\
31 \\
11 \\
-\end{array}$ & $\begin{array}{l}43 \\
41 \\
12 \\
11 \\
\end{array}$ & \\
\hline $\begin{array}{l}1481 \\
1481(2) \\
1573 \\
1662\end{array}$ & $\frac{20}{10}$ & $\frac{9}{15}$ & $\frac{27}{3}$ & \\
\hline
\end{tabular}

(1) BLUE TRACER PARTICLES WERE PLACED AS BED MATERIAL 10 METERS DOWNSTREAM FROM SECTION 1695 ON MAY 19, 1979.

(2) SMALL OVERFLOW CHANNEL ADJACENT TO SECTION.

(3) BYPASS CHANNEL. SEE FIGURE 3.

(4) SECTIONS 0043 THRU 0516 MEASURED ON 10-09. 
TABLE 7.- NUMBER OF ORANGE TRACER PARTICLES(1) BETWEEN 0.25 AND 8.00 MILLIMETERS, PER 100 GRAMS OF BED MATERIAL, AS A FUNCTION OF DATE AND SECTION, EAST FORK RIVER, WYOMING, 1979

\begin{tabular}{|c|c|c|c|c|c|c|c|c|c|}
\hline \multirow{2}{*}{$\begin{array}{l}\text { SECTION } \\
\text { NUMBER }\end{array}$} & \multicolumn{9}{|c|}{ DATE } \\
\hline & $5-20$ & $5-21$ & $5-23$ & $5-24$ & $5-25$ & $5-26$ & $5-27$ & $5-28$ & $5-30$ \\
\hline $\begin{array}{l}0043 \\
0075 \\
0137 \\
0220 \\
0301\end{array}$ & $\begin{array}{r}-- \\
-\overline{6} \\
135\end{array}$ & $\begin{array}{r}-\frac{2}{2} \\
1 \frac{13}{86}\end{array}$ & $\begin{array}{r}--0 \\
0 \\
0 \\
38\end{array}$ & $\begin{array}{r}33 \\
33 \\
2 \\
13 \\
71\end{array}$ & $\begin{array}{r}13 \\
37 \\
12 \\
28 \\
142\end{array}$ & $\begin{array}{r}2 \\
34 \\
13 \\
29 \\
61\end{array}$ & $\begin{array}{r}-7 \\
54 \\
38 \\
31\end{array}$ & $\begin{array}{l}12 \\
37 \\
61 \\
62 \\
19\end{array}$ & $\begin{array}{r}37 \\
58 \\
43 \\
27 \\
6\end{array}$ \\
\hline $\begin{array}{l}0301(2) \\
0421\end{array}$ & $209^{8}$ & $\frac{11}{73}$ & $\begin{array}{r}260 \\
76\end{array}$ & $\begin{array}{r}12 \\
7\end{array}$ & $\begin{array}{l}57 \\
10\end{array}$ & $\begin{array}{r}243 \\
10\end{array}$ & 82 & $-\overline{1}$ & $-\frac{-}{1}$ \\
\hline
\end{tabular}

\begin{tabular}{|c|c|c|c|c|c|c|c|c|c|}
\hline \multirow{2}{*}{$\begin{array}{l}\text { SECTION } \\
\text { NUMBER }\end{array}$} & \multicolumn{9}{|c|}{ DATE } \\
\hline & $5-31$ & $6-01$ & $6-02$ & $6-03$ & $6-04$ & $6-05$ & $6-06$ & $6-07$ & $6-08$ \\
\hline $\begin{array}{l}0043 \\
0075 \\
0137 \\
0220 \\
0301\end{array}$ & $\begin{array}{r}14 \\
29 \\
33 \\
23 \\
5\end{array}$ & $\begin{array}{r}15 \\
27 \\
13 \\
10 \\
2\end{array}$ & $\begin{array}{l}20 \\
49 \\
14 \\
18 \\
10\end{array}$ & $\begin{array}{l}14 \\
67 \\
12 \\
18 \\
17\end{array}$ & $\begin{array}{r}34 \\
26 \\
3 \\
12 \\
26\end{array}$ & $\begin{array}{l}29 \\
29 \\
11 \\
17 \\
18\end{array}$ & $\begin{array}{l}23 \\
20 \\
2 \\
11 \\
18\end{array}$ & $\begin{array}{l}10 \\
36 \\
14 \\
22 \\
12\end{array}$ & $\begin{array}{r}30 \\
31 \\
4 \\
9 \\
27\end{array}$ \\
\hline $\begin{array}{l}0301(2) \\
0421\end{array}$ & $-\overline{3}$ & $\overline{1 I}$ & $\overline{156}$ & $\overline{19}$ & $\overline{26}$ & $\begin{array}{r}8 \\
23\end{array}$ & $\frac{18}{24}$ & $\begin{array}{r}43 \\
4\end{array}$ & $\overline{I I}$ \\
\hline
\end{tabular}

\begin{tabular}{|c|c|c|c|c|c|c|c|c|c|}
\hline \multirow{2}{*}{$\begin{array}{l}\text { SECTION } \\
\text { NUMBER }\end{array}$} & \multicolumn{9}{|c|}{ DATE } \\
\hline & $6-09$ & $6-10$ & $6-11$ & $6-12$ & $6-13$ & $6-14$ & $6-15$ & $6-16$ & $6-17$ \\
\hline $\begin{array}{l}0043 \\
0075 \\
0137 \\
0220 \\
0301\end{array}$ & $\begin{array}{r}16 \\
20 \\
0 \\
2 \\
1\end{array}$ & $\begin{array}{l}23 \\
21 \\
22 \\
23 \\
19\end{array}$ & $\begin{array}{r}36 \\
19 \\
22 \\
8 \\
23\end{array}$ & $\begin{array}{l}24 \\
18 \\
37 \\
13 \\
24\end{array}$ & $\begin{array}{r}11 \\
17 \\
9 \\
11 \\
16\end{array}$ & $\begin{array}{l}15 \\
11 \\
19 \\
15 \\
10\end{array}$ & $\begin{array}{l}24 \\
10 \\
25 \\
23 \\
18\end{array}$ & $\begin{array}{l}10 \\
12 \\
14 \\
23 \\
20\end{array}$ & $\begin{array}{l}14 \\
20 \\
16 \\
17 \\
37\end{array}$ \\
\hline $\begin{array}{l}0301(2) \\
0421\end{array}$ & $-\overline{2}$ & $-m$ & $1 \overline{14}$ & $\begin{array}{l}18 \\
51\end{array}$ & $\begin{array}{l}19 \\
49\end{array}$ & $\begin{array}{l}23 \\
52\end{array}$ & $\begin{array}{l}31 \\
10\end{array}$ & $3 \frac{1}{9}$ & $\begin{array}{l}24 \\
16\end{array}$ \\
\hline
\end{tabular}


TABLE 7.- NUMBER OF ORANGE TRACER PARTICLES(1) BETWEEN 0.25 AND 8.00 MILLIMETERS, PER 100 GRAMS OF BED MATERIAL, AS A FUNCTION OF DATE AND SECTION, EAST FORK RIVER, WYOMING, 1979--CONTINUED

\begin{tabular}{|c|c|c|c|c|c|c|c|c|c|}
\hline \multirow{2}{*}{$\begin{array}{l}\text { SECTION } \\
\text { NUMBER }\end{array}$} & \multicolumn{9}{|c|}{ DATE } \\
\hline & $6-18$ & $6-19$ & $6-20$ & $6-21$ & $6-22$ & $6-23$ & $6-24$ & $6-25$ & $6-26$ \\
\hline $\begin{array}{l}0043 \\
0075 \\
0137 \\
0220 \\
0301\end{array}$ & $\begin{array}{l}15 \\
12 \\
23 \\
11 \\
11\end{array}$ & $\begin{array}{r}18 \\
9 \\
18 \\
11 \\
22\end{array}$ & $\begin{array}{l}25 \\
12 \\
16 \\
23 \\
38\end{array}$ & $\begin{array}{r}22 \\
10 \\
15 \\
4 \\
14\end{array}$ & $\begin{array}{l}26 \\
16 \\
18 \\
17 \\
50\end{array}$ & $\begin{array}{r}22 \\
19 \\
9 \\
6 \\
41\end{array}$ & $\begin{array}{l}18 \\
18 \\
23 \\
14 \\
94\end{array}$ & $\begin{array}{r}12 \\
8 \\
6 \\
14 \\
62\end{array}$ & $\begin{array}{l}17 \\
17 \\
16 \\
19 \\
87\end{array}$ \\
\hline $\begin{array}{l}0301(2) \\
0421\end{array}$ & $\overline{46}$ & $\overline{112}$ & $\overline{70}$ & $2 \overline{65}$ & $\overline{121}$ & $\overline{79}$ & $\overline{19}$ & $1 \overline{05}$ & $\overline{43}$ \\
\hline
\end{tabular}

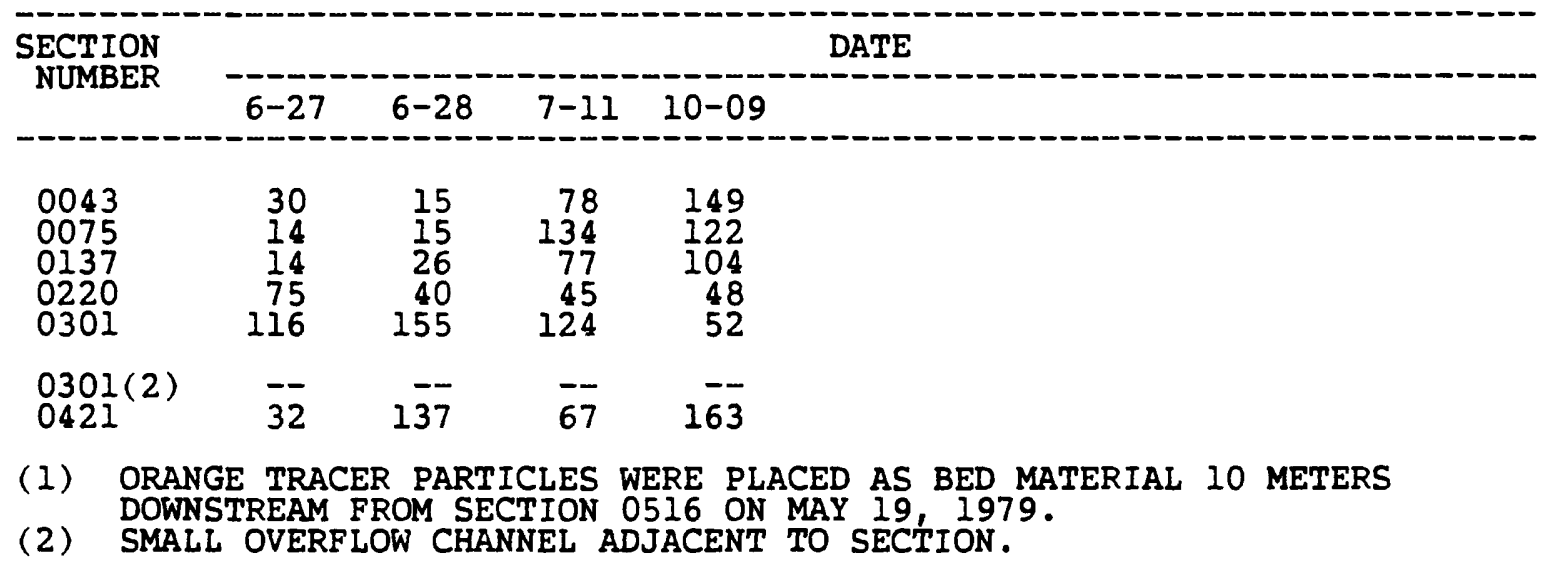


TABLE 8.- NUMBER OF PINK TRACER PARTICLES(1) BETWEEN 0.25 AND 8.00 MILLIMETERS, PER 100 GRAMS OF BEDLOAD, AS A FUNCTION OF DATE AND SECTION, EAST FORK RIVER, WYOMING, 1980

\begin{tabular}{|c|c|c|c|c|c|c|c|c|c|}
\hline \multirow{2}{*}{$\begin{array}{l}\text { SECTION } \\
\text { NUMBER }\end{array}$} & \multicolumn{9}{|c|}{ DATE } \\
\hline & $5-16$ & $5-19$ & $5-21$ & $5-22$ & $5-24$ & $5-26$ & $5-27$ & $5-28$ & $5-29$ \\
\hline $\begin{array}{l}0043 \\
0075 \\
0137 \\
0178 \\
0220\end{array}$ & $\begin{array}{l}0++ \\
0+ \\
0 \\
0 \\
0+\end{array}$ & $\begin{array}{l}0++ \\
0 \\
0+++ \\
0 \\
0\end{array}$ & $\begin{array}{l}0+ \\
0 \\
0++ \\
0 \\
0\end{array}$ & $\begin{array}{l}-- \\
=- \\
=- \\
=-\end{array}$ & $\begin{array}{l}-- \\
-- \\
-- \\
-- \\
--\end{array}$ & $\begin{array}{l}0 \\
4+ \\
0 \\
0 \\
0\end{array}$ & $\begin{array}{l}0+ \\
0+ \\
0 \\
0 \\
0+\end{array}$ & $\begin{array}{l}0++ \\
0 \\
0 \\
3 \\
0\end{array}$ & $\begin{array}{l}-- \\
-- \\
-- \\
--\end{array}$ \\
\hline $\begin{array}{l}0257 \\
0301 \\
0348 \\
0421 \\
0460\end{array}$ & $\begin{array}{l}0+ \\
0+ \\
0 \\
0 \\
0\end{array}$ & $\begin{array}{l}0 \\
0 \\
0 \\
0 \\
0++\end{array}$ & $\begin{array}{l}0 \\
0 \\
0 \\
2 \\
3+\end{array}$ & $\begin{array}{l}-- \\
-- \\
-- \\
-- \\
--\end{array}$ & $\begin{array}{l}-- \\
=- \\
=- \\
--\end{array}$ & $\begin{array}{l}2 \\
0+ \\
1 \\
0 \\
1\end{array}$ & $\begin{array}{l}0 \\
0 \\
0 \\
0 \\
0++\end{array}$ & $\begin{array}{l}0++ \\
0 \\
0 \\
0 \\
0+\end{array}$ & $\begin{array}{l}-- \\
-- \\
-- \\
--\end{array}$ \\
\hline $\begin{array}{l}0516 \\
0556 \\
0602 \\
0653 \\
0708\end{array}$ & $\begin{array}{l}0 \\
0+++ \\
3 \\
0+ \\
0\end{array}$ & $\begin{array}{l}1++ \\
1 \\
0 \\
0+ \\
0\end{array}$ & $\begin{array}{l}0 \\
0 \\
1 \\
0+ \\
0\end{array}$ & $\begin{array}{l}-- \\
-- \\
-- \\
-- \\
--\end{array}$ & $\begin{array}{l}-- \\
=- \\
=- \\
=-\end{array}$ & $\begin{array}{l}0 \\
0 \\
0 \\
0 \\
0\end{array}$ & $\begin{array}{l}0++ \\
0+ \\
0+ \\
0 \\
0\end{array}$ & $\begin{array}{l}0+ \\
0 \\
0 \\
0+ \\
3\end{array}$ & $\begin{array}{l}-- \\
-- \\
-- \\
-\overline{0}\end{array}$ \\
\hline $\begin{array}{l}0757 \\
0808 \\
0853 \\
0898 \\
0940\end{array}$ & $\begin{array}{l}0 \\
0 \\
3 \\
0++ \\
0\end{array}$ & $\begin{array}{l}0 \\
0 \\
3 \\
3+ \\
3+\end{array}$ & $\begin{array}{l}3 \\
0 \\
0 \\
0 \\
1\end{array}$ & $\begin{array}{l}-- \\
=- \\
-- \\
-- \\
--\end{array}$ & $\begin{array}{l}-- \\
-- \\
-- \\
-- \\
--\end{array}$ & $\begin{array}{l}0 \\
0 \\
0 \\
0 \\
0\end{array}$ & $\begin{array}{l}0 \\
0 \\
0 \\
0 \\
0\end{array}$ & $\begin{array}{l}0 \\
1 \\
0 \\
0 \\
0\end{array}$ & $\begin{array}{l}-- \\
=- \\
-- \\
\overline{0++}\end{array}$ \\
\hline $\begin{array}{l}0985 \\
1038 \\
1077 \\
1120 \\
1155\end{array}$ & $\begin{array}{l}0 \\
1+ \\
0 \\
0+ \\
0+++\end{array}$ & $\begin{array}{l}0 \\
1 \\
4+ \\
8+ \\
0+++\end{array}$ & $\begin{array}{l}0 \\
0 \\
0 \\
0 \\
3\end{array}$ & $\begin{array}{r}-- \\
1 \\
0 \\
0 \\
0\end{array}$ & $\begin{array}{c}-\overline{0} \\
1 \\
2 \\
1\end{array}$ & $\begin{array}{l}0 \\
=- \\
=- \\
-- \\
--\end{array}$ & $\begin{array}{l}0+ \\
0+ \\
3+ \\
3+ \\
0 \\
4++\end{array}$ & $\begin{array}{l}0+ \\
0++ \\
0 \\
0 \\
0+\end{array}$ & $\begin{array}{l}-- \\
=- \\
-- \\
--\end{array}$ \\
\hline $\begin{array}{l}1202 \\
1241 \\
1284 \\
1315 \\
1360\end{array}$ & $\begin{array}{c}10 \\
1 \\
0 \\
0 \\
0+\end{array}$ & $\begin{array}{l}1+ \\
4 \\
1 \\
8 \\
4+\end{array}$ & $\begin{array}{c}5 \\
0 \\
1 \\
0 \\
14+\end{array}$ & $\begin{array}{l}0 \\
0 \\
0 \\
3 \\
0\end{array}$ & $\begin{array}{r}2 \\
4 \\
7 \\
10 \\
19\end{array}$ & $\begin{array}{l}=- \\
=- \\
=- \\
-- \\
--\end{array}$ & $\begin{array}{l}3 \\
9++ \\
1 \\
1 \\
0++\end{array}$ & $\begin{array}{l}2+ \\
1 \\
4 \\
0+ \\
1\end{array}$ & $\begin{array}{l}-\overline{14} \\
-- \\
--\end{array}$ \\
\hline $\begin{array}{l}1396 \\
1400(2) \\
1425 \\
1481 \\
1533\end{array}$ & $\begin{array}{c}0 \\
-\frac{0}{0++} \\
0++ \\
3++\end{array}$ & $\begin{array}{l}-\frac{3}{5} \\
5 \\
0+++\end{array}$ & $\begin{array}{l}2 \\
0++ \\
0 \\
3 \\
7\end{array}$ & $\begin{array}{l}0 \\
3 \\
0 \\
5 \\
3\end{array}$ & $\begin{array}{r}4 \\
10 \\
1 \\
7 \\
9\end{array}$ & $\begin{array}{l}=- \\
=- \\
=- \\
--\end{array}$ & $\begin{array}{l}3 \\
0 \\
1+ \\
2 \\
6\end{array}$ & $\begin{array}{l}2 \\
7+ \\
1 \\
5 \\
0\end{array}$ & $\begin{array}{l}-- \\
-- \\
-- \\
-- \\
--\end{array}$ \\
\hline $\begin{array}{l}1573 \\
1610 \\
1662 \\
1695 \\
1730\end{array}$ & $\begin{array}{l}0+++ \\
0++ \\
11 \\
11+ \\
7++\end{array}$ & $\begin{array}{c}0+++ \\
13 \\
9+ \\
6+ \\
44\end{array}$ & $\begin{array}{r}14 \\
5 \\
4 \\
29 \\
12\end{array}$ & $\begin{array}{r}3 \\
4 \\
3 \\
8 \\
14\end{array}$ & $\begin{array}{r}3 \\
10 \\
13 \\
17 \\
31\end{array}$ & $\begin{array}{l}-- \\
-- \\
-- \\
--\end{array}$ & $\begin{array}{l}2 \\
12+ \\
19 \\
17 \\
16\end{array}$ & $\begin{array}{l}1+ \\
6 \\
13 \\
26 \\
3++\end{array}$ & $\begin{array}{l}=- \\
=- \\
=- \\
--\end{array}$ \\
\hline $\begin{array}{l}1766 \\
1800 \\
1830\end{array}$ & $\begin{array}{c}25 \\
6 \\
7+\end{array}$ & $\begin{array}{c}12 \\
7 \\
26+\end{array}$ & $\begin{array}{l}12 \\
35 \\
63\end{array}$ & $\begin{array}{l}15 \\
24 \\
27\end{array}$ & $\begin{array}{l}10 \\
21 \\
15\end{array}$ & $\begin{array}{l}-- \\
=- \\
--\end{array}$ & $\begin{array}{l}12+ \\
27 \\
42\end{array}$ & $\begin{array}{l}28+ \\
24 \\
39\end{array}$ & $\begin{array}{l}0+++ \\
=-\end{array}$ \\
\hline
\end{tabular}


TABLE 8.- NUMBER OF PINK TRACER PARTICLES(1) BETWEEN 0.25 AND 8.00 MILLIMETERS, PER 100 GRAMS OF BEDLOAD, AS A FUNCTION OF DATE AND SECTION, EAST FORK RIVER, WYOMING, 1980--CONTINUED

\begin{tabular}{|c|c|c|c|c|c|c|c|c|c|}
\hline \multirow{2}{*}{$\begin{array}{l}\text { SECTION } \\
\text { NUMBER }\end{array}$} & \multicolumn{9}{|c|}{ DATE } \\
\hline & $5-30$ & $6-01$ & $6-03$ & $6-05$ & $6-07$ & $6-09$ & $6-10$ & $6-11$ & $6-12$ \\
\hline $\begin{array}{l}0043 \\
0075 \\
0137 \\
0178 \\
0220\end{array}$ & $\begin{array}{l}0+++ \\
0+++ \\
0+ \\
0 \\
0+\end{array}$ & $\begin{array}{l}0++ \\
0 \\
0++ \\
0 \\
0++\end{array}$ & $\begin{array}{l}0+++ \\
0 \\
0++ \\
0++ \\
0++\end{array}$ & $\begin{array}{l}0+++ \\
0 \\
0+++ \\
0 \\
0\end{array}$ & $\begin{array}{l}0+++ \\
0 \\
0 \\
0 \\
0\end{array}$ & $\begin{array}{l}0 \\
0 \\
1 \\
0 \\
2\end{array}$ & $\begin{array}{l}2 \\
2 \\
0 \\
0 \\
0\end{array}$ & $\begin{array}{r}0 \\
11 \\
0 \\
0 \\
1\end{array}$ & $\begin{array}{l}0 \\
0 \\
0 \\
0 \\
0\end{array}$ \\
\hline $\begin{array}{l}0257 \\
0301 \\
0348 \\
0421 \\
0460\end{array}$ & $\begin{array}{l}0+ \\
0 \\
0 \\
0 \\
0++\end{array}$ & $\begin{array}{l}0++ \\
0+ \\
0 \\
0 \\
0++\end{array}$ & $\begin{array}{l}0 \\
0 \\
0 \\
0 \\
0+++\end{array}$ & $\begin{array}{l}0 \\
0 \\
0 \\
0+ \\
0++\end{array}$ & $\begin{array}{l}0 \\
0 \\
0 \\
0 \\
0++\end{array}$ & $\begin{array}{l}0 \\
0 \\
0 \\
0 \\
0\end{array}$ & $\begin{array}{l}0 \\
0 \\
0 \\
0 \\
0\end{array}$ & $\begin{array}{l}0 \\
1 \\
1 \\
0 \\
1\end{array}$ & $\begin{array}{l}0 \\
0 \\
0 \\
0 \\
0\end{array}$ \\
\hline $\begin{array}{l}0516 \\
0556 \\
0602 \\
0653 \\
0708\end{array}$ & $\begin{array}{l}3++ \\
0++ \\
6 \\
0++ \\
1+\end{array}$ & $\begin{array}{l}0+++ \\
0++ \\
4+ \\
1 \\
3++\end{array}$ & $\begin{array}{l}0+++ \\
2++ \\
0+ \\
0 \\
0\end{array}$ & $\begin{array}{l}0+++ \\
0+ \\
0 \\
0 \\
0\end{array}$ & $\begin{array}{l}0++ \\
1+ \\
0 \\
0 \\
0\end{array}$ & $\begin{array}{l}0 \\
0 \\
0 \\
0 \\
0\end{array}$ & $\begin{array}{l}0 \\
0 \\
0 \\
0 \\
0\end{array}$ & $\begin{array}{l}\frac{1}{1} \\
\frac{1}{1} \\
\frac{1}{2}\end{array}$ & $\begin{array}{l}0 \\
1 \\
0 \\
1 \\
0\end{array}$ \\
\hline $\begin{array}{l}0757 \\
0808 \\
0853 \\
0898 \\
0940\end{array}$ & $\begin{array}{l}0 \\
1 \\
0 \\
0 \\
2+\end{array}$ & $\begin{array}{l}0+ \\
0 \\
0 \\
2 \\
0+\end{array}$ & $\begin{array}{l}0 \\
0 \\
0 \\
0++ \\
0++\end{array}$ & $\begin{array}{l}0 \\
0 \\
0+++ \\
0++ \\
0+\end{array}$ & $\begin{array}{l}0 \\
0 \\
0 \\
0++ \\
0\end{array}$ & $\begin{array}{l}0 \\
2 \\
1 \\
3 \\
4\end{array}$ & $\begin{array}{c}0+ \\
0 \\
18 \\
7 \\
1\end{array}$ & $\begin{array}{r}19 \\
6 \\
5 \\
8 \\
1\end{array}$ & $\begin{array}{r}10 \\
0 \\
1 \\
1 \\
0\end{array}$ \\
\hline $\begin{array}{l}0985 \\
1038 \\
1077 \\
1120 \\
1155\end{array}$ & $\begin{array}{l}0++ \\
0++ \\
0+ \\
0+ \\
0+\end{array}$ & $\begin{array}{l}0+++ \\
0+++ \\
0+++ \\
0++ \\
0++\end{array}$ & $\begin{array}{l}0++ \\
0+++ \\
4 \\
0++ \\
1++\end{array}$ & $\begin{array}{l}0+++ \\
0+++ \\
0 \\
0+ \\
0+++\end{array}$ & $\begin{array}{c}0++ \\
0+++ \\
1 \\
10 \\
0\end{array}$ & $\begin{array}{l}4 \\
0 \\
3 \\
0 \\
1\end{array}$ & $\begin{array}{l}8 \\
3 \\
7 \\
2 \\
2\end{array}$ & $\begin{array}{l}3 \\
1 \\
0 \\
5 \\
6\end{array}$ & $\begin{array}{l}1 \\
0 \\
1 \\
1 \\
1\end{array}$ \\
\hline $\begin{array}{l}1202 \\
1241 \\
1284 \\
1315 \\
1360\end{array}$ & $\begin{array}{l}0++ \\
0+ \\
0 \\
4 \\
3\end{array}$ & $\begin{array}{l}0++ \\
5 \\
3 \\
2 \\
0++\end{array}$ & $\begin{array}{l}6 \\
6 \\
1 \\
2 \\
5+\end{array}$ & $\begin{array}{l}1 \\
0 \\
0 \\
2+ \\
5\end{array}$ & $\begin{array}{r}4 \\
6 \\
5 \\
10 \\
8\end{array}$ & $\begin{array}{l}1 \\
0 \\
0 \\
0 \\
6\end{array}$ & $\begin{array}{l}1 \\
0 \\
6 \\
4 \\
7\end{array}$ & $\begin{array}{r}1 \\
4 \\
10 \\
3 \\
8\end{array}$ & $\begin{array}{r}5 \\
4 \\
3 \\
3 \\
11\end{array}$ \\
\hline $\begin{array}{l}1396 \\
1400(2) \\
1425 \\
1481 \\
1533\end{array}$ & $\begin{array}{l}5 \\
0+++ \\
2+ \\
3 \\
8\end{array}$ & $\frac{0}{\frac{1}{8}+}$ & $\begin{array}{c}-\frac{5}{2+} \\
6 \\
0\end{array}$ & $\begin{array}{r}-\frac{1}{1} \\
\frac{1}{5} \\
6\end{array}$ & $\begin{array}{l}5 \\
0+++ \\
5 \\
5 \\
5\end{array}$ & $\begin{array}{l}7 \\
4 \\
2 \\
4 \\
9\end{array}$ & $\begin{array}{r}2 \\
9 \\
8 \\
14 \\
8\end{array}$ & $\begin{array}{r}9 \\
10 \\
6 \\
8 \\
4\end{array}$ & $\begin{array}{r}5 \\
26 \\
4 \\
15 \\
10\end{array}$ \\
\hline $\begin{array}{l}1573 \\
1610 \\
1662 \\
1695 \\
1730\end{array}$ & $\begin{array}{c}7 \\
4+ \\
19 \\
13++ \\
0++\end{array}$ & $\begin{array}{l}3+ \\
3 \\
2+ \\
9++ \\
0+++\end{array}$ & $\begin{array}{c}8+ \\
0+ \\
21 \\
8++ \\
9++\end{array}$ & $\begin{array}{c}0++ \\
3+ \\
10+ \\
10++ \\
0+++\end{array}$ & $\begin{array}{l}5^{++} \\
29 \\
21 \\
37 \\
24\end{array}$ & $\begin{array}{r}8 \\
7 \\
14 \\
21 \\
13\end{array}$ & $\begin{array}{l}18 \\
15 \\
10 \\
18 \\
16\end{array}$ & $\begin{array}{l}9 \\
15 \\
15 \\
29 \\
21\end{array}$ & $\begin{array}{l}17 \\
14 \\
21 \\
17 \\
30\end{array}$ \\
\hline $\begin{array}{l}1766 \\
1800 \\
1830\end{array}$ & $\begin{array}{l}32^{7+++} \\
0\end{array}$ & $\begin{array}{l}26++ \\
33 \\
19++\end{array}$ & $\begin{array}{c}42 \\
9++ \\
7+++\end{array}$ & $\begin{array}{l}37 \\
45 \\
0+++\end{array}$ & $\begin{array}{l}18 \\
16 \\
23+\end{array}$ & $\begin{array}{l}13 \\
13 \\
36\end{array}$ & $\begin{array}{r}12 \\
7 \\
21\end{array}$ & $\begin{array}{l}22 \\
14 \\
18\end{array}$ & $\begin{array}{l}15 \\
21 \\
21\end{array}$ \\
\hline
\end{tabular}


TABLE 8.- NUMBER OF PINK TRACER PARTICLES(1) BETWEEN 0.25 AND 8.00 MILLIMETERS, PER 100 GRAMS OF BEDLOAD, AS A FUNCTION OF DATE AND SECTION, EAST FORK RIVER, WYOMING, 1980--CONTINUED

\begin{tabular}{|c|c|c|c|c|c|c|c|c|c|}
\hline \multirow{2}{*}{$\begin{array}{l}\text { SECTION } \\
\text { NUMBER }\end{array}$} & \multicolumn{9}{|c|}{ DATE } \\
\hline & $6-13$ & $6-14$ & $6-15$ & $6-16$ & $6-17$ & $6-18$ & $6-19$ & $6-20$ & $6-21$ \\
\hline $\begin{array}{l}0043 \\
0075 \\
0137 \\
0178 \\
0220\end{array}$ & $\begin{array}{l}0 \\
0 \\
0 \\
0 \\
0\end{array}$ & $\begin{array}{l}0 \\
0 \\
0 \\
1 \\
0\end{array}$ & $\begin{array}{l}0 \\
0 \\
0 \\
1 \\
2\end{array}$ & $\begin{array}{l}=- \\
=- \\
=- \\
--\end{array}$ & $\begin{array}{l}0 \\
0 \\
0 \\
0 \\
0\end{array}$ & $\begin{array}{l}0 \\
0 \\
0 \\
0 \\
0\end{array}$ & $\begin{array}{l}0 \\
0 \\
0 \\
0 \\
0\end{array}$ & $\begin{array}{l}0 \\
0 \\
0 \\
0 \\
0\end{array}$ & $\begin{array}{l}0 \\
0 \\
0 \\
0 \\
0\end{array}$ \\
\hline $\begin{array}{l}0257 \\
0301 \\
0348 \\
0421 \\
0460\end{array}$ & $\begin{array}{l}0 \\
0 \\
0 \\
0 \\
0\end{array}$ & $\begin{array}{l}0 \\
1 \\
1 \\
0 \\
0\end{array}$ & $\begin{array}{l}1 \\
0 \\
0 \\
0 \\
0\end{array}$ & $\begin{array}{r}1 \\
0 \\
0 \\
0 \\
--\end{array}$ & $\begin{array}{l}0 \\
0 \\
0 \\
1 \\
0\end{array}$ & $\begin{array}{l}0 \\
0 \\
0 \\
0 \\
0\end{array}$ & $\begin{array}{l}0 \\
0 \\
0 \\
0 \\
0\end{array}$ & $\begin{array}{r}2 \\
1 \\
0 \\
2 \\
10\end{array}$ & $\begin{array}{l}0 \\
0 \\
0 \\
2 \\
0\end{array}$ \\
\hline $\begin{array}{l}0516 \\
0556 \\
0602 \\
0653 \\
0708\end{array}$ & $\begin{array}{l}0 \\
0 \\
0 \\
7 \\
3\end{array}$ & $\begin{array}{l}0 \\
1 \\
0 \\
7 \\
0\end{array}$ & $\begin{array}{l}2 \\
2 \\
1 \\
5 \\
1\end{array}$ & $\begin{array}{l}-- \\
-- \\
-- \\
-\overline{0}\end{array}$ & $\begin{array}{l}0 \\
1 \\
8 \\
3 \\
1\end{array}$ & $\begin{array}{l}0 \\
1 \\
1 \\
2 \\
0\end{array}$ & $\begin{array}{l}2 \\
2 \\
1 \\
0 \\
0\end{array}$ & $\begin{array}{l}1 \\
1 \\
0 \\
1 \\
0\end{array}$ & $\begin{array}{l}0 \\
0 \\
0 \\
0 \\
1\end{array}$ \\
\hline $\begin{array}{l}0757 \\
0808 \\
0853 \\
0898 \\
0940\end{array}$ & $\begin{array}{l}6 \\
2 \\
2 \\
2 \\
0\end{array}$ & $\begin{array}{l}2 \\
0 \\
2 \\
0 \\
2\end{array}$ & $\begin{array}{l}1 \\
4 \\
1 \\
0 \\
1\end{array}$ & $\begin{array}{r}0 \\
0 \\
0 \\
---\end{array}$ & $\begin{array}{l}1 \\
1 \\
1 \\
0 \\
0\end{array}$ & $\begin{array}{l}0 \\
1 \\
0 \\
0 \\
0\end{array}$ & $\begin{array}{l}0 \\
2 \\
0 \\
0 \\
0\end{array}$ & $\begin{array}{l}2 \\
2 \\
4 \\
0 \\
0\end{array}$ & $\begin{array}{l}1 \\
3 \\
0 \\
2 \\
1\end{array}$ \\
\hline $\begin{array}{l}0985 \\
1038 \\
1077 \\
1120 \\
1155\end{array}$ & $\begin{array}{l}0 \\
3 \\
0 \\
0 \\
0\end{array}$ & $\begin{array}{l}0 \\
2 \\
0 \\
0 \\
1\end{array}$ & $\begin{array}{l}0 \\
1 \\
0 \\
1 \\
0\end{array}$ & $\begin{array}{l}=- \\
=- \\
=- \\
--\end{array}$ & $\begin{array}{l}1 \\
2 \\
2 \\
0 \\
7\end{array}$ & $\begin{array}{l}0 \\
0 \\
0 \\
1 \\
7\end{array}$ & $\begin{array}{r}1 \\
4 \\
2 \\
15 \\
6\end{array}$ & $\begin{array}{r}8 \\
2 \\
6 \\
10 \\
6\end{array}$ & $\begin{array}{l}3 \\
2 \\
5 \\
7 \\
6\end{array}$ \\
\hline $\begin{array}{l}1202 \\
1241 \\
1284 \\
1315 \\
1360\end{array}$ & $\begin{array}{l}0 \\
0 \\
6 \\
4 \\
2\end{array}$ & $\begin{array}{r}0 \\
2 \\
11 \\
4 \\
11\end{array}$ & $\begin{array}{r}3 \\
4 \\
7 \\
12 \\
11\end{array}$ & $\begin{array}{l}-\overline{11} \\
13 \\
17 \\
12\end{array}$ & $\begin{array}{r}10 \\
9 \\
14 \\
9 \\
13\end{array}$ & $\begin{array}{r}3 \\
1 \\
2 \\
12 \\
8\end{array}$ & $\begin{array}{r}3 \\
3 \\
4 \\
10 \\
13\end{array}$ & $\begin{array}{r}9 \\
7 \\
20 \\
9 \\
6\end{array}$ & $\begin{array}{r}4 \\
7 \\
8 \\
16 \\
12\end{array}$ \\
\hline $\begin{array}{l}1396 \\
1400(2) \\
1425 \\
1481 \\
1533\end{array}$ & $\begin{array}{r}3 \\
9 \\
1 \\
9 \\
17\end{array}$ & $\begin{array}{r}3 \\
18 \\
7 \\
6 \\
7\end{array}$ & $\begin{array}{l}8 \\
13 \\
16 \\
18 \\
10\end{array}$ & $\begin{array}{r}10 \\
19 \\
5 \\
9 \\
--\end{array}$ & $\begin{array}{l}17 \\
17 \\
3 \\
16 \\
15\end{array}$ & $\begin{array}{r}9 \\
9 \\
7 \\
19 \\
15\end{array}$ & $\begin{array}{r}4 \\
19 \\
7 \\
12 \\
15\end{array}$ & $\begin{array}{r}7 \\
12 \\
6 \\
11 \\
23\end{array}$ & $\begin{array}{r}9 \\
17 \\
18 \\
20 \\
17\end{array}$ \\
\hline $\begin{array}{l}1573 \\
1610 \\
1662 \\
1695 \\
1730\end{array}$ & $\begin{array}{r}9 \\
4 \\
21 \\
14 \\
14\end{array}$ & $\begin{array}{l}14 \\
13 \\
22 \\
14 \\
28\end{array}$ & $\begin{array}{l}10 \\
16 \\
24 \\
10 \\
10\end{array}$ & $\begin{array}{l}-- \\
=- \\
--\end{array}$ & $\begin{array}{l}16 \\
19 \\
16 \\
17 \\
15\end{array}$ & $\begin{array}{l}11 \\
18 \\
16 \\
15 \\
18\end{array}$ & $\begin{array}{l}23 \\
16 \\
26 \\
8 \\
18\end{array}$ & $\begin{array}{l}8 \\
27 \\
11 \\
22 \\
13\end{array}$ & $\begin{array}{r}19 \\
25 \\
20 \\
21 \\
6\end{array}$ \\
\hline $\begin{array}{l}1766 \\
1800 \\
1830\end{array}$ & $\begin{array}{r}8 \\
16 \\
21\end{array}$ & $\begin{array}{r}0 \\
20 \\
19\end{array}$ & $\begin{array}{l}15 \\
36 \\
26\end{array}$ & $\begin{array}{l}-- \\
--\end{array}$ & $\begin{array}{l}13 \\
30 \\
19\end{array}$ & $\begin{array}{l}15 \\
25 \\
18\end{array}$ & $\begin{array}{l}21 \\
22 \\
19\end{array}$ & $\begin{array}{l}18 \\
15 \\
18\end{array}$ & $\begin{array}{l}12 \\
13 \\
16\end{array}$ \\
\hline
\end{tabular}


TABLE 8.- NUMBER OF PINK TRACER PARTICLES(1) BETWEEN 0.25 AND 8.00 MILLIMETERS, PER 100 GRAMS OF BEDLOAD, AS A FUNCTION OF DATE AND SECTION, EAST FORK RIVER, WYOMING, 1980--CONTINUED

\begin{tabular}{|c|c|c|c|c|c|c|c|c|c|}
\hline SECTION & & & & & DATE & & & & \\
\hline & $6-22$ & $6-23$ & $6-25$ & $6-27$ & $6-29$ & $7-01$ & $7-03$ & $7-05$ & $7-07$ \\
\hline $\begin{array}{l}0043 \\
0075 \\
0137 \\
0178 \\
0220\end{array}$ & $\begin{array}{l}=- \\
=- \\
=- \\
--\end{array}$ & $\begin{array}{l}0 \\
3 \\
0 \\
0 \\
3\end{array}$ & $\begin{array}{r}0 \\
0 \\
21 \\
1 \\
1\end{array}$ & $\begin{array}{l}1 \\
1 \\
1 \\
0 \\
0\end{array}$ & $\begin{array}{l}=- \\
=- \\
=- \\
-=\end{array}$ & $\begin{array}{l}=- \\
=- \\
=- \\
--\end{array}$ & $\begin{array}{l}= \\
- \\
-- \\
-\end{array}$ & $\begin{array}{l}=- \\
-\overline{-} \\
-\overline{-}\end{array}$ & $\begin{array}{l}= \\
= \\
=\end{array}$ \\
\hline $\begin{array}{l}0257 \\
0301 \\
0348 \\
0421 \\
0460\end{array}$ & $\begin{array}{l}=- \\
=- \\
=- \\
--\end{array}$ & $\begin{array}{l}0 \\
2 \\
2 \\
0 \\
3\end{array}$ & $\begin{array}{r}0 \\
0 \\
11 \\
1 \\
0\end{array}$ & $\begin{array}{l}0 \\
1 \\
3 \\
0 \\
1\end{array}$ & $\begin{array}{l}-- \\
-\overline{2} \\
--\end{array}$ & $\begin{array}{l}=- \\
=- \\
=- \\
--\end{array}$ & $\begin{array}{l}=- \\
=- \\
=-\end{array}$ & $\begin{array}{l}= \\
-\overline{-} \\
-\end{array}$ & $\begin{array}{l}= \\
-- \\
-- \\
-\end{array}$ \\
\hline $\begin{array}{l}0516 \\
0556 \\
0602 \\
0653 \\
0708\end{array}$ & $\begin{array}{l}=- \\
=- \\
=- \\
--\end{array}$ & $\begin{array}{l}0 \\
0 \\
2 \\
1 \\
4\end{array}$ & $\begin{array}{r}\frac{1}{3} \\
1 \\
17 \\
0\end{array}$ & $\begin{array}{l}0 \\
6 \\
2 \\
3 \\
3\end{array}$ & $\begin{array}{l}= \\
= \\
=-\end{array}$ & $\begin{array}{l}=- \\
=- \\
--\end{array}$ & $\begin{array}{l}=- \\
\overline{-} \\
=-\end{array}$ & $\begin{array}{l}=- \\
=- \\
=-\end{array}$ & $\begin{array}{l}= \\
=- \\
=\end{array}$ \\
\hline $\begin{array}{l}0757 \\
0808 \\
0853 \\
0898 \\
0940\end{array}$ & $\begin{array}{r}-\overline{0} \\
1 \\
1 \frac{1}{3}\end{array}$ & $\begin{array}{l}0 \\
0 \\
0 \\
7 \\
1\end{array}$ & $\begin{array}{l}0 \\
1 \\
1 \\
6 \\
0\end{array}$ & $\begin{array}{l}1 \\
2 \\
2 \\
3 \\
1\end{array}$ & $\begin{array}{l}-\overline{2} \\
-- \\
--\end{array}$ & $\begin{array}{l}=- \\
=- \\
=-\end{array}$ & $\begin{array}{l}= \\
= \\
= \\
-\end{array}$ & $\begin{array}{l}= \\
=- \\
= \\
=\end{array}$ & $\begin{array}{l}= \\
=- \\
=-\end{array}$ \\
\hline $\begin{array}{l}0985 \\
1038 \\
1077 \\
1120 \\
1155\end{array}$ & $\begin{array}{r}8 \\
--- \\
--\end{array}$ & $\begin{array}{r}2 \\
5 \\
4 \\
10 \\
3\end{array}$ & $\begin{array}{r}5 \\
25 \\
25 \\
5 \\
9\end{array}$ & $\begin{array}{l}6 \\
11 \\
17 \\
17 \\
11\end{array}$ & $\begin{array}{l}=- \\
=- \\
=- \\
--\end{array}$ & $\begin{array}{l}=- \\
=- \\
--\end{array}$ & $\begin{array}{l}=- \\
=- \\
=- \\
--\end{array}$ & $\begin{array}{l}-\overline{-} \\
=- \\
--\end{array}$ & $\begin{array}{l}-= \\
=- \\
-- \\
--\end{array}$ \\
\hline $\begin{array}{l}1202 \\
1241 \\
1284 \\
1315 \\
1360\end{array}$ & $\begin{array}{l}-- \\
-- \\
=-\end{array}$ & $\begin{array}{r}16 \\
7 \\
10 \\
9 \\
8\end{array}$ & $\begin{array}{r}8 \\
15 \\
21 \\
26 \\
13\end{array}$ & $\begin{array}{l}11 \\
18 \\
25 \\
27 \\
20\end{array}$ & $\begin{array}{l}-= \\
-= \\
-- \\
--\end{array}$ & $\begin{array}{l}=- \\
=- \\
=-\end{array}$ & $\begin{array}{l}=- \\
=- \\
=-\end{array}$ & $\begin{array}{l}=- \\
=- \\
=-\end{array}$ & $\begin{array}{l}=- \\
=- \\
=- \\
-=\end{array}$ \\
\hline $\begin{array}{l}1396 \\
1400(2) \\
1425 \\
1481 \\
1533\end{array}$ & $\begin{array}{l}=- \\
=- \\
=- \\
=-\end{array}$ & $\begin{array}{r}6 \\
7 \\
19 \\
18 \\
9\end{array}$ & $\begin{array}{r}8 \\
24 \\
14 \\
21 \\
16\end{array}$ & $\begin{array}{r}15 \\
0 \\
14 \\
28 \\
24\end{array}$ & $\begin{array}{l}-- \\
-\overline{-} \\
--\end{array}$ & $\begin{array}{l}-\overline{-} \\
=- \\
=-\end{array}$ & $\begin{array}{l}\overline{-} \\
\overline{14}\end{array}$ & $\begin{array}{l}\overline{-} \\
\overline{-} \\
\overline{13}\end{array}$ & $\begin{array}{l}=- \\
=- \\
\overline{1}\end{array}$ \\
\hline $\begin{array}{l}1573 \\
1610 \\
1662 \\
1695 \\
1730\end{array}$ & $\begin{array}{l}= \\
-\overline{-} \\
--\end{array}$ & $\begin{array}{l}13 \\
21 \\
17 \\
12 \\
17\end{array}$ & $\begin{array}{r}28 \\
28 \\
15 \\
14 \\
6\end{array}$ & $\begin{array}{l}22 \\
29 \\
29 \\
21 \\
17\end{array}$ & $\begin{array}{l}-- \\
-- \\
-- \\
--\end{array}$ & $\begin{array}{l}20 \\
-- \\
--\end{array}$ & $\begin{array}{l}= \\
=- \\
-=\end{array}$ & $\begin{array}{l}17 \\
19+ \\
=- \\
=-\end{array}$ & $\begin{array}{l}15 \\
0+++ \\
-= \\
=-\end{array}$ \\
\hline $\begin{array}{l}1766 \\
1800 \\
1830\end{array}$ & $\begin{array}{l}-- \\
--\end{array}$ & $\begin{array}{l}21 \\
16 \\
20\end{array}$ & $\begin{array}{l}17 \\
21 \\
22\end{array}$ & $\begin{array}{l}21 \\
25 \\
18\end{array}$ & $\begin{array}{l}-- \\
--\end{array}$ & $\begin{array}{l}-- \\
--\end{array}$ & $=$ & $=-$ & $\begin{array}{l}-\overline{-} \\
--\end{array}$ \\
\hline
\end{tabular}


TABLE 8.- NUMBER OF PINK TRACER PARTICLES(1) BETWEEN 0.25 AND 8.00 MILLIMETERS, PER 100 GRAMS OF BEDLOAD, AS A FUNCTION OF DATE AND SECTION, EAST FORK RIVER, WYOMING, 1980--CONTINUED

(1) PINK TRACER PARTICLES WERE PLACED AS BED MATERIAL 10 METERS

DOWNSTREAM FROM SECTION 3047 ON MAY 18, 1979.

(2) BYPASS CHANNEL. SEE FIGURE 3.

+ MASS OF SAMPLE ANALYZED WAS BETWEEN 50 AND 100 GRAMS; NUMBER OF TRACER PARTICLES ADJUSTED TO AN EQU IVALENT 100-GRAM SAMPLE; TRACER PARTICLES ADJUSTED TO AN EQU IVALENT 100

+ MASS OF SAMPLE ANALYZED WAS BETWEEN 10 AND 50 GRAMS; NUMBER OF TRACER PARTICLES ADJUSTED TO AN EQUIVALENT 100-GRAM SAMPLE; CARE SHOULD BE TAKEN IN USING THE EXTRAPOLATED NUMBERS.

+++ MASS OF SAMPLE ANALYZED WAS BETWEEN 0 AND 10 GRAMS: NUMBER OF TRACER PARTICLES ADJUSTED TO AN EOUIVALENT 100-GRAM SAMPLE; EXTRAPOLATED NUMBERS ARE NOT CONSIDERED RELIABLE. 
TABLE 9.- NUMBER OF BLUE TRACER PARTICLES( 1 ) BETWEEN 0.25 AND 8.00 MILLIMETERS, PER 100 GRAMS OF BEDLOAD, AS A FUNCTION OF DATE AND SECTION, EAST FORK RIVER, WYOMING, 1980

\begin{tabular}{|c|c|c|c|c|c|c|c|c|c|}
\hline \multirow{2}{*}{$\begin{array}{l}\text { SECTION } \\
\text { NUMBER }\end{array}$} & \multicolumn{9}{|c|}{ DATE } \\
\hline & $5-16$ & $5-19$ & $5-21$ & $5-22$ & $5-24$ & $5-26$ & $5-27$ & $5-28$ & $5-29$ \\
\hline $\begin{array}{l}0043 \\
0075 \\
0137 \\
0178 \\
0220\end{array}$ & $\begin{array}{l}0++ \\
8+ \\
9 \\
8 \\
5+\end{array}$ & $\begin{array}{l}1++ \\
10 \\
0+++ \\
12 \\
18\end{array}$ & $\begin{array}{l}7^{+} \\
8^{7++} \\
17 \\
15\end{array}$ & $\begin{array}{l}-- \\
=- \\
=- \\
--\end{array}$ & $\begin{array}{l}-- \\
-- \\
=- \\
--\end{array}$ & $\begin{array}{l}3 \\
2+ \\
4 \\
2 \\
0\end{array}$ & $\begin{array}{l}2+ \\
0+ \\
0 \\
3 \\
4+\end{array}$ & $\begin{array}{l}0++ \\
0 \\
3 \\
0 \\
2\end{array}$ & $\begin{array}{l}\overline{18} \\
\overline{-} \\
--\end{array}$ \\
\hline $\begin{array}{l}0257 \\
0301 \\
0348 \\
0421 \\
0460\end{array}$ & $\begin{array}{l}24+ \\
15+ \\
3 \\
11 \\
10\end{array}$ & $\begin{array}{l}19 \\
11 \\
7 \\
18 \\
4++\end{array}$ & $\begin{array}{c}7 \\
6 \\
3 \\
19 \\
17+\end{array}$ & $\begin{array}{l}-- \\
=- \\
--\end{array}$ & $\begin{array}{l}=- \\
=- \\
=- \\
--\end{array}$ & $\begin{array}{c}7 \\
2+ \\
9 \\
14 \\
16\end{array}$ & $\begin{array}{l}8 \\
5 \\
4 \\
7 \\
0++\end{array}$ & $\begin{array}{c}7++ \\
14 \\
8 \\
5 \\
4+\end{array}$ & $\begin{array}{l}-\overline{11} \\
\overline{-} \\
--\end{array}$ \\
\hline $\begin{array}{l}0516 \\
0556 \\
0602 \\
0653 \\
0708\end{array}$ & $\begin{array}{l}30 \\
8+++ \\
46 \\
26+ \\
53\end{array}$ & $\begin{array}{l}10++ \\
39 \\
41 \\
53+ \\
32\end{array}$ & $\begin{array}{l}27 \\
26 \\
23 \\
23+ \\
29\end{array}$ & $\begin{array}{l}=- \\
=- \\
=-\end{array}$ & $\begin{array}{l}-- \\
-- \\
=- \\
--\end{array}$ & $\begin{array}{r}19 \\
8 \\
16 \\
34 \\
43\end{array}$ & $\begin{array}{l}2++ \\
12+ \\
32+ \\
52 \\
36\end{array}$ & $\begin{array}{l}10+ \\
12 \\
24 \\
42+ \\
60^{+}\end{array}$ & $\begin{array}{l}-- \\
\overline{--} \\
\overline{32}\end{array}$ \\
\hline $\begin{array}{l}0757 \\
0808 \\
0853 \\
0898 \\
0940\end{array}$ & $\begin{array}{l}46 \\
51 \\
34 \\
0++ \\
37\end{array}$ & $\begin{array}{l}32 \\
33 \\
25 \\
39+ \\
19+\end{array}$ & $\begin{array}{l}45 \\
32 \\
31 \\
14 \\
31\end{array}$ & $\begin{array}{l}-- \\
-- \\
-- \\
--\end{array}$ & $\begin{array}{l}-- \\
=- \\
=- \\
--\end{array}$ & $\begin{array}{l}34 \\
41 \\
55 \\
24 \\
21\end{array}$ & $\begin{array}{l}50 \\
38 \\
18 \\
27 \\
39\end{array}$ & $\begin{array}{l}37 \\
49 \\
32 \\
29 \\
19\end{array}$ & $\begin{array}{l}-- \\
\overline{--} \\
\overline{21++}\end{array}$ \\
\hline $\begin{array}{l}0985 \\
1038 \\
1077 \\
1120 \\
1155\end{array}$ & $\begin{array}{l}46 \\
31+ \\
29 \\
17+ \\
0+++\end{array}$ & $\begin{array}{l}36 \\
46 \\
29+ \\
14+ \\
0+++\end{array}$ & $\begin{array}{l}26 \\
46 \\
21 \\
17 \\
16\end{array}$ & $\begin{array}{r}-\overline{3} \\
23 \\
11 \\
6\end{array}$ & $\begin{array}{l}--\overline{25} \\
28 \\
18 \\
25\end{array}$ & $\begin{array}{l}38 \\
=- \\
-- \\
--\end{array}$ & $\begin{array}{l}15+ \\
15+ \\
20+ \\
17 \\
20++\end{array}$ & $\begin{array}{l}12+ \\
0++ \\
20 \\
16 \\
10+\end{array}$ & $\begin{array}{l}-- \\
=- \\
=- \\
--\end{array}$ \\
\hline $\begin{array}{l}1202 \\
1241 \\
1284 \\
1315 \\
1360\end{array}$ & $\begin{array}{r}16 \\
9 \\
5 \\
4 \\
5+\end{array}$ & $\begin{array}{l}8+ \\
7 \\
2 \\
3 \\
3+\end{array}$ & $\begin{array}{c}21 \\
14 \\
3 \\
0 \\
0+\end{array}$ & $\begin{array}{r}10 \\
12 \\
3 \\
7 \\
1\end{array}$ & $\begin{array}{r}22 \\
22 \\
7 \\
29 \\
2\end{array}$ & $\begin{array}{l}=- \\
=- \\
=- \\
--\end{array}$ & $\begin{array}{l}2 \frac{1}{2++} \\
2 \\
4 \\
7++\end{array}$ & $\begin{array}{c}1+ \\
9 \\
11 \\
0+ \\
6\end{array}$ & $\begin{array}{l}-- \\
-- \\
--\end{array}$ \\
\hline $\begin{array}{l}1396 \\
1400(2) \\
1425 \\
1481 \\
1533\end{array}$ & $\begin{array}{c}2 \\
-\frac{2}{4++} \\
6++ \\
0++\end{array}$ & $\begin{array}{l}-0 \\
-4 \\
2 \\
0+++\end{array}$ & $\begin{array}{l}2 \\
0++ \\
6 \\
2 \\
0\end{array}$ & $\begin{array}{r}0 \\
19 \\
1 \\
1 \\
3\end{array}$ & $\begin{array}{r}1 \\
19 \\
4 \\
3 \\
5\end{array}$ & $\begin{array}{l}-- \\
=- \\
=- \\
--\end{array}$ & $\begin{array}{c}4 \\
10 \\
2+ \\
6 \\
3\end{array}$ & $\begin{array}{c}10 \\
15+ \\
5 \\
2 \\
2\end{array}$ & $\begin{array}{l}-- \\
-- \\
-- \\
--\end{array}$ \\
\hline $\begin{array}{l}1573 \\
1610 \\
1662\end{array}$ & $\begin{array}{l}0+++ \\
4++ \\
0\end{array}$ & $\begin{array}{l}0+++ \\
2 \\
2+\end{array}$ & $\begin{array}{l}4 \\
0 \\
0\end{array}$ & $\begin{array}{l}4 \\
3 \\
4\end{array}$ & $\begin{array}{r}13 \\
3 \\
6\end{array}$ & $\begin{array}{l}-- \\
--\end{array}$ & $\begin{array}{l}5 \\
2+ \\
0\end{array}$ & $\begin{array}{l}5+ \\
0 \\
0\end{array}$ & $\begin{array}{l}-- \\
--\end{array}$ \\
\hline
\end{tabular}


TABLE 9.- NUMBER OF BLUE TRACER PARTICLES(1) BETWEEN 0.25 AND 8.00 MILLIMETERS, PER 100 GRAMS OF BEDLOAD, AS A FUNCTION OF DATE AND SECTION, EAST FORK RIVER, WYOMING, 1980--CONTINUED

\begin{tabular}{|c|c|c|c|c|c|c|c|c|c|}
\hline \multirow{2}{*}{$\begin{array}{c}\text { SECTION } \\
\text { NUMBER }\end{array}$} & \multicolumn{9}{|c|}{ DATE } \\
\hline & $5-30$ & $6-01$ & $6-03$ & $6-05$ & $6-07$ & $6-09$ & $6-10$ & $6-11$ & $6-12$ \\
\hline $\begin{array}{l}0043 \\
0075 \\
0137 \\
0178 \\
0220\end{array}$ & $\begin{array}{l}0+++ \\
0+++ \\
4+ \\
4 \\
7 .+\end{array}$ & $\begin{array}{c}4++ \\
2 \\
2++ \\
10 \\
7++\end{array}$ & $\begin{array}{l}0+++ \\
7 \\
4++ \\
0++ \\
3++\end{array}$ & $\begin{array}{l}0+++ \\
3 \\
0+++ \\
12 \\
25\end{array}$ & $\begin{array}{l}0+++ \\
0 \\
9 \\
21 \\
19\end{array}$ & $\begin{array}{r}6 \\
12 \\
16 \\
16 \\
13\end{array}$ & $\begin{array}{r}11 \\
4 \\
15 \\
8 \\
9\end{array}$ & $\begin{array}{r}4 \\
6 \\
18 \\
14 \\
10\end{array}$ & $\begin{array}{r}11 \\
0 \\
8 \\
10 \\
8\end{array}$ \\
\hline $\begin{array}{l}0257 \\
0301 \\
0348 \\
0421 \\
0460\end{array}$ & $\begin{array}{c}9+ \\
8 \\
27 \\
17 \\
6++\end{array}$ & $\begin{array}{l}9++ \\
20+ \\
18 \\
29 \\
12++\end{array}$ & $\begin{array}{l}23 \\
21 \\
30 \\
14 \\
0+++\end{array}$ & $\begin{array}{l}18 \\
16 \\
25 \\
2+ \\
4++\end{array}$ & $\begin{array}{l}14 \\
26 \\
25 \\
29 \\
0++\end{array}$ & $\begin{array}{l}9 \\
16 \\
20 \\
11 \\
11\end{array}$ & $\begin{array}{r}6 \\
11 \\
7 \\
25 \\
16\end{array}$ & $\begin{array}{r}5 \\
9 \\
8 \\
25 \\
26\end{array}$ & $\begin{array}{l}20 \\
16 \\
3 \\
21 \\
23\end{array}$ \\
\hline $\begin{array}{l}0516 \\
0556 \\
0602 \\
0653 \\
0708\end{array}$ & $\begin{array}{l}18++ \\
13++ \\
36 \\
31++ \\
65+\end{array}$ & $\begin{array}{c}12+++ \\
6++ \\
118+ \\
50 \\
64++\end{array}$ & $\begin{array}{l}20+++ \\
11++ \\
27+ \\
46 \\
24\end{array}$ & $\begin{array}{l}5+++ \\
8+ \\
50 \\
46 \\
33\end{array}$ & $\begin{array}{l}2++ \\
24+ \\
46 \\
45 \\
29\end{array}$ & $\begin{array}{r}6 \\
36 \\
32 \\
29 \\
34\end{array}$ & $\begin{array}{l}14 \\
31 \\
39 \\
38 \\
19\end{array}$ & $\begin{array}{l}25 \\
24 \\
14 \\
13 \\
19\end{array}$ & $\begin{array}{r}21 \\
19 \\
13 \\
8 \\
14\end{array}$ \\
\hline $\begin{array}{l}0757 \\
0808 \\
0853 \\
0898 \\
0940\end{array}$ & $\begin{array}{l}37 \\
27 \\
23 \\
27 \\
0+\end{array}$ & $\begin{array}{l}38+ \\
29 \\
19 \\
25 \\
30+\end{array}$ & $\begin{array}{l}44 \\
32 \\
25 \\
17++ \\
11++\end{array}$ & $\begin{array}{l}37 \\
30 \\
0+++ \\
22++ \\
21+\end{array}$ & $\begin{array}{l}29 \\
16 \\
20 \\
15++ \\
32\end{array}$ & $\begin{array}{r}34 \\
0 \\
44 \\
19 \\
12\end{array}$ & $\begin{array}{l}9+ \\
36 \\
23 \\
26 \\
27\end{array}$ & $\begin{array}{l}10 \\
10 \\
30 \\
9 \\
16\end{array}$ & $\begin{array}{l}24 \\
23 \\
23 \\
21 \\
31\end{array}$ \\
\hline $\begin{array}{l}0985 \\
1038 \\
1077 \\
1120 \\
1155\end{array}$ & $\begin{array}{l}20++ \\
27++ \\
26+ \\
5+ \\
56+\end{array}$ & $\begin{array}{c}0+++ \\
0+++ \\
0+++ \\
17++ \\
2++\end{array}$ & $\begin{array}{c}5++ \\
18+++ \\
7 \\
5++ \\
3++\end{array}$ & $\begin{array}{l}0+++ \\
0+++ \\
7 \\
4+ \\
0+++\end{array}$ & $\begin{array}{c}20++ \\
0+++ \\
6 \\
29 \\
17\end{array}$ & $\begin{array}{r}20 \\
6 \\
4 \\
1 \\
15\end{array}$ & $\begin{array}{l}39 \\
21 \\
13 \\
14 \\
20\end{array}$ & $\begin{array}{r}18 \\
9 \\
1 \\
21 \\
22\end{array}$ & $\begin{array}{l}19 \\
13 \\
15 \\
14 \\
16\end{array}$ \\
\hline $\begin{array}{l}1202 \\
1241 \\
1284 \\
1315 \\
1360\end{array}$ & $\begin{array}{l}9++ \\
6+ \\
9 \\
5 \\
0\end{array}$ & $\begin{array}{l}3++ \\
11 \\
12 \\
4 \\
0++\end{array}$ & $\begin{array}{c}11 \\
12 \\
2 \\
9 \\
2+\end{array}$ & $\begin{array}{c}12 \\
7 \\
5 \\
3+ \\
3\end{array}$ & $\begin{array}{r}7 \\
9 \\
2 \\
11 \\
3\end{array}$ & $\begin{array}{l}7 \\
5 \\
0 \\
5 \\
0\end{array}$ & $\begin{array}{r}8 \\
3 \\
9 \\
10 \\
3\end{array}$ & $\begin{array}{r}8 \\
12 \\
6 \\
6 \\
3\end{array}$ & $\begin{array}{r}11 \\
12 \\
2 \\
6 \\
1\end{array}$ \\
\hline $\begin{array}{l}1396 \\
1400(2) \\
1425 \\
1481 \\
1533\end{array}$ & $\begin{array}{l}7 \\
0+++ \\
1+ \\
4 \\
3\end{array}$ & $\begin{array}{c}0 \\
-\frac{4}{4} \\
5+ \\
3\end{array}$ & $\begin{array}{c}-2 \\
-0+ \\
3 \\
0\end{array}$ & $\begin{array}{r}1 \\
-\frac{1}{0} \\
2 \\
1\end{array}$ & $\begin{array}{l}1 \\
0+++ \\
9 \\
1 \\
4\end{array}$ & $\begin{array}{l}0 \\
0 \\
0 \\
0 \\
0\end{array}$ & $\begin{array}{l}1 \\
7 \\
3 \\
1 \\
1\end{array}$ & $\begin{array}{l}1 \\
6 \\
3 \\
3 \\
0\end{array}$ & $\begin{array}{l}2 \\
5 \\
1 \\
2 \\
0\end{array}$ \\
\hline $\begin{array}{l}1573 \\
1610 \\
1662\end{array}$ & $\begin{array}{l}3 \\
3+ \\
0\end{array}$ & $\begin{array}{l}3+ \\
0 \\
0+\end{array}$ & $\begin{array}{l}4+ \\
0+ \\
0\end{array}$ & $\begin{array}{c}16++ \\
3+ \\
0+\end{array}$ & $\begin{array}{l}0++ \\
0 \\
0\end{array}$ & $\begin{array}{l}0 \\
0 \\
0\end{array}$ & $\begin{array}{l}1 \\
1 \\
0\end{array}$ & $\begin{array}{l}\frac{1}{2} \\
2\end{array}$ & $\begin{array}{l}1 \\
1 \\
0\end{array}$ \\
\hline
\end{tabular}


TABLE 9.- NUMBER OF BLUE TRACER PARTICLES(I) BETWEEN 0.25 AND 8.00 MILLIMETERS, PER 100 GRAMS OF BEDLOAD, AS A FUNCTION OF DATE AND SECTION, EAST FORK RIVER, WYOMING; 1980--CONTINUED

\begin{tabular}{|c|c|c|c|c|c|c|c|c|c|}
\hline \multirow{2}{*}{$\begin{array}{l}\text { SECTION } \\
\text { NUMBER }\end{array}$} & \multicolumn{9}{|c|}{ DATE } \\
\hline & $6-13$ & $6-14$ & $6-15$ & $6-16$ & $6-17$ & $6-18$ & $6-19$ & $6-20$ & $6-21$ \\
\hline $\begin{array}{l}0043 \\
0075 \\
0137 \\
0178 \\
0220\end{array}$ & $\begin{array}{r}6 \\
22 \\
7 \\
8 \\
14\end{array}$ & $\begin{array}{l}14 \\
12 \\
12 \\
10 \\
13\end{array}$ & $\begin{array}{r}6 \\
15 \\
10 \\
17 \\
25\end{array}$ & $\begin{array}{l}-- \\
z- \\
--\end{array}$ & $\begin{array}{r}9 \\
14 \\
20 \\
20 \\
21\end{array}$ & $\begin{array}{l}18 \\
24 \\
17 \\
20 \\
20\end{array}$ & $\begin{array}{l}19 \\
16 \\
20 \\
24 \\
26\end{array}$ & $\begin{array}{r}6 \\
17 \\
10 \\
16 \\
9\end{array}$ & $\begin{array}{r}9 \\
14 \\
19 \\
17 \\
18\end{array}$ \\
\hline $\begin{array}{l}0257 \\
0301 \\
0348 \\
0421 \\
0460\end{array}$ & $\begin{array}{l}13 \\
16 \\
38 \\
25 \\
20\end{array}$ & $\begin{array}{l}13 \\
29 \\
25 \\
32 \\
13\end{array}$ & $\begin{array}{l}11 \\
29 \\
26 \\
13 \\
15\end{array}$ & $\begin{array}{l}19 \\
12 \\
33 \\
18 \\
--\end{array}$ & $\begin{array}{l}20 \\
12 \\
23 \\
19 \\
21\end{array}$ & $\begin{array}{l}17 \\
19 \\
17 \\
30 \\
10\end{array}$ & $\begin{array}{l}31 \\
24 \\
22 \\
20 \\
13\end{array}$ & $\begin{array}{l}11 \\
11 \\
16 \\
23 \\
24\end{array}$ & $\begin{array}{l}13 \\
22 \\
28 \\
21 \\
17\end{array}$ \\
\hline $\begin{array}{l}0516 \\
0556 \\
0602 \\
0653 \\
0708\end{array}$ & $\begin{array}{r}19 \\
8 \\
19 \\
11 \\
29\end{array}$ & $\begin{array}{r}9 \\
8 \\
20 \\
29 \\
18\end{array}$ & $\begin{array}{l}18 \\
18 \\
20 \\
22 \\
28\end{array}$ & $\begin{array}{l}\overline{-} \\
\overline{--} \\
\overline{18}\end{array}$ & $\begin{array}{r}99 \\
34 \\
30 \\
12 \\
20\end{array}$ & $\begin{array}{l}14 \\
19 \\
32 \\
33 \\
18\end{array}$ & $\begin{array}{l}19 \\
22 \\
22 \\
21 \\
12\end{array}$ & $\begin{array}{l}26 \\
27 \\
22 \\
14 \\
10\end{array}$ & $\begin{array}{r}25 \\
19 \\
9 \\
14 \\
11\end{array}$ \\
\hline $\begin{array}{l}0757 \\
0808 \\
0853 \\
0898 \\
0940\end{array}$ & $\begin{array}{l}31 \\
27 \\
27 \\
36 \\
26\end{array}$ & $\begin{array}{l}18 \\
22 \\
13 \\
31 \\
13\end{array}$ & $\begin{array}{r}14 \\
27 \\
9 \\
22 \\
20\end{array}$ & $\begin{array}{l}15 \\
14 \\
19 \\
--\end{array}$ & $\begin{array}{l}20 \\
35 \\
18 \\
7 \\
12\end{array}$ & $\begin{array}{r}15 \\
26 \\
5 \\
7 \\
16\end{array}$ & $\begin{array}{r}7 \\
22 \\
10 \\
30 \\
11\end{array}$ & $\begin{array}{l}15 \\
12 \\
0 \\
17 \\
15\end{array}$ & $\begin{array}{l}19 \\
17 \\
16 \\
11 \\
16\end{array}$ \\
\hline $\begin{array}{l}0985 \\
1038 \\
1077 \\
1120 \\
1155\end{array}$ & $\begin{array}{r}19 \\
11 \\
12 \\
8 \\
9\end{array}$ & $\begin{array}{r}20 \\
8 \\
19 \\
0 \\
10\end{array}$ & $\begin{array}{l}12 \\
11 \\
18 \\
7 \\
16\end{array}$ & $\begin{array}{l}-- \\
-\overline{-} \\
-\overline{-}\end{array}$ & $\begin{array}{r}15 \\
21 \\
14 \\
15 \\
4\end{array}$ & $\begin{array}{r}13 \\
21 \\
36 \\
10 \\
8\end{array}$ & $\begin{array}{r}14 \\
12 \\
13 \\
8 \\
10\end{array}$ & $\begin{array}{r}11 \\
11 \\
8 \\
6 \\
9\end{array}$ & $\begin{array}{r}14 \\
11 \\
8 \\
0 \\
4\end{array}$ \\
\hline $\begin{array}{l}1202 \\
1241 \\
1284 \\
1315 \\
1360\end{array}$ & $\begin{array}{r}12 \\
18 \\
1 \\
4 \\
0\end{array}$ & $\begin{array}{r}14 \\
11 \\
11 \\
2 \\
2\end{array}$ & $\begin{array}{r}11 \\
12 \\
5 \\
3 \\
0\end{array}$ & $\begin{array}{r}-- \\
15 \\
6 \\
3 \\
3\end{array}$ & $\begin{array}{l}3 \\
5 \\
5 \\
4 \\
2\end{array}$ & $\begin{array}{l}4 \\
6 \\
4 \\
1 \\
0\end{array}$ & $\begin{array}{l}5 \\
0 \\
2 \\
1 \\
0\end{array}$ & $\begin{array}{l}0 \\
3 \\
3 \\
1 \\
0\end{array}$ & $\begin{array}{l}6 \\
3 \\
1 \\
1 \\
0\end{array}$ \\
\hline $\begin{array}{l}1396 \\
1400(2) \\
1425 \\
1481 \\
1533\end{array}$ & $\begin{array}{l}1 \\
0 \\
0 \\
1 \\
1\end{array}$ & $\begin{array}{l}1 \\
1 \\
0 \\
0 \\
0\end{array}$ & $\begin{array}{l}2 \\
0 \\
0 \\
1 \\
1\end{array}$ & $\begin{array}{r}0 \\
4 \\
0 \\
0 \\
--\end{array}$ & $\begin{array}{l}3 \\
3 \\
1 \\
0 \\
0\end{array}$ & $\begin{array}{l}0 \\
1 \\
0 \\
0 \\
0\end{array}$ & $\begin{array}{l}0 \\
0 \\
0 \\
0 \\
1\end{array}$ & $\begin{array}{l}0 \\
0 \\
0 \\
0 \\
0\end{array}$ & $\begin{array}{l}0 \\
1 \\
0 \\
0 \\
0\end{array}$ \\
\hline $\begin{array}{l}1573 \\
1610 \\
1662\end{array}$ & $\begin{array}{l}0 \\
1 \\
0\end{array}$ & $\begin{array}{l}1 \\
0 \\
0\end{array}$ & $\begin{array}{l}0 \\
0 \\
0\end{array}$ & $\begin{array}{l}-- \\
--\end{array}$ & $\begin{array}{l}0 \\
0 \\
0\end{array}$ & $\begin{array}{l}0 \\
0 \\
0\end{array}$ & $\begin{array}{l}0 \\
0 \\
0\end{array}$ & $\begin{array}{r}0 \\
0 \\
11\end{array}$ & $\begin{array}{l}0 \\
0 \\
0\end{array}$ \\
\hline
\end{tabular}


TABLE 9.- NUMBER OF BLUE TRACER PARTICLES(1) BETWEEN 0.25 AND 8.00

MILL IMETERS, PER 100 GRAMS OF BEDLOAD, AS A FUNCTION OF DATE AND SECTION, EAST FORK RIVER, WYOMING, 1980--CONTINUED

\begin{tabular}{|c|c|c|c|c|c|c|c|c|c|}
\hline \multirow{2}{*}{$\begin{array}{l}\text { SECTION } \\
\text { NUMBER }\end{array}$} & \multicolumn{9}{|c|}{ DATE } \\
\hline & $6-22$ & $6-23$ & $6-25$ & $6-27$ & $6-29$ & $7-0 I$ & $7-03$ & $7-05$ & $7-07$ \\
\hline $\begin{array}{l}0043 \\
0075 \\
0137 \\
0178 \\
0220\end{array}$ & $\begin{array}{l}-- \\
=- \\
=- \\
--\end{array}$ & $\begin{array}{r}26 \\
6 \\
18 \\
18 \\
41\end{array}$ & $\begin{array}{r}10 \\
12 \\
28 \\
7 \\
29\end{array}$ & $\begin{array}{l}21 \\
11 \\
26 \\
21 \\
13\end{array}$ & $\begin{array}{l}-- \\
-- \\
-- \\
-- \\
--\end{array}$ & $\begin{array}{l}=- \\
=- \\
=- \\
--\end{array}$ & $\begin{array}{l}-- \\
=- \\
=- \\
--\end{array}$ & $\begin{array}{l}=- \\
=- \\
z- \\
--\end{array}$ & $\begin{array}{l}=- \\
=- \\
=- \\
--\end{array}$ \\
\hline $\begin{array}{l}0257 \\
0301 \\
0348 \\
0421 \\
0460\end{array}$ & $\begin{array}{l}-- \\
=- \\
-- \\
--\end{array}$ & $\begin{array}{r}0 \\
25 \\
26 \\
15 \\
33\end{array}$ & $\begin{array}{r}29 \\
14 \\
8 \\
16 \\
11\end{array}$ & $\begin{array}{l}14 \\
23 \\
14 \\
25 \\
27\end{array}$ & $\begin{array}{l}-- \\
27 \\
--\end{array}$ & $\begin{array}{l}-- \\
-- \\
=- \\
--\end{array}$ & $\begin{array}{l}=- \\
=- \\
=-\end{array}$ & $\begin{array}{l}-- \\
=- \\
=- \\
--\end{array}$ & $\begin{array}{l}=- \\
=- \\
=- \\
--\end{array}$ \\
\hline $\begin{array}{l}0516 \\
0556 \\
0602 \\
0653 \\
0708\end{array}$ & $\begin{array}{l}-- \\
=- \\
=- \\
--\end{array}$ & $\begin{array}{l}18 \\
10 \\
10 \\
11 \\
15\end{array}$ & $\begin{array}{l}25 \\
18 \\
19 \\
1 \\
20\end{array}$ & $\begin{array}{l}28 \\
33 \\
13 \\
30 \\
11\end{array}$ & $\begin{array}{l}=- \\
=- \\
=- \\
--\end{array}$ & $\begin{array}{l}-- \\
=- \\
=- \\
--\end{array}$ & $\begin{array}{l}-- \\
=- \\
=- \\
--\end{array}$ & $\begin{array}{l}=- \\
\overline{-} \\
\overline{-}\end{array}$ & $\begin{array}{l}=- \\
=- \\
=- \\
--\end{array}$ \\
\hline $\begin{array}{l}0757 \\
0808 \\
0853 \\
0898 \\
0940\end{array}$ & $\begin{array}{l}-\overline{10} \\
13 \\
20 \\
17\end{array}$ & $\begin{array}{r}15 \\
19 \\
11 \\
12 \\
9\end{array}$ & $\begin{array}{r}17 \\
24 \\
17 \\
14 \\
8\end{array}$ & $\begin{array}{r}24 \\
20 \\
0 \\
0 \\
0\end{array}$ & $\begin{array}{l}20 \\
=- \\
--\end{array}$ & $\begin{array}{l}-- \\
=- \\
-- \\
--\end{array}$ & $\begin{array}{l}=- \\
=- \\
=- \\
--\end{array}$ & $\begin{array}{l}=- \\
=- \\
=-\end{array}$ & $\begin{array}{l}-- \\
=- \\
=- \\
--\end{array}$ \\
\hline $\begin{array}{l}0985 \\
1038 \\
1077 \\
1120 \\
1155\end{array}$ & $\begin{array}{l}11 \\
5 \\
-- \\
--\end{array}$ & $\begin{array}{l}8 \\
2 \\
2 \\
7 \\
2\end{array}$ & $\begin{array}{r}10 \\
6 \\
9 \\
2 \\
4\end{array}$ & $\begin{array}{r}0 \\
17 \\
9 \\
3 \\
1\end{array}$ & $\begin{array}{l}-- \\
=- \\
-- \\
--\end{array}$ & $\begin{array}{l}-- \\
=- \\
=- \\
--\end{array}$ & $\begin{array}{l}=- \\
z- \\
z-\end{array}$ & $\begin{array}{l}-- \\
=- \\
=- \\
--\end{array}$ & $\begin{array}{l}-- \\
=- \\
z- \\
--\end{array}$ \\
\hline $\begin{array}{l}1202 \\
1241 \\
1284 \\
1315 \\
1360\end{array}$ & $\begin{array}{l}=- \\
=- \\
=- \\
--\end{array}$ & $\begin{array}{l}5 \\
2 \\
1 \\
2 \\
0\end{array}$ & $\begin{array}{r}12 \\
0 \\
1 \\
1 \\
0\end{array}$ & $\begin{array}{l}0 \\
2 \\
3 \\
6 \\
0\end{array}$ & $\begin{array}{l}-- \\
=- \\
=- \\
--\end{array}$ & $\begin{array}{l}-- \\
=- \\
=- \\
--\end{array}$ & $\begin{array}{l}-- \\
-- \\
=- \\
--\end{array}$ & $\begin{array}{l}-- \\
-- \\
-- \\
-- \\
--\end{array}$ & $\begin{array}{l}-- \\
=- \\
=- \\
--\end{array}$ \\
\hline $\begin{array}{l}1396 \\
1400(2) \\
1425 \\
1481 \\
1533\end{array}$ & $\begin{array}{l}-- \\
=- \\
-- \\
--\end{array}$ & $\begin{array}{l}0 \\
0 \\
0 \\
0 \\
0\end{array}$ & $\begin{array}{l}0 \\
1 \\
0 \\
0 \\
5\end{array}$ & $\begin{array}{l}1 \\
0 \\
1 \\
0 \\
0\end{array}$ & $\begin{array}{l}-- \\
=- \\
=- \\
--\end{array}$ & $\begin{array}{l}-- \\
-- \\
-- \\
--\end{array}$ & $\begin{array}{l}=- \\
=- \\
-\overline{0}\end{array}$ & $\begin{array}{l}=- \\
=- \\
-\overline{0}\end{array}$ & $\begin{array}{l}=- \\
=- \\
\overline{0}+\end{array}$ \\
\hline $\begin{array}{l}1573 \\
1610 \\
1662\end{array}$ & $\begin{array}{l}-- \\
--\end{array}$ & $\begin{array}{l}0 \\
0 \\
0\end{array}$ & $\begin{array}{l}0 \\
0 \\
0\end{array}$ & $\begin{array}{l}0 \\
0 \\
1\end{array}$ & $\begin{array}{l}-- \\
--\end{array}$ & -- & $\begin{array}{l}-- \\
--\end{array}$ & $\begin{array}{c}0 \\
0+ \\
--\end{array}$ & $\underbrace{0}_{--}++$ \\
\hline
\end{tabular}


TABLE 9.- NUMBER OF BLUE TRACER PARTICLES(I) BETWEEN 0.25 AND 8.00 MILLIMETERS, PER 100 GRAMS OF BEDLOAD, AS A FUNCTION OF DATE AND SECTION, EAST FORK RIVER, WYOMING, 1980--CONTINUED

(1) BLUE TRACER PARTICLES WERE PLACED AS BED MATERIAL 10 METERS DOWNSTREAM FROM SECTION 1695 ON MAY 19, 1979.

(2) BYPASS CHANNEL. SEE FIGURE 3.

+ MASS OF SAMPLE ANALYZED WAS BETWEEN 50 AND 100 GRAMS; NUMBER OF TRACER PARTICLES ADJUSTED TO AN EOUIVALENT 100-GRAM SAMPLE; EXTRAPOLATED NUMBERS ARE CONSIDERED REL IABLE.

++ MASS OF SAMPLE ANALYZED WAS BETWEEN 10 AND 50 GRAMS; NUMBER OF TRACER PARTICLES ADJUSTED TO AN EQUIVALENT 100-GRAM SAMPLE; CARE SHOULD BE TAKEN IN USING THE EXTRAPOLATED NUMBERS.

+++ MASS OF SAMPLE ANALYZED WAS BETWEEN 0 AND 10 GRAMS: NUMBER OF TRACER PARTICLES ADJUSTED TO AN EQUIVALENT 100-GRAM SAMPLE; EXTRAPOLATED NUMBERS ARE NOT CONSIDERED RELIABLE. 
TABLE 10.- NUMBER OF ORANGE TRACER PARTICLES(1) BETWEEN 0.25 AND 8.00 MILLIMETERS, PER 100 GRAMS OF BEDLOAD, AS A FUNCTION OF DATE AND SECTION, EAST FORK RIVER, WYOMING, 1980

\begin{tabular}{|c|c|c|c|c|c|c|c|c|c|}
\hline \multirow{2}{*}{$\begin{array}{r}\text { SECTION } \\
\text { NUMBER }\end{array}$} & \multicolumn{9}{|c|}{ DATE } \\
\hline & $5-16$ & $5-19$ & $5-21$ & $5-22$ & $5-24$ & $5-26$ & $5-27$ & $5-28$ & $5-29$ \\
\hline $\begin{array}{l}0043 \\
0075 \\
0137 \\
0178 \\
0220\end{array}$ & $\begin{array}{l}29++ \\
8+ \\
29 \\
11 \\
11+\end{array}$ & $\begin{array}{l}23++ \\
14 \\
0+++ \\
25 \\
15\end{array}$ & $\begin{array}{c}50+ \\
30 \\
6++ \\
9 \\
8\end{array}$ & $\begin{array}{l}-- \\
=- \\
=- \\
--\end{array}$ & $\begin{array}{l}-- \\
-- \\
=- \\
--\end{array}$ & $\begin{array}{c}32 \\
12+ \\
9 \\
6 \\
2\end{array}$ & $\begin{array}{c}26+ \\
2+ \\
6 \\
8 \\
2+\end{array}$ & $\begin{array}{c}18++ \\
12 \\
7 \\
6 \\
5\end{array}$ & $\begin{array}{l}-\overline{4} \\
-- \\
--\end{array}$ \\
\hline $\begin{array}{l}0257 \\
0301 \\
0348 \\
0421 \\
0460\end{array}$ & $\begin{array}{c}10+ \\
18+ \\
8 \\
16 \\
4\end{array}$ & $\begin{array}{l}5 \\
6 \\
4 \\
2 \\
0++\end{array}$ & $\begin{array}{l}4 \\
3 \\
3 \\
2 \\
1+\end{array}$ & $\begin{array}{l}-- \\
=- \\
=- \\
--\end{array}$ & $\begin{array}{l}-- \\
=- \\
=- \\
--\end{array}$ & $\begin{array}{l}4 \\
6+ \\
0 \\
0 \\
0\end{array}$ & $\begin{array}{l}0 \\
0 \\
1 \\
1 \\
0++\end{array}$ & $\begin{array}{l}0++ \\
0 \\
0 \\
0 \\
0+\end{array}$ & $\begin{array}{l}-- \\
-- \\
--\end{array}$ \\
\hline \multirow{2}{*}{$\begin{array}{l}\text { SECTION } \\
\text { NUMBER }\end{array}$} & \multicolumn{9}{|c|}{ DATE } \\
\hline & $5-30$ & $6-01$ & $6-03$ & $6-05$ & $6-07$ & $6-09$ & $6-10$ & $6-11$ & $6-12$ \\
\hline $\begin{array}{l}0043 \\
0075 \\
0137 \\
0178 \\
0220\end{array}$ & $\begin{array}{c}120+++ \\
0+++ \\
4+ \\
7 \\
6+\end{array}$ & $\begin{array}{c}17++ \\
12 \\
7++ \\
2 \\
0++\end{array}$ & $\begin{array}{l}0+++ \\
5 \\
1++ \\
0++ \\
0++\end{array}$ & $\begin{array}{c}0+++ \\
13 \\
0+++ \\
2 \\
3\end{array}$ & $\begin{array}{l}0+++ \\
0 \\
4 \\
1 \\
3\end{array}$ & $\begin{array}{r}10 \\
7 \\
3 \\
1 \\
1\end{array}$ & $\begin{array}{r}11 \\
8 \\
0 \\
1 \\
3\end{array}$ & $\begin{array}{l}6 \\
0 \\
6 \\
2 \\
3\end{array}$ & $\begin{array}{l}8 \\
0 \\
3 \\
4 \\
5\end{array}$ \\
\hline $\begin{array}{l}0257 \\
0301 \\
0348 \\
0421 \\
0460\end{array}$ & $\begin{array}{l}0+ \\
0 \\
0 \\
0 \\
0++\end{array}$ & $\begin{array}{l}3++ \\
3+ \\
1 \\
1 \\
0++\end{array}$ & $\begin{array}{l}0 \\
0 \\
0 \\
1 \\
0+++\end{array}$ & $\begin{array}{l}1 \\
0 \\
1 \\
0+ \\
0++\end{array}$ & $\begin{array}{l}4 \\
4 \\
1 \\
0 \\
0++\end{array}$ & $\begin{array}{l}9 \\
4 \\
0 \\
2 \\
4\end{array}$ & $\begin{array}{l}1 \\
3 \\
0 \\
0 \\
0\end{array}$ & $\begin{array}{l}2 \\
1 \\
0 \\
0 \\
0\end{array}$ & $\begin{array}{l}2 \\
1 \\
0 \\
0 \\
0\end{array}$ \\
\hline \multirow{2}{*}{$\begin{array}{l}\text { SECTION } \\
\text { NUMBER }\end{array}$} & \multicolumn{9}{|c|}{ DATE } \\
\hline & $6-13$ & $6-14$ & $6-15$ & $6-16$ & $6-17$ & $6-18$ & $6-19$ & $6-20$ & $6-21$ \\
\hline $\begin{array}{l}0043 \\
0075 \\
0137 \\
0178 \\
0220\end{array}$ & $\begin{array}{r}2 \\
5 \\
5 \\
8 \\
11\end{array}$ & $\begin{array}{l}9 \\
5 \\
8 \\
5 \\
0\end{array}$ & $\begin{array}{l}5 \\
4 \\
2 \\
2 \\
2\end{array}$ & $\begin{array}{l}-- \\
=- \\
=- \\
=-\end{array}$ & $\begin{array}{r}14 \\
7 \\
3 \\
1 \\
4\end{array}$ & $\begin{array}{r}10 \\
4 \\
7 \\
6 \\
0\end{array}$ & $\begin{array}{l}7 \\
6 \\
3 \\
4 \\
0\end{array}$ & $\begin{array}{l}5 \\
5 \\
4 \\
2 \\
0\end{array}$ & $\begin{array}{l}1 \\
0 \\
0 \\
0 \\
0\end{array}$ \\
\hline $\begin{array}{l}0257 \\
0301 \\
0348 \\
0421 \\
0460\end{array}$ & $\begin{array}{r}11 \\
7 \\
13 \\
4 \\
8\end{array}$ & $\begin{array}{l}1 \\
1 \\
0 \\
0 \\
0\end{array}$ & $\begin{array}{l}1 \\
2 \\
0 \\
0 \\
0\end{array}$ & $\begin{array}{r}4 \\
0 \\
0 \\
1 \\
-\end{array}$ & $\begin{array}{l}1 \\
0 \\
0 \\
0 \\
0\end{array}$ & $\begin{array}{l}4 \\
3 \\
0 \\
0 \\
0\end{array}$ & $\begin{array}{l}0 \\
0 \\
0 \\
0 \\
0\end{array}$ & $\begin{array}{l}0 \\
0 \\
0 \\
0 \\
0\end{array}$ & $\begin{array}{l}0 \\
1 \\
0 \\
0 \\
0\end{array}$ \\
\hline
\end{tabular}


TABLE 10.- NUMBER OF ORANGE TRACER PARTICLES(1) BETWEEN 0.25 AND 8.00

MILLIMETERS, PER 100 GRAMS OF BEDLOAD, AS A FUNCTION OF DATE AND SECTION, EAST FORK RIVER, WYOMING, 1980--CONTINUED

\begin{tabular}{|c|c|c|c|c|c|c|c|c|c|}
\hline \multirow{2}{*}{$\begin{array}{l}\text { SECTION } \\
\text { NUMBER }\end{array}$} & \multicolumn{9}{|c|}{ DATE } \\
\hline & $6-22$ & $6-23$ & $6-25$ & $6-27$ & $6-29$ & $7-01$ & $7-03$ & $7-05$ & $7-07$ \\
\hline $\begin{array}{l}0043 \\
0075 \\
0137 \\
0178 \\
0220\end{array}$ & $\begin{array}{l}-- \\
=- \\
=- \\
--\end{array}$ & $\begin{array}{l}5 \\
2 \\
0 \\
2 \\
2\end{array}$ & $\begin{array}{l}3 \\
2 \\
2 \\
1 \\
1\end{array}$ & $\begin{array}{r}7 \\
1 \\
10 \\
10 \\
15\end{array}$ & $\begin{array}{l}-- \\
=- \\
=- \\
--\end{array}$ & $\begin{array}{l}-- \\
-- \\
-- \\
--\end{array}$ & $\begin{array}{l}= \\
=- \\
=- \\
--\end{array}$ & $\begin{array}{l}-- \\
-- \\
-- \\
--\end{array}$ & $\begin{array}{l}-- \\
=- \\
=- \\
--\end{array}$ \\
\hline $\begin{array}{l}0257 \\
0301 \\
0348 \\
0421 \\
0460\end{array}$ & $\begin{array}{l}=- \\
=- \\
=-\end{array}$ & $\begin{array}{l}0 \\
0 \\
0 \\
0 \\
0\end{array}$ & $\begin{array}{l}2 \\
0 \\
0 \\
0 \\
0\end{array}$ & $\begin{array}{r}10 \\
7 \\
5 \\
8 \\
15\end{array}$ & $\begin{array}{l}-- \\
-\overline{0} \\
--\end{array}$ & $\begin{array}{l}-- \\
-- \\
=- \\
--\end{array}$ & $\begin{array}{l}=- \\
=- \\
=-\end{array}$ & $\begin{array}{l}=- \\
=- \\
=- \\
--\end{array}$ & $\begin{array}{l}=- \\
=- \\
=-\end{array}$ \\
\hline
\end{tabular}

(1) ORANGE TRACER PARTICLES WERE PLACED AS BED MATERIAL 10 METERS DOWNSTREAM FROM SECTION 0516 ON MAY $19,1979$.

$+\quad$ MASS OF SAMPLE ANALYZED WAS BETWEEN 50 AND 100 GRAMS; NUMBER OF TRACER PARTICLES ADJUSTED TO AN EQUIVALENT 100-GRAM SAMPLE; EXTRAPOLATED NUMBERS ARE CONSIDERED RELIABLE.

+ MASS OF SAMPLE ANALYZED WAS BETWEEN 10 AND 50 GRAMS; NUMBER OF TRACER PARTICLES ADJUSTED TO AN EQUIVALENT 100-GRAM SAMPLE; CARE SHOULD BE TAKEN IN USING THE EXTRAPOLATED NUMBERS.

+++ MASS OF SAMPLE ANALYZED WAS BETWEEN 0 AND 10 GRAMS: NUMBER OF TRACER PARTICLES ADJUSTED TO AN EQUIVALENT 100-GRAM SAMPLE; EXTRAPOLATED NUMBERS ARE NOT CONSIDERED RELIABLE. 
TABLE 11.- NUMBER OF GREEN TRACER PARTICLES(1) BETWEEN 0.25 AND 8.00 MILLIMETERS, PER 100 GRAMS OF BEDLOAD, AS A FUNCTION OF DATE AND SECTION, EAST FORK RIVER, WYOMING, 1980

\begin{tabular}{|c|c|c|c|c|c|c|c|c|c|}
\hline \multirow{2}{*}{$\begin{array}{l}\text { SECTION } \\
\text { NUMBER }\end{array}$} & \multicolumn{9}{|c|}{ DATE } \\
\hline & $6-01$ & $6-03$ & $6-05$ & $6-07$ & $6-09$ & $6-10$ & $6-11$ & $6-12$ & $6-13$ \\
\hline $\begin{array}{l}0043 \\
0075 \\
0137 \\
0178 \\
0220\end{array}$ & $\begin{array}{l}0++ \\
0 \\
0++ \\
0 \\
0++\end{array}$ & $\begin{array}{l}0+++ \\
0 \\
0++ \\
0++ \\
0++\end{array}$ & $\begin{array}{l}0+++ \\
0 \\
0+++ \\
0 \\
0\end{array}$ & $\begin{array}{l}0+++ \\
0 \\
0 \\
0 \\
0\end{array}$ & $\begin{array}{l}0 \\
2 \\
1 \\
0 \\
1\end{array}$ & $\begin{array}{l}0 \\
0 \\
0 \\
0 \\
0\end{array}$ & $\begin{array}{r}0 \\
12 \\
0 \\
0 \\
0\end{array}$ & $\begin{array}{l}0 \\
0 \\
1 \\
0 \\
0\end{array}$ & $\begin{array}{l}0 \\
0 \\
0 \\
0 \\
0\end{array}$ \\
\hline $\begin{array}{l}0257 \\
0301 \\
0348 \\
0421 \\
0460\end{array}$ & $\begin{array}{l}0++ \\
0+ \\
0 \\
0 \\
0++\end{array}$ & $\begin{array}{l}0 \\
0 \\
0 \\
0 \\
0+++\end{array}$ & $\begin{array}{l}0 \\
0 \\
0 \\
0+ \\
0++\end{array}$ & $\begin{array}{l}0 \\
0 \\
0 \\
0 \\
0++\end{array}$ & $\begin{array}{l}0 \\
0 \\
0 \\
0 \\
0\end{array}$ & $\begin{array}{l}0 \\
.0 \\
0 \\
0 \\
0\end{array}$ & $\begin{array}{l}0 \\
0 \\
0 \\
0 \\
0\end{array}$ & $\begin{array}{l}0 \\
0 \\
0 \\
0 \\
0\end{array}$ & $\begin{array}{l}0 \\
0 \\
0 \\
0 \\
0\end{array}$ \\
\hline $\begin{array}{l}0516 \\
0556 \\
0602 \\
0653 \\
0708\end{array}$ & $\begin{array}{l}0+++ \\
0++ \\
0+ \\
0 \\
0++\end{array}$ & $\begin{array}{l}0+++ \\
0++ \\
0+ \\
0 \\
0\end{array}$ & $\begin{array}{l}0+++ \\
0+ \\
0 \\
0 \\
0\end{array}$ & $\begin{array}{l}0++ \\
0+ \\
0 \\
0 \\
0\end{array}$ & $\begin{array}{l}0 \\
0 \\
0 \\
0 \\
0\end{array}$ & $\begin{array}{l}0 \\
0 \\
0 \\
0 \\
0\end{array}$ & $\begin{array}{l}0 \\
0 \\
0 \\
0 \\
1\end{array}$ & $\begin{array}{l}0 \\
0 \\
0 \\
2 \\
0\end{array}$ & $\begin{array}{l}0 \\
0 \\
0 \\
0 \\
0\end{array}$ \\
\hline $\begin{array}{l}0757 \\
0808 \\
0853 \\
0898 \\
0940\end{array}$ & $\begin{array}{l}0+ \\
0 \\
0 \\
1 \\
0+\end{array}$ & $\begin{array}{l}0 \\
0 \\
0 \\
0++ \\
0++\end{array}$ & $\begin{array}{l}0 \\
0 \\
0+++ \\
0++ \\
0+\end{array}$ & $\begin{array}{l}0 \\
0 \\
0 \\
0++ \\
0\end{array}$ & $\begin{array}{l}0 \\
3 \\
0 \\
5 \\
3\end{array}$ & $\begin{array}{l}0+ \\
0 \\
3 \\
3 \\
0\end{array}$ & $\begin{array}{r}15 \\
3 \\
0 \\
5 \\
0\end{array}$ & $\begin{array}{l}2 \\
1 \\
0 \\
0 \\
0\end{array}$ & $\begin{array}{l}0 \\
0 \\
0 \\
0 \\
1\end{array}$ \\
\hline $\begin{array}{l}0985 \\
1038 \\
1077 \\
1120 \\
1155\end{array}$ & $\begin{array}{l}0+++ \\
0+++ \\
0+++ \\
0++ \\
0++\end{array}$ & $\begin{array}{l}0++ \\
0+++ \\
0 \\
0++ \\
0++\end{array}$ & $\begin{array}{l}0+++ \\
0+++ \\
0 \\
0+ \\
0+++\end{array}$ & $\begin{array}{l}0++ \\
0+++ \\
0 \\
0 \\
0\end{array}$ & $\begin{array}{l}0 \\
0 \\
1 \\
0 \\
0\end{array}$ & $\begin{array}{l}0 \\
0 \\
0 \\
0 \\
0\end{array}$ & $\begin{array}{l}0 \\
0 \\
0 \\
3 \\
4\end{array}$ & $\begin{array}{l}0 \\
0 \\
1 \\
0 \\
0\end{array}$ & $\begin{array}{l}0 \\
0 \\
0 \\
0 \\
0\end{array}$ \\
\hline $\begin{array}{l}1202 \\
1241 \\
1284 \\
1315 \\
1360\end{array}$ & $\begin{array}{l}0++ \\
0 \\
0 \\
0 \\
0++\end{array}$ & $\begin{array}{l}0 \\
0 \\
0 \\
1 \\
0+\end{array}$ & $\begin{array}{l}0 \\
0 \\
0 \\
0+ \\
0\end{array}$ & $\begin{array}{l}0 \\
0 \\
0 \\
0 \\
2\end{array}$ & $\begin{array}{r}0 \\
0 \\
0 \\
12 \\
48\end{array}$ & $\begin{array}{r}0 \\
8 \\
22 \\
21 \\
35\end{array}$ & $\begin{array}{r}3 \\
12 \\
50 \\
33 \\
25\end{array}$ & $\begin{array}{r}9 \\
4 \\
25 \\
18 \\
34\end{array}$ & $\begin{array}{r}2 \\
2 \\
47 \\
15 \\
22\end{array}$ \\
\hline $\begin{array}{l}1396 \\
1400(2) \\
1425 \\
1481 \\
1533\end{array}$ & $\begin{array}{c}0 \\
-0 \\
0+ \\
0 \\
0\end{array}$ & $\begin{array}{c}-0 \\
-0+ \\
0 \\
0\end{array}$ & $\begin{array}{r}-1 \\
0 \\
0 \\
7\end{array}$ & $\begin{array}{l}1 \\
0+++ \\
7 \\
3 \\
7\end{array}$ & $\begin{array}{r}34 \\
23 \\
21 \\
24 \\
128\end{array}$ & $\begin{array}{l}32 \\
75 \\
10 \\
55 \\
33\end{array}$ & $\begin{array}{l}19 \\
89 \\
30 \\
34 \\
12\end{array}$ & $\begin{array}{r}20 \\
104 \\
15 \\
18 \\
7\end{array}$ & $\begin{array}{r}5 \\
60 \\
6 \\
11 \\
1\end{array}$ \\
\hline $\begin{array}{l}1573 \\
1610\end{array}$ & $3^{0+}$ & $\begin{array}{l}0+ \\
0+\end{array}$ & $\begin{array}{l}0++ \\
0+\end{array}$ & $\begin{array}{c}185++ \\
15\end{array}$ & $\begin{array}{l}77 \\
34\end{array}$ & $\begin{array}{r}108 \\
0\end{array}$ & $\begin{array}{l}6 \\
0\end{array}$ & $\begin{array}{l}2 \\
0\end{array}$ & $\begin{array}{l}0 \\
0\end{array}$ \\
\hline
\end{tabular}


TABLE 11.- NUMBER OF GREEN TRACER PARTICLES(1) BETWEEN 0.25 AND 8.00 MILL IMETERS, PER 100 GRAMS OF BEDLOAD, AS A FUNCTION OF DATE AND SECTION, EAST FORK RIVER, WYOMING, 1980--CONTINUED

\begin{tabular}{|c|c|c|c|c|c|c|c|c|c|}
\hline \multirow{2}{*}{$\begin{array}{l}\text { SECTION } \\
\text { NUMBER }\end{array}$} & \multicolumn{9}{|c|}{ DATE } \\
\hline & $6-14$ & $6-15$ & $6-16$ & $6-17$ & $6-18$ & $6-19$ & $6-20$ & $6-21$ & $6-22$ \\
\hline $\begin{array}{l}0043 \\
0075 \\
0137 \\
0178 \\
0220\end{array}$ & $\begin{array}{l}2 \\
0 \\
0 \\
0 \\
0\end{array}$ & $\begin{array}{l}1 \\
0 \\
0 \\
0 \\
1\end{array}$ & $\begin{array}{l}-- \\
-- \\
=- \\
--\end{array}$ & $\begin{array}{l}0 \\
1 \\
1 \\
0 \\
0\end{array}$ & $\begin{array}{l}0 \\
0 \\
0 \\
0 \\
0\end{array}$ & $\begin{array}{l}0 \\
0 \\
0 \\
0 \\
0\end{array}$ & $\begin{array}{l}0 \\
0 \\
0 \\
0 \\
0\end{array}$ & $\begin{array}{l}0 \\
0 \\
0 \\
0 \\
0\end{array}$ & $\begin{array}{l}-z \\
-z \\
--\end{array}$ \\
\hline $\begin{array}{l}0257 \\
0301 \\
0348 \\
0421 \\
0460\end{array}$ & $\begin{array}{l}1 \\
0 \\
0 \\
0 \\
0\end{array}$ & $\begin{array}{l}0 \\
0 \\
0 \\
0 \\
0\end{array}$ & $\begin{array}{r}0 \\
0 \\
0 \\
0 \\
--\end{array}$ & $\begin{array}{l}0 \\
0 \\
0 \\
0 \\
0\end{array}$ & $\begin{array}{l}0 \\
0 \\
0 \\
0 \\
0\end{array}$ & $\begin{array}{l}0 \\
0 \\
0 \\
0 \\
0\end{array}$ & $\begin{array}{l}0 \\
0 \\
0 \\
0 \\
0\end{array}$ & $\begin{array}{l}0 \\
0 \\
0 \\
0 \\
0\end{array}$ & $\begin{array}{l}- \\
-z \\
-z\end{array}$ \\
\hline $\begin{array}{l}0516 \\
0556 \\
0602 \\
0653 \\
0708\end{array}$ & $\begin{array}{l}0 \\
0 \\
0 \\
0 \\
0\end{array}$ & $\begin{array}{l}0 \\
0 \\
0 \\
0 \\
0\end{array}$ & $\begin{array}{l}-- \\
=- \\
-\overline{0}\end{array}$ & $\begin{array}{l}0 \\
0 \\
1 \\
9 \\
0\end{array}$ & $\begin{array}{l}0 \\
0 \\
0 \\
0 \\
0\end{array}$ & $\begin{array}{l}0 \\
0 \\
0 \\
0 \\
0\end{array}$ & $\begin{array}{l}0 \\
0 \\
0 \\
0 \\
0\end{array}$ & $\begin{array}{l}0 \\
0 \\
0 \\
0 \\
0\end{array}$ & $\begin{array}{l}-- \\
-z \\
-\end{array}$ \\
\hline $\begin{array}{l}0757 \\
0808 \\
0853 \\
0898 \\
0940\end{array}$ & $\begin{array}{l}0 \\
0 \\
0 \\
0 \\
0\end{array}$ & $\begin{array}{l}0 \\
0 \\
0 \\
0 \\
0\end{array}$ & $\begin{array}{r}0 \\
0 \\
0 \\
-- \\
--\end{array}$ & $\begin{array}{l}0 \\
4 \\
0 \\
0 \\
0\end{array}$ & $\begin{array}{l}0 \\
1 \\
0 \\
0 \\
0\end{array}$ & $\begin{array}{l}0 \\
1 \\
0 \\
0 \\
1\end{array}$ & $\begin{array}{l}2 \\
3 \\
2 \\
0 \\
0\end{array}$ & $\begin{array}{l}2 \\
4 \\
0 \\
0 \\
1\end{array}$ & $\begin{array}{r}- \\
0 \\
0 \\
13 \\
7\end{array}$ \\
\hline $\begin{array}{l}0985 \\
1038 \\
1077 \\
1120 \\
1155\end{array}$ & $\begin{array}{l}0 \\
0 \\
0 \\
0 \\
0\end{array}$ & $\begin{array}{l}0 \\
0 \\
1 \\
0 \\
0\end{array}$ & $\begin{array}{l}-- \\
-- \\
--\end{array}$ & $\begin{array}{l}0 \\
4 \\
1 \\
4 \\
5\end{array}$ & $\begin{array}{r}0 \\
3 \\
0 \\
4 \\
26\end{array}$ & $\begin{array}{r}0 \\
2 \\
0 \\
12 \\
5\end{array}$ & $\begin{array}{l}18 \\
3 \\
10 \\
20 \\
15\end{array}$ & $\begin{array}{r}4 \\
3 \\
9 \\
13 \\
9\end{array}$ & $\begin{array}{r}7 \\
17 \\
-- \\
--\end{array}$ \\
\hline $\begin{array}{l}1202 \\
1241 \\
1284 \\
1315 \\
1360\end{array}$ & $\begin{array}{r}0 \\
6 \\
29 \\
20 \\
18\end{array}$ & $\begin{array}{r}2 \\
14 \\
18 \\
21 \\
9\end{array}$ & $\begin{array}{l}-\overline{17} \\
21 \\
29 \\
11\end{array}$ & $\begin{array}{r}7 \\
23 \\
21 \\
16 \\
3\end{array}$ & $\begin{array}{r}5 \\
12 \\
4 \\
21 \\
10\end{array}$ & $\begin{array}{r}9 \\
10 \\
9 \\
16 \\
4\end{array}$ & $\begin{array}{r}15 \\
10 \\
16 \\
5 \\
1\end{array}$ & $\begin{array}{r}8 \\
11 \\
4 \\
9 \\
4\end{array}$ & $\begin{array}{l}-z \\
-z \\
--\end{array}$ \\
\hline $\begin{array}{l}1396 \\
1400(2) \\
1425 \\
1481 \\
1533\end{array}$ & $\begin{array}{r}5 \\
36 \\
3 \\
11 \\
0\end{array}$ & $\begin{array}{r}1 \\
18 \\
6 \\
2 \\
1\end{array}$ & $\begin{array}{r}7 \\
22 \\
3 \\
4 \\
--\end{array}$ & $\begin{array}{r}0 \\
19 \\
1 \\
1 \\
1\end{array}$ & $\begin{array}{r}9 \\
12 \\
2 \\
5 \\
8\end{array}$ & $\begin{array}{r}5 \\
26 \\
5 \\
5 \\
12\end{array}$ & $\begin{array}{r}2 \\
18 \\
7 \\
17 \\
3\end{array}$ & $\begin{array}{r}6 \\
12 \\
2 \\
1 \\
2\end{array}$ & $\begin{array}{l}-- \\
-z \\
--\end{array}$ \\
\hline $\begin{array}{l}1573 \\
1610\end{array}$ & $\begin{array}{l}1 \\
0\end{array}$ & $\begin{array}{l}1 \\
0\end{array}$ & $=$ & $\begin{array}{l}3 \\
0\end{array}$ & $\begin{array}{l}8 \\
0\end{array}$ & $\begin{array}{l}5 \\
0\end{array}$ & $\begin{array}{l}1 \\
0\end{array}$ & $\begin{array}{l}1 \\
0\end{array}$ & $=$ \\
\hline
\end{tabular}


TABLE 11.- NUMBER OF GREEN TRACER PARTICLES(1) BETWEEN 0.25 AND 8.00

MILLIMETERS, PER 100 GRAMS OF BEDLOAD, AS A FUNCTION OF DATE AND SECTION, EAST FORK RIVER, WYOMING, 1980--CONTINUED

\begin{tabular}{|c|c|c|c|c|c|c|c|c|}
\hline \multirow{2}{*}{$\begin{array}{l}\text { SECTION } \\
\text { NUMBER }\end{array}$} & \multicolumn{8}{|c|}{ DATE } \\
\hline & $6-23$ & $6-25$ & $6-27$ & $6-29$ & $7-01$ & $7-03$ & $7-05$ & $7-07$ \\
\hline $\begin{array}{l}0043 \\
0075 \\
0137 \\
0178 \\
0220\end{array}$ & $\begin{array}{l}0 \\
0 \\
0 \\
0 \\
0\end{array}$ & $\begin{array}{l}0 \\
0 \\
0 \\
9 \\
0\end{array}$ & $\begin{array}{l}0 \\
0 \\
0 \\
0 \\
0\end{array}$ & $\begin{array}{l}-- \\
=- \\
=- \\
--\end{array}$ & $\begin{array}{l}=- \\
=- \\
=- \\
--\end{array}$ & $\begin{array}{l}=- \\
=- \\
=-\end{array}$ & $\begin{array}{l}=- \\
=- \\
=- \\
--\end{array}$ & $\begin{array}{l}=- \\
=- \\
=- \\
--\end{array}$ \\
\hline $\begin{array}{l}0257 \\
0301 \\
0348 \\
0421 \\
0460\end{array}$ & $\begin{array}{l}0 \\
0 \\
2 \\
1 \\
5\end{array}$ & $\begin{array}{l}0 \\
0 \\
0 \\
0 \\
0\end{array}$ & $\begin{array}{l}0 \\
0 \\
2 \\
1 \\
0\end{array}$ & $\begin{array}{l}-- \\
-\overline{3} \\
--\end{array}$ & $\begin{array}{l}=- \\
=- \\
=- \\
--\end{array}$ & $\begin{array}{l}-- \\
=- \\
-- \\
--\end{array}$ & $\begin{array}{l}=- \\
=- \\
--\end{array}$ & $\begin{array}{l}=- \\
=- \\
=- \\
=-\end{array}$ \\
\hline $\begin{array}{l}0516 \\
0556 \\
0602 \\
0653 \\
0708\end{array}$ & $\begin{array}{l}0 \\
0 \\
0 \\
1 \\
1\end{array}$ & $\begin{array}{l}2 \\
2 \\
3 \\
0 \\
0\end{array}$ & $\begin{array}{l}3 \\
4 \\
3 \\
2 \\
5\end{array}$ & $\begin{array}{l}=- \\
=- \\
=- \\
--\end{array}$ & $\begin{array}{l}=- \\
=- \\
=- \\
--\end{array}$ & $\begin{array}{l}-- \\
-- \\
-- \\
--\end{array}$ & $\begin{array}{l}=- \\
=- \\
=- \\
--\end{array}$ & $\begin{array}{l}=- \\
=- \\
=-\end{array}$ \\
\hline $\begin{array}{l}0757 \\
0808 \\
0853 \\
0898 \\
0940\end{array}$ & $\begin{array}{l}2 \\
0 \\
6 \\
5 \\
0\end{array}$ & $\begin{array}{l}0 \\
3 \\
1 \\
3 \\
0\end{array}$ & $\begin{array}{l}3 \\
3 \\
2 \\
7 \\
2\end{array}$ & $\begin{array}{l}--\overline{3} \\
-- \\
-- \\
--\end{array}$ & $\begin{array}{l}-- \\
=- \\
=- \\
--\end{array}$ & $\begin{array}{l}-- \\
=- \\
=- \\
--\end{array}$ & $\begin{array}{l}-- \\
=- \\
=- \\
--\end{array}$ & $\begin{array}{l}=- \\
=- \\
-- \\
--\end{array}$ \\
\hline $\begin{array}{l}0985 \\
1038 \\
1077 \\
1120 \\
1155\end{array}$ & $\begin{array}{r}3 \\
12 \\
10 \\
10 \\
3\end{array}$ & $\begin{array}{r}7 \\
12 \\
12 \\
8 \\
9\end{array}$ & $\begin{array}{r}15 \\
11 \\
10 \\
3 \\
2\end{array}$ & $\begin{array}{l}-- \\
=- \\
=- \\
--\end{array}$ & $\begin{array}{l}-- \\
=- \\
=- \\
--\end{array}$ & $\begin{array}{l}-- \\
-z \\
--\end{array}$ & $\begin{array}{l}-- \\
=- \\
=- \\
--\end{array}$ & $\begin{array}{l}-- \\
=- \\
=- \\
--\end{array}$ \\
\hline $\begin{array}{l}1202 \\
1241 \\
1284 \\
1315 \\
1360\end{array}$ & $\begin{array}{r}17 \\
3 \\
9 \\
8 \\
0\end{array}$ & $\begin{array}{l}4 \\
2 \\
3 \\
5 \\
0\end{array}$ & $\begin{array}{l}2 \\
3 \\
2 \\
3 \\
1\end{array}$ & $\begin{array}{l}=- \\
=- \\
=- \\
--\end{array}$ & $\begin{array}{l}=- \\
=- \\
=- \\
=-\end{array}$ & $\begin{array}{l}-- \\
=- \\
=- \\
--\end{array}$ & $\begin{array}{l}-- \\
=- \\
=- \\
--\end{array}$ & $\begin{array}{l}-- \\
=- \\
=- \\
--\end{array}$ \\
\hline $\begin{array}{l}1396 \\
1400(2) \\
1425 \\
1481 \\
1533\end{array}$ & $\begin{array}{l}0 \\
6 \\
0 \\
1 \\
0\end{array}$ & $\begin{array}{l}0 \\
4 \\
0 \\
0 \\
1\end{array}$ & $\begin{array}{l}0 \\
3 \\
0 \\
2 \\
4\end{array}$ & $\begin{array}{l}=- \\
=- \\
=- \\
--\end{array}$ & $\begin{array}{l}-- \\
=- \\
=- \\
--\end{array}$ & $\begin{array}{l}=- \\
\overline{-} \\
-\overline{2}\end{array}$ & $\begin{array}{l}-- \\
\overline{--} \\
-\overline{3}\end{array}$ & $\begin{array}{l}=- \\
\overline{--} \\
\overline{0}+\end{array}$ \\
\hline $\begin{array}{l}1573 \\
1610\end{array}$ & $\begin{array}{l}0 \\
0\end{array}$ & $\begin{array}{l}2 \\
0\end{array}$ & $\begin{array}{r}10 \\
0\end{array}$ & $=$ & $-\frac{-}{1}$ & $=-$ & 2 & $\begin{array}{l}0 \\
0++\end{array}$ \\
\hline
\end{tabular}


TABLE 11.- NUMBER OF GREEN TRACER PARTICLES(1) BETWEEN 0.25 AND 8.00 MILL IMETERS, PER 100 GRAMS OF BEDLOAD, AS A FUNCTION OF DATE AND SECTION, EAST FORK RIVER, WYOMING, 1980--CONTINUED

(1) GREEN TRACER PARTICLES WERE PLACED AS BED MATERIAL 42 METERS DOWNSTREAM FROM SECTION 1662 ON MAY 31, 1980.

(2) BYPASS CHANNEL. SEE FIGURE 3.

+ MASS OF SAMPLE ANALYZED WAS BETWEEN 50 AND 100 GRAMS; NUMBER OF TRACER PARTICLES ADJUSTED TO AN EQUIVALENT 100-GRAM SAMPLE; EXTRAPOLATED NUMBERS ARE CONS IDERED REL IABLE.

+ MASS OF SAMPLE ANALYZED WAS BETWEEN 10 AND 50 GRAMS; NUMBER OF TRACER PARTICLES ADJUSTED TO AN EQUIVALENT 100-GRAM SAMPLE; CARE SHOULD BE TAKEN IN USING THE EXTRAPOLATED NUMBERS.

++ MASS OF SAMPLE ANALYZED WAS BETWEEN 0 AND 10 GRAMS: NUMBER OF TRACER PARTICLES ADJUSTED TO AN EQUIVALENT 100-GRAM SAMPLE; EXTRAPOLATED NUMBERS ARE NOT CONSIDERED REL IABLE. 
TABLE 12.- NUMBER OF PINK TRACER PARTICLES(1), PER 100 GRAMS OF BED MATERIAL, EAST FORK RIVER, WYOMING, 1979

SECTION 0043 SECTION 0075

\begin{tabular}{|c|c|c|c|c|c|c|c|c|c|c|}
\hline \multirow[t]{2}{*}{ DATE } & \multicolumn{3}{|c|}{$\begin{array}{rll}\text { NUMBER OF } & \text { PINK } \\
\text { PARTICLES } & \text { BY } & \text { SIZE }\end{array}$} & \multicolumn{2}{|c|}{$\begin{array}{l}\text { TRACER } \\
\text { CLASS (MM) }\end{array}$} & \multicolumn{5}{|c|}{$\begin{array}{l}\text { NUMBER OF PINK TRACER } \\
\text { PARTICLES BY SIZE CLASS (MM) }\end{array}$} \\
\hline & $\begin{array}{l}0.25 \\
\text { TO } \\
0.50\end{array}$ & $\begin{array}{r}0.50 \\
\text { TO } \\
1.00\end{array}$ & $\begin{array}{r}1.00 \\
\text { TO } \\
2.00\end{array}$ & $\begin{array}{c}2.00 \\
\text { TO } \\
4.00\end{array}$ & $\begin{array}{l}4.00 \\
\text { TO } \\
8.00\end{array}$ & $\begin{array}{r}0.25 \\
\text { TO } \\
0.50\end{array}$ & $\begin{array}{r}0.50 \\
\text { T0 } \\
1.00\end{array}$ & $\begin{array}{l}1.00 \\
\text { TO } \\
2.00\end{array}$ & $\begin{array}{r}2.00 \\
\mathrm{TO} \\
4.00\end{array}$ & $\begin{array}{r}4.00 \\
\text { TO } \\
8.00\end{array}$ \\
\hline $\begin{array}{l}5-20 \\
5-21 \\
5-23 \\
5-24 \\
5-25\end{array}$ & $-\frac{-}{0}$ & $\begin{array}{r}--\overline{0} \\
-0 \\
0\end{array}$ & $\begin{array}{r}-- \\
-\frac{-}{0} \\
0 \\
0\end{array}$ & $\begin{array}{r}--\overline{0} \\
-\frac{1}{0} \\
0\end{array}$ & $\begin{array}{r}-- \\
-0 \\
0 \\
0\end{array}$ & $\begin{array}{r}-- \\
- \\
0 \\
0 \\
0\end{array}$ & $\begin{array}{c}-- \\
-0 \\
0 \\
0\end{array}$ & $\begin{array}{c}-- \\
-\overline{0} \\
0 \\
0\end{array}$ & $\begin{array}{c}-- \\
-\overline{0} \\
0 \\
0\end{array}$ & $\begin{array}{c}-- \\
-\overline{0} \\
0 \\
0\end{array}$ \\
\hline $\begin{array}{l}5-26 \\
5-27 \\
5-28 \\
5-30 \\
5-31\end{array}$ & $\begin{array}{r}-0 \\
-\frac{0}{0} \\
0 \\
0\end{array}$ & $\begin{array}{r}0 \\
-0 \\
0 \\
0 \\
0\end{array}$ & $\begin{array}{r}0 \\
-0 \\
0 \\
0\end{array}$ & $\begin{array}{r}-0 \\
-0 \\
0 \\
0\end{array}$ & $\begin{array}{r}0 \\
-0 \\
0 \\
0\end{array}$ & $\begin{array}{l}0 \\
0 \\
0 \\
0 \\
0\end{array}$ & $\begin{array}{l}0 \\
0 \\
0 \\
0 \\
0\end{array}$ & $\begin{array}{l}0 \\
0 \\
0 \\
0 \\
0\end{array}$ & $\begin{array}{l}0 \\
0 \\
0 \\
0 \\
0\end{array}$ & $\begin{array}{l}0 \\
0 \\
0 \\
0 \\
0\end{array}$ \\
\hline $\begin{array}{l}6-01 \\
6-02 \\
6-03 \\
6-04 \\
6-05\end{array}$ & $\begin{array}{l}0 \\
0 \\
0 \\
0 \\
0\end{array}$ & $\begin{array}{l}0 \\
0 \\
0 \\
0 \\
0\end{array}$ & $\begin{array}{l}0 \\
0 \\
0 \\
1 \\
0\end{array}$ & $\begin{array}{l}0 \\
0 \\
0 \\
0 \\
0\end{array}$ & $\begin{array}{l}0 \\
0 \\
0 \\
0 \\
0\end{array}$ & $\begin{array}{l}0 \\
0 \\
0 \\
0 \\
0\end{array}$ & $\begin{array}{l}0 \\
0 \\
0 \\
0 \\
0\end{array}$ & $\begin{array}{l}0 \\
0 \\
0 \\
0 \\
0\end{array}$ & $\begin{array}{l}0 \\
0 \\
0 \\
0 \\
0\end{array}$ & $\begin{array}{l}0 \\
0 \\
0 \\
0 \\
0\end{array}$ \\
\hline $\begin{array}{l}6-06 \\
6-07 \\
6-08 \\
6-09 \\
6-10\end{array}$ & $\begin{array}{l}0 \\
0 \\
0 \\
0 \\
0\end{array}$ & $\begin{array}{l}0 \\
0 \\
0 \\
0 \\
0\end{array}$ & $\begin{array}{l}0 \\
0 \\
0 \\
0 \\
0\end{array}$ & $\begin{array}{l}0 \\
0 \\
0 \\
0 \\
0\end{array}$ & $\begin{array}{l}0 \\
0 \\
0 \\
0 \\
0\end{array}$ & $\begin{array}{l}1 \\
1 \\
0 \\
0 \\
0\end{array}$ & $\begin{array}{l}1 \\
1 \\
0 \\
0 \\
0\end{array}$ & $\begin{array}{l}0 \\
0 \\
0 \\
0 \\
0\end{array}$ & $\begin{array}{l}0 \\
0 \\
0 \\
0 \\
0\end{array}$ & $\begin{array}{l}0 \\
0 \\
0 \\
0 \\
0\end{array}$ \\
\hline $\begin{array}{l}6-11 \\
6-12 \\
6-13 \\
6-14 \\
6-15\end{array}$ & $\begin{array}{l}0 \\
0 \\
0 \\
0 \\
0\end{array}$ & $\begin{array}{l}0 \\
0 \\
0 \\
0 \\
0\end{array}$ & $\begin{array}{l}0 \\
0 \\
0 \\
0 \\
0\end{array}$ & $\begin{array}{l}0 \\
0 \\
0 \\
0 \\
0\end{array}$ & $\begin{array}{l}0 \\
0 \\
0 \\
0 \\
0\end{array}$ & $\begin{array}{l}0 \\
0 \\
0 \\
0 \\
0\end{array}$ & $\begin{array}{l}0 \\
0 \\
0 \\
0 \\
0\end{array}$ & $\begin{array}{l}0 \\
0 \\
0 \\
0 \\
0\end{array}$ & $\begin{array}{l}0 \\
0 \\
0 \\
0 \\
0\end{array}$ & $\begin{array}{l}0 \\
0 \\
0 \\
0 \\
0\end{array}$ \\
\hline $\begin{array}{l}6-16 \\
6-17 \\
6-18 \\
6-19 \\
6-20\end{array}$ & $\begin{array}{l}0 \\
0 \\
0 \\
0 \\
0\end{array}$ & $\begin{array}{l}0 \\
0 \\
0 \\
0 \\
0\end{array}$ & $\begin{array}{l}0 \\
0 \\
0 \\
0 \\
0\end{array}$ & $\begin{array}{l}0 \\
0 \\
0 \\
0 \\
0\end{array}$ & $\begin{array}{l}0 \\
0 \\
0 \\
0 \\
0\end{array}$ & $\begin{array}{l}0 \\
0 \\
0 \\
0 \\
0\end{array}$ & $\begin{array}{l}0 \\
0 \\
0 \\
0 \\
0\end{array}$ & $\begin{array}{l}0 \\
0 \\
0 \\
0 \\
0\end{array}$ & $\begin{array}{l}0 \\
0 \\
0 \\
0 \\
0\end{array}$ & $\begin{array}{l}0 \\
0 \\
0 \\
0 \\
0\end{array}$ \\
\hline $\begin{array}{l}6-21 \\
6-22 \\
6-23 \\
6-24 \\
6-25\end{array}$ & $\begin{array}{l}0 \\
0 \\
0 \\
0 \\
0\end{array}$ & $\begin{array}{l}0 \\
0 \\
0 \\
0 \\
0\end{array}$ & $\begin{array}{l}0 \\
0 \\
0 \\
0 \\
0\end{array}$ & $\begin{array}{l}0 \\
0 \\
0 \\
0 \\
0\end{array}$ & $\begin{array}{l}0 \\
0 \\
0 \\
0 \\
0\end{array}$ & $\begin{array}{l}0 \\
0 \\
0 \\
0 \\
0\end{array}$ & $\begin{array}{l}0 \\
0 \\
0 \\
0 \\
0\end{array}$ & $\begin{array}{l}0 \\
0 \\
0 \\
0 \\
0\end{array}$ & $\begin{array}{l}0 \\
0 \\
0 \\
0 \\
0\end{array}$ & $\begin{array}{l}0 \\
0 \\
0 \\
0 \\
0\end{array}$ \\
\hline $\begin{array}{r}6-26 \\
6-27 \\
6-28 \\
7-11 \\
10-09\end{array}$ & $\begin{array}{l}0 \\
0 \\
0 \\
0 \\
1\end{array}$ & $\begin{array}{l}0 \\
0 \\
0 \\
0 \\
1\end{array}$ & $\begin{array}{l}0 \\
0 \\
0 \\
0 \\
0\end{array}$ & $\begin{array}{l}0 \\
0 \\
0 \\
0 \\
0\end{array}$ & $\begin{array}{l}0 \\
0 \\
0 \\
0 \\
0\end{array}$ & $\begin{array}{l}0 \\
0 \\
0 \\
0 \\
0\end{array}$ & $\begin{array}{l}0 \\
0 \\
0 \\
0 \\
0\end{array}$ & $\begin{array}{l}0 \\
0 \\
0 \\
0 \\
1\end{array}$ & $\begin{array}{l}0 \\
0 \\
0 \\
0 \\
0\end{array}$ & $\begin{array}{l}0 \\
0 \\
0 \\
0 \\
0\end{array}$ \\
\hline
\end{tabular}


TABLE 12.- NUMBER OF PINK TRACER PARTICLES(1), PER 100 GRAMS OF BED MATERIAL, EAST FORK RIVER, WYOMING, 1979--CONTINUED

SECTION 0137

\begin{tabular}{|c|c|c|c|c|c|c|c|c|c|c|}
\hline \multirow[t]{2}{*}{ DATE } & \multicolumn{2}{|c|}{$\begin{array}{r}\text { NUMBER } 0 \\
\text { PARTICLES B }\end{array}$} & $\begin{array}{l}\text { PINK } \\
\text { SIZE }\end{array}$ & \multicolumn{2}{|c|}{$\begin{array}{l}\text { TRACER } \\
\text { CLASS (MM) }\end{array}$} & \multicolumn{5}{|c|}{$\begin{array}{r}\text { NUMBER OF PINK TRACER } \\
\text { PARTICLES BY SIZE CLASS (MM) }\end{array}$} \\
\hline & $\begin{array}{l}0.25 \\
\text { To } \\
0.50\end{array}$ & $\begin{array}{l}0.50 \\
\text { TO } \\
1.00\end{array}$ & $\begin{array}{l}1.00 \\
\text { TO } \\
2.00\end{array}$ & $\begin{array}{l}2.00 \\
\text { TO } \\
4.00\end{array}$ & $\begin{array}{l}4.00 \\
\text { TO } \\
8.00\end{array}$ & $\begin{array}{r}0.25 \\
\text { TO } \\
0.50\end{array}$ & $\begin{array}{r}0.50 \\
\text { TO } \\
1.00\end{array}$ & $\begin{array}{r}1.00 \\
\mathrm{TO} \\
2.00\end{array}$ & $\begin{array}{r}2.00 \\
\text { TO } \\
4.00\end{array}$ & $\begin{array}{r}4.00 \\
\text { TO } \\
8.00\end{array}$ \\
\hline $\begin{array}{l}5-20 \\
5-21 \\
5-23 \\
5-24 \\
5-25\end{array}$ & $\begin{array}{r}-- \\
0 \\
0 \\
0 \\
0\end{array}$ & $\begin{array}{l}-- \\
0 \\
0 \\
0 \\
0\end{array}$ & $\begin{array}{c}-- \\
0 \\
0 \\
0 \\
0\end{array}$ & $\begin{array}{l}-- \\
0 \\
0 \\
0 \\
0\end{array}$ & $\begin{array}{l}-\overline{0} \\
0 \\
0 \\
0\end{array}$ & $\begin{array}{l}0 \\
0 \\
0 \\
0 \\
0\end{array}$ & $\begin{array}{l}0 \\
0 \\
0 \\
0 \\
0\end{array}$ & $\begin{array}{l}0 \\
0 \\
0 \\
0 \\
0\end{array}$ & $\begin{array}{l}0 \\
0 \\
0 \\
0 \\
0\end{array}$ & $\begin{array}{l}0 \\
0 \\
0 \\
0 \\
0\end{array}$ \\
\hline $\begin{array}{l}5-26 \\
5-27 \\
5-28 \\
5-30 \\
5-31\end{array}$ & $\begin{array}{l}0 \\
0 \\
0 \\
0 \\
0\end{array}$ & $\begin{array}{l}0 \\
0 \\
0 \\
0 \\
0\end{array}$ & $\begin{array}{l}0 \\
0 \\
0 \\
0 \\
0\end{array}$ & $\begin{array}{l}0 \\
0 \\
0 \\
0 \\
0\end{array}$ & $\begin{array}{l}0 \\
0 \\
0 \\
0 \\
0\end{array}$ & $\begin{array}{l}0 \\
0 \\
0 \\
0 \\
0\end{array}$ & $\begin{array}{l}0 \\
0 \\
0 \\
0 \\
0\end{array}$ & $\begin{array}{l}0 \\
0 \\
0 \\
0 \\
0\end{array}$ & $\begin{array}{l}0 \\
0 \\
0 \\
0 \\
0\end{array}$ & $\begin{array}{l}0 \\
0 \\
0 \\
0 \\
0\end{array}$ \\
\hline $\begin{array}{l}6-01 \\
6-02 \\
6-03 \\
6-04 \\
6-05\end{array}$ & $\begin{array}{l}0 \\
0 \\
0 \\
0 \\
0\end{array}$ & $\begin{array}{l}0 \\
0 \\
0 \\
1 \\
0\end{array}$ & $\begin{array}{l}0 \\
0 \\
0 \\
0 \\
0\end{array}$ & $\begin{array}{l}0 \\
0 \\
0 \\
0 \\
0\end{array}$ & $\begin{array}{l}0 \\
0 \\
0 \\
0 \\
0\end{array}$ & $\begin{array}{l}0 \\
0 \\
0 \\
0 \\
0\end{array}$ & $\begin{array}{l}0 \\
0 \\
0 \\
0 \\
0\end{array}$ & $\begin{array}{l}0 \\
0 \\
0 \\
0 \\
0\end{array}$ & $\begin{array}{l}0 \\
0 \\
0 \\
0 \\
0\end{array}$ & $\begin{array}{l}0 \\
0 \\
0 \\
0 \\
0\end{array}$ \\
\hline $\begin{array}{r}6-06 \\
6-07 \\
6-08 \\
6-09 \\
6-10\end{array}$ & $\begin{array}{l}0 \\
0 \\
0 \\
0 \\
0\end{array}$ & $\begin{array}{l}0 \\
0 \\
0 \\
0 \\
0\end{array}$ & $\begin{array}{l}1 \\
0 \\
0 \\
0 \\
0\end{array}$ & $\begin{array}{l}0 \\
0 \\
0 \\
0 \\
0\end{array}$ & $\begin{array}{l}0 \\
0 \\
0 \\
0 \\
0\end{array}$ & $\begin{array}{l}0 \\
0 \\
0 \\
0 \\
0\end{array}$ & $\begin{array}{l}0 \\
0 \\
0 \\
0 \\
0\end{array}$ & $\begin{array}{l}0 \\
0 \\
0 \\
0 \\
0\end{array}$ & $\begin{array}{l}0 \\
0 \\
0 \\
0 \\
0\end{array}$ & $\begin{array}{l}0 \\
0 \\
0 \\
0 \\
0\end{array}$ \\
\hline $\begin{array}{l}6-11 \\
6-12 \\
6-13 \\
6-14 \\
6-15\end{array}$ & $\begin{array}{l}0 \\
0 \\
0 \\
0 \\
0\end{array}$ & $\begin{array}{l}0 \\
0 \\
0 \\
0 \\
0\end{array}$ & $\begin{array}{l}0 \\
0 \\
0 \\
0 \\
0\end{array}$ & $\begin{array}{l}0 \\
0 \\
0 \\
0 \\
0\end{array}$ & $\begin{array}{l}0 \\
0 \\
0 \\
0 \\
0\end{array}$ & $\begin{array}{l}0 \\
0 \\
0 \\
0 \\
0\end{array}$ & $\begin{array}{l}0 \\
0 \\
0 \\
0 \\
1\end{array}$ & $\begin{array}{l}0 \\
0 \\
0 \\
0 \\
0\end{array}$ & $\begin{array}{l}0 \\
0 \\
0 \\
0 \\
0\end{array}$ & $\begin{array}{l}0 \\
0 \\
0 \\
0 \\
0\end{array}$ \\
\hline $\begin{array}{l}6-16 \\
6-17 \\
6-18 \\
6-19 \\
6-20\end{array}$ & $\begin{array}{l}0 \\
0 \\
0 \\
0 \\
0\end{array}$ & $\begin{array}{l}0 \\
0 \\
0 \\
0 \\
0\end{array}$ & $\begin{array}{l}0 \\
0 \\
0 \\
0 \\
0\end{array}$ & $\begin{array}{l}0 \\
0 \\
0 \\
0 \\
0\end{array}$ & $\begin{array}{l}0 \\
0 \\
0 \\
0 \\
0\end{array}$ & $\begin{array}{l}0 \\
0 \\
0 \\
0 \\
0\end{array}$ & $\begin{array}{l}0 \\
0 \\
0 \\
0 \\
0\end{array}$ & $\begin{array}{l}0 \\
0 \\
0 \\
0 \\
0\end{array}$ & $\begin{array}{l}0 \\
0 \\
0 \\
0 \\
0\end{array}$ & $\begin{array}{l}0 \\
0 \\
0 \\
0 \\
0\end{array}$ \\
\hline $\begin{array}{l}6-21 \\
6-22 \\
6-23 \\
6-24 \\
6-25\end{array}$ & $\begin{array}{l}1 \\
0 \\
0 \\
0 \\
0\end{array}$ & $\begin{array}{l}1 \\
0 \\
0 \\
0 \\
0\end{array}$ & $\begin{array}{l}0 \\
0 \\
0 \\
0 \\
0\end{array}$ & $\begin{array}{l}0 \\
0 \\
0 \\
0 \\
0\end{array}$ & $\begin{array}{l}0 \\
0 \\
0 \\
0 \\
0\end{array}$ & $\begin{array}{l}1 \\
0 \\
0 \\
0 \\
0\end{array}$ & $\begin{array}{l}0 \\
0 \\
0 \\
0 \\
0\end{array}$ & $\begin{array}{l}0 \\
0 \\
0 \\
0 \\
0\end{array}$ & $\begin{array}{l}0 \\
0 \\
0 \\
0 \\
0\end{array}$ & $\begin{array}{l}0 \\
0 \\
0 \\
0 \\
0\end{array}$ \\
\hline $\begin{array}{r}6-26 \\
6-27 \\
6-28 \\
7-11 \\
10-09\end{array}$ & $\begin{array}{l}0 \\
0 \\
0 \\
0 \\
0\end{array}$ & $\begin{array}{l}0 \\
0 \\
0 \\
0 \\
0\end{array}$ & $\begin{array}{l}0 \\
0 \\
0 \\
0 \\
0\end{array}$ & $\begin{array}{l}0 \\
0 \\
0 \\
0 \\
0\end{array}$ & $\begin{array}{l}0 \\
0 \\
0 \\
0 \\
0\end{array}$ & $\begin{array}{l}0 \\
0 \\
0 \\
0 \\
0\end{array}$ & $\begin{array}{l}0 \\
0 \\
0 \\
0 \\
0\end{array}$ & $\begin{array}{l}0 \\
0 \\
0 \\
0 \\
0\end{array}$ & $\begin{array}{l}0 \\
0 \\
0 \\
0 \\
0\end{array}$ & $\begin{array}{l}0 \\
0 \\
0 \\
0 \\
0\end{array}$ \\
\hline
\end{tabular}


TABLE 12.- NUMBER OF PINK TRACER PARTICLES(1), PER 100 GRAMS OF BED MATERIAL, EAST FORK RIVER, WYOMING, 1979--CONTINUED

SECTION 0301

SECTION 0301(2)

\begin{tabular}{|c|c|c|c|c|c|c|c|c|c|c|}
\hline \multirow[t]{2}{*}{ DATE } & \multicolumn{5}{|c|}{$\begin{array}{l}\text { NUMBER OF PINK TRACER } \\
\text { PARTICLES BY SIZE CLASS (MM) }\end{array}$} & \multicolumn{5}{|c|}{$\begin{array}{l}\text { NUMBER OF PINK TRACER } \\
\text { PARTICLES BY SIZE CLASS (MM) }\end{array}$} \\
\hline & $\begin{array}{l}0.25 \\
\text { TO } \\
0.50\end{array}$ & $\begin{array}{l}0.50 \\
\text { TO } \\
1.00\end{array}$ & $\begin{array}{l}1.00 \\
2.00\end{array}$ & $\begin{array}{l}2.00 \\
\text { TO } \\
4.00\end{array}$ & $\begin{array}{l}4.00 \\
\text { TO } \\
8.00\end{array}$ & $\begin{array}{l}0.25 \\
\text { TO } \\
0.50\end{array}$ & $\begin{array}{r}0.50 \\
\text { TO } \\
1.00\end{array}$ & $\begin{array}{r}1.00 \\
\text { TO } \\
2.00\end{array}$ & $\begin{array}{r}2.00 \\
\text { TO } \\
4.00\end{array}$ & $\begin{array}{r}4.00 \\
\text { TO } \\
8.00\end{array}$ \\
\hline $\begin{array}{l}5-20 \\
5-21 \\
5-23 \\
5-24 \\
5-25\end{array}$ & $\begin{array}{l}0 \\
0 \\
0 \\
0 \\
0\end{array}$ & $\begin{array}{l}0 \\
0 \\
0 \\
0 \\
0\end{array}$ & $\begin{array}{l}0 \\
0 \\
0 \\
0 \\
0\end{array}$ & $\begin{array}{l}0 \\
0 \\
0 \\
0 \\
0\end{array}$ & $\begin{array}{l}0 \\
0 \\
0 \\
0 \\
0\end{array}$ & $\begin{array}{l}0 \\
0 \\
0 \\
0 \\
0\end{array}$ & $\begin{array}{l}0 \\
0 \\
0 \\
0 \\
0\end{array}$ & $\begin{array}{l}0 \\
0 \\
0 \\
0 \\
0\end{array}$ & $\begin{array}{l}0 \\
0 \\
0 \\
0 \\
0\end{array}$ & $\begin{array}{l}0 \\
0 \\
0 \\
0 \\
0\end{array}$ \\
\hline $\begin{array}{l}5-26 \\
5-27 \\
5-28 \\
5-30 \\
5-31\end{array}$ & $\begin{array}{l}0 \\
0 \\
0 \\
0 \\
0\end{array}$ & $\begin{array}{l}0 \\
0 \\
0 \\
0 \\
0\end{array}$ & $\begin{array}{l}0 \\
0 \\
0 \\
0 \\
0\end{array}$ & $\begin{array}{l}0 \\
0 \\
0 \\
0 \\
0\end{array}$ & $\begin{array}{l}0 \\
0 \\
0 \\
0 \\
0\end{array}$ & $\begin{array}{r}0 \\
0 \\
-- \\
--\end{array}$ & $\begin{array}{r}0 \\
0 \\
-- \\
--\end{array}$ & $\begin{array}{r}0 \\
0 \\
--- \\
--\end{array}$ & $\begin{array}{r}0 \\
0 \\
-- \\
--\end{array}$ & $\begin{array}{r}0 \\
0 \\
-- \\
--\end{array}$ \\
\hline $\begin{array}{l}6-01 \\
6-02 \\
6-03 \\
6-04 \\
6-05\end{array}$ & $\begin{array}{l}0 \\
0 \\
0 \\
0 \\
0\end{array}$ & $\begin{array}{l}0 \\
0 \\
0 \\
0 \\
0\end{array}$ & $\begin{array}{l}0 \\
0 \\
0 \\
0 \\
0\end{array}$ & $\begin{array}{l}0 \\
0 \\
0 \\
0 \\
0\end{array}$ & $\begin{array}{l}0 \\
0 \\
0 \\
0 \\
0\end{array}$ & $\begin{array}{l}-= \\
=- \\
-\overline{0}\end{array}$ & $\begin{array}{l}=- \\
=- \\
0\end{array}$ & $\begin{array}{l}=- \\
=- \\
-\overline{0}\end{array}$ & $\begin{array}{l}-- \\
-\overline{-} \\
-\overline{0}\end{array}$ & $\begin{array}{l}=- \\
=- \\
-\overline{0}\end{array}$ \\
\hline $\begin{array}{l}6-06 \\
6-07 \\
6-08 \\
6-09 \\
6-10\end{array}$ & $\begin{array}{l}0 \\
0 \\
0 \\
0 \\
0\end{array}$ & $\begin{array}{l}0 \\
0 \\
0 \\
0 \\
0\end{array}$ & $\begin{array}{l}0 \\
0 \\
0 \\
0 \\
0\end{array}$ & $\begin{array}{l}0 \\
0 \\
0 \\
0 \\
0\end{array}$ & $\begin{array}{l}0 \\
0 \\
0 \\
0 \\
0\end{array}$ & $\begin{array}{r}0 \\
0 \\
-- \\
--\end{array}$ & $\begin{array}{l}0 \\
0 \\
-- \\
--\end{array}$ & $\begin{array}{r}0 \\
0 \\
-- \\
--\end{array}$ & $\begin{array}{c}0 \\
0 \\
-- \\
--\end{array}$ & $\begin{array}{r}0 \\
0 \\
-- \\
--\end{array}$ \\
\hline $\begin{array}{l}6-11 \\
6-12 \\
6-13 \\
6-14 \\
6-15\end{array}$ & $\begin{array}{l}0 \\
0 \\
0 \\
0 \\
0\end{array}$ & $\begin{array}{l}0 \\
0 \\
0 \\
0 \\
0\end{array}$ & $\begin{array}{l}0 \\
0 \\
0 \\
0 \\
0\end{array}$ & $\begin{array}{l}0 \\
0 \\
0 \\
0 \\
0\end{array}$ & $\begin{array}{l}0 \\
0 \\
0 \\
0 \\
0\end{array}$ & $\begin{array}{c}-- \\
0 \\
0 \\
0 \\
0\end{array}$ & $\begin{array}{l}-- \\
0 \\
0 \\
0 \\
0\end{array}$ & $\begin{array}{c}-- \\
0 \\
0 \\
0 \\
0\end{array}$ & $\begin{array}{c}-\overline{0} \\
0 \\
0 \\
0\end{array}$ & $\begin{array}{c}-- \\
0 \\
0 \\
0 \\
0\end{array}$ \\
\hline $\begin{array}{l}6-16 \\
6-17 \\
6-18 \\
6-19 \\
6-20\end{array}$ & $\begin{array}{l}0 \\
0 \\
0 \\
0 \\
0\end{array}$ & $\begin{array}{l}0 \\
0 \\
0 \\
0 \\
0\end{array}$ & $\begin{array}{l}0 \\
0 \\
0 \\
0 \\
0\end{array}$ & $\begin{array}{l}0 \\
0 \\
0 \\
0 \\
0\end{array}$ & $\begin{array}{l}0 \\
0 \\
0 \\
0 \\
0\end{array}$ & $\begin{array}{r}0 \\
0 \\
-- \\
--\end{array}$ & $\begin{array}{r}0 \\
0 \\
-- \\
--\end{array}$ & $\begin{array}{l}0 \\
0 \\
-- \\
-- \\
--\end{array}$ & $\begin{array}{r}0 \\
0 \\
-- \\
--\end{array}$ & $\begin{array}{r}0 \\
0 \\
-- \\
--\end{array}$ \\
\hline $\begin{array}{l}6-21 \\
6-22 \\
6-23 \\
6-24 \\
6-25\end{array}$ & $\begin{array}{l}0 \\
0 \\
0 \\
0 \\
0\end{array}$ & $\begin{array}{l}0 \\
2 \\
0 \\
0 \\
0\end{array}$ & $\begin{array}{l}0 \\
0 \\
0 \\
0 \\
0\end{array}$ & $\begin{array}{l}0 \\
0 \\
0 \\
0 \\
0\end{array}$ & $\begin{array}{l}0 \\
0 \\
0 \\
0 \\
0\end{array}$ & $\begin{array}{l}-- \\
-\overline{-} \\
--\end{array}$ & $\begin{array}{l}=- \\
=- \\
=-\end{array}$ & $\begin{array}{l}=- \\
=- \\
-\overline{-} \\
--\end{array}$ & $\begin{array}{l}-- \\
-- \\
-- \\
--\end{array}$ & $\begin{array}{l}=- \\
=- \\
=-\end{array}$ \\
\hline $\begin{array}{r}6-26 \\
6-27 \\
6-28 \\
7-11 \\
10-09\end{array}$ & $\begin{array}{l}0 \\
0 \\
0 \\
0 \\
0\end{array}$ & $\begin{array}{l}0 \\
0 \\
0 \\
0 \\
0\end{array}$ & $\begin{array}{l}0 \\
0 \\
0 \\
0 \\
0\end{array}$ & $\begin{array}{l}0 \\
0 \\
0 \\
0 \\
0\end{array}$ & $\begin{array}{l}0 \\
0 \\
0 \\
0 \\
0\end{array}$ & $\begin{array}{l}- \\
-- \\
-- \\
--\end{array}$ & $\begin{array}{l}=- \\
=- \\
=-\end{array}$ & $\begin{array}{l}-- \\
-- \\
--\end{array}$ & $\begin{array}{l}-- \\
-- \\
-- \\
--\end{array}$ & $\begin{array}{l}-- \\
-\square \\
--\end{array}$ \\
\hline
\end{tabular}


TABLE 12.- NUMBER OF PINK TRACER PARTICLES(1), PER 100 GRAMS OF BED MATERIAL, EAST FORK RIVER, WYOMING, 1979--CONTINUED

\begin{tabular}{|c|c|c|c|c|c|c|c|c|c|c|}
\hline \multirow[t]{2}{*}{ DATE } & \multicolumn{3}{|c|}{$\begin{array}{rll}\text { NUMBER OF } & \text { PINK } \\
\text { PARTICLES } & \text { BY } & \text { SIZE }\end{array}$} & \multicolumn{2}{|c|}{$\begin{array}{l}\text { TRACER } \\
\text { CLASS (MM) }\end{array}$} & \multicolumn{5}{|c|}{$\begin{array}{l}\text { NUMBER OF PINK TRACER } \\
\text { PARTICLES BY SIZE CLASS (MM) }\end{array}$} \\
\hline & $\begin{array}{l}0.25 \\
\text { TO } \\
0.50\end{array}$ & $\begin{array}{l}0.50 \\
\text { TO } \\
1.00\end{array}$ & $\begin{array}{l}1.00 \\
2.00 \\
\text { TO }\end{array}$ & $\begin{array}{l}2.00 \\
\text { TO } \\
4.00\end{array}$ & $\begin{array}{l}4.00 \\
\text { TO } \\
8.00\end{array}$ & $\begin{array}{l}0.25 \\
\text { TO } \\
0.50\end{array}$ & $\begin{array}{r}0.50 \\
\text { TO } \\
1.00\end{array}$ & $\begin{array}{l}1.00 \\
2.00\end{array}$ & $\begin{array}{r}2.00 \\
\text { TO } \\
4.00\end{array}$ & $\begin{array}{r}4.00 \\
\text { TO } \\
8.00\end{array}$ \\
\hline $\begin{array}{l}5-20 \\
5-21 \\
5-23 \\
5-24 \\
5-25\end{array}$ & $\begin{array}{l}0 \\
0 \\
0 \\
0 \\
0\end{array}$ & $\begin{array}{l}0 \\
0 \\
0 \\
0 \\
0\end{array}$ & $\begin{array}{l}0 \\
0 \\
0 \\
0 \\
0\end{array}$ & $\begin{array}{l}0 \\
0 \\
0 \\
0 \\
0\end{array}$ & $\begin{array}{l}0 \\
0 \\
0 \\
0 \\
0\end{array}$ & $\begin{array}{l}=- \\
=- \\
=-\end{array}$ & $\begin{array}{l}-- \\
=- \\
=-\end{array}$ & $\begin{array}{l}=- \\
=- \\
=-\end{array}$ & $\begin{array}{l}=- \\
=- \\
=-\end{array}$ & $\begin{array}{l}-- \\
-- \\
--\end{array}$ \\
\hline $\begin{array}{l}5-26 \\
5-27 \\
5-28 \\
5-30 \\
5-31\end{array}$ & $\begin{array}{l}0 \\
0 \\
0 \\
0 \\
0\end{array}$ & $\begin{array}{l}0 \\
0 \\
0 \\
0 \\
0\end{array}$ & $\begin{array}{l}0 \\
0 \\
0 \\
0 \\
0\end{array}$ & $\begin{array}{l}0 \\
0 \\
0 \\
0 \\
0\end{array}$ & $\begin{array}{l}0 \\
0 \\
0 \\
0 \\
0\end{array}$ & $\overline{-}$ & $\begin{array}{c}-- \\
-\overline{0} \\
0\end{array}$ & $\begin{array}{l}=- \\
-\overline{0} \\
0\end{array}$ & $=$ & $\begin{array}{c}-- \\
-\overline{0} \\
0\end{array}$ \\
\hline $\begin{array}{l}6-01 \\
6-02 \\
6-03 \\
6-04 \\
6-05\end{array}$ & $\begin{array}{l}0 \\
0 \\
0 \\
0 \\
0\end{array}$ & $\begin{array}{l}0 \\
0 \\
0 \\
0 \\
0\end{array}$ & $\begin{array}{l}0 \\
0 \\
0 \\
0 \\
0\end{array}$ & $\begin{array}{l}0 \\
0 \\
0 \\
0 \\
0\end{array}$ & $\begin{array}{l}0 \\
0 \\
0 \\
0 \\
0\end{array}$ & $\begin{array}{l}0 \\
0 \\
0 \\
0 \\
0\end{array}$ & $\begin{array}{l}0 \\
0 \\
0 \\
0 \\
0\end{array}$ & $\begin{array}{l}0 \\
0 \\
0 \\
0 \\
0\end{array}$ & $\begin{array}{l}0 \\
0 \\
0 \\
0 \\
0\end{array}$ & $\begin{array}{l}0 \\
0 \\
0 \\
0 \\
0\end{array}$ \\
\hline $\begin{array}{l}6-06 \\
6-07 \\
6-08 \\
6-09 \\
6-10\end{array}$ & $\begin{array}{l}0 \\
0 \\
0 \\
0 \\
0\end{array}$ & $\begin{array}{l}0 \\
0 \\
0 \\
0 \\
0\end{array}$ & $\begin{array}{l}0 \\
0 \\
0 \\
0 \\
0\end{array}$ & $\begin{array}{l}0 \\
0 \\
0 \\
0 \\
0\end{array}$ & $\begin{array}{l}0 \\
0 \\
0 \\
0 \\
0\end{array}$ & $\begin{array}{l}0 \\
0 \\
0 \\
0 \\
0\end{array}$ & $\begin{array}{l}0 \\
0 \\
0 \\
0 \\
0\end{array}$ & $\begin{array}{l}0 \\
0 \\
0 \\
0 \\
0\end{array}$ & $\begin{array}{l}0 \\
0 \\
0 \\
0 \\
0\end{array}$ & $\begin{array}{l}0 \\
0 \\
0 \\
0 \\
0\end{array}$ \\
\hline $\begin{array}{l}6-11 \\
6-12 \\
6-13 \\
6-14 \\
6-15\end{array}$ & $\begin{array}{l}0 \\
0 \\
0 \\
0 \\
0\end{array}$ & $\begin{array}{l}0 \\
0 \\
0 \\
0 \\
0\end{array}$ & $\begin{array}{l}0 \\
0 \\
0 \\
0 \\
0\end{array}$ & $\begin{array}{l}0 \\
0 \\
0 \\
0 \\
0\end{array}$ & $\begin{array}{l}0 \\
0 \\
0 \\
0 \\
0\end{array}$ & $\begin{array}{l}0 \\
0 \\
0 \\
0 \\
0\end{array}$ & $\begin{array}{l}0 \\
0 \\
0 \\
0 \\
0\end{array}$ & $\begin{array}{l}0 \\
0 \\
0 \\
0 \\
0\end{array}$ & $\begin{array}{l}0 \\
0 \\
0 \\
0 \\
0\end{array}$ & $\begin{array}{l}0 \\
0 \\
0 \\
0 \\
0\end{array}$ \\
\hline $\begin{array}{l}6-16 \\
6-17 \\
6-18 \\
6-19 \\
6-20\end{array}$ & $\begin{array}{l}1 \\
0 \\
0 \\
0 \\
0\end{array}$ & $\begin{array}{l}0 \\
0 \\
0 \\
0 \\
0\end{array}$ & $\begin{array}{l}0 \\
0 \\
0 \\
0 \\
0\end{array}$ & $\begin{array}{l}0 \\
0 \\
0 \\
0 \\
0\end{array}$ & $\begin{array}{l}0 \\
0 \\
0 \\
0 \\
0\end{array}$ & $\begin{array}{l}0 \\
1 \\
0 \\
0 \\
0\end{array}$ & $\begin{array}{l}0 \\
0 \\
0 \\
0 \\
0\end{array}$ & $\begin{array}{l}0 \\
0 \\
0 \\
0 \\
0\end{array}$ & $\begin{array}{l}0 \\
0 \\
0 \\
0 \\
0\end{array}$ & $\begin{array}{l}0 \\
0 \\
0 \\
0 \\
0\end{array}$ \\
\hline $\begin{array}{l}6-21 \\
6-22 \\
6-23 \\
6-24 \\
6-25\end{array}$ & $\begin{array}{l}0 \\
0 \\
0 \\
0 \\
0\end{array}$ & $\begin{array}{l}0 \\
0 \\
0 \\
0 \\
0\end{array}$ & $\begin{array}{l}0 \\
0 \\
0 \\
0 \\
0\end{array}$ & $\begin{array}{l}0 \\
0 \\
0 \\
0 \\
0\end{array}$ & $\begin{array}{l}0 \\
0 \\
0 \\
0 \\
0\end{array}$ & $\begin{array}{r}0 \\
0 \\
0 \\
0 \\
--\end{array}$ & $\begin{array}{r}0 \\
0 \\
0 \\
0 \\
--\end{array}$ & $\begin{array}{r}0 \\
0 \\
0 \\
0 \\
-\end{array}$ & $\begin{array}{r}0 \\
0 \\
0 \\
0 \\
--\end{array}$ & $\begin{array}{r}0 \\
0 \\
0 \\
0 \\
--\end{array}$ \\
\hline $\begin{array}{r}6-26 \\
6-27 \\
6-28 \\
7-11 \\
10-09\end{array}$ & $\begin{array}{l}0 \\
0 \\
0 \\
0 \\
0\end{array}$ & $\begin{array}{l}0 \\
0 \\
0 \\
0 \\
1\end{array}$ & $\begin{array}{l}0 \\
0 \\
0 \\
0 \\
0\end{array}$ & $\begin{array}{l}0 \\
0 \\
0 \\
0 \\
0\end{array}$ & $\begin{array}{l}0 \\
0 \\
0 \\
0 \\
0\end{array}$ & $\begin{array}{r}-- \\
2 \\
2 \\
0\end{array}$ & $\begin{array}{r}-- \\
-\overline{0} \\
0 \\
0\end{array}$ & $\begin{array}{c}-- \\
-0 \\
0 \\
0\end{array}$ & $\begin{array}{c}-- \\
-0 \\
0 \\
0\end{array}$ & $\begin{array}{c}-- \\
-\overline{0} \\
0 \\
0\end{array}$ \\
\hline
\end{tabular}


TABLE 12.- NUMBER OF PINK TRACER PARTICLES(1), PER 100 GRAMS OF BED MATERIAL, EAST FORK RIVER, WYOMING, 1979--CONTINUED

\begin{tabular}{|c|c|c|c|c|c|c|c|c|c|c|}
\hline \multirow{2}{*}{ DATE } & \multicolumn{3}{|c|}{$\begin{array}{rll}\text { NUMBER OF } & \text { PINK } \\
\text { PARTICLES } & \text { BY } & \text { SIZE }\end{array}$} & \multicolumn{2}{|c|}{$\begin{array}{l}\text { TRACER } \\
\text { CLASS (MM) }\end{array}$} & \multicolumn{5}{|c|}{$\begin{array}{l}\text { NUMBER OF PINK TRACER } \\
\text { PARTICLES BY SIZE CLASS (MM) }\end{array}$} \\
\hline & $\begin{array}{l}0.25 \\
\text { TO } \\
0.50\end{array}$ & $\begin{array}{l}0.50 \\
\text { TO } \\
1.00\end{array}$ & $\begin{array}{l}1.00 \\
2.00 \\
\text { TO }\end{array}$ & $\begin{array}{l}2.00 \\
\text { TO } \\
4.00\end{array}$ & $\begin{array}{l}4.00 \\
\text { TO } \\
8.00\end{array}$ & $\begin{array}{r}0.25 \\
\text { TO } \\
0.50\end{array}$ & $\begin{array}{r}0.50 \\
\text { TO } \\
1.00\end{array}$ & $\begin{array}{l}1.00 \\
2.00\end{array}$ & $\begin{array}{r}2.00 \\
\text { TO } \\
4.00\end{array}$ & $\begin{array}{r}4.00 \\
\text { TO } \\
8.00\end{array}$ \\
\hline $5-20$ & -- & -- & -- & -- & -- & -- & -- & -- & -- & -- \\
\hline $\begin{array}{l}5-21 \\
5-23\end{array}$ & $=-$ & $=$ & $\overline{--}$ & $=-$ & $=$ & $=-$ & $=$ & $=-$ & $=$ & - \\
\hline $5-24$ & -- & -- & -- & -- & -- & -- & -- & -- & & - \\
\hline $5-25$ & -- & - & - & - & -- & -- & -- & -- & -- & -- \\
\hline $5-26$ & -- & - & -- & - & -- & -- & -- & -- & -- & -- \\
\hline $\begin{array}{l}5-21 \\
5-28\end{array}$ & -- & $=$ & $=$ & $=$ & $=$ & $=-$ & $=$ & $=-$ & $=-$ & $=-$ \\
\hline $5-30$ & 0 & 0 & 0 & 0 & 0 & 0 & 0 & 0 & 0 & 0 \\
\hline $\begin{array}{l}6-01 \\
6-02 \\
6-03 \\
6-04 \\
6-05\end{array}$ & $\begin{array}{l}0 \\
0 \\
0 \\
0 \\
0\end{array}$ & $\begin{array}{l}0 \\
0 \\
0 \\
0 \\
0\end{array}$ & $\begin{array}{l}0 \\
0 \\
0 \\
0 \\
0\end{array}$ & $\begin{array}{l}0 \\
0 \\
0 \\
0 \\
0\end{array}$ & $\begin{array}{l}0 \\
0 \\
0 \\
0 \\
0\end{array}$ & $\begin{array}{l}0 \\
0 \\
0 \\
0 \\
0\end{array}$ & $\begin{array}{l}0 \\
0 \\
0 \\
0 \\
0\end{array}$ & $\begin{array}{l}0 \\
0 \\
0 \\
0 \\
0\end{array}$ & $\begin{array}{l}0 \\
0 \\
0 \\
0 \\
0\end{array}$ & $\begin{array}{l}0 \\
0 \\
0 \\
0 \\
0\end{array}$ \\
\hline $\begin{array}{l}6-06 \\
6-07 \\
6-08 \\
6-09 \\
6-10\end{array}$ & $\begin{array}{l}0 \\
0 \\
0 \\
0 \\
0\end{array}$ & $\begin{array}{l}0 \\
0 \\
0 \\
0 \\
0\end{array}$ & $\begin{array}{l}0 \\
0 \\
0 \\
0 \\
0\end{array}$ & $\begin{array}{l}0 \\
0 \\
0 \\
0 \\
0\end{array}$ & $\begin{array}{l}0 \\
0 \\
0 \\
0 \\
0\end{array}$ & $\begin{array}{l}0 \\
0 \\
0 \\
0 \\
0\end{array}$ & $\begin{array}{l}0 \\
0 \\
0 \\
0 \\
0\end{array}$ & $\begin{array}{l}0 \\
0 \\
0 \\
0 \\
0\end{array}$ & $\begin{array}{l}0 \\
0 \\
0 \\
0 \\
0\end{array}$ & $\begin{array}{l}0 \\
0 \\
0 \\
0 \\
0\end{array}$ \\
\hline $\begin{array}{l}6-11 \\
6-12 \\
6-13 \\
6-14 \\
6-15\end{array}$ & $\begin{array}{l}0 \\
0 \\
0 \\
0 \\
0\end{array}$ & $\begin{array}{l}0 \\
0 \\
0 \\
0 \\
0\end{array}$ & $\begin{array}{l}0 \\
0 \\
0 \\
0 \\
0\end{array}$ & $\begin{array}{l}0 \\
0 \\
0 \\
0 \\
0\end{array}$ & $\begin{array}{l}0 \\
0 \\
0 \\
0 \\
0\end{array}$ & $\begin{array}{l}0 \\
0 \\
0 \\
0 \\
0\end{array}$ & $\begin{array}{l}0 \\
0 \\
0 \\
0 \\
0\end{array}$ & $\begin{array}{l}0 \\
0 \\
0 \\
0 \\
0\end{array}$ & $\begin{array}{l}0 \\
0 \\
0 \\
0 \\
0\end{array}$ & $\begin{array}{l}0 \\
0 \\
0 \\
0 \\
0\end{array}$ \\
\hline $\begin{array}{l}6-16 \\
6-17 \\
6-18 \\
6-19 \\
6-20\end{array}$ & $\begin{array}{l}0 \\
0 \\
0 \\
0 \\
0\end{array}$ & $\begin{array}{l}1 \\
0 \\
0 \\
0 \\
0\end{array}$ & $\begin{array}{l}0 \\
0 \\
0 \\
0 \\
0\end{array}$ & $\begin{array}{l}0 \\
0 \\
0 \\
0 \\
0\end{array}$ & $\begin{array}{l}0 \\
0 \\
0 \\
0 \\
0\end{array}$ & $\begin{array}{l}0 \\
0 \\
0 \\
0 \\
0\end{array}$ & $\begin{array}{l}0 \\
0 \\
0 \\
0 \\
0\end{array}$ & $\begin{array}{l}0 \\
0 \\
0 \\
0 \\
0\end{array}$ & $\begin{array}{l}0 \\
0 \\
0 \\
0 \\
0\end{array}$ & $\begin{array}{l}0 \\
0 \\
0 \\
0 \\
0\end{array}$ \\
\hline $\begin{array}{l}6-21 \\
6-22 \\
6-23 \\
6-24 \\
6-25\end{array}$ & $\begin{array}{r}0 \\
0 \\
0 \\
0 \\
--\end{array}$ & $\begin{array}{r}0 \\
0 \\
0 \\
0 \\
--\end{array}$ & $\begin{array}{r}0 \\
0 \\
0 \\
0 \\
--\end{array}$ & $\begin{array}{r}0 \\
0 \\
0 \\
0 \\
--\end{array}$ & $\begin{array}{r}0 \\
0 \\
0 \\
0 \\
--\end{array}$ & $\begin{array}{l}0 \\
0 \\
0 \\
0 \\
0\end{array}$ & $\begin{array}{l}0 \\
0 \\
0 \\
0 \\
0\end{array}$ & $\begin{array}{l}0 \\
0 \\
0 \\
0 \\
0\end{array}$ & $\begin{array}{l}0 \\
0 \\
0 \\
0 \\
0\end{array}$ & $\begin{array}{l}0 \\
0 \\
0 \\
0 \\
0\end{array}$ \\
\hline $\begin{array}{r}6-26 \\
6-27 \\
6-28 \\
7-11 \\
10-08\end{array}$ & $\begin{array}{r}-0 \\
-0 \\
2 \\
0\end{array}$ & $\begin{array}{r}0 \\
-1 \\
0 \\
0 \\
0\end{array}$ & $\begin{array}{r}-0 \\
-0 \\
0 \\
0\end{array}$ & $\begin{array}{r}0 \\
-1 \\
0 \\
0 \\
0\end{array}$ & $\begin{array}{r}0 \\
-\frac{1}{0} \\
0 \\
0\end{array}$ & $\begin{array}{l}0 \\
0 \\
0 \\
1 \\
3\end{array}$ & $\begin{array}{l}0 \\
0 \\
0 \\
0 \\
0\end{array}$ & $\begin{array}{l}0 \\
0 \\
0 \\
0 \\
0\end{array}$ & $\begin{array}{l}0 \\
0 \\
0 \\
0 \\
0\end{array}$ & $\begin{array}{l}0 \\
0 \\
0 \\
0 \\
0\end{array}$ \\
\hline
\end{tabular}


TABLE 12.- NUMBER OF PINK TRACER PARTICLES(1), PER 100 GRAMS OF BED MATERIAL, EAST FORK RIVER, WYOMING, 1979--CONTINUED

SECTION 0808

SECTION 0808(2)

\begin{tabular}{|c|c|c|c|c|c|c|c|c|c|c|}
\hline \multirow{2}{*}{ DATE } & \multicolumn{5}{|c|}{$\begin{array}{l}\text { NUMBER OF PINK TRACER } \\
\text { PARTICLES BY SIZE CLASS (MM) }\end{array}$} & \multicolumn{5}{|c|}{$\begin{array}{l}\text { NUMBER OF PINK TRACER } \\
\text { PARTICLES BY SIZE CLASS (MM) }\end{array}$} \\
\hline & $\begin{array}{l}0.25 \\
\text { TO } \\
0.50\end{array}$ & $\begin{array}{l}0.50 \\
\text { TO } \\
1.00\end{array}$ & $\begin{array}{l}1.00 \\
2.00\end{array}$ & $\begin{array}{l}2.00 \\
\text { TO } \\
4.00\end{array}$ & $\begin{array}{l}4.00 \\
\text { TO } \\
8.00\end{array}$ & $\begin{array}{r}0.25 \\
\text { T0 } \\
0.50\end{array}$ & $\begin{array}{r}0.50 \\
\text { TO } \\
1.00\end{array}$ & $\begin{array}{r}1.00 \\
\text { TO } \\
2.00\end{array}$ & $\begin{array}{r}2.00 \\
\text { TO } \\
4.00\end{array}$ & $\begin{array}{r}4.00 \\
\text { TO } \\
8.00\end{array}$ \\
\hline $\begin{array}{l}5-20 \\
5-21 \\
5-23 \\
5-24 \\
5-25\end{array}$ & $\begin{array}{l}-- \\
=- \\
=-\end{array}$ & $\begin{array}{l}-- \\
=- \\
=-\end{array}$ & $\begin{array}{l}-- \\
-- \\
-- \\
--\end{array}$ & $\begin{array}{l}=- \\
=- \\
=-\end{array}$ & $\begin{array}{l}-- \\
=- \\
--\end{array}$ & $\begin{array}{l}-- \\
\overline{-} \\
--\end{array}$ & $\begin{array}{l}-- \\
-- \\
-\end{array}$ & $\begin{array}{l}=- \\
=- \\
=-\end{array}$ & $\begin{array}{l}-- \\
=- \\
=-\end{array}$ & $\begin{array}{l}-- \\
-- \\
--\end{array}$ \\
\hline $\begin{array}{l}5-26 \\
5-27 \\
5-28 \\
5-30 \\
5-31\end{array}$ & $\begin{array}{c}-- \\
-\overline{0} \\
0 \\
0\end{array}$ & $\begin{array}{c}-- \\
-\overline{0} \\
0 \\
0\end{array}$ & $\begin{array}{c}-- \\
-\overline{0} \\
0 \\
0\end{array}$ & $\begin{array}{c}-- \\
-\overline{0} \\
0 \\
0\end{array}$ & $\begin{array}{c}-- \\
-\overline{0} \\
0 \\
0\end{array}$ & $\begin{array}{l}-- \\
=- \\
=-\end{array}$ & $\begin{array}{l}=- \\
\overline{-} \\
=-\end{array}$ & $\begin{array}{l}-- \\
\overline{--} \\
-\end{array}$ & $\begin{array}{l}=- \\
=- \\
=- \\
--\end{array}$ & $\overline{-}=$ \\
\hline $\begin{array}{l}6-01 \\
6-02 \\
6-03 \\
6-04 \\
6-05\end{array}$ & $\begin{array}{l}0 \\
0 \\
0 \\
0 \\
0\end{array}$ & $\begin{array}{l}0 \\
0 \\
0 \\
0 \\
0\end{array}$ & $\begin{array}{l}0 \\
0 \\
0 \\
0 \\
0\end{array}$ & $\begin{array}{l}0 \\
0 \\
0 \\
0 \\
0\end{array}$ & $\begin{array}{l}0 \\
0 \\
0 \\
0 \\
0\end{array}$ & $\begin{array}{c}-- \\
-\overline{0} \\
0\end{array}$ & $\begin{array}{c}-- \\
-\overline{0} \\
0\end{array}$ & $\overline{--}$ & $\overline{--}$ & $\overline{-}$ \\
\hline $\begin{array}{l}6-06 \\
6-07 \\
6-08 \\
6-09 \\
6-10\end{array}$ & $\begin{array}{l}0 \\
0 \\
0 \\
0 \\
0\end{array}$ & $\begin{array}{l}0 \\
0 \\
0 \\
0 \\
0\end{array}$ & $\begin{array}{l}0 \\
0 \\
0 \\
0 \\
0\end{array}$ & $\begin{array}{l}0 \\
0 \\
0 \\
0 \\
0\end{array}$ & $\begin{array}{l}0 \\
0 \\
0 \\
0 \\
0\end{array}$ & $\begin{array}{r}0 \\
0 \\
0 \\
-- \\
--\end{array}$ & $\begin{array}{r}0 \\
0 \\
0 \\
--\end{array}$ & $\begin{array}{r}0 \\
0 \\
0 \\
-- \\
--\end{array}$ & $\begin{array}{r}0 \\
0 \\
0 \\
-- \\
--\end{array}$ & $\begin{array}{r}0 \\
0 \\
0 \\
-- \\
--\end{array}$ \\
\hline $\begin{array}{l}6-11 \\
6-12 \\
6-13 \\
6-14 \\
6-15\end{array}$ & $\begin{array}{l}0 \\
0 \\
0 \\
0 \\
0\end{array}$ & $\begin{array}{l}0 \\
0 \\
0 \\
0 \\
0\end{array}$ & $\begin{array}{l}0 \\
0 \\
0 \\
0 \\
0\end{array}$ & $\begin{array}{l}0 \\
0 \\
0 \\
0 \\
0\end{array}$ & $\begin{array}{l}0 \\
0 \\
0 \\
0 \\
0\end{array}$ & $\begin{array}{l}-\overline{0} \\
0 \\
0 \\
0\end{array}$ & $\begin{array}{l}-- \\
0 \\
0 \\
0 \\
0\end{array}$ & $\begin{array}{l}-- \\
0 \\
0 \\
0 \\
0\end{array}$ & $\begin{array}{c}-\overline{0} \\
0 \\
0 \\
0\end{array}$ & $\begin{array}{c}-\overline{0} \\
0 \\
0 \\
0\end{array}$ \\
\hline $\begin{array}{l}6-16 \\
6-17 \\
6-18 \\
6-19 \\
6-20\end{array}$ & $\begin{array}{l}3 \\
2 \\
0 \\
0 \\
0\end{array}$ & $\begin{array}{l}1 \\
0 \\
0 \\
0 \\
0\end{array}$ & $\begin{array}{l}0 \\
0 \\
0 \\
0 \\
0\end{array}$ & $\begin{array}{l}0 \\
0 \\
0 \\
0 \\
0\end{array}$ & $\begin{array}{l}0 \\
0 \\
0 \\
0 \\
0\end{array}$ & $\begin{array}{r}0 \\
0 \\
0 \\
--\end{array}$ & $\begin{array}{r}0 \\
0 \\
0 \\
-- \\
--\end{array}$ & $\begin{array}{r}0 \\
0 \\
0 \\
-- \\
--\end{array}$ & $\begin{array}{r}0 \\
0 \\
0 \\
--\end{array}$ & $\begin{array}{r}0 \\
0 \\
0 \\
--\end{array}$ \\
\hline $\begin{array}{l}6-21 \\
6-22 \\
6-23 \\
6-24 \\
6-25\end{array}$ & $\begin{array}{r}0 \\
0 \\
0 \\
0 \\
--\end{array}$ & $\begin{array}{r}0 \\
0 \\
0 \\
0 \\
--\end{array}$ & $\begin{array}{r}0 \\
0 \\
0 \\
0 \\
--\end{array}$ & $\begin{array}{r}0 \\
0 \\
0 \\
0 \\
--\end{array}$ & $\begin{array}{l}0 \\
0 \\
0 \\
0 \\
-\end{array}$ & $\begin{array}{l}=- \\
=- \\
=- \\
--\end{array}$ & $\begin{array}{l}-\overline{-} \\
\overline{-} \\
\overline{-}\end{array}$ & $\begin{array}{l}-\overline{-} \\
\overline{-} \\
\overline{-}\end{array}$ & $\begin{array}{l}-- \\
=- \\
=-\end{array}$ & $\begin{array}{l}-- \\
\overline{-} \\
=-\end{array}$ \\
\hline $\begin{array}{r}6-26 \\
6-27 \\
6-28 \\
7-11 \\
10-08\end{array}$ & $\begin{array}{c}-- \\
-1 \\
0 \\
1\end{array}$ & $\begin{array}{c}-- \\
-\overline{0} \\
0 \\
1\end{array}$ & $\begin{array}{c}-- \\
-0 \\
0 \\
0\end{array}$ & $\begin{array}{c}-- \\
-\overline{0} \\
0 \\
0\end{array}$ & $\begin{array}{c}-- \\
-\overline{0} \\
0 \\
0\end{array}$ & $\begin{array}{l}-- \\
=- \\
=-\end{array}$ & $\begin{array}{l}-- \\
-- \\
--\end{array}$ & $\begin{array}{l}-- \\
-- \\
--\end{array}$ & $\begin{array}{l}-- \\
=- \\
=-\end{array}$ & $\begin{array}{l}-- \\
\overline{-} \\
--\end{array}$ \\
\hline
\end{tabular}


TABLE 12.- NUMBER OF PINK TRACER PARTICLES(1), PER 100 GRAMS OF BED MATERIAL, EAST FORK RIVER, WYOMING, 1979--CONTINUED

SECTION 0898

SECTION 0985

\begin{tabular}{|c|c|c|c|c|c|c|c|c|c|c|}
\hline \multirow[t]{2}{*}{ DATE } & \multicolumn{5}{|c|}{$\begin{array}{r}\text { NUMBER OF PINK TRACER } \\
\text { PARTICLES BY SIZE CLASS (MM) }\end{array}$} & \multicolumn{5}{|c|}{$\begin{array}{l}\text { NUMBER OF PINR TRACER } \\
\text { PARTICLES BY SIZE CLASS (MM) }\end{array}$} \\
\hline & $\begin{array}{c}0.25 \\
\text { TO } \\
0.50\end{array}$ & $\begin{array}{l}0.50 \\
\text { TO } \\
1.00\end{array}$ & $\begin{array}{l}1.00 \\
2.00 \\
2.00\end{array}$ & $\begin{array}{l}2.00 \\
\text { TO } \\
4.00\end{array}$ & $\begin{array}{l}4.00 \\
\text { TO } \\
8.00\end{array}$ & $\begin{array}{r}0.25 \\
\text { TO } \\
0.50\end{array}$ & $\begin{array}{r}0.50 \\
\text { TO } \\
1.00\end{array}$ & $\begin{array}{l}1.00 \\
\text { TO } \\
2.00\end{array}$ & $\begin{array}{r}2.00 \\
\text { TO } \\
4.00\end{array}$ & $\begin{array}{r}4.00 \\
\text { TO } \\
8.00\end{array}$ \\
\hline $5-20$ & -- & -- & -- & -- & -- & - & - & -- & - & -- \\
\hline $\begin{array}{l}5-21 \\
5-23\end{array}$ & $=$ & $\overline{--}$ & $\overline{-}$ & $=$ & $\overline{-}$ & $=$ & $=$ & $=$ & $=$ & -- \\
\hline $5-24$ & -- & -- & - & -- & -- & -- & - & - & - & 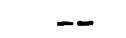 \\
\hline $5-25$ & -- & -- & -- & -- & -- & - & -- & - & -- & - \\
\hline $5-26$ & -- & -- & -- & - & -- & $-\overline{0}$ & $-\overline{0}$ & - & $-\overline{0}$ & $-\overline{0}$ \\
\hline $5-27$ & $-\overline{0}$ & -- & $-\overline{0}$ & - & $-\overline{0}$ & 0 & 0 & 0 & 0 & 0 \\
\hline $\begin{array}{l}5-28 \\
5-30\end{array}$ & $\begin{array}{l}0 \\
0\end{array}$ & 0 & $\begin{array}{l}0 \\
0\end{array}$ & 0 & 0 & 0 & 0 & 0 & 0 & 0 \\
\hline $5-31$ & 0 & 0 & 0 & 0 & 0 & 0 & 0 & 0 & 0 & 0 \\
\hline $6-01$ & 0 & 0 & 0 & 0 & 0 & 0 & 0 & 0 & 0 & 0 \\
\hline $\begin{array}{l}6-02 \\
6-03\end{array}$ & $\begin{array}{l}0 \\
0\end{array}$ & $\begin{array}{l}0 \\
0\end{array}$ & 0 & 0 & O & 0 & $\begin{array}{l}0 \\
0\end{array}$ & 0 & $\begin{array}{l}0 \\
0\end{array}$ & 0 \\
\hline $\begin{array}{l}6-04 \\
6-05\end{array}$ & 0 & 0 & 0 & 0 & 0 & 0 & O & 0 & 0 & 0 \\
\hline & & & & & & & & & & \\
\hline $6-06$ & 0 & 0 & 0 & 0 & 0 & 0 & 0 & 0 & 0 & 0 \\
\hline $\begin{array}{l}6-07 \\
6-08\end{array}$ & $\begin{array}{l}0 \\
0\end{array}$ & $\begin{array}{l}0 \\
0\end{array}$ & $\begin{array}{l}0 \\
0\end{array}$ & $\begin{array}{l}0 \\
0\end{array}$ & $\begin{array}{l}0 \\
0\end{array}$ & $\begin{array}{l}0 \\
0\end{array}$ & $\begin{array}{l}0 \\
0\end{array}$ & $\begin{array}{l}0 \\
0\end{array}$ & $\begin{array}{l}0 \\
0\end{array}$ & $\begin{array}{l}0 \\
0\end{array}$ \\
\hline $6-09$ & 0 & 0 & 0 & 0 & 0 & 0 & 0 & 0 & 0 & 0 \\
\hline $6-10$ & 0 & 0 & 0 & 0 & 0 & 0 & 0 & 0 & 0 & 0 \\
\hline $6-11$ & 0 & 0 & 0 & 0 & 0 & 0 & 0 & 0 & 0 & 0 \\
\hline $\begin{array}{l}6-12 \\
6-13\end{array}$ & 0 & 0 & 0 & 0 & 0 & 0 & 0 & 0 & 0 & 0 \\
\hline $\begin{array}{l}0-13 \\
6-14\end{array}$ & 0 & 0 & 0 & 0 & $\begin{array}{l}0 \\
0\end{array}$ & 0 & 0 & 0 & 0 & 0 \\
\hline $6-15$ & 0 & 0 & 0 & 0 & 0 & 0 & 0 & 0 & 0 & 0 \\
\hline & 0 & 0 & 0 & 0 & 0 & 0 & 0 & 0 & 0 & 0 \\
\hline $\begin{array}{l}6-17 \\
6-18\end{array}$ & 0 & 0 & 0 & 0 & 0 & $\begin{array}{l}0 \\
0\end{array}$ & $\begin{array}{l}0 \\
0\end{array}$ & $\begin{array}{l}0 \\
0\end{array}$ & $\begin{array}{l}0 \\
0\end{array}$ & $\begin{array}{l}0 \\
0\end{array}$ \\
\hline $6-19$ & 0 & 0 & 0 & 0 & 0 & 0 & 0 & 0 & 0 & 0 \\
\hline $6-20$ & 0 & 0 & 0 & 0 & 0 & 0 & 0 & 0 & 0 & 0 \\
\hline & 0 & 0 & 0 & 0 & 0 & 3 & 0 & 0 & 0 & 0 \\
\hline $\begin{array}{l}6-22 \\
6-23\end{array}$ & $\begin{array}{l}0 \\
0\end{array}$ & $\begin{array}{l}0 \\
0\end{array}$ & $\begin{array}{l}0 \\
0\end{array}$ & 0 & $\begin{array}{l}0 \\
0\end{array}$ & $\begin{array}{l}0 \\
0\end{array}$ & $\begin{array}{l}0 \\
0\end{array}$ & 0 & 0 & 0 \\
\hline $6-24$ & 0 & 0 & 0 & 0 & 0 & 0 & 0 & 0 & 0 & 0 \\
\hline $6-25$ & -- & -- & -- & -- & -- & -- & - & -- & -- & - \\
\hline $6-26$ & -- & -- & -- & - & - & -- & -- & -- & -- & -- \\
\hline $6-27$ & -- & $-\overline{-}$ & $-\overline{0}$ & $-\overline{-}$ & $-\overline{0}$ & $-\overline{-}$ & $-\overline{0}$ & $-\overline{0}$ & $-\overline{0}$ & $-\overline{0}$ \\
\hline $\begin{array}{l}6-28 \\
7-11\end{array}$ & $\begin{array}{l}0 \\
0\end{array}$ & $\begin{array}{l}0 \\
0\end{array}$ & $\begin{array}{l}0 \\
0\end{array}$ & $\begin{array}{l}0 \\
0\end{array}$ & $\begin{array}{l}0 \\
0\end{array}$ & $\begin{array}{l}0 \\
0\end{array}$ & $\begin{array}{l}0 \\
0\end{array}$ & $\begin{array}{l}0 \\
0\end{array}$ & $\begin{array}{l}0 \\
0\end{array}$ & $\begin{array}{l}0 \\
0\end{array}$ \\
\hline $10-08$ & 0 & 0 & 0 & 0 & 0 & 0 & 0 & 0 & 0 & 0 \\
\hline
\end{tabular}


TABLE 12.- NUMBER OF PINK TRACER PARTICLES(1), PER 100 GRAMS OF BED MATERIAL, EAST FORK RIVER, WYOMING, 1979--CONTINUED

SECTION 1077 SECTION 1155

\begin{tabular}{|c|c|c|c|c|c|c|c|c|c|c|}
\hline \multirow[t]{2}{*}{ DATE } & \multicolumn{2}{|c|}{$\begin{array}{r}\text { NUMBER OF } \\
\text { PARTICLES By }\end{array}$} & $\begin{array}{l}\text { PINK } \\
\text { SIZE }\end{array}$ & $\begin{array}{l}\text { TRACER } \\
\text { CLASS }\end{array}$ & (MM) & \multicolumn{2}{|c|}{$\begin{array}{r}\text { NUMBER } \\
\text { PARTICLES }\end{array}$} & $\begin{array}{ll}\text { OF } & \text { PINK } \\
\text { BY } & \text { SIZE }\end{array}$ & \multicolumn{2}{|c|}{$\begin{array}{l}\text { TRACER } \\
\text { CLASS (MM) }\end{array}$} \\
\hline & $\begin{array}{l}0.25 \\
\text { T. } \\
0.50\end{array}$ & $\begin{array}{l}0.50 \\
\text { TO } \\
? .00\end{array}$ & $\begin{array}{l}1.00 \\
\text { TO } \\
2.00\end{array}$ & $\begin{array}{l}2.00 \\
\text { TO } \\
4.00\end{array}$ & $\begin{array}{l}4.00 \\
\text { TO } \\
8.00\end{array}$ & $\begin{array}{r}0.25 \\
\text { TO } \\
-0.50\end{array}$ & $\begin{array}{r}0.50 \\
\text { TO } \\
1.00\end{array}$ & $\begin{array}{r}1.00 \\
\text { TO } \\
2.00\end{array}$ & $\begin{array}{r}2.00 \\
\text { TO } \\
4.00\end{array}$ & $\begin{array}{r}4.00 \\
\text { TO } \\
8.00\end{array}$ \\
\hline $\begin{array}{l}5-20 \\
5-21 \\
5-23 \\
5-24 \\
5-25\end{array}$ & $\begin{array}{l}=- \\
=- \\
=-\end{array}$ & $\begin{array}{l}-- \\
-- \\
-- \\
--\end{array}$ & $\begin{array}{l}=- \\
=- \\
=-\end{array}$ & $\begin{array}{l}-- \\
-- \\
-- \\
--\end{array}$ & $\begin{array}{l}-- \\
=- \\
=- \\
--\end{array}$ & $\begin{array}{l}=- \\
-- \\
-\overline{0}\end{array}$ & $\begin{array}{l}=- \\
\overline{-} \\
-\overline{0}\end{array}$ & $\begin{array}{l}-\overline{-} \\
\overline{-} \\
-\overline{0}\end{array}$ & $\begin{array}{l}-\overline{-} \\
\overline{-} \\
-\overline{0}\end{array}$ & $\begin{array}{l}-z \\
-\overline{-} \\
-\overline{0}\end{array}$ \\
\hline $\begin{array}{l}5-26 \\
5-27 \\
5-28 \\
5-30 \\
5-31\end{array}$ & $\begin{array}{l}0 \\
0 \\
0 \\
0 \\
0\end{array}$ & $\begin{array}{l}0 \\
0 \\
0 \\
0 \\
0\end{array}$ & $\begin{array}{l}0 \\
0 \\
0 \\
0 \\
0\end{array}$ & $\begin{array}{l}0 \\
0 \\
0 \\
0 \\
0\end{array}$ & $\begin{array}{l}0 \\
0 \\
0 \\
0 \\
0\end{array}$ & $\begin{array}{l}0 \\
0 \\
0 \\
2 \\
0\end{array}$ & $\begin{array}{l}0 \\
0 \\
0 \\
1 \\
0\end{array}$ & $\begin{array}{l}0 \\
0 \\
0 \\
0 \\
0\end{array}$ & $\begin{array}{l}0 \\
0 \\
0 \\
0 \\
0\end{array}$ & $\begin{array}{l}0 \\
0 \\
0 \\
0 \\
0\end{array}$ \\
\hline $\begin{array}{l}6-01 \\
6-02 \\
6-03 \\
6-04 \\
6-05\end{array}$ & $\begin{array}{l}0 \\
0 \\
0 \\
0 \\
0\end{array}$ & $\begin{array}{l}0 \\
0 \\
0 \\
0 \\
0\end{array}$ & $\begin{array}{l}0 \\
0 \\
0 \\
0 \\
0\end{array}$ & $\begin{array}{l}0 \\
0 \\
0 \\
0 \\
0\end{array}$ & $\begin{array}{l}0 \\
0 \\
0 \\
0 \\
0\end{array}$ & $\begin{array}{l}0 \\
0 \\
0 \\
0 \\
0\end{array}$ & $\begin{array}{l}0 \\
0 \\
0 \\
0 \\
0\end{array}$ & $\begin{array}{l}0 \\
0 \\
0 \\
0 \\
0\end{array}$ & $\begin{array}{l}0 \\
0 \\
0 \\
0 \\
0\end{array}$ & $\begin{array}{l}0 \\
0 \\
0 \\
0 \\
0\end{array}$ \\
\hline $\begin{array}{l}6-06 \\
6-07 \\
6-08 \\
6-09 \\
6-10\end{array}$ & $\begin{array}{l}0 \\
0 \\
0 \\
0 \\
0\end{array}$ & $\begin{array}{l}0 \\
0 \\
0 \\
0 \\
0\end{array}$ & $\begin{array}{l}0 \\
0 \\
0 \\
0 \\
0\end{array}$ & $\begin{array}{l}0 \\
0 \\
0 \\
0 \\
0\end{array}$ & $\begin{array}{l}0 \\
0 \\
0 \\
0 \\
0\end{array}$ & $\begin{array}{l}0 \\
0 \\
0 \\
0 \\
0\end{array}$ & $\begin{array}{l}0 \\
0 \\
0 \\
0 \\
0\end{array}$ & $\begin{array}{l}0 \\
0 \\
0 \\
0 \\
0\end{array}$ & $\begin{array}{l}0 \\
0 \\
0 \\
0 \\
0\end{array}$ & $\begin{array}{l}0 \\
0 \\
0 \\
0 \\
0\end{array}$ \\
\hline $\begin{array}{l}6-11 \\
6-12 \\
6-13 \\
6-14 \\
6-15\end{array}$ & $\begin{array}{l}0 \\
0 \\
0 \\
0 \\
0\end{array}$ & $\begin{array}{l}0 \\
0 \\
0 \\
0 \\
0\end{array}$ & $\begin{array}{l}0 \\
0 \\
0 \\
0 \\
0\end{array}$ & $\begin{array}{l}0 \\
0 \\
0 \\
0 \\
0\end{array}$ & $\begin{array}{l}0 \\
0 \\
0 \\
0 \\
0\end{array}$ & $\begin{array}{l}0 \\
0 \\
0 \\
0 \\
0\end{array}$ & $\begin{array}{l}0 \\
0 \\
0 \\
0 \\
0\end{array}$ & $\begin{array}{l}0 \\
0 \\
0 \\
0 \\
0\end{array}$ & $\begin{array}{l}0 \\
0 \\
0 \\
0 \\
0\end{array}$ & $\begin{array}{l}0 \\
0 \\
0 \\
0 \\
0\end{array}$ \\
\hline $\begin{array}{l}6-16 \\
6-17 \\
6-18 \\
6-19 \\
6-20\end{array}$ & $\begin{array}{l}0 \\
0 \\
0 \\
0 \\
0\end{array}$ & $\begin{array}{l}1 \\
0 \\
0 \\
0 \\
0\end{array}$ & $\begin{array}{l}0 \\
0 \\
0 \\
0 \\
0\end{array}$ & $\begin{array}{l}0 \\
0 \\
0 \\
0 \\
0\end{array}$ & $\begin{array}{l}0 \\
0 \\
0 \\
0 \\
0\end{array}$ & $\begin{array}{l}0 \\
1 \\
4 \\
0 \\
0\end{array}$ & $\begin{array}{l}0 \\
0 \\
0 \\
0 \\
0\end{array}$ & $\begin{array}{l}0 \\
0 \\
0 \\
0 \\
0\end{array}$ & $\begin{array}{l}0 \\
0 \\
0 \\
0 \\
0\end{array}$ & $\begin{array}{l}0 \\
0 \\
0 \\
0 \\
0\end{array}$ \\
\hline $\begin{array}{l}6-21 \\
6-22 \\
6-23 \\
6-24 \\
6-25\end{array}$ & $\begin{array}{l}0 \\
0 \\
0 \\
0 \\
0\end{array}$ & $\begin{array}{l}0 \\
0 \\
0 \\
0 \\
0\end{array}$ & $\begin{array}{l}0 \\
0 \\
0 \\
0 \\
0\end{array}$ & $\begin{array}{l}0 \\
0 \\
0 \\
0 \\
0\end{array}$ & $\begin{array}{l}0 \\
0 \\
0 \\
0 \\
0\end{array}$ & $\begin{array}{l}1 \\
0 \\
0 \\
0 \\
0\end{array}$ & $\begin{array}{l}0 \\
0 \\
0 \\
0 \\
0\end{array}$ & $\begin{array}{l}0 \\
0 \\
0 \\
0 \\
0\end{array}$ & $\begin{array}{l}0 \\
0 \\
0 \\
0 \\
0\end{array}$ & $\begin{array}{l}0 \\
0 \\
0 \\
0 \\
0\end{array}$ \\
\hline $\begin{array}{r}6-26 \\
6-27 \\
6-28 \\
7-11 \\
10-08\end{array}$ & $\begin{array}{r}-0 \\
-0 \\
1 \\
0\end{array}$ & $\begin{array}{r}0 \\
-0 \\
0 \\
0 \\
0\end{array}$ & $\begin{array}{r}0 \\
-0 \\
0 \\
0\end{array}$ & $\begin{array}{r}-0 \\
-0 \\
0 \\
0\end{array}$ & $\begin{array}{r}0 \\
-0 \\
0 \\
0 \\
0\end{array}$ & $\begin{array}{l}0 \\
0 \\
0 \\
2 \\
1\end{array}$ & $\begin{array}{l}0 \\
0 \\
0 \\
0 \\
0\end{array}$ & $\begin{array}{l}0 \\
0 \\
0 \\
0 \\
0\end{array}$ & $\begin{array}{l}0 \\
0 \\
0 \\
0 \\
0\end{array}$ & $\begin{array}{l}0 \\
0 \\
0 \\
0 \\
0\end{array}$ \\
\hline
\end{tabular}


TABLE 12.- NUMBER OF PINK TRACER PARTICLES(1), PER 100 GRAMS OF BED MATERIAL, EAST FORK RIVER, WYOMING, 1979--CONTINUED

\begin{tabular}{|c|c|c|c|c|c|c|c|c|c|c|}
\hline \multirow[t]{2}{*}{ DATE } & \multicolumn{3}{|c|}{$\begin{aligned} & \text { NUMBER OF } \text { PINK } \\
& \text { PARTICLES } \text { BY } \\
& \text { SIZE }\end{aligned}$} & \multicolumn{2}{|c|}{$\begin{array}{l}\text { TRACER } \\
\text { CLASS (MM) }\end{array}$} & \multicolumn{5}{|c|}{$\begin{array}{l}\text { NUMBER OF PINK TRACER } \\
\text { PARTICLES BY SIZE CLASS (MM) }\end{array}$} \\
\hline & $\begin{array}{c}0.25 \\
\text { TO } \\
0.50\end{array}$ & $\begin{array}{c}0.50 \\
\text { TOO } \\
1.00\end{array}$ & $\begin{array}{l}1.00 \\
2.00 \\
\text { TO }\end{array}$ & $\begin{array}{l}2.00 \\
\text { TO } \\
4.00\end{array}$ & $\begin{array}{l}4.00 \\
\text { TO } \\
8.00\end{array}$ & $\begin{array}{l}0.25 \\
\text { TO } \\
0.50\end{array}$ & $\begin{array}{r}0.50 \\
\text { TO } \\
1.00\end{array}$ & $\begin{array}{r}1.00 \\
\text { TO } \\
2.00\end{array}$ & $\begin{array}{r}2.00 \\
\text { TO } \\
4.00\end{array}$ & $\begin{array}{r}4.00 \\
\text { TO } \\
8.00\end{array}$ \\
\hline $\begin{array}{l}5-20 \\
5-21 \\
5-23 \\
5-24 \\
5-25\end{array}$ & $\begin{array}{c}-- \\
-- \\
-0 \\
0\end{array}$ & $\begin{array}{c}-- \\
-- \\
0 \\
0\end{array}$ & $\begin{array}{l}-- \\
-\overline{0} \\
0\end{array}$ & $\begin{array}{c}-- \\
-\overline{0} \\
0\end{array}$ & $\begin{array}{c}-- \\
-- \\
-0 \\
0\end{array}$ & $\begin{array}{r}0 \\
-0 \\
0 \\
0\end{array}$ & $\begin{array}{r}0 \\
-0 \\
0 \\
0\end{array}$ & $\begin{array}{r}0 \\
-0 \\
0 \\
0\end{array}$ & $\begin{array}{r}0 \\
-0 \\
0 \\
0\end{array}$ & $\begin{array}{r}0 \\
-\frac{1}{0} \\
0 \\
0\end{array}$ \\
\hline $\begin{array}{l}5-26 \\
5-27 \\
5-28 \\
5-30 \\
5-31\end{array}$ & $\begin{array}{l}0 \\
0 \\
0 \\
0 \\
0\end{array}$ & $\begin{array}{l}0 \\
0 \\
0 \\
0 \\
0\end{array}$ & $\begin{array}{l}0 \\
0 \\
0 \\
0 \\
0\end{array}$ & $\begin{array}{l}0 \\
0 \\
0 \\
0 \\
0\end{array}$ & $\begin{array}{l}0 \\
0 \\
0 \\
0 \\
0\end{array}$ & $\begin{array}{l}0 \\
0 \\
0 \\
0 \\
0\end{array}$ & $\begin{array}{l}0 \\
0 \\
0 \\
0 \\
0\end{array}$ & $\begin{array}{l}0 \\
0 \\
0 \\
0 \\
0\end{array}$ & $\begin{array}{l}0 \\
0 \\
0 \\
0 \\
0\end{array}$ & $\begin{array}{l}0 \\
0 \\
0 \\
0 \\
0\end{array}$ \\
\hline $\begin{array}{l}6-01 \\
6-02 \\
6-03 \\
6-04 \\
6-05\end{array}$ & $\begin{array}{l}0 \\
0 \\
0 \\
0 \\
0\end{array}$ & $\begin{array}{l}0 \\
0 \\
0 \\
0 \\
0\end{array}$ & $\begin{array}{l}0 \\
0 \\
0 \\
0 \\
0\end{array}$ & $\begin{array}{l}0 \\
0 \\
0 \\
0 \\
0\end{array}$ & $\begin{array}{l}0 \\
0 \\
0 \\
0 \\
0\end{array}$ & $\begin{array}{l}0 \\
0 \\
0 \\
0 \\
0\end{array}$ & $\begin{array}{l}0 \\
0 \\
0 \\
0 \\
0\end{array}$ & $\begin{array}{l}0 \\
0 \\
0 \\
0 \\
0\end{array}$ & $\begin{array}{l}0 \\
0 \\
0 \\
0 \\
0\end{array}$ & $\begin{array}{l}0 \\
0 \\
0 \\
0 \\
0\end{array}$ \\
\hline $\begin{array}{l}6-06 \\
6-07 \\
6-08 \\
6-09 \\
6-10\end{array}$ & $\begin{array}{l}0 \\
0 \\
0 \\
0 \\
0\end{array}$ & $\begin{array}{l}0 \\
0 \\
0 \\
0 \\
0\end{array}$ & $\begin{array}{l}0 \\
0 \\
0 \\
0 \\
0\end{array}$ & $\begin{array}{l}0 \\
0 \\
0 \\
0 \\
0\end{array}$ & $\begin{array}{l}0 \\
0 \\
0 \\
0 \\
0\end{array}$ & $\begin{array}{l}0 \\
0 \\
0 \\
0 \\
0\end{array}$ & $\begin{array}{l}0 \\
0 \\
0 \\
0 \\
0\end{array}$ & $\begin{array}{l}0 \\
0 \\
0 \\
0 \\
0\end{array}$ & $\begin{array}{l}0 \\
0 \\
0 \\
0 \\
0\end{array}$ & $\begin{array}{l}0 \\
0 \\
0 \\
0 \\
0\end{array}$ \\
\hline $\begin{array}{l}6-11 \\
6-12 \\
6-13 \\
6-14 \\
6-15\end{array}$ & $\begin{array}{l}0 \\
0 \\
0 \\
0 \\
0\end{array}$ & $\begin{array}{l}0 \\
0 \\
0 \\
0 \\
0\end{array}$ & $\begin{array}{l}0 \\
0 \\
0 \\
0 \\
0\end{array}$ & $\begin{array}{l}0 \\
0 \\
0 \\
0 \\
0\end{array}$ & $\begin{array}{l}0 \\
0 \\
0 \\
0 \\
0\end{array}$ & $\begin{array}{l}0 \\
0 \\
0 \\
0 \\
0\end{array}$ & $\begin{array}{l}0 \\
0 \\
0 \\
0 \\
0\end{array}$ & $\begin{array}{l}0 \\
0 \\
0 \\
0 \\
0\end{array}$ & $\begin{array}{l}0 \\
0 \\
0 \\
0 \\
0\end{array}$ & $\begin{array}{l}0 \\
0 \\
0 \\
0 \\
0\end{array}$ \\
\hline $\begin{array}{l}6-16 \\
6-17 \\
6-18 \\
6-19 \\
6-20\end{array}$ & $\begin{array}{l}0 \\
5 \\
0 \\
3 \\
0\end{array}$ & $\begin{array}{l}0 \\
0 \\
0 \\
0 \\
0\end{array}$ & $\begin{array}{l}0 \\
0 \\
0 \\
0 \\
0\end{array}$ & $\begin{array}{l}0 \\
0 \\
0 \\
0 \\
0\end{array}$ & $\begin{array}{l}0 \\
0 \\
0 \\
0 \\
0\end{array}$ & $\begin{array}{l}0 \\
0 \\
0 \\
0 \\
0\end{array}$ & $\begin{array}{l}0 \\
0 \\
0 \\
0 \\
0\end{array}$ & $\begin{array}{l}0 \\
0 \\
0 \\
0 \\
0\end{array}$ & $\begin{array}{l}0 \\
0 \\
0 \\
0 \\
0\end{array}$ & $\begin{array}{l}0 \\
0 \\
0 \\
0 \\
0\end{array}$ \\
\hline $\begin{array}{l}6-21 \\
6-22 \\
6-23 \\
6-24 \\
6-25\end{array}$ & $\begin{array}{l}3 \\
0 \\
0 \\
0 \\
0\end{array}$ & $\begin{array}{l}0 \\
0 \\
0 \\
0 \\
0\end{array}$ & $\begin{array}{l}0 \\
0 \\
0 \\
0 \\
0\end{array}$ & $\begin{array}{l}0 \\
0 \\
0 \\
0 \\
0\end{array}$ & $\begin{array}{l}0 \\
0 \\
0 \\
0 \\
0\end{array}$ & $\begin{array}{l}0 \\
0 \\
0 \\
0 \\
0\end{array}$ & $\begin{array}{l}0 \\
0 \\
0 \\
0 \\
0\end{array}$ & $\begin{array}{l}0 \\
0 \\
0 \\
0 \\
0\end{array}$ & $\begin{array}{l}0 \\
0 \\
0 \\
0 \\
0\end{array}$ & $\begin{array}{l}0 \\
0 \\
0 \\
0 \\
0\end{array}$ \\
\hline $\begin{array}{r}6-26 \\
6-27 \\
6-28 \\
7-11 \\
10-08\end{array}$ & $\begin{array}{l}0 \\
0 \\
2 \\
0 \\
2\end{array}$ & $\begin{array}{l}0 \\
0 \\
0 \\
0 \\
1\end{array}$ & $\begin{array}{l}0 \\
0 \\
0 \\
0 \\
0\end{array}$ & $\begin{array}{l}0 \\
0 \\
0 \\
0 \\
0\end{array}$ & $\begin{array}{l}0 \\
0 \\
0 \\
0 \\
0\end{array}$ & $\begin{array}{l}0 \\
0 \\
0 \\
0 \\
2\end{array}$ & $\begin{array}{l}0 \\
0 \\
0 \\
0 \\
1\end{array}$ & $\begin{array}{l}0 \\
0 \\
0 \\
0 \\
0\end{array}$ & $\begin{array}{l}0 \\
0 \\
0 \\
0 \\
0\end{array}$ & $\begin{array}{l}0 \\
0 \\
0 \\
0 \\
0\end{array}$ \\
\hline
\end{tabular}


TABLE 12.- NUMBER OF PINK TRACER PARTICLES(1), PER 100 GRAMS OF BED MATERIAL, EAST FORK RIVER, WYOMING, 1979--CONTINUED

\begin{tabular}{|c|c|c|c|c|c|c|c|c|c|c|}
\hline \multirow[t]{2}{*}{ DATE } & \multicolumn{5}{|c|}{$\begin{aligned} \text { NUMBER OF } & \text { PINK TRACER } \\
\text { PARTICLES BY } & \text { SIZE CLASS (MM) }\end{aligned}$} & \multicolumn{5}{|c|}{$\begin{aligned} & \text { NUMBEE OF } \text { PINK TRACER } \\
& \text { PARTICLES BY SIZE CLASS (MM) }\end{aligned}$} \\
\hline & $\begin{array}{c}0.25 \\
\text { TO } \\
0.50\end{array}$ & $\begin{array}{l}0.50 \\
\text { TO } \\
1.00\end{array}$ & $\begin{array}{l}1.00 \\
\text { TO } \\
2.00\end{array}$ & $\begin{array}{l}2.00 \\
\text { TO } \\
4.00\end{array}$ & $\begin{array}{l}4.00 \\
\text { TO } \\
8.00\end{array}$ & $\begin{array}{r}0.25 \\
\text { TO } \\
0.50\end{array}$ & $\begin{array}{r}0.50 \\
\text { TO } \\
1.00\end{array}$ & $\begin{array}{r}1.00 \\
\text { TO } \\
2.00\end{array}$ & $\begin{array}{r}2.00 \\
\text { TO } \\
4.00\end{array}$ & $\begin{array}{r}4.00 \\
\mathrm{TO} \\
8.00\end{array}$ \\
\hline $\begin{array}{l}5-20 \\
5-21 \\
5-23 \\
5-24 \\
5-25\end{array}$ & $\begin{array}{l}0 \\
0 \\
0 \\
0 \\
0\end{array}$ & $\begin{array}{l}0 \\
0 \\
0 \\
0 \\
0\end{array}$ & $\begin{array}{l}0 \\
0 \\
0 \\
0 \\
0\end{array}$ & $\begin{array}{l}0 \\
0 \\
0 \\
0 \\
0\end{array}$ & $\begin{array}{l}0 \\
0 \\
0 \\
0 \\
0\end{array}$ & $\begin{array}{l}\overline{-} \\
\overline{-} \\
\overline{0}\end{array}$ & $\begin{array}{l}\overline{-} \\
\overline{-} \\
\overline{0}\end{array}$ & $\begin{array}{l}\overline{-} \\
\overline{-}\end{array}$ & $\begin{array}{l}-- \\
\overline{0} \\
-\overline{0}\end{array}$ & $\begin{array}{l}-- \\
-- \\
-\overline{0}\end{array}$ \\
\hline $\begin{array}{l}5-26 \\
5-27 \\
5-28 \\
5-30 \\
5-31\end{array}$ & $\begin{array}{l}0 \\
0 \\
0 \\
0 \\
0\end{array}$ & $\begin{array}{l}0 \\
0 \\
0 \\
0 \\
0\end{array}$ & $\begin{array}{l}0 \\
0 \\
0 \\
0 \\
0\end{array}$ & $\begin{array}{l}0 \\
0 \\
0 \\
0 \\
0\end{array}$ & $\begin{array}{l}0 \\
0 \\
0 \\
0 \\
0\end{array}$ & $\begin{array}{r}0 \\
- \\
-- \\
0\end{array}$ & $\begin{array}{c}0 \\
0 \\
-- \\
-0\end{array}$ & $\begin{array}{r}0 \\
0 \\
-- \\
-0\end{array}$ & $\begin{array}{r}0 \\
-- \\
-0\end{array}$ & $\begin{array}{r}0 \\
0 \\
-- \\
-0\end{array}$ \\
\hline $\begin{array}{l}6-01 \\
6-02 \\
6-03 \\
6-04 \\
6-05\end{array}$ & $\begin{array}{l}0 \\
0 \\
0 \\
0 \\
0\end{array}$ & $\begin{array}{l}0 \\
0 \\
0 \\
0 \\
0\end{array}$ & $\begin{array}{l}0 \\
0 \\
0 \\
0 \\
0\end{array}$ & $\begin{array}{l}0 \\
0 \\
0 \\
0 \\
0\end{array}$ & $\begin{array}{l}0 \\
0 \\
0 \\
0 \\
0\end{array}$ & $\begin{array}{l}0 \\
0 \\
0 \\
0 \\
0\end{array}$ & $\begin{array}{l}0 \\
0 \\
0 \\
0 \\
0\end{array}$ & $\begin{array}{l}0 \\
0 \\
0 \\
0 \\
0\end{array}$ & $\begin{array}{l}0 \\
0 \\
0 \\
0 \\
0\end{array}$ & $\begin{array}{l}0 \\
0 \\
0 \\
0 \\
0\end{array}$ \\
\hline $\begin{array}{l}6-06 \\
6-07 \\
6-08 \\
6-09 \\
6-10\end{array}$ & $\begin{array}{l}0 \\
0 \\
0 \\
0 \\
0\end{array}$ & $\begin{array}{l}0 \\
0 \\
0 \\
0 \\
0\end{array}$ & $\begin{array}{l}0 \\
0 \\
0 \\
0 \\
0\end{array}$ & $\begin{array}{l}0 \\
0 \\
0 \\
0 \\
0\end{array}$ & $\begin{array}{l}0 \\
0 \\
0 \\
0 \\
0\end{array}$ & $\begin{array}{l}0 \\
0 \\
0 \\
0 \\
0\end{array}$ & $\begin{array}{l}0 \\
0 \\
0 \\
0 \\
0\end{array}$ & $\begin{array}{l}0 \\
0 \\
0 \\
0 \\
0\end{array}$ & $\begin{array}{l}0 \\
0 \\
0 \\
0 \\
0\end{array}$ & $\begin{array}{l}0 \\
0 \\
0 \\
0 \\
0\end{array}$ \\
\hline $\begin{array}{l}6-11 \\
6-12 \\
6-13 \\
6-14 \\
6-15\end{array}$ & $\begin{array}{l}0 \\
0 \\
0 \\
0 \\
0\end{array}$ & $\begin{array}{l}0 \\
0 \\
0 \\
0 \\
0\end{array}$ & $\begin{array}{l}0 \\
0 \\
0 \\
0 \\
0\end{array}$ & $\begin{array}{l}0 \\
0 \\
0 \\
0 \\
0\end{array}$ & $\begin{array}{l}0 \\
0 \\
0 \\
0 \\
0\end{array}$ & $\begin{array}{l}0 \\
0 \\
0 \\
0 \\
0\end{array}$ & $\begin{array}{l}0 \\
0 \\
0 \\
0 \\
0\end{array}$ & $\begin{array}{l}0 \\
0 \\
0 \\
0 \\
0\end{array}$ & $\begin{array}{l}0 \\
0 \\
0 \\
0 \\
0\end{array}$ & $\begin{array}{l}0 \\
0 \\
0 \\
0 \\
0\end{array}$ \\
\hline $\begin{array}{l}6-16 \\
6-17 \\
6-18 \\
6-19 \\
6-20\end{array}$ & $\begin{array}{l}0 \\
0 \\
0 \\
0 \\
0\end{array}$ & $\begin{array}{l}0 \\
0 \\
0 \\
0 \\
0\end{array}$ & $\begin{array}{l}0 \\
0 \\
0 \\
0 \\
0\end{array}$ & $\begin{array}{l}0 \\
0 \\
0 \\
0 \\
0\end{array}$ & $\begin{array}{l}0 \\
0 \\
0 \\
0 \\
0\end{array}$ & $\begin{array}{r}0 \\
1 \\
0 \\
0 \\
--\end{array}$ & $\begin{array}{r}0 \\
0 \\
0 \\
0 \\
--\end{array}$ & $\begin{array}{r}0 \\
0 \\
0 \\
0 \\
--\end{array}$ & $\begin{array}{r}0 \\
0 \\
0 \\
0 \\
--\end{array}$ & $\begin{array}{r}0 \\
0 \\
0 \\
0 \\
--\end{array}$ \\
\hline $\begin{array}{l}6-21 \\
6-22 \\
6-23 \\
6-24 \\
6-25\end{array}$ & $\begin{array}{l}0 \\
0 \\
0 \\
0 \\
0\end{array}$ & $\begin{array}{l}0 \\
0 \\
0 \\
0 \\
0\end{array}$ & $\begin{array}{l}0 \\
0 \\
0 \\
0 \\
0\end{array}$ & $\begin{array}{l}0 \\
0 \\
0 \\
0 \\
0\end{array}$ & $\begin{array}{l}0 \\
0 \\
0 \\
0 \\
0\end{array}$ & $\begin{array}{l}-- \\
\overline{--} \\
\overline{--}\end{array}$ & $\begin{array}{l}=- \\
\overline{--} \\
=-\end{array}$ & $\begin{array}{l}=- \\
=- \\
=-\end{array}$ & $\begin{array}{l}=- \\
=- \\
-- \\
--\end{array}$ & $\begin{array}{l}=- \\
-- \\
=-\end{array}$ \\
\hline $\begin{array}{r}6-26 \\
6-27 \\
6-28 \\
7-11 \\
10-08\end{array}$ & $\begin{array}{l}0 \\
0 \\
0 \\
2 \\
1\end{array}$ & $\begin{array}{l}0 \\
0 \\
0 \\
1 \\
0\end{array}$ & $\begin{array}{l}0 \\
0 \\
0 \\
0 \\
0\end{array}$ & $\begin{array}{l}0 \\
0 \\
0 \\
0 \\
0\end{array}$ & $\begin{array}{l}0 \\
0 \\
0 \\
0 \\
0\end{array}$ & $\begin{array}{l}=- \\
\overline{--} \\
\overline{-}\end{array}$ & $\begin{array}{l}=- \\
\overline{--} \\
\overline{--}\end{array}$ & $\begin{array}{l}=- \\
\overline{--} \\
\overline{--}\end{array}$ & $\begin{array}{l}-- \\
-- \\
-- \\
--\end{array}$ & $\begin{array}{l}-- \\
=- \\
=- \\
--\end{array}$ \\
\hline
\end{tabular}


TABLE 12.- NUMBER OF PINK TRACER PARTICLES(1), PER 100 GRAMS OF BED MATERIAL, EAST FORK RIVER, WYOMING, 1979--CONTINUED

SECTION 1481

SECTION 1481(2)

\begin{tabular}{|c|c|c|c|c|c|c|c|c|c|c|}
\hline \multirow[t]{2}{*}{ DATE } & \multicolumn{2}{|c|}{$\begin{aligned} \text { NUMBER } & 0 \\
\text { PARTICLES } & B\end{aligned}$} & $\begin{array}{l}\text { PINK } \\
\text { SIZE }\end{array}$ & \multicolumn{2}{|c|}{$\begin{array}{l}\text { TPACER } \\
\text { CLASS (MM) }\end{array}$} & \multicolumn{5}{|c|}{$\begin{aligned} \text { NUMBER OF } & \text { PINK TRACER } \\
\text { PARTICLES BY } & \text { SIZE CLASS (MM) }\end{aligned}$} \\
\hline & $\begin{array}{c}0.25 \\
\text { TO } \\
0.50\end{array}$ & $\begin{array}{l}0.50 \\
\text { TO } \\
1.00\end{array}$ & $\begin{array}{l}1.00 \\
\text { TO } \\
2.00\end{array}$ & $\begin{array}{l}2.00 \\
\text { TO } \\
4.00\end{array}$ & $\begin{array}{l}4.00 \\
\text { TO } \\
8.00\end{array}$ & $\begin{array}{l}0.25 \\
\text { TO } \\
0.50\end{array}$ & $\begin{array}{r}0.50 \\
\text { TO } \\
1.00\end{array}$ & $\begin{array}{l}1.00 \\
2.00\end{array}$ & $\begin{array}{r}2.00 \\
\text { TO } \\
4.00\end{array}$ & $\begin{array}{r}4.00 \\
\text { TO } \\
8.00\end{array}$ \\
\hline $\begin{array}{l}5-20 \\
5-21 \\
5-23 \\
5-24 \\
5-25\end{array}$ & $\begin{array}{l}0 \\
0 \\
0 \\
0 \\
1\end{array}$ & $\begin{array}{l}0 \\
0 \\
0 \\
0 \\
0\end{array}$ & $\begin{array}{l}0 \\
0 \\
0 \\
0 \\
0\end{array}$ & $\begin{array}{l}0 \\
0 \\
0 \\
0 \\
0\end{array}$ & $\begin{array}{l}0 \\
0 \\
0 \\
0 \\
0\end{array}$ & $\begin{array}{l}0 \\
0 \\
1 \\
0 \\
0\end{array}$ & $\begin{array}{l}0 \\
0 \\
0 \\
0 \\
0\end{array}$ & $\begin{array}{l}0 \\
0 \\
0 \\
0 \\
0\end{array}$ & $\begin{array}{l}0 \\
0 \\
0 \\
0 \\
0\end{array}$ & $\begin{array}{l}0 \\
0 \\
0 \\
0 \\
0\end{array}$ \\
\hline $\begin{array}{l}5-26 \\
5-27 \\
5-28 \\
5-30 \\
5-31\end{array}$ & $\begin{array}{l}0 \\
0 \\
0 \\
0 \\
0\end{array}$ & $\begin{array}{l}0 \\
0 \\
0 \\
0 \\
0\end{array}$ & $\begin{array}{l}0 \\
0 \\
0 \\
0 \\
0\end{array}$ & $\begin{array}{l}0 \\
0 \\
0 \\
0 \\
0\end{array}$ & $\begin{array}{l}0 \\
0 \\
0 \\
0 \\
0\end{array}$ & $\begin{array}{r}0 \\
0 \\
-0 \\
0 \\
0\end{array}$ & $\begin{array}{r}0 \\
-0 \\
-0 \\
0\end{array}$ & $\begin{array}{l}0 \\
0 \\
0 \\
0\end{array}$ & $\begin{array}{r}0 \\
-0 \\
0 \\
0\end{array}$ & $\begin{array}{r}0 \\
0 \\
-0 \\
0 \\
0\end{array}$ \\
\hline $\begin{array}{l}6-01 \\
6-02 \\
6-03 \\
6-04 \\
6-05\end{array}$ & $\begin{array}{l}0 \\
0 \\
0 \\
0 \\
0\end{array}$ & $\begin{array}{l}0 \\
0 \\
0 \\
0 \\
0\end{array}$ & $\begin{array}{l}0 \\
0 \\
0 \\
0 \\
0\end{array}$ & $\begin{array}{l}0 \\
0 \\
0 \\
0 \\
0\end{array}$ & $\begin{array}{l}0 \\
0 \\
0 \\
0 \\
0\end{array}$ & $\begin{array}{l}0 \\
0 \\
0 \\
0 \\
0\end{array}$ & $\begin{array}{l}0 \\
0 \\
0 \\
0 \\
0\end{array}$ & $\begin{array}{l}0 \\
0 \\
0 \\
0 \\
0\end{array}$ & $\begin{array}{l}0 \\
0 \\
0 \\
0 \\
0\end{array}$ & $\begin{array}{l}0 \\
0 \\
0 \\
0 \\
0\end{array}$ \\
\hline $\begin{array}{l}6-06 \\
6-07 \\
6-08 \\
6-09 \\
6-10\end{array}$ & $\begin{array}{l}0 \\
0 \\
0 \\
0 \\
0\end{array}$ & $\begin{array}{l}0 \\
0 \\
0 \\
0 \\
0\end{array}$ & $\begin{array}{l}0 \\
0 \\
0 \\
0 \\
0\end{array}$ & $\begin{array}{l}0 \\
0 \\
0 \\
0 \\
0\end{array}$ & $\begin{array}{l}0 \\
0 \\
0 \\
0 \\
0\end{array}$ & $\begin{array}{r}0 \\
0 \\
- \\
0 \\
0\end{array}$ & $\begin{array}{r}0 \\
0 \\
- \\
0 \\
0\end{array}$ & $\begin{array}{r}0 \\
0 \\
-0 \\
0 \\
0\end{array}$ & $\begin{array}{r}0 \\
0 \\
-0 \\
0 \\
0\end{array}$ & $\begin{array}{r}0 \\
0 \\
-0 \\
0 \\
0\end{array}$ \\
\hline $\begin{array}{l}6-11 \\
6-12 \\
6-13 \\
6-14 \\
6-15\end{array}$ & $\begin{array}{l}0 \\
0 \\
0 \\
0 \\
0\end{array}$ & $\begin{array}{l}0 \\
0 \\
0 \\
0 \\
0\end{array}$ & $\begin{array}{l}0 \\
0 \\
0 \\
0 \\
0\end{array}$ & $\begin{array}{l}0 \\
0 \\
0 \\
0 \\
0\end{array}$ & $\begin{array}{l}0 \\
0 \\
0 \\
0 \\
0\end{array}$ & $\begin{array}{l}0 \\
0 \\
0 \\
1 \\
0\end{array}$ & $\begin{array}{l}0 \\
0 \\
0 \\
0 \\
0\end{array}$ & $\begin{array}{l}0 \\
0 \\
0 \\
0 \\
0\end{array}$ & $\begin{array}{l}0 \\
0 \\
0 \\
0 \\
0\end{array}$ & $\begin{array}{l}0 \\
0 \\
0 \\
0 \\
0\end{array}$ \\
\hline $\begin{array}{l}6-16 \\
6-17 \\
6-18 \\
6-19 \\
6-20\end{array}$ & $\begin{array}{l}3 \\
0 \\
0 \\
0 \\
0\end{array}$ & $\begin{array}{l}1 \\
0 \\
0 \\
0 \\
0\end{array}$ & $\begin{array}{l}0 \\
0 \\
0 \\
0 \\
0\end{array}$ & $\begin{array}{l}0 \\
0 \\
0 \\
0 \\
0\end{array}$ & $\begin{array}{l}0 \\
0 \\
0 \\
0 \\
0\end{array}$ & $\begin{array}{r}3 \\
1 \\
0 \\
0 \\
--\end{array}$ & $\begin{array}{r}1 \\
0 \\
0 \\
0 \\
--\end{array}$ & $\begin{array}{r}0 \\
0 \\
0 \\
0 \\
--\end{array}$ & $\begin{array}{r}0 \\
0 \\
0 \\
0 \\
--\end{array}$ & $\begin{array}{r}0 \\
0 \\
0 \\
0 \\
--\end{array}$ \\
\hline $\begin{array}{l}6-21 \\
6-22 \\
6-23 \\
6-24 \\
6-25\end{array}$ & $\begin{array}{l}0 \\
2 \\
0 \\
0 \\
0\end{array}$ & $\begin{array}{l}0 \\
0 \\
0 \\
0 \\
0\end{array}$ & $\begin{array}{l}0 \\
0 \\
0 \\
0 \\
0\end{array}$ & $\begin{array}{l}0 \\
0 \\
0 \\
0 \\
0\end{array}$ & $\begin{array}{l}0 \\
0 \\
0 \\
0 \\
0\end{array}$ & $\begin{array}{l}=- \\
=- \\
=- \\
--\end{array}$ & $\begin{array}{l}=- \\
=- \\
-- \\
--\end{array}$ & $\begin{array}{l}-- \\
-- \\
-- \\
--\end{array}$ & $\begin{array}{l}=- \\
=- \\
--\end{array}$ & $\begin{array}{l}-- \\
=- \\
z- \\
--\end{array}$ \\
\hline $\begin{array}{r}6-26 \\
6-27 \\
6-28 \\
7-11 \\
10-08\end{array}$ & $\begin{array}{c}-- \\
0 \\
0 \\
0 \\
0\end{array}$ & $\begin{array}{c}-- \\
0 \\
0 \\
0 \\
0\end{array}$ & $\begin{array}{c}-- \\
0 \\
0 \\
0 \\
0\end{array}$ & $\begin{array}{c}-- \\
0 \\
0 \\
0 \\
0\end{array}$ & $\begin{array}{r}- \\
0 \\
0 \\
0 \\
0\end{array}$ & $\begin{array}{l}=- \\
=- \\
=- \\
--\end{array}$ & $\begin{array}{l}=- \\
=- \\
--\end{array}$ & $=$ & $\begin{array}{l}=- \\
=- \\
=- \\
=-\end{array}$ & $\begin{array}{l}-- \\
=- \\
=- \\
=- \\
--\end{array}$ \\
\hline
\end{tabular}


TABLE 12.- NUMBER OF PINK TRACER PARTICLES(1), PER 100 GRAMS OF BED MATERIAL, EAST FORK RIVER, WYOMING, 1979--CONTINUED SECTION 1573 SECTION 1662

\begin{tabular}{|c|c|c|c|c|c|c|c|c|c|c|}
\hline \multirow[t]{2}{*}{ DATE } & \multicolumn{3}{|c|}{$\begin{array}{r}\text { NUMBER OF } \\
\text { PARTICLES BY SIR }\end{array}$} & \multicolumn{2}{|c|}{$\begin{array}{l}\text { TRACER } \\
\text { CLASS (MM) }\end{array}$} & \multicolumn{5}{|c|}{$\begin{array}{r}\text { NUMBER OF PINK TRACER } \\
\text { PARTICLES BY SIZE CLASS (MM) }\end{array}$} \\
\hline & $\begin{array}{l}0.25 \\
\text { TOO } \\
0.50\end{array}$ & $\begin{array}{l}0.50 \\
\text { TO } \\
1.00\end{array}$ & $\begin{array}{l}1.00 \\
\text { TO } \\
2.00\end{array}$ & $\begin{array}{l}2.00 \\
\text { TO } \\
4.00\end{array}$ & $\begin{array}{l}4.00 \\
\text { TO } \\
8.00\end{array}$ & $\begin{array}{l}0.25 \\
\text { TO } \\
0.50\end{array}$ & $\begin{array}{r}0.50 \\
\text { TO } \\
1.00\end{array}$ & $\begin{array}{r}1.00 \\
\text { TO } \\
2.00\end{array}$ & $\begin{array}{r}2.00 \\
\text { TO } \\
4.00\end{array}$ & $\begin{array}{r}4.00 \\
\text { TO } \\
8.00\end{array}$ \\
\hline $\begin{array}{l}5-20 \\
5-21 \\
5-23 \\
5-24 \\
5-25\end{array}$ & $\begin{array}{l}0 \\
0 \\
0 \\
0 \\
0\end{array}$ & $\begin{array}{l}0 \\
0 \\
1 \\
0 \\
0\end{array}$ & $\begin{array}{l}0 \\
0 \\
0 \\
0 \\
0\end{array}$ & $\begin{array}{l}0 \\
0 \\
0 \\
0 \\
0\end{array}$ & $\begin{array}{l}0 \\
0 \\
0 \\
0 \\
0\end{array}$ & $\begin{array}{l}0 \\
0 \\
0 \\
0 \\
5\end{array}$ & $\begin{array}{l}0 \\
0 \\
0 \\
0 \\
0\end{array}$ & $\begin{array}{l}0 \\
0 \\
0 \\
0 \\
0\end{array}$ & $\begin{array}{l}0 \\
0 \\
0 \\
0 \\
0\end{array}$ & $\begin{array}{l}0 \\
0 \\
0 \\
0 \\
0\end{array}$ \\
\hline $\begin{array}{l}5-26 \\
5-27 \\
5-28 \\
5-30 \\
5-31\end{array}$ & $\begin{array}{l}0 \\
0 \\
0 \\
0 \\
0\end{array}$ & $\begin{array}{l}0 \\
0 \\
0 \\
0 \\
0\end{array}$ & $\begin{array}{l}0 \\
0 \\
0 \\
0 \\
0\end{array}$ & $\begin{array}{l}0 \\
0 \\
0 \\
0 \\
0\end{array}$ & $\begin{array}{l}0 \\
0 \\
0 \\
0 \\
0\end{array}$ & $\begin{array}{l}0 \\
0 \\
0 \\
0 \\
0\end{array}$ & $\begin{array}{l}0 \\
0 \\
0 \\
0 \\
0\end{array}$ & $\begin{array}{l}0 \\
0 \\
0 \\
0 \\
0\end{array}$ & $\begin{array}{l}0 \\
0 \\
0 \\
0 \\
0\end{array}$ & $\begin{array}{l}0 \\
0 \\
0 \\
0 \\
0\end{array}$ \\
\hline $\begin{array}{l}6-01 \\
6-02 \\
6-03 \\
6-04 \\
6-05\end{array}$ & $\begin{array}{l}0 \\
0 \\
0 \\
0 \\
0\end{array}$ & $\begin{array}{l}0 \\
0 \\
0 \\
0 \\
0\end{array}$ & $\begin{array}{l}0 \\
0 \\
0 \\
0 \\
0\end{array}$ & $\begin{array}{l}0 \\
0 \\
0 \\
0 \\
0\end{array}$ & $\begin{array}{l}0 \\
0 \\
0 \\
0 \\
0\end{array}$ & $\begin{array}{l}0 \\
0 \\
0 \\
0 \\
0\end{array}$ & $\begin{array}{l}0 \\
0 \\
0 \\
0 \\
0\end{array}$ & $\begin{array}{l}0 \\
0 \\
0 \\
0 \\
0\end{array}$ & $\begin{array}{l}0 \\
0 \\
0 \\
0 \\
0\end{array}$ & $\begin{array}{l}0 \\
0 \\
0 \\
0 \\
0\end{array}$ \\
\hline $\begin{array}{l}6-06 \\
6-07 \\
6-08 \\
6-09 \\
6-10\end{array}$ & $\begin{array}{l}0 \\
0 \\
0 \\
0 \\
0\end{array}$ & $\begin{array}{l}0 \\
0 \\
0 \\
0 \\
0\end{array}$ & $\begin{array}{l}0 \\
0 \\
0 \\
0 \\
0 \\
0\end{array}$ & $\begin{array}{l}0 \\
0 \\
0 \\
0 \\
0\end{array}$ & $\begin{array}{l}0 \\
0 \\
0 \\
0 \\
0\end{array}$ & $\begin{array}{l}0 \\
0 \\
0 \\
0 \\
0\end{array}$ & $\begin{array}{l}0 \\
0 \\
0 \\
0 \\
0\end{array}$ & $\begin{array}{l}0 \\
0 \\
0 \\
0 \\
0\end{array}$ & $\begin{array}{l}0 \\
0 \\
0 \\
0 \\
0\end{array}$ & $\begin{array}{l}0 \\
0 \\
0 \\
0 \\
0\end{array}$ \\
\hline $\begin{array}{l}6-11 \\
6-12 \\
6-13 \\
6-14 \\
6-15\end{array}$ & $\begin{array}{l}0 \\
0 \\
0 \\
2 \\
0\end{array}$ & $\begin{array}{l}0 \\
0 \\
0 \\
0 \\
0\end{array}$ & $\begin{array}{l}0 \\
0 \\
0 \\
0 \\
0\end{array}$ & $\begin{array}{l}0 \\
0 \\
0 \\
0 \\
0\end{array}$ & $\begin{array}{l}0 \\
0 \\
0 \\
0 \\
0\end{array}$ & $\begin{array}{l}0 \\
0 \\
0 \\
4 \\
0\end{array}$ & $\begin{array}{l}0 \\
0 \\
0 \\
0 \\
0\end{array}$ & $\begin{array}{l}0 \\
0 \\
0 \\
0 \\
0\end{array}$ & $\begin{array}{l}0 \\
0 \\
0 \\
0 \\
0\end{array}$ & $\begin{array}{l}0 \\
0 \\
0 \\
0 \\
0\end{array}$ \\
\hline $\begin{array}{l}6-16 \\
6-17 \\
6-18 \\
6-19 \\
6-20\end{array}$ & $\begin{array}{l}0 \\
0 \\
0 \\
2 \\
0\end{array}$ & $\begin{array}{l}0 \\
0 \\
0 \\
0 \\
0\end{array}$ & $\begin{array}{l}0 \\
0 \\
0 \\
0 \\
0\end{array}$ & $\begin{array}{l}0 \\
0 \\
0 \\
0 \\
0\end{array}$ & $\begin{array}{l}0 \\
0 \\
0 \\
0 \\
0\end{array}$ & $\begin{array}{l}2 \\
3 \\
6 \\
0 \\
3\end{array}$ & $\begin{array}{l}0 \\
0 \\
0 \\
0 \\
0\end{array}$ & $\begin{array}{l}0 \\
0 \\
0 \\
0 \\
0\end{array}$ & $\begin{array}{l}0 \\
0 \\
0 \\
0 \\
0\end{array}$ & $\begin{array}{l}0 \\
0 \\
0 \\
0 \\
0\end{array}$ \\
\hline $\begin{array}{l}6-21 \\
6-22 \\
6-23 \\
6-24 \\
6-25\end{array}$ & $\begin{array}{l}0 \\
0 \\
0 \\
0 \\
0\end{array}$ & $\begin{array}{l}0 \\
0 \\
0 \\
0 \\
0\end{array}$ & $\begin{array}{l}0 \\
0 \\
0 \\
0 \\
0\end{array}$ & $\begin{array}{l}0 \\
0 \\
0 \\
0 \\
0\end{array}$ & $\begin{array}{l}0 \\
0 \\
0 \\
0 \\
0\end{array}$ & $\begin{array}{r}6 \\
5 \\
0 \\
0 \\
--\end{array}$ & $\begin{array}{r}4 \\
0 \\
0 \\
0 \\
--\end{array}$ & $\begin{array}{r}0 \\
0 \\
0 \\
0 \\
--\end{array}$ & $\begin{array}{r}0 \\
0 \\
0 \\
0 \\
--\end{array}$ & $\begin{array}{r}0 \\
0 \\
0 \\
0 \\
--\end{array}$ \\
\hline $\begin{array}{r}6-26 \\
6-27 \\
6-28 \\
7-11 \\
10-08\end{array}$ & $\begin{array}{l}0 \\
2 \\
0 \\
0 \\
0\end{array}$ & $\begin{array}{l}0 \\
0 \\
0 \\
1 \\
0\end{array}$ & $\begin{array}{l}0 \\
0 \\
0 \\
0 \\
0\end{array}$ & $\begin{array}{l}0 \\
0 \\
0 \\
0 \\
0\end{array}$ & $\begin{array}{l}0 \\
0 \\
0 \\
0 \\
0\end{array}$ & $\begin{array}{l}0 \\
2 \\
0 \\
4 \\
1\end{array}$ & $\begin{array}{l}0 \\
0 \\
0 \\
2 \\
2\end{array}$ & $\begin{array}{l}0 \\
0 \\
0 \\
0 \\
0\end{array}$ & $\begin{array}{l}0 \\
0 \\
0 \\
0 \\
0\end{array}$ & $\begin{array}{l}0 \\
0 \\
0 \\
0 \\
0\end{array}$ \\
\hline
\end{tabular}


TABLE 12.- NUMBER OF PINK TRACER PARTICLES(1), PER 100 GRAMS OF BED MATERIAL, EAST FORK RIVER, WYOMING, 1979--CONTINUED

SECTION 1695

SECTION 1766

\begin{tabular}{|c|c|c|c|c|c|c|c|c|c|c|}
\hline \multirow[t]{2}{*}{ DATE } & \multicolumn{3}{|c|}{$\begin{array}{rll}\text { NUMBER OF } & \text { PINK } \\
\text { PARTICLES } & \text { BY } & \text { SIZE }\end{array}$} & \multicolumn{2}{|c|}{$\begin{array}{l}\text { TRACER } \\
\text { CLASS (MM) }\end{array}$} & \multicolumn{2}{|c|}{$\begin{array}{r}\text { NUMBER } \\
\text { PARTICLES }\end{array}$} & $\begin{array}{ll}\text { OF } & \text { PIN } \\
\text { BY } & \text { SIZ }\end{array}$ & \multicolumn{2}{|c|}{$\begin{array}{l}\text { TRACER } \\
\text { CLASS (MM) }\end{array}$} \\
\hline & $\begin{array}{l}0.25 \\
\text { TO } \\
0.50\end{array}$ & $\begin{array}{l}0.50 \\
\text { TO } \\
1.00\end{array}$ & $\begin{array}{l}1.00 \\
2.00\end{array}$ & $\begin{array}{l}2.00 \\
\text { TO } \\
4.00\end{array}$ & $\begin{array}{l}4.00 \\
\text { TO } \\
8.00\end{array}$ & $\begin{array}{l}0.25 \\
\text { TO } \\
0.50\end{array}$ & $\begin{array}{r}0.50 \\
\text { TO } \\
1.00\end{array}$ & $\begin{array}{r}1.00 \\
\text { TO } \\
2.00\end{array}$ & $\begin{array}{r}2.00 \\
\mathrm{TO} \\
4.00\end{array}$ & $\begin{array}{l}4.00 \\
\text { TO } \\
8.00\end{array}$ \\
\hline $\begin{array}{l}5-20 \\
5-21 \\
5-23 \\
5-24 \\
5-25\end{array}$ & $\begin{array}{l}-- \\
=- \\
=-\end{array}$ & $\begin{array}{l}-- \\
=- \\
--\end{array}$ & $\begin{array}{l}-- \\
=- \\
--\end{array}$ & $\begin{array}{l}=- \\
\overline{-} \\
=-\end{array}$ & $\begin{array}{l}=- \\
=- \\
=-\end{array}$ & $\begin{array}{l}=- \\
=- \\
--\end{array}$ & $\begin{array}{l}=- \\
\overline{--} \\
z\end{array}$ & $\begin{array}{l}=- \\
\overline{-} \\
\overline{-}\end{array}$ & $\begin{array}{l}-- \\
=- \\
--\end{array}$ & $\begin{array}{l}m \\
=- \\
=- \\
--\end{array}$ \\
\hline $\begin{array}{l}5-26 \\
5-27 \\
5-28 \\
5-30 \\
5-31\end{array}$ & $\overline{--}$ & $\begin{array}{c}-- \\
-- \\
-0 \\
0\end{array}$ & $\overline{--}$ & $\overline{--}$ & $\begin{array}{c}-- \\
\overline{--} \\
0 \\
0\end{array}$ & $\begin{array}{c}-- \\
-- \\
0 \\
0\end{array}$ & $\begin{array}{c}-- \\
-- \\
0 \\
0\end{array}$ & $\begin{array}{c}\overline{-} \\
\overline{0} \\
0\end{array}$ & $\overline{--}$ & $\begin{array}{c}-- \\
-- \\
-0 \\
0\end{array}$ \\
\hline $\begin{array}{l}6-01 \\
6-02 \\
6-03 \\
6-04 \\
6-05\end{array}$ & $\begin{array}{l}0 \\
0 \\
0 \\
0 \\
0\end{array}$ & $\begin{array}{l}0 \\
0 \\
0 \\
0 \\
0\end{array}$ & $\begin{array}{l}0 \\
0 \\
0 \\
0 \\
0\end{array}$ & $\begin{array}{l}0 \\
0 \\
0 \\
0 \\
0\end{array}$ & $\begin{array}{l}0 \\
0 \\
0 \\
0 \\
0\end{array}$ & $\begin{array}{l}0 \\
2 \\
2 \\
0 \\
1\end{array}$ & $\begin{array}{l}0 \\
1 \\
0 \\
0 \\
0\end{array}$ & $\begin{array}{l}0 \\
0 \\
0 \\
0 \\
0\end{array}$ & $\begin{array}{l}0 \\
0 \\
0 \\
0 \\
0\end{array}$ & $\begin{array}{l}0 \\
0 \\
0 \\
0 \\
0\end{array}$ \\
\hline $\begin{array}{l}6-06 \\
6-07 \\
6-08 \\
6-09 \\
6-10\end{array}$ & $\begin{array}{l}1 \\
1 \\
0 \\
2 \\
0\end{array}$ & $\begin{array}{l}0 \\
0 \\
0 \\
0 \\
0\end{array}$ & $\begin{array}{l}0 \\
0 \\
0 \\
0 \\
0\end{array}$ & $\begin{array}{l}0 \\
0 \\
0 \\
0 \\
0\end{array}$ & $\begin{array}{l}0 \\
0 \\
0 \\
0 \\
0\end{array}$ & $\begin{array}{l}0 \\
0 \\
1 \\
\frac{1}{2}\end{array}$ & $\begin{array}{l}0 \\
0 \\
0 \\
0 \\
0\end{array}$ & $\begin{array}{l}0 \\
0 \\
0 \\
0 \\
0\end{array}$ & $\begin{array}{l}0 \\
0 \\
0 \\
0 \\
0\end{array}$ & $\begin{array}{l}0 \\
0 \\
0 \\
0 \\
0\end{array}$ \\
\hline $\begin{array}{l}6-11 \\
6-12 \\
6-13 \\
6-14 \\
6-15\end{array}$ & $\begin{array}{l}1 \\
4 \\
7 \\
3 \\
2\end{array}$ & $\begin{array}{l}0 \\
0 \\
0 \\
0 \\
1\end{array}$ & $\begin{array}{l}0 \\
0 \\
0 \\
0 \\
0\end{array}$ & $\begin{array}{l}0 \\
0 \\
0 \\
0 \\
0\end{array}$ & $\begin{array}{l}0 \\
0 \\
0 \\
0 \\
0\end{array}$ & $\begin{array}{l}0 \\
1 \\
0 \\
1 \\
0\end{array}$ & $\begin{array}{l}0 \\
0 \\
0 \\
0 \\
0\end{array}$ & $\begin{array}{l}0 \\
0 \\
0 \\
0 \\
0\end{array}$ & $\begin{array}{l}0 \\
0 \\
0 \\
0 \\
0\end{array}$ & $\begin{array}{l}0 \\
0 \\
0 \\
0 \\
0\end{array}$ \\
\hline $\begin{array}{l}6-16 \\
6-17 \\
6-18 \\
6-19 \\
6-20\end{array}$ & $\begin{array}{l}3 \\
4 \\
3 \\
2 \\
2\end{array}$ & $\begin{array}{l}0 \\
0 \\
0 \\
0 \\
0\end{array}$ & $\begin{array}{l}0 \\
0 \\
0 \\
0 \\
0\end{array}$ & $\begin{array}{l}0 \\
0 \\
0 \\
0 \\
0\end{array}$ & $\begin{array}{l}0 \\
0 \\
0 \\
0 \\
0\end{array}$ & $\begin{array}{l}\frac{1}{3} \\
1 \\
7 \\
2\end{array}$ & $\begin{array}{l}0 \\
1 \\
0 \\
0 \\
2\end{array}$ & $\begin{array}{l}0 \\
0 \\
0 \\
0 \\
0\end{array}$ & $\begin{array}{l}0 \\
0 \\
0 \\
0 \\
0\end{array}$ & $\begin{array}{l}0 \\
0 \\
0 \\
0 \\
0\end{array}$ \\
\hline $\begin{array}{l}6-21 \\
6-22 \\
6-23 \\
6-24 \\
6-25\end{array}$ & $\begin{array}{l}1 \\
0 \\
3 \\
2 \\
3\end{array}$ & $\begin{array}{l}1 \\
0 \\
1 \\
1 \\
0\end{array}$ & $\begin{array}{l}0 \\
0 \\
0 \\
0 \\
0\end{array}$ & $\begin{array}{l}0 \\
0 \\
0 \\
0 \\
0\end{array}$ & $\begin{array}{l}0 \\
0 \\
0 \\
0 \\
0\end{array}$ & $\begin{array}{l}3 \\
3 \\
0 \\
0 \\
0\end{array}$ & $\begin{array}{l}0 \\
1 \\
0 \\
0 \\
2\end{array}$ & $\begin{array}{l}0 \\
0 \\
0 \\
0 \\
0\end{array}$ & $\begin{array}{l}0 \\
0 \\
0 \\
0 \\
0\end{array}$ & $\begin{array}{l}0 \\
0 \\
0 \\
0 \\
0\end{array}$ \\
\hline $\begin{array}{r}6-26 \\
6-27 \\
6-28 \\
7-11 \\
10-08\end{array}$ & $\begin{array}{l}0 \\
4 \\
4 \\
1 \\
3\end{array}$ & $\begin{array}{l}0 \\
0 \\
0 \\
0 \\
4\end{array}$ & $\begin{array}{l}0 \\
0 \\
0 \\
0 \\
0\end{array}$ & $\begin{array}{l}0 \\
0 \\
0 \\
0 \\
0\end{array}$ & $\begin{array}{l}0 \\
0 \\
0 \\
0 \\
0\end{array}$ & $\begin{array}{l}2 \\
1 \\
1 \\
0 \\
2\end{array}$ & $\begin{array}{l}2 \\
2 \\
2 \\
0 \\
1\end{array}$ & $\begin{array}{l}0 \\
0 \\
0 \\
0 \\
0\end{array}$ & $\begin{array}{l}0 \\
0 \\
0 \\
0 \\
0\end{array}$ & $\begin{array}{l}0 \\
0 \\
0 \\
0 \\
0\end{array}$ \\
\hline
\end{tabular}


TABLE 12.- NUMBER OF PINK TRACER PARTICLES(1), PER 100 GRAMS OF BED MATERIAL, EAST FORK RIVER, WYOMING, 1979--CONTINUED

\begin{tabular}{|c|c|c|c|c|c|c|c|c|c|c|}
\hline \multirow[t]{2}{*}{ DATE } & \multicolumn{3}{|c|}{ 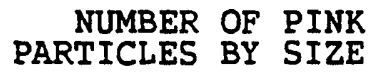 } & \multicolumn{2}{|c|}{$\begin{array}{l}\text { TRACER } \\
\text { CLASS (MM) }\end{array}$} & \multicolumn{5}{|c|}{$\begin{array}{l}\text { NUMBER OF PINK TRACKR } \\
\text { PARTICLES BY SIZE CLASS (MM) }\end{array}$} \\
\hline & $\begin{array}{l}0.25 \\
\text { TO } \\
0.50\end{array}$ & $\begin{array}{r}0.50 \\
\text { TO } \\
1.00\end{array}$ & $\begin{array}{l}1.00 \\
\text { TO } \\
2.00\end{array}$ & $\begin{array}{l}2.00 \\
\text { TO } \\
4.00\end{array}$ & $\begin{array}{l}4.00 \\
\text { TO } \\
8.00\end{array}$ & $\begin{array}{r}0.25 \\
\text { TO } \\
0.50\end{array}$ & $\begin{array}{r}0.50 \\
\text { TO } \\
1.00\end{array}$ & $\begin{array}{l}1.00 \\
\text { TO } \\
2.00\end{array}$ & $\begin{array}{r}2.00 \\
\text { TO } \\
4.00\end{array}$ & $\begin{array}{r}4.00 \\
\text { TO } \\
8.00\end{array}$ \\
\hline $\begin{array}{l}5-20 \\
5-21\end{array}$ & $=-$ & $=-$ & $=$ & $=$ & $=$ & $=-$ & $=-$ & $=$ & $=$ & $=-$ \\
\hline $5-23$ & -- & -- & -- & -- & -- & -- & -- & -- & -- & -- \\
\hline $\begin{array}{l}5-24 \\
5-25\end{array}$ & $\overline{--}$ & $=-$ & $=$ & $=-$ & $=$ & $\overline{--}$ & $=-$ & $=$ & $=-$ & $=-$ \\
\hline $5-26$ & -- & -- & -- & - & -- & -- & -- & -- & -- & -- \\
\hline $\begin{array}{l}5-27 \\
5-28\end{array}$ & $=$ & $=$ & $=$ & $=$ & $\overline{-}$ & $=$ & $\overline{-}$ & - & $=$ & $\overline{-}$ \\
\hline $5-30$ & 0 & 0 & 0 & 0 & 0 & 0 & 0 & 0 & 0 & 0 \\
\hline $\begin{array}{l}6-01 \\
6-02 \\
6-03 \\
6-04 \\
6-05\end{array}$ & $\begin{array}{l}0 \\
0 \\
2 \\
2 \\
4\end{array}$ & $\begin{array}{l}0 \\
0 \\
0 \\
0 \\
0\end{array}$ & $\begin{array}{l}0 \\
0 \\
0 \\
0 \\
0\end{array}$ & $\begin{array}{l}0 \\
0 \\
0 \\
0 \\
0\end{array}$ & $\begin{array}{l}0 \\
0 \\
0 \\
0 \\
0\end{array}$ & $\begin{array}{l}0 \\
0 \\
2 \\
0 \\
0\end{array}$ & $\begin{array}{l}0 \\
0 \\
1 \\
0 \\
0\end{array}$ & $\begin{array}{l}0 \\
0 \\
0 \\
0 \\
0\end{array}$ & $\begin{array}{l}0 \\
0 \\
0 \\
0 \\
0\end{array}$ & $\begin{array}{l}0 \\
0 \\
0 \\
0 \\
0\end{array}$ \\
\hline $\begin{array}{l}6-06 \\
6-07 \\
6-08 \\
6-09 \\
6-10\end{array}$ & $\begin{array}{l}3 \\
2 \\
0 \\
1 \\
0\end{array}$ & $\begin{array}{l}0 \\
0 \\
0 \\
0 \\
0\end{array}$ & $\begin{array}{l}0 \\
0 \\
0 \\
0 \\
0\end{array}$ & $\begin{array}{l}0 \\
0 \\
0 \\
0 \\
0\end{array}$ & $\begin{array}{l}0 \\
0 \\
0 \\
0 \\
0\end{array}$ & $\begin{array}{l}1 \\
0 \\
0 \\
2 \\
0\end{array}$ & $\begin{array}{l}0 \\
0 \\
0 \\
0 \\
0\end{array}$ & $\begin{array}{l}0 \\
0 \\
0 \\
0 \\
0\end{array}$ & $\begin{array}{l}0 \\
0 \\
0 \\
0 \\
0\end{array}$ & $\begin{array}{l}0 \\
0 \\
0 \\
0 \\
0\end{array}$ \\
\hline $\begin{array}{l}6-11 \\
6-12 \\
6-13 \\
6-14 \\
6-15\end{array}$ & $\begin{array}{l}7 \\
0 \\
4 \\
4 \\
2\end{array}$ & $\begin{array}{l}0 \\
0 \\
0 \\
1 \\
0\end{array}$ & $\begin{array}{l}0 \\
0 \\
0 \\
0 \\
0\end{array}$ & $\begin{array}{l}0 \\
0 \\
0 \\
0 \\
0\end{array}$ & $\begin{array}{l}0 \\
0 \\
0 \\
0 \\
0\end{array}$ & $\begin{array}{l}5 \\
0 \\
0 \\
1 \\
6\end{array}$ & $\begin{array}{l}1 \\
0 \\
0 \\
0 \\
3\end{array}$ & $\begin{array}{l}0 \\
0 \\
0 \\
0 \\
0\end{array}$ & $\begin{array}{l}0 \\
0 \\
0 \\
0 \\
0\end{array}$ & $\begin{array}{l}0 \\
0 \\
0 \\
0 \\
0\end{array}$ \\
\hline $\begin{array}{l}6-16 \\
6-17 \\
6-18 \\
6-19 \\
6-20\end{array}$ & $\begin{array}{l}1 \\
5 \\
6 \\
5 \\
4\end{array}$ & $\begin{array}{l}1 \\
1 \\
4 \\
1 \\
1\end{array}$ & $\begin{array}{l}0 \\
0 \\
0 \\
0 \\
0\end{array}$ & $\begin{array}{l}0 \\
0 \\
0 \\
0 \\
0\end{array}$ & $\begin{array}{l}0 \\
0 \\
0 \\
0 \\
0\end{array}$ & $\begin{array}{r}2 \\
8 \\
1 \\
15 \\
8\end{array}$ & $\begin{array}{r}2 \\
3 \\
2 \\
7 \\
10\end{array}$ & $\begin{array}{l}0 \\
0 \\
0 \\
0 \\
0\end{array}$ & $\begin{array}{l}0 \\
0 \\
0 \\
0 \\
0\end{array}$ & $\begin{array}{l}0 \\
0 \\
0 \\
0 \\
0\end{array}$ \\
\hline $\begin{array}{l}6-21 \\
6-22 \\
6-23 \\
6-24 \\
6-25\end{array}$ & $\begin{array}{l}3 \\
3 \\
5 \\
2 \\
3\end{array}$ & $\begin{array}{l}1 \\
1 \\
3 \\
0 \\
1\end{array}$ & $\begin{array}{l}0 \\
0 \\
0 \\
0 \\
0\end{array}$ & $\begin{array}{l}0 \\
0 \\
0 \\
0 \\
0\end{array}$ & $\begin{array}{l}0 \\
0 \\
0 \\
0 \\
0\end{array}$ & $\begin{array}{r}20 \\
11 \\
10 \\
6 \\
--\end{array}$ & $\begin{array}{r}9 \\
10 \\
3 \\
4 \\
--\end{array}$ & $\begin{array}{r}0 \\
0 \\
0 \\
0 \\
--\end{array}$ & $\begin{array}{r}0 \\
0 \\
0 \\
0 \\
--\end{array}$ & $\begin{array}{r}0 \\
0 \\
0 \\
0 \\
--\end{array}$ \\
\hline $6-26$ & 0 & 1 & 0 & 0 & 0 & $=$ & - & - & - & - \\
\hline $\begin{array}{r}0 \\
6-28 \\
7-11 \\
10-08\end{array}$ & $\begin{array}{l}1 \\
0 \\
0\end{array}$ & $\begin{array}{l}0 \\
0 \\
0\end{array}$ & $\begin{array}{l}0 \\
0 \\
0\end{array}$ & $\begin{array}{l}0 \\
0 \\
0\end{array}$ & $\begin{array}{l}0 \\
0 \\
0\end{array}$ & $\frac{11}{1}$ & $\begin{array}{l}7 \\
4 \\
4\end{array}$ & $\begin{array}{l}0 \\
0 \\
0\end{array}$ & $\begin{array}{l}0 \\
0 \\
0\end{array}$ & $\begin{array}{l}0 \\
0 \\
0\end{array}$ \\
\hline
\end{tabular}


TABLE 12.- NUMBER OF PINK TRACER PARTICLES(1), PER 100 GRAMS OF BED MATERIAL, EAST FORK RIVER, WYOMING, 1979--CONTINUED

\begin{tabular}{|c|c|c|c|c|c|c|c|c|c|c|}
\hline \multirow[t]{2}{*}{ DATE } & \multicolumn{2}{|c|}{$\begin{array}{r}\text { NUMBER O: } \\
\text { PARTICLES }\end{array}$} & $\begin{array}{l}\text { PINK } \\
\text { SIZE }\end{array}$ & \multicolumn{2}{|c|}{$\begin{array}{l}\text { TRACER } \\
\text { CLASS (MM) }\end{array}$} & \multicolumn{2}{|c|}{$\begin{array}{r}\text { NUMBER } \\
\text { PARTICLES }\end{array}$} & \multicolumn{3}{|c|}{$\begin{array}{l}\text { OF PTNK TRACER } \\
\text { BY } \text { SIZE CLASS (MM) }\end{array}$} \\
\hline & $\begin{array}{l}0.25 \\
\text { TO } \\
0.50\end{array}$ & $\begin{array}{l}0.50 \\
\text { TO } \\
1.00\end{array}$ & $\begin{array}{l}1.00 \\
\text { TO } \\
2.00\end{array}$ & $\begin{array}{l}2.00 \\
\text { TO } \\
4.00\end{array}$ & $\begin{array}{l}4.00 \\
\text { TO } \\
8.00\end{array}$ & $\begin{array}{l}0.25 \\
\text { TO } \\
0.50\end{array}$ & $\begin{array}{r}0.50 \\
\text { TO } \\
1.00\end{array}$ & $\begin{array}{l}1.00 \\
\text { TO } \\
2.00\end{array}$ & $\begin{array}{r}2.00 \\
\text { TO } \\
4.00\end{array}$ & $\begin{array}{r}4.00 \\
\text { TO } \\
8.00\end{array}$ \\
\hline $\begin{array}{l}5-20 \\
5-21 \\
5-23 \\
5-24 \\
5-25\end{array}$ & $\begin{array}{l}=- \\
=- \\
=- \\
--\end{array}$ & $\begin{array}{l}=- \\
=- \\
=- \\
--\end{array}$ & $\begin{array}{l}=- \\
=- \\
--\end{array}$ & $\begin{array}{l}=- \\
=- \\
=-\end{array}$ & $\begin{array}{l}=- \\
=- \\
=-\end{array}$ & $\begin{array}{l}=- \\
=- \\
=-\end{array}$ & $\begin{array}{l}=- \\
\overline{-} \\
=-\end{array}$ & $\begin{array}{l}=- \\
\overline{-} \\
=-\end{array}$ & $\begin{array}{l}-- \\
=- \\
=- \\
--\end{array}$ & $\begin{array}{l}= \\
=- \\
=-\end{array}$ \\
\hline $\begin{array}{l}5-26 \\
5-27 \\
5-28 \\
5-30 \\
5-31\end{array}$ & $\begin{array}{l}-- \\
-\overline{0} \\
0\end{array}$ & $\overline{--}$ & $\begin{array}{c}-- \\
-- \\
-0 \\
0\end{array}$ & $\begin{array}{c}-- \\
-- \\
-\overline{0} \\
0\end{array}$ & $\begin{array}{c}-- \\
-\overline{0} \\
0\end{array}$ & $\begin{array}{c}-- \\
\overline{0} \\
0\end{array}$ & $\overline{--}$ & $\begin{array}{c}\overline{-} \\
\overline{0} \\
0\end{array}$ & $\begin{array}{c}-- \\
\overline{-} \\
\overline{0} \\
0\end{array}$ & $\overline{-}$ \\
\hline $\begin{array}{l}6-01 \\
6-02 \\
6-03 \\
6-04 \\
6-05\end{array}$ & $\begin{array}{l}0 \\
0 \\
0 \\
1 \\
0\end{array}$ & $\begin{array}{l}0 \\
0 \\
0 \\
0 \\
0\end{array}$ & $\begin{array}{l}0 \\
0 \\
0 \\
0 \\
0\end{array}$ & $\begin{array}{l}0 \\
0 \\
0 \\
0 \\
0\end{array}$ & $\begin{array}{l}0 \\
0 \\
0 \\
0 \\
0\end{array}$ & $\begin{array}{l}0 \\
0 \\
0 \\
0 \\
1\end{array}$ & $\begin{array}{l}0 \\
0 \\
0 \\
1 \\
0\end{array}$ & $\begin{array}{l}0 \\
0 \\
0 \\
0 \\
0\end{array}$ & $\begin{array}{l}0 \\
0 \\
0 \\
0 \\
0\end{array}$ & $\begin{array}{l}0 \\
0 \\
0 \\
0 \\
0\end{array}$ \\
\hline $\begin{array}{l}6-06 \\
6-07 \\
6-08 \\
6-09 \\
6-10\end{array}$ & $\begin{array}{l}0 \\
0 \\
0 \\
0 \\
1\end{array}$ & $\begin{array}{l}0 \\
0 \\
0 \\
0 \\
0\end{array}$ & $\begin{array}{l}0 \\
0 \\
0 \\
0 \\
0\end{array}$ & $\begin{array}{l}0 \\
0 \\
0 \\
0 \\
0\end{array}$ & $\begin{array}{l}0 \\
0 \\
0 \\
0 \\
0\end{array}$ & $\begin{array}{l}3 \\
4 \\
1 \\
0 \\
0\end{array}$ & $\begin{array}{l}0 \\
1 \\
0 \\
0 \\
0\end{array}$ & $\begin{array}{l}0 \\
0 \\
0 \\
0 \\
0\end{array}$ & $\begin{array}{l}0 \\
0 \\
0 \\
0 \\
0\end{array}$ & $\begin{array}{l}0 \\
0 \\
0 \\
0 \\
0\end{array}$ \\
\hline $\begin{array}{l}6-11 \\
6-12 \\
6-13 \\
6-14 \\
6-15\end{array}$ & $\begin{array}{l}1 \\
1 \\
0 \\
0 \\
0\end{array}$ & $\begin{array}{l}0 \\
0 \\
0 \\
1 \\
0\end{array}$ & $\begin{array}{l}0 \\
0 \\
0 \\
0 \\
0\end{array}$ & $\begin{array}{l}0 \\
0 \\
0 \\
0 \\
0\end{array}$ & $\begin{array}{l}0 \\
0 \\
0 \\
0 \\
0\end{array}$ & $\begin{array}{r}3 \\
0 \\
0 \\
16 \\
2\end{array}$ & $\begin{array}{l}1 \\
0 \\
0 \\
2 \\
3\end{array}$ & $\begin{array}{l}0 \\
0 \\
0 \\
0 \\
0\end{array}$ & $\begin{array}{l}0 \\
0 \\
0 \\
0 \\
0\end{array}$ & $\begin{array}{l}0 \\
0 \\
0 \\
0 \\
0\end{array}$ \\
\hline $\begin{array}{l}6-16 \\
6-17 \\
6-18 \\
6-19 \\
6-20\end{array}$ & $\begin{array}{l}3 \\
2 \\
2 \\
0 \\
2\end{array}$ & $\begin{array}{l}1 \\
2 \\
3 \\
1 \\
0\end{array}$ & $\begin{array}{l}0 \\
0 \\
0 \\
0 \\
0\end{array}$ & $\begin{array}{l}0 \\
0 \\
0 \\
0 \\
0\end{array}$ & $\begin{array}{l}0 \\
0 \\
0 \\
0 \\
0\end{array}$ & $\begin{array}{l}6 \\
8 \\
3 \\
1 \\
6\end{array}$ & $\begin{array}{r}5 \\
3 \\
13 \\
2 \\
2\end{array}$ & $\begin{array}{l}0 \\
0 \\
0 \\
0 \\
0\end{array}$ & $\begin{array}{l}0 \\
0 \\
0 \\
0 \\
0\end{array}$ & $\begin{array}{l}0 \\
0 \\
0 \\
0 \\
0\end{array}$ \\
\hline $\begin{array}{l}6-21 \\
6-22 \\
6-23 \\
6-24 \\
6-25\end{array}$ & $\begin{array}{l}0 \\
5 \\
2 \\
3 \\
1\end{array}$ & $\begin{array}{l}2 \\
2 \\
2 \\
6 \\
0\end{array}$ & $\begin{array}{l}0 \\
0 \\
0 \\
0 \\
1\end{array}$ & $\begin{array}{l}0 \\
0 \\
0 \\
0 \\
0\end{array}$ & $\begin{array}{l}0 \\
0 \\
0 \\
0 \\
0\end{array}$ & $\begin{array}{l}2 \\
0 \\
0 \\
4 \\
3\end{array}$ & $\begin{array}{l}\frac{1}{1} \\
\frac{1}{2} \\
\frac{1}{2}\end{array}$ & $\begin{array}{l}0 \\
0 \\
0 \\
0 \\
0\end{array}$ & $\begin{array}{l}0 \\
0 \\
0 \\
0 \\
0\end{array}$ & $\begin{array}{l}0 \\
0 \\
0 \\
0 \\
0\end{array}$ \\
\hline $\begin{array}{r}6-26 \\
6-27 \\
6-28 \\
7-11 \\
10-08\end{array}$ & $\begin{array}{l}2 \\
9 \\
4 \\
0 \\
0\end{array}$ & $\begin{array}{l}2 \\
3 \\
2 \\
1 \\
5\end{array}$ & $\begin{array}{l}1 \\
0 \\
1 \\
0 \\
1\end{array}$ & $\begin{array}{l}0 \\
0 \\
0 \\
0 \\
0\end{array}$ & $\begin{array}{l}0 \\
0 \\
0 \\
0 \\
0\end{array}$ & $=-$ & $\begin{array}{r}-- \\
-3 \\
2 \\
1\end{array}$ & $\begin{array}{c}-- \\
-\overline{0} \\
0 \\
0\end{array}$ & $\begin{array}{c}-- \\
-0 \\
0 \\
0\end{array}$ & $\begin{array}{c}-- \\
-\overline{0} \\
0 \\
0\end{array}$ \\
\hline
\end{tabular}


TABLE 12.- NUMBER OF PINK TRACER PARTICLES(1), PER 100 GRAMS OF BED MATERIAL, EAST FORK RIVER, WYOMING, 1979--CONTINUED

\begin{tabular}{|c|c|c|c|c|c|c|c|c|c|c|}
\hline \multirow[t]{2}{*}{ DATE } & \multicolumn{5}{|c|}{$\begin{aligned} \text { NUMBER OF } & \text { PINK TRACER } \\
\text { PARTICLES BY } & \text { SIZE CLASS (MM) }\end{aligned}$} & \multicolumn{5}{|c|}{$\begin{array}{l}\text { NUMBER OF PINK TRACER } \\
\text { PARTICLES BY SIZE CLASS (MM) }\end{array}$} \\
\hline & $\begin{array}{l}0.25 \\
\text { TO } \\
0.50\end{array}$ & $\begin{array}{l}0.50 \\
\text { TO } \\
1.00\end{array}$ & $\begin{array}{l}1.00 \\
\text { TO } \\
2.00\end{array}$ & $\begin{array}{l}2.00 \\
\text { TO } \\
4.00\end{array}$ & $\begin{array}{l}4.00 \\
\text { TO } \\
8.00\end{array}$ & $\begin{array}{r}0.25 \\
\text { TO } \\
0.50\end{array}$ & $\begin{array}{r}0.50 \\
\text { TO } \\
1.00\end{array}$ & $\begin{array}{r}1.00 \\
\text { TO } \\
2.00\end{array}$ & $\begin{array}{r}2.00 \\
\text { TO } \\
4.00\end{array}$ & $\begin{array}{r}4.00 \\
\text { TO } \\
8.00\end{array}$ \\
\hline $\begin{array}{l}5-20 \\
5-21 \\
5-23 \\
5-24 \\
5-25\end{array}$ & $\begin{array}{l}=- \\
=- \\
=- \\
=-\end{array}$ & $\begin{array}{l}-- \\
-- \\
-- \\
-- \\
--\end{array}$ & $\begin{array}{l}=- \\
=- \\
=- \\
--\end{array}$ & $\begin{array}{l}-- \\
-- \\
-- \\
-- \\
--\end{array}$ & $\begin{array}{l}-- \\
-- \\
-- \\
-- \\
--\end{array}$ & $\begin{array}{l}-- \\
-- \\
-- \\
-- \\
--\end{array}$ & $\begin{array}{l}-- \\
-- \\
-- \\
-- \\
--\end{array}$ & $\begin{array}{l}-- \\
-- \\
-- \\
--\end{array}$ & $\begin{array}{l}-- \\
=- \\
-- \\
--\end{array}$ & $\begin{array}{l}-- \\
=- \\
-- \\
--\end{array}$ \\
\hline $\begin{array}{l}5-26 \\
5-27 \\
5-28 \\
5-30 \\
5-31\end{array}$ & $\begin{array}{c}-- \\
-- \\
0 \\
0 \\
0\end{array}$ & $\begin{array}{c}-- \\
-\overline{0} \\
1 \\
0\end{array}$ & $\begin{array}{c}-- \\
-- \\
0 \\
0 \\
0\end{array}$ & $\begin{array}{c}-- \\
-\overline{0} \\
0 \\
0\end{array}$ & $\begin{array}{c}-- \\
-\overline{0} \\
0 \\
0\end{array}$ & $\begin{array}{l}-\overline{0} \\
0 \\
0 \\
0\end{array}$ & $\begin{array}{l}-\overline{0} \\
0 \\
0 \\
0\end{array}$ & $\begin{array}{l}- \\
0 \\
0 \\
0 \\
0\end{array}$ & $\begin{array}{c}-\overline{0} \\
0 \\
0 \\
0\end{array}$ & $\begin{array}{l}- \\
0 \\
0 \\
0 \\
0\end{array}$ \\
\hline $\begin{array}{l}6-01 \\
6-02 \\
6-03 \\
6-04 \\
6-05\end{array}$ & $\begin{array}{l}0 \\
0 \\
0 \\
0 \\
0\end{array}$ & $\begin{array}{l}0 \\
0 \\
0 \\
0 \\
1\end{array}$ & $\begin{array}{l}0 \\
0 \\
0 \\
0 \\
0\end{array}$ & $\begin{array}{l}0 \\
0 \\
0 \\
0 \\
0\end{array}$ & $\begin{array}{l}0 \\
0 \\
0 \\
0 \\
0\end{array}$ & $\begin{array}{l}1 \\
1 \\
4 \\
3 \\
1\end{array}$ & $\begin{array}{l}0 \\
0 \\
2 \\
3 \\
2\end{array}$ & $\begin{array}{l}0 \\
0 \\
0 \\
0 \\
0\end{array}$ & $\begin{array}{l}0 \\
0 \\
0 \\
0 \\
0\end{array}$ & $\begin{array}{l}0 \\
0 \\
0 \\
0 \\
0\end{array}$ \\
\hline $\begin{array}{l}6-06 \\
6-07 \\
6-08 \\
6-09 \\
6-10\end{array}$ & $\begin{array}{r}1 \\
4 \\
9 \\
8 \\
10\end{array}$ & $\begin{array}{l}1 \\
0 \\
0 \\
2 \\
0\end{array}$ & $\begin{array}{l}0 \\
0 \\
0 \\
0 \\
0\end{array}$ & $\begin{array}{l}0 \\
0 \\
0 \\
0 \\
0\end{array}$ & $\begin{array}{l}0 \\
0 \\
0 \\
0 \\
0\end{array}$ & $\begin{array}{r}2 \\
13 \\
1 \\
2 \\
9\end{array}$ & $\begin{array}{l}2 \\
0 \\
2 \\
1 \\
4\end{array}$ & $\begin{array}{l}0 \\
0 \\
0 \\
0 \\
0\end{array}$ & $\begin{array}{l}0 \\
0 \\
0 \\
0 \\
0\end{array}$ & $\begin{array}{l}0 \\
0 \\
0 \\
0 \\
0\end{array}$ \\
\hline $\begin{array}{l}6-11 \\
6-12 \\
6-13 \\
6-14 \\
6-15\end{array}$ & $\begin{array}{l}2 \\
0 \\
7 \\
7 \\
8\end{array}$ & $\begin{array}{r}0 \\
1 \\
5 \\
4 \\
13\end{array}$ & $\begin{array}{l}0 \\
0 \\
0 \\
0 \\
0\end{array}$ & $\begin{array}{l}0 \\
0 \\
0 \\
0 \\
0\end{array}$ & $\begin{array}{l}0 \\
0 \\
0 \\
0 \\
0\end{array}$ & $\begin{array}{r}4 \\
2 \\
19 \\
9 \\
5\end{array}$ & $\begin{array}{l}3 \\
2 \\
5 \\
6 \\
6\end{array}$ & $\begin{array}{l}0 \\
0 \\
0 \\
0 \\
1\end{array}$ & $\begin{array}{l}0 \\
0 \\
0 \\
0 \\
0\end{array}$ & $\begin{array}{l}0 \\
0 \\
0 \\
0 \\
0\end{array}$ \\
\hline $\begin{array}{l}6-16 \\
6-17 \\
6-18 \\
6-19 \\
6-20\end{array}$ & $\begin{array}{r}10 \\
11 \\
4 \\
3 \\
0\end{array}$ & $\begin{array}{r}13 \\
13 \\
3 \\
8 \\
4\end{array}$ & $\begin{array}{l}0 \\
1 \\
0 \\
0 \\
0\end{array}$ & $\begin{array}{l}0 \\
0 \\
0 \\
0 \\
0\end{array}$ & $\begin{array}{l}0 \\
0 \\
0 \\
0 \\
0\end{array}$ & $\begin{array}{r}23 \\
7 \\
3 \\
8 \\
5\end{array}$ & $\begin{array}{r}6 \\
14 \\
15 \\
18 \\
22\end{array}$ & $\begin{array}{l}2 \\
0 \\
4 \\
2 \\
1\end{array}$ & $\begin{array}{l}0 \\
1 \\
0 \\
0 \\
0\end{array}$ & $\begin{array}{l}0 \\
0 \\
0 \\
0 \\
0\end{array}$ \\
\hline $\begin{array}{l}6-21 \\
6-22 \\
6-23 \\
6-24 \\
6-25\end{array}$ & $\begin{array}{r}3 \\
2 \\
0 \\
2 \\
--\end{array}$ & $\begin{array}{r}3 \\
3 \\
8 \\
10 \\
--\end{array}$ & $\begin{array}{r}0 \\
1 \\
2 \\
0 \\
--\end{array}$ & $\begin{array}{r}0 \\
0 \\
0 \\
0 \\
--\end{array}$ & $\begin{array}{r}0 \\
0 \\
0 \\
0 \\
--\end{array}$ & $\begin{array}{r}0 \\
5 \\
2 \\
11 \\
--\end{array}$ & $\begin{array}{r}6 \\
9 \\
16 \\
19 \\
\end{array}$ & $\begin{array}{r}2 \\
1 \\
0 \\
2 \\
--\end{array}$ & $\begin{array}{r}0 \\
0 \\
0 \\
0 \\
--\end{array}$ & $\begin{array}{r}0 \\
0 \\
0 \\
0 \\
--\end{array}$ \\
\hline $\begin{array}{l}6-26 \\
6-27 \\
6-28 \\
7-11 \\
10-08(4)\end{array}$ & $\frac{--}{-\frac{1}{1}}$ & $\begin{array}{r}-- \\
-\overline{5} \\
8 \\
13\end{array}$ & $\begin{array}{r}-- \\
-\overline{0} \\
0 \\
2\end{array}$ & $\begin{array}{l}-- \\
-\overline{0} \\
0 \\
0\end{array}$ & $\begin{array}{r}-- \\
-\overline{0} \\
0 \\
0\end{array}$ & $\begin{array}{c}-- \\
-- \\
4 \\
1\end{array}$ & $\begin{array}{r}-- \\
19 \\
2 \\
18\end{array}$ & $\begin{array}{r}-- \\
-\overline{2} \\
3 \\
3\end{array}$ & $\begin{array}{c}-- \\
-\overline{1} \\
0 \\
0\end{array}$ & $\begin{array}{c}-- \\
-\overline{0} \\
0 \\
0\end{array}$ \\
\hline
\end{tabular}


TABLE 12.- NUMBER OF PINK TRACER PARTICLES(1), PER 100 GRAMS OF BED MATERIAL, EAST FORK RIVER, WYOMING, 1979--CONTINUED

\begin{tabular}{|c|c|c|c|c|c|c|c|c|c|c|}
\hline \multirow[t]{2}{*}{ DATE } & \multicolumn{2}{|c|}{$\begin{array}{r}\text { NUMBER } \\
\text { PARTICLES }\end{array}$} & $\begin{array}{l}\text { PINK } \\
\text { SIZE }\end{array}$ & \multicolumn{2}{|c|}{$\begin{array}{l}\text { TRACER } \\
\text { CLASS (MM) }\end{array}$} & \multicolumn{2}{|c|}{$\begin{array}{r}\text { NUMBER } \\
\text { PARTICLES }\end{array}$} & $\begin{array}{ll}\partial F & \text { PIN } \\
\text { BY } & \text { SIZ }\end{array}$ & \multicolumn{2}{|c|}{$\begin{array}{l}\text { TRACER } \\
\text { CLASS }(\mathrm{MM})\end{array}$} \\
\hline & $\begin{array}{c}0.25 \\
\text { TO } \\
0.50\end{array}$ & $\begin{array}{c}0.50 \\
\text { TO } \\
1.00\end{array}$ & $\begin{array}{l}1.00 \\
2.00\end{array}$ & $\begin{array}{l}2.00 \\
\text { TO } \\
4.00\end{array}$ & $\begin{array}{l}4.00 \\
\text { TO } \\
8.00\end{array}$ & $\begin{array}{r}0.25 \\
\text { TO } \\
0.50\end{array}$ & $\begin{array}{r}0.50 \\
\text { TO } \\
1.00\end{array}$ & $\begin{array}{r}1.00 \\
\text { TO } \\
2.00\end{array}$ & $\begin{array}{r}2.00 \\
\text { TO } \\
4.00\end{array}$ & $\begin{array}{l}4.00 \\
\text { TO } \\
8.00\end{array}$ \\
\hline $5-20$ & -- & -- & -- & -- & -- & -- & -- & -- & -- & -- \\
\hline $\begin{array}{l}5-21 \\
5-23\end{array}$ & $=-$ & $=-$ & $=-$ & $=$ & -- & $=-$ & $=-$ & $=-$ & $=-$ & $=-$ \\
\hline $5-24$ & -- & -- & -- & -- & -- & -- & -- & & & -- \\
\hline $5-25$ & -- & -- & -- & -- & -- & 0 & 0 & 0 & 0 & 0 \\
\hline $\begin{array}{l}5-26 \\
5-27 \\
5-28 \\
5-30 \\
5-31\end{array}$ & $\begin{array}{l}0 \\
0 \\
0 \\
0 \\
2\end{array}$ & $\begin{array}{l}0 \\
0 \\
0 \\
0 \\
0\end{array}$ & $\begin{array}{l}0 \\
1 \\
0 \\
0 \\
0\end{array}$ & $\begin{array}{l}0 \\
0 \\
0 \\
0 \\
0\end{array}$ & $\begin{array}{l}0 \\
0 \\
0 \\
0 \\
0\end{array}$ & $\begin{array}{l}0 \\
0 \\
0 \\
1 \\
0\end{array}$ & $\begin{array}{l}0 \\
0 \\
0 \\
1 \\
1\end{array}$ & $\begin{array}{l}0 \\
0 \\
0 \\
0 \\
0\end{array}$ & $\begin{array}{l}0 \\
0 \\
0 \\
0 \\
0\end{array}$ & $\begin{array}{l}0 \\
0 \\
0 \\
0 \\
0\end{array}$ \\
\hline $\begin{array}{l}6-01 \\
6-02 \\
6-03 \\
6-04 \\
6-05\end{array}$ & $\begin{array}{l}0 \\
0 \\
0 \\
0 \\
1\end{array}$ & $\begin{array}{l}1 \\
2 \\
0 \\
2 \\
1\end{array}$ & $\begin{array}{l}0 \\
0 \\
0 \\
0 \\
0\end{array}$ & $\begin{array}{l}0 \\
0 \\
0 \\
0 \\
0\end{array}$ & $\begin{array}{l}0 \\
0 \\
0 \\
0 \\
0\end{array}$ & $\begin{array}{l}1 \\
2 \\
0 \\
0 \\
0\end{array}$ & $\begin{array}{l}2 \\
3 \\
1 \\
0 \\
0\end{array}$ & $\begin{array}{l}0 \\
0 \\
0 \\
0 \\
0\end{array}$ & $\begin{array}{l}0 \\
0 \\
0 \\
0 \\
0\end{array}$ & $\begin{array}{l}0 \\
0 \\
0 \\
0 \\
0\end{array}$ \\
\hline $\begin{array}{l}6-06 \\
6-07 \\
6-08 \\
6-09 \\
6-10\end{array}$ & $\begin{array}{r}6 \\
10 \\
5 \\
7 \\
25\end{array}$ & $\begin{array}{r}2 \\
3 \\
10 \\
17 \\
34\end{array}$ & $\begin{array}{l}0 \\
0 \\
0 \\
0 \\
3\end{array}$ & $\begin{array}{l}0 \\
0 \\
0 \\
0 \\
0\end{array}$ & $\begin{array}{l}0 \\
0 \\
0 \\
0 \\
0\end{array}$ & $\begin{array}{r}23 \\
7 \\
5 \\
3 \\
10\end{array}$ & $\begin{array}{r}99 \\
23 \\
22 \\
33 \\
42\end{array}$ & $\begin{array}{l}0 \\
1 \\
2 \\
2 \\
3\end{array}$ & $\begin{array}{l}0 \\
0 \\
0 \\
1 \\
1\end{array}$ & $\begin{array}{l}0 \\
0 \\
0 \\
0 \\
0\end{array}$ \\
\hline $\begin{array}{l}6-11 \\
6-12 \\
6-13 \\
6-14 \\
6-15\end{array}$ & $\begin{array}{r}12 \\
1 \\
8 \\
12 \\
2\end{array}$ & $\begin{array}{l}26 \\
13 \\
27 \\
15 \\
11\end{array}$ & $\begin{array}{l}0 \\
2 \\
0 \\
2 \\
4\end{array}$ & $\begin{array}{l}0 \\
0 \\
0 \\
0 \\
0\end{array}$ & $\begin{array}{l}0 \\
0 \\
0 \\
0 \\
0\end{array}$ & $\begin{array}{r}5 \\
1 \\
5 \\
5 \\
10\end{array}$ & $\begin{array}{r}59 \\
23 \\
8 \\
6 \\
23\end{array}$ & $\begin{array}{l}5 \\
8 \\
0 \\
0 \\
5\end{array}$ & $\begin{array}{l}2 \\
0 \\
0 \\
0 \\
0\end{array}$ & $\begin{array}{l}0 \\
0 \\
0 \\
0 \\
0\end{array}$ \\
\hline $\begin{array}{l}6-16 \\
6-17 \\
6-18 \\
6-19 \\
6-20\end{array}$ & $\begin{array}{r}11 \\
4 \\
4 \\
8 \\
6\end{array}$ & $\begin{array}{l}23 \\
34 \\
44 \\
39 \\
58\end{array}$ & $\begin{array}{r}9 \\
5 \\
11 \\
11 \\
12\end{array}$ & $\begin{array}{l}0 \\
0 \\
1 \\
2 \\
0\end{array}$ & $\begin{array}{l}0 \\
0 \\
0 \\
0 \\
0\end{array}$ & $\begin{array}{l}1 \\
1 \\
8 \\
1 \\
1\end{array}$ & $\begin{array}{l}25 \\
25 \\
14 \\
23 \\
30\end{array}$ & $\begin{array}{r}12 \\
10 \\
6 \\
11 \\
13\end{array}$ & $\begin{array}{r}5 \\
4 \\
4 \\
14 \\
3\end{array}$ & $\begin{array}{l}0 \\
1 \\
0 \\
0 \\
0\end{array}$ \\
\hline $\begin{array}{l}6-21 \\
6-22 \\
6-23 \\
6-24 \\
6-25\end{array}$ & $\begin{array}{r}3 \\
4 \\
6 \\
5 \\
10\end{array}$ & $\begin{array}{l}47 \\
65 \\
62 \\
37 \\
34\end{array}$ & $\begin{array}{r}14 \\
9 \\
14 \\
18 \\
14\end{array}$ & $\begin{array}{l}1 \\
0 \\
1 \\
4 \\
3\end{array}$ & $\begin{array}{l}0 \\
0 \\
0 \\
0 \\
0\end{array}$ & $\begin{array}{l}1 \\
2 \\
1 \\
2 \\
2\end{array}$ & $\begin{array}{l}28 \\
45 \\
37 \\
20 \\
18\end{array}$ & $\begin{array}{l}10 \\
11 \\
14 \\
9 \\
17\end{array}$ & $\begin{array}{l}3 \\
5 \\
6 \\
6 \\
5\end{array}$ & $\begin{array}{l}0 \\
0 \\
0 \\
0 \\
0\end{array}$ \\
\hline $\begin{array}{r}6-26 \\
6-27 \\
6-28 \\
7-11 \\
10-07\end{array}$ & $\begin{array}{r}10 \\
7 \\
6 \\
0 \\
9\end{array}$ & $\begin{array}{l}44 \\
54 \\
35 \\
13 \\
43\end{array}$ & $\begin{array}{r}9 \\
15 \\
18 \\
11 \\
18\end{array}$ & $\begin{array}{l}1 \\
0 \\
5 \\
5 \\
0\end{array}$ & $\begin{array}{l}0 \\
0 \\
0 \\
0 \\
0\end{array}$ & $\begin{array}{l}5 \\
5 \\
7 \\
1 \\
5\end{array}$ & $\begin{array}{l}29 \\
23 \\
23 \\
17 \\
27\end{array}$ & $\begin{array}{l}25 \\
14 \\
11 \\
12 \\
12\end{array}$ & $\begin{array}{r}6 \\
3 \\
10 \\
3 \\
5\end{array}$ & $\begin{array}{l}0 \\
0 \\
1 \\
0 \\
0\end{array}$ \\
\hline
\end{tabular}


TABLE 12.- NUMBER OF PINK TRACER PARTICLES(1), PER 100 GRAMS OF BED MATERIAL, EAST FORK RIVER, WYOMING, 1979--CONTINUED

\begin{tabular}{|c|c|c|c|c|c|c|c|c|c|c|}
\hline \multirow[t]{2}{*}{ DATE } & \multicolumn{3}{|c|}{ 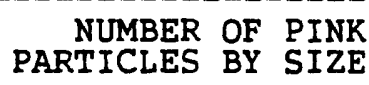 } & \multicolumn{2}{|c|}{$\begin{array}{l}\text { TRACER } \\
\text { CLASS (MM) }\end{array}$} & \multicolumn{5}{|c|}{$\begin{array}{l}\text { NUMBER OF PINK TRACER } \\
\text { PARTICLES BY SIZE CLASS (MM) }\end{array}$} \\
\hline & $\begin{array}{l}0.25 \\
\text { TO } \\
0.50\end{array}$ & $\begin{array}{l}0.50 \\
\text { TO } \\
1.00\end{array}$ & $\begin{array}{l}1.00 \\
\text { TO } \\
2.00\end{array}$ & $\begin{array}{l}2.00 \\
\text { TO } \\
4.00\end{array}$ & $\begin{array}{c}4.00 \\
\text { TO } \\
8.00\end{array}$ & $\begin{array}{l}0.25 \\
\text { TO } \\
0.50\end{array}$ & $\begin{array}{r}0.50 \\
\text { TO } \\
1.00\end{array}$ & $\begin{array}{r}1.00 \\
\text { TO } \\
2.00\end{array}$ & $\begin{array}{r}2.00 \\
\text { TO } \\
4.00\end{array}$ & $\begin{array}{r}4.00 \\
\text { TO } \\
8.00\end{array}$ \\
\hline $\begin{array}{l}5-20 \\
5-21 \\
5-23 \\
5-24 \\
5-25\end{array}$ & $\begin{array}{c}-- \\
-- \\
0 \\
1\end{array}$ & $\begin{array}{c}-- \\
-- \\
0 \\
0\end{array}$ & $\begin{array}{c}-- \\
-\overline{0} \\
0\end{array}$ & $\begin{array}{l}-- \\
-- \\
0 \\
0\end{array}$ & $\begin{array}{l}-- \\
-- \\
-\overline{0} \\
0\end{array}$ & $\begin{array}{r}-- \\
-\overline{0} \\
0 \\
1\end{array}$ & $\begin{array}{c}-- \\
-\overline{0} \\
0 \\
0\end{array}$ & $\begin{array}{c}-- \\
-\overline{0} \\
0 \\
0\end{array}$ & $\begin{array}{c}-- \\
-\overline{0} \\
0 \\
0\end{array}$ & $\begin{array}{c}-- \\
-\overline{0} \\
0 \\
0\end{array}$ \\
\hline $\begin{array}{l}5-26 \\
5-27 \\
5-28 \\
5-30 \\
5-31\end{array}$ & $\begin{array}{l}0 \\
0 \\
0 \\
0 \\
0\end{array}$ & $\begin{array}{r}1 \\
1 \\
3 \\
4 \\
10\end{array}$ & $\begin{array}{l}0 \\
0 \\
1 \\
0 \\
5\end{array}$ & $\begin{array}{l}0 \\
0 \\
0 \\
1 \\
2\end{array}$ & $\begin{array}{l}0 \\
0 \\
0 \\
1 \\
0\end{array}$ & $\begin{array}{l}0 \\
1 \\
0 \\
0 \\
2\end{array}$ & $\begin{array}{l}2 \\
1 \\
0 \\
4 \\
4\end{array}$ & $\begin{array}{l}0 \\
0 \\
0 \\
1 \\
1\end{array}$ & $\begin{array}{l}0 \\
0 \\
0 \\
0 \\
1\end{array}$ & $\begin{array}{l}0 \\
0 \\
0 \\
0 \\
0\end{array}$ \\
\hline $\begin{array}{l}6-01 \\
6-02 \\
6-03 \\
6-04 \\
6-05\end{array}$ & $\begin{array}{r}5 \\
0 \\
6 \\
6 \\
11\end{array}$ & $\begin{array}{l}20 \\
15 \\
13 \\
16 \\
33\end{array}$ & $\begin{array}{l}4 \\
1 \\
4 \\
4 \\
8\end{array}$ & $\begin{array}{l}2 \\
4 \\
1 \\
2 \\
1\end{array}$ & $\begin{array}{l}0 \\
1 \\
0 \\
0 \\
0\end{array}$ & $\begin{array}{r}1 \\
9 \\
29 \\
6 \\
3\end{array}$ & $\begin{array}{r}9 \\
12 \\
16 \\
54 \\
58\end{array}$ & $\begin{array}{r}1 \\
1 \\
1 \\
5 \\
20\end{array}$ & $\begin{array}{l}0 \\
0 \\
1 \\
0 \\
4\end{array}$ & $\begin{array}{l}0 \\
0 \\
0 \\
0 \\
0\end{array}$ \\
\hline $\begin{array}{l}6-06 \\
6-07 \\
6-08 \\
6-09 \\
6-10\end{array}$ & $\begin{array}{l}0 \\
0 \\
2 \\
1 \\
4\end{array}$ & $\begin{array}{r}0 \\
43 \\
54 \\
49 \\
68\end{array}$ & $\begin{array}{l}0 \\
14 \\
15 \\
15 \\
19\end{array}$ & $\begin{array}{l}0 \\
1 \\
1 \\
2 \\
3\end{array}$ & $\begin{array}{l}0 \\
0 \\
0 \\
0 \\
0\end{array}$ & $\begin{array}{l}1 \\
0 \\
0 \\
8 \\
7\end{array}$ & $\begin{array}{l}18 \\
10 \\
34 \\
48 \\
41\end{array}$ & $\begin{array}{r}19 \\
25 \\
17 \\
16 \\
8\end{array}$ & $\begin{array}{r}10 \\
6 \\
8 \\
7 \\
10\end{array}$ & $\begin{array}{l}2 \\
1 \\
0 \\
0 \\
1\end{array}$ \\
\hline $\begin{array}{l}6-11 \\
6-12 \\
6-13 \\
6-14 \\
6-15\end{array}$ & $\begin{array}{l}4 \\
4 \\
9 \\
4 \\
0\end{array}$ & $\begin{array}{l}41 \\
47 \\
46 \\
46 \\
23\end{array}$ & $\begin{array}{r}20 \\
16 \\
30 \\
10 \\
8\end{array}$ & $\begin{array}{r}4 \\
1 \\
7 \\
3 \\
10\end{array}$ & $\begin{array}{l}0 \\
0 \\
0 \\
0 \\
1\end{array}$ & $\begin{array}{l}9 \\
9 \\
2 \\
1 \\
2\end{array}$ & $\begin{array}{r}44 \\
30 \\
51 \\
5 \\
18\end{array}$ & $\begin{array}{r}31 \\
12 \\
28 \\
9 \\
10\end{array}$ & $\begin{array}{l}0 \\
1 \\
5 \\
5 \\
2\end{array}$ & $\begin{array}{l}0 \\
0 \\
0 \\
1 \\
1\end{array}$ \\
\hline $\begin{array}{l}6-16 \\
6-17 \\
6-18 \\
6-19 \\
6-20\end{array}$ & $\begin{array}{l}0 \\
4 \\
3 \\
0 \\
1\end{array}$ & $\begin{array}{l}41 \\
10 \\
26 \\
18 \\
13\end{array}$ & $\begin{array}{l}25 \\
16 \\
11 \\
11 \\
13\end{array}$ & $\begin{array}{l}0 \\
6 \\
9 \\
5 \\
5\end{array}$ & $\begin{array}{l}0 \\
0 \\
0 \\
3 \\
2\end{array}$ & $\begin{array}{l}2 \\
3 \\
4 \\
1 \\
1\end{array}$ & $\begin{array}{l}4 \\
11 \\
20 \\
16 \\
12\end{array}$ & $\begin{array}{r}3 \\
10 \\
8 \\
4 \\
11\end{array}$ & $\begin{array}{l}1 \\
6 \\
4 \\
2 \\
4\end{array}$ & $\begin{array}{l}1 \\
0 \\
1 \\
1 \\
0\end{array}$ \\
\hline $\begin{array}{l}6-21 \\
6-22 \\
6-23 \\
6-24 \\
6-25\end{array}$ & $\begin{array}{l}2 \\
1 \\
0 \\
3 \\
3\end{array}$ & $\begin{array}{l}14 \\
14 \\
13 \\
10 \\
10\end{array}$ & $\begin{array}{r}10 \\
3 \\
14 \\
12 \\
14\end{array}$ & $\begin{array}{l}9 \\
2 \\
5 \\
8 \\
5\end{array}$ & $\begin{array}{l}1 \\
0 \\
0 \\
2 \\
1\end{array}$ & $\begin{array}{r}3 \\
0 \\
3 \\
16 \\
36\end{array}$ & $\begin{array}{r}9 \\
16 \\
19 \\
7 \\
22\end{array}$ & $\begin{array}{r}4 \\
4 \\
8 \\
11 \\
7\end{array}$ & $\begin{array}{l}1 \\
5 \\
3 \\
1 \\
4\end{array}$ & $\begin{array}{l}1 \\
0 \\
0 \\
0 \\
0\end{array}$ \\
\hline $\begin{array}{r}6-26 \\
6-27 \\
6-28 \\
7-11 \\
10-07\end{array}$ & $\begin{array}{c}-- \\
-\overline{1} \\
7 \\
8\end{array}$ & $\begin{array}{r}-- \\
-\overline{5} \\
\frac{1}{23}\end{array}$ & $\begin{array}{r}-- \\
-\overline{9} \\
7 \\
14\end{array}$ & $\begin{array}{r}-- \\
-- \\
3 \\
2\end{array}$ & $\frac{--}{-1}$ & $\begin{array}{r}41 \\
48 \\
54 \\
6 \\
3\end{array}$ & $\begin{array}{l}15 \\
37 \\
64 \\
55 \\
21\end{array}$ & $\begin{array}{r}5 \\
2 \\
4 \\
12 \\
4\end{array}$ & $\begin{array}{l}3 \\
0 \\
0 \\
1 \\
1\end{array}$ & $\begin{array}{l}0 \\
0 \\
0 \\
0 \\
0\end{array}$ \\
\hline
\end{tabular}


TABLE 12.- NUMBER OF PINK TRACER PARTICLES(1), PER 100 GRAMS OF BED MATERIAL, EAST FORK RIVER, WYOMING, 1979--CONTINUED

\begin{tabular}{|c|c|c|c|c|c|c|c|c|c|c|}
\hline DATE & $\begin{array}{r}\text { NL } \\
\text { PART }\end{array}$ & $\begin{array}{l}\text { YBER } \\
\text { CLES }\end{array}$ & $\begin{array}{ll}\text { PINK } \\
\text { SIZE }\end{array}$ & $\begin{array}{l}\text { TRACE } \\
\text { CLASS }\end{array}$ & (MM) & PAR' & $\begin{array}{l}\text { JMBER } \\
\text { ICLES }\end{array}$ & $\begin{array}{ll}\text { DF } & \text { PIN } \\
\text { BY } & \text { SIZ }\end{array}$ & $\begin{array}{l}\text { TRAC } \\
\text { CLAS }\end{array}$ & (MM) \\
\hline$-\cdots$ & $\begin{array}{l}0.25 \\
\text { TO } \\
0.50\end{array}$ & $\begin{array}{l}0.50 \\
\text { TO } \\
1.00\end{array}$ & $\begin{array}{l}1.00 \\
2.00 \\
2.00\end{array}$ & $\begin{array}{l}2.00 \\
\text { TO } \\
4.00\end{array}$ & $\begin{array}{l}4.00 \\
\text { TO } \\
8.00\end{array}$ & $\begin{array}{r}0.25 \\
\text { TO } \\
0.50\end{array}$ & $\begin{array}{r}0.50 \\
\text { TO } \\
1.00\end{array}$ & $\begin{array}{r}1.00 \\
\text { TO } \\
2.00\end{array}$ & $\begin{array}{r}2.00 \\
\text { TO } \\
4.00\end{array}$ & $\begin{array}{l}4.00 \\
\text { TO } \\
8.00\end{array}$ \\
\hline $\begin{array}{l}5-20 \\
5-21 \\
5-23 \\
5-24 \\
5-25\end{array}$ & $\begin{array}{r}-- \\
0 \\
0 \\
2 \\
0\end{array}$ & $\begin{array}{c}-- \\
0 \\
1 \\
0 \\
0\end{array}$ & $\begin{array}{r}--\overline{0} \\
0 \\
0 \\
0\end{array}$ & $\begin{array}{c}--\overline{0} \\
0 \\
0 \\
0\end{array}$ & $\begin{array}{c}-- \\
0 \\
0 \\
0 \\
0\end{array}$ & $\begin{array}{r}-- \\
0 \\
0 \\
2 \\
0\end{array}$ & $\begin{array}{r}--\overline{0} \\
0 \\
4 \\
0\end{array}$ & $\begin{array}{r}--\overline{0} \\
0 \\
1 \\
1\end{array}$ & $\begin{array}{c}-- \\
0 \\
0 \\
0 \\
0\end{array}$ & $\begin{array}{c}-- \\
0 \\
0 \\
0 \\
0\end{array}$ \\
\hline $\begin{array}{l}5-26 \\
5-27 \\
5-28 \\
5-30 \\
5-31\end{array}$ & $\begin{array}{l}0 \\
0 \\
0 \\
0 \\
1\end{array}$ & $\begin{array}{r}0 \\
1 \\
1 \\
0 \\
11\end{array}$ & $\begin{array}{l}0 \\
1 \\
0 \\
0 \\
3\end{array}$ & $\begin{array}{l}0 \\
0 \\
0 \\
0 \\
0\end{array}$ & $\begin{array}{l}0 \\
0 \\
0 \\
0 \\
0\end{array}$ & $\begin{array}{r}0 \\
1 \frac{1}{2} \\
0 \\
2\end{array}$ & $\begin{array}{l}6 \\
32 \\
62 \\
19 \\
24\end{array}$ & $\begin{array}{r}1 \\
10 \\
4 \\
22 \\
24\end{array}$ & $\begin{array}{l}0 \\
0 \\
0 \\
6 \\
4\end{array}$ & $\begin{array}{l}0 \\
0 \\
0 \\
2 \\
1\end{array}$ \\
\hline $\begin{array}{l}6-01 \\
6-02 \\
6-03 \\
6-04 \\
6-05\end{array}$ & $\begin{array}{l}6 \\
8 \\
2 \\
3 \\
6\end{array}$ & $\begin{array}{l}65 \\
46 \\
36 \\
50 \\
43\end{array}$ & $\begin{array}{l}13 \\
27 \\
27 \\
20 \\
28\end{array}$ & $\begin{array}{l}3 \\
5 \\
8 \\
4 \\
4\end{array}$ & $\begin{array}{l}0 \\
0 \\
2 \\
1 \\
0\end{array}$ & $\begin{array}{r}5 \\
11 \\
4 \\
\frac{1}{3}\end{array}$ & $\begin{array}{r}55 \\
74 \\
26 \\
16 \\
7\end{array}$ & $\begin{array}{r}31 \\
15 \\
12 \\
9 \\
6\end{array}$ & $\begin{array}{r}4 \\
3 \\
10 \\
4 \\
5\end{array}$ & $\begin{array}{l}0 \\
1 \\
0 \\
0 \\
0\end{array}$ \\
\hline $\begin{array}{l}6-06 \\
6-07 \\
6-08 \\
6-09 \\
6-10\end{array}$ & $\begin{array}{l}3 \\
0 \\
0 \\
6 \\
5\end{array}$ & $\begin{array}{l}54 \\
24 \\
12 \\
26 \\
47\end{array}$ & $\begin{array}{l}12 \\
13 \\
17 \\
13 \\
22\end{array}$ & $\begin{array}{l}4 \\
5 \\
3 \\
2 \\
2\end{array}$ & $\begin{array}{l}0 \\
1 \\
0 \\
0 \\
1\end{array}$ & $\begin{array}{l}0 \\
0 \\
0 \\
1 \\
4\end{array}$ & $\begin{array}{r}7 \\
3 \\
6 \\
19 \\
41\end{array}$ & $\begin{array}{r}9 \\
8 \\
4 \\
11 \\
13\end{array}$ & $\begin{array}{l}4 \\
0 \\
1 \\
2 \\
4\end{array}$ & $\begin{array}{l}0 \\
0 \\
1 \\
0 \\
0\end{array}$ \\
\hline $\begin{array}{l}6-11 \\
6-12 \\
6-13 \\
6-14 \\
6-15\end{array}$ & $\begin{array}{l}6 \\
4 \\
0 \\
2 \\
7\end{array}$ & $\begin{array}{l}26 \\
31 \\
23 \\
27 \\
24\end{array}$ & $\begin{array}{r}8 \\
16 \\
17 \\
6 \\
6\end{array}$ & $\begin{array}{l}4 \\
2 \\
5 \\
1 \\
3\end{array}$ & $\begin{array}{l}0 \\
0 \\
0 \\
0 \\
0\end{array}$ & $\begin{array}{l}2 \\
5 \\
0 \\
4 \\
1\end{array}$ & $\begin{array}{r}6 \\
11 \\
11 \\
20 \\
21\end{array}$ & $\begin{array}{r}1 \\
4 \\
3 \\
11 \\
9\end{array}$ & $\begin{array}{l}3 \\
6 \\
5 \\
5 \\
1\end{array}$ & $\begin{array}{l}0 \\
0 \\
1 \\
0 \\
0\end{array}$ \\
\hline $\begin{array}{l}6-16 \\
6-17 \\
6-18 \\
6-19 \\
6-20\end{array}$ & $\begin{array}{l}2 \\
6 \\
1 \\
4 \\
4\end{array}$ & $\begin{array}{l}19 \\
32 \\
12 \\
41 \\
22\end{array}$ & $\begin{array}{r}10 \\
6 \\
2 \\
8 \\
8\end{array}$ & $\begin{array}{l}2 \\
0 \\
3 \\
0 \\
2\end{array}$ & $\begin{array}{l}0 \\
0 \\
0 \\
0 \\
0\end{array}$ & $\begin{array}{l}\frac{1}{1} \\
\frac{1}{2} \\
\frac{1}{4}\end{array}$ & $\begin{array}{r}13 \\
9 \\
5 \\
16 \\
28\end{array}$ & $\begin{array}{r}10 \\
12 \\
2 \\
12 \\
8\end{array}$ & $\begin{array}{l}4 \\
4 \\
1 \\
0 \\
0\end{array}$ & $\begin{array}{l}0 \\
2 \\
0 \\
0 \\
0\end{array}$ \\
\hline $\begin{array}{l}6-21 \\
6-22 \\
6-23 \\
6-24 \\
6-25\end{array}$ & $\begin{array}{r}1 \\
5 \\
26 \\
19 \\
9\end{array}$ & $\begin{array}{l}11 \\
21 \\
44 \\
36 \\
37\end{array}$ & $\begin{array}{r}5 \\
7 \\
8 \\
16 \\
10\end{array}$ & $\begin{array}{l}4 \\
1 \\
1 \\
4 \\
4\end{array}$ & $\begin{array}{l}0 \\
0 \\
0 \\
1 \\
0\end{array}$ & $\begin{array}{r}5 \\
17 \\
17 \\
8 \\
22\end{array}$ & $\begin{array}{l}33 \\
59 \\
43 \\
48 \\
22\end{array}$ & $\begin{array}{r}18 \\
9 \\
8 \\
4 \\
4\end{array}$ & $\begin{array}{l}5 \\
1 \\
1 \\
3 \\
5\end{array}$ & $\begin{array}{l}1 \\
0 \\
0 \\
0 \\
1\end{array}$ \\
\hline $\begin{array}{r}6-26 \\
6-27 \\
6-28 \\
7-11 \\
10-07\end{array}$ & $\begin{array}{r}6 \\
22 \\
15 \\
10 \\
0\end{array}$ & $\begin{array}{l}56 \\
34 \\
46 \\
21 \\
12\end{array}$ & $\begin{array}{r}16 \\
7 \\
10 \\
8 \\
7\end{array}$ & $\begin{array}{l}0 \\
3 \\
5 \\
3 \\
0\end{array}$ & $\begin{array}{l}0 \\
1 \\
0 \\
0 \\
0\end{array}$ & $\begin{array}{r}-- \\
15 \\
7\end{array}$ & $\begin{array}{l}-- \\
\overline{28} \\
12 \\
10\end{array}$ & $\begin{array}{r}-- \\
-\overline{4} \\
5 \\
4\end{array}$ & $\begin{array}{r}-- \\
- \\
3 \\
2 \\
2\end{array}$ & $\begin{array}{c}-- \\
-0 \\
0 \\
1\end{array}$ \\
\hline
\end{tabular}


TABLE 12. - NUMBER OF PINK TRACER PARTICLES(1), PER 100 GRAMS OF BED MATERIAL, EAST FORK RIVER, WYOMING, 1979--CONTINUED

\begin{tabular}{|c|c|c|c|c|c|c|c|c|c|c|}
\hline \multirow[t]{2}{*}{ DATE } & \multicolumn{5}{|c|}{$\begin{aligned} & \text { NUMBER OF PINK TRACER } \\
& \text { PARTICLES BY } \text { SIZE CLASS (MM) }\end{aligned}$} & \multicolumn{5}{|c|}{$\begin{array}{l}\text { NUMBER OF PINK TRACER } \\
\text { PARTICLES BY SIZE CLASS (MM) }\end{array}$} \\
\hline & $\begin{array}{l}0.25 \\
\text { TO } \\
0.50\end{array}$ & $\begin{array}{c}0.50 \\
\text { TO } \\
1.00\end{array}$ & $\begin{array}{l}1.00 \\
\text { TO } \\
2.00\end{array}$ & $\begin{array}{l}2.00 \\
\text { TO } \\
4.00\end{array}$ & $\begin{array}{l}4.00 \\
\text { TO } \\
8.00\end{array}$ & $\begin{array}{r}0.25 \\
\text { TO } \\
0.50\end{array}$ & $\begin{array}{r}0.50 \\
\text { TO } \\
1.00\end{array}$ & $\begin{array}{l}1.00 \\
\text { TO } \\
2.00\end{array}$ & $\begin{array}{r}2.00 \\
\text { TO } \\
4.00\end{array}$ & $\begin{array}{r}4.00 \\
\text { TO } \\
8.00\end{array}$ \\
\hline $\begin{array}{l}5-20 \\
5-21 \\
5-23 \\
5-24 \\
5-25\end{array}$ & $\begin{array}{l}0 \\
2 \\
1 \\
1 \\
1\end{array}$ & $\begin{array}{r}0 \\
4 \\
26 \\
19 \\
20\end{array}$ & $\begin{array}{r}0 \\
4 \\
82 \\
14 \\
24\end{array}$ & $\begin{array}{r}0 \\
0 \\
21 \\
7 \\
13\end{array}$ & $\begin{array}{l}0 \\
0 \\
1 \\
1 \\
1\end{array}$ & $\begin{array}{r}19 \\
14 \\
1 \\
0 \\
0\end{array}$ & $\begin{array}{r}59 \\
34 \\
17 \\
7 \\
7\end{array}$ & $\begin{array}{l}49 \\
79 \\
24 \\
25 \\
13\end{array}$ & $\begin{array}{r}6 \\
18 \\
11 \\
14 \\
5\end{array}$ & $\begin{array}{l}0 \\
3 \\
4 \\
0 \\
1\end{array}$ \\
\hline $\begin{array}{l}5-26 \\
5-27 \\
5-28 \\
5-30 \\
5-31\end{array}$ & $\begin{array}{l}1 \\
0 \\
0 \\
0 \\
0\end{array}$ & $\begin{array}{r}27 \\
9 \\
6 \\
2 \\
1\end{array}$ & $\begin{array}{r}25 \\
10 \\
12 \\
4 \\
2\end{array}$ & $\begin{array}{l}4 \\
3 \\
7 \\
2 \\
3\end{array}$ & $\begin{array}{l}2 \\
0 \\
0 \\
0 \\
1\end{array}$ & $\begin{array}{l}0 \\
0 \\
0 \\
0 \\
0\end{array}$ & $\begin{array}{l}5 \\
6 \\
3 \\
3 \\
2\end{array}$ & $\begin{array}{l}7 \\
2 \\
6 \\
3 \\
3\end{array}$ & $\begin{array}{l}0 \\
2 \\
1 \\
3 \\
1\end{array}$ & $\begin{array}{l}0 \\
0 \\
0 \\
0 \\
0\end{array}$ \\
\hline $\begin{array}{l}6-01 \\
6-02 \\
6-03 \\
6-04 \\
6-05\end{array}$ & $\begin{array}{l}0 \\
0 \\
3 \\
0 \\
0\end{array}$ & $\begin{array}{r}4 \\
10 \\
7 \\
5 \\
4\end{array}$ & $\begin{array}{l}2 \\
4 \\
4 \\
0 \\
8\end{array}$ & $\begin{array}{l}5 \\
2 \\
3 \\
2 \\
5\end{array}$ & $\begin{array}{l}1 \\
0 \\
2 \\
0 \\
1\end{array}$ & $\begin{array}{l}0 \\
0 \\
0 \\
0 \\
0\end{array}$ & $\begin{array}{l}1 \\
1 \\
\frac{1}{3} \\
5\end{array}$ & $\begin{array}{l}2 \\
2 \\
1 \\
1 \\
0\end{array}$ & $\begin{array}{l}1 \\
0 \\
0 \\
0 \\
0\end{array}$ & $\begin{array}{l}0 \\
0 \\
0 \\
0 \\
1\end{array}$ \\
\hline $\begin{array}{l}6-06 \\
6-07 \\
6-08 \\
6-09 \\
6-10\end{array}$ & $\begin{array}{l}0 \\
1 \\
0 \\
1 \\
0\end{array}$ & $\begin{array}{r}11 \\
20 \\
13 \\
6 \\
6\end{array}$ & $\begin{array}{r}16 \\
26 \\
2 \\
3 \\
6\end{array}$ & $\begin{array}{l}6 \\
3 \\
2 \\
5 \\
1\end{array}$ & $\begin{array}{l}0 \\
0 \\
0 \\
0 \\
0\end{array}$ & $\begin{array}{l}0 \\
0 \\
0 \\
0 \\
1\end{array}$ & $\begin{array}{l}0 \\
1 \\
2 \\
3 \\
6\end{array}$ & $\begin{array}{l}0 \\
2 \\
1 \\
1 \\
2\end{array}$ & $\begin{array}{l}0 \\
0 \\
1 \\
1 \\
0\end{array}$ & $\begin{array}{l}0 \\
0 \\
0 \\
0 \\
0\end{array}$ \\
\hline $\begin{array}{l}6-11 \\
6-12 \\
6-13 \\
6-14 \\
6-15\end{array}$ & $\begin{array}{l}2 \\
3 \\
0 \\
7 \\
0\end{array}$ & $\begin{array}{r}8 \\
4 \\
8 \\
51 \\
13\end{array}$ & $\begin{array}{r}7 \\
2 \\
13 \\
7 \\
6\end{array}$ & $\begin{array}{l}0 \\
3 \\
5 \\
2 \\
3\end{array}$ & $\begin{array}{l}0 \\
0 \\
1 \\
0 \\
1\end{array}$ & $\begin{array}{l}0 \\
0 \\
1 \\
0 \\
0\end{array}$ & $\begin{array}{l}6 \\
3 \\
8 \\
3 \\
4\end{array}$ & $\begin{array}{l}4 \\
1 \\
4 \\
2 \\
0\end{array}$ & $\begin{array}{l}1 \\
1 \\
0 \\
1 \\
0\end{array}$ & $\begin{array}{l}0 \\
0 \\
0 \\
0 \\
1\end{array}$ \\
\hline $\begin{array}{l}6-16 \\
6-17 \\
6-18 \\
6-19 \\
6-20\end{array}$ & $\begin{array}{l}1 \\
0 \\
0 \\
1 \\
0\end{array}$ & $\begin{array}{l}5 \\
4 \\
7 \\
2 \\
6\end{array}$ & $\begin{array}{l}5 \\
7 \\
6 \\
2 \\
1\end{array}$ & $\begin{array}{l}2 \\
2 \\
2 \\
2 \\
1\end{array}$ & $\begin{array}{l}1 \\
0 \\
0 \\
0 \\
1\end{array}$ & $\begin{array}{l}0 \\
0 \\
0 \\
0 \\
0\end{array}$ & $\begin{array}{l}0 \\
1 \\
0 \\
2 \\
2\end{array}$ & $\begin{array}{l}1 \\
1 \\
1 \\
1 \\
0\end{array}$ & $\begin{array}{l}1 \\
0 \\
0 \\
0 \\
1\end{array}$ & $\begin{array}{l}0 \\
0 \\
0 \\
0 \\
0\end{array}$ \\
\hline $\begin{array}{l}6-21 \\
6-22 \\
6-23 \\
6-24 \\
6-25\end{array}$ & $\begin{array}{r}1 \\
0 \\
1 \\
1 \\
--\end{array}$ & $\begin{array}{r}0 \\
1 \\
0 \\
1 \\
--\end{array}$ & $\begin{array}{r}4 \\
0 \\
0 \\
2 \\
--\end{array}$ & $\begin{array}{r}2 \\
1 \\
3 \\
2 \\
--\end{array}$ & $\begin{array}{r}0 \\
0 \\
0 \\
1 \\
--\end{array}$ & $\begin{array}{r}0 \\
0 \\
0 \\
0 \\
--\end{array}$ & $\begin{array}{r}0 \\
1 \\
0 \\
0 \\
--\end{array}$ & $\begin{array}{r}0 \\
1 \\
0 \\
0 \\
--\end{array}$ & $\begin{array}{r}0 \\
0 \\
1 \\
1 \\
-\end{array}$ & $\begin{array}{r}0 \\
0 \\
0 \\
1 \\
--\end{array}$ \\
\hline $\begin{array}{r}6-26 \\
6-27 \\
6-28 \\
7-11 \\
10-07\end{array}$ & $\begin{array}{r}-- \\
-\overline{2} \\
0 \\
0\end{array}$ & $\begin{array}{r}-- \\
-- \\
3 \\
2 \\
4\end{array}$ & $\begin{array}{r}-- \\
-- \\
3 \\
0 \\
0\end{array}$ & $\begin{array}{r}-- \\
-\overline{1} \\
0 \\
2\end{array}$ & $\begin{array}{r}-- \\
-\overline{1} \\
0 \\
0\end{array}$ & $\begin{array}{r}-- \\
-- \\
0 \\
0\end{array}$ & $\begin{array}{r}-- \\
-\overline{1} \\
\frac{1}{2}\end{array}$ & $\begin{array}{c}-- \\
-1 \\
1 \\
0\end{array}$ & $\begin{array}{r}-- \\
-0 \\
1 \\
0\end{array}$ & $\begin{array}{c}-- \\
-\overline{0} \\
0 \\
0\end{array}$ \\
\hline
\end{tabular}


TABLE 12.- NUMBER OF PINK TRACER PARTICLES(1), PER 100 GRAMS OF BED MATERIAL, EAST FORK RIVER, WYOMING; 1979--CONTINUED

(1) PINK TRACER PARTICLES PLACED AS BED MATERIAL 10 METERS DOWNSTREAM

OF SECTION 3047 ON MAY 18, 1979.

(2) SMALL OVERFLOW CHANNEL ADJACENT TO SECTION.

(3) BYPASS CHANNEL. SEE FIGURE 3

(4) SECTION 2278 MEASURED ON 10-07. 
TABLE 13.- NUMBER OF BLUE TRACER PARTICLES(1), PER 100 GRAMS OF BED MATERIAL, EAST FORK RIVER, WYOMING, 1979

SECTION 0043

SECTION 0075

\begin{tabular}{|c|c|c|c|c|c|c|c|c|c|c|}
\hline \multirow[t]{2}{*}{ DATE } & \multicolumn{5}{|c|}{$\begin{aligned} \text { NUMBER OF } & \text { BLUE TRACER } \\
\text { PARTICLES BY } & \text { SIZE CLASS (MM) }\end{aligned}$} & \multicolumn{5}{|c|}{$\begin{array}{rll}\text { NUMBER OF } & \text { BLUE TRACER } \\
\text { PARTICLES BY } & \text { SIZE CLASS (MM) }\end{array}$} \\
\hline & $\begin{array}{l}0.25 \\
\text { TO } \\
0.50\end{array}$ & $\begin{array}{l}0.50 \\
\text { TO } \\
1.00\end{array}$ & $\begin{array}{l}1.00 \\
\text { TO } \\
2.00\end{array}$ & $\begin{array}{l}2.00 \\
\text { TO } \\
4.00\end{array}$ & $\begin{array}{l}4.00 \\
\text { TO } \\
8.00\end{array}$ & $\begin{array}{r}0.25 \\
\text { TO } \\
0.50\end{array}$ & $\begin{array}{r}0.50 \\
\text { TO } \\
1.00\end{array}$ & $\begin{array}{r}1.00 \\
\text { TO } \\
2.00\end{array}$ & $\begin{array}{r}2.00 \\
\text { TO } \\
4.00\end{array}$ & $\begin{array}{r}4.00 \\
\text { TO } \\
8.00\end{array}$ \\
\hline $\begin{array}{l}5-20 \\
5-21 \\
5-23 \\
5-24 \\
5-25\end{array}$ & $\begin{array}{r}--\overline{0} \\
-\frac{1}{0} \\
0\end{array}$ & $\begin{array}{r}-- \\
-\frac{-}{0} \\
0\end{array}$ & $\begin{array}{r}-\frac{-}{0} \\
-\frac{1}{0} \\
0\end{array}$ & $\begin{array}{r}-- \\
-\frac{0}{0} \\
0\end{array}$ & $\begin{array}{r}-- \\
-\frac{0}{0} \\
0\end{array}$ & $\begin{array}{c}-- \\
-\overline{0} \\
0 \\
0\end{array}$ & $\begin{array}{c}-- \\
-\overline{0} \\
0 \\
0\end{array}$ & $\begin{array}{r}-- \\
-\overline{0} \\
0 \\
0\end{array}$ & $\begin{array}{c}-- \\
-\overline{0} \\
0 \\
0\end{array}$ & $\begin{array}{c}-- \\
-\overline{0} \\
0 \\
0\end{array}$ \\
\hline $\begin{array}{l}5-26 \\
5-27 \\
5-28 \\
5-30 \\
5-31\end{array}$ & $\begin{array}{r}-0 \\
-0 \\
0 \\
0\end{array}$ & $\begin{array}{r}0 \\
-\frac{1}{0} \\
2 \\
0\end{array}$ & $\begin{array}{r}-0 \\
-0 \\
0 \\
0\end{array}$ & $\begin{array}{r}0 \\
-\frac{0}{0} \\
0 \\
0\end{array}$ & $\begin{array}{r}0 \\
-\frac{0}{0} \\
0 \\
0\end{array}$ & $\begin{array}{l}0 \\
0 \\
0 \\
4 \\
0\end{array}$ & $\begin{array}{l}0 \\
0 \\
0 \\
0 \\
0\end{array}$ & $\begin{array}{l}0 \\
0 \\
0 \\
0 \\
0\end{array}$ & $\begin{array}{l}0 \\
0 \\
0 \\
0 \\
0\end{array}$ & $\begin{array}{l}0 \\
0 \\
0 \\
0 \\
0\end{array}$ \\
\hline $\begin{array}{l}6-01 \\
6-02 \\
6-03 \\
6-04 \\
6-05\end{array}$ & $\begin{array}{l}0 \\
0 \\
0 \\
0 \\
1\end{array}$ & $\begin{array}{l}0 \\
0 \\
0 \\
1 \\
0\end{array}$ & $\begin{array}{l}0 \\
0 \\
0 \\
0 \\
0\end{array}$ & $\begin{array}{l}0 \\
0 \\
0 \\
0 \\
0\end{array}$ & $\begin{array}{l}0 \\
0 \\
0 \\
0 \\
0\end{array}$ & $\begin{array}{l}0 \\
0 \\
0 \\
0 \\
0\end{array}$ & $\begin{array}{l}0 \\
0 \\
0 \\
1 \\
0\end{array}$ & $\begin{array}{l}0 \\
0 \\
0 \\
0 \\
0\end{array}$ & $\begin{array}{l}0 \\
0 \\
0 \\
0 \\
0\end{array}$ & $\begin{array}{l}0 \\
0 \\
0 \\
0 \\
0\end{array}$ \\
\hline $\begin{array}{l}6-06 \\
6-07 \\
6-08 \\
6-09 \\
6-10\end{array}$ & $\begin{array}{l}0 \\
0 \\
0 \\
2 \\
0\end{array}$ & $\begin{array}{l}0 \\
1 \\
1 \\
0 \\
0\end{array}$ & $\begin{array}{l}0 \\
0 \\
0 \\
0 \\
0\end{array}$ & $\begin{array}{l}0 \\
0 \\
0 \\
0 \\
0\end{array}$ & $\begin{array}{l}0 \\
0 \\
0 \\
0 \\
0\end{array}$ & $\begin{array}{l}2 \\
0 \\
2 \\
6 \\
1\end{array}$ & $\begin{array}{l}0 \\
0 \\
1 \\
\frac{1}{2}\end{array}$ & $\begin{array}{l}0 \\
0 \\
0 \\
0 \\
0\end{array}$ & $\begin{array}{l}0 \\
0 \\
0 \\
0 \\
0\end{array}$ & $\begin{array}{l}0 \\
0 \\
0 \\
0 \\
0\end{array}$ \\
\hline $\begin{array}{l}6-11 \\
6-12 \\
6-13 \\
6-14 \\
6-15\end{array}$ & $\begin{array}{l}5 \\
1 \\
0 \\
0 \\
1\end{array}$ & $\begin{array}{l}0 \\
0 \\
0 \\
1 \\
0\end{array}$ & $\begin{array}{l}0 \\
0 \\
0 \\
0 \\
0\end{array}$ & $\begin{array}{l}0 \\
0 \\
0 \\
0 \\
0\end{array}$ & $\begin{array}{l}0 \\
0 \\
0 \\
0 \\
0\end{array}$ & $\begin{array}{l}0 \\
3 \\
0 \\
0 \\
2\end{array}$ & $\begin{array}{l}0 \\
1 \\
0 \\
1 \\
0\end{array}$ & $\begin{array}{l}0 \\
0 \\
0 \\
0 \\
0\end{array}$ & $\begin{array}{l}0 \\
0 \\
0 \\
0 \\
0\end{array}$ & $\begin{array}{l}0 \\
0 \\
0 \\
0 \\
0\end{array}$ \\
\hline $\begin{array}{l}6-16 \\
6-17 \\
6-18 \\
6-19 \\
6-20\end{array}$ & $\begin{array}{l}0 \\
1 \\
0 \\
1 \\
5\end{array}$ & $\begin{array}{l}0 \\
0 \\
0 \\
0 \\
1\end{array}$ & $\begin{array}{l}0 \\
0 \\
0 \\
0 \\
0\end{array}$ & $\begin{array}{l}0 \\
0 \\
0 \\
0 \\
0\end{array}$ & $\begin{array}{l}0 \\
0 \\
0 \\
0 \\
0\end{array}$ & $\begin{array}{l}2 \\
4 \\
3 \\
4 \\
0\end{array}$ & $\begin{array}{l}1 \\
0 \\
0 \\
0 \\
0\end{array}$ & $\begin{array}{l}0 \\
0 \\
0 \\
0 \\
0\end{array}$ & $\begin{array}{l}0 \\
0 \\
0 \\
0 \\
0\end{array}$ & $\begin{array}{l}0 \\
0 \\
0 \\
0 \\
0\end{array}$ \\
\hline $\begin{array}{l}6-21 \\
6-22 \\
6-23 \\
6-24 \\
6-25\end{array}$ & $\begin{array}{l}0 \\
4 \\
4 \\
3 \\
1\end{array}$ & $\begin{array}{l}1 \\
2 \\
1 \\
0 \\
0\end{array}$ & $\begin{array}{l}0 \\
0 \\
0 \\
0 \\
0\end{array}$ & $\begin{array}{l}0 \\
0 \\
0 \\
0 \\
0\end{array}$ & $\begin{array}{l}0 \\
0 \\
0 \\
0 \\
0\end{array}$ & $\begin{array}{l}3 \\
0 \\
0 \\
0 \\
0\end{array}$ & $\begin{array}{l}0 \\
0 \\
2 \\
1 \\
1\end{array}$ & $\begin{array}{l}0 \\
0 \\
0 \\
0 \\
0\end{array}$ & $\begin{array}{l}0 \\
0 \\
0 \\
0 \\
0\end{array}$ & $\begin{array}{l}0 \\
0 \\
0 \\
0 \\
0\end{array}$ \\
\hline $\begin{array}{r}6-26 \\
6-27 \\
6-28 \\
7-11 \\
10-09\end{array}$ & $\begin{array}{l}2 \\
1 \\
3 \\
2 \\
2\end{array}$ & $\begin{array}{l}0 \\
0 \\
0 \\
0 \\
1\end{array}$ & $\begin{array}{l}0 \\
0 \\
0 \\
0 \\
0\end{array}$ & $\begin{array}{l}0 \\
0 \\
0 \\
0 \\
0\end{array}$ & $\begin{array}{l}0 \\
0 \\
0 \\
0 \\
0\end{array}$ & $\begin{array}{l}2 \\
1 \\
4 \\
3 \\
2\end{array}$ & $\begin{array}{l}0 \\
0 \\
0 \\
2 \\
0\end{array}$ & $\begin{array}{l}0 \\
0 \\
0 \\
0 \\
0\end{array}$ & $\begin{array}{l}0 \\
0 \\
0 \\
0 \\
0\end{array}$ & $\begin{array}{l}0 \\
0 \\
0 \\
0 \\
0\end{array}$ \\
\hline
\end{tabular}


TABLE 13.- NUMBER OF BLUE TRACER PARTICLES(1), PER 100 GRAMS OF BED MATERIAL, EAST FORK RIVER, WYOMING, 1979--CONTINUED

\begin{tabular}{|c|c|c|c|c|c|c|c|c|c|c|}
\hline \multirow[t]{2}{*}{ DATE } & \multicolumn{5}{|c|}{$\begin{aligned} \text { NUMBER OF } & \text { BLUE TRACER } \\
\text { PARTICLES BY } & \text { SIZE CLASS (MM) }\end{aligned}$} & \multicolumn{5}{|c|}{$\begin{array}{l}\text { NUABER OF BLUE TRACER } \\
\text { PARTICLES BY SIZE CLASS (MM) }\end{array}$} \\
\hline & $\begin{array}{l}0.25 \\
\text { TO } \\
0.50\end{array}$ & $\begin{array}{l}0.50 \\
\text { TO } \\
1.00\end{array}$ & $\begin{array}{l}1.00 \\
2.00\end{array}$ & $\begin{array}{l}2.00 \\
\text { TO } \\
4.00\end{array}$ & $\begin{array}{l}4.00 \\
\text { TO } \\
8.00\end{array}$ & $\begin{array}{l}0.25 \\
\text { TO } \\
0.50\end{array}$ & $\begin{array}{r}0.50 \\
\text { TO } \\
1.00\end{array}$ & $\begin{array}{r}1.00 \\
\text { TO } \\
2.00\end{array}$ & $\begin{array}{r}2.00 \\
\mathrm{TO} \\
4.00\end{array}$ & $\begin{array}{r}4.00 \\
\text { TO } \\
8.00\end{array}$ \\
\hline $\begin{array}{l}5-20 \\
5-21 \\
5-23 \\
5-24 \\
5-25\end{array}$ & $\begin{array}{c}-- \\
0 \\
0 \\
0 \\
0\end{array}$ & $\begin{array}{c}-- \\
0 \\
0 \\
0 \\
0\end{array}$ & $\begin{array}{c}-\overline{0} \\
0 \\
0 \\
0\end{array}$ & $\begin{array}{c}-\overline{0} \\
0 \\
0 \\
0\end{array}$ & $\begin{array}{c}-\overline{0} \\
0 \\
0 \\
0\end{array}$ & $\begin{array}{l}0 \\
0 \\
0 \\
0 \\
0\end{array}$ & $\begin{array}{l}0 \\
0 \\
0 \\
0 \\
0\end{array}$ & $\begin{array}{l}0 \\
0 \\
0 \\
0 \\
0\end{array}$ & $\begin{array}{l}0 \\
0 \\
0 \\
0 \\
0\end{array}$ & $\begin{array}{l}0 \\
0 \\
0 \\
0 \\
0\end{array}$ \\
\hline $\begin{array}{l}5-26 \\
5-27 \\
5-28 \\
5-30 \\
5-31\end{array}$ & $\begin{array}{l}0 \\
0 \\
0 \\
0 \\
0\end{array}$ & $\begin{array}{l}0 \\
0 \\
0 \\
0 \\
0\end{array}$ & $\begin{array}{l}0 \\
0 \\
0 \\
1 \\
0\end{array}$ & $\begin{array}{l}0 \\
0 \\
0 \\
0 \\
0\end{array}$ & $\begin{array}{l}0 \\
0 \\
0 \\
0 \\
0\end{array}$ & $\begin{array}{l}0 \\
0 \\
0 \\
0 \\
0\end{array}$ & $\begin{array}{l}0 \\
0 \\
0 \\
0 \\
1\end{array}$ & $\begin{array}{l}0 \\
0 \\
0 \\
0 \\
0\end{array}$ & $\begin{array}{l}0 \\
1 \\
0 \\
0 \\
0\end{array}$ & $\begin{array}{l}0 \\
0 \\
0 \\
0 \\
0\end{array}$ \\
\hline $\begin{array}{l}6-01 \\
6-02 \\
6-03 \\
6-04 \\
6-05\end{array}$ & $\begin{array}{l}0 \\
0 \\
0 \\
0 \\
0\end{array}$ & $\begin{array}{l}0 \\
0 \\
0 \\
0 \\
0\end{array}$ & $\begin{array}{l}0 \\
0 \\
0 \\
0 \\
0\end{array}$ & $\begin{array}{l}0 \\
0 \\
0 \\
0 \\
0\end{array}$ & $\begin{array}{l}0 \\
0 \\
0 \\
0 \\
0\end{array}$ & $\begin{array}{l}0 \\
0 \\
0 \\
0 \\
0\end{array}$ & $\begin{array}{l}0 \\
0 \\
0 \\
1 \\
0\end{array}$ & $\begin{array}{l}0 \\
0 \\
0 \\
0 \\
0\end{array}$ & $\begin{array}{l}0 \\
0 \\
0 \\
0 \\
0\end{array}$ & $\begin{array}{l}0 \\
0 \\
0 \\
0 \\
0\end{array}$ \\
\hline $\begin{array}{l}6-06 \\
6-07 \\
6-08 \\
6-09 \\
6-10\end{array}$ & $\begin{array}{l}0 \\
0 \\
0 \\
0 \\
0\end{array}$ & $\begin{array}{l}0 \\
0 \\
0 \\
0 \\
2\end{array}$ & $\begin{array}{l}1 \\
0 \\
0 \\
0 \\
0\end{array}$ & $\begin{array}{l}0 \\
0 \\
0 \\
0 \\
0\end{array}$ & $\begin{array}{l}0 \\
0 \\
0 \\
0 \\
0\end{array}$ & $\begin{array}{l}1 \\
0 \\
0 \\
0 \\
0\end{array}$ & $\begin{array}{l}0 \\
0 \\
0 \\
0 \\
0\end{array}$ & $\begin{array}{l}0 \\
0 \\
0 \\
0 \\
0\end{array}$ & $\begin{array}{l}0 \\
0 \\
0 \\
0 \\
0\end{array}$ & $\begin{array}{l}0 \\
0 \\
0 \\
0 \\
0\end{array}$ \\
\hline $\begin{array}{l}6-11 \\
6-12 \\
6-13 \\
6-14 \\
6-15\end{array}$ & $\begin{array}{l}0 \\
3 \\
0 \\
1 \\
0\end{array}$ & $\begin{array}{l}0 \\
3 \\
0 \\
0 \\
1\end{array}$ & $\begin{array}{l}0 \\
0 \\
0 \\
0 \\
0\end{array}$ & $\begin{array}{l}0 \\
0 \\
0 \\
0 \\
0\end{array}$ & $\begin{array}{l}0 \\
0 \\
0 \\
0 \\
0\end{array}$ & $\begin{array}{l}1 \\
1 \\
0 \\
3 \\
1\end{array}$ & $\begin{array}{l}0 \\
1 \\
0 \\
0 \\
0\end{array}$ & $\begin{array}{l}0 \\
0 \\
0 \\
0 \\
0\end{array}$ & $\begin{array}{l}0 \\
0 \\
0 \\
0 \\
0\end{array}$ & $\begin{array}{l}0 \\
0 \\
0 \\
0 \\
0\end{array}$ \\
\hline $\begin{array}{l}6-16 \\
6-17 \\
6-18 \\
6-19 \\
6-20\end{array}$ & $\begin{array}{l}1 \\
0 \\
1 \\
1 \\
0\end{array}$ & $\begin{array}{l}0 \\
0 \\
1 \\
0 \\
0\end{array}$ & $\begin{array}{l}0 \\
0 \\
0 \\
0 \\
0\end{array}$ & $\begin{array}{l}0 \\
0 \\
0 \\
0 \\
0\end{array}$ & $\begin{array}{l}0 \\
0 \\
0 \\
0 \\
0\end{array}$ & $\begin{array}{l}0 \\
2 \\
1 \\
1 \\
4\end{array}$ & $\begin{array}{l}0 \\
0 \\
1 \\
0 \\
2\end{array}$ & $\begin{array}{l}0 \\
0 \\
0 \\
0 \\
0\end{array}$ & $\begin{array}{l}0 \\
0 \\
0 \\
0 \\
0\end{array}$ & $\begin{array}{l}0 \\
0 \\
0 \\
0 \\
0\end{array}$ \\
\hline $\begin{array}{l}6-21 \\
6-22 \\
6-23 \\
6-24 \\
6-25\end{array}$ & $\begin{array}{l}0 \\
0 \\
0 \\
1 \\
0\end{array}$ & $\begin{array}{l}0 \\
0 \\
2 \\
1 \\
0\end{array}$ & $\begin{array}{l}0 \\
0 \\
0 \\
0 \\
0\end{array}$ & $\begin{array}{l}0 \\
0 \\
0 \\
0 \\
0\end{array}$ & $\begin{array}{l}0 \\
0 \\
0 \\
0 \\
0\end{array}$ & $\begin{array}{l}2 \\
1 \\
1 \\
0 \\
0\end{array}$ & $\begin{array}{l}0 \\
2 \\
2 \\
2 \\
0\end{array}$ & $\begin{array}{l}0 \\
0 \\
0 \\
0 \\
0\end{array}$ & $\begin{array}{l}0 \\
0 \\
0 \\
0 \\
0\end{array}$ & $\begin{array}{l}0 \\
0 \\
0 \\
0 \\
0\end{array}$ \\
\hline $\begin{array}{r}6-26 \\
6-27 \\
6-28 \\
7-11 \\
10-09\end{array}$ & $\begin{array}{l}0 \\
1 \\
1 \\
1 \\
1\end{array}$ & $\begin{array}{l}1 \\
0 \\
0 \\
0 \\
0\end{array}$ & $\begin{array}{l}0 \\
0 \\
0 \\
0 \\
0\end{array}$ & $\begin{array}{l}0 \\
0 \\
0 \\
0 \\
0\end{array}$ & $\begin{array}{l}0 \\
0 \\
0 \\
0 \\
0\end{array}$ & $\begin{array}{l}8 \\
2 \\
1 \\
0 \\
1\end{array}$ & $\begin{array}{l}3 \\
0 \\
0 \\
0 \\
0\end{array}$ & $\begin{array}{l}0 \\
0 \\
0 \\
0 \\
0\end{array}$ & $\begin{array}{l}0 \\
0 \\
0 \\
0 \\
0\end{array}$ & $\begin{array}{l}0 \\
0 \\
0 \\
0 \\
0\end{array}$ \\
\hline
\end{tabular}


TABLE 13.- NUMBER OF BLUE TRACER PARTICLES(1), PER 100 GRAMS OF BED MATERIAL, EAST FORK RIVER, WYOMING, 1979--CONTINUED

\begin{tabular}{|c|c|c|c|c|c|}
\hline \multirow[t]{2}{*}{ DATE } & \multicolumn{2}{|c|}{$\begin{array}{r}\text { NUMBER } \\
\text { PARTICLES }\end{array}$} & $\begin{array}{l}F \text { BLUE } \\
\text { Y }\end{array}$ & \multicolumn{2}{|c|}{$\begin{array}{l}\text { TRACER } \\
\text { CLASS (MM) }\end{array}$} \\
\hline & $\begin{array}{l}0.25 \\
\text { TO } \\
0.50\end{array}$ & $\begin{array}{l}0.50 \\
\text { TO } \\
1.00\end{array}$ & $\begin{array}{l}1.00 \\
\text { TO } \\
2.00\end{array}$ & $\begin{array}{l}2.00 \\
\text { TO } \\
4.00\end{array}$ & $\begin{array}{l}4.00 \\
8.00 \\
8.00\end{array}$ \\
\hline
\end{tabular}

$0.250 .50 \quad 1.00 \quad 2.00 \quad 4.00$

$\begin{array}{rrrrr}\text { TO } & \text { TO } & \text { TO } & \text { TO } & \text { TO } \\ 0.50 & 1.00 & 2.00 & 4.00 & 8.00\end{array}$

$\begin{array}{llllll}5-20 & 0 & 0 & 0 & 0 & 0 \\ 5-21 & 1 & 0 & 0 & 0 & 0 \\ 5-23 & 0 & 0 & 0 & 0 & 0 \\ 5-24 & 0 & 0 & 0 & 0 & 0 \\ 5-25 & 0 & 0 & 0 & 0 & 0\end{array}$

$\begin{array}{lllll}0 & 0 & 0 & 0 & 0 \\ 0 & 0 & 0 & 0 & 0 \\ 0 & 0 & 0 & 0 & 0 \\ 0 & 0 & 0 & 0 & 0 \\ 0 & 0 & 0 & 0 & 0\end{array}$

$\begin{array}{llllll}5-26 & 0 & 0 & 0 & 0 & 0 \\ 5-27 & 0 & 0 & 0 & 0 & 0 \\ 5-28 & 0 & 0 & 0 & 0 & 0 \\ 5-30 & 0 & 0 & 0 & 0 & 0 \\ 5-31 & 0 & 0 & 0 & 0 & 0\end{array}$

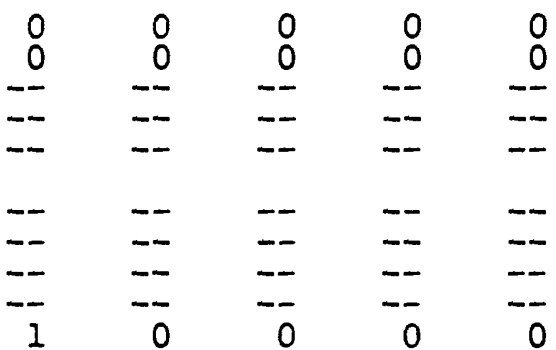

$6-01$
$6-02$
$6-03$
$6-04$

$\begin{array}{llll}0 & 0 & 0 & 0 \\ 0 & 0 & 0 & 0 \\ 0 & 0 & 0 & 0 \\ 0 & 0 & 0 & 0 \\ 0 & 0 & 0 & 0\end{array}$

$6-06$

$6-07$

$6-08$

6-09

$\begin{array}{llll}1 & 0 & 0 & 0 \\ 0 & 0 & 0 & 0 \\ 1 & 0 & 0 & 0 \\ 0 & 0 & 0 & 0 \\ 0 & 0 & 0 & 0\end{array}$

$\begin{array}{rrrrr}2 & 0 & 0 & 0 & 0 \\ 3 & 1 & 0 & 0 & 0 \\ -- & -- & -- & -- & =\end{array}$

$6-11$

6-12

$6-13$

$6-14$

$\begin{array}{ll}0 & 0 \\ 0 & 0 \\ 0 & 0 \\ 0 & 0 \\ 0 & 0\end{array}$

6-15

$\begin{array}{ll}0 & 1 \\ 0 & 0 \\ 0 & 1 \\ 0 & 0 \\ 1 & 0\end{array}$

6-16

6-17

6-18

$6-19$

$\begin{array}{ll}\frac{1}{1} & 0 \\ 0 & 1 \\ 0 & 0 \\ 0 & 1\end{array}$

$\begin{array}{llll}0 & 0 & 0 & 0 \\ 1 & 0 & 0 & 0 \\ 0 & 0 & 0 & 0 \\ 0 & 0 & 0 & 0 \\ 1 & 0 & 0 & 0\end{array}$

$\begin{array}{cc}- & - \\ -0 & 0 \\ 5 & 1 \\ 1 & 1 \\ 4 & 1\end{array}$

$-\overline{0}$
0
0
0

$-\overline{0}$
0
0
0

-
0
0
0
0

$\begin{array}{rrrrr}0 & 0 & 0 & 0 & 0 \\ 3 & 0 & 0 & 0 & 0 \\ -- & -- & -- & -- & =- \\ -- & -- & -- & -- & --\end{array}$

$6-21$

6-23

$6-24$

0
0
1
4
10

$\begin{array}{ll}0 & 0 \\ 0 & 0 \\ 0 & 0 \\ 2 & 0 \\ 2 & 0\end{array}$

$\begin{array}{ll}0 & 0 \\ 0 & 0 \\ 0 & 0 \\ 0 & 0 \\ 0 & 0\end{array}$

$\begin{array}{ll}4 & 0 \\ 1 & 0 \\ 6 & 0 \\ 4 & 0 \\ 1 & 0\end{array}$

$\begin{array}{ll}0 & 0 \\ 0 & 0 \\ 0 & 0 \\ 0 & 0 \\ 0 & 0\end{array}$

$\begin{array}{lllll}\square- & = & = & = & = \\ z & = & = & = & = \\ z & \square & = & = & =\end{array}$

$6-26$
$6-27$
$6-28$
$7-11$
$10-09$

$\begin{array}{ll}0 & 0 \\ 0 & 0 \\ 0 & 0 \\ 1 & 2 \\ 2 & 1\end{array}$

$\begin{array}{ll}0 & 0 \\ 0 & 0 \\ 0 & 0 \\ 0 & 0 \\ 0 & 0\end{array}$

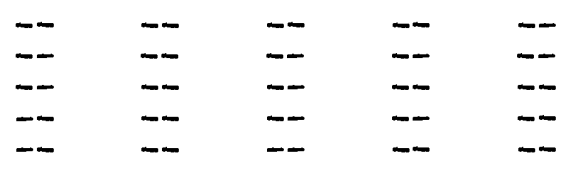


TABLE 13.- NUMBER OF BLUE TRACER PARTICLES(1), PER 100 GRAMS OF BED MATERIAL, EAST FORK RIVER, WYOMING, 1979--CONTINUED SECTION 0421

SECTION 0516

\begin{tabular}{|c|c|c|c|c|c|c|c|c|c|c|}
\hline \multirow[t]{2}{*}{ DATE } & \multicolumn{2}{|c|}{$\begin{array}{r}\text { NUMBER } \\
\text { PARTICLES }\end{array}$} & $\begin{array}{l}\text { BLUE } \\
\text { SIZE }\end{array}$ & \multicolumn{2}{|c|}{$\begin{array}{l}\text { TRACER } \\
\text { CLASS (MM) }\end{array}$} & \multicolumn{2}{|c|}{$\begin{array}{r}\text { NUMBER } \\
\text { PARTICLES }\end{array}$} & \multicolumn{3}{|c|}{$\begin{array}{l}\text { DF BLUE TRACER } \\
\text { BY SIZE CLASS (MM) }\end{array}$} \\
\hline & $\begin{array}{l}0.25 \\
\text { TO } \\
0.50\end{array}$ & $\begin{array}{l}0.50 \\
\text { TO } \\
1.00\end{array}$ & $\begin{array}{l}1.00 \\
2.00 \\
\text { TO }\end{array}$ & $\begin{array}{l}2.00 \\
\text { TO } \\
4.00\end{array}$ & $\begin{array}{l}4.00 \\
\text { T. } \\
8.00\end{array}$ & $\begin{array}{r}0.25 \\
\text { TO } \\
0.50\end{array}$ & $\begin{array}{r}0.50 \\
\text { TO } \\
1.00\end{array}$ & $\begin{array}{l}1.00 \\
\text { TO } \\
2.00\end{array}$ & $\begin{array}{r}2.00 \\
\text { TO } \\
4.00\end{array}$ & $\begin{array}{r}4.00 \\
\text { TO } \\
8.00\end{array}$ \\
\hline $\begin{array}{l}5-20 \\
5-21 \\
5-23 \\
5-24 \\
5-25\end{array}$ & $\begin{array}{l}0 \\
0 \\
0 \\
0 \\
0\end{array}$ & $\begin{array}{l}0 \\
0 \\
0 \\
0 \\
0\end{array}$ & $\begin{array}{l}0 \\
0 \\
0 \\
0 \\
0\end{array}$ & $\begin{array}{l}0 \\
0 \\
0 \\
0 \\
0\end{array}$ & $\begin{array}{l}0 \\
0 \\
0 \\
0 \\
0\end{array}$ & $\begin{array}{l}=- \\
=- \\
--\end{array}$ & $\begin{array}{l}=- \\
=- \\
=-\end{array}$ & $\begin{array}{l}=- \\
=- \\
=-\end{array}$ & $\begin{array}{l}=- \\
=- \\
--\end{array}$ & $\begin{array}{l}=- \\
=- \\
=- \\
--\end{array}$ \\
\hline $\begin{array}{l}5-26 \\
5-27 \\
5-28 \\
5-30 \\
5-31\end{array}$ & $\begin{array}{l}1 \\
0 \\
0 \\
0 \\
0\end{array}$ & $\begin{array}{l}0 \\
0 \\
0 \\
0 \\
0\end{array}$ & $\begin{array}{l}0 \\
0 \\
0 \\
0 \\
0\end{array}$ & $\begin{array}{l}0 \\
0 \\
0 \\
0 \\
0\end{array}$ & $\begin{array}{l}0 \\
0 \\
0 \\
0 \\
0\end{array}$ & $\overline{--}$ & $\begin{array}{l}\overline{-} \\
\overline{0} \\
0\end{array}$ & $\begin{array}{c}-- \\
\overline{0} \\
0\end{array}$ & $\overline{--}$ & $\begin{array}{l}-- \\
-- \\
0 \\
0\end{array}$ \\
\hline $\begin{array}{l}6-01 \\
6-02 \\
6-03 \\
6-04 \\
6-05\end{array}$ & $\begin{array}{l}0 \\
0 \\
0 \\
0 \\
1\end{array}$ & $\begin{array}{l}0 \\
0 \\
0 \\
0 \\
0\end{array}$ & $\begin{array}{l}0 \\
0 \\
0 \\
0 \\
0\end{array}$ & $\begin{array}{l}0 \\
0 \\
0 \\
0 \\
0\end{array}$ & $\begin{array}{l}0 \\
0 \\
0 \\
0 \\
0\end{array}$ & $\begin{array}{l}0 \\
1 \\
0 \\
0 \\
2\end{array}$ & $\begin{array}{l}0 \\
0 \\
0 \\
0 \\
0\end{array}$ & $\begin{array}{l}0 \\
0 \\
0 \\
0 \\
0\end{array}$ & $\begin{array}{l}0 \\
0 \\
0 \\
0 \\
0\end{array}$ & $\begin{array}{l}0 \\
0 \\
0 \\
0 \\
0 \\
0\end{array}$ \\
\hline $\begin{array}{l}6-06 \\
6-07 \\
6-08 \\
6-09 \\
6-10\end{array}$ & $\begin{array}{l}2 \\
3 \\
2 \\
0 \\
3\end{array}$ & $\begin{array}{l}0 \\
3 \\
0 \\
0 \\
0\end{array}$ & $\begin{array}{l}0 \\
0 \\
0 \\
0 \\
0\end{array}$ & $\begin{array}{l}0 \\
0 \\
0 \\
0 \\
0\end{array}$ & $\begin{array}{l}0 \\
0 \\
0 \\
0 \\
0\end{array}$ & $\begin{array}{l}7 \\
4 \\
5 \\
2 \\
2\end{array}$ & $\begin{array}{l}5 \\
0 \\
0 \\
2 \\
2\end{array}$ & $\begin{array}{l}0 \\
0 \\
0 \\
0 \\
0\end{array}$ & $\begin{array}{l}0 \\
0 \\
0 \\
0 \\
0\end{array}$ & $\begin{array}{l}0 \\
0 \\
0 \\
0 \\
0\end{array}$ \\
\hline $\begin{array}{l}6-11 \\
6-12 \\
6-13 \\
6-14 \\
6-15\end{array}$ & $\begin{array}{l}2 \\
1 \\
1 \\
5 \\
3\end{array}$ & $\begin{array}{l}1 \\
0 \\
0 \\
2 \\
0\end{array}$ & $\begin{array}{l}0 \\
0 \\
0 \\
0 \\
0\end{array}$ & $\begin{array}{l}0 \\
0 \\
0 \\
0 \\
0\end{array}$ & $\begin{array}{l}0 \\
0 \\
0 \\
0 \\
0\end{array}$ & $\begin{array}{r}3 \\
10 \\
0 \\
0 \\
3\end{array}$ & $\begin{array}{l}0 \\
2 \\
0 \\
0 \\
0\end{array}$ & $\begin{array}{l}0 \\
0 \\
0 \\
0 \\
0\end{array}$ & $\begin{array}{l}0 \\
0 \\
0 \\
0 \\
0\end{array}$ & $\begin{array}{l}0 \\
0 \\
0 \\
0 \\
0\end{array}$ \\
\hline $\begin{array}{l}6-16 \\
6-17 \\
6-18 \\
6-19 \\
6-20\end{array}$ & $\begin{array}{l}3 \\
1 \\
3 \\
1 \\
0\end{array}$ & $\begin{array}{l}0 \\
0 \\
1 \\
0 \\
0\end{array}$ & $\begin{array}{l}0 \\
0 \\
0 \\
0 \\
0\end{array}$ & $\begin{array}{l}0 \\
0 \\
0 \\
0 \\
0\end{array}$ & $\begin{array}{l}0 \\
0 \\
0 \\
0 \\
0\end{array}$ & $\begin{array}{l}3 \\
2 \\
0 \\
8 \\
0\end{array}$ & $\begin{array}{l}3 \\
1 \\
0 \\
1 \\
1\end{array}$ & $\begin{array}{l}0 \\
0 \\
0 \\
0 \\
0\end{array}$ & $\begin{array}{l}0 \\
0 \\
0 \\
0 \\
0\end{array}$ & $\begin{array}{l}0 \\
0 \\
0 \\
0 \\
0\end{array}$ \\
\hline $\begin{array}{l}6-21 \\
6-22 \\
6-23 \\
6-24 \\
6-25\end{array}$ & $\begin{array}{l}0 \\
0 \\
1 \\
0 \\
0\end{array}$ & $\begin{array}{l}0 \\
0 \\
1 \\
1 \\
0\end{array}$ & $\begin{array}{l}0 \\
0 \\
0 \\
0 \\
0\end{array}$ & $\begin{array}{l}0 \\
0 \\
0 \\
0 \\
0\end{array}$ & $\begin{array}{l}0 \\
0 \\
0 \\
0 \\
0\end{array}$ & $\begin{array}{r}2 \\
2 \\
5 \\
5 \\
-\end{array}$ & $\begin{array}{r}0 \\
0 \\
3 \\
3 \\
--\end{array}$ & $\begin{array}{l}0 \\
0 \\
0 \\
1 \\
-\end{array}$ & $\begin{array}{r}0 \\
0 \\
0 \\
0 \\
-\end{array}$ & $\begin{array}{r}0 \\
0 \\
0 \\
0 \\
--\end{array}$ \\
\hline $\begin{array}{r}6-26 \\
6-27 \\
6-28 \\
7-11 \\
10-09\end{array}$ & $\begin{array}{l}1 \\
1 \\
4 \\
0 \\
2\end{array}$ & $\begin{array}{l}1 \\
0 \\
1 \\
0 \\
0\end{array}$ & $\begin{array}{l}0 \\
0 \\
0 \\
0 \\
0\end{array}$ & $\begin{array}{l}0 \\
0 \\
0 \\
0 \\
0\end{array}$ & $\begin{array}{l}0 \\
0 \\
0 \\
0 \\
0\end{array}$ & $\begin{array}{r}-- \\
1 \\
3 \\
4\end{array}$ & $\begin{array}{r}-- \\
-\frac{1}{2} \\
5\end{array}$ & $\begin{array}{c}-- \\
-0 \\
0 \\
0\end{array}$ & $\begin{array}{c}-- \\
-0 \\
0 \\
0\end{array}$ & $\begin{array}{c}-- \\
-\overline{0} \\
0 \\
0\end{array}$ \\
\hline
\end{tabular}


TABLE 13.- NUMBER OF BLUE TRACER PARTICLES(1), PER 100 GRAMS OF BED MATERIAL, EAST FORK RIVER, WYOMING, 1979--CONTINUED

SECTION 0602

SECTION 0708

\begin{tabular}{|c|c|c|c|c|c|c|c|c|c|c|}
\hline \multirow[t]{2}{*}{ DATE } & \multicolumn{5}{|c|}{$\begin{array}{l}\text { NUMBER OF BIUE TRACER } \\
\text { PARTICLES BY SIZE CLASS (MM) }\end{array}$} & \multicolumn{5}{|c|}{$\begin{array}{r}\text { NUMBER OF } \text { BLUE TRACER } \\
\text { PARTICLES BY SIZE CLASS (MM) }\end{array}$} \\
\hline & $\begin{array}{l}0.25 \\
\text { TO } \\
0.50\end{array}$ & $\begin{array}{l}0.50 \\
\text { TO } \\
1.00\end{array}$ & $\begin{array}{l}1.00 \\
\text { TO } \\
2.00\end{array}$ & $\begin{array}{l}2.00 \\
\text { TO } \\
4.00\end{array}$ & $\begin{array}{l}4.00 \\
\text { To } \\
8.00\end{array}$ & $\begin{array}{r}0.25 \\
\text { TO } \\
0.50\end{array}$ & $\begin{array}{r}0.50 \\
\text { TO } \\
1.00\end{array}$ & $\begin{array}{l}1.00 \\
\text { TO } \\
2.00\end{array}$ & $\begin{array}{r}2.00 \\
\text { TO } \\
4.00\end{array}$ & $\begin{array}{r}4.00 \\
\text { TO } \\
8.00\end{array}$ \\
\hline $\begin{array}{l}5-20 \\
5-21 \\
5-23 \\
5-24 \\
5-25\end{array}$ & $\begin{array}{l}=- \\
=- \\
=- \\
--\end{array}$ & $\begin{array}{l}-- \\
=- \\
-- \\
--\end{array}$ & $\begin{array}{l}=- \\
=- \\
=- \\
--\end{array}$ & $\begin{array}{l}=- \\
-- \\
--\end{array}$ & $\begin{array}{l}-= \\
=- \\
-- \\
--\end{array}$ & $\begin{array}{l}-- \\
-- \\
--\end{array}$ & $\begin{array}{l}-- \\
-- \\
-- \\
--\end{array}$ & $\begin{array}{l}-- \\
-- \\
--\end{array}$ & $\begin{array}{l}-- \\
-- \\
-- \\
--\end{array}$ & $\begin{array}{l}=- \\
-- \\
-- \\
--\end{array}$ \\
\hline $\begin{array}{l}5-26 \\
5-27 \\
5-28 \\
5-30 \\
5-31\end{array}$ & $\begin{array}{c}-- \\
\overline{--} \\
\overline{0} \\
0\end{array}$ & $\begin{array}{c}-- \\
-- \\
0 \\
0\end{array}$ & $\begin{array}{c}-- \\
-- \\
-\overline{0} \\
0\end{array}$ & $\begin{array}{c}-- \\
-- \\
-\overline{0} \\
0\end{array}$ & $\begin{array}{c}-- \\
-- \\
0 \\
0\end{array}$ & $\overline{--}$ & $\begin{array}{c}-- \\
-\overline{0} \\
0\end{array}$ & $\begin{array}{l}-- \\
\overline{0} \\
0\end{array}$ & $\begin{array}{l}-- \\
-\overline{0} \\
0 \\
0\end{array}$ & $\begin{array}{c}-- \\
-- \\
0 \\
0\end{array}$ \\
\hline $\begin{array}{l}6-01 \\
6-02 \\
6-03 \\
6-04 \\
6-05\end{array}$ & $\begin{array}{r}0 \\
2 \\
0 \\
11 \\
5\end{array}$ & $\begin{array}{l}0 \\
0 \\
0 \\
0 \\
3\end{array}$ & $\begin{array}{l}0 \\
0 \\
0 \\
1 \\
0\end{array}$ & $\begin{array}{l}0 \\
0 \\
0 \\
0 \\
0\end{array}$ & $\begin{array}{l}0 \\
0 \\
0 \\
0 \\
0\end{array}$ & $\begin{array}{l}2 \\
5 \\
0 \\
0 \\
0\end{array}$ & $\begin{array}{l}0 \\
1 \\
0 \\
0 \\
0\end{array}$ & $\begin{array}{l}0 \\
0 \\
0 \\
0 \\
0\end{array}$ & $\begin{array}{l}0 \\
0 \\
0 \\
0 \\
0\end{array}$ & $\begin{array}{l}0 \\
0 \\
0 \\
0 \\
0\end{array}$ \\
\hline $\begin{array}{l}6-06 \\
6-07 \\
6-08 \\
6-09 \\
6-10\end{array}$ & $\begin{array}{l}6 \\
0 \\
1 \\
\frac{1}{3}\end{array}$ & $\begin{array}{l}0 \\
0 \\
2 \\
0 \\
0\end{array}$ & $\begin{array}{l}0 \\
0 \\
0 \\
0 \\
0\end{array}$ & $\begin{array}{l}0 \\
0 \\
0 \\
0 \\
0\end{array}$ & $\begin{array}{l}0 \\
0 \\
0 \\
0 \\
0\end{array}$ & $\begin{array}{r}0 \\
3 \\
16 \\
33 \\
4\end{array}$ & $\begin{array}{l}0 \\
0 \\
5 \\
8 \\
0\end{array}$ & $\begin{array}{l}0 \\
0 \\
0 \\
0 \\
0\end{array}$ & $\begin{array}{l}0 \\
0 \\
0 \\
0 \\
0\end{array}$ & $\begin{array}{l}0 \\
0 \\
0 \\
0 \\
0\end{array}$ \\
\hline $\begin{array}{l}6-11 \\
6-12 \\
6-13 \\
6-14 \\
6-15\end{array}$ & $\begin{array}{l}8 \\
5 \\
0 \\
2 \\
3\end{array}$ & $\begin{array}{l}1 \\
0 \\
0 \\
0 \\
1\end{array}$ & $\begin{array}{l}0 \\
0 \\
0 \\
0 \\
0\end{array}$ & $\begin{array}{l}0 \\
0 \\
0 \\
0 \\
0\end{array}$ & $\begin{array}{l}0 \\
0 \\
0 \\
0 \\
0\end{array}$ & $\begin{array}{l}7 \\
5 \\
0 \\
4 \\
4\end{array}$ & $\begin{array}{l}3 \\
0 \\
0 \\
1 \\
1\end{array}$ & $\begin{array}{l}0 \\
0 \\
0 \\
0 \\
0\end{array}$ & $\begin{array}{l}0 \\
0 \\
0 \\
0 \\
0\end{array}$ & $\begin{array}{l}0 \\
0 \\
0 \\
0 \\
0\end{array}$ \\
\hline $\begin{array}{l}6-16 \\
6-17 \\
6-18 \\
6-19 \\
6-20\end{array}$ & $\begin{array}{r}8 \\
0 \\
7 \\
4 \\
17\end{array}$ & $\begin{array}{l}4 \\
0 \\
3 \\
4 \\
5\end{array}$ & $\begin{array}{l}0 \\
0 \\
0 \\
0 \\
0\end{array}$ & $\begin{array}{l}0 \\
0 \\
0 \\
0 \\
0\end{array}$ & $\begin{array}{l}0 \\
0 \\
0 \\
0 \\
0\end{array}$ & $\begin{array}{r}5 \\
4 \\
4 \\
8 \\
19\end{array}$ & $\begin{array}{l}0 \\
4 \\
3 \\
6 \\
5\end{array}$ & $\begin{array}{l}0 \\
0 \\
0 \\
0 \\
0\end{array}$ & $\begin{array}{l}0 \\
0 \\
0 \\
0 \\
0\end{array}$ & $\begin{array}{l}0 \\
0 \\
0 \\
0 \\
0\end{array}$ \\
\hline $\begin{array}{l}6-21 \\
6-22 \\
6-23 \\
6-24 \\
6-25\end{array}$ & $\begin{array}{r}16 \\
6 \\
11 \\
12 \\
--\end{array}$ & $\begin{array}{r}4 \\
0 \\
5 \\
2 \\
--\end{array}$ & $\begin{array}{r}0 \\
0 \\
0 \\
0 \\
--\end{array}$ & $\begin{array}{r}0 \\
0 \\
0 \\
0 \\
--\end{array}$ & $\begin{array}{r}0 \\
0 \\
0 \\
0 \\
--\end{array}$ & $\begin{array}{r}20 \\
9 \\
13 \\
14 \\
19\end{array}$ & $\begin{array}{r}12 \\
1 \\
1 \\
2 \\
5\end{array}$ & $\begin{array}{l}0 \\
0 \\
0 \\
0 \\
0\end{array}$ & $\begin{array}{l}0 \\
0 \\
0 \\
0 \\
0\end{array}$ & $\begin{array}{l}0 \\
0 \\
0 \\
0 \\
0\end{array}$ \\
\hline $\begin{array}{r}6-26 \\
6-27 \\
6-28 \\
7-11 \\
10-08\end{array}$ & $\begin{array}{r}19 \\
-\frac{8}{8} \\
11 \\
6\end{array}$ & $\begin{array}{r}-1 \\
3 \\
6 \\
7\end{array}$ & $\begin{array}{r}-0 \\
-0 \\
0 \\
0\end{array}$ & $\begin{array}{r}0 \\
-0 \\
0 \\
0 \\
0\end{array}$ & $\begin{array}{r}-0 \\
-0 \\
0 \\
0\end{array}$ & $\begin{array}{r}11 \\
10 \\
7 \\
8 \\
3\end{array}$ & $\begin{array}{r}10 \\
8 \\
9 \\
1 \\
8\end{array}$ & $\begin{array}{l}0 \\
2 \\
0 \\
0 \\
0\end{array}$ & $\begin{array}{l}0 \\
0 \\
0 \\
0 \\
0\end{array}$ & $\begin{array}{l}0 \\
0 \\
0 \\
0 \\
0\end{array}$ \\
\hline
\end{tabular}


TABLE 13.- NUMBER OF BLUE TRACER PARTICLES(1), PER 100 GRAMS OF BED MATERIAL, EAST FORK RIVER, WYOMING, 1979--CONTINUED

\begin{tabular}{|c|c|c|c|c|c|c|c|c|c|c|}
\hline \multirow[t]{2}{*}{ DATE } & \multicolumn{5}{|c|}{$\begin{array}{r}\text { NUMBER OF } \text { BLUE TRACER } \\
\text { PARTICLES BY SIZE CLASS (MM) }\end{array}$} & \multicolumn{5}{|c|}{$\begin{array}{l}\text { NUMBER OF BLUE TRACER } \\
\text { PARTICLES BY SIZE CLASS (MM) }\end{array}$} \\
\hline & $\begin{array}{l}0.25 \\
\text { TO } \\
0.50\end{array}$ & $\begin{array}{l}0.50 \\
\text { TO } \\
1.00\end{array}$ & $\begin{array}{l}1.00 \\
\text { TO } \\
2.00\end{array}$ & $\begin{array}{l}2.00 \\
\text { TO } \\
4.00\end{array}$ & $\begin{array}{l}4.00 \\
\text { TO } \\
8.00\end{array}$ & $\begin{array}{l}0.25 \\
\text { TO } \\
0.50\end{array}$ & $\begin{array}{r}0.50 \\
\text { TO } \\
1.00\end{array}$ & $\begin{array}{r}1.00 \\
\text { TO } \\
2.00\end{array}$ & $\begin{array}{r}2.00 \\
\text { TO } \\
4.00\end{array}$ & $\begin{array}{l}4.00 \\
\mathrm{TO} \\
8.00\end{array}$ \\
\hline $\begin{array}{l}5-20 \\
5-21 \\
5-23 \\
5-24 \\
5-25\end{array}$ & $\begin{array}{l}=- \\
=- \\
=- \\
=-\end{array}$ & $\begin{array}{l}-- \\
-- \\
=- \\
=-\end{array}$ & $\begin{array}{l}=- \\
=- \\
=- \\
--\end{array}$ & $\begin{array}{l}-- \\
=- \\
-- \\
--\end{array}$ & $\begin{array}{l}-- \\
=- \\
=- \\
--\end{array}$ & $\begin{array}{l}=- \\
=- \\
-- \\
--\end{array}$ & $\begin{array}{l}-- \\
=- \\
-- \\
--\end{array}$ & $\begin{array}{l}=- \\
=- \\
-- \\
--\end{array}$ & $\begin{array}{l}=- \\
=- \\
=- \\
--\end{array}$ & $\begin{array}{l}-- \\
=- \\
-- \\
--\end{array}$ \\
\hline $\begin{array}{l}5-26 \\
5-27 \\
5-28 \\
5-30 \\
5-31\end{array}$ & $\begin{array}{c}-- \\
-\overline{0} \\
0 \\
0\end{array}$ & $\begin{array}{c}-- \\
-\overline{0} \\
0 \\
0\end{array}$ & $\begin{array}{c}-- \\
-\overline{0} \\
0 \\
0\end{array}$ & $\begin{array}{c}-- \\
-\overline{0} \\
0 \\
0\end{array}$ & $\begin{array}{c}-- \\
-\overline{0} \\
0 \\
0\end{array}$ & $\begin{array}{l}=- \\
=- \\
=- \\
--\end{array}$ & $\begin{array}{l}=- \\
z- \\
=-\end{array}$ & $\begin{array}{l}=- \\
=- \\
=- \\
--\end{array}$ & $\begin{array}{l}=- \\
=- \\
=- \\
--\end{array}$ & $\begin{array}{l}-- \\
=- \\
=- \\
--\end{array}$ \\
\hline $\begin{array}{l}6-01 \\
6-02 \\
6-03 \\
6-04 \\
6-05\end{array}$ & $\begin{array}{l}0 \\
1 \\
0 \\
2 \\
5\end{array}$ & $\begin{array}{l}0 \\
1 \\
0 \\
0 \\
0\end{array}$ & $\begin{array}{l}0 \\
0 \\
0 \\
0 \\
0\end{array}$ & $\begin{array}{l}0 \\
0 \\
0 \\
0 \\
0\end{array}$ & $\begin{array}{l}0 \\
0 \\
0 \\
0 \\
0\end{array}$ & $\begin{array}{l}-- \\
-- \\
4 \\
3\end{array}$ & $\begin{array}{l}-- \\
-- \\
-\overline{0} \\
2\end{array}$ & $\begin{array}{l}-- \\
-- \\
-0 \\
0\end{array}$ & $\begin{array}{l}-- \\
-- \\
-\overline{0} \\
0\end{array}$ & $\begin{array}{c}-- \\
\overline{-} \\
0 \\
0\end{array}$ \\
\hline $\begin{array}{l}6-06 \\
6-07 \\
6-08 \\
6-09 \\
6-10\end{array}$ & $\begin{array}{r}3 \\
11 \\
4 \\
8 \\
0\end{array}$ & $\begin{array}{l}0 \\
1 \\
2 \\
1 \\
3\end{array}$ & $\begin{array}{l}0 \\
0 \\
0 \\
0 \\
0\end{array}$ & $\begin{array}{l}0 \\
0 \\
0 \\
0 \\
0\end{array}$ & $\begin{array}{l}0 \\
0 \\
0 \\
0 \\
0\end{array}$ & $\begin{array}{r}0 \\
23 \\
46 \\
- \\
--\end{array}$ & $\begin{array}{r}0 \\
2 \\
6 \\
-- \\
--\end{array}$ & $\begin{array}{r}0 \\
0 \\
0 \\
-- \\
--\end{array}$ & $\begin{array}{r}0 \\
0 \\
0 \\
---\end{array}$ & $\begin{array}{r}0 \\
0 \\
0 \\
-- \\
--\end{array}$ \\
\hline $\begin{array}{l}6-11 \\
6-12 \\
6-13 \\
6-14 \\
6-15\end{array}$ & $\begin{array}{l}9 \\
6 \\
4 \\
7 \\
8\end{array}$ & $\begin{array}{l}8 \\
1 \\
0 \\
0 \\
8\end{array}$ & $\begin{array}{l}0 \\
0 \\
0 \\
0 \\
0\end{array}$ & $\begin{array}{l}0 \\
0 \\
0 \\
1 \\
0\end{array}$ & $\begin{array}{l}0 \\
0 \\
0 \\
0 \\
0\end{array}$ & $\begin{array}{r}17 \\
9 \\
16 \\
8\end{array}$ & $\begin{array}{r}-- \\
\frac{1}{2} \\
4 \\
0\end{array}$ & $\begin{array}{l}- \\
0 \\
0 \\
0 \\
0\end{array}$ & $\begin{array}{l}- \\
0 \\
0 \\
0 \\
0\end{array}$ & $\begin{array}{c}-- \\
0 \\
0 \\
0 \\
0\end{array}$ \\
\hline $\begin{array}{l}6-16 \\
6-17 \\
6-18 \\
6-19 \\
6-20\end{array}$ & $\begin{array}{r}8 \\
9 \\
11 \\
7 \\
4\end{array}$ & $\begin{array}{r}11 \\
\frac{1}{1} \\
2 \frac{1}{7}\end{array}$ & $\begin{array}{l}3 \\
0 \\
0 \\
0 \\
3\end{array}$ & $\begin{array}{l}0 \\
0 \\
0 \\
0 \\
0\end{array}$ & $\begin{array}{l}0 \\
0 \\
0 \\
0 \\
0\end{array}$ & $\begin{array}{r}11 \\
5 \\
3 \\
-- \\
--\end{array}$ & $\begin{array}{r}0 \\
4 \\
14 \\
--\end{array}$ & $\begin{array}{r}0 \\
0 \\
0 \\
-- \\
--\end{array}$ & $\begin{array}{r}0 \\
0 \\
0 \\
---\end{array}$ & $\begin{array}{r}0 \\
0 \\
0 \\
-- \\
--\end{array}$ \\
\hline $\begin{array}{l}6-21 \\
6-22 \\
6-23 \\
6-24 \\
6-25\end{array}$ & $\begin{array}{r}30 \\
9 \\
11 \\
18 \\
--\end{array}$ & $\begin{array}{r}7 \\
0 \\
7 \\
18 \\
--\end{array}$ & $\begin{array}{r}1 \\
0 \\
0 \\
7 \\
--\end{array}$ & $\begin{array}{r}0 \\
0 \\
0 \\
0 \\
--\end{array}$ & $\begin{array}{r}0 \\
0 \\
0 \\
0 \\
--\end{array}$ & $\begin{array}{l}=- \\
=- \\
=- \\
--\end{array}$ & $\begin{array}{l}=- \\
\overline{--} \\
\overline{--}\end{array}$ & $\begin{array}{l}=- \\
=- \\
--\end{array}$ & $\begin{array}{l}=- \\
=- \\
=- \\
=-\end{array}$ & $\begin{array}{l}=- \\
=- \\
=- \\
=-\end{array}$ \\
\hline $\begin{array}{r}6-26 \\
6-27 \\
6-28 \\
7-11 \\
10-08\end{array}$ & $\begin{array}{r}-- \\
10 \\
2 \\
0\end{array}$ & $\begin{array}{c}-- \\
-- \\
8 \\
1 \\
4\end{array}$ & $\begin{array}{r}-- \\
-\overline{0} \\
0 \\
1\end{array}$ & $\begin{array}{r}-- \\
-0 \\
0 \\
0\end{array}$ & $\begin{array}{c}-- \\
-\overline{0} \\
0 \\
0\end{array}$ & $\begin{array}{l}=- \\
=- \\
=- \\
--\end{array}$ & $\begin{array}{l}=- \\
=- \\
=- \\
--\end{array}$ & $\begin{array}{l}=- \\
=- \\
=- \\
--\end{array}$ & $\begin{array}{l}-- \\
=- \\
=- \\
--\end{array}$ & $\begin{array}{l}-- \\
=- \\
-- \\
-- \\
--\end{array}$ \\
\hline
\end{tabular}


TABLE 13.- NUMBER OF BLUE TRACER PARTICLES(1), PER 100 GRAMS OF BED MATERIAL, EAST FORK RIVER, WYOMING, 1979--CONTINUED

\begin{tabular}{|c|c|c|c|c|c|c|c|c|c|c|}
\hline \multirow[t]{2}{*}{ DATE } & \multicolumn{5}{|c|}{$\begin{array}{l}\text { NUMBER OF } \text { BLUE TRACER } \\
\text { PARTICLES BY SIZE CLASS (MM) }\end{array}$} & \multicolumn{5}{|c|}{$\begin{array}{r}\text { NUMBER OF BLUE TRACER } \\
\text { PARTICLES BY SIZE CLASS (MM) }\end{array}$} \\
\hline & $\begin{array}{l}0.25 \\
\text { TO } \\
0.50\end{array}$ & $\begin{array}{l}0.50 \\
\text { TO } \\
1.00\end{array}$ & $\begin{array}{l}1.00 \\
\text { TO } \\
2.00\end{array}$ & $\begin{array}{l}2.00 \\
\text { TO } \\
4.00\end{array}$ & $\begin{array}{c}4.00 \\
\text { TO } \\
8.00\end{array}$ & $\begin{array}{r}0.25 \\
\text { TO } \\
0.50\end{array}$ & $\begin{array}{r}0.50 \\
\text { TO } \\
1.00\end{array}$ & $\begin{array}{l}1.00 \\
\text { TO } \\
2.00\end{array}$ & $\begin{array}{r}2.00 \\
\text { TO } \\
4.00\end{array}$ & $\begin{array}{r}4.00 \\
\text { TO } \\
8.00\end{array}$ \\
\hline $\begin{array}{l}5-20 \\
5-21 \\
5-23 \\
5-24 \\
5-25\end{array}$ & $\begin{array}{l}=- \\
\overline{--} \\
--\end{array}$ & $\begin{array}{l}=- \\
=- \\
=-\end{array}$ & $\begin{array}{l}-- \\
-- \\
-- \\
--\end{array}$ & $\begin{array}{l}-- \\
-- \\
-- \\
--\end{array}$ & $\begin{array}{l}=- \\
=- \\
=-\end{array}$ & $\begin{array}{l}\overline{-} \\
\overline{--} \\
\overline{-}\end{array}$ & $\begin{array}{l}=- \\
=- \\
=-\end{array}$ & $\begin{array}{l}-- \\
=- \\
=-\end{array}$ & $\begin{array}{l}=- \\
\overline{-} \\
\overline{--}\end{array}$ & $\begin{array}{l}\overline{-} \\
\overline{-} \\
\overline{-}\end{array}$ \\
\hline $\begin{array}{l}5-26 \\
5-27 \\
5-28 \\
5-30 \\
5-31\end{array}$ & $\begin{array}{c}-- \\
-\overline{0} \\
0 \\
0\end{array}$ & $\begin{array}{c}-- \\
-\overline{0} \\
0 \\
0\end{array}$ & $\begin{array}{c}-- \\
-\overline{0} \\
0 \\
0\end{array}$ & $\begin{array}{c}-- \\
-\overline{0} \\
0 \\
0\end{array}$ & $\begin{array}{c}-- \\
-0 \\
0 \\
0\end{array}$ & $\begin{array}{c}-- \\
0 \\
0 \\
0 \\
0\end{array}$ & $\begin{array}{c}-\overline{0} \\
0 \\
0 \\
0\end{array}$ & $\begin{array}{l}-0 \\
0 \\
0 \\
0\end{array}$ & $\begin{array}{c}-- \\
0 \\
0 \\
0 \\
0\end{array}$ & $\begin{array}{c}-\overline{0} \\
0 \\
0 \\
0\end{array}$ \\
\hline $\begin{array}{l}6-01 \\
6-02 \\
6-03 \\
6-04 \\
6-05\end{array}$ & $\begin{array}{l}2 \\
0 \\
0 \\
0 \\
3\end{array}$ & $\begin{array}{l}1 \\
0 \\
0 \\
0 \\
0\end{array}$ & $\begin{array}{l}0 \\
0 \\
0 \\
0 \\
0\end{array}$ & $\begin{array}{l}0 \\
0 \\
0 \\
0 \\
0\end{array}$ & $\begin{array}{l}0 \\
0 \\
0 \\
0 \\
0\end{array}$ & $\begin{array}{l}0 \\
0 \\
0 \\
1 \\
0\end{array}$ & $\begin{array}{l}0 \\
1 \\
0 \\
0 \\
0\end{array}$ & $\begin{array}{l}0 \\
0 \\
0 \\
0 \\
0\end{array}$ & $\begin{array}{l}0 \\
0 \\
0 \\
0 \\
0\end{array}$ & $\begin{array}{l}0 \\
0 \\
0 \\
0 \\
0\end{array}$ \\
\hline $\begin{array}{l}6-06 \\
6-07 \\
6-08 \\
6-09 \\
6-10\end{array}$ & $\begin{array}{r}5 \\
5 \\
1 \\
7 \\
17\end{array}$ & $\begin{array}{l}1 \\
1 \\
0 \\
2 \\
7\end{array}$ & $\begin{array}{l}0 \\
0 \\
0 \\
1 \\
0\end{array}$ & $\begin{array}{l}0 \\
0 \\
0 \\
0 \\
0\end{array}$ & $\begin{array}{l}0 \\
0 \\
0 \\
0 \\
0\end{array}$ & $\begin{array}{r}0 \\
36 \\
10 \\
2 \\
11\end{array}$ & $\begin{array}{r}2 \\
18 \\
3 \\
1 \\
2\end{array}$ & $\begin{array}{l}0 \\
0 \\
0 \\
0 \\
0\end{array}$ & $\begin{array}{l}0 \\
0 \\
0 \\
0 \\
0\end{array}$ & $\begin{array}{l}0 \\
0 \\
0 \\
0 \\
0\end{array}$ \\
\hline $\begin{array}{l}6-11 \\
6-12 \\
6-13 \\
6-14 \\
6-15\end{array}$ & $\begin{array}{r}9 \\
4 \\
5 \\
12 \\
4\end{array}$ & $\begin{array}{l}7 \\
1 \\
4 \\
6 \\
2\end{array}$ & $\begin{array}{l}0 \\
0 \\
0 \\
0 \\
1\end{array}$ & $\begin{array}{l}0 \\
0 \\
0 \\
0 \\
0\end{array}$ & $\begin{array}{l}0 \\
0 \\
0 \\
0 \\
0\end{array}$ & $\begin{array}{r}17 \\
4 \\
12 \\
2 \\
8\end{array}$ & $\begin{array}{r}6 \\
0 \\
4 \\
3 \\
13\end{array}$ & $\begin{array}{l}0 \\
1 \\
0 \\
0 \\
0\end{array}$ & $\begin{array}{l}0 \\
0 \\
0 \\
0 \\
0\end{array}$ & $\begin{array}{l}0 \\
0 \\
0 \\
0 \\
0\end{array}$ \\
\hline $\begin{array}{l}6-16 \\
6-17 \\
6-18 \\
6-19 \\
6-20\end{array}$ & $\begin{array}{r}8 \\
7 \\
1 \\
34 \\
15\end{array}$ & $\begin{array}{r}16 \\
2 \\
15 \\
13 \\
20\end{array}$ & $\begin{array}{l}1 \\
0 \\
2 \\
0 \\
0\end{array}$ & $\begin{array}{l}0 \\
0 \\
0 \\
0 \\
0\end{array}$ & $\begin{array}{l}0 \\
0 \\
0 \\
0 \\
0\end{array}$ & $\begin{array}{r}22 \\
14 \\
0 \\
0 \\
3\end{array}$ & $\begin{array}{r}24 \\
7 \\
4 \\
1 \\
6\end{array}$ & $\begin{array}{l}1 \\
0 \\
0 \\
0 \\
2\end{array}$ & $\begin{array}{l}1 \\
0 \\
0 \\
0 \\
0\end{array}$ & $\begin{array}{l}0 \\
0 \\
0 \\
0 \\
0\end{array}$ \\
\hline $\begin{array}{l}6-21 \\
6-22 \\
6-23 \\
6-24 \\
6-25\end{array}$ & $\begin{array}{r}28 \\
4 \\
16 \\
25 \\
--\end{array}$ & $\begin{array}{r}36 \\
6 \\
17 \\
31 \\
-\end{array}$ & $\begin{array}{r}2 \\
0 \\
0 \\
4 \\
--\end{array}$ & $\begin{array}{r}0 \\
0 \\
0 \\
0 \\
--\end{array}$ & $\begin{array}{r}0 \\
0 \\
0 \\
0 \\
--\end{array}$ & $\begin{array}{r}11 \\
18 \\
5 \\
14 \\
--\end{array}$ & $\begin{array}{r}14 \\
5 \\
9 \\
4 \\
--\end{array}$ & $\begin{array}{r}3 \\
1 \\
0 \\
0 \\
-\end{array}$ & $\begin{array}{r}1 \\
0 \\
1 \\
0 \\
--\end{array}$ & $\begin{array}{r}0 \\
0 \\
0 \\
0 \\
--\end{array}$ \\
\hline $\begin{array}{r}6-26 \\
6-27 \\
6-28 \\
7-11 \\
10-08\end{array}$ & $\begin{array}{r}-- \\
\overline{10} \\
3\end{array}$ & $\begin{array}{l}-- \\
\overline{16} \\
20 \\
13\end{array}$ & $\begin{array}{r}-- \\
-\frac{1}{3} \\
\frac{1}{3}\end{array}$ & \begin{tabular}{c}
$--\overline{0}$ \\
\hdashline 0 \\
1 \\
0
\end{tabular} & $\begin{array}{c}-- \\
\overline{0} \\
0 \\
0\end{array}$ & $\begin{array}{r}-- \\
\overline{13} \\
8 \\
1\end{array}$ & $\begin{array}{r}-- \\
-5 \\
\frac{1}{3}\end{array}$ & $\begin{array}{r}-- \\
-\overline{3} \\
0 \\
0\end{array}$ & $\begin{array}{c}-- \\
-0 \\
0 \\
0\end{array}$ & $\begin{array}{c}-- \\
-\overline{0} \\
0 \\
0\end{array}$ \\
\hline
\end{tabular}


TABLE 13.- NUMBER OF BLUE TRACER PARTICLES(1), PER 100 GRAMS OF BED MATERIAL, EAST FORK RIVER, WYOMING, 1979--CONTINUED SECTION 1077

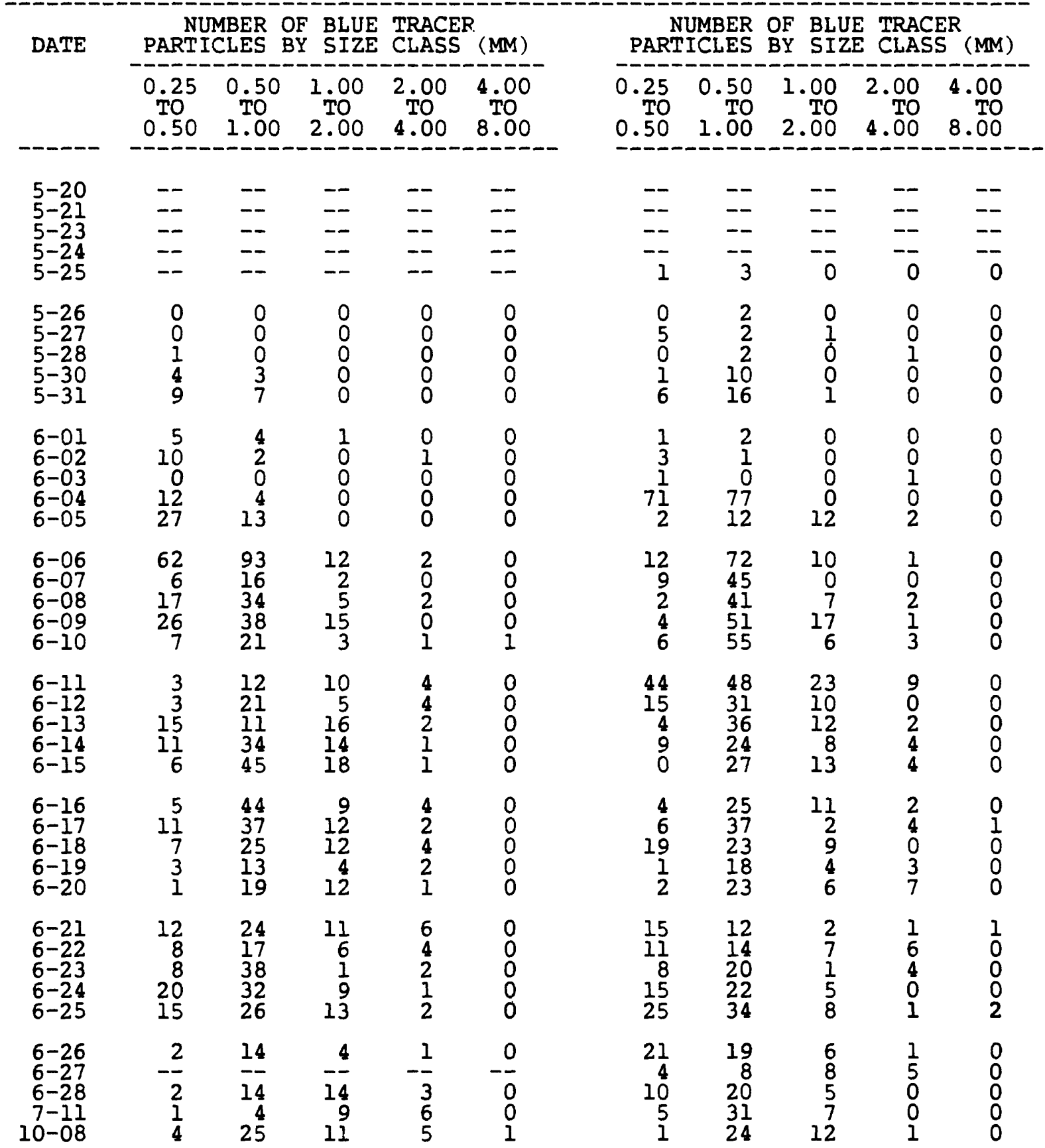


TABLE 13.- NUMBER OF BLUE TRACER PARTICLES(1), PER 100 GRAMS OF BED MATERIAL, EAST FORK RIVER, WYOMING, 1979--CONTINUED

DATE

NUMBER OF BLUE TRACER

PARTICLES BY SIZE CLASS (MM)

$0.25 \quad 0.50 \quad 1.00 \quad 2.00 \quad 4.00$

$\begin{array}{lllll}\text { TO } & \text { TO } & \text { TO } & \text { TO } & \text { TO } \\ 0.50 & 1.00 & 2.00 & 4.00 & 8.00\end{array}$

$$
0.50
$$

$.00 \quad 4.00 \quad 8.00$

$\begin{array}{cccccc}5-20 & -- & -- & -- & -- & -- \\ 5-21 & -- & -- & -- & -- & -- \\ 5-23 & -\overline{3} & \overline{10} & -\overline{0} & -\overline{0} & - \\ 5-24 & 13 & 10 & 0 & 2 & 0 \\ 5-25 & 2 & 11 & 0\end{array}$

$\begin{array}{llllll}5-26 & 1 & 24 & 2 & 0 & 0\end{array}$

$5-26$
$5-27$
$5-28$

$5-30$

$6 \quad 13$

$\frac{1}{14}$

1
38

$6-01$
$6-02$

$6-03$

$6-04$
$6-05$

13
12

43

33

14
48
23

2
1
0

1
0
1
8

0

$6-06$

6-07

$6-08$

6-09

6-10

$\begin{array}{lll}10 & 0 & 0 \\ 20 & 7 & 0 \\ 11 & 3 & 0 \\ 15 & 1 & 0 \\ 12 & 3 & 0\end{array}$

$\begin{array}{rr}45 & 13 \\ 39 & 23 \\ 42 & 17 \\ 27 & 3 \\ 24 & 5\end{array}$

6-11

$6-12$
$6-13$

$6-14$

6-15

5

2

12

23

8
6
9
7
10

$\begin{array}{ll}7 & 1 \\ 3 & 1 \\ 1 & 0 \\ 4 & 0 \\ 4 & 0\end{array}$

$6-16$

$6-17$
$6-18$

$6-19$

2

$6-20$

29

25
15
35
24

7
4
8
10
11

$\begin{array}{ll}5 & 0 \\ 4 & 0 \\ 3 & 0 \\ 5 & 0 \\ 2 & 0\end{array}$

$6-21$

$6-23$

$6-24$

$6-25$

$\begin{array}{rr}27 & 3 \\ 14 & 6 \\ 18 & 3 \\ 6 & 3 \\ 9 & 8\end{array}$

$\begin{array}{ll}4 & 0 \\ 0 & 0 \\ 4 & 0 \\ 0 & 0 \\ 1 & 0\end{array}$

$\begin{array}{ll}1 & 1 \\ 1 & 0 \\ 2 & 0 \\ 2 & 0 \\ 3 & 0\end{array}$

$6-26$
$6-27$
$6-28$

$7-11$

10-08

NUMBEF. OF BLUE TRACER

PARTICLES BY SIZE CLASS (MM)

$0.250 .50 \quad 1.00 \quad 2.00 \quad 4.00$

$\begin{array}{rrrrr}\text { TO } & \text { TO } & \text { TO } & \text { TO } & \text { TO } \\ 0.50 & 1.00 & 2.00 & 4.00 & 8.00\end{array}$

$\begin{array}{rrrrr}31 & 0 & 0 & 0 & 0 \\ -5 & -\frac{7}{7} & -\frac{1}{0} & -\frac{0}{0} \\ 6 & 27 & 2 & 0 & 0 \\ 6 & 34 & 9 & 0 & 0\end{array}$

$\begin{array}{rrrrr}6 & 88 & 18 & 0 & 0 \\ 6 & 35 & 23 & 4 & 0 \\ 0 & 6 & 9 & 4 & 1 \\ 3 & 45 & 16 & 7 & 1 \\ 1 & 45 & 24 & 6 & 1\end{array}$

$\begin{array}{lllll}12 & 22 & 12 & 3 & 1 \\ 13 & 46 & 13 & 2 & 0\end{array}$

$\begin{array}{rrrrr}13 & 46 & 13 & 2 & 0 \\ 0 & 14 & 7 & 3 & 1 \\ 9 & 19 & 27 & 5 & 0 \\ 5 & 51 & 11 & 1 & 0\end{array}$

$\begin{array}{rrrrr}5 & 44 & 24 & 3 & 0 \\ 4 & 30 & 25 & 2 & 0 \\ 0 & 14 & 6 & 0 & 0 \\ 3 & 13 & 5 & 1 & 0 \\ 8 & 20 & 7 & 2 & 0\end{array}$

$\begin{array}{rrrrr}3 & 18 & 9 & 1 & 0 \\ 4 & 13 & 5 & 2 & 0 \\ 6 & 9 & 1 & 2 & 0 \\ 12 & 16 & 11 & 1 & 1 \\ 8 & 45 & 4 & 0 & 0\end{array}$

$\begin{array}{rrrrr}5 & 13 & 6 & 2 & 0 \\ 2 & 16 & 9 & 4 & 0 \\ 6 & 17 & 12 & 4 & 0 \\ 0 & 17 & 5 & 4 & 0 \\ 5 & 2 & 6 & 0 & 2\end{array}$

$\begin{array}{rrrrr}0 & 2 & 3 & 1 & 1 \\ 2 & 5 & 3 & 2 & 0 \\ 0 & 1 & 4 & 5 & 0 \\ 12 & 7 & 1 & 2 & 1 \\ 2 & 6 & 6 & 0 & 0\end{array}$

$\begin{array}{lllll}6 & 3 & 5 & 4 & 1 \\ 0 & 3 & 0 & 0 & 0 \\ 9 & 7 & 9 & 6 & 0 \\ 3 & 4 & 3 & 2 & 0 \\ 1 & 3 & 2 & 1 & 1\end{array}$ 
TABLE 13.- NUMBER OF BLUE TRACER PARTICLES(1), PER 100 GRAMS OF BED MATERIAL, EAST FORK RIVER, WYOMING, 1979--CONTINUED

SECTION $1400(3)$

\begin{tabular}{|c|c|c|c|c|c|c|c|c|c|c|}
\hline \multirow[t]{2}{*}{ DATE } & \multicolumn{2}{|c|}{$\begin{aligned} \text { NUMBER } & 0 \\
\text { PARTICLES } & B\end{aligned}$} & $\begin{array}{l}\text { BLUE } \\
\text { SIZE }\end{array}$ & \multicolumn{2}{|c|}{$\begin{array}{l}\text { TRACER } \\
\text { CLASS (MM) }\end{array}$} & \multicolumn{2}{|c|}{$\begin{array}{r}\text { NUMBER } \\
\text { PARTICLES }\end{array}$} & \multicolumn{3}{|c|}{$\begin{array}{ll}\text { OF } & \text { BLUE TRACER } \\
\text { BY } & \text { SIZE CLASS (MM) }\end{array}$} \\
\hline & $\begin{array}{l}0.25 \\
\text { TO } \\
0.50\end{array}$ & $\begin{array}{l}0.50 \\
\text { TO } \\
1.00\end{array}$ & $\begin{array}{l}1.00 \\
\text { TO } \\
2.00\end{array}$ & $\begin{array}{l}2.00 \\
\text { TO } \\
4.00\end{array}$ & $\begin{array}{l}4.00 \\
\text { TO } \\
8.00\end{array}$ & $\begin{array}{l}0.25 \\
\text { TO } \\
0.50\end{array}$ & $\begin{array}{r}0.50 \\
\text { TO } \\
1.00\end{array}$ & $\begin{array}{r}1.00 \\
\text { TO } \\
2.00\end{array}$ & $\begin{array}{r}2.00 \\
\mathrm{TO} \\
4.00\end{array}$ & $\begin{array}{r}4.00 \\
\text { TO } \\
8.00\end{array}$ \\
\hline $\begin{array}{l}5-20 \\
5-21 \\
5-23 \\
5-24 \\
5-25\end{array}$ & $\begin{array}{r}23 \\
10 \\
7 \\
7 \\
0\end{array}$ & $\begin{array}{r}5 \\
32 \\
3 \\
73 \\
37\end{array}$ & $\begin{array}{r}0 \\
0 \\
0 \\
40 \\
32\end{array}$ & $\begin{array}{l}0 \\
0 \\
0 \\
4 \\
7\end{array}$ & $\begin{array}{l}0 \\
0 \\
0 \\
1 \\
2\end{array}$ & $\begin{array}{l}-- \\
-- \\
-- \\
-\overline{2}\end{array}$ & $\begin{array}{l}-- \\
\overline{--} \\
150\end{array}$ & $\begin{array}{l}-- \\
-- \\
\overline{29}\end{array}$ & $\begin{array}{l}-- \\
-- \\
-- \\
-\overline{4}\end{array}$ & $\begin{array}{l}-- \\
\overline{-} \\
-\overline{2}\end{array}$ \\
\hline $\begin{array}{l}5-26 \\
5-27 \\
5-28 \\
5-30 \\
5-31\end{array}$ & $\begin{array}{l}2 \\
1 \\
1 \\
1 \\
7\end{array}$ & $\begin{array}{r}11 \\
10 \\
6 \\
8 \\
18\end{array}$ & $\begin{array}{r}10 \\
10 \\
14 \\
10 \\
4\end{array}$ & $\begin{array}{l}8 \\
8 \\
4 \\
3 \\
1\end{array}$ & $\begin{array}{l}2 \\
1 \\
0 \\
0 \\
1\end{array}$ & $\begin{array}{r}10 \\
-- \\
-7\end{array}$ & $\begin{array}{l}36 \\
48 \\
-- \\
-50\end{array}$ & $\begin{array}{l}31 \\
67 \\
-- \\
29\end{array}$ & $-\frac{4}{5}$ & $\begin{array}{r}2 \\
-- \\
-2\end{array}$ \\
\hline $\begin{array}{l}6-01 \\
6-02 \\
6-03 \\
6-04 \\
6-05\end{array}$ & $\begin{array}{l}0 \\
9 \\
3 \\
3 \\
2\end{array}$ & $\begin{array}{r}6 \\
70 \\
16 \\
3 \\
1\end{array}$ & $\begin{array}{r}1 \\
28 \\
9 \\
0 \\
0\end{array}$ & $\begin{array}{l}2 \\
2 \\
0 \\
0 \\
0\end{array}$ & $\begin{array}{l}0 \\
0 \\
0 \\
0 \\
0\end{array}$ & $\begin{array}{r}15 \\
2 \\
12 \\
24 \\
4\end{array}$ & $\begin{array}{r}49 \\
3 \\
42 \\
30 \\
101\end{array}$ & $\begin{array}{r}21 \\
4 \\
22 \\
11 \\
32\end{array}$ & $\begin{array}{l}2 \\
3 \\
9 \\
3 \\
1\end{array}$ & $\begin{array}{l}0 \\
1 \\
1 \\
0 \\
0\end{array}$ \\
\hline $\begin{array}{l}6-06 \\
6-07 \\
6-08 \\
6-09 \\
6-10\end{array}$ & $\begin{array}{r}1 \\
1 \\
7 \\
10 \\
28\end{array}$ & $\begin{array}{l}13 \\
5 \\
61 \\
11 \\
50\end{array}$ & $\begin{array}{r}4 \\
7 \\
10 \\
8 \\
3\end{array}$ & $\begin{array}{l}1 \\
0 \\
3 \\
1 \\
1\end{array}$ & $\begin{array}{l}0 \\
0 \\
1 \\
0 \\
0\end{array}$ & $\begin{array}{r}75 \\
4 \\
0 \\
5 \\
4\end{array}$ & $\begin{array}{r}116 \\
40 \\
9 \\
30 \\
3\end{array}$ & $\begin{array}{r}8 \\
1 \frac{1}{8} \\
11 \\
2\end{array}$ & $\begin{array}{l}0 \\
1 \\
1 \\
1 \\
0\end{array}$ & $\begin{array}{l}0 \\
0 \\
1 \\
0 \\
0\end{array}$ \\
\hline $\begin{array}{l}6-11 \\
6-12 \\
6-13 \\
6-14 \\
6-15\end{array}$ & $\begin{array}{r}3 \\
1 \\
1 \frac{1}{3} \\
3 \\
0\end{array}$ & $\begin{array}{r}6 \\
7 \\
9 \\
7 \\
10\end{array}$ & $\begin{array}{l}3 \\
4 \\
4 \\
5 \\
4\end{array}$ & $\begin{array}{l}\frac{1}{1} \\
\frac{1}{2} \\
\frac{1}{2}\end{array}$ & $\begin{array}{l}1 \\
0 \\
0 \\
0 \\
0\end{array}$ & $\begin{array}{r}7 \\
11 \\
14 \\
13 \\
2\end{array}$ & $\begin{array}{l}46 \\
27 \\
47 \\
41 \\
22\end{array}$ & $\begin{array}{l}12 \\
11 \\
11 \\
11 \\
15\end{array}$ & $\begin{array}{l}4 \\
4 \\
2 \\
2 \\
1\end{array}$ & $\begin{array}{l}0 \\
1 \\
0 \\
0 \\
0\end{array}$ \\
\hline $\begin{array}{l}6-16 \\
6-17 \\
6-18 \\
6-19 \\
6-20\end{array}$ & $\begin{array}{l}1 \\
1 \\
0 \\
0 \\
2\end{array}$ & $\begin{array}{r}10 \\
4 \\
1 \\
5 \\
7\end{array}$ & $\begin{array}{l}7 \\
2 \\
0 \\
4 \\
1\end{array}$ & $\begin{array}{l}4 \\
2 \\
2 \\
1 \\
4\end{array}$ & $\begin{array}{l}0 \\
0 \\
1 \\
0 \\
0\end{array}$ & $\begin{array}{r}0 \\
3 \\
5 \\
3 \\
--\end{array}$ & $\begin{array}{r}0 \\
16 \\
21 \\
14 \\
-\end{array}$ & $\begin{array}{r}0 \\
10 \\
4 \\
6 \\
--\end{array}$ & $\begin{array}{r}0 \\
2 \\
2 \\
1 \\
--\end{array}$ & $\begin{array}{r}0 \\
1 \\
0 \\
1 \\
--\end{array}$ \\
\hline $\begin{array}{l}6-21 \\
6-22 \\
6-23 \\
6-24 \\
6-25\end{array}$ & $\begin{array}{l}1 \\
3 \\
0 \\
1 \\
5\end{array}$ & $\begin{array}{l}5 \\
3 \\
0 \\
5 \\
5\end{array}$ & $\begin{array}{l}0 \\
0 \\
4 \\
5 \\
4\end{array}$ & $\begin{array}{l}1 \\
0 \\
4 \\
0 \\
1\end{array}$ & $\begin{array}{l}0 \\
0 \\
0 \\
0 \\
0\end{array}$ & $\begin{array}{l}-- \\
-\overline{-} \\
-\overline{-} \\
--\end{array}$ & $\begin{array}{l}=- \\
=- \\
=-\end{array}$ & $\begin{array}{l}=- \\
=- \\
=\end{array}$ & $\begin{array}{l}-- \\
\overline{-} \\
--\end{array}$ & $\begin{array}{l}-- \\
-- \\
--\end{array}$ \\
\hline $\begin{array}{r}6-26 \\
6-27 \\
6-28 \\
7-11 \\
10-08\end{array}$ & $\begin{array}{l}0 \\
4 \\
3 \\
1 \\
0\end{array}$ & $\begin{array}{l}5 \\
5 \\
3 \\
8 \\
1\end{array}$ & $\begin{array}{l}3 \\
2 \\
3 \\
2 \\
1\end{array}$ & $\begin{array}{l}2 \\
3 \\
2 \\
0 \\
0\end{array}$ & $\begin{array}{l}0 \\
0 \\
0 \\
0 \\
0\end{array}$ & $\begin{array}{l}-- \\
=- \\
-- \\
--\end{array}$ & $\begin{array}{l}=- \\
=- \\
=-\end{array}$ & $\begin{array}{l}=- \\
=- \\
=-\end{array}$ & $\begin{array}{l}- \\
=- \\
=- \\
-\end{array}$ & $\begin{array}{l}=- \\
=- \\
=-\end{array}$ \\
\hline
\end{tabular}


TABLE 13.- NUMBER OF BLUE TRACER PARTICLES(1), PER 100 GRAMS OF BED MATERIAL, EAST FORK RIVER, WYOMING, 1979--CONTINUED

SECTION 1481

SECTION 1481(2)

\begin{tabular}{|c|c|c|c|c|c|c|c|c|c|c|}
\hline \multirow[t]{2}{*}{ DATE } & \multicolumn{5}{|c|}{$\begin{array}{l}\text { NUMBER OF BLUE TRACER } \\
\text { PARTICLES BY SIZE CLASS (MM) }\end{array}$} & \multicolumn{5}{|c|}{$\begin{array}{l}\text { NUMBER OF BLUE TRACER } \\
\text { PARTICLES BY SIZE CLASS (MM) }\end{array}$} \\
\hline & $\begin{array}{r}0.25 \\
\text { TO } \\
0.50\end{array}$ & $\begin{array}{l}0.50 \\
\text { TO } \\
1.00\end{array}$ & $\begin{array}{l}1.00 \\
2.00\end{array}$ & $\begin{array}{l}2.00 \\
\text { TO } \\
4.00\end{array}$ & $\begin{array}{l}4.00 \\
\text { TO } \\
8.00\end{array}$ & $\begin{array}{r}0.25 \\
\text { TO } \\
0.50\end{array}$ & $\begin{array}{l}0.50 \\
\text { TO } \\
1.00\end{array}$ & $\begin{array}{r}1.00 \\
\text { TO } \\
2.00\end{array}$ & $\begin{array}{r}2.00 \\
\text { TO } \\
4.00\end{array}$ & $\begin{array}{r}4.00 \\
\text { TO } \\
8.00\end{array}$ \\
\hline $\begin{array}{l}5-20 \\
5-21 \\
5-23 \\
5-24 \\
5-25\end{array}$ & $\begin{array}{r}10 \\
44 \\
9 \\
2 \\
1\end{array}$ & $\begin{array}{r}9 \\
49 \\
96 \\
48 \\
25\end{array}$ & $\begin{array}{r}0 \\
0 \\
44 \\
41 \\
24\end{array}$ & $\begin{array}{r}0 \\
0 \\
8 \\
15 \\
15\end{array}$ & $\begin{array}{l}0 \\
0 \\
2 \\
3 \\
3\end{array}$ & $\begin{array}{r}7 \\
20 \\
17 \\
8 \\
2\end{array}$ & $\begin{array}{r}0 \\
32 \\
164 \\
136 \\
65\end{array}$ & $\begin{array}{r}0 \\
0 \\
65 \\
64 \\
43\end{array}$ & $\begin{array}{r}0 \\
0 \\
3 \\
21 \\
10\end{array}$ & $\begin{array}{l}0 \\
0 \\
0 \\
3 \\
1\end{array}$ \\
\hline $\begin{array}{l}5-26 \\
5-27 \\
5-28 \\
5-30 \\
5-31\end{array}$ & $\begin{array}{r}32 \\
0 \\
0 \\
0 \\
0\end{array}$ & $\begin{array}{r}41 \\
8 \\
3 \\
4 \\
5\end{array}$ & $\begin{array}{r}16 \\
10 \\
2 \\
5 \\
4\end{array}$ & $\begin{array}{l}6 \\
9 \\
3 \\
3 \\
1\end{array}$ & $\begin{array}{l}3 \\
0 \\
0 \\
0 \\
1\end{array}$ & $\begin{array}{r}3 \\
0 \\
-\frac{1}{0} \\
0\end{array}$ & $\begin{array}{r}24 \\
12 \\
10 \\
6\end{array}$ & $\begin{array}{r}23 \\
14 \\
-\frac{1}{4}\end{array}$ & $\begin{array}{r}9 \\
-7 \\
-3 \\
3\end{array}$ & $\begin{array}{r}0 \\
-\frac{0}{1} \\
0\end{array}$ \\
\hline $\begin{array}{l}6-01 \\
6-02 \\
6-03 \\
6-04 \\
6-05\end{array}$ & $\begin{array}{l}1 \\
0 \\
0 \\
3 \\
2\end{array}$ & $\begin{array}{r}7 \\
8 \\
6 \\
2 \\
23\end{array}$ & $\begin{array}{l}3 \\
5 \\
3 \\
2 \\
6\end{array}$ & $\begin{array}{l}3 \\
2 \\
4 \\
1 \\
1\end{array}$ & $\begin{array}{l}0 \\
0 \\
0 \\
0 \\
0\end{array}$ & $\begin{array}{l}3 \\
1 \\
0 \\
1 \\
0\end{array}$ & $\begin{array}{r}11 \\
8 \\
4 \\
6 \\
11\end{array}$ & $\begin{array}{l}1 \\
1 \\
9 \\
3 \\
5\end{array}$ & $\begin{array}{l}1 \\
0 \\
6 \\
1 \\
1\end{array}$ & $\begin{array}{l}0 \\
0 \\
0 \\
0 \\
0\end{array}$ \\
\hline $\begin{array}{l}6-06 \\
6-07 \\
6-08 \\
6-09 \\
6-10\end{array}$ & $\begin{array}{l}5 \\
1 \\
1 \\
2 \\
4\end{array}$ & $\begin{array}{l}12 \\
13 \\
23 \\
7 \\
31\end{array}$ & $\begin{array}{r}12 \\
10 \\
8 \\
3 \\
13\end{array}$ & $\begin{array}{l}4 \\
2 \\
1 \\
3 \\
3\end{array}$ & $\begin{array}{l}2 \\
0 \\
0 \\
0 \\
0\end{array}$ & $\begin{array}{r}0 \\
1 \\
-3 \\
3 \\
1\end{array}$ & $\frac{73}{43}$ & $\begin{array}{r}12 \\
16 \\
-\frac{7}{7} \\
2\end{array}$ & $\begin{array}{r}\frac{1}{4} \\
-\frac{1}{0} \\
3\end{array}$ & $\begin{array}{r}1 \\
-0 \\
0 \\
0\end{array}$ \\
\hline $\begin{array}{l}6-11 \\
6-12 \\
6-13 \\
6-14 \\
6-15\end{array}$ & $\begin{array}{r}1 \\
0 \\
11 \\
2 \\
0\end{array}$ & $\begin{array}{r}12 \\
5 \\
16 \\
7 \\
5\end{array}$ & $\begin{array}{l}9 \\
7 \\
7 \\
6 \\
1\end{array}$ & $\begin{array}{l}0 \\
2 \\
4 \\
0 \\
1\end{array}$ & $\begin{array}{l}0 \\
0 \\
0 \\
2 \\
0\end{array}$ & $\begin{array}{r}0 \\
15 \\
8 \\
9 \\
0\end{array}$ & $\begin{array}{l}14 \\
37 \\
32 \\
31 \\
5\end{array}$ & $\begin{array}{r}4 \\
15 \\
7 \\
14 \\
4\end{array}$ & $\begin{array}{l}0 \\
1 \\
1 \\
0 \\
1\end{array}$ & $\begin{array}{l}0 \\
1 \\
0 \\
0 \\
0\end{array}$ \\
\hline $\begin{array}{l}6-16 \\
6-17 \\
6-18 \\
6-19 \\
6-20\end{array}$ & $\begin{array}{l}3 \\
2 \\
3 \\
2 \\
4\end{array}$ & $\begin{array}{r}6 \\
7 \\
11 \\
13 \\
3\end{array}$ & $\begin{array}{l}4 \\
1 \\
6 \\
2 \\
4\end{array}$ & $\begin{array}{l}1 \\
1 \\
0 \\
2 \\
0\end{array}$ & $\begin{array}{l}2 \\
1 \\
0 \\
0 \\
0\end{array}$ & $\begin{array}{r}2 \\
0 \\
5 \\
1 \\
--\end{array}$ & $\begin{array}{r}8 \\
5 \\
14 \\
5 \\
--\end{array}$ & $\begin{array}{r}6 \\
3 \\
1 \\
1 \\
-\end{array}$ & $\begin{array}{r}6 \\
0 \\
0 \\
1 \\
--\end{array}$ & $\begin{array}{r}0 \\
0 \\
0 \\
0 \\
--\end{array}$ \\
\hline $\begin{array}{l}6-21 \\
6-22 \\
6-23 \\
6-24 \\
6-25\end{array}$ & $\begin{array}{l}0 \\
0 \\
1 \\
3 \\
5\end{array}$ & $\begin{array}{l}6 \\
3 \\
5 \\
3 \\
4\end{array}$ & $\begin{array}{l}3 \\
2 \\
7 \\
4 \\
2\end{array}$ & $\begin{array}{l}0 \\
2 \\
6 \\
2 \\
3\end{array}$ & $\begin{array}{l}0 \\
2 \\
3 \\
0 \\
0\end{array}$ & $\begin{array}{l}-- \\
-- \\
=- \\
--\end{array}$ & $\begin{array}{l}-- \\
-- \\
z- \\
--\end{array}$ & $\begin{array}{l}-- \\
-- \\
=- \\
--\end{array}$ & $\begin{array}{l}-- \\
=- \\
=- \\
--\end{array}$ & $\begin{array}{l}=- \\
\overline{--} \\
=-\end{array}$ \\
\hline $\begin{array}{r}6-26 \\
6-27 \\
6-28 \\
7-11 \\
10-08\end{array}$ & $\begin{array}{r}-- \\
6 \\
2 \\
6 \\
0\end{array}$ & $\begin{array}{r}-- \\
8 \\
3 \\
13 \\
2\end{array}$ & $\begin{array}{r}-- \\
4 \\
2 \\
3 \\
2\end{array}$ & $\begin{array}{r}-- \\
1 \\
2 \\
5 \\
1\end{array}$ & $\begin{array}{c}-- \\
1 \\
0 \\
0 \\
0\end{array}$ & $\begin{array}{l}=- \\
=- \\
-- \\
--\end{array}$ & $\begin{array}{l}-- \\
-- \\
-- \\
--\end{array}$ & $\begin{array}{l}=- \\
=- \\
=- \\
--\end{array}$ & $\begin{array}{l}-- \\
-- \\
-- \\
--\end{array}$ & $\begin{array}{l}=- \\
=- \\
=- \\
--\end{array}$ \\
\hline
\end{tabular}


TABLE 13.- NUMBER OF BLUE TRACER PARTICLES(1), PER 100 GRAMS OF BED MATERIAL, EAST FORK RIVER, WYOMING, 1979--CONTINUED

\begin{tabular}{|c|c|c|c|c|c|c|c|c|c|c|}
\hline \multirow[t]{2}{*}{ DATE } & \multicolumn{5}{|c|}{$\begin{array}{r}\text { NUMBER OF } \text { BLUE TRACER } \\
\text { PARTICLES BY SIZE CLASS (MM) }\end{array}$} & \multicolumn{5}{|c|}{$\begin{array}{l}\text { NUMBER OF BLUE TRACER } \\
\text { PARTICLES BY SIZE CLASS (MM) }\end{array}$} \\
\hline & $\begin{array}{c}0.25 \\
\text { TO } \\
0.50\end{array}$ & $\begin{array}{l}0.50 \\
\text { TO } \\
1.00\end{array}$ & $\begin{array}{l}1.00 \\
\text { TO } \\
2.00\end{array}$ & $\begin{array}{l}2.00 \\
\text { TO } \\
4.00\end{array}$ & $\begin{array}{l}4.00 \\
\text { TO } \\
8.00\end{array}$ & $\begin{array}{l}0.25 \\
\text { TO } \\
0.50\end{array}$ & $\begin{array}{r}0.50 \\
\text { TO } \\
1.00\end{array}$ & $\begin{array}{l}1.00 \\
2.00\end{array}$ & $\begin{array}{r}2.00 \\
\mathrm{TO} \\
4.00\end{array}$ & $\begin{array}{r}4.00 \\
\text { TO } \\
8.00\end{array}$ \\
\hline $\begin{array}{l}5-20 \\
5-21 \\
5-23 \\
5-24 \\
5-25\end{array}$ & $\begin{array}{r}30 \\
76 \\
10 \\
8 \\
2\end{array}$ & $\begin{array}{r}45 \\
152 \\
76 \\
28 \\
13\end{array}$ & $\begin{array}{r}0 \\
57 \\
26 \\
21 \\
18\end{array}$ & $\begin{array}{l}0 \\
3 \\
7 \\
5 \\
5\end{array}$ & $\begin{array}{l}0 \\
0 \\
3 \\
3 \\
0\end{array}$ & $\begin{array}{r}72 \\
26 \\
1 \\
0 \\
0\end{array}$ & $\begin{array}{r}108 \\
148 \\
4 \\
1 \\
3\end{array}$ & $\begin{array}{r}21 \\
66 \\
16 \\
1 \\
0\end{array}$ & $\begin{array}{r}17 \\
18 \\
2 \\
0 \\
0\end{array}$ & $\begin{array}{l}4 \\
2 \\
0 \\
0 \\
0\end{array}$ \\
\hline $\begin{array}{l}5-26 \\
5-27 \\
5-28 \\
5-30 \\
5-31\end{array}$ & $\begin{array}{l}1 \\
0 \\
0 \\
1 \\
0\end{array}$ & $\begin{array}{l}6 \\
1 \\
2 \\
4 \\
1\end{array}$ & $\begin{array}{l}8 \\
3 \\
2 \\
0 \\
0\end{array}$ & $\begin{array}{l}0 \\
2 \\
1 \\
0 \\
0\end{array}$ & $\begin{array}{l}0 \\
0 \\
0 \\
0 \\
0\end{array}$ & $\begin{array}{l}1 \\
0 \\
0 \\
1 \\
0\end{array}$ & $\begin{array}{l}6 \\
1 \\
0 \\
1 \\
0\end{array}$ & $\begin{array}{l}1 \\
0 \\
0 \\
0 \\
0\end{array}$ & $\begin{array}{l}0 \\
0 \\
0 \\
0 \\
0\end{array}$ & $\begin{array}{l}0 \\
0 \\
0 \\
0 \\
0\end{array}$ \\
\hline $\begin{array}{l}6-01 \\
6-02 \\
6-03 \\
6-04 \\
6-05\end{array}$ & $\begin{array}{r}1 \\
0 \\
0 \\
1 \\
16\end{array}$ & $\begin{array}{r}3 \\
5 \\
6 \\
10 \\
50\end{array}$ & $\begin{array}{l}2 \\
0 \\
3 \\
5 \\
4\end{array}$ & $\begin{array}{l}1 \\
0 \\
1 \\
8 \\
3\end{array}$ & $\begin{array}{l}0 \\
0 \\
0 \\
3 \\
0\end{array}$ & $\begin{array}{l}0 \\
0 \\
0 \\
0 \\
0\end{array}$ & $\begin{array}{l}0 \\
0 \\
0 \\
0 \\
0\end{array}$ & $\begin{array}{l}0 \\
0 \\
0 \\
0 \\
0\end{array}$ & $\begin{array}{l}0 \\
0 \\
0 \\
0 \\
0\end{array}$ & $\begin{array}{l}0 \\
0 \\
0 \\
0 \\
0\end{array}$ \\
\hline $\begin{array}{l}6-06 \\
6-07 \\
6-08 \\
6-09 \\
6-10\end{array}$ & $\begin{array}{l}0 \\
0 \\
1 \\
7 \\
0\end{array}$ & $\begin{array}{r}15 \\
2 \\
8 \\
14 \\
3\end{array}$ & $\begin{array}{r}16 \\
3 \\
2 \\
3 \\
4\end{array}$ & $\begin{array}{l}3 \\
2 \\
0 \\
0 \\
1\end{array}$ & $\begin{array}{l}0 \\
0 \\
0 \\
0 \\
0\end{array}$ & $\begin{array}{l}0 \\
0 \\
0 \\
2 \\
1\end{array}$ & $\begin{array}{l}1 \\
2 \\
0 \\
2 \\
0\end{array}$ & $\begin{array}{l}0 \\
0 \\
0 \\
0 \\
0\end{array}$ & $\begin{array}{l}0 \\
0 \\
0 \\
0 \\
0\end{array}$ & $\begin{array}{l}0 \\
0 \\
0 \\
0 \\
0\end{array}$ \\
\hline $\begin{array}{l}6-11 \\
6-12 \\
6-13 \\
6-14 \\
6-15\end{array}$ & $\begin{array}{l}0 \\
3 \\
0 \\
2 \\
0\end{array}$ & $\begin{array}{l}5 \\
1 \\
0 \\
\frac{1}{3}\end{array}$ & $\begin{array}{l}1 \\
1 \\
0 \\
0 \\
1\end{array}$ & $\begin{array}{l}2 \\
1 \\
0 \\
0 \\
0\end{array}$ & $\begin{array}{l}0 \\
0 \\
0 \\
0 \\
0\end{array}$ & $\begin{array}{l}0 \\
2 \\
0 \\
0 \\
0\end{array}$ & $\begin{array}{l}0 \\
0 \\
0 \\
2 \\
0\end{array}$ & $\begin{array}{l}0 \\
0 \\
0 \\
0 \\
0\end{array}$ & $\begin{array}{l}0 \\
0 \\
0 \\
0 \\
0\end{array}$ & $\begin{array}{l}0 \\
0 \\
0 \\
0 \\
0\end{array}$ \\
\hline $\begin{array}{l}6-16 \\
6-17 \\
6-18 \\
6-19 \\
6-20\end{array}$ & $\begin{array}{l}0 \\
1 \\
0 \\
0 \\
0\end{array}$ & $\begin{array}{l}2 \\
0 \\
0 \\
2 \\
2\end{array}$ & $\begin{array}{l}1 \\
0 \\
2 \\
0 \\
2\end{array}$ & $\begin{array}{l}0 \\
0 \\
0 \\
0 \\
0\end{array}$ & $\begin{array}{l}0 \\
0 \\
0 \\
0 \\
0\end{array}$ & $\begin{array}{l}0 \\
0 \\
3 \\
1 \\
0\end{array}$ & $\begin{array}{l}0 \\
0 \\
0 \\
0 \\
1\end{array}$ & $\begin{array}{l}0 \\
0 \\
0 \\
0 \\
0\end{array}$ & $\begin{array}{l}0 \\
0 \\
0 \\
0 \\
0\end{array}$ & $\begin{array}{l}0 \\
0 \\
0 \\
0 \\
0\end{array}$ \\
\hline $\begin{array}{l}6-21 \\
6-22 \\
6-23 \\
6-24 \\
6-25\end{array}$ & $\begin{array}{l}0 \\
1 \\
0 \\
1 \\
1\end{array}$ & $\begin{array}{l}4 \\
1 \\
1 \\
1 \\
0\end{array}$ & $\begin{array}{l}3 \\
5 \\
0 \\
1 \\
1\end{array}$ & $\begin{array}{l}1 \\
0 \\
0 \\
3 \\
0\end{array}$ & $\begin{array}{l}0 \\
0 \\
0 \\
0 \\
1\end{array}$ & $\begin{array}{r}0 \\
0 \\
1 \\
0 \\
-\end{array}$ & $\begin{array}{r}0 \\
0 \\
0 \\
0 \\
--\end{array}$ & $\begin{array}{r}0 \\
0 \\
0 \\
0 \\
--\end{array}$ & $\begin{array}{r}0 \\
0 \\
0 \\
0 \\
- \\
-\end{array}$ & $\begin{array}{r}0 \\
0 \\
0 \\
0 \\
- \\
-\end{array}$ \\
\hline $\begin{array}{r}6-26 \\
6-27 \\
6-28 \\
7-11 \\
10-08\end{array}$ & $\begin{array}{l}0 \\
2 \\
5 \\
0 \\
0\end{array}$ & $\begin{array}{l}2 \\
5 \\
2 \\
3 \\
1\end{array}$ & $\begin{array}{l}0 \\
1 \\
4 \\
0 \\
0\end{array}$ & $\begin{array}{l}1 \\
0 \\
4 \\
0 \\
0\end{array}$ & $\begin{array}{l}0 \\
2 \\
0 \\
0 \\
0\end{array}$ & $\begin{array}{l}0 \\
0 \\
2 \\
0 \\
1\end{array}$ & $\begin{array}{l}0 \\
0 \\
0 \\
0 \\
1\end{array}$ & $\begin{array}{l}0 \\
0 \\
0 \\
0 \\
0\end{array}$ & $\begin{array}{l}0 \\
0 \\
0 \\
0 \\
0\end{array}$ & $\begin{array}{l}0 \\
0 \\
0 \\
0 \\
0\end{array}$ \\
\hline
\end{tabular}


TABLE 13.- NUMBER OF BLUE TRACER PARTICLES(1), PER 100 GRAMS OF BED MATERIAL, EAST FORK RIVER, WYOMING, 1979--CONTINUED

(1) BLUE TRACER PARTICLES PLACED AS BED MATERIAL 10 METERS DOWNSTREAM OF SECTION 1695 ON MAY $19,1979$.

(2) SMALL OVERFLOW CHANNEL ADJACENT TO SECTION.

(3) BYPASS CHANNEL. SEE FIGURE 3. 
TABLE 14.- NUMBER OF ORANGE TRACER PARTICLES(1), PER 100 GRAMS OF BED MATERIAL, EAST FORK RIVER, WYOMING, 1979

\begin{tabular}{|c|c|c|c|c|c|c|c|c|c|c|}
\hline \multirow[t]{2}{*}{ DATE } & \multicolumn{5}{|c|}{$\begin{array}{l}\text { NUMBER OF ORANGE TRACER } \\
\text { PARTICLES BY SIZE CLASS (MM) }\end{array}$} & \multicolumn{5}{|c|}{$\begin{array}{l}\text { NUMBER OF ORANGE TRACER } \\
\text { PARTICLES BY SIZE CLASS (MM) }\end{array}$} \\
\hline & $\begin{array}{r}0.25 \\
\text { T0 } \\
0.50\end{array}$ & $\begin{array}{l}0.50 \\
\text { To } \\
1.00\end{array}$ & $\begin{array}{l}1.00 \\
\text { To } \\
2.00\end{array}$ & $\begin{array}{l}2.00 \\
\text { TO } \\
4.00\end{array}$ & $\begin{array}{l}4.00 \\
\text { TO } \\
8.00\end{array}$ & $\begin{array}{r}0.25 \\
\text { TO } \\
0.50\end{array}$ & $\begin{array}{r}0.50 \\
\text { To } \\
1.00\end{array}$ & $\begin{array}{r}1.00 \\
2.00\end{array}$ & $\begin{array}{r}2.00 \\
\text { TO } \\
4.00\end{array}$ & $\begin{array}{r}4.00 \\
\text { TO } \\
8.00\end{array}$ \\
\hline $\begin{array}{l}5-20 \\
5-21 \\
5-23 \\
5-24 \\
5-25\end{array}$ & $-\frac{--}{2}$ & $\begin{array}{r}--\overline{0} \\
-\overline{0} \\
9\end{array}$ & $\begin{array}{r}--\overline{0} \\
-0 \\
0\end{array}$ & $\begin{array}{r}-- \\
-0 \\
0 \\
0\end{array}$ & $\begin{array}{r}-- \\
-0 \\
0 \\
0\end{array}$ & $\begin{array}{r}-- \\
-\overline{0} \\
30 \\
27\end{array}$ & $\begin{array}{r}-- \\
-0 \\
3 \\
10\end{array}$ & $\begin{array}{c}-- \\
-\overline{0} \\
0 \\
0\end{array}$ & $\begin{array}{c}-- \\
-0 \\
0 \\
0\end{array}$ & $\begin{array}{c}-- \\
-\overline{0} \\
0 \\
0\end{array}$ \\
\hline $\begin{array}{l}5-26 \\
5-27 \\
5-28 \\
5-30 \\
5-31\end{array}$ & $\begin{array}{r}-\frac{1}{4} \\
-1 \frac{1}{2}\end{array}$ & $\begin{array}{r}0 \\
-\frac{0}{2} \\
19 \\
12\end{array}$ & $\begin{array}{r}-1 \\
-\frac{1}{5} \\
6 \\
0\end{array}$ & $\begin{array}{c}-0 \\
\frac{1}{1} \\
0\end{array}$ & $\begin{array}{r}0 \\
-0 \\
0 \\
0\end{array}$ & $\begin{array}{r}20 \\
1 \\
20 \\
9 \\
1\end{array}$ & $\begin{array}{r}13 \\
4 \\
15 \\
39 \\
22\end{array}$ & $\begin{array}{l}1 \\
2 \\
2 \\
9 \\
3\end{array}$ & $\begin{array}{l}0 \\
0 \\
0 \\
1 \\
3\end{array}$ & $\begin{array}{l}0 \\
0 \\
0 \\
0 \\
0\end{array}$ \\
\hline $\begin{array}{l}6-01 \\
6-02 \\
6-03 \\
6-04 \\
6-05\end{array}$ & $\begin{array}{r}3 \\
13 \\
8 \\
8 \\
6\end{array}$ & $\begin{array}{r}11 \\
4 \\
5 \\
19 \\
22\end{array}$ & $\begin{array}{l}1 \\
3 \\
1 \\
7 \\
1\end{array}$ & $\begin{array}{l}0 \\
0 \\
0 \\
0 \\
0\end{array}$ & $\begin{array}{l}0 \\
0 \\
0 \\
0 \\
0\end{array}$ & $\begin{array}{r}8 \\
13 \\
40 \\
5 \\
1\end{array}$ & $\begin{array}{l}18 \\
27 \\
24 \\
17 \\
19\end{array}$ & $\begin{array}{l}1 \\
7 \\
3 \\
3 \\
8\end{array}$ & $\begin{array}{l}0 \\
2 \\
0 \\
1 \\
1\end{array}$ & $\begin{array}{l}0 \\
0 \\
0 \\
0 \\
0\end{array}$ \\
\hline $\begin{array}{l}6-06 \\
6-07 \\
6-08 \\
6-09 \\
6-10\end{array}$ & $\begin{array}{l}6 \\
0 \\
0 \\
3 \\
2\end{array}$ & $\begin{array}{r}11 \\
7 \\
17 \\
9 \\
15\end{array}$ & $\begin{array}{l}5 \\
2 \\
9 \\
4 \\
6\end{array}$ & $\begin{array}{l}1 \\
1 \\
4 \\
0 \\
0\end{array}$ & $\begin{array}{l}0 \\
0 \\
0 \\
0 \\
0\end{array}$ & $\begin{array}{l}1 \\
7 \\
3 \\
6 \\
7\end{array}$ & $\begin{array}{l}12 \\
25 \\
26 \\
11 \\
13\end{array}$ & $\begin{array}{l}6 \\
3 \\
1 \\
2 \\
1\end{array}$ & $\begin{array}{l}1 \\
1 \\
1 \\
1 \\
0\end{array}$ & $\begin{array}{l}0 \\
0 \\
0 \\
0 \\
0\end{array}$ \\
\hline $\begin{array}{l}6-11 \\
6-12 \\
6-13 \\
6-14 \\
6-15\end{array}$ & $\begin{array}{r}10 \\
5 \\
0 \\
3 \\
7\end{array}$ & $\begin{array}{r}20 \\
14 \\
3 \\
6 \\
15\end{array}$ & $\begin{array}{l}2 \\
5 \\
6 \\
4 \\
2\end{array}$ & $\begin{array}{l}3 \\
0 \\
2 \\
2 \\
0\end{array}$ & $\begin{array}{l}1 \\
0 \\
0 \\
0 \\
0\end{array}$ & $\begin{array}{l}1 \\
0 \\
1 \\
4 \\
1\end{array}$ & $\begin{array}{r}14 \\
10 \\
14 \\
4 \\
7\end{array}$ & $\begin{array}{l}2 \\
5 \\
2 \\
3 \\
2\end{array}$ & $\begin{array}{l}2 \\
3 \\
0 \\
0 \\
0\end{array}$ & $\begin{array}{l}0 \\
0 \\
0 \\
0 \\
0\end{array}$ \\
\hline $\begin{array}{l}6-16 \\
6-17 \\
6-18 \\
6-19 \\
6-20\end{array}$ & $\begin{array}{l}0 \\
1 \\
2 \\
2 \\
4\end{array}$ & $\begin{array}{r}5 \\
6 \\
9 \\
13 \\
17\end{array}$ & $\begin{array}{l}3 \\
6 \\
3 \\
1 \\
4\end{array}$ & $\begin{array}{l}2 \\
1 \\
1 \\
2 \\
0\end{array}$ & $\begin{array}{l}0 \\
0 \\
0 \\
0 \\
0\end{array}$ & $\begin{array}{l}4 \\
5 \\
5 \\
1 \\
0\end{array}$ & $\begin{array}{r}5 \\
14 \\
7 \\
3 \\
9\end{array}$ & $\begin{array}{l}2 \\
1 \\
0 \\
3 \\
1\end{array}$ & $\begin{array}{l}1 \\
0 \\
0 \\
2 \\
2\end{array}$ & $\begin{array}{l}0 \\
0 \\
0 \\
0 \\
0\end{array}$ \\
\hline $\begin{array}{l}6-21 \\
6-22 \\
6-23 \\
6-24 \\
6-25\end{array}$ & $\begin{array}{l}0 \\
2 \\
1 \\
0 \\
1\end{array}$ & $\begin{array}{r}18 \\
21 \\
14 \\
10 \\
8\end{array}$ & $\begin{array}{l}3 \\
2 \\
6 \\
7 \\
2\end{array}$ & $\begin{array}{l}\frac{1}{1} \\
\frac{1}{1} \\
1\end{array}$ & $\begin{array}{l}0 \\
0 \\
0 \\
0 \\
0\end{array}$ & $\begin{array}{l}0 \\
0 \\
1 \\
1 \\
0\end{array}$ & $\begin{array}{r}8 \\
9 \\
8 \\
11 \\
8\end{array}$ & $\begin{array}{l}2 \\
3 \\
8 \\
3 \\
0\end{array}$ & $\begin{array}{l}0 \\
3 \\
2 \\
3 \\
0\end{array}$ & $\begin{array}{l}0 \\
1 \\
0 \\
0 \\
0\end{array}$ \\
\hline $\begin{array}{r}6-26 \\
6-27 \\
6-28 \\
7-11 \\
10-09\end{array}$ & $\begin{array}{r}1 \\
4 \\
2 \\
25 \\
31\end{array}$ & $\begin{array}{r}9 \\
16 \\
11 \\
49 \\
110\end{array}$ & $\begin{array}{l}5 \\
7 \\
2 \\
2 \\
6\end{array}$ & $\begin{array}{l}2 \\
3 \\
0 \\
2 \\
2\end{array}$ & $\begin{array}{l}0 \\
0 \\
0 \\
0 \\
0\end{array}$ & $\begin{array}{r}1 \\
5 \\
2 \\
24 \\
12\end{array}$ & $\begin{array}{r}11 \\
11 \\
101 \\
85\end{array}$ & $\begin{array}{r}1 \\
6 \\
2 \\
9 \\
23\end{array}$ & $\begin{array}{l}4 \\
0 \\
0 \\
0 \\
2\end{array}$ & $\begin{array}{l}0 \\
0 \\
0 \\
0 \\
0\end{array}$ \\
\hline
\end{tabular}


TABLE 14.- NUMBER OF ORANGE TRACER PARTICLES(1) PER 100 GRAMS OF BED MATERIAL, EAST FORK RIVER, WYOMING, 1979--CONTINUED

SECTION 0137

SECTION 0220

\begin{tabular}{|c|c|c|c|c|c|c|c|c|c|c|}
\hline \multirow[t]{2}{*}{ DATE } & \multicolumn{5}{|c|}{$\begin{array}{l}\text { NUMBER OF ORANGE TRACER } \\
\text { PARTICLES BY SIZE CLASS (MM) }\end{array}$} & \multicolumn{5}{|c|}{$\begin{array}{l}\text { NUMBER OF ORANGE TRACER } \\
\text { PARTICLES BY SIZE CLASS (MM) }\end{array}$} \\
\hline & $\begin{array}{l}0.25 \\
\text { TO } \\
0.50\end{array}$ & $\begin{array}{l}0.50 \\
\text { TO } \\
1.00\end{array}$ & $\begin{array}{l}1.00 \\
2.00 \\
2.00\end{array}$ & $\begin{array}{l}2.00 \\
\text { TO } \\
4.00\end{array}$ & $\begin{array}{l}4.00 \\
\text { TO } \\
8.00\end{array}$ & $\begin{array}{r}0.25 \\
\text { TO } \\
0.50\end{array}$ & $\begin{array}{r}0.50 \\
\text { TO } \\
1.00\end{array}$ & $\begin{array}{r}1.00 \\
\text { TO } \\
2.00\end{array}$ & $\begin{array}{r}2.00 \\
\text { TO } \\
4.00\end{array}$ & $\begin{array}{r}4.00 \\
\text { TO } \\
8.00\end{array}$ \\
\hline $\begin{array}{l}5-20 \\
5-21 \\
5-23 \\
5-24 \\
5-25\end{array}$ & $\begin{array}{r}-- \\
2 \\
0 \\
0 \\
6\end{array}$ & $\begin{array}{c}-- \\
0 \\
0 \\
1 \\
6\end{array}$ & $\begin{array}{c}-\overline{0} \\
0 \\
0 \\
0\end{array}$ & $\begin{array}{c}-\overline{0} \\
0 \\
1 \\
0\end{array}$ & $\begin{array}{l}-0 \\
0 \\
0 \\
0\end{array}$ & $\begin{array}{r}6 \\
13 \\
0 \\
3 \\
4\end{array}$ & $\begin{array}{r}0 \\
0 \\
0 \\
7 \\
21\end{array}$ & $\begin{array}{l}0 \\
0 \\
0 \\
2 \\
3\end{array}$ & $\begin{array}{l}0 \\
0 \\
0 \\
1 \\
0\end{array}$ & $\begin{array}{l}0 \\
0 \\
0 \\
0 \\
0\end{array}$ \\
\hline $\begin{array}{l}5-26 \\
5-27 \\
5-28 \\
5-30 \\
5-31\end{array}$ & $\begin{array}{r}4 \\
13 \\
13 \\
7 \\
3\end{array}$ & $\begin{array}{r}8 \\
37 \\
45 \\
30 \\
26\end{array}$ & $\begin{array}{l}1 \\
4 \\
3 \\
4 \\
4\end{array}$ & $\begin{array}{l}0 \\
0 \\
0 \\
2 \\
0\end{array}$ & $\begin{array}{l}0 \\
0 \\
0 \\
0 \\
0\end{array}$ & $\begin{array}{r}7 \\
3 \\
8 \\
7 \\
14\end{array}$ & $\begin{array}{r}17 \\
27 \\
49 \\
19 \\
5\end{array}$ & $\begin{array}{l}4 \\
7 \\
5 \\
1 \\
1\end{array}$ & $\begin{array}{l}1 \\
1 \\
0 \\
0 \\
3\end{array}$ & $\begin{array}{l}0 \\
0 \\
0 \\
0 \\
0\end{array}$ \\
\hline $\begin{array}{l}6-01 \\
6-02 \\
6-03 \\
6-04 \\
6-05\end{array}$ & $\begin{array}{l}0 \\
1 \\
2 \\
1 \\
4\end{array}$ & $\begin{array}{r}11 \\
8 \\
3 \\
2 \\
5\end{array}$ & $\begin{array}{l}1 \\
5 \\
5 \\
0 \\
2\end{array}$ & $\begin{array}{l}1 \\
0 \\
2 \\
0 \\
0\end{array}$ & $\begin{array}{l}0 \\
0 \\
0 \\
0 \\
0\end{array}$ & $\begin{array}{r}1 \\
1 \\
10 \\
2 \\
2\end{array}$ & $\begin{array}{r}5 \\
14 \\
7 \\
6 \\
11\end{array}$ & $\begin{array}{l}4 \\
2 \\
1 \\
4 \\
4\end{array}$ & $\begin{array}{l}0 \\
1 \\
0 \\
0 \\
0\end{array}$ & $\begin{array}{l}0 \\
0 \\
0 \\
0 \\
0\end{array}$ \\
\hline $\begin{array}{l}6-06 \\
6-07 \\
6-08 \\
6-09 \\
6-10\end{array}$ & $\begin{array}{l}0 \\
2 \\
0 \\
0 \\
1\end{array}$ & $\begin{array}{r}2 \\
7 \\
2 \\
0 \\
11\end{array}$ & $\begin{array}{l}0 \\
3 \\
1 \\
0 \\
6\end{array}$ & $\begin{array}{l}0 \\
2 \\
1 \\
0 \\
4\end{array}$ & $\begin{array}{l}0 \\
0 \\
0 \\
0 \\
0\end{array}$ & $\begin{array}{l}0 \\
2 \\
0 \\
0 \\
0\end{array}$ & $\begin{array}{r}3 \\
10 \\
8 \\
2 \\
12\end{array}$ & $\begin{array}{l}5 \\
8 \\
1 \\
0 \\
6\end{array}$ & $\begin{array}{l}2 \\
2 \\
0 \\
0 \\
2\end{array}$ & $\begin{array}{l}1 \\
0 \\
0 \\
0 \\
3\end{array}$ \\
\hline $\begin{array}{l}6-11 \\
6-12 \\
6-13 \\
6-14 \\
6-15\end{array}$ & $\begin{array}{l}3 \\
5 \\
1 \\
4 \\
7\end{array}$ & $\begin{array}{l}11 \\
24 \\
7 \\
12 \\
11\end{array}$ & $\begin{array}{l}5 \\
8 \\
1 \\
3 \\
6\end{array}$ & $\begin{array}{l}3 \\
0 \\
0 \\
0 \\
1\end{array}$ & $\begin{array}{l}0 \\
0 \\
0 \\
0 \\
0\end{array}$ & $\begin{array}{l}0 \\
1 \\
0 \\
2 \\
2\end{array}$ & $\begin{array}{r}5 \\
8 \\
7 \\
9 \\
17\end{array}$ & $\begin{array}{l}2 \\
2 \\
2 \\
4 \\
3\end{array}$ & $\begin{array}{l}1 \\
2 \\
2 \\
0 \\
1\end{array}$ & $\begin{array}{l}0 \\
0 \\
0 \\
0 \\
0\end{array}$ \\
\hline $\begin{array}{l}6-16 \\
6-17 \\
6-18 \\
6-19 \\
6-20\end{array}$ & $\begin{array}{l}3 \\
1 \\
2 \\
1 \\
0\end{array}$ & $\begin{array}{r}4 \\
11 \\
8 \\
13 \\
13\end{array}$ & $\begin{array}{l}4 \\
3 \\
8 \\
4 \\
3\end{array}$ & $\begin{array}{l}2 \\
1 \\
4 \\
0 \\
0\end{array}$ & $\begin{array}{l}1 \\
0 \\
1 \\
0 \\
0\end{array}$ & $\begin{array}{l}3 \\
1 \\
1 \\
2 \\
1\end{array}$ & $\begin{array}{r}14 \\
9 \\
8 \\
7 \\
17\end{array}$ & $\begin{array}{l}6 \\
5 \\
1 \\
2 \\
4\end{array}$ & $\begin{array}{l}0 \\
1 \\
1 \\
0 \\
1\end{array}$ & $\begin{array}{l}0 \\
1 \\
0 \\
0 \\
0\end{array}$ \\
\hline $\begin{array}{l}6-21 \\
6-22 \\
6-23 \\
6-24 \\
6-25\end{array}$ & $\begin{array}{l}1 \\
0 \\
0 \\
2 \\
1\end{array}$ & $\begin{array}{r}13 \\
10 \\
5 \\
15 \\
3\end{array}$ & $\begin{array}{l}0 \\
6 \\
3 \\
5 \\
2\end{array}$ & $\begin{array}{l}1 \\
2 \\
1 \\
0 \\
0\end{array}$ & $\begin{array}{l}0 \\
0 \\
0 \\
1 \\
0\end{array}$ & $\begin{array}{l}0 \\
1 \\
0 \\
1 \\
3\end{array}$ & $\begin{array}{l}2 \\
6 \\
4 \\
9 \\
4\end{array}$ & $\begin{array}{l}\frac{1}{7} \\
\frac{1}{3} \\
7\end{array}$ & $\begin{array}{l}1 \\
3 \\
1 \\
0 \\
0\end{array}$ & $\begin{array}{l}0 \\
0 \\
0 \\
1 \\
0\end{array}$ \\
\hline $\begin{array}{r}6-26 \\
6-27 \\
6-28 \\
7-11 \\
10-09\end{array}$ & $\begin{array}{r}1 \\
5 \\
12 \\
6 \\
10\end{array}$ & $\begin{array}{r}9 \\
4 \\
8 \\
48 \\
74\end{array}$ & $\begin{array}{r}4 \\
2 \\
4 \\
23 \\
16\end{array}$ & $\begin{array}{l}2 \\
2 \\
1 \\
0 \\
4\end{array}$ & $\begin{array}{l}0 \\
1 \\
1 \\
0 \\
0\end{array}$ & $\begin{array}{r}4 \\
21 \\
7 \\
7 \\
8\end{array}$ & $\begin{array}{l}10 \\
46 \\
25 \\
19 \\
30\end{array}$ & $\begin{array}{r}4 \\
7 \\
6 \\
12 \\
7\end{array}$ & $\begin{array}{l}1 \\
1 \\
2 \\
7 \\
2\end{array}$ & $\begin{array}{l}0 \\
0 \\
0 \\
0 \\
1\end{array}$ \\
\hline
\end{tabular}


TABLE 14.- NUMBER OF ORANGE TRACER PARTICLES(1), PER 100 GRAMS OF BED MATERIAL, EAST FORK RIVER, WYOMING, 1979--CONTINUED

SECTION 0301

NUMBER OF ORANGE TRACER

DATE

PARTICLES BY SIZE CLASS (MM)

$0.250 .50 \quad 1.00 \quad 2.00 \quad 4.00$

$\begin{array}{lllll}\text { TO } & \text { TO } & \text { TO } & \text { TO } & \text { TO } \\ 0.50 & 1.00 & 2.00 & 4.00 & 8.00\end{array}$

$0.50 \quad 1.00 \quad 2.00 \quad 4.00 \quad 8.00$

$\begin{array}{rrrrrr}5-20 & 127 & 8 & 0 & 0 & 0 \\ 5-21 & 173 & 13 & 0 & 0 & 0 \\ 5-23 & 11 & 20 & 6 & 1 & 0 \\ 5-24 & 14 & 39 & 10 & 8 & 0 \\ 5-25 & 21 & 103 & 12 & 6 & 0\end{array}$

$5-26$
$5-27$
$5-28$

$5-30$

5-31

6-01

$6-02$

$6-03$

$6-04$
$6-05$

$6-06$

6-07

$6-08$

$6-09$
$6-10$

$6-11$

$6-1 \frac{1}{2}$

6-13

6-14

6-15

6-16

6-17

6-18

$6-19$

6-20

$6-21$

$6-22$

$6-24$

$6-25$

6-26

$6-27$
$6-28$

$7-11$
$10-09$
SECTION 0301(2)

NUMBER OF ORANGE TRACER

PARTICLES BY SIZE CLASS (MM)

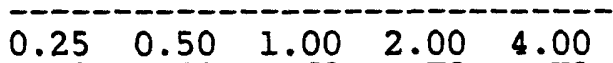

$\begin{array}{rrrrr}0.50 & 1.00 & 2.00 & 4.00 & 8.00\end{array}$

$\begin{array}{rrrll}5 & 2 & 1 & 0 & 0 \\ 10 & 1 & 0 & 0 & 0 \\ 148 & 112 & 0 & 0 & 0 \\ 6 & 5 & 1 & 0 & 0 \\ 17 & 35 & 5 & 0 & 0\end{array}$

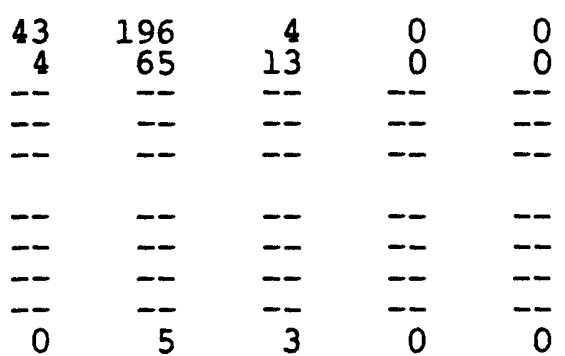

$\begin{array}{rrrrr}1 & 9 & 7 & 1 & 0 \\ 8 & 34 & 1 & 0 & 0 \\ -- & -- & -- & -- & -- \\ -- & -- & -- & -- & -- \\ -- & -- & -- & -- & -- \\ 2 & 13 & 2 & 1 & 0 \\ 2 & 13 & 3 & 1 & 0 \\ 2 & 13 & 8 & 0 & 0 \\ 6 & 21 & 4 & 0 & 0\end{array}$

$\begin{array}{rrrrr}3 & 25 & 3 & 0 & 0 \\ 2 & 15 & 6 & 1 & 0 \\ -- & -- & -- & -- & -- \\ -- & -- & -- & -- & --\end{array}$

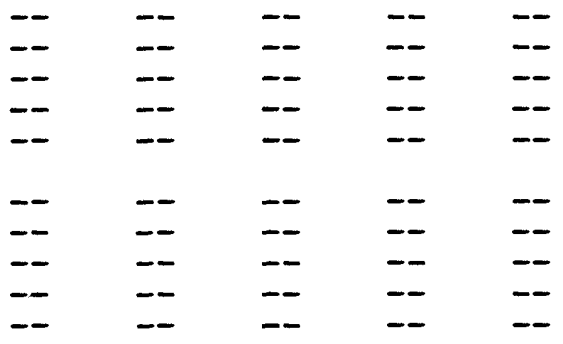


TABLE 14.- NUMBER OF ORANGE TRACER PARTICLES(1), PER 100 GRAMS OF BED MATERIAL, EAST FORK RIVER, WYOMING, 1979--CONTINUED

SECTION 0421

\begin{tabular}{|c|c|c|c|c|c|}
\hline \multirow[t]{2}{*}{ DATE } & \multicolumn{5}{|c|}{$\begin{array}{l}\text { NUMBER OF ORANGE TRACER } \\
\text { PARTICLES BY SIZE CLASS (MM) }\end{array}$} \\
\hline & $\begin{array}{l}0.25 \\
\text { TO } \\
0.50\end{array}$ & $\begin{array}{l}0.50 \\
\text { TO } \\
1.00\end{array}$ & $\begin{array}{l}1.00 \\
2.00\end{array}$ & $\begin{array}{l}2.00 \\
\text { TO } \\
4.00\end{array}$ & $\begin{array}{l}4.00 \\
\text { TO } \\
8.00\end{array}$ \\
\hline $\begin{array}{l}5-20 \\
5-21 \\
5-23 \\
5-24 \\
5-25\end{array}$ & $\begin{array}{r}179 \\
23 \\
23 \\
4 \\
1\end{array}$ & $\begin{array}{r}29 \\
46 \\
35 \\
0 \\
5\end{array}$ & $\begin{array}{r}1 \\
4 \\
13 \\
0 \\
4\end{array}$ & $\begin{array}{l}0 \\
0 \\
5 \\
2 \\
0\end{array}$ & $\begin{array}{l}0 \\
0 \\
0 \\
1 \\
0\end{array}$ \\
\hline $\begin{array}{l}5-26 \\
5-27 \\
5-28 \\
5-30 \\
5-31\end{array}$ & $\begin{array}{l}9 \\
0 \\
0 \\
0 \\
0\end{array}$ & $\begin{array}{l}1 \\
0 \\
0 \\
1 \\
2\end{array}$ & $\begin{array}{l}0 \\
0 \\
1 \\
0 \\
1\end{array}$ & $\begin{array}{l}0 \\
0 \\
0 \\
0 \\
0\end{array}$ & $\begin{array}{l}0 \\
0 \\
0 \\
0 \\
0\end{array}$ \\
\hline $\begin{array}{l}6-01 \\
6-02 \\
6-03 \\
6-04 \\
6-05\end{array}$ & $\begin{array}{r}0 \\
12 \\
0 \\
2 \\
2\end{array}$ & $\begin{array}{r}7 \\
105 \\
10 \\
14 \\
11\end{array}$ & $\begin{array}{r}4 \\
34 \\
6 \\
4 \\
10\end{array}$ & $\begin{array}{l}0 \\
5 \\
2 \\
6 \\
0\end{array}$ & $\begin{array}{l}0 \\
0 \\
1 \\
0 \\
0\end{array}$ \\
\hline $\begin{array}{l}6-06 \\
6-07 \\
6-08 \\
6-09 \\
6-10\end{array}$ & $\begin{array}{l}2 \\
1 \\
3 \\
0 \\
3\end{array}$ & $\begin{array}{r}10 \\
1 \\
4 \\
2 \\
42\end{array}$ & $\begin{array}{l}8 \\
2 \\
.4 \\
0 \\
8\end{array}$ & $\begin{array}{l}3 \\
0 \\
0 \\
0 \\
3\end{array}$ & $\begin{array}{l}1 \\
0 \\
0 \\
0 \\
0\end{array}$ \\
\hline $\begin{array}{l}6-11 \\
6-12 \\
6-13 \\
6-14 \\
6-15\end{array}$ & $\begin{array}{r}21 \\
12 \\
4 \\
2 \\
0\end{array}$ & $\begin{array}{r}87 \\
29 \\
31 \\
24 \\
8\end{array}$ & $\begin{array}{r}6 \\
8 \\
10 \\
20 \\
2\end{array}$ & $\begin{array}{l}0 \\
2 \\
3 \\
6 \\
0\end{array}$ & $\begin{array}{l}0 \\
0 \\
1 \\
0 \\
0\end{array}$ \\
\hline $\begin{array}{l}6-16 \\
6-17 \\
6-18 \\
6-19 \\
6-20\end{array}$ & $\begin{array}{l}1 \\
2 \\
2 \\
3 \\
3\end{array}$ & $\begin{array}{r}5 \\
8 \\
35 \\
88 \\
38\end{array}$ & $\begin{array}{r}2 \\
2 \\
7 \\
14 \\
21\end{array}$ & $\begin{array}{l}0 \\
4 \\
2 \\
6 \\
7\end{array}$ & $\begin{array}{l}1 \\
0 \\
0 \\
1 \\
1\end{array}$ \\
\hline $\begin{array}{l}6-21 \\
6-22 \\
6-23 \\
6-24 \\
6-25\end{array}$ & $\begin{array}{l}8 \\
3 \\
1 \\
1 \\
2\end{array}$ & $\begin{array}{r}183 \\
76 \\
45 \\
11 \\
48\end{array}$ & $\begin{array}{r}69 \\
33 \\
31 \\
5 \\
40\end{array}$ & $\begin{array}{r}5 \\
8 \\
2 \\
2 \\
13\end{array}$ & $\begin{array}{l}0 \\
1 \\
0 \\
0 \\
2\end{array}$ \\
\hline $\begin{array}{r}6-26 \\
6-27 \\
6-28 \\
7-11 \\
10-09\end{array}$ & $\begin{array}{r}0 \\
5 \\
60 \\
13 \\
42\end{array}$ & $\begin{array}{r}28 \\
8 \\
71 \\
42 \\
81\end{array}$ & $\begin{array}{r}13 \\
2 \\
4 \\
10 \\
22\end{array}$ & $\begin{array}{r}2 \\
1 \\
2 \\
1 \\
16\end{array}$ & $\begin{array}{l}0 \\
0 \\
0 \\
1 \\
2\end{array}$ \\
\hline
\end{tabular}


TABLE 14.- NUMBER OF ORANGE TRACER PARTICLES(1), PER 100 GRAMS OF BED MATERIAL, EAST FORK RIVER, WYOMING, 1979--CONTINUED

(1) ORANGE TRACER PARTICLES PLACED AS BED MATERIAL 10 METERS DOWNSTREAM

(2) SMALL OVERFLOW CHANNEL ADJACENT TO SECTION. 
TABLE 15.- NUMBER OF PINK TRACER PARTICLES(1), PER 100 GRAMS OF BEDLOAD SAMPLE, COMPOSITED FROM CROSS-CHANNEL SAMPLING POSITIONS SPACED AT I-METER INTERVALS ACROSS THE CHANNEL WIDTH, EAST FORK RIVER, WYOMING, 1980

SECTION 0043

\begin{tabular}{|c|c|c|c|c|c|c|c|}
\hline \multirow{3}{*}{ DATE } & \multirow{3}{*}{$\begin{array}{c}\text { DRY } \\
\text { MASS } \\
\text { OF } \\
\text { SAMPLE (2) } \\
\text { (G) }\end{array}$} & \multicolumn{6}{|c|}{ NUMBER OF PINK TRACER PARTICLES } \\
\hline & & \multirow[b]{2}{*}{ TOTAL } & \multicolumn{5}{|c|}{ BY SIZE CLASS (MM) } \\
\hline & & & $\begin{array}{l}0.25 \\
\text { TO } \\
0.50\end{array}$ & $\begin{array}{l}0.50 \\
\text { TO } \\
1.00\end{array}$ & $\begin{array}{l}1.00 \\
2.00\end{array}$ & $\begin{array}{l}2.00 \\
\text { TO } \\
4.00\end{array}$ & $\begin{array}{l}4.00 \\
\text { TO } \\
8.00\end{array}$ \\
\hline $\begin{array}{l}5-16 \\
5-19 \\
5-21 \\
5-22\end{array}$ & $\begin{array}{l}59++ \\
75++ \\
54+ \\
--\end{array}$ & $\begin{array}{r}0 \\
0 \\
0 \\
--\end{array}$ & $\begin{array}{r}0 \\
0 \\
0 \\
--\end{array}$ & $\begin{array}{r}0 \\
0 \\
0 \\
--\end{array}$ & $\begin{array}{r}0 \\
0 \\
0\end{array}$ & $\begin{array}{r}0 \\
0 \\
0\end{array}$ & $\begin{array}{r}0 \\
0 \\
0 \\
-0\end{array}$ \\
\hline $5-24$ & - & - & -- & - & - & $\overline{-}$ & -- \\
\hline $\begin{array}{l}5-26 \\
5-27 \\
5-28 \\
5-29\end{array}$ & $\begin{array}{c}371 \\
84+ \\
28++\end{array}$ & $\begin{array}{r}0 \\
0 \\
0 \\
-0\end{array}$ & $\begin{array}{r}0 \\
0 \\
0\end{array}$ & $\begin{array}{r}0 \\
0 \\
0 \\
-\end{array}$ & $\begin{array}{l}0 \\
0 \\
0\end{array}$ & $\begin{array}{l}0 \\
0 \\
0\end{array}$ & $\begin{array}{l}0 \\
0 \\
0\end{array}$ \\
\hline $\begin{array}{l}5-29 \\
5-30\end{array}$ & $5+++$ & $\overline{0}$ & $\overline{0}$ & $\overline{0}$ & $\overline{0}$ & $\overline{0}$ & $\overline{0}$ \\
\hline $\begin{array}{l}6-01 \\
6-03 \\
6-05 \\
6-07 \\
6-09\end{array}$ & $\begin{array}{c}81++ \\
0+++ \\
0+++ \\
111^{+++} \\
303^{++}\end{array}$ & $\begin{array}{l}0 \\
0 \\
0 \\
0 \\
0\end{array}$ & $\begin{array}{l}0 \\
0 \\
0 \\
0 \\
0\end{array}$ & $\begin{array}{l}0 \\
0 \\
0 \\
0 \\
0\end{array}$ & $\begin{array}{l}0 \\
0 \\
0 \\
0 \\
0\end{array}$ & $\begin{array}{l}0 \\
0 \\
0 \\
0 \\
0\end{array}$ & $\begin{array}{l}0 \\
0 \\
0 \\
0 \\
0\end{array}$ \\
\hline $\begin{array}{l}6-10 \\
6-11 \\
6-12 \\
6-13 \\
6-14\end{array}$ & $\begin{array}{l}3071 \\
3889 \\
2659 \\
1640 \\
1030\end{array}$ & $\begin{array}{l}2 \\
0 \\
0 \\
0 \\
0\end{array}$ & $\begin{array}{l}2 \\
0 \\
0 \\
0 \\
0\end{array}$ & $\begin{array}{l}0 \\
0 \\
0 \\
0 \\
0\end{array}$ & $\begin{array}{l}0 \\
0 \\
0 \\
0 \\
0\end{array}$ & $\begin{array}{l}0 \\
0 \\
0 \\
0 \\
0\end{array}$ & $\begin{array}{l}0 \\
0 \\
0 \\
0 \\
0\end{array}$ \\
\hline $6-15$ & 984 & 0 & 0 & 0 & 0 & 0 & 0 \\
\hline $\begin{array}{l}6-17 \\
6-18 \\
6-19\end{array}$ & $\begin{array}{r}383 \\
320 \\
1123\end{array}$ & $\begin{array}{l}0 \\
0 \\
0\end{array}$ & $\begin{array}{l}0 \\
0 \\
0\end{array}$ & $\begin{array}{l}0 \\
0 \\
0\end{array}$ & $\begin{array}{l}0 \\
0 \\
0\end{array}$ & $\begin{array}{l}0 \\
0 \\
0\end{array}$ & $\begin{array}{l}0 \\
0 \\
0\end{array}$ \\
\hline $\begin{array}{l}6-20 \\
6-21\end{array}$ & $\begin{array}{l}271 \\
764\end{array}$ & $\begin{array}{l}0 \\
0\end{array}$ & $\begin{array}{l}0 \\
0\end{array}$ & $\begin{array}{l}0 \\
0\end{array}$ & $\begin{array}{l}0 \\
0\end{array}$ & $\begin{array}{l}0 \\
0\end{array}$ & $\begin{array}{l}0 \\
0\end{array}$ \\
\hline $\begin{array}{l}6-23 \\
6-25\end{array}$ & $\begin{array}{r}585 \\
1008\end{array}$ & $\begin{array}{l}0 \\
0\end{array}$ & $\begin{array}{l}0 \\
0\end{array}$ & $\begin{array}{l}0 \\
0\end{array}$ & $\begin{array}{l}0 \\
0\end{array}$ & 0 & 0 \\
\hline $6-27$ & 486 & 1 & 1 & 0 & 0 & 0 & 0 \\
\hline $\begin{array}{l}0-29 \\
7-01\end{array}$ & $\overline{-}$ & $\overline{-}$ & $\overline{--}$ & $\overline{--}$ & $\overline{--}$ & $\overline{-}$ & $\overline{--}$ \\
\hline $7-03$ & - & -- & - & -- & -- & -- & -- \\
\hline $7-05$ & -- & -- & - & - & -- & -- & -- \\
\hline $7-07$ & -- & -- & -- & -- & -- & -- & -- \\
\hline
\end{tabular}


TABLE 15.- NUMBER OF PINK TRACER PARTICLES(1), PER 100 GRAMS OF BEDLOAD SAMPLE, COMPOSITED FROM CROSS-CHANNEL SAMPLING POSITIONS SPACED AT 1-METER INTERVALS ACROSS THE CHANNEL WIDTH, EAST FORK RIVER, WYOMING, 1980

SECTION 0075

\begin{tabular}{|c|c|c|c|c|c|c|c|}
\hline \multirow{3}{*}{ DATE } & \multirow{3}{*}{$\begin{array}{c}\text { DRY } \\
\text { MASS } \\
\text { OF } \\
\text { SAMPLE(2) } \\
\text { (G) }\end{array}$} & \multicolumn{6}{|c|}{ NUMBER OF PINK TRACER PARTICLES } \\
\hline & & \multirow[b]{2}{*}{ TOTAL } & \multicolumn{5}{|c|}{ BY SIZE CLASS (MM) } \\
\hline & & & $\begin{array}{l}0.25 \\
\text { TO } \\
0.50\end{array}$ & $\begin{array}{l}0.50 \\
\text { To } \\
1.00\end{array}$ & $\begin{array}{l}1.00 \\
2.00\end{array}$ & $\begin{array}{l}2.00 \\
\mathrm{TO} \\
4.00\end{array}$ & $\begin{array}{l}4.00 \\
8.00 \\
8.00\end{array}$ \\
\hline $\begin{array}{l}5-16 \\
5-19 \\
5-21 \\
5-22 \\
5-24\end{array}$ & $\begin{array}{r}161+ \\
346 \\
1581 \\
=-\end{array}$ & $\begin{array}{r}0 \\
0 \\
0 \\
-- \\
--\end{array}$ & $\begin{array}{r}0 \\
0 \\
0 \\
-- \\
--\end{array}$ & $\begin{array}{r}0 \\
0 \\
0 \\
--\end{array}$ & $\begin{array}{r}0 \\
0 \\
0 \\
-- \\
--\end{array}$ & $\begin{array}{r}0 \\
0 \\
0 \\
--\end{array}$ & $\begin{array}{r}0 \\
0 \\
0 \\
--\end{array}$ \\
\hline $\begin{array}{l}5-26 \\
5-27 \\
5-28 \\
5-29 \\
5-30\end{array}$ & $\begin{array}{l}51+ \\
127+ \\
127 \\
237 \\
19+++\end{array}$ & $\begin{array}{l}4 \\
0 \\
0 \\
0 \\
0\end{array}$ & $\begin{array}{l}2 \\
0 \\
0 \\
0 \\
0\end{array}$ & $\begin{array}{l}2 \\
0 \\
0 \\
0 \\
0\end{array}$ & $\begin{array}{l}0 \\
0 \\
0 \\
0 \\
0\end{array}$ & $\begin{array}{l}0 \\
0 \\
0 \\
0 \\
0\end{array}$ & $\begin{array}{l}0 \\
0 \\
0 \\
0 \\
0\end{array}$ \\
\hline $\begin{array}{l}6-01 \\
6-03 \\
6-05 \\
6-07 \\
6-09\end{array}$ & $\begin{array}{r}1907 \\
900 \\
283 \\
742 \\
1650\end{array}$ & $\begin{array}{l}0 \\
0 \\
0 \\
0 \\
0\end{array}$ & $\begin{array}{l}0 \\
0 \\
0 \\
0 \\
0\end{array}$ & $\begin{array}{l}0 \\
0 \\
0 \\
0 \\
0\end{array}$ & $\begin{array}{l}0 \\
0 \\
0 \\
0 \\
0\end{array}$ & $\begin{array}{l}0 \\
0 \\
0 \\
0 \\
0\end{array}$ & $\begin{array}{l}0 \\
0 \\
0 \\
0 \\
0\end{array}$ \\
\hline $\begin{array}{l}6-10 \\
6-11 \\
6-12 \\
6-13 \\
6-14\end{array}$ & $\begin{array}{l}3128 \\
1416 \\
2786 \\
1735 \\
1897\end{array}$ & $\begin{array}{r}2 \\
11 \\
0 \\
0 \\
0\end{array}$ & $\begin{array}{l}\frac{1}{2} \\
0 \\
0 \\
0\end{array}$ & $\begin{array}{l}1 \\
9 \\
0 \\
0 \\
0\end{array}$ & $\begin{array}{l}0 \\
0 \\
0 \\
0 \\
0\end{array}$ & $\begin{array}{l}0 \\
0 \\
0 \\
0 \\
0\end{array}$ & $\begin{array}{l}0 \\
0 \\
0 \\
0 \\
0\end{array}$ \\
\hline $6-15$ & 651 & 0 & 0 & 0 & 0 & 0 & 0 \\
\hline $\begin{array}{l}6-17 \\
6-18 \\
6-19\end{array}$ & $\begin{array}{r}900 \\
1969 \\
2818\end{array}$ & $\begin{array}{l}0 \\
0 \\
0\end{array}$ & $\begin{array}{l}0 \\
0 \\
0\end{array}$ & $\begin{array}{l}0 \\
0 \\
0\end{array}$ & $\begin{array}{l}0 \\
0 \\
0\end{array}$ & $\begin{array}{l}0 \\
0 \\
0\end{array}$ & $\begin{array}{l}0 \\
0 \\
0\end{array}$ \\
\hline $\begin{array}{l}6-20 \\
6-21\end{array}$ & $\begin{array}{l}5583 \\
4777\end{array}$ & $\begin{array}{l}0 \\
0\end{array}$ & $\begin{array}{l}0 \\
0\end{array}$ & $\begin{array}{l}0 \\
0\end{array}$ & $\begin{array}{l}0 \\
0\end{array}$ & $\begin{array}{l}0 \\
0\end{array}$ & $\begin{array}{l}0 \\
0\end{array}$ \\
\hline $\begin{array}{l}6-23 \\
6-25\end{array}$ & $\begin{array}{l}1409 \\
1558\end{array}$ & $\begin{array}{l}3 \\
0\end{array}$ & $\begin{array}{l}3 \\
0\end{array}$ & $\begin{array}{l}0 \\
0\end{array}$ & $\begin{array}{l}0 \\
0\end{array}$ & $\begin{array}{l}0 \\
0\end{array}$ & $\begin{array}{l}0 \\
0\end{array}$ \\
\hline $\begin{array}{l}6-27 \\
6-29 \\
7-01 \\
7-03 \\
7-05\end{array}$ & $\begin{array}{r}2302 \\
=- \\
=- \\
=-\end{array}$ & $\begin{array}{l}1 \\
=- \\
=-\end{array}$ & $\begin{array}{l}0 \\
=- \\
=-\end{array}$ & $\begin{array}{l}1 \\
-- \\
--\end{array}$ & $\begin{array}{l}0 \\
-- \\
=- \\
--\end{array}$ & $\begin{array}{l}0 \\
=- \\
=-\end{array}$ & $\begin{array}{l}0 \\
-- \\
-- \\
--\end{array}$ \\
\hline $7-07$ & -- & -- & -- & -- & -- & -- & -- \\
\hline
\end{tabular}


TABLE 15.- NUMBER OF PINK TRACER PARTICLES(1), PER 100 GRAMS OF BEDLOAD SAMPLE, COMPOSITED FROM CROSS-CHANNEL SAMPLING POSITIONS SPACED AT 1-METER INTERVALS ACROSS THE CHANNEL WIDTH, EAST FORK RIVER, WYOMING, 1980

SECTION 0137

\begin{tabular}{|c|c|c|c|c|c|c|c|}
\hline \multirow{3}{*}{\multicolumn{2}{|c|}{$\begin{array}{lc}\text { DATE } & \text { DRY } \\
& \text { MASS } \\
\text { OF } & \text { SAMPLE (2) } \\
& \text { (G) }\end{array}$}} & \multicolumn{6}{|c|}{ NUMBER OF PINK TRACER PARTICLES } \\
\hline & & \multirow[b]{2}{*}{ TOTAL } & \multicolumn{5}{|c|}{ BY SIZE CLASS (MM) } \\
\hline & & & $\begin{array}{r}0.25 \\
\text { To } \\
0.50\end{array}$ & $\begin{array}{l}0.50 \\
\text { TO } \\
1.00\end{array}$ & $\begin{array}{l}1.00 \\
2.00 \\
\text { TO }\end{array}$ & $\begin{array}{l}2.00 \\
\text { TO } \\
4.00\end{array}$ & $\begin{array}{r}4.00 \\
\text { TO } \\
8.00\end{array}$ \\
\hline $\begin{array}{l}5-16 \\
5-19 \\
5-21 \\
5-22 \\
5-24\end{array}$ & $\begin{array}{c}225 \\
56+++ \\
113++ \\
=- \\
--\end{array}$ & $\begin{array}{r}0 \\
0 \\
0 \\
--\end{array}$ & $\begin{array}{r}0 \\
0 \\
0 \\
-- \\
--\end{array}$ & $\begin{array}{r}0 \\
0 \\
0 \\
--\end{array}$ & $\begin{array}{r}0 \\
0 \\
0 \\
-- \\
--\end{array}$ & $\begin{array}{r}0 \\
0 \\
0 \\
-- \\
--\end{array}$ & $\begin{array}{r}0 \\
0 \\
0 \\
-- \\
--\end{array}$ \\
\hline $\begin{array}{l}5-26 \\
5-27 \\
5-28 \\
5-20\end{array}$ & $\begin{array}{r}1360 \\
1794 \\
135\end{array}$ & $\begin{array}{l}0 \\
0 \\
0\end{array}$ & $\begin{array}{l}0 \\
0 \\
0 \\
-0\end{array}$ & $\begin{array}{l}0 \\
0 \\
0 \\
-0\end{array}$ & $\begin{array}{l}0 \\
0 \\
0 \\
-\end{array}$ & $\begin{array}{l}0 \\
0 \\
0\end{array}$ & $\begin{array}{l}0 \\
0 \\
0\end{array}$ \\
\hline $5-30$ & $99+$ & 0 & 0 & 0 & 0 & 0 & 0 \\
\hline $\begin{array}{l}6-01 \\
6-03 \\
6-05 \\
6-07 \\
6-09\end{array}$ & $\begin{array}{c}41++ \\
73++ \\
0+++ \\
588 \\
1099\end{array}$ & $\begin{array}{l}0 \\
0 \\
0 \\
0 \\
1\end{array}$ & $\begin{array}{l}0 \\
0 \\
0 \\
0 \\
0\end{array}$ & $\begin{array}{l}0 \\
0 \\
0 \\
0 \\
1\end{array}$ & $\begin{array}{l}0 \\
0 \\
0 \\
0 \\
0\end{array}$ & $\begin{array}{l}0 \\
0 \\
0 \\
0 \\
0\end{array}$ & $\begin{array}{l}0 \\
0 \\
0 \\
0 \\
0\end{array}$ \\
\hline $\begin{array}{l}6-10 \\
6-11 \\
6-12 \\
6-13 \\
6-14\end{array}$ & $\begin{array}{r}1907 \\
1139 \\
517 \\
1648 \\
3009\end{array}$ & $\begin{array}{l}0 \\
0 \\
0 \\
0 \\
0\end{array}$ & $\begin{array}{l}0 \\
0 \\
0 \\
0 \\
0\end{array}$ & $\begin{array}{l}0 \\
0 \\
0 \\
0 \\
0\end{array}$ & $\begin{array}{l}0 \\
0 \\
0 \\
0 \\
0\end{array}$ & $\begin{array}{l}0 \\
0 \\
0 \\
0 \\
0\end{array}$ & $\begin{array}{l}0 \\
0 \\
0 \\
0 \\
0\end{array}$ \\
\hline $6-15$ & 2497 & 0 & 0 & 0 & 0 & 0 & 0 \\
\hline $\begin{array}{l}6-17 \\
6-18 \\
6-19\end{array}$ & $\begin{array}{l}3116 \\
3204 \\
2346\end{array}$ & $\begin{array}{l}0 \\
0 \\
0\end{array}$ & $\begin{array}{l}0 \\
0 \\
0\end{array}$ & $\begin{array}{l}0 \\
0 \\
0\end{array}$ & $\begin{array}{l}0 \\
0 \\
0\end{array}$ & $\begin{array}{l}0 \\
0 \\
0\end{array}$ & $\begin{array}{l}0 \\
0 \\
0\end{array}$ \\
\hline $\begin{array}{l}6-20 \\
6-21\end{array}$ & $\begin{array}{l}2042 \\
3752\end{array}$ & $\begin{array}{l}0 \\
0\end{array}$ & $\begin{array}{l}0 \\
0\end{array}$ & $\begin{array}{l}0 \\
0\end{array}$ & $\begin{array}{l}0 \\
0\end{array}$ & $\begin{array}{l}0 \\
0\end{array}$ & $\begin{array}{l}0 \\
0\end{array}$ \\
\hline $\begin{array}{l}6-23 \\
6-25\end{array}$ & $\begin{array}{l}2398 \\
3614\end{array}$ & $\begin{array}{l}0 \\
1\end{array}$ & $\begin{array}{l}0 \\
1\end{array}$ & $\begin{array}{l}0 \\
0\end{array}$ & $\begin{array}{l}0 \\
0\end{array}$ & $\begin{array}{l}0 \\
0\end{array}$ & $\begin{array}{l}0 \\
0\end{array}$ \\
\hline $6-27$ & 4050 & 1 & $\underline{0}$ & 1 & 0 & 0 & 0 \\
\hline $\begin{array}{l}0-29 \\
7-01\end{array}$ & -- & - & $\overline{-}$ & $=$ & -- & 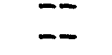 & $\overline{-}$ \\
\hline $\begin{array}{l}7-03 \\
7-05\end{array}$ & $\overline{-}$ & $=$ & -- & -- & -- & $\overline{-}$ & $=$ \\
\hline $7-07$ & -- & -- & -- & -- & -- & -- & -- \\
\hline
\end{tabular}


TABLE 15.- NUMBER OF PINK TRACER PARTICLES(1), PER 100 GRAMS OF BEDLOAD SAMPLE, COMPOSITED FROM CROSS-CHANNEL SAMPLING POSITIONS SPACED AT 1-METER INTERVALS ACROSS THE CHANNEL WIDTH, EAST FORK RIVER, WYOMING, 1980

SECTION 0178

\begin{tabular}{|c|c|c|c|c|c|c|c|}
\hline \multirow{3}{*}{\multicolumn{2}{|c|}{$\begin{array}{cc} & \\
\text { DATE } & \text { DRY } \\
& \text { MASS } \\
\text { OF } & \\
& \text { SAMPLE (2) } \\
& \text { (G) }\end{array}$}} & \multicolumn{6}{|c|}{ NUMBER OF PINK TRACER PARTICLES } \\
\hline & & \multirow[b]{2}{*}{ TOTAL } & \multicolumn{5}{|c|}{ BY SIZE CLASS (MM) } \\
\hline & & & $\begin{array}{l}0.25 \\
\text { To } \\
0.50\end{array}$ & $\begin{array}{r}0.50 \\
\text { TO } \\
1.00\end{array}$ & $\begin{array}{l}1.00 \\
\text { TO } \\
2.00\end{array}$ & $\begin{array}{l}2.00 \\
\text { TO } \\
4.00\end{array}$ & $\begin{array}{l}4.00 \\
\text { TO } \\
8.00\end{array}$ \\
\hline $\begin{array}{l}5-16 \\
5-19 \\
5-21 \\
5-22 \\
5-24\end{array}$ & $\begin{array}{r}335 \\
1172 \\
430 \\
--\end{array}$ & $\begin{array}{r}0 \\
0 \\
0 \\
-- \\
--\end{array}$ & $\begin{array}{r}0 \\
0 \\
0 \\
-- \\
--\end{array}$ & $\begin{array}{r}0 \\
0 \\
0 \\
-- \\
--\end{array}$ & $\begin{array}{r}0 \\
0 \\
0 \\
-- \\
--\end{array}$ & $\begin{array}{r}0 \\
0 \\
0 \\
--\end{array}$ & $\begin{array}{r}0 \\
0 \\
0 \\
-- \\
--\end{array}$ \\
\hline $\begin{array}{l}5-26 \\
5-27 \\
5-28\end{array}$ & $\begin{array}{r}2416 \\
677 \\
446\end{array}$ & $\begin{array}{l}0 \\
0 \\
3\end{array}$ & $\begin{array}{l}0 \\
0 \\
3\end{array}$ & $\begin{array}{l}0 \\
0 \\
0\end{array}$ & $\begin{array}{l}0 \\
0 \\
0\end{array}$ & $\begin{array}{l}0 \\
0 \\
0\end{array}$ & $\begin{array}{l}0 \\
0 \\
0\end{array}$ \\
\hline $5-30$ & 470 & 0 & 0 & 0 & 0 & 0 & 0 \\
\hline $\begin{array}{l}6-01 \\
6-03 \\
6-05 \\
6-07 \\
6-09\end{array}$ & $\begin{array}{c}521 \\
18++ \\
689 \\
1624 \\
2378\end{array}$ & $\begin{array}{l}0 \\
0 \\
0 \\
0 \\
0\end{array}$ & $\begin{array}{l}0 \\
0 \\
0 \\
0 \\
0\end{array}$ & $\begin{array}{l}0 \\
0 \\
0 \\
0 \\
0\end{array}$ & $\begin{array}{l}0 \\
0 \\
0 \\
0 \\
0\end{array}$ & $\begin{array}{l}0 \\
0 \\
0 \\
0 \\
0\end{array}$ & $\begin{array}{l}0 \\
0 \\
0 \\
0 \\
0\end{array}$ \\
\hline $\begin{array}{l}6-10 \\
6-11 \\
6-12 \\
6-13 \\
6-14\end{array}$ & $\begin{array}{l}6609 \\
4737 \\
1694 \\
5416 \\
3955\end{array}$ & $\begin{array}{l}0 \\
0 \\
0 \\
0 \\
1\end{array}$ & $\begin{array}{l}0 \\
0 \\
0 \\
0 \\
1\end{array}$ & $\begin{array}{l}0 \\
0 \\
0 \\
0 \\
0\end{array}$ & $\begin{array}{l}0 \\
0 \\
0 \\
0 \\
0\end{array}$ & $\begin{array}{l}0 \\
0 \\
0 \\
0 \\
0\end{array}$ & $\begin{array}{l}0 \\
0 \\
0 \\
0 \\
0\end{array}$ \\
\hline $6-15$ & 3251 & 1 & 0 & 1 & 0 & 0 & 0 \\
\hline $\begin{array}{l}6-17 \\
6-18 \\
6-19\end{array}$ & $\begin{array}{l}1273 \\
3439 \\
3696\end{array}$ & $\begin{array}{l}0 \\
0 \\
0\end{array}$ & $\begin{array}{l}0 \\
0 \\
0\end{array}$ & $\begin{array}{l}0 \\
0 \\
0\end{array}$ & $\begin{array}{l}0 \\
0 \\
0\end{array}$ & $\begin{array}{l}0 \\
0 \\
0\end{array}$ & $\begin{array}{l}0 \\
0 \\
0\end{array}$ \\
\hline $\begin{array}{l}6-20 \\
6-21\end{array}$ & $\begin{array}{l}5908 \\
5074\end{array}$ & $\begin{array}{l}0 \\
0\end{array}$ & $\begin{array}{l}0 \\
0\end{array}$ & $\begin{array}{l}0 \\
0\end{array}$ & $\begin{array}{l}0 \\
0\end{array}$ & $\begin{array}{l}0 \\
0\end{array}$ & $\begin{array}{l}0 \\
0\end{array}$ \\
\hline $\begin{array}{l}6-23 \\
6-25\end{array}$ & $\begin{array}{l}4771 \\
1510\end{array}$ & $\begin{array}{r}0 \\
2 I\end{array}$ & $\begin{array}{l}0 \\
8\end{array}$ & $\begin{array}{l}0 \\
9\end{array}$ & $\begin{array}{l}0 \\
4\end{array}$ & $\begin{array}{l}0 \\
0\end{array}$ & $\begin{array}{l}0 \\
0\end{array}$ \\
\hline $6-27$ & 3035 & 0 & 0 & 0 & 0 & 0 & 0 \\
\hline $\begin{array}{l}6-29 \\
7-01\end{array}$ & $=$ & $\overline{-}$ & $\overline{--}$ & $=$ & $=-$ & $=$ & $=$ \\
\hline $7-0 \overline{3}$ & - & -- & - & -- & - & -- & -- \\
\hline $7-05$ & -- & -- & - & -- & -- & -- & -- \\
\hline $7-07$ & -- & -- & - & -- & -- & -- & - \\
\hline
\end{tabular}


TABLE 15.- NUMBER OF PINK TRACER PARTICLES(1), PER 100 GRAMS OF BEDLOAD SAMPLE, COMPOSITED FROM CROSS-CHANNEL SAMPLING POSITIONS SPACED AT 1-METER INTERVALS ACROSS THE CHANNEL WIDTH, EAST FORK RIVER, WYOMING, 1980

SECTION 0220

\begin{tabular}{|c|c|c|c|c|c|c|c|}
\hline \multirow{3}{*}{ DATE } & \multirow{3}{*}{$\begin{array}{c}\text { DRY } \\
\text { MASS } \\
\text { OF } \\
\text { SAMPLE (2) } \\
\text { (G) }\end{array}$} & & BER & INK 7 & ZER PA & [CLES & \\
\hline & & & \multicolumn{5}{|c|}{ BY SIZE CLASS (MM) } \\
\hline & & TOTAL & $\begin{array}{l}0.25 \\
\text { TO } \\
0.50\end{array}$ & $\begin{array}{l}0.50 \\
\text { TO } \\
1.00\end{array}$ & $\begin{array}{l}1.00 \\
\text { TO } \\
2.00\end{array}$ & $\begin{array}{l}2.00 \\
\text { TO } \\
4.00\end{array}$ & $\begin{array}{l}4.00 \\
\text { TO } \\
8.00\end{array}$ \\
\hline $\begin{array}{l}5-16 \\
5-19 \\
5-21 \\
5-22 \\
5-24\end{array}$ & $\begin{array}{c}144+ \\
512 \\
5907 \\
-- \\
--\end{array}$ & $\begin{array}{r}0 \\
0 \\
0 \\
-- \\
--\end{array}$ & $\begin{array}{r}0 \\
0 \\
0 \\
-- \\
--\end{array}$ & $\begin{array}{r}0 \\
0 \\
0 \\
-- \\
--\end{array}$ & $\begin{array}{r}0 \\
0 \\
0 \\
-- \\
--\end{array}$ & $\begin{array}{r}0 \\
0 \\
0 \\
-- \\
--\end{array}$ & $\begin{array}{r}0 \\
0 \\
0 \\
--\end{array}$ \\
\hline $\begin{array}{l}5-26 \\
5-27 \\
5-28 \\
5-29 \\
5-30\end{array}$ & $\begin{array}{l}871 \\
83+ \\
673 \\
\overline{50} \\
150+\end{array}$ & $\begin{array}{l}0 \\
0 \\
0 \\
-\frac{0}{0}\end{array}$ & $\begin{array}{r}0 \\
0 \\
0 \\
-0 \\
0\end{array}$ & $\begin{array}{r}0 \\
0 \\
0 \\
-- \\
0\end{array}$ & $\begin{array}{r}0 \\
0 \\
0 \\
-0 \\
0\end{array}$ & $\begin{array}{r}0 \\
0 \\
0 \\
-\frac{1}{0}\end{array}$ & $\begin{array}{r}0 \\
0 \\
0 \\
-0 \\
0\end{array}$ \\
\hline $\begin{array}{l}6-01 \\
6-03 \\
6-05 \\
6-07 \\
6-09\end{array}$ & $\begin{array}{c}44++ \\
67++ \\
569 \\
758 \\
4213\end{array}$ & $\begin{array}{l}0 \\
0 \\
0 \\
0 \\
2\end{array}$ & $\begin{array}{l}0 \\
0 \\
0 \\
0 \\
0\end{array}$ & $\begin{array}{l}0 \\
0 \\
0 \\
0 \\
2\end{array}$ & $\begin{array}{l}0 \\
0 \\
0 \\
0 \\
0\end{array}$ & $\begin{array}{l}0 \\
0 \\
0 \\
0 \\
0\end{array}$ & $\begin{array}{l}0 \\
0 \\
0 \\
0 \\
0\end{array}$ \\
\hline $\begin{array}{l}6-10 \\
6-11 \\
6-12 \\
6-13 \\
6-14\end{array}$ & $\begin{array}{l}6882 \\
5760 \\
3938 \\
4401 \\
5375\end{array}$ & $\begin{array}{l}0 \\
1 \\
0 \\
0 \\
0\end{array}$ & $\begin{array}{l}0 \\
0 \\
0 \\
0 \\
0\end{array}$ & $\begin{array}{l}0 \\
0 \\
0 \\
0 \\
0\end{array}$ & $\begin{array}{l}0 \\
1 \\
0 \\
0 \\
0\end{array}$ & $\begin{array}{l}0 \\
0 \\
0 \\
0 \\
0\end{array}$ & $\begin{array}{l}0 \\
0 \\
0 \\
0 \\
0\end{array}$ \\
\hline $6-15$ & 241 & 2 & 2 & 0 & 0 & 0 & 0 \\
\hline $\begin{array}{l}6-17 \\
6-18 \\
6-19\end{array}$ & $\begin{array}{r}946 \\
3574 \\
6083\end{array}$ & $\begin{array}{l}0 \\
0 \\
0\end{array}$ & $\begin{array}{l}0 \\
0 \\
0\end{array}$ & $\begin{array}{l}0 \\
0 \\
0\end{array}$ & $\begin{array}{l}0 \\
0 \\
0\end{array}$ & $\begin{array}{l}0 \\
0 \\
0\end{array}$ & $\begin{array}{l}0 \\
0 \\
0\end{array}$ \\
\hline $\begin{array}{l}6-20 \\
6-21\end{array}$ & $\begin{array}{l}6451 \\
3627\end{array}$ & $\begin{array}{l}0 \\
0\end{array}$ & $\begin{array}{l}0 \\
0\end{array}$ & $\begin{array}{l}0 \\
0\end{array}$ & $\begin{array}{l}0 \\
0\end{array}$ & $\begin{array}{l}0 \\
0\end{array}$ & $\begin{array}{l}0 \\
0\end{array}$ \\
\hline $\begin{array}{l}6-23 \\
6-25\end{array}$ & $\begin{array}{l}1357 \\
3244\end{array}$ & $\begin{array}{l}3 \\
1\end{array}$ & $\begin{array}{l}2 \\
0\end{array}$ & $\frac{1}{1}$ & 0 & $\begin{array}{l}0 \\
0\end{array}$ & $\begin{array}{l}0 \\
0\end{array}$ \\
\hline $6-27$ & 1327 & 0 & 0 & 0 & 0 & 0 & 0 \\
\hline $\begin{array}{l}6-29 \\
7-01\end{array}$ & $=$ & $=$ & $=-$ & -- & $=$ & -- & -- \\
\hline $7-0 \overline{3}$ & -- & -- & -- & -- & -- & -- & - \\
\hline $7-05$ & -- & -- & -- & - & -- & -- & -- \\
\hline $7-07$ & -- & -- & -- & -- & -- & -- & -- \\
\hline
\end{tabular}


TABLE 15.- NUMBER OF PINK TRACER PARTICLES(I), PER IO0 GRAMS OF BEDLOAD SAMPLE, COMPOSITED FROM CROSS-CHANNEL SAMPLING POSITIONS SPACED AT I-METER INTERVALS ACROSS THE CHANNEL WIDTH, EAST FORK RIVER, WYOMING, 1980

SECTION 0257

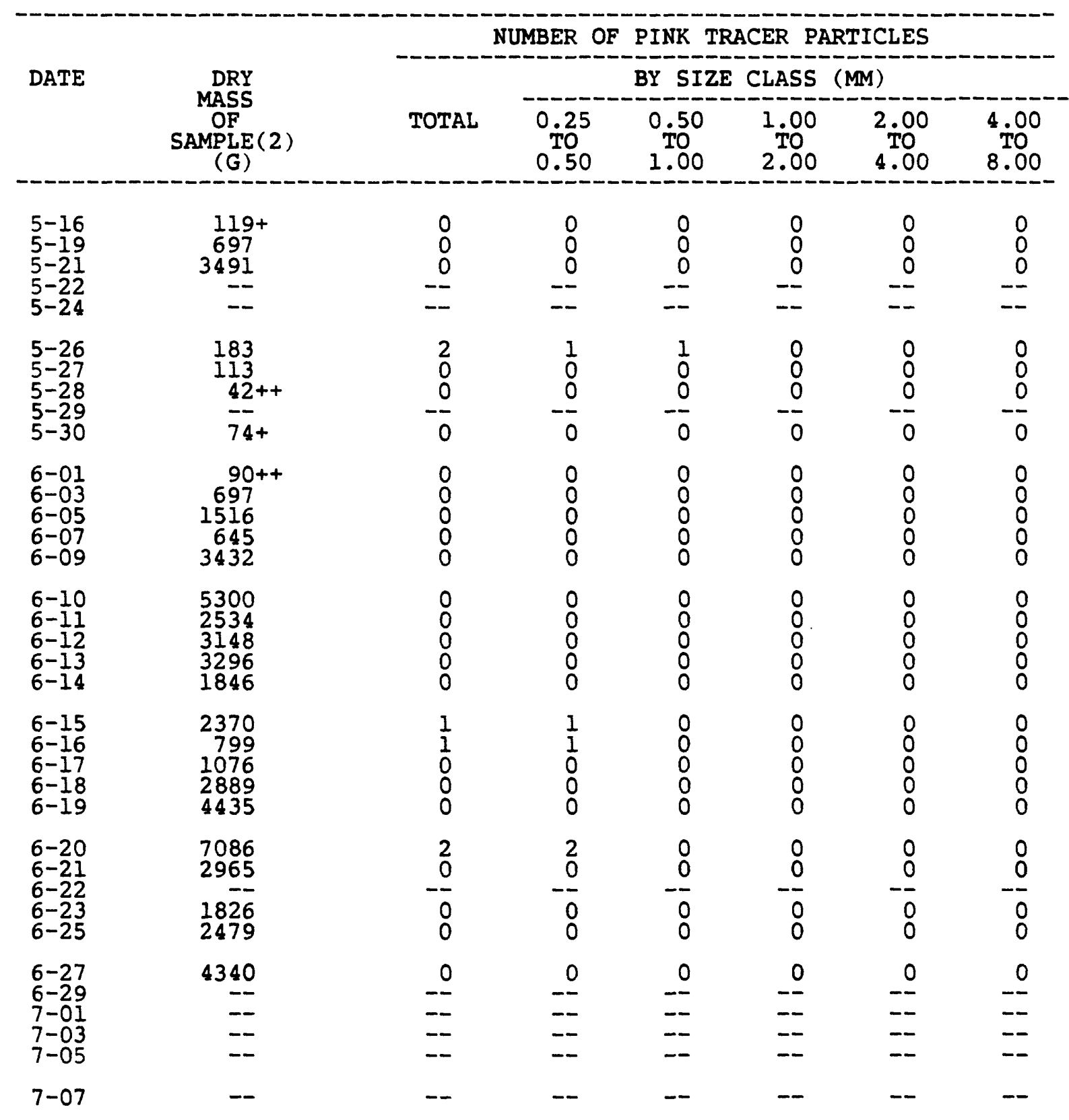


TABLE 15.- NUMBER OF PINK TRACER PARTICLES(1), PER 100 GRAMS OF BEDLOAD SAMPLE, COMPOSITED FROM CROSS-CHANNEL SAMPLING POSITIONS SPACED AT 1-METER INTERVALS ACROSS THE CHANNEL WIDTH, EAST FORK RIVER, WYOMING, 1980

SECTION $0257(3)$

\begin{tabular}{|c|c|c|c|c|c|c|c|}
\hline \multirow{3}{*}{ DATE } & \multirow{3}{*}{$\begin{array}{c}\text { DRY } \\
\text { MASS } \\
\text { OF } \\
\text { SAMPLE (2) } \\
\text { (G) }\end{array}$} & \multicolumn{6}{|c|}{ NUMBER OF PINK TRACER PARTICLES } \\
\hline & & \multirow[b]{2}{*}{ TOTAL } & \multicolumn{5}{|c|}{ BY SIZE CLASS (MM) } \\
\hline & & & $\begin{array}{l}0.25 \\
\text { TO } \\
0.50\end{array}$ & $\begin{array}{l}0.50 \\
\text { TO } \\
1.00\end{array}$ & $\begin{array}{l}1.00 \\
\text { TO } \\
2.00\end{array}$ & $\begin{array}{l}2.00 \\
\text { TO } \\
4.00\end{array}$ & $\begin{array}{l}4.00 \\
\text { TO } \\
8.00\end{array}$ \\
\hline $5-16$ & -- & - & -- & -- & -- & -- & -- \\
\hline $5-19$ & $\overline{-}$ & $\overline{-}$ & $\overline{-}$ & -- & -- & - & -- \\
\hline $5-22$ & - & - & -- & $\ldots$ & - & $\ldots$ & - \\
\hline $5-24$ & -- & - & -- & -- & -- & -- & -- \\
\hline $5-26$ & -- & - & -- & -- & -- & -- & - \\
\hline $\begin{array}{l}5-27 \\
5\end{array}$ & $=$ & -- & - & -- & -- & -- & -- \\
\hline $5-29$ & -- & -- & -- & -- & - & -- & -- \\
\hline $5-30$ & -- & -- & - & - & -- & -- & -- \\
\hline $6-01$ & -- & -- & -- & -- & -- & -- & -- \\
\hline $6-03$ & - & -- & -- & -- & -- & -- & - \\
\hline $6-05$ & - & $=$ & -- & -- & -- & -- & - \\
\hline $6-09$ & 314 & 0 & 0 & 0 & 0 & 0 & 0 \\
\hline $6-10$ & 820 & 0 & 0 & 0 & 0 & 0 & 0 \\
\hline $6-12$ & 1057 & 0 & 0 & 0 & 0 & 0 & 0 \\
\hline $\begin{array}{l}6-13 \\
6-14\end{array}$ & $\begin{array}{l}528 \\
634\end{array}$ & 0 & 0 & 0 & 0 & 0 & 0 \\
\hline & & & & & & & \\
\hline & 588 & 0 & 0 & 0 & 0 & 0 & 0 \\
\hline $\begin{array}{l}0-16 \\
6-17\end{array}$ & $=$ & $=$ & $=$ & $=$ & $=$ & $=$ & $=$ \\
\hline $6-18$ & 276 & 4 & 2 & 2 & 0 & 0 & 0 \\
\hline $6-19$ & 1102 & 0 & 0 & 0 & 0 & 0 & 0 \\
\hline $6-20$ & 298 & 4 & 3 & 1 & 0 & 0 & 0 \\
\hline $6-21$ & 287 & 0 & 0 & 0 & 0 & 0 & 0 \\
\hline $\begin{array}{l}0-22 \\
6-23\end{array}$ & $=$ & $=$ & $=$ & $=$ & $=$ & $=$ & $=$ \\
\hline $6-25$ & 598 & 0 & 0 & 0 & 0 & 0 & 0 \\
\hline & 320 & 5 & 1 & 4 & 0 & 0 & 0 \\
\hline $6-29$ & $\overline{-}$ & $\overline{-}$ & $=$ & - & $=$ & $=$ & $=$ \\
\hline $7-0 \frac{1}{3}$ & - & - & - & - & - & - & - \\
\hline $7-05$ & -- & -- & - & - & - & -- & - \\
\hline $7-07$ & -- & -- & - & - & -- & -- & -- \\
\hline
\end{tabular}


TABLE 15.- NUMBER OF PINK TRACER PARTICLES(1), PER 100 GRAMS OF BEDLOAD SAMPLE, COMPOSITED FROM CROSS-CHANNEL SAMPLING POSITIONS SPACED AT 1-METER INTERVALS ACROSS THE CHANNEL WIDTH, EAST FORK RIVER, WYOMING, 1980

SECTION 0301

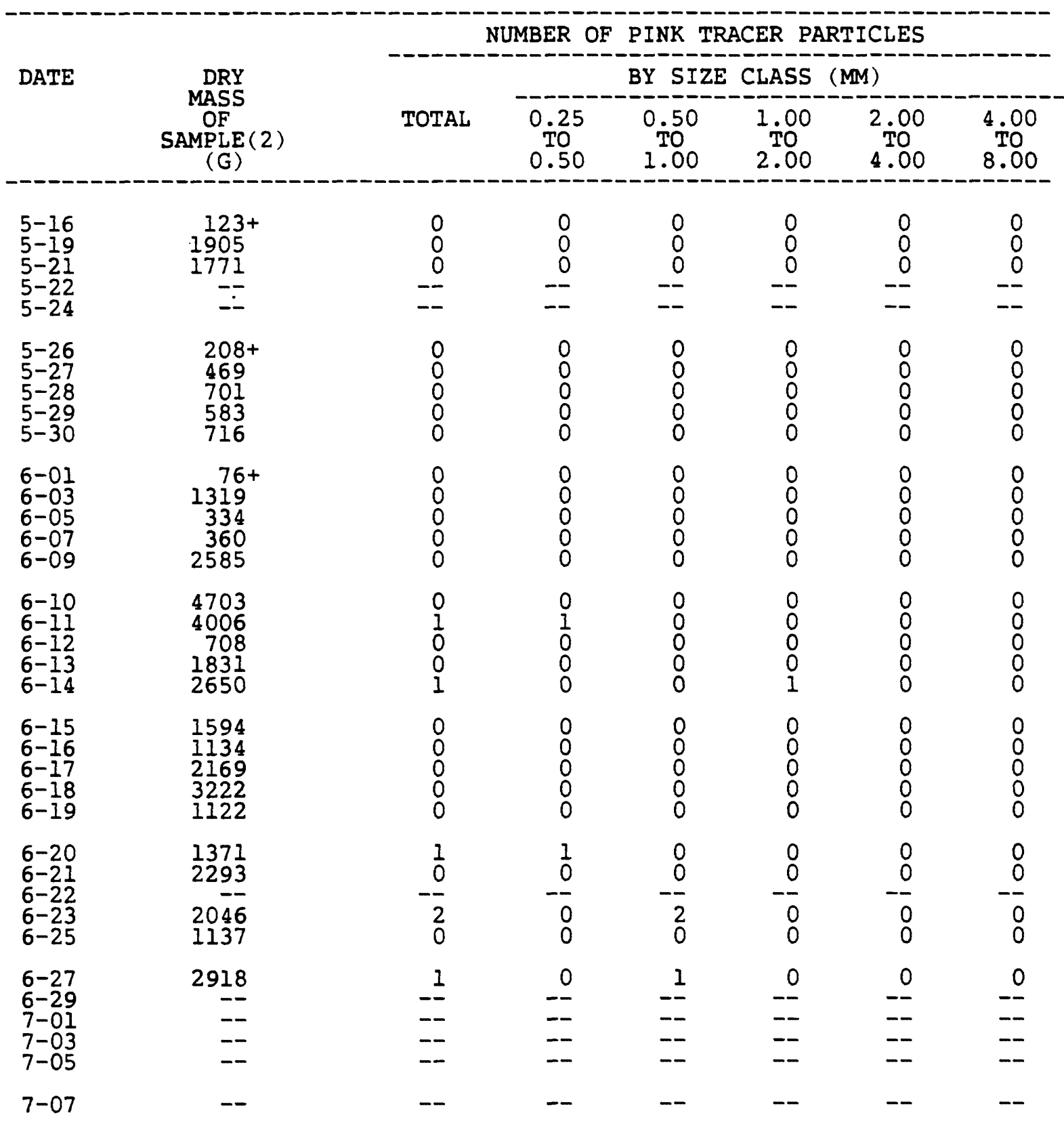


TABLE 15.- NUMBER OF PINK TRACER PARTICLES(1), PER 100 GRAMS OF BEDLOAD SAMPLE, COMPOSITED FROM CROSS-CHANNEL SAMPLING POSITIONS

SPACED'AT I-METER INTERVALS ACROSS THE CHANNEL WIDTH,

EAST FORK RIVER, WYOMING, 1980

$$
\text { SECTION } 0301(3)
$$

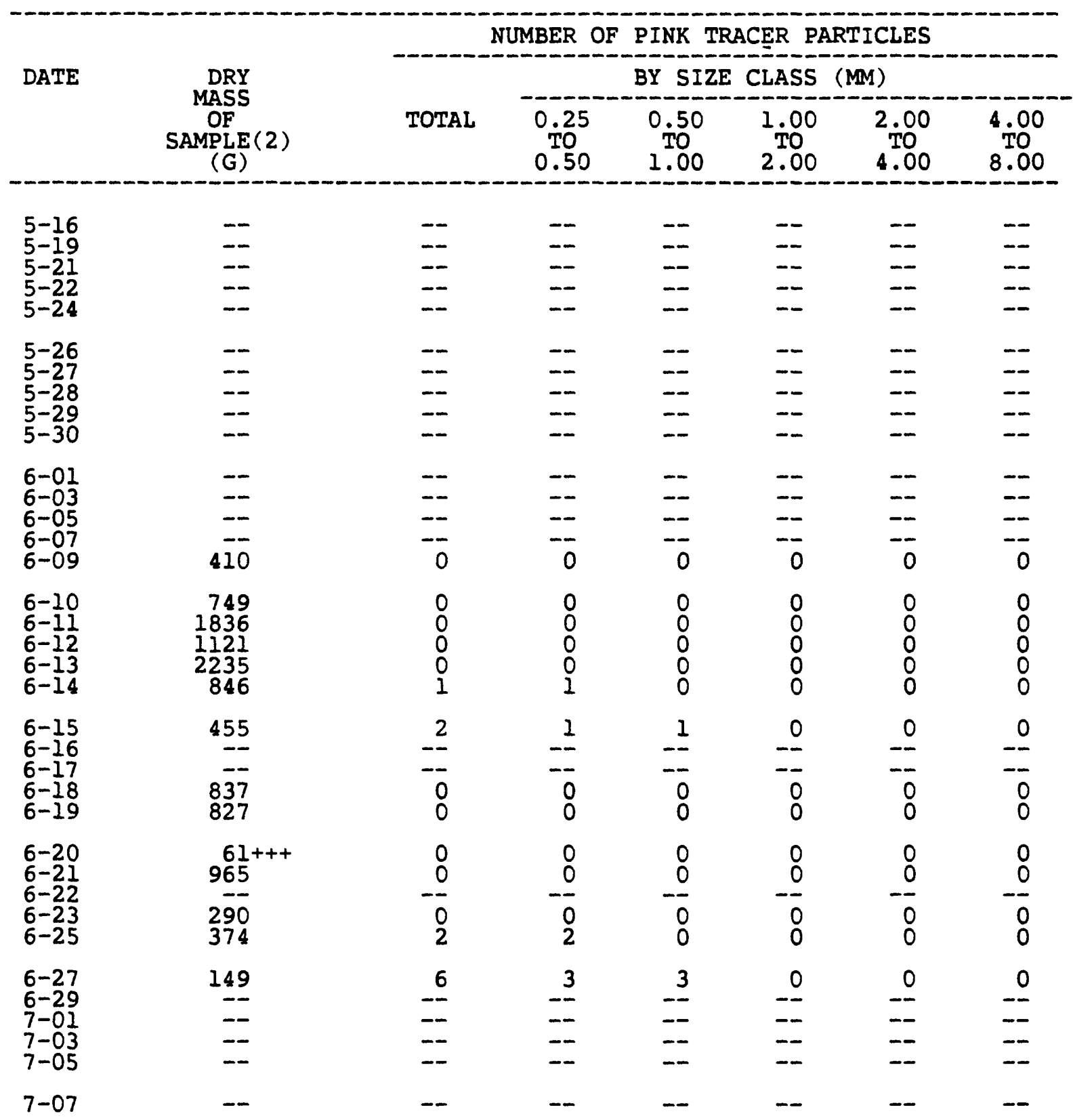


TABLE 15.- NUMBER OF PINK TRACER PARTICLES(1), PER 100 GRAMS OF BEDLOAD SAMPLE, COMPOSITED FROM CROSS-CHANNEL SAMPLING POSITIONS SPACED AT I-METER INTERVALS ACROSS THE CHANNEL WIDTH, EAST FORK RIVER, WYOMING, 1980

SECTION 0348

\begin{tabular}{|c|c|c|c|c|c|c|c|}
\hline \multirow{3}{*}{ DATE } & \multirow{3}{*}{$\begin{array}{c}\text { DRY } \\
\text { MASS } \\
\text { OF } \\
\text { SAMPLE (2) } \\
\text { (G) }\end{array}$} & \multicolumn{6}{|c|}{ NUMBER OF PINK TRACER PARTICLES } \\
\hline & & \multirow[b]{2}{*}{ TOTAL } & \multicolumn{5}{|c|}{ BY SIZE CLASS (MM) } \\
\hline & & & $\begin{array}{l}0.250 \\
\text { TO } \\
0.50\end{array}$ & $\begin{array}{l}0.50 \\
\text { TO } \\
1.00\end{array}$ & $\begin{array}{l}1.00 \\
\text { TO } \\
2.00\end{array}$ & $\begin{array}{l}2.00 \\
\text { To } \\
4.00\end{array}$ & $\begin{array}{l}4.00 \\
\text { TO } \\
8.00\end{array}$ \\
\hline $\begin{array}{l}5-16 \\
5-19 \\
5-21 \\
5-27\end{array}$ & $\begin{array}{r}1385 \\
719 \\
1217\end{array}$ & $\begin{array}{r}0 \\
0 \\
0 \\
-0\end{array}$ & $\begin{array}{r}0 \\
0 \\
0 \\
-0\end{array}$ & $\begin{array}{r}0 \\
0 \\
0 \\
-2\end{array}$ & $\begin{array}{r}0 \\
0 \\
0 \\
-\end{array}$ & $\begin{array}{r}0 \\
0 \\
0\end{array}$ & $\begin{array}{r}0 \\
0 \\
0 \\
-0\end{array}$ \\
\hline $\begin{array}{l}5-22 \\
5-24\end{array}$ & $\overline{--}$ & $\overline{-}$ & $\overline{--}$ & $\overline{-}$ & $\overline{-}$ & $\overline{-}$ & $\overline{-}$ \\
\hline $\begin{array}{l}5-26 \\
5-27 \\
5-28\end{array}$ & $\begin{array}{r}471 \\
1592 \\
1893\end{array}$ & $\begin{array}{l}1 \\
0 \\
0\end{array}$ & $\begin{array}{l}1 \\
0 \\
0\end{array}$ & $\begin{array}{l}0 \\
0 \\
0\end{array}$ & $\begin{array}{l}0 \\
0 \\
0\end{array}$ & $\begin{array}{l}0 \\
0 \\
0\end{array}$ & $\begin{array}{l}0 \\
0 \\
0\end{array}$ \\
\hline $5-30$ & 1540 & 0 & 0 & 0 & 0 & 0 & 0 \\
\hline $\begin{array}{l}6-01 \\
6-03 \\
6-05 \\
6-07 \\
6-09\end{array}$ & $\begin{array}{r}872 \\
407 \\
701 \\
214 \\
1115\end{array}$ & $\begin{array}{l}0 \\
0 \\
0 \\
0 \\
0\end{array}$ & $\begin{array}{l}0 \\
0 \\
0 \\
0 \\
0\end{array}$ & $\begin{array}{l}0 \\
0 \\
0 \\
0 \\
0\end{array}$ & $\begin{array}{l}0 \\
0 \\
0 \\
0 \\
0\end{array}$ & $\begin{array}{l}0 \\
0 \\
0 \\
0 \\
0\end{array}$ & $\begin{array}{l}0 \\
0 \\
0 \\
0 \\
0\end{array}$ \\
\hline $\begin{array}{l}6-10 \\
6-11 \\
6-12 \\
6-13 \\
6-14\end{array}$ & $\begin{array}{l}1871 \\
2608 \\
218 \\
1290 \\
6798\end{array}$ & $\begin{array}{l}0 \\
1 \\
0 \\
0 \\
1\end{array}$ & $\begin{array}{l}0 \\
0 \\
0 \\
0 \\
1\end{array}$ & $\begin{array}{l}0 \\
1 \\
0 \\
0 \\
0\end{array}$ & $\begin{array}{l}0 \\
0 \\
0 \\
0 \\
0\end{array}$ & $\begin{array}{l}0 \\
0 \\
0 \\
0 \\
0\end{array}$ & $\begin{array}{l}0 \\
0 \\
0 \\
0 \\
0\end{array}$ \\
\hline $\begin{array}{l}6-15 \\
6-16 \\
6-17 \\
6-18 \\
6-19\end{array}$ & $\begin{array}{l}3561 \\
1007 \\
4185 \\
7816 \\
5678\end{array}$ & $\begin{array}{l}0 \\
0 \\
0 \\
0 \\
0\end{array}$ & $\begin{array}{l}0 \\
0 \\
0 \\
0 \\
0\end{array}$ & $\begin{array}{l}0 \\
0 \\
0 \\
0 \\
0\end{array}$ & $\begin{array}{l}0 \\
0 \\
0 \\
0 \\
0\end{array}$ & $\begin{array}{l}0 \\
0 \\
0 \\
0 \\
0\end{array}$ & $\begin{array}{l}0 \\
0 \\
0 \\
0 \\
0\end{array}$ \\
\hline $\begin{array}{l}6-20 \\
6-21\end{array}$ & $\begin{array}{l}2601 \\
2274\end{array}$ & $\begin{array}{l}0 \\
0\end{array}$ & $\begin{array}{l}0 \\
0\end{array}$ & $\begin{array}{l}0 \\
0\end{array}$ & $\begin{array}{l}0 \\
0\end{array}$ & $\begin{array}{l}0 \\
0\end{array}$ & $\begin{array}{l}0 \\
0\end{array}$ \\
\hline $\begin{array}{l}6-22 \\
6-23 \\
6-25\end{array}$ & $\begin{array}{l}1858 \\
3862\end{array}$ & 2 & $\begin{array}{l}2 \\
1\end{array}$ & $\begin{array}{l}0 \\
7\end{array}$ & $\begin{array}{l}0 \\
2\end{array}$ & $\begin{array}{l}0 \\
1\end{array}$ & $\begin{array}{l}0 \\
0\end{array}$ \\
\hline $\begin{array}{l}6-27 \\
6-29\end{array}$ & $\begin{array}{l}2790 \\
2728\end{array}$ & $\begin{array}{l}3 \\
2\end{array}$ & $\begin{array}{l}2 \\
0\end{array}$ & $\frac{1}{1}$ & $\begin{array}{l}0 \\
1\end{array}$ & $\begin{array}{l}0 \\
0\end{array}$ & $\begin{array}{l}0 \\
0\end{array}$ \\
\hline $\begin{array}{l}7-01 \\
7-03\end{array}$ & $\overline{--}$ & $\overline{--}$ & $=$ & - & $\overline{-}$ & - & $=$ \\
\hline $7-05$ & - & -- & -- & - & -- & -- & -- \\
\hline $7-07$ & - & -- & -- & -- & -- & -- & - \\
\hline
\end{tabular}


TABLE 15.- NUMBER OF PINK TRACER PARTICLES(1), PER 100 GRAMS OF BEDLOAD SAMPLE, COMPOSITED FROM CROSS-CHANNEL SAMPLING POSITIONS SPACED AT 1-METER INTERVALS ACROSS THE CHANNEL WIDTH, EAST FORK RIVER, WYOMING, 1980

\section{SECTION 0421}

\begin{tabular}{|c|c|c|c|c|c|c|c|}
\hline \multirow{3}{*}{ DATE } & \multirow{3}{*}{$\begin{array}{c}\text { DRY } \\
\text { MASS } \\
\text { OF } \\
\text { SAMPLE (2) } \\
\text { (G) }\end{array}$} & \multicolumn{6}{|c|}{ NUMBER OF PINK TRACER PARTICLES } \\
\hline & & \multirow[b]{2}{*}{ TOTAL } & \multicolumn{3}{|c|}{ BY SIZE CLASS } & \multicolumn{2}{|c|}{ (MM) } \\
\hline & & & $\begin{array}{c}0.25 \\
\text { TO } \\
0.50\end{array}$ & $\begin{array}{l}0.50 \\
\text { To } \\
1.00\end{array}$ & $\begin{array}{l}1.00 \\
2.00 \\
2.00\end{array}$ & $\begin{array}{l}2.00 \\
\text { TO } \\
4.00\end{array}$ & $\begin{array}{l}4.00 \\
\text { TO } \\
8.00\end{array}$ \\
\hline $\begin{array}{l}5-16 \\
5-19 \\
5-21 \\
5-22 \\
5-24\end{array}$ & $\begin{array}{r}242 \\
777 \\
612 \\
-- \\
--\end{array}$ & $\begin{array}{r}0 \\
0 \\
2 \\
-- \\
--\end{array}$ & $\begin{array}{r}0 \\
0 \\
2 \\
-- \\
--\end{array}$ & $\begin{array}{r}0 \\
0 \\
0 \\
-- \\
--\end{array}$ & $\begin{array}{r}0 \\
0 \\
0 \\
-- \\
--\end{array}$ & $\begin{array}{r}0 \\
0 \\
0 \\
-- \\
--\end{array}$ & $\begin{array}{r}0 \\
0 \\
0 \\
-- \\
--\end{array}$ \\
\hline $\begin{array}{l}5-26 \\
5-27 \\
5-28 \\
5-29\end{array}$ & $\begin{array}{r}3173 \\
896 \\
561 \\
\end{array}$ & $\begin{array}{r}0 \\
0 \\
0 \\
--\end{array}$ & $\begin{array}{r}0 \\
0 \\
0 \\
--\end{array}$ & $\begin{array}{r}0 \\
0 \\
0 \\
-\end{array}$ & $\begin{array}{r}0 \\
0 \\
0 \\
-\end{array}$ & $\begin{array}{r}0 \\
0 \\
0 \\
--\end{array}$ & $\begin{array}{r}0 \\
0 \\
0 \\
--\end{array}$ \\
\hline $5-30$ & 277 & 0 & 0 & 0 & 0 & 0 & 0 \\
\hline $\begin{array}{l}6-01 \\
6-03 \\
6-05 \\
6-07 \\
6-09\end{array}$ & $\begin{array}{l}563 \\
384 \\
88+ \\
376 \\
958\end{array}$ & $\begin{array}{l}0 \\
0 \\
0 \\
0 \\
0\end{array}$ & $\begin{array}{l}0 \\
0 \\
0 \\
0 \\
0\end{array}$ & $\begin{array}{l}0 \\
0 \\
0 \\
0 \\
0\end{array}$ & $\begin{array}{r}0 \\
0 \\
-\quad 0 \\
0 \\
0 \\
0\end{array}$ & $\begin{array}{l}0 \\
0 \\
0 \\
0 \\
0 .\end{array}$ & $\begin{array}{l}0 \\
0 \\
0 \\
0 \\
0\end{array}$ \\
\hline $\begin{array}{l}6-10 \\
6-11 \\
6-12 \\
6-13 \\
6-14\end{array}$ & $\begin{array}{l}4947 \\
4214 \\
2586 \\
5444 \\
4647\end{array}$ & $\begin{array}{l}0 \\
0 \\
0 \\
0 \\
0\end{array}$ & $\begin{array}{l}0 \\
0 \\
0 \\
0 \\
0\end{array}$ & $\begin{array}{l}0 \\
0 \\
0 \\
0 \\
0\end{array}$ & $\begin{array}{l}0 \\
0 \\
0 \\
0 \\
0\end{array}$ & $\begin{array}{l}0 \\
0 \\
0 \\
0 \\
0\end{array}$ & $\begin{array}{l}0 \\
0 \\
0 \\
0 \\
0\end{array}$ \\
\hline $\begin{array}{l}6-15 \\
6-16 \\
6-17 \\
6-18 \\
6-19\end{array}$ & $\begin{array}{l}4003 \\
3030 \\
3022 \\
1824 \\
1450\end{array}$ & $\begin{array}{l}0 \\
0 \\
1 \\
0 \\
0\end{array}$ & $\begin{array}{l}0 \\
0 \\
1 \\
0 \\
0\end{array}$ & $\begin{array}{l}0 \\
0 \\
0 \\
0 \\
0\end{array}$ & $\begin{array}{l}0 \\
0 \\
0 \\
0 \\
0\end{array}$ & $\begin{array}{l}0 \\
0 \\
0 \\
0 \\
0\end{array}$ & $\begin{array}{l}0 \\
0 \\
0 \\
0 \\
0\end{array}$ \\
\hline $\begin{array}{l}6-20 \\
6-21\end{array}$ & $\begin{array}{l}2879 \\
9086\end{array}$ & $\begin{array}{l}2 \\
2\end{array}$ & $\begin{array}{l}1 \\
0\end{array}$ & $\frac{1}{2}$ & $\begin{array}{l}0 \\
0\end{array}$ & $\begin{array}{l}0 \\
0\end{array}$ & $\begin{array}{l}0 \\
0\end{array}$ \\
\hline $\begin{array}{l}6-23 \\
6-25\end{array}$ & $\begin{array}{l}1446 \\
3306\end{array}$ & $\begin{array}{l}0 \\
1\end{array}$ & $\begin{array}{l}0 \\
0\end{array}$ & $\begin{array}{l}0 \\
1\end{array}$ & $\begin{array}{l}0 \\
0\end{array}$ & $\begin{array}{l}0 \\
0\end{array}$ & $\begin{array}{l}0 \\
0\end{array}$ \\
\hline $6-27$ & 1800 & 0 & 0 & 0 & 0 & 0 & 0 \\
\hline $7-01$ & $\overline{--}$ & $\overline{--}$ & $\overline{--}$ & $\overline{--}$ & $\overline{-}$ & - & - \\
\hline $\begin{array}{l}7-03 \\
7-05\end{array}$ & $\overline{--}$ & $\overline{--}$ & $\overline{-}$ & $\overline{--}$ & $\overline{-}$ & $\overline{-}$ & $\overline{-}$ \\
\hline $7-07$ & -- & -- & -- & -- & -- & -- & -- \\
\hline
\end{tabular}


TABLE 15.- NUMBER OF PINK TRACER PARTICLES(1), PER 100 GRAMS OF BEDLOAD SAMPLE, COMPOSITED FROM CROSS-CHANNEL SAMPLING POSITIONS SPACED AT 1-METER INTERVALS ACROSS THE CHANNEL WIDTH, EAST FORK RIVER, WYOMING, 1980

SECTION 0460

\begin{tabular}{|c|c|c|c|c|c|c|c|}
\hline \multirow{3}{*}{ DATE } & \multirow{3}{*}{$\begin{array}{c}\text { DRY } \\
\text { MASS } \\
\text { OF } \\
\text { SAMPLE (2) } \\
\text { (G) }\end{array}$} & \multicolumn{6}{|c|}{ NUMBER OF PINK TRACER PARTICLES } \\
\hline & & \multirow[b]{2}{*}{ TOTAL } & \multicolumn{5}{|c|}{ BY SIZE CLASS (MM) } \\
\hline & & & $\begin{array}{l}0.25 \\
\text { TO } \\
0.50\end{array}$ & $\begin{array}{l}0.50 \\
\text { To } \\
1.00\end{array}$ & $\begin{array}{l}1.00 \\
2.00 \\
2.00\end{array}$ & $\begin{array}{l}2.00 \\
\text { TO } \\
4.00\end{array}$ & $\begin{array}{l}4.00 \\
\text { TO } \\
8.00\end{array}$ \\
\hline $\begin{array}{l}5-16 \\
5-19 \\
5-21 \\
5-22 \\
5-24\end{array}$ & $\begin{array}{l}233 \\
104++ \\
153+ \\
---\end{array}$ & $\begin{array}{r}0 \\
0 \\
3 \\
-- \\
--\end{array}$ & $\begin{array}{r}0 \\
0 \\
3 \\
-- \\
--\end{array}$ & $\begin{array}{r}0 \\
0 \\
0 \\
-- \\
--\end{array}$ & $\begin{array}{r}0 \\
0 \\
0 \\
-- \\
--\end{array}$ & $\begin{array}{r}0 \\
0 \\
0 \\
-- \\
--\end{array}$ & $\begin{array}{r}0 \\
0 \\
0 \\
-- \\
--\end{array}$ \\
\hline $\begin{array}{l}5-26 \\
5-27 \\
5-28 \\
5-29 \\
5-30\end{array}$ & $\begin{array}{c}1436 \\
39++ \\
190+ \\
-16++\end{array}$ & $\begin{array}{r}1 \\
0 \\
0 \\
-0\end{array}$ & $\begin{array}{r}0 \\
0 \\
0 \\
-0\end{array}$ & $\begin{array}{r}1 \\
0 \\
0 \\
-0\end{array}$ & $\begin{array}{r}0 \\
0 \\
0 \\
-0 \\
0\end{array}$ & $\begin{array}{r}0 \\
0 \\
0 \\
-0 \\
0\end{array}$ & $\begin{array}{r}0 \\
0 \\
0 \\
-- \\
0\end{array}$ \\
\hline $\begin{array}{l}6-01 \\
6-03 \\
6-05 \\
6-07 \\
6-09\end{array}$ & $\begin{array}{c}25++ \\
10+++ \\
24++ \\
43++ \\
2147\end{array}$ & $\begin{array}{l}0 \\
0 \\
0 \\
0 \\
0\end{array}$ & $\begin{array}{l}0 \\
0 \\
0 \\
0 \\
0\end{array}$ & $\begin{array}{l}0 \\
0 \\
0 \\
0 \\
0\end{array}$ & $\begin{array}{l}0 \\
0 \\
0 \\
0 \\
0\end{array}$ & $\begin{array}{l}0 \\
0 \\
0 \\
0 \\
0\end{array}$ & $\begin{array}{l}0 \\
0 \\
0 \\
0 \\
0\end{array}$ \\
\hline $\begin{array}{l}6-10 \\
6-11 \\
6-12 \\
6-13 \\
6-14\end{array}$ & $\begin{array}{l}6109 \\
8822 \\
7920 \\
3616 \\
4080\end{array}$ & $\begin{array}{l}0 \\
1 \\
0 \\
0 \\
0\end{array}$ & $\begin{array}{l}0 \\
0 \\
0 \\
0 \\
0\end{array}$ & $\begin{array}{l}0 \\
1 \\
0 \\
0 \\
0\end{array}$ & $\begin{array}{l}0 \\
0 \\
0 \\
0 \\
0\end{array}$ & $\begin{array}{l}0 \\
0 \\
0 \\
0 \\
0\end{array}$ & $\begin{array}{l}0 \\
0 \\
0 \\
0 \\
0\end{array}$ \\
\hline $6-15$ & 2730 & 0 & 0 & 0 & 0 & $\underline{0}$ & 0 \\
\hline $\begin{array}{l}6-17 \\
6-18 \\
6-19\end{array}$ & $\begin{array}{r}952 \\
1870 \\
2636\end{array}$ & $\begin{array}{l}0 \\
0 \\
0\end{array}$ & $\begin{array}{l}0 \\
0 \\
0\end{array}$ & $\begin{array}{l}0 \\
0 \\
0\end{array}$ & $\begin{array}{l}0 \\
0 \\
0\end{array}$ & $\begin{array}{l}0 \\
0 \\
0\end{array}$ & $\begin{array}{l}0 \\
0 \\
0\end{array}$ \\
\hline $\begin{array}{l}6-20 \\
6-21\end{array}$ & $\begin{array}{l}2850 \\
4894\end{array}$ & $\begin{array}{r}10 \\
0\end{array}$ & $\begin{array}{l}6 \\
0\end{array}$ & $\begin{array}{l}2 \\
0\end{array}$ & $\begin{array}{l}1 \\
0\end{array}$ & $\begin{array}{l}1 \\
0\end{array}$ & $\begin{array}{l}0 \\
0\end{array}$ \\
\hline $\begin{array}{l}6-23 \\
6-25\end{array}$ & $\begin{array}{l}1697 \\
1719\end{array}$ & $\begin{array}{l}3 \\
0\end{array}$ & $\begin{array}{l}3 \\
0\end{array}$ & $\begin{array}{l}0 \\
0\end{array}$ & $\begin{array}{l}0 \\
0\end{array}$ & $\begin{array}{l}0 \\
0\end{array}$ & $\begin{array}{l}0 \\
0\end{array}$ \\
\hline $6-27$ & 2173 & 1 & 0 & 1 & 0 & 0 & 0 \\
\hline $\begin{array}{l}6-29 \\
7-01\end{array}$ & $\overline{-}$ & $\overline{-}$ & $\overline{--}$ & 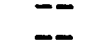 & $\overline{--}$ & $\overline{-}$ & $\overline{-}$ \\
\hline $7-03$ & -- & -- & -- & -- & -- & - & -- \\
\hline $7-05$ & -- & -- & -- & -- & -- & -- & -- \\
\hline $7-07$ & -- & -- & -- & -- & -- & -- & - \\
\hline
\end{tabular}


TABLE 15.- NUMBER OF PINK TRACER PARTICLES(1), PER 100 GRAMS OF BEDLOAD SAMPLE, COMPOSITED FROM CROSS-CHANNEL SAMPLING POSITIONS SPACED AT I-METER INTERVALS ACROSS THE CHANNEL WIDTH, EAST FORK RIVER, WYOMING, 1980

SECTION 0516

\begin{tabular}{|c|c|c|c|c|c|c|c|}
\hline \multirow[t]{2}{*}{ DATE } & \multirow{2}{*}{$\begin{array}{c}\text { DRY } \\
\text { MASS } \\
\text { OF } \\
\text { SAMPLE (2) } \\
\text { (G) }\end{array}$} & \multicolumn{6}{|c|}{ NUMBER OF PINK TRACER PARTICLES } \\
\hline & & TOTAL & $\begin{array}{l}0.25 \\
\text { TO } \\
0.50\end{array}$ & $\begin{array}{l}0.50 \\
\text { TO } \\
1.00\end{array}$ & $\begin{array}{l}1.00 \\
\text { TO } \\
2.00\end{array}$ & $\begin{array}{l}2.00 \\
\text { TO } \\
4.00\end{array}$ & $\begin{array}{l}4.00 \\
8.00 \\
8.00\end{array}$ \\
\hline $\begin{array}{l}5-16 \\
5-19 \\
5-21 \\
5-22 \\
5-24\end{array}$ & $\begin{array}{c}238 \\
104++ \\
3254 \\
=- \\
--\end{array}$ & $\begin{array}{r}0 \\
1 \\
0 \\
---\end{array}$ & $\begin{array}{r}0 \\
1 \\
0 \\
-- \\
--\end{array}$ & $\begin{array}{r}0 \\
0 \\
0 \\
-- \\
--\end{array}$ & $\begin{array}{r}0 \\
0 \\
0 \\
-- \\
--\end{array}$ & $\begin{array}{r}0 \\
0 \\
0 \\
-- \\
--\end{array}$ & $\begin{array}{r}0 \\
0 \\
0 \\
-- \\
--\end{array}$ \\
\hline $\begin{array}{l}5-26 \\
5-27 \\
5-28 \\
5-29 \\
5-30\end{array}$ & $\begin{array}{c}1122 \\
45++ \\
177+ \\
-+ \\
40++\end{array}$ & $\begin{array}{r}0 \\
0 \\
0 \\
-- \\
3\end{array}$ & $\begin{array}{r}0 \\
0 \\
0 \\
-\frac{-}{3}\end{array}$ & $\begin{array}{r}0 \\
0 \\
0 \\
-- \\
0\end{array}$ & $\begin{array}{r}0 \\
0 \\
0 \\
-- \\
0\end{array}$ & $\begin{array}{r}0 \\
0 \\
0 \\
-- \\
0\end{array}$ & $\begin{array}{r}0 \\
0 \\
0 \\
-- \\
0\end{array}$ \\
\hline $\begin{array}{l}6-01 \\
6-03 \\
6-05 \\
6-07 \\
6-09\end{array}$ & $\begin{array}{r}17+++ \\
5+++ \\
20+++ \\
43++ \\
3573^{++}\end{array}$ & $\begin{array}{l}0 \\
0 \\
0 \\
0 \\
0\end{array}$ & $\begin{array}{l}0 \\
0 \\
0 \\
0 \\
0\end{array}$ & $\begin{array}{l}0 \\
0 \\
0 \\
0 \\
0\end{array}$ & $\begin{array}{l}0 \\
0 \\
0 \\
0 \\
0\end{array}$ & $\begin{array}{l}0 \\
0 \\
0 \\
0 \\
0\end{array}$ & $\begin{array}{l}0 \\
0 \\
0 \\
0 \\
0\end{array}$ \\
\hline $\begin{array}{l}6-10 \\
6-11 \\
6-12 \\
6-13 \\
6-14\end{array}$ & $\begin{array}{l}5819 \\
7742 \\
4199 \\
2145 \\
8739\end{array}$ & $\begin{array}{l}0 \\
1 \\
0 \\
0 \\
0\end{array}$ & $\begin{array}{l}0 \\
1 \\
0 \\
0 \\
0\end{array}$ & $\begin{array}{l}0 \\
0 \\
0 \\
0 \\
0\end{array}$ & $\begin{array}{l}0 \\
0 \\
0 \\
0 \\
0\end{array}$ & $\begin{array}{l}0 \\
0 \\
0 \\
0 \\
0\end{array}$ & $\begin{array}{l}0 \\
0 \\
0 \\
0 \\
0\end{array}$ \\
\hline $\begin{array}{l}6-15 \\
6-16 \\
6-17 \\
6-18 \\
6-19\end{array}$ & $\begin{array}{r}2323 \\
-- \\
739 \\
3247 \\
4233\end{array}$ & $\begin{array}{r}2 \\
-\frac{2}{0} \\
0 \\
2\end{array}$ & $\begin{array}{r}1 \\
-\frac{1}{0} \\
0 \\
0\end{array}$ & $\begin{array}{r}-\frac{1}{0} \\
0 \\
0 \\
2\end{array}$ & $\begin{array}{r}-\frac{0}{0} \\
0 \\
0 \\
0\end{array}$ & $\begin{array}{r}-0 \\
-0 \\
0 \\
0\end{array}$ & $\begin{array}{r}-0 \\
-0 \\
0 \\
0\end{array}$ \\
\hline $\begin{array}{l}6-20 \\
6-21 \\
6-22 \\
6-23 \\
6-25\end{array}$ & $\begin{array}{r}3247 \\
3537 \\
-- \\
1833 \\
2312\end{array}$ & $\begin{array}{r}1 \\
-\frac{1}{0} \\
0 \\
1\end{array}$ & $\begin{array}{r}1 \\
-\frac{1}{0} \\
0 \\
1\end{array}$ & $\begin{array}{r}0 \\
0 \\
-\frac{1}{0} \\
0\end{array}$ & $\begin{array}{r}0 \\
-0 \\
-0 \\
0\end{array}$ & $\begin{array}{r}0 \\
-0 \\
-\frac{1}{0} \\
0\end{array}$ & $\begin{array}{r}0 \\
-0 \\
-0 \\
0\end{array}$ \\
\hline $\begin{array}{l}6-27 \\
6-29 \\
7-01 \\
7-03 \\
7-05\end{array}$ & $\begin{array}{r}1577 \\
=- \\
=- \\
=-\end{array}$ & $\begin{array}{l}0 \\
-- \\
-- \\
--\end{array}$ & $\begin{array}{l}0 \\
-- \\
=- \\
-- \\
--\end{array}$ & $\begin{array}{l}0 \\
=- \\
=- \\
--\end{array}$ & $\begin{array}{l}0 \\
=- \\
=- \\
=-\end{array}$ & $\begin{array}{l}0 \\
-- \\
-- \\
--\end{array}$ & $\begin{array}{l}0 \\
=- \\
=- \\
--\end{array}$ \\
\hline $7-07$ & -- & -- & -- & -- & -- & -- & -- \\
\hline
\end{tabular}


TABLE 15.- NUMBER OF PINK TRACER PARTICLES(1), PER 100 GRAMS OF BEDLOAD SAMPLE, COMPOSITED FROM CROSS-CHANNEL SAMPLING POSITIONS SPACED AT I-METER INTERVALS ACROSS THE CHANNEL WIDTH, EAST FORK RIVER, WYOMING, 1980

SECTION 0556

\begin{tabular}{|c|c|c|c|c|c|c|c|}
\hline \multirow{3}{*}{ DATE } & \multirow{3}{*}{$\begin{array}{c}\text { DRY } \\
\text { MASS } \\
\text { OF } \\
\text { SAMPLE (2) } \\
(G)\end{array}$} & \multicolumn{6}{|c|}{ NUMBER OF PINK TRACER PARTICLES } \\
\hline & & \multirow[b]{2}{*}{ TOTAL } & \multicolumn{4}{|c|}{ BY SIZE CLASS (MM) } & \multirow[b]{2}{*}{$\begin{array}{l}4.00 \\
. \text { TO } \\
8.00\end{array}$} \\
\hline & & & $\begin{array}{l}0.25 \\
\text { TO } \\
0.50\end{array}$ & $\begin{array}{l}0.50 \\
\text { TO } \\
1.00\end{array}$ & $\begin{array}{l}1.00 \\
\text { TO } \\
2.00\end{array}$ & $\begin{array}{l}2.00 \\
\text { TO } \\
4.00\end{array}$ & \\
\hline $\begin{array}{l}5-16 \\
5-19 \\
5-21 \\
5-22 \\
5-24\end{array}$ & $\begin{array}{c}12+++ \\
272 \\
1800 \\
-- \\
--\end{array}$ & $\begin{array}{r}0 \\
1 \\
0 \\
-- \\
--\end{array}$ & $\begin{array}{r}0 \\
0 \\
0 \\
-- \\
--\end{array}$ & $\begin{array}{r}0 \\
1 \\
0 \\
-- \\
--\end{array}$ & $\begin{array}{r}0 \\
0 \\
0 \\
-- \\
--\end{array}$ & $\begin{array}{r}0 \\
0 \\
0 \\
-- \\
--\end{array}$ & $\begin{array}{r}0 \\
0 \\
0 \\
-- \\
--\end{array}$ \\
\hline $\begin{array}{l}5-26 \\
5-27 \\
5-28\end{array}$ & $\begin{array}{l}840 \\
58+ \\
207\end{array}$ & $\begin{array}{l}0 \\
0 \\
0\end{array}$ & $\begin{array}{l}0 \\
0 \\
0\end{array}$ & $\begin{array}{l}0 \\
0 \\
0\end{array}$ & $\begin{array}{l}0 \\
0 \\
0\end{array}$ & $\begin{array}{l}0 \\
0 \\
0\end{array}$ & $\begin{array}{l}0 \\
0 \\
0\end{array}$ \\
\hline $\begin{array}{l}5-29 \\
5-30\end{array}$ & $\overline{62++}$ & $-\overline{0}$ & $\overline{0}$ & $-\overline{0}$ & $-\overline{0}$ & $-\overline{0}$ & $-\overline{0}$ \\
\hline $\begin{array}{l}6-01 \\
6-03 \\
6-05 \\
6-07 \\
6-09\end{array}$ & $\begin{array}{c}77++ \\
53++ \\
93+ \\
103+ \\
5100^{+}\end{array}$ & $\begin{array}{l}0 \\
2 \\
0 \\
1 \\
0\end{array}$ & $\begin{array}{l}0 \\
2 \\
0 \\
1 \\
0\end{array}$ & $\begin{array}{l}0 \\
0 \\
0 \\
0 \\
0\end{array}$ & $\begin{array}{l}0 \\
0 \\
0 \\
0 \\
0\end{array}$ & $\begin{array}{l}0 \\
0 \\
0 \\
0 \\
0\end{array}$ & $\begin{array}{l}0 \\
0 \\
0 \\
0 \\
0\end{array}$ \\
\hline $\begin{array}{l}6-10 \\
6-11 \\
6-12 \\
6-13 \\
6-14\end{array}$ & $\begin{array}{l}5747 \\
2974 \\
9117 \\
3194 \\
3427\end{array}$ & $\begin{array}{l}0 \\
1 \\
1 \\
0 \\
1\end{array}$ & $\begin{array}{l}0 \\
0 \\
1 \\
0 \\
1\end{array}$ & $\begin{array}{l}0 \\
1 \\
0 \\
0 \\
0\end{array}$ & $\begin{array}{l}0 \\
0 \\
0 \\
0 \\
0\end{array}$ & $\begin{array}{l}0 \\
0 \\
0 \\
0 \\
0\end{array}$ & $\begin{array}{l}0 \\
0 \\
0 \\
0 \\
0\end{array}$ \\
\hline $6-15$ & 1217 & 2 & 0 & 2 & 0 & 0 & 0 \\
\hline $\begin{array}{l}6-17 \\
6-18 \\
6-19\end{array}$ & $\begin{array}{l}1354 \\
3938 \\
2389\end{array}$ & $\begin{array}{l}1 \\
1 \\
2\end{array}$ & $\begin{array}{l}0 \\
1 \\
2\end{array}$ & $\begin{array}{l}1 \\
0 \\
0\end{array}$ & $\begin{array}{l}0 \\
0 \\
0\end{array}$ & $\begin{array}{l}0 \\
0 \\
0\end{array}$ & $\begin{array}{l}0 \\
0 \\
0\end{array}$ \\
\hline $\begin{array}{l}6-20 \\
6-21\end{array}$ & $\begin{array}{l}5650 \\
2083\end{array}$ & $\begin{array}{l}1 \\
0\end{array}$ & $\begin{array}{l}1 \\
0\end{array}$ & $\begin{array}{l}0 \\
0\end{array}$ & $\begin{array}{l}0 \\
0\end{array}$ & $\begin{array}{l}0 \\
0\end{array}$ & $\begin{array}{l}0 \\
0\end{array}$ \\
\hline $\begin{array}{l}6-22 \\
6-23 \\
6-25\end{array}$ & $\begin{array}{r}3676 \\
872\end{array}$ & $\begin{array}{l}0 \\
3\end{array}$ & $\begin{array}{l}0 \\
2\end{array}$ & $\begin{array}{l}0 \\
1\end{array}$ & $\begin{array}{l}0 \\
0\end{array}$ & $\begin{array}{l}0 \\
0\end{array}$ & $\begin{array}{l}0 \\
0\end{array}$ \\
\hline $6-27$ & 2150 & 6 & 6 & 0 & 0 & 0 & 0 \\
\hline $\begin{array}{l}6-29 \\
7-01\end{array}$ & $\overline{--}$ & $\overline{--}$ & $\overline{--}$ & $\overline{--}$ & $\overline{--}$ & $\overline{--}$ & $\overline{--}$ \\
\hline $7-0 \frac{1}{3}$ & -- & -- & -- & -- & -- & -- & -- \\
\hline $7-05$ & -- & -- & -- & - & & - & -- \\
\hline $7-07$ & -- & -- & -- & -- & -- & -- & -- \\
\hline
\end{tabular}


TABLE 15.- NUMBER OF PINK TRACER PARTICLES(1), PER 100 GRAMS OF BEDLOAD SAMPLE, COMPOSITED FROM CROSS-CHANNEL SAMPLING POSITIONS SPACED AT I-METER INTERVALS ACROSS THE CHANNEL WIDTH, EAST FORK RIVER, WYOMING, 1980

SECTION 0602

\begin{tabular}{|c|c|c|c|c|c|c|c|}
\hline \multirow{3}{*}{ DATE } & \multirow{3}{*}{$\begin{array}{c}\text { DRY } \\
\text { MASS } \\
\text { OF } \\
\text { SAMPLE (2) } \\
(G)\end{array}$} & \multicolumn{6}{|c|}{ NUMBER OF PINK TRACER PARTICLES } \\
\hline & & \multirow[b]{2}{*}{ TOTAL } & \multicolumn{4}{|c|}{ BY SIZE CLASS (MM) } & \\
\hline & & & $\begin{array}{l}0.25 \\
. \mathrm{TO} \\
0.50\end{array}$ & $\begin{array}{l}0.50 \\
\text { TO } \\
1.00\end{array}$ & $\begin{array}{l}1.00 \\
\text { TO } \\
2.00\end{array}$ & $\begin{array}{l}2.00 \\
\text { TO } \\
4.00\end{array}$ & $\begin{array}{l}4.00 \\
\text { TO } \\
8.00\end{array}$ \\
\hline $\begin{array}{l}5-16 \\
5-19 \\
5-21 \\
5-22 \\
5-24\end{array}$ & $\begin{array}{r}136 \\
1010 \\
1054 \\
--\end{array}$ & $\begin{array}{r}3 \\
0 \\
1 \\
-- \\
--\end{array}$ & $\begin{array}{r}3 \\
0 \\
1 \\
--\end{array}$ & $\begin{array}{r}0 \\
0 \\
0 \\
--\end{array}$ & $\begin{array}{r}0 \\
0 \\
0 \\
-- \\
--\end{array}$ & $\begin{array}{r}0 \\
0 \\
0 \\
-- \\
--\end{array}$ & $\begin{array}{r}0 \\
0 \\
0 \\
-- \\
--\end{array}$ \\
\hline $\begin{array}{l}5-26 \\
5-27 \\
5-28\end{array}$ & $\begin{array}{c}1299 \\
167+ \\
112\end{array}$ & $\begin{array}{l}0 \\
0 \\
0\end{array}$ & $\begin{array}{l}0 \\
0 \\
0\end{array}$ & $\begin{array}{l}0 \\
0 \\
0\end{array}$ & $\begin{array}{l}0 \\
0 \\
0\end{array}$ & $\begin{array}{l}0 \\
0 \\
0\end{array}$ & $\begin{array}{l}0 \\
0 \\
0\end{array}$ \\
\hline $5-30$ & 474 & 6 & 5 & 1 & 0 & 0 & 0 \\
\hline $\begin{array}{l}6-01 \\
6-03 \\
6-05 \\
6-07 \\
6-09\end{array}$ & $\begin{array}{r}56+ \\
162+ \\
921 \\
1320 \\
5773\end{array}$ & $\begin{array}{l}4 \\
0 \\
0 \\
0 \\
0\end{array}$ & $\begin{array}{l}4 \\
0 \\
0 \\
0 \\
0\end{array}$ & $\begin{array}{l}0 \\
0 \\
0 \\
0 \\
0\end{array}$ & $\begin{array}{l}0 \\
0 \\
0 \\
0 \\
0\end{array}$ & $\begin{array}{l}0 \\
0 \\
0 \\
0 \\
0\end{array}$ & $\begin{array}{l}0 \\
0 \\
0 \\
0 \\
0\end{array}$ \\
\hline $\begin{array}{l}6-10 \\
6-11 \\
6-12 \\
6-13 \\
6-14\end{array}$ & $\begin{array}{l}4233 \\
5076 \\
2864 \\
3765 \\
2492\end{array}$ & $\begin{array}{l}0 \\
1 \\
0 \\
0 \\
0\end{array}$ & $\begin{array}{l}0 \\
1 \\
0 \\
0 \\
0\end{array}$ & $\begin{array}{l}0 \\
0 \\
0 \\
0 \\
0\end{array}$ & $\begin{array}{l}0 \\
0 \\
0 \\
0 \\
0\end{array}$ & $\begin{array}{l}0 \\
0 \\
0 \\
0 \\
0\end{array}$ & $\begin{array}{l}0 \\
0 \\
0 \\
0 \\
0\end{array}$ \\
\hline $6-15$ & 1838 & 1 & 1 & 0 & 0 & 0 & 0 \\
\hline $\begin{array}{l}6-17 \\
6-18 \\
6-19\end{array}$ & $\begin{array}{l}2006 \\
1637 \\
5043\end{array}$ & $\begin{array}{l}8 \\
1 \\
1\end{array}$ & $\begin{array}{l}6 \\
0 \\
0\end{array}$ & $\begin{array}{l}2 \\
1 \\
1\end{array}$ & $\begin{array}{l}0 \\
0 \\
0\end{array}$ & $\begin{array}{l}0 \\
0 \\
0\end{array}$ & $\begin{array}{l}0 \\
0 \\
0\end{array}$ \\
\hline $\begin{array}{l}6-20 \\
6-21\end{array}$ & $\begin{array}{l}7174 \\
2827\end{array}$ & $\begin{array}{l}0 \\
0\end{array}$ & $\begin{array}{l}0 \\
0\end{array}$ & $\begin{array}{l}0 \\
0\end{array}$ & $\begin{array}{l}0 \\
0\end{array}$ & $\begin{array}{l}0 \\
0\end{array}$ & $\begin{array}{l}0 \\
0\end{array}$ \\
\hline $\begin{array}{l}6-23 \\
6-25\end{array}$ & $\begin{array}{l}3194 \\
4332\end{array}$ & $\begin{array}{l}2 \\
1\end{array}$ & $\frac{1}{1}$ & $\begin{array}{l}1 \\
0\end{array}$ & $\begin{array}{l}0 \\
0\end{array}$ & $\begin{array}{l}0 \\
0\end{array}$ & $\begin{array}{l}0 \\
0\end{array}$ \\
\hline $6-27$ & 1490 & 2 & 2 & 0 & 0 & 0 & 0 \\
\hline $\begin{array}{l}0-29 \\
7-01\end{array}$ & $=$ & $=$ & $=$ & $=$ & $=$ & $=$ & $=$ \\
\hline $7-0 \overline{3}$ & -- & -- & - & - & -- & -- & -- \\
\hline $7-05$ & -- & -- & -- & -- & - & -- & -- \\
\hline $7-07$ & -- & -- & -- & -- & -- & -- & -- \\
\hline
\end{tabular}


TABLE 15.- NUMBER OF PINK TRACER PARTICLES(1), PER 100 GRAMS OF BEDLOAD SAMPLE, COMPOSITED FROM CROSS-CHANNEL SAMPLING POSITIONS SPACED AT 1-METER INTERVALS ACROSS THE CHANNEL WIDTH, EAST FORK RIVER, WYOMING, 1980

SECTION 0653

\begin{tabular}{|c|c|c|c|c|c|c|c|}
\hline \multirow{3}{*}{ DATE } & \multirow{3}{*}{$\begin{array}{c}\text { DRY } \\
\text { MASS } \\
\text { OF } \\
\text { SAMPLE (2) } \\
\text { (G) }\end{array}$} & \multicolumn{6}{|c|}{ NUMBER OF PINK TRACER PARTICLES } \\
\hline & & \multirow[b]{2}{*}{ TOTAL } & \multicolumn{5}{|c|}{ BY SIZE CLASS (MM) } \\
\hline & & & $\begin{array}{c}0.25 \\
\text { TO } \\
0.50\end{array}$ & $\begin{array}{l}0.50 \\
\text { TO } \\
1.00\end{array}$ & $\begin{array}{l}1.00 \\
2.00 \\
2.00\end{array}$ & $\begin{array}{l}2.00 \\
\text { TO } \\
4.00\end{array}$ & $\begin{array}{c}4.00 \\
\text { TOO } \\
8.00\end{array}$ \\
\hline $\begin{array}{l}5-16 \\
5-19 \\
5-21 \\
5-22\end{array}$ & $\begin{array}{l}148+ \\
168+ \\
153+\end{array}$ & $\begin{array}{r}0 \\
0 \\
0 \\
--\end{array}$ & $\begin{array}{r}0 \\
0 \\
0 \\
--\end{array}$ & $\begin{array}{r}0 \\
0 \\
0 \\
--\end{array}$ & $\begin{array}{r}0 \\
0 \\
0 \\
-\end{array}$ & $\begin{array}{r}0 \\
0 \\
0 \\
--\end{array}$ & $\begin{array}{r}0 \\
0 \\
0 \\
--\end{array}$ \\
\hline $5-24$ & -- & - & - & - & -- & -- & -- \\
\hline $\begin{array}{l}5-26 \\
5-27 \\
5-28\end{array}$ & $\begin{array}{l}393 \\
491 \\
108+\end{array}$ & $\begin{array}{l}0 \\
0 \\
0\end{array}$ & $\begin{array}{l}0 \\
0 \\
0\end{array}$ & $\begin{array}{l}0 \\
0 \\
0\end{array}$ & $\begin{array}{l}0 \\
0 \\
0\end{array}$ & $\begin{array}{l}0 \\
0 \\
0\end{array}$ & $\begin{array}{l}0 \\
0 \\
0\end{array}$ \\
\hline $\begin{array}{l}5-29 \\
5-30\end{array}$ & $\overline{83}++$ & $\overline{0}$ & $-\overline{0}$ & $\overline{0}$ & $\overline{0}$ & $-\overline{0}$ & $\overline{0}$ \\
\hline $\begin{array}{l}6-01 \\
6-03 \\
6-05 \\
6-07 \\
6-09\end{array}$ & $\begin{array}{r}372 \\
439 \\
490 \\
2330 \\
3768\end{array}$ & $\begin{array}{l}1 \\
0 \\
0 \\
0 \\
0\end{array}$ & $\begin{array}{l}1 \\
0 \\
0 \\
0 \\
0\end{array}$ & $\begin{array}{l}0 \\
0 \\
0 \\
0 \\
0\end{array}$ & $\begin{array}{l}0 \\
0 \\
0 \\
0 \\
0\end{array}$ & $\begin{array}{l}0 \\
0 \\
0 \\
0 \\
0\end{array}$ & $\begin{array}{l}0 \\
0 \\
0 \\
0 \\
0\end{array}$ \\
\hline $\begin{array}{l}6-10 \\
6-11 \\
6-12 \\
6-13 \\
6-14\end{array}$ & $\begin{array}{r}15664 \\
3692 \\
348 \\
427 \\
257\end{array}$ & $\begin{array}{l}0 \\
1 \\
1 \\
7 \\
8\end{array}$ & $\begin{array}{l}0 \\
1 \\
1 \\
6 \\
4\end{array}$ & $\begin{array}{l}0 \\
0 \\
0 \\
1 \\
4\end{array}$ & $\begin{array}{l}0 \\
0 \\
0 \\
0 \\
0\end{array}$ & $\begin{array}{l}0 \\
0 \\
0 \\
0 \\
0\end{array}$ & $\begin{array}{l}0 \\
0 \\
0 \\
0 \\
0\end{array}$ \\
\hline $\begin{array}{l}6-15 \\
6-16\end{array}$ & 622 & 5 & 1 & -2 & $\underline{0}$ & 0 & 0 \\
\hline $\begin{array}{l}6-17 \\
6-18 \\
6-19\end{array}$ & $\begin{array}{l}2105 \\
3735 \\
4034\end{array}$ & $\begin{array}{l}3 \\
2 \\
0\end{array}$ & $\begin{array}{l}3 \\
1 \\
0\end{array}$ & $\begin{array}{l}0 \\
1 \\
0\end{array}$ & $\begin{array}{l}0 \\
0 \\
0\end{array}$ & $\begin{array}{l}0 \\
0 \\
0\end{array}$ & $\begin{array}{l}0 \\
0 \\
0\end{array}$ \\
\hline $\begin{array}{l}6-20 \\
6-21\end{array}$ & $\begin{array}{l}1922 \\
2686\end{array}$ & $\frac{1}{0}$ & $\begin{array}{l}0 \\
0\end{array}$ & $\frac{1}{0}$ & $\begin{array}{l}0 \\
0\end{array}$ & $\begin{array}{l}0 \\
0\end{array}$ & $\begin{array}{l}0 \\
0\end{array}$ \\
\hline $\begin{array}{l}6-23 \\
6-25\end{array}$ & $\begin{array}{l}1278 \\
1894\end{array}$ & 17 & $\frac{1}{0}$ & $\begin{array}{r}0 \\
13\end{array}$ & $\begin{array}{l}0 \\
3\end{array}$ & $\begin{array}{l}0 \\
1\end{array}$ & $\begin{array}{l}0 \\
0\end{array}$ \\
\hline $6-27$ & 2210 & 3 & 0 & 3 & 0 & 0 & 0 \\
\hline $\begin{array}{l}6-29 \\
7-01\end{array}$ & $\overline{--}$ & $\overline{--}$ & $\overline{--}$ & $\overline{--}$ & $\overline{--}$ & $\overline{--}$ & $\overline{--}$ \\
\hline $7-03$ & -- & -- & -- & -- & - & - & -- \\
\hline $7-05$ & - & -- & -- & -- & -- & -- & -- \\
\hline $7-07$ & -- & -- & -- & -- & -- & -- & -- \\
\hline
\end{tabular}


TABLE 15.- NUMBER OF PINK TRACER PARTICLES(1), PER 100 GRAMS OF BEDLOAD SAMPLE, COMPOSITED FROM CROSS-CHANNEL SAMPLING POSITIONS SPACED AT 1-METER INTERVALS ACROSS THE CHANNEL WIDTH, EAST FORK RIVER, WYOMING, 1980

SECTION 0653(3)

\begin{tabular}{|c|c|c|c|c|c|c|c|}
\hline \multirow[t]{2}{*}{ DATE } & \multirow{2}{*}{$\begin{array}{c}\text { DRY } \\
\text { MASS } \\
\text { OF } \\
\text { SAMPLE (2) } \\
\text { (G) }\end{array}$} & \multicolumn{6}{|c|}{$\begin{array}{l}\text { NUMBER OF PINK TRACER PARTICLES } \\
\text { BY SIZE CLASS (MM) }\end{array}$} \\
\hline & & TOTAL & $\begin{array}{l}0.25 \\
\text { TO } \\
0.50\end{array}$ & $\begin{array}{l}0.50 \\
\text { TO } \\
1.00\end{array}$ & $\begin{array}{l}1.00 \\
2.00\end{array}$ & $\begin{array}{l}2.00 \\
400\end{array}$ & $\begin{array}{l}4.00 \\
\text { TO } \\
8.00\end{array}$ \\
\hline $\begin{array}{l}5-16 \\
5-19 \\
5-21 \\
5-22 \\
5-24\end{array}$ & $\begin{array}{l}-- \\
-- \\
--\end{array}$ & $\begin{array}{l}-- \\
z- \\
z- \\
--\end{array}$ & $\begin{array}{l}-- \\
=- \\
--\end{array}$ & $\begin{array}{l}-- \\
-- \\
-- \\
--\end{array}$ & $\begin{array}{l}-- \\
-- \\
-- \\
--\end{array}$ & $\begin{array}{l}-- \\
-- \\
-- \\
--\end{array}$ & $\begin{array}{l}=- \\
=- \\
=- \\
--\end{array}$ \\
\hline $\begin{array}{l}5-26 \\
5-27 \\
5-28 \\
5-29 \\
5-30\end{array}$ & $\begin{array}{l}=- \\
=- \\
=-\end{array}$ & $\begin{array}{l}-- \\
=- \\
=-\end{array}$ & $\begin{array}{l}=- \\
=- \\
z-\end{array}$ & $\begin{array}{l}-- \\
=- \\
=- \\
--\end{array}$ & $\begin{array}{l}-- \\
=- \\
=- \\
--\end{array}$ & $\begin{array}{l}-- \\
-- \\
-- \\
--\end{array}$ & $\begin{array}{l}-- \\
=- \\
=- \\
--\end{array}$ \\
\hline $\begin{array}{l}6-01 \\
6-03 \\
6-05 \\
6-07 \\
6-09\end{array}$ & $\begin{array}{l}\overline{-} \\
\overline{-5} \\
\overline{61+}\end{array}$ & $\begin{array}{l}-- \\
-- \\
-- \\
0\end{array}$ & $\begin{array}{l}-- \\
=- \\
-\overline{0}\end{array}$ & $\begin{array}{l}=- \\
=- \\
-\overline{0}\end{array}$ & $\begin{array}{l}-- \\
\overline{--} \\
-\overline{0}\end{array}$ & $\begin{array}{l}-- \\
-- \\
-\overline{0}\end{array}$ & $\begin{array}{l}-- \\
-- \\
-- \\
-\overline{0}\end{array}$ \\
\hline $\begin{array}{l}6-10 \\
6-11 \\
6-12 \\
6-13 \\
6-14\end{array}$ & $\begin{array}{l}283 \\
26- \\
266 \\
24+++ \\
190\end{array}$ & $\begin{array}{r}-0 \\
-0 \\
4 \\
6\end{array}$ & $\begin{array}{r}-\frac{0}{0} \\
4 \\
6\end{array}$ & $\begin{array}{r}-0 \\
-0 \\
0 \\
0\end{array}$ & $\begin{array}{r}0 \\
-\frac{0}{0} \\
0 \\
0\end{array}$ & $\begin{array}{r}0 \\
- \\
0 \\
0 \\
0\end{array}$ & $\begin{array}{r}0 \\
-\frac{0}{0} \\
0 \\
0\end{array}$ \\
\hline $\begin{array}{l}6-15 \\
6-16 \\
6-17 \\
6-18 \\
6-19\end{array}$ & \begin{tabular}{l}
$33++$ \\
\hdashline- \\
$38++$ \\
$0+++$
\end{tabular} & $\begin{array}{r}0 \\
-- \\
-0 \\
0\end{array}$ & $\begin{array}{c}0 \\
-- \\
-0 \\
0\end{array}$ & $\begin{array}{c}0 \\
-- \\
-0 \\
0\end{array}$ & $\begin{array}{c}0 \\
-- \\
-\overline{0} \\
0\end{array}$ & $\begin{array}{c}0 \\
-- \\
-0 \\
0\end{array}$ & $\begin{array}{r}0 \\
-- \\
-0 \\
0\end{array}$ \\
\hline $\begin{array}{l}6-20 \\
6-21 \\
6-22 \\
6-23 \\
6-25\end{array}$ & $\begin{array}{l}371 \\
71+ \\
\overline{--} \\
22+++\end{array}$ & $\begin{array}{r}5 \\
0 \\
-- \\
-0\end{array}$ & $\frac{1}{0}$ & $\begin{array}{r}4 \\
-\frac{1}{0} \\
-\overline{0}\end{array}$ & $\begin{array}{r}0 \\
0 \\
-- \\
-0\end{array}$ & $\begin{array}{r}0 \\
0 \\
-- \\
-0\end{array}$ & $\begin{array}{r}0 \\
0 \\
-- \\
-0\end{array}$ \\
\hline $\begin{array}{l}6-27 \\
6-29 \\
7-01 \\
7-03 \\
7-05\end{array}$ & $\begin{array}{l}6+++ \\
-- \\
-- \\
--\end{array}$ & $\begin{array}{l}0 \\
-- \\
-- \\
--\end{array}$ & $\begin{array}{l}0 \\
-- \\
=- \\
--\end{array}$ & $\begin{array}{l}0 \\
-- \\
-- \\
-- \\
--\end{array}$ & $\begin{array}{l}0 \\
-- \\
-- \\
--\end{array}$ & $\begin{array}{l}0 \\
-- \\
-- \\
--\end{array}$ & $\begin{array}{l}0 \\
-- \\
-- \\
--\end{array}$ \\
\hline $7-07$ & -- & -- & -- & -- & -- & -- & -- \\
\hline
\end{tabular}


TABLE 15.- NUMBER OF PINK TRACER PARTICLES(1), PER 100 GRAMS OF BEDLOAD SAMPLE, COMPOSITED FROM CROSS-CHANNEL SAMPLING POSITIONS SPACED AT 1-METER INTERVALS ACROSS THE CHANNEL WIDTH, EAST FORK RIVER, WYOMING, 1980

SECTION 0708

\begin{tabular}{|c|c|c|c|c|c|c|c|}
\hline \multirow{3}{*}{ DATE } & \multirow{3}{*}{$\begin{array}{c}\text { DRY } \\
\text { MASS } \\
\text { OF } \\
\text { SAMPLE (2) } \\
(G)\end{array}$} & \multicolumn{2}{|c|}{ NUMBER 0} & \multicolumn{3}{|c|}{ PINK TRACER PARTICLES } & \\
\hline & & \multirow[b]{2}{*}{ TOTAL } & \multicolumn{5}{|c|}{ BY SIZE CLASS (MM) } \\
\hline & & & $\begin{array}{l}0.25 \\
\text { TO } \\
0.50\end{array}$ & $\begin{array}{l}0.50 \\
\text { TO } \\
1.00\end{array}$ & $\begin{array}{l}1.00 \\
2.00 \\
2.00\end{array}$ & $\begin{array}{l}2.00 \\
\text { TO } \\
4.00\end{array}$ & $\begin{array}{l}4.00 \\
\text { TO } \\
8.00\end{array}$ \\
\hline $\begin{array}{l}5-16 \\
5-19 \\
5-21 \\
5-22\end{array}$ & $\begin{array}{r}841 \\
395 \\
3726 \\
\end{array}$ & $\begin{array}{r}0 \\
0 \\
0 \\
--\end{array}$ & $\begin{array}{r}0 \\
0 \\
0 \\
-\end{array}$ & $\begin{array}{r}0 \\
0 \\
0 \\
--\end{array}$ & $\begin{array}{r}0 \\
0 \\
0 \\
--\end{array}$ & $\begin{array}{r}0 \\
0 \\
0 \\
-\end{array}$ & $\begin{array}{r}0 \\
0 \\
0 \\
--\end{array}$ \\
\hline $5-24$ & -- & -- & -- & -- & -- & -- & -- \\
\hline $\begin{array}{l}5-26 \\
5-27 \\
5-28 \\
5-29 \\
5-30\end{array}$ & $\begin{array}{l}513 \\
215 \\
288 \\
422 \\
88+\end{array}$ & $\begin{array}{l}0 \\
0 \\
3 \\
0 \\
1\end{array}$ & $\begin{array}{l}0 \\
0 \\
3 \\
0 \\
0\end{array}$ & $\begin{array}{l}0 \\
0 \\
0 \\
0 \\
0\end{array}$ & $\begin{array}{l}0 \\
0 \\
0 \\
0 \\
1\end{array}$ & $\begin{array}{l}0 \\
0 \\
0 \\
0 \\
0\end{array}$ & $\begin{array}{l}0 \\
0 \\
0 \\
0 \\
0\end{array}$ \\
\hline $\begin{array}{l}6-01 \\
6-03 \\
6-05 \\
6-07 \\
6-09\end{array}$ & $\begin{array}{c}39++ \\
909 \\
702 \\
1550 \\
3638\end{array}$ & $\begin{array}{l}3 \\
0 \\
0 \\
0 \\
0\end{array}$ & $\begin{array}{l}3 \\
0 \\
0 \\
0 \\
0\end{array}$ & $\begin{array}{l}0 \\
0 \\
0 \\
0 \\
0\end{array}$ & $\begin{array}{l}0 \\
0 \\
0 \\
0 \\
0\end{array}$ & $\begin{array}{l}0 \\
0 \\
0 \\
0 \\
0\end{array}$ & $\begin{array}{l}0 \\
0 \\
0 \\
0 \\
0\end{array}$ \\
\hline $\begin{array}{l}6-10 \\
6-11 \\
6-12 \\
6-13 \\
6-14\end{array}$ & $\begin{array}{r}7438 \\
1025 \\
583 \\
1330 \\
2343\end{array}$ & $\begin{array}{l}0 \\
2 \\
0 \\
3 \\
0\end{array}$ & $\begin{array}{l}0 \\
2 \\
0 \\
3 \\
0\end{array}$ & $\begin{array}{l}0 \\
0 \\
0 \\
0 \\
0\end{array}$ & $\begin{array}{l}0 \\
0 \\
0 \\
0 \\
0\end{array}$ & $\begin{array}{l}0 \\
0 \\
0 \\
0 \\
0\end{array}$ & $\begin{array}{l}0 \\
0 \\
0 \\
0 \\
0\end{array}$ \\
\hline $\begin{array}{l}6-15 \\
6-16 \\
6-17 \\
6-18 \\
6-19\end{array}$ & $\begin{array}{r}862 \\
884 \\
1457 \\
3737 \\
3122\end{array}$ & $\begin{array}{l}1 \\
0 \\
1 \\
0 \\
0\end{array}$ & $\begin{array}{l}1 \\
0 \\
0 \\
0 \\
0\end{array}$ & $\begin{array}{l}0 \\
0 \\
1 \\
0 \\
0\end{array}$ & $\begin{array}{l}0 \\
0 \\
0 \\
0 \\
0\end{array}$ & $\begin{array}{l}0 \\
0 \\
0 \\
0 \\
0\end{array}$ & $\begin{array}{l}0 \\
0 \\
0 \\
0 \\
0\end{array}$ \\
\hline $\begin{array}{l}6-20 \\
6-21\end{array}$ & $\begin{array}{r}10382 \\
1174\end{array}$ & $\begin{array}{l}0 \\
1\end{array}$ & $\begin{array}{l}0 \\
0\end{array}$ & $\begin{array}{l}0 \\
1\end{array}$ & $\begin{array}{l}0 \\
0\end{array}$ & $\begin{array}{l}0 \\
0\end{array}$ & $\begin{array}{l}0 \\
0\end{array}$ \\
\hline $\begin{array}{l}6-23 \\
6-25\end{array}$ & $\begin{array}{l}997 \\
721\end{array}$ & $\begin{array}{l}4 \\
0\end{array}$ & $\begin{array}{l}1 \\
0\end{array}$ & $\begin{array}{l}3 \\
0\end{array}$ & $\begin{array}{l}0 \\
0\end{array}$ & $\begin{array}{l}0 \\
0\end{array}$ & $\begin{array}{l}0 \\
0\end{array}$ \\
\hline $6-27$ & 2107 & 3 & 3 & 0 & 0 & 0 & 0 \\
\hline $7-01$ & $=$ & -- & $\overline{--}$ & $\overline{--}$ & $\overline{--}$ & - & - \\
\hline $\begin{array}{l}7-03 \\
7-05\end{array}$ & $\overline{--}$ & $\overline{--}$ & $\overline{--}$ & $=$ & $\overline{--}$ & $\overline{--}$ & $\overline{--}$ \\
\hline $7-07$ & -- & -- & -- & - & -- & -- & -- \\
\hline
\end{tabular}


TABLE 15.- NUMBER OF PINK TRACER PARTICLES(1), PER 100 GRAMS OF BEDLOAD SAMPLE, COMPOSITED FROM CROSS-CHANNEL SAMPLING POSITIONS

SPACED AT 1-METER INTERVALS ACROSS THE CHANNEL WIDTH,

EAST FORK RIVER, WYOMING, 1980

SECTION 0757

\begin{tabular}{|c|c|c|c|c|c|c|c|}
\hline \multirow{3}{*}{ DATE } & \multirow{3}{*}{$\begin{array}{c}\text { DRY } \\
\text { MASS } \\
\text { OF } \\
\text { SAMPLE (2) } \\
\text { (G) }\end{array}$} & \multicolumn{6}{|c|}{ NUMBER OF PINK TRACER PARTICLES } \\
\hline & & \multirow[b]{2}{*}{ TOTAL } & \multicolumn{5}{|c|}{ BY SIZE CLASS (MM) } \\
\hline & & & $\begin{array}{l}0.25 \\
\text { TO } \\
0.50\end{array}$ & $\begin{array}{l}0.50 \\
\text { TO } \\
1.00\end{array}$ & $\begin{array}{l}1.00 \\
2.00\end{array}$ & $\begin{array}{l}2.00 \\
\text { TO } \\
4.00\end{array}$ & $\begin{array}{l}4.00 \\
\text { TO } \\
8.00\end{array}$ \\
\hline $\begin{array}{l}5-16 \\
5-19 \\
5-21\end{array}$ & $\begin{array}{l}450 \\
463 \\
613\end{array}$ & $\begin{array}{l}0 \\
0 \\
3\end{array}$ & $\begin{array}{l}0 \\
0 \\
3\end{array}$ & $\begin{array}{l}0 \\
0 \\
0\end{array}$ & $\begin{array}{l}0 \\
0 \\
0\end{array}$ & $\begin{array}{l}0 \\
0 \\
0\end{array}$ & $\begin{array}{l}0 \\
0 \\
0\end{array}$ \\
\hline $\begin{array}{l}5-22 \\
5-24\end{array}$ & $=$ & 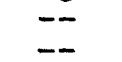 & -- & $\overline{-}$ & -- & 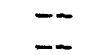 & $\overline{--}$ \\
\hline $\begin{array}{l}5-26 \\
5-27 \\
5-28\end{array}$ & $\begin{array}{l}6427 \\
3063 \\
1324\end{array}$ & $\begin{array}{l}0 \\
0 \\
0\end{array}$ & $\begin{array}{l}0 \\
0 \\
0\end{array}$ & $\begin{array}{l}0 \\
0 \\
0\end{array}$ & $\begin{array}{l}0 \\
0 \\
0\end{array}$ & $\begin{array}{l}0 \\
0 \\
0\end{array}$ & $\begin{array}{l}0 \\
0 \\
0\end{array}$ \\
\hline $5-30$ & 1695 & 0 & 0 & 0 & 0 & 0 & 0 \\
\hline $\begin{array}{l}6-01 \\
6-03 \\
6-05 \\
6-07 \\
6-09\end{array}$ & $\begin{array}{c}1578+ \\
448 \\
384 \\
501 \\
716\end{array}$ & $\begin{array}{l}0 \\
0 \\
0 \\
0 \\
0\end{array}$ & $\begin{array}{l}0 \\
0 \\
0 \\
0 \\
0\end{array}$ & $\begin{array}{l}0 \\
0 \\
0 \\
0 \\
0\end{array}$ & $\begin{array}{l}0 \\
0 \\
0 \\
0 \\
0\end{array}$ & $\begin{array}{l}0 \\
0 \\
0 \\
0 \\
0\end{array}$ & $\begin{array}{l}0 \\
0 \\
0 \\
0 \\
0\end{array}$ \\
\hline $\begin{array}{l}6-10 \\
6-11 \\
6-12 \\
6-13 \\
6-14\end{array}$ & $\begin{array}{l}68+ \\
320 \\
2425 \\
1014 \\
3202\end{array}$ & $\begin{array}{r}0 \\
19 \\
10 \\
6 \\
2\end{array}$ & $\begin{array}{r}0 \\
18 \\
8 \\
5 \\
2\end{array}$ & $\begin{array}{l}0 \\
1 \\
2 \\
1 \\
0\end{array}$ & $\begin{array}{l}0 \\
0 \\
0 \\
0 \\
0\end{array}$ & $\begin{array}{l}0 \\
0 \\
0 \\
0 \\
0\end{array}$ & $\begin{array}{l}0 \\
0 \\
0 \\
0 \\
0\end{array}$ \\
\hline $\begin{array}{l}6-15 \\
6-16 \\
6-17 \\
6-18 \\
6-19\end{array}$ & $\begin{array}{l}1515 \\
3208 \\
5436 \\
6123 \\
2003\end{array}$ & $\begin{array}{l}1 \\
0 \\
1 \\
0 \\
0\end{array}$ & $\begin{array}{l}0 \\
0 \\
0 \\
0 \\
0\end{array}$ & $\begin{array}{l}1 \\
0 \\
1 \\
0 \\
0\end{array}$ & $\begin{array}{l}0 \\
0 \\
0 \\
0 \\
0\end{array}$ & $\begin{array}{l}0 \\
0 \\
0 \\
0 \\
0\end{array}$ & $\begin{array}{l}0 \\
0 \\
0 \\
0 \\
0\end{array}$ \\
\hline $\begin{array}{l}6-20 \\
6-21\end{array}$ & $\begin{array}{l}2586 \\
1847\end{array}$ & $\begin{array}{l}2 \\
I\end{array}$ & $\begin{array}{l}2 \\
0\end{array}$ & $\begin{array}{l}0 \\
1\end{array}$ & $\begin{array}{l}0 \\
0\end{array}$ & $\begin{array}{l}0 \\
0\end{array}$ & $\begin{array}{l}0 \\
0\end{array}$ \\
\hline $\begin{array}{l}6-23 \\
6-25\end{array}$ & $\begin{array}{l}1505 \\
2895\end{array}$ & $\begin{array}{l}0 \\
0\end{array}$ & $\begin{array}{l}0 \\
0\end{array}$ & $\begin{array}{l}0 \\
0\end{array}$ & $\begin{array}{l}0 \\
0\end{array}$ & 0 & $\begin{array}{l}0 \\
0\end{array}$ \\
\hline $6-27$ & 1785 & 1 & 1 & 0 & 0 & 0 & 0 \\
\hline $\begin{array}{l}6-29 \\
7-01\end{array}$ & $\overline{--}$ & $\overline{--}$ & $\overline{--}$ & $\overline{--}$ & $\overline{--}$ & $=$ & $\overline{--}$ \\
\hline $7-03$ & -- & -- & -- & -- & -- & -- & - \\
\hline $7-05$ & -- & -- & -- & -- & -- & -- & -- \\
\hline $7-07$ & -- & -- & -- & -- & -- & -- & -- \\
\hline
\end{tabular}


TABLE 15.- NUMBER OF PINK TRACER PARTICLES(1), PER 100 GRAMS OF BEDLOAD SAMPLE, COMPOSITED FROM CROSS-CHANNEL SAMPLING POSITIONS SPACED AT 1-METER INTERVALS ACROSS THE CHANNEL WIDTH, EAST FORK RIVER, WYOMING, 1980

SECTION 0808

\begin{tabular}{|c|c|c|c|c|c|c|c|}
\hline \multirow{3}{*}{ DATE } & \multirow{3}{*}{$\begin{array}{c}\text { DRY } \\
\text { MASS } \\
\text { OF } \\
\text { SAMPLE (2) } \\
\text { (G) }\end{array}$} & \multicolumn{6}{|c|}{ NUMBER OF PINK TRACER PARTICLES } \\
\hline & & \multirow[b]{2}{*}{ TOTAL } & \multicolumn{5}{|c|}{ BY SIZE CLASS (MM) } \\
\hline & & & $\begin{array}{l}0.25 \\
\text { TO } \\
0.50\end{array}$ & $\begin{array}{c}0.50 \\
\text { TO } \\
1.00\end{array}$ & $\begin{array}{l}1.00 \\
\text { TO } \\
2.00\end{array}$ & $\begin{array}{l}2.00 \\
\text { TO } \\
4.00\end{array}$ & $\begin{array}{c}4.00 \\
\text { TO } \\
8.00\end{array}$ \\
\hline $\begin{array}{l}5-16 \\
5-19 \\
5-21\end{array}$ & $\begin{array}{l}573 \\
894 \\
314\end{array}$ & $\begin{array}{l}0 \\
0 \\
0\end{array}$ & $\begin{array}{l}0 \\
0 \\
0\end{array}$ & $\begin{array}{l}0 \\
0 \\
0\end{array}$ & $\begin{array}{l}0 \\
0 \\
0\end{array}$ & $\begin{array}{l}0 \\
0 \\
0\end{array}$ & $\begin{array}{l}0 \\
0 \\
0\end{array}$ \\
\hline $\begin{array}{l}5-22 \\
5-24\end{array}$ & -- & $\overline{-}$ & $=$ & $\overline{-}$ & $\overline{--}$ & $\overline{-}$ & $\overline{-}$ \\
\hline $\begin{array}{l}5-26 \\
5-27 \\
5-28\end{array}$ & $\begin{array}{l}3784 \\
2360 \\
2445\end{array}$ & $\begin{array}{l}0 \\
0 \\
1\end{array}$ & $\begin{array}{l}0 \\
0 \\
0\end{array}$ & $\begin{array}{l}0 \\
0 \\
1\end{array}$ & $\begin{array}{l}0 \\
0 \\
0\end{array}$ & $\begin{array}{l}0 \\
0 \\
0\end{array}$ & $\begin{array}{l}0 \\
0 \\
0\end{array}$ \\
\hline $5-30$ & 1203 & 1 & 1 & 0 & 0 & 0 & 0 \\
\hline $\begin{array}{l}6-01 \\
6-03 \\
6-05 \\
6-07 \\
6-09\end{array}$ & $\begin{array}{l}573 \\
196 \\
292 \\
119 \\
358\end{array}$ & $\begin{array}{l}0 \\
0 \\
0 \\
0 \\
3\end{array}$ & $\begin{array}{l}0 \\
0 \\
0 \\
0 \\
2\end{array}$ & $\begin{array}{l}0 \\
0 \\
0 \\
0 \\
1\end{array}$ & $\begin{array}{l}0 \\
0 \\
0 \\
0 \\
0\end{array}$ & $\begin{array}{l}0 \\
0 \\
0 \\
0 \\
0\end{array}$ & $\begin{array}{l}0 \\
0 \\
0 \\
0 \\
0\end{array}$ \\
\hline $\begin{array}{l}6-10 \\
6-11 \\
6-12 \\
6-13 \\
6-14\end{array}$ & $\begin{array}{l}52+++ \\
598 \\
1683 \\
1907 \\
7240\end{array}$ & $\begin{array}{l}2 \\
6 \\
0 \\
1 \\
0\end{array}$ & $\begin{array}{l}0 \\
4 \\
0 \\
1 \\
0\end{array}$ & $\begin{array}{l}2 \\
2 \\
0 \\
0 \\
0\end{array}$ & $\begin{array}{l}0 \\
0 \\
0 \\
0 \\
0\end{array}$ & $\begin{array}{l}0 \\
0 \\
0 \\
0 \\
0\end{array}$ & $\begin{array}{l}0 \\
0 \\
0 \\
0 \\
0\end{array}$ \\
\hline $\begin{array}{l}6-15 \\
6-16 \\
6-17 \\
6-18 \\
6-19\end{array}$ & $\begin{array}{l}2905 \\
5512 \\
5656 \\
2381 \\
1544\end{array}$ & $\begin{array}{l}4 \\
0 \\
1 \\
1 \\
2\end{array}$ & $\begin{array}{l}2 \\
0 \\
0 \\
0 \\
0\end{array}$ & $\begin{array}{l}2 \\
0 \\
1 \\
1 \\
2\end{array}$ & $\begin{array}{l}0 \\
0 \\
0 \\
0 \\
0\end{array}$ & $\begin{array}{l}0 \\
0 \\
0 \\
0 \\
0\end{array}$ & $\begin{array}{l}0 \\
0 \\
0 \\
0 \\
0\end{array}$ \\
\hline $\begin{array}{l}6-20 \\
6-21 \\
6-22 \\
6-23 \\
6-25\end{array}$ & $\begin{array}{r}6034 \\
2623 \\
2266 \\
651 \\
2859\end{array}$ & $\begin{array}{l}2 \\
3 \\
1 \\
0 \\
1\end{array}$ & $\begin{array}{l}0 \\
3 \\
1 \\
0 \\
1\end{array}$ & $\begin{array}{l}0 \\
0 \\
0 \\
0 \\
0\end{array}$ & $\begin{array}{l}2 \\
0 \\
0 \\
0 \\
0\end{array}$ & $\begin{array}{l}0 \\
0 \\
0 \\
0 \\
0\end{array}$ & $\begin{array}{l}0 \\
0 \\
0 \\
0 \\
0\end{array}$ \\
\hline $\begin{array}{l}6-27 \\
6-29\end{array}$ & $\begin{array}{l}2745 \\
2692\end{array}$ & $\begin{array}{l}2 \\
2\end{array}$ & $\begin{array}{l}0 \\
0\end{array}$ & $\begin{array}{l}2 \\
2\end{array}$ & $\begin{array}{l}0 \\
0\end{array}$ & $\begin{array}{l}0 \\
0\end{array}$ & $\begin{array}{l}0 \\
0\end{array}$ \\
\hline $\begin{array}{r}7-01 \\
7-03\end{array}$ & $\overline{--}+$ & $=$ & $=$ & $=$ & $=$ & $=$ & $=$ \\
\hline $7-05$ & -- & -- & - & - & - & - & - \\
\hline $7-07$ & - & -- & -- & -- & -- & -- & - \\
\hline
\end{tabular}


TABLE 15.- NUMBER OF PINK TRACER PARTICLES( 1 ), PER 100 GRAMS OF BEDLOAD SAMPLE, COMPOSITED FROM CROSS-CHANNEL SAMPLING POSITIONS SPACED AT 1-METER INTERVALS ACROSS THE CHANNEL WIDTH, EAST FORK RIVER, WYOMING, 1980

SECTION 0808(3)

\begin{tabular}{|c|c|c|c|c|c|c|c|}
\hline \multirow{3}{*}{ DATE } & \multirow{3}{*}{$\begin{array}{c}\text { DRY } \\
\text { MASS } \\
\text { OF } \\
\text { SAMPLE (2) } \\
(G)\end{array}$} & \multicolumn{6}{|c|}{ NUMBER OF PINK TRACER PARTICLES } \\
\hline & & \multirow[b]{2}{*}{ TOTAL } & \multicolumn{5}{|c|}{ BY SIZE CLASS (MM) } \\
\hline & & & $\begin{array}{l}0.25 \\
\text { TO } \\
0.50\end{array}$ & $\begin{array}{l}0.50 \\
\text { TO } \\
1.00\end{array}$ & $\begin{array}{l}1.00 \\
\text { TO } \\
2.00\end{array}$ & $\begin{array}{l}2.00 \\
\text { TO } \\
4.00\end{array}$ & $\begin{array}{l}4.00 \\
\text { TO } \\
8.00\end{array}$ \\
\hline $5-16$ & -- & -- & -- & - & - & - & -- \\
\hline $\begin{array}{l}5-19 \\
5-21\end{array}$ & $\overline{--}$ & $\overline{--}$ & $\overline{-}$ & $\overline{-}$ & $\overline{--}$ & -- & $\ddot{--}$ \\
\hline $\begin{array}{l}5-21 \\
5-22\end{array}$ & - & - & - & - & - & - & - \\
\hline $5-2 \overline{4}$ & -- & -- & -- & - & - & - & -- \\
\hline $5-26$ & - & -- & -- & - & -- & - & -- \\
\hline $5-27$ & $=$ & -- & -- & -- & -- & -- & -- \\
\hline $\begin{array}{l}5-28 \\
5-29\end{array}$ & - & $=$ & - & $=$ & $=$ & - & $=$ \\
\hline $5-30$ & -- & -- & -- & -- & - & -- & -- \\
\hline $6-01$ & -- & -- & -- & $\cdots$ & -- & -- & -- \\
\hline $6-03$ & - & -- & -- & $=$ & -- & -- & - \\
\hline $6-07$ & $\ldots$ & $\ldots$ & $=$ & $\ldots$ & $\ldots$ & $\ldots$ & $=$ \\
\hline $6-09$ & $85+$ & 0 & 0 & 0 & 0 & 0 & 0 \\
\hline $\begin{array}{l}6-10 \\
6-11\end{array}$ & $\begin{array}{l}677 \\
49++\end{array}$ & $\begin{array}{l}0 \\
0\end{array}$ & $\begin{array}{l}0 \\
0\end{array}$ & $\begin{array}{l}0 \\
0\end{array}$ & $\begin{array}{l}0 \\
0\end{array}$ & $\begin{array}{l}0 \\
0\end{array}$ & $\begin{array}{l}0 \\
0\end{array}$ \\
\hline $\begin{array}{l}6-12 \\
6-13\end{array}$ & $\overline{\overline{6}}$ & $\overline{3}$ & $\overline{7}$ & - & $\overline{0}$ & $\overline{0}$ & $-\overline{0}$ \\
\hline $6-14$ & 371 & $\begin{array}{r}14 \\
2\end{array}$ & 10 & $\frac{1}{2}$ & 0 & 0 & 0 \\
\hline & & 1 & 1 & 0 & 0 & 0 & 0 \\
\hline $\begin{array}{l}6-16 \\
6-17\end{array}$ & $\begin{array}{l}103 \\
25++\end{array}$ & $\begin{array}{l}0 \\
0\end{array}$ & $\begin{array}{l}0 \\
0\end{array}$ & $\begin{array}{l}0 \\
0\end{array}$ & $\begin{array}{l}0 \\
0\end{array}$ & $\begin{array}{l}0 \\
0\end{array}$ & $\begin{array}{l}0 \\
0\end{array}$ \\
\hline $6-18$ & 726 & 2 & 0 & 2 & 0 & 0 & 0 \\
\hline & $34++$ & & 0 & 0 & 0 & 0 & 0 \\
\hline $6-20$ & $0+++$ & 0 & 0 & 0 & 0 & 0 & 0 \\
\hline $\begin{array}{l}6-21 \\
6-22\end{array}$ & $\begin{array}{l}17++ \\
68+\end{array}$ & $\begin{array}{l}0 \\
0\end{array}$ & $\begin{array}{l}0 \\
0\end{array}$ & $\begin{array}{l}0 \\
0\end{array}$ & $\begin{array}{l}0 \\
0\end{array}$ & $\begin{array}{l}0 \\
0\end{array}$ & $\begin{array}{l}0 \\
0\end{array}$ \\
\hline $6-23$ & $52+$ & 0 & 0 & 0 & 0 & 0 & 0 \\
\hline & & & & & & & \\
\hline $6-27$ & 124 & 3 & 0 & 3 & 0 & 0 & 0 \\
\hline $\begin{array}{l}6-29 \\
7-01\end{array}$ & $=$ & $\overline{--}$ & $=$ & $=$ & $=$ & $=$ & $=$ \\
\hline $7-03$ & - & - & $=$ & $=$ & - & $\ldots$ & $\ldots$ \\
\hline $7-05$ & -- & -- & -- & -- & -- & -- & -- \\
\hline $7-07$ & -- & -- & -- & -- & -- & -- & -- \\
\hline
\end{tabular}


TABLE 15.- NUMBER OF PINK TRACER PARTICLES(1), PER 100 GRAMS OF BEDLOAD SAMPLE, COMPOSITED FROM CROSS-CHANNEL SAMPLING POSITIONS SPACED AT 1-METER INTERVALS ACROSS THE CHANNEL WIDTH, EAST FORK RIVER, WYOMING, 1980

SECTION 0853

\begin{tabular}{|c|c|c|c|c|c|c|c|}
\hline \multirow{3}{*}{\multicolumn{2}{|c|}{$\begin{array}{cc}\text { DATE } & \text { DRY } \\
& \text { MASS } \\
\text { OF } \\
\text { SAMPLE (2) } \\
& (\mathrm{G})\end{array}$}} & \multicolumn{6}{|c|}{ NUMBER OF PINK TRACER PARTICLES } \\
\hline & & \multirow[b]{2}{*}{ TOTAL } & \multicolumn{5}{|c|}{ BY SIZE CLASS (MM) } \\
\hline & & & $\begin{array}{l}0.25 \\
\text { TO } \\
0.50\end{array}$ & $\begin{array}{l}0.50 \\
\text { TO } \\
1.00\end{array}$ & $\begin{array}{l}1.00 \\
\text { TO } \\
2.00\end{array}$ & $\begin{array}{c}2.00 \\
\text { TO } \\
4.00\end{array}$ & $\begin{array}{l}4.00 \\
\text { TO } \\
8.00\end{array}$ \\
\hline $\begin{array}{l}5-16 \\
5-19 \\
5-21\end{array}$ & $\begin{array}{l}284 \\
459 \\
827\end{array}$ & $\begin{array}{l}3 \\
3 \\
0\end{array}$ & $\begin{array}{l}3 \\
2 \\
0\end{array}$ & $\begin{array}{l}0 \\
1 \\
0\end{array}$ & $\begin{array}{l}0 \\
0 \\
0\end{array}$ & $\begin{array}{l}0 \\
0 \\
0\end{array}$ & $\begin{array}{l}0 \\
0 \\
0\end{array}$ \\
\hline $\begin{array}{l}5-22 \\
5-24\end{array}$ & - & $\overline{--}$ & $\overline{--}$ & $\overline{--}$ & $\overline{--}$ & $\overline{--}$ & - \\
\hline $\begin{array}{l}5-26 \\
5-27 \\
5-28\end{array}$ & $\begin{array}{r}2765 \\
834 \\
1822\end{array}$ & $\begin{array}{l}0 \\
0 \\
0\end{array}$ & $\begin{array}{l}0 \\
0 \\
0\end{array}$ & $\begin{array}{l}0 \\
0 \\
0\end{array}$ & $\begin{array}{l}0 \\
0 \\
0\end{array}$ & $\begin{array}{l}0 \\
0 \\
0\end{array}$ & $\begin{array}{l}0 \\
0 \\
0\end{array}$ \\
\hline $5-30$ & 550 & 0 & 0 & 0 & 0 & 0 & 0 \\
\hline $\begin{array}{l}6-01 \\
6-03 \\
6-05 \\
6-07 \\
6-09\end{array}$ & $\begin{array}{c}242 \\
217 \\
4+++ \\
170 \\
322\end{array}$ & $\begin{array}{l}0 \\
0 \\
0 \\
0 \\
1\end{array}$ & $\begin{array}{l}0 \\
0 \\
0 \\
0 \\
1\end{array}$ & $\begin{array}{l}0 \\
0 \\
0 \\
0 \\
0\end{array}$ & $\begin{array}{l}0 \\
0 \\
0 \\
0 \\
0\end{array}$ & $\begin{array}{l}0 \\
0 \\
0 \\
0 \\
0\end{array}$ & $\begin{array}{l}0 \\
0 \\
0 \\
0 \\
0\end{array}$ \\
\hline $\begin{array}{l}6-10 \\
6-11 \\
6-12 \\
6-13 \\
6-14\end{array}$ & $\begin{array}{r}847 \\
2219 \\
6895 \\
6089 \\
5264\end{array}$ & $\begin{array}{r}18 \\
5 \\
1 \\
2 \\
2\end{array}$ & $\begin{array}{r}18 \\
3 \\
0 \\
2 \\
2\end{array}$ & $\begin{array}{l}0 \\
2 \\
1 \\
0 \\
0\end{array}$ & $\begin{array}{l}0 \\
0 \\
0 \\
0 \\
0\end{array}$ & $\begin{array}{l}0 \\
0 \\
0 \\
0 \\
0\end{array}$ & $\begin{array}{l}0 \\
0 \\
0 \\
0 \\
0\end{array}$ \\
\hline $\begin{array}{l}6-15 \\
6-16 \\
6-17 \\
6-18 \\
6-19\end{array}$ & $\begin{array}{l}1825 \\
4440 \\
3923 \\
3082 \\
3537\end{array}$ & $\begin{array}{l}1 \\
0 \\
1 \\
0 \\
0\end{array}$ & $\begin{array}{l}0 \\
0 \\
0 \\
0 \\
0\end{array}$ & $\begin{array}{l}1 \\
0 \\
1 \\
0 \\
0\end{array}$ & $\begin{array}{l}0 \\
0 \\
0 \\
0 \\
0\end{array}$ & $\begin{array}{l}0 \\
0 \\
0 \\
0 \\
0\end{array}$ & $\begin{array}{l}0 \\
0 \\
0 \\
0 \\
0\end{array}$ \\
\hline $\begin{array}{l}6-20 \\
6-21 \\
6-22 \\
6-23 \\
6-25\end{array}$ & $\begin{array}{r}628 \\
1390 \\
3195 \\
1491 \\
711\end{array}$ & $\begin{array}{l}4 \\
0 \\
1 \\
0 \\
1\end{array}$ & $\begin{array}{l}0 \\
0 \\
0 \\
0 \\
0\end{array}$ & $\begin{array}{l}4 \\
0 \\
0 \\
0 \\
1\end{array}$ & $\begin{array}{l}0 \\
0 \\
1 \\
0 \\
0\end{array}$ & $\begin{array}{l}0 \\
0 \\
0 \\
0 \\
0\end{array}$ & $\begin{array}{l}0 \\
0 \\
0 \\
0 \\
0\end{array}$ \\
\hline $6-27$ & 2440 & 2 & 0 & 2 & 0 & 0 & 0 \\
\hline $\begin{array}{l}6-29 \\
7-01\end{array}$ & $\overline{--}$ & $\overline{--}$ & $\overline{--}$ & $\overline{--}$ & $\overline{--}$ & - & -- \\
\hline $7-03$ & -- & -- & $\overline{--}$ & $\overline{--}$ & -- & $\overline{-}$ & $\overline{--}$ \\
\hline & & & & & & & \\
\hline $7-07$ & -- & -- & -- & -- & -- & -- & -- \\
\hline
\end{tabular}


TABLE 15.- NUMBER OF PINK TRACER PARTICLES(1), PER 100 GRAMS OF BEDLOAD SAMPLE, COMPOSITED FROM CROSS-CHANNEL SAMPLING POSITIONS SPACED AT 1-METER INTERVALS ACROSS THE CHANNEL WIDTH, EAST FORK RIVER, WYOMING, 1980

SECTION 0898

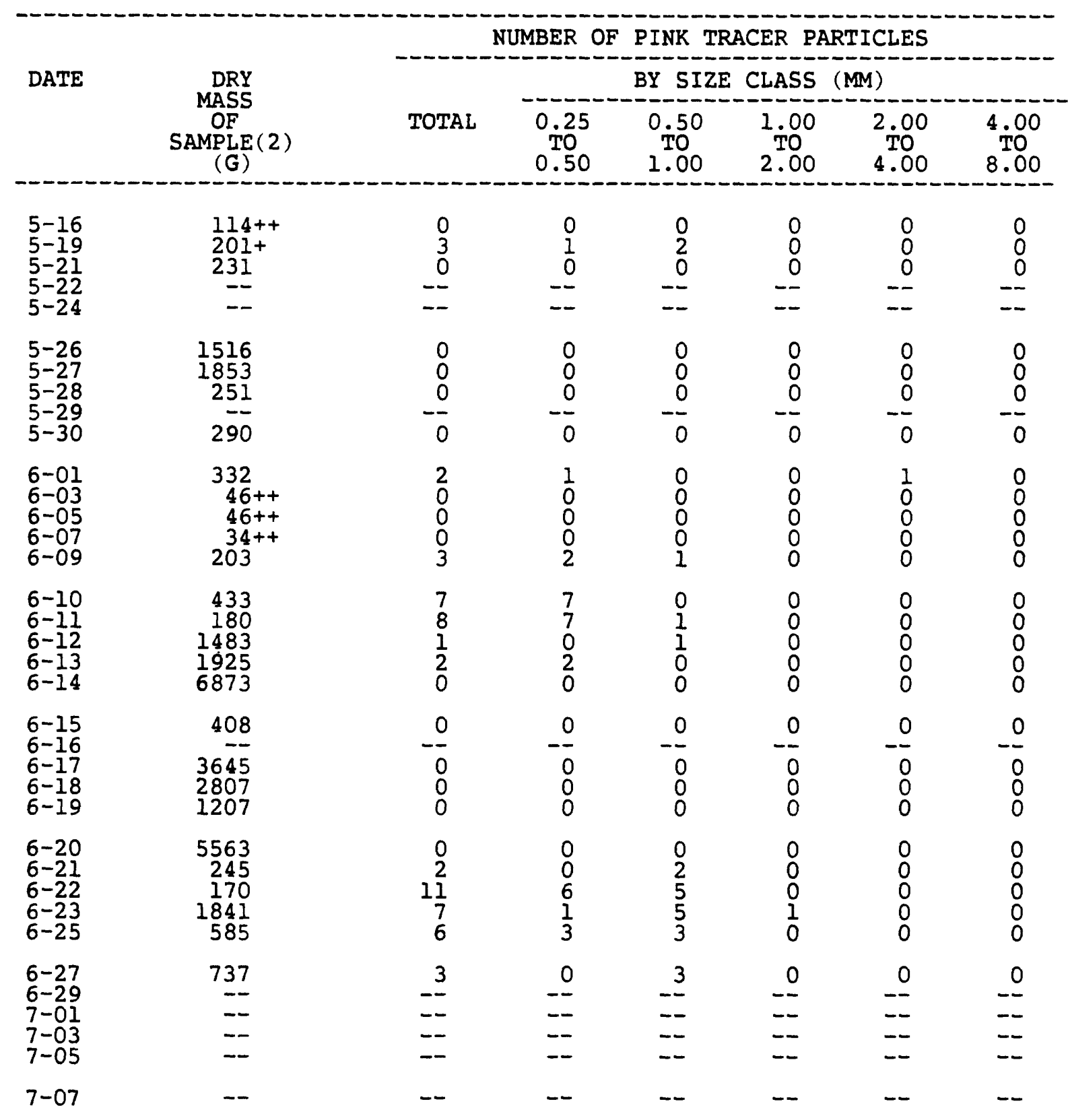


TABLE 15.- NUMBER OF PINK TRACER PARTICLES(1), PER 100 GRAMS OF BEDLOAD SAMPLE, COMPOSITED FROM CROSS-CHANNEL SAMPLING POSITIONS SPACED AT I-METER INTERVALS ACROSS THE CHANNEL WIDTH, EAST FORK RIVER, WYOMING, 1980

\section{SECTION 0940}

\begin{tabular}{|c|c|c|c|c|c|c|c|}
\hline \multirow{3}{*}{ DATE } & \multirow{3}{*}{$\begin{array}{c}\text { DRY } \\
\text { MASS } \\
\text { OF } \\
\text { SAMPLE (2) } \\
\text { (G) }\end{array}$} & \multicolumn{6}{|c|}{ NUMBER OF PINK TRACER PARTICLES } \\
\hline & & \multirow[b]{2}{*}{ TOTAL } & \multicolumn{5}{|c|}{ BY SIZE CLASS (MM) } \\
\hline & & & $\begin{array}{l}0.25 \\
\text { TO } \\
0.50\end{array}$ & $\begin{array}{l}0.50 \\
\text { TO } \\
1.00\end{array}$ & $\begin{array}{l}1.00 \\
\text { TO } \\
2.00\end{array}$ & $\begin{array}{l}2.00 \\
\text { TO } \\
4.00\end{array}$ & $\begin{array}{l}4.00 \\
\text { TO } \\
8.00\end{array}$ \\
\hline $\begin{array}{l}5-16 \\
5-19 \\
5-21\end{array}$ & $\begin{array}{l}412 \\
113+ \\
1047\end{array}$ & $\begin{array}{l}0 \\
3 \\
1\end{array}$ & $\begin{array}{l}0 \\
3 \\
1\end{array}$ & $\begin{array}{l}0 \\
0 \\
0\end{array}$ & $\begin{array}{l}0 \\
0 \\
0\end{array}$ & $\begin{array}{l}0 \\
0 \\
0\end{array}$ & $\begin{array}{l}0 \\
0 \\
0\end{array}$ \\
\hline $\begin{array}{l}5-22 \\
5-24\end{array}$ & $\overline{--}$ & -- & $\overline{-}$ & $\overline{--}$ & $\overline{-}$ & $\overline{--}$ & $\overline{--}$ \\
\hline $\begin{array}{l}5-26 \\
5-27 \\
5-28 \\
5-29 \\
5-30\end{array}$ & $\begin{array}{c}3564 \\
1729 \\
803 \\
43++ \\
89+\end{array}$ & $\begin{array}{l}0 \\
0 \\
0 \\
0 \\
2\end{array}$ & $\begin{array}{l}0 \\
0 \\
0 \\
0 \\
0\end{array}$ & $\begin{array}{l}0 \\
0 \\
0 \\
0 \\
1\end{array}$ & $\begin{array}{l}0 \\
0 \\
0 \\
0 \\
1\end{array}$ & $\begin{array}{l}0 \\
0 \\
0 \\
0 \\
0\end{array}$ & $\begin{array}{l}0 \\
0 \\
0 \\
0 \\
0\end{array}$ \\
\hline $\begin{array}{l}6-01 \\
6-03 \\
6-05 \\
6-07 \\
6-09\end{array}$ & $\begin{array}{c}108+ \\
73++ \\
73+ \\
134 \\
555\end{array}$ & $\begin{array}{l}0 \\
0 \\
0 \\
0 \\
4\end{array}$ & $\begin{array}{l}0 \\
0 \\
0 \\
0 \\
3\end{array}$ & $\begin{array}{l}0 \\
0 \\
0 \\
0 \\
1\end{array}$ & $\begin{array}{l}0 \\
0 \\
0 \\
0 \\
0\end{array}$ & $\begin{array}{l}0 \\
0 \\
0 \\
0 \\
0\end{array}$ & $\begin{array}{l}0 \\
0 \\
0 \\
0 \\
0\end{array}$ \\
\hline $\begin{array}{l}6-10 \\
6-11 \\
6-12 \\
6-13 \\
6-14\end{array}$ & $\begin{array}{r}714 \\
5615 \\
6832 \\
5258 \\
2459\end{array}$ & $\begin{array}{l}1 \\
1 \\
0 \\
0 \\
2\end{array}$ & $\begin{array}{l}0 \\
1 \\
0 \\
0 \\
0\end{array}$ & $\begin{array}{l}1 \\
0 \\
0 \\
0 \\
2\end{array}$ & $\begin{array}{l}0 \\
0 \\
0 \\
0 \\
0\end{array}$ & $\begin{array}{l}0 \\
0 \\
0 \\
0 \\
0\end{array}$ & $\begin{array}{l}0 \\
0 \\
0 \\
0 \\
0\end{array}$ \\
\hline $6-15$ & 2313 & 1 & 0 & 1 & 0 & 0 & 0 \\
\hline $\begin{array}{l}6-17 \\
6-18 \\
6-19\end{array}$ & $\begin{array}{l}1205 \\
1785 \\
1143\end{array}$ & $\begin{array}{l}0 \\
0 \\
0\end{array}$ & $\begin{array}{l}0 \\
0 \\
0\end{array}$ & $\begin{array}{l}0 \\
0 \\
0\end{array}$ & $\begin{array}{l}0 \\
0 \\
0\end{array}$ & $\begin{array}{l}0 \\
0 \\
0\end{array}$ & $\begin{array}{l}0 \\
0 \\
0\end{array}$ \\
\hline $\begin{array}{l}6-20 \\
6-21 \\
6-22 \\
6-23 \\
6-25\end{array}$ & $\begin{array}{l}1735 \\
6301 \\
2855 \\
3197 \\
1877\end{array}$ & $\begin{array}{l}0 \\
1 \\
3 \\
1 \\
0\end{array}$ & $\begin{array}{l}0 \\
1 \\
1 \\
0 \\
0\end{array}$ & $\begin{array}{l}0 \\
0 \\
2 \\
1 \\
0\end{array}$ & $\begin{array}{l}0 \\
0 \\
0 \\
0 \\
0\end{array}$ & $\begin{array}{l}0 \\
0 \\
0 \\
0 \\
0\end{array}$ & $\begin{array}{l}0 \\
0 \\
0 \\
0 \\
0\end{array}$ \\
\hline $6-27$ & 1569 & 1 & 1 & 0 & 0 & 0 & 0 \\
\hline $\begin{array}{l}6-29 \\
7-01\end{array}$ & $=$ & $\overline{-}$ & $\overline{--}$ & $\overline{-}$ & $\overline{-}$ & $\overline{-}$ & $\overline{--}$ \\
\hline $7-0 \overline{3}$ & -- & -- & -- & -- & -- & -- & -- \\
\hline $7-05$ & -- & -- & - & -- & - & -- & - \\
\hline $7-07$ & -- & -- & -- & -- & -- & -- & -- \\
\hline
\end{tabular}


TABLE 15.- NUMBER OF PINK TRACER PARTICLES(1), PER 100 GRAMS OF BEDLOAD SAMPLE, COMPOSITED FROM CROSS-CHANNEL SAMPLING POSITIONS SPACED AT I-METER INTERVALS ACROSS THE CHANNEL WIDTH, EAST FORK RIVER, WYOMING, 1980

SECTION 0985

\begin{tabular}{|c|c|c|c|c|c|c|c|}
\hline \multirow{3}{*}{ DATE } & \multirow{3}{*}{$\begin{array}{c}\text { DRY } \\
\text { MASS } \\
\text { OF } \\
\text { SAMPLE (2) } \\
\text { (G) }\end{array}$} & \multicolumn{6}{|c|}{ NUMBER OF PINK TRACER PARTICLES } \\
\hline & & \multirow[b]{2}{*}{ TOTAL } & \multicolumn{5}{|c|}{ BY SIZE CLASS (MM) } \\
\hline & & & $\begin{array}{l}0.25 \\
\text { TO } \\
0.50\end{array}$ & $\begin{array}{l}0.50 \\
\text { TO } \\
1.00\end{array}$ & $\begin{array}{l}1.00 \\
2.00 \\
2.00\end{array}$ & $\begin{array}{l}2.00 \\
\text { TO } \\
4.00\end{array}$ & $\begin{array}{l}4.00 \\
\text { TO } \\
8.00\end{array}$ \\
\hline $\begin{array}{l}5-16 \\
5-19 \\
5-21 \\
5-22\end{array}$ & $\begin{array}{r}344 \\
323 \\
2789 \\
\end{array}$ & $\begin{array}{r}0 \\
0 \\
0 \\
--\end{array}$ & $\begin{array}{r}0 \\
0 \\
0 \\
--\end{array}$ & $\begin{array}{r}0 \\
0 \\
0 \\
-\end{array}$ & $\begin{array}{r}0 \\
0 \\
0 \\
--\end{array}$ & $\begin{array}{r}0 \\
0 \\
0 \\
-\end{array}$ & $\begin{array}{r}0 \\
0 \\
0 \\
--\end{array}$ \\
\hline $5-24$ & -- & -- & -- & -- & - & -- & -- \\
\hline $\begin{array}{l}5-26 \\
5-27 \\
5-28 \\
5-29\end{array}$ & $\begin{array}{c}3289 \\
73+ \\
105+ \\
-\end{array}$ & $\begin{array}{r}0 \\
0 \\
0 \\
--\end{array}$ & $\begin{array}{r}0 \\
0 \\
0 \\
--\end{array}$ & $\begin{array}{r}0 \\
0 \\
0 \\
--\end{array}$ & $\begin{array}{r}0 \\
0 \\
0 \\
--\end{array}$ & $\begin{array}{r}0 \\
0 \\
0 \\
-\end{array}$ & $\begin{array}{r}0 \\
0 \\
0 \\
--\end{array}$ \\
\hline $5-30$ & $10++$ & 0 & 0 & 0 & 0 & 0 & 0 \\
\hline $\begin{array}{l}6-01 \\
6-03 \\
6-05 \\
6-07 \\
6-09\end{array}$ & $\begin{array}{r}5+++ \\
39++ \\
199+++ \\
50++ \\
1844\end{array}$ & $\begin{array}{l}0 \\
0 \\
0 \\
0 \\
4\end{array}$ & $\begin{array}{l}0 \\
0 \\
0 \\
0 \\
4\end{array}$ & $\begin{array}{l}0 \\
0 \\
0 \\
0 \\
0\end{array}$ & $\begin{array}{l}0 \\
0 \\
0 \\
0 \\
0\end{array}$ & $\begin{array}{l}0 \\
0 \\
0 \\
0 \\
0\end{array}$ & $\begin{array}{l}0 \\
0 \\
0 \\
0 \\
0\end{array}$ \\
\hline $\begin{array}{l}6-10 \\
6-11 \\
6-12 \\
6-13 \\
6-14\end{array}$ & $\begin{array}{l}5658 \\
3740 \\
4765 \\
8303 \\
7534\end{array}$ & $\begin{array}{l}8 \\
3 \\
1 \\
0 \\
0\end{array}$ & $\begin{array}{l}7 \\
3 \\
0 \\
0 \\
0\end{array}$ & $\begin{array}{l}1 \\
0 \\
1 \\
0 \\
0\end{array}$ & $\begin{array}{l}0 \\
0 \\
0 \\
0 \\
0\end{array}$ & $\begin{array}{l}0 \\
0 \\
0 \\
0 \\
0\end{array}$ & $\begin{array}{l}0 \\
0 \\
0 \\
0 \\
0\end{array}$ \\
\hline $6-15$ & 3829 & 0 & $\underline{0}$ & $\underline{0}$ & $\underline{0}$ & 0 & 0 \\
\hline $\begin{array}{l}6-17 \\
6-18 \\
6-19\end{array}$ & $\begin{array}{r}954 \\
3865 \\
1696\end{array}$ & $\begin{array}{l}1 \\
0 \\
1\end{array}$ & $\begin{array}{l}0 \\
0 \\
0\end{array}$ & $\begin{array}{l}0 \\
0 \\
1\end{array}$ & $\begin{array}{l}1 \\
0 \\
0\end{array}$ & $\begin{array}{l}0 \\
0 \\
0\end{array}$ & $\begin{array}{l}0 \\
0 \\
0\end{array}$ \\
\hline $\begin{array}{l}6-20 \\
6-21 \\
6-22 \\
6-23 \\
6-25\end{array}$ & $\begin{array}{r}1955 \\
10233 \\
2284 \\
3294 \\
2383\end{array}$ & $\begin{array}{l}8 \\
3 \\
4 \\
2 \\
5\end{array}$ & $\begin{array}{l}6 \\
0 \\
2 \\
0 \\
2\end{array}$ & $\begin{array}{l}2 \\
3 \\
1 \\
2 \\
3\end{array}$ & $\begin{array}{l}0 \\
0 \\
1 \\
0 \\
0\end{array}$ & $\begin{array}{l}0 \\
0 \\
0 \\
0 \\
0\end{array}$ & $\begin{array}{l}0 \\
0 \\
0 \\
0 \\
0\end{array}$ \\
\hline $6-27$ & 2130 & 6 & 2 & 4 & $\underline{0}$ & 0 & 0 \\
\hline $7-01$ & -- & - & - & - & - & - & - \\
\hline $\begin{array}{l}7-03 \\
7-05\end{array}$ & -- & $\overline{--}$ & $\overline{--}$ & $\overline{-}$ & $\overline{-}$ & $\bar{m}$ & $\overline{-}$ \\
\hline $7-07$ & -- & -- & -- & -- & -- & -- & -- \\
\hline
\end{tabular}


TABLE 15.- NUMBER OF PINK TRACER PARTICLES(I), PER 100 GRAMS OF BEDLOAD SAMPLE, COMPOSITED FROM CROSS-CHANNEL SAMPLING POSITIONS SPACED AT I-METER INTERVALS ACROSS THE CHANNEL WIDTH, EAST FORK RIVER, WYOMING, 1980

SECTION 1038

\begin{tabular}{|c|c|c|c|c|c|c|c|}
\hline \multirow[t]{2}{*}{ DATE } & \multirow{2}{*}{$\begin{array}{c}\text { DRY } \\
\text { MASS } \\
\text { OF } \\
\text { SAMPLE (2) } \\
(\mathbf{G})\end{array}$} & & \multicolumn{5}{|c|}{ BY SIZE CLASS (MM) } \\
\hline & & TOTAL & $\begin{array}{l}0.25 \\
\text { TO } \\
0.50\end{array}$ & $\begin{array}{l}0.50 \\
\text { TO } \\
1.00\end{array}$ & $\begin{array}{l}1.00 \\
\text { TO } \\
2.00\end{array}$ & $\begin{array}{l}2.00 \\
\text { TO } \\
4.00\end{array}$ & $\begin{array}{l}4.00 \\
\text { TO } \\
8.00\end{array}$ \\
\hline $\begin{array}{l}5-16 \\
5-19 \\
5-21 \\
5-22 \\
5-24\end{array}$ & $\begin{array}{c}153+ \\
700 \\
3042 \\
2365 \\
4864\end{array}$ & $\begin{array}{l}1 \\
1 \\
0 \\
1 \\
0\end{array}$ & $\begin{array}{l}1 \\
1 \\
0 \\
0 \\
0\end{array}$ & $\begin{array}{l}0 \\
0 \\
0 \\
1 \\
0\end{array}$ & $\begin{array}{l}0 \\
0 \\
0 \\
0 \\
0\end{array}$ & $\begin{array}{l}0 \\
0 \\
0 \\
0 \\
0\end{array}$ & $\begin{array}{l}0 \\
0 \\
0 \\
0 \\
0\end{array}$ \\
\hline $\begin{array}{l}5-26 \\
5-27 \\
5-28 \\
5-29 \\
5-30\end{array}$ & $\begin{array}{l}1 \overline{8+} \\
18++ \\
\overline{26++}\end{array}$ & $\begin{array}{r}--\overline{0} \\
0 \\
-\frac{0}{0}\end{array}$ & $\begin{array}{r}--\overline{0} \\
0 \\
-0\end{array}$ & $\begin{array}{r}-\overline{0} \\
0 \\
-0 \\
0\end{array}$ & $\begin{array}{r}-- \\
0 \\
-0 \\
0\end{array}$ & $\begin{array}{r}-\overline{0} \\
-0 \\
-0\end{array}$ & $\begin{array}{r}-\overline{0} \\
-0 \\
-0\end{array}$ \\
\hline $\begin{array}{l}6-01 \\
6-03 \\
6-05 \\
6-07 \\
6-09\end{array}$ & $\begin{array}{r}5+++ \\
17+++ \\
0+++ \\
0+++ \\
1865\end{array}$ & $\begin{array}{l}0 \\
0 \\
0 \\
0 \\
0\end{array}$ & $\begin{array}{l}0 \\
0 \\
0 \\
0 \\
0\end{array}$ & $\begin{array}{l}0 \\
0 \\
0 \\
0 \\
0\end{array}$ & $\begin{array}{l}0 \\
0 \\
0 \\
0 \\
0\end{array}$ & $\begin{array}{l}0 \\
0 \\
0 \\
0 \\
0\end{array}$ & $\begin{array}{l}0 \\
0 \\
0 \\
0 \\
0\end{array}$ \\
\hline $\begin{array}{l}6-10 \\
6-11 \\
6-12 \\
6-13 \\
6-14\end{array}$ & $\begin{array}{r}6434 \\
7545 \\
12301 \\
1950 \\
2923\end{array}$ & $\begin{array}{l}3 \\
1 \\
0 \\
3 \\
2\end{array}$ & $\begin{array}{l}1 \\
0 \\
0 \\
0 \\
0\end{array}$ & $\begin{array}{l}2 \\
0 \\
0 \\
1 \\
2\end{array}$ & $\begin{array}{l}0 \\
1 \\
0 \\
2 \\
0\end{array}$ & $\begin{array}{l}0 \\
0 \\
0 \\
0 \\
0\end{array}$ & $\begin{array}{l}0 \\
0 \\
0 \\
0 \\
0\end{array}$ \\
\hline $\begin{array}{l}6-15 \\
6-16 \\
6-17 \\
6-18 \\
6-19\end{array}$ & $\begin{array}{r}238 \\
-- \\
468 \\
1679 \\
249\end{array}$ & $\begin{array}{r}-\frac{1}{2} \\
0 \\
4\end{array}$ & $\begin{array}{r}-1 \\
-1 \\
0 \\
4\end{array}$ & $\begin{array}{r}-\frac{0}{1} \\
\frac{1}{0} \\
0\end{array}$ & $\begin{array}{r}-0 \\
-0 \\
0 \\
0\end{array}$ & $\begin{array}{r}-0 \\
-0 \\
0 \\
0\end{array}$ & $\begin{array}{r}0 \\
-\frac{0}{0} \\
0 \\
0\end{array}$ \\
\hline $\begin{array}{l}6-20 \\
6-21 \\
6-22 \\
6-23 \\
6-25\end{array}$ & $\begin{array}{r}1361 \\
2079 \\
1187 \\
2267 \\
809\end{array}$ & $\begin{array}{r}2 \\
2 \\
8 \\
5 \\
25\end{array}$ & $\begin{array}{l}0 \\
0 \\
5 \\
3 \\
6\end{array}$ & $\begin{array}{r}2 \\
2 \\
3 \\
2 \\
15\end{array}$ & $\begin{array}{l}0 \\
0 \\
0 \\
0 \\
4\end{array}$ & $\begin{array}{l}0 \\
0 \\
0 \\
0 \\
0\end{array}$ & $\begin{array}{l}0 \\
0 \\
0 \\
0 \\
0\end{array}$ \\
\hline $\begin{array}{l}6-27 \\
6-29 \\
7-01 \\
7-03 \\
7-05\end{array}$ & $\begin{array}{r}2291 \\
=- \\
=- \\
=-\end{array}$ & $\begin{array}{l}11 \\
=- \\
--\end{array}$ & $\begin{array}{l}3 \\
--- \\
-- \\
--\end{array}$ & $\begin{array}{l}7 \\
-- \\
-- \\
--\end{array}$ & $\begin{array}{l}1 \\
-- \\
-- \\
--\end{array}$ & $\begin{array}{l}0 \\
-- \\
-- \\
--\end{array}$ & $\begin{array}{l}0 \\
-- \\
-- \\
--\end{array}$ \\
\hline $7-07$ & -- & -- & -- & -- & -- & -- & -- \\
\hline
\end{tabular}


TABLE 15.- NUMBER OF PINK TRACER PARTICLES(1), PER 100 GRAMS OF BEDLOAD SAMPLE, COMPOSITED FROM CROSS-CHANNEL SAMPLING POSITIONS SPACED AT 1-METER INTERVALS ACROSS THE CHANNEL WIDTH, EAST FORK RIVER, WYOMING, 1980

SECTION 1077

\begin{tabular}{|c|c|c|c|c|c|c|c|}
\hline \multirow{3}{*}{ DATE } & \multirow{3}{*}{$\begin{array}{c}\text { DRY } \\
\text { MASS } \\
\text { OF } \\
\text { SAMPLE (2) } \\
\text { (G) }\end{array}$} & \multicolumn{6}{|c|}{ NUMBER OF PINK TRACER PARTICLES } \\
\hline & & \multirow[b]{2}{*}{ TOTAL } & \multicolumn{5}{|c|}{ BY SIZE CLASS (MM) } \\
\hline & & & $\begin{array}{l}0.25 \\
\text { TO } \\
0.50\end{array}$ & $\begin{array}{l}0.50 \\
\text { TO } \\
1.00\end{array}$ & $\begin{array}{l}1.00 \\
\text { TO } \\
2.00\end{array}$ & $\begin{array}{l}2.00 \\
\text { TO } \\
4.00\end{array}$ & $\begin{array}{l}4.00 \\
\text { TO } \\
8.00\end{array}$ \\
\hline $\begin{array}{l}5-16 \\
5-19 \\
5-21 \\
5-22 \\
5-24\end{array}$ & $\begin{array}{l}488 \\
141+ \\
1036 \\
6690 \\
2093\end{array}$ & $\begin{array}{l}0 \\
4 \\
0 \\
0 \\
1\end{array}$ & $\begin{array}{l}0 \\
4 \\
0 \\
0 \\
1\end{array}$ & $\begin{array}{l}0 \\
0 \\
0 \\
0 \\
0\end{array}$ & $\begin{array}{l}0 \\
0 \\
0 \\
0 \\
0\end{array}$ & $\begin{array}{l}0 \\
0 \\
0 \\
0 \\
0\end{array}$ & $\begin{array}{l}0 \\
0 \\
0 \\
0 \\
0\end{array}$ \\
\hline $\begin{array}{l}5-26 \\
5-27 \\
5-28 \\
5-20\end{array}$ & $\begin{array}{r}\overline{66}+ \\
247\end{array}$ & $\begin{array}{r}-- \\
0 \\
-\end{array}$ & $\begin{array}{r}-\overline{3} \\
0\end{array}$ & $\begin{array}{r}-0 \\
0\end{array}$ & $\begin{array}{r}-- \\
0 \\
0\end{array}$ & $\overline{0}$ & $\begin{array}{r}--\overline{0} \\
0\end{array}$ \\
\hline $5-30$ & $93+$ & 0 & 0 & 0 & 0 & 0 & 0 \\
\hline $\begin{array}{l}6-01 \\
6-03 \\
6-05 \\
6-07 \\
6-09\end{array}$ & $\begin{array}{l}4^{4++} \\
368 \\
443 \\
861 \\
6847\end{array}$ & $\begin{array}{l}0 \\
4 \\
0 \\
1 \\
3\end{array}$ & $\begin{array}{l}0 \\
3 \\
0 \\
1 \\
3\end{array}$ & $\begin{array}{l}0 \\
1 \\
0 \\
0 \\
0\end{array}$ & $\begin{array}{l}0 \\
0 \\
0 \\
0 \\
0\end{array}$ & $\begin{array}{l}0 \\
0 \\
0 \\
0 \\
0\end{array}$ & $\begin{array}{l}0 \\
0 \\
0 \\
0 \\
0\end{array}$ \\
\hline $\begin{array}{l}6-10 \\
6-11 \\
6-12 \\
6-13 \\
6-14\end{array}$ & $\begin{array}{l}5686 \\
7026 \\
5955 \\
4795 \\
3167\end{array}$ & $\begin{array}{l}7 \\
0 \\
1 \\
0 \\
0\end{array}$ & $\begin{array}{l}6 \\
0 \\
1 \\
0 \\
0\end{array}$ & $\begin{array}{l}1 \\
0 \\
0 \\
0 \\
0\end{array}$ & $\begin{array}{l}0 \\
0 \\
0 \\
0 \\
0\end{array}$ & $\begin{array}{l}0 \\
0 \\
0 \\
0 \\
0\end{array}$ & $\begin{array}{l}0 \\
0 \\
0 \\
0 \\
0\end{array}$ \\
\hline $\begin{array}{l}6-15 \\
6-16\end{array}$ & 1830 & 0 & 0 & 0 & 0 & 0 & 0 \\
\hline $\begin{array}{l}6-17 \\
6-18 \\
6-19\end{array}$ & $\begin{array}{l}1193 \\
3263 \\
4561\end{array}$ & $\begin{array}{l}2 \\
0 \\
2\end{array}$ & $\begin{array}{l}1 \\
0 \\
1\end{array}$ & $\begin{array}{l}1 \\
0 \\
1\end{array}$ & $\begin{array}{l}0 \\
0 \\
0\end{array}$ & $\begin{array}{l}0 \\
0 \\
0\end{array}$ & $\begin{array}{l}0 \\
0 \\
0\end{array}$ \\
\hline $\begin{array}{l}6-20 \\
6-21 \\
6-22\end{array}$ & $\begin{array}{l}5961 \\
2481 \\
\end{array}$ & $\begin{array}{r}6 \\
5\end{array}$ & $\begin{array}{l}6 \\
1\end{array}$ & $\begin{array}{l}0 \\
2\end{array}$ & $\begin{array}{l}0 \\
2\end{array}$ & $\begin{array}{l}0 \\
0\end{array}$ & $\begin{array}{l}0 \\
0\end{array}$ \\
\hline $\begin{array}{l}6-23 \\
6-25\end{array}$ & $\begin{array}{r}5700 \\
878\end{array}$ & 25 & $\begin{array}{l}3 \\
0\end{array}$ & 20 & $\begin{array}{l}0 \\
5\end{array}$ & $\begin{array}{l}0 \\
0\end{array}$ & $\begin{array}{l}0 \\
0\end{array}$ \\
\hline $\begin{array}{l}6-27 \\
6-29\end{array}$ & 2988 & 17 & 6 & 11 & $\underline{-0}$ & $\underline{0}$ & 0 \\
\hline $7-01$ & -- & - & -- & - & - & - & - \\
\hline $\begin{array}{l}7-03 \\
7-05\end{array}$ & $=$ & $=$ & $=-$ & -- & $\overline{--}$ & $\overline{--}$ & $\overline{--}$ \\
\hline $7-07$ & -- & -- & -- & -- & -- & -- & -- \\
\hline
\end{tabular}


TABLE 15.- NUMBER OF PINK TRACER PARTICLES(1), PER 100 GRAMS OF BEDLOAD SAMPLE, COMPOSITED FROM CROSS-CHANNEL SAMPLING POSITIONS SPACED AT 1-METER INTERVALS ACROSS THE CHANNEL WIDTH, EAST FORK RIVER, WYOMING, 1980

SECTION 1120

\begin{tabular}{|c|c|c|c|c|c|c|c|}
\hline \multirow{3}{*}{ DATE } & \multirow{3}{*}{$\begin{array}{c}\text { DRY } \\
\text { MASS } \\
\text { OF } \\
\text { SAMPLE (2) } \\
(G)\end{array}$} & \multicolumn{6}{|c|}{ NUMBER OF PINK TRACER PARTICLES } \\
\hline & & \multirow[b]{2}{*}{ TOTAL } & \multicolumn{5}{|c|}{ BY SIZE CLASS (MM) } \\
\hline & & & $\begin{array}{l}0.25 \\
\text { TO } \\
0.50\end{array}$ & $\begin{array}{l}0.50 \\
\text { To. } \\
1.00\end{array}$ & $\begin{array}{l}1.00 \\
\text { TO } \\
2.00\end{array}$ & $\begin{array}{l}2.00 \\
\text { TO } \\
4.00\end{array}$ & $\begin{array}{c}4.00 \\
\text { TO } \\
8.00\end{array}$ \\
\hline $\begin{array}{l}5-16 \\
5-19 \\
5-21 \\
5-22 \\
5-24\end{array}$ & $\begin{array}{r}72+ \\
273+ \\
3540 \\
5580 \\
2052\end{array}$ & $\begin{array}{l}0 \\
8 \\
0 \\
0 \\
2\end{array}$ & $\begin{array}{l}0 \\
8 \\
0 \\
0 \\
1\end{array}$ & $\begin{array}{l}0 \\
0 \\
0 \\
0 \\
1\end{array}$ & $\begin{array}{l}0 \\
0 \\
0 \\
0 \\
0\end{array}$ & $\begin{array}{l}0 \\
0 \\
0 \\
0 \\
0\end{array}$ & $\begin{array}{l}0 \\
0 \\
0 \\
0 \\
0\end{array}$ \\
\hline $\begin{array}{l}5-26 \\
5-27 \\
5-28\end{array}$ & $\begin{array}{l}300 \\
273\end{array}$ & $\begin{array}{r}-- \\
0 \\
0\end{array}$ & $\begin{array}{r}--\overline{0} \\
0\end{array}$ & $\begin{array}{r}--\overline{0} \\
0\end{array}$ & $\begin{array}{r}-- \\
0 \\
0\end{array}$ & $\begin{array}{r}--\overline{0} \\
0\end{array}$ & $\begin{array}{r}-- \\
0 \\
0\end{array}$ \\
\hline $5-30$ & $103+$ & 0 & 0 & 0 & 0 & 0 & 0 \\
\hline $\begin{array}{l}6-01 \\
6-03 \\
6-05 \\
6-07 \\
6-09\end{array}$ & $\begin{array}{r}77++ \\
84++ \\
90+ \\
1350 \\
6295\end{array}$ & $\begin{array}{r}0 \\
0 \\
0 \\
10 \\
0\end{array}$ & $\begin{array}{l}0 \\
0 \\
0 \\
9 \\
0\end{array}$ & $\begin{array}{l}0 \\
0 \\
0 \\
1 \\
0\end{array}$ & $\begin{array}{l}0 \\
0 \\
0 \\
0 \\
0\end{array}$ & $\begin{array}{l}0 \\
0 \\
0 \\
0 \\
0\end{array}$ & $\begin{array}{l}0 \\
0 \\
0 \\
0 \\
0\end{array}$ \\
\hline $\begin{array}{l}6-10 \\
6-11 \\
6-12 \\
6-13 \\
6-14\end{array}$ & $\begin{array}{r}5355 \\
2111 \\
1473 \\
537 \\
1581\end{array}$ & $\begin{array}{l}2 \\
5 \\
1 \\
0 \\
0\end{array}$ & $\begin{array}{l}1 \\
2 \\
1 \\
0 \\
0\end{array}$ & $\begin{array}{l}1 \\
3 \\
0 \\
0 \\
0\end{array}$ & $\begin{array}{l}0 \\
0 \\
0 \\
0 \\
0\end{array}$ & $\begin{array}{l}0 \\
0 \\
0 \\
0 \\
0\end{array}$ & $\begin{array}{l}0 \\
0 \\
0 \\
0 \\
0\end{array}$ \\
\hline $6-15$ & 2074 & 1 & 0 & 1 & 0 & 0 & 0 \\
\hline $\begin{array}{l}6-17 \\
6-18 \\
6-19\end{array}$ & $\begin{array}{l}1501 \\
4293 \\
2789\end{array}$ & $\begin{array}{r}0 \\
1 \\
15\end{array}$ & $\begin{array}{l}0 \\
1 \\
9\end{array}$ & $\begin{array}{l}0 \\
0 \\
6\end{array}$ & $\begin{array}{l}0 \\
0 \\
0\end{array}$ & $\begin{array}{l}0 \\
0 \\
0\end{array}$ & $\begin{array}{l}0 \\
0 \\
0\end{array}$ \\
\hline $\begin{array}{l}6-20 \\
6-21\end{array}$ & $\begin{array}{l}3904 \\
2718\end{array}$ & $\begin{array}{r}10 \\
7\end{array}$ & $\begin{array}{l}5 \\
1\end{array}$ & $\begin{array}{l}4 \\
3\end{array}$ & $\frac{1}{3}$ & $\begin{array}{l}0 \\
0\end{array}$ & $\begin{array}{l}0 \\
0\end{array}$ \\
\hline $\begin{array}{l}6-23 \\
6-25\end{array}$ & $\begin{array}{l}2504 \\
2506\end{array}$ & $\begin{array}{r}10 \\
5\end{array}$ & $\begin{array}{l}5 \\
0\end{array}$ & $\begin{array}{l}3 \\
5\end{array}$ & $\begin{array}{l}2 \\
0\end{array}$ & $\begin{array}{l}0 \\
0\end{array}$ & 0 \\
\hline $6-27$ & 4237 & 17 & 2 & 10 & 5 & 0 & 0 \\
\hline $\begin{array}{l}6-29 \\
7-01\end{array}$ & -- & -- & $\overline{-}$ & $\overline{-}$ & $\overline{--}$ & $\overline{--}$ & - \\
\hline $7-03$ & -- & -- & -- & -- & -- & -- & -- \\
\hline $7-05$ & -- & -- & -- & -- & -- & $-\cdots$ & $-\infty$ \\
\hline $7-07$ & -- & -- & -- & -- & -- & -- & -- \\
\hline
\end{tabular}


TABLE 15.- NUMBER OF PINK TRACER PARTICLES(1), PER 100 GRAMS OF BEDLOAD SAMPLE, COMPOSITED FROM CROSS-CHANNEL SAMPLING POSITIONS SPACED AT 1-METER INTERVALS ACROSS THE CHANNEL WIDTH,

EAST FORK RIVER, WYOMING, 1980

SECTION 1155

\begin{tabular}{|c|c|c|c|c|c|c|c|}
\hline \multirow{3}{*}{ DATE } & \multirow{3}{*}{$\begin{array}{c}\text { DRY } \\
\text { MASS } \\
\text { OF } \\
\text { SAMPLE (2) } \\
\text { (G) }\end{array}$} & \multicolumn{6}{|c|}{ NUMBER OF PINK TRACER PARTICLES } \\
\hline & & \multirow[b]{2}{*}{ TOTAL } & \multicolumn{5}{|c|}{ BY SIZE CLASS (MM) } \\
\hline & & & $\begin{array}{l}0.25 \\
\text { TO } \\
0.50\end{array}$ & $\begin{array}{l}0.50 \\
\text { TO } \\
1.00\end{array}$ & $\begin{array}{l}1.00 \\
2.00 \\
\text { TO }\end{array}$ & $\begin{array}{l}2.00 \\
\text { TO } \\
4.00\end{array}$ & $\begin{array}{l}4.00 \\
\text { TO } \\
8.00\end{array}$ \\
\hline $\begin{array}{l}5-16 \\
5-19 \\
5-21 \\
5-22 \\
5-24\end{array}$ & $\begin{array}{r}17+++ \\
73+++ \\
2437 \\
2980 \\
2253\end{array}$ & $\begin{array}{l}0 \\
0 \\
3 \\
0 \\
1\end{array}$ & $\begin{array}{l}0 \\
0 \\
1 \\
0 \\
1\end{array}$ & $\begin{array}{l}0 \\
0 \\
2 \\
0 \\
0\end{array}$ & $\begin{array}{l}0 \\
0 \\
0 \\
0 \\
0\end{array}$ & $\begin{array}{l}0 \\
0 \\
0 \\
0 \\
0\end{array}$ & $\begin{array}{l}0 \\
0 \\
0 \\
0 \\
0\end{array}$ \\
\hline $\begin{array}{l}5-26 \\
5-27 \\
5-28 \\
5-29\end{array}$ & $\begin{array}{l}\overline{25++} \\
51+\end{array}$ & $\begin{array}{r}- \\
0 \\
-\end{array}$ & $\begin{array}{r}-\overline{4} \\
0 \\
-\end{array}$ & $\begin{array}{r}-\overline{0} \\
0 \\
-\end{array}$ & $\begin{array}{r}-\overline{0} \\
0 \\
--\end{array}$ & $\begin{array}{r}-\overline{0} \\
0 \\
-\end{array}$ & $\begin{array}{r}--\overline{0} \\
0 \\
-\end{array}$ \\
\hline $5-30$ & $52+$ & 0 & 0 & 0 & 0 & 0 & 0 \\
\hline $\begin{array}{l}6-01 \\
6-03 \\
6-05 \\
6-07 \\
6-09\end{array}$ & $\begin{array}{c}46++ \\
87++ \\
23+++ \\
341 \\
2849\end{array}$ & $\begin{array}{l}0 \\
1 \\
0 \\
0 \\
1\end{array}$ & $\begin{array}{l}0 \\
0 \\
0 \\
0 \\
0\end{array}$ & $\begin{array}{l}0 \\
1 \\
0 \\
0 \\
1\end{array}$ & $\begin{array}{l}0 \\
0 \\
0 \\
0 \\
0\end{array}$ & $\begin{array}{l}0 \\
0 \\
0 \\
0 \\
0\end{array}$ & $\begin{array}{l}0 \\
0 \\
0 \\
0 \\
0\end{array}$ \\
\hline $\begin{array}{l}6-10 \\
6-11 \\
6-12 \\
6-13 \\
6-14\end{array}$ & $\begin{array}{r}4178 \\
1008 \\
776 \\
526 \\
2043\end{array}$ & $\begin{array}{l}2 \\
6 \\
1 \\
0 \\
1\end{array}$ & $\begin{array}{l}1 \\
5 \\
0 \\
0 \\
0\end{array}$ & $\begin{array}{l}1 \\
1 \\
0 \\
0 \\
0\end{array}$ & $\begin{array}{l}0 \\
0 \\
1 \\
0 \\
1\end{array}$ & $\begin{array}{l}0 \\
0 \\
0 \\
0 \\
0\end{array}$ & $\begin{array}{l}0 \\
0 \\
0 \\
0 \\
0\end{array}$ \\
\hline $6-15$ & 957 & 0 & 0 & 0 & 0 & 0 & 0 \\
\hline $\begin{array}{l}6-17 \\
6-18 \\
6-19\end{array}$ & $\begin{array}{r}659 \\
2916 \\
3706\end{array}$ & $\begin{array}{l}7 \\
7 \\
6\end{array}$ & $\begin{array}{l}6 \\
3 \\
2\end{array}$ & $\begin{array}{l}0 \\
4 \\
4\end{array}$ & $\begin{array}{l}0 \\
0 \\
0\end{array}$ & $\begin{array}{l}1 \\
0 \\
0\end{array}$ & $\begin{array}{l}0 \\
0 \\
0\end{array}$ \\
\hline $\begin{array}{l}6-20 \\
6-21\end{array}$ & $\begin{array}{l}1301 \\
2023\end{array}$ & $\begin{array}{l}6 \\
6\end{array}$ & $\frac{1}{1}$ & $\frac{4}{2}$ & $\frac{1}{3}$ & $\begin{array}{l}0 \\
0\end{array}$ & $\begin{array}{l}0 \\
0\end{array}$ \\
\hline $\begin{array}{l}6-23 \\
6-25\end{array}$ & $\begin{array}{r}1384 \\
629\end{array}$ & $\begin{array}{l}3 \\
9\end{array}$ & $\begin{array}{l}0 \\
1\end{array}$ & $\begin{array}{l}3 \\
8\end{array}$ & $\begin{array}{l}0 \\
0\end{array}$ & $\begin{array}{l}0 \\
0\end{array}$ & $\begin{array}{l}0 \\
0\end{array}$ \\
\hline $6-27$ & 3473 & 11 & 0 & 7 & 4 & 0 & 0 \\
\hline $7-01$ & - & - & - & - & - & - & - \\
\hline $7-03$ & - & - & -- & -- & -- & -- & - \\
\hline $7-05$ & -- & -- & -- & -- & - & -- & -- \\
\hline $7-07$ & -- & -- & -- & -- & -- & -- & - \\
\hline
\end{tabular}


TABLE 15.- NUMBER OF PINK TRACER PARTICLES(1), PER 100 GRAMS OF BEDLOAD SAMPLE, COMPOSITED FROM CROSS-CHANNEL SAMPLING POSITIONS SPACED AT 1-METER INTERVALS ACROSS THE CHANNEL WIDTH, EAST FORK RIVER, WYOMING, 1980

\section{SECTION 1202}

\begin{tabular}{|c|c|c|c|c|c|c|c|}
\hline \multirow{3}{*}{\multicolumn{2}{|c|}{$\begin{array}{lc}\text { DATE } & \text { DRY } \\
& \text { MASS } \\
\text { OF } \\
& \text { SAMPLE (2) } \\
& (\mathrm{G})\end{array}$}} & \multicolumn{6}{|c|}{ NUMBER OF PINK TRACER PARTICLES } \\
\hline & & \multirow[b]{2}{*}{ TOTAL } & \multicolumn{5}{|c|}{ BY SIZE CLASS (MM) } \\
\hline & & & $\begin{array}{l}0.25 \\
\text { TO } \\
0.50\end{array}$ & $\begin{array}{c}0.50 \\
\text { TO } \\
1.00\end{array}$ & $\begin{array}{l}1.00 \\
2.00 \\
\text { TO }\end{array}$ & $\begin{array}{l}2.00 \\
\text { TO } \\
4.00\end{array}$ & $\begin{array}{l}4.00 \\
\text { TO } \\
8.00\end{array}$ \\
\hline $\begin{array}{l}5-16 \\
5-19 \\
5-21 \\
5-22 \\
5-24\end{array}$ & $\begin{array}{c}323 \\
251+ \\
2833 \\
6017 \\
1764\end{array}$ & $\begin{array}{r}10 \\
1 \\
5 \\
0 \\
2\end{array}$ & $\begin{array}{r}10 \\
1 \\
5 \\
0 \\
2\end{array}$ & $\begin{array}{l}0 \\
0 \\
0 \\
0 \\
0\end{array}$ & $\begin{array}{l}0 \\
0 \\
0 \\
0 \\
0\end{array}$ & $\begin{array}{l}0 \\
0 \\
0 \\
0 \\
0\end{array}$ & $\begin{array}{l}0 \\
0 \\
0 \\
0 \\
0\end{array}$ \\
\hline $\begin{array}{l}5-26 \\
5-27 \\
5-28 \\
5-29 \\
5-30\end{array}$ & $\begin{array}{l}\frac{402}{113+} \\
\overline{35++}\end{array}$ & $\begin{array}{r}-\overline{3} \\
-\frac{1}{0}\end{array}$ & $\begin{array}{r}--\overline{2} \\
-\frac{2}{0}\end{array}$ & $-\frac{-}{1}$ & $\begin{array}{r}-\overline{0} \\
0 \\
-0\end{array}$ & $\begin{array}{r}-- \\
0 \\
-0 \\
0\end{array}$ & $\begin{array}{r}-- \\
0 \\
0 \\
-0\end{array}$ \\
\hline $\begin{array}{l}6-01 \\
6-03 \\
6-05 \\
6-07 \\
6-09\end{array}$ & $\begin{array}{l}102++ \\
419 \\
1372 \\
1767 \\
7316\end{array}$ & $\begin{array}{l}0 \\
6 \\
1 \\
4 \\
1\end{array}$ & $\begin{array}{l}0 \\
3 \\
0 \\
1 \\
0\end{array}$ & $\begin{array}{l}0 \\
3 \\
1 \\
3 \\
1\end{array}$ & $\begin{array}{l}0 \\
0 \\
0 \\
0 \\
0\end{array}$ & $\begin{array}{l}0 \\
0 \\
0 \\
0 \\
0\end{array}$ & $\begin{array}{l}0 \\
0 \\
0 \\
0 \\
0\end{array}$ \\
\hline $\begin{array}{l}6-10 \\
6-11 \\
6-12 \\
6-13 \\
6-14\end{array}$ & $\begin{array}{r}4322 \\
1660 \\
907 \\
729 \\
2204\end{array}$ & $\begin{array}{l}1 \\
1 \\
5 \\
0 \\
0\end{array}$ & $\begin{array}{l}0 \\
0 \\
2 \\
0 \\
0\end{array}$ & $\begin{array}{l}\frac{1}{1} \\
\frac{1}{2} \\
0 \\
0\end{array}$ & $\begin{array}{l}0 \\
0 \\
1 \\
0 \\
0\end{array}$ & $\begin{array}{l}0 \\
0 \\
0 \\
0 \\
0\end{array}$ & $\begin{array}{l}0 \\
0 \\
0 \\
0 \\
0\end{array}$ \\
\hline $6-15$ & 917 & 3 & 1 & 2 & 0 & 0 & 0 \\
\hline $\begin{array}{l}0-10 \\
6-17 \\
6-18 \\
6-19\end{array}$ & $\begin{array}{l}1171 \\
1103 \\
4168\end{array}$ & $\begin{array}{r}10 \\
3 \\
3\end{array}$ & $\begin{array}{l}2 \\
2 \\
1\end{array}$ & $\begin{array}{l}6 \\
1 \\
0\end{array}$ & $\begin{array}{l}2 \\
0 \\
2\end{array}$ & $\begin{array}{l}0 \\
0 \\
0\end{array}$ & $\begin{array}{l}0 \\
0 \\
0\end{array}$ \\
\hline $\begin{array}{l}6-20 \\
6-21\end{array}$ & $\begin{array}{l}2102 \\
4380\end{array}$ & $\begin{array}{l}9 \\
4\end{array}$ & $\begin{array}{l}2 \\
0\end{array}$ & $\begin{array}{l}7 \\
4\end{array}$ & $\begin{array}{l}0 \\
0\end{array}$ & $\begin{array}{l}0 \\
0\end{array}$ & $\begin{array}{l}0 \\
0\end{array}$ \\
\hline $\begin{array}{l}6-22 \\
6-23 \\
6-25\end{array}$ & $\begin{array}{l}2561 \\
5151\end{array}$ & $\begin{array}{r}16 \\
8\end{array}$ & 10 & 6 & 1 & 0 & 0 \\
\hline $6-27$ & 2402 & 11 & 1 & 7 & 3 & $\underline{-0}$ & $\underline{0}$ \\
\hline $\begin{array}{l}0-29 \\
7-01\end{array}$ & - & - & - & - & - & - & - \\
\hline $\begin{array}{l}7-03 \\
7-05\end{array}$ & $\overline{-}$ & -- & $=$ & $\overline{-}$ & $\overline{-}$ & $\overline{-}$ & $\overline{--}$ \\
\hline $7-07$ & -- & -- & - & -- & -- & -- & -- \\
\hline
\end{tabular}


TABLE 15.- NUMBER OF PINK TRACER PARTICLES(1), PER 100 GRAMS OF BEDLOAD SAMPLE, COMPOSITED FROM CROSS-CHANNEL SAMPLING POSITIONS SPACED AT 1-METER INTERVALS ACROSS THE CHANNEL WIDTH, EAST FORK RIVER, WYOMING, 1980

SECTION 1241

\begin{tabular}{|c|c|c|c|c|c|c|c|}
\hline \multirow{3}{*}{ DATE } & \multirow{3}{*}{$\begin{array}{c}\text { DRY } \\
\text { MASS } \\
\text { OF } \\
\text { SAMPLE (2) } \\
\text { (G) }\end{array}$} & \multicolumn{6}{|c|}{ NUMBER OF PINK TRACER PARTICLES } \\
\hline & & \multirow[b]{2}{*}{ TOTAL } & \multicolumn{5}{|c|}{ BY SIZE CLASS (MM) } \\
\hline & & & $\begin{array}{l}0.25 \\
\text { TO } \\
0.50\end{array}$ & $\begin{array}{l}0.50 \\
\text { TO } \\
1.00\end{array}$ & $\begin{array}{l}1.00 \\
\text { TO } \\
2.00\end{array}$ & $\begin{array}{l}2.00 \\
\text { TO } \\
4.00\end{array}$ & $\begin{array}{r}4.00 \\
\text { TO } \\
8.00\end{array}$ \\
\hline $\begin{array}{l}5-16 \\
5-19 \\
5-21 \\
5-22 \\
5-24\end{array}$ & $\begin{array}{r}909 \\
359 \\
1312 \\
3206 \\
510\end{array}$ & $\begin{array}{l}1 \\
4 \\
0 \\
0 \\
4\end{array}$ & $\begin{array}{l}1 \\
3 \\
0 \\
0 \\
4\end{array}$ & $\begin{array}{l}0 \\
1 \\
0 \\
0 \\
0\end{array}$ & $\begin{array}{l}0 \\
0 \\
0 \\
0 \\
0\end{array}$ & $\begin{array}{l}0 \\
0 \\
0 \\
0 \\
0\end{array}$ & $\begin{array}{l}0 \\
0 \\
0 \\
0 \\
0\end{array}$ \\
\hline $\begin{array}{l}5-26 \\
5-27 \\
5-28 \\
5-29 \\
5-30\end{array}$ & $\begin{array}{l}\overline{56++} \\
170 \\
660 \\
101+\end{array}$ & $\begin{array}{r}-- \\
9 \\
1 \\
14 \\
0\end{array}$ & $\begin{array}{r}-\frac{-}{7} \\
1 \\
1 \frac{1}{1} \\
0\end{array}$ & $\begin{array}{r}-- \\
2 \\
0 \\
3 \\
0\end{array}$ & $\begin{array}{l}-- \\
0 \\
0 \\
0 \\
0\end{array}$ & $\begin{array}{r}-- \\
0 \\
0 \\
0 \\
0\end{array}$ & $\begin{array}{l}-- \\
0 \\
0 \\
0 \\
0\end{array}$ \\
\hline $\begin{array}{l}6-01 \\
6-03 \\
6-05 \\
6-07 \\
6-09\end{array}$ & $\begin{array}{r}1777 \\
452 \\
759 \\
1177 \\
1501\end{array}$ & $\begin{array}{l}5 \\
6 \\
0 \\
6 \\
0\end{array}$ & $\begin{array}{l}3 \\
2 \\
0 \\
5 \\
0\end{array}$ & $\begin{array}{l}2 \\
4 \\
0 \\
1 \\
0\end{array}$ & $\begin{array}{l}0 \\
0 \\
0 \\
0 \\
0\end{array}$ & $\begin{array}{l}0 \\
0 \\
0 \\
0 \\
0\end{array}$ & $\begin{array}{l}0 \\
0 \\
0 \\
0 \\
0\end{array}$ \\
\hline $\begin{array}{l}6-10 \\
6-11 \\
6-12 \\
6-13 \\
6-14\end{array}$ & $\begin{array}{r}690 \\
945 \\
324 \\
308 \\
1093\end{array}$ & $\begin{array}{l}0 \\
4 \\
4 \\
0 \\
2\end{array}$ & $\begin{array}{l}0 \\
3 \\
3 \\
0 \\
2\end{array}$ & $\begin{array}{l}0 \\
1 \\
1 \\
0 \\
0\end{array}$ & $\begin{array}{l}0 \\
0 \\
0 \\
0 \\
0\end{array}$ & $\begin{array}{l}0 \\
0 \\
0 \\
0 \\
0\end{array}$ & $\begin{array}{l}0 \\
0 \\
0 \\
0 \\
0\end{array}$ \\
\hline $\begin{array}{l}6-15 \\
6-16 \\
6-17 \\
6-18 \\
6-19\end{array}$ & $\begin{array}{l}1350 \\
301 \\
1431 \\
3603 \\
1291\end{array}$ & $\begin{array}{r}4 \\
11 \\
9 \\
\frac{1}{3}\end{array}$ & $\begin{array}{l}\frac{1}{3} \\
2 \\
0 \\
3\end{array}$ & $\begin{array}{l}2 \\
7 \\
7 \\
0 \\
0\end{array}$ & $\begin{array}{l}1 \\
1 \\
0 \\
1 \\
0\end{array}$ & $\begin{array}{l}0 \\
0 \\
0 \\
0 \\
0\end{array}$ & $\begin{array}{l}0 \\
0 \\
0 \\
0 \\
0\end{array}$ \\
\hline $\begin{array}{l}6-20 \\
6-21 \\
6-22\end{array}$ & $\begin{array}{l}2036 \\
6118 \\
\end{array}$ & $\begin{array}{r}7 \\
7 \\
-2\end{array}$ & $\frac{1}{3}$ & $\begin{array}{r}4 \\
4 \\
-\end{array}$ & $\begin{array}{l}1 \\
0\end{array}$ & $\begin{array}{l}1 \\
0\end{array}$ & $\begin{array}{l}0 \\
0\end{array}$ \\
\hline $\begin{array}{l}6-23 \\
6-25\end{array}$ & $\begin{array}{r}453 \\
5117\end{array}$ & $\begin{array}{r}7 \\
15\end{array}$ & $\overline{2}$ & 11 & $\frac{1}{3}$ & $\overline{0}$ & 0 \\
\hline $\begin{array}{l}6-27 \\
6-29\end{array}$ & 1604 & 18 & -3 & 10 & -4 & -1 & -0 \\
\hline $7-0 \frac{1}{3}$ & -- & -- & -- & - & - & -- & -- \\
\hline $\begin{array}{l}7-03 \\
7-05\end{array}$ & -- & -- & -- & -- & -- & -- & -- \\
\hline $7-07$ & -- & -- & -- & -- & -- & -- & -- \\
\hline
\end{tabular}


TABLE 15.- NUMBER OF PINK TRACER PARTICLES(1), PER 100 GRAMS OF BEDLOAD SAMPLE, COMPOSITED FROM CROSS-CHANNEL SAMPLING POSITIONS SPACED AT 1-METER INTERVALS ACROSS THE CHANNEL WIDTH, EAST FORK RIVER, WYOMING, 1980

SECTION 1284

\begin{tabular}{|c|c|c|c|c|c|c|c|}
\hline \multirow{3}{*}{\multicolumn{2}{|c|}{$\begin{array}{cc}\text { DRY } \\
\text { DATE } \\
\text { OFS } \\
\text { SAMPLE (2) } \\
\text { (G) }\end{array}$}} & \multicolumn{6}{|c|}{ NUMBER OF PINK TRACER PARTICLES } \\
\hline & & \multirow[b]{2}{*}{ TOTAL } & \multicolumn{5}{|c|}{ BY SIZE CLASS (MM) } \\
\hline & & & $\begin{array}{c}0.25 \\
\text { TO } \\
0.50\end{array}$ & $\begin{array}{l}0.50 \\
\text { TO } \\
1.00\end{array}$ & $\begin{array}{l}1.00 \\
2.00\end{array}$ & $\begin{array}{l}2.00 \\
\text { TO } \\
4.00\end{array}$ & $\begin{array}{l}4.00 \\
\text { TO } \\
8.00\end{array}$ \\
\hline $\begin{array}{l}5-16 \\
5-19 \\
5-21 \\
5-22 \\
5-24\end{array}$ & $\begin{array}{r}3816 \\
1486 \\
1193 \\
1704 \\
763\end{array}$ & $\begin{array}{l}0 \\
1 \\
1 \\
0 \\
7\end{array}$ & $\begin{array}{l}0 \\
1 \\
1 \\
0 \\
7\end{array}$ & $\begin{array}{l}0 \\
0 \\
0 \\
0 \\
0\end{array}$ & $\begin{array}{l}0 \\
0 \\
0 \\
0 \\
0\end{array}$ & $\begin{array}{l}0 \\
0 \\
0 \\
0 \\
0\end{array}$ & $\begin{array}{l}0 \\
0 \\
0 \\
0 \\
0\end{array}$ \\
\hline $\begin{array}{l}5-26 \\
5-27 \\
5-28\end{array}$ & $\begin{array}{l}14 \overline{59} \\
1247\end{array}$ & $\begin{array}{r}-- \\
0 \\
4\end{array}$ & $\begin{array}{r}-- \\
0 \\
4\end{array}$ & $\begin{array}{r}--\overline{0} \\
0\end{array}$ & $\begin{array}{r}--\overline{0} \\
0\end{array}$ & $\begin{array}{r}-- \\
0 \\
0\end{array}$ & $\begin{array}{r}-- \\
0 \\
0\end{array}$ \\
\hline $\begin{array}{l}5-29 \\
5-30\end{array}$ & 1345 & 0 & 0 & 0 & 0 & 0 & 0 \\
\hline $\begin{array}{l}6-01 \\
6-03 \\
6-05 \\
6-07 \\
6-09\end{array}$ & $\begin{array}{r}1178 \\
741 \\
944 \\
969 \\
2293\end{array}$ & $\begin{array}{l}3 \\
1 \\
0 \\
5 \\
0\end{array}$ & $\begin{array}{l}0 \\
0 \\
0 \\
3 \\
0\end{array}$ & $\begin{array}{l}3 \\
1 \\
0 \\
2 \\
0\end{array}$ & $\begin{array}{l}0 \\
0 \\
0 \\
0 \\
0\end{array}$ & $\begin{array}{l}0 \\
0 \\
0 \\
0 \\
0\end{array}$ & $\begin{array}{l}0 \\
0 \\
0 \\
0 \\
0\end{array}$ \\
\hline $\begin{array}{l}6-10 \\
6-11 \\
6-12 \\
6-13 \\
6-14\end{array}$ & $\begin{array}{r}1907 \\
879 \\
374 \\
470 \\
897\end{array}$ & $\begin{array}{r}6 \\
10 \\
3 \\
6 \\
11\end{array}$ & $\begin{array}{l}4 \\
8 \\
1 \\
4 \\
8\end{array}$ & $\begin{array}{l}\frac{1}{2} \\
2 \\
2 \\
3\end{array}$ & $\begin{array}{l}0 \\
0 \\
0 \\
0 \\
0\end{array}$ & $\begin{array}{l}1 \\
0 \\
0 \\
0 \\
0\end{array}$ & $\begin{array}{l}0 \\
0 \\
0 \\
0 \\
0\end{array}$ \\
\hline $\begin{array}{l}6-15 \\
6-16 \\
6-17 \\
6-18 \\
6-19\end{array}$ & $\begin{array}{l}2970 \\
1347 \\
6911 \\
2817 \\
2731\end{array}$ & $\begin{array}{r}7 \\
13 \\
14 \\
2 \\
4\end{array}$ & $\begin{array}{l}2 \\
6 \\
3 \\
1 \\
2\end{array}$ & $\begin{array}{l}5 \\
7 \\
9 \\
1 \\
0\end{array}$ & $\begin{array}{l}0 \\
0 \\
2 \\
0 \\
2\end{array}$ & $\begin{array}{l}0 \\
0 \\
0 \\
0 \\
0\end{array}$ & $\begin{array}{l}0 \\
0 \\
0 \\
0 \\
0\end{array}$ \\
\hline $\begin{array}{l}6-20 \\
6-21\end{array}$ & $\begin{array}{l}1814 \\
1452\end{array}$ & $\begin{array}{r}20 \\
8\end{array}$ & $\begin{array}{l}6 \\
2\end{array}$ & $\begin{array}{r}12 \\
4\end{array}$ & $\begin{array}{l}2 \\
1\end{array}$ & $\begin{array}{l}0 \\
0\end{array}$ & $\begin{array}{l}0 \\
1\end{array}$ \\
\hline $\begin{array}{l}6-22 \\
6-23 \\
6-25\end{array}$ & $\begin{array}{l}24 \overline{30} \\
2306\end{array}$ & $\frac{10}{21}$ & 3 & $\begin{array}{r}6 \\
18\end{array}$ & $\frac{1}{2}$ & 0 & 0 \\
\hline $6-27$ & 3246 & 25 & 4 & 16 & 4 & 1 & 0 \\
\hline $\begin{array}{l}6-29 \\
7-01\end{array}$ & $\overline{--}$ & $\overline{--}$ & $\overline{--}$ & $\overline{--}$ & $\overline{-}$ & $=$ & $\overline{--}$ \\
\hline $\begin{array}{l}7-03 \\
7-05\end{array}$ & $\overline{-}$ & $\overline{--}$ & $\overline{--}$ & $\overline{--}$ & $\overline{-}$ & $\overline{--}$ & $\overline{-}$ \\
\hline $7-07$ & -- & -- & -- & -- & - & -- & - \\
\hline
\end{tabular}


TABLE 15.- NUMBER OF PINK TRACER PARTICLES(1), PER 100 GRAMS OF BEDLOAD SAMPLE, COMPOSITED FROM CROSS-CHANKEL SAMPLING POSITIONS SPACED AT I-METER INTERVALS ACROSS THE CHANNEL WIDTH, EAST FORK RIVER, WYOMING, 1980

SECTION 1315

\begin{tabular}{|c|c|c|c|c|c|c|c|}
\hline \multirow{3}{*}{ DATE } & \multirow{3}{*}{$\begin{array}{c}\text { DRY } \\
\text { MASS } \\
\text { OF } \\
\text { SAMPLE (2) } \\
(G)\end{array}$} & \multicolumn{6}{|c|}{ NUMBER OF PINK TRACER PARTICLES } \\
\hline & & \multirow[b]{2}{*}{ TOTAL } & \multicolumn{5}{|c|}{ BY SIZE CLASS (MM) } \\
\hline & & & $\begin{array}{l}0.25 \\
\text { TO } \\
0.50\end{array}$ & $\begin{array}{l}0.50 \\
\text { TO } \\
1.00\end{array}$ & $\begin{array}{l}1.00 \\
2.00 \\
2.00\end{array}$ & $\begin{array}{l}2.00 \\
\text { TO } \\
4.00\end{array}$ & $\begin{array}{l}4.00 \\
\text { TO } \\
8.00\end{array}$ \\
\hline $\begin{array}{l}5-16 \\
5-19 \\
5-21 \\
5-22 \\
5-24\end{array}$ & $\begin{array}{r}803 \\
351 \\
374 \\
684 \\
1499\end{array}$ & $\begin{array}{r}0 \\
8 \\
0 \\
3 \\
10\end{array}$ & $\begin{array}{r}0 \\
8 \\
0 \\
3 \\
10\end{array}$ & $\begin{array}{l}0 \\
0 \\
0 \\
0 \\
0\end{array}$ & $\begin{array}{l}0 \\
0 \\
0 \\
0 \\
0\end{array}$ & $\begin{array}{l}0 \\
0 \\
0 \\
0 \\
0\end{array}$ & $\begin{array}{l}0 \\
0 \\
0 \\
0 \\
0\end{array}$ \\
\hline $\begin{array}{l}5-26 \\
5-27 \\
5-28 \\
5-29 \\
5-30\end{array}$ & $\begin{array}{c}12 \overline{3} \\
9 \overline{9}+ \\
12 \overline{5}\end{array}$ & $-\frac{-}{1}$ & $\begin{array}{r}-- \\
0 \\
-\frac{1}{1}\end{array}$ & $-\frac{-}{1}$ & $\begin{array}{r}-\overline{0} \\
0 \\
-1\end{array}$ & $\begin{array}{r}-\overline{0} \\
-\frac{0}{0}\end{array}$ & $\begin{array}{r}-\overline{0} \\
0 \\
-0\end{array}$ \\
\hline $\begin{array}{l}6-01 \\
6-03 \\
6-05 \\
6-07 \\
6-09\end{array}$ & $\begin{array}{c}1241 \\
217 \\
98+ \\
249 \\
483\end{array}$ & $\begin{array}{r}2 \\
2 \\
2 \\
10 \\
0\end{array}$ & $\begin{array}{l}2 \\
0 \\
2 \\
3 \\
0\end{array}$ & $\begin{array}{l}0 \\
0 \\
0 \\
7 \\
0\end{array}$ & $\begin{array}{l}0 \\
2 \\
0 \\
0 \\
0\end{array}$ & $\begin{array}{l}0 \\
0 \\
0 \\
0 \\
0\end{array}$ & $\begin{array}{l}0 \\
0 \\
0 \\
0 \\
0\end{array}$ \\
\hline $\begin{array}{l}6-10 \\
6-11 \\
6-12 \\
6-13 \\
6-14\end{array}$ & $\begin{array}{r}2803 \\
4062 \\
842 \\
846 \\
2194\end{array}$ & $\begin{array}{l}4 \\
3 \\
3 \\
4 \\
4\end{array}$ & $\begin{array}{l}4 \\
3 \\
1 \\
\frac{1}{2}\end{array}$ & $\begin{array}{l}0 \\
0 \\
2 \\
3 \\
1\end{array}$ & $\begin{array}{l}0 \\
0 \\
0 \\
0 \\
1\end{array}$ & $\begin{array}{l}0 \\
0 \\
0 \\
0 \\
0\end{array}$ & $\begin{array}{l}0 \\
0 \\
0 \\
0 \\
0\end{array}$ \\
\hline $\begin{array}{l}6-15 \\
6-16 \\
6-17 \\
6-18 \\
6-19\end{array}$ & $\begin{array}{l}2768 \\
3105 \\
5091 \\
2403 \\
2294\end{array}$ & $\begin{array}{r}12 \\
17 \\
9 \\
12 \\
10\end{array}$ & $\begin{array}{l}4 \\
8 \\
2 \\
2 \\
2\end{array}$ & $\begin{array}{l}8 \\
9 \\
5 \\
7 \\
6\end{array}$ & $\begin{array}{l}0 \\
0 \\
2 \\
3 \\
2\end{array}$ & $\begin{array}{l}0 \\
0 \\
0 \\
0 \\
0\end{array}$ & $\begin{array}{l}0 \\
0 \\
0 \\
0 \\
0\end{array}$ \\
\hline $\begin{array}{l}6-20 \\
6-21\end{array}$ & $\begin{array}{l}2348 \\
2830\end{array}$ & $\begin{array}{r}99 \\
16\end{array}$ & $\begin{array}{l}2 \\
6\end{array}$ & $\begin{array}{l}4 \\
8\end{array}$ & $\begin{array}{l}2 \\
2\end{array}$ & 1 & $\begin{array}{l}0 \\
0\end{array}$ \\
\hline $\begin{array}{l}6-23 \\
6-25\end{array}$ & $\begin{array}{l}3316 \\
6606\end{array}$ & $2 \frac{9}{26}$ & $\frac{1}{2}$ & $\begin{array}{r}6 \\
18\end{array}$ & $\frac{1}{3}$ & $\frac{1}{3}$ & $\begin{array}{l}0 \\
0\end{array}$ \\
\hline $6-27$ & 2276 & 27 & 3 & 22 & 2 & 0 & 0 \\
\hline $7-01$ & - & - & - & - & - & - & - \\
\hline $\begin{array}{l}7-03 \\
7-05\end{array}$ & -- & -- & -- & -- & -- & $-\overline{-}$ & $\overline{--}$ \\
\hline & & & & & & & \\
\hline $7-07$ & -- & -- & -- & -- & -- & -- & -- \\
\hline
\end{tabular}


TABLE 15.- NUMBER OF PINK TRACER PARTICLES(1), PER 100 GRAMS OF BEDLOAD SAMPLE, COMPOSITED FROM CROSS-CHANNEL SAMPL ING POSITIONS SPACED AT I-METER INTERVALS ACROSS THE CHANNEL WIDTH, EAST FORK RIVER, WYOMING, 1980

SECTION $1360(4)$

\begin{tabular}{|c|c|c|c|c|c|c|c|}
\hline \multirow{3}{*}{ DATE } & \multirow{3}{*}{$\begin{array}{c}\text { DRY } \\
\text { MASS } \\
\text { OF } \\
\text { SAMPLE (2) } \\
\text { (G) }\end{array}$} & \multicolumn{6}{|c|}{ NUMBER OF PINK TRACER PARTICLES } \\
\hline & & \multirow[b]{2}{*}{ TOTAL } & \multicolumn{5}{|c|}{ BY SIZE CLASS (MM) } \\
\hline & & & $\begin{array}{c}0.25 \\
\text { TO } \\
0.50\end{array}$ & $\begin{array}{l}0.50 \\
\text { TO } \\
1.00\end{array}$ & $\begin{array}{l}1.00 \\
\text { TO } \\
2.00\end{array}$ & $\begin{array}{l}2.00 \\
\text { TO } \\
4.00\end{array}$ & $\begin{array}{l}4.00 \\
\text { TO } \\
8.00\end{array}$ \\
\hline $\begin{array}{l}5-16 \\
5-19 \\
5-21 \\
5-22 \\
5-24\end{array}$ & $\begin{array}{r}114+ \\
255+ \\
197+ \\
1553 \\
1286\end{array}$ & $\begin{array}{r}0 \\
4 \\
14 \\
0 \\
19\end{array}$ & $\begin{array}{r}0 \\
3 \\
13 \\
0 \\
17\end{array}$ & $\begin{array}{l}0 \\
1 \\
1 \\
0 \\
2\end{array}$ & $\begin{array}{l}0 \\
0 \\
0 \\
0 \\
0\end{array}$ & $\begin{array}{l}0 \\
0 \\
0 \\
0 \\
0\end{array}$ & $\begin{array}{l}0 \\
0 \\
0 \\
0 \\
0\end{array}$ \\
\hline $\begin{array}{l}5-26 \\
5-27 \\
5-28 \\
5-29 \\
5-30\end{array}$ & $\frac{4 \overline{4}++}{1042}$ & $\begin{array}{r}-\overline{0} \\
-1 \\
-\frac{3}{3}\end{array}$ & $\begin{array}{r}-\overline{0} \\
0 \\
-\overline{0}\end{array}$ & $\begin{array}{c}--\overline{0} \\
-\frac{1}{1}\end{array}$ & $\begin{array}{r}-\overline{0} \\
0 \\
-\overline{0}\end{array}$ & $\begin{array}{r}-\overline{0} \\
-\frac{1}{2}\end{array}$ & $\begin{array}{r}-\overline{0} \\
-0 \\
-0\end{array}$ \\
\hline $\begin{array}{l}6-01 \\
6-03 \\
6-05 \\
6-07 \\
6-09\end{array}$ & $\begin{aligned} & 31++ \\
& 235+ \\
& 147 \\
& 535 \\
& 1049\end{aligned}$ & $\begin{array}{l}0 \\
5 \\
5 \\
8 \\
6\end{array}$ & $\begin{array}{l}0 \\
2 \\
0 \\
5 \\
1\end{array}$ & $\begin{array}{l}0 \\
2 \\
5 \\
3 \\
5\end{array}$ & $\begin{array}{l}0 \\
1 \\
0 \\
0 \\
0\end{array}$ & $\begin{array}{l}0 \\
0 \\
0 \\
0 \\
0\end{array}$ & $\begin{array}{l}0 \\
0 \\
0 \\
0 \\
0\end{array}$ \\
\hline $\begin{array}{l}6-10 \\
6-11 \\
6-12 \\
6-13 \\
6-14\end{array}$ & $\begin{array}{r}1192 \\
1011 \\
419 \\
841 \\
612\end{array}$ & $\begin{array}{r}7 \\
8 \\
11 \\
2 \\
11\end{array}$ & $\begin{array}{l}2 \\
3 \\
5 \\
0 \\
4\end{array}$ & $\begin{array}{l}5 \\
4 \\
6 \\
2 \\
6\end{array}$ & $\begin{array}{l}0 \\
0 \\
0 \\
0 \\
1\end{array}$ & $\begin{array}{l}0 \\
1 \\
0 \\
0 \\
0\end{array}$ & $\begin{array}{l}0 \\
0 \\
0 \\
0 \\
0\end{array}$ \\
\hline $\begin{array}{l}6-15 \\
6-16 \\
6-17 \\
6-18 \\
6-19\end{array}$ & $\begin{array}{r}3832 \\
1042 \\
588 \\
520 \\
1603\end{array}$ & $\begin{array}{r}11 \\
12 \\
13 \\
8 \\
13\end{array}$ & $\begin{array}{l}3 \\
2 \\
2 \\
4 \\
1\end{array}$ & $\begin{array}{r}6 \\
7 \\
10 \\
4 \\
10\end{array}$ & $\begin{array}{l}2 \\
2 \\
1 \\
0 \\
2\end{array}$ & $\begin{array}{l}0 \\
1 \\
0 \\
0 \\
0\end{array}$ & $\begin{array}{l}0 \\
0 \\
0 \\
0 \\
0\end{array}$ \\
\hline $\begin{array}{l}6-20 \\
6-21\end{array}$ & $\begin{array}{r}391 \\
1253\end{array}$ & $\begin{array}{r}6 \\
12\end{array}$ & $\begin{array}{l}0 \\
2\end{array}$ & $\frac{1}{7}$ & $\begin{array}{l}5 \\
2\end{array}$ & $\begin{array}{l}0 \\
1\end{array}$ & $\begin{array}{l}0 \\
0\end{array}$ \\
\hline $\begin{array}{l}6-23 \\
6-25\end{array}$ & $\begin{array}{l}265 \\
651\end{array}$ & $\begin{array}{r}8 \\
13\end{array}$ & $\begin{array}{l}4 \\
5\end{array}$ & $\begin{array}{l}2 \\
6\end{array}$ & 2 & $\begin{array}{l}0 \\
0\end{array}$ & $\begin{array}{l}0 \\
0\end{array}$ \\
\hline $6-27$ & 870 & 20 & 7 & 10 & 2 & 1 & 0 \\
\hline $7-01$ & - & - & - & - & - & - & - \\
\hline $\begin{array}{l}7-03 \\
7-05\end{array}$ & - & $\overline{--}$ & $\overline{--}$ & $\overline{--}$ & $\overline{z-}$ & 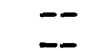 & $\overline{--}$ \\
\hline $7-07$ & -- & -- & -- & -- & -- & -- & -- \\
\hline
\end{tabular}


TABLE 15.- NUMBER OF PINK TRACER PARTICLES(1), PER 100 GRAMS OF BEDLOAD SAMPLE, COMPOSITED FROM CROSS-CHANNEL SAMPLING POSITIONS SPACED AT I-METER INTERVALS ACROSS THE CHANNEL WIDTH, EAST FORK RIVER, WYOMING, 1980

SECTION 1396(4)

\begin{tabular}{|c|c|c|c|c|c|c|c|}
\hline \multirow{3}{*}{\multicolumn{2}{|c|}{$\begin{array}{cc} & \\
\text { DATE } & \text { DRY } \\
& \text { MASS } \\
\text { OF } & \\
& \text { SAMPLE (2) } \\
& (\mathrm{G})\end{array}$}} & \multicolumn{6}{|c|}{ NUMBER OF PINK TRACER PARTICLES } \\
\hline & & \multirow[b]{2}{*}{ TOTAL } & \multicolumn{5}{|c|}{ BY SIZE CLASS (MM) } \\
\hline & & & $\begin{array}{l}0.25 \\
\text { TO } \\
0.50\end{array}$ & $\begin{array}{c}0.50 \\
\text { TO } \\
1.00\end{array}$ & $\begin{array}{l}1.00 \\
\text { TO } \\
2.00\end{array}$ & $\begin{array}{l}2.00 \\
\text { TO } \\
4.00\end{array}$ & $\begin{array}{l}4.00 \\
\text { TO } \\
8.00\end{array}$ \\
\hline $\begin{array}{l}5-16 \\
5-19 \\
5-21 \\
5-22 \\
5-24\end{array}$ & $\begin{array}{r}331 \\
318 \\
1166 \\
589 \\
1626\end{array}$ & $\begin{array}{l}0 \\
3 \\
2 \\
0 \\
4\end{array}$ & $\begin{array}{l}0 \\
3 \\
2 \\
0 \\
2\end{array}$ & $\begin{array}{l}0 \\
0 \\
0 \\
0 \\
2\end{array}$ & $\begin{array}{l}0 \\
0 \\
0 \\
0 \\
0\end{array}$ & $\begin{array}{l}0 \\
0 \\
0 \\
0 \\
0\end{array}$ & $\begin{array}{l}0 \\
0 \\
0 \\
0 \\
0\end{array}$ \\
\hline $\begin{array}{l}5-26 \\
5-27 \\
5-28\end{array}$ & $\begin{array}{r}\overline{32} \\
439\end{array}$ & $\begin{array}{r}- \\
3 \\
2\end{array}$ & $\begin{array}{r}-- \\
3 \\
0\end{array}$ & $\begin{array}{r}-\overline{0} \\
2\end{array}$ & $\begin{array}{r}-- \\
0 \\
0\end{array}$ & $\begin{array}{r}-- \\
0 \\
0\end{array}$ & $\begin{array}{r}-- \\
0 \\
0\end{array}$ \\
\hline $5-30$ & 398 & 5 & 0 & 5 & 0 & 0 & 0 \\
\hline $\begin{array}{l}6-01 \\
6-03 \\
6-05 \\
6-07 \\
6-09\end{array}$ & $\begin{array}{l}178 \\
472 \\
407 \\
532 \\
493\end{array}$ & $\begin{array}{l}0 \\
5 \\
1 \\
5 \\
7\end{array}$ & $\begin{array}{l}0 \\
0 \\
1 \\
2 \\
5\end{array}$ & $\begin{array}{l}0 \\
4 \\
0 \\
1 \\
2\end{array}$ & $\begin{array}{l}0 \\
1 \\
0 \\
2 \\
0\end{array}$ & $\begin{array}{l}0 \\
0 \\
0 \\
0 \\
0\end{array}$ & $\begin{array}{l}0 \\
0 \\
0 \\
0 \\
0\end{array}$ \\
\hline $\begin{array}{l}6-10 \\
6-11 \\
6-12 \\
6-13 \\
6-14\end{array}$ & $\begin{array}{r}2231 \\
1028 \\
467 \\
651 \\
2493\end{array}$ & $\begin{array}{l}2 \\
9 \\
5 \\
3 \\
3\end{array}$ & $\begin{array}{l}1 \\
3 \\
1 \\
0 \\
0\end{array}$ & $\begin{array}{l}0 \\
4 \\
3 \\
2 \\
3\end{array}$ & $\begin{array}{l}1 \\
1 \\
1 \\
1 \\
0\end{array}$ & $\begin{array}{l}0 \\
1 \\
0 \\
0 \\
0\end{array}$ & $\begin{array}{l}0 \\
0 \\
0 \\
0 \\
0\end{array}$ \\
\hline $\begin{array}{l}6-15 \\
6-16 \\
6-17 \\
6-18 \\
6-19\end{array}$ & $\begin{array}{r}1830 \\
1400 \\
751 \\
1513 \\
1126\end{array}$ & $\begin{array}{r}8 \\
10 \\
17 \\
9 \\
4\end{array}$ & $\begin{array}{l}2 \\
4 \\
8 \\
1 \\
0\end{array}$ & $\begin{array}{l}5 \\
5 \\
7 \\
5 \\
3\end{array}$ & $\begin{array}{l}1 \\
1 \\
2 \\
2 \\
1\end{array}$ & $\begin{array}{l}0 \\
0 \\
0 \\
1 \\
0\end{array}$ & $\begin{array}{l}0 \\
0 \\
0 \\
0 \\
0\end{array}$ \\
\hline $\begin{array}{l}6-20 \\
6-21\end{array}$ & $\begin{array}{l}432 \\
855\end{array}$ & $\begin{array}{l}7 \\
9\end{array}$ & $\begin{array}{l}1 \\
0\end{array}$ & $\begin{array}{l}5 \\
6\end{array}$ & $\frac{1}{1}$ & $\begin{array}{l}0 \\
2\end{array}$ & $\begin{array}{l}0 \\
0\end{array}$ \\
\hline $\begin{array}{l}6-23 \\
6-25\end{array}$ & $\begin{array}{l}754 \\
487\end{array}$ & 8 & $\begin{array}{l}0 \\
2\end{array}$ & $\begin{array}{r}-\overline{4} \\
5\end{array}$ & $\frac{1}{0}$ & $\frac{1}{1}$ & $\begin{array}{l}\overline{0} \\
0\end{array}$ \\
\hline $6-27$ & 1773 & 15 & 2 & 8 & 3 & 2 & 0 \\
\hline $7-01$ & - & - & $\overline{--}$ & - & $\overline{--}$ & $\overline{--}$ & $\overline{-}$ \\
\hline $7-0 \overline{3}$ & -- & -- & -- & -- & -- & -- & -- \\
\hline $7-05$ & -- & - & -- & -- & -- & -- & -- \\
\hline $7-07$ & -- & -- & -- & -- & -- & -- & -- \\
\hline
\end{tabular}


TABLE 15.- NUMBER OF PINK TRACER PARTICLES(1), PER 100 GRAMS OF BEDLOAD SAMPLE, COMPOSITED FROM CROSS-CHANNEL SAMPLING POSITIONS SPACED AT 1-METER INTERVALS ACROSS THE CHANNEL WIDTH, EAST FORK RIVER, WYOMING, 1980

$$
\text { SECTION } 1400(5)
$$

\begin{tabular}{|c|c|c|c|c|c|c|c|}
\hline \multirow{3}{*}{ DATE } & \multirow{3}{*}{$\begin{array}{c}\text { DRY } \\
\text { MASS } \\
\text { OF } \\
\text { SAMPLE (2) } \\
\text { (G) }\end{array}$} & \multicolumn{6}{|c|}{ NUMBER OF PINK TRACER PARTICLES } \\
\hline & & \multirow[b]{2}{*}{ TOTAL } & \multicolumn{5}{|c|}{ BY SIZE CLASS (MM) } \\
\hline & & & $\begin{array}{c}0.25 \\
\text { TO } \\
0.50\end{array}$ & $\begin{array}{l}0.50 \\
\text { TO } \\
1.00\end{array}$ & $\begin{array}{l}1.00 \\
\text { TO } \\
2.00\end{array}$ & $\begin{array}{l}2.00 \\
\text { TOO } \\
4.00\end{array}$ & $\begin{array}{l}4.00 \\
\text { TO } \\
8.00\end{array}$ \\
\hline $5-16$ & -- & -- & - & - & - & -- & - \\
\hline $\begin{array}{l}5-19 \\
5-21\end{array}$ & $\overline{37+++}$ & $-\overline{0}$ & $-\overline{0}$ & $-\overline{0}$ & $\overline{0}$ & $-\overline{0}$ & $-\overline{0}$ \\
\hline $\begin{array}{l}5-22 \\
5-24\end{array}$ & $\begin{array}{r}352 \\
1306\end{array}$ & $\begin{array}{r}3 \\
10\end{array}$ & $\begin{array}{r}3 \\
10\end{array}$ & $\begin{array}{l}0 \\
0\end{array}$ & $\begin{array}{l}0 \\
0\end{array}$ & $\begin{array}{l}0 \\
0\end{array}$ & $\begin{array}{l}0 \\
0\end{array}$ \\
\hline $5-26$ & -- & -- & -- & -- & - & - & -- \\
\hline $5-27$ & 362 & 0 & 0 & 0 & 0 & 0 & 0 \\
\hline $\begin{array}{l}5-28 \\
5-29\end{array}$ & $87+$ & -7 & 7 & $-\underline{0}$ & 0 & 0 & - \\
\hline $5-30$ & $0+++$ & 0 & 0 & 0 & 0 & 0 & 0 \\
\hline $6-01$ & -- & -- & -- & -- & - & -- & - \\
\hline $\begin{array}{l}6-03 \\
6-05\end{array}$ & $\overline{-}$ & $\overline{--}$ & $\overline{--}$ & $\overline{--}$ & $\overline{--}$ & -- & $\overline{--}$ \\
\hline $6-07$ & $0+++$ & 0 & 0 & 0 & 0 & 0 & 0 \\
\hline $6-09$ & 1103 & 4 & 0 & 3 & 1 & 0 & 0 \\
\hline $6-10$ & 877 & 9 & 7 & 2 & 0 & 0 & 0 \\
\hline $\begin{array}{l}6-1 \frac{1}{2} \\
6-12\end{array}$ & $\begin{array}{l}867 \\
371\end{array}$ & $\begin{array}{l}10 \\
26\end{array}$ & $\begin{array}{r}3 \\
13\end{array}$ & $\begin{array}{r}6 \\
13\end{array}$ & $\frac{1}{0}$ & 0 & 0 \\
\hline $6-13$ & 1218 & 9 & 2 & 5 & 2 & 0 & 0 \\
\hline $6-14$ & 1182 & 18 & 3 & 13 & 2 & 0 & 0 \\
\hline $6-15$ & 2624 & 13 & 3 & 8 & 2 & 0 & 0 \\
\hline $\begin{array}{l}6-16 \\
6-17\end{array}$ & $\begin{array}{r}620 \\
\end{array}$ & 19 & 8 & 11 & $\begin{array}{l}0 \\
3\end{array}$ & O & 0 \\
\hline $6-18$ & 799 & $\begin{array}{r}11 \\
9\end{array}$ & $\begin{array}{l}4 \\
3\end{array}$ & 2 & 4 & 0 & 0 \\
\hline $6-19$ & 1020 & 19 & 8 & 10 & $i$ & 0 & 0 \\
\hline $6-20$ & 351 & 12 & 6 & 4 & 2 & 0 & 0 \\
\hline $\begin{array}{l}6-21 \\
6-22\end{array}$ & 2623 & 17 & 1 & 10 & 5 & 1 & 0 \\
\hline $6-23$ & 908 & 7 & 1 & 6 & 0 & 0 & 0 \\
\hline & 265 & 24 & 5 & 18 & 0 & & 0 \\
\hline $6-27$ & 1030 & 0 & 0 & 0 & 0 & 0 & 0 \\
\hline $6-29$ & -- & -- & - & -- & -- & -- & -- \\
\hline $\begin{array}{l}7-01 \\
7-03\end{array}$ & $=-$ & $=$ & $=$ & $=-$ & $=-$ & $\overline{-}$ & $=$ \\
\hline $7-05$ & -- & -- & -- & -- & -- & -- & -- \\
\hline $7-07$ & -- & -- & -- & -- & -- & -- & -- \\
\hline
\end{tabular}


TABLE 15.- NUMBER OF PINK TRACER PARTICLES(1), PER 100 GRAMS OF BEDLOAD SAMPLE, COMPOSITED FROM CROSS-CHANNEL SAMPLING POSITIONS SPACED AT 1-METER INTERVALS ACROSS THE CHANNEL WIDTH, EAST FORK RIVER, WYOMING, 1980

SECTION 1425(4)

\begin{tabular}{|c|c|c|c|c|c|c|c|}
\hline \multirow{3}{*}{ DATE } & \multirow{3}{*}{$\begin{array}{c}\text { DRY } \\
\text { MASS } \\
\text { OF } \\
\text { SAMPLE (2) } \\
\text { (G) } \\
\end{array}$} & \multicolumn{6}{|c|}{ NUMBER OF PINK TRACER PARTICLES } \\
\hline & & \multirow[b]{2}{*}{ TOTAL } & \multicolumn{5}{|c|}{ BY SIZE CLASS (MM) } \\
\hline & & & $\begin{array}{l}0.25 \\
\text { TO } \\
0.50\end{array}$ & $\begin{array}{l}0.50 \\
\text { TO } \\
1.00\end{array}$ & $\begin{array}{l}1.00 \\
\text { TO } \\
2.00\end{array}$ & $\begin{array}{l}2.00 \\
\text { TO } \\
4.00\end{array}$ & $\begin{array}{l}4.00 \\
\text { TO } \\
8.00\end{array}$ \\
\hline $\begin{array}{l}5-16 \\
5-19 \\
5-21 \\
5-22 \\
5-24\end{array}$ & $\begin{array}{l}55++ \\
500 \\
404 \\
1002 \\
1445\end{array}$ & $\begin{array}{l}0 \\
5 \\
0 \\
0 \\
1\end{array}$ & $\begin{array}{l}0 \\
5 \\
0 \\
0 \\
0\end{array}$ & $\begin{array}{l}0 \\
0 \\
0 \\
0 \\
1\end{array}$ & $\begin{array}{l}0 \\
0 \\
0 \\
0 \\
0\end{array}$ & $\begin{array}{l}0 \\
0 \\
0 \\
0 \\
0\end{array}$ & $\begin{array}{l}0 \\
0 \\
0 \\
0 \\
0\end{array}$ \\
\hline $\begin{array}{l}5-26 \\
5-27 \\
5-28 \\
5-29 \\
5-30\end{array}$ & $\begin{array}{c}1 \overline{56+} \\
589 \\
\overline{85}+\end{array}$ & $-\frac{-}{1}$ & $-\frac{1}{0}$ & $\begin{array}{r}-\overline{0} \\
-1 \\
-0\end{array}$ & $\begin{array}{r}-\overline{0} \\
-\frac{1}{2}\end{array}$ & $\begin{array}{r}-- \\
0 \\
-0 \\
0\end{array}$ & $\begin{array}{r}-0 \\
0 \\
-0\end{array}$ \\
\hline $\begin{array}{l}6-01 \\
6-03 \\
6-05 \\
6-07 \\
6-09\end{array}$ & $\begin{array}{l}793 \\
193+ \\
209 \\
935 \\
621\end{array}$ & $\begin{array}{l}\frac{1}{2} \\
1 \\
\frac{1}{5} \\
2\end{array}$ & $\begin{array}{l}0 \\
0 \\
0 \\
0 \\
2\end{array}$ & $\begin{array}{l}\frac{1}{2} \\
1 \\
5 \\
0\end{array}$ & $\begin{array}{l}0 \\
0 \\
0 \\
0 \\
0\end{array}$ & $\begin{array}{l}0 \\
0 \\
0 \\
0 \\
0\end{array}$ & $\begin{array}{l}0 \\
0 \\
0 \\
0 \\
0\end{array}$ \\
\hline $\begin{array}{l}6-10 \\
6-11 \\
6-12 \\
6-13 \\
6-14\end{array}$ & $\begin{array}{r}1337 \\
257 \\
181 \\
126 \\
1223\end{array}$ & $\begin{array}{l}8 \\
6 \\
4 \\
1 \\
7\end{array}$ & $\begin{array}{l}3 \\
4 \\
0 \\
0 \\
3\end{array}$ & $\begin{array}{l}5 \\
2 \\
2 \\
1 \\
3\end{array}$ & $\begin{array}{l}0 \\
0 \\
1 \\
0 \\
1\end{array}$ & $\begin{array}{l}0 \\
0 \\
1 \\
0 \\
0\end{array}$ & $\begin{array}{l}0 \\
0 \\
0 \\
0 \\
0\end{array}$ \\
\hline $\begin{array}{l}6-15 \\
6-16 \\
6-17 \\
6-18 \\
6-19\end{array}$ & $\begin{array}{l}197 \\
146 \\
177 \\
807 \\
186\end{array}$ & $\begin{array}{r}16 \\
5 \\
3 \\
7 \\
7\end{array}$ & $\begin{array}{l}5 \\
1 \\
2 \\
2 \\
2\end{array}$ & $\begin{array}{r}10 \\
2 \\
1 \\
4 \\
5\end{array}$ & $\begin{array}{l}1 \\
2 \\
0 \\
1 \\
0\end{array}$ & $\begin{array}{l}0 \\
0 \\
0 \\
0 \\
0\end{array}$ & $\begin{array}{l}0 \\
0 \\
0 \\
0 \\
0\end{array}$ \\
\hline $\begin{array}{l}6-20 \\
6-21 \\
6-22 \\
6-23 \\
6-25\end{array}$ & $\begin{array}{l}368 \\
228 \\
-7 \\
258 \\
212\end{array}$ & $\begin{array}{l}6 \\
18 \\
19 \\
19\end{array}$ & $\begin{array}{r}0 \\
-\frac{3}{3} \\
1 \frac{1}{3}\end{array}$ & $\frac{13}{18}$ & $-\frac{1}{2}$ & $\begin{array}{r}1 \\
-\frac{1}{0} \\
0\end{array}$ & $\begin{array}{r}0 \\
0 \\
-0 \\
0\end{array}$ \\
\hline $\begin{array}{l}6-27 \\
6-29 \\
7-01 \\
7-03 \\
7-05\end{array}$ & $\begin{array}{r}664 \\
-- \\
=- \\
--\end{array}$ & $\begin{array}{l}14 \\
=- \\
=-\end{array}$ & $\begin{array}{l}3 \\
-- \\
-- \\
--\end{array}$ & $\begin{array}{l}7 \\
-- \\
-- \\
--\end{array}$ & $\begin{array}{l}3 \\
-- \\
--\end{array}$ & $\begin{array}{l}1 \\
=- \\
=- \\
--\end{array}$ & $\begin{array}{l}0 \\
=- \\
=- \\
--\end{array}$ \\
\hline $7-07$ & -- & -- & -- & -- & -- & -- & -- \\
\hline
\end{tabular}


TABLE 15.- NUMBER OF PINK TRACER PARTICLES(1), PER 100 GRAMS OF BEDLOAD SAMPLE, COMPOSITED FROM CROSS-CHANNEL SAMPLING POSITIONS SPACED AT 1-METER INTERVALS ACROSS THE CHANNEL WIDTH, EAST FORK RIVER, WYOMING, 1980

SECTION 1481

\begin{tabular}{|c|c|c|c|c|c|c|c|}
\hline \multirow{3}{*}{ DATE } & \multirow{3}{*}{$\begin{array}{c}\text { DRY } \\
\text { MASS } \\
\text { OF } \\
\text { SAMPLE (2) } \\
\text { (G) }\end{array}$} & \multicolumn{6}{|c|}{ NUMBER OF PINK TRACER PARTICLES } \\
\hline & & \multirow[b]{2}{*}{ TOTAL } & \multicolumn{5}{|c|}{ BY SIZE CLASS (MM) } \\
\hline & & & $\begin{array}{c}0.25 \\
\text { TO } \\
0.50\end{array}$ & $\begin{array}{l}0.50 \\
\text { TO } \\
1.00\end{array}$ & $\begin{array}{l}1.00 \\
\text { To } \\
2.00\end{array}$ & $\begin{array}{l}2.00 \\
\text { TO } \\
4.00\end{array}$ & $\begin{array}{c}4.00 \\
\text { TO } \\
8.00\end{array}$ \\
\hline $\begin{array}{l}5-16 \\
5-19 \\
5-21 \\
5-22 \\
5-24\end{array}$ & $\begin{array}{l}177^{++} \\
320 \\
329 \\
2322 \\
3171\end{array}$ & $\begin{array}{l}0 \\
5 \\
4 \\
5 \\
7\end{array}$ & $\begin{array}{l}0 \\
2 \\
3 \\
5 \\
5\end{array}$ & $\begin{array}{l}0 \\
3 \\
1 \\
0 \\
1\end{array}$ & $\begin{array}{l}0 \\
0 \\
0 \\
0 \\
1\end{array}$ & $\begin{array}{l}0 \\
0 \\
0 \\
0 \\
0\end{array}$ & $\begin{array}{l}0 \\
0 \\
0 \\
0 \\
0\end{array}$ \\
\hline $\begin{array}{l}5-26 \\
5-27 \\
5-28\end{array}$ & $\begin{array}{l}4 \overline{36} \\
29 \overline{5}\end{array}$ & $\begin{array}{r}-- \\
2 \\
5\end{array}$ & $\begin{array}{r}-\overline{0} \\
0\end{array}$ & $\begin{array}{r}-- \\
2 \\
5\end{array}$ & $\begin{array}{r}-\overline{0} \\
0\end{array}$ & $\begin{array}{r}-\overline{0} \\
0\end{array}$ & $\begin{array}{r}-- \\
0 \\
0\end{array}$ \\
\hline $5-30$ & 436 & 3 & 1 & 1 & 1 & 0 & 0 \\
\hline $\begin{array}{l}6-01 \\
6-03 \\
6-05 \\
6-07 \\
6-09\end{array}$ & $\begin{array}{l}187+ \\
235 \\
197 \\
389 \\
2148\end{array}$ & $\begin{array}{l}8 \\
6 \\
5 \\
5 \\
4\end{array}$ & $\begin{array}{l}3 \\
2 \\
0 \\
1 \\
1\end{array}$ & $\begin{array}{l}5 \\
3 \\
2 \\
4 \\
3\end{array}$ & $\begin{array}{l}0 \\
1 \\
3 \\
0 \\
0\end{array}$ & $\begin{array}{l}0 \\
0 \\
0 \\
0 \\
0\end{array}$ & $\begin{array}{l}0 \\
0 \\
0 \\
0 \\
0\end{array}$ \\
\hline $\begin{array}{l}6-10 \\
6-11 \\
6-12 \\
6-13 \\
6-14\end{array}$ & $\begin{array}{r}2785 \\
1497 \\
685 \\
767 \\
1433\end{array}$ & $\begin{array}{r}11 \\
6 \\
17 \\
10 \\
5\end{array}$ & $\begin{array}{l}3 \\
1 \\
6 \\
3 \\
3\end{array}$ & $\begin{array}{r}7 \\
4 \\
10 \\
7 \\
1\end{array}$ & $\begin{array}{l}0 \\
1 \\
1 \\
0 \\
1\end{array}$ & $\begin{array}{l}1 \\
0 \\
0 . \\
0 \\
0\end{array}$ & $\begin{array}{l}0 \\
0 \\
0 \\
0 \\
0\end{array}$ \\
\hline $\begin{array}{l}6-15 \\
6-16 \\
6-17 \\
6-18 \\
6-19\end{array}$ & $\begin{array}{r}2451 \\
824 \\
674 \\
2022 \\
1665\end{array}$ & $\begin{array}{l}13 \\
10 \\
8 \\
16 \\
11\end{array}$ & $\begin{array}{l}1 \\
4 \\
1 \\
2 \\
0\end{array}$ & $\begin{array}{l}8 \\
1 \\
5 \\
8 \\
2\end{array}$ & $\begin{array}{l}4 \\
5 \\
1 \\
5 \\
7\end{array}$ & $\begin{array}{l}0 \\
0 \\
1 \\
1 \\
2\end{array}$ & $\begin{array}{l}0 \\
0 \\
0 \\
0 \\
0\end{array}$ \\
\hline $\begin{array}{l}6-20 \\
6-21\end{array}$ & $\begin{array}{l}1146 \\
1233\end{array}$ & $\begin{array}{l}11 \\
15\end{array}$ & $\frac{1}{3}$ & $\begin{array}{l}5 \\
7\end{array}$ & $\begin{array}{l}3 \\
4\end{array}$ & $\begin{array}{l}2 \\
0\end{array}$ & $\begin{array}{l}0 \\
1\end{array}$ \\
\hline $\begin{array}{l}6-23 \\
6-25\end{array}$ & $\begin{array}{r}776 \\
1216\end{array}$ & $\frac{14}{21}$ & $\frac{1}{2}$ & $\begin{array}{r}8 \\
11\end{array}$ & 4 & $\frac{1}{1}$ & $\begin{array}{l}0 \\
0\end{array}$ \\
\hline $6-27$ & 1479 & 29 & 5 & 23 & 1 & 0 & 0 \\
\hline $\begin{array}{l}6-29 \\
7-01\end{array}$ & $\overline{--}$ & $\overline{-}$ & $\overline{--}$ & $\overline{-}$ & $\overline{--}$ & $\overline{-}$ & $\overline{--}$ \\
\hline $\begin{array}{l}7-03 \\
7-05\end{array}$ & $\overline{--}$ & $\overline{-}$ & $\overline{--}$ & $\overline{--}$ & $\overline{--}$ & $\overline{-}$ & $\overline{--}$ \\
\hline $7-07$ & -- & -- & -- & -- & -- & -- & -- \\
\hline
\end{tabular}


TABLE 15.- NUMBER OF PINK TRACER PARTICLES(1), PER 100 GRAMS OF BEDLOAD SAMPLE, COMPOSITED FROM CROSS-CHANNEL SAMPLING POSITIONS SPACED AT I-METER INTERVALS ACROSS THE CHANNEL WIDTH,

EAST FORK RIVER, WYOMING, 1980

SECTION $1481(3)$

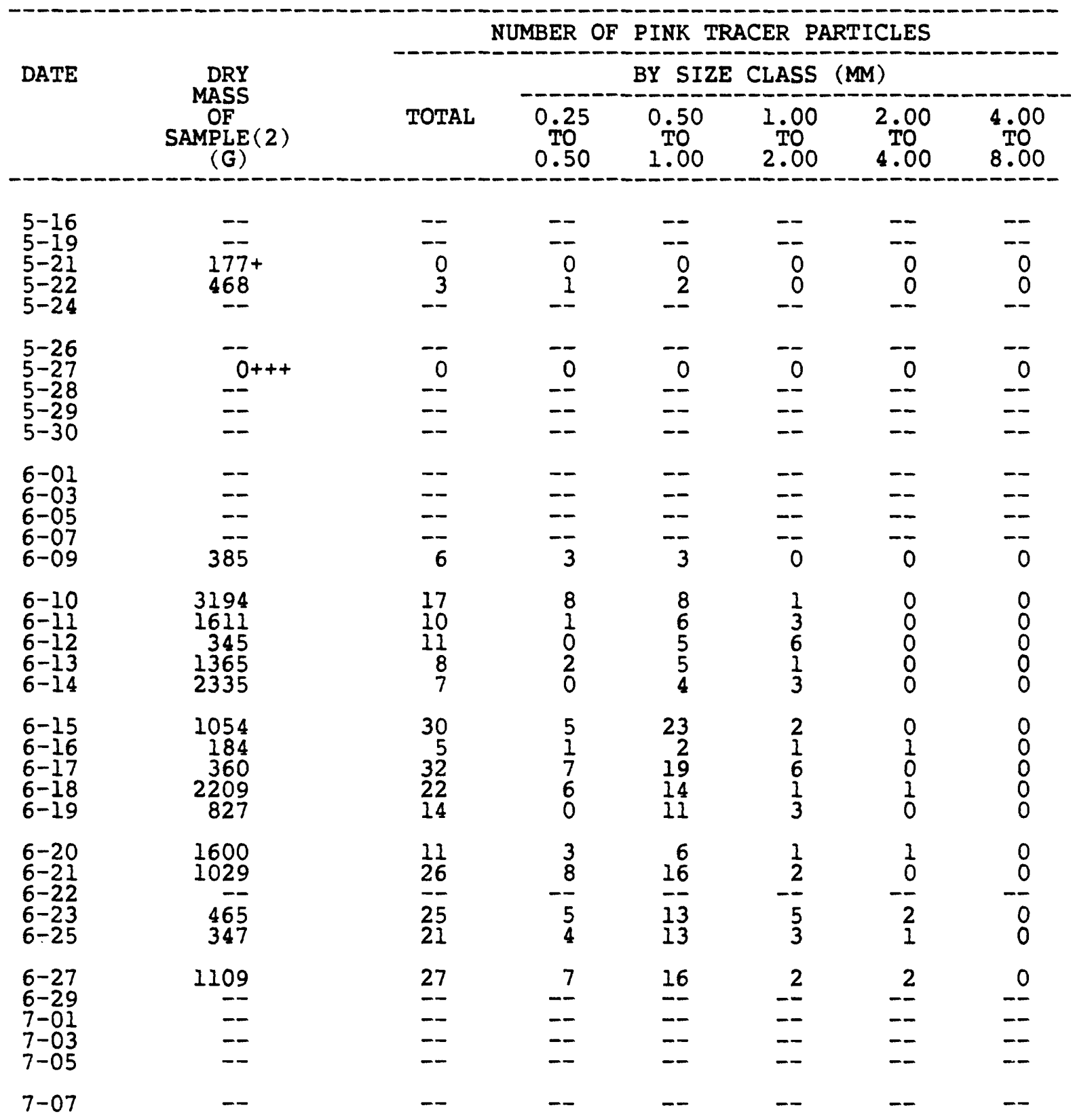


TABLE 15.- NUMBER OF PINK TRACER PARTICLES(1), PER 100 GRAMS OF BEDLOAD SAMPLE, COMPOSITED FROM CROSS-CHANNEL SAMPLING POSITIONS SPACED AT 1-METER INTERVALS ACROSS THE CHANNEL WIDTH, EAST FORK RIVER, WYOMING, 1980

SECTION 1533

\begin{tabular}{|c|c|c|c|c|c|c|c|}
\hline \multirow{3}{*}{\multicolumn{2}{|c|}{$\begin{array}{cc} & \\
\text { DATE } & \text { DRY } \\
& \text { MASS } \\
\text { OF } \\
\text { SAMPLE(2) } \\
\\
\text { (G) }\end{array}$}} & \multicolumn{6}{|c|}{ NUMBER OF PINK TRACER PARTICLES } \\
\hline & & \multirow[b]{2}{*}{ TOTAL } & \multicolumn{5}{|c|}{ BY SIZE CLASS (MM) } \\
\hline & & & $\begin{array}{l}0.25 \\
\text { TO } \\
0.50\end{array}$ & $\begin{array}{l}0.50 \\
\text { TO } \\
1.00\end{array}$ & $\begin{array}{l}1.00 \\
\text { To } \\
2.00\end{array}$ & $\begin{array}{l}2.00 \\
\text { TO } \\
4.00\end{array}$ & $\begin{array}{l}4.00 \\
\text { TO } \\
8.00\end{array}$ \\
\hline $\begin{array}{l}5-16 \\
5-19 \\
5-21 \\
5-22 \\
5-24\end{array}$ & $\begin{array}{c}72++ \\
22+++ \\
256 \\
2210 \\
1455\end{array}$ & $\begin{array}{l}3 \\
0 \\
7 \\
3 \\
9\end{array}$ & $\begin{array}{l}3 \\
0 \\
6 \\
3 \\
7\end{array}$ & $\begin{array}{l}0 \\
0 \\
1 \\
0 \\
2\end{array}$ & $\begin{array}{l}0 \\
0 \\
0 \\
0 \\
0\end{array}$ & $\begin{array}{l}0 \\
0 \\
0 \\
0 \\
0\end{array}$ & $\begin{array}{l}0 \\
0 \\
0 \\
0 \\
0\end{array}$ \\
\hline $\begin{array}{l}5-26 \\
5-27 \\
5-28\end{array}$ & $\begin{array}{l}11 \overline{39} \\
1385\end{array}$ & $\begin{array}{r}-\overline{6} \\
0\end{array}$ & $\overline{1}$ & $\begin{array}{r}-\overline{3} \\
0\end{array}$ & $\begin{array}{r}-- \\
0\end{array}$ & $-\frac{1}{0}$ & $\begin{array}{c}-\overline{0} \\
0\end{array}$ \\
\hline $5-30$ & 249 & 8 & 1 & 5 & 2 & 0 & 0 \\
\hline $\begin{array}{l}6-01 \\
6-03 \\
6-05 \\
6-07 \\
6-09\end{array}$ & $\begin{array}{l}452 \\
169 \\
521 \\
342 \\
979\end{array}$ & $\begin{array}{l}6 \\
0 \\
6 \\
5 \\
9\end{array}$ & $\begin{array}{l}2 \\
0 \\
1 \\
1 \\
6\end{array}$ & $\begin{array}{l}4 \\
0 \\
3 \\
1 \\
3\end{array}$ & $\begin{array}{l}0 \\
0 \\
1 \\
3 \\
0\end{array}$ & $\begin{array}{l}0 \\
0 \\
0 \\
0 \\
0\end{array}$ & $\begin{array}{l}0 \\
0 \\
1 \\
0 \\
0\end{array}$ \\
\hline $\begin{array}{l}6-10 \\
6-11 \\
6-12 \\
6-13 \\
6-14\end{array}$ & $\begin{array}{l}4669 \\
1344 \\
1319 \\
3564 \\
3557\end{array}$ & $\begin{array}{r}8 \\
4 \\
10 \\
17 \\
7\end{array}$ & $\begin{array}{l}3 \\
1 \\
4 \\
3 \\
1\end{array}$ & $\begin{array}{r}3 \\
2 \\
5 \\
11 \\
4\end{array}$ & $\begin{array}{l}2 \\
1 \\
0 \\
3 \\
1\end{array}$ & $\begin{array}{l}0 \\
0 \\
1 \\
0 \\
1\end{array}$ & $\begin{array}{l}0 \\
0 \\
0 \\
0 \\
0\end{array}$ \\
\hline $6-15$ & 2663 & 10 & 1 & 5 & 3 & 1 & 0 \\
\hline $\begin{array}{l}6-17 \\
6-18 \\
6-19\end{array}$ & $\begin{array}{r}2076 \\
4222 \\
745\end{array}$ & $\begin{array}{l}15 \\
15 \\
15\end{array}$ & $\begin{array}{l}5 \\
3 \\
3\end{array}$ & $\begin{array}{l}8 \\
9 \\
8\end{array}$ & $\begin{array}{l}\frac{1}{3} \\
2\end{array}$ & $\begin{array}{l}1 \\
0 \\
2\end{array}$ & $\begin{array}{l}0 \\
0 \\
0\end{array}$ \\
\hline $\begin{array}{l}6-20 \\
6-21\end{array}$ & $\begin{array}{l}1383 \\
2034\end{array}$ & $\begin{array}{l}23 \\
17\end{array}$ & $\begin{array}{l}7 \\
3\end{array}$ & $\begin{array}{l}14 \\
11\end{array}$ & $\begin{array}{l}2 \\
1\end{array}$ & $\begin{array}{l}0 \\
2\end{array}$ & $\begin{array}{l}0 \\
0\end{array}$ \\
\hline $\begin{array}{l}6-23 \\
6-25\end{array}$ & $\begin{array}{l}1253 \\
1756\end{array}$ & $\begin{array}{r}9 \\
16\end{array}$ & $\frac{1}{0}$ & $\begin{array}{r}5 \\
10\end{array}$ & $\begin{array}{l}3 \\
5\end{array}$ & $\begin{array}{l}0 \\
1\end{array}$ & $\begin{array}{l}0 \\
0\end{array}$ \\
\hline $6-27$ & 3070 & 24 & 3 & 15 & 5 & 1 & 0 \\
\hline $\begin{array}{l}6-29 \\
7-01\end{array}$ & $\overline{--}$ & $\overline{--}$ & $\overline{--}$ & $\overline{--}$ & $\overline{--}$ & $\overline{--}$ & $\overline{--}$ \\
\hline $7-0 \overline{3}$ & 1043 & 14 & 2 & 6 & 4 & 2 & 0 \\
\hline & & & & & & & \\
\hline $7-07$ & $81+$ & 1 & 0 & 1 & 0 & 0 & 0 \\
\hline
\end{tabular}


TABLE 15.- NUMBER OF PINK TRACER PARTICLES(1), PER 100 GRAMS OF BEDLOAD SAMPLE, COMPOSITED FROM CROSS-CHANNEL SAMPLING POSITIONS SPACED AT 1-METER INTERVALS ACROSS THE CHANNEL WIDTH, EAST FORK RIVER, WYOMING, 1980

SECTICH 1573

\begin{tabular}{|c|c|c|c|c|c|c|c|}
\hline \multirow{3}{*}{ DATE } & \multirow{3}{*}{$\begin{array}{c}\text { DRY } \\
\text { MASS } \\
\text { OF } \\
\text { SAMPLE (2) } \\
\text { (G) }\end{array}$} & \multicolumn{6}{|c|}{ NUMBER OF PINK TRACER PARTICLES } \\
\hline & & \multirow[b]{2}{*}{ TOTAL } & \multicolumn{5}{|c|}{ BY SIZE CLASS (MM) } \\
\hline & & & $\begin{array}{l}0.25 \\
\text { TO } \\
0.50\end{array}$ & $\begin{array}{l}0.50 \\
\text { TO } \\
1.00\end{array}$ & $\begin{array}{l}1.00 \\
\text { TO } \\
2.00\end{array}$ & $\begin{array}{l}2.00 \\
\text { TO } \\
4.00\end{array}$ & $\begin{array}{l}4.00 \\
\text { TO } \\
8.00\end{array}$ \\
\hline $\begin{array}{l}5-16 \\
5-19 \\
5-21 \\
5-22 \\
5-24\end{array}$ & $\begin{array}{l}0+++ \\
109+++ \\
223 \\
5886 \\
3327\end{array}$ & $\begin{array}{r}0 \\
0 \\
14 \\
3 \\
3\end{array}$ & $\begin{array}{r}0 \\
0 \\
13 \\
3 \\
2\end{array}$ & $\begin{array}{l}0 \\
0 \\
1 \\
0 \\
1\end{array}$ & $\begin{array}{l}0 \\
0 \\
0 \\
0 \\
0\end{array}$ & $\begin{array}{l}0 \\
0 \\
0 \\
0 \\
0\end{array}$ & $\begin{array}{l}0 \\
0 \\
0 \\
0 \\
0\end{array}$ \\
\hline $\begin{array}{l}5-26 \\
5-27 \\
5-28 \\
5-20\end{array}$ & $\begin{array}{r}10 \overline{8} \\
102+\end{array}$ & $\begin{array}{r}-\overline{2} \\
1\end{array}$ & $\begin{array}{r}-- \\
0 \\
0\end{array}$ & $\begin{array}{r}-\overline{2} \\
1\end{array}$ & $\begin{array}{r}-- \\
0 \\
0\end{array}$ & $\begin{array}{r}-- \\
0\end{array}$ & $\begin{array}{r}-- \\
0\end{array}$ \\
\hline $5-30$ & 471 & 7 & 2 & 5 & 0 & 0 & 0 \\
\hline $\begin{array}{l}6-01 \\
6-03 \\
6-05 \\
6-07 \\
6-09\end{array}$ & $\begin{array}{c}93+ \\
110+ \\
45++ \\
59++ \\
2507\end{array}$ & $\begin{array}{l}3 \\
8 \\
0 \\
5 \\
8\end{array}$ & $\begin{array}{l}0 \\
1 \\
0 \\
0 \\
4\end{array}$ & $\begin{array}{l}3 \\
7 \\
0 \\
5 \\
3\end{array}$ & $\begin{array}{l}0 \\
0 \\
0 \\
0 \\
1\end{array}$ & $\begin{array}{l}0 \\
0 \\
0 \\
0 \\
0\end{array}$ & $\begin{array}{l}0 \\
0 \\
0 \\
0 \\
0\end{array}$ \\
\hline $\begin{array}{l}6-10 \\
6-11 \\
6-12 \\
6-13 \\
6-14\end{array}$ & $\begin{array}{l}2398 \\
1189 \\
4094 \\
3490 \\
5136\end{array}$ & $\begin{array}{r}18 \\
9 \\
17 \\
9 \\
14\end{array}$ & $\begin{array}{l}6 \\
4 \\
5 \\
1 \\
4\end{array}$ & $\begin{array}{r}10 \\
4 \\
10 \\
6 \\
8\end{array}$ & $\begin{array}{l}2 \\
1 \\
2 \\
2 \\
2\end{array}$ & $\begin{array}{l}0 \\
0 \\
0 \\
0 \\
0\end{array}$ & $\begin{array}{l}0 \\
0 \\
0 \\
0 \\
0\end{array}$ \\
\hline $6-15$ & 1507 & 10 & 1 & 6 & 2 & 1 & 0 \\
\hline $\begin{array}{l}6-17 \\
6-18 \\
6-19\end{array}$ & $\begin{array}{l}5589 \\
3202 \\
1359\end{array}$ & $\begin{array}{l}16 \\
11 \\
23\end{array}$ & $\begin{array}{l}2 \\
\frac{1}{2}\end{array}$ & $\begin{array}{r}10 \\
6 \\
14\end{array}$ & $\begin{array}{l}3 \\
4 \\
6\end{array}$ & $\begin{array}{l}1 \\
0 \\
0\end{array}$ & $\begin{array}{l}0 \\
0 \\
1\end{array}$ \\
\hline $\begin{array}{l}6-20 \\
6-21\end{array}$ & $\begin{array}{l}4272 \\
3339\end{array}$ & $\begin{array}{r}8 \\
19\end{array}$ & $\frac{1}{5}$ & $\begin{array}{r}5 \\
10\end{array}$ & $\frac{1}{3}$ & $\begin{array}{l}0 \\
1\end{array}$ & $\begin{array}{l}1 \\
0\end{array}$ \\
\hline $\begin{array}{l}6-23 \\
6-25\end{array}$ & $\begin{array}{l}1849 \\
2192\end{array}$ & $\begin{array}{l}13 \\
28\end{array}$ & $\begin{array}{l}2 \\
6\end{array}$ & 25 & 3 & $\begin{array}{l}2 \\
0\end{array}$ & $\begin{array}{l}1 \\
0\end{array}$ \\
\hline $\begin{array}{l}6-27 \\
6-29\end{array}$ & 2560 & 22 & -3 & 13 & -5 & 1 & - \\
\hline $7-01$ & -- & -- & -- & -- & -- & -- & -- \\
\hline $7-05$ & $2 \overline{69}$ & $\overline{17}$ & $\overline{4}$ & $\overline{7}$ & $\overline{4}$ & $\overline{2}$ & $\overline{0}$ \\
\hline $7-07$ & 393 & 15 & 3 & 9 & 2 & 1 & 0 \\
\hline
\end{tabular}


TABLE 15.- NUMBER OF PINK TRACER PARTICLES(1), PER 100 GRAMS OF BEDLOAD SAMPLE, COMPOSITED FROM CROSS-CHANNEL SAMPLING POSITIONS SPACED AT 1-METER INTERVALS ACROSS THE CHANNEL WIDTH, EAST FORK RIVER, WYOMING, 1980

SECTION 1610

\begin{tabular}{|c|c|c|c|c|c|c|c|}
\hline \multirow{3}{*}{ DATE } & \multirow{3}{*}{$\begin{array}{c}\text { DRY } \\
\text { MASS } \\
\text { OF } \\
\text { SAMPLE }(2) \\
(G)\end{array}$} & \multicolumn{6}{|c|}{ NUMBER OF PINK TRACER PARTICLES } \\
\hline & & \multirow[b]{2}{*}{ TOTAL } & \multicolumn{3}{|c|}{ BY SIZE CLASS } & $(\mathbf{M M})$ & \multirow[b]{2}{*}{$\begin{array}{l}4.00 \\
\text { TO } \\
8.00\end{array}$} \\
\hline & & & $\begin{array}{l}0.25 \\
\text { TO } \\
0.50\end{array}$ & $\begin{array}{l}0.50 \\
\text { TO } \\
1.00\end{array}$ & $\begin{array}{l}1.00 \\
\text { TO } \\
2.00\end{array}$ & $\begin{array}{l}2.00 \\
\text { TO } \\
4.00\end{array}$ & \\
\hline $\begin{array}{l}5-16 \\
5-19 \\
5-21 \\
5-22 \\
5-24\end{array}$ & $\begin{array}{l}25++ \\
312 \\
1752 \\
4024 \\
2137\end{array}$ & $\begin{array}{r}0 \\
13 \\
5 \\
4 \\
10\end{array}$ & $\begin{array}{r}0 \\
12 \\
3 \\
3 \\
7\end{array}$ & $\begin{array}{l}0 \\
1 \\
2 \\
1 \\
3\end{array}$ & $\begin{array}{l}0 \\
0 \\
0 \\
0 \\
0\end{array}$ & $\begin{array}{l}0 \\
0 \\
0 \\
0 \\
0\end{array}$ & $\begin{array}{l}0 \\
0 \\
0 \\
0 \\
0\end{array}$ \\
\hline $\begin{array}{l}5-26 \\
5-27 \\
5-28\end{array}$ & $\begin{array}{l}1 \overline{89}+ \\
1153\end{array}$ & $\frac{-\overline{2}}{6}$ & $\frac{--}{1}$ & $\overline{11}$ & $\begin{array}{r}--\overline{0} \\
3\end{array}$ & $\begin{array}{r}-- \\
0 \\
0\end{array}$ & $\begin{array}{r}-\overline{0} \\
0\end{array}$ \\
\hline $5-30$ & $76+$ & 4 & 0 & 1 & 3 & 0 & 0 \\
\hline $\begin{array}{l}6-01 \\
6-03 \\
6-05 \\
6-07 \\
6-09\end{array}$ & $\begin{array}{c}286 \\
162+ \\
92+ \\
1082 \\
2704\end{array}$ & $\begin{array}{l}3 \\
0 \\
3 \\
9 \\
7\end{array}$ & $\begin{array}{l}0 \\
0 \\
0 \\
0 \\
1\end{array}$ & $\begin{array}{l}3 \\
0 \\
0 \\
7 \\
5\end{array}$ & $\begin{array}{l}0 \\
0 \\
1 \\
2 \\
1\end{array}$ & $\begin{array}{l}0 \\
0 \\
2 \\
0 \\
0\end{array}$ & $\begin{array}{l}0 \\
0 \\
0 \\
0 \\
0\end{array}$ \\
\hline $\begin{array}{l}6-10 \\
6-11 \\
6-12 \\
6-13 \\
6-14\end{array}$ & $\begin{array}{l}7137 \\
4062 \\
1592 \\
1856 \\
2864\end{array}$ & $\begin{array}{l}15 \\
15 \\
14 \\
4 \\
13\end{array}$ & $\begin{array}{l}2 \\
4 \\
4 \\
1 \\
3\end{array}$ & $\begin{array}{r}11 \\
8 \\
6 \\
1 \\
8\end{array}$ & $\begin{array}{l}1 \\
3 \\
3 \\
2 \\
2\end{array}$ & $\begin{array}{l}1 \\
0 \\
1 \\
0 \\
0\end{array}$ & $\begin{array}{l}0 \\
0 \\
0 \\
0 \\
0\end{array}$ \\
\hline $6-15$ & 3503 & 16 & 1 & 11 & 3 & 1 & 0 \\
\hline $\begin{array}{l}6-16 \\
6-17 \\
6-18 \\
6-19\end{array}$ & $\begin{array}{l}4084 \\
2445 \\
1694\end{array}$ & $\begin{array}{l}19 \\
18 \\
16\end{array}$ & $\begin{array}{l}3 \\
2 \\
2\end{array}$ & $\begin{array}{l}10 \\
10 \\
11\end{array}$ & $\begin{array}{l}5 \\
5 \\
2\end{array}$ & $\frac{1}{1}$ & $\begin{array}{l}0 \\
0 \\
0\end{array}$ \\
\hline $\begin{array}{l}6-20 \\
6-21\end{array}$ & $\begin{array}{l}1253 \\
2001\end{array}$ & $\begin{array}{l}27 \\
25\end{array}$ & $\begin{array}{l}6 \\
3\end{array}$ & $\begin{array}{l}15 \\
19\end{array}$ & $\begin{array}{l}5 \\
3\end{array}$ & $\begin{array}{l}1 \\
0\end{array}$ & $\begin{array}{l}0 \\
0\end{array}$ \\
\hline $\begin{array}{l}6-23 \\
6-25\end{array}$ & $\begin{array}{r}1393 \\
553\end{array}$ & 28 & 2 & $\begin{array}{l}14 \\
16\end{array}$ & $\begin{array}{l}5 \\
2\end{array}$ & $\begin{array}{l}0 \\
3\end{array}$ & $\begin{array}{l}0 \\
0\end{array}$ \\
\hline $6-27$ & 1604 & 29 & 4 & 19 & 6 & 0 & 0 \\
\hline $7-01$ & 1661 & 20 & 2 & 11 & 6 & 1 & 0 \\
\hline $7-05$ & $374+$ & 19 & 3 & 14 & 1 & 1 & 0 \\
\hline $7-07$ & $6+++$ & 0 & 0 & 0 & 0 & 0 & 0 \\
\hline
\end{tabular}


TABLE 15.- NUMBER OF PINK TRACER PARTICLES(1), PER 100 GRAMS OF BEDLOAD SAMPLE, COMPOSITED FROM CROSS-CHANNEL SAMPLING POSITIONS SPACED AT 1-METER INTERVALS ACROSS THE CHANNEL WIDTH, EAST FORK RIVER, WYOMING, 1980

SECTION 1662

\begin{tabular}{|c|c|c|c|c|c|c|c|}
\hline \multirow{3}{*}{ DATE } & \multirow{3}{*}{$\begin{array}{c}\text { DRY } \\
\text { MASS } \\
\text { OF } \\
\text { SAMPLE (2) } \\
\text { (G) }\end{array}$} & \multicolumn{6}{|c|}{ NUMBER OF PINK TRACER PARTICLES } \\
\hline & & \multirow[b]{2}{*}{ TOTAL } & \multicolumn{5}{|c|}{ BY SIZE CLASS (MM) } \\
\hline & & & $\begin{array}{l}0.25 \\
\text { TO } \\
0.50\end{array}$ & $\begin{array}{l}0.50 \\
\text { TO } \\
1.00\end{array}$ & $\begin{array}{l}1.00 \\
\text { TO } \\
2.00\end{array}$ & $\begin{array}{l}2.00 \\
\text { TO } \\
4.00\end{array}$ & $\begin{array}{l}4.00 \\
\text { TO } \\
8.00\end{array}$ \\
\hline $\begin{array}{l}5-16 \\
5-19 \\
5-21 \\
5-22 \\
5-24\end{array}$ & $\begin{array}{l}199 \\
282+ \\
677 \\
1722 \\
5059\end{array}$ & $\begin{array}{r}11 \\
9 \\
4 \\
3 \\
13\end{array}$ & $\begin{array}{r}8 \\
7 \\
2 \\
1 \\
11\end{array}$ & $\begin{array}{l}3 \\
2 \\
2 \\
2 \\
2\end{array}$ & $\begin{array}{l}0 \\
0 \\
0 \\
0 \\
0\end{array}$ & $\begin{array}{l}0 \\
0 \\
0 \\
0 \\
0\end{array}$ & $\begin{array}{l}0 \\
0 \\
0 \\
0 \\
0\end{array}$ \\
\hline $\begin{array}{l}5-26 \\
5-27 \\
5-28\end{array}$ & $\begin{array}{r}10 \overline{77} \\
910\end{array}$ & $\begin{array}{l}\overline{19} \\
13\end{array}$ & $\begin{array}{r}-\overline{5} \\
0\end{array}$ & $\begin{array}{r}-\overline{9} \\
9\end{array}$ & $-\frac{-}{4}$ & $-\frac{1}{0}$ & $\begin{array}{c}--\overline{0} \\
0\end{array}$ \\
\hline $5-30$ & 365 & 19 & 3 & 10 & 6 & 0 & 0 \\
\hline $\begin{array}{l}6-01 \\
6-03 \\
6-05 \\
6-07 \\
6-09\end{array}$ & $\begin{array}{c}144+ \\
280 \\
96+ \\
393 \\
3919\end{array}$ & $\begin{array}{l}2 \\
21 \\
10 \\
21 \\
14\end{array}$ & $\begin{array}{l}0 \\
2 \\
2 \\
9 \\
2\end{array}$ & $\begin{array}{r}0 \\
16 \\
7 \\
10 \\
9\end{array}$ & $\begin{array}{l}2 \\
3 \\
1 \\
\frac{1}{2} \\
3\end{array}$ & $\begin{array}{l}0 \\
0 \\
0 \\
0 \\
0\end{array}$ & $\begin{array}{l}0 \\
0 \\
0 \\
0 \\
0\end{array}$ \\
\hline $\begin{array}{l}6-10 \\
6-11 \\
6-12 \\
6-13 \\
6-14\end{array}$ & $\begin{array}{r}1830 \\
490 \\
2588 \\
1221 \\
1830\end{array}$ & $\begin{array}{l}10 \\
15 \\
21 \\
21 \\
22\end{array}$ & $\begin{array}{l}6 \\
1 \\
2 \\
3 \\
2\end{array}$ & $\begin{array}{r}2 \\
9 \\
14 \\
14 \\
16\end{array}$ & $\begin{array}{l}2 \\
5 \\
3 \\
4 \\
4\end{array}$ & $\begin{array}{l}0 \\
0 \\
2 \\
0 \\
0\end{array}$ & $\begin{array}{l}0 \\
0 \\
0 \\
0 \\
0\end{array}$ \\
\hline $6-15$ & 1372 & 24 & 5 & 11 & 7 & 1 & 0 \\
\hline $\begin{array}{l}6-10 \\
6-17 \\
6-18 \\
6-19\end{array}$ & $\begin{array}{l}3344 \\
3394 \\
3121\end{array}$ & $\begin{array}{l}16 \\
16 \\
26\end{array}$ & $\frac{1}{1}$ & $\begin{array}{l}13 \\
11 \\
17\end{array}$ & $\begin{array}{l}2 \\
4 \\
8\end{array}$ & $\begin{array}{l}0 \\
0 \\
0\end{array}$ & $\begin{array}{l}0 \\
0 \\
0\end{array}$ \\
\hline $\begin{array}{l}6-20 \\
6-21\end{array}$ & $\begin{array}{l}1823 \\
3246\end{array}$ & $\frac{11}{20}$ & $\begin{array}{l}5 \\
2\end{array}$ & $\begin{array}{r}6 \\
12\end{array}$ & $\begin{array}{l}0 \\
6\end{array}$ & $\begin{array}{l}0 \\
0\end{array}$ & $\begin{array}{l}0 \\
0\end{array}$ \\
\hline $\begin{array}{l}6-23 \\
6-25 \\
6-25\end{array}$ & $\begin{array}{l}2408 \\
2133\end{array}$ & $\begin{array}{l}17 \\
15\end{array}$ & $\begin{array}{l}0 \\
1\end{array}$ & $\begin{array}{l}16 \\
10\end{array}$ & $\frac{1}{3}$ & $\begin{array}{l}0 \\
1\end{array}$ & $\begin{array}{l}0 \\
0\end{array}$ \\
\hline $\begin{array}{l}6-27 \\
6-20\end{array}$ & 2454 & 29 & -3 & 23 & 3 & $\underline{0}$ & $\underline{0}$ \\
\hline $7-01$ & -- & - & -- & - & -- & -- & - \\
\hline $\begin{array}{l}7-03 \\
7-05\end{array}$ & $\overline{--}$ & $\overline{-}$ & $\overline{--}$ & $\overline{--}$ & $\overline{--}$ & $\overline{--}$ & $\overline{--}$ \\
\hline $7-07$ & -- & -- & -- & -- & -- & -- & -- \\
\hline
\end{tabular}


TABLE 15.- NUMBER OF PINK TRACER PARTICLES(1), PER 100 GRAMS OF BEDLOAD SAMPLE, COMPOSITED FROM CROSS-CHANNEL SAMPLING POSITIONS SPACED AT 1-METER INTERVALS ACROSS THE CHANNEL WIDTH, EAST FORK RIVER, WYOMING, 1980

SECTION 1695

\begin{tabular}{|c|c|c|c|c|c|c|c|}
\hline \multirow{3}{*}{ DATE } & \multirow{3}{*}{$\begin{array}{c}\text { DRY } \\
\text { MASS } \\
\text { OF } \\
\text { SAMPLE (2) } \\
\text { (G) }\end{array}$} & \multicolumn{6}{|c|}{ NUMBER OF PINK TRACER PARTICLES } \\
\hline & & \multirow[b]{2}{*}{ TOTAL } & \multicolumn{5}{|c|}{ BY SIZE CLASS (MM) } \\
\hline & & & $\begin{array}{c}0.25 \\
\text { TO } \\
0.50\end{array}$ & $\begin{array}{l}0.50 \\
\text { TO } \\
1.00\end{array}$ & $\begin{array}{l}1.00 \\
\text { TO } \\
2.00\end{array}$ & $\begin{array}{l}2.00 \\
\text { TO } \\
4.00\end{array}$ & $\begin{array}{l}4.00 \\
\text { TO } \\
8.00\end{array}$ \\
\hline $\begin{array}{l}5-16 \\
5-19 \\
5-21 \\
5-22 \\
5-24\end{array}$ & $\begin{array}{l}191+ \\
158+ \\
3357 \\
5239 \\
6226\end{array}$ & $\begin{array}{r}11 \\
6 \\
29 \\
8 \\
17\end{array}$ & $\begin{array}{r}5 \\
3 \\
20 \\
2 \\
3\end{array}$ & $\begin{array}{r}6 \\
3 \\
8 \\
5 \\
14\end{array}$ & $\begin{array}{l}0 \\
0 \\
1 \\
1 \\
0\end{array}$ & $\begin{array}{l}0 \\
0 \\
0 \\
0 \\
0\end{array}$ & $\begin{array}{l}0 \\
0 \\
0 \\
0 \\
0\end{array}$ \\
\hline $\begin{array}{l}5-26 \\
5-27 \\
5-28 \\
5-29 \\
5-30\end{array}$ & $\begin{array}{l}20 \overline{2} \\
403 \\
\overline{67++}\end{array}$ & $\begin{array}{l}-\overline{17} \\
\frac{26}{13}\end{array}$ & $\begin{array}{r}-\overline{2} \\
-\frac{2}{3}\end{array}$ & $\frac{11}{22}$ & $\begin{array}{r}--\frac{3}{2} \\
-\frac{1}{1}\end{array}$ & $-\frac{1}{0}$ & $\begin{array}{r}-- \\
0 \\
-\frac{0}{0}\end{array}$ \\
\hline $\begin{array}{l}6-01 \\
6-03 \\
6-05 \\
6-07 \\
6-09\end{array}$ & $\begin{array}{c}22++ \\
25++ \\
50++ \\
300 \\
3922\end{array}$ & $\begin{array}{r}9 \\
8 \\
10 \\
37 \\
21\end{array}$ & $\begin{array}{r}0 \\
0 \\
0 \\
17 \\
3\end{array}$ & $\begin{array}{r}9 \\
8 \\
10 \\
19 \\
15\end{array}$ & $\begin{array}{l}0 \\
0 \\
0 \\
\frac{1}{3}\end{array}$ & $\begin{array}{l}0 \\
0 \\
0 \\
0 \\
0\end{array}$ & $\begin{array}{l}0 \\
0 \\
0 \\
0 \\
0\end{array}$ \\
\hline $\begin{array}{l}6-10 \\
6-11 \\
6-12 \\
6-13 \\
6-14\end{array}$ & $\begin{array}{l}4128 \\
3102 \\
2957 \\
2864 \\
7796\end{array}$ & $\begin{array}{l}18 \\
29 \\
17 \\
14 \\
14\end{array}$ & $\begin{array}{l}2 \\
8 \\
0 \\
1 \\
0\end{array}$ & $\begin{array}{r}15 \\
21 \\
13 \\
7 \\
12\end{array}$ & $\begin{array}{l}1 \\
0 \\
4 \\
5 \\
2\end{array}$ & $\begin{array}{l}0 \\
0 \\
0 \\
1 \\
0\end{array}$ & $\begin{array}{l}0 \\
0 \\
0 \\
0 \\
0\end{array}$ \\
\hline $6-15$ & 6608 & 10 & $\underline{0}$ & 5 & 5 & $\underline{0}$ & 0 \\
\hline $\begin{array}{l}6-17 \\
6-18 \\
6-19\end{array}$ & $\begin{array}{l}2172 \\
1774 \\
8146\end{array}$ & $\begin{array}{r}17 \\
15 \\
8\end{array}$ & $\begin{array}{l}1 \\
3 \\
0\end{array}$ & $\begin{array}{r}12 \\
12 \\
6\end{array}$ & $\begin{array}{l}4 \\
0 \\
2\end{array}$ & $\begin{array}{l}0 \\
0 \\
0\end{array}$ & $\begin{array}{l}0 \\
0 \\
0\end{array}$ \\
\hline $\begin{array}{l}6-20 \\
6-21\end{array}$ & $\begin{array}{l}8676 \\
5780\end{array}$ & $\begin{array}{l}22 \\
21\end{array}$ & $\begin{array}{l}2 \\
1\end{array}$ & $\begin{array}{l}16 \\
10\end{array}$ & $\begin{array}{l}4 \\
9\end{array}$ & $\begin{array}{l}0 \\
1\end{array}$ & $\begin{array}{l}0 \\
0\end{array}$ \\
\hline $\begin{array}{l}6-23 \\
6-25\end{array}$ & $\begin{array}{l}4387 \\
2589\end{array}$ & $\begin{array}{l}12 \\
14\end{array}$ & $\frac{1}{0}$ & $\frac{4}{7}$ & $\begin{array}{l}4 \\
7\end{array}$ & $\begin{array}{l}3 \\
0\end{array}$ & $\begin{array}{l}0 \\
0\end{array}$ \\
\hline $6-27$ & 1937 & 21 & 2 & 15 & 2 & 2 & 0 \\
\hline $\begin{array}{l}6-29 \\
7-01\end{array}$ & $\overline{--}$ & $\overline{--}$ & $\overline{--}$ & $\overline{--}$ & $\overline{--}$ & $\overline{--}$ & -- \\
\hline $\begin{array}{l}7-03 \\
7-05\end{array}$ & $=$ & $\overline{--}$ & $=$ & $=$ & $\overline{--}$ & -- & -- \\
\hline & & & & & & & \\
\hline $1-01$ & - & - & - & - & - & - & - \\
\hline
\end{tabular}


TABLE 15.- NUMBER OF PINK TRACER PARTICLES(I), PER 100 GRAMS OF BEDLOAD SAMPLE, COMPOSITED FROM CROSS-CHANNEL SAMPLING POSITIONS SPACED AT I-METER INTERVALS ACROSS THE CHANNEL WIDTH, EAST FORK RIVER, WYOMING, 1980

SECTION 1730

\begin{tabular}{|c|c|c|c|c|c|c|c|}
\hline \multirow{3}{*}{ DATE } & \multirow{3}{*}{$\begin{array}{c}\text { DRY } \\
\text { MASS } \\
\text { OF } \\
\text { SAMPLE (2) } \\
\text { (G) }\end{array}$} & \multicolumn{6}{|c|}{ NUMBER OF PINK TRACER PARTICLES } \\
\hline & & \multirow[b]{2}{*}{ TOTAL } & \multicolumn{5}{|c|}{ BY SIZE CLASS (MM) } \\
\hline & & & $\begin{array}{l}0.25 \\
\text { TO } \\
0.50\end{array}$ & $\begin{array}{c}0.50 \\
\text { TO } \\
1.00\end{array}$ & $\begin{array}{l}1.00 \\
2.00\end{array}$ & $\begin{array}{l}2.00 \\
\mathrm{~T} 0 \\
4.00\end{array}$ & $\begin{array}{l}4.00 \\
\text { TO } \\
8.00\end{array}$ \\
\hline $\begin{array}{l}5-16 \\
5-19 \\
5-21 \\
5-22 \\
5-24\end{array}$ & $\begin{array}{c}42++ \\
411 \\
1942 \\
3183 \\
2906\end{array}$ & $\begin{array}{l}7 \\
44 \\
12 \\
14 \\
31\end{array}$ & $\begin{array}{r}0 \\
35 \\
7 \\
7 \\
3\end{array}$ & $\begin{array}{r}5 \\
8 \\
5 \\
5 \\
22\end{array}$ & $\begin{array}{l}2 \\
1 \\
0 \\
2 \\
5\end{array}$ & $\begin{array}{l}0 \\
0 \\
0 \\
0 \\
1\end{array}$ & $\begin{array}{l}0 \\
0 \\
0 \\
0 \\
0\end{array}$ \\
\hline $\begin{array}{l}5-26 \\
5-27 \\
5-28\end{array}$ & $\begin{array}{l}4 \overline{67} \\
32++\end{array}$ & $\frac{-}{16}$ & $\begin{array}{r}--2 \\
0\end{array}$ & $\frac{-\overline{2}}{3}$ & $-\frac{1}{1}$ & $\frac{-}{0}$ & $\begin{array}{r}-- \\
0 \\
0\end{array}$ \\
\hline $\begin{array}{l}5-29 \\
5-30\end{array}$ & $\overline{18++}$ & $\overline{0}$ & 0 & 0 & 0 & 0 & 0 \\
\hline $\begin{array}{l}6-01 \\
6-03 \\
6-05 \\
6-07 \\
6-09\end{array}$ & $\begin{array}{c}0+++ \\
23++ \\
8+++ \\
922 \\
1693\end{array}$ & $\begin{array}{r}0 \\
9 \\
0 \\
24 \\
13\end{array}$ & $\begin{array}{l}0 \\
0 \\
0 \\
9 \\
2\end{array}$ & $\begin{array}{r}0 \\
9 \\
0 \\
13 \\
10\end{array}$ & $\begin{array}{l}0 \\
0 \\
0 \\
2 \\
0\end{array}$ & $\begin{array}{l}0 \\
0 \\
0 \\
0 \\
1\end{array}$ & $\begin{array}{l}0 \\
0 \\
0 \\
0 \\
0\end{array}$ \\
\hline $\begin{array}{l}6-10 \\
6-11 \\
6-12 \\
6-13 \\
6-14\end{array}$ & $\begin{array}{l}2532 \\
8766 \\
1893 \\
2690 \\
3680\end{array}$ & $\begin{array}{l}16 \\
21 \\
30 \\
14 \\
28\end{array}$ & $\begin{array}{l}2 \\
1 \\
3 \\
2 \\
2\end{array}$ & $\begin{array}{l}13 \\
10 \\
25 \\
6 \\
20\end{array}$ & $\begin{array}{r}1 \\
10 \\
2 \\
5 \\
4\end{array}$ & $\begin{array}{l}0 \\
0 \\
0 \\
1 \\
2\end{array}$ & $\begin{array}{l}0 \\
0 \\
0 \\
0 \\
0\end{array}$ \\
\hline $6-15$ & 1568 & 10 & 0 & 5 & 3 & 2 & 0 \\
\hline $\begin{array}{l}6-17 \\
6-18 \\
6-19\end{array}$ & $\begin{array}{l}1568 \\
4989 \\
4585\end{array}$ & $\begin{array}{l}15 \\
18 \\
18\end{array}$ & $\begin{array}{l}0 \\
2 \\
0\end{array}$ & $\begin{array}{l}14 \\
12 \\
11\end{array}$ & $\begin{array}{l}1 \\
4 \\
7\end{array}$ & $\begin{array}{l}0 \\
0 \\
0\end{array}$ & $\begin{array}{l}0 \\
0 \\
0\end{array}$ \\
\hline $\begin{array}{l}6-20 \\
6-21\end{array}$ & $\begin{array}{l}5776 \\
1653\end{array}$ & $\begin{array}{r}13 \\
6\end{array}$ & $\begin{array}{l}0 \\
0\end{array}$ & $\begin{array}{l}7 \\
1\end{array}$ & $\begin{array}{l}4 \\
5\end{array}$ & $\begin{array}{l}2 \\
0\end{array}$ & $\begin{array}{l}0 \\
0\end{array}$ \\
\hline $\begin{array}{l}6-22 \\
6-23 \\
6-25\end{array}$ & $\begin{array}{l}16 \overline{8} \\
1300\end{array}$ & $\begin{array}{r}\overline{17} \\
6\end{array}$ & $\overline{0}$ & $\begin{array}{r}12 \\
2\end{array}$ & $\begin{array}{l}5 \\
3\end{array}$ & $\begin{array}{l}\overline{0} \\
0\end{array}$ & $\overline{0}$ \\
\hline $6-27$ & 2285 & 17 & 0 & 10 & 4 & 3 & 0 \\
\hline $\begin{array}{l}6-29 \\
7-01\end{array}$ & $-\overline{-}$ & -- & $\overline{-}$ & -- & $\overline{-}$ & -- & $=$ \\
\hline $7-03$ & -- & -- & -- & -- & -- & -- & -- \\
\hline & & & & & & & \\
\hline $7-07$ & -- & -- & -- & -- & -- & -- & -- \\
\hline
\end{tabular}


TABLE 15.- NUMBER OF PINK TRACER PARTICLES(1), PER 100 GRAMS OF BEDLOAD SAMPLE, COMPOSITED FROM CROSS-CHANNEL SAMPLING POSITIONS

SPACED AT 1-METER INTERVALS ACROSS THE CHANNEL WIDTH,

EAST FORK RIVER, WYOMING, 1980

SECTION 1766

\begin{tabular}{|c|c|c|c|c|c|c|c|}
\hline \multirow{3}{*}{ DATE } & \multirow{3}{*}{$\begin{array}{c}\text { DRY } \\
\text { MASS } \\
\text { OF } \\
\text { SAMPLE (2) } \\
\text { (G) }\end{array}$} & \multicolumn{6}{|c|}{ NUMBER OF PINK TRACER PARTICLES } \\
\hline & & \multirow[b]{2}{*}{ TOTAL } & \multicolumn{5}{|c|}{ BY SIZE CLASS (MM) } \\
\hline & & & $\begin{array}{c}0.25 \\
\text { TO } \\
0.50\end{array}$ & $\begin{array}{l}0.50 \\
\text { TO } \\
1.00\end{array}$ & $\begin{array}{l}1.00 \\
\text { TO } \\
2.00\end{array}$ & $\begin{array}{l}2.00 \\
\text { TO } \\
4.00\end{array}$ & $\begin{array}{l}4.00 \\
\text { TO } \\
8.00\end{array}$ \\
\hline $\begin{array}{l}5-16 \\
5-19 \\
5-21 \\
5-22 \\
5-24\end{array}$ & $\begin{array}{r}505 \\
1355 \\
1588 \\
3585 \\
6386\end{array}$ & $\begin{array}{l}25 \\
12 \\
12 \\
15 \\
10\end{array}$ & $\begin{array}{l}7 \\
5 \\
6 \\
8 \\
2\end{array}$ & $\begin{array}{r}18 \\
7 \\
6 \\
6 \\
8\end{array}$ & $\begin{array}{l}0 \\
0 \\
0 \\
1 \\
0\end{array}$ & $\begin{array}{l}0 \\
0 \\
0 \\
0 \\
0\end{array}$ & $\begin{array}{l}0 \\
0 \\
0 \\
0 \\
0\end{array}$ \\
\hline $\begin{array}{l}5-26 \\
5-27 \\
5-28 \\
5-29 \\
5-30\end{array}$ & $\begin{array}{c}-\overline{152+} \\
78+ \\
17+++ \\
15+++\end{array}$ & $\begin{array}{r}\overline{12} \\
28 \\
0 \\
7\end{array}$ & $\begin{array}{r}-- \\
12 \\
0 \\
7\end{array}$ & $\begin{array}{r}-- \\
8 \\
16 \\
0 \\
0\end{array}$ & $\begin{array}{r}-- \\
2 \\
0 \\
0 \\
0\end{array}$ & $\begin{array}{r}-- \\
0 \\
0 \\
0 \\
0\end{array}$ & $\begin{array}{l}-- \\
0 \\
0 \\
0 \\
0\end{array}$ \\
\hline $\begin{array}{l}6-01 \\
6-03 \\
6-05 \\
6-07 \\
6-09\end{array}$ & $\begin{array}{l}74++ \\
354 \\
349 \\
630 \\
1104\end{array}$ & $\begin{array}{l}26 \\
42 \\
37 \\
18 \\
13\end{array}$ & $\begin{array}{r}4 \\
8 \\
15 \\
2 \\
1\end{array}$ & $\begin{array}{l}19 \\
32 \\
19 \\
11 \\
7\end{array}$ & $\begin{array}{l}3 \\
2 \\
3 \\
4 \\
5\end{array}$ & $\begin{array}{l}0 \\
0 \\
0 \\
1 \\
0\end{array}$ & $\begin{array}{l}0 \\
0 \\
0 \\
0 \\
0\end{array}$ \\
\hline $\begin{array}{l}6-10 \\
6-11 \\
6-12 \\
6-13 \\
6-14\end{array}$ & $\begin{array}{l}6609 \\
6272 \\
5793 \\
1769 \\
3874\end{array}$ & $\begin{array}{r}12 \\
22 \\
15 \\
8 \\
27\end{array}$ & $\begin{array}{l}1 \\
5 \\
0 \\
1 \\
1\end{array}$ & $\begin{array}{r}8 \\
14 \\
11 \\
1 \\
18\end{array}$ & $\begin{array}{l}3 \\
3 \\
2 \\
5 \\
8\end{array}$ & $\begin{array}{l}0 \\
0 \\
2 \\
1 \\
0\end{array}$ & $\begin{array}{l}0 \\
0 \\
0 \\
0 \\
0\end{array}$ \\
\hline $6-15$ & 4197 & 15 & 2 & 10 & 2 & 1 & 0 \\
\hline $\begin{array}{l}6-17 \\
6-18 \\
6-19\end{array}$ & $\begin{array}{l}1338 \\
2494 \\
4630\end{array}$ & $\begin{array}{l}13 \\
15 \\
21\end{array}$ & $\begin{array}{l}0 \\
0 \\
1\end{array}$ & $\begin{array}{l}12 \\
12 \\
12\end{array}$ & $\begin{array}{l}\frac{1}{3} \\
6\end{array}$ & $\begin{array}{l}0 \\
0 \\
2\end{array}$ & $\begin{array}{l}0 \\
0 \\
0\end{array}$ \\
\hline $\begin{array}{l}6-20 \\
6-21\end{array}$ & $\begin{array}{l}4529 \\
3319\end{array}$ & $\begin{array}{l}18 \\
12\end{array}$ & $\begin{array}{l}0 \\
0\end{array}$ & $\begin{array}{l}8 \\
3\end{array}$ & $\begin{array}{r}10 \\
9\end{array}$ & $\begin{array}{l}0 \\
0\end{array}$ & $\begin{array}{l}0 \\
0\end{array}$ \\
\hline $\begin{array}{l}6-23 \\
6-25\end{array}$ & $\begin{array}{l}2870 \\
2131\end{array}$ & $\begin{array}{l}21 \\
17\end{array}$ & $\frac{1}{0}$ & $\begin{array}{r}13 \\
8\end{array}$ & $\begin{array}{l}7 \\
5\end{array}$ & $\begin{array}{l}0 \\
1\end{array}$ & $\begin{array}{l}0 \\
3\end{array}$ \\
\hline $6-27$ & 1767 & 21 & 0 & 14 & 6 & 1 & 0 \\
\hline $7-01$ & - & - & - & - & - & - & - \\
\hline $\begin{array}{l}7-03 \\
7-05\end{array}$ & -- & $\overline{-}$ & $\overline{--}$ & $=$ & $\overline{--}$ & $\overline{--}$ & $\overline{--}$ \\
\hline $7-07$ & -- & -- & -- & -- & -- & - & -- \\
\hline
\end{tabular}


TABLE 15.- NUMBER OF PINK TRACER PARTICLES(1), PER 100 GRAMS OF BEDLOAD SAMPLE, COMPOSITED FROM CROSS-CHANNEL SAMPLING POSITIONS SPACED AT 1-METER INTERVALS ACROSS THE CHANNEL WIDTH, EAST FORK RIVER, WYOMING, 1980

SECTION 1800

\begin{tabular}{|c|c|c|c|c|c|c|c|}
\hline \multirow{3}{*}{ DATE } & \multirow{3}{*}{$\begin{array}{c}\text { DRY } \\
\text { MASS } \\
\text { OF } \\
\text { SAMPLE (2) } \\
\text { (G) }\end{array}$} & \multicolumn{6}{|c|}{ NUMBER OF PINK TRACER PARTICLES } \\
\hline & & & & BY SI2 & CLASS & & \\
\hline & & TOTAL & $\begin{array}{l}0.25 \\
. \text { TO } \\
0.50\end{array}$ & $\begin{array}{l}0.50 \\
\text { TO } \\
1.00\end{array}$ & $\begin{array}{r}1.00 \\
\text { TO } \\
2.00\end{array}$ & $\begin{array}{l}2.00 \\
\text { TO } \\
4.00\end{array}$ & $\begin{array}{r}4.00 \\
\text { TO } \\
8.00\end{array}$ \\
\hline $\begin{array}{l}5-16 \\
5-19 \\
5-21 \\
5-22 \\
5-24\end{array}$ & $\begin{array}{r}1300 \\
152 \\
420 \\
1243 \\
2871\end{array}$ & $\begin{array}{r}6 \\
7 \\
35 \\
24 \\
21\end{array}$ & $\begin{array}{r}1 \\
3 \\
25 \\
14 \\
1\end{array}$ & $\begin{array}{r}3 \\
4 \\
10 \\
10 \\
11\end{array}$ & $\begin{array}{l}2 \\
0 \\
0 \\
0 \\
9\end{array}$ & $\begin{array}{l}0 \\
0 \\
0 \\
0 \\
0\end{array}$ & $\begin{array}{l}0 \\
0 \\
0 \\
0 \\
0\end{array}$ \\
\hline $\begin{array}{l}5-26 \\
5-27 \\
5-28 \\
5-29 \\
5-30\end{array}$ & $\begin{array}{r}8 \overline{66} \\
1008 \\
-\overline{132}\end{array}$ & $\begin{array}{l}\overline{27} \\
24 \\
\overline{32}\end{array}$ & $\begin{array}{r}-\frac{7}{7} \\
-\frac{5}{8}\end{array}$ & $\begin{array}{l}\overline{19} \\
17 \\
\overline{23}\end{array}$ & $-\frac{-}{2}$ & $\begin{array}{r}-0 \\
0 \\
-1\end{array}$ & $\begin{array}{r}-- \\
0 \\
-0 \\
0\end{array}$ \\
\hline $\begin{array}{l}6-01 \\
6-03 \\
6-05 \\
6-07 \\
6-09\end{array}$ & $\begin{array}{l}575 \\
91++ \\
385 \\
514 \\
1354\end{array}$ & $\begin{array}{l}33 \\
9 \\
45 \\
16 \\
13\end{array}$ & $\begin{array}{r}11 \\
0 \\
12 \\
5 \\
3\end{array}$ & $\begin{array}{r}21 \\
9 \\
30 \\
7 \\
8\end{array}$ & $\begin{array}{l}1 \\
0 \\
3 \\
4 \\
2\end{array}$ & $\begin{array}{l}0 \\
0 \\
0 \\
0 \\
0\end{array}$ & $\begin{array}{l}0 \\
0 \\
0 \\
0 \\
0\end{array}$ \\
\hline $\begin{array}{l}6-10 \\
6-11 \\
6-12 \\
6-13 \\
6-14\end{array}$ & $\begin{array}{l}3791 \\
6242 \\
3693 \\
2782 \\
1616\end{array}$ & $\begin{array}{r}7 \\
14 \\
21 \\
16 \\
20\end{array}$ & $\begin{array}{l}1 \\
1 \\
5 \\
0 \\
3\end{array}$ & $\begin{array}{r}4 \\
5 \\
12 \\
11 \\
16\end{array}$ & $\begin{array}{l}2 \\
3 \\
3 \\
2 \\
1\end{array}$ & $\begin{array}{l}0 \\
5 \\
1 \\
3 \\
0\end{array}$ & $\begin{array}{l}0 \\
0 \\
0 \\
0 \\
0\end{array}$ \\
\hline $\begin{array}{l}6-15 \\
6-16 \\
6-17 \\
6-18 \\
6-19\end{array}$ & $\begin{array}{r}1843 \\
1908 \\
3679 \\
2045\end{array}$ & $\begin{array}{l}36 \\
-30 \\
30 \\
22\end{array}$ & $\begin{array}{r}-\frac{3}{2} \\
2 \\
2\end{array}$ & $\begin{array}{l}28 \\
--2 \\
17 \\
19\end{array}$ & $\begin{array}{r}-3 \\
-7 \\
5 \\
1\end{array}$ & $\begin{array}{r}-1 \\
0 \\
1 \\
0\end{array}$ & $\begin{array}{r}-1 \\
0 \\
0 \\
0\end{array}$ \\
\hline $\begin{array}{l}6-20 \\
6-21 \\
6-22 \\
6-23 \\
6-25\end{array}$ & $\begin{array}{r}3961 \\
3071 \\
-1 \\
4646 \\
4199\end{array}$ & $\begin{array}{l}15 \\
13 \\
-16 \\
16 \\
21\end{array}$ & $\begin{array}{r}0 \\
0 \\
-0 \\
0 \\
0\end{array}$ & $\begin{array}{r}14 \\
-\frac{8}{8} \\
10\end{array}$ & $-\frac{1}{1}$ & $\begin{array}{r}0 \\
4 \\
-3 \\
3 \\
5\end{array}$ & $\begin{array}{r}0 \\
0 \\
-\frac{-}{0} \\
0\end{array}$ \\
\hline $\begin{array}{l}6-27 \\
6-29 \\
7-01 \\
7-03 \\
7-05\end{array}$ & $\begin{array}{r}2148 \\
=- \\
=- \\
=-\end{array}$ & $\begin{array}{l}25 \\
-- \\
-- \\
--\end{array}$ & $\begin{array}{l}0 \\
-- \\
=- \\
--\end{array}$ & $\begin{array}{l}15 \\
=- \\
-- \\
--\end{array}$ & $\begin{array}{l}8 \\
-- \\
-- \\
--\end{array}$ & $\begin{array}{l}2 \\
-= \\
--\end{array}$ & $\begin{array}{l}0 \\
-- \\
-- \\
--\end{array}$ \\
\hline $7-07$ & -- & -- & -- & -- & -- & -- & - \\
\hline
\end{tabular}


TABLE 15.- NUMBER OF PINK TRACER PARTICLES(1), PER 100 GRAMS OF BEDLOAD SAMPLE, COMPOSITED FROM CROSS-CHANNEL SAMPLING POSITIONS SPACED AT 1-METER INTERVALS ACROSS THE CHANNEL WIDTH, EAST FORK RIVER, WYOMING, 1980

SECTION 18.30

\begin{tabular}{|c|c|c|c|c|c|c|c|}
\hline \multirow{3}{*}{ DATE } & \multirow{3}{*}{$\begin{array}{c}\text { DRY } \\
\text { MASS } \\
\text { OF } \\
\text { SAMPLE (2) } \\
\text { (G) }\end{array}$} & \multicolumn{6}{|c|}{ NUMBER OF PINK TRACER PARTICLES } \\
\hline & & \multirow[b]{2}{*}{ TOTAL } & \multicolumn{5}{|c|}{ BY SIZE CLASS (MM) } \\
\hline & & & $\begin{array}{l}0.25 \\
\text { TO } \\
0.50\end{array}$ & $\begin{array}{l}0.50 \\
\text { TO } \\
1.00\end{array}$ & $\begin{array}{l}1.00 \\
2.00\end{array}$ & $\begin{array}{l}2.00 \\
\text { TO } \\
4.00\end{array}$ & $\begin{array}{l}4.00 \\
\text { TO } \\
8.00\end{array}$ \\
\hline $\begin{array}{l}5-16 \\
5-19 \\
5-21 \\
5-22 \\
5-24\end{array}$ & $\begin{array}{l}131+ \\
249+ \\
533 \\
2592 \\
5459\end{array}$ & $\begin{array}{l}7 \\
26 \\
63 \\
27 \\
15\end{array}$ & $\begin{array}{r}0 \\
13 \\
50 \\
15 \\
1\end{array}$ & $\begin{array}{r}5 \\
12 \\
10 \\
12 \\
7\end{array}$ & $\begin{array}{l}2 \\
1 \\
3 \\
0 \\
6\end{array}$ & $\begin{array}{l}0 \\
0 \\
0 \\
0 \\
1\end{array}$ & $\begin{array}{l}0 \\
0 \\
0 \\
0 \\
0\end{array}$ \\
\hline $\begin{array}{l}5-26 \\
5-27 \\
5-28 \\
5-29\end{array}$ & $\begin{array}{r}4 \overline{28} \\
289 \\
-\end{array}$ & $\begin{array}{l}-\overline{42} \\
39 \\
-\end{array}$ & $\begin{array}{l}-\overline{8} \\
18 \\
13\end{array}$ & $\begin{array}{l}21 \\
24\end{array}$ & $\begin{array}{r}-- \\
2 \\
-2\end{array}$ & $\begin{array}{r}-- \\
-1 \\
--\end{array}$ & $\begin{array}{r}-- \\
0 \\
-\end{array}$ \\
\hline $5-30$ & 116 & 0 & 0 & 0 & 0 & 0 & 0 \\
\hline $\begin{array}{l}6-01 \\
6-03 \\
6-05 \\
6-07 \\
6-09\end{array}$ & $\begin{array}{c}27^{++} \\
15^{+++} \\
12+++ \\
83+ \\
2040^{++}\end{array}$ & $\begin{array}{r}19 \\
7 \\
0 \\
23 \\
36\end{array}$ & $\begin{array}{l}4 \\
7 \\
0 \\
5 \\
9\end{array}$ & $\begin{array}{r}15 \\
0 \\
0 \\
17 \\
25\end{array}$ & $\begin{array}{l}0 \\
0 \\
0 \\
1 \\
2\end{array}$ & $\begin{array}{l}0 \\
0 \\
0 \\
0 \\
0\end{array}$ & $\begin{array}{l}0 \\
0 \\
0 \\
0 \\
0\end{array}$ \\
\hline $\begin{array}{l}6-10 \\
6-11 \\
6-12 \\
6-13 \\
6-14\end{array}$ & $\begin{array}{l}5918 \\
1623 \\
3014 \\
706 \\
2159\end{array}$ & $\begin{array}{l}21 \\
18 \\
21 \\
21 \\
19\end{array}$ & $\begin{array}{l}2 \\
1 \\
\frac{1}{1} \\
\frac{1}{2}\end{array}$ & $\begin{array}{l}14 \\
9 \\
16 \\
16 \\
10\end{array}$ & $\begin{array}{l}4 \\
6 \\
4 \\
4 \\
7\end{array}$ & $\begin{array}{l}1 \\
2 \\
0 \\
0 \\
0\end{array}$ & $\begin{array}{l}0 \\
0 \\
0 \\
0 \\
0\end{array}$ \\
\hline $\begin{array}{l}6-15 \\
6-16 \\
6-17 \\
6-18 \\
6-19\end{array}$ & $\begin{array}{r}5217 \\
-- \\
661 \\
2528 \\
4246\end{array}$ & $\begin{array}{l}26 \\
\overline{19} \\
19 \\
19\end{array}$ & $\begin{array}{r}-\frac{2}{1} \\
0 \\
0\end{array}$ & $\frac{17}{17}$ & $\begin{array}{r}-\frac{5}{7} \\
5 \\
6\end{array}$ & $\begin{array}{r}-2 \\
0 \\
0 \\
4\end{array}$ & $\begin{array}{r}-0 \\
-0 \\
0 \\
0\end{array}$ \\
\hline $\begin{array}{l}6-20 \\
6-21\end{array}$ & $\begin{array}{l}4132 \\
3768\end{array}$ & $\begin{array}{l}18 \\
16\end{array}$ & $\begin{array}{r}0 \\
4 \\
-1\end{array}$ & $1 \frac{1}{7}$ & $\begin{array}{r}5 \\
4 \\
\end{array}$ & $\begin{array}{l}2 \\
0\end{array}$ & $\begin{array}{l}0 \\
1\end{array}$ \\
\hline $\begin{array}{l}6-23 \\
6-25\end{array}$ & $\begin{array}{l}4215 \\
4124\end{array}$ & $\begin{array}{l}20 \\
22\end{array}$ & $\begin{array}{l}3 \\
0\end{array}$ & $\begin{array}{r}8 \\
10\end{array}$ & $\begin{array}{r}5 \\
12\end{array}$ & $\begin{array}{l}4 \\
0\end{array}$ & $\begin{array}{l}0 \\
0\end{array}$ \\
\hline $\begin{array}{l}6-27 \\
6-29\end{array}$ & 2146 & 18 & -1 & 10 & -6 & -2 & -1 \\
\hline $7-01$ & -- & -- & -- & -- & -- & -- & -- \\
\hline $\begin{array}{l}7-03 \\
7-05\end{array}$ & $=$ & $=$ & $=-$ & $=$ & -- & -- & $=$ \\
\hline $7-07$ & -- & -- & -- & -- & -- & -- & -- \\
\hline
\end{tabular}


TABLE 15.- NUMBER OF PINK TRACER PARTICLES(1), PER 100 GRAMS OF BEDLOAD SAMPLE, COMPOSITED FROM CROSS-CHANNEL SAMPLING POSITIONS SPACED AT 1-METER INTERVALS ACROSS THE CHANNEL WIDTH, EAST FORK RIVER, WYOMING, 1980--CONTINUED

(1) PINK TRACER PARTICLES PLACED AS BED MATERIAL 10 METERS DOWNSTREAM OF SECTION 3047 ON MAY $18,1979$.

(2) REPRESENTS TOTAL MASS, IN GRAMS, RETAINED IN BEDLOAD SAMPLER AS MEASURED IN THE FIELD! FOR SUBSEOUENT ANALYSIS OF NUMBER AND SIZE OF TRACER, PARTICLES SMALLER THAN $0.25 \mathrm{MM}$ AND LARGER THAN $8.0 \mathrm{MM}$ WERE DISCARDED. REMAINING SAMPLES WEIGHING MORE THAN 100 GRAMS WERE CUT TO A STANDARD 100 GRAM-PORTION; SAMPLES WEIGHING LESS THAN 100 GRAMS WERE ANALYZED INTACT.

(3) SMALL OVERFLOW CHANNEL ADJACENT TO SECTION.

(4) DOES NOT INCLUDE TRACER PARTICLES MEASURED IN TRANSPORT AT SECTION 1400, A BYPASS CHANNEL COMMON TO SECTIONS 1360, 1396, AND 1425. SEE FIGURE 3.

(5) BYPASS CHANNELL. SEE FIGURE 3.

+ MASS OF SAMPLE ANALYZED WAS BETWEEN 50 AND 100 GRAMS; NUMBER OF TRACER PARTICLES ADJUSTED TO AN EQUIVALENT 100-GRAM SAMPLE; EXTRAPOLATED NUMBERS ARE CONSIDERED REL IABLE.

+ MASS OF SAMPLE ANALYZED WAS BETWEEN 10 AND 50 GRAMS; NUMBER OF TRACER PARTICLES ADJUSTED TO AN EQUIVALENT 100-GRAM SAMPLE; CARE SHOULD BE TAKEN IN USING THE EXTRAPOLATED NUMBERS.

++ MASS OF SAMPLE ANALYZED WAS BETWEEN 0 AND 10 GRAMS : NUMBER OF TRACER PARTICLES ADJUSTED TO AN EQU IVALENT 100-GRAM SAMPLE; EXTRAPOLATED NUMBERS ARE NOT CONSIDERED RELIABLE. 
TABLE 16.- NUMBER OF BLUE TRACER PARTICLES(1), PER 100 GRAMS OF BEDLOAD SAMPLE, COMPOSITED FROM CROSS-CHANNEL SAMPLING POSITIONS SPACED AT I-METER INTERVALS ACROSS THE CHANNEL WIDTH, EAST FORK RIVER, WYOMING, 1980

SECTION 0043

\begin{tabular}{|c|c|c|c|c|c|c|c|}
\hline \multirow{3}{*}{ DATE } & \multirow{3}{*}{$\begin{array}{c}\text { DRY } \\
\text { MASS } \\
\text { OF } \\
\text { SAMPLE (2) } \\
(\mathrm{G})\end{array}$} & \multicolumn{6}{|c|}{ NUMBER OF BLUE TRACER PARTICLES } \\
\hline & & \multirow[b]{2}{*}{ TOTAL } & \multicolumn{5}{|c|}{ BY SIZE CLASS (MM) } \\
\hline & & & $\begin{array}{l}0.25 \\
\mathrm{TO} \\
0.50\end{array}$ & $\begin{array}{l}0.50 \\
\text { TO } \\
1.00\end{array}$ & $\begin{array}{l}1.00 \\
2.00\end{array}$ & $\begin{array}{l}2.00 \\
\text { TO } \\
4.00\end{array}$ & $\begin{array}{l}4.00 \\
\text { TO } \\
8.00\end{array}$ \\
\hline $\begin{array}{l}5-16 \\
5-19 \\
5-21\end{array}$ & $\begin{array}{l}59++ \\
75++ \\
54+\end{array}$ & $\begin{array}{l}0 \\
1 \\
7\end{array}$ & $\begin{array}{l}0 \\
1 \\
6\end{array}$ & $\begin{array}{l}0 \\
0 \\
1\end{array}$ & $\begin{array}{l}0 \\
0 \\
0\end{array}$ & $\begin{array}{l}0 \\
0 \\
0\end{array}$ & $\begin{array}{l}0 \\
0 \\
0\end{array}$ \\
\hline $\begin{array}{l}5-22 \\
5-24\end{array}$ & $=$ & $\therefore$ & 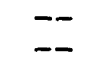 & $\therefore$ & - & $=$ & -- \\
\hline $\begin{array}{l}5-26 \\
5-27 \\
5-28 \\
5-29\end{array}$ & $\begin{array}{c}371 \\
84+ \\
28++ \\
--\end{array}$ & $\begin{array}{r}3 \\
2 \\
0 \\
-\end{array}$ & $\begin{array}{r}1 \\
0 \\
0 \\
--\end{array}$ & $\begin{array}{r}2 \\
2 \\
0 \\
--\end{array}$ & $\begin{array}{r}0 \\
0 \\
0 \\
--\end{array}$ & $\begin{array}{r}0 \\
0 \\
0 \\
--\end{array}$ & $\begin{array}{l}0 \\
0 \\
0 \\
-2\end{array}$ \\
\hline $5-30$ & $5+++$ & 0 & 0 & 0 & 0 & 0 & 0 \\
\hline $\begin{array}{l}6-01 \\
6-03 \\
6-05 \\
6-07 \\
6-09\end{array}$ & $\begin{array}{c}81++ \\
0+++ \\
0+++ \\
111^{+++} \\
303\end{array}$ & $\begin{array}{l}4 \\
0 \\
0 \\
0 \\
6\end{array}$ & $\begin{array}{l}1 \\
0 \\
0 \\
0 \\
2\end{array}$ & $\begin{array}{l}3 \\
0 \\
0 \\
0 \\
3\end{array}$ & $\begin{array}{l}0 \\
0 \\
0 \\
0 \\
1\end{array}$ & $\begin{array}{l}0 \\
0 \\
0 \\
0 \\
0\end{array}$ & $\begin{array}{l}0 \\
0 \\
0 \\
0 \\
0\end{array}$ \\
\hline $\begin{array}{l}6-10 \\
6-11 \\
6-12 \\
6-13 \\
6-14\end{array}$ & $\begin{array}{l}3071 \\
3889 \\
2659 \\
1640 \\
1030\end{array}$ & $\begin{array}{r}11 \\
4 \\
11 \\
6 \\
14\end{array}$ & $\begin{array}{l}5 \\
0 \\
1 \\
1 \\
1\end{array}$ & $\begin{array}{l}6 \\
2 \\
9 \\
5 \\
9\end{array}$ & $\begin{array}{l}0 \\
2 \\
1 \\
0 \\
2\end{array}$ & $\begin{array}{l}0 \\
0 \\
0 \\
0 \\
2\end{array}$ & $\begin{array}{l}0 \\
0 \\
0 \\
0 \\
0\end{array}$ \\
\hline $6-15$ & 984 & 6 & 2 & 4 & 0 & 0 & 0 \\
\hline $\begin{array}{l}6-17 \\
6-18 \\
6-19\end{array}$ & $\begin{array}{r}383 \\
320 \\
1123\end{array}$ & $\begin{array}{l}9 \\
18 \\
19\end{array}$ & $\begin{array}{l}3 \\
3 \\
1\end{array}$ & $\begin{array}{r}5 \\
13 \\
12\end{array}$ & $\begin{array}{l}\frac{1}{2} \\
5\end{array}$ & $\begin{array}{l}0 \\
0 \\
1\end{array}$ & $\begin{array}{l}0 \\
0 \\
0\end{array}$ \\
\hline $\begin{array}{l}6-20 \\
6-21\end{array}$ & $\begin{array}{l}271 \\
764\end{array}$ & $\begin{array}{l}6 \\
9\end{array}$ & $\begin{array}{l}0 \\
0\end{array}$ & $\begin{array}{l}6 \\
6\end{array}$ & $\begin{array}{l}0 \\
3\end{array}$ & $\begin{array}{l}0 \\
0\end{array}$ & $\begin{array}{l}0 \\
0\end{array}$ \\
\hline $\begin{array}{l}6-23 \\
6-25\end{array}$ & $\begin{array}{r}585 \\
1008\end{array}$ & $\begin{array}{l}26 \\
10\end{array}$ & $\begin{array}{l}7 \\
3\end{array}$ & $\begin{array}{r}15 \\
3\end{array}$ & $\begin{array}{l}3 \\
4\end{array}$ & $\frac{1}{0}$ & $\begin{array}{l}0 \\
0\end{array}$ \\
\hline $6-27$ & 486 & 21 & 0 & 13 & 7 & 1 & 0 \\
\hline $\begin{array}{l}6-29 \\
7-01\end{array}$ & $\overline{--}$ & $\overline{--}$ & - & $\overline{-}$ & -- & -- & -- \\
\hline $\begin{array}{l}7-03 \\
7-05\end{array}$ & $=$ & 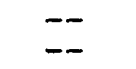 & $=$ & $=$ & $=$ & $=$ & $=$ \\
\hline $7-07$ & -- & -- & -- & -- & -- & -- & -- \\
\hline
\end{tabular}


TABLE 16.- NUMBER OF BLUE TRACER PARTICLES(1), PER 100 GRAMS OF BEDLOAD SAMPLE, COMPOSITED FROM CROSS-CHANNEL SAMPLING POSITIONS SPACED AT I-METER INTERVALS ACROSS THE CHANNEL WIDTH, EAST FORK RIVER, WYOMING, I980

SECTION 0075

\begin{tabular}{|c|c|c|c|c|c|c|c|}
\hline \multirow{3}{*}{ DATE } & \multirow{3}{*}{$\begin{array}{c}\text { DRY } \\
\text { MASS } \\
\text { OF } \\
\text { SAMPLE (2) } \\
\text { (G) }\end{array}$} & \multicolumn{6}{|c|}{ NUMBER OF BLUE TRACER PARTICLES } \\
\hline & & \multirow[b]{2}{*}{ TOTAL } & \multicolumn{5}{|c|}{ BY SIZE CLASS (MM) } \\
\hline & & & $\begin{array}{c}0.25 \\
\text { TO } \\
0.50\end{array}$ & $\begin{array}{l}0.50 \\
\text { TO } \\
1.00\end{array}$ & $\begin{array}{l}1.00 \\
2.00\end{array}$ & $\begin{array}{l}2.00 \\
\text { TO } \\
4.00\end{array}$ & $\begin{array}{c}4.00 \\
\text { TO } \\
8.00\end{array}$ \\
\hline $\begin{array}{l}5-16 \\
5-19 \\
5-21 \\
5-22 \\
5-24\end{array}$ & $\begin{array}{c}161+ \\
346 \\
1581 \\
-- \\
--\end{array}$ & $\begin{array}{r}8 \\
10 \\
8 \\
--\end{array}$ & $\begin{array}{r}7 \\
10 \\
4 \\
--\end{array}$ & $\begin{array}{r}1 \\
0 \\
-1 \\
--\end{array}$ & $\begin{array}{r}0 \\
0 \\
0 \\
-- \\
--\end{array}$ & $\begin{array}{r}0 \\
0 \\
0 \\
-- \\
--\end{array}$ & $\begin{array}{r}0 \\
0 \\
0 \\
-- \\
--\end{array}$ \\
\hline $\begin{array}{l}5-26 \\
5-27 \\
5-28 \\
5-29 \\
5-30\end{array}$ & $\begin{array}{l}51+ \\
127+ \\
127 \\
237 \\
19+++\end{array}$ & $\begin{array}{r}2 \\
0 \\
0 \\
18 \\
0\end{array}$ & $\begin{array}{l}2 \\
0 \\
0 \\
3 \\
0\end{array}$ & $\begin{array}{r}0 \\
0 \\
0 \\
15 \\
0\end{array}$ & $\begin{array}{l}0 \\
0 \\
0 \\
0 \\
0\end{array}$ & $\begin{array}{l}0 \\
0 \\
0 \\
0 \\
0\end{array}$ & $\begin{array}{l}0 \\
0 \\
0 \\
0 \\
0\end{array}$ \\
\hline $\begin{array}{l}6-01 \\
6-03 \\
6-05 \\
6-07 \\
6-09\end{array}$ & $\begin{array}{r}1907 \\
900 \\
283 \\
742 \\
1650\end{array}$ & $\begin{array}{r}2 \\
7 \\
3 \\
0 \\
12\end{array}$ & $\begin{array}{l}1 \\
2 \\
0 \\
0 \\
2\end{array}$ & $\begin{array}{l}1 \\
5 \\
3 \\
0 \\
8\end{array}$ & $\begin{array}{l}0 \\
0 \\
0 \\
0 \\
2\end{array}$ & $\begin{array}{l}0 \\
0 \\
0 \\
0 \\
0\end{array}$ & $\begin{array}{l}0 \\
0 \\
0 \\
0 \\
0\end{array}$ \\
\hline $\begin{array}{l}6-10 \\
6-11 \\
6-12 \\
6-13 \\
6-14\end{array}$ & $\begin{array}{l}3128 \\
1416 \\
2786 \\
1735 \\
1897\end{array}$ & $\begin{array}{r}4 \\
6 \\
0 \\
22 \\
12\end{array}$ & $\begin{array}{r}1 \\
2 \\
0 \\
10 \\
3\end{array}$ & $\begin{array}{r}3 \\
3 \\
0 \\
12 \\
7\end{array}$ & $\begin{array}{l}0 \\
1 \\
0 \\
0 \\
2\end{array}$ & $\begin{array}{l}0 \\
0 \\
0 \\
0 \\
0\end{array}$ & $\begin{array}{l}0 \\
0 \\
0 \\
0 \\
0\end{array}$ \\
\hline $6-15$ & 651 & 15 & 4 & 10 & 1 & 0 & 0 \\
\hline $\begin{array}{l}6-17 \\
6-18 \\
6-19\end{array}$ & $\begin{array}{r}900 \\
1969 \\
2818\end{array}$ & $\begin{array}{l}14 \\
24 \\
16\end{array}$ & $\begin{array}{l}4 \\
2 \\
1\end{array}$ & $\begin{array}{r}8 \\
18 \\
14\end{array}$ & $\begin{array}{l}2 \\
4 \\
1\end{array}$ & $\begin{array}{l}0 \\
0 \\
0\end{array}$ & $\begin{array}{l}0 \\
0 \\
0\end{array}$ \\
\hline $\begin{array}{l}6-20 \\
6-21 \\
6-25\end{array}$ & $\begin{array}{l}5583 \\
4777 \\
\end{array}$ & $\begin{array}{l}17 \\
14\end{array}$ & $\begin{array}{l}3 \\
1\end{array}$ & $\begin{array}{l}12 \\
10\end{array}$ & $\begin{array}{r}2 \\
0 \\
\end{array}$ & $\begin{array}{l}0 \\
3\end{array}$ & $\begin{array}{l}0 \\
0\end{array}$ \\
\hline $\begin{array}{l}6-23 \\
6-25\end{array}$ & $\begin{array}{l}1409 \\
1558\end{array}$ & $\begin{array}{r}6 \\
12\end{array}$ & $\begin{array}{l}2 \\
3\end{array}$ & $\begin{array}{l}3 \\
5\end{array}$ & $\frac{1}{3}$ & $\begin{array}{l}0 \\
0\end{array}$ & $\begin{array}{l}0 \\
I\end{array}$ \\
\hline $\begin{array}{l}6-27 \\
6-29\end{array}$ & 2302 & 11 & -1 & -6 & -3 & -2 & $-\underline{0}$ \\
\hline $7-01$ & -- & -- & -- & - & -- & -- & -- \\
\hline $\begin{array}{l}7-03 \\
7-05\end{array}$ & $\overline{--}$ & $\overline{--}$ & $\overline{--}$ & $\overline{--}$ & $=$ & $\overline{--}$ & $\overline{--}$ \\
\hline $7-07$ & -- & -- & -- & -- & -- & -- & -- \\
\hline
\end{tabular}


TABLE 16.- NUMBER OF BLUE TRACER PARTICLES(1), PER 100 GRAMS OF BEDLOAD SAMPLE, COMPOSITED FROM CROSS-CHANNEL SAMPLING POSITIONS SPACED AT 1-METER INTERVALS ACROSS THE CHANNEL WIDTH, EAST FORK RIVER, WYOMING, 1980

$$
\text { SECTION } 0137
$$

\begin{tabular}{|c|c|c|c|c|c|c|c|}
\hline \multirow{3}{*}{ DATE } & \multirow{3}{*}{$\begin{array}{c}\text { DRY } \\
\text { MASS } \\
\text { OF } \\
\text { SAMPLE (2) } \\
(G)\end{array}$} & \multicolumn{6}{|c|}{ NUMBER OF BLUE TRACER PARTICLES } \\
\hline & & \multirow[b]{2}{*}{ TOTAL } & \multicolumn{5}{|c|}{ BY SIZE CLASS (MM) } \\
\hline & & & $\begin{array}{l}0.25 \\
\text { TO } \\
0.50\end{array}$ & $\begin{array}{l}0.50 \\
\text { TO } \\
1.00\end{array}$ & $\begin{array}{l}1.00 \\
\text { TO } \\
2.00\end{array}$ & $\begin{array}{l}2.00 \\
\text { TO } \\
4.00\end{array}$ & $\begin{array}{l}4.00 \\
\text { TO } \\
8.00\end{array}$ \\
\hline $\begin{array}{l}5-16 \\
5-19 \\
5-21 \\
5-22 \\
5-24\end{array}$ & $\begin{array}{l}225 \\
56+++ \\
113++ \\
=-\end{array}$ & $\begin{array}{r}9 \\
0 \\
7 \\
-- \\
--\end{array}$ & $\begin{array}{r}8 \\
0 \\
5 \\
-- \\
--\end{array}$ & $\begin{array}{r}1 \\
0 \\
2 \\
-- \\
--\end{array}$ & $\begin{array}{r}0 \\
0 \\
0 \\
-- \\
--\end{array}$ & $\begin{array}{r}0 \\
0 \\
0 \\
-- \\
--\end{array}$ & $\begin{array}{r}0 \\
0 \\
0 \\
-- \\
--\end{array}$ \\
\hline $\begin{array}{l}5-26 \\
5-27 \\
5-28 \\
5-29\end{array}$ & $\begin{array}{r}1360 \\
1794 \\
135 \\
\end{array}$ & $\begin{array}{r}4 \\
0 \\
3 \\
-\end{array}$ & $\begin{array}{l}1 \\
0 \\
3\end{array}$ & $\begin{array}{l}1 \\
0 \\
0\end{array}$ & $\begin{array}{l}2 \\
0 \\
0\end{array}$ & $\begin{array}{l}0 \\
0 \\
0\end{array}$ & $\begin{array}{l}0 \\
0 \\
0\end{array}$ \\
\hline $5-30$ & $99+$ & 4 & 2 & 2 & 0 & 0 & 0 \\
\hline $\begin{array}{l}6-01 \\
6-03 \\
6-05 \\
6-07 \\
6-09\end{array}$ & $\begin{array}{c}41++ \\
73++ \\
0+++ \\
588 \\
1099\end{array}$ & $\begin{array}{r}2 \\
4 \\
0 \\
9 \\
16\end{array}$ & $\begin{array}{l}0 \\
0 \\
0 \\
1 \\
4\end{array}$ & $\begin{array}{r}2 \\
0 \\
0 \\
5 \\
12\end{array}$ & $\begin{array}{l}0 \\
4 \\
0 \\
3 \\
0\end{array}$ & $\begin{array}{l}0 \\
0 \\
0 \\
0 \\
0\end{array}$ & $\begin{array}{l}0 \\
0 \\
0 \\
0 \\
0\end{array}$ \\
\hline $\begin{array}{l}6-10 \\
6-11 \\
6-12 \\
6-13 \\
6-14\end{array}$ & $\begin{array}{r}1907 \\
1139 \\
517 \\
1648 \\
3009\end{array}$ & $\begin{array}{r}15 \\
18 \\
8 \\
7 \\
12\end{array}$ & $\begin{array}{l}0 \\
4 \\
0 \\
1 \\
3\end{array}$ & $\begin{array}{r}12 \\
10 \\
4 \\
5 \\
7\end{array}$ & $\begin{array}{l}3 \\
4 \\
3 \\
1 \\
2\end{array}$ & $\begin{array}{l}0 \\
0 \\
1 \\
0 \\
0\end{array}$ & $\begin{array}{l}0 \\
0 \\
0 \\
0 \\
0\end{array}$ \\
\hline $6-15$ & 2497 & 10 & 3 & 7 & 0 & 0 & 0 \\
\hline $\begin{array}{l}0-10 \\
6-17 \\
6-18 \\
6-19\end{array}$ & $\begin{array}{l}3116 \\
3204 \\
2346\end{array}$ & $\begin{array}{l}20 \\
17 \\
20\end{array}$ & $\begin{array}{l}3 \\
0 \\
0\end{array}$ & $\begin{array}{l}15 \\
14 \\
15\end{array}$ & $\begin{array}{l}2 \\
2 \\
5\end{array}$ & $\begin{array}{l}0 \\
1 \\
0\end{array}$ & $\begin{array}{l}0 \\
0 \\
0\end{array}$ \\
\hline $\begin{array}{l}6-20 \\
6-21\end{array}$ & $\begin{array}{l}2042 \\
3752\end{array}$ & $\begin{array}{l}10 \\
19\end{array}$ & $\begin{array}{l}2 \\
1\end{array}$ & $\begin{array}{r}7 \\
13\end{array}$ & $\frac{1}{5}$ & $\begin{array}{l}0 \\
0\end{array}$ & $\begin{array}{l}0 \\
0\end{array}$ \\
\hline $\begin{array}{l}6-22 \\
6-23 \\
6-25\end{array}$ & $\begin{array}{l}2398 \\
3614\end{array}$ & $\begin{array}{l}18 \\
28\end{array}$ & $\begin{array}{l}0 \\
6\end{array}$ & $\begin{array}{l}14 \\
15\end{array}$ & $\begin{array}{l}2 \\
6\end{array}$ & $\begin{array}{l}2 \\
1\end{array}$ & $\begin{array}{l}0 \\
0\end{array}$ \\
\hline $6-27$ & 4050 & 26 & 3 & 11 & 10 & 2 & 0 \\
\hline $7-01$ & - & - & - & - & -- & - & - \\
\hline $\begin{array}{l}7-03 \\
7-05\end{array}$ & $=$ & $\overline{--}$ & $\overline{--}$ & $\overline{-}$ & $\overline{-}$ & $\overline{--}$ & -- \\
\hline $7-07$ & -- & -- & -- & -- & -- & -- & -- \\
\hline
\end{tabular}


TABLE 16.- NUMBER OF BLUE TRACER PARTICLES(1), PER 100 GRAMS OF BEDLOAD SAMPLE, COMPOSITED FROM CROSS-CHANNEL SAMPLING POSITIONS SPACED AT 1-METER INTERVALS ACROSS THE CHANNEL WIDTH, EAST FORK RIVER, WYOMING, 1980

SECTION 0178

\begin{tabular}{|c|c|c|c|c|c|c|c|}
\hline \multirow{3}{*}{ DATE } & \multirow{3}{*}{$\begin{array}{c}\text { DRY } \\
\text { MASS } \\
\text { OF } \\
\text { SAMPLE (2) } \\
\text { (G) }\end{array}$} & \multicolumn{6}{|c|}{ NUMBER OF BLUE TRACER PARTICLES } \\
\hline & & \multirow[b]{2}{*}{ TOTAL } & \multicolumn{5}{|c|}{ BY SIZE CLASS (MM) } \\
\hline & & & $\begin{array}{l}0.25 \\
\text { TO } \\
0.50\end{array}$ & $\begin{array}{r}0.50 \\
\text { TO } \\
1.00\end{array}$ & $\begin{array}{l}1.00 \\
2.00\end{array}$ & $\begin{array}{l}2.00 \\
\text { TO } \\
4.00\end{array}$ & $\begin{array}{l}4.00 \\
\text { TO } \\
8.00\end{array}$ \\
\hline $\begin{array}{l}5-16 \\
5-19 \\
5-21 \\
5-22 \\
5-24\end{array}$ & $\begin{array}{r}335 \\
1172 \\
430 \\
-- \\
--\end{array}$ & $\begin{array}{r}8 \\
12 \\
17 \\
-- \\
--\end{array}$ & $\begin{array}{r}8 \\
6 \\
11 \\
--\end{array}$ & $\begin{array}{r}0 \\
6 \\
6 \\
--\end{array}$ & $\begin{array}{r}0 \\
0 \\
0 \\
--\end{array}$ & $\begin{array}{r}0 \\
0 \\
0 \\
--\end{array}$ & $\begin{array}{r}0 \\
0 \\
0 \\
--\end{array}$ \\
\hline $\begin{array}{l}5-26 \\
5-27 \\
5-28 \\
5-29\end{array}$ & $\begin{array}{r}2416 \\
677 \\
446 \\
\end{array}$ & $\begin{array}{r}2 \\
3 \\
0 \\
--\end{array}$ & $\begin{array}{r}2 \\
1 \\
0 \\
-\end{array}$ & $\begin{array}{r}0 \\
2 \\
0 \\
-\end{array}$ & $\begin{array}{r}0 \\
0 \\
0 \\
-\end{array}$ & $\begin{array}{r}0 \\
0 \\
0 \\
-\end{array}$ & $\begin{array}{l}0 \\
0 \\
0 \\
-\end{array}$ \\
\hline $5-30$ & 470 & 4 & 0 & 3 & 1 & 0 & 0 \\
\hline $\begin{array}{l}6-01 \\
6-03 \\
6-05 \\
6-07 \\
6-09\end{array}$ & $\begin{array}{c}521 \\
18++ \\
689 \\
1624 \\
2378\end{array}$ & $\begin{array}{r}10 \\
0 \\
12 \\
21 \\
16\end{array}$ & $\begin{array}{l}1 \\
0 \\
3 \\
1 \\
0\end{array}$ & $\begin{array}{r}2 \\
0 \\
8 \\
19 \\
11\end{array}$ & $\begin{array}{l}7 \\
0 \\
1 \\
\frac{1}{3}\end{array}$ & $\begin{array}{l}0 \\
0 \\
0 \\
0 \\
2\end{array}$ & $\begin{array}{l}0 \\
0 \\
0 \\
0 \\
0\end{array}$ \\
\hline $\begin{array}{l}6-10 \\
6-11 \\
6-12 \\
6-13 \\
6-14\end{array}$ & $\begin{array}{l}6609 \\
4737 \\
1694 \\
5416 \\
3955\end{array}$ & $\begin{array}{r}8 \\
14 \\
10 \\
8 \\
10\end{array}$ & $\begin{array}{l}\frac{1}{3} \\
1 \\
4 \\
3\end{array}$ & $\begin{array}{l}4 \\
5 \\
6 \\
4 \\
4\end{array}$ & $\begin{array}{l}3 \\
5 \\
3 \\
0 \\
3\end{array}$ & $\begin{array}{l}0 \\
1 \\
0 \\
0 \\
0\end{array}$ & $\begin{array}{l}0 \\
0 \\
0 \\
0 \\
0\end{array}$ \\
\hline $6-15$ & 3251 & 17 & 2 & 9 & 6 & 0 & 0 \\
\hline $\begin{array}{l}6-17 \\
6-18 \\
6-19\end{array}$ & $\begin{array}{l}1273 \\
3439 \\
3696\end{array}$ & $\begin{array}{l}20 \\
20 \\
24\end{array}$ & $\begin{array}{l}3 \\
4 \\
2\end{array}$ & $\begin{array}{l}11 \\
11 \\
16\end{array}$ & $\begin{array}{l}5 \\
4 \\
6\end{array}$ & $\begin{array}{l}\frac{1}{1} \\
0\end{array}$ & $\begin{array}{l}0 \\
0 \\
0\end{array}$ \\
\hline $\begin{array}{l}6-20 \\
6-21\end{array}$ & $\begin{array}{l}5908 \\
5074\end{array}$ & $\begin{array}{l}16 \\
17\end{array}$ & $\begin{array}{l}0 \\
5\end{array}$ & $\begin{array}{r}12 \\
5\end{array}$ & $\begin{array}{l}2 \\
6\end{array}$ & $\begin{array}{l}2 \\
1\end{array}$ & $\begin{array}{l}0 \\
0\end{array}$ \\
\hline $\begin{array}{l}6-23 \\
6-25\end{array}$ & $\begin{array}{l}4771 \\
1510\end{array}$ & $\begin{array}{r}18 \\
7\end{array}$ & $\begin{array}{l}3 \\
2\end{array}$ & 11 & 3 & $\frac{1}{0}$ & $\begin{array}{l}0 \\
0\end{array}$ \\
\hline $6-27$ & 3035 & 21 & 0 & 9 & 11 & 1 & 0 \\
\hline $7-01$ & $=$ & $=$ & - & $=$ & - & - & - \\
\hline $\begin{array}{l}7-03 \\
7-05\end{array}$ & -- & $\overline{--}$ & $\overline{--}$ & $\overline{--}$ & $\overline{--}$ & $\overline{-}$ & $\overline{--}$ \\
\hline $7-07$ & -- & -- & -- & -- & -- & -- & -- \\
\hline
\end{tabular}


TABLE 16.- NUMBER OF BLUE TRACER PARTICLES(1), PER 100 GRAMS OF BEDLOAD SAMPLE, COMPOSITED FROM CROSS-CHANNEL SAMPLING POSITIONS SPACED AT 1-METER INTERVALS ACROSS THE CHANNEL WIDTH, EAST FORK RIVER, WYOMING, 1980

SECTION 0220

\begin{tabular}{|c|c|c|c|c|c|c|c|}
\hline \multirow{3}{*}{ DATE } & \multirow{3}{*}{$\begin{array}{c}\text { DRY } \\
\text { MASS } \\
\text { OF } \\
\text { SAMPLE (2) } \\
\text { (G) }\end{array}$} & \multicolumn{6}{|c|}{ NUMBER OF BLUE TRACER PARTICLES } \\
\hline & & \multirow[b]{2}{*}{ TOTAL } & \multicolumn{5}{|c|}{ BY SIZE CLASS (MM) } \\
\hline & & & $\begin{array}{c}0.25 \\
\text { TO } \\
0.50\end{array}$ & $\begin{array}{l}0.50 \\
\text { TO } \\
1.00\end{array}$ & $\begin{array}{l}1.00 \\
\text { TO } \\
2.00\end{array}$ & $\begin{array}{l}2.00 \\
\text { TO } \\
4.00\end{array}$ & $\begin{array}{l}4.00 \\
\text { TO } \\
8.00\end{array}$ \\
\hline $\begin{array}{l}5-16 \\
5-19 \\
5-21 \\
5-22 \\
5-24\end{array}$ & $\begin{array}{c}144+ \\
512 \\
5907 \\
-- \\
--\end{array}$ & $\begin{array}{l}5 \\
18 \\
15 \\
--\end{array}$ & $\begin{array}{r}0 \\
9 \\
9 \\
-- \\
--\end{array}$ & $\begin{array}{r}5 \\
9 \\
6 \\
-- \\
--\end{array}$ & $\begin{array}{r}0 \\
0 \\
0 \\
-- \\
--\end{array}$ & $\begin{array}{r}0 \\
0 \\
0 \\
-- \\
--\end{array}$ & $\begin{array}{r}0 \\
0 \\
0 \\
-- \\
--\end{array}$ \\
\hline $\begin{array}{l}5-26 \\
5-27 \\
5-28 \\
5-20\end{array}$ & $\begin{array}{c}871 \\
873+ \\
673 \\
\end{array}$ & $\begin{array}{r}0 \\
4 \\
2 \\
-2\end{array}$ & $\begin{array}{l}0 \\
0 \\
0\end{array}$ & $\begin{array}{l}0 \\
4 \\
1\end{array}$ & $\begin{array}{l}0 \\
0 \\
1\end{array}$ & $\begin{array}{l}0 \\
0 \\
0\end{array}$ & $\begin{array}{r}0 \\
0 \\
0 \\
-0\end{array}$ \\
\hline $5-30$ & $150+$ & 7 & 2 & 5 & 0 & 0 & 0 \\
\hline $\begin{array}{l}6-01 \\
6-03 \\
6-05 \\
6-07 \\
6-09\end{array}$ & $\begin{array}{c}44++ \\
67++ \\
569 \\
758 \\
4213\end{array}$ & $\begin{array}{r}7 \\
3 \\
25 \\
19 \\
13\end{array}$ & $\begin{array}{l}0 \\
0 \\
3 \\
2 \\
0\end{array}$ & $\begin{array}{r}0 \\
3 \\
18 \\
11 \\
6\end{array}$ & $\begin{array}{l}7 \\
0 \\
4 \\
6 \\
5\end{array}$ & $\begin{array}{l}0 \\
0 \\
0 \\
0 \\
2\end{array}$ & $\begin{array}{l}0 \\
0 \\
0 \\
0 \\
0\end{array}$ \\
\hline $\begin{array}{l}6-10 \\
6-11 \\
6-12 \\
6-13 \\
6-14\end{array}$ & $\begin{array}{l}6882 \\
5760 \\
3938 \\
4401 \\
5375\end{array}$ & $\begin{array}{r}9 \\
10 \\
8 \\
14 \\
13\end{array}$ & $\begin{array}{l}2 \\
4 \\
2 \\
6 \\
3\end{array}$ & $\begin{array}{l}6 \\
3 \\
4 \\
5 \\
9\end{array}$ & $\begin{array}{l}1 \\
2 \\
2 \\
3 \\
1\end{array}$ & $\begin{array}{l}0 \\
1 \\
0 \\
0 \\
0\end{array}$ & $\begin{array}{l}0 \\
0 \\
0 \\
0 \\
0\end{array}$ \\
\hline $6-15$ & 241 & 25 & 13 & 11 & 1 & 0 & 0 \\
\hline $\begin{array}{l}6-17 \\
6-18 \\
6-19\end{array}$ & $\begin{array}{r}946 \\
3574 \\
6083\end{array}$ & $\begin{array}{l}21 \\
20 \\
26\end{array}$ & $\begin{array}{l}3 \\
3 \\
2\end{array}$ & $\begin{array}{r}13 \\
8 \\
14\end{array}$ & $\begin{array}{l}5 \\
9 \\
9\end{array}$ & $\begin{array}{l}0 \\
0 \\
1\end{array}$ & $\begin{array}{l}0 \\
0 \\
0\end{array}$ \\
\hline $\begin{array}{l}6-20 \\
6-21\end{array}$ & $\begin{array}{l}6451 \\
3627\end{array}$ & $\begin{array}{r}9 \\
18\end{array}$ & $\begin{array}{l}0 \\
0\end{array}$ & $\begin{array}{r}2 \\
11\end{array}$ & $\begin{array}{l}6 \\
7\end{array}$ & $\frac{1}{0}$ & $\begin{array}{l}0 \\
0\end{array}$ \\
\hline $\begin{array}{l}6-23 \\
6-25\end{array}$ & $\begin{array}{l}1357 \\
3244\end{array}$ & $\begin{array}{l}4 \frac{1}{29} \\
29\end{array}$ & $\begin{array}{l}9 \\
7\end{array}$ & $\begin{array}{l}20 \\
17\end{array}$ & 11 & $\frac{1}{1}$ & $\begin{array}{l}0 \\
0\end{array}$ \\
\hline $6-27$ & 1327 & 13 & 2 & 8 & 1 & 2 & 0 \\
\hline $7-01$ & - & - & - & -- & - & - & - \\
\hline $7-0 \overline{3}$ & -- & -- & -- & -- & -- & -- & -- \\
\hline $7-05$ & -- & - & -- & -- & -- & -- & -- \\
\hline $7-07$ & -- & -- & -- & -- & -- & -- & -- \\
\hline
\end{tabular}


TABLE 16.- NUMBER OF BLUE TRACER PARTICLES(1), PER 100 GRAMS OF BEDLOAD SAMPLE, COMPOSITED FROM CROSS-CHANNEL SAMPLING POSITIONS SPACED AT 1-METER INTERVALS ACROSS THE CHANNEL WIDTH, EAST FORK RIVER, WYOMING, 1980

SECTION C257

\begin{tabular}{|c|c|c|c|c|c|c|c|}
\hline \multirow{3}{*}{ DATE } & \multirow{3}{*}{$\begin{array}{c}\text { DRY } \\
\text { MASS } \\
\text { OF } \\
\text { SAMPLE (2) } \\
\text { (G) }\end{array}$} & \multicolumn{6}{|c|}{ NUMBER OF BLUE TRACER PARTICLES } \\
\hline & & \multirow[b]{2}{*}{ TOTAL } & \multicolumn{5}{|c|}{ BY SIZE CLASS (MM) } \\
\hline & & & $\begin{array}{c}0.25 \\
\text { TO } \\
0.50\end{array}$ & $\begin{array}{l}0.50 \\
\text { TO } \\
1.00\end{array}$ & $\begin{array}{l}1.00 \\
\text { TO } \\
2.00\end{array}$ & $\begin{array}{l}2.00 \\
\text { TO } \\
4.00\end{array}$ & $\begin{array}{l}4.00 \\
\text { TO } \\
8.00\end{array}$ \\
\hline $\begin{array}{l}5-16 \\
5-19 \\
5-21 \\
5-22 \\
5-24\end{array}$ & $\begin{array}{r}119+ \\
697 \\
3491 \\
-= \\
--\end{array}$ & $\begin{array}{r}24 \\
19 \\
7 \\
-- \\
--\end{array}$ & $\begin{array}{r}10 \\
13 \\
4 \\
-- \\
--\end{array}$ & $\begin{array}{r}14 \\
6 \\
3 \\
-- \\
--\end{array}$ & $\begin{array}{r}0 \\
0 \\
0 \\
-- \\
--\end{array}$ & $\begin{array}{r}0 \\
0 \\
0 \\
-- \\
--\end{array}$ & $\begin{array}{r}0 \\
0 \\
0 \\
-- \\
--\end{array}$ \\
\hline $\begin{array}{l}5-26 \\
5-27 \\
5-28 \\
5-29 \\
5-30\end{array}$ & $\begin{array}{l}183 \\
113 \\
42++ \\
-74+\end{array}$ & $\begin{array}{r}7 \\
8 \\
7 \\
-9\end{array}$ & $\begin{array}{r}4 \\
0 \\
5 \\
-1 \\
0\end{array}$ & $\begin{array}{r}3 \\
2 \\
0 \\
-1 \\
4\end{array}$ & $\begin{array}{r}0 \\
5 \\
2 \\
- \\
5\end{array}$ & $\begin{array}{r}0 \\
1 \\
0 \\
-0\end{array}$ & $\begin{array}{r}0 \\
0 \\
0 \\
-0\end{array}$ \\
\hline $\begin{array}{l}6-01 \\
6-03 \\
6-05 \\
6-07 \\
6-09\end{array}$ & $\begin{array}{c}90++ \\
697 \\
1516 \\
645 \\
3432\end{array}$ & $\begin{array}{r}99 \\
23 \\
18 \\
14 \\
9\end{array}$ & $\begin{array}{l}1 \\
0 \\
\frac{1}{1} \\
\frac{1}{2}\end{array}$ & $\begin{array}{l}8 \\
19 \\
10 \\
10 \\
7\end{array}$ & $\begin{array}{l}0 \\
4 \\
6 \\
3 \\
0\end{array}$ & $\begin{array}{l}0 \\
0 \\
1 \\
0 \\
0\end{array}$ & $\begin{array}{l}0 \\
0 \\
0 \\
0 \\
0\end{array}$ \\
\hline $\begin{array}{l}6-10 \\
6-11 \\
6-12 \\
6-13 \\
6-14\end{array}$ & $\begin{array}{l}5300 \\
2534 \\
3148 \\
3296 \\
1846\end{array}$ & $\begin{array}{r}6 \\
5 \\
17 \\
10 \\
6\end{array}$ & $\begin{array}{l}0 \\
1 \\
5 \\
3 \\
2\end{array}$ & $\begin{array}{r}5 \\
4 \\
10 \\
7 \\
3\end{array}$ & $\begin{array}{l}1 \\
0 \\
2 \\
0 \\
1\end{array}$ & $\begin{array}{l}0 \\
0 \\
0 \\
0 \\
0\end{array}$ & $\begin{array}{l}0 \\
0 \\
0 \\
0 \\
0\end{array}$ \\
\hline $\begin{array}{l}6-15 \\
6-16 \\
6-17 \\
6-18 \\
6-19\end{array}$ & $\begin{array}{r}2370 \\
799 \\
1076 \\
2889 \\
4435\end{array}$ & $\begin{array}{r}7 \\
19 \\
20 \\
15 \\
28\end{array}$ & $\begin{array}{l}1 \\
6 \\
3 \\
0 \\
1\end{array}$ & $\begin{array}{r}4 \\
12 \\
11 \\
8 \\
17\end{array}$ & $\begin{array}{l}2 \\
1 \\
6 \\
6 \\
9\end{array}$ & $\begin{array}{l}0 \\
0 \\
0 \\
1 \\
1\end{array}$ & $\begin{array}{l}0 \\
0 \\
0 \\
0 \\
0\end{array}$ \\
\hline $\begin{array}{l}6-20 \\
6-21\end{array}$ & $\begin{array}{l}7086 \\
2965\end{array}$ & $\begin{array}{l}10 \\
12\end{array}$ & $\frac{1}{2}$ & $\begin{array}{l}5 \\
8\end{array}$ & $\begin{array}{l}3 \\
2\end{array}$ & $\begin{array}{l}1 \\
0\end{array}$ & $\begin{array}{l}0 \\
0\end{array}$ \\
\hline $\begin{array}{l}6-23 \\
6-25\end{array}$ & $\begin{array}{l}1826 \\
2479\end{array}$ & $\begin{array}{r}0 \\
29\end{array}$ & $\begin{array}{r}0 \\
10\end{array}$ & $\begin{array}{r}0 \\
18\end{array}$ & o & $\begin{array}{l}0 \\
0\end{array}$ & $\begin{array}{l}0 \\
0\end{array}$ \\
\hline $6-27$ & 4340 & 13 & 0 & 9 & 4 & 0 & 0 \\
\hline $7-01$ & - & - & -- & -- & - & - & - \\
\hline $\begin{array}{l}7-03 \\
7-05\end{array}$ & $\overline{--}$ & $=$ & $\overline{--}$ & $\overline{--}$ & $=$ & $=$ & $\overline{--}$ \\
\hline $7-07$ & -- & -- & -- & -- & -- & -- & -- \\
\hline
\end{tabular}


TABLE 16.- NUMBER OF BLUE TRACER PARTICLES(1), PER 100 GRAMS OF BEDLOAD SAMPLE, COMPOSITED FROM CROSS-CHANNEL SAMPLING POSITIONS SPACED AT 1-METER INTERVALS ACROSS THE CHANNEL WIDTH, EAST FORK RIVER, WYOMING, 1980

SECTION 0257(3)

\begin{tabular}{|c|c|c|c|c|c|c|c|}
\hline \multirow{3}{*}{\multicolumn{2}{|c|}{$\begin{array}{cc}\text { DATE } & \text { DRY } \\
& \text { MASS } \\
\text { OF } & \text { SAMPLE (2) } \\
& \text { (G) }\end{array}$}} & \multicolumn{6}{|c|}{ NUMBER OF BLUE TRACER PARTICLES } \\
\hline & & \multirow[b]{2}{*}{ TOTAL } & \multicolumn{3}{|c|}{ BY SIZE CLASS } & \multicolumn{2}{|c|}{$(\mathrm{MM})$} \\
\hline & & & $\begin{array}{r}0.25 \\
\text { TO } \\
0.50\end{array}$ & $\begin{array}{l}0.50 \\
\text { To } \\
1.00\end{array}$ & $\begin{array}{l}1.00 \\
2.00\end{array}$ & $\begin{array}{l}2.00 \\
\text { To } \\
4.00\end{array}$ & $\begin{array}{c}4.00 \\
\text { To } \\
8.00\end{array}$ \\
\hline \multirow{4}{*}{$\begin{array}{l}5-16 \\
5-19 \\
5-21 \\
5-22 \\
5-24\end{array}$} & -- & -- & -- & -- & -- & -- & -- \\
\hline & $=$ & $\overline{--}$ & $\overline{-}$ & $=$ & $=$ & $\overline{-}$ & $=$ \\
\hline & -- & -- & -- & - & -- & -- & -- \\
\hline & -- & -- & -- & -- & -- & -- & -- \\
\hline \multirow{4}{*}{$\begin{array}{l}5-26 \\
5-27 \\
5-28 \\
5-29 \\
5-30\end{array}$} & -- & -- & -- & -- & -- & -- & -- \\
\hline & -- & -- & -- & -- & -- & -- & -- \\
\hline & $=$ & $\overline{-}$ & $\overline{--}$ & $=$ & $=$ & $=$ & $\Xi$ \\
\hline & -- & -- & -- & -- & -- & -- & $\therefore$ \\
\hline \multirow{4}{*}{$\begin{array}{l}6-01 \\
6-03 \\
6-05 \\
6-07 \\
6-09\end{array}$} & -- & -- & -- & -- & -- & -- & -- \\
\hline & -- & -- & -- & -- & -- & $=$ & -- \\
\hline & $\overline{-}$ & $=-$ & $=$ & $=$ & $=$ & $=$ & $=$ \\
\hline & 314 & 0 & 0 & 0 & 0 & 0 & 0 \\
\hline \multirow{3}{*}{$\begin{array}{l}6-10 \\
6-11 \\
6-12 \\
6-13 \\
6-14\end{array}$} & 820 & 7 & 4 & 2 & 1 & 0 & 0 \\
\hline & 1057 & 27 & 6 & 20 & 1 & 0 & 0 \\
\hline & $\begin{array}{l}528 \\
634\end{array}$ & $\begin{array}{l}33 \\
34\end{array}$ & $\begin{array}{l}15 \\
14\end{array}$ & $\frac{16}{20}$ & $\overline{2}_{0}$ & $\begin{array}{l}0 \\
0\end{array}$ & $\begin{array}{l}0 \\
0\end{array}$ \\
\hline \multirow{4}{*}{$\begin{array}{l}6-15 \\
6-16 \\
6-17 \\
6-18 \\
6-19\end{array}$} & 588 & 27 & 12 & 15 & 0 & 0 & 0 \\
\hline & $\overline{-}$ & - & $=-$ & $=$ & $=$ & -- & $=$ \\
\hline & $2 \overline{7 \overline{6}}$ & $4 \overline{0}$ & $\overline{12}$ & $\overline{26}$ & 2 & 0 & 0 \\
\hline & 1102 & 41 & 1 & 38 & 1 & 1 & 0 \\
\hline \multirow{4}{*}{$\begin{array}{l}6-20 \\
6-21 \\
6-22 \\
6-23 \\
6-25\end{array}$} & 298 & 26 & 7 & 18 & 1 & 0 & 0 \\
\hline & 281 & 20 & - & 10 & - & -- & -- \\
\hline & $5 \overline{98}$ & $\overline{31}$ & $-\overline{6}$ & $\overline{22}$ & $-\overline{3}$ & $-\overline{0}$ & $-\overline{0}$ \\
\hline & & & & & & & \\
\hline \multirow{4}{*}{$\begin{array}{l}6-27 \\
6-29 \\
7-01 \\
7-03 \\
7-05\end{array}$} & 320 & 25 & -5 & 19 & -1 & 1 & 0 \\
\hline & $=$ & $\overline{-}$ & $=$ & $=$ & $=$ & $=$ & $=$ \\
\hline & -- & -- & -- & - & -- & -- & - \\
\hline & -- & -- & -- & & -- & -- & - \\
\hline $7-07$ & -- & -- & -- & -- & -- & -- & -- \\
\hline
\end{tabular}


TABLE 16.- NUMBER OF BLUE TRACER PARTICLES(1), PER 100 GRAMS OF BEDLOAD SAMPLE, COMPOSITED FROM CROSS-CHANNEL SAMPLING POSITIONS SPACED AT 1-METER INTERVALS ACROSS THE CHANNEL WIDTH,

EAST FORK RIVER, WYOMING, 1980

SECTION 0301

\begin{tabular}{|c|c|c|c|c|c|c|c|}
\hline \multirow{3}{*}{ DATE } & \multirow{3}{*}{$\begin{array}{c}\text { DRY } \\
\text { MASS } \\
\text { OF } \\
\text { SAMPLE (2) } \\
\text { (G) }\end{array}$} & \multicolumn{6}{|c|}{ NUMBER OF BLUE TRACER PARTICLES } \\
\hline & & \multirow[b]{2}{*}{ TOTAL } & \multicolumn{4}{|c|}{ BY SIZE CLASS (MM) } & \multirow[b]{2}{*}{$\begin{array}{r}4.00 \\
\text { TO } \\
8.00\end{array}$} \\
\hline & & & $\begin{array}{l}0.25 \\
\text { TO } \\
0.50\end{array}$ & $\begin{array}{r}0.50 \\
\text { TO } \\
1.00\end{array}$ & $\begin{array}{l}1.00 \\
\text { TO } \\
2.00\end{array}$ & $\begin{array}{l}2.00 \\
\text { TO } \\
4.00\end{array}$ & \\
\hline $\begin{array}{l}5-16 \\
5-19 \\
5-21 \\
5-22 \\
5-24\end{array}$ & $\begin{array}{c}123+ \\
1905 \\
1771 \\
- \\
-\end{array}$ & $\begin{array}{r}15 \\
11 \\
6 \\
--\end{array}$ & $\begin{array}{r}5 \\
6 \\
2 \\
-- \\
--\end{array}$ & $\begin{array}{r}10 \\
5 \\
3 \\
-- \\
--\end{array}$ & $\begin{array}{r}0 \\
0 \\
1 \\
-- \\
--\end{array}$ & $\begin{array}{r}0 \\
0 \\
0 \\
-- \\
--\end{array}$ & $\begin{array}{r}0 \\
0 \\
0 \\
-- \\
--\end{array}$ \\
\hline $\begin{array}{l}5-26 \\
5-27 \\
5-28 \\
5-29 \\
5-30\end{array}$ & $\begin{array}{l}208+ \\
469 \\
701 \\
583 \\
716\end{array}$ & $\begin{array}{r}2 \\
5 \\
14 \\
11 \\
8\end{array}$ & $\begin{array}{l}0 \\
1 \\
3 \\
2 \\
1\end{array}$ & $\begin{array}{l}0 \\
2 \\
4 \\
6 \\
5\end{array}$ & $\begin{array}{l}2 \\
2 \\
6 \\
3 \\
2\end{array}$ & $\begin{array}{l}0 \\
0 \\
1 \\
0 \\
0\end{array}$ & $\begin{array}{l}0 \\
0 \\
0 \\
0 \\
0\end{array}$ \\
\hline $\begin{array}{l}6-01 \\
6-03 \\
6-05 \\
6-07 \\
6-09\end{array}$ & $\begin{array}{r}76+ \\
1319 \\
334 \\
360 \\
2585\end{array}$ & $\begin{array}{l}20 \\
21 \\
16 \\
26 \\
16\end{array}$ & $\begin{array}{l}1 \\
1 \\
5 \\
6 \\
2\end{array}$ & $\begin{array}{l}15 \\
15 \\
10 \\
19 \\
11\end{array}$ & $\begin{array}{l}4 \\
5 \\
1 \\
\frac{1}{3}\end{array}$ & $\begin{array}{l}0 \\
0 \\
0 \\
0 \\
0\end{array}$ & $\begin{array}{l}0 \\
0 \\
0 \\
0 \\
0\end{array}$ \\
\hline $\begin{array}{l}6-10 \\
6-11 \\
6-12 \\
6-13 \\
6-14\end{array}$ & $\begin{array}{r}4703 \\
4006 \\
708 \\
1831 \\
2650\end{array}$ & $\begin{array}{r}9 \\
9 \\
15 \\
13 \\
27\end{array}$ & $\begin{array}{l}0 \\
4 \\
4 \\
5 \\
6\end{array}$ & $\begin{array}{r}4 \\
4 \\
9 \\
7 \\
18\end{array}$ & $\begin{array}{l}5 \\
1 \\
2 \\
1 \\
2\end{array}$ & $\begin{array}{l}0 \\
0 \\
0 \\
0 \\
1\end{array}$ & $\begin{array}{l}0 \\
0 \\
0 \\
0 \\
0\end{array}$ \\
\hline $\begin{array}{l}6-15 \\
6-16 \\
6-17 \\
6-18 \\
6-19\end{array}$ & $\begin{array}{l}1594 \\
1134 \\
2169 \\
3222 \\
1122\end{array}$ & $\begin{array}{l}24 \\
12 \\
12 \\
12 \\
17\end{array}$ & $\begin{array}{l}7 \\
1 \\
2 \\
1 \\
0\end{array}$ & $\begin{array}{r}14 \\
9 \\
5 \\
5 \\
10\end{array}$ & $\begin{array}{l}3 \\
2 \\
4 \\
5 \\
7\end{array}$ & $\begin{array}{l}0 \\
0 \\
1 \\
1 \\
0\end{array}$ & $\begin{array}{l}0 \\
0 \\
0 \\
0 \\
0\end{array}$ \\
\hline $\begin{array}{l}6-20 \\
6-21\end{array}$ & $\begin{array}{l}1371 \\
2293\end{array}$ & $\begin{array}{l}10 \\
15\end{array}$ & $\begin{array}{l}0 \\
0\end{array}$ & $\begin{array}{l}7 \\
9\end{array}$ & $\begin{array}{l}3 \\
6\end{array}$ & $\begin{array}{l}0 \\
0\end{array}$ & $\begin{array}{l}0 \\
0\end{array}$ \\
\hline $\begin{array}{l}6-23 \\
6-25\end{array}$ & $\begin{array}{l}2046 \\
1137\end{array}$ & $\begin{array}{r}23 \\
8\end{array}$ & $\frac{1}{1}$ & $\begin{array}{r}17 \\
2\end{array}$ & $\begin{array}{l}5 \\
0\end{array}$ & $\begin{array}{l}0 \\
5\end{array}$ & $\begin{array}{l}0 \\
0\end{array}$ \\
\hline $6-27$ & 2918 & 23 & 1 & 14 & 7 & 1 & 0 \\
\hline $\begin{array}{l}6-29 \\
7-01\end{array}$ & $\overline{--}$ & $\overline{--}$ & $\overline{--}$ & $\overline{--}$ & $\overline{--}$ & $\overline{--}$ & $=-$ \\
\hline $7-0 \overline{3}$ & -- & -- & -- & -- & -- & -- & -- \\
\hline $7-05$ & -- & - & -- & -- & -- & -- & -- \\
\hline $7-07$ & -- & -- & -- & -- & -- & -- & -- \\
\hline
\end{tabular}


TABLE 16. - NUMBER OF BLUE TRACER PARTICLES (1), PER 100 GRAMS OF BEDLOAD SAMPLE, COMPOSITED FROM CROSS-CHANNEL SAMPLING POSITIONS

SPACED AT I-METER INTERVALS ACROSS THE CHANNEL WIDTH,

EAST FORK RIVER, WYOMING, 1980

SECTION $0301(3)$

\begin{tabular}{|c|c|c|c|c|c|c|c|}
\hline \multirow{3}{*}{ DATE } & \multirow{3}{*}{$\begin{array}{c}\text { DRY } \\
\text { MASS } \\
\text { OF } \\
\text { SAMPLE (2) } \\
\text { (G) }\end{array}$} & & MBER & BLUE ? & CER PI & ICLES & \\
\hline & & \multirow[b]{2}{*}{ TOTAL } & \multicolumn{5}{|c|}{ BY SIZE CLASS (MM) } \\
\hline & & & $\begin{array}{l}0.25 \\
\text { TO } \\
0.50\end{array}$ & $\begin{array}{l}0.50 \\
\text { TO } \\
1.00\end{array}$ & $\begin{array}{l}1.00 \\
\text { TO } \\
2.00\end{array}$ & $\begin{array}{l}2.00 \\
4.00\end{array}$ & $\begin{array}{l}4.00 \\
\text { TO } \\
8.00\end{array}$ \\
\hline $5-16$ & -- & -- & -- & -- & -- & - & - \\
\hline $\begin{array}{l}5-19 \\
5-27\end{array}$ & $=-$ & -- & - & - & -- & -- & - \\
\hline $5-22$ & -- & $\ldots$ & - & - & - & -. & $-\infty$ \\
\hline $5-24$ & -- & -- & -- & -- & - & -- & -- \\
\hline $5-26$ & -- & -- & - & - & -- & -- & - \\
\hline $5-27$ & -- & -- & -- & -- & -- & -- & -- \\
\hline $5-29$ & -- & -- & -- & -- & - & - & -- \\
\hline $5-30$ & -. & -- & - & -- & - & - & -- \\
\hline $6-01$ & - & -- & - & - & -- & -- & -- \\
\hline $6-03$ & -- & -- & -- & -- & -- & -- & -- \\
\hline $\begin{array}{l}6-05 \\
6-07\end{array}$ & $\overline{-}$ & $\overline{--}$ & $=$ & $\overline{--}$ & $\overline{-}$ & $=$ & - \\
\hline $6-09$ & 410 & 14 & 5 & 8 & 1 & 0 & 0 \\
\hline $6-10$ & .749 & 22 & 9 & 13 & 0 & 0 & 0 \\
\hline $6-12$ & $\begin{array}{l}1850 \\
1121\end{array}$ & 17 & $\begin{array}{l}3 \\
1\end{array}$ & $\begin{array}{r}6 \\
14\end{array}$ & $\frac{1}{2}$ & $\begin{array}{l}0 \\
0\end{array}$ & $\begin{array}{l}0 \\
0\end{array}$ \\
\hline $\begin{array}{l}6-13 \\
6-14\end{array}$ & $\begin{array}{r}2235 \\
846\end{array}$ & $\begin{array}{l}19 \\
34\end{array}$ & 6 & 10 & 3 & 0 & 0 \\
\hline & & & & & & & \\
\hline $6-15$ & 455 & 48 & 17 & 30 & 1 & 0 & 0 \\
\hline $\begin{array}{l}6-16 \\
6-17\end{array}$ & $\overline{--}$ & $\overline{--}$ & $=$ & $=$ & $=$ & $\overline{--}$ & $=$ \\
\hline $6-18$ & 837 & 47 & 7 & 37 & 3 & 0 & 0 \\
\hline $6-19$ & 827 & 34 & 2 & 27 & 5 & 0 & 0 \\
\hline $6-20$ & $6 \frac{1}{5}+++$ & 38 & $\begin{array}{l}5 \\
3\end{array}$ & 25 & 6 & 2 & 0 \\
\hline $6-22$ & & $=-$ & -2 & & & & \\
\hline $6-23$ & 290 & 39 & 5 & 31 & 3 & 0 & 0 \\
\hline $6-25$ & 374 & 32 & 11 & 21 & 0 & 0 & 0 \\
\hline & 149 & 30 & 4 & 23 & 3 & 0 & 0 \\
\hline $\begin{array}{l}6-29 \\
7-07\end{array}$ & $\overline{-}$ & $=$ & $=$ & $=$ & $=$ & $=$ & $=$ \\
\hline $7-0 \frac{1}{3}$ & - & - & - & - & - & - & - \\
\hline $7-05$ & -- & -- & - & & -- & - & -- \\
\hline $7-07$ & -- & -- & -- & -- & -- & - & - \\
\hline
\end{tabular}


TABLE 16.- NUMBER OF BLUE TRACER PARTICLES(1), PER 100 GRAMS OF BEDLOAD SAMPLE, COMPOSITED FROM CROSS-CHANNEL SAMPLING POSITIONS SPACED AT 1-METER INTERVALS ACROSS THE CHANNEL WIDTH, EAST FORK RIVER, WYOMING, 1980

SECTION 0348

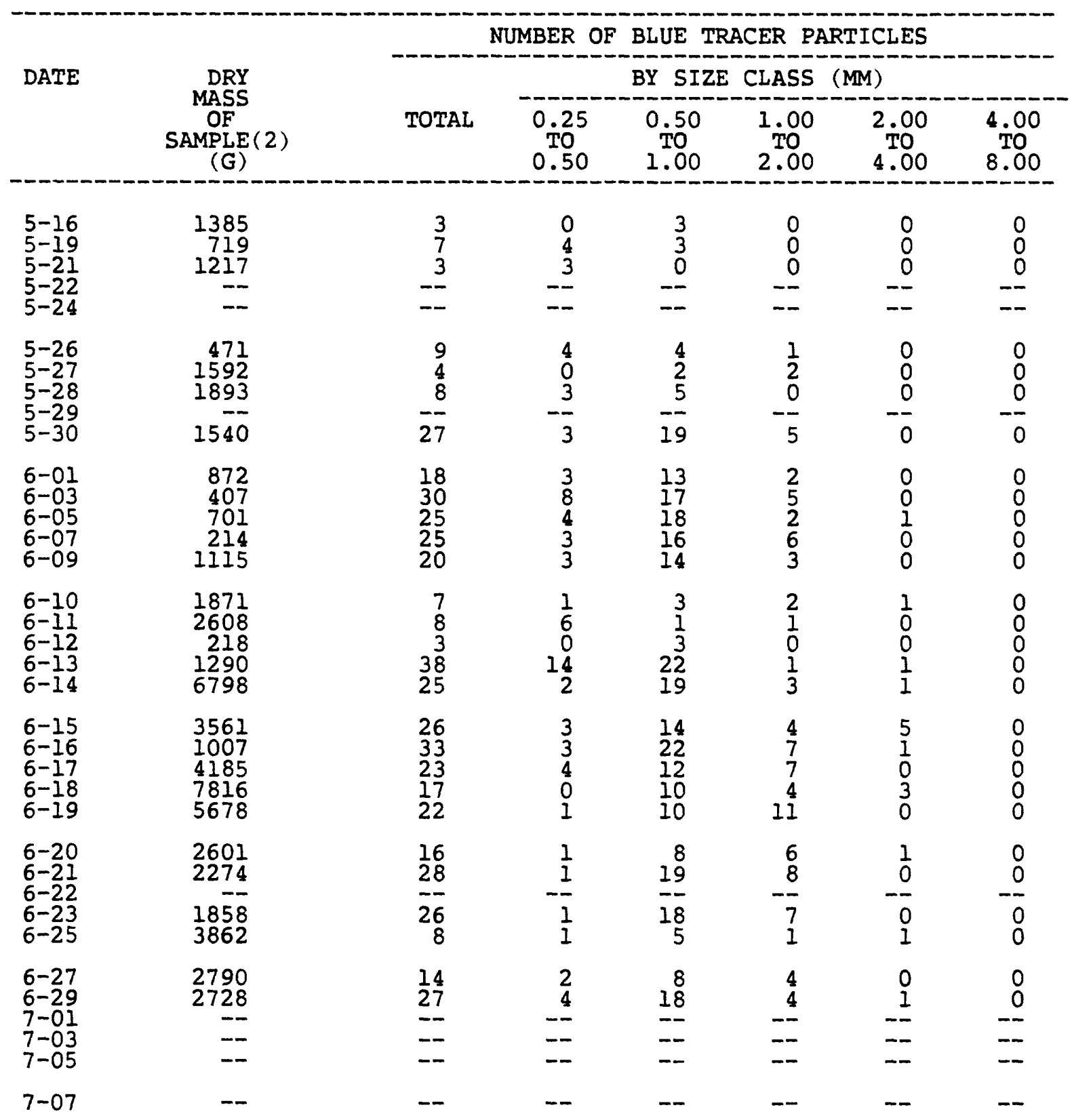


TABLE 16.- NUMBER OF BLUE TRACER PARTICLES(1), PER 100 GRAMS OF BEDLOAD SAMPLE, COMPOSITED FROM CROSS-CHANNEL SAMPLING POSITIONS SPACED AT I-METER INTERVALS ACROSS THE CHANNEL WIDTH, EAST FORK RIVER, WYOMING, 1980

\section{SECTION 0421}

\begin{tabular}{|c|c|c|c|c|c|c|c|}
\hline \multirow{3}{*}{ DATE } & \multirow{3}{*}{$\begin{array}{c}\text { DRY } \\
\text { MASS } \\
\text { OF } \\
\text { SAMPLE (2) } \\
\text { (G) }\end{array}$} & \multicolumn{6}{|c|}{ NUMBER OF BLUE TRACER PARTICLES } \\
\hline & & \multirow[b]{2}{*}{ TOTAL } & \multicolumn{5}{|c|}{ BY SIZE CLASS (MM) } \\
\hline & & & $\begin{array}{l}0.25 \\
\text { TO } \\
0.50\end{array}$ & $\begin{array}{l}0.50 \\
\text { TO. } \\
1.00\end{array}$ & $\begin{array}{l}1.00 \\
\text { TO } \\
2.00\end{array}$ & $\begin{array}{l}2.00 \\
\text { TO } \\
4.00\end{array}$ & $\begin{array}{l}4.00 \\
\text { TO } \\
8.00\end{array}$ \\
\hline $\begin{array}{l}5-16 \\
5-19 \\
5-21 \\
5-22\end{array}$ & $\begin{array}{l}242 \\
777 \\
612 \\
-\end{array}$ & $\begin{array}{l}11 \\
18 \\
19 \\
-1\end{array}$ & $\begin{array}{r}6 \\
6 \\
8 \\
--\end{array}$ & $\begin{array}{r}5 \\
10 \\
11 \\
-\end{array}$ & $\begin{array}{r}0 \\
2 \\
0 \\
--\end{array}$ & $\begin{array}{r}0 \\
0 \\
0 \\
-\end{array}$ & $\begin{array}{r}0 \\
0 \\
0 \\
-\end{array}$ \\
\hline $5-24$ & -- & -- & -- & -- & - & -- & -- \\
\hline $\begin{array}{l}5-26 \\
5-27 \\
5-28\end{array}$ & $\begin{array}{r}3173 \\
896 \\
561\end{array}$ & $\begin{array}{r}14 \\
7 \\
5\end{array}$ & $\begin{array}{l}0 \\
2 \\
0\end{array}$ & $\begin{array}{l}6 \\
3 \\
1\end{array}$ & $\begin{array}{l}8 \\
2 \\
3\end{array}$ & $\begin{array}{l}0 \\
0 \\
1\end{array}$ & $\begin{array}{l}0 \\
0 \\
0\end{array}$ \\
\hline $5-30$ & 277 & 17 & 5 & 12 & 0 & 0 & 0 \\
\hline $\begin{array}{l}6-01 \\
6-03 \\
6-05 \\
6-07 \\
6-09\end{array}$ & $\begin{array}{l}563 \\
384 \\
88+ \\
376 \\
958\end{array}$ & $\begin{array}{r}29 \\
14 \\
2 \\
29 \\
11\end{array}$ & $\begin{array}{l}6 \\
3 \\
0 \\
7 \\
2\end{array}$ & $\begin{array}{r}22 \\
10 \\
2 \\
18 \\
7\end{array}$ & $\begin{array}{l}1 \\
1 \\
0 \\
4 \\
2\end{array}$ & $\begin{array}{l}0 \\
0 \\
0 \\
0 \\
0\end{array}$ & $\begin{array}{l}0 \\
0 \\
0 \\
0 \\
0\end{array}$ \\
\hline $\begin{array}{l}6-10 \\
6-11 \\
6-12 \\
6-13 \\
6-14\end{array}$ & $\begin{array}{l}4947 \\
4214 \\
2586 \\
5444 \\
4647\end{array}$ & $\begin{array}{l}25 \\
25 \\
21 \\
25 \\
32\end{array}$ & $\begin{array}{r}12 \\
1 \\
3 \\
3 \\
2\end{array}$ & $\begin{array}{l}11 \\
22 \\
16 \\
13 \\
22\end{array}$ & $\begin{array}{l}1 \\
2 \\
2 \\
8 \\
7\end{array}$ & $\begin{array}{l}1 \\
0 \\
0 \\
1 \\
0\end{array}$ & $\begin{array}{l}0 \\
0 \\
0 \\
0 \\
1\end{array}$ \\
\hline $\begin{array}{l}6-15 \\
6-16 \\
6-17 \\
6-18 \\
6-19\end{array}$ & $\begin{array}{l}4003 \\
3030 \\
3022 \\
1824 \\
1450\end{array}$ & $\begin{array}{l}13 \\
18 \\
19 \\
30 \\
20\end{array}$ & $\begin{array}{l}1 \\
1 \\
3 \\
3 \\
4\end{array}$ & $\begin{array}{r}5 \\
9 \\
11 \\
22 \\
8\end{array}$ & $\begin{array}{l}7 \\
5 \\
2 \\
5 \\
4\end{array}$ & $\begin{array}{l}0 \\
3 \\
3 \\
0 \\
3\end{array}$ & $\begin{array}{l}0 \\
0 \\
0 \\
0 \\
1\end{array}$ \\
\hline $\begin{array}{l}6-20 \\
6-21\end{array}$ & $\begin{array}{l}2879 \\
9086\end{array}$ & $\begin{array}{l}23 \\
21\end{array}$ & $\begin{array}{r}10 \\
3\end{array}$ & 11 & $\frac{1}{7}$ & $\frac{1}{2}$ & $\begin{array}{l}0 \\
0\end{array}$ \\
\hline $\begin{array}{l}6-23 \\
6-25\end{array}$ & $\begin{array}{l}1446 \\
3306\end{array}$ & $\begin{array}{l}15 \\
16\end{array}$ & $\begin{array}{l}2 \\
1\end{array}$ & $\begin{array}{r}8 \\
12\end{array}$ & $\begin{array}{l}5 \\
3\end{array}$ & $\begin{array}{l}0 \\
0\end{array}$ & $\begin{array}{l}0 \\
0\end{array}$ \\
\hline $6-27$ & 1800 & 25 & 3 & 18 & 3 & 1 & 0 \\
\hline $7-01$ & - & - & -- & -- & - & - & -- \\
\hline $7-03$ & - & -- & -- & -- & -- & -- & -- \\
\hline $7-05$ & -- & -- & -- & -- & -- & -- & -- \\
\hline $7-07$ & - & - & -- & -- & -- & -- & - \\
\hline
\end{tabular}


TABLE 26.- NUMBER OF BLUE TRACER PARTICLES(1), PER 100 GRAMS OF BEDLOAD SAMPLE, COMPOSITED FROM CROSS-CHANNEL SAMPLING POSITIONS SPACED AT I-METER INTERVALS ACROSS THE CHANNEL WIDTH,

EAST FORK RIVER, WYOMING, 1980

\section{SECTION 0460}

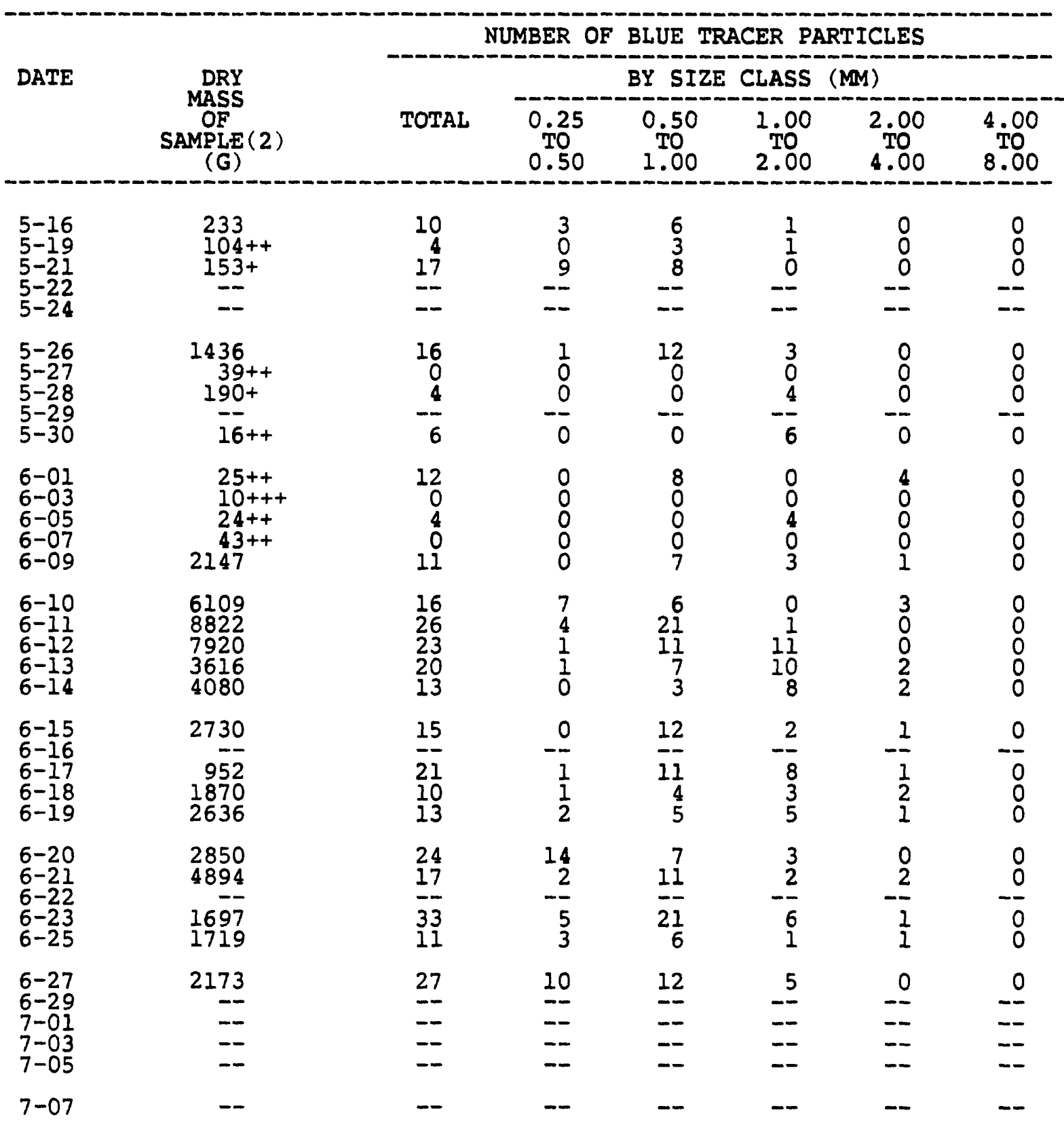


TABLE 16.- NUMBER OF BLUE TRACER PARTICLES(1), PER 100 GRAMS OF BEDLOAD SAMPLE, COMPOSITED FROM CROSS-CHANNEL SAMPLING POSITIONS

SPACED AT 1-METER INTERVALS ACROSS THE CHANNEL WIDTH,

EAST FORK RIVER, WYOMING, 1980

$$
\text { SECTION } 0516
$$

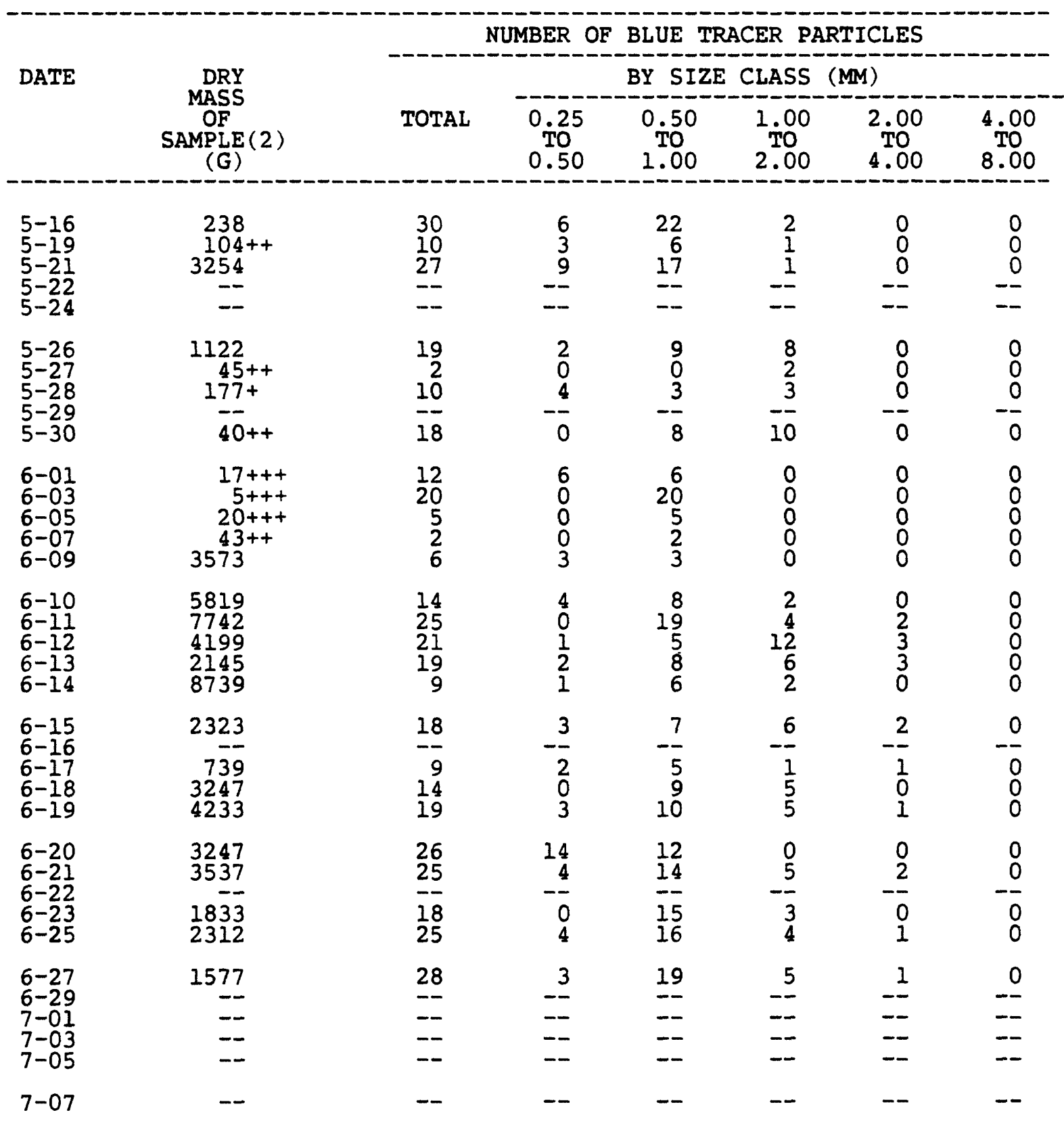


TABLE 16.- NUMBER OF BLUE TRACER PARTICLES(1), PER 100 GRAMS OF BEDLOAD SAMPLE, COMPOSITED FROM CROSS-CHANNEL SAMPLING POSITIONS SPACED AT 1-METER INTERVALS ACROSS THE CHANNEL WIDTH, EAST FORK RIVER, WYOMING, 1980

SECTION 0556

\begin{tabular}{|c|c|c|c|c|c|c|c|}
\hline \multirow{3}{*}{ DATE } & \multirow{3}{*}{$\begin{array}{c}\text { DRY } \\
\text { MASS } \\
\text { OF } \\
\text { SAMPLE (2) } \\
\text { (G) }\end{array}$} & \multicolumn{6}{|c|}{ NUMBER OF BLUE TRACER PARTICLES } \\
\hline & & \multirow[b]{2}{*}{ TOTAL } & \multicolumn{5}{|c|}{ BY SIZE CLASS (MM) } \\
\hline & & & $\begin{array}{l}0.25 \\
\text { TिO } \\
0.50\end{array}$ & $\begin{array}{r}0.50 \\
\text { TO } \\
1.00\end{array}$ & $\begin{array}{l}1.00 \\
2.00\end{array}$ & $\begin{array}{l}2.00 \\
\text { TO } \\
4.00\end{array}$ & $\begin{array}{l}4.00 \\
\text { TO } \\
8.00\end{array}$ \\
\hline $\begin{array}{l}5-16 \\
5-19 \\
5-21 \\
5-22 \\
5-24\end{array}$ & $\begin{array}{l}12+++ \\
272 \\
1800 \\
-- \\
--\end{array}$ & $\begin{array}{l}8 \\
39 \\
26 \\
--\end{array}$ & $\begin{array}{r}0 \\
9 \\
5 \\
-- \\
--\end{array}$ & $\begin{array}{l}8 \\
24 \\
19 \\
--\end{array}$ & $\begin{array}{r}0 \\
6 \\
1 \\
-- \\
--\end{array}$ & $\begin{array}{r}0 \\
0 \\
1 \\
-- \\
--\end{array}$ & $\begin{array}{r}0 \\
0 \\
0 \\
-- \\
--\end{array}$ \\
\hline $\begin{array}{l}5-26 \\
5-27 \\
5-28\end{array}$ & $\begin{array}{c}840 \\
58+ \\
207\end{array}$ & $\begin{array}{r}8 \\
12 \\
12\end{array}$ & $\begin{array}{l}2 \\
2 \\
6\end{array}$ & $\begin{array}{l}4 \\
8 \\
5\end{array}$ & $\begin{array}{l}2 \\
2 \\
1\end{array}$ & $\begin{array}{l}0 \\
0 \\
0\end{array}$ & $\begin{array}{l}0 \\
0 \\
0\end{array}$ \\
\hline $5-30$ & $62++$ & 13 & 3 & 8 & 2 & 0 & 0 \\
\hline $\begin{array}{l}6-01 \\
6-03 \\
6-05 \\
6-07 \\
6-09\end{array}$ & $\begin{array}{c}77++ \\
53++ \\
93+ \\
103+ \\
5100\end{array}$ & $\begin{array}{r}6 \\
11 \\
8 \\
24 \\
36\end{array}$ & $\begin{array}{l}0 \\
2 \\
3 \\
7 \\
7\end{array}$ & $\begin{array}{r}6 \\
7 \\
3 \\
16 \\
22\end{array}$ & $\begin{array}{l}0 \\
2 \\
2 \\
1 \\
5\end{array}$ & $\begin{array}{l}0 \\
0 \\
0 \\
0 \\
2\end{array}$ & $\begin{array}{l}0 \\
0 \\
0 \\
0 \\
0\end{array}$ \\
\hline $\begin{array}{l}6-10 \\
6-11 \\
6-12 \\
6-13 \\
6-14\end{array}$ & $\begin{array}{l}5747 \\
2974 \\
9117 \\
3194 \\
3427\end{array}$ & $\begin{array}{r}31 \\
24 \\
19 \\
8 \\
8\end{array}$ & $\begin{array}{l}3 \\
3 \\
0 \\
3 \\
0\end{array}$ & $\begin{array}{r}21 \\
11 \\
12 \\
2 \\
5\end{array}$ & $\begin{array}{l}7 \\
8 \\
7 \\
3 \\
2\end{array}$ & $\begin{array}{l}0 \\
2 \\
0 \\
0 \\
1\end{array}$ & $\begin{array}{l}0 \\
0 \\
0 \\
0 \\
0\end{array}$ \\
\hline $6-15$ & 1217 & 18 & 3 & 13 & 2 & 0 & 0 \\
\hline $\begin{array}{l}6-17 \\
6-18 \\
6-19\end{array}$ & $\begin{array}{l}1354 \\
3938 \\
2389\end{array}$ & $\begin{array}{l}34 \\
19 \\
22\end{array}$ & $\begin{array}{r}11 \\
2 \\
2\end{array}$ & $\begin{array}{l}22 \\
14 \\
17\end{array}$ & $\begin{array}{l}\frac{1}{2} \\
2\end{array}$ & $\begin{array}{l}0 \\
1 \\
1\end{array}$ & $\begin{array}{l}0 \\
0 \\
0\end{array}$ \\
\hline $\begin{array}{l}6-20 \\
6-21\end{array}$ & $\begin{array}{l}5650 \\
2083\end{array}$ & $\begin{array}{l}27 \\
19\end{array}$ & $\begin{array}{l}7 \\
0\end{array}$ & $\begin{array}{l}17 \\
11\end{array}$ & $\frac{1}{7}$ & $\begin{array}{l}2 \\
1\end{array}$ & $\begin{array}{l}0 \\
0\end{array}$ \\
\hline $\begin{array}{l}6-22 \\
6-23 \\
6-25\end{array}$ & $\begin{array}{r}3676 \\
872\end{array}$ & $\begin{array}{l}10 \\
18\end{array}$ & $\begin{array}{l}4 \\
9\end{array}$ & $\begin{array}{l}4 \\
7\end{array}$ & $\begin{array}{l}2 \\
1\end{array}$ & $\begin{array}{l}0 \\
1\end{array}$ & $\begin{array}{l}0 \\
0\end{array}$ \\
\hline $6-27$ & 2150 & 33 & 9 & 18 & 5 & 1 & 0 \\
\hline $7-01$ & $=$ & - & $=$ & $=$ & $=$ & $=$ & $=$ \\
\hline $7-0 \overline{3}$ & - & -- & -- & -- & -- & -- & -- \\
\hline $7-05$ & -- & -- & -- & -- & - & -- & -- \\
\hline $7-07$ & -- & -- & - & -- & -- & -- & -- \\
\hline
\end{tabular}


TABLE 16.- NUMBER OF BLUE TRACER PARTICLES(1), PER 100 GRAMS OF BEDLOAD SAMPLE, COMPOSITED FROM CROSS-CHANNEL SAMPLING POSITIONS SPACED AT I-METER INTERVALS ACROSS THE CHANNEL WIDTH, EAST FORK RIVER, WYOMING, 1980

$$
\text { SECTION } 0602
$$

\begin{tabular}{|c|c|c|c|c|c|c|c|}
\hline \multirow{3}{*}{ DATE } & \multirow{3}{*}{$\begin{array}{c}\text { DRY } \\
\text { MASS } \\
\text { OF } \\
\text { SAMPLE (2) } \\
\text { (G) }\end{array}$} & \multicolumn{6}{|c|}{ NUMBER OF BLUE TRACER PARTICLES } \\
\hline & & \multirow[b]{2}{*}{ TOTAL } & \multicolumn{5}{|c|}{ BY SIZE CLASS (MM) } \\
\hline & & & $\begin{array}{l}0.25 \\
\text { TO } \\
0.50\end{array}$ & $\begin{array}{l}0.50 \\
\text { TOO } \\
1.00\end{array}$ & $\begin{array}{l}1.00 \\
2.00 \\
2.00\end{array}$ & $\begin{array}{l}2.00 \\
\text { TO } \\
4.00\end{array}$ & $\begin{array}{c}4.00 \\
\text { TO } \\
8.00\end{array}$ \\
\hline $\begin{array}{l}5-16 \\
5-19 \\
5-21 \\
5-22 \\
5-24\end{array}$ & $\begin{array}{r}136 \\
1010 \\
1054 \\
- \\
-\end{array}$ & $\begin{array}{l}46 \\
41 \\
23 \\
-- \\
--\end{array}$ & $\begin{array}{r}15 \\
7 \\
4 \\
--\end{array}$ & $\begin{array}{l}26 \\
19 \\
18 \\
--\end{array}$ & $\begin{array}{r}5 \\
15 \\
1 \\
--\end{array}$ & $\begin{array}{r}0 \\
0 \\
0 \\
--\end{array}$ & $\begin{array}{r}0 \\
0 \\
0 \\
--\end{array}$ \\
\hline $\begin{array}{l}5-26 \\
5-27 \\
5-28 \\
5-29 \\
5-30\end{array}$ & $\begin{array}{l}1299 \\
167+ \\
112 \\
\frac{-1}{474}\end{array}$ & $\begin{array}{l}16 \\
32 \\
24 \\
\frac{3}{36}\end{array}$ & $\begin{array}{r}1 \\
14 \\
-\frac{1}{23}\end{array}$ & $\begin{array}{l}10 \\
17 \\
20 \\
-12\end{array}$ & $\begin{array}{r}5 \\
1 \\
0 \\
-1\end{array}$ & $\begin{array}{r}0 \\
0 \\
0 \\
-0\end{array}$ & $\begin{array}{r}0 \\
0 \\
0 \\
-0\end{array}$ \\
\hline $\begin{array}{l}6-01 \\
6-03 \\
6-05 \\
6-07 \\
6-09\end{array}$ & $\begin{array}{r}56+ \\
162+ \\
921 \\
1320 \\
5773\end{array}$ & $\begin{array}{r}118 \\
27 \\
50 \\
46 \\
32\end{array}$ & $\begin{array}{r}39 \\
10 \\
26 \\
15 \\
4\end{array}$ & $\begin{array}{l}72 \\
15 \\
22 \\
28 \\
20\end{array}$ & $\begin{array}{l}1 \\
2 \\
2 \\
2 \\
8\end{array}$ & $\begin{array}{l}6 \\
0 \\
0 \\
1 \\
0\end{array}$ & $\begin{array}{l}0 \\
0 \\
0 \\
0 \\
0\end{array}$ \\
\hline $\begin{array}{l}6-10 \\
6-11 \\
6-12 \\
6-13 \\
6-14\end{array}$ & $\begin{array}{l}4233 \\
5076 \\
2864 \\
3765 \\
2492\end{array}$ & $\begin{array}{l}39 \\
14 \\
13 \\
19 \\
20\end{array}$ & $\begin{array}{l}8 \\
1 \\
2 \\
5 \\
6\end{array}$ & $\begin{array}{r}25 \\
4 \\
7 \\
8 \\
12\end{array}$ & $\begin{array}{l}5 \\
7 \\
4 \\
5 \\
1\end{array}$ & $\begin{array}{l}1 \\
2 \\
0 \\
1 \\
1\end{array}$ & $\begin{array}{l}0 \\
0 \\
0 \\
0 \\
0\end{array}$ \\
\hline $6-15$ & 1838 & 20 & 5 & 10 & 5 & 0 & 0 \\
\hline $\begin{array}{l}0-10 \\
6-17 \\
6-18 \\
6-19\end{array}$ & $\begin{array}{l}2006 \\
1637 \\
5043\end{array}$ & $\begin{array}{l}30 \\
32 \\
22\end{array}$ & $\begin{array}{l}2 \\
7 \\
4\end{array}$ & $\begin{array}{l}23 \\
24 \\
14\end{array}$ & $\begin{array}{l}4 \\
1 \\
4\end{array}$ & $\begin{array}{l}1 \\
0 \\
0\end{array}$ & $\begin{array}{l}0 \\
0 \\
0\end{array}$ \\
\hline $\begin{array}{l}6-20 \\
6-21\end{array}$ & $\begin{array}{l}7174 \\
2827\end{array}$ & $\begin{array}{r}22 \\
9\end{array}$ & $\begin{array}{l}1 \\
0\end{array}$ & $\begin{array}{r}10 \\
2\end{array}$ & $\begin{array}{l}8 \\
3\end{array}$ & $\begin{array}{l}3 \\
0\end{array}$ & $\begin{array}{l}0 \\
4\end{array}$ \\
\hline $\begin{array}{l}6-23 \\
6-25\end{array}$ & $\begin{array}{l}3194 \\
4332\end{array}$ & $\begin{array}{l}10 \\
19\end{array}$ & $\begin{array}{l}2 \\
3\end{array}$ & $\begin{array}{r}6 \\
10\end{array}$ & $\frac{1}{6}$ & $\begin{array}{l}1 \\
0\end{array}$ & $\begin{array}{l}0 \\
0\end{array}$ \\
\hline $6-27$ & 1490 & 13 & 0 & 10 & 1 & 2 & 0 \\
\hline $\begin{array}{l}6-29 \\
7-01\end{array}$ & $\overline{-}$ & $\overline{--}$ & $\overline{-}$ & $\overline{-}$ & $\overline{--}$ & $\overline{-}$ & $\overline{-}$ \\
\hline $7-03$ & -- & -- & -- & -- & -- & -- & -- \\
\hline $7-05$ & -- & -- & - & -- & -- & -- & -- \\
\hline $7-07$ & -- & -- & - & -- & -- & - & -- \\
\hline
\end{tabular}


TABLE 16.- NUMBER OF BLUE TRACER PARTICLES(1), PER 100 GRAMS OF BEDLOAD SAMPLE, COMPOSITED FROM CROSS-CHANNEL SAMPLING POSITIONS SPACED AT 1-METER INTERVALS ACROSS THE CHANNEL WIDTH, EAST FORK RIVER, WYOMING, 1980

SECTION 0653

\begin{tabular}{|c|c|c|c|c|c|c|c|}
\hline \multirow{3}{*}{ DATE } & \multirow{3}{*}{$\begin{array}{c}\text { DRY } \\
\text { MASS } \\
\text { OF } \\
\text { SAMPLE (2) } \\
\text { (G) }\end{array}$} & & ABER & BLUE ? & CER PI & ICLES & \\
\hline & & & \multicolumn{5}{|c|}{ BY SIZE CLASS (MM) } \\
\hline & & TOTAL & $\begin{array}{c}0.25 \\
\text { TO } \\
0.50\end{array}$ & $\begin{array}{r}0.50 \\
\text { TO } \\
1.00\end{array}$ & $\begin{array}{l}1.00 \\
\text { TOO } \\
2.00\end{array}$ & $\begin{array}{l}2.00 \\
\text { TO } \\
4.00\end{array}$ & $\begin{array}{l}4.00 \\
\text { TO } \\
8.00\end{array}$ \\
\hline $\begin{array}{l}5-16 \\
5-19 \\
5-21 \\
5-22 \\
5-24\end{array}$ & $\begin{array}{l}148+ \\
168+ \\
153+ \\
--\end{array}$ & $\begin{array}{l}26 \\
53 \\
23 \\
-- \\
--\end{array}$ & $\begin{array}{r}\frac{1}{3} \\
11 \\
--\end{array}$ & $\begin{array}{l}22 \\
37 \\
10 \\
-- \\
--\end{array}$ & $\begin{array}{r}3 \\
13 \\
2 \\
--\end{array}$ & $\begin{array}{r}0 \\
0 \\
0 \\
-- \\
--\end{array}$ & $\begin{array}{r}0 \\
0 \\
0 \\
-- \\
--\end{array}$ \\
\hline $\begin{array}{l}5-26 \\
5-27 \\
5-28 \\
5-29\end{array}$ & $\begin{array}{l}393 \\
491 \\
108+\end{array}$ & $\begin{array}{l}34 \\
52 \\
42\end{array}$ & $\begin{array}{r}11 \\
9 \\
12\end{array}$ & $\begin{array}{l}22 \\
39 \\
26 \\
--\end{array}$ & $\begin{array}{l}0 \\
4 \\
4\end{array}$ & $\begin{array}{l}1 \\
0 \\
0\end{array}$ & $\begin{array}{l}0 \\
0 \\
0\end{array}$ \\
\hline $5-30$ & $83++$ & 31 & 4 & 24 & 3 & 0 & 0 \\
\hline $\begin{array}{l}6-01 \\
6-03 \\
6-05 \\
6-07 \\
6-09\end{array}$ & $\begin{array}{r}372 \\
439 \\
490 \\
2330 \\
3768\end{array}$ & $\begin{array}{l}50 \\
46 \\
46 \\
45 \\
29\end{array}$ & $\begin{array}{r}11 \\
8 \\
10 \\
6 \\
4\end{array}$ & $\begin{array}{l}34 \\
32 \\
30 \\
30 \\
20\end{array}$ & $\begin{array}{l}4 \\
6 \\
6 \\
7 \\
4\end{array}$ & $\begin{array}{l}1 \\
0 \\
0 \\
2 \\
1\end{array}$ & $\begin{array}{l}0 \\
0 \\
0 \\
0 \\
0\end{array}$ \\
\hline $\begin{array}{l}6-10 \\
6-11 \\
6-12 \\
6-13 \\
6-14\end{array}$ & $\begin{array}{r}15664 \\
3692 \\
348 \\
427 \\
257\end{array}$ & $\begin{array}{l}38 \\
13 \\
15 \\
11 \\
36\end{array}$ & $\begin{array}{r}2 \\
1 \\
4 \\
6 \\
20\end{array}$ & $\begin{array}{r}23 \\
8 \\
9 \\
3 \\
15\end{array}$ & $\begin{array}{r}10 \\
3 \\
1 \\
2 \\
1\end{array}$ & $\begin{array}{l}3 \\
1 \\
1 \\
0 \\
0\end{array}$ & $\begin{array}{l}0 \\
0 \\
0 \\
0 \\
0\end{array}$ \\
\hline $6-15$ & 622 & 23 & 3 & 16 & 4 & 0 & 0 \\
\hline $\begin{array}{l}6-17 \\
6-18 \\
6-19\end{array}$ & $\begin{array}{l}2105 \\
3735 \\
4034\end{array}$ & $\begin{array}{l}12 \\
33 \\
21\end{array}$ & $\begin{array}{l}3 \\
2 \\
3\end{array}$ & $\begin{array}{r}7 \\
25 \\
11\end{array}$ & $\begin{array}{l}2 \\
4 \\
7\end{array}$ & $\begin{array}{l}0 \\
2 \\
0\end{array}$ & $\begin{array}{l}0 \\
0 \\
0\end{array}$ \\
\hline $\begin{array}{l}6-20 \\
6-21\end{array}$ & $\begin{array}{l}1922 \\
2686\end{array}$ & $\begin{array}{l}11 \\
14\end{array}$ & $\begin{array}{l}0 \\
0\end{array}$ & $\begin{array}{l}1 \\
5\end{array}$ & $\frac{4}{3}$ & $\begin{array}{l}6 \\
6\end{array}$ & $\begin{array}{l}0 \\
0\end{array}$ \\
\hline $\begin{array}{l}6-23 \\
6-25\end{array}$ & $\begin{array}{l}1278 \\
1894\end{array}$ & 11 & $\begin{array}{l}0 \\
0\end{array}$ & $\begin{array}{l}7 \\
0\end{array}$ & $\begin{array}{l}3 \\
1\end{array}$ & $\begin{array}{l}1 \\
0\end{array}$ & $\begin{array}{l}0 \\
0\end{array}$ \\
\hline $6-27$ & 2210 & 30 & 1 & 23 & 6 & 0 & 0 \\
\hline $\begin{array}{l}6-29 \\
7-01\end{array}$ & $\overline{--}$ & $\overline{--}$ & $\overline{--}$ & $\overline{--}$ & $=$ & $\overline{--}$ & $\overline{--}$ \\
\hline $7-03$ & -- & -- & -- & -- & -- & - & -- \\
\hline $7-05$ & -- & -- & -- & & -- & -- & -- \\
\hline $7-07$ & -- & -- & -- & -- & -- & -- & -- \\
\hline
\end{tabular}


TABLE 16.- NUMBER OF BLUE TRACER PARTICLES(1), PER 100 GRAMS OF BEDLOAD SAMPLE, COMPOSITED FROM CROSS-CHANNEL SAMPLING POSITIONS SPACED AT 1-METER INTERVALS ACROSS THE CHANNEL WIDTH, EAST FORK RIVER, WYOMING, 1980

SECTION 0653(3)

\begin{tabular}{|c|c|c|c|c|c|c|c|}
\hline \multirow{3}{*}{ DATE } & \multirow{3}{*}{$\begin{array}{c}\text { DRY } \\
\text { MASS } \\
\text { OF } \\
\text { SAMPLE (2) } \\
\text { (G) }\end{array}$} & \multicolumn{6}{|c|}{ NUMBER OF BLUE TRACER PARTICLES } \\
\hline & & \multirow[b]{2}{*}{ TOTAL } & \multicolumn{5}{|c|}{ BY SIZE CLASS (MM) } \\
\hline & & & $\begin{array}{l}0.25 \\
\text { TO } \\
0.50\end{array}$ & $\begin{array}{l}0.50 \\
\text { TO } \\
1.00\end{array}$ & $\begin{array}{l}1.00 \\
2.00 \\
2.00\end{array}$ & $\begin{array}{l}2.00 \\
\text { TO } \\
4.00\end{array}$ & $\begin{array}{l}4.00 \\
\text { TO } \\
8.00\end{array}$ \\
\hline $5-16$ & $\overline{--}$ & -- & - & - & $\overline{--}$ & -- & -- \\
\hline $\begin{array}{l}5-19 \\
5-21\end{array}$ & - & - & - & - & - & - & - \\
\hline $5-22$ & -- & -- & -- & -- & -- & -- & -- \\
\hline & -- & & - & -- & -- & -- & -- \\
\hline $5-26$ & -- & -- & -- & - & -- & -- & -- \\
\hline $\begin{array}{l}5-27 \\
5-28\end{array}$ & $\overline{--}$ & $\overline{--}$ & -- & $=$ & $\overline{-}$ & $\overline{--}$ & $\overline{-}$ \\
\hline $5-29$ & -- & -- & -- & -- & -- & -- & -- \\
\hline $5-30$ & -- & -- & -- & -- & -- & -- & -- \\
\hline $6-01$ & -- & -- & -- & -- & -- & -- & -- \\
\hline $6-03$ & -- & -- & -- & -- & -- & -- & -- \\
\hline $\begin{array}{l}6-05 \\
6-07\end{array}$ & $=-$ & $=-$ & $\overline{-}$ & $=$ & $=$ & $=-$ & $=$ \\
\hline $6-09$ & $61+$ & 7 & 0 & 5 & 0 & 2 & 0 \\
\hline $6-10$ & 283 & 11 & 3 & 8 & 0 & 0 & 0 \\
\hline $6-12$ & 266 & 0 & 0 & 0 & 0 & 0 & 0 \\
\hline $\begin{array}{l}6-13 \\
6-14\end{array}$ & $\begin{array}{l}24+++ \\
190\end{array}$ & $\begin{array}{l}25 \\
20\end{array}$ & $\begin{array}{r}17 \\
9\end{array}$ & $\begin{array}{l}8 \\
7\end{array}$ & $\begin{array}{l}0 \\
3\end{array}$ & $\begin{array}{l}0 \\
1\end{array}$ & $\begin{array}{l}0 \\
0\end{array}$ \\
\hline $6-15$ & $33++$ & 3 & 3 & 0 & 0 & 0 & 0 \\
\hline $6-16$ & & -- & -- & -- & -- & -- & -- \\
\hline $6-17$ & $\overline{3}+1$ & $\overline{-}$ & -- & $-\overline{2}$ & $-\overline{0}$ & $-\overline{0}$ & $-\overline{0}$ \\
\hline $\begin{array}{l}6-18 \\
6-19\end{array}$ & $\begin{array}{c}38++ \\
0+++\end{array}$ & $\begin{array}{l}5 \\
0\end{array}$ & 0 & 2 & 0 & 0 & 0 \\
\hline $6-20$ & 371 & 27 & 7 & 16 & 3 & 1 & 0 \\
\hline $\begin{array}{l}6-21 \\
6-22\end{array}$ & $71+$ & 25 & -4 & 20 & 1 & -1 & -0 \\
\hline $6-23$ & -- & -- & -- & -- & -- & -- & -- \\
\hline $6-25$ & $22+++$ & 9 & 0 & 5 & 4 & 0 & 0 \\
\hline $6-27$ & $6+++$ & 0 & 0 & 0 & 0 & 0 & 0 \\
\hline $6-29$ & -- & -- & -- & -- & -- & -- & - \\
\hline $7-03$ & - & -- & $=$ & - & -- & - & -- \\
\hline $7-05$ & -- & -- & -- & -- & -- & -- & -- \\
\hline $7-07$ & -- & -- & -- & -- & -- & -- & -- \\
\hline
\end{tabular}


TABLE 16.- NUMBER OF BLUE TRACER PARTICLES(1), PER 100 GRAMS OF BEDLOAD SAMPLE, COMPOSITED FROM CROSS-CHANNEL SAMPLING POSITIONS SPACED AT 1-METER INTERVALS ACROSS THE CHANNEL WIDTH, EAST FORK RIVER, WYOMING, 1980

SECTION 0708

\begin{tabular}{|c|c|c|c|c|c|c|c|}
\hline \multirow{3}{*}{ DATE } & \multirow{3}{*}{$\begin{array}{c}\text { DRY } \\
\text { MASS } \\
\text { OF } \\
\text { SAMPLE (2) } \\
(G)\end{array}$} & \multicolumn{6}{|c|}{ NUMBER OF BLUE TRACER PARTICLES } \\
\hline & & \multirow[b]{2}{*}{ TOTAL } & \multicolumn{4}{|c|}{ BY SIZE CLASS (MM) } & \multirow[b]{2}{*}{$\begin{array}{l}4.00 \\
\text { TO } \\
8.00\end{array}$} \\
\hline & & & $\begin{array}{l}0.25 \\
\text { TO } \\
0.50\end{array}$ & $\begin{array}{l}0.50 \\
\text { TO } \\
1.00\end{array}$ & $\begin{array}{l}1.00 \\
\text { TO } \\
2.00\end{array}$ & $\begin{array}{l}2.00 \\
\text { TO } \\
4.00\end{array}$ & \\
\hline $\begin{array}{l}5-16 \\
5-19 \\
5-21 \\
5-22\end{array}$ & $\begin{array}{r}841 \\
395 \\
3726 \\
--\end{array}$ & $\begin{array}{l}53 \\
32 \\
29 \\
--\end{array}$ & $\begin{array}{r}4 \\
4 \\
4 \\
--\end{array}$ & $\begin{array}{l}36 \\
16 \\
16 \\
-\end{array}$ & $\begin{array}{r}13 \\
12 \\
8 \\
--\end{array}$ & $\begin{array}{r}0 \\
0 \\
1 \\
-1\end{array}$ & $\begin{array}{r}0 \\
0 \\
0 \\
--\end{array}$ \\
\hline $5-24$ & -- & - & -- & -- & -- & -- & -- \\
\hline $\begin{array}{l}5-26 \\
5-27 \\
5-28 \\
5-29 \\
5-30\end{array}$ & $\begin{array}{l}513 \\
215 \\
288 \\
422 \\
88+\end{array}$ & $\begin{array}{l}43 \\
36 \\
60 \\
32 \\
65\end{array}$ & $\begin{array}{r}4 \\
5 \\
14 \\
2 \\
12\end{array}$ & $\begin{array}{l}35 \\
26 \\
43 \\
21 \\
46\end{array}$ & $\begin{array}{l}3 \\
5 \\
3 \\
8 \\
7\end{array}$ & $\begin{array}{l}1 \\
0 \\
0 \\
1 \\
0\end{array}$ & $\begin{array}{l}0 \\
0 \\
0 \\
0 \\
0\end{array}$ \\
\hline $\begin{array}{l}6-01 \\
6-03 \\
6-05 \\
6-07 \\
6-09\end{array}$ & $\begin{array}{c}39++ \\
909 \\
702 \\
1550 \\
3638\end{array}$ & $\begin{array}{l}64 \\
24 \\
33 \\
29 \\
34\end{array}$ & $\begin{array}{r}8 \\
0 \\
12 \\
4 \\
0\end{array}$ & $\begin{array}{l}43 \\
16 \\
10 \\
19 \\
24\end{array}$ & $\begin{array}{r}11 \\
5 \\
11 \\
6 \\
9\end{array}$ & $\begin{array}{l}2 \\
3 \\
0 \\
0 \\
1\end{array}$ & $\begin{array}{l}0 \\
0 \\
0 \\
0 \\
0\end{array}$ \\
\hline $\begin{array}{l}6-10 \\
6-11 \\
6-12 \\
6-13 \\
6-14\end{array}$ & $\begin{array}{l}7438 \\
1025 \\
583 \\
1330 \\
2343\end{array}$ & $\begin{array}{l}19 \\
19 \\
14 \\
29 \\
18\end{array}$ & $\begin{array}{l}2 \\
1 \\
1 \\
9 \\
3\end{array}$ & $\begin{array}{l}11 \\
16 \\
6 \\
16 \\
11\end{array}$ & $\begin{array}{l}5 \\
2 \\
4 \\
2 \\
2\end{array}$ & $\begin{array}{l}0 \\
0 \\
3 \\
2 \\
2\end{array}$ & $\begin{array}{l}1 \\
0 \\
0 \\
0 \\
0\end{array}$ \\
\hline $\begin{array}{l}6-15 \\
6-16 \\
6-17 \\
6-18 \\
6-19\end{array}$ & $\begin{array}{r}862 \\
884 \\
1457 \\
3737 \\
3122\end{array}$ & $\begin{array}{l}28 \\
18 \\
20 \\
18 \\
12\end{array}$ & $\begin{array}{l}5 \\
7 \\
1 \\
0 \\
0\end{array}$ & $\begin{array}{r}20 \\
7 \\
16 \\
12 \\
7\end{array}$ & $\begin{array}{l}3 \\
4 \\
3 \\
4 \\
4\end{array}$ & $\begin{array}{l}0 \\
0 \\
0 \\
2 \\
1\end{array}$ & $\begin{array}{l}0 \\
0 \\
0 \\
0 \\
0\end{array}$ \\
\hline $\begin{array}{l}6-20 \\
6-21\end{array}$ & $\begin{array}{r}10382 \\
1174\end{array}$ & $\begin{array}{l}10 \\
11\end{array}$ & $\frac{1}{2}$ & $\begin{array}{l}3 \\
3\end{array}$ & $\begin{array}{l}5 \\
4\end{array}$ & $\begin{array}{l}0 \\
2\end{array}$ & 1 \\
\hline $\begin{array}{l}6-23 \\
6-25\end{array}$ & $\begin{array}{l}997 \\
721\end{array}$ & $\frac{15}{20}$ & $\begin{array}{l}5 \\
0\end{array}$ & $\begin{array}{r}5 \\
14\end{array}$ & $\begin{array}{l}5 \\
5\end{array}$ & $\begin{array}{l}0 \\
1\end{array}$ & 0 \\
\hline $6-27$ & 2107 & 11 & 1 & 7 & 2 & 1 & 0 \\
\hline $\begin{array}{l}0-29 \\
7-01\end{array}$ & -- & -- & -- & -- & - & $\overline{--}$ & -- \\
\hline $7-03$ & - & -- & -- & -- & -- & -- & -- \\
\hline $7-05$ & -- & -- & -- & -- & -- & -- & -- \\
\hline $7-07$ & -- & -- & -- & -- & -- & -- & -- \\
\hline
\end{tabular}


TABLE 16.- NUMBER OF BLUE TRACER PARTICLES(1), PER 100 GRAMS OF BEDLOAD SAMPLE, COMPOSITED FROM CROSS-CHANNEL SAMPLING POSITIONS

SPACED AT I-METER INTERVALS ACROSS THE CHANNEL WIDTH,

EAST FORK RIVER, WYOMING, 1980

SECTION 0757

\begin{tabular}{|c|c|c|c|c|c|c|c|}
\hline \multirow{3}{*}{\multicolumn{2}{|c|}{$\begin{array}{cc}\text { DATE } & \text { DRY } \\
& \text { MASS } \\
\text { OF } & \\
& \text { SAMPLE (2) } \\
& \text { (G) }\end{array}$}} & \multicolumn{6}{|c|}{ NUMBER OF BLUE TRACER PARTICLES } \\
\hline & & \multirow[b]{2}{*}{ TOTAL } & \multicolumn{4}{|c|}{ BY SIZE CLASS (MM) } & \multirow[b]{2}{*}{$\begin{array}{l}4.00 \\
\text { TO } \\
8.00\end{array}$} \\
\hline & & & $\begin{array}{l}0.25 \\
\text { TO } \\
0.50\end{array}$ & $\begin{array}{l}0.50 \\
\text { TO } \\
1.00\end{array}$ & $\begin{array}{l}1.00 \\
\text { TO } \\
2.00\end{array}$ & $\begin{array}{l}2.00 \\
\text { TO } \\
4.00\end{array}$ & \\
\hline $\begin{array}{l}5-16 \\
5-19 \\
5-21 \\
5-22 \\
5-24\end{array}$ & $\begin{array}{r}450 \\
463 \\
613 \\
-- \\
--\end{array}$ & $\begin{array}{l}46 \\
32 \\
45 \\
-- \\
--\end{array}$ & $\begin{array}{r}3 \\
2 \\
9 \\
---\end{array}$ & $\begin{array}{l}30 \\
17 \\
25 \\
-- \\
--\end{array}$ & $\begin{array}{r}13 \\
13 \\
8 \\
---\end{array}$ & $\begin{array}{r}0 \\
0 \\
3 \\
-- \\
--\end{array}$ & $\begin{array}{r}0 \\
0 \\
0 \\
---\end{array}$ \\
\hline \multirow{2}{*}{$\begin{array}{l}5-26 \\
5-27 \\
5-28 \\
5-29 \\
5-30\end{array}$} & $\begin{array}{l}6427 \\
3063 \\
1324\end{array}$ & $\begin{array}{l}34 \\
50 \\
37\end{array}$ & $\begin{array}{l}1 \\
4 \\
3\end{array}$ & $\begin{array}{l}26 \\
33 \\
29\end{array}$ & $\begin{array}{r}5 \\
11 \\
4\end{array}$ & $\begin{array}{l}1 \\
2 \\
1\end{array}$ & $\begin{array}{l}1 \\
0 \\
0\end{array}$ \\
\hline & 1695 & 37 & $\overline{5}$ & $\overline{20}$ & $\overline{12}$ & 0 & $\overline{0}$ \\
\hline $\begin{array}{l}6-01 \\
6-03 \\
6-05 \\
6-07 \\
6-09\end{array}$ & $\begin{array}{c}1578+ \\
448 \\
384 \\
501 \\
716\end{array}$ & $\begin{array}{l}38 \\
44 \\
37 \\
29 \\
34\end{array}$ & $\begin{array}{r}3 \\
8 \\
10 \\
4 \\
2\end{array}$ & $\begin{array}{l}25 \\
31 \\
21 \\
21 \\
25\end{array}$ & $\begin{array}{l}8 \\
3 \\
5 \\
4 \\
7\end{array}$ & $\begin{array}{l}1 \\
2 \\
1 \\
0 \\
0\end{array}$ & $\begin{array}{l}1 \\
0 \\
0 \\
0 \\
0\end{array}$ \\
\hline $\begin{array}{l}6-10 \\
6-11 \\
6-12 \\
6-13 \\
6-14\end{array}$ & $\begin{array}{r}68+ \\
320 \\
2425 \\
1014 \\
3202\end{array}$ & $\begin{array}{l}99 \\
10 \\
24 \\
31 \\
18\end{array}$ & $\begin{array}{r}3 \\
3 \\
4 \\
15 \\
3\end{array}$ & $\begin{array}{r}1 \\
16 \\
16 \\
14 \\
11\end{array}$ & $\begin{array}{l}5 \\
1 \\
4 \\
1 \\
4\end{array}$ & $\begin{array}{l}0 \\
0 \\
0 \\
1 \\
0\end{array}$ & $\begin{array}{l}0 \\
0 \\
0 \\
0 \\
0\end{array}$ \\
\hline $\begin{array}{l}6-15 \\
6-16 \\
6-17 \\
6-18 \\
6-19\end{array}$ & $\begin{array}{l}1515 \\
3208 \\
5436 \\
6123 \\
2003\end{array}$ & $\begin{array}{r}14 \\
15 \\
20 \\
15 \\
7\end{array}$ & $\begin{array}{l}2 \\
5 \\
2 \\
2 \\
0\end{array}$ & $\begin{array}{r}12 \\
4 \\
16 \\
6 \\
7\end{array}$ & $\begin{array}{l}0 \\
3 \\
2 \\
4 \\
0\end{array}$ & $\begin{array}{l}0 \\
3 \\
0 \\
3 \\
0\end{array}$ & $\begin{array}{l}0 \\
0 \\
0 \\
0 \\
0\end{array}$ \\
\hline $\begin{array}{l}6-20 \\
6-21 \\
6-22\end{array}$ & $\begin{array}{l}2586 \\
1847\end{array}$ & $\begin{array}{l}15 \\
19 \\
--\end{array}$ & $\begin{array}{r}1 \\
-2\end{array}$ & $\begin{array}{r}12 \\
9\end{array}$ & $\begin{array}{r}1 \\
4 \\
-2\end{array}$ & $\begin{array}{r}0 \\
2 \\
-2\end{array}$ & $\begin{array}{r}1 \\
0\end{array}$ \\
\hline $\begin{array}{l}6-22 \\
6-23 \\
6-25\end{array}$ & $\begin{array}{l}1505 \\
2895\end{array}$ & $\overline{15}$ & $\overline{1}$ & $\begin{array}{r}9 \\
13\end{array}$ & $\overline{2}$ & $\overline{3}$ & $\begin{array}{l}0 \\
0\end{array}$ \\
\hline $\begin{array}{l}6-27 \\
6-20\end{array}$ & 1785 & 24 & 1 & 18 & 4 & 1 & $\underline{0}$ \\
\hline $7-01$ & -- & -- & -- & -- & -- & -- & -- \\
\hline $\begin{array}{l}7-03 \\
7-05\end{array}$ & -- & -- & -- & -- & -- & -- & $\overline{--}$ \\
\hline $7-07$ & -- & -- & -- & -- & -- & -- & -- \\
\hline
\end{tabular}


TABLE 16.- NUMBER OF BLUE TRACER PARTICLES(1), PER 100 GRAMS OF BEDLOAD SAMPLE, COMPOSITED FROM CROSS-CHANNEL SAMPLING POSITIONS SPACED AT I-METER INTERVALS ACROSS THE CHANNEL WIDTH, EAST FORK RIVER, WYOMING, 1980

SECTION 0803

\begin{tabular}{|c|c|c|c|c|c|c|c|}
\hline \multirow{3}{*}{ DATE } & \multirow{3}{*}{$\begin{array}{c}\text { DRY } \\
\text { MASS } \\
\text { OF } \\
\text { SAMPLE (2) } \\
\text { (G) }\end{array}$} & \multicolumn{6}{|c|}{ NUMBER OF BLUE TRACER PARTICLES } \\
\hline & & \multirow[b]{2}{*}{ TOTAL } & \multicolumn{5}{|c|}{ BY SIZE CLASS (MM) } \\
\hline & & & $\begin{array}{r}0.25 \\
\text { TO } \\
0.50\end{array}$ & $\begin{array}{l}0.50 \\
\text { To } \\
1.00\end{array}$ & $\begin{array}{l}1.00 \\
2.00\end{array}$ & $\begin{array}{l}2.00 \\
\text { TO } \\
4.00\end{array}$ & $\begin{array}{l}4.00 \\
\text { TOO } \\
8.00\end{array}$ \\
\hline $\begin{array}{l}5-16 \\
5-19 \\
5-21 \\
5-22 \\
5-24\end{array}$ & $\begin{array}{r}573 \\
894 \\
314 \\
-- \\
--\end{array}$ & $\begin{array}{l}51 \\
33 \\
32 \\
--\end{array}$ & $\begin{array}{r}\frac{1}{3} \\
6 \\
--\end{array}$ & $\begin{array}{l}32 \\
14 \\
15 \\
-- \\
--\end{array}$ & $\begin{array}{l}17 \\
12 \\
10 \\
--\end{array}$ & $\begin{array}{r}1 \\
4 \\
-1 \\
---\end{array}$ & $\begin{array}{r}0 \\
0 \\
0 \\
--\end{array}$ \\
\hline $\begin{array}{l}5-26 \\
5-27 \\
5-28\end{array}$ & $\begin{array}{l}3784 \\
2360 \\
2445\end{array}$ & $\begin{array}{l}41 \\
38 \\
49\end{array}$ & $\begin{array}{l}4 \\
0 \\
3\end{array}$ & $\begin{array}{l}26 \\
16 \\
31\end{array}$ & $\begin{array}{r}8 \\
20 \\
13\end{array}$ & $\begin{array}{l}3 \\
2 \\
2\end{array}$ & $\begin{array}{l}0 \\
0 \\
0\end{array}$ \\
\hline $5-30$ & 1203 & 27 & 3 & 14 & 7 & 3 & 0 \\
\hline $\begin{array}{l}6-01 \\
6-03 \\
6-05 \\
6-07 \\
6-09\end{array}$ & $\begin{array}{l}573 \\
196 \\
292 \\
1199 \\
358\end{array}$ & $\begin{array}{l}29 \\
32 \\
30 \\
16 \\
24\end{array}$ & $\begin{array}{l}3 \\
2 \\
7 \\
\frac{1}{2}\end{array}$ & $\begin{array}{l}16 \\
26 \\
14 \\
7 \\
14\end{array}$ & $\begin{array}{l}6 \\
4 \\
8 \\
7 \\
7\end{array}$ & $\begin{array}{l}4 \\
0 \\
1 \\
\frac{1}{1} \\
1\end{array}$ & $\begin{array}{l}0 \\
0 \\
0 \\
0 \\
0\end{array}$ \\
\hline $\begin{array}{l}6-10 \\
6-11 \\
6-12 \\
6-13 \\
6-14\end{array}$ & $\begin{aligned} & 52+++ \\
& 598 \\
& 1683 \\
& 1907 \\
& 7240\end{aligned}$ & $\begin{array}{l}10 \\
10 \\
23 \\
28 \\
21\end{array}$ & $\begin{array}{l}2 \\
1 \\
5 \\
8 \\
4\end{array}$ & $\begin{array}{r}6 \\
5 \\
14 \\
18 \\
14\end{array}$ & $\begin{array}{l}2 \\
2 \\
3 \\
2 \\
2\end{array}$ & $\begin{array}{l}0 \\
2 \\
1 \\
0 \\
1\end{array}$ & $\begin{array}{l}0 \\
0 \\
0 \\
0 \\
0\end{array}$ \\
\hline $\begin{array}{l}6-15 \\
6-16 \\
6-17 \\
6-18 \\
6-19\end{array}$ & $\begin{array}{l}2905 \\
5512 \\
5656 \\
2381 \\
1544\end{array}$ & $\begin{array}{l}26 \\
14 \\
35 \\
24 \\
22\end{array}$ & $\begin{array}{l}1 \\
0 \\
2 \\
1 \\
7\end{array}$ & $\begin{array}{l}18 \\
4 \\
19 \\
17 \\
11\end{array}$ & $\begin{array}{r}7 \\
6 \\
13 \\
5 \\
4\end{array}$ & $\begin{array}{l}0 \\
4 \\
1 \\
1 \\
0\end{array}$ & $\begin{array}{l}0 \\
0 \\
0 \\
0 \\
0\end{array}$ \\
\hline $\begin{array}{l}6-20 \\
6-21 \\
6-22 \\
6-23 \\
6-25\end{array}$ & $\begin{array}{l}6034 \\
2623 \\
2266 \\
651 \\
2859\end{array}$ & $\begin{array}{l}12 \\
17 \\
17 \\
18 \\
24\end{array}$ & $\begin{array}{l}2 \\
0 \\
1 \\
0 \\
1\end{array}$ & $\begin{array}{r}6 \\
14 \\
12 \\
13 \\
17\end{array}$ & $\begin{array}{l}3 \\
3 \\
3 \\
3 \\
5\end{array}$ & $\begin{array}{l}\frac{1}{0} \\
\frac{1}{1} \\
\frac{1}{1}\end{array}$ & $\begin{array}{l}0 \\
0 \\
0 \\
1 \\
0\end{array}$ \\
\hline $\begin{array}{l}6-27 \\
6-29 \\
7-01 \\
7-03 \\
7-05\end{array}$ & $\begin{array}{r}2745 \\
2692 \\
- \\
-- \\
--\end{array}$ & $\begin{array}{l}20 \\
20 \\
-- \\
--\end{array}$ & $\begin{array}{r}3 \\
1 \\
-- \\
--\end{array}$ & $\begin{array}{l}16 \\
16 \\
-- \\
-- \\
--\end{array}$ & $\begin{array}{r}0 \\
2 \\
-- \\
--\end{array}$ & $\begin{array}{r}\frac{1}{1} \\
---\end{array}$ & $\begin{array}{r}0 \\
0 \\
-- \\
--\end{array}$ \\
\hline $7-07$ & -- & -- & -- & -- & -- & -- & -- \\
\hline
\end{tabular}


TABLE 16.- NUMBER OF BLUE TRACER PARTICLES(1), PER 100 GRAMS OF BEDLOAD SAMPLE, COMPOSITED FROM CROSS-CHANNEL SAMPLING POSITIONS SPACED AT 1-METER INTERVALS ACROSS THE CHANNEL WIDTH, EAST FORK RIVER, WYOMING, 1980

SECTION $0808(3)$

\begin{tabular}{|c|c|c|c|c|c|c|c|}
\hline \multirow{3}{*}{ DATE } & \multirow{3}{*}{$\begin{array}{c}\text { DRY } \\
\text { MASS } \\
\text { OF } \\
\text { SAMPLE (2) } \\
\text { (G) }\end{array}$} & \multicolumn{6}{|c|}{ NUMBER OF BLUE TRACER PARTICLES } \\
\hline & & \multirow[b]{2}{*}{ TOTAL } & \multicolumn{5}{|c|}{ BY SIZE CLASS (MM) } \\
\hline & & & $\begin{array}{l}0.25 \\
\text { TO } \\
0.50\end{array}$ & $\begin{array}{l}0.50 \\
\text { TO } \\
1.00\end{array}$ & $\begin{array}{l}1.00 \\
\text { TO } \\
2.00\end{array}$ & $\begin{array}{l}2.00 \\
\text { TO } \\
4.00\end{array}$ & $\begin{array}{c}4.00 \\
\text { TO } \\
8.00\end{array}$ \\
\hline $5-16$ & -- & -- & -- & -- & - & $\overline{--}$ & -- \\
\hline $\begin{array}{l}5-19 \\
5-21\end{array}$ & - & $\overline{--}$ & $\overline{--}$ & - & - & $\overline{--}$ & $\overline{--}$ \\
\hline $5-22$ & -- & -- & -- & -- & -- & -- & -- \\
\hline & - & - & -- & -- & -- & - & - \\
\hline $5-26$ & -- & -- & -- & -- & -- & -- & -- \\
\hline $\begin{array}{l}5-27 \\
5-28\end{array}$ & $\overline{--}$ & $\overline{--}$ & $\overline{--}$ & $\overline{--}$ & $\overline{-}$ & $\overline{--}$ & $\overline{--}$ \\
\hline $5-29$ & - & - & - & - & - & $=$ & $=$ \\
\hline $5-30$ & -- & -- & -- & -- & -- & -- & -- \\
\hline $6-01$ & -- & -- & -- & -- & -- & -- & -- \\
\hline $6-03$ & -- & -- & -- & - & - & -- & -- \\
\hline $6-05$ & -- & -- & -- & -- & - & - & -- \\
\hline 6-09 & $85+$ & 29 & 2 & 26 & 1 & 0 & 0 \\
\hline $6-10$ & 677 & 38 & 13 & 23 & 1 & $\frac{1}{0}$ & 0 \\
\hline $6-12$ & & & $=-$ & $=$ & -- & - & \\
\hline $\begin{array}{l}6-13 \\
6-14\end{array}$ & $\begin{array}{l}462 \\
371\end{array}$ & $\begin{array}{l}22 \\
46\end{array}$ & $\begin{array}{r}7 \\
11\end{array}$ & $\begin{array}{l}15 \\
32\end{array}$ & $\begin{array}{l}0 \\
3\end{array}$ & $\begin{array}{l}0 \\
0\end{array}$ & $\begin{array}{l}0 \\
0\end{array}$ \\
\hline $6-15$ & 504 & 34 & 1 & 33 & 0 & 0 & 0 \\
\hline $\begin{array}{l}6-16 \\
6-17\end{array}$ & 103 & 21 & 7 & 13 & 1 & 0 & 0 \\
\hline $6-18$ & 726 & $3 i$ & 4 & 23 & 4 & 0 & 0 \\
\hline $6-19$ & $34++$ & 0 & 0 & 0 & 0 & 0 & 0 \\
\hline & & & & & & & 0 \\
\hline $6-21$ & $17++$ & $\begin{array}{r}0 \\
75\end{array}$ & 0 & 0 & 0 & $\begin{array}{l}0 \\
0\end{array}$ & $\begin{array}{l}0 \\
0\end{array}$ \\
\hline $\begin{array}{l}6-22 \\
6-23\end{array}$ & $\begin{array}{l}68+ \\
52+\end{array}$ & $\begin{array}{l}15 \\
17\end{array}$ & $\begin{array}{l}6 \\
6\end{array}$ & $\begin{array}{l}9 \\
9\end{array}$ & $\begin{array}{l}0 \\
2\end{array}$ & $\begin{array}{l}0 \\
0\end{array}$ & $\begin{array}{l}0 \\
0\end{array}$ \\
\hline $6-25$ & $34+++$ & 12 & 0 & 12 & 0 & 0 & 0 \\
\hline & 124 & 20 & 5 & 14 & 1 & 0 & 0 \\
\hline $6-29$ & - & & & - & & - & - \\
\hline $\begin{array}{l}7-01 \\
7-03\end{array}$ & $=$ & $=$ & $=$ & $=$ & $=$ & $=$ & $=$ \\
\hline $7-05$ & -- & -- & -- & -- & -- & -- & -- \\
\hline $7-07$ & -- & -- & -- & -- & -- & -- & -- \\
\hline
\end{tabular}


TABLE 16.- NUMBER OF BLUE TRACER PARTICLES(1), PER 100 GRAMS OF BEDLOAD SAMPLE, COMPOSITED FROM CROSS-CHANNEL SAMPLING POSITIONS SPACED AT 1-METER INTERVALS ACROSS THE CHANNEL WIDTH, EAST FORK RIVER, WYOMING, 1980

SECTION 0853

\begin{tabular}{|c|c|c|c|c|c|c|c|}
\hline \multirow{3}{*}{ DATE } & \multirow{3}{*}{$\begin{array}{c}\text { DRY } \\
\text { MASS } \\
\text { OF } \\
\text { SAMPLE (2) } \\
\text { (G) }\end{array}$} & \multicolumn{6}{|c|}{ NUMBER OF BLUE TRACER PARTICLES } \\
\hline & & \multirow[b]{2}{*}{ TOTAL } & \multicolumn{5}{|c|}{ BY SIZE CLASS (MM) } \\
\hline & & & $\begin{array}{c}0.25 \\
\text { TO } \\
0.50\end{array}$ & $\begin{array}{l}0.50 \\
\text { TO } \\
1.00\end{array}$ & $\begin{array}{l}1.00 \\
2.00 \\
2.00\end{array}$ & $\begin{array}{l}2.00 \\
\text { TO } \\
4.00\end{array}$ & $\begin{array}{l}4.00 \\
8.00 \\
8.00\end{array}$ \\
\hline $\begin{array}{l}5-16 \\
5-19 \\
5-21 \\
5-22 \\
5-24\end{array}$ & $\begin{array}{r}284 \\
459 \\
827 \\
--\end{array}$ & $\begin{array}{l}34 \\
25 \\
31 \\
-- \\
--\end{array}$ & $\begin{array}{r}2 \\
0 \\
1 \\
-- \\
--\end{array}$ & $\begin{array}{r}22 \\
8 \\
16 \\
--\end{array}$ & $\begin{array}{r}10 \\
16 \\
9 \\
--\end{array}$ & $\begin{array}{r}0 \\
1 \\
4 \\
-- \\
--\end{array}$ & $\begin{array}{r}0 \\
0 \\
1 \\
-- \\
--\end{array}$ \\
\hline $\begin{array}{l}5-26 \\
5-27 \\
5-28\end{array}$ & $\begin{array}{r}2765 \\
834 \\
1822\end{array}$ & $\begin{array}{l}55 \\
18 \\
32\end{array}$ & $\begin{array}{l}5 \\
1 \\
1\end{array}$ & $\begin{array}{r}31 \\
8 \\
18\end{array}$ & $\begin{array}{r}18 \\
8 \\
13\end{array}$ & $\begin{array}{l}1 \\
1 \\
0\end{array}$ & $\begin{array}{l}0 \\
0 \\
0\end{array}$ \\
\hline $5-30$ & 550 & 23 & 5 & 16 & 1 & 1 & 0 \\
\hline $\begin{array}{l}6-01 \\
6-03 \\
6-05 \\
6-07 \\
6-09\end{array}$ & $\begin{array}{l}242 \\
217 \\
4+++ \\
170 \\
322\end{array}$ & $\begin{array}{r}19 \\
25 \\
0 \\
20 \\
44\end{array}$ & $\begin{array}{l}1 \\
2 \\
0 \\
2 \\
9\end{array}$ & $\begin{array}{r}10 \\
17 \\
0 \\
11 \\
25\end{array}$ & $\begin{array}{l}7 \\
4 \\
0 \\
6 \\
9\end{array}$ & $\begin{array}{l}1 \\
2 \\
0 \\
0 \\
1\end{array}$ & $\begin{array}{l}0 \\
0 \\
0 \\
1 \\
0\end{array}$ \\
\hline $\begin{array}{l}6-10 \\
6-11 \\
6-12 \\
6-13 \\
6-14\end{array}$ & $\begin{array}{r}847 \\
2219 \\
6895 \\
6089 \\
5264\end{array}$ & $\begin{array}{l}23 \\
30 \\
23 \\
27 \\
13\end{array}$ & $\begin{array}{r}6 \\
3 \\
4 \\
11 \\
\frac{1}{3}\end{array}$ & $\begin{array}{r}12 \\
22 \\
10 \\
9 \\
6\end{array}$ & $\begin{array}{l}3 \\
4 \\
8 \\
7 \\
2\end{array}$ & $\begin{array}{l}2 \\
1 \\
1 \\
0 \\
2\end{array}$ & $\begin{array}{l}0 \\
0 \\
0 \\
0 \\
0\end{array}$ \\
\hline $\begin{array}{l}6-15 \\
6-16 \\
6-17 \\
6-18 \\
6-19\end{array}$ & $\begin{array}{l}1825 \\
4440 \\
3923 \\
3082 \\
3537\end{array}$ & $\begin{array}{r}9 \\
19 \\
18 \\
5 \\
10\end{array}$ & $\begin{array}{l}0 \\
4 \\
1 \\
1 \\
0\end{array}$ & $\begin{array}{r}7 \\
11 \\
11 \\
3 \\
6\end{array}$ & $\begin{array}{l}2 \\
4 \\
3 \\
\frac{1}{3}\end{array}$ & $\begin{array}{l}0 \\
0 \\
3 \\
0 \\
1\end{array}$ & $\begin{array}{l}0 \\
0 \\
0 \\
0 \\
0\end{array}$ \\
\hline $\begin{array}{l}6-20 \\
6-21 \\
6-22 \\
6-23 \\
6-25\end{array}$ & $\begin{array}{r}628 \\
1390 \\
3195 \\
1491 \\
711\end{array}$ & $\begin{array}{l}12 \\
16 \\
13 \\
11 \\
17\end{array}$ & $\begin{array}{l}4 \\
3 \\
3 \\
1 \\
2\end{array}$ & $\begin{array}{r}5 \\
9 \\
5 \\
8 \\
10\end{array}$ & $\begin{array}{l}3 \\
4 \\
4 \\
2 \\
4\end{array}$ & $\begin{array}{l}0 \\
0 \\
1 \\
0 \\
1\end{array}$ & $\begin{array}{l}0 \\
0 \\
0 \\
0 \\
0\end{array}$ \\
\hline $6-27$ & 2440 & 18 & 1 & 12 & 3 & 2 & 0 \\
\hline $\begin{array}{l}0-29 \\
7-01\end{array}$ & $=$ & $=$ & $=$ & $=$ & $=$ & $=-$ & $=$ \\
\hline $7-0 \overline{3}$ & -- & -- & -- & -- & -- & -- & -- \\
\hline $7-05$ & -- & -- & -- & - & -- & - & -- \\
\hline $7-07$ & -- & -- & -- & -- & -- & -- & -- \\
\hline
\end{tabular}


TABLE 16.- NUMBER OF BLUE TRACER PARTICLES(1), PER 100 GRAMS OF BEDLOAD SAMPLE, COMPOSITED FROM CROSS-CHANNEL SAMPLING POSITIONS SPACED AT 1-METER INTERVALS ACROSS THE CHANNEL WIDTH,

EAST FORK RIVER, WYOMING, 1980

$$
\text { SECTION } 0898
$$

\begin{tabular}{|c|c|c|c|c|c|c|c|}
\hline \multirow{3}{*}{ DATE } & \multirow{3}{*}{$\begin{array}{c}\text { DRY } \\
\text { MASS } \\
\text { OF } \\
\text { SAMPLE (2) } \\
\text { (G) }\end{array}$} & \multicolumn{6}{|c|}{ NUMBER OF BLUE TRACER PARTICLES } \\
\hline & & \multirow[b]{2}{*}{ TOTAL } & \multicolumn{5}{|c|}{ BY SIZE CLASS (MM) } \\
\hline & & & $\begin{array}{l}0.25 \\
\text { TO } \\
0.50\end{array}$ & $\begin{array}{l}0.50 \\
\text { TO } \\
1.00\end{array}$ & $\begin{array}{l}1.00 \\
\text { TO } \\
2.00\end{array}$ & $\begin{array}{l}2.00 \\
\text { TO } \\
4.00\end{array}$ & $\begin{array}{l}4.00 \\
\text { TO } \\
8.00\end{array}$ \\
\hline $\begin{array}{l}5-16 \\
5-19 \\
5-21 \\
5-22 \\
5-24\end{array}$ & $\begin{array}{l}114++ \\
201+ \\
231 \\
-- \\
--\end{array}$ & $\begin{array}{l}0 \\
39 \\
14 \\
--\end{array}$ & $\begin{array}{r}0 \\
5 \\
3 \\
-- \\
--\end{array}$ & $\begin{array}{r}0 \\
19 \\
4 \\
--\end{array}$ & $\begin{array}{r}0 \\
14 \\
5 \\
--\end{array}$ & $\begin{array}{r}0 \\
1 \\
1 \\
---\end{array}$ & $\begin{array}{r}0 \\
0 \\
1 \\
-- \\
--\end{array}$ \\
\hline $\begin{array}{l}5-26 \\
5-27 \\
5-28 \\
5-29 \\
5-30\end{array}$ & $\begin{array}{r}1516 \\
1853 \\
251 \\
290\end{array}$ & $\begin{array}{l}24 \\
27 \\
29 \\
-9 \\
27\end{array}$ & $\begin{array}{r}\frac{1}{3} \\
-\frac{4}{3}\end{array}$ & $\begin{array}{l}11 \\
11 \\
15 \\
\frac{16}{16}\end{array}$ & $\begin{array}{r}10 \\
9 \\
6 \\
-7\end{array}$ & $\begin{array}{r}2 \\
4 \\
-4 \\
-1\end{array}$ & $\begin{array}{r}0 \\
0 \\
0 \\
-\frac{1}{0}\end{array}$ \\
\hline $\begin{array}{l}6-01 \\
6-03 \\
6-05 \\
6-07 \\
6-09\end{array}$ & $\begin{array}{c}332 \\
46++ \\
46++ \\
34++ \\
203\end{array}$ & $\begin{array}{l}25 \\
17 \\
22 \\
15 \\
19\end{array}$ & $\begin{array}{l}5 \\
7 \\
4 \\
0 \\
9\end{array}$ & $\begin{array}{r}15 \\
10 \\
13 \\
12 \\
9\end{array}$ & $\begin{array}{l}4 \\
0 \\
5 \\
3 \\
1\end{array}$ & $\begin{array}{l}1 \\
0 \\
0 \\
0 \\
0\end{array}$ & $\begin{array}{l}0 \\
0 \\
0 \\
0 \\
0\end{array}$ \\
\hline $\begin{array}{l}6-10 \\
6-11 \\
6-12 \\
6-13 \\
6-14\end{array}$ & $\begin{array}{r}433 \\
180 \\
1483 \\
1925 \\
6873\end{array}$ & $\begin{array}{r}26 \\
9 \\
21 \\
36 \\
31\end{array}$ & $\begin{array}{r}7 \\
2 \\
4 \\
14 \\
6\end{array}$ & $\begin{array}{r}15 \\
3 \\
7 \\
21 \\
17\end{array}$ & $\begin{array}{r}4 \\
2 \\
10 \\
1 \\
6\end{array}$ & $\begin{array}{l}0 \\
2 \\
0 \\
0 \\
2\end{array}$ & $\begin{array}{l}0 \\
0 \\
0 \\
0 \\
0\end{array}$ \\
\hline $\begin{array}{l}6-15 \\
6-16 \\
6-17 \\
6-18 \\
6-19\end{array}$ & $\begin{array}{r}408 \\
36-7 \\
2807 \\
1207\end{array}$ & $\begin{array}{r}22 \\
-\frac{7}{7} \\
30\end{array}$ & $\begin{array}{r}-\frac{2}{1} \\
0 \\
5\end{array}$ & $\begin{array}{r}16 \\
-\frac{2}{2} \\
5 \\
12\end{array}$ & $\begin{array}{r}-\frac{2}{3} \\
11 \\
11\end{array}$ & $-\frac{2}{1}$ & $\begin{array}{r}-0 \\
-0 \\
0 \\
0\end{array}$ \\
\hline $\begin{array}{l}6-20 \\
6-21 \\
6-22 \\
6-23 \\
6-25\end{array}$ & $\begin{array}{r}5563 \\
245 \\
170 \\
1841 \\
585\end{array}$ & $\begin{array}{l}17 \\
11 \\
20 \\
12 \\
14\end{array}$ & $\begin{array}{l}2 \\
1 \\
3 \\
3 \\
2\end{array}$ & $\begin{array}{r}13 \\
6 \\
17 \\
5 \\
10\end{array}$ & $\begin{array}{l}2 \\
4 \\
0 \\
4 \\
2\end{array}$ & $\begin{array}{l}0 \\
0 \\
0 \\
0 \\
0\end{array}$ & $\begin{array}{l}0 \\
0 \\
0 \\
0 \\
0\end{array}$ \\
\hline $\begin{array}{l}6-27 \\
6-29 \\
7-01 \\
7-03 \\
7-05\end{array}$ & $\begin{array}{r}737 \\
=- \\
=- \\
--\end{array}$ & $\begin{array}{l}12 \\
=- \\
--\end{array}$ & $\begin{array}{l}0 \\
-- \\
-- \\
--\end{array}$ & $\begin{array}{l}8 \\
-- \\
--\end{array}$ & $\begin{array}{l}3 \\
-- \\
-- \\
--\end{array}$ & $\begin{array}{l}0 \\
=- \\
=-\end{array}$ & $\begin{array}{l}1 \\
=- \\
=-\end{array}$ \\
\hline $7-07$ & - & -- & -- & -- & -- & -- & -- \\
\hline
\end{tabular}


TABLE 16.- NUMBER OF BLUE TRACER PARTICLES(1), PER 100 GRAMS OF BEDLOAD SAMPLE, COMPOSITED FROM CROSS-CHANNEL SAMPLING POSITIONS SPACED AT 1-METER INTERVALS ACROSS THE CHANNEL WIDTH, EAST FORK RIVER, WYOMING, 1980

SECTION 0940

\begin{tabular}{|c|c|c|c|c|c|c|c|}
\hline \multirow{3}{*}{ DATE } & \multirow{3}{*}{$\begin{array}{c}\text { DRY } \\
\text { MASS } \\
\text { OF } \\
\text { SAMPLE (2) } \\
\text { (G) }\end{array}$} & \multicolumn{6}{|c|}{ NUMBER OF BLUE TRACER PARTICLES } \\
\hline & & \multirow[b]{2}{*}{ TOTAL } & \multicolumn{5}{|c|}{ BY SIZE CLASS (MM) } \\
\hline & & & $\begin{array}{l}0.25 \\
\text { TO } \\
0.50\end{array}$ & $\begin{array}{l}0.50 \\
\text { TO } \\
1.00\end{array}$ & $\begin{array}{l}1.00 \\
\text { TO } \\
2.00\end{array}$ & $\begin{array}{l}2.00 \\
\text { TO } \\
4.00\end{array}$ & $\begin{array}{l}4.00 \\
\text { TO } \\
8.00\end{array}$ \\
\hline $\begin{array}{l}5-16 \\
5-19 \\
5-21 \\
5-22\end{array}$ & $\begin{array}{c}412 \\
113+ \\
1047 \\
--\end{array}$ & $\begin{array}{l}37 \\
19 \\
31 \\
-\end{array}$ & $\begin{array}{r}2 \\
0 \\
3 \\
--\end{array}$ & $\begin{array}{l}25 \\
16 \\
24 \\
-\end{array}$ & $\begin{array}{r}9 \\
3 \\
2 \\
-\end{array}$ & $\begin{array}{r}1 \\
0 \\
2 \\
-\end{array}$ & $\begin{array}{r}0 \\
0 \\
0 \\
--\end{array}$ \\
\hline $5-24$ & -- & -- & -- & -- & -- & -- & -- \\
\hline $\begin{array}{l}5-26 \\
5-27 \\
5-28 \\
5-29 \\
5-30\end{array}$ & $\begin{array}{c}3564 \\
1729 \\
803 \\
43++ \\
89+\end{array}$ & $\begin{array}{l}21 \\
39 \\
19 \\
21 \\
0\end{array}$ & $\begin{array}{l}1 \\
1 \\
1 \\
0 \\
0\end{array}$ & $\begin{array}{r}9 \\
21 \\
13 \\
7 \\
0\end{array}$ & $\begin{array}{r}9 \\
16 \\
4 \\
9 \\
0\end{array}$ & $\begin{array}{l}2 \\
1 \\
1 \\
5 \\
0\end{array}$ & $\begin{array}{l}0 \\
0 \\
0 \\
0 \\
0\end{array}$ \\
\hline $\begin{array}{l}6-01 \\
6-03 \\
6-05 \\
6-07 \\
6-09\end{array}$ & $\begin{array}{c}108+ \\
73++ \\
73+ \\
134 \\
555\end{array}$ & $\begin{array}{l}30 \\
11 \\
21 \\
32 \\
12\end{array}$ & $\begin{array}{l}6 \\
3 \\
3 \\
4 \\
2\end{array}$ & $\begin{array}{r}21 \\
8 \\
12 \\
28 \\
6\end{array}$ & $\begin{array}{l}3 \\
0 \\
6 \\
0 \\
2\end{array}$ & $\begin{array}{l}0 \\
0 \\
0 \\
0 \\
2\end{array}$ & $\begin{array}{l}0 \\
0 \\
0 \\
0 \\
0\end{array}$ \\
\hline $\begin{array}{l}6-10 \\
6-11 \\
6-12 \\
6-13 \\
6-14\end{array}$ & $\begin{array}{r}714 \\
5615 \\
6832 \\
5258 \\
2459\end{array}$ & $\begin{array}{l}27 \\
16 \\
31 \\
26 \\
13\end{array}$ & $\begin{array}{l}5 \\
0 \\
2 \\
6 \\
1\end{array}$ & $\begin{array}{r}15 \\
10 \\
26 \\
16 \\
3\end{array}$ & $\begin{array}{l}7 \\
3 \\
1 \\
4 \\
8\end{array}$ & $\begin{array}{l}0 \\
3 \\
2 \\
0 \\
1\end{array}$ & $\begin{array}{l}0 \\
0 \\
0 \\
0 \\
0\end{array}$ \\
\hline $6-15$ & 2313 & 20 & 3 & 14 & 3 & 0 & 0 \\
\hline $\begin{array}{l}6-17 \\
6-18 \\
6-19\end{array}$ & $\begin{array}{l}1205 \\
1785 \\
1143\end{array}$ & $\begin{array}{l}12 \\
16 \\
11\end{array}$ & $\begin{array}{l}1 \\
0 \\
1\end{array}$ & $\begin{array}{r}8 \\
10 \\
5\end{array}$ & $\begin{array}{l}3 \\
1 \\
4\end{array}$ & $\begin{array}{l}0 \\
5 \\
1\end{array}$ & $\begin{array}{l}0 \\
0 \\
0\end{array}$ \\
\hline $\begin{array}{l}6-20 \\
6-21 \\
6-22 \\
6-23 \\
6-25\end{array}$ & $\begin{array}{l}1735 \\
6301 \\
2855 \\
3197 \\
1877\end{array}$ & $\begin{array}{r}15 \\
16 \\
17 \\
9 \\
8\end{array}$ & $\begin{array}{l}2 \\
2 \\
7 \\
0 \\
0\end{array}$ & $\begin{array}{r}8 \\
10 \\
10 \\
6 \\
4\end{array}$ & $\begin{array}{l}3 \\
3 \\
0 \\
1 \\
2\end{array}$ & $\begin{array}{l}2 \\
1 \\
0 \\
2 \\
1\end{array}$ & $\begin{array}{l}0 \\
0 \\
0 \\
0 \\
1\end{array}$ \\
\hline $6-27$ & 1569 & 6 & 0 & 3 & 2 & 0 & 1 \\
\hline $\begin{array}{l}6-29 \\
7-01\end{array}$ & -- & $\overline{--}$ & $\overline{--}$ & $\overline{--}$ & $\overline{--}$ & $\overline{-}$ & $\overline{--}$ \\
\hline $\begin{array}{l}7-03 \\
7-05\end{array}$ & -- & $\overline{--}$ & $\overline{--}$ & $\overline{--}$ & $=$ & $\overline{--}$ & $\overline{-}$ \\
\hline $7-07$ & -- & -- & -- & -- & -- & -- & -- \\
\hline
\end{tabular}


TABLE 16.- NUMBER OF BLUE TRACER PARTICLES(1), PER 100 GRAMS OF BEDLOAD SAMPLE, COMPOSITED FROM CROSS-CHANNEL SAMPLING POSITIONS SPACED AT 1-METER INTERVALS ACROSS THE CHANNEL WIDTH, EAST FORK RIVER, WYOMING, 1980 SECTION 0985

\begin{tabular}{|c|c|c|c|c|c|c|c|}
\hline \multirow{3}{*}{ DATE } & \multirow{3}{*}{$\begin{array}{c}\text { DRY } \\
\text { MASS } \\
\text { OF } \\
\text { SAMPLE (2) } \\
\text { (G) }\end{array}$} & \multicolumn{6}{|c|}{ NUMBER OF BLUE TRACER PARTICLES } \\
\hline & & \multirow[b]{2}{*}{ TOTAL } & \multicolumn{3}{|c|}{ BY SIZE CLASS } & \multicolumn{2}{|c|}{$(\mathbf{M M})$} \\
\hline & & & $\begin{array}{l}0.25 \\
\text { TO } \\
0.50\end{array}$ & $\begin{array}{l}0.50 \\
\text { TO } \\
1.00\end{array}$ & $\begin{array}{l}1.00 \\
2.00\end{array}$ & $\begin{array}{l}2.00 \\
\text { TO } \\
4.00\end{array}$ & $\begin{array}{l}4.00 \\
\text { TO } \\
8.00\end{array}$ \\
\hline $\begin{array}{l}5-16 \\
5-19 \\
5-21 \\
5-22\end{array}$ & $\begin{array}{r}344 \\
323 \\
2789 \\
\end{array}$ & $\begin{array}{l}46 \\
36 \\
26 \\
--\end{array}$ & $\begin{array}{r}5 \\
2 \\
7 \\
--\end{array}$ & $\begin{array}{l}20 \\
20 \\
17 \\
--\end{array}$ & $\begin{array}{r}17 \\
11 \\
1\end{array}$ & $\begin{array}{r}4 \\
3 \\
1 \\
--\end{array}$ & $\begin{array}{r}0 \\
0 \\
0 \\
--\end{array}$ \\
\hline $5-24$ & - & - & - & - & -- & -- & -- \\
\hline $\begin{array}{l}5-26 \\
5-27 \\
5-28\end{array}$ & $\begin{array}{c}3289 \\
73+ \\
105+\end{array}$ & $\begin{array}{l}38 \\
15 \\
12\end{array}$ & $\begin{array}{l}\frac{1}{1} \\
\frac{1}{2}\end{array}$ & $\begin{array}{r}17 \\
4 \\
6\end{array}$ & $\begin{array}{r}18 \\
7 \\
1\end{array}$ & $\begin{array}{l}2 \\
2 \\
3\end{array}$ & $\begin{array}{l}0 \\
1 \\
0\end{array}$ \\
\hline $\begin{array}{l}5-29 \\
5-30\end{array}$ & $\overline{10++}$ & $2 \overline{0}$ & $\overline{0}$ & $\overline{20}$ & $\overline{0}$ & $\overline{0}$ & $\overline{0}$ \\
\hline $\begin{array}{l}6-01 \\
6-03 \\
6-05 \\
6-07 \\
6-09\end{array}$ & $\begin{array}{c}5+++ \\
39++ \\
19+++ \\
50++ \\
1844\end{array}$ & $\begin{array}{r}0 \\
5 \\
0 \\
20 \\
20\end{array}$ & $\begin{array}{l}0 \\
0 \\
0 \\
2 \\
2\end{array}$ & $\begin{array}{r}0 \\
5 \\
0 \\
144 \\
13\end{array}$ & $\begin{array}{l}0 \\
0 \\
0 \\
4 \\
3\end{array}$ & $\begin{array}{l}0 \\
0 \\
0 \\
0 \\
1\end{array}$ & $\begin{array}{l}0 \\
0 \\
0 \\
0 \\
1\end{array}$ \\
\hline $\begin{array}{l}6-10 \\
6-11 \\
6-12 \\
6-13 \\
6-14\end{array}$ & $\begin{array}{l}5658 \\
3740 \\
4765 \\
8303 \\
7534\end{array}$ & $\begin{array}{l}39 \\
18 \\
19 \\
19 \\
20\end{array}$ & $\begin{array}{l}9 \\
2 \\
3 \\
3 \\
3\end{array}$ & $\begin{array}{l}21 \\
12 \\
11 \\
10 \\
13\end{array}$ & $\begin{array}{l}7 \\
2 \\
4 \\
5 \\
4\end{array}$ & $\begin{array}{l}2 \\
2 \\
1 \\
1 \\
0\end{array}$ & $\begin{array}{l}0 \\
0 \\
0 \\
0 \\
0\end{array}$ \\
\hline $6-15$ & 3829 & 12 & 2 & 7 & 2 & 1 & 0 \\
\hline $\begin{array}{l}6-10 \\
6-17 \\
6-18 \\
6-19\end{array}$ & $\begin{array}{r}954 \\
3865 \\
1696\end{array}$ & $\begin{array}{l}15 \\
13 \\
14\end{array}$ & $\begin{array}{l}2 \\
0 \\
1\end{array}$ & $\begin{array}{l}10 \\
10 \\
10\end{array}$ & $\begin{array}{l}2 \\
2 \\
3\end{array}$ & $\begin{array}{l}1 \\
1 \\
0\end{array}$ & $\begin{array}{l}0 \\
0 \\
0\end{array}$ \\
\hline $\begin{array}{l}6-20 \\
6-21 \\
6-22 \\
6-23 \\
6-25\end{array}$ & $\begin{array}{r}1955 \\
10233 \\
2284 \\
3294 \\
2383\end{array}$ & $\begin{array}{l}11 \\
14 \\
11 \\
8 \\
10\end{array}$ & $\begin{array}{l}5 \\
4 \\
3 \\
1 \\
0\end{array}$ & $\begin{array}{l}3 \\
8 \\
6 \\
5 \\
8\end{array}$ & $\begin{array}{l}3 \\
2 \\
1 \\
1 \\
1\end{array}$ & $\begin{array}{l}0 \\
0 \\
1 \\
1 \\
1\end{array}$ & $\begin{array}{l}0 \\
0 \\
0 \\
0 \\
0\end{array}$ \\
\hline $6-27$ & 2130 & 12 & 0 & 5 & 3 & 4 & 0 \\
\hline $\begin{array}{l}6-29 \\
7-07\end{array}$ & $\overline{--}$ & $\overline{-}$ & $=$ & $z$ & $\overline{--}$ & -- & $\overline{--}$ \\
\hline $7-03$ & -- & -- & -- & -- & -- & -- & - \\
\hline $7-05$ & -- & -- & -- & -- & -- & -- & -- \\
\hline $7-07$ & -- & -- & -- & -- & -- & -- & -- \\
\hline
\end{tabular}


TABLE 16.- NUMBER OF BLUE TRACER PARTICLES(1), PER 100 GRAMS OF BEDLOAD SAMPLE, COMPOSITED FROM CROSS-CHANNEL SAMPLING POSITIONS SPACED AT 1-METER INTERVALS ACROSS THE CHANNEL WIDTH,

EAST FORK RIVER, WYOMING, 1980

SECTION 1038

\begin{tabular}{|c|c|c|c|c|c|c|c|}
\hline \multirow{3}{*}{\multicolumn{2}{|c|}{$\begin{array}{lc}\text { DATE } & \text { DRY } \\
\text { MASS } & \text { OF } \\
& \text { SAMPLE (2) } \\
& (\mathrm{G})\end{array}$}} & \multicolumn{6}{|c|}{ NUMBER OF BLUE TRACER PARTICLES } \\
\hline & & \multirow[b]{2}{*}{ TOTAL } & \multicolumn{5}{|c|}{ BY SIZE CLASS (MM) } \\
\hline & & & $\begin{array}{l}0.25 \\
\text { TO } \\
0.50\end{array}$ & $\begin{array}{l}0.50 \\
\text { TO } \\
1.00\end{array}$ & $\begin{array}{l}1.00 \\
\text { TO } \\
2.00\end{array}$ & $\begin{array}{l}2.00 \\
\text { TO } \\
4.00\end{array}$ & $\begin{array}{l}4.00 \\
\text { TO } \\
8.00\end{array}$ \\
\hline $\begin{array}{l}5-16 \\
5-19 \\
5-21 \\
5-22 \\
5-24\end{array}$ & $\begin{array}{l}153+ \\
700 \\
3042 \\
2365 \\
4864\end{array}$ & $\begin{array}{l}31 \\
46 \\
46 \\
35 \\
25\end{array}$ & $\begin{array}{r}0 \\
1 \frac{1}{7} \\
3 \\
5\end{array}$ & $\begin{array}{l}19 \\
30 \\
32 \\
24 \\
11\end{array}$ & $\begin{array}{r}12 \\
3 \\
6 \\
7 \\
7\end{array}$ & $\begin{array}{l}0 \\
\frac{1}{1} \\
\frac{1}{1} \\
2\end{array}$ & $\begin{array}{l}0 \\
1 \\
0 \\
0 \\
0\end{array}$ \\
\hline $\begin{array}{l}5-26 \\
5-27 \\
5-28 \\
5-20\end{array}$ & $\begin{array}{c}\overline{118+} \\
18++\end{array}$ & $\begin{array}{r}-\overline{5} \\
0\end{array}$ & $\begin{array}{r}-- \\
0\end{array}$ & $\begin{array}{r}-\overline{9} \\
0\end{array}$ & $--\overline{4}$ & -- & $\begin{array}{r}-- \\
0 \\
0\end{array}$ \\
\hline $5-30$ & $26++$ & 27 & 0 & 27 & 0 & 0 & 0 \\
\hline $\begin{array}{l}6-01 \\
6-03 \\
6-05 \\
6-07 \\
6-09\end{array}$ & $\begin{array}{r}5+++ \\
17+++ \\
0+++ \\
0+++ \\
1865\end{array}$ & $\begin{array}{r}0 \\
18 \\
0 \\
0 \\
6\end{array}$ & $\begin{array}{l}0 \\
0 \\
0 \\
0 \\
1\end{array}$ & $\begin{array}{r}0 \\
12 \\
0 \\
0 \\
3\end{array}$ & $\begin{array}{l}0 \\
6 \\
0 \\
0 \\
2\end{array}$ & $\begin{array}{l}0 \\
0 \\
0 \\
0 \\
0\end{array}$ & $\begin{array}{l}0 \\
0 \\
0 \\
0 \\
0\end{array}$ \\
\hline $\begin{array}{l}6-10 \\
6-11 \\
6-12 \\
6-13 \\
6-14\end{array}$ & $\begin{array}{r}6434 \\
7545 \\
12301 \\
1950 \\
2923\end{array}$ & $\begin{array}{r}21 \\
19 \\
13 \\
11 \\
8\end{array}$ & $\begin{array}{l}5 \\
1 \\
2 \\
1 \\
1\end{array}$ & $\begin{array}{r}13 \\
6 \\
7 \\
7 \\
6\end{array}$ & $\begin{array}{l}3 \\
2 \\
4 \\
2 \\
1\end{array}$ & $\begin{array}{l}0 \\
0 \\
0 \\
1 \\
0\end{array}$ & $\begin{array}{l}0 \\
0 \\
0 \\
0 \\
0\end{array}$ \\
\hline $6-15$ & 238 & 11 & 4 & 7 & 0 & 0 & 0 \\
\hline $\begin{array}{l}6-17 \\
6-18 \\
6-19\end{array}$ & $\begin{array}{r}468 \\
1679 \\
249\end{array}$ & $\begin{array}{l}21 \\
21 \\
12\end{array}$ & $\begin{array}{r}10 \\
2 \\
3\end{array}$ & $1 \frac{9}{8}$ & $\begin{array}{l}\frac{1}{7} \\
1\end{array}$ & $\begin{array}{l}1 \\
1 \\
0\end{array}$ & $\begin{array}{l}0 \\
0 \\
0\end{array}$ \\
\hline $\begin{array}{l}6-20 \\
6-21 \\
6-22 \\
6-23 \\
6-25\end{array}$ & $\begin{array}{r}1361 \\
2079 \\
1187 \\
2267 \\
809\end{array}$ & $\begin{array}{r}11 \\
11 \\
5 \\
2 \\
6\end{array}$ & $\begin{array}{l}1 \\
3 \\
0 \\
0 \\
0\end{array}$ & $\begin{array}{l}7 \\
5 \\
3 \\
0 \\
5\end{array}$ & $\begin{array}{l}3 \\
2 \\
1 \\
1 \\
0\end{array}$ & $\begin{array}{l}0 \\
1 \\
1 \\
1 \\
1\end{array}$ & $\begin{array}{l}0 \\
0 \\
0 \\
0 \\
0\end{array}$ \\
\hline $6-27$ & 2291 & 17 & $\underline{2}$ & 13 & 1 & 1 & $\underline{0}$ \\
\hline $7-01$ & - & - & - & - & - & - & - \\
\hline $\begin{array}{l}7-03 \\
7-05\end{array}$ & $=$ & $=$ & $=$ & $=$ & $=$ & $=$ & $=$ \\
\hline $7-07$ & -- & -- & -- & -- & -- & -- & -- \\
\hline
\end{tabular}


TABLE 16.- NUMBER OF BLUE TRACER PARTICLES(1), PER 100 GRAMS OF BEDLOAD SAMPLE, COMPOSITED FROM CROSS-CHANNEL SAMPLING POSITIONS

SPACED AT I-METER INTERVALS ACROSS THE CHANNEL WIDTH,

EAST FORK RIVER, WYOMING, 1980

SECTION 1077

\begin{tabular}{|c|c|c|c|c|c|c|c|}
\hline \multirow{3}{*}{ DATE } & \multirow{3}{*}{$\begin{array}{c}\text { DRY } \\
\text { MASS } \\
\text { OF } \\
\text { SAMPLE (2) } \\
(G)\end{array}$} & \multicolumn{6}{|c|}{ NUMBER OF BLUE TRACER PARTICLES } \\
\hline & & \multirow[b]{2}{*}{ TOTAL } & \multicolumn{5}{|c|}{ BY SIZE CLASS (MM) } \\
\hline & & & $\begin{array}{l}0.25 \\
\text { TO } \\
0.50\end{array}$ & $\begin{array}{r}0.50 \\
\text { TO } \\
1.00\end{array}$ & $\begin{array}{l}1.00 \\
\text { To } \\
2.00\end{array}$ & $\begin{array}{r}2.00 \\
\text { TO } \\
4.00\end{array}$ & $\begin{array}{r}4.00 \\
\text { TO } \\
8.00\end{array}$ \\
\hline $\begin{array}{l}5-16 \\
5-19 \\
5-21 \\
5-22 \\
5-24\end{array}$ & $\begin{array}{l}488 \\
141+ \\
1036 \\
6690 \\
2093\end{array}$ & $\begin{array}{l}29 \\
29 \\
21 \\
23 \\
28\end{array}$ & $\begin{array}{r}4 \\
6 \\
7 \\
1 \\
11\end{array}$ & $\begin{array}{r}22 \\
16 \\
6 \\
13 \\
13\end{array}$ & $\begin{array}{l}3 \\
4 \\
6 \\
7 \\
4\end{array}$ & $\begin{array}{l}0 \\
3 \\
1 \\
1 \\
0\end{array}$ & $\begin{array}{l}0 \\
0 \\
1 \\
1 \\
0\end{array}$ \\
\hline $\begin{array}{l}5-26 \\
5-27 \\
5-28\end{array}$ & $\begin{array}{c}\overline{66}+ \\
247\end{array}$ & $\begin{array}{l}\overline{20} \\
20\end{array}$ & $\begin{array}{r}--\overline{2} \\
0\end{array}$ & $\begin{array}{l}-\overline{1} \\
12\end{array}$ & $\begin{array}{r}-- \\
3 \\
3\end{array}$ & $\begin{array}{r}-\overline{0} \\
0\end{array}$ & $\begin{array}{r}-- \\
0 \\
0\end{array}$ \\
\hline $5-30$ & $93+$ & 26 & 2 & 23 & 1 & 0 & 0 \\
\hline $\begin{array}{l}6-01 \\
6-03 \\
6-05 \\
6-07 \\
6-09\end{array}$ & $\begin{array}{l}4^{4+++} \\
368 \\
443 \\
861 \\
6847\end{array}$ & $\begin{array}{l}0 \\
7 \\
7 \\
6 \\
4\end{array}$ & $\begin{array}{l}0 \\
0 \\
0 \\
0 \\
2\end{array}$ & $\begin{array}{l}0 \\
7 \\
4 \\
2 \\
2\end{array}$ & $\begin{array}{l}0 \\
0 \\
3 \\
0 \\
0\end{array}$ & $\begin{array}{l}0 \\
0 \\
0 \\
2 \\
0\end{array}$ & $\begin{array}{l}0 \\
0 \\
0 \\
2 \\
0\end{array}$ \\
\hline $\begin{array}{l}6-10 \\
6-11 \\
6-12 \\
6-13 \\
6-14\end{array}$ & $\begin{array}{l}5686 \\
7026 \\
5955 \\
4795 \\
3167\end{array}$ & $\begin{array}{r}13 \\
1 \\
15 \\
12 \\
19\end{array}$ & $\begin{array}{l}3 \\
0 \\
2 \\
0 \\
3\end{array}$ & $\begin{array}{r}3 \\
1 \\
9 \\
10 \\
11\end{array}$ & $\begin{array}{l}5 \\
0 \\
4 \\
0 \\
5\end{array}$ & $\begin{array}{l}1 \\
0 \\
0 \\
2 \\
0\end{array}$ & $\begin{array}{l}1 \\
0 \\
0 \\
0 \\
0\end{array}$ \\
\hline $6-15$ & 1830 & 18 & 2 & 12 & 3 & 1 & 0 \\
\hline $\begin{array}{l}6-17 \\
6-18 \\
6-19\end{array}$ & $\begin{array}{l}1193 \\
3263 \\
4561\end{array}$ & $\begin{array}{l}14 \\
36 \\
13\end{array}$ & $\begin{array}{l}2 \\
7 \\
4\end{array}$ & $\begin{array}{r}8 \\
25 \\
7\end{array}$ & $\begin{array}{l}1 \\
4 \\
2\end{array}$ & $\begin{array}{l}2 \\
0 \\
0\end{array}$ & $\begin{array}{l}1 \\
0 \\
0\end{array}$ \\
\hline $\begin{array}{l}6-20 \\
6-21\end{array}$ & $\begin{array}{l}5961 \\
2481\end{array}$ & $\begin{array}{r}8 \\
8\end{array}$ & $\begin{array}{l}2 \\
0\end{array}$ & $\begin{array}{l}5 \\
4\end{array}$ & $\frac{1}{4}$ & $\begin{array}{l}0 \\
0\end{array}$ & $\begin{array}{l}0 \\
0\end{array}$ \\
\hline $\begin{array}{l}6-23 \\
6-25\end{array}$ & $\begin{array}{r}5700 \\
878\end{array}$ & $\begin{array}{l}2 \\
9\end{array}$ & $\begin{array}{l}0 \\
0\end{array}$ & $\begin{array}{l}0 \\
6\end{array}$ & $\begin{array}{l}2 \\
3\end{array}$ & $\begin{array}{l}0 \\
0\end{array}$ & $\begin{array}{l}0 \\
0\end{array}$ \\
\hline $6-27$ & 2988 & $\underline{9}$ & 1 & 5 & 3 & $\underline{0}$ & $\underline{0}$ \\
\hline $\begin{array}{l}6-29 \\
7-01\end{array}$ & -- & $\overline{--}$ & - & $\overline{--}$ & $\overline{--}$ & $\overline{--}$ & $\overline{--}$ \\
\hline $\begin{array}{l}7-03 \\
7-05\end{array}$ & $\overline{--}$ & $\overline{--}$ & -- & $\overline{--}$ & $\overline{--}$ & $\overline{--}$ & -- \\
\hline $7-07$ & -- & -- & -- & -- & -- & -- & -- \\
\hline
\end{tabular}


TABLE 16.- NUMBER OF BLUE TRACER PARTICLES(1), PER 100 GRAMS OF BEDLOAD SAMPLE, COMPOSITED FROM CROSS-CHANNEL SAMPLING POSITIONS SPACED AT 1-METER INTERVALS ACROSS THE CHANNEL WIDTH, EAST FORK RIVER, WYOMING, 1980

SECTION 1120

\begin{tabular}{|c|c|c|c|c|c|c|c|}
\hline \multirow{3}{*}{ DATE } & \multirow{3}{*}{$\begin{array}{c}\text { DRY } \\
\text { MASS } \\
\text { OF } \\
\text { SAMPLE (2) } \\
\text { (G) }\end{array}$} & \multicolumn{6}{|c|}{ NUMBER OF BLUE TRACER PARTICLES } \\
\hline & & \multirow[b]{2}{*}{ TOTAL } & \multicolumn{5}{|c|}{ BY SIZE CLASS (MM) } \\
\hline & & & $\begin{array}{l}0.25 \\
\text { TOO } \\
0.50\end{array}$ & $\begin{array}{l}0.50 \\
\text { TO } \\
1.00\end{array}$ & $\begin{array}{l}1.00 \\
\text { TO } \\
2.00\end{array}$ & $\begin{array}{l}2.00 \\
\text { TO } \\
4.00\end{array}$ & $\begin{array}{l}4.00 \\
\text { TO } \\
8.00\end{array}$ \\
\hline $\begin{array}{l}5-16 \\
5-19 \\
5-21 \\
5-22 \\
5-24\end{array}$ & $\begin{array}{c}72+ \\
273+ \\
3540 \\
5580 \\
2052\end{array}$ & $\begin{array}{l}17 \\
14 \\
17 \\
11 \\
18\end{array}$ & $\begin{array}{l}0 \\
2 \\
3 \\
0 \\
5\end{array}$ & $\begin{array}{r}10 \\
10 \\
9 \\
6 \\
9\end{array}$ & $\begin{array}{l}4 \\
2 \\
4 \\
3 \\
4\end{array}$ & $\begin{array}{l}3 \\
0 \\
1 \\
2 \\
0\end{array}$ & $\begin{array}{l}0 \\
0 \\
0 \\
0 \\
0\end{array}$ \\
\hline $\begin{array}{l}5-26 \\
5-27 \\
5-28\end{array}$ & $\begin{array}{l}3 \overline{0} \\
273\end{array}$ & $\begin{array}{l}-- \\
17 \\
16\end{array}$ & $\begin{array}{r}-\pi \\
2\end{array}$ & $\frac{11}{11}$ & $-\frac{-}{2}$ & $-\frac{-}{1}$ & $\begin{array}{r}-\overline{0} \\
0\end{array}$ \\
\hline $5-30$ & $103+$ & 5 & 1 & 4 & 0 & 0 & 0 \\
\hline $\begin{array}{l}6-01 \\
6-03 \\
6-05 \\
6-07 \\
6-09\end{array}$ & $\begin{array}{r}77++ \\
84++ \\
90+ \\
1350 \\
6295\end{array}$ & $\begin{array}{r}17 \\
5 \\
4 \\
29 \\
1\end{array}$ & $\begin{array}{r}1 \\
0 \\
0 \\
14 \\
0\end{array}$ & $\begin{array}{r}11 \\
5 \\
1 \\
15 \\
0\end{array}$ & $\begin{array}{l}5 \\
0 \\
3 \\
0 \\
1\end{array}$ & $\begin{array}{l}0 \\
0 \\
0 \\
0 \\
0\end{array}$ & $\begin{array}{l}0 \\
0 \\
0 \\
0 \\
0\end{array}$ \\
\hline $\begin{array}{l}6-10 \\
6-11 \\
6-12 \\
6-13 \\
6-14\end{array}$ & $\begin{array}{r}5355 \\
2111 \\
1473 \\
537 \\
1581\end{array}$ & $\begin{array}{r}14 \\
21 \\
14 \\
8 \\
0\end{array}$ & $\begin{array}{l}6 \\
6 \\
4 \\
0 \\
0\end{array}$ & $\begin{array}{r}7 \\
13 \\
5 \\
3 \\
0\end{array}$ & $\begin{array}{l}1 \\
2 \\
5 \\
5 \\
0\end{array}$ & $\begin{array}{l}0 \\
0 \\
0 \\
0 \\
0\end{array}$ & $\begin{array}{l}0 \\
0 \\
0 \\
0 \\
0\end{array}$ \\
\hline $6-15$ & 2074 & 7 & 1 & 3 & 3 & 0 & 0 \\
\hline $\begin{array}{l}6-17 \\
6-18 \\
6-19\end{array}$ & $\begin{array}{l}1501 \\
4293 \\
2789\end{array}$ & $\begin{array}{r}15 \\
10 \\
8\end{array}$ & $\begin{array}{l}3 \\
0 \\
0\end{array}$ & $\begin{array}{r}10 \\
7 \\
7\end{array}$ & $\begin{array}{l}2 \\
3 \\
1\end{array}$ & $\begin{array}{l}0 \\
0 \\
0\end{array}$ & $\begin{array}{l}0 \\
0 \\
0\end{array}$ \\
\hline $\begin{array}{l}6-20 \\
6-21\end{array}$ & $\begin{array}{l}3904 \\
2718\end{array}$ & $\begin{array}{l}6 \\
0\end{array}$ & $\frac{1}{0}$ & $\begin{array}{l}4 \\
0\end{array}$ & $\frac{1}{0}$ & $\begin{array}{l}0 \\
0\end{array}$ & $\begin{array}{l}0 \\
0\end{array}$ \\
\hline $\begin{array}{l}6-23 \\
6-25 \\
6-25\end{array}$ & $\begin{array}{l}2504 \\
2506\end{array}$ & 7 & $\begin{array}{l}3 \\
0\end{array}$ & $\begin{array}{l}3 \\
1\end{array}$ & $\frac{1}{1}$ & $\begin{array}{l}0 \\
0\end{array}$ & $\begin{array}{l}0 \\
0\end{array}$ \\
\hline $6-27$ & 4237 & 3 & 0 & 2 & 1 & 0 & 0 \\
\hline $\begin{array}{l}6-29 \\
7-01\end{array}$ & $\overline{--}$ & $\overline{-}$ & $\overline{--}$ & $\overline{--}$ & - & $\overline{-}$ & $\overline{-}$ \\
\hline $\begin{array}{l}7-03 \\
7-05\end{array}$ & $\overline{--}$ & $\overline{--}$ & $\overline{-}$ & $\overline{-}$ & $=$ & $\overline{-}$ & $\overline{--}$ \\
\hline $7-07$ & -- & -- & -- & -- & -- & -- & -- \\
\hline
\end{tabular}


TABLE 16.- NUMBER OF BLUE TRACER PARTICLES(1), PER 100 GRAMS OF BEDLOAD SAMPLE, COMPOSITED FROM CROSS-CHANNEL SAMPLING POSITIONS SPACED AT 1-METER INTERVALS ACROSS THE CHANNEL WIDTH, EAST FORK RIVER, WYOMING, 1980

SECTION 1155

\begin{tabular}{|c|c|c|c|c|c|c|c|}
\hline \multirow{3}{*}{ DATE } & \multirow{3}{*}{$\begin{array}{c}\text { DRY } \\
\text { MASS } \\
\text { OF } \\
\text { SAMPLE (2) } \\
(\mathrm{G})\end{array}$} & \multicolumn{6}{|c|}{ NUMBER OF BLUE TRACER PARTICLES } \\
\hline & & \multirow[b]{2}{*}{ TOTAL } & \multicolumn{5}{|c|}{ BY SIZE CLASS (MM) } \\
\hline & & & $\begin{array}{l}0.25 \\
\text { TO } \\
0.50\end{array}$ & $\begin{array}{l}0.50 \\
\text { TO } \\
1.00\end{array}$ & $\begin{array}{l}1.00 \\
2.00 \\
\text { TO }\end{array}$ & $\begin{array}{l}2.00 \\
\text { TO } \\
4.00\end{array}$ & $\begin{array}{l}4.00 \\
\text { To } \\
8.00\end{array}$ \\
\hline $\begin{array}{l}5-16 \\
5-19 \\
5-21 \\
5-22 \\
5-24\end{array}$ & $\begin{array}{r}177^{+++} \\
73^{+++} \\
2437 \\
2980 \\
2253\end{array}$ & $\begin{array}{r}0 \\
0 \\
16 \\
6 \\
25\end{array}$ & $\begin{array}{l}0 \\
0 \\
1 \\
2 \\
8\end{array}$ & $\begin{array}{r}0 \\
0 \\
11 \\
4 \\
9\end{array}$ & $\begin{array}{l}0 \\
0 \\
2 \\
0 \\
7\end{array}$ & $\begin{array}{l}0 \\
0 \\
2 \\
0 \\
1\end{array}$ & $\begin{array}{l}0 \\
0 \\
0 \\
0 \\
0\end{array}$ \\
\hline $\begin{array}{l}5-26 \\
5-27 \\
5-28 \\
5-29 \\
5-30\end{array}$ & $\begin{array}{l}\overline{25}++ \\
51+ \\
\overline{52+}\end{array}$ & $\begin{array}{l}-\overline{20} \\
10 \\
\overline{56}\end{array}$ & $\begin{array}{r}--\overline{0} \\
-0 \\
-0\end{array}$ & $\begin{array}{r}20 \\
42\end{array}$ & $\begin{array}{r}-\overline{0} \\
-\overline{0} \\
-\frac{1}{2}\end{array}$ & $\begin{array}{r}-\overline{0} \\
-\frac{-}{2}\end{array}$ & $\begin{array}{r}-\overline{0} \\
-\frac{1}{0}\end{array}$ \\
\hline $\begin{array}{l}6-01 \\
6-03 \\
6-05 \\
6-07 \\
6-09\end{array}$ & $\begin{array}{c}46++ \\
87^{++} \\
23+++ \\
341 \\
2849\end{array}$ & $\begin{array}{r}2 \\
3 \\
0 \\
17 \\
15\end{array}$ & $\begin{array}{l}0 \\
0 \\
0 \\
4 \\
5\end{array}$ & $\begin{array}{r}2 \\
2 \\
0 \\
10 \\
10\end{array}$ & $\begin{array}{l}0 \\
1 \\
0 \\
2 \\
0\end{array}$ & $\begin{array}{l}0 \\
0 \\
0 \\
1 \\
0\end{array}$ & $\begin{array}{l}0 \\
0 \\
0 \\
0 \\
0\end{array}$ \\
\hline $\begin{array}{l}6-10 \\
6-11 \\
6-12 \\
6-13 \\
6-14\end{array}$ & $\begin{array}{r}4178 \\
1008 \\
776 \\
526 \\
2043\end{array}$ & $\begin{array}{l}20 \\
22 \\
16 \\
9 \\
10\end{array}$ & $\begin{array}{r}10 \\
1 \\
4 \\
1\end{array}$ & $\begin{array}{r}14 \\
10 \\
12 \\
1 \\
6\end{array}$ & $\begin{array}{l}4 \\
\frac{1}{3} \\
\frac{1}{3}\end{array}$ & $\begin{array}{l}1 \\
1 \\
0 \\
3 \\
0\end{array}$ & $\begin{array}{l}0 \\
0 \\
0 \\
0 \\
0\end{array}$ \\
\hline $\begin{array}{l}6-15 \\
6-16 \\
6-17 \\
6-18 \\
6-19\end{array}$ & $\begin{array}{r}957 \\
-- \\
659 \\
2916 \\
3706\end{array}$ & $\begin{array}{r}16 \\
-4 \\
8 \\
10\end{array}$ & $\begin{array}{r}-\frac{1}{0} \\
0 \\
2 \\
1\end{array}$ & $\begin{array}{r}10 \\
-\frac{1}{2} \\
2 \\
6\end{array}$ & $-\frac{3}{2}$ & $\begin{array}{r}-\frac{2}{0} \\
0 \\
0 \\
0\end{array}$ & $\begin{array}{c}0 \\
-\frac{1}{0} \\
0 \\
0\end{array}$ \\
\hline $\begin{array}{l}6-20 \\
6-21\end{array}$ & $\begin{array}{l}1301 \\
2023\end{array}$ & $\begin{array}{l}9 \\
4\end{array}$ & $\begin{array}{l}2 \\
0\end{array}$ & $\frac{1}{3}$ & $\begin{array}{l}5 \\
1\end{array}$ & $\begin{array}{l}1 \\
0\end{array}$ & $\begin{array}{l}0 \\
0\end{array}$ \\
\hline $\begin{array}{l}0-23 \\
6-25\end{array}$ & $\begin{array}{r}1384 \\
629\end{array}$ & $\overline{2}$ & 0 & 2 & 0 & $\begin{array}{l}0 \\
1\end{array}$ & $\begin{array}{l}0 \\
0\end{array}$ \\
\hline $6-27$ & 3473 & 1 & 0 & 1 & 0 & 0 & 0 \\
\hline $\begin{array}{l}6-29 \\
7-01\end{array}$ & $\overline{--}$ & $\overline{--}$ & $\overline{--}$ & - & $\overline{--}$ & $\overline{--}$ & $\overline{-}$ \\
\hline $\begin{array}{l}7-03 \\
7-05\end{array}$ & $\overline{--}$ & $\overline{--}$ & -- & $\overline{--}$ & $\overline{--}$ & $z$ & -- \\
\hline $7-07$ & -- & -- & -- & -- & -- & -- & -- \\
\hline
\end{tabular}


TABLE 16.- NUMBER OF BLUE TRACER PARTICLES(1), PER 100 GRAMS OF BEDLOAD SAMPLE, COMPOSITED FROM CROSS-CHANNEL SAMPLING POSITIONS SPACED AT 1-METER INTERVALS ACROSS THE CHANNEL WIDTH, EAST FORK RIVER, WYOMING, 1980

SECTION 1202

\begin{tabular}{|c|c|c|c|c|c|c|c|}
\hline \multirow{3}{*}{ DATE } & \multirow{3}{*}{$\begin{array}{c}\text { DRY } \\
\text { MASS } \\
\text { OF } \\
\text { SAMPLE (2) } \\
\text { (G) }\end{array}$} & \multicolumn{6}{|c|}{ NUMBER OF BLUE TRACER PARTICLES } \\
\hline & & \multirow[b]{2}{*}{ TOTAL } & \multicolumn{5}{|c|}{ BY SIZE CLASS (MM) } \\
\hline & & & $\begin{array}{c}0.25 \\
\text { T0 } \\
0.50\end{array}$ & $\begin{array}{l}0.50 \\
\text { TO } \\
1.00\end{array}$ & $\begin{array}{l}1.00 \\
\text { TO } \\
2.00\end{array}$ & $\begin{array}{l}2.00 \\
\text { TO } \\
4.00\end{array}$ & $\begin{array}{l}4.00 \\
\text { TO } \\
8.00\end{array}$ \\
\hline $\begin{array}{l}5-16 \\
5-19 \\
5-21 \\
5-22 \\
5-24\end{array}$ & $\begin{array}{l}323 \\
251+ \\
2833 \\
6017 \\
1764\end{array}$ & $\begin{array}{l}16 \\
8 \\
21 \\
10 \\
22\end{array}$ & $\begin{array}{l}7 \\
3 \\
7 \\
0 \\
6\end{array}$ & $\begin{array}{r}8 \\
1 \\
11 \\
5 \\
12\end{array}$ & $\begin{array}{l}0 \\
2 \\
1 \\
4 \\
4\end{array}$ & $\begin{array}{l}1 \\
2 \\
2 \\
1 \\
0\end{array}$ & $\begin{array}{l}0 \\
0 \\
0 \\
0 \\
0\end{array}$ \\
\hline $\begin{array}{l}5-26 \\
5-27 \\
5-28 \\
5-29\end{array}$ & $\begin{array}{l}\overline{402} \\
113+\end{array}$ & $\begin{array}{r}-\overline{1} \\
-1\end{array}$ & $\begin{array}{r}-- \\
0 \\
--\end{array}$ & $\begin{array}{r}-- \\
16 \\
--\end{array}$ & $\begin{array}{r}-- \\
1 \\
--\end{array}$ & $\begin{array}{r}-- \\
0 \\
- \\
-\end{array}$ & $\begin{array}{r}-- \\
0 \\
-\end{array}$ \\
\hline $5-30$ & $35++$ & 9 & 0 & 9 & 0 & 0 & 0 \\
\hline $\begin{array}{l}6-01 \\
6-03 \\
6-05 \\
6-07 \\
6-09\end{array}$ & $\begin{array}{l}102++ \\
419 \\
1372 \\
1767 \\
7316\end{array}$ & $\begin{array}{r}3 \\
11 \\
12 \\
7 \\
7\end{array}$ & $\begin{array}{l}0 \\
2 \\
3 \\
0 \\
1\end{array}$ & $\begin{array}{l}3 \\
7 \\
7 \\
6 \\
3\end{array}$ & $\begin{array}{l}0 \\
2 \\
2 \\
1 \\
3\end{array}$ & $\begin{array}{l}0 \\
0 \\
0 \\
0 \\
0\end{array}$ & $\begin{array}{l}0 \\
0 \\
0 \\
0 \\
0\end{array}$ \\
\hline $\begin{array}{l}6-10 \\
6-11 \\
6-12 \\
6-13 \\
6-14\end{array}$ & $\begin{array}{r}4322 \\
1660 \\
907 \\
729 \\
2204\end{array}$ & $\begin{array}{r}8 \\
8 \\
11 \\
12 \\
14\end{array}$ & $\begin{array}{l}1 \\
3 \\
2 \\
3 \\
2\end{array}$ & $\begin{array}{l}5 \\
4 \\
4 \\
2 \\
5\end{array}$ & $\begin{array}{l}1 \\
1 \\
3 \\
6 \\
4\end{array}$ & $\begin{array}{l}1 \\
0 \\
2 \\
1 \\
3\end{array}$ & $\begin{array}{l}0 \\
0 \\
0 \\
0 \\
0\end{array}$ \\
\hline $\begin{array}{l}6-15 \\
6-16\end{array}$ & 917 & 11 & -2 & -7 & 1 & 1 & 0 \\
\hline $\begin{array}{l}6-17 \\
6-18 \\
6-19\end{array}$ & $\begin{array}{l}1171 \\
1103 \\
4168\end{array}$ & $\begin{array}{l}3 \\
4 \\
5\end{array}$ & $\begin{array}{l}1 \\
1 \\
0\end{array}$ & $\begin{array}{l}1 \\
0 \\
2\end{array}$ & $\begin{array}{l}1 \\
3 \\
2\end{array}$ & $\begin{array}{l}0 \\
0 \\
1\end{array}$ & $\begin{array}{l}0 \\
0 \\
0\end{array}$ \\
\hline $\begin{array}{l}6-20 \\
6-21 \\
6-25\end{array}$ & $\begin{array}{l}2102 \\
4380\end{array}$ & $\begin{array}{r}0 \\
6 \\
-\end{array}$ & $\begin{array}{l}0 \\
1\end{array}$ & $\begin{array}{l}0 \\
5\end{array}$ & $\begin{array}{l}0 \\
0\end{array}$ & $\begin{array}{l}0 \\
0\end{array}$ & $\begin{array}{l}0 \\
0\end{array}$ \\
\hline $\begin{array}{l}6-23 \\
6-25\end{array}$ & $\begin{array}{l}2561 \\
5151\end{array}$ & $\begin{array}{r}5 \\
12\end{array}$ & $\begin{array}{l}0 \\
0\end{array}$ & $\begin{array}{r}5 \\
10\end{array}$ & $\begin{array}{l}0 \\
1\end{array}$ & $\begin{array}{l}0 \\
1\end{array}$ & $\begin{array}{l}0 \\
0\end{array}$ \\
\hline $6-27$ & 2402 & $\underline{-1}$ & 0 & 0 & 0 & 0 & 0 \\
\hline $\begin{array}{l}0-29 \\
7-01\end{array}$ & - & - & $\overline{--}$ & - & $\overline{--}$ & $\overline{-}$ & - \\
\hline $\begin{array}{l}7-03 \\
7-05\end{array}$ & $\overline{--}$ & $\overline{--}$ & $\overline{--}$ & $\overline{--}$ & $\overline{--}$ & $\overline{--}$ & $\overline{--}$ \\
\hline $7-07$ & -- & -- & -- & -- & -- & -- & -- \\
\hline
\end{tabular}


TABLE 16.- NUMBER OF BLUE TRACER PARTICLES(1), PER 100 GRAMS OF BEDLOAD SAMPLE, COMPOSITED FROM CROSS-CHANNEL SAMPLING POSITIONS SPACED AT 1-METER INTERVALS ACROSS THE CHANNEL WIDTH, EAST FORK RIVER, WYOMING, 1980

SECTION 1241

\begin{tabular}{|c|c|c|c|c|c|c|c|}
\hline \multirow[t]{2}{*}{ DATE } & \multirow{2}{*}{$\begin{array}{c}\text { DRY } \\
\text { MASS } \\
\text { OF } \\
\text { SAMPLE (2) } \\
\text { (G) }\end{array}$} & \multicolumn{6}{|c|}{ NUMBER OF BLUE TRACER PARTICLES } \\
\hline & & TOTAL & $\begin{array}{l}0.25 \\
\text { TO } \\
0.50\end{array}$ & $\begin{array}{l}0.50 \\
\text { TO } \\
1.00\end{array}$ & $\begin{array}{l}1.00 \\
\text { TO } \\
2.00\end{array}$ & $\begin{array}{l}2.00 \\
\text { TO } \\
4.00\end{array}$ & $\begin{array}{l}4.00 \\
\text { TO } \\
8.00\end{array}$ \\
\hline $\begin{array}{l}5-16 \\
5-19 \\
5-21 \\
5-22 \\
5-24\end{array}$ & $\begin{array}{r}909 \\
359 \\
1312 \\
3206 \\
510\end{array}$ & $\begin{array}{r}9 \\
7 \\
14 \\
12 \\
22\end{array}$ & $\begin{array}{l}0 \\
2 \\
5 \\
2 \\
8\end{array}$ & $\begin{array}{r}4 \\
2 \\
8 \\
2 \\
12\end{array}$ & $\begin{array}{l}4 \\
2 \\
1 \\
4 \\
1\end{array}$ & $\begin{array}{l}1 \\
1 \\
0 \\
4 \\
1\end{array}$ & $\begin{array}{l}0 \\
0 \\
0 \\
0 \\
0\end{array}$ \\
\hline $\begin{array}{l}5-26 \\
5-27 \\
5-28 \\
5-29 \\
5-30\end{array}$ & $\begin{array}{l}-\overline{56++} \\
170 \\
660 \\
101+\end{array}$ & $\begin{array}{r}-- \\
2 \\
9 \\
3 \\
6\end{array}$ & $\begin{array}{r}-- \\
0 \\
3 \\
2 \\
0\end{array}$ & $\begin{array}{r}-- \\
2 \\
5 \\
1 \\
1\end{array}$ & $\begin{array}{l}-- \\
0 \\
1 \\
0 \\
4\end{array}$ & $\begin{array}{l}-- \\
0 \\
0 \\
0 \\
1\end{array}$ & $\begin{array}{l}-- \\
0 \\
0 \\
0 \\
0\end{array}$ \\
\hline $\begin{array}{l}6-01 \\
6-03 \\
6-05 \\
6-07 \\
6-09\end{array}$ & $\begin{array}{r}1777 \\
452 \\
759 \\
1177 \\
1501\end{array}$ & $\begin{array}{r}11 \\
12 \\
7 \\
9 \\
5\end{array}$ & $\begin{array}{l}0 \\
2 \\
0 \\
0 \\
2\end{array}$ & $\begin{array}{l}6 \\
7 \\
2 \\
5 \\
1\end{array}$ & $\begin{array}{l}4 \\
3 \\
4 \\
4 \\
2\end{array}$ & $\begin{array}{l}1 \\
0 \\
1 \\
0 \\
0\end{array}$ & $\begin{array}{l}0 \\
0 \\
0 \\
0 \\
0\end{array}$ \\
\hline $\begin{array}{l}6-10 \\
6-11 \\
6-12 \\
6-13 \\
6-14\end{array}$ & $\begin{array}{r}690 \\
945 \\
324 \\
308 \\
1093\end{array}$ & $\begin{array}{l}3 \\
12 \\
12 \\
18 \\
11\end{array}$ & $\begin{array}{l}2 \\
5 \\
1 \\
4 \\
5\end{array}$ & $\begin{array}{r}0 \\
6 \\
6 \\
11 \\
5\end{array}$ & $\begin{array}{l}1 \\
1 \\
4 \\
1 \\
1\end{array}$ & $\begin{array}{l}0 \\
0 \\
1 \\
1 \\
0\end{array}$ & $\begin{array}{l}0 \\
0 \\
0 \\
1 \\
0\end{array}$ \\
\hline $\begin{array}{l}6-15 \\
6-16 \\
6-17 \\
6-18 \\
6-19\end{array}$ & $\begin{array}{l}1350 \\
301 \\
1431 \\
3603 \\
1291\end{array}$ & $\begin{array}{r}12 \\
15 \\
5 \\
6 \\
0\end{array}$ & $\begin{array}{l}0 \\
4 \\
0 \\
1 \\
0\end{array}$ & $\begin{array}{l}7 \\
9 \\
2 \\
1 \\
0\end{array}$ & $\begin{array}{l}4 \\
2 \\
1 \\
3 \\
0\end{array}$ & $\begin{array}{l}1 \\
0 \\
2 \\
1 \\
0\end{array}$ & $\begin{array}{l}0 \\
0 \\
0 \\
0 \\
0\end{array}$ \\
\hline $\begin{array}{l}6-20 \\
6-21\end{array}$ & $\begin{array}{l}2036 \\
6118\end{array}$ & $\begin{array}{l}3 \\
3\end{array}$ & $\begin{array}{l}0 \\
0\end{array}$ & $\frac{2}{2}$ & $\frac{1}{1}$ & $\begin{array}{l}0 \\
0\end{array}$ & $\begin{array}{l}0 \\
0\end{array}$ \\
\hline $\begin{array}{l}6-22 \\
6-23 \\
6-25\end{array}$ & $\begin{array}{r}45 \overline{3} \\
5117\end{array}$ & $\overline{2}$ & $\begin{array}{l}0 \\
0\end{array}$ & $\overline{2}$ & $\begin{array}{l}0 \\
0\end{array}$ & 0 & $\begin{array}{l}0 \\
0\end{array}$ \\
\hline $\begin{array}{l}6-27 \\
6-29 \\
7-01 \\
7-03 \\
7-05\end{array}$ & $\begin{array}{r}1604 \\
=- \\
=-\end{array}$ & $\begin{array}{l}2 \\
-- \\
--\end{array}$ & $\begin{array}{l}0 \\
-- \\
-- \\
--\end{array}$ & $\begin{array}{l}2 \\
-- \\
--\end{array}$ & $\begin{array}{l}0 \\
-= \\
--\end{array}$ & $\begin{array}{l}0 \\
-- \\
--\end{array}$ & $\begin{array}{l}0 \\
-- \\
--\end{array}$ \\
\hline $7-07$ & -- & -- & -- & -- & -- & -- & -- \\
\hline
\end{tabular}


TABLE 16.- NUMBER OF BLUE TRACER PARTICLES(1), PER 100 GRAMS OF BEDLOAD SAMPLE, COMPOSITED FROM CROSS-CHANNEL SAMPLING POSITIONS SPACED AT 1-METER INTERVALS ACROSS THE CHANNEL WIDTH,

EAST FORK RIVER, WYOMING, 1980

SECTION 1284

\begin{tabular}{|c|c|c|c|c|c|c|c|}
\hline \multirow{3}{*}{ DATE } & \multirow{3}{*}{$\begin{array}{c}\text { DRY } \\
\text { MASS } \\
\text { OF } \\
\text { SAMPLE (2) } \\
\text { (G) }\end{array}$} & \multicolumn{6}{|c|}{ NUMBER OF BLUE TRACER PARTICLES } \\
\hline & & \multirow[b]{2}{*}{ TOTAL } & \multicolumn{5}{|c|}{ BY SIZE CLASS (MM) } \\
\hline & & & $\begin{array}{c}0.25 \\
\text { TO } \\
0.50\end{array}$ & $\begin{array}{l}0.50 \\
\text { TO } \\
1.00\end{array}$ & $\begin{array}{l}1.00 \\
2.00 \\
\text { TO }\end{array}$ & $\begin{array}{l}2.00 \\
\text { TO } \\
4.00\end{array}$ & $\begin{array}{c}4.00 \\
\text { TO } \\
8.00\end{array}$ \\
\hline $\begin{array}{l}5-16 \\
5-19 \\
5-21 \\
5-22 \\
5-24\end{array}$ & $\begin{array}{l}3816 \\
1486 \\
1193 \\
1704 \\
763\end{array}$ & $\begin{array}{l}5 \\
2 \\
3 \\
3 \\
7\end{array}$ & $\begin{array}{l}0 \\
1 \\
0 \\
0 \\
4\end{array}$ & $\begin{array}{l}3 \\
1 \\
3 \\
0 \\
3\end{array}$ & $\begin{array}{l}2 \\
0 \\
0 \\
0 \\
0\end{array}$ & $\begin{array}{l}0 \\
0 \\
0 \\
3 \\
0\end{array}$ & $\begin{array}{l}0 \\
0 \\
0 \\
0 \\
0\end{array}$ \\
\hline $\begin{array}{l}5-26 \\
5-27 \\
5-28\end{array}$ & $\begin{array}{l}1459 \\
1247\end{array}$ & $\begin{array}{r}-- \\
11\end{array}$ & $\begin{array}{r}-- \\
0 \\
4\end{array}$ & $\begin{array}{r}-\frac{-}{2} \\
4\end{array}$ & $\begin{array}{r}-- \\
0 \\
1\end{array}$ & $\begin{array}{r}-- \\
0 \\
2\end{array}$ & $\begin{array}{r}-- \\
0 \\
0\end{array}$ \\
\hline $5-30$ & 1345 & 9 & 2 & 4 & 3 & 0 & 0 \\
\hline $\begin{array}{l}6-01 \\
6-03 \\
6-05 \\
6-07 \\
6-09\end{array}$ & $\begin{array}{r}1178 \\
741 \\
944 \\
969 \\
2293\end{array}$ & $\begin{array}{r}12 \\
2 \\
5 \\
2 \\
0\end{array}$ & $\begin{array}{l}2 \\
0 \\
1 \\
0 \\
0\end{array}$ & $\begin{array}{l}7 \\
1 \\
4 \\
0 \\
0\end{array}$ & $\begin{array}{l}3 \\
1 \\
0 \\
2 \\
0\end{array}$ & $\begin{array}{l}0 \\
0 \\
0 \\
0 \\
0\end{array}$ & $\begin{array}{l}0 \\
0 \\
0 \\
0 \\
0\end{array}$ \\
\hline $\begin{array}{l}6-10 \\
6-11 \\
6-12 \\
6-13 \\
6-14\end{array}$ & $\begin{array}{r}1907 \\
879 \\
374 \\
470 \\
897\end{array}$ & $\begin{array}{r}9 \\
6 \\
2 \\
1 \\
11\end{array}$ & $\begin{array}{l}7 \\
2 \\
0 \\
1 \\
3\end{array}$ & $\begin{array}{l}1 \\
3 \\
2 \\
0 \\
8\end{array}$ & $\begin{array}{l}1 \\
1 \\
0 \\
0 \\
0\end{array}$ & $\begin{array}{l}0 \\
0 \\
0 \\
0 \\
0\end{array}$ & $\begin{array}{l}0 \\
0 \\
0 \\
0 \\
0\end{array}$ \\
\hline $\begin{array}{l}6-15 \\
6-16 \\
6-17 \\
6-18 \\
6-19\end{array}$ & $\begin{array}{l}2970 \\
1347 \\
6911 \\
2817 \\
2731\end{array}$ & $\begin{array}{l}5 \\
6 \\
5 \\
4 \\
2\end{array}$ & $\begin{array}{l}2 \\
1 \\
0 \\
1 \\
1\end{array}$ & $\begin{array}{l}1 \\
4 \\
2 \\
1 \\
1\end{array}$ & $\begin{array}{l}2 \\
1 \\
2 \\
1 \\
0\end{array}$ & $\begin{array}{l}0 \\
0 \\
1 \\
1 \\
0\end{array}$ & $\begin{array}{l}0 \\
0 \\
0 \\
0 \\
0\end{array}$ \\
\hline $\begin{array}{l}6-20 \\
6-21\end{array}$ & $\begin{array}{l}1814 \\
1452\end{array}$ & $\begin{array}{l}3 \\
1\end{array}$ & $\begin{array}{l}0 \\
1\end{array}$ & $\begin{array}{l}1 \\
0\end{array}$ & $\begin{array}{l}2 \\
0\end{array}$ & $\begin{array}{l}0 \\
0\end{array}$ & $\begin{array}{l}0 \\
0\end{array}$ \\
\hline $\begin{array}{l}6-23 \\
6-25\end{array}$ & $\begin{array}{l}2430 \\
2306\end{array}$ & $\frac{1}{1}$ & $\begin{array}{l}0 \\
0\end{array}$ & $\frac{1}{1}$ & $\begin{array}{l}0 \\
0\end{array}$ & $\begin{array}{l}0 \\
0\end{array}$ & $\begin{array}{l}0 \\
0\end{array}$ \\
\hline $6-27$ & 3246 & 3 & 0 & 3 & 0 & 0 & 0 \\
\hline $7-01$ & -- & 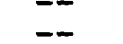 & $=$ & - & - & $=$ & $=$ \\
\hline $7-03$ & -- & -- & - & -- & -- & -- & -- \\
\hline $7-05$ & -- & -- & -- & -- & -- & -- & -- \\
\hline $7-07$ & -- & -- & - & -- & - & - & - \\
\hline
\end{tabular}


TABLE 16.- NUMBER OF BLUE TRACER PARTICLES(1), PER 100 GRAMS OF BEDLOAD SAMPLE, COMPOSITED FROM CROSS-CHANNEL SAMPLING POSITIONS SPACED AT 1-METER INTERVALS ACROSS THE CHANNEL WIDTH, EAST FORK RIVER, WYOMING, 1980

SECTION 1315

\begin{tabular}{|c|c|c|c|c|c|c|c|}
\hline \multirow{3}{*}{ DATE } & \multirow{3}{*}{$\begin{array}{c}\text { DRY } \\
\text { MASS } \\
\text { OF } \\
\text { SAMPLE (2) } \\
\text { (G) }\end{array}$} & \multicolumn{6}{|c|}{ NUMBER OF BLUE TRACER PARTICLES } \\
\hline & & \multirow[b]{2}{*}{ TOTAL } & \multicolumn{5}{|c|}{ BY SIZE CLASS (MM) } \\
\hline & & & $\begin{array}{l}0.25 \\
\text { To } \\
0.50\end{array}$ & $\begin{array}{l}0.50 \\
\text { TO } \\
1.00\end{array}$ & $\begin{array}{l}1.00 \\
2.00\end{array}$ & $\begin{array}{l}2.00 \\
\text { TO } \\
4.00\end{array}$ & $\begin{array}{l}4.00 \\
\text { TO } \\
8.00\end{array}$ \\
\hline $\begin{array}{l}5-16 \\
5-19 \\
5-21 \\
5-22 \\
5-24\end{array}$ & $\begin{array}{r}803 \\
351 \\
374 \\
684 \\
1499\end{array}$ & $\begin{array}{r}4 \\
3 \\
0 \\
7 \\
29\end{array}$ & $\begin{array}{l}1 \\
2 \\
0 \\
2 \\
4\end{array}$ & $\begin{array}{r}2 \\
0 \\
0 \\
4 \\
17\end{array}$ & $\begin{array}{l}1 \\
1 \\
0 \\
1 \\
8\end{array}$ & $\begin{array}{l}0 \\
0 \\
0 \\
0 \\
0\end{array}$ & $\begin{array}{l}0 \\
0 \\
0 \\
0 \\
0\end{array}$ \\
\hline $\begin{array}{l}5-26 \\
5-27 \\
5-28 \\
5-20\end{array}$ & $\begin{array}{r}12 \overline{63} \\
99+\end{array}$ & $\begin{array}{r}- \\
0 \\
0\end{array}$ & $\begin{array}{r}-- \\
0 \\
-0\end{array}$ & $\begin{array}{r}-- \\
0 \\
-\end{array}$ & $\begin{array}{r}-- \\
0 \\
-\end{array}$ & $\begin{array}{r}-\overline{0} \\
0\end{array}$ & $\begin{array}{r}--\overline{0} \\
0\end{array}$ \\
\hline $5-30$ & 1205 & 5 & 0 & 4 & 0 & 1 & 0 \\
\hline $\begin{array}{l}6-01 \\
6-03 \\
6-05 \\
6-07 \\
6-09\end{array}$ & $\begin{array}{c}1241 \\
217 \\
98+ \\
249 \\
483\end{array}$ & $\begin{array}{r}4 \\
9 \\
3 \\
11 \\
5\end{array}$ & $\begin{array}{l}0 \\
3 \\
0 \\
3 \\
3\end{array}$ & $\begin{array}{l}\frac{1}{5} \\
2 \\
7 \\
2\end{array}$ & $\begin{array}{l}3 \\
1 \\
1 \\
\frac{1}{1} \\
0\end{array}$ & $\begin{array}{l}0 \\
0 \\
0 \\
0 \\
0\end{array}$ & $\begin{array}{l}0 \\
0 \\
0 \\
0 \\
0\end{array}$ \\
\hline $\begin{array}{l}6-10 \\
6-11 \\
6-12 \\
6-13 \\
6-14\end{array}$ & $\begin{array}{r}2803 \\
4062 \\
842 \\
846 \\
2194\end{array}$ & $\begin{array}{r}10 \\
6 \\
6 \\
4 \\
2\end{array}$ & $\begin{array}{l}3 \\
0 \\
0 \\
1 \\
0\end{array}$ & $\begin{array}{l}7 \\
4 \\
3 \\
2 \\
1\end{array}$ & $\begin{array}{l}0 \\
1 \\
3 \\
1 \\
1\end{array}$ & $\begin{array}{l}0 \\
1 \\
0 \\
0 \\
0\end{array}$ & $\begin{array}{l}0 \\
0 \\
0 \\
0 \\
0\end{array}$ \\
\hline $\begin{array}{l}6-15 \\
6-16 \\
6-17 \\
6-18 \\
6-19\end{array}$ & $\begin{array}{l}2768 \\
3105 \\
5091 \\
2403 \\
2294\end{array}$ & $\begin{array}{l}3 \\
3 \\
4 \\
1 \\
1\end{array}$ & $\begin{array}{l}0 \\
1 \\
1 \\
0 \\
0\end{array}$ & $\begin{array}{l}2 \\
2 \\
1 \\
\frac{1}{1}\end{array}$ & $\begin{array}{l}0 \\
0 \\
1 \\
0 \\
0\end{array}$ & $\begin{array}{l}0 \\
0 \\
1 \\
0 \\
0\end{array}$ & $\begin{array}{l}1 \\
0 \\
0 \\
0 \\
0\end{array}$ \\
\hline $\begin{array}{l}6-20 \\
6-21\end{array}$ & $\begin{array}{l}2348 \\
2830\end{array}$ & $\frac{1}{1}$ & $\begin{array}{l}0 \\
0\end{array}$ & $\frac{1}{1}$ & $\begin{array}{l}0 \\
0\end{array}$ & $\begin{array}{l}0 \\
0 \\
-\end{array}$ & $\begin{array}{l}0 \\
0\end{array}$ \\
\hline $\begin{array}{l}6-23 \\
6-25\end{array}$ & $\begin{array}{l}3316 \\
6606\end{array}$ & $\begin{array}{l}2 \\
1\end{array}$ & $\begin{array}{l}0 \\
0\end{array}$ & $\begin{array}{l}0 \\
1\end{array}$ & $\begin{array}{l}0 \\
0\end{array}$ & $\begin{array}{l}2 \\
0\end{array}$ & $\begin{array}{l}0 \\
0\end{array}$ \\
\hline $\begin{array}{l}6-27 \\
6-20\end{array}$ & 2276 & 6 & -2 & -4 & -0 & -1 & -2 \\
\hline $7-01$ & -- & -- & -- & -- & -- & -- & - \\
\hline $\begin{array}{l}7-0 \overline{3} \\
7-05\end{array}$ & $=$ & $=$ & $=$ & $=$ & $=$ & $=$ & $=$ \\
\hline $7-07$ & -- & -- & -- & -- & -- & -- & -- \\
\hline
\end{tabular}


TABLE 16.- NUMBER OF BLUE TRACER PARTICLES(1), PER 100 GRAMS OF BEDLOAD SAMPLE, COMPOSITED FROM CROSS-CHANNEL SAMPLING POSITIONS SPACED AT I-METER INTERVALS ACROSS THE CHANNEL WIDTH, EAST FORK RIVER, WYOMING, 1980

SECTION $1360(4)$

\begin{tabular}{|c|c|c|c|c|c|c|c|}
\hline \multirow{3}{*}{ DATE } & \multirow{3}{*}{$\begin{array}{c}\text { DRY } \\
\text { MASS } \\
\text { OF } \\
\text { SAMPLE (2) } \\
\text { (G) }\end{array}$} & \multicolumn{6}{|c|}{ NUMBER OF BLUE TRACER PARTICLES } \\
\hline & & \multirow[b]{2}{*}{ TOTAL } & \multicolumn{5}{|c|}{ BY SIZE CLASS (MM) } \\
\hline & & & $\begin{array}{l}0.25 \\
\text { TO } \\
0.50\end{array}$ & $\begin{array}{l}0.50 \\
\text { TO } \\
1.00\end{array}$ & $\begin{array}{l}1.00 \\
2.00\end{array}$ & $\begin{array}{l}2.00 \\
\text { TO } \\
4.00\end{array}$ & $\begin{array}{l}4.00 \\
\text { TO } \\
8.00\end{array}$ \\
\hline $\begin{array}{l}5-16 \\
5-19 \\
5-21 \\
5-22 \\
5-24\end{array}$ & $\begin{array}{r}114+ \\
255+ \\
197+ \\
1553 \\
1286\end{array}$ & $\begin{array}{l}5 \\
3 \\
0 \\
1 \\
2\end{array}$ & $\begin{array}{l}0 \\
2 \\
0 \\
0 \\
0\end{array}$ & $\begin{array}{l}4 \\
1 \\
0 \\
0 \\
2\end{array}$ & $\begin{array}{l}1 \\
0 \\
0 \\
1 \\
0\end{array}$ & $\begin{array}{l}0 \\
0 \\
0 \\
0 \\
0\end{array}$ & $\begin{array}{l}0 \\
0 \\
0 \\
0 \\
0\end{array}$ \\
\hline $\begin{array}{l}5-26 \\
5-27 \\
5-28 \\
5-29\end{array}$ & 1042 & $\begin{array}{r}--7 \\
6 \\
--\end{array}$ & $\begin{array}{r}-- \\
0 \\
--\end{array}$ & $\begin{array}{r}- \\
0 \\
-4\end{array}$ & $\begin{array}{r}- \\
0 \\
1\end{array}$ & $\begin{array}{r}-- \\
7 \\
-1\end{array}$ & $\begin{array}{r}- \\
0 \\
-\end{array}$ \\
\hline $5-30$ & 724 & 0 & 0 & 0 & 0 & 0 & 0 \\
\hline $\begin{array}{l}6-01 \\
6-03 \\
6-05 \\
6-07 \\
6-09\end{array}$ & $\begin{array}{l}31++ \\
235+ \\
147 \\
535 \\
1049\end{array}$ & $\begin{array}{l}0 \\
2 \\
3 \\
3 \\
0\end{array}$ & $\begin{array}{l}0 \\
0 \\
0 \\
0 \\
0\end{array}$ & $\begin{array}{l}0 \\
1 \\
3 \\
0 \\
0\end{array}$ & $\begin{array}{l}0 \\
1 \\
0 \\
2 \\
0\end{array}$ & $\begin{array}{l}0 \\
0 \\
0 \\
1 \\
0\end{array}$ & $\begin{array}{l}0 \\
0 \\
0 \\
0 \\
0\end{array}$ \\
\hline $\begin{array}{l}6-10 \\
6-11 \\
6-12 \\
6-13 \\
6-14\end{array}$ & $\begin{array}{r}1192 \\
1011 \\
419 \\
841 \\
612\end{array}$ & $\begin{array}{l}3 \\
3 \\
1 \\
0 \\
2\end{array}$ & $\begin{array}{l}2 \\
0 \\
1 \\
0 \\
0\end{array}$ & $\begin{array}{l}1 \\
1 \\
0 \\
0 \\
2\end{array}$ & $\begin{array}{l}0 \\
1 \\
0 \\
0 \\
0\end{array}$ & $\begin{array}{l}0 \\
1 \\
0 \\
0 \\
0\end{array}$ & $\begin{array}{l}0 \\
0 \\
0 \\
0 \\
0\end{array}$ \\
\hline $\begin{array}{l}6-15 \\
6-16 \\
6-17 \\
6-18 \\
6-19\end{array}$ & $\begin{array}{r}3832 \\
1042 \\
588 \\
520 \\
1603\end{array}$ & $\begin{array}{l}0 \\
3 \\
2 \\
0 \\
0\end{array}$ & $\begin{array}{l}0 \\
0 \\
1 \\
0 \\
0\end{array}$ & $\begin{array}{l}0 \\
2 \\
1 \\
0 \\
0\end{array}$ & $\begin{array}{l}0 \\
1 \\
0 \\
0 \\
0\end{array}$ & $\begin{array}{l}0 \\
0 \\
0 \\
0 \\
0\end{array}$ & $\begin{array}{l}0 \\
0 \\
0 \\
0 \\
0\end{array}$ \\
\hline $\begin{array}{l}6-20 \\
6-21\end{array}$ & $\begin{array}{r}391 \\
1253\end{array}$ & $\begin{array}{l}0 \\
0\end{array}$ & $\begin{array}{l}0 \\
0\end{array}$ & $\begin{array}{l}0 \\
0\end{array}$ & $\begin{array}{l}0 \\
0\end{array}$ & $\begin{array}{l}0 \\
0\end{array}$ & $\begin{array}{l}0 \\
0\end{array}$ \\
\hline $\begin{array}{l}6-23 \\
6-25\end{array}$ & $\begin{array}{l}265 \\
651\end{array}$ & $\begin{array}{l}0 \\
0\end{array}$ & 0 & $\begin{array}{l}0 \\
0\end{array}$ & $\begin{array}{l}0 \\
0\end{array}$ & $\begin{array}{l}0 \\
0\end{array}$ & $\begin{array}{l}0 \\
0\end{array}$ \\
\hline $6-27$ & 870 & 0 & 0 & 0 & 0 & 0 & 0 \\
\hline $\begin{array}{l}6-29 \\
7-01\end{array}$ & -- & -- & -- & -- & -- & $=$ & $\overline{--}$ \\
\hline $7-03$ & -- & -- & -- & -- & -- & -- & -- \\
\hline $7-05$ & -- & -- & -- & -- & -- & -- & -- \\
\hline $7-07$ & -- & -- & -- & -- & -- & -- & -- \\
\hline
\end{tabular}


TABLE 16.- NUMBER OF BLUE TRACER PARTICLES(1), PER 100 GRAMS OF BEDLOAD SAMPLE, COMPOSITED FROM CROSS-CHANNEL SAMPLING POSITIONS SPACED AT 1-METER INTERVALS ACROSS THE CHANNEL WIDTH, EAST FORK RIVER, WYOMING, 1980

SECTION 1396(4)

\begin{tabular}{|c|c|c|c|c|c|c|c|}
\hline \multirow{3}{*}{ DATE } & \multirow{3}{*}{$\begin{array}{c}\text { DRY } \\
\text { MASS } \\
\text { OF } \\
\text { SAMPLE (2) } \\
\text { (G) }\end{array}$} & \multicolumn{6}{|c|}{ NUMBER OF BLUE TRACER PARTICLES } \\
\hline & & \multirow[b]{2}{*}{ TOTAL } & \multicolumn{5}{|c|}{ BY SIZE CLASS (MM) } \\
\hline & & & $\begin{array}{l}0.25 \\
\text { TO } \\
0.50\end{array}$ & $\begin{array}{l}0.50 \\
\text { TO } \\
1.00\end{array}$ & $\begin{array}{l}1.00 \\
\text { TO } \\
2.00\end{array}$ & $\begin{array}{l}2.00 \\
\text { TO } \\
4.00\end{array}$ & $\begin{array}{c}4.00 \\
\text { TO } \\
8.00\end{array}$ \\
\hline $\begin{array}{l}5-16 \\
5-19 \\
5-21 \\
5-22 \\
5-24\end{array}$ & $\begin{array}{r}331 \\
318 \\
1166 \\
589 \\
1626\end{array}$ & $\begin{array}{l}2 \\
0 \\
2 \\
0 \\
1\end{array}$ & $\begin{array}{l}0 \\
0 \\
0 \\
0 \\
1\end{array}$ & $\begin{array}{l}2 \\
0 \\
2 \\
0 \\
0\end{array}$ & $\begin{array}{l}0 \\
0 \\
0 \\
0 \\
0\end{array}$ & $\begin{array}{l}0 \\
0 \\
0 \\
0 \\
0\end{array}$ & $\begin{array}{l}0 \\
0 \\
0 \\
0 \\
0\end{array}$ \\
\hline $\begin{array}{l}5-26 \\
5-27 \\
5-28\end{array}$ & $\begin{array}{r}2 \overline{2} \\
439\end{array}$ & 10 & $-\frac{-}{1}$ & $\begin{array}{r}-- \\
\frac{7}{7}\end{array}$ & $-\frac{-}{3}$ & $\begin{array}{c}-- \\
0 \\
0\end{array}$ & $\begin{array}{r}-- \\
0 \\
0\end{array}$ \\
\hline $5-30$ & 398 & 7 & 1 & 3 & 3 & 0 & 0 \\
\hline $\begin{array}{l}6-01 \\
6-03 \\
6-05 \\
6-07 \\
6-09\end{array}$ & $\begin{array}{l}178 \\
472 \\
407 \\
532 \\
493\end{array}$ & $\begin{array}{l}0 \\
2 \\
1 \\
1 \\
0\end{array}$ & $\begin{array}{l}0 \\
0 \\
1 \\
0 \\
0\end{array}$ & $\begin{array}{l}0 \\
2 \\
0 \\
1 \\
0\end{array}$ & $\begin{array}{l}0 \\
0 \\
0 \\
0 \\
0\end{array}$ & $\begin{array}{l}0 \\
0 \\
0 \\
0 \\
0\end{array}$ & $\begin{array}{l}0 \\
0 \\
0 \\
0 \\
0\end{array}$ \\
\hline $\begin{array}{l}6-10 \\
6-11 \\
6-12 \\
6-13 \\
6-14\end{array}$ & $\begin{array}{r}2231 \\
1028 \\
467 \\
651 \\
2493\end{array}$ & $\begin{array}{l}1 \\
\frac{1}{2} \\
1 \\
1\end{array}$ & $\begin{array}{l}0 \\
0 \\
0 \\
0 \\
0\end{array}$ & $\begin{array}{l}0 \\
1 \\
0 \\
0 \\
1\end{array}$ & $\begin{array}{l}1 \\
0 \\
2 \\
0 \\
0\end{array}$ & $\begin{array}{l}0 \\
0 \\
0 \\
1 \\
0\end{array}$ & $\begin{array}{l}0 \\
0 \\
0 \\
0 \\
0\end{array}$ \\
\hline $\begin{array}{l}6-15 \\
6-16 \\
6-17 \\
6-18 \\
6-19\end{array}$ & $\begin{array}{l}1830 \\
1400 \\
751 \\
1513 \\
1126\end{array}$ & $\begin{array}{l}2 \\
0 \\
3 \\
0 \\
0\end{array}$ & $\begin{array}{l}0 \\
0 \\
0 \\
0 \\
0\end{array}$ & $\begin{array}{l}0 \\
0 \\
1 \\
0 \\
0\end{array}$ & $\begin{array}{l}1 \\
0 \\
1 \\
0 \\
0\end{array}$ & $\begin{array}{l}1 \\
0 \\
1 \\
0 \\
0\end{array}$ & $\begin{array}{l}0 \\
0 \\
0 \\
0 \\
0\end{array}$ \\
\hline $\begin{array}{l}6-20 \\
6-21\end{array}$ & $\begin{array}{l}432 \\
855\end{array}$ & $\begin{array}{l}0 \\
0\end{array}$ & $\begin{array}{l}0 \\
0\end{array}$ & $\begin{array}{l}0 \\
0\end{array}$ & $\begin{array}{l}0 \\
0\end{array}$ & $\begin{array}{l}0 \\
0\end{array}$ & $\begin{array}{l}0 \\
0\end{array}$ \\
\hline $\begin{array}{l}6-23 \\
6-25\end{array}$ & $\begin{array}{l}754 \\
487\end{array}$ & $\begin{array}{l}0 \\
0\end{array}$ & $\begin{array}{l}0 \\
0\end{array}$ & $\begin{array}{l}0 \\
0\end{array}$ & $\begin{array}{l}0 \\
0\end{array}$ & $\begin{array}{l}0 \\
0\end{array}$ & $\begin{array}{l}0 \\
0\end{array}$ \\
\hline $6-27$ & 1773 & 1 & 0 & 0 & 1 & 0 & $\underline{0}$ \\
\hline $\begin{array}{l}0-29 \\
7-01\end{array}$ & - & $\overline{-}$ & - & - & - & - & - \\
\hline $\begin{array}{l}7-03 \\
7-05\end{array}$ & $=$ & -- & $=$ & $=$ & $=-$ & -- & $=-$ \\
\hline $7-07$ & -- & -- & -- & -- & -- & -- & -- \\
\hline
\end{tabular}


TABLE 16.- NUMBER OF BLUE TRACER PARTICLES(1), PER 100 GRAMS OF BEDLOAD SAMPLE, COMPOSITED FROM CROSS-CHANNEL SAMPLING POSITIONS SPACED AT 1-METER INTERVALS ACROSS THE CHANNEL WIDTH, EAST FORK RIVER, WYOMING, 1980

SECTION $1400(5)$

\begin{tabular}{|c|c|c|c|c|c|c|c|}
\hline \multirow{3}{*}{ DATE } & \multirow{3}{*}{$\begin{array}{c}\text { DRY } \\
\text { MASS } \\
\text { OF } \\
\text { SAMPLE (2) } \\
\text { (G) }\end{array}$} & \multicolumn{6}{|c|}{ NUMBER OF BLUE TRACER PARTICLES } \\
\hline & & \multirow[b]{2}{*}{ TOTAL } & \multicolumn{5}{|c|}{ BY SIZE CLASS (MM) } \\
\hline & & & $\begin{array}{l}0.25 \\
\text { TO } \\
0.50\end{array}$ & $\begin{array}{l}0.50 \\
\text { TO } \\
1.00\end{array}$ & $\begin{array}{l}1.00 \\
\text { TO } \\
2.00\end{array}$ & $\begin{array}{l}2.00 \\
\text { TO } \\
4.00\end{array}$ & $\begin{array}{l}4.00 \\
\text { TO } \\
8.00\end{array}$ \\
\hline $\begin{array}{l}5-16 \\
5-19 \\
5-21 \\
5-22 \\
5-24\end{array}$ & $\begin{array}{l}-- \\
\overline{37}+++ \\
352 \\
1306\end{array}$ & $\begin{array}{r}-- \\
-\overline{0} \\
19 \\
19\end{array}$ & $\begin{array}{c}-- \\
-- \\
0 \\
1 \\
7\end{array}$ & $\begin{array}{r}-- \\
-\overline{0} \\
16 \\
8\end{array}$ & $\begin{array}{c}-- \\
-\overline{0} \\
2 \\
4\end{array}$ & $\begin{array}{c}-- \\
-\overline{0} \\
0 \\
0\end{array}$ & $\begin{array}{c}-- \\
-\overline{0} \\
0 \\
0\end{array}$ \\
\hline $\begin{array}{l}5-26 \\
5-27 \\
5-28 \\
5-29 \\
5-30\end{array}$ & $\begin{array}{c}3 \overline{62} \\
87+ \\
-\overline{0+++}\end{array}$ & $\begin{array}{l}-\overline{10} \\
15 \\
-\frac{1}{0}\end{array}$ & $\begin{array}{r}-\frac{3}{5} \\
-\frac{1}{0}\end{array}$ & $\begin{array}{r}--\overline{6} \\
-\frac{5}{0}\end{array}$ & $-\frac{-}{1}$ & $\begin{array}{r}-\overline{0} \\
-\frac{1}{0}\end{array}$ & $\begin{array}{r}-\overline{0} \\
-\frac{0}{0}\end{array}$ \\
\hline $\begin{array}{l}6-01 \\
6-03 \\
6-05 \\
6-07 \\
6-09\end{array}$ & $1103^{--}$ & $\begin{array}{l}-- \\
-- \\
-\overline{0} \\
0\end{array}$ & $\begin{array}{l}-- \\
-- \\
-\overline{0} \\
0\end{array}$ & $\begin{array}{l}-- \\
-- \\
-0 \\
0\end{array}$ & $\begin{array}{l}-- \\
-- \\
-\overline{0} \\
0\end{array}$ & $\begin{array}{l}-- \\
-- \\
-\overline{0} \\
0\end{array}$ & $\begin{array}{l}-- \\
-- \\
-\overline{0} \\
0\end{array}$ \\
\hline $\begin{array}{l}6-10 \\
6-11 \\
6-12 \\
6-13 \\
6-14\end{array}$ & $\begin{array}{r}877 \\
867 \\
371 \\
1218 \\
1182\end{array}$ & $\begin{array}{l}7 \\
6 \\
5 \\
0 \\
1\end{array}$ & $\begin{array}{l}4 \\
1 \\
1 \\
0 \\
0\end{array}$ & $\begin{array}{l}2 \\
4 \\
2 \\
0 \\
1\end{array}$ & $\begin{array}{l}1 \\
1 \\
2 \\
0 \\
0\end{array}$ & $\begin{array}{l}0 \\
0 \\
0 \\
0 \\
0\end{array}$ & $\begin{array}{l}0 \\
0 \\
0 \\
0 \\
0\end{array}$ \\
\hline $\begin{array}{l}6-15 \\
6-16 \\
6-17 \\
6-18 \\
6-19\end{array}$ & $\begin{array}{r}2624 \\
620 \\
1053 \\
799 \\
1020\end{array}$ & $\begin{array}{l}0 \\
4 \\
3 \\
1 \\
0\end{array}$ & $\begin{array}{l}0 \\
0 \\
0 \\
0 \\
0\end{array}$ & $\begin{array}{l}0 \\
2 \\
2 \\
0 \\
0\end{array}$ & $\begin{array}{l}0 \\
2 \\
1 \\
1 \\
0\end{array}$ & $\begin{array}{l}0 \\
0 \\
0 \\
0 \\
0\end{array}$ & $\begin{array}{l}0 \\
0 \\
0 \\
0 \\
0\end{array}$ \\
\hline $\begin{array}{l}6-20 \\
6-21 \\
6-22 \\
6-23 \\
6-25\end{array}$ & $\begin{array}{r}351 \\
262 \frac{1}{3} \\
-\frac{1}{908} \\
265\end{array}$ & $\begin{array}{r}0 \\
1 \\
-0 \\
0 \\
1\end{array}$ & $\begin{array}{r}0 \\
1 \\
-\frac{1}{0} \\
0\end{array}$ & $\begin{array}{r}0 \\
0 \\
-\frac{1}{0} \\
1\end{array}$ & $\begin{array}{r}0 \\
0 \\
-0 \\
0 \\
0\end{array}$ & $\begin{array}{r}0 \\
-\frac{0}{0} \\
0 \\
0\end{array}$ & $\begin{array}{r}0 \\
0 \\
-0 \\
0 \\
0\end{array}$ \\
\hline $\begin{array}{l}6-27 \\
6-29 \\
7-01 \\
7-03 \\
7-05\end{array}$ & $\begin{array}{r}1030 \\
=- \\
=- \\
=-\end{array}$ & $\begin{array}{l}0 \\
-- \\
-- \\
-- \\
--\end{array}$ & $\begin{array}{l}0 \\
-- \\
-- \\
-- \\
--\end{array}$ & $\begin{array}{l}0 \\
-- \\
=- \\
--\end{array}$ & $\begin{array}{l}0 \\
-- \\
-- \\
--\end{array}$ & $\begin{array}{l}0 \\
-- \\
=- \\
--\end{array}$ & $\begin{array}{l}0 \\
=- \\
=- \\
--\end{array}$ \\
\hline $7-07$ & -- & -- & -- & -- & -- & -- & -- \\
\hline
\end{tabular}


TABLE 16.- NUMBER OF BLUE TRACER PARTICLES(1), PER 100 GRAMS OF BEDLOAD SAMPLE, COMPOSITED FROM CROSS-CHANNEL SAMPLING POSITIONS SPACED AT I-METER INTERVALS ACROSS THE CHANNEL WIDTH, EAST FORK RIVER, WYOMING, 1980

SECTION $1425(4)$

\begin{tabular}{|c|c|c|c|c|c|c|c|}
\hline \multirow{3}{*}{ DATE } & \multirow{3}{*}{$\begin{array}{c}\text { DRY } \\
\text { MASS } \\
\text { OF } \\
\text { SAMPLE (2) } \\
\text { (G) }\end{array}$} & \multicolumn{6}{|c|}{ NUMBER OF BLUE TRACER PARTICLES } \\
\hline & & \multirow[b]{2}{*}{ TCTAL } & \multicolumn{5}{|c|}{ BY SIZE CLASS (MM) } \\
\hline & & & $\begin{array}{r}0.25 \\
\text { TO } \\
0.50\end{array}$ & $\begin{array}{l}0.50 \\
\text { TO } \\
1.00\end{array}$ & $\begin{array}{l}1.00 \\
\text { TO } \\
2.00\end{array}$ & $\begin{array}{l}2.00 \\
\text { TO } \\
4.00\end{array}$ & $\begin{array}{l}4.00 \\
\text { TO } \\
8.00\end{array}$ \\
\hline $\begin{array}{l}5-16 \\
5-19 \\
5-21 \\
5-22 \\
5-24\end{array}$ & $\begin{array}{l}55++ \\
500 \\
404 \\
1002 \\
1445\end{array}$ & $\begin{array}{l}4 \\
4 \\
6 \\
1 \\
4\end{array}$ & $\begin{array}{l}2 \\
1 \\
3 \\
0 \\
3\end{array}$ & $\begin{array}{l}2 \\
3 \\
2 \\
1 \\
0\end{array}$ & $\begin{array}{l}0 \\
0 \\
1 \\
0 \\
0\end{array}$ & $\begin{array}{l}0 \\
0 \\
0 \\
0 \\
1\end{array}$ & $\begin{array}{l}0 \\
0 \\
0 \\
0 \\
0\end{array}$ \\
\hline $\begin{array}{l}5-26 \\
5-27 \\
5-28 \\
5-29\end{array}$ & $\begin{array}{l}15 \overline{1} \\
158+ \\
59\end{array}$ & $\begin{array}{r}-- \\
2 \\
5 \\
--\end{array}$ & $\begin{array}{r}-\overline{0} \\
0 \\
-\end{array}$ & $\begin{array}{r}-- \\
2 \\
5 \\
--\end{array}$ & $\begin{array}{r}-- \\
0 \\
0 \\
--\end{array}$ & $\begin{array}{r}-- \\
0 \\
0 \\
--\end{array}$ & $\begin{array}{r}-- \\
0 \\
0 \\
--\end{array}$ \\
\hline $5-30$ & $85+$ & 1 & 0 & 1 & 0 & 0 & 0 \\
\hline $\begin{array}{l}6-01 \\
6-03 \\
6-05 \\
6-07 \\
6-09\end{array}$ & $\begin{array}{l}793 \\
193+ \\
209 \\
935 \\
621\end{array}$ & $\begin{array}{l}4 \\
0 \\
0 \\
9 \\
0\end{array}$ & $\begin{array}{l}0 \\
0 \\
0 \\
2 \\
0\end{array}$ & $\begin{array}{l}3 \\
0 \\
0 \\
1 \\
0\end{array}$ & $\begin{array}{l}1 \\
0 \\
0 \\
6 \\
0\end{array}$ & $\begin{array}{l}0 \\
0 \\
0 \\
0 \\
0\end{array}$ & $\begin{array}{l}0 \\
0 \\
0 \\
0 \\
0\end{array}$ \\
\hline $\begin{array}{l}6-10 \\
6-11 \\
6-12 \\
6-13 \\
6-14\end{array}$ & $\begin{array}{r}1337 \\
257 \\
181 \\
126 \\
1223\end{array}$ & $\begin{array}{l}3 \\
3 \\
1 \\
0 \\
0\end{array}$ & $\begin{array}{l}1 \\
1 \\
1 \\
0 \\
0\end{array}$ & $\begin{array}{l}0 \\
0 \\
0 \\
0 \\
0\end{array}$ & $\begin{array}{l}0 \\
2 \\
0 \\
0 \\
0\end{array}$ & $\begin{array}{l}2 \\
0 \\
0 \\
0 \\
0\end{array}$ & $\begin{array}{l}0 \\
0 \\
0 \\
0 \\
0\end{array}$ \\
\hline $\begin{array}{l}6-15 \\
6-16 \\
6-17 \\
6-18 \\
6-19\end{array}$ & $\begin{array}{l}197 \\
146 \\
177 \\
807 \\
186\end{array}$ & $\begin{array}{l}0 \\
0 \\
1 \\
0 \\
0\end{array}$ & $\begin{array}{l}0 \\
0 \\
0 \\
0 \\
0\end{array}$ & $\begin{array}{l}0 \\
0 \\
1 \\
0 \\
0\end{array}$ & $\begin{array}{l}0 \\
0 \\
0 \\
0 \\
0\end{array}$ & $\begin{array}{l}0 \\
0 \\
0 \\
0 \\
0\end{array}$ & $\begin{array}{l}0 \\
0 \\
0 \\
0 \\
0\end{array}$ \\
\hline $\begin{array}{l}6-20 \\
6-21\end{array}$ & $\begin{array}{l}368 \\
228\end{array}$ & $\begin{array}{l}0 \\
0\end{array}$ & $\begin{array}{l}0 \\
0\end{array}$ & $\begin{array}{l}0 \\
0\end{array}$ & $\begin{array}{r}0 \\
0 \\
-\end{array}$ & $\begin{array}{l}0 \\
0 \\
-\end{array}$ & $\begin{array}{l}0 \\
0\end{array}$ \\
\hline $\begin{array}{l}6-22 \\
6-23 \\
6-25\end{array}$ & $\begin{array}{l}2 \overline{8} \\
212\end{array}$ & $\overline{0}$ & 0 & $\begin{array}{l}\overline{0} \\
0\end{array}$ & $\begin{array}{l}0 \\
0\end{array}$ & 0 & $\overline{0}$ \\
\hline $6-27$ & 664 & 1 & -0 & -1 & $-\underline{0}$ & $\underline{0}$ & 1 \\
\hline $\begin{array}{l}6-29 \\
7-01\end{array}$ & $\overline{--}$ & - & $\overline{--}$ & - & $\overline{-}$ & - & - \\
\hline $\begin{array}{l}7-03 \\
7-05\end{array}$ & $\overline{--}$ & $\overline{--}$ & $=$ & $\overline{--}$ & -- & -- & $\overline{--}$ \\
\hline $7-07$ & -- & -- & -- & -- & -- & -- & -- \\
\hline
\end{tabular}


TABLE 16.- NUMBER OF BLUE TRACER PARTICLES(1), PER 100 GRAMS OF BEDLOAD SAMPLE, COMPOSITED FROM CROSS-CHANNEL SAMPLING POSITIONS SPACED AT 1-METER INTERVALS ACROSS THE CHANNEL WIDTH,

EAST FORK RIVER, WYOMING, 1980

SECTION 1481

\begin{tabular}{|c|c|c|c|c|c|c|c|}
\hline \multirow{3}{*}{ DATE } & \multirow{3}{*}{$\begin{array}{c}\text { DRY } \\
\text { MASS } \\
\text { OF } \\
\text { SAMPLE (2) } \\
\text { (G) }\end{array}$} & \multicolumn{6}{|c|}{ NUMBER OF BLUE TRACER PARTICLES } \\
\hline & & \multirow[b]{2}{*}{ TOTAL } & \multicolumn{5}{|c|}{ BY SIZE CLASS (MM) } \\
\hline & & & $\begin{array}{l}0.25 \\
\text { TO } \\
0.50\end{array}$ & $\begin{array}{l}0.50 \\
\text { TO } \\
1.00\end{array}$ & $\begin{array}{l}1.00 \\
\text { TO } \\
2.00\end{array}$ & $\begin{array}{l}2.00 \\
\text { TO } \\
4.00\end{array}$ & $\begin{array}{l}4.00 \\
\text { TO } \\
8.00\end{array}$ \\
\hline $\begin{array}{l}5-16 \\
5-19 \\
5-21 \\
5-22 \\
5-24\end{array}$ & $\begin{array}{l}3^{17++} \\
320 \\
329 \\
2322 \\
3171\end{array}$ & $\begin{array}{l}6 \\
2 \\
2 \\
1 \\
3\end{array}$ & $\begin{array}{l}0 \\
0 \\
1 \\
0 \\
0\end{array}$ & $\begin{array}{l}0 \\
2 \\
1 \\
1 \\
2\end{array}$ & $\begin{array}{l}6 \\
0 \\
0 \\
0 \\
1\end{array}$ & $\begin{array}{l}0 \\
0 \\
0 \\
0 \\
0\end{array}$ & $\begin{array}{l}0 \\
0 \\
0 \\
0 \\
0\end{array}$ \\
\hline $\begin{array}{l}5-26 \\
5-27 \\
5-28 \\
5-29\end{array}$ & $\begin{array}{l}4 \overline{36} \\
295 \\
-\end{array}$ & $\begin{array}{r}-- \\
2 \\
--\end{array}$ & $\begin{array}{r}-- \\
1 \\
-\end{array}$ & $\begin{array}{r}-- \\
0 \\
-\end{array}$ & $\frac{1}{2}$ & $\begin{array}{r}-- \\
0 \\
-\end{array}$ & $-\overline{0}$ \\
\hline $5-30$ & 436 & 4 & 0 & 4 & 0 & 0 & 0 \\
\hline $\begin{array}{l}6-01 \\
6-03 \\
6-05 \\
6-07 \\
6-09\end{array}$ & $\begin{array}{r}187+ \\
235 \\
197 \\
389 \\
2148\end{array}$ & $\begin{array}{l}5 \\
3 \\
2 \\
1 \\
0\end{array}$ & $\begin{array}{l}0 \\
0 \\
0 \\
0 \\
0\end{array}$ & $\begin{array}{l}3 \\
0 \\
0 \\
0 \\
0\end{array}$ & $\begin{array}{l}2 \\
3 \\
2 \\
1 \\
0\end{array}$ & $\begin{array}{l}0 \\
0 \\
0 \\
0 \\
0\end{array}$ & $\begin{array}{l}0 \\
0 \\
0 \\
0 \\
0\end{array}$ \\
\hline $\begin{array}{l}6-10 \\
6-11 \\
6-12 \\
6-13 \\
6-14\end{array}$ & $\begin{array}{r}2785 \\
1497 \\
685 \\
767 \\
1433\end{array}$ & $\begin{array}{l}1 \\
2 \\
2 \\
1 \\
0\end{array}$ & $\begin{array}{l}0 \\
1 \\
0 \\
0 \\
0\end{array}$ & $\begin{array}{l}\frac{1}{1} \\
\frac{1}{2} \\
1 \\
0\end{array}$ & $\begin{array}{l}0 \\
0 \\
0 \\
0 \\
0\end{array}$ & $\begin{array}{l}0 \\
0 \\
0 \\
0 \\
0\end{array}$ & $\begin{array}{l}0 \\
0 \\
0 \\
0 \\
0\end{array}$ \\
\hline $\begin{array}{l}6-15 \\
6-16 \\
6-17 \\
6-18 \\
6-19\end{array}$ & $\begin{array}{r}2451 \\
824 \\
674 \\
2022 \\
1665\end{array}$ & $\begin{array}{l}1 \\
0 \\
0 \\
0 \\
0\end{array}$ & $\begin{array}{l}0 \\
0 \\
0 \\
0 \\
0\end{array}$ & $\begin{array}{l}0 \\
0 \\
0 \\
0 \\
0\end{array}$ & $\begin{array}{l}0 \\
0 \\
0 \\
0 \\
0\end{array}$ & $\begin{array}{l}1 \\
0 \\
0 \\
0 \\
0\end{array}$ & $\begin{array}{l}0 \\
0 \\
0 \\
0 \\
0\end{array}$ \\
\hline $\begin{array}{l}6-20 \\
6-21 \\
6-27\end{array}$ & $\begin{array}{l}1146 \\
1233\end{array}$ & $\begin{array}{l}0 \\
0\end{array}$ & $\begin{array}{l}0 \\
0\end{array}$ & $\begin{array}{l}0 \\
0\end{array}$ & $\begin{array}{l}0 \\
0\end{array}$ & $\begin{array}{l}0 \\
0\end{array}$ & $\begin{array}{l}0 \\
0\end{array}$ \\
\hline $\begin{array}{l}6-23 \\
6-25\end{array}$ & $\begin{array}{r}776 \\
1216\end{array}$ & $\begin{array}{l}0 \\
0\end{array}$ & $\begin{array}{l}0 \\
0\end{array}$ & $\begin{array}{l}0 \\
0\end{array}$ & $\begin{array}{l}0 \\
0\end{array}$ & $\begin{array}{l}0 \\
0\end{array}$ & $\begin{array}{l}0 \\
0\end{array}$ \\
\hline $\begin{array}{l}6-27 \\
6-29\end{array}$ & 1479 & $\underline{0}$ & $\underline{0}$ & -0 & $\underline{0}$ & $\underline{0}$ & $\underline{0}$ \\
\hline $7-01$ & -- & -- & - & - & - & - & - \\
\hline $\begin{array}{l}7-03 \\
7-05\end{array}$ & $\overline{--}$ & $\overline{--}$ & $\overline{-}$ & $\overline{-}$ & $=$ & $=$ & $\overline{-}$ \\
\hline $7-07$ & -- & -- & -- & - & -- & -- & -- \\
\hline
\end{tabular}


TABLE 16.- NUMBER OF BLUE TRACER PARTICLES(1), PER 100 GRAMS OF BEDLOAD SAMPLE, COMPOSITED FROM CROSS-CHANNEL SAMPLING POSITIONS SPACED AT I-METER INTERVALS ACROSS THE CHANNEL WIDTH, EAST FORK RIVER, WYOMING, 1980

SECTION 1481(3)

\begin{tabular}{|c|c|c|c|c|c|c|c|}
\hline \multirow{3}{*}{ DATE } & \multirow{3}{*}{$\begin{array}{c}\text { DRY } \\
\text { MASS } \\
\text { OF } \\
\text { SAMPLE (2) } \\
\text { (G) }\end{array}$} & \multicolumn{6}{|c|}{ NUMBER OF BLUE TRACER PARTICLES } \\
\hline & & & & BY SIZ & CLASS & & \\
\hline & & TOTAL & $\begin{array}{l}0.25 \\
\text { TO } \\
0.50\end{array}$ & $\begin{array}{l}0.50 \\
\text { TO } \\
1.00\end{array}$ & $\begin{array}{l}1.00 \\
\text { TO } \\
2.00\end{array}$ & $\begin{array}{l}2.00 \\
\text { TO } \\
4.00\end{array}$ & $\begin{array}{l}4.00 \\
\text { TO } \\
8.00\end{array}$ \\
\hline $\begin{array}{l}5-16 \\
5-19 \\
5-21 \\
5-22 \\
5-24\end{array}$ & $\begin{array}{l}-- \\
17 \overline{7}+ \\
468 \\
--\end{array}$ & $\begin{array}{r}-- \\
-\overline{2} \\
4 \\
--\end{array}$ & $\begin{array}{r}-- \\
-0 \\
0 \\
--\end{array}$ & $\begin{array}{r}-- \\
-\overline{2} \\
4 \\
--\end{array}$ & $\begin{array}{r}-- \\
-\overline{0} \\
0 \\
--\end{array}$ & $\begin{array}{r}-- \\
-\overline{0} \\
0\end{array}$ & $\begin{array}{l}-- \\
-\overline{0} \\
0\end{array}$ \\
\hline $\begin{array}{l}5-26 \\
5-27 \\
5-28 \\
5-29 \\
5-30\end{array}$ & $\begin{array}{l}-\overline{0+++} \\
-- \\
=-\end{array}$ & $\begin{array}{l}-- \\
--- \\
--\end{array}$ & $=-\overline{0}$ & $\begin{array}{l}-- \\
-- \\
--\end{array}$ & $\begin{array}{l}-- \\
-- \\
--\end{array}$ & $\begin{array}{l}-\overline{0} \\
-- \\
--\end{array}$ & $\begin{array}{c}-- \\
-- \\
--\end{array}$ \\
\hline $\begin{array}{l}6-01 \\
6-03 \\
6-05 \\
6-07 \\
6-09\end{array}$ & $\overline{-}$ & $\begin{array}{c}-- \\
-- \\
0\end{array}$ & $\begin{array}{l}-- \\
-- \\
-\overline{0}\end{array}$ & $\begin{array}{c}-- \\
\overline{-} \\
\overline{0}\end{array}$ & $\overline{--}$ & $\overline{--}$ & $\overline{--}$ \\
\hline $\begin{array}{l}6-10 \\
6-11 \\
6-12 \\
6-13 \\
6-14\end{array}$ & $\begin{array}{l}3194 \\
1611 \\
345 \\
1365 \\
2335\end{array}$ & $\begin{array}{l}1 \\
5 \\
2 \\
1 \\
1\end{array}$ & $\begin{array}{l}0 \\
2 \\
1 \\
0 \\
0\end{array}$ & $\begin{array}{l}\frac{1}{2} \\
1 \\
\frac{1}{1}\end{array}$ & $\begin{array}{l}0 \\
1 \\
0 \\
0 \\
0\end{array}$ & $\begin{array}{l}0 \\
0 \\
0 \\
0 \\
0\end{array}$ & $\begin{array}{l}0 \\
0 \\
0 \\
0 \\
0\end{array}$ \\
\hline $\begin{array}{l}6-15 \\
6-16 \\
6-17 \\
6-18 \\
6-19\end{array}$ & $\begin{array}{r}1054 \\
184 \\
360 \\
2209 \\
827\end{array}$ & $\begin{array}{l}0 \\
0 \\
0 \\
0 \\
0\end{array}$ & $\begin{array}{l}0 \\
0 \\
0 \\
0 \\
0\end{array}$ & $\begin{array}{l}0 \\
0 \\
0 \\
0 \\
0\end{array}$ & $\begin{array}{l}0 \\
0 \\
0 \\
0 \\
0\end{array}$ & $\begin{array}{l}0 \\
0 \\
0 \\
0 \\
0\end{array}$ & $\begin{array}{l}0 \\
0 \\
0 \\
0 \\
0\end{array}$ \\
\hline $\begin{array}{l}6-20 \\
6-21\end{array}$ & $\begin{array}{l}1600 \\
1029\end{array}$ & $\begin{array}{l}0 \\
0\end{array}$ & $\begin{array}{l}0 \\
0\end{array}$ & $\begin{array}{l}0 \\
0\end{array}$ & $\begin{array}{l}0 \\
0\end{array}$ & $\begin{array}{l}0 \\
0\end{array}$ & $\begin{array}{l}0 \\
0\end{array}$ \\
\hline $\begin{array}{l}6-23 \\
6-25\end{array}$ & $\begin{array}{l}465 \\
347\end{array}$ & $\begin{array}{l}0 \\
0\end{array}$ & $\begin{array}{l}0 \\
0\end{array}$ & $\begin{array}{l}0 \\
0\end{array}$ & $\begin{array}{l}0 \\
0\end{array}$ & $\begin{array}{l}0 \\
0\end{array}$ & $\begin{array}{l}0 \\
0\end{array}$ \\
\hline $\begin{array}{l}6-27 \\
6-29 \\
7-01 \\
7-03 \\
7-05\end{array}$ & $\begin{array}{r}1109 \\
=- \\
=-\end{array}$ & $\begin{array}{l}0 \\
-- \\
-- \\
--\end{array}$ & $\begin{array}{l}0 \\
=- \\
=-\end{array}$ & $\begin{array}{l}0 \\
-- \\
-- \\
--\end{array}$ & $\begin{array}{l}0 \\
-- \\
--\end{array}$ & $\begin{array}{l}0 \\
- \\
-\end{array}$ & $\begin{array}{l}0 \\
=- \\
-- \\
--\end{array}$ \\
\hline $7-07$ & -- & -- & - & -- & -- & -- & -- \\
\hline
\end{tabular}


TABLE 16.- NUMBER OF BLUE TRACER PARTICLES(1), PER 100 GRAMS OF BEDLOAD SAMPLE, COMPOSITED FROM CROSS-CHANNEL SAMPLING POSITIONS

SPACED AT 1-METER INTERVALS ACROSS THE CHANNEL WIDTH,

EAST FORK RIVER, WYOMING, 1980

SECTION 1533

\begin{tabular}{|c|c|c|c|c|c|c|c|}
\hline \multirow{3}{*}{ DATE } & \multirow{3}{*}{$\begin{array}{c}\text { DRY } \\
\text { MASS } \\
\text { OF } \\
\text { SAMPLE (2) } \\
\text { (G) }\end{array}$} & \multicolumn{6}{|c|}{ NUMBER OF BLUE TRACER PARTICLES } \\
\hline & & \multirow[b]{2}{*}{ TOTAL } & \multicolumn{5}{|c|}{ BY SIZE CLASS (MM) } \\
\hline & & & $\begin{array}{l}0.25 \\
\text { TO } \\
0.50\end{array}$ & $\begin{array}{l}0.50 \\
\text { TO } \\
1.00\end{array}$ & $\begin{array}{l}1.00 \\
\text { TO } \\
2.00\end{array}$ & $\begin{array}{l}2.00 \\
\text { TO } \\
4.00\end{array}$ & $\begin{array}{l}4.00 \\
\text { TO } \\
8.00\end{array}$ \\
\hline $\begin{array}{l}5-16 \\
5-19 \\
5-21 \\
5-22 \\
5-24\end{array}$ & $\begin{array}{l}72++ \\
22+++ \\
256 \\
2210 \\
1455\end{array}$ & $\begin{array}{l}0 \\
0 \\
0 \\
3 \\
5\end{array}$ & $\begin{array}{l}0 \\
0 \\
0 \\
2 \\
0\end{array}$ & $\begin{array}{l}0 \\
0 \\
0 \\
1 \\
5\end{array}$ & $\begin{array}{l}0 \\
0 \\
0 \\
0 \\
0\end{array}$ & $\begin{array}{l}0 \\
0 \\
0 \\
0 \\
0\end{array}$ & $\begin{array}{l}0 \\
0 \\
0 \\
0 \\
0\end{array}$ \\
\hline $\begin{array}{l}5-26 \\
5-27 \\
5-28 \\
5-29\end{array}$ & $\begin{array}{r}11 \overline{39} \\
1385\end{array}$ & $\begin{array}{r}-- \\
3 \\
--\end{array}$ & $\begin{array}{r}-\overline{0} \\
0 \\
--\end{array}$ & $-\frac{-}{2}$ & $\begin{array}{r}--\overline{0} \\
0 \\
-\end{array}$ & $\begin{array}{r}-\overline{2} \\
0 \\
--\end{array}$ & $\begin{array}{r}-\overline{0} \\
0 \\
--\end{array}$ \\
\hline $5-30$ & 249 & 3 & 1 & 2 & 0 & 0 & 0 \\
\hline $\begin{array}{l}6-01 \\
6-03 \\
6-05 \\
6-07 \\
6-09\end{array}$ & $\begin{array}{l}452 \\
169 \\
521 \\
342 \\
979\end{array}$ & $\begin{array}{l}3 \\
0 \\
1 \\
4 \\
0\end{array}$ & $\begin{array}{l}0 \\
0 \\
0 \\
1 \\
0\end{array}$ & $\begin{array}{l}3 \\
0 \\
0 \\
3 \\
0\end{array}$ & $\begin{array}{l}0 \\
0 \\
0 \\
0 \\
0\end{array}$ & $\begin{array}{l}0 \\
0 \\
1 \\
0 \\
0\end{array}$ & $\begin{array}{l}0 \\
0 \\
0 \\
0 \\
0\end{array}$ \\
\hline $\begin{array}{l}6-10 \\
6-11 \\
6-12 \\
6-13 \\
6-14\end{array}$ & $\begin{array}{l}4669 \\
1344 \\
1319 \\
3564 \\
3557\end{array}$ & $\begin{array}{l}1 \\
0 \\
0 \\
1 \\
0\end{array}$ & $\begin{array}{l}0 \\
0 \\
0 \\
0 \\
0\end{array}$ & $\begin{array}{l}1 \\
0 \\
0 \\
0 \\
0\end{array}$ & $\begin{array}{l}0 \\
0 \\
0 \\
1 \\
0\end{array}$ & $\begin{array}{l}0 \\
0 \\
0 \\
0 \\
0\end{array}$ & $\begin{array}{l}0 \\
0 \\
0 \\
0 \\
0\end{array}$ \\
\hline $\begin{array}{l}6-15 \\
6-16\end{array}$ & 2663 & 1 & $\underline{0}$ & 0 & 0 & 1 & 0 \\
\hline $\begin{array}{l}6-17 \\
6-18 \\
6-19\end{array}$ & $\begin{array}{r}2076 \\
4222 \\
745\end{array}$ & $\begin{array}{l}0 \\
0 \\
1\end{array}$ & $\begin{array}{l}0 \\
0 \\
0\end{array}$ & $\begin{array}{l}0 \\
0 \\
1\end{array}$ & $\begin{array}{l}0 \\
0 \\
0\end{array}$ & $\begin{array}{l}0 \\
0 \\
0\end{array}$ & $\begin{array}{l}0 \\
0 \\
0\end{array}$ \\
\hline $\begin{array}{l}6-20 \\
6-21\end{array}$ & $\begin{array}{l}1383 \\
2034\end{array}$ & $\begin{array}{l}0 \\
0\end{array}$ & $\begin{array}{l}0 \\
0\end{array}$ & $\begin{array}{l}0 \\
0\end{array}$ & $\begin{array}{l}0 \\
0\end{array}$ & $\begin{array}{l}0 \\
0\end{array}$ & $\begin{array}{l}0 \\
0\end{array}$ \\
\hline $\begin{array}{l}6-23 \\
6-25\end{array}$ & $\begin{array}{l}1253 \\
1756\end{array}$ & $\begin{array}{l}0 \\
5\end{array}$ & $\begin{array}{l}0 \\
1\end{array}$ & $\begin{array}{l}0 \\
3\end{array}$ & $\begin{array}{l}0 \\
1\end{array}$ & $\begin{array}{l}0 \\
0\end{array}$ & $\begin{array}{l}0 \\
0\end{array}$ \\
\hline $6-27$ & 3070 & 0 & 0 & 0 & 0 & 0 & 0 \\
\hline $\begin{array}{l}0-29 \\
7-01\end{array}$ & -- & - & - & - & - & - & - \\
\hline $\begin{array}{l}7-03 \\
7-05\end{array}$ & $\begin{array}{l}1043 \\
1247\end{array}$ & $\begin{array}{l}0 \\
0\end{array}$ & $\begin{array}{l}0 \\
0\end{array}$ & $\begin{array}{l}0 \\
0\end{array}$ & $\begin{array}{l}0 \\
0\end{array}$ & $\begin{array}{l}0 \\
0\end{array}$ & $\begin{array}{l}0 \\
0\end{array}$ \\
\hline $7-07$ & $81+$ & 0 & 0 & 0 & 0 & 0 & 0 \\
\hline
\end{tabular}


TABLE 16.- NUMBER OF BLUE TRACER PARTICLES(1), PER 100 GRAMS OF BEDLOAD SAMPLE, COMPOSITED FROM CROSS-CHANNEL SAMPLING POSITIONS

SPACED AT 1-METER INTERVALS ACROSS THE CHANNEL WIDTH,

EAST FORK RIVER, WYOMING, 1980

SECTION 1573

\begin{tabular}{|c|c|c|c|c|c|c|c|}
\hline \multirow{3}{*}{ DATE } & \multirow{3}{*}{$\begin{array}{c}\text { DRY } \\
\text { MASS } \\
\text { OF } \\
\text { SAMPLE (2) } \\
(\mathrm{G})\end{array}$} & \multicolumn{6}{|c|}{ NUMBER OF BLUE TRACER PARTICLES } \\
\hline & & \multirow[b]{2}{*}{ TOTAL } & \multicolumn{5}{|c|}{ BY SIZE CLASS (MM) } \\
\hline & & & $\begin{array}{l}0.25 \\
\text { TO } \\
0.50\end{array}$ & $\begin{array}{l}0.50 \\
\text { TO } \\
1.00\end{array}$ & $\begin{array}{l}1.00 \\
2.00\end{array}$ & $\begin{array}{l}2.00 \\
\text { TO } \\
4.00\end{array}$ & $\begin{array}{l}4.00 \\
\text { TO } \\
8.00\end{array}$ \\
\hline $\begin{array}{l}5-16 \\
5-19 \\
5-21 \\
5-22 \\
5-24\end{array}$ & $\begin{array}{c}0+++ \\
109+++ \\
223 \\
5886 \\
3327\end{array}$ & $\begin{array}{r}0 \\
0 \\
4 \\
4 \\
13\end{array}$ & $\begin{array}{l}0 \\
0 \\
3 \\
2 \\
2\end{array}$ & $\begin{array}{l}0 \\
0 \\
1 \\
1 \\
5\end{array}$ & $\begin{array}{l}0 \\
0 \\
0 \\
1 \\
5\end{array}$ & $\begin{array}{l}0 \\
0 \\
0 \\
0 \\
1\end{array}$ & $\begin{array}{l}0 \\
0 \\
0 \\
0 \\
0\end{array}$ \\
\hline $\begin{array}{l}5-26 \\
5-27 \\
5-28\end{array}$ & $\begin{array}{c}-\overline{-} \\
1058 \\
102+\end{array}$ & $\begin{array}{r}-- \\
5 \\
5\end{array}$ & $\begin{array}{r}--\overline{0} \\
0\end{array}$ & $-\frac{-}{2}$ & $\begin{array}{r}--\overline{3} \\
4\end{array}$ & $\begin{array}{r}-- \\
0 \\
0\end{array}$ & $\begin{array}{r}-- \\
0\end{array}$ \\
\hline $5-30$ & $47 \overline{1}$ & 3 & 3 & 0 & 0 & 0 & 0 \\
\hline $\begin{array}{l}6-01 \\
6-03 \\
6-05 \\
6-07 \\
6-09\end{array}$ & $\begin{array}{c}93+ \\
110+ \\
45++ \\
59++ \\
2507\end{array}$ & $\begin{array}{r}3 \\
4 \\
16 \\
0 \\
0\end{array}$ & $\begin{array}{l}1 \\
1 \\
2 \\
0 \\
0\end{array}$ & $\begin{array}{l}2 \\
1 \\
7 \\
0 \\
0\end{array}$ & $\begin{array}{l}0 \\
2 \\
7 \\
0 \\
0\end{array}$ & $\begin{array}{l}0 \\
0 \\
0 \\
0 \\
0\end{array}$ & $\begin{array}{l}0 \\
0 \\
0 \\
0 \\
0\end{array}$ \\
\hline $\begin{array}{l}6-10 \\
6-11 \\
6-12 \\
6-13 \\
6-14\end{array}$ & $\begin{array}{l}2398 \\
1189 \\
4094 \\
3490 \\
5136\end{array}$ & $\begin{array}{l}1 \\
1 \\
1 \\
0 \\
1\end{array}$ & $\begin{array}{l}0 \\
0 \\
0 \\
0 \\
0\end{array}$ & $\begin{array}{l}1 \\
0 \\
1 \\
0 \\
1\end{array}$ & $\begin{array}{l}0 \\
0 \\
0 \\
0 \\
0\end{array}$ & $\begin{array}{l}0 \\
1 \\
0 \\
0 \\
0\end{array}$ & $\begin{array}{l}0 \\
0 \\
0 \\
0 \\
0\end{array}$ \\
\hline $6-15$ & 1507 & 0 & 0 & 0 & 0 & 0 & 0 \\
\hline $\begin{array}{l}6-10 \\
6-17 \\
6-18 \\
6-19\end{array}$ & $\begin{array}{l}5589 \\
3202 \\
1359\end{array}$ & $\begin{array}{l}0 \\
0 \\
0\end{array}$ & $\begin{array}{l}0 \\
0 \\
0\end{array}$ & $\begin{array}{l}0 \\
0 \\
0\end{array}$ & $\begin{array}{l}0 \\
0 \\
0\end{array}$ & $\begin{array}{l}0 \\
0 \\
0\end{array}$ & $\begin{array}{l}0 \\
0 \\
0\end{array}$ \\
\hline $\begin{array}{l}6-20 \\
6-21\end{array}$ & $\begin{array}{l}4272 \\
3339\end{array}$ & $\begin{array}{l}0 \\
0\end{array}$ & $\begin{array}{l}0 \\
0\end{array}$ & $\begin{array}{l}0 \\
0\end{array}$ & $\begin{array}{l}0 \\
0\end{array}$ & $\begin{array}{l}0 \\
0\end{array}$ & $\begin{array}{l}0 \\
0\end{array}$ \\
\hline $\begin{array}{l}6-23 \\
6-25\end{array}$ & $\begin{array}{l}1849 \\
2192\end{array}$ & $\begin{array}{l}0 \\
0\end{array}$ & $\begin{array}{l}0 \\
0\end{array}$ & $\begin{array}{l}0 \\
0\end{array}$ & $\begin{array}{l}0 \\
0\end{array}$ & $\begin{array}{l}0 \\
0\end{array}$ & $\begin{array}{l}0 \\
0\end{array}$ \\
\hline $\begin{array}{l}6-27 \\
6-20\end{array}$ & 2560 & $\underline{-0}$ & $\underline{0}$ & $\underline{0}$ & $\underline{-0}$ & $\underline{0}$ & $\underline{0}$ \\
\hline $7-01$ & -- & - & - & -- & -- & -- & -- \\
\hline $\begin{array}{l}7-03 \\
7-05\end{array}$ & $2 \overline{69}$ & $\overline{0}$ & $\overline{0}$ & $\overline{0}$ & $\overline{0}$ & $\overline{0}$ & $\overline{0}$ \\
\hline $7-07$ & 393 & 0 & 0 & 0 & 0 & 0 & 0 \\
\hline
\end{tabular}


TABLE 16.- NUMBER OF BLUE TRACER PARTICLES(1), PER 100 GRAMS OF BEDLOAD SAMPLE, COMPOSITED FROM CROSS-CHANNEL SAMPLING POSITIONS

SPACED AT 1-METER INTERVALS ACROSS THE CHANNEL WIDTH,

EAST FORK RIVER, WYOMING, 1980

SECTION 16.10

\begin{tabular}{|c|c|c|c|c|c|c|c|}
\hline \multirow{3}{*}{ DATE } & \multirow{3}{*}{$\begin{array}{c}\text { DRY } \\
\text { MASS } \\
\text { OF } \\
\text { SAMPLE (2) } \\
\text { (G) }\end{array}$} & \multicolumn{6}{|c|}{ NUMBER OF BLUE TRACER PARTICLES } \\
\hline & & \multirow[b]{2}{*}{ TOTAL } & \multicolumn{5}{|c|}{ BY SIZE CLASS (MM) } \\
\hline & & & $\begin{array}{c}0.25 \\
\text { TO } \\
0.50\end{array}$ & $\begin{array}{l}0.50 \\
\text { TO } \\
1.00\end{array}$ & $\begin{array}{l}1.00 \\
\text { TO } \\
2.00\end{array}$ & $\begin{array}{l}2.00 \\
\text { TO } \\
4.00\end{array}$ & $\begin{array}{l}4.00 \\
\text { TO } \\
8.00\end{array}$ \\
\hline $\begin{array}{l}5-16 \\
5-19 \\
5-21 \\
5-22 \\
5-24\end{array}$ & $\begin{array}{l}25++ \\
312 \\
1752 \\
4024 \\
2137\end{array}$ & $\begin{array}{l}4 \\
2 \\
0 \\
3 \\
3\end{array}$ & $\begin{array}{l}0 \\
1 \\
0 \\
0 \\
0\end{array}$ & $\begin{array}{l}4 \\
0 \\
0 \\
3 \\
1\end{array}$ & $\begin{array}{l}0 \\
1 \\
0 \\
0 \\
2\end{array}$ & $\begin{array}{l}0 \\
0 \\
0 \\
0 \\
0\end{array}$ & $\begin{array}{l}0 \\
0 \\
0 \\
0 \\
0\end{array}$ \\
\hline $\begin{array}{l}5-26 \\
5-27 \\
5-28 \\
5-29 \\
5-30\end{array}$ & $\begin{array}{c}1 \overline{89+} \\
1153 \\
\frac{76+}{76+}\end{array}$ & $\begin{array}{r}-- \\
2 \\
-\frac{0}{3}\end{array}$ & $\begin{array}{r}-\overline{0} \\
0 \\
-\frac{1}{0}\end{array}$ & $\begin{array}{r}--\overline{2} \\
-\frac{0}{0}\end{array}$ & $\begin{array}{r}--\overline{0} \\
0 \\
-\frac{1}{3}\end{array}$ & $\begin{array}{r}-\overline{0} \\
-0 \\
-0\end{array}$ & $\begin{array}{r}-- \\
0 \\
-0 \\
0\end{array}$ \\
\hline $\begin{array}{l}6-01 \\
6-03 \\
6-05 \\
6-07 \\
6-09\end{array}$ & $\begin{array}{l}286 \\
162+ \\
92+ \\
1082 \\
2704\end{array}$ & $\begin{array}{l}0 \\
0 \\
3 \\
0 \\
0\end{array}$ & $\begin{array}{l}0 \\
0 \\
0 \\
0 \\
0\end{array}$ & $\begin{array}{l}0 \\
0 \\
2 \\
0 \\
0\end{array}$ & $\begin{array}{l}0 \\
0 \\
1 \\
0 \\
0\end{array}$ & $\begin{array}{l}0 \\
0 \\
0 \\
0 \\
0\end{array}$ & $\begin{array}{l}0 \\
0 \\
0 \\
0 \\
0\end{array}$ \\
\hline $\begin{array}{l}6-10 \\
6-11 \\
6-12 \\
6-13 \\
6-14\end{array}$ & $\begin{array}{l}7137 \\
4062 \\
1592 \\
1856 \\
2864\end{array}$ & $\begin{array}{l}1 \\
2 \\
1 \\
1 \\
0\end{array}$ & $\begin{array}{l}0 \\
1 \\
0 \\
0 \\
0\end{array}$ & $\begin{array}{l}1 \\
1 \\
1 \\
1 \\
0\end{array}$ & $\begin{array}{l}0 \\
0 \\
0 \\
0 \\
0\end{array}$ & $\begin{array}{l}0 \\
0 \\
0 \\
0 \\
0\end{array}$ & $\begin{array}{l}0 \\
0 \\
0 \\
0 \\
0\end{array}$ \\
\hline $6-15$ & 3503 & 0 & 0 & 0 & 0 & 0 & 0 \\
\hline $\begin{array}{l}6-17 \\
6-18 \\
6-19\end{array}$ & $\begin{array}{l}4084 \\
2445 \\
1694\end{array}$ & $\begin{array}{l}0 \\
0 \\
0\end{array}$ & $\begin{array}{l}0 \\
0 \\
0\end{array}$ & $\begin{array}{l}0 \\
0 \\
0\end{array}$ & $\begin{array}{l}0 \\
0 \\
0\end{array}$ & $\begin{array}{l}0 \\
0 \\
0\end{array}$ & $\begin{array}{l}0 \\
0 \\
0\end{array}$ \\
\hline $\begin{array}{l}6-20 \\
6-21\end{array}$ & $\begin{array}{l}1253 \\
2001\end{array}$ & $\begin{array}{l}0 \\
0\end{array}$ & $\begin{array}{l}0 \\
0\end{array}$ & $\begin{array}{l}0 \\
0\end{array}$ & $\begin{array}{l}0 \\
0\end{array}$ & $\begin{array}{l}0 \\
0\end{array}$ & $\begin{array}{l}0 \\
0\end{array}$ \\
\hline $\begin{array}{l}6-23 \\
6-25\end{array}$ & $\begin{array}{r}1393 \\
553\end{array}$ & $\begin{array}{l}0 \\
0\end{array}$ & $\begin{array}{l}0 \\
0\end{array}$ & $\begin{array}{l}0 \\
0\end{array}$ & $\begin{array}{l}0 \\
0\end{array}$ & $\begin{array}{l}0 \\
0\end{array}$ & $\begin{array}{l}0 \\
0\end{array}$ \\
\hline $6-27$ & 1604 & 0 & 0 & 0 & 0 & 0 & 0 \\
\hline $7-01$ & 1661 & 0 & 0 & 0 & 0 & 0 & 0 \\
\hline $\begin{array}{l}7-03 \\
7-05\end{array}$ & $3 \overline{4}+$ & $\overline{0}$ & $\overline{0}$ & $\overline{0}$ & $\overline{0}$ & $\overline{0}$ & $\overline{0}$ \\
\hline $7-07$ & $6+++$ & 0 & 0 & 0 & 0 & 0 & 0 \\
\hline
\end{tabular}


TABLE 16.- NUMBER OF BLUE TRACER PARTICLES(1), PER 100 GRAMS OF BEDLOAD SAMPLE, COMPOSITED FROM CROSS-CHANNEL SAMPLING POSITIONS SPACED AT I-METER INTERVALS ACROSS THE CHANNEL WIDTH, EAST FORK RIVER, WYOMING, 1980

SECTION 1662

\begin{tabular}{|c|c|c|c|c|c|c|c|}
\hline \multirow{3}{*}{ DATE } & \multirow{3}{*}{$\begin{array}{c}\text { DRY } \\
\text { MASS } \\
\text { OF } \\
\text { SAMPLE (2) } \\
\text { (G) }\end{array}$} & \multicolumn{6}{|c|}{ NUMBER OF BLUE TRACER PARTICLES } \\
\hline & & \multirow[b]{2}{*}{ TOTAL } & \multicolumn{5}{|c|}{ BY SIZE CLASS (MM) } \\
\hline & & & $\begin{array}{l}0.25 \\
\text { TO } \\
0.50\end{array}$ & $\begin{array}{l}0.50 \\
\text { TO } \\
1.00\end{array}$ & $\begin{array}{l}1.00 \\
\text { TO } \\
2.00\end{array}$ & $\begin{array}{l}2.00 \\
\text { TO } \\
4.00\end{array}$ & $\begin{array}{c}4.00 \\
\text { TO } \\
8.00\end{array}$ \\
\hline $\begin{array}{l}5-16 \\
5-19 \\
5-21 \\
5-22 \\
5-24\end{array}$ & $\begin{array}{l}199 \\
282+ \\
677 \\
1722 \\
5059\end{array}$ & $\begin{array}{l}0 \\
2 \\
0 \\
4 \\
6\end{array}$ & $\begin{array}{l}0 \\
1 \\
0 \\
0 \\
3\end{array}$ & $\begin{array}{l}0 \\
1 \\
0 \\
3 \\
1\end{array}$ & $\begin{array}{l}0 \\
0 \\
0 \\
0 \\
1\end{array}$ & $\begin{array}{l}0 \\
0 \\
0 \\
0 \\
1\end{array}$ & $\begin{array}{l}0 \\
0 \\
0 \\
1 \\
0\end{array}$ \\
\hline $\begin{array}{l}5-26 \\
5-27 \\
5-28\end{array}$ & $\begin{array}{r}10 \overline{7} \\
910\end{array}$ & $\begin{array}{c}--\overline{0} \\
0\end{array}$ & $\begin{array}{r}-- \\
0 \\
0\end{array}$ & $\begin{array}{r}-\overline{0} \\
0\end{array}$ & $\begin{array}{r}--\overline{0} \\
0\end{array}$ & $\begin{array}{r}-\overline{0} \\
0\end{array}$ & $\begin{array}{r}-\overline{0} \\
0\end{array}$ \\
\hline $5-30$ & 365 & 0 & 0 & 0 & 0 & 0 & 0 \\
\hline $\begin{array}{l}6-01 \\
6-03 \\
6-05 \\
6-07 \\
6-09\end{array}$ & $\begin{array}{c}144+ \\
280 \\
96+ \\
393 \\
3919\end{array}$ & $\begin{array}{l}0 \\
0 \\
0 \\
0 \\
0\end{array}$ & $\begin{array}{l}0 \\
0 \\
0 \\
0 \\
0\end{array}$ & $\begin{array}{l}0 \\
0 \\
0 \\
0 \\
0\end{array}$ & $\begin{array}{l}0 \\
0 \\
0 \\
0 \\
0\end{array}$ & $\begin{array}{l}0 \\
0 \\
0 \\
0 \\
0\end{array}$ & $\begin{array}{l}0 \\
0 \\
0 \\
0 \\
0\end{array}$ \\
\hline $\begin{array}{l}6-10 \\
6-11 \\
6-12 \\
6-13 \\
6-14\end{array}$ & $\begin{array}{r}1830 \\
490 \\
2588 \\
1221 \\
1830\end{array}$ & $\begin{array}{l}0 \\
2 \\
0 \\
0 \\
0\end{array}$ & $\begin{array}{l}0 \\
0 \\
0 \\
0 \\
0\end{array}$ & $\begin{array}{l}0 \\
1 \\
0 \\
0 \\
0\end{array}$ & $\begin{array}{l}0 \\
0 \\
0 \\
0 \\
0\end{array}$ & $\begin{array}{l}0 \\
1 \\
0 \\
0 \\
0\end{array}$ & $\begin{array}{l}0 \\
0 \\
0 \\
0 \\
0\end{array}$ \\
\hline $\begin{array}{l}6-15 \\
6-16\end{array}$ & 1372 & 0 & 0 & 0 & 0 & 0 & 0 \\
\hline $\begin{array}{l}6-17 \\
6-18 \\
6-19\end{array}$ & $\begin{array}{l}3344 \\
3394 \\
3121\end{array}$ & $\begin{array}{l}0 \\
0 \\
0\end{array}$ & $\begin{array}{l}0 \\
0 \\
0\end{array}$ & $\begin{array}{l}0 \\
0 \\
0\end{array}$ & $\begin{array}{l}0 \\
0 \\
0\end{array}$ & $\begin{array}{l}0 \\
0 \\
0\end{array}$ & $\begin{array}{l}0 \\
0 \\
0\end{array}$ \\
\hline $\begin{array}{l}6-20 \\
6-21\end{array}$ & $\begin{array}{l}1823 \\
3246\end{array}$ & 11 & $\begin{array}{l}4 \\
0\end{array}$ & $\begin{array}{l}5 \\
0\end{array}$ & $\begin{array}{l}2 \\
0\end{array}$ & $\begin{array}{l}0 \\
0\end{array}$ & $\begin{array}{l}0 \\
0\end{array}$ \\
\hline $\begin{array}{l}6-23 \\
6-25\end{array}$ & $\begin{array}{l}2408 \\
2133\end{array}$ & $\begin{array}{l}0 \\
0\end{array}$ & $\begin{array}{l}0 \\
0\end{array}$ & $\begin{array}{l}0 \\
0\end{array}$ & $\begin{array}{l}0 \\
0\end{array}$ & $\begin{array}{l}0 \\
0\end{array}$ & $\begin{array}{l}0 \\
0\end{array}$ \\
\hline $6-27$ & 2454 & 1 & 0 & 1 & 0 & 0 & $\underline{0}$ \\
\hline $\begin{array}{l}0-29 \\
7-01\end{array}$ & - & - & - & - & -- & - & -- \\
\hline $\begin{array}{l}7-03 \\
7-05\end{array}$ & $=$ & $\overline{--}$ & $\overline{--}$ & $\overline{--}$ & $\overline{-}$ & -- & $\overline{--}$ \\
\hline $7-07$ & -- & -- & -- & -- & -- & -- & -- \\
\hline
\end{tabular}


TABLE 16.- NUMBER OF BLUE TRACER PARTICLES(1), PER 100 GRAMS OF BEDLOAD SAMPLE, COMPOSITED FROM CROSS-CHANNEL SAMPLING POSITIONS SPACED AT 1-METER INTERVALS ACROSS THE CHANNEL WIDTH, EAST FORK RIVER, WYOMING, 1980--CONTINUED

(1) BLUE TRACER PARTICLES PLACED AS BED MATERIAL 10 METERS DOWNSTREAM OF SECTION 1695 ON MAY 19, 1979.

(2) REPRESENTS TOTAL MASS, IN' GRAMS; RETAINED IN BEDLOAD SAMPLER AS MEASURED IN THE FIELD. FOR SUBSEQUENT ANALYSIS OF NUMBER AND SIZE OF TRACER, PARTICLES SMALLER THAN 0.25 MM AND LARGER THAN $8.0 \mathrm{MM}$ WERE DISCARDED. REMAINING SAMPLES WEIGHING MORE THAN 100 GRAMS WERE CUT TO A STANDARD 100 GRAM-PORTION; SAMPLES WEIGHING LESS THAN 100 GRAMS WERE ANALYZED INTACT.

(3) SMALL OVERFLOW CHANNEL ADJACENT TO SECTION.

(4) DOES NOT INCLUDE TRACER PARTICLES MEASURED IN TRANSPORT AT SECTION 1400 , A BYPASS CHANNEL COMMON TO SECTIONS 1360, 1396, AND 1425.

(5) BYPASS CHANNEL. SEE FIGURE 3

+ MASS OF SAMPLE ANALYZED WAS BETWEEN 50 AND 100 GRAMS; NUMBER OF TRACER PARTICLES ADJUSTED TO AN EQUIVALENT 100-GRAM SAMPLE; EXTRAPOL ATED NUMBERS ARE CONSIDERED REL IABLE.

$+\quad$ MASS OF SAMPLE ANALYZED WAS BETWEEN 10 AND 50 GRAMS; NUMBER OF TRACER PARTICLES ADJUSTED TO AN EQUIVALENT 100-GRAM SAMPLE; CARE SHOULD BE TAKEN IN USING THE EXTRAPOLATED NUMBERS.

++ MASS OF SAMPLE ANALYZED WAS BETWEEN 0 AND 10 GRAMS: NUMBER OF TRACER PARTICLES ADJUSTED TO AN EQUIVALENT 100-GRAM SAMPLE; EXTRAPOLATED NUMBERS ARE NOT CONSIDERED RELIABLE. 
TABLE 17.- NUMBER OF ORANGE TRACER PARTICLES(1), PER 100 GRAMS OF BEDLOAD SAMPLE, COMPOSITED FROM CROSS-CHANNEL SAMPLING POSITIONS SPACED AT 1-METER INTERVALS ACROSS THE CHANNEL WIDTH, EAST FORK RIVER, WYOMING, 1980

SECTION 0043

\begin{tabular}{|c|c|c|c|c|c|c|c|}
\hline \multirow{3}{*}{ DATE } & \multirow{3}{*}{$\begin{array}{c}\text { DRY } \\
\text { MASS } \\
\text { OF } \\
\text { SAMPLE (2) } \\
\text { (G) }\end{array}$} & \multicolumn{6}{|c|}{ NUMBER OF ORANGE TRACER PARTICLES } \\
\hline & & \multirow[b]{2}{*}{ TOTAL } & \multicolumn{5}{|c|}{ BY SIZE CLASS (MM) } \\
\hline & & & $\begin{array}{l}0.25 \\
\text { To } \\
0.50\end{array}$ & $\begin{array}{l}0.50 \\
\text { TO } \\
1.00\end{array}$ & $\begin{array}{l}1.00 \\
\text { TO } \\
2.00\end{array}$ & $\begin{array}{l}2.00 \\
\text { TO } \\
4.00\end{array}$ & $\begin{array}{l}4.00 \\
\text { TO } \\
8.00\end{array}$ \\
\hline $\begin{array}{l}5-16 \\
5-19 \\
5-21 \\
5-22\end{array}$ & $\begin{array}{l}59++ \\
75++ \\
54+ \\
--\end{array}$ & $\begin{array}{l}29 \\
23 \\
50 \\
-\end{array}$ & $\begin{array}{r}0 \\
12 \\
17 \\
-\end{array}$ & $\begin{array}{l}27 \\
11 \\
24\end{array}$ & $\begin{array}{r}2 \\
0 \\
9 \\
-\end{array}$ & $\begin{array}{r}0 \\
0 \\
0 \\
-\end{array}$ & $\begin{array}{r}0 \\
0 \\
0 \\
-\end{array}$ \\
\hline $5-24$ & -- & - & -- & -- & -- & -- & -- \\
\hline $\begin{array}{l}5-26 \\
5-27 \\
5-28\end{array}$ & $\begin{array}{c}371 \\
84+ \\
28++\end{array}$ & $\begin{array}{l}32 \\
26 \\
18\end{array}$ & $\begin{array}{l}9 \\
6 \\
4\end{array}$ & $\begin{array}{l}20 \\
18 \\
14\end{array}$ & $\begin{array}{l}1 \\
2 \\
0\end{array}$ & $\begin{array}{l}2 \\
0 \\
0\end{array}$ & $\begin{array}{l}0 \\
0 \\
0\end{array}$ \\
\hline $\begin{array}{l}5-29 \\
5-30\end{array}$ & $5+++$ & $1 \overline{20}$ & 20 & 100 & 0 & $\overline{0}$ & $\overline{0}$ \\
\hline $\begin{array}{l}6-01 \\
6-03 \\
6-05 \\
6-07 \\
6-09\end{array}$ & $\begin{array}{r}81++ \\
0+++ \\
0+++ \\
111+++ \\
303\end{array}$ & $\begin{array}{r}17 \\
0 \\
0 \\
0 \\
10\end{array}$ & $\begin{array}{l}5 \\
0 \\
0 \\
0 \\
3\end{array}$ & $\begin{array}{r}11 \\
0 \\
0 \\
0 \\
5\end{array}$ & $\begin{array}{l}1 \\
0 \\
0 \\
0 \\
2\end{array}$ & $\begin{array}{l}0 \\
0 \\
0 \\
0 \\
0\end{array}$ & $\begin{array}{l}0 \\
0 \\
0 \\
0 \\
0\end{array}$ \\
\hline $\begin{array}{l}6-10 \\
6-11 \\
6-12 \\
6-13 \\
6-14\end{array}$ & $\begin{array}{l}3071 \\
3889 \\
2659 \\
1640 \\
1030\end{array}$ & $\begin{array}{r}11 \\
6 \\
8 \\
2 \\
9\end{array}$ & $\begin{array}{l}1 \\
2 \\
0 \\
0 \\
3\end{array}$ & $\begin{array}{l}8 \\
2 \\
5 \\
0 \\
6\end{array}$ & $\begin{array}{l}1 \\
2 \\
1 \\
0 \\
0\end{array}$ & $\begin{array}{l}1 \\
0 \\
2 \\
2 \\
0\end{array}$ & $\begin{array}{l}0 \\
0 \\
0 \\
0 \\
0\end{array}$ \\
\hline $6-15$ & 984 & 5 & 1 & 4 & 0 & 0 & 0 \\
\hline $\begin{array}{l}6-17 \\
6-18 \\
6-19\end{array}$ & $\begin{array}{r}383 \\
320 \\
1123\end{array}$ & $\begin{array}{r}14 \\
10 \\
7\end{array}$ & $\begin{array}{l}6 \\
3 \\
0\end{array}$ & $\begin{array}{l}7 \\
7 \\
7\end{array}$ & $\begin{array}{l}0 \\
0 \\
0\end{array}$ & $\begin{array}{l}1 \\
0 \\
0\end{array}$ & $\begin{array}{l}0 \\
0 \\
0\end{array}$ \\
\hline $\begin{array}{l}6-20 \\
6-21\end{array}$ & $\begin{array}{l}271 \\
764\end{array}$ & $\begin{array}{l}5 \\
1\end{array}$ & $\begin{array}{l}0 \\
0\end{array}$ & $\begin{array}{l}5 \\
0\end{array}$ & $\begin{array}{l}0 \\
1\end{array}$ & $\begin{array}{l}0 \\
0\end{array}$ & $\begin{array}{l}0 \\
0\end{array}$ \\
\hline $\begin{array}{l}6-23 \\
6-25\end{array}$ & $\begin{array}{r}585 \\
1008\end{array}$ & $\begin{array}{l}5 \\
3\end{array}$ & $\frac{1}{2}$ & $\frac{3}{1}$ & $\begin{array}{l}0 \\
0\end{array}$ & $\begin{array}{l}1 \\
0\end{array}$ & $\begin{array}{l}0 \\
0\end{array}$ \\
\hline $6-27$ & 486 & 7 & 0 & 4 & 3 & 0 & 0 \\
\hline $\begin{array}{l}6-29 \\
7-01\end{array}$ & $\overline{-}$ & $\overline{-}$ & $\overline{--}$ & $\overline{--}$ & $\overline{--}$ & $\overline{--}$ & $\overline{--}$ \\
\hline $7-0 \overline{3}$ & - & - & -- & -- & -- & -- & -- \\
\hline $7-05$ & -- & - & -- & -- & -- & -- & -- \\
\hline $7-07$ & -- & -- & -- & -- & -- & -- & -- \\
\hline
\end{tabular}


TABLE 17.- NUMBER OF ORANGE TRACER PARTICLES(1), PER 100 GRAMS OF BEDLOAD SAMPLE, COMPOSITED FROM CROSS-CHANNEL SAMPLING POSITIONS SPACED AT 1-METER INTERVALS ACROSS THE CHANNEL WIDTH, EAST FORK RIVER, WYOMING, 1980

SECTION 0075

\begin{tabular}{|c|c|c|c|c|c|c|c|}
\hline \multirow[t]{2}{*}{ DATE } & \multirow{2}{*}{$\begin{array}{c}\text { DRY } \\
\text { MASS } \\
\text { OF } \\
\text { SAMPLE (2) } \\
\text { (G) }\end{array}$} & \multicolumn{6}{|c|}{$\begin{array}{l}\text { NUMBER OF ORANGE TRACER PARTICLES } \\
\text { BY SIZE CLASS (MM) }\end{array}$} \\
\hline & & TOTAL & $\begin{array}{l}0.25 \\
\text { TO } \\
0.50\end{array}$ & $\begin{array}{l}0.50 \\
\text { TO } \\
1.00\end{array}$ & $\begin{array}{l}1.00 \\
2.00\end{array}$ & $\begin{array}{l}2.00 \\
4.00\end{array}$ & $\begin{array}{l}4.00 \\
\text { TO } \\
8.00\end{array}$ \\
\hline $\begin{array}{l}5-16 \\
5-19 \\
5-21 \\
5-22 \\
5-24\end{array}$ & $\begin{array}{c}161+ \\
346 \\
1581 \\
-- \\
--\end{array}$ & $\begin{array}{r}8 \\
14 \\
30 \\
-- \\
--\end{array}$ & $\begin{array}{r}3 \\
7 \\
7 \\
-- \\
--\end{array}$ & $\begin{array}{r}5 \\
3 \\
17 \\
-- \\
--\end{array}$ & $\begin{array}{r}0 \\
1 \\
5 \\
-- \\
--\end{array}$ & $\begin{array}{r}0 \\
3 \\
1 \\
-- \\
--\end{array}$ & $\begin{array}{r}0 \\
0 \\
0 \\
-- \\
--\end{array}$ \\
\hline $\begin{array}{l}5-26 \\
5-27 \\
5-28 \\
5-29 \\
5-30\end{array}$ & $\begin{array}{l}51+ \\
127+ \\
127 \\
237 \\
19+++\end{array}$ & $\begin{array}{r}12 \\
2 \\
12 \\
4 \\
0\end{array}$ & $\begin{array}{l}6 \\
0 \\
1 \\
0 \\
0\end{array}$ & $\begin{array}{r}4 \\
1 \\
10 \\
2 \\
0\end{array}$ & $\begin{array}{l}2 \\
1 \\
1 \\
2 \\
0\end{array}$ & $\begin{array}{l}0 \\
0 \\
0 \\
0 \\
0\end{array}$ & $\begin{array}{l}0 \\
0 \\
0 \\
0 \\
0\end{array}$ \\
\hline $\begin{array}{l}6-01 \\
6-03 \\
6-05 \\
6-07 \\
6-09\end{array}$ & $\begin{array}{r}1907 \\
900 \\
283 \\
742 \\
1650\end{array}$ & $\begin{array}{r}12 \\
5 \\
13 \\
0 \\
7\end{array}$ & $\begin{array}{l}4 \\
0 \\
1 \\
0 \\
1\end{array}$ & $\begin{array}{l}7 \\
2 \\
8 \\
0 \\
6\end{array}$ & $\begin{array}{l}1 \\
3 \\
4 \\
0 \\
0\end{array}$ & $\begin{array}{l}0 \\
0 \\
0 \\
0 \\
0\end{array}$ & $\begin{array}{l}0 \\
0 \\
0 \\
0 \\
0\end{array}$ \\
\hline $\begin{array}{l}6-10 \\
6-11 \\
6-12 \\
6-13 \\
6-14\end{array}$ & $\begin{array}{l}3128 \\
1416 \\
2786 \\
1735 \\
1897\end{array}$ & $\begin{array}{l}8 \\
0 \\
0 \\
5 \\
5\end{array}$ & $\begin{array}{l}2 \\
0 \\
0 \\
1 \\
1\end{array}$ & $\begin{array}{l}4 \\
0 \\
0 \\
2 \\
2\end{array}$ & $\begin{array}{l}1 \\
0 \\
0 \\
2 \\
2\end{array}$ & $\begin{array}{l}1 \\
0 \\
0 \\
0 \\
0\end{array}$ & $\begin{array}{l}0 \\
0 \\
0 \\
0 \\
0\end{array}$ \\
\hline $\begin{array}{l}6-15 \\
6-16 \\
6-17 \\
6-18 \\
6-19\end{array}$ & $\begin{array}{r}651 \\
-- \\
900 \\
1969 \\
2818\end{array}$ & $\begin{array}{r}-\frac{4}{7} \\
4 \\
6\end{array}$ & $\begin{array}{r}-0 \\
-\frac{1}{2} \\
2 \\
3\end{array}$ & $\begin{array}{r}-\frac{3}{5} \\
\frac{2}{3}\end{array}$ & $\begin{array}{r}-\frac{1}{0} \\
0 \\
0\end{array}$ & $\begin{array}{r}-0 \\
-0 \\
0 \\
0\end{array}$ & $\begin{array}{r}0 \\
-\frac{0}{0} \\
0 \\
0\end{array}$ \\
\hline $\begin{array}{l}6-20 \\
6-21 \\
6-22 \\
6-23 \\
6-25\end{array}$ & $\begin{array}{r}5583 \\
4777 \\
1409 \\
1558\end{array}$ & $\begin{array}{r}5 \\
0 \\
-\frac{1}{2} \\
2\end{array}$ & $\begin{array}{r}1 \\
-1 \\
\frac{1}{0}\end{array}$ & $\begin{array}{r}3 \\
-0 \\
-1 \\
0\end{array}$ & $\begin{array}{r}1 \\
-0 \\
0 \\
0\end{array}$ & $\begin{array}{r}0 \\
-0 \\
-0 \\
2\end{array}$ & $\begin{array}{r}0 \\
0 \\
-\frac{1}{0} \\
0\end{array}$ \\
\hline $\begin{array}{l}6-27 \\
6-29 \\
7-01 \\
7-03 \\
7-05\end{array}$ & $\begin{array}{r}2302 \\
=- \\
=- \\
=-\end{array}$ & $\begin{array}{l}1 \\
-- \\
--\end{array}$ & $\begin{array}{l}0 \\
-- \\
-- \\
--\end{array}$ & $\begin{array}{l}1 \\
-- \\
-- \\
--\end{array}$ & $\begin{array}{l}0 \\
-- \\
-- \\
--\end{array}$ & $\begin{array}{l}0 \\
-- \\
--\end{array}$ & $\begin{array}{l}0 \\
-- \\
-- \\
--\end{array}$ \\
\hline $7-07$ & -- & -- & -- & -- & -- & -- & -- \\
\hline
\end{tabular}


TABLE 17.- NUMBER OF ORANGE TRACER PARTICLES(1), PER 100 GRAMS OF BEDLOAD SAMPLE, COMPOSITED FROM CROSS-CHANNEL SAMPLING POSITIONS SPACED AT 1-METER INTERVALS ACROSS THE CHANNEL WIDTH, EAST FORK RIVER, WYOMING, 1980

SECTION 0137

\begin{tabular}{|c|c|c|c|c|c|c|c|}
\hline \multirow{3}{*}{ DATE } & \multirow{3}{*}{$\begin{array}{c}\text { DRY } \\
\text { MASS } \\
\text { OF } \\
\text { SAMPLE (2) } \\
\text { (G) }\end{array}$} & \multicolumn{6}{|c|}{ NUMBER OF ORANGE TRACER PARTICLES } \\
\hline & & & & BY SI & CLASS & & \\
\hline & & TOTAL & $\begin{array}{c}0.25 \\
\text { TO } \\
0.50\end{array}$ & $\begin{array}{l}0.50 \\
\text { TO } \\
1.00\end{array}$ & $\begin{array}{l}1.00 \\
\text { TO } \\
2.00\end{array}$ & $\begin{array}{l}2.00 \\
\text { TO } \\
4.00\end{array}$ & $\begin{array}{l}4.00 \\
\text { TO } \\
8.00\end{array}$ \\
\hline $\begin{array}{l}5-16 \\
5-19 \\
5-21 \\
5-22 \\
5-24\end{array}$ & $\begin{array}{l}225 \\
56+++ \\
113++ \\
=- \\
--\end{array}$ & $\begin{array}{r}29 \\
0 \\
6 \\
--\end{array}$ & $\begin{array}{r}15 \\
0 \\
3 \\
-- \\
--\end{array}$ & $\begin{array}{r}10 \\
0 \\
3 \\
--\end{array}$ & $\begin{array}{r}2 \\
0 \\
0 \\
-- \\
--\end{array}$ & $\begin{array}{r}2 \\
0 \\
0 \\
-- \\
--\end{array}$ & $\begin{array}{r}0 \\
0 \\
0 \\
-- \\
--\end{array}$ \\
\hline $\begin{array}{l}5-26 \\
5-27 \\
5-28 \\
5-29 \\
5-30\end{array}$ & $\begin{array}{r}1360 \\
1794 \\
135 \\
\overline{99+}\end{array}$ & $\begin{array}{r}9 \\
6 \\
7 \\
-4\end{array}$ & $\begin{array}{r}0 \\
0 \\
-1 \\
-3\end{array}$ & $\begin{array}{r}6 \\
4 \\
2 \\
-0\end{array}$ & $\begin{array}{r}3 \\
2 \\
2 \\
-1\end{array}$ & $\begin{array}{r}0 \\
0 \\
2 \\
-2 \\
0\end{array}$ & $\begin{array}{r}0 \\
0 \\
0 \\
-0\end{array}$ \\
\hline $\begin{array}{l}6-01 \\
6-03 \\
6-05 \\
6-07 \\
6-09\end{array}$ & $\begin{array}{c}41++ \\
73++ \\
0+++ \\
588 \\
1099\end{array}$ & $\begin{array}{l}7 \\
1 \\
0 \\
4 \\
3\end{array}$ & $\begin{array}{l}0 \\
0 \\
0 \\
0 \\
3\end{array}$ & $\begin{array}{l}7 \\
0 \\
0 \\
3 \\
0\end{array}$ & $\begin{array}{l}0 \\
1 \\
0 \\
0 \\
0\end{array}$ & $\begin{array}{l}0 \\
0 \\
0 \\
1 \\
0\end{array}$ & $\begin{array}{l}0 \\
0 \\
0 \\
0 \\
0\end{array}$ \\
\hline $\begin{array}{l}6-10 \\
6-11 \\
6-12 \\
6-13 \\
6-14\end{array}$ & $\begin{array}{r}1907 \\
1139 \\
517 \\
1648 \\
3009\end{array}$ & $\begin{array}{l}0 \\
6 \\
3 \\
5 \\
8\end{array}$ & $\begin{array}{l}0 \\
3 \\
1 \\
1 \\
1\end{array}$ & $\begin{array}{l}0 \\
3 \\
1 \\
4 \\
3\end{array}$ & $\begin{array}{l}0 \\
0 \\
0 \\
0 \\
2\end{array}$ & $\begin{array}{l}0 \\
0 \\
1 \\
0 \\
2\end{array}$ & $\begin{array}{l}0 \\
0 \\
0 \\
0 \\
0\end{array}$ \\
\hline $\begin{array}{l}6-15 \\
6-16 \\
6-17 \\
6-18 \\
6-19\end{array}$ & $\begin{array}{r}2497 \\
3116 \\
3204 \\
2346\end{array}$ & $\begin{array}{r}-2 \\
-3 \\
7 \\
3\end{array}$ & $\begin{array}{r}0 \\
-0 \\
0 \\
0\end{array}$ & $\begin{array}{r}-0 \\
-\frac{1}{2} \\
6 \\
2\end{array}$ & $-\frac{2}{1}$ & 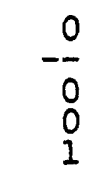 & $\begin{array}{r}-0 \\
-0 \\
0 \\
0\end{array}$ \\
\hline $\begin{array}{l}6-20 \\
6-21\end{array}$ & $\begin{array}{l}2042 \\
3752\end{array}$ & 4 & $\begin{array}{l}0 \\
0\end{array}$ & $\begin{array}{l}3 \\
0\end{array}$ & $\frac{1}{0}$ & $\begin{array}{l}0 \\
0\end{array}$ & $\begin{array}{l}0 \\
0\end{array}$ \\
\hline $\begin{array}{l}6-23 \\
6-25\end{array}$ & $\begin{array}{l}2398 \\
3614\end{array}$ & 0 & $\begin{array}{l}0 \\
0\end{array}$ & $\begin{array}{l}0 \\
1\end{array}$ & 0 & $\begin{array}{l}0 \\
0\end{array}$ & $\begin{array}{l}0 \\
0\end{array}$ \\
\hline $\begin{array}{l}6-27 \\
6-29 \\
7-01 \\
7-03 \\
7-05\end{array}$ & $\begin{array}{r}4050 \\
=- \\
=- \\
=-\end{array}$ & $\begin{array}{l}10 \\
=- \\
=-\end{array}$ & $\begin{array}{l}1 \\
-- \\
--\end{array}$ & $\begin{array}{l}5 \\
-- \\
--\end{array}$ & $\begin{array}{l}4 \\
-- \\
--\end{array}$ & $\begin{array}{l}0 \\
-- \\
--\end{array}$ & $\begin{array}{l}0 \\
-- \\
--\end{array}$ \\
\hline $7-07$ & -- & - & -- & -- & - & -- & - \\
\hline
\end{tabular}


TABLE 17.- NUMBER OF ORANGE TRACER PARTICLES(1), PER 100 GRAMS OF BEDLOAD SAMPLE, COMPOSITED FROM CROSS-CHANNEL SAMPLING POSITIONS

SPACED AT 1-METER INTERVALS ACROSS THE CHANNEL WIDTH,

EAST FORK RIVER, WYOMING, 1980

\section{SECTION 0178}

\begin{tabular}{|c|c|c|c|c|c|c|c|}
\hline \multirow{3}{*}{ DATE } & \multirow{3}{*}{$\begin{array}{c}\text { DRY } \\
\text { MASS } \\
\text { OF } \\
\text { SAMPLE (2) } \\
\text { (G) }\end{array}$} & \multicolumn{6}{|c|}{ NUMBER OF ORANGE TRACER PARTICLES } \\
\hline & & \multirow[b]{2}{*}{ TOTAL } & \multicolumn{5}{|c|}{ BY SIZE CLASS (MM) } \\
\hline & & & $\begin{array}{c}0.25 \\
\text { TO } \\
0.50\end{array}$ & $\begin{array}{c}0.50 \\
\text { TO } \\
1.00\end{array}$ & $\begin{array}{l}1.00 \\
\text { TO } \\
2.00\end{array}$ & $\begin{array}{l}2.00 \\
\text { TO } \\
4.00\end{array}$ & $\begin{array}{c}4.00 \\
\text { TO } \\
8.00\end{array}$ \\
\hline $\begin{array}{l}5-16 \\
5-19 \\
5-21 \\
5-22 \\
5-24\end{array}$ & $\begin{array}{r}335 \\
1172 \\
430 \\
-- \\
--\end{array}$ & $\begin{array}{r}11 \\
25 \\
9 \\
-- \\
--\end{array}$ & $\begin{array}{r}1 \\
1 \frac{1}{7} \\
--\end{array}$ & $\begin{array}{r}8 \\
13 \\
1 \\
-- \\
--\end{array}$ & $\begin{array}{r}1 \\
0 \\
1 \\
-- \\
--\end{array}$ & $\begin{array}{r}1 \\
1 \\
0 \\
--\end{array}$ & $\begin{array}{r}0 \\
0 \\
0 \\
-- \\
--\end{array}$ \\
\hline $\begin{array}{l}5-26 \\
5-27 \\
5-28\end{array}$ & $\begin{array}{r}2416 \\
677 \\
446\end{array}$ & $\begin{array}{l}6 \\
8 \\
6\end{array}$ & $\begin{array}{l}0 \\
3 \\
1\end{array}$ & $\begin{array}{l}4 \\
2 \\
2\end{array}$ & $\begin{array}{l}0 \\
3 \\
3\end{array}$ & $\begin{array}{l}2 \\
0 \\
0\end{array}$ & $\begin{array}{l}0 \\
0 \\
0\end{array}$ \\
\hline $5-30$ & 470 & 7 & 2 & 4 & 1 & 0 & 0 \\
\hline $\begin{array}{l}6-01 \\
6-03 \\
6-05 \\
6-07 \\
6-09\end{array}$ & $\begin{array}{c}521 \\
18++ \\
689 \\
1624 \\
2378\end{array}$ & $\begin{array}{l}2 \\
0 \\
2 \\
1 \\
1\end{array}$ & $\begin{array}{l}0 \\
0 \\
1 \\
0 \\
0\end{array}$ & $\begin{array}{l}0 \\
0 \\
1 \\
1 \\
1\end{array}$ & $\begin{array}{l}0 \\
0 \\
0 \\
0 \\
0\end{array}$ & $\begin{array}{l}0 \\
0 \\
0 \\
0 \\
0\end{array}$ & $\begin{array}{l}2 \\
0 \\
0 \\
0 \\
0\end{array}$ \\
\hline $\begin{array}{l}6-10 \\
6-11 \\
6-12 \\
6-13 \\
6-14\end{array}$ & $\begin{array}{l}6609 \\
4737 \\
1694 \\
5416 \\
3955\end{array}$ & $\begin{array}{l}1 \\
2 \\
4 \\
8 \\
5\end{array}$ & $\begin{array}{l}0 \\
0 \\
0 \\
4 \\
0\end{array}$ & $\begin{array}{l}0 \\
0 \\
4 \\
3 \\
4\end{array}$ & $\begin{array}{l}1 \\
2 \\
0 \\
1 \\
1\end{array}$ & $\begin{array}{l}0 \\
0 \\
0 \\
0 \\
0\end{array}$ & $\begin{array}{l}0 \\
0 \\
0 \\
0 \\
0\end{array}$ \\
\hline $6-15$ & 3251 & 2 & 0 & 2 & 0 & 0 & 0 \\
\hline $\begin{array}{l}6-17 \\
6-18 \\
6-19\end{array}$ & $\begin{array}{l}1273 \\
3439 \\
3696\end{array}$ & $\begin{array}{l}1 \\
6 \\
4\end{array}$ & $\begin{array}{l}0 \\
1 \\
1\end{array}$ & $\begin{array}{l}0 \\
4 \\
2\end{array}$ & $\begin{array}{l}1 \\
1 \\
0\end{array}$ & $\begin{array}{l}0 \\
0 \\
1\end{array}$ & $\begin{array}{l}0 \\
0 \\
0\end{array}$ \\
\hline $\begin{array}{l}6-20 \\
6-21\end{array}$ & $\begin{array}{l}5908 \\
5074\end{array}$ & $\begin{array}{l}2 \\
0\end{array}$ & $\begin{array}{l}0 \\
0\end{array}$ & $\begin{array}{l}0 \\
0\end{array}$ & $\begin{array}{l}0 \\
0\end{array}$ & $\begin{array}{l}2 \\
0\end{array}$ & $\begin{array}{l}0 \\
0\end{array}$ \\
\hline $\begin{array}{l}6-23 \\
6-25\end{array}$ & $\begin{array}{l}4771 \\
1510\end{array}$ & $\begin{array}{l}2 \\
1\end{array}$ & $\begin{array}{l}0 \\
0\end{array}$ & $\begin{array}{l}0 \\
1\end{array}$ & $\begin{array}{l}2 \\
0\end{array}$ & $\begin{array}{l}0 \\
0\end{array}$ & $\begin{array}{l}0 \\
0\end{array}$ \\
\hline $6-27$ & 3035 & 10 & 1 & 7 & 1 & 1 & 0 \\
\hline $7-01$ & - & $=$ & - & $=$ & $=$ & $=$ & $=$ \\
\hline $7-0 \overline{3}$ & -- & -- & -- & - & -- & - & -- \\
\hline $7-05$ & - & - & - & - & - & - & - \\
\hline $7-07$ & -- & -- & -- & - & -- & -- & -- \\
\hline
\end{tabular}


TABLE 17.- NUMBER OF ORANGE TRACER PARTICLES(1), PER 100 GRAMS OF BEDLOAD SAMPLE, COMPOSITED FROM CROSS-CHANNEL SAMPLING POSITIONS

SPACED AT 1-METER INTERVALS ACROSS THE CHANNEL WIDTH,

EAST FORK RIVER, WYOMING, 1980

$$
\text { SECTION } 2220
$$

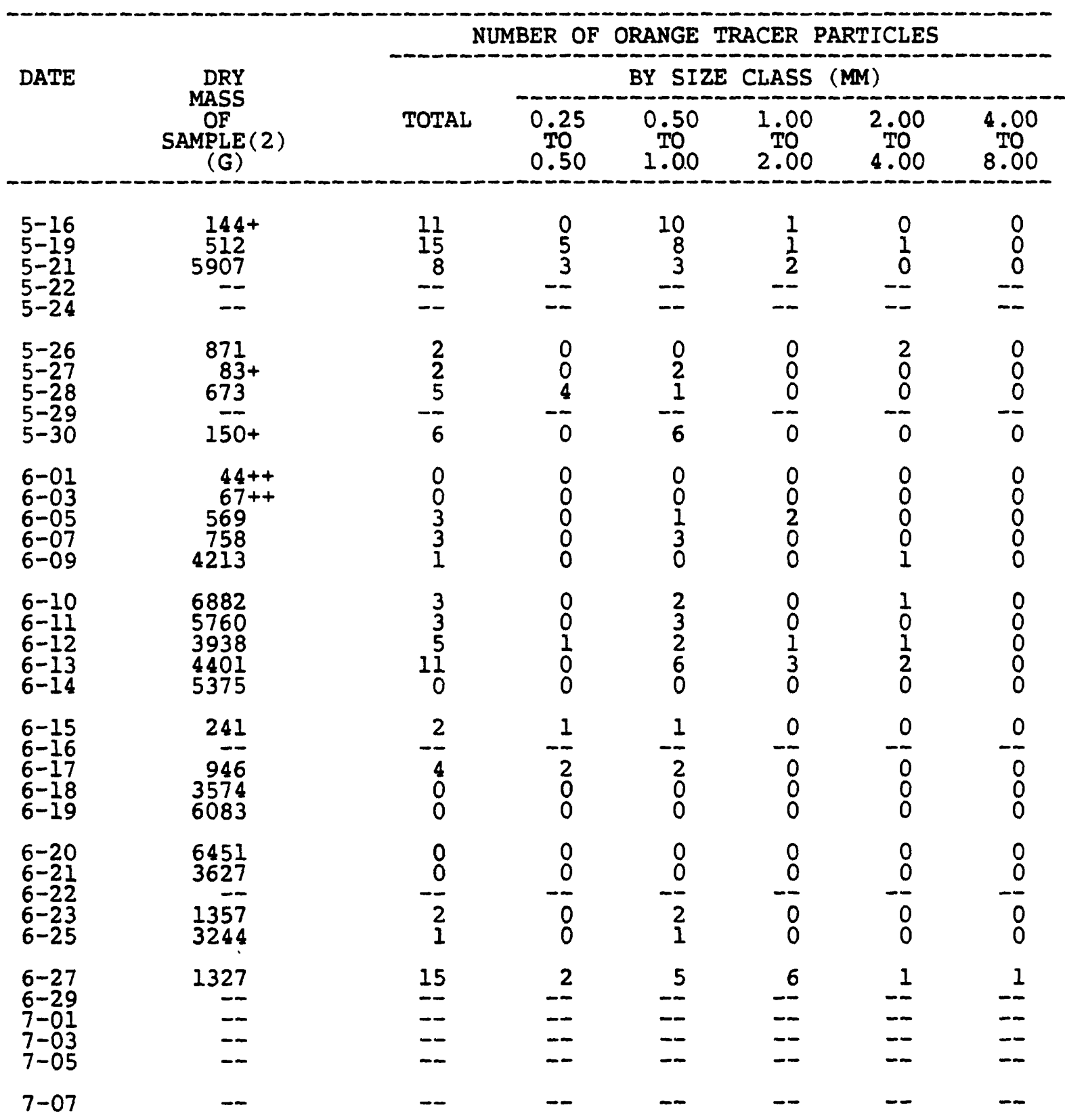


TABLE 17.- NUMBER OF ORANGE TRACER PARTICLES(1), PER 100 GRAMS OF BEDLOAD SAMPLE, COMPOSITED FROM CROSS-CHANNEL SAMPLING POSITIONS SPACED AT 1-METER INTERVALS ACROSS THE CHANNEL WIDTH, EAST FORK RIVER, WYOMING, 1980

SECTION 0257

\begin{tabular}{|c|c|c|c|c|c|c|c|}
\hline \multirow[t]{2}{*}{ DATE } & \multirow{2}{*}{$\begin{array}{c}\text { DRY } \\
\text { MASS } \\
\text { OF } \\
\text { SAMPLE (2) } \\
(\mathrm{G})\end{array}$} & \multicolumn{6}{|c|}{ NUMBER OF ORANGE TRACER PARTICLES } \\
\hline & & TOTAL & $\begin{array}{l}0.25 \\
\text { TO } \\
0.50\end{array}$ & $\begin{array}{r}0.50 \\
\text { TO } \\
1.00\end{array}$ & $\begin{array}{l}1.00 \\
\text { TO } \\
2.00\end{array}$ & $\begin{array}{l}2.00 \\
\text { TO } \\
4.00\end{array}$ & $\begin{array}{l}4.00 \\
\text { TO } \\
8.00\end{array}$ \\
\hline $\begin{array}{l}5-16 \\
5-19 \\
5-21 \\
5-22 \\
5-24\end{array}$ & $\begin{array}{c}119+ \\
697 \\
3491 \\
-- \\
--\end{array}$ & $\begin{array}{r}10 \\
5 \\
4 \\
-- \\
--\end{array}$ & $\begin{array}{r}7 \\
\frac{1}{3} \\
-- \\
--\end{array}$ & $\begin{array}{r}3 \\
1 \\
0 \\
---\end{array}$ & $\begin{array}{r}0 \\
3 \\
1 \\
-- \\
--\end{array}$ & $\begin{array}{r}0 \\
0 \\
0 \\
-- \\
--\end{array}$ & $\begin{array}{r}0 \\
0 \\
0 \\
-- \\
--\end{array}$ \\
\hline $\begin{array}{l}5-26 \\
5-27 \\
5-28 \\
5-29 \\
5-30\end{array}$ & $\begin{array}{l}183 \\
113 \\
42++ \\
74+\end{array}$ & $\begin{array}{r}4 \\
0 \\
0 \\
-0\end{array}$ & $\begin{array}{r}0 \\
0 \\
0 \\
-0\end{array}$ & $\begin{array}{r}0 \\
0 \\
0 \\
-0\end{array}$ & $\begin{array}{r}4 \\
0 \\
0 \\
-\frac{1}{0}\end{array}$ & $\begin{array}{r}0 \\
0 \\
0 \\
-0 \\
0\end{array}$ & $\begin{array}{r}0 \\
0 \\
0 \\
- \\
0\end{array}$ \\
\hline $\begin{array}{l}6-01 \\
6-03 \\
6-05 \\
6-07 \\
6-09\end{array}$ & $\begin{array}{c}90++ \\
697 \\
1516 \\
645 \\
3432\end{array}$ & $\begin{array}{l}3 \\
0 \\
1 \\
4 \\
9\end{array}$ & $\begin{array}{l}0 \\
0 \\
0 \\
2 \\
2\end{array}$ & $\begin{array}{l}3 \\
0 \\
0 \\
2 \\
7\end{array}$ & $\begin{array}{l}0 \\
0 \\
1 \\
0 \\
0\end{array}$ & $\begin{array}{l}0 \\
0 \\
0 \\
0 \\
0\end{array}$ & $\begin{array}{l}0 \\
0 \\
0 \\
0 \\
0\end{array}$ \\
\hline $\begin{array}{l}6-10 \\
6-11 \\
6-12 \\
6-13 \\
6-14\end{array}$ & $\begin{array}{l}5300 \\
2534 \\
3148 \\
3296 \\
1846\end{array}$ & $\begin{array}{r}1 \\
2 \\
3 \\
10 \\
1\end{array}$ & $\begin{array}{l}0 \\
0 \\
1 \\
5 \\
0\end{array}$ & $\begin{array}{l}1 \\
2 \\
2 \\
3 \\
1\end{array}$ & $\begin{array}{l}0 \\
0 \\
0 \\
2 \\
0\end{array}$ & $\begin{array}{l}0 \\
0 \\
0 \\
0 \\
0\end{array}$ & $\begin{array}{l}0 \\
0 \\
0 \\
0 \\
0\end{array}$ \\
\hline $\begin{array}{l}6-15 \\
6-16 \\
6-17 \\
6-18 \\
6-19\end{array}$ & $\begin{array}{r}2370 \\
799 \\
1076 \\
2889 \\
4435\end{array}$ & $\begin{array}{l}1 \\
4 \\
1 \\
3 \\
0\end{array}$ & $\begin{array}{l}0 \\
3 \\
0 \\
0 \\
0\end{array}$ & $\begin{array}{l}1 \\
1 \\
1 \\
0 \\
0\end{array}$ & $\begin{array}{l}0 \\
0 \\
0 \\
3 \\
0\end{array}$ & $\begin{array}{l}0 \\
0 \\
0 \\
0 \\
0\end{array}$ & $\begin{array}{l}0 \\
0 \\
0 \\
0 \\
0\end{array}$ \\
\hline $\begin{array}{l}6-20 \\
6-21\end{array}$ & $\begin{array}{l}7086 \\
2965\end{array}$ & $\begin{array}{l}0 \\
0\end{array}$ & $\begin{array}{l}0 \\
0\end{array}$ & $\begin{array}{l}0 \\
0\end{array}$ & $\begin{array}{l}0 \\
0\end{array}$ & $\begin{array}{l}0 \\
0\end{array}$ & $\begin{array}{l}0 \\
0\end{array}$ \\
\hline $\begin{array}{l}6-23 \\
6-25\end{array}$ & $\begin{array}{l}1826 \\
2479\end{array}$ & $\begin{array}{l}0 \\
3\end{array}$ & $\begin{array}{l}0 \\
3\end{array}$ & $\begin{array}{l}0 \\
0\end{array}$ & $\begin{array}{l}0 \\
0\end{array}$ & $\begin{array}{l}0 \\
0\end{array}$ & $\begin{array}{l}0 \\
0\end{array}$ \\
\hline $\begin{array}{l}6-27 \\
6-29 \\
7-01 \\
7-03 \\
7-05\end{array}$ & $\begin{array}{r}4340 \\
=- \\
=- \\
--\end{array}$ & $\begin{array}{l}10 \\
-- \\
-- \\
--\end{array}$ & $\begin{array}{l}0 \\
-- \\
-- \\
--\end{array}$ & $\begin{array}{l}5 \\
-- \\
-- \\
--\end{array}$ & $\begin{array}{l}5 \\
-- \\
-- \\
--\end{array}$ & $\begin{array}{l}0 \\
-- \\
-- \\
--\end{array}$ & $\begin{array}{l}0 \\
-- \\
-- \\
--\end{array}$ \\
\hline $7-07$ & -- & -- & -- & -- & -- & -- & -- \\
\hline
\end{tabular}


TABLE 17.- NUMBER OF ORANGE TRACER PARTICLES(1), PER 100 GRAMS OF BEDLOAD SAMPLE, COMPOSITED FROM CROSS-CHANNEL SAMPLING POSITIONS SPACED AT 1-METER INTERVALS ACROSS THE CHANNEL WIDTH, EAST FORK RIVER, WYOMING, 1980

SECTION $0257(3)$

\begin{tabular}{|c|c|c|c|c|c|c|c|}
\hline \multirow{3}{*}{ DATE } & \multirow{3}{*}{$\begin{array}{c}\text { DRY } \\
\text { MASS } \\
\text { OF } \\
\text { SAMPLE (2) } \\
\text { (G) }\end{array}$} & \multicolumn{6}{|c|}{ NUMBER OF ORANGE TRACER PARTICLES } \\
\hline & & \multirow[b]{2}{*}{ TOTAL } & \multicolumn{5}{|c|}{ BY SIZE CLASS (MM) } \\
\hline & & & $\begin{array}{c}0.25 \\
\text { TO } \\
0.50\end{array}$ & $\begin{array}{l}0.50 \\
\text { TO } \\
1.00\end{array}$ & $\begin{array}{l}1.00 \\
2.00 \\
\text { TO }\end{array}$ & $\begin{array}{l}2.00 \\
\text { TO } \\
4.00\end{array}$ & $\begin{array}{c}4.00 \\
\text { TO } \\
8.00\end{array}$ \\
\hline $5-16$ & -- & - & -- & -- & - & -- & - \\
\hline $\begin{array}{l}5-19 \\
5-21\end{array}$ & $=$ & $\overline{-}$ & $\overline{-}$ & $\overline{--}$ & $\overline{-}$ & $\overline{-}$ & $\overline{--}$ \\
\hline $5-22$ & - & -- & - & -- & -- & -- & -- \\
\hline $5-24$ & -- & -- & -- & - & - & -- & - \\
\hline $5-26$ & -- & - & -- & - & - & - & -- \\
\hline $5-27$ & $=$ & $=$ & $=$ & $=$ & -- & -- & $=$ \\
\hline $5-29$ & - & -- & -- & - & -- & -- & -- \\
\hline $5-30$ & -- & -- & -- & -- & -- & -- & - \\
\hline $6-01$ & - & -- & -- & - & -- & -- & -- \\
\hline $6-03$ & - & -- & -- & - & -- & -- & -- \\
\hline $6-07$ & -- & -- & - & -- & - & -- & - \\
\hline $6-09$ & 314 & 0 & 0 & 0 & 0 & 0 & 0 \\
\hline $6-10$ & 820 & 11 & 1 & 8 & 2 & 0 & 0 \\
\hline $6-12$ & 1057 & 0 & 0 & 0 & 0 & 0 & 0 \\
\hline $\begin{array}{l}6-13 \\
6-14\end{array}$ & $\begin{array}{l}528 \\
634\end{array}$ & 20 & $\begin{array}{r}15 \\
0\end{array}$ & $\begin{array}{l}5 \\
2\end{array}$ & $\begin{array}{l}0 \\
0\end{array}$ & $\begin{array}{l}0 \\
1\end{array}$ & $\begin{array}{l}0 \\
0\end{array}$ \\
\hline $6-15$ & 588 & 2 & 1 & 1 & 0 & 0 & 0 \\
\hline $6-16$ & -- & -- & -- & -- & -- & - & - \\
\hline $6-18$ & 276 & 10 & 3 & 6 & 1 & 0 & 0 \\
\hline $6-19$ & 1102 & 0 & 0 & 0 & 0 & 0 & 0 \\
\hline $6-20$ & 298 & 3 & 2 & 1 & 0 & 0 & 0 \\
\hline $6-21$ & 287 & 3 & 2 & 1 & 0 & 0 & 0 \\
\hline $6-23$ & - & - & -- & - & -- & -- & -- \\
\hline $6-25$ & 598 & 0 & 0 & 0 & 0 & 0 & 0 \\
\hline $6-27$ & 320 & 15 & 0 & 14 & 1 & 0 & 0 \\
\hline $\begin{array}{l}6-29 \\
7-01\end{array}$ & $=$ & $=$ & $=$ & $=$ & $=-$ & $=$ & - \\
\hline $7-03$ & -- & -- & -- & -- & -- & -- & - \\
\hline $7-05$ & -- & -- & -- & -- & -- & -- & \\
\hline $7-07$ & -- & -- & -- & -- & -- & -- & - \\
\hline
\end{tabular}


TABLE 17.- NUMBER OF ORANGE TRACER PARTICLES(1), PER 100 GRAMS OF BEDLOAD SAMPLE, COMPOSITED FROM CROSS-CHANNEL SAMPLING POSITIONS SPACED AT I-METER INTERVALS ACROSS THE CHANNEL WIDTH, EAST FORK RIVER, WYOMING, 1980

SECTION 0301

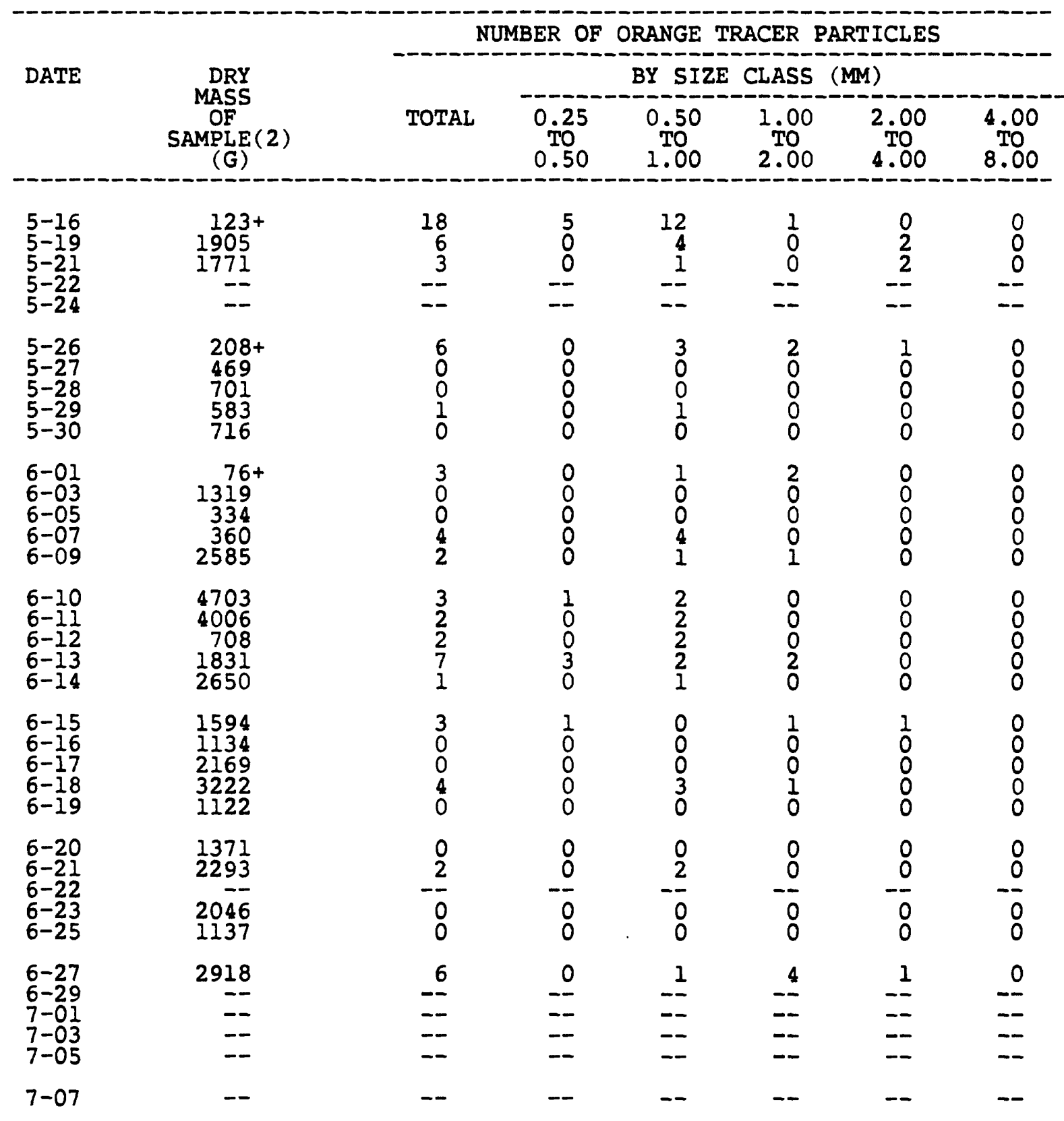


TABLE 17.- NUMBER OF ORANGE TRACER PARTICLES(1), PER 100 GRAMS OF BEDLOAD SAMPLE, COMPOSITED FROM CROSS-CHANNEL SAMPLING POSITIONS SPACED AT I-METER INTERVALS ACROSS THE CHANNEL WIDTH, EAST FORK RIVER, WYOMING, 1980

$$
\text { SECTION 0301(3) }
$$

\begin{tabular}{|c|c|c|c|c|c|c|c|}
\hline \multirow{3}{*}{ DATE } & \multirow{3}{*}{$\begin{array}{c}\text { DRY } \\
\text { MASS } \\
\text { OF } \\
\text { SAMPLE (2) } \\
\text { (G) }\end{array}$} & \multicolumn{6}{|c|}{ NUMBER OF ORANGE TRACER PARTICLES } \\
\hline & & \multirow[b]{2}{*}{ TOTAL } & \multicolumn{5}{|c|}{ BY SIZE CLASS (MM) } \\
\hline & & & $\begin{array}{l}0.25 \\
\text { TO } \\
0.50\end{array}$ & $\begin{array}{l}0.50 \\
\text { TO } \\
1.00\end{array}$ & $\begin{array}{l}1.00 \\
\text { TO } \\
2.00\end{array}$ & $\begin{array}{l}2.00 \\
\text { TO } \\
4.00\end{array}$ & $\begin{array}{l}4.00 \\
\text { TO } \\
8.00\end{array}$ \\
\hline $5-16$ & -- & -- & - & -- & -- & -- & -- \\
\hline $\begin{array}{l}5-19 \\
5-21\end{array}$ & -- & - & - & - & $\overline{--}$ & $\overline{--}$ & $\overline{--}$ \\
\hline $5-22$ & -- & -- & -- & -- & -- & -- & -- \\
\hline $5-24$ & -- & -- & -- & -- & -- & -- & -- \\
\hline $5-26$ & -- & -- & -- & -- & -- & -- & -- \\
\hline $\begin{array}{l}5-27 \\
5-28\end{array}$ & 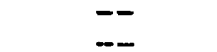 & $\overline{--}$ & -- & $\overline{--}$ & $\ddot{--}$ & -- & $=-$ \\
\hline $5-29$ & -- & -- & -- & -- & -- & ... & -- \\
\hline $5-30$ & -- & -- & -- & -- & -- & -- & -- \\
\hline $6-01$ & -- & -- & -- & -- & - & -- & -- \\
\hline $6-03$ & $\overline{-}$ & $=$ & -- & $\overline{-}$ & $=$ & -- & -- \\
\hline $\begin{array}{l}6-05 \\
6-07\end{array}$ & -- & -- & -- & -- & -- & - & - \\
\hline $6-09$ & 410 & 14 & 1 & 9 & 4 & 0 & 0 \\
\hline $6-10$ & 749 & 4 & 3 & 1 & 0 & 0 & 0 \\
\hline $6-11$ & 1836 & 0 & 0 & 0 & 0 & 0 & 0 \\
\hline $6-13$ & $\begin{array}{l}1 \frac{1}{2}<\frac{1}{2} \\
2235\end{array}$ & $\frac{1}{7}$ & 2 & 4 & $\frac{1}{1}$ & 0 & 0 \\
\hline $6-14$ & 846 & 3 & 0 & 3 & $\overline{0}$ & 0 & 0 \\
\hline $6-15$ & 455 & 0 & 0 & 0 & 0 & 0 & 0 \\
\hline $6-16$ & -- & -- & -- & -- & -- & -- & -- \\
\hline $\begin{array}{l}0-1 / \\
6-18\end{array}$ & 837 & 0 & 0 & 0 & 0 & 0 & 0 \\
\hline $6-19$ & 827 & 0 & 0 & 0 & 0 & 0 & 0 \\
\hline $6-20$ & $61+++$ & 0 & 0 & 0 & 0 & 0 & 0 \\
\hline $\begin{array}{l}6-21 \\
6-22\end{array}$ & 965 & -2 & -2 & -- & 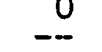 & 0 & \\
\hline $6-23$ & 290 & 0 & 0 & 0 & 0 & 0 & 0 \\
\hline & & & & & & & \\
\hline & 149 & 17 & 6 & 11 & 0 & 0 & 0 \\
\hline $\begin{array}{r}6-29 \\
7-01\end{array}$ & $=-$ & $=-$ & - & $=$ & $=$ & $=$ & $=$ \\
\hline $7-03$ & $\ldots$ & -- & -- & -- & -- & -- & -- \\
\hline $7-05$ & -- & -- & -- & -- & -- & -- & -- \\
\hline $7-07$ & -- & -- & -- & -- & -- & -- & -- \\
\hline
\end{tabular}


TABLE 17.- NUMBER OF ORANGE TRACER PARTICLES(1), PER 100 GRAMS OF BEDLOAD SAMPLE, COMPOSITED FROM CROSS-CHANNEL SAMPLING POSITIONS SPACED AT I-METER INTERVALS ACROSS THE CHANNEL WIDTH, EAST FORK RIVER, WYOMING, 1980

\section{SECTION 0348}

\begin{tabular}{|c|c|c|c|c|c|c|c|}
\hline \multirow{3}{*}{\multicolumn{2}{|c|}{$\begin{array}{cc}\text { DATE } & \text { DRY } \\
& \text { MASS } \\
\text { OF } \\
\text { SAMPLE (2) } \\
& (\mathrm{G})\end{array}$}} & \multicolumn{6}{|c|}{ NUMBER OF ORANGE TRACER PARTICLES } \\
\hline & & \multirow[b]{2}{*}{ TOTAL } & \multicolumn{5}{|c|}{ BY SIZE CLASS (MM) } \\
\hline & & & $\begin{array}{c}0.25 \\
\text { TO } \\
0.50\end{array}$ & $\begin{array}{l}0.50 \\
\text { TO } \\
1.00\end{array}$ & $\begin{array}{c}1.00 \\
\text { TO } \\
2.00\end{array}$ & $\begin{array}{l}2.00 \\
\text { TO } \\
4.00\end{array}$ & $\begin{array}{l}4.00 \\
\text { TO } \\
8.00\end{array}$ \\
\hline $\begin{array}{l}5-16 \\
5-19 \\
5-21\end{array}$ & $\begin{array}{r}1385 \\
719 \\
1217\end{array}$ & $\begin{array}{l}8 \\
4 \\
3\end{array}$ & $\begin{array}{l}1 \\
0 \\
0\end{array}$ & $\begin{array}{l}4 \\
0 \\
3\end{array}$ & $\begin{array}{l}3 \\
2 \\
0\end{array}$ & $\begin{array}{l}0 \\
2 \\
0\end{array}$ & $\begin{array}{l}0 \\
0 \\
0\end{array}$ \\
\hline $\begin{array}{l}5-22 \\
5-24\end{array}$ & $\overline{-}$ & $=$ & $=$ & $=$ & $\overline{-}$ & $=$ & $=$ \\
\hline $\begin{array}{l}5-26 \\
5-27 \\
5-28\end{array}$ & $\begin{array}{r}471 \\
1592 \\
1893\end{array}$ & $\begin{array}{l}0 \\
1 \\
0\end{array}$ & $\begin{array}{l}0 \\
0 \\
0\end{array}$ & $\begin{array}{l}0 \\
0 \\
0\end{array}$ & $\begin{array}{l}0 \\
1 \\
0\end{array}$ & $\begin{array}{l}0 \\
0 \\
0\end{array}$ & $\begin{array}{l}0 \\
0 \\
0\end{array}$ \\
\hline $5-30$ & 1540 & 0 & 0 & 0 & 0 & 0 & 0 \\
\hline $\begin{array}{l}6-01 \\
6-03 \\
6-05 \\
6-07 \\
6-09\end{array}$ & $\begin{array}{r}872 \\
407 \\
701 \\
214 \\
1115\end{array}$ & $\begin{array}{l}1 \\
0 \\
1 \\
1 \\
0\end{array}$ & $\begin{array}{l}0 \\
0 \\
0 \\
0 \\
0\end{array}$ & $\begin{array}{l}0 \\
0 \\
1 \\
1 \\
0\end{array}$ & $\begin{array}{l}1 \\
0 \\
0 \\
0 \\
0\end{array}$ & $\begin{array}{l}0 \\
0 \\
0 \\
0 \\
0\end{array}$ & $\begin{array}{l}0 \\
0 \\
0 \\
0 \\
0\end{array}$ \\
\hline $\begin{array}{l}6-10 \\
6-11 \\
6-12 \\
6-13 \\
6-14\end{array}$ & $\begin{array}{r}1871 \\
2608 \\
218 \\
1290 \\
6798\end{array}$ & $\begin{array}{r}0 \\
0 \\
0 \\
13 \\
0\end{array}$ & $\begin{array}{l}0 \\
0 \\
0 \\
2 \\
0\end{array}$ & $\begin{array}{r}0 \\
0 \\
0 \\
11 \\
0\end{array}$ & $\begin{array}{l}0 \\
0 \\
0 \\
0 \\
0\end{array}$ & $\begin{array}{l}0 \\
0 \\
0 \\
0 \\
0\end{array}$ & $\begin{array}{l}0 \\
0 \\
0 \\
0 \\
0\end{array}$ \\
\hline $\begin{array}{l}6-15 \\
6-16 \\
6-17 \\
6-18 \\
6-19\end{array}$ & $\begin{array}{l}3561 \\
1007 \\
4185 \\
7816 \\
5678\end{array}$ & $\begin{array}{l}0 \\
0 \\
0 \\
0 \\
0\end{array}$ & $\begin{array}{l}0 \\
0 \\
0 \\
0 \\
0\end{array}$ & $\begin{array}{l}0 \\
0 \\
0 \\
0 \\
0\end{array}$ & $\begin{array}{l}0 \\
0 \\
0 \\
0 \\
0\end{array}$ & $\begin{array}{l}0 \\
0 \\
0 \\
0 \\
0\end{array}$ & $\begin{array}{l}0 \\
0 \\
0 \\
0 \\
0\end{array}$ \\
\hline $\begin{array}{l}6-20 \\
6-21\end{array}$ & $\begin{array}{l}2601 \\
2274\end{array}$ & $\begin{array}{l}0 \\
0\end{array}$ & $\begin{array}{l}0 \\
0\end{array}$ & $\begin{array}{l}0 \\
0\end{array}$ & $\begin{array}{l}0 \\
0\end{array}$ & $\begin{array}{l}0 \\
0\end{array}$ & $\begin{array}{l}0 \\
0\end{array}$ \\
\hline $\begin{array}{l}0-23 \\
6-25 \\
6-25\end{array}$ & $\begin{array}{l}1858 \\
3862\end{array}$ & $\begin{array}{l}0 \\
0\end{array}$ & 0 & $\begin{array}{l}0 \\
0\end{array}$ & $\begin{array}{l}0 \\
0\end{array}$ & $\begin{array}{l}0 \\
0\end{array}$ & $\begin{array}{l}0 \\
0\end{array}$ \\
\hline $\begin{array}{l}6-27 \\
6-29\end{array}$ & $\begin{array}{l}2790 \\
2728\end{array}$ & $\begin{array}{l}5 \\
0\end{array}$ & $\begin{array}{l}2 \\
0\end{array}$ & $\begin{array}{l}0 \\
0\end{array}$ & $\begin{array}{l}3 \\
0\end{array}$ & $\begin{array}{l}0 \\
0\end{array}$ & $\begin{array}{l}0 \\
0\end{array}$ \\
\hline $\begin{array}{l}7-01 \\
7-03\end{array}$ & $=$ & $=$ & $=$ & $=$ & $=$ & $=$ & $\overline{--}$ \\
\hline $7-05$ & -- & -- & -- & - & - & - & - \\
\hline $7-07$ & -- & -- & -- & -- & - & - & - \\
\hline
\end{tabular}


TABLE 17.- NUMBER OF ORANGE TRACER PARTICLES(1), PER 100 GRAMS OF BEDLOAD SAMPLE, COMPOSITED FROM CROSS-CHANNEL SAMPLING POSITIONS

SPACED AT 1-METER INTERVALS ACROSS THE CHANNEL WIDTH,

EAST FORK RIVER, WYOMING, 1980

SECTION 0421

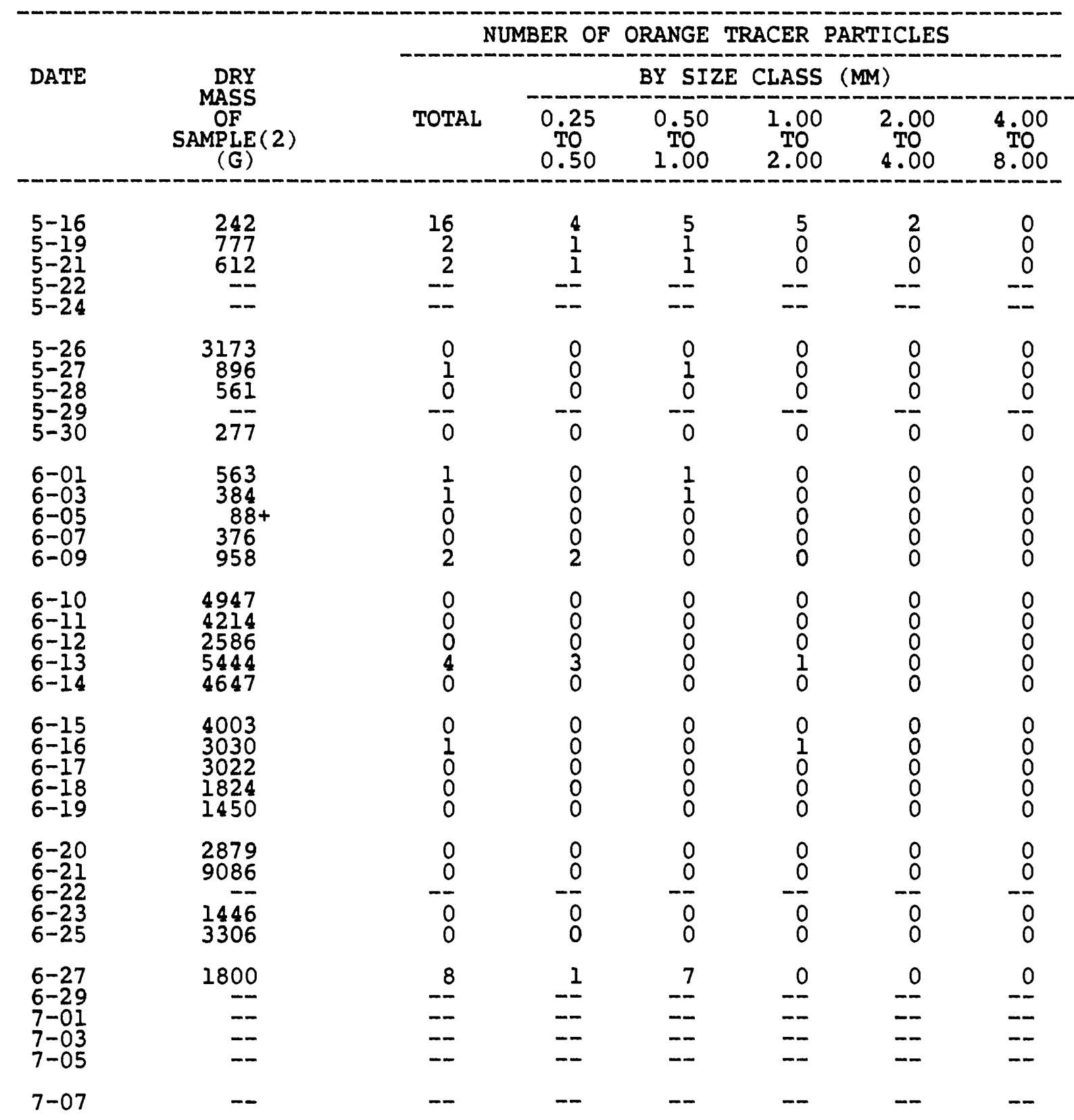


TABLE 17.- NUMBER OF ORANGE TRACER PARTICLES(1), PER 100 GRAMS OF BEDLOAD SAMPLE, COMPOSITED FROM CROSS-CHANNEL SAMPLING POSITIONS SPACED AT I-METER INTERVALS ACROSS THE CHANNEL WIDTH, EAST FORK RIVER, WYOMING, 1980

\section{SECTION 0460}

\begin{tabular}{|c|c|c|c|c|c|c|c|}
\hline \multirow{3}{*}{ DATE } & \multirow{3}{*}{$\begin{array}{c}\text { DRY } \\
\text { MASS } \\
\text { OF } \\
\text { SAMPLE (2) } \\
\text { (G) }\end{array}$} & \multicolumn{6}{|c|}{ NUMBER OF ORANGE TRACER PARTICLES } \\
\hline & & \multirow[b]{2}{*}{ TOTAL } & \multicolumn{5}{|c|}{ BY SIZE CLASS (MM) } \\
\hline & & & $\begin{array}{l}0.25 \\
\text { TO } \\
0.50\end{array}$ & $\begin{array}{l}0.50 \\
\text { TO } \\
1.00\end{array}$ & $\begin{array}{l}1.00 \\
\text { TO } \\
2.00\end{array}$ & $\begin{array}{l}2.00 \\
\text { TO } \\
4.00\end{array}$ & $\begin{array}{r}4.00 \\
\text { TO } \\
8.00\end{array}$ \\
\hline $\begin{array}{l}5-16 \\
5-19 \\
5-21 \\
5-22 \\
5-24\end{array}$ & $\begin{array}{l}233 \\
104++ \\
153^{+} \\
=- \\
--\end{array}$ & $\begin{array}{r}4 \\
0 \\
1 \\
-- \\
--\end{array}$ & $\begin{array}{r}2 \\
0 \\
0 \\
-- \\
--\end{array}$ & $\begin{array}{r}2 \\
0 \\
1 \\
-- \\
--\end{array}$ & $\begin{array}{r}0 \\
0 \\
0 \\
-- \\
--\end{array}$ & $\begin{array}{r}0 \\
0 \\
0 \\
-- \\
--\end{array}$ & $\begin{array}{r}0 \\
0 \\
0 \\
-- \\
--\end{array}$ \\
\hline $\begin{array}{l}5-26 \\
5-27 \\
5-28 \\
5-20\end{array}$ & $\begin{array}{c}1436 \\
39++ \\
190+\end{array}$ & $\begin{array}{l}0 \\
0 \\
0\end{array}$ & $\begin{array}{l}0 \\
0 \\
0 \\
-\end{array}$ & $\begin{array}{l}0 \\
0 \\
0 \\
-\end{array}$ & $\begin{array}{l}0 \\
0 \\
0\end{array}$ & $\begin{array}{l}0 \\
0 \\
0\end{array}$ & $\begin{array}{l}0 \\
0 \\
0\end{array}$ \\
\hline $5-30$ & $16++$ & 0 & 0 & 0 & 0 & 0 & 0 \\
\hline $\begin{array}{l}6-01 \\
6-03 \\
6-05 \\
6-07 \\
6-09\end{array}$ & $\begin{array}{r}25++ \\
10+++ \\
24++ \\
43^{++} \\
2147^{++}\end{array}$ & $\begin{array}{l}0 \\
0 \\
0 \\
0 \\
4\end{array}$ & $\begin{array}{l}0 \\
0 \\
0 \\
0 \\
4\end{array}$ & $\begin{array}{l}0 \\
0 \\
0 \\
0 \\
0\end{array}$ & $\begin{array}{l}0 \\
0 \\
0 \\
0 \\
0\end{array}$ & $\begin{array}{l}0 \\
0 \\
0 \\
0 \\
0\end{array}$ & $\begin{array}{l}0 \\
0 \\
0 \\
0 \\
0\end{array}$ \\
\hline $\begin{array}{l}6-10 \\
6-11 \\
6-12 \\
6-13 \\
6-14\end{array}$ & $\begin{array}{l}6109 \\
8822 \\
7920 \\
3616 \\
4080\end{array}$ & $\begin{array}{l}0 \\
0 \\
0 \\
8 \\
0\end{array}$ & $\begin{array}{l}0 \\
0 \\
0 \\
0 \\
0\end{array}$ & $\begin{array}{l}0 \\
0 \\
0 \\
3 \\
0\end{array}$ & $\begin{array}{l}0 \\
0 \\
0 \\
5 \\
0\end{array}$ & $\begin{array}{l}0 \\
0 \\
0 \\
0 \\
0\end{array}$ & $\begin{array}{l}0 \\
0 \\
0 \\
0 \\
0\end{array}$ \\
\hline $6-15$ & 2730 & 0 & 0 & 0 & 0 & 0 & 0 \\
\hline $\begin{array}{l}6-17 \\
6-18 \\
6-19\end{array}$ & $\begin{array}{r}952 \\
1870 \\
2636\end{array}$ & $\begin{array}{l}0 \\
0 \\
0\end{array}$ & $\begin{array}{l}0 \\
0 \\
0\end{array}$ & $\begin{array}{l}0 \\
0 \\
0\end{array}$ & $\begin{array}{l}0 \\
0 \\
0\end{array}$ & $\begin{array}{l}0 \\
0 \\
0\end{array}$ & $\begin{array}{l}0 \\
0 \\
0\end{array}$ \\
\hline $\begin{array}{l}6-20 \\
6-21\end{array}$ & $\begin{array}{l}2850 \\
4894\end{array}$ & $\begin{array}{l}0 \\
0\end{array}$ & $\begin{array}{l}0 \\
0\end{array}$ & $\begin{array}{l}0 \\
0\end{array}$ & $\begin{array}{l}0 \\
0\end{array}$ & $\begin{array}{l}0 \\
0\end{array}$ & $\begin{array}{l}0 \\
0\end{array}$ \\
\hline $\begin{array}{l}6-23 \\
6-25\end{array}$ & $\begin{array}{l}1697 \\
1719\end{array}$ & $\begin{array}{l}0 \\
0\end{array}$ & $\begin{array}{l}0 \\
0\end{array}$ & $\begin{array}{l}0 \\
0\end{array}$ & $\begin{array}{l}0 \\
0\end{array}$ & $\begin{array}{l}0 \\
0\end{array}$ & $\begin{array}{l}0 \\
0\end{array}$ \\
\hline $6-27$ & 2173 & 15 & 5 & 7 & 3 & $\underline{0}$ & $\underline{0}$ \\
\hline $7-01$ & - & - & - & - & $\overline{-}$ & $\overline{-}$ & $\overline{--}$ \\
\hline $7-0 \overline{3}$ & -- & -- & -- & -- & -- & -- & -- \\
\hline $7-05$ & -- & -- & -- & -- & -- & -- & -- \\
\hline $7-07$ & -- & -- & -- & -- & -- & -- & -- \\
\hline
\end{tabular}


TABLE 17.- NUMBER OF ORANGE TRACER PARTICLES(1), PER 100 GRAMS OF BEDLOAD SAMPLE, COMPOSITED FROM CROSS-CHANNEL SAMPLING POSITIONS SPACED AT I-METER INTERVALS ACROSS THE CHANNEL WIDTH, EAST FORK RIVER, WYOMING, I980--CONTINUED

(1) ORANGE TRACER PARTICLES PLACED AS BED MATERIAL 10 METERS DOWNSTREAM

OF SECTION 0516 ON MAY I9, 1979.

(2) REPRESENTS TOTAL MASS, IN GRAMS; RETAINED IN BEDLOAD SAMPLER AS MEASURED IN THE FIELD! FOR SUBSÉOUENT ANALYSIS OF NUMBER AND SIZE OF TRACER, PARTICLES SMALLER THAN 0.25 MM AND LARGER THAN $8.0 \mathrm{MM}$ WERE DISCARDED. REMAINING SAMPLES WEIGHING MORE THAN 100 GRAMS WERE CUT TO A STANDARD 100 GRAM-PORTION; SAMPLES WEIGHING LESS THAN 100 GRAMS WERE ANALYZED INTACT.

(3) SMALL OVERFLOW CHANNEL ADJACENT TO SECTION.

$+\quad$ MASS OF SAMPLE ANALYZED WAS BETWEEN 50 AND 100 GRAMS; NUMBER OF TRACER PARTICLES ADJUSTED TO AN EQUIVALENT IOO-GRAM SAMPLE; EXTRAPOLATED NUMBERS ARE CONSIDERED RELIABLE.

+ MASS OF SAMPLE ANALYZED WAS BETWEEN IO AND 50 GRAMS; NUMBER OF TRACER PARTICLES ADJUSTED TO AN EQUIVALENT 100-GRAM SAMPLE; CARE SHOULD BE TAKEN IN USING THE EXTRAPOLATED NUMBERS.

++ MASS OF SAMPLE ANALYZED WAS BETWEEN 0 AND 10 GRAMS: NUMBER OF TRACER PARTICLES ADJUSTED TO AN EQUIVALENT IOO-GRAM SAMPLE; EXTRAPOLATED NUMBERS ARE NOT CONSIDERED RELIABLE. 
TABLE 18.- NUMBER OF GREEN TRACER PARTICLES(1), PER 100 GRAMS OF BEDLOAD SAMPLE, COMPOSITED FROM CROSS-CHANNEL SAMPLING POSITIONS SPACED AT 1-METER INTERVALS ACROSS THE CHANNEL WIDTH, EAST FORK RIVER, WYOMING, 1980

\section{SECTION 0043}

\begin{tabular}{|c|c|c|c|c|c|c|c|}
\hline \multirow{3}{*}{ DATE } & \multirow{3}{*}{$\begin{array}{c}\text { DRY } \\
\text { MASS } \\
\text { OF } \\
\text { SAMPLE (2) } \\
\text { (G) }\end{array}$} & \multicolumn{6}{|c|}{ NUMBER OF GREEN TRACER PARTICLES } \\
\hline & & \multirow[b]{2}{*}{ TOTAL } & \multicolumn{4}{|c|}{ BY SIZE CLASS (MM) } & \multirow[b]{2}{*}{$\begin{array}{l}4.00 \\
\text { TO } \\
8.00\end{array}$} \\
\hline & & & $\begin{array}{l}0.25 \\
\text { TO } \\
0.50\end{array}$ & $\begin{array}{l}0.50 \\
\text { TO } \\
1.00\end{array}$ & $\begin{array}{l}1.00 \\
\text { TO } \\
2.00\end{array}$ & $\begin{array}{l}2.00 \\
\text { TO } \\
4.00\end{array}$ & \\
\hline $\begin{array}{l}5-16 \\
5-19 \\
5-21 \\
5-22 \\
5-24\end{array}$ & $\begin{array}{l}59++ \\
75++ \\
54+ \\
-- \\
--\end{array}$ & $\begin{array}{r}0 \\
0 \\
0 \\
--\end{array}$ & $\begin{array}{r}0 \\
0 \\
0 \\
--\end{array}$ & $\begin{array}{r}0 \\
0 \\
0 \\
--\end{array}$ & $\begin{array}{r}0 \\
0 \\
0 \\
-- \\
--\end{array}$ & $\begin{array}{r}0 \\
0 \\
0 \\
-- \\
--\end{array}$ & $\begin{array}{r}0 \\
0 \\
0 \\
-- \\
--\end{array}$ \\
\hline $\begin{array}{l}5-26 \\
5-27 \\
5-28 \\
5-29 \\
5-30\end{array}$ & $\begin{array}{c}371 \\
84+ \\
28++ \\
-5+++\end{array}$ & $\begin{array}{r}0 \\
0 \\
11 \\
0\end{array}$ & $\begin{array}{r}0 \\
0 \\
0 \\
-0\end{array}$ & $\begin{array}{r}0 \\
0 \\
11 \\
-0\end{array}$ & $\begin{array}{r}0 \\
0 \\
0 \\
-0\end{array}$ & $\begin{array}{r}0 \\
0 \\
0 \\
-0\end{array}$ & $\begin{array}{r}0 \\
0 \\
0 \\
-0\end{array}$ \\
\hline $\begin{array}{l}6-01 \\
6-03 \\
6-05 \\
6-07 \\
6-09\end{array}$ & $\begin{array}{c}81++ \\
0+++ \\
0+++ \\
111+++ \\
303\end{array}$ & $\begin{array}{l}0 \\
0 \\
0 \\
0 \\
0\end{array}$ & $\begin{array}{l}0 \\
0 \\
0 \\
0 \\
0\end{array}$ & $\begin{array}{l}0 \\
0 \\
0 \\
0 \\
0\end{array}$ & $\begin{array}{l}0 \\
0 \\
0 \\
0 \\
0\end{array}$ & $\begin{array}{l}0 \\
0 \\
0 \\
0 \\
0\end{array}$ & $\begin{array}{l}0 \\
0 \\
0 \\
0 \\
0\end{array}$ \\
\hline $\begin{array}{l}6-10 \\
6-11 \\
6-12 \\
6-13 \\
6-14\end{array}$ & $\begin{array}{l}3071 \\
3889 \\
2659 \\
1640 \\
1030\end{array}$ & $\begin{array}{l}0 \\
0 \\
0 \\
0 \\
2\end{array}$ & $\begin{array}{l}0 \\
0 \\
0 \\
0 \\
2\end{array}$ & $\begin{array}{l}0 \\
0 \\
0 \\
0 \\
0\end{array}$ & $\begin{array}{l}0 \\
0 \\
0 \\
0 \\
0\end{array}$ & $\begin{array}{l}0 \\
0 \\
0 \\
0 \\
0\end{array}$ & $\begin{array}{l}0 \\
0 \\
0 \\
0 \\
0\end{array}$ \\
\hline $\begin{array}{l}6-15 \\
6-16\end{array}$ & 984 & -1 & 1 & 0 & $\underline{0}$ & $\underline{0}$ & $\underline{0}$ \\
\hline $\begin{array}{l}6-17 \\
6-18 \\
6-19\end{array}$ & $\begin{array}{r}383 \\
320 \\
1123\end{array}$ & $\begin{array}{l}0 \\
0 \\
0\end{array}$ & $\begin{array}{l}0 \\
0 \\
0\end{array}$ & $\begin{array}{l}0 \\
0 \\
0\end{array}$ & $\begin{array}{l}0 \\
0 \\
0\end{array}$ & $\begin{array}{l}0 \\
0 \\
0\end{array}$ & $\begin{array}{l}0 \\
0 \\
0\end{array}$ \\
\hline $\begin{array}{l}6-20 \\
6-21 \\
6-22\end{array}$ & $\begin{array}{l}271 \\
764\end{array}$ & $\begin{array}{r}0 \\
0 \\
-\end{array}$ & $\begin{array}{l}0 \\
0\end{array}$ & $\begin{array}{l}0 \\
0\end{array}$ & $\begin{array}{l}0 \\
0\end{array}$ & $\begin{array}{l}0 \\
0\end{array}$ & $\begin{array}{l}0 \\
0\end{array}$ \\
\hline $\begin{array}{l}6-23 \\
6-25\end{array}$ & $\begin{array}{r}585 \\
1008\end{array}$ & $\begin{array}{l}0 \\
0\end{array}$ & $\begin{array}{l}0 \\
0\end{array}$ & $\begin{array}{l}0 \\
0\end{array}$ & $\begin{array}{l}0 \\
0\end{array}$ & $\begin{array}{l}0 \\
0\end{array}$ & $\begin{array}{l}0 \\
0\end{array}$ \\
\hline $\begin{array}{l}6-27 \\
6-29\end{array}$ & 486 & -0 & -0 & -0 & -0 & -0 & -0 \\
\hline $\begin{array}{l}7-01 \\
7-03\end{array}$ & $\overline{-}$ & - & - & $\overline{--}$ & - & - & - \\
\hline $7-05$ & - & - & - & - & - & - & - \\
\hline $7-07$ & -- & -- & - & -- & -- & -- & -- \\
\hline
\end{tabular}


TABLE 18.- NUMBER OF GREEN TRACER PARTICLES(1), PER 100 GRAMS OF BEDLOAD SAMPLE, COMPOSITED FROM CROSS-CHANNEL SAMPLING POSITIONS SPACED AT 1-METER INTERVALS ACROSS THE CHANNEL WIDTH, EAST FORK RIVER, WYOMING, 1980

SECTION 0075

\begin{tabular}{|c|c|c|c|c|c|c|c|}
\hline \multirow{3}{*}{ DATE } & \multirow{3}{*}{$\begin{array}{c}\text { DRY } \\
\text { MASS } \\
\text { OF } \\
\text { SAMPLE (2) } \\
\text { (G) }\end{array}$} & \multicolumn{6}{|c|}{ NUMBER OF GREEN TRACER PARTICLES } \\
\hline & & \multirow[b]{2}{*}{ TOTAL } & \multicolumn{4}{|c|}{ BY SIZE CLASS (MM) } & \multirow[b]{2}{*}{$\begin{array}{l}4.00 \\
\text { TO } \\
8.00\end{array}$} \\
\hline & & & $\begin{array}{l}0.25 \\
\text { TO } \\
0.50\end{array}$ & $\begin{array}{c}0.50 \\
\text { TO } \\
1.00\end{array}$ & $\begin{array}{l}1.00 \\
2.00 \\
2.00\end{array}$ & $\begin{array}{l}2.00 \\
\text { TO } \\
4.00\end{array}$ & \\
\hline $\begin{array}{l}5-16 \\
5-19 \\
5-21\end{array}$ & $\begin{array}{c}161+ \\
346 \\
1581\end{array}$ & $\begin{array}{l}0 \\
0 \\
0\end{array}$ & $\begin{array}{l}0 \\
0 \\
0\end{array}$ & $\begin{array}{l}0 \\
0 \\
0\end{array}$ & $\begin{array}{l}0 \\
0 \\
0\end{array}$ & $\begin{array}{l}0 \\
0 \\
0\end{array}$ & $\begin{array}{l}0 \\
0 \\
0\end{array}$ \\
\hline $\begin{array}{l}5-22 \\
5-24\end{array}$ & $=$ & $\overline{--}$ & $=$ & $\overline{-}$ & $\overline{--}$ & $=$ & $=$ \\
\hline $\begin{array}{l}5-26 \\
5-27 \\
5-28 \\
5-29 \\
5-30\end{array}$ & $\begin{array}{l}51+ \\
127^{+} \\
127 \\
237 \\
19+++\end{array}$ & $\begin{array}{l}2 \\
0 \\
0 \\
0 \\
0\end{array}$ & $\begin{array}{l}2 \\
0 \\
0 \\
0 \\
0\end{array}$ & $\begin{array}{l}0 \\
0 \\
0 \\
0 \\
0\end{array}$ & $\begin{array}{l}0 \\
0 \\
0 \\
0 \\
0\end{array}$ & $\begin{array}{l}0 \\
0 \\
0 \\
0 \\
0\end{array}$ & $\begin{array}{l}0 \\
0 \\
0 \\
0 \\
0\end{array}$ \\
\hline $\begin{array}{l}6-01 \\
6-03 \\
6-05 \\
6-07 \\
6-09\end{array}$ & $\begin{array}{r}1907 \\
900 \\
283 \\
742 \\
1650\end{array}$ & $\begin{array}{l}0 \\
0 \\
0 \\
0 \\
2\end{array}$ & $\begin{array}{l}0 \\
0 \\
0 \\
0 \\
1\end{array}$ & $\begin{array}{l}0 \\
0 \\
0 \\
0 \\
1\end{array}$ & $\begin{array}{l}0 \\
0 \\
0 \\
0 \\
0\end{array}$ & $\begin{array}{l}0 \\
0 \\
0 \\
0 \\
0\end{array}$ & $\begin{array}{l}0 \\
0 \\
0 \\
0 \\
0\end{array}$ \\
\hline $\begin{array}{l}6-10 \\
6-11 \\
6-12 \\
6-13 \\
6-14\end{array}$ & $\begin{array}{l}3128 \\
1416 \\
2786 \\
1735 \\
1897\end{array}$ & $\begin{array}{r}0 \\
12 \\
0 \\
0 \\
0\end{array}$ & $\begin{array}{l}0 \\
0 \\
0 \\
0 \\
0\end{array}$ & $\begin{array}{l}0 \\
8 \\
0 \\
0 \\
0\end{array}$ & $\begin{array}{l}0 \\
4 \\
0 \\
0 \\
0\end{array}$ & $\begin{array}{l}0 \\
0 \\
0 \\
0 \\
0\end{array}$ & $\begin{array}{l}0 \\
0 \\
0 \\
0 \\
0\end{array}$ \\
\hline $6-15$ & 651 & 0 & 0 & 0 & 0 & 0 & 0 \\
\hline $\begin{array}{l}6-17 \\
6-18 \\
6-19\end{array}$ & $\begin{array}{r}900 \\
1969 \\
2818\end{array}$ & $\begin{array}{l}1 \\
0 \\
0\end{array}$ & $\begin{array}{l}1 \\
0 \\
0\end{array}$ & $\begin{array}{l}0 \\
0 \\
0\end{array}$ & $\begin{array}{l}0 \\
0 \\
0\end{array}$ & $\begin{array}{l}0 \\
0 \\
0\end{array}$ & $\begin{array}{l}0 \\
0 \\
0\end{array}$ \\
\hline $\begin{array}{l}6-20 \\
6-21\end{array}$ & $\begin{array}{l}5583 \\
4777\end{array}$ & $\begin{array}{l}0 \\
0\end{array}$ & $\begin{array}{l}0 \\
0\end{array}$ & $\begin{array}{l}0 \\
0\end{array}$ & $\begin{array}{l}0 \\
0\end{array}$ & $\begin{array}{l}0 \\
0\end{array}$ & $\begin{array}{l}0 \\
0\end{array}$ \\
\hline $\begin{array}{l}6-23 \\
6-25\end{array}$ & $\begin{array}{l}1409 \\
1558\end{array}$ & $\begin{array}{l}0 \\
0\end{array}$ & $\begin{array}{l}0 \\
0\end{array}$ & $\begin{array}{l}0 \\
0\end{array}$ & $\begin{array}{l}0 \\
0\end{array}$ & $\begin{array}{l}0 \\
0\end{array}$ & $\begin{array}{l}0 \\
0\end{array}$ \\
\hline $\begin{array}{l}6-27 \\
6-20\end{array}$ & 2302 & $\underline{0}$ & $\underline{0}$ & $\underline{0}$ & $\underline{0}$ & $\underline{0}$ & $\underline{0}$ \\
\hline $7-0 \frac{1}{2}$ & -- & -- & -- & -- & -- & -- & -- \\
\hline $\begin{array}{l}7-03 \\
7-05\end{array}$ & $=$ & $=$ & $\overline{-}$ & $\overline{-}$ & $-\overline{-}$ & $\overline{--}$ & $\overline{-}$ \\
\hline $7-07$ & -- & - & -- & -- & -- & -- & -- \\
\hline
\end{tabular}


TABLE 18.- NUMBER OF GREEN TRACER PARTICLES(1), PER 100 GRAMS OF BEDLOAD SAMPLE, COMPOSITED FROM CROSS-CHANNEL SAMPLING POSITIONS SPACED AT I-METER INTERVALS ACROSS THE CHANNEL WIDTH, EAST FORK RIVER, WYOMING, 1980

SECTION 0137

\begin{tabular}{|c|c|c|c|c|c|c|c|}
\hline \multirow{3}{*}{ DATE } & \multirow{3}{*}{$\begin{array}{c}\text { DRY } \\
\text { MASS } \\
\text { OF } \\
\text { SAMPLE (2) } \\
\text { (G) }\end{array}$} & \multicolumn{6}{|c|}{ NUMBER OF GREEN TRACER PARTICLES } \\
\hline & & \multirow[b]{2}{*}{ TOTAL } & \multicolumn{5}{|c|}{ BY SIZE CLASS (MM) } \\
\hline & & & $\begin{array}{l}0.25 \\
\text { TO } \\
0.50\end{array}$ & $\begin{array}{l}0.50 \\
\text { TO } \\
1.00\end{array}$ & $\begin{array}{l}1.00 \\
\text { TO } \\
2.00\end{array}$ & $\begin{array}{l}2.00 \\
\text { TO } \\
4.00\end{array}$ & $\begin{array}{l}4.00 \\
\text { TO } \\
8.00\end{array}$ \\
\hline $\begin{array}{l}5-16 \\
5-19 \\
5-21\end{array}$ & $\begin{array}{l}225 \\
56+++ \\
113++\end{array}$ & $\begin{array}{l}0 \\
0 \\
0\end{array}$ & $\begin{array}{l}0 \\
0 \\
0\end{array}$ & $\begin{array}{l}0 \\
0 \\
0\end{array}$ & $\begin{array}{l}0 \\
0 \\
0\end{array}$ & $\begin{array}{l}0 \\
0 \\
0\end{array}$ & $\begin{array}{l}0 \\
0 \\
0\end{array}$ \\
\hline $\begin{array}{l}5-22 \\
5-24\end{array}$ & $=$ & $=$ & 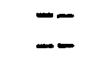 & $=$ & $=$ & $=$ & $=$ \\
\hline $\begin{array}{l}5-26 \\
5-27 \\
5-28 \\
5-20\end{array}$ & $\begin{array}{r}1360 \\
1794 \\
135 \\
\end{array}$ & $\begin{array}{l}0 \\
0 \\
0\end{array}$ & $\begin{array}{l}0 \\
0 \\
0\end{array}$ & $\begin{array}{l}0 \\
0 \\
0 \\
-\end{array}$ & $\begin{array}{l}0 \\
0 \\
0\end{array}$ & $\begin{array}{l}0 \\
0 \\
0\end{array}$ & $\begin{array}{r}0 \\
0 \\
0\end{array}$ \\
\hline $5-30$ & $99+$ & 0 & 0 & 0 & 0 & 0 & 0 \\
\hline $\begin{array}{l}6-01 \\
6-03 \\
6-05 \\
6-07 \\
6-09\end{array}$ & $\begin{array}{c}41++ \\
73++ \\
0+++ \\
588 \\
1099\end{array}$ & $\begin{array}{l}0 \\
0 \\
0 \\
0 \\
1\end{array}$ & $\begin{array}{l}0 \\
0 \\
0 \\
0 \\
1\end{array}$ & $\begin{array}{l}0 \\
0 \\
0 \\
0 \\
0\end{array}$ & $\begin{array}{l}0 \\
0 \\
0 \\
0 \\
0\end{array}$ & $\begin{array}{l}0 \\
0 \\
0 \\
0 \\
0\end{array}$ & $\begin{array}{l}0 \\
0 \\
0 \\
0 \\
0\end{array}$ \\
\hline $\begin{array}{l}6-10 \\
6-11 \\
6-12 \\
6-13 \\
6-14\end{array}$ & $\begin{array}{l}1907 \\
1139 \\
517 \\
1648 \\
3009\end{array}$ & $\begin{array}{l}0 \\
0 \\
1 \\
0 \\
0\end{array}$ & $\begin{array}{l}0 \\
0 \\
0 \\
0 \\
0\end{array}$ & $\begin{array}{l}0 \\
0 \\
1 \\
0 \\
0\end{array}$ & $\begin{array}{l}0 \\
0 \\
0 \\
0 \\
0\end{array}$ & $\begin{array}{l}0 \\
0 \\
0 \\
0 \\
0\end{array}$ & $\begin{array}{l}0 \\
0 \\
0 \\
0 \\
0\end{array}$ \\
\hline $6-15$ & 2497 & 0 & 0 & 0 & 0 & 0 & 0 \\
\hline $\begin{array}{l}6-17 \\
6-18 \\
6-19\end{array}$ & $\begin{array}{l}3116 \\
3204 \\
2346\end{array}$ & $\begin{array}{l}1 \\
0 \\
0\end{array}$ & $\begin{array}{l}1 \\
0 \\
0\end{array}$ & $\begin{array}{l}0 \\
0 \\
0\end{array}$ & $\begin{array}{l}0 \\
0 \\
0\end{array}$ & $\begin{array}{l}0 \\
0 \\
0\end{array}$ & $\begin{array}{l}0 \\
0 \\
0\end{array}$ \\
\hline $\begin{array}{l}6-20 \\
6-21\end{array}$ & $\begin{array}{l}2042 \\
3752\end{array}$ & $\begin{array}{l}0 \\
0\end{array}$ & $\begin{array}{l}0 \\
0\end{array}$ & $\begin{array}{l}0 \\
0\end{array}$ & $\begin{array}{l}0 \\
0\end{array}$ & $\begin{array}{l}0 \\
0\end{array}$ & $\begin{array}{l}0 \\
0\end{array}$ \\
\hline $\begin{array}{l}6-23 \\
6-25\end{array}$ & $\begin{array}{l}2398 \\
3614\end{array}$ & $\begin{array}{l}0 \\
0\end{array}$ & $\begin{array}{l}0 \\
0\end{array}$ & $\begin{array}{l}0 \\
0\end{array}$ & $\begin{array}{l}0 \\
0\end{array}$ & $\begin{array}{l}0 \\
0\end{array}$ & $\begin{array}{l}0 \\
0\end{array}$ \\
\hline $6-27$ & 4050 & 0 & 0 & 0 & 0 & 0 & 0 \\
\hline $7-01$ & - & - & - & - & $\overline{-}$ & - & $\overline{-}$ \\
\hline $7-03$ & -- & -- & -- & -- & -- & - & -- \\
\hline $7-05$ & -- & -- & -- & -- & -- & -- & -- \\
\hline $7-07$ & -- & - & -- & -- & - & -- & -- \\
\hline
\end{tabular}


TABLE 18.- NUMBER OF GREEN TRACER PARTICLES(1), PER 100 GRAMS OF BEDLOAD SAMPLE, COMPOSITED FROM CROSS-CHANNEL SAMPLING POSITIONS SPACED AT 1-METER INTERVALS ACROSS THE CHANNEL WIDTH, EAST FORK RIVER, WYOMING, 1980

SECTION 0178

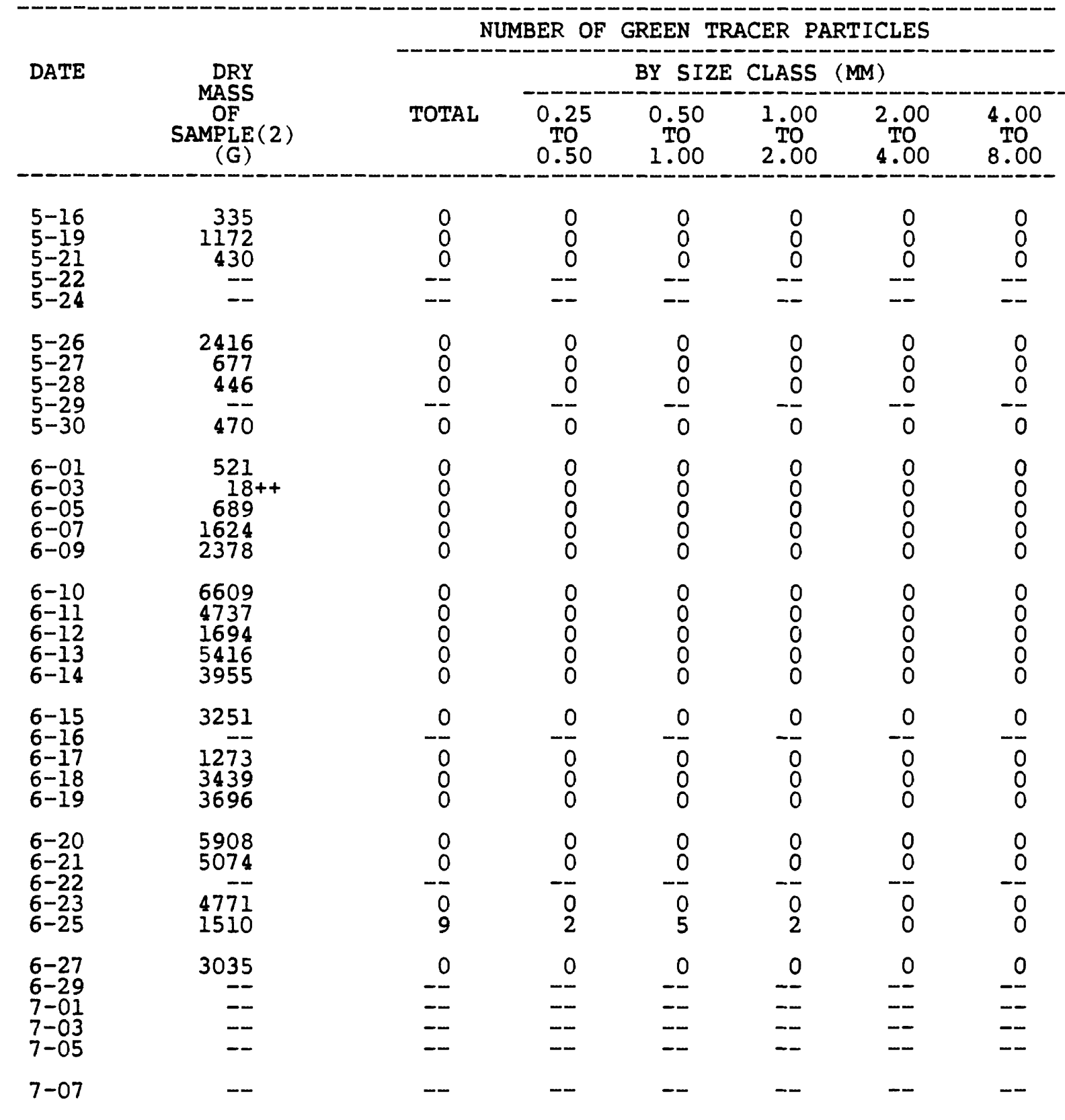


TABLE 18.- NUMBER OF GREEN TRACER PARTICLES(1), PER 100 GRAMS OF BEDLOAD SAMPLE, COMPOSITED FROM CROSS-CHANNEL SAMPLING POSITIONS SPACED AT 1-METER INTERVALS ACROSS THE CHANNEL WIDTH, EAST FORK RIVER, WYOMING, 1980

SECTION 0220

\begin{tabular}{|c|c|c|c|c|c|c|c|}
\hline \multirow{3}{*}{ DATE } & \multirow{3}{*}{$\begin{array}{c}\text { DRY } \\
\text { MASS } \\
\text { OF } \\
\text { SAMPLE (2) } \\
\text { (G) }\end{array}$} & \multicolumn{6}{|c|}{ NUMBER OF GREEN TRACER PARTICLES } \\
\hline & & \multirow[b]{2}{*}{ TOTAL } & \multicolumn{4}{|c|}{ BY SIZE CLASS (MM) } & \multirow[b]{2}{*}{$\begin{array}{l}4.00 \\
\text { TO } \\
8.00\end{array}$} \\
\hline & & & $\begin{array}{l}0.25 \\
\text { TO } \\
0.50\end{array}$ & $\begin{array}{l}0.50 \\
\text { TO } \\
1.00\end{array}$ & $\begin{array}{l}1.00 \\
2.00\end{array}$ & $\begin{array}{l}2.00 \\
\text { TO } \\
4.00\end{array}$ & \\
\hline $\begin{array}{l}5-16 \\
5-19 \\
5-21\end{array}$ & $\begin{array}{c}144+ \\
512 \\
5907\end{array}$ & $\begin{array}{l}0 \\
0 \\
0\end{array}$ & $\begin{array}{l}0 \\
0 \\
0\end{array}$ & $\begin{array}{l}0 \\
0 \\
0\end{array}$ & $\begin{array}{l}0 \\
0 \\
0\end{array}$ & $\begin{array}{l}0 \\
0 \\
0\end{array}$ & $\begin{array}{l}0 \\
0 \\
0\end{array}$ \\
\hline $\begin{array}{l}5-22 \\
5-24\end{array}$ & $=$ & $=$ & $=$ & $=$ & $=$ & $=$ & $=$ \\
\hline $\begin{array}{l}5-26 \\
5-27 \\
5-28\end{array}$ & $\begin{array}{c}871 \\
83+ \\
673\end{array}$ & $\begin{array}{l}0 \\
0 \\
0\end{array}$ & $\begin{array}{l}0 \\
0 \\
0\end{array}$ & $\begin{array}{l}0 \\
0 \\
0\end{array}$ & $\begin{array}{l}0 \\
0 \\
0\end{array}$ & $\begin{array}{l}0 \\
0 \\
0\end{array}$ & $\begin{array}{l}0 \\
0 \\
0\end{array}$ \\
\hline $\begin{array}{l}5-29 \\
5-30\end{array}$ & $150+$ & 0 & $\overline{0}$ & $\overline{0}$ & $\overline{0}$ & $\overline{0}$ & $\overline{0}$ \\
\hline $\begin{array}{l}6-01 \\
6-03 \\
6-05 \\
6-07 \\
6-09\end{array}$ & $\begin{array}{c}44++ \\
67++ \\
569 \\
758 \\
4213\end{array}$ & $\begin{array}{l}0 \\
0 \\
0 \\
0 \\
1\end{array}$ & $\begin{array}{l}0 \\
0 \\
0 \\
0 \\
0\end{array}$ & $\begin{array}{l}0 \\
0 \\
0 \\
0 \\
1\end{array}$ & $\begin{array}{l}0 \\
0 \\
0 \\
0 \\
0\end{array}$ & $\begin{array}{l}0 \\
0 \\
0 \\
0 \\
0\end{array}$ & $\begin{array}{l}0 \\
0 \\
0 \\
0 \\
0\end{array}$ \\
\hline $\begin{array}{l}6-10 \\
6-11 \\
6-12 \\
6-13 \\
6-14\end{array}$ & $\begin{array}{l}6882 \\
5760 \\
3938 \\
4401 \\
5375\end{array}$ & $\begin{array}{l}0 \\
0 \\
0 \\
0 \\
0\end{array}$ & $\begin{array}{l}0 \\
0 \\
0 \\
0 \\
0\end{array}$ & $\begin{array}{l}0 \\
0 \\
0 \\
0 \\
0\end{array}$ & $\begin{array}{l}0 \\
0 \\
0 \\
0 \\
0\end{array}$ & $\begin{array}{l}0 \\
0 \\
0 \\
0 \\
0\end{array}$ & $\begin{array}{l}0 \\
0 \\
0 \\
0 \\
0\end{array}$ \\
\hline $6-15$ & 241 & 1 & 0 & 1 & 0 & 0 & 0 \\
\hline $\begin{array}{l}0-10 \\
6-17 \\
6-18 \\
6-19\end{array}$ & $\begin{array}{r}946 \\
3574 \\
6083\end{array}$ & $\begin{array}{l}0 \\
0 \\
0\end{array}$ & $\begin{array}{l}0 \\
0 \\
0\end{array}$ & $\begin{array}{l}0 \\
0 \\
0\end{array}$ & $\begin{array}{l}0 \\
0 \\
0\end{array}$ & $\begin{array}{l}0 \\
0 \\
0\end{array}$ & $\begin{array}{l}0 \\
0 \\
0\end{array}$ \\
\hline $\begin{array}{l}6-20 \\
6-21\end{array}$ & $\begin{array}{l}6451 \\
3627\end{array}$ & $\begin{array}{l}0 \\
0\end{array}$ & $\begin{array}{l}0 \\
0\end{array}$ & $\begin{array}{l}0 \\
0\end{array}$ & $\begin{array}{l}0 \\
0\end{array}$ & $\begin{array}{l}0 \\
0\end{array}$ & $\begin{array}{l}0 \\
0\end{array}$ \\
\hline $\begin{array}{l}6-23 \\
6-25\end{array}$ & $\begin{array}{l}1357 \\
3244\end{array}$ & 0 & $\begin{array}{l}0 \\
0\end{array}$ & $\begin{array}{l}0 \\
0\end{array}$ & $\begin{array}{l}0 \\
0\end{array}$ & 0 & $\begin{array}{l}0 \\
0\end{array}$ \\
\hline $6-27$ & 1327 & 0 & 0 & 0 & 0 & 0 & 0 \\
\hline $7-01$ & - & - & - & - & - & - & - \\
\hline $\begin{array}{l}7-03 \\
7-05\end{array}$ & $\overline{--}$ & $\overline{--}$ & $\overline{--}$ & $\overline{--}$ & $\overline{--}$ & $\overline{-}$ & $\overline{-}$ \\
\hline $7-07$ & -- & -- & -- & -- & -- & -- & -- \\
\hline
\end{tabular}


TABLE 18.- NUMBER OF GREEN TRACER PARTICLES(1), PER 100 GRAMS OF BEDLOAD SAMPLE, COMPOSITED FROM CROSS-CHANNEL SAMPLING POSITIONS SPACED AT 1-METER INTERVALS ACROSS THE CHANNEL WIDTH, EAST FORK RIVER, WYOMING, 1980

SECTION 0257

\begin{tabular}{|c|c|c|c|c|c|c|c|}
\hline \multirow{3}{*}{\multicolumn{2}{|c|}{$\begin{array}{cc} & \\
\text { DATE } & \text { DRY } \\
& \text { MASS } \\
\text { OF } \\
\text { SAMPLE (2) } \\
& (\mathrm{G})\end{array}$}} & \multicolumn{6}{|c|}{ NUMBER OF GREEN TRACER PARTICLES } \\
\hline & & \multirow[b]{2}{*}{ TOTAL } & \multicolumn{5}{|c|}{ BY SIZE CLASS (MM) } \\
\hline & & & $\begin{array}{l}0.25 \\
\text { TO } \\
0.50\end{array}$ & $\begin{array}{l}0.50 \\
\text { TO } \\
1.00\end{array}$ & $\begin{array}{l}1.00 \\
2.00\end{array}$ & $\begin{array}{l}2.00 \\
\text { TO } \\
4.00\end{array}$ & $\begin{array}{l}4.00 \\
\text { TO } \\
8.00\end{array}$ \\
\hline $\begin{array}{l}5-16 \\
5-19 \\
5-21 \\
5-22 \\
5-24\end{array}$ & $\begin{array}{r}119+ \\
697 \\
3491 \\
-- \\
--\end{array}$ & $\begin{array}{r}0 \\
0 \\
0 \\
-- \\
--\end{array}$ & $\begin{array}{r}0 \\
0 \\
0 \\
-- \\
--\end{array}$ & $\begin{array}{r}0 \\
0 \\
0 \\
-- \\
--\end{array}$ & $\begin{array}{r}0 \\
0 \\
0 \\
-- \\
--\end{array}$ & $\begin{array}{r}0 \\
0 \\
0 \\
-- \\
--\end{array}$ & $\begin{array}{r}0 \\
0 \\
0 \\
-- \\
--\end{array}$ \\
\hline $\begin{array}{l}5-26 \\
5-27 \\
5-28 \\
5-29\end{array}$ & $\begin{array}{l}183 \\
113 \\
42++ \\
--\end{array}$ & $\begin{array}{r}0 \\
0 \\
0 \\
--\end{array}$ & $\begin{array}{l}0 \\
0 \\
0 \\
-\end{array}$ & $\begin{array}{l}0 \\
0 \\
0 \\
-\end{array}$ & $\begin{array}{r}0 \\
0 \\
0 \\
-\end{array}$ & $\begin{array}{l}0 \\
0 \\
0 \\
-\end{array}$ & $\begin{array}{r}0 \\
0 \\
0 \\
--\end{array}$ \\
\hline $5-30$ & $74+$ & 0 & 0 & 0 & 0 & 0 & 0 \\
\hline $\begin{array}{l}6-01 \\
6-03 \\
6-05 \\
6-07 \\
6-09\end{array}$ & $\begin{array}{c}90++ \\
697 \\
1516 \\
645 \\
3432\end{array}$ & $\begin{array}{l}0 \\
0 \\
0 \\
0 \\
0\end{array}$ & $\begin{array}{l}0 \\
0 \\
0 \\
0 \\
0\end{array}$ & $\begin{array}{l}0 \\
0 \\
0 \\
0 \\
0\end{array}$ & $\begin{array}{l}0 \\
0 \\
0 \\
0 \\
0\end{array}$ & $\begin{array}{l}0 \\
0 \\
0 \\
0 \\
0\end{array}$ & $\begin{array}{l}0 \\
0 \\
0 \\
0 \\
0\end{array}$ \\
\hline $\begin{array}{l}6-10 \\
6-11 \\
6-12 \\
6-13 \\
6-14\end{array}$ & $\begin{array}{l}5300 \\
2534 \\
3148 \\
3296 \\
1846\end{array}$ & $\begin{array}{l}0 \\
0 \\
0 \\
0 \\
1\end{array}$ & $\begin{array}{l}0 \\
0 \\
0 \\
0 \\
0\end{array}$ & $\begin{array}{l}0 \\
0 \\
0 \\
0 \\
1\end{array}$ & $\begin{array}{l}0 \\
0 \\
0 \\
0 \\
0\end{array}$ & $\begin{array}{l}0 \\
0 \\
0 \\
0 \\
0\end{array}$ & $\begin{array}{l}0 \\
0 \\
0 \\
0 \\
0\end{array}$ \\
\hline $\begin{array}{l}6-15 \\
6-16 \\
6-17 \\
6-18 \\
6-19\end{array}$ & $\begin{array}{r}2370 \\
799 \\
1076 \\
2889 \\
4435\end{array}$ & $\begin{array}{l}0 \\
0 \\
0 \\
0 \\
0\end{array}$ & $\begin{array}{l}0 \\
0 \\
0 \\
0 \\
0\end{array}$ & $\begin{array}{l}0 \\
0 \\
0 \\
0 \\
0\end{array}$ & $\begin{array}{l}0 \\
0 \\
0 \\
0 \\
0\end{array}$ & $\begin{array}{l}0 \\
0 \\
0 \\
0 \\
0\end{array}$ & $\begin{array}{l}0 \\
0 \\
0 \\
0 \\
0\end{array}$ \\
\hline $\begin{array}{l}6-20 \\
6-21 \\
6-27\end{array}$ & $\begin{array}{l}7086 \\
2965 \\
\end{array}$ & $\begin{array}{l}0 \\
0\end{array}$ & $\begin{array}{l}0 \\
0\end{array}$ & $\begin{array}{l}0 \\
0\end{array}$ & $\begin{array}{l}0 \\
0\end{array}$ & $\begin{array}{l}0 \\
0\end{array}$ & $\begin{array}{l}0 \\
0\end{array}$ \\
\hline $\begin{array}{l}6-23 \\
6-25\end{array}$ & $\begin{array}{l}1826 \\
2479\end{array}$ & 0 & $\begin{array}{l}0 \\
0\end{array}$ & 0 & 0 & $\begin{array}{l}0 \\
0\end{array}$ & $\begin{array}{l}0 \\
0\end{array}$ \\
\hline $6-27$ & 4340 & 0 & 0 & 0 & 0 & 0 & 0 \\
\hline $\begin{array}{l}6-29 \\
7-01\end{array}$ & $\overline{--}$ & $\overline{--}$ & $\overline{--}$ & $\overline{-}$ & -- & $\overline{--}$ & $\overline{-}$ \\
\hline $\begin{array}{l}7-03 \\
7-05\end{array}$ & $=$ & $=$ & $=$ & -- & $=$ & $=$ & $=$ \\
\hline $7-07$ & -- & -- & -- & -- & -- & -- & -- \\
\hline
\end{tabular}


TABLE 18.- NUMBER OF GREEN TRACER PARTICLES(1), PER 100 GRAMS OF BEDLOAD SAMPLE, COMPOSITED FROM CROSS-CHANNEL SAMPLING POSITIONS SPACED AT 1-METER INTERVALS ACROSS THE CHANNEL WIDTH, EAST FORK RIVER, WYOMING, 1980

SECTION 0257 (3)

\begin{tabular}{|c|c|c|c|c|c|c|c|}
\hline \multirow{3}{*}{ DATE } & \multirow{3}{*}{$\begin{array}{c}\text { DRY } \\
\text { MASS } \\
\text { OF } \\
\text { SAMPLE (2) } \\
\text { (G) }\end{array}$} & \multicolumn{6}{|c|}{ NUMBER OF GREEN TRACER PARTICLES } \\
\hline & & \multirow[b]{2}{*}{ TOTAL } & \multicolumn{5}{|c|}{ BY SIZE CLASS (MM) } \\
\hline & & & $\begin{array}{l}0.25 \\
\text { TO } \\
0.50\end{array}$ & $\begin{array}{c}0.50 \\
\text { TO } \\
1.00\end{array}$ & $\begin{array}{l}1.00 \\
2.00 \\
2.00\end{array}$ & $\begin{array}{l}2.00 \\
\text { TO } \\
4.00\end{array}$ & $\begin{array}{l}4.00 \\
\text { TO } \\
8.00\end{array}$ \\
\hline $5-16$ & - & -- & -- & - & - & - & -- \\
\hline $\begin{array}{l}5-19 \\
5-21\end{array}$ & $\overline{-}$ & $\overline{--}$ & $\bar{z}$ & $\overline{--}$ & $\overline{-}$ & 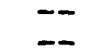 & $\overline{--}$ \\
\hline $5-2 \overline{2}$ & -- & -- & - & -- & -- & -- & -- \\
\hline $5-24$ & - & -- & - & - & - & -- & -- \\
\hline $5-26$ & - & -- & -- & -- & -- & -- & -- \\
\hline $\begin{array}{l}5-27 \\
5-28\end{array}$ & $\overline{-}$ & $\overline{--}$ & $\overline{--}$ & $\overline{--}$ & $=$ & $\overline{-}$ & $=$ \\
\hline $5-29$ & -- & - & - & -- & - & - & -- \\
\hline $5-30$ & - & - & -- & -- & - & - & - \\
\hline $6-01$ & -- & -- & -- & -- & -- & -- & -- \\
\hline $6-03$ & $=$ & $\overline{--}$ & $\overline{--}$ & $\overline{--}$ & $\overline{-}$ & $=$ & $=-$ \\
\hline $6-07$ & -- & -- & - & -- & - & - & -- \\
\hline $6-09$ & 314 & 0 & 0 & 0 & 0 & 0 & 0 \\
\hline $6-10$ & 820 & 0 & 0 & 0 & 0 & 0 & 0 \\
\hline $6-12$ & 1057 & 0 & 0 & 0 & 0 & 0 & 0 \\
\hline $\begin{array}{l}6-13 \\
6-14\end{array}$ & $\begin{array}{l}528 \\
634\end{array}$ & $\begin{array}{l}0 \\
2\end{array}$ & $\begin{array}{l}0 \\
1\end{array}$ & $\begin{array}{l}0 \\
1\end{array}$ & $\begin{array}{l}0 \\
0\end{array}$ & $\begin{array}{l}0 \\
0\end{array}$ & $\begin{array}{l}0 \\
0\end{array}$ \\
\hline $6-15$ & 588 & 0 & 0 & 0 & 0 & 0 & 0 \\
\hline $6-16$ & - & - & - & - & -- & - & - \\
\hline $6-17$ & $\overline{-}$ & $-\overline{2}$ & $-\overline{-}$ & $-\overline{0}$ & $-\overline{0}$ & $-\overline{0}$ & $-\overline{0}$ \\
\hline $6-19$ & 1102 & 0 & 0 & 0 & 0 & 0 & 0 \\
\hline $6-20$ & 298 & 0 & 0 & 0 & 0 & 0 & 0 \\
\hline $\begin{array}{l}6-21 \\
6-22\end{array}$ & 287 & 0 & 0 & -- & -0 & 0 & - \\
\hline $6-23$ & -- & - & - & -- & - & -- & - \\
\hline $6-25$ & 598 & 0 & 0 & 0 & 0 & 0 & 0 \\
\hline $6-27$ & 320 & 0 & 0 & 0 & 0 & 0 & 0 \\
\hline $6-29$ & -- & - & -- & -- & -- & $=$ & - \\
\hline $7-03$ & - & -- & - & - & -- & -- & $=$ \\
\hline $7-05$ & -- & -- & -- & -- & -- & -- & -- \\
\hline $7-07$ & -- & -- & -- & -- & -- & -- & - \\
\hline
\end{tabular}


TABLE 18.- NUMBER OF GREEN TRACER PARTICLES(1), PER 100 GRAMS OF BEDLOAD SAMPLE, COMPOSITED FROM CROSS-CHANNEL SAMPLING POSITIONS SPACED AT 1-METER INTERVALS ACROSS THE CHANNEL WIDTH, EAST FORK RIVER, WYOMING, 1980

SECTION 0301

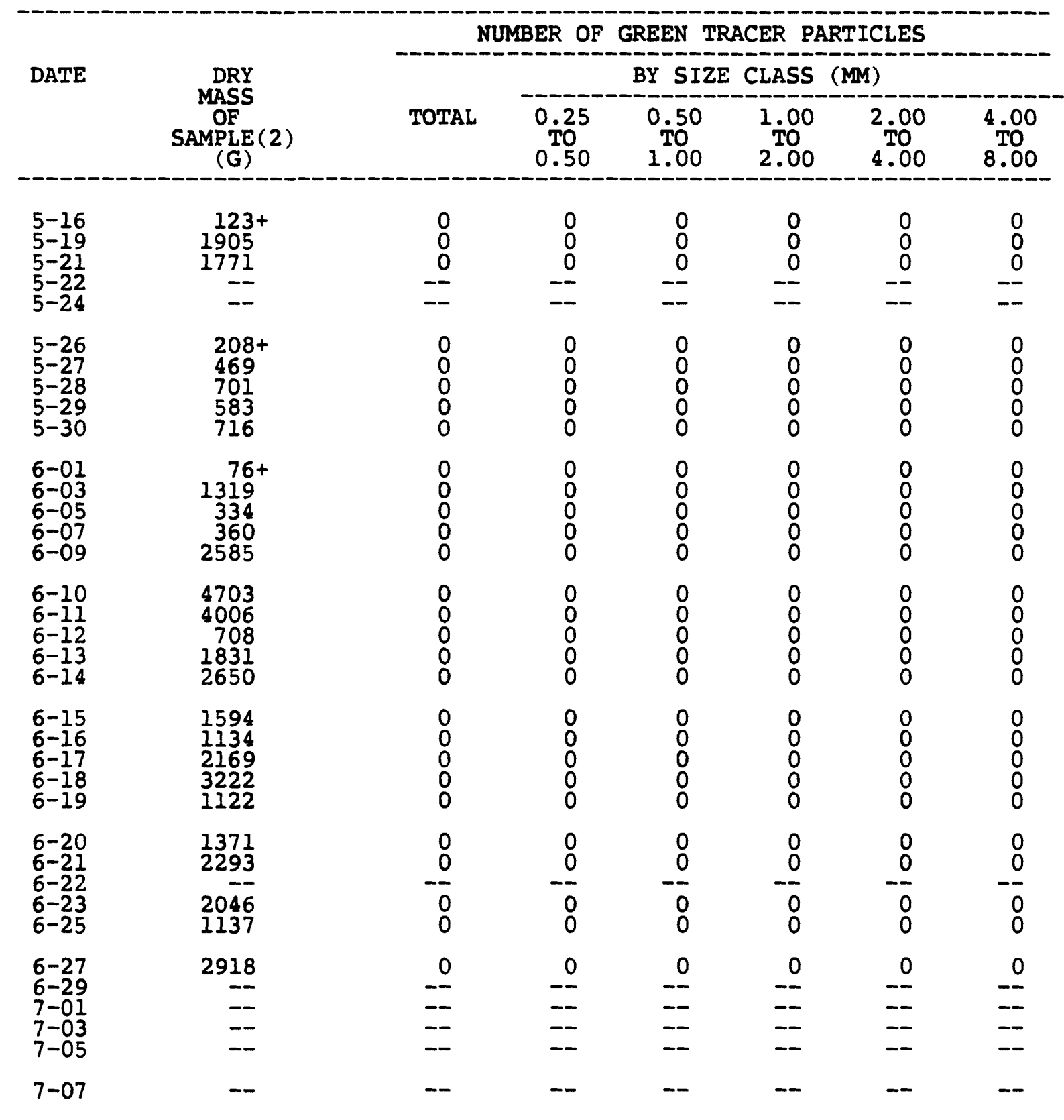


TABLE 18.- NUMBER OF GREEN TRACER PARTICLES(1), PER 100 GRAMS OF BEDLOAD SAMPLE, COMPOSITED FROM CROSS-CHANNEL SAMPLING POSITIONS SPACED AT 1-METER INTERVALS ACROSS THE CHANNEL WIDTH, EAST FORK RIVER, WYOMING, 1980

SECTION 0301(3)

\begin{tabular}{|c|c|c|c|c|c|c|c|}
\hline \multirow{3}{*}{ DATE } & \multirow{3}{*}{$\begin{array}{c}\text { DRY } \\
\text { MASS } \\
\text { OF } \\
\text { SAMPLE (2) } \\
\text { (G) }\end{array}$} & \multicolumn{6}{|c|}{ NUMBER OF GREEN TRACER PARTICLES } \\
\hline & & \multirow[b]{2}{*}{ TOTAL } & \multicolumn{5}{|c|}{ BY SIZE CLASS (MM) } \\
\hline & & & $\begin{array}{l}0.25 \\
\text { TO } \\
0.50\end{array}$ & $\begin{array}{l}0.50 \\
\text { To } \\
1.00\end{array}$ & $\begin{array}{l}1.00 \\
\text { TO } \\
2.00\end{array}$ & $\begin{array}{l}2.00 \\
\text { TO } \\
4.00\end{array}$ & $\begin{array}{l}4.00 \\
\text { To } \\
8.00\end{array}$ \\
\hline $\begin{array}{l}5-16 \\
5-10\end{array}$ & $\overline{--}$ & $\overline{--}$ & $\overline{-}$ & $=$ & -- & $\overline{--}$ & $\overline{--}$ \\
\hline $\begin{array}{l}5-19 \\
5-21\end{array}$ & $\overline{-}$ & $\overline{--}$ & $\overline{-}$ & -- & - & $=$ & $\overline{-}$ \\
\hline $\begin{array}{l}5-22 \\
5-24\end{array}$ & $\overline{--}$ & $\overline{--}$ & $\overline{--}$ & $\overline{--}$ & $\overline{--}$ & $\overline{--}$ & $\overline{--}$ \\
\hline $5-26$ & -- & -- & -- & -- & -- & -- & - \\
\hline $5-27$ & - & -- & -- & -- & -- & -- & - \\
\hline $\begin{array}{l}5-28 \\
5-20\end{array}$ & $\overline{--}$ & $\overline{--}$ & $\overline{--}$ & $\overline{-}$ & $\overline{--}$ & $\overline{--}$ & $\overline{-}$ \\
\hline $5-30$ & - & - & - & - & - & - & - \\
\hline $6-01$ & -- & -- & -- & -- & -- & -- & -- \\
\hline $6-03$ & -- & -- & - & - & -- & -- & -- \\
\hline $\begin{array}{l}6-05 \\
6-07\end{array}$ & $=$ & $=$ & $=$ & $=$ & $=$ & $=$ & $=$ \\
\hline $6 \div 09$ & 410 & 0 & 0 & 0 & 0 & 0 & 0 \\
\hline $\begin{array}{l}6-10 \\
6-11 \\
6-12 \\
6-13 \\
6-14\end{array}$ & $\begin{array}{r}749 \\
1836 \\
1121 \\
2235 \\
846\end{array}$ & $\begin{array}{l}2 \\
0 \\
0 \\
0 \\
0\end{array}$ & $\begin{array}{l}2 \\
0 \\
0 \\
0 \\
0\end{array}$ & $\begin{array}{l}0 \\
0 \\
0 \\
0 \\
0\end{array}$ & $\begin{array}{l}0 \\
0 \\
0 \\
0 \\
0\end{array}$ & $\begin{array}{l}0 \\
0 \\
0 \\
0 \\
0\end{array}$ & $\begin{array}{l}0 \\
0 \\
0 \\
0 \\
0\end{array}$ \\
\hline $6-15$ & 455 & 0 & 0 & 0 & 0 & 0 & 0 \\
\hline $\begin{array}{l}6-16 \\
6-17\end{array}$ & $\overline{-}$ & $=$ & $\overline{-}$ & $=$ & $=-$ & $=$ & $=$ \\
\hline $6-18$ & 837 & 0 & 0 & 0 & 0 & 0 & 0 \\
\hline $6-19$ & 827 & 0 & 0 & 0 & 0 & 0 & 0 \\
\hline $\begin{array}{l}6-20 \\
6-21\end{array}$ & $\begin{array}{l}61+++ \\
965\end{array}$ & $\begin{array}{l}0 \\
0\end{array}$ & $\begin{array}{l}0 \\
0\end{array}$ & $\begin{array}{l}0 \\
0\end{array}$ & $\begin{array}{l}0 \\
0\end{array}$ & $\begin{array}{l}0 \\
0\end{array}$ & $\begin{array}{l}0 \\
0\end{array}$ \\
\hline $6-23$ & 290 & 2 & 0 & 2 & 0 & 0 & 0 \\
\hline & & & & & & & \\
\hline $6-27$ & 149 & 5 & 5 & 0 & 0 & 0 & 0 \\
\hline $\begin{array}{l}6-29 \\
7-01\end{array}$ & $\overline{-}$ & $=$ & $=$ & $=$ & $=$ & $=$ & $=$ \\
\hline $7-03$ & -- & -- & -- & -- & -- & -- & -- \\
\hline $7-05$ & -- & - & -- & -- & -- & -- & -- \\
\hline $7-07$ & -- & -- & -- & -- & -- & - & -- \\
\hline
\end{tabular}


TABLE 18.- NUMBER OF GREEN TRACER PARTICLES(1), PER 100 GRAMS OF BEDLOAD SAMPLE, COMPOSITED FROM CROSS-CHANNEL SAMPLING POSITIONS SPACED AT 1-METER INTERVALS ACROSS THE CHANNEL WIDTH, EAST FORK RIVER, WYOMING, 1980

SECTION 0348

\begin{tabular}{|c|c|c|c|c|c|c|c|}
\hline \multirow[t]{2}{*}{ DATE } & \multirow{2}{*}{$\begin{array}{c}\text { DRY } \\
\text { MASS } \\
\text { OF } \\
\text { SAMPLE (2) } \\
(G)\end{array}$} & - & BER O & $\frac{\text { REEN }}{\text { BY SI }}$ & CER P & $\begin{array}{l}\text { ICLES } \\
M)\end{array}$ & \\
\hline & & TOTAL & $\begin{array}{l}0.25 \\
\text { TO } \\
0.50\end{array}$ & $\begin{array}{l}0.50 \\
\text { TO } \\
1.00\end{array}$ & $\begin{array}{l}1.00 \\
\text { TO } \\
2.00\end{array}$ & $\begin{array}{l}2.00 \\
\text { TO } \\
4.00\end{array}$ & $\begin{array}{c}4.00 \\
\mathrm{TO} \\
8.00\end{array}$ \\
\hline $\begin{array}{l}5-16 \\
5-19 \\
5-21 \\
5-22 \\
5-24\end{array}$ & $\begin{array}{r}1385 \\
719 \\
1217 \\
-- \\
--\end{array}$ & $\begin{array}{r}0 \\
0 \\
0 \\
---\end{array}$ & $\begin{array}{r}0 \\
0 \\
0 \\
-- \\
--\end{array}$ & $\begin{array}{r}0 \\
0 \\
0 \\
-- \\
--\end{array}$ & $\begin{array}{r}0 \\
0 \\
0 \\
-- \\
--\end{array}$ & $\begin{array}{r}0 \\
0 \\
0 \\
-- \\
--\end{array}$ & $\begin{array}{r}0 \\
0 \\
0 \\
-- \\
--\end{array}$ \\
\hline $\begin{array}{l}5-26 \\
5-27 \\
5-28\end{array}$ & $\begin{array}{r}471 \\
1592 \\
1893\end{array}$ & $\begin{array}{l}0 \\
0 \\
0\end{array}$ & $\begin{array}{l}0 \\
0 \\
0\end{array}$ & $\begin{array}{l}0 \\
0 \\
0\end{array}$ & $\begin{array}{l}0 \\
0 \\
0\end{array}$ & $\begin{array}{l}0 \\
0 \\
0\end{array}$ & $\begin{array}{l}0 \\
0 \\
0\end{array}$ \\
\hline $5-30$ & 1540 & 0 & 0 & 0 & 0 & 0 & 0 \\
\hline $\begin{array}{l}6-01 \\
6-03 \\
6-05 \\
6-07 \\
6-09\end{array}$ & $\begin{array}{r}872 \\
407 \\
701 \\
214 \\
1115\end{array}$ & $\begin{array}{l}0 \\
0 \\
0 \\
0 \\
0\end{array}$ & $\begin{array}{l}0 \\
0 \\
0 \\
0 \\
0\end{array}$ & $\begin{array}{l}0 \\
0 \\
0 \\
0 \\
0\end{array}$ & $\begin{array}{l}0 \\
0 \\
0 \\
0 \\
0\end{array}$ & $\begin{array}{l}0 \\
0 \\
0 \\
0 \\
0\end{array}$ & $\begin{array}{l}0 \\
0 \\
0 \\
0 \\
0\end{array}$ \\
\hline $\begin{array}{l}6-10 \\
6-11 \\
6-12 \\
6-13 \\
6-14\end{array}$ & $\begin{array}{l}1871 \\
2608 \\
218 \\
1290 \\
6798\end{array}$ & $\begin{array}{l}0 \\
0 \\
0 \\
0 \\
0\end{array}$ & $\begin{array}{l}0 \\
0 \\
0 \\
0 \\
0\end{array}$ & $\begin{array}{l}0 \\
0 \\
0 \\
0 \\
0\end{array}$ & $\begin{array}{l}0 \\
0 \\
0 \\
0 \\
0\end{array}$ & $\begin{array}{l}0 \\
0 \\
0 \\
0 \\
0\end{array}$ & $\begin{array}{l}0 \\
0 \\
0 \\
0 \\
0\end{array}$ \\
\hline $\begin{array}{l}6-15 \\
6-16 \\
6-17 \\
6-18 \\
6-19\end{array}$ & $\begin{array}{l}3561 \\
1007 \\
4185 \\
7816 \\
5678\end{array}$ & $\begin{array}{l}0 \\
0 \\
0 \\
0 \\
0\end{array}$ & $\begin{array}{l}0 \\
0 \\
0 \\
0 \\
0\end{array}$ & $\begin{array}{l}0 \\
0 \\
0 \\
0 \\
0\end{array}$ & $\begin{array}{l}0 \\
0 \\
0 \\
0 \\
0\end{array}$ & $\begin{array}{l}0 \\
0 \\
0 \\
0 \\
0\end{array}$ & $\begin{array}{l}0 \\
0 \\
0 \\
0 \\
0\end{array}$ \\
\hline $\begin{array}{l}6-20 \\
6-21\end{array}$ & $\begin{array}{l}2601 \\
2274\end{array}$ & $\begin{array}{l}0 \\
0\end{array}$ & $\begin{array}{l}0 \\
0\end{array}$ & $\begin{array}{l}0 \\
0\end{array}$ & $\begin{array}{l}0 \\
0\end{array}$ & $\begin{array}{l}0 \\
0\end{array}$ & $\begin{array}{l}0 \\
0\end{array}$ \\
\hline $\begin{array}{l}6-23 \\
6-25\end{array}$ & $\begin{array}{l}1858 \\
3862\end{array}$ & 2 & 2 & $\begin{array}{l}0 \\
0\end{array}$ & $\begin{array}{l}0 \\
0\end{array}$ & $\begin{array}{l}0 \\
0\end{array}$ & 0 \\
\hline $\begin{array}{l}6-27 \\
6-29 \\
7-01 \\
7-03 \\
7-05\end{array}$ & $\begin{array}{r}2790 \\
2728 \\
-- \\
-- \\
--\end{array}$ & $\begin{array}{r}2 \\
3 \\
-- \\
--\end{array}$ & $\begin{array}{r}2 \\
3 \\
-- \\
--\end{array}$ & $\begin{array}{c}0 \\
0 \\
-- \\
--\end{array}$ & $\begin{array}{l}0 \\
0 \\
-- \\
--\end{array}$ & $\begin{array}{l}0 \\
0 \\
-- \\
--\end{array}$ & $\begin{array}{r}0 \\
0 \\
-- \\
--\end{array}$ \\
\hline $7-07$ & - & -- & - & -- & -- & -- & -- \\
\hline
\end{tabular}


TABLE 18.- NUMBER OF GREEN TRACER PARTICLES(1), PER 100 GRAMS OF BEDLOAD SAMPLE, COMPOSITED FROM CROSS-CHANNEL SAMPLING POSITIONS SPACED AT 1-METER INTERVALS ACROSS THE CHANNEL WIDTH,

EAST FORK RIVER, WYOMING, 1980

SECTION 0421

\begin{tabular}{|c|c|c|c|c|c|c|c|}
\hline \multirow{3}{*}{ DATE } & \multirow{3}{*}{$\begin{array}{c}\text { DRY } \\
\text { MASS } \\
\text { OF } \\
\text { SAMPLE (2) } \\
\text { (G) }\end{array}$} & \multicolumn{6}{|c|}{ NUMBER OF GREEN TRACER PARTICLES } \\
\hline & & \multirow[b]{2}{*}{ TOTAL } & \multicolumn{5}{|c|}{ BY SIZE CLASS (MM) } \\
\hline & & & $\begin{array}{l}0.25 \\
\text { TO } \\
0.50\end{array}$ & $\begin{array}{l}0.50 \\
\text { TO } \\
1.00\end{array}$ & $\begin{array}{l}1.00 \\
\text { TO } \\
2.00\end{array}$ & $\begin{array}{l}2.00 \\
\text { T० } \\
4.00\end{array}$ & $\begin{array}{l}4.00 \\
\text { TO } \\
8.00\end{array}$ \\
\hline $\begin{array}{l}5-16 \\
5-19 \\
5-21 \\
5-22 \\
5-24\end{array}$ & $\begin{array}{l}242 \\
777 \\
612 \\
-2 \\
--\end{array}$ & $\begin{array}{r}0 \\
0 \\
0 \\
-- \\
--\end{array}$ & $\begin{array}{r}0 \\
0 \\
0 \\
--\end{array}$ & $\begin{array}{r}0 \\
0 \\
0 \\
-- \\
--\end{array}$ & $\begin{array}{r}0 \\
0 \\
0 \\
-- \\
--\end{array}$ & $\begin{array}{r}0 \\
0 \\
0 \\
-- \\
--\end{array}$ & $\begin{array}{r}0 \\
0 \\
0 \\
-- \\
--\end{array}$ \\
\hline $\begin{array}{l}5-26 \\
5-27 \\
5-28\end{array}$ & $\begin{array}{r}3173 \\
896 \\
561\end{array}$ & $\begin{array}{l}0 \\
0 \\
0\end{array}$ & $\begin{array}{l}0 \\
0 \\
0\end{array}$ & $\begin{array}{l}0 \\
0 \\
0\end{array}$ & $\begin{array}{l}0 \\
0 \\
0\end{array}$ & $\begin{array}{l}0 \\
0 \\
0 .\end{array}$ & $\begin{array}{l}0 \\
0 \\
0\end{array}$ \\
\hline $5-30$ & 277 & 0 & 0 & 0 & 0 & 0 & 0 \\
\hline $\begin{array}{l}6-01 \\
6-03 \\
6-05 \\
6-07 \\
6-09\end{array}$ & $\begin{array}{l}563 \\
384 \\
88+ \\
376 \\
958\end{array}$ & $\begin{array}{l}0 \\
0 \\
0 \\
0 \\
0\end{array}$ & $\begin{array}{l}0 \\
0 \\
0 \\
0 \\
0\end{array}$ & $\begin{array}{l}0 \\
0 \\
0 \\
0 \\
0\end{array}$ & $\begin{array}{l}0 \\
0 \\
0 \\
0 \\
0\end{array}$ & $\begin{array}{l}0 \\
0 \\
0 \\
0 \\
0\end{array}$ & $\begin{array}{l}0 \\
0 \\
0 \\
0 \\
0\end{array}$ \\
\hline $\begin{array}{l}6-10 \\
6-11 \\
6-12 \\
6-13 \\
6-14\end{array}$ & $\begin{array}{l}4947 \\
4214 \\
2586 \\
5444 \\
4647\end{array}$ & $\begin{array}{l}0 \\
0 \\
0 \\
0 \\
0\end{array}$ & $\begin{array}{l}0 \\
0 \\
0 \\
0 \\
0\end{array}$ & $\begin{array}{l}0 \\
0 \\
0 \\
0 \\
0\end{array}$ & $\begin{array}{l}0 \\
0 \\
0 \\
0 \\
0\end{array}$ & $\begin{array}{l}0 \\
0 \\
0 \\
0 \\
0\end{array}$ & $\begin{array}{l}0 \\
0 \\
0 \\
0 \\
0\end{array}$ \\
\hline $\begin{array}{l}6-15 \\
6-16 \\
6-17 \\
6-18 \\
6-19\end{array}$ & $\begin{array}{l}4003 \\
3030 \\
3022 \\
1824 \\
1450\end{array}$ & $\begin{array}{l}0 \\
0 \\
0 \\
0 \\
0\end{array}$ & $\begin{array}{l}0 \\
0 \\
0 \\
0 \\
0\end{array}$ & $\begin{array}{l}0 \\
0 \\
0 \\
0 \\
0\end{array}$ & $\begin{array}{l}0 \\
0 \\
0 \\
0 \\
0\end{array}$ & $\begin{array}{l}0 \\
0 \\
0 \\
0 \\
0\end{array}$ & $\begin{array}{l}0 \\
0 \\
0 \\
0 \\
0\end{array}$ \\
\hline $\begin{array}{l}6-20 \\
6-21\end{array}$ & $\begin{array}{l}2879 \\
9086\end{array}$ & $\begin{array}{l}0 \\
0\end{array}$ & $\begin{array}{l}0 \\
0\end{array}$ & $\begin{array}{l}0 \\
0\end{array}$ & $\begin{array}{l}0 \\
0\end{array}$ & $\begin{array}{l}0 \\
0\end{array}$ & $\begin{array}{l}0 \\
0\end{array}$ \\
\hline $\begin{array}{l}6-23 \\
6-25\end{array}$ & $\begin{array}{l}1446 \\
3306\end{array}$ & $\begin{array}{l}1 \\
0\end{array}$ & $\begin{array}{l}0 \\
0\end{array}$ & $\begin{array}{l}1 \\
0\end{array}$ & $\begin{array}{l}0 \\
0\end{array}$ & $\begin{array}{l}0 \\
0\end{array}$ & $\begin{array}{l}0 \\
0\end{array}$ \\
\hline $6-27$ & 1800 & 1 & 1 & 0 & 0 & 0 & 0 \\
\hline $\begin{array}{l}6-29 \\
7-01\end{array}$ & $\overline{--}$ & $\overline{--}$ & $\overline{--}$ & $\overline{--}$ & $\overline{--}$ & $\overline{-}$ & $\overline{--}$ \\
\hline $7-0 \overline{3}$ & -- & -- & -- & -- & -- & -- & -- \\
\hline $7-05$ & -- & -- & -- & - & -- & - & -- \\
\hline $7-07$ & -- & -- & -- & -- & -- & -- & -- \\
\hline
\end{tabular}


TABLE 18.- NUMBER OF GREEN TRACER PARTICLES(1), PER 100 GRAMS OF BEDLOAD SAMPLE, COMPOSITED FROM CROSS-CHANNEL SAMPLING POSITIONS SPACED AT 1-METER INTERVALS ACROSS THE CHANNEL WIDTH, EAST FORK RIVER, WYOMING, 1980

SECTION 0460

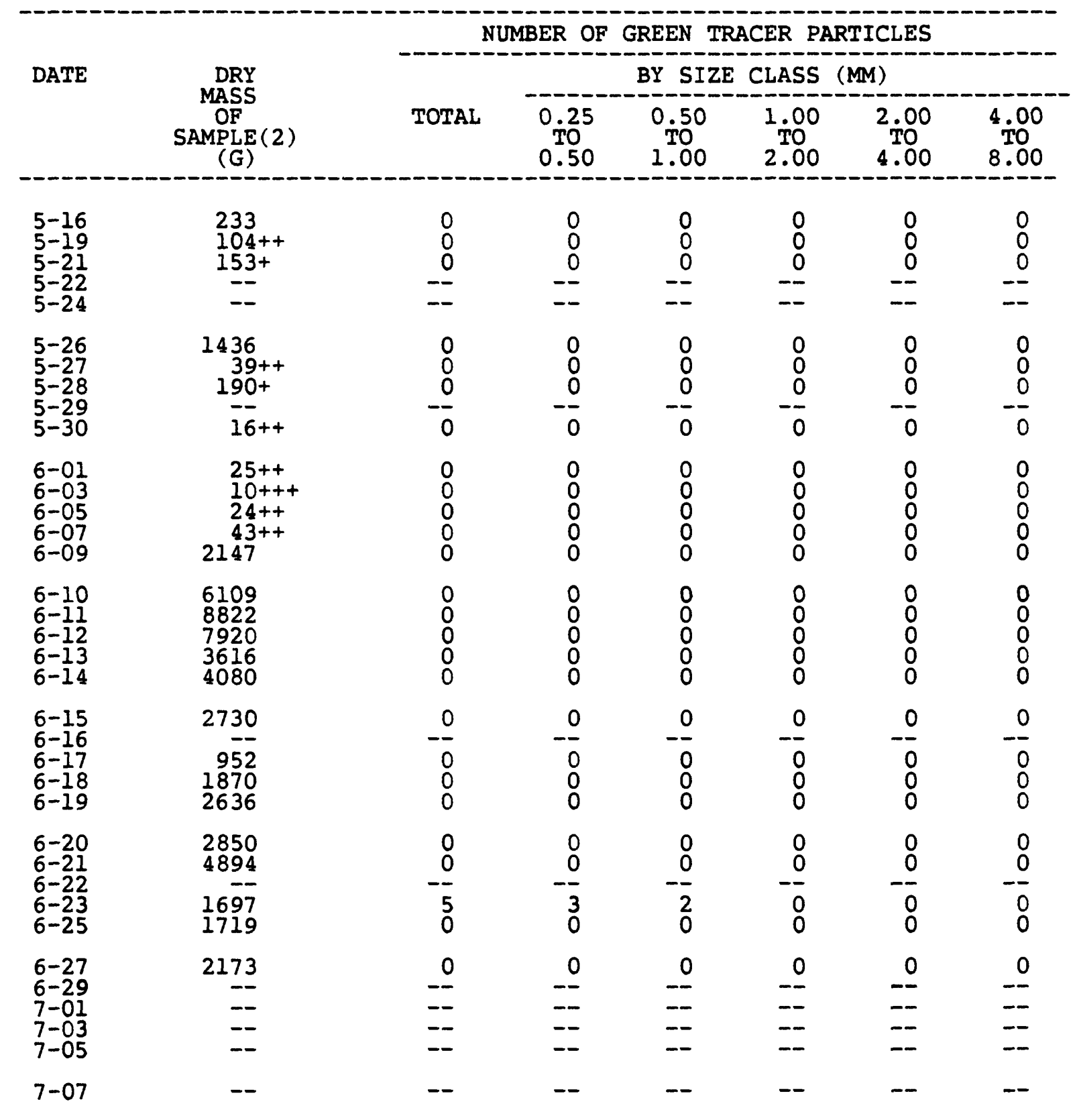


TABLE 18.- NUMBER OF GREEN TRACER PARTICLES(1), PER 100 GRAMS OF BEDLOAD SAMPLE, COMPOSITED FROM CROSS-CHANNEL SAMPLING POSITIONS SPACED AT I-METER INTERVALS ACROSS THE CHANNEL WIDTH, EAST FORK RIVER, WYOMING, 1980

\section{SECTION 0516}

\begin{tabular}{|c|c|c|c|c|c|c|c|}
\hline \multirow{3}{*}{ DATE } & \multirow{3}{*}{$\begin{array}{c}\text { DRY } \\
\text { MASS } \\
\text { OF } \\
\text { SAMPLE (2) } \\
\text { (G) }\end{array}$} & \multicolumn{6}{|c|}{ NUMBER OF GREEN TRACER PARTICLES } \\
\hline & & \multirow[b]{2}{*}{ TOTAL } & \multicolumn{5}{|c|}{ BY SIZE CLASS (MM) } \\
\hline & & & $\begin{array}{l}0.25 \\
. \mathrm{TO} \\
0.50\end{array}$ & $\begin{array}{l}0.50 \\
\text { TO } \\
1.00\end{array}$ & $\begin{array}{l}1.00 \\
\text { TO } \\
2.00\end{array}$ & $\begin{array}{l}2.00 \\
\text { TO } \\
4.00\end{array}$ & $\begin{array}{l}4.00 \\
\text { TO } \\
8.00\end{array}$ \\
\hline $\begin{array}{l}5-16 \\
5-19 \\
5-21 \\
5-22 \\
5-24\end{array}$ & $\begin{array}{c}238 \\
104++ \\
3254 \\
- \\
-\end{array}$ & $\begin{array}{r}0 \\
0 \\
0 \\
-- \\
--\end{array}$ & $\begin{array}{r}0 \\
0 \\
0 \\
-- \\
--\end{array}$ & $\begin{array}{r}0 \\
0 \\
0 \\
-- \\
--\end{array}$ & $\begin{array}{r}0 \\
0 \\
0 \\
--\end{array}$ & $\begin{array}{r}0 \\
0 \\
0 \\
-- \\
--\end{array}$ & $\begin{array}{r}0 \\
0 \\
0 \\
-- \\
--\end{array}$ \\
\hline $\begin{array}{l}5-26 \\
5-27 \\
5-28 \\
5-29 \\
5-30\end{array}$ & $\begin{array}{c}1122 \\
45++ \\
177+ \\
40++\end{array}$ & $\begin{array}{r}0 \\
0 \\
0 \\
-0\end{array}$ & $\begin{array}{r}0 \\
0 \\
0 \\
-0 \\
0\end{array}$ & $\begin{array}{r}0 \\
0 \\
0 \\
-- \\
0\end{array}$ & $\begin{array}{r}0 \\
0 \\
0 \\
-0 \\
0\end{array}$ & $\begin{array}{r}0 \\
0 \\
0 \\
-0\end{array}$ & $\begin{array}{r}0 \\
0 \\
0 \\
-0\end{array}$ \\
\hline $\begin{array}{l}6-01 \\
6-03 \\
6-05 \\
6-07 \\
6-09\end{array}$ & $\begin{array}{r}17+++ \\
5+++ \\
20+++ \\
43++ \\
3573^{++}\end{array}$ & $\begin{array}{l}0 \\
0 \\
0 \\
0 \\
0\end{array}$ & $\begin{array}{l}0 \\
0 \\
0 \\
0 \\
0\end{array}$ & $\begin{array}{l}0 \\
0 \\
0 \\
0 \\
0\end{array}$ & $\begin{array}{l}0 \\
0 \\
0 \\
0 \\
0\end{array}$ & $\begin{array}{l}0 \\
0 \\
0 \\
0 \\
0\end{array}$ & $\begin{array}{l}0 \\
0 \\
0 \\
0 \\
0\end{array}$ \\
\hline $\begin{array}{l}6-10 \\
6-11 \\
6-12 \\
6-13 \\
6-14\end{array}$ & $\begin{array}{l}5819 \\
7742 \\
4199 \\
2145 \\
8739\end{array}$ & $\begin{array}{l}0 \\
0 \\
0 \\
0 \\
0\end{array}$ & $\begin{array}{l}0 \\
0 \\
0 \\
0 \\
0\end{array}$ & $\begin{array}{l}0 \\
0 \\
0 \\
0 \\
0\end{array}$ & $\begin{array}{l}0 \\
0 \\
0 \\
0 \\
0\end{array}$ & $\begin{array}{l}0 \\
0 \\
0 \\
0 \\
0\end{array}$ & $\begin{array}{l}0 \\
0 \\
0 \\
0 \\
0\end{array}$ \\
\hline $\begin{array}{l}6-15 \\
6-16 \\
6-17 \\
6-18 \\
6-19\end{array}$ & $\begin{array}{r}2323 \\
739 \\
3247 \\
4233\end{array}$ & $\begin{array}{r}0 \\
-10 \\
0 \\
0\end{array}$ & $\begin{array}{r}0 \\
-0 \\
0 \\
0\end{array}$ & $\begin{array}{r}0 \\
-0 \\
0 \\
0\end{array}$ & $\begin{array}{r}0 \\
-\frac{0}{0} \\
0 \\
0\end{array}$ & $\begin{array}{r}0 \\
-\frac{0}{0} \\
0 \\
0\end{array}$ & $\begin{array}{r}-0 \\
-\frac{0}{0} \\
0 \\
0\end{array}$ \\
\hline $\begin{array}{l}6-20 \\
6-21\end{array}$ & $\begin{array}{l}3247 \\
3537\end{array}$ & $\begin{array}{l}0 \\
0\end{array}$ & $\begin{array}{l}0 \\
0\end{array}$ & $\begin{array}{l}0 \\
0\end{array}$ & $\begin{array}{l}0 \\
0\end{array}$ & $\begin{array}{l}0 \\
0\end{array}$ & $\begin{array}{l}0 \\
0\end{array}$ \\
\hline $\begin{array}{l}6-23 \\
6-25\end{array}$ & $\begin{array}{l}1833 \\
2312\end{array}$ & $\begin{array}{l}0 \\
2\end{array}$ & $\begin{array}{l}0 \\
2\end{array}$ & $\begin{array}{l}0 \\
0\end{array}$ & $\begin{array}{l}0 \\
0\end{array}$ & $\begin{array}{l}0 \\
0\end{array}$ & $\begin{array}{l}0 \\
0\end{array}$ \\
\hline $\begin{array}{l}6-27 \\
6-29 \\
7-01 \\
7-03 \\
7-05\end{array}$ & $\begin{array}{r}1577 \\
=- \\
-- \\
--\end{array}$ & $\begin{array}{l}3 \\
-- \\
-- \\
--\end{array}$ & $\begin{array}{l}1 \\
-- \\
-- \\
--\end{array}$ & $\begin{array}{l}2 \\
-- \\
-- \\
--\end{array}$ & $\begin{array}{l}0 \\
-- \\
-- \\
--\end{array}$ & $\begin{array}{l}0 \\
-- \\
-- \\
--\end{array}$ & $\begin{array}{l}0 \\
-- \\
-- \\
--\end{array}$ \\
\hline $7-07$ & -- & -- & -- & -- & -- & -- & -- \\
\hline
\end{tabular}


TABLE 18.- NUMBER OF GREEN TRACER PARTICLES(1), PER 100 GRAMS OF BEDLOAD SAMPLE, COMPOSITED FROM CROSS-CHANNEL SAMPLING POSITIONS SPACED AT 1-METER INTERVALS ACROSS THE CHANNEL WIDTH, EAST FORK RIVER, WYOMING, 1980

SECTION 0556

\begin{tabular}{|c|c|c|c|c|c|c|c|}
\hline \multirow{3}{*}{ DATE } & \multirow{3}{*}{$\begin{array}{c}\text { DRY } \\
\text { MASS } \\
\text { OF } \\
\text { SAMPLE (2) } \\
\text { (G) }\end{array}$} & \multicolumn{6}{|c|}{ NUMBER OF GREEN TRACER PARTICLES } \\
\hline & & \multirow[b]{2}{*}{ TOTAL } & \multicolumn{4}{|c|}{ BY SIZE CLASS (MM) } & \\
\hline & & & $\begin{array}{c}0.25 \\
\text { TO } \\
0.50\end{array}$ & $\begin{array}{l}0.50 \\
\text { To } \\
1.00\end{array}$ & $\begin{array}{l}1.00 \\
\text { TO } \\
2.00\end{array}$ & $\begin{array}{l}2.00 \\
\text { To } \\
4.00\end{array}$ & $\begin{array}{l}4.00 \\
\text { TO } \\
8.00\end{array}$ \\
\hline $\begin{array}{l}5-16 \\
5-19 \\
5-21 \\
5-22 \\
5-24\end{array}$ & $\begin{array}{c}12+++ \\
272 \\
1800 \\
- \\
-\end{array}$ & $\begin{array}{r}0 \\
0 \\
0 \\
--\end{array}$ & $\begin{array}{l}0 \\
0 \\
0 \\
--\end{array}$ & $\begin{array}{l}0 \\
0 \\
0 \\
--\end{array}$ & $\begin{array}{r}0 \\
0 \\
0 \\
--\end{array}$ & $\begin{array}{r}0 \\
0 \\
0 \\
-\infty\end{array}$ & $\begin{array}{r}0 \\
0 \\
0 \\
--\end{array}$ \\
\hline $\begin{array}{l}5-26 \\
5-27 \\
5-28 \\
5-29 \\
5-30\end{array}$ & $\begin{array}{l}840 \\
58+ \\
207 \\
\frac{62++}{62+}\end{array}$ & $\begin{array}{r}0 \\
0 \\
0 \\
-0\end{array}$ & $\begin{array}{r}0 \\
0 \\
0 \\
-0\end{array}$ & $\begin{array}{r}0 \\
0 \\
0 \\
-0 \\
0\end{array}$ & $\begin{array}{r}0 \\
0 \\
0 \\
-0\end{array}$ & $\begin{array}{r}0 \\
0 \\
0 \\
-0\end{array}$ & $\begin{array}{r}0 \\
0 \\
0 \\
-0\end{array}$ \\
\hline $\begin{array}{l}6-01 \\
6-03 \\
6-05 \\
6-07 \\
6-09\end{array}$ & $\begin{array}{c}77++ \\
53++ \\
93+ \\
103+ \\
5100^{+}\end{array}$ & $\begin{array}{l}0 \\
0 \\
0 \\
0 \\
0\end{array}$ & $\begin{array}{l}0 \\
0 \\
0 \\
0 \\
0\end{array}$ & $\begin{array}{l}0 \\
0 \\
0 \\
0 \\
0\end{array}$ & $\begin{array}{l}0 \\
0 \\
0 \\
0 \\
0\end{array}$ & $\begin{array}{l}0 \\
0 \\
0 \\
0 \\
0\end{array}$ & $\begin{array}{l}0 \\
0 \\
0 \\
0 \\
0\end{array}$ \\
\hline $\begin{array}{l}6-10 \\
6-11 \\
6-12 \\
6-13 \\
6-14\end{array}$ & $\begin{array}{l}5747 \\
2974 \\
9117 \\
3194 \\
3427\end{array}$ & $\begin{array}{l}0 \\
0 \\
0 \\
0 \\
0\end{array}$ & $\begin{array}{l}0 \\
0 \\
0 \\
0 \\
0\end{array}$ & $\begin{array}{l}0 \\
0 \\
0 \\
0 \\
0\end{array}$ & $\begin{array}{l}0 \\
0 \\
0 \\
0 \\
0\end{array}$ & $\begin{array}{l}0 \\
0 \\
0 \\
0 \\
0\end{array}$ & $\begin{array}{l}0 \\
0 \\
0 \\
0 \\
0\end{array}$ \\
\hline $\begin{array}{l}6-15 \\
6-16 \\
6-17 \\
6-18 \\
6-19\end{array}$ & $\begin{array}{l}1217 \\
1354 \\
3938 \\
2389\end{array}$ & $\begin{array}{c}0 \\
-\frac{0}{0} \\
0 \\
0\end{array}$ & $\begin{array}{c}0 \\
-0 \\
0 \\
0\end{array}$ & $\begin{array}{r}0 \\
-0 \\
0 \\
0\end{array}$ & $\begin{array}{r}-0 \\
0 \\
0 \\
0\end{array}$ & $\begin{array}{r}-0 \\
-0 \\
0 \\
0\end{array}$ & $\begin{array}{c}0 \\
0 \\
0 \\
0\end{array}$ \\
\hline $\begin{array}{l}6-20 \\
6-21\end{array}$ & $\begin{array}{l}5650 \\
2083\end{array}$ & $\begin{array}{l}0 \\
0\end{array}$ & $\begin{array}{l}0 \\
0\end{array}$ & $\begin{array}{l}0 \\
0\end{array}$ & $\begin{array}{l}0 \\
0\end{array}$ & $\begin{array}{l}0 \\
0\end{array}$ & $\begin{array}{l}0 \\
0\end{array}$ \\
\hline $\begin{array}{l}6-22 \\
6-23 \\
6-25\end{array}$ & $\begin{array}{r}3676 \\
872\end{array}$ & $\begin{array}{l}0 \\
2\end{array}$ & $\begin{array}{l}0 \\
2\end{array}$ & 0 & 0 & $\begin{array}{l}0 \\
0\end{array}$ & $\begin{array}{l}0 \\
0\end{array}$ \\
\hline $6-27$ & 2150 & 4 & 3 & 1 & 0 & 0 & 0 \\
\hline $\begin{array}{l}6-29 \\
7-01\end{array}$ & $\overline{-}$ & $\overline{-}$ & $\overline{-\infty}$ & $\overline{-}$ & $\overline{-}$ & $\overline{-}$ & $\overline{-}$ \\
\hline $7-03$ & - & - & -- & - & - & $-\infty$ & - \\
\hline $7-05$ & -- & -- & - & - & -- & -- & -- \\
\hline $7-07$ & -- & $-\infty$ & -- & -- & - & -- & - \\
\hline
\end{tabular}


TABLE 18.- NUMBER OF GREEN TRACER PARTICLES(1), PER 100 GRAMS OF BEDLOAD SAMPLE, COMPOSITED FROM CROSS-CHANNEL SAMPLING POSITIONS SPACED AT 1-METER INTERVALS ACROSS THE CHANNEL WIDTH, EAST FORK RIVER, WYOMING, 1980

SECTION 0602

\begin{tabular}{|c|c|c|c|c|c|c|c|}
\hline \multirow{3}{*}{ DATE } & \multirow{3}{*}{$\begin{array}{c}\text { DRY } \\
\text { MASS } \\
\text { OF } \\
\text { SAMPLE (2) } \\
\text { (G) }\end{array}$} & \multicolumn{6}{|c|}{ NUMBER OF GREEN TRACER PARTICLES } \\
\hline & & \multirow[b]{2}{*}{ TOTAL } & \multicolumn{5}{|c|}{ BY SIZE CLASS (MM) } \\
\hline & & & $\begin{array}{c}0.25 \\
\text { TO } \\
0.50\end{array}$ & $\begin{array}{r}0.50 \\
\text { TO } \\
1.00\end{array}$ & $\begin{array}{l}1.00 \\
\text { TO } \\
2.00\end{array}$ & $\begin{array}{l}2.00 \\
\text { TO } \\
4.00\end{array}$ & $\begin{array}{l}4.00 \\
\text { TO } \\
8.00\end{array}$ \\
\hline $\begin{array}{l}5-16 \\
5-19 \\
5-21\end{array}$ & $\begin{array}{r}136 \\
1010 \\
1054\end{array}$ & $\begin{array}{l}0 \\
0 \\
0\end{array}$ & $\begin{array}{l}0 \\
0 \\
0\end{array}$ & $\begin{array}{l}0 \\
0 \\
0\end{array}$ & $\begin{array}{l}0 \\
0 \\
0\end{array}$ & $\begin{array}{l}0 \\
0 \\
0\end{array}$ & $\begin{array}{l}0 \\
0 \\
0\end{array}$ \\
\hline $\begin{array}{l}5-22 \\
5-24\end{array}$ & - & - & - & - & - & - & - \\
\hline $\begin{array}{l}5-26 \\
5-27 \\
5-28\end{array}$ & $\begin{array}{c}1299 \\
167+ \\
112\end{array}$ & $\begin{array}{l}0 \\
0 \\
0\end{array}$ & $\begin{array}{l}0 \\
0 \\
0\end{array}$ & $\begin{array}{l}0 \\
0 \\
0\end{array}$ & $\begin{array}{l}0 \\
0 \\
0\end{array}$ & $\begin{array}{l}0 \\
0 \\
0\end{array}$ & $\begin{array}{l}0 \\
0 \\
0\end{array}$ \\
\hline $\begin{array}{l}5-29 \\
5-30\end{array}$ & 474 & $\overline{0}$ & $\overline{0}$ & $\overline{0}$ & $\overline{0}$ & $\overline{0}$ & $\overline{0}$ \\
\hline $\begin{array}{l}6-01 \\
6-03 \\
6-05 \\
6-07 \\
6-09\end{array}$ & $\begin{array}{r}56+ \\
162+ \\
921 \\
1320 \\
5773\end{array}$ & $\begin{array}{l}0 \\
0 \\
0 \\
0 \\
0\end{array}$ & $\begin{array}{l}0 \\
0 \\
0 \\
0 \\
0\end{array}$ & $\begin{array}{l}0 \\
0 \\
0 \\
0 \\
0\end{array}$ & $\begin{array}{l}0 \\
0 \\
0 \\
0 \\
0\end{array}$ & $\begin{array}{l}0 \\
0 \\
0 \\
0 \\
0\end{array}$ & $\begin{array}{l}0 \\
0 \\
0 \\
0 \\
0\end{array}$ \\
\hline $\begin{array}{l}6-10 \\
6-11 \\
6-12 \\
6-13 \\
6-14\end{array}$ & $\begin{array}{l}4233 \\
5076 \\
2864 \\
3765 \\
2492\end{array}$ & $\begin{array}{l}0 \\
0 \\
0 \\
0 \\
0\end{array}$ & $\begin{array}{l}0 \\
0 \\
0 \\
0 \\
0\end{array}$ & $\begin{array}{l}0 \\
0 \\
0 \\
0 \\
0\end{array}$ & $\begin{array}{l}0 \\
0 \\
0 \\
0 \\
0\end{array}$ & $\begin{array}{l}0 \\
0 \\
0 \\
0 \\
0\end{array}$ & $\begin{array}{l}0 \\
0 \\
0 \\
0 \\
0\end{array}$ \\
\hline $6-15$ & 1838 & 0 & 0 & 0 & 0 & 0 & 0 \\
\hline $\begin{array}{l}6-17 \\
6-18 \\
6-19\end{array}$ & $\begin{array}{l}2006 \\
1637 \\
5043\end{array}$ & $\begin{array}{l}1 \\
0 \\
0\end{array}$ & $\begin{array}{l}1 \\
0 \\
0\end{array}$ & $\begin{array}{l}0 \\
0 \\
0\end{array}$ & $\begin{array}{l}0 \\
0 \\
0\end{array}$ & $\begin{array}{l}0 \\
0 \\
0\end{array}$ & $\begin{array}{l}0 \\
0 \\
0\end{array}$ \\
\hline $\begin{array}{l}6-20 \\
6-21\end{array}$ & $\begin{array}{l}7174 \\
2827\end{array}$ & $\begin{array}{l}0 \\
0\end{array}$ & $\begin{array}{l}0 \\
0\end{array}$ & $\begin{array}{l}0 \\
0\end{array}$ & $\begin{array}{l}0 \\
0\end{array}$ & $\begin{array}{l}0 \\
0\end{array}$ & $\begin{array}{l}0 \\
0\end{array}$ \\
\hline $\begin{array}{l}6-23 \\
6-25\end{array}$ & $\begin{array}{l}3194 \\
4332\end{array}$ & $\begin{array}{l}0 \\
3\end{array}$ & $\begin{array}{l}0 \\
3\end{array}$ & $\begin{array}{l}0 \\
0\end{array}$ & $\begin{array}{l}0 \\
0\end{array}$ & $\begin{array}{l}0 \\
0\end{array}$ & $\begin{array}{l}0 \\
0\end{array}$ \\
\hline $6-27$ & 1490 & 3 & 1 & 2 & 0 & 0 & 0 \\
\hline $\begin{array}{l}0-29 \\
7-01\end{array}$ & $-\overline{-}$ & $\overline{--}$ & $\overline{--}$ & $\overline{--}$ & -- & $\overline{--}$ & - \\
\hline $\begin{array}{l}7-03 \\
7-05\end{array}$ & $=$ & -- & $=$ & $=$ & -- & $=$ & 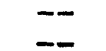 \\
\hline $7-07$ & -- & -- & -- & -- & -- & -- & -- \\
\hline
\end{tabular}


TABLE 18.- NUMBER OF GREEN TRACER PARTICLES(1), PER 100 GRAMS OF BEDLOAD SAMPLE, COMPOSITED FROM CROSS-CHANNEL SAMPLING POSITIONS SPACED AT 1-METER INTERVALS ACROSS THE CHANNEL WIDTH, EAST FORK RIVER, WYOMING, 1980

SECTION 0653

\begin{tabular}{|c|c|c|c|c|c|c|c|}
\hline \multirow[t]{2}{*}{ DATE } & \multirow{2}{*}{$\begin{array}{c}\text { DRY } \\
\text { MASS } \\
\text { OF } \\
\text { SAMPLE (2) } \\
\text { (G) }\end{array}$} & \multicolumn{5}{|c|}{ NUMBER OF GREEN TRACER PARTICLES } & \\
\hline & & TOTAL & $\begin{array}{l}0.25 \\
\text { TO } \\
0.50\end{array}$ & $\begin{array}{l}0.50 \\
\text { TO } \\
1.00\end{array}$ & $\begin{array}{l}1.00 \\
\text { To } \\
2.00\end{array}$ & $\begin{array}{l}2.00 \\
\text { TO } \\
4.00\end{array}$ & $\begin{array}{l}4.00 \\
\text { TO } \\
8.00\end{array}$ \\
\hline $\begin{array}{l}5-16 \\
5-19 \\
5-21 \\
5-22 \\
5-24\end{array}$ & $\begin{array}{l}148+ \\
168+ \\
153+ \\
--\end{array}$ & $\begin{array}{r}0 \\
0 \\
0 \\
-- \\
--\end{array}$ & $\begin{array}{r}0 \\
0 \\
0 \\
-- \\
--\end{array}$ & $\begin{array}{r}0 \\
0 \\
0 \\
-- \\
--\end{array}$ & $\begin{array}{r}0 \\
0 \\
0 \\
-- \\
--\end{array}$ & $\begin{array}{r}0 \\
0 \\
0 \\
-- \\
--\end{array}$ & $\begin{array}{r}0 \\
0 \\
0 \\
-- \\
--\end{array}$ \\
\hline $\begin{array}{l}5-26 \\
5-27 \\
5-28 \\
5-29 \\
5-30\end{array}$ & $\begin{array}{l}393 \\
491 \\
108+ \\
-- \\
83++\end{array}$ & $\begin{array}{r}0 \\
0 \\
0 \\
-0 \\
0\end{array}$ & $\begin{array}{r}0 \\
0 \\
0 \\
-0\end{array}$ & $\begin{array}{r}0 \\
0 \\
0 \\
-- \\
0\end{array}$ & $\begin{array}{r}0 \\
0 \\
0 \\
-0 \\
0\end{array}$ & $\begin{array}{r}0 \\
0 \\
0 \\
-0\end{array}$ & $\begin{array}{r}0 \\
0 \\
0 \\
-0\end{array}$ \\
\hline $\begin{array}{l}6-01 \\
6-03 \\
6-05 \\
6-07 \\
6-09\end{array}$ & $\begin{array}{r}372 \\
439 \\
490 \\
2330 \\
3768\end{array}$ & $\begin{array}{l}0 \\
0 \\
0 \\
0 \\
0\end{array}$ & $\begin{array}{l}0 \\
0 \\
0 \\
0 \\
0\end{array}$ & $\begin{array}{l}0 \\
0 \\
0 \\
0 \\
0\end{array}$ & $\begin{array}{l}0 \\
0 \\
0 \\
0 \\
0\end{array}$ & $\begin{array}{l}0 \\
0 \\
0 \\
0 \\
0\end{array}$ & $\begin{array}{l}0 \\
0 \\
0 \\
0 \\
0\end{array}$ \\
\hline $\begin{array}{l}6-10 \\
6-11 \\
6-12 \\
6-13 \\
6-14\end{array}$ & $\begin{array}{r}15664 \\
3692 \\
348 \\
427 \\
257\end{array}$ & $\begin{array}{l}0 \\
0 \\
3 \\
0 \\
0\end{array}$ & $\begin{array}{l}0 \\
0 \\
3 \\
0 \\
0\end{array}$ & $\begin{array}{l}0 \\
0 \\
0 \\
0 \\
0\end{array}$ & $\begin{array}{l}0 \\
0 \\
0 \\
0 \\
0\end{array}$ & $\begin{array}{l}0 \\
0 \\
0 \\
0 \\
0\end{array}$ & $\begin{array}{l}0 \\
0 \\
0 \\
0 \\
0\end{array}$ \\
\hline $\begin{array}{l}6-15 \\
6-16 \\
6-17 \\
6-18 \\
6-19\end{array}$ & $\begin{array}{r}622 \\
-- \\
2105 \\
3735 \\
4034\end{array}$ & $\begin{array}{r}0 \\
-- \\
9 \\
0 \\
0\end{array}$ & $\begin{array}{r}-0 \\
-\frac{6}{6} \\
0 \\
0\end{array}$ & $\begin{array}{r}0 \\
-- \\
3 \\
0 \\
0\end{array}$ & $\begin{array}{r}-0 \\
-0 \\
0 \\
0\end{array}$ & $\begin{array}{r}-0 \\
-0 \\
0 \\
0\end{array}$ & $\begin{array}{r}0 \\
-0 \\
0 \\
0 \\
0\end{array}$ \\
\hline $\begin{array}{l}6-20 \\
6-21 \\
6-22 \\
6-23 \\
6-25\end{array}$ & $\begin{array}{r}1922 \\
2686 \\
-- \\
1278 \\
1894\end{array}$ & $\begin{array}{r}0 \\
-0 \\
-1 \\
0\end{array}$ & $\begin{array}{r}0 \\
0 \\
-\frac{1}{1} \\
0\end{array}$ & $\begin{array}{r}0 \\
0 \\
-- \\
0 \\
0\end{array}$ & $\begin{array}{r}0 \\
0 \\
-0 \\
0 \\
0\end{array}$ & $\begin{array}{r}0 \\
0 \\
-0 \\
0 \\
0\end{array}$ & $\begin{array}{r}0 \\
0 \\
-0 \\
0 \\
0\end{array}$ \\
\hline $\begin{array}{l}6-27 \\
6-29 \\
7-01 \\
7-03 \\
7-05\end{array}$ & $\begin{array}{r}2210 \\
=- \\
= \\
=\end{array}$ & $\begin{array}{l}2 \\
-- \\
-- \\
--\end{array}$ & $\begin{array}{l}2 \\
-- \\
-- \\
--\end{array}$ & $\begin{array}{l}0 \\
-- \\
--\end{array}$ & $\begin{array}{l}0 \\
-- \\
=- \\
--\end{array}$ & $\begin{array}{l}0 \\
-- \\
=- \\
--\end{array}$ & $\begin{array}{l}0 \\
-- \\
=- \\
--\end{array}$ \\
\hline $7-07$ & -- & -- & -- & -- & -- & -- & -- \\
\hline
\end{tabular}


TABLE 18.- NUMBER OF GREEN TRACER PARTICLES(1), PER 100 GRAMS OF BEDLOAD SAMPLE, COMPOSITED FROM CROSS-CHANNEL SAMPLING POSITIONS SPACED AT 1-METER INTERVALS ACROSS THE CHANNEL WIDTH, EAST FORK RIVER, WYOMING, 1980

SECTION 0653(3)

\begin{tabular}{|c|c|c|c|c|c|c|c|}
\hline \multirow{3}{*}{ DATE } & \multirow{3}{*}{$\begin{array}{c}\text { DRY } \\
\text { MASS } \\
\text { OF } \\
\text { SAMPLE (2) } \\
(\mathbf{G})\end{array}$} & \multicolumn{6}{|c|}{ NUMBER OF GREEN TRACER PARTICLES } \\
\hline & & \multirow[b]{2}{*}{ TOTAL } & \multicolumn{5}{|c|}{ BY SIZE CLASS (MM) } \\
\hline & & & $\begin{array}{l}0.25 \\
\text { To } \\
0.50\end{array}$ & $\begin{array}{l}0.50 \\
\text { TO } \\
1.00\end{array}$ & $\begin{array}{l}1.00 \\
2.00\end{array}$ & $\begin{array}{l}2.00 \\
\text { TO } \\
4.00\end{array}$ & $\begin{array}{l}4.00 \\
\text { TO } \\
8.00\end{array}$ \\
\hline $5-16$ & - & -- & - & - & -- & -- & -- \\
\hline $5-19$ & - & -- & -- & -- & -- & -- & -- \\
\hline $\begin{array}{l}5-2 \frac{1}{2} \\
5-22\end{array}$ & $=$ & $=$ & $=$ & $=$ & $\overline{--}$ & $=$ & $=$ \\
\hline $5-24$ & -- & -- & -- & - & -- & - & -- \\
\hline $5-26$ & - & -- & - & - & -- & - & - \\
\hline $5-27$ & - & -- & - & - & -- & -- & - \\
\hline $\begin{array}{l}5-28 \\
5-29\end{array}$ & $\overline{-}$ & $=$ & $=$ & $=$ & $=$ & $=$ & $=$ \\
\hline $5-30$ & - & -- & - & - & -- & -- & -- \\
\hline $6-01$ & - & - & - & - & -- & - & - \\
\hline $6-03$ & $\overline{-}$ & $\overline{--}$ & $\overline{--}$ & $\overline{-}$ & $\overline{--}$ & -- & -- \\
\hline $\begin{array}{l}-4 \\
6-05 \\
6-07\end{array}$ & - & $=$ & $=$ & $\overline{-}$ & $-\overline{-}$ & $=$ & -- \\
\hline $6-09$ & $61+$ & 0 & 0 & 0 & 0 & 0 & 0 \\
\hline $6-10$ & 283 & a. & 0 & 0 & 0 & 0 & 0 \\
\hline $6-12$ & 266 & 0 & 0 & 0 & 0 & 0 & 0 \\
\hline $\begin{array}{l}6-13 \\
6-14\end{array}$ & $190^{24++}$ & $\begin{array}{l}0 \\
0\end{array}$ & $\begin{array}{l}0 \\
0\end{array}$ & $\begin{array}{l}0 \\
0\end{array}$ & $\begin{array}{l}0 \\
0\end{array}$ & $\begin{array}{l}0 \\
0\end{array}$ & $\begin{array}{l}0 \\
0\end{array}$ \\
\hline $6-15$ & $33++$ & 0 & 0 & 0 & 0 & 0 & 0 \\
\hline $\begin{array}{l}6-16 \\
6-17\end{array}$ & $\overline{-}$ & $=$ & $=$ & $=$ & -- & -- & $=$ \\
\hline $6-18$ & $38++$ & 0 & 0 & 0 & 0 & 0 & 0 \\
\hline $6-19$ & $0+++$ & 0 & 0 & 0 & 0 & 0 & 0 \\
\hline $6-20$ & 371 & 0 & 0 & 0 & 0 & 0 & 0 \\
\hline $6-21$ & $71+$ & 0 & 0 & 0 & 0 & 0 & 0 \\
\hline $\begin{array}{l}6-22 \\
6-23\end{array}$ & $=$ & $=$ & $=$ & $=$ & $=$ & $=$ & $\overline{--}$ \\
\hline $6-25$ & $22+++$ & 0 & 0 & 0 & 0 & 0 & 0 \\
\hline $6-27$ & $6+++$ & 0 & 0 & 0 & 0 & 0 & 0 \\
\hline $\begin{array}{l}6-29 \\
7-07\end{array}$ & $=$ & $=$ & $=$ & $=$ & $=$ & $=$ & $=$ \\
\hline $7-03$ & -- & -- & - & -- & -- & -- & -- \\
\hline $7-05$ & - & -- & - & -- & -- & - & -- \\
\hline $7-07$ & -- & - & - & -- & - & -- & -- \\
\hline
\end{tabular}


TABLE 18.- NUMBER OF GREEN TRACER PARTICLES(1), PER 100 GRAMS OF BEDLOAD SAMPLE, COMPOSITED FROM CROSS-CHANNEL SAMPLING POSITIONS SPACED AT 1-METER INTERVALS ACROSS THE CHANNEL WIDTH, EAST FORK RIVER, WYOMING, 1980

SECTION 0708

\begin{tabular}{|c|c|c|c|c|c|c|c|}
\hline \multirow[t]{2}{*}{ DATE } & \multirow{2}{*}{$\begin{array}{c}\text { DRY } \\
\text { MASS } \\
\text { OF } \\
\text { SAMPLE (2) } \\
\text { (G) }\end{array}$} & \multicolumn{6}{|c|}{ NUMBER OF GREEN TRACER PARTICLES } \\
\hline & & TOTAL & $\begin{array}{l}0.25 \\
\text { TO } \\
0.50\end{array}$ & $\begin{array}{l}0.50 \\
\text { TO } \\
1.00\end{array}$ & $\begin{array}{l}1.00 \\
\text { TO } \\
2.00\end{array}$ & $\begin{array}{l}2.00 \\
\text { TO } \\
4.00\end{array}$ & $\begin{array}{l}4.00 \\
\text { TO } \\
8.00\end{array}$ \\
\hline $\begin{array}{l}5-16 \\
5-19 \\
5-21 \\
5-22 \\
5-24\end{array}$ & $\begin{array}{r}841 \\
395 \\
3726 \\
-- \\
--\end{array}$ & $\begin{array}{r}0 \\
0 \\
0 \\
--\end{array}$ & $\begin{array}{r}0 \\
0 \\
0 \\
-- \\
--\end{array}$ & $\begin{array}{r}0 \\
0 \\
0 \\
--\end{array}$ & $\begin{array}{r}0 \\
0 \\
0 \\
-- \\
--\end{array}$ & $\begin{array}{r}0 \\
0 \\
0 \\
-- \\
--\end{array}$ & $\begin{array}{r}0 \\
0 \\
0 \\
--\end{array}$ \\
\hline $\begin{array}{l}5-26 \\
5-27 \\
5-28 \\
5-29 \\
5-30\end{array}$ & $\begin{array}{l}513 \\
215 \\
288 \\
422 \\
88+\end{array}$ & $\begin{array}{l}0 \\
0 \\
0 \\
0 \\
0\end{array}$ & $\begin{array}{l}0 \\
0 \\
0 \\
0 \\
0\end{array}$ & $\begin{array}{l}0 \\
0 \\
0 \\
0 \\
0\end{array}$ & $\begin{array}{l}0 \\
0 \\
0 \\
0 \\
0\end{array}$ & $\begin{array}{l}0 \\
0 \\
0 \\
0 \\
0\end{array}$ & $\begin{array}{l}0 \\
0 \\
0 \\
0 \\
0\end{array}$ \\
\hline $\begin{array}{l}6-01 \\
6-03 \\
6-05 \\
6-07 \\
6-09\end{array}$ & $\begin{array}{l}39++ \\
909 \\
702 \\
1550 \\
3638\end{array}$ & $\begin{array}{l}0 \\
0 \\
0 \\
0 \\
0\end{array}$ & $\begin{array}{l}0 \\
0 \\
0 \\
0 \\
0\end{array}$ & $\begin{array}{l}0 \\
0 \\
0 \\
0 \\
0\end{array}$ & $\begin{array}{l}0 \\
0 \\
0 \\
0 \\
0\end{array}$ & $\begin{array}{l}0 \\
0 \\
0 \\
0 \\
0\end{array}$ & $\begin{array}{l}0 \\
0 \\
0 \\
0 \\
0\end{array}$ \\
\hline $\begin{array}{l}6-10 \\
6-11 \\
6-12 \\
6-13 \\
6-14\end{array}$ & $\begin{array}{r}7438 \\
1025 \\
583 \\
1330 \\
2343\end{array}$ & $\begin{array}{l}0 \\
1 \\
0 \\
0 \\
0\end{array}$ & $\begin{array}{l}0 \\
1 \\
0 \\
0 \\
0\end{array}$ & $\begin{array}{l}0 \\
0 \\
0 \\
0 \\
0\end{array}$ & $\begin{array}{l}0 \\
0 \\
0 \\
0 \\
0\end{array}$ & $\begin{array}{l}0 \\
0 \\
0 \\
0 \\
0\end{array}$ & $\begin{array}{l}0 \\
0 \\
0 \\
0 \\
0\end{array}$ \\
\hline $\begin{array}{l}6-15 \\
6-16 \\
6-17 \\
6-18 \\
6-19\end{array}$ & $\begin{array}{r}862 \\
884 \\
1457 \\
3737 \\
3122\end{array}$ & $\begin{array}{l}0 \\
0 \\
0 \\
0 \\
0\end{array}$ & $\begin{array}{l}0 \\
0 \\
0 \\
0 \\
0\end{array}$ & $\begin{array}{l}0 \\
0 \\
0 \\
0 \\
0\end{array}$ & $\begin{array}{l}0 \\
0 \\
0 \\
0 \\
0\end{array}$ & $\begin{array}{l}0 \\
0 \\
0 \\
0 \\
0\end{array}$ & $\begin{array}{l}0 \\
0 \\
0 \\
0 \\
0\end{array}$ \\
\hline $\begin{array}{l}6-20 \\
6-21\end{array}$ & $\begin{array}{r}10382 \\
1174\end{array}$ & $\begin{array}{l}0 \\
0\end{array}$ & $\begin{array}{l}0 \\
0\end{array}$ & $\begin{array}{l}0 \\
0\end{array}$ & $\begin{array}{l}0 \\
0\end{array}$ & $\begin{array}{l}0 \\
0\end{array}$ & $\begin{array}{l}0 \\
0\end{array}$ \\
\hline $\begin{array}{l}6-22 \\
6-23 \\
6-25\end{array}$ & $\begin{array}{l}997 \\
721\end{array}$ & 1 & 1 & 0 & $\begin{array}{l}0 \\
0\end{array}$ & $\begin{array}{l}0 \\
0\end{array}$ & $\begin{array}{l}0 \\
0\end{array}$ \\
\hline $\begin{array}{l}6-27 \\
6-29 \\
7-01 \\
7-03 \\
7-05\end{array}$ & $\begin{array}{r}2107 \\
=- \\
=- \\
=-\end{array}$ & $\begin{array}{l}5 \\
=- \\
--\end{array}$ & $\begin{array}{l}4 \\
=- \\
--\end{array}$ & $\begin{array}{l}1 \\
=- \\
--\end{array}$ & $\begin{array}{l}0 \\
=- \\
=- \\
--\end{array}$ & $\begin{array}{l}0 \\
=- \\
=- \\
--\end{array}$ & $\begin{array}{l}0 \\
=- \\
=- \\
--\end{array}$ \\
\hline $7-07$ & -- & -- & -- & -- & -- & -- & -- \\
\hline
\end{tabular}


TABLE 18.- NUMBER OF GREEN TRACER PARTICLES(1), PER 100 GRAMS OF BEDLOAD SAMPLE, COMPOSITED FROM CROSS-CHANNEL SAMPLING POSITIONS SPACED AT 1-METER INTERVALS ACROSS THE CHANNEL WIDTH, EAST FORK RIVER, WYOMING, 1980

SECTION 0757

\begin{tabular}{|c|c|c|c|c|c|c|c|}
\hline \multirow[t]{2}{*}{ DATE } & \multirow{2}{*}{$\begin{array}{c}\text { DRY } \\
\text { MASS } \\
\text { OF } \\
\text { SAMPLE (2) } \\
\text { (G) }\end{array}$} & \multicolumn{6}{|c|}{ NUMBER OF GREEN TRACER PARTICLES } \\
\hline & & TOTAL & $\begin{array}{l}0.25 \\
\text { TO } \\
0.50\end{array}$ & $\begin{array}{l}0.50 \\
\text { TO } \\
1.00\end{array}$ & $\begin{array}{l}1.00 \\
2.00\end{array}$ & $\begin{array}{l}2.00 \\
\text { TO } \\
4.00\end{array}$ & $\begin{array}{l}4.00 \\
\text { TO } \\
8.00\end{array}$ \\
\hline $\begin{array}{l}5-16 \\
5-19 \\
5-21 \\
5-22 \\
5-24\end{array}$ & $\begin{array}{r}450 \\
463 \\
613 \\
-- \\
--\end{array}$ & $\begin{array}{r}0 \\
0 \\
0 \\
-- \\
--\end{array}$ & $\begin{array}{r}0 \\
0 \\
0 \\
--\end{array}$ & $\begin{array}{r}0 \\
0 \\
0 \\
---\end{array}$ & $\begin{array}{r}0 \\
0 \\
0 \\
--\end{array}$ & $\begin{array}{r}0 \\
0 \\
0 \\
--\end{array}$ & $\begin{array}{r}0 \\
0 \\
0 \\
--\end{array}$ \\
\hline $\begin{array}{l}5-26 \\
5-27 \\
5-28 \\
5-20\end{array}$ & $\begin{array}{l}6427 \\
3063 \\
1324\end{array}$ & $\begin{array}{l}0 \\
0 \\
0\end{array}$ & $\begin{array}{l}0 \\
0 \\
0\end{array}$ & $\begin{array}{l}0 \\
0 \\
0\end{array}$ & $\begin{array}{l}0 \\
0 \\
0\end{array}$ & $\begin{array}{l}0 \\
0 \\
0\end{array}$ & $\begin{array}{l}0 \\
0 \\
0\end{array}$ \\
\hline $5-30$ & 1695 & 0 & 0 & 0 & 0 & 0 & 0 \\
\hline $\begin{array}{l}6-01 \\
6-03 \\
6-05 \\
6-07 \\
6-09\end{array}$ & $\begin{array}{c}1578+ \\
448 \\
384 \\
501 \\
716\end{array}$ & $\begin{array}{l}0 \\
0 \\
0 \\
0 \\
0\end{array}$ & $\begin{array}{l}0 \\
0 \\
0 \\
0 \\
0\end{array}$ & $\begin{array}{l}0 \\
0 \\
0 \\
0 \\
0\end{array}$ & $\begin{array}{l}0 \\
0 \\
0 \\
0 \\
0\end{array}$ & $\begin{array}{l}0 \\
0 \\
0 \\
0 \\
0\end{array}$ & $\begin{array}{l}0 \\
0 \\
0 \\
0 \\
0\end{array}$ \\
\hline $\begin{array}{l}6-10 \\
6-11 \\
6-12 \\
6-13 \\
6-14\end{array}$ & $\begin{array}{l}68+ \\
320 \\
2425 \\
1014 \\
3202\end{array}$ & $\begin{array}{r}0 \\
15 \\
2 \\
0 \\
0\end{array}$ & $\begin{array}{r}0 \\
13 \\
2 \\
0 \\
0\end{array}$ & $\begin{array}{l}0 \\
2 \\
0 \\
0 \\
0\end{array}$ & $\begin{array}{l}0 \\
0 \\
0 \\
0 \\
0\end{array}$ & $\begin{array}{l}0 \\
0 \\
0 \\
0 \\
0\end{array}$ & $\begin{array}{l}0 \\
0 \\
0 \\
0 \\
0\end{array}$ \\
\hline $\begin{array}{l}6-15 \\
6-16 \\
6-17 \\
6-18 \\
6-19\end{array}$ & $\begin{array}{l}1515 \\
3208 \\
5436 \\
6123 \\
2003\end{array}$ & $\begin{array}{l}0 \\
0 \\
0 \\
0 \\
0\end{array}$ & $\begin{array}{l}0 \\
0 \\
0 \\
0 \\
0\end{array}$ & $\begin{array}{l}0 \\
0 \\
0 \\
0 \\
0\end{array}$ & $\begin{array}{l}0 \\
0 \\
0 \\
0 \\
0\end{array}$ & $\begin{array}{l}0 \\
0 \\
0 \\
0 \\
0\end{array}$ & $\begin{array}{l}0 \\
0 \\
0 \\
0 \\
0\end{array}$ \\
\hline $\begin{array}{l}6-20 \\
6-21 \\
6-27\end{array}$ & $\begin{array}{l}2586 \\
1847\end{array}$ & $\begin{array}{l}2 \\
2\end{array}$ & $\begin{array}{l}2 \\
2\end{array}$ & $\begin{array}{l}0 \\
0\end{array}$ & $\begin{array}{l}0 \\
0\end{array}$ & $\begin{array}{l}0 \\
0\end{array}$ & $\begin{array}{l}0 \\
0\end{array}$ \\
\hline $\begin{array}{l}6-23 \\
6-25\end{array}$ & $\begin{array}{l}1505 \\
2895\end{array}$ & 2 & $\begin{array}{l}2 \\
0\end{array}$ & 0 & 0 & 0 & $\begin{array}{l}0 \\
0\end{array}$ \\
\hline $\begin{array}{l}6-27 \\
6-29\end{array}$ & 1785 & -3 & -1 & -3 & $\underline{0}$ & $\underline{0}$ & $\underline{0}$ \\
\hline $7-01$ & -- & - & - & -- & - & - & - \\
\hline $7-05$ & -- & $\overline{-}$ & $=$ & $\overline{--}$ & $\overline{--}$ & $=$ & $=$ \\
\hline $7-07$ & -- & -- & -- & -- & -- & -- & -- \\
\hline
\end{tabular}


TABLE 18. - NUMBER OF GREEN TRACER PARTICLES (1), PER 100 GRAMS OF BEDLOAD SAMPLE, COMPOSITED FROM CROSS-CHANNEL SAMPLING POSITIONS SPACED AT I-METER INTERVALS ACROSS THE CHANNEL WIDTH, EAST FORK RIVER, WYOMING, 1980

SECTION 0808

\begin{tabular}{|c|c|c|c|c|c|c|c|}
\hline \multirow{3}{*}{\multicolumn{2}{|c|}{$\begin{array}{cc} & \\
\text { DATE } & \text { DRY } \\
& \text { MASS } \\
\text { OF } & \text { SAMPLE (2) } \\
& \text { (G) }\end{array}$}} & \multicolumn{6}{|c|}{ NUMBER OF GREEN TRACER PARTICLES } \\
\hline & & \multirow[b]{2}{*}{ TOTAL } & \multicolumn{5}{|c|}{ BY SIZE CLASS (MM) } \\
\hline & & & $\begin{array}{l}0.25 \\
\text { TO } \\
0.50\end{array}$ & $\begin{array}{l}0.50 \\
\text { TO } \\
1.00\end{array}$ & $\begin{array}{l}1.00 \\
\text { TO } \\
2.00\end{array}$ & $\begin{array}{l}2.00 \\
\text { TO } \\
4.00\end{array}$ & $\begin{array}{l}4.00 \\
\text { TO } \\
8.00\end{array}$ \\
\hline $\begin{array}{l}5-16 \\
5-19 \\
5-21\end{array}$ & $\begin{array}{l}573 \\
894 \\
314\end{array}$ & $\begin{array}{l}0 \\
0 \\
0\end{array}$ & $\begin{array}{l}0 \\
0 \\
0\end{array}$ & $\begin{array}{l}0 \\
0 \\
0\end{array}$ & $\begin{array}{l}0 \\
0 \\
0\end{array}$ & $\begin{array}{l}0 \\
0 \\
0\end{array}$ & $\begin{array}{l}0 \\
0 \\
0\end{array}$ \\
\hline $\begin{array}{l}5-22 \\
5-24\end{array}$ & $\overline{--}$ & - & - & - & - & -- & - \\
\hline $\begin{array}{l}5-26 \\
5-27 \\
5-28\end{array}$ & $\begin{array}{l}3784 \\
2360 \\
2445\end{array}$ & $\begin{array}{l}0 \\
0 \\
0\end{array}$ & $\begin{array}{l}0 \\
0 \\
0\end{array}$ & $\begin{array}{l}0 \\
0 \\
0\end{array}$ & $\begin{array}{l}0 \\
0 \\
0\end{array}$ & $\begin{array}{l}0 \\
0 \\
0\end{array}$ & $\begin{array}{l}0 \\
0 \\
0\end{array}$ \\
\hline $5-30$ & 1203 & 0 & 0 & 0 & 0 & 0 & 0 \\
\hline $\begin{array}{l}6-01 \\
6-03 \\
6-05 \\
6-07 \\
6-09\end{array}$ & $\begin{array}{l}573 \\
196 \\
292 \\
119 \\
358\end{array}$ & $\begin{array}{l}0 \\
0 \\
0 \\
0 \\
4\end{array}$ & $\begin{array}{l}0 \\
0 \\
0 \\
0 \\
3\end{array}$ & $\begin{array}{l}0 \\
0 \\
0 \\
0 \\
1\end{array}$ & $\begin{array}{l}0 \\
0 \\
0 \\
0 \\
0\end{array}$ & $\begin{array}{l}0 \\
0 \\
0 \\
0 \\
0\end{array}$ & $\begin{array}{l}0 \\
0 \\
0 \\
0 \\
0\end{array}$ \\
\hline $\begin{array}{l}6-10 \\
6-11 \\
6-12 \\
6-13 \\
6-14\end{array}$ & $\begin{array}{l}52+++ \\
598 \\
1683 \\
1907 \\
7240\end{array}$ & $\begin{array}{l}0 \\
3 \\
1 \\
0 \\
0\end{array}$ & $\begin{array}{l}0 \\
3 \\
1 \\
0 \\
0\end{array}$ & $\begin{array}{l}0 \\
0 \\
0 \\
0 \\
0\end{array}$ & $\begin{array}{l}0 \\
0 \\
0 \\
0 \\
0\end{array}$ & $\begin{array}{l}0 \\
0 \\
0 \\
0 \\
0\end{array}$ & $\begin{array}{l}0 \\
0 \\
0 \\
0 \\
0\end{array}$ \\
\hline $\begin{array}{l}6-15 \\
6-16 \\
6-17 \\
6-18 \\
6-19\end{array}$ & $\begin{array}{l}2905 \\
5512 \\
5656 \\
2381 \\
1544\end{array}$ & $\begin{array}{l}0 \\
0 \\
4 \\
1 \\
1\end{array}$ & $\begin{array}{l}0 \\
0 \\
3 \\
1 \\
1\end{array}$ & $\begin{array}{l}0 \\
0 \\
1 \\
0 \\
0\end{array}$ & $\begin{array}{l}0 \\
0 \\
0 \\
0 \\
0\end{array}$ & $\begin{array}{l}0 \\
0 \\
0 \\
0 \\
0\end{array}$ & $\begin{array}{l}0 \\
0 \\
0 \\
0 \\
0\end{array}$ \\
\hline $\begin{array}{l}6-20 \\
6-21 \\
6-22 \\
6-23 \\
6-25\end{array}$ & $\begin{array}{r}6034 \\
2623 \\
2266 \\
651 \\
2859\end{array}$ & $\begin{array}{l}3 \\
4 \\
0 \\
0 \\
3\end{array}$ & $\begin{array}{l}3 \\
0 \\
0 \\
0 \\
3\end{array}$ & $\begin{array}{l}0 \\
2 \\
0 \\
0 \\
0\end{array}$ & $\begin{array}{l}0 \\
2 \\
0 \\
0 \\
0\end{array}$ & $\begin{array}{l}0 \\
0 \\
0 \\
0 \\
0\end{array}$ & $\begin{array}{l}0 \\
0 \\
0 \\
0 \\
0\end{array}$ \\
\hline $\begin{array}{l}6-27 \\
6-29\end{array}$ & $\begin{array}{l}2745 \\
2692\end{array}$ & $\begin{array}{l}3 \\
3\end{array}$ & $\frac{1}{1}$ & $\frac{2}{2}$ & $\begin{array}{l}0 \\
0\end{array}$ & $\begin{array}{l}0 \\
0\end{array}$ & $\begin{array}{l}0 \\
0\end{array}$ \\
\hline $\begin{array}{l}7-01 \\
7-03\end{array}$ & -- & $\overline{-}$ & $=$ & - & $=$ & $\overline{-}$ & $=$ \\
\hline $7-05$ & - & - & - & - & - & -- & - \\
\hline $7-07$ & -- & - & -- & -- & -- & -- & -- \\
\hline
\end{tabular}


TABLE 18.- NUMBER OF GREEN TRACER PARTICLES(1), PER 100 GRAMS OF BEDLOAD SAMPLE, COMPOSITED FROM CROSS-CHANNEL SAMPLING POSITIONS SPACED AT I-METER INTERVALS ACROSS THE CHANNEL WIDTH, EAST FORK RIVER, WYOMING, 1980

SECTION $0808(3)$

\begin{tabular}{|c|c|c|c|c|c|c|c|}
\hline \multirow{3}{*}{ DATE } & \multirow{3}{*}{$\begin{array}{c}\text { DRY } \\
\text { MASS } \\
\text { OF } \\
\text { SAMPLE (2) } \\
\text { (G) }\end{array}$} & \multicolumn{6}{|c|}{ NUMBER OF GREEN TRACER PARTICLES } \\
\hline & & \multirow[b]{2}{*}{ TOTAL } & \multicolumn{5}{|c|}{ BY SIZE CLASS (MM) } \\
\hline & & & $\begin{array}{c}0.25 \\
\text { TO } \\
0.50\end{array}$ & $\begin{array}{l}0.50 \\
\text { TO } \\
1.00\end{array}$ & $\begin{array}{l}1.00 \\
\text { TO } \\
2.00\end{array}$ & $\begin{array}{l}2.00 \\
\text { TO } \\
4.00\end{array}$ & $\begin{array}{l}4.00 \\
\text { TO } \\
8.00\end{array}$ \\
\hline $5-16$ & -- & -- & -- & - & -- & -- & -- \\
\hline $5-19$ & -- & -- & -- & -- & -- & - & -- \\
\hline $\begin{array}{l}5-21 \\
5-22\end{array}$ & $\overline{--}$ & $\overline{--}$ & $\overline{--}$ & $\overline{--}$ & $-\overline{-}$ & $\overline{-}$ & $\overline{--}$ \\
\hline $5-24$ & -- & -- & -- & -- & -- & -- & -- \\
\hline $5-26$ & -- & -- & -- & -- & -- & -- & -- \\
\hline $5-27$ & $\overline{--}$ & -- & -- & - & -- & -- & - \\
\hline $5-29$ & -- & -- & -- & - & -- & -- & -- \\
\hline $5-30$ & -- & -- & -- & -- & -- & -- & -- \\
\hline $6-01$ & -- & -- & -- & -- & -- & -- & -- \\
\hline $6-03$ & -- & -- & -- & -- & -- & -- & -- \\
\hline $\begin{array}{l}0-05 \\
6-07\end{array}$ & $=-$ & $=-$ & $=$ & $=$ & $=$ & -- & -- \\
\hline $6-09$ & $85+$ & 0 & 0 & 0 & 0 & 0 & 0 \\
\hline $\begin{array}{l}6-10 \\
6-11\end{array}$ & $\begin{array}{l}677 \\
49++\end{array}$ & $\begin{array}{l}0 \\
0\end{array}$ & $\begin{array}{l}0 \\
0\end{array}$ & $\begin{array}{l}0 \\
0\end{array}$ & $\begin{array}{l}0 \\
0\end{array}$ & $\begin{array}{l}0 \\
0\end{array}$ & $\begin{array}{l}0 \\
0\end{array}$ \\
\hline $6-13$ & 462 & 3 & 3 & 0 & 0 & 0 & 0 \\
\hline $6-14$ & 371 & $\hat{0}$ & 0 & 0 & 0 & 0 & 0 \\
\hline $6-15$ & 504 & 0 & 0 & 0 & 0 & 0 & 0 \\
\hline $\begin{array}{l}6-16 \\
6-17\end{array}$ & 103 & 0 & 0 & 0 & 0 & 0 & 0 \\
\hline $6-18$ & 726 & 0 & 0 & 0 & 0 & 0 & 0 \\
\hline $6-19$ & $34++$ & 0 & 0 & 0 & 0 & 0 & 0 \\
\hline $6-20$ & $0+++$ & 0 & 0 & 0 & 0 & 0 & 0 \\
\hline $6-21$ & $17++$ & 0 & 0 & 0 & 0 & 0 & 0 \\
\hline $\begin{array}{l}6-22 \\
6-23\end{array}$ & $\begin{array}{l}68+ \\
52+\end{array}$ & $\begin{array}{l}0 \\
0\end{array}$ & $\begin{array}{l}0 \\
0\end{array}$ & 0 & 0 & $\begin{array}{l}0 \\
0\end{array}$ & 0 \\
\hline $6-25$ & $34+++$ & 0 & 0 & 0 & 0 & 0 & 0 \\
\hline $6-27$ & 124 & 6 & 4 & 2 & 0 & 0 & 0 \\
\hline $6-29$ & -- & -- & -- & -- & -- & - & - \\
\hline $7-0 \frac{1}{3}$ & - & $=$ & - & $=$ & $=$ & - & $=$ \\
\hline $7-05$ & -- & -- & -- & -- & -- & -- & -- \\
\hline $7-07$ & -- & -- & -- & -- & -- & -- & -- \\
\hline
\end{tabular}


TABLE 18.- NUMBER OF GREEN TRACER PARTICLES(1), PER 100 GRAMS OF BEDLOAD SAMPLE, COMPOSITED FROM CROSS-CHANNEL SAMPLING POSITIONS SPACED AT 1-METER INTERVALS ACROSS THE CHANNEL WIDTH, EAST FORK RIVER, WYOMING, 1980

\section{SECTION 0853}

\begin{tabular}{|c|c|c|c|c|c|c|c|}
\hline \multicolumn{8}{|c|}{ NUMBER OF GREEN TRACER PARTICLES } \\
\hline \multirow[t]{2}{*}{ DATE } & \multirow{2}{*}{$\begin{array}{c}\text { DRY } \\
\text { MASS } \\
\text { OF } \\
\text { SAMPLE (2) } \\
\text { (G) }\end{array}$} & & \multicolumn{5}{|c|}{ BY SIZE CLASS (MM) } \\
\hline & & TOTAL & $\begin{array}{l}0.25 \\
\text { TO } \\
0.50\end{array}$ & $\begin{array}{l}0.50 \\
\text { TO } \\
1.00\end{array}$ & $\begin{array}{l}1.00 \\
2.00\end{array}$ & $\begin{array}{l}2.00 \\
\text { TO } \\
4.00\end{array}$ & $\begin{array}{l}4.00 \\
\text { TO } \\
8.00\end{array}$ \\
\hline $\begin{array}{l}5-16 \\
5-19 \\
5-21\end{array}$ & $\begin{array}{l}284 \\
459 \\
827\end{array}$ & $\begin{array}{l}0 \\
0 \\
0\end{array}$ & $\begin{array}{l}0 \\
0 \\
0\end{array}$ & $\begin{array}{l}0 \\
0 \\
0\end{array}$ & $\begin{array}{l}0 \\
0 \\
0\end{array}$ & $\begin{array}{l}0 \\
0 \\
0\end{array}$ & $\begin{array}{l}0 \\
0 \\
0\end{array}$ \\
\hline $\begin{array}{l}5-22 \\
5-24\end{array}$ & $=$ & $=$ & $=$ & $=$ & $=$ & $=$ & $=$ \\
\hline $\begin{array}{l}5-26 \\
5-27 \\
5-28\end{array}$ & $\begin{array}{r}2765 \\
834 \\
1822\end{array}$ & $\begin{array}{l}0 \\
0 \\
0\end{array}$ & $\begin{array}{l}0 \\
0 \\
0\end{array}$ & $\begin{array}{l}0 \\
0 \\
0\end{array}$ & $\begin{array}{l}0 \\
0 \\
0\end{array}$ & $\begin{array}{l}0 \\
0 \\
0\end{array}$ & $\begin{array}{l}0 \\
0 \\
0\end{array}$ \\
\hline $5-30$ & 550 & 0 & 0 & 0 & 0 & 0 & 0 \\
\hline $\begin{array}{l}6-01 \\
6-03 \\
6-05 \\
6-07 \\
6-09\end{array}$ & $\begin{array}{l}242 \\
217 \\
4+++ \\
170 \\
322\end{array}$ & $\begin{array}{l}0 \\
0 \\
0 \\
0 \\
0\end{array}$ & $\begin{array}{l}0 \\
0 \\
0 \\
0 \\
0\end{array}$ & $\begin{array}{l}0 \\
0 \\
0 \\
0 \\
0\end{array}$ & $\begin{array}{l}0 \\
0 \\
0 \\
0 \\
0\end{array}$ & $\begin{array}{l}0 \\
0 \\
0 \\
0 \\
0\end{array}$ & $\begin{array}{l}0 \\
0 \\
0 \\
0 \\
0\end{array}$ \\
\hline $\begin{array}{l}6-10 \\
6-11 \\
6-12 \\
6-13 \\
6-14\end{array}$ & $\begin{array}{r}847 \\
2219 \\
6895 \\
6089 \\
5264\end{array}$ & $\begin{array}{l}3 \\
0 \\
0 \\
0 \\
0\end{array}$ & $\begin{array}{l}1 \\
0 \\
0 \\
0 \\
0\end{array}$ & $\begin{array}{l}2 \\
0 \\
0 \\
0 \\
0\end{array}$ & $\begin{array}{l}0 \\
0 \\
0 \\
0 \\
0\end{array}$ & $\begin{array}{l}0 \\
0 \\
0 \\
0 \\
0\end{array}$ & $\begin{array}{l}0 \\
0 \\
0 \\
0 \\
0\end{array}$ \\
\hline $\begin{array}{l}6-15 \\
6-16 \\
6-17 \\
6-18 \\
6-19\end{array}$ & $\begin{array}{l}1825 \\
4440 \\
3923 \\
3082 \\
3537\end{array}$ & $\begin{array}{l}0 \\
0 \\
0 \\
0 \\
0\end{array}$ & $\begin{array}{l}0 \\
0 \\
0 \\
0 \\
0\end{array}$ & $\begin{array}{l}0 \\
0 \\
0 \\
0 \\
0\end{array}$ & $\begin{array}{l}0 \\
0 \\
0 \\
0 \\
0\end{array}$ & $\begin{array}{l}0 \\
0 \\
0 \\
0 \\
0\end{array}$ & $\begin{array}{l}0 \\
0 \\
0 \\
0 \\
0\end{array}$ \\
\hline $\begin{array}{l}6-20 \\
6-21 \\
6-22 \\
6-23 \\
6-25\end{array}$ & $\begin{array}{r}628 \\
1390 \\
3195 \\
1491 \\
711\end{array}$ & $\begin{array}{l}2 \\
0 \\
0 \\
6 \\
1\end{array}$ & $\begin{array}{l}2 \\
0 \\
0 \\
6 \\
0\end{array}$ & $\begin{array}{l}0 \\
0 \\
0 \\
0 \\
1\end{array}$ & $\begin{array}{l}0 \\
0 \\
0 \\
0 \\
0\end{array}$ & $\begin{array}{l}0 \\
0 \\
0 \\
0 \\
0\end{array}$ & $\begin{array}{l}0 \\
0 \\
0 \\
0 \\
0\end{array}$ \\
\hline $6-27$ & 2440 & 2 & 0 & 2 & 0 & 0 & 0 \\
\hline $\begin{array}{l}6-29 \\
7-01\end{array}$ & $\overline{--}$ & $\overline{-}$ & $\overline{--}$ & $\overline{-}$ & $\overline{-}$ & $\overline{--}$ & $\overline{--}$ \\
\hline $7-03$ & -- & -- & -- & -- & - & -- & -- \\
\hline $7-05$ & - & -- & -- & -- & -- & -- & -- \\
\hline $7-07$ & -- & -- & -- & -- & -- & -- & -- \\
\hline
\end{tabular}


TABLE 18.- NUMBER OF GREEN TRACER PARTICLES(1), PER 100 GRAMS OF BEDLOAD SAMPLE, COMPOSITED FROM CROSS-CHANNEL SAMPLING POSITIONS SPACED AT I-METER INTERVALS ACROSS THE CHANNEL WIDTH, EAST FORK RIVER, WYOMING, 1980

SECTION 0898

\begin{tabular}{|c|c|c|c|c|c|c|c|}
\hline \multirow{3}{*}{ DATE } & \multirow{3}{*}{$\begin{array}{c}\text { DRY } \\
\text { MASS } \\
\text { OF } \\
\text { SAMPLE (2) } \\
(G)\end{array}$} & \multicolumn{6}{|c|}{ NUMBER OF GREEN TRACER PARTICLES } \\
\hline & & \multirow[b]{2}{*}{ TOTAL } & \multicolumn{4}{|c|}{ BY SIZE CLASS (MM) } & \multirow[b]{2}{*}{$\begin{array}{l}4.00 \\
\text { TO } \\
8.00\end{array}$} \\
\hline & & & $\begin{array}{l}0.25 \\
\text { TO } \\
0.50\end{array}$ & $\begin{array}{l}0.50 \\
\text { To } \\
1.00\end{array}$ & $\begin{array}{l}1.00 \\
2.00\end{array}$ & $\begin{array}{l}2.00 \\
\text { To } \\
4.00\end{array}$ & \\
\hline $\begin{array}{l}5-16 \\
5-19 \\
5-21 \\
5-22 \\
5-24\end{array}$ & $\begin{array}{l}114++ \\
201+ \\
231 \\
- \\
-\end{array}$ & $\begin{array}{r}0 \\
0 \\
0 \\
--\end{array}$ & $\begin{array}{r}0 \\
0 \\
0 \\
-- \\
--\end{array}$ & $\begin{array}{r}0 \\
0 \\
0 \\
-- \\
--\end{array}$ & $\begin{array}{r}0 \\
0 \\
0 \\
-- \\
--\end{array}$ & $\begin{array}{r}0 \\
0 \\
0 \\
--\end{array}$ & $\begin{array}{r}0 \\
0 \\
0 \\
-- \\
--\end{array}$ \\
\hline $\begin{array}{l}5-26 \\
5-27 \\
5-28 \\
5-29\end{array}$ & $\begin{array}{r}1516 \\
1853 \\
251 \\
-\end{array}$ & $\begin{array}{l}0 \\
0 \\
0 \\
-\end{array}$ & $\begin{array}{l}0 \\
0 \\
0 \\
-\end{array}$ & $\begin{array}{l}0 \\
0 \\
0 \\
-\end{array}$ & $\begin{array}{l}0 \\
0 \\
0 \\
-\end{array}$ & $\begin{array}{r}0 \\
0 \\
0 \\
-\end{array}$ & $\begin{array}{l}0 \\
0 \\
0 \\
-\end{array}$ \\
\hline $5-30$ & 290 & 0 & 0 & 0 & 0 & 0 & 0 \\
\hline $\begin{array}{l}6-01 \\
6-03 \\
6-05 \\
6-07 \\
6-09\end{array}$ & $\begin{array}{c}332 \\
46++ \\
46++ \\
34++ \\
203\end{array}$ & $\begin{array}{l}1 \\
0 \\
0 \\
0 \\
5\end{array}$ & $\begin{array}{l}1 \\
0 \\
0 \\
0 \\
4\end{array}$ & $\begin{array}{l}0 \\
0 \\
0 \\
0 \\
1\end{array}$ & $\begin{array}{l}0 \\
0 \\
0 \\
0 \\
0\end{array}$ & $\begin{array}{l}0 \\
0 \\
0 \\
0 \\
0\end{array}$ & $\begin{array}{l}0 \\
0 \\
0 \\
0 \\
0\end{array}$ \\
\hline $\begin{array}{l}6-10 \\
6-11 \\
6-12 \\
6-13 \\
6-14\end{array}$ & $\begin{array}{r}433 \\
180 \\
1483 \\
1925 \\
6873\end{array}$ & $\begin{array}{l}3 \\
5 \\
0 \\
0 \\
0\end{array}$ & $\begin{array}{l}3 \\
4 \\
0 \\
0 \\
0\end{array}$ & $\begin{array}{l}0 \\
1 \\
0 \\
0 \\
0\end{array}$ & $\begin{array}{l}0 \\
0 \\
0 \\
0 \\
0\end{array}$ & $\begin{array}{l}0 \\
0 \\
0 \\
0 \\
0\end{array}$ & $\begin{array}{l}0 \\
0 \\
0 \\
0 \\
0\end{array}$ \\
\hline $6-15$ & 408 & 0 & 0 & 0 & 0 & 0 & 0 \\
\hline $\begin{array}{l}6-17 \\
6-18 \\
6-19\end{array}$ & $\begin{array}{l}3645 \\
2807 \\
1207\end{array}$ & $\begin{array}{l}0 \\
0 \\
0\end{array}$ & $\begin{array}{l}0 \\
0 \\
0\end{array}$ & $\begin{array}{l}0 \\
0 \\
0\end{array}$ & $\begin{array}{l}0 \\
0 \\
0\end{array}$ & $\begin{array}{l}0 \\
0 \\
0\end{array}$ & $\begin{array}{l}0 \\
0 \\
0\end{array}$ \\
\hline $\begin{array}{l}6-20 \\
6-21 \\
6-22 \\
6-23 \\
6-25\end{array}$ & $\begin{array}{r}5563 \\
245 \\
170 \\
1841 \\
585\end{array}$ & $\begin{array}{r}0 \\
0 \\
13 \\
5 \\
3\end{array}$ & $\begin{array}{r}0 \\
0 \\
12 \\
3 \\
2\end{array}$ & $\begin{array}{l}0 \\
0 \\
1 \\
1 \\
1\end{array}$ & $\begin{array}{l}0 \\
0 \\
0 \\
1 \\
0\end{array}$ & $\begin{array}{l}0 \\
0 \\
0 \\
0 \\
0\end{array}$ & $\begin{array}{l}0 \\
0 \\
0 \\
0 \\
0\end{array}$ \\
\hline $6-27$ & 737 & 7 & 2 & 5 & 0 & 0 & 0 \\
\hline $\begin{array}{l}6-29 \\
7-01\end{array}$ & $\overline{-}$ & $\overline{-}$ & $\overline{-}$ & $\overline{-}$ & $\overline{-}$ & $\overline{-}$ & $\overline{-}$ \\
\hline $7-0 \overline{3}$ & -- & -- & - & - & - & -- & -- \\
\hline I-US & -- & -- & -- & -- & -- & - & -- \\
\hline $7-07$ & -- & -- & -- & -- & -- & - & - \\
\hline
\end{tabular}


TABLE 18.- NUMBER OF GREEN TRACER PARTICLES(1), PER 100 GRAMS OF BEDLOAD SAMPLE, COMPOSITED FROM CROSS-CHANNEL SAMPLING POSITIONS SPACED AT 1-METER INTERVALS ACROSS THE CHANNEL WIDTH, EAST FORK RIVER, WYOMING, 1980

SECTION 0940

\begin{tabular}{|c|c|c|c|c|c|c|c|}
\hline \multirow{3}{*}{ DATE } & \multirow{3}{*}{$\begin{array}{c}\text { DRY } \\
\text { MASS } \\
\text { OF } \\
\text { SAMPLE(2) } \\
\text { (G) }\end{array}$} & \multicolumn{6}{|c|}{ NUMBER OF GREEN TRACER PARTICLES } \\
\hline & & \multirow[b]{2}{*}{ TOTAL } & \multicolumn{5}{|c|}{ BY SIZE CLASS (MM) } \\
\hline & & & $\begin{array}{c}0.25 \\
\text { TO } \\
0.50\end{array}$ & $\begin{array}{l}0.50 \\
1.00\end{array}$ & $\begin{array}{l}1.00 \\
2.00\end{array}$ & $\begin{array}{l}2.00 \\
\text { TO } \\
4.00\end{array}$ & $\begin{array}{l}4.00 \\
\text { TO } \\
8.00\end{array}$ \\
\hline $\begin{array}{l}5-16 \\
5-19 \\
5-21 \\
5-22 \\
5-24\end{array}$ & $\begin{array}{c}412 \\
113+ \\
1047 \\
=-\end{array}$ & $\begin{array}{r}0 \\
0 \\
0 \\
-- \\
--\end{array}$ & $\begin{array}{r}0 \\
0 \\
0 \\
--\end{array}$ & $\begin{array}{r}0 \\
0 \\
0 \\
-- \\
--\end{array}$ & $\begin{array}{r}0 \\
0 \\
0 \\
-- \\
--\end{array}$ & $\begin{array}{r}0 \\
0 \\
0 \\
-- \\
--\end{array}$ & $\begin{array}{r}0 \\
0 \\
0 \\
-- \\
--\end{array}$ \\
\hline $\begin{array}{l}5-26 \\
5-27 \\
5-28 \\
5-29 \\
5-30\end{array}$ & $\begin{array}{c}3564 \\
1729 \\
803 \\
43++ \\
89+\end{array}$ & $\begin{array}{l}0 \\
0 \\
0 \\
0 \\
0\end{array}$ & $\begin{array}{l}0 \\
0 \\
0 \\
0 \\
0\end{array}$ & $\begin{array}{l}0 \\
0 \\
0 \\
0 \\
0\end{array}$ & $\begin{array}{l}0 \\
0 \\
0 \\
0 \\
0\end{array}$ & $\begin{array}{l}0 \\
0 \\
0 \\
0 \\
0\end{array}$ & $\begin{array}{l}0 \\
0 \\
0 \\
0 \\
0\end{array}$ \\
\hline $\begin{array}{l}6-01 \\
6-03 \\
6-05 \\
6-07 \\
6-09\end{array}$ & $\begin{array}{c}108+ \\
73++ \\
73+ \\
134 \\
555\end{array}$ & $\begin{array}{l}0 \\
0 \\
0 \\
0 \\
3\end{array}$ & $\begin{array}{l}0 \\
0 \\
0 \\
0 \\
3\end{array}$ & $\begin{array}{l}0 \\
0 \\
0 \\
0 \\
0\end{array}$ & $\begin{array}{l}0 \\
0 \\
0 \\
0 \\
0\end{array}$ & $\begin{array}{l}0 \\
0 \\
0 \\
0 \\
0\end{array}$ & $\begin{array}{l}0 \\
0 \\
0 \\
0 \\
0\end{array}$ \\
\hline $\begin{array}{l}6-10 \\
6-11 \\
6-12 \\
6-13 \\
6-14\end{array}$ & $\begin{array}{r}714 \\
5615 \\
6832 \\
5258 \\
2459\end{array}$ & $\begin{array}{l}0 \\
0 \\
0 \\
1 \\
0\end{array}$ & $\begin{array}{l}0 \\
0 \\
0 \\
1 \\
0\end{array}$ & $\begin{array}{l}0 \\
0 \\
0 \\
0 \\
0\end{array}$ & $\begin{array}{l}0 \\
0 \\
0 \\
0 \\
0\end{array}$ & $\begin{array}{l}0 \\
0 \\
0 \\
0 \\
0\end{array}$ & $\begin{array}{l}0 \\
0 \\
0 \\
0 \\
0\end{array}$ \\
\hline $6-15$ & 2313 & 0 & 0 & 0 & 0 & 0 & 0 \\
\hline $\begin{array}{l}6-17 \\
6-18 \\
6-19\end{array}$ & $\begin{array}{l}1205 \\
1785 \\
1143\end{array}$ & $\begin{array}{l}0 \\
0 \\
1\end{array}$ & $\begin{array}{l}0 \\
0 \\
1\end{array}$ & $\begin{array}{l}0 \\
0 \\
0\end{array}$ & $\begin{array}{l}0 \\
0 \\
0\end{array}$ & $\begin{array}{l}0 \\
0 \\
0\end{array}$ & $\begin{array}{l}0 \\
0 \\
0\end{array}$ \\
\hline $\begin{array}{l}6-20 \\
6-21 \\
6-22 \\
6-23 \\
6-25\end{array}$ & $\begin{array}{l}1735 \\
6301 \\
2855 \\
3197 \\
1877\end{array}$ & $\begin{array}{l}0 \\
1 \\
7 \\
0 \\
0\end{array}$ & $\begin{array}{l}0 \\
1 \\
7 \\
0 \\
0\end{array}$ & $\begin{array}{l}0 \\
0 \\
0 \\
0 \\
0\end{array}$ & $\begin{array}{l}0 \\
0 \\
0 \\
0 \\
0\end{array}$ & $\begin{array}{l}0 \\
0 \\
0 \\
0 \\
0\end{array}$ & $\begin{array}{l}0 \\
0 \\
0 \\
0 \\
0\end{array}$ \\
\hline $\begin{array}{l}6-27 \\
6-29 \\
7-01 \\
7-03 \\
7-05\end{array}$ & $\begin{array}{r}1569 \\
=- \\
=-\end{array}$ & $\begin{array}{l}2 \\
=- \\
=- \\
--\end{array}$ & $\begin{array}{l}0 \\
-- \\
--\end{array}$ & $\begin{array}{l}2 \\
-- \\
--\end{array}$ & $\begin{array}{l}0 \\
=- \\
=-\end{array}$ & $\begin{array}{l}0 \\
=- \\
=-\end{array}$ & $\begin{array}{l}0 \\
=- \\
=-\end{array}$ \\
\hline $7-07$ & -- & -- & -- & -- & -- & -- & -- \\
\hline
\end{tabular}


TABLE 18.- NUMBER OF GREEN TRACER PARTICLES(1), PER 100 GRAMS OF BEDLOAD SAMPLE, COMPOSITED FROM CROSS-CHANNEL SAMPLING POSITIONS SPACED AT 1-METER INTERVALS ACROSS THE CHANNEL WIDTH, EAST FORK RIVER, WYOMING, 1980

\section{SECTION 0985}

\begin{tabular}{|c|c|c|c|c|c|c|c|c|}
\hline \multirow{3}{*}{\multicolumn{2}{|c|}{$\begin{array}{lc}\text { DATE } & \text { DRY } \\
& \text { MASS } \\
& \text { OF } \\
& \text { SAMPLE (2) } \\
& (\mathrm{G}) \\
& -\end{array}$}} & & \multicolumn{6}{|c|}{ NUMBER OF GREEN TRACER PARTICLES } \\
\hline & & & \multirow[b]{2}{*}{ TOTAL } & \multicolumn{4}{|c|}{ BY SIZE CLASS (MM) } & \multirow[b]{2}{*}{$\begin{array}{r}4.00 \\
\text { TO } \\
8.00\end{array}$} \\
\hline & & & & $\begin{array}{l}0.25 \\
\mathrm{TO} \\
0.50\end{array}$ & $\begin{array}{l}0.50 \\
\text { To } \\
1.00\end{array}$ & $\begin{array}{l}1.00 \\
\text { TO } \\
2.00\end{array}$ & $\begin{array}{l}2.00 \\
\text { TO } \\
4.00\end{array}$ & \\
\hline $\begin{array}{l}5-16 \\
5-19 \\
5-21 \\
5-22 \\
5-24\end{array}$ & $\begin{array}{r}344 \\
323 \\
2789 \\
-- \\
--\end{array}$ & & $\begin{array}{r}0 \\
0 \\
0 \\
--\end{array}$ & $\begin{array}{r}0 \\
0 \\
0 \\
--\end{array}$ & $\begin{array}{r}0 \\
0 \\
0 \\
-- \\
--\end{array}$ & $\begin{array}{r}0 \\
0 \\
0 \\
-- \\
--\end{array}$ & $\begin{array}{r}0 \\
0 \\
0 \\
-- \\
--\end{array}$ & $\begin{array}{r}0 \\
0 \\
0 \\
-- \\
--\end{array}$ \\
\hline $\begin{array}{l}5-26 \\
5-27 \\
5-28 \\
5-29\end{array}$ & $\begin{array}{c}3289 \\
73+ \\
105+ \\
--\end{array}$ & & $\begin{array}{r}0 \\
0 \\
0 \\
--\end{array}$ & $\begin{array}{r}0 \\
0 \\
0 \\
--\end{array}$ & $\begin{array}{r}0 \\
0 \\
0 \\
-0\end{array}$ & $\begin{array}{r}0 \\
0 \\
0 \\
-0\end{array}$ & $\begin{array}{r}0 \\
0 \\
0 \\
--\end{array}$ & $\begin{array}{r}0 \\
0 \\
0 \\
-0\end{array}$ \\
\hline $5-30$ & $10++$ & & 0 & 0 & 0 & 0 & 0 & 0 \\
\hline $\begin{array}{l}6-01 \\
6-03 \\
6-05 \\
6-07 \\
6-09\end{array}$ & $\begin{array}{c}5+++ \\
39++ \\
19+++ \\
50++ \\
1844\end{array}$ & & $\begin{array}{l}0 \\
0 \\
0 \\
0 \\
0\end{array}$ & $\begin{array}{l}0 \\
0 \\
0 \\
0 \\
0\end{array}$ & $\begin{array}{l}0 \\
0 \\
0 \\
0 \\
0\end{array}$ & $\begin{array}{l}0 \\
0 \\
0 \\
0 \\
0\end{array}$ & $\begin{array}{l}0 \\
0 \\
0 \\
0 \\
0\end{array}$ & $\begin{array}{l}0 \\
0 \\
0 \\
0 \\
0\end{array}$ \\
\hline $\begin{array}{l}6-10 \\
6-11 \\
6-12 \\
6-13 \\
6-14\end{array}$ & $\begin{array}{l}5658 \\
3740 \\
4765 \\
8303 \\
7534\end{array}$ & o & $\begin{array}{l}0 \\
0 \\
0 \\
0 \\
0\end{array}$ & $\begin{array}{l}0 \\
0 \\
0 \\
0 \\
0\end{array}$ & $\begin{array}{l}0 \\
0 \\
0 \\
0 \\
0\end{array}$ & $\begin{array}{l}0 \\
0 \\
0 \\
0 \\
0\end{array}$ & $\begin{array}{l}0 \\
0 \\
0 \\
0 \\
0\end{array}$ & $\begin{array}{l}0 \\
0 \\
0 \\
0 \\
0\end{array}$ \\
\hline $6-15$ & 3829 & & 0 & 0 & 0 & $\underline{0}$ & 0 & 0 \\
\hline $\begin{array}{l}6-17 \\
6-18 \\
6-19\end{array}$ & $\begin{array}{r}954 \\
3865 \\
1696\end{array}$ & & $\begin{array}{l}0 \\
0 \\
0\end{array}$ & $\begin{array}{l}0 \\
0 \\
0\end{array}$ & $\begin{array}{l}0 \\
0 \\
0\end{array}$ & $\begin{array}{l}0 \\
0 \\
0\end{array}$ & $\begin{array}{l}0 \\
0 \\
0\end{array}$ & $\begin{array}{l}0 \\
0 \\
0\end{array}$ \\
\hline $\begin{array}{l}6-20 \\
6-21 \\
6-22 \\
6-23 \\
6-25\end{array}$ & $\begin{array}{r}1955 \\
10233 \\
2284 \\
3294 \\
2383\end{array}$ & & $\begin{array}{r}18 \\
4 \\
7 \\
3 \\
7\end{array}$ & $\begin{array}{r}13 \\
4 \\
4 \\
0 \\
6\end{array}$ & $\begin{array}{l}5 \\
0 \\
3 \\
3 \\
1\end{array}$ & $\begin{array}{l}0 \\
0 \\
0 \\
0 \\
0\end{array}$ & $\begin{array}{l}0 \\
0 \\
0 \\
0 \\
0\end{array}$ & $\begin{array}{l}0 \\
0 \\
0 \\
0 \\
0\end{array}$ \\
\hline $6-27$ & 2130 & & 15 & 5 & 10 & 0 & 0 & 0 \\
\hline $\begin{array}{l}0-29 \\
7-01\end{array}$ & $-\overline{-}$ & & $-\overline{-}$ & -- & $\overline{--}$ & $\overline{--}$ & - & $\overline{--}$ \\
\hline $7-0 \overline{3}$ & -- & & -- & -- & -- & -- & -- & -- \\
\hline $7-05$ & -- & & -- & -- & -- & -- & -- & -- \\
\hline $7-07$ & -- & & -- & -- & - & -- & -- & -- \\
\hline
\end{tabular}


TABLE 18.- NUMBER OF GREEN TRACER PARTICLES(1), PER 100 GRAMS OF BEDLOAD SAMPLE, COMPOSITED FROM CROSS-CHANNEL SAMPLING POSITIONS SPACED AT 1-METER INTERVALS ACROSS THE CHANNEL WIDTH, EAST FORK RIVER, WYOMING, 1980

SECTION 1038

\begin{tabular}{|c|c|c|c|c|c|c|c|}
\hline \multirow{3}{*}{\multicolumn{2}{|c|}{ 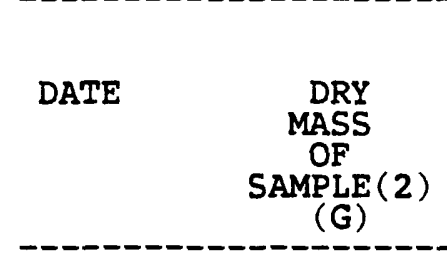 }} & \multicolumn{6}{|c|}{ NUMBER OF GREEN TRACER PARTICLES } \\
\hline & & \multirow[b]{2}{*}{ TOTAL } & \multicolumn{5}{|c|}{ BY SIZE CLASS (MM) } \\
\hline & & & $\begin{array}{l}0.25 \\
\text { TO } \\
0.50\end{array}$ & $\begin{array}{l}0.50 \\
\text { TO. } \\
1.00\end{array}$ & $\begin{array}{l}1.00 \\
2.00 \\
\text { TO }\end{array}$ & $\begin{array}{l}2.00 \\
\text { TO } \\
4.00\end{array}$ & $\begin{array}{c}4.00 \\
\text { TO } \\
8.00\end{array}$ \\
\hline $\begin{array}{l}5-16 \\
5-19 \\
5-21 \\
5-22 \\
5-24\end{array}$ & $\begin{array}{c}153+ \\
700 \\
3042 \\
2365 \\
4864\end{array}$ & $\begin{array}{l}0 \\
0 \\
0 \\
0 \\
0\end{array}$ & $\begin{array}{l}0 \\
0 \\
0 \\
0 \\
0\end{array}$ & $\begin{array}{l}0 \\
0 \\
0 \\
0 \\
0\end{array}$ & $\begin{array}{l}0 \\
0 \\
0 \\
0 \\
0\end{array}$ & $\begin{array}{l}0 \\
0 \\
0 \\
0 \\
0\end{array}$ & $\begin{array}{l}0 \\
0 \\
0 \\
0 \\
0\end{array}$ \\
\hline $\begin{array}{l}5-26 \\
5-27 \\
5-28\end{array}$ & $\begin{array}{c}1 \overline{8}+ \\
18++\end{array}$ & $\begin{array}{r}-\overline{0} \\
0\end{array}$ & $\begin{array}{l}-\overline{0} \\
0\end{array}$ & $\begin{array}{r}--\overline{0} \\
0\end{array}$ & $\begin{array}{r}-\overline{0} \\
0\end{array}$ & $\begin{array}{r}-\overline{0} \\
0\end{array}$ & $\begin{array}{c}-- \\
0\end{array}$ \\
\hline $5-30$ & $26++$ & 0 & 0 & 0 & 0 & 0 & 0 \\
\hline $\begin{array}{l}6-01 \\
6-03 \\
6-05 \\
6-07 \\
6-09\end{array}$ & $\begin{array}{r}5+++ \\
17+++ \\
0+++ \\
0+++ \\
1865\end{array}$ & $\begin{array}{l}0 \\
0 \\
0 \\
0 \\
0\end{array}$ & $\begin{array}{l}0 \\
0 \\
0 \\
0 \\
0\end{array}$ & $\begin{array}{l}0 \\
0 \\
0 \\
0 \\
0\end{array}$ & $\begin{array}{l}0 \\
0 \\
0 \\
0 \\
0\end{array}$ & $\begin{array}{l}0 \\
0 \\
0 \\
0 \\
0\end{array}$ & $\begin{array}{l}0 \\
0 \\
0 \\
0 \\
0\end{array}$ \\
\hline $\begin{array}{l}6-10 \\
6-11 \\
6-12 \\
6-13 \\
6-14\end{array}$ & $\begin{array}{r}6434 \\
7545 \\
12301 \\
1950 \\
2923\end{array}$ & $\begin{array}{l}0 \\
0 \\
0 \\
0 \\
0\end{array}$ & $\begin{array}{l}0 \\
0 \\
0 \\
0 \\
0\end{array}$ & $\begin{array}{l}0 \\
0 \\
0 \\
0 \\
0\end{array}$ & $\begin{array}{l}0 \\
0 \\
0 \\
0 \\
0\end{array}$ & $\begin{array}{l}0 \\
0 \\
0 \\
0 \\
0\end{array}$ & $\begin{array}{l}0 \\
0 \\
0 \\
0 \\
0\end{array}$ \\
\hline $6-15$ & 238 & 0 & 0 & 0 & 0 & 0 & 0 \\
\hline $\begin{array}{l}6-17 \\
6-18 \\
6-19\end{array}$ & $\begin{array}{r}468 \\
1679 \\
249\end{array}$ & $\begin{array}{l}4 \\
3 \\
2\end{array}$ & $\begin{array}{l}4 \\
0 \\
0\end{array}$ & $\begin{array}{l}0 \\
0 \\
2\end{array}$ & $\begin{array}{l}0 \\
3 \\
0\end{array}$ & $\begin{array}{l}0 \\
0 \\
0\end{array}$ & $\begin{array}{l}0 \\
0 \\
0\end{array}$ \\
\hline $\begin{array}{l}6-20 \\
6-21 \\
6-22 \\
6-23 \\
6-25\end{array}$ & $\begin{array}{r}1361 \\
2079 \\
1187 \\
2267 \\
809\end{array}$ & $\begin{array}{r}3 \\
3 \\
17 \\
12 \\
12\end{array}$ & $\begin{array}{r}\frac{1}{2} \\
\frac{11}{11} \\
6\end{array}$ & $\begin{array}{l}2 \\
1 \\
6 \\
1 \\
6\end{array}$ & $\begin{array}{l}0 \\
0 \\
0 \\
0 \\
0\end{array}$ & $\begin{array}{l}0 \\
0 \\
0 \\
0 \\
0\end{array}$ & $\begin{array}{l}0 \\
0 \\
0 \\
0 \\
0\end{array}$ \\
\hline $6-27$ & 2291 & 11 & 1 & 9 & 1 & 0 & 0 \\
\hline $\begin{array}{l}0-2 y \\
7-01\end{array}$ & - & - & - & - & - & - & - \\
\hline $\begin{array}{l}7-03 \\
7-05\end{array}$ & $\overline{--}$ & $\overline{-}$ & $\overline{--}$ & $\overline{-}$ & $\overline{-}$ & $\overline{-}$ & $=$ \\
\hline $7-07$ & -- & -- & -- & -- & -- & -- & -- \\
\hline
\end{tabular}


TABLE 18.- NUMBER OF GREEN TRACER PARTICLES(1), PER 100 GRAMS OF BEDLOAD SAMPLE, COMPOSITED FROM CROSS-CHANNEL SAMPLING POSITIONS SPACED AT I-METER INTERVALS ACROSS THE CHANNEL WIDTH, EAST FORK RIVER, WYOMING, 1980

SECTION 1077

\begin{tabular}{|c|c|c|c|c|c|c|c|}
\hline \multirow{3}{*}{ DATE } & \multirow{3}{*}{$\begin{array}{c}\text { DRY } \\
\text { MASS } \\
\text { OF } \\
\text { SAMPLE (2) } \\
\text { (G) }\end{array}$} & \multicolumn{6}{|c|}{ NUMBER OF GREEN TRACER PARTICLES } \\
\hline & & \multirow[b]{2}{*}{ TOTAL } & \multicolumn{5}{|c|}{ BY SIZE CLASS (MM) } \\
\hline & & & $\begin{array}{l}0.25 \\
\text { To } \\
0.50\end{array}$ & $\begin{array}{l}0.50 \\
\text { TO } \\
1.00\end{array}$ & $\begin{array}{l}1.00 \\
2.00\end{array}$ & $\begin{array}{l}2.00 \\
\text { TO } \\
4.00\end{array}$ & $\begin{array}{l}4.00 \\
\text { TO } \\
8.00\end{array}$ \\
\hline $\begin{array}{l}5-16 \\
5-19 \\
5-21 \\
5-22 \\
5-24\end{array}$ & $\begin{array}{l}488 \\
141+ \\
1036 \\
6690 \\
2093\end{array}$ & $\begin{array}{l}0 \\
0 \\
0 \\
0 \\
0\end{array}$ & $\begin{array}{l}0 \\
0 \\
0 \\
0 \\
0\end{array}$ & $\begin{array}{l}0 \\
0 \\
0 \\
0 \\
0\end{array}$ & $\begin{array}{l}0 \\
0 \\
0 \\
0 \\
0\end{array}$ & $\begin{array}{l}0 \\
0 \\
0 \\
0 \\
0\end{array}$ & $\begin{array}{l}0 \\
0 \\
0 \\
0 \\
0\end{array}$ \\
\hline $\begin{array}{l}5-26 \\
5-27 \\
5-28 \\
5-29 \\
5-30\end{array}$ & $\begin{array}{c}\overline{6 \overline{6}+} \\
24 \overline{7} \\
\overline{93+}\end{array}$ & $\begin{array}{r}-\overline{0} \\
0 \\
-0\end{array}$ & $\begin{array}{r}--\overline{0} \\
-\frac{1}{0}\end{array}$ & $\begin{array}{r}--\overline{0} \\
0 \\
-0\end{array}$ & $\begin{array}{r}--\overline{0} \\
-\frac{0}{0}\end{array}$ & $\begin{array}{r}-- \\
0 \\
-\frac{0}{0}\end{array}$ & $\begin{array}{r}-- \\
0 \\
-0\end{array}$ \\
\hline $\begin{array}{l}6-01 \\
6-03 \\
6-05 \\
6-07 \\
6-09\end{array}$ & $\begin{array}{l}368^{4+++} \\
443 \\
861 \\
6847\end{array}$ & $\begin{array}{l}0 \\
0 \\
0 \\
0 \\
1\end{array}$ & $\begin{array}{l}0 \\
0 \\
0 \\
0 \\
1\end{array}$ & $\begin{array}{l}0 \\
0 \\
0 \\
0 \\
0\end{array}$ & $\begin{array}{l}0 \\
0 \\
0 \\
0 \\
0\end{array}$ & $\begin{array}{l}0 \\
0 \\
0 \\
0 \\
0\end{array}$ & $\begin{array}{l}0 \\
0 \\
0 \\
0 \\
0\end{array}$ \\
\hline $\begin{array}{l}6-10 \\
6-11 \\
6-12 \\
6-13 \\
6-14\end{array}$ & $\begin{array}{l}5686 \\
7026 \\
5955 \\
4795 \\
3167\end{array}$ & $\begin{array}{l}0 \\
0 \\
1 \\
0 \\
0\end{array}$ & $\begin{array}{l}0 \\
0 \\
1 \\
0 \\
0\end{array}$ & $\begin{array}{l}0 \\
0 \\
0 \\
0 \\
0\end{array}$ & $\begin{array}{l}0 \\
0 \\
0 \\
0 \\
0\end{array}$ & $\begin{array}{l}0 \\
0 \\
0 \\
0 \\
0\end{array}$ & $\begin{array}{l}0 \\
0 \\
0 \\
0 \\
0\end{array}$ \\
\hline $6-15$ & 1830 & 1 & 1 & 0 & 0 & 0 & 0 \\
\hline $\begin{array}{l}6-17 \\
6-18 \\
6-19\end{array}$ & $\begin{array}{l}1193 \\
3263 \\
4561\end{array}$ & $\begin{array}{l}1 \\
0 \\
0\end{array}$ & $\begin{array}{l}1 \\
0 \\
0\end{array}$ & $\begin{array}{l}0 \\
0 \\
0\end{array}$ & $\begin{array}{l}0 \\
0 \\
0\end{array}$ & $\begin{array}{l}0 \\
0 \\
0\end{array}$ & $\begin{array}{l}0 \\
0 \\
0\end{array}$ \\
\hline $\begin{array}{l}6-20 \\
6-21\end{array}$ & $\begin{array}{l}5961 \\
2481\end{array}$ & $\begin{array}{r}10 \\
9\end{array}$ & $\begin{array}{l}6 \\
6\end{array}$ & $\frac{4}{3}$ & $\begin{array}{l}0 \\
0\end{array}$ & $\begin{array}{l}0 \\
0\end{array}$ & $\begin{array}{l}0 \\
0\end{array}$ \\
\hline $\begin{array}{l}6-22 \\
6-23 \\
6-25\end{array}$ & $\begin{array}{r}5700 \\
878\end{array}$ & $\begin{array}{l}10 \\
12\end{array}$ & 4 & $\begin{array}{r}6 \\
10\end{array}$ & $\begin{array}{l}0 \\
1\end{array}$ & $\begin{array}{l}0 \\
0\end{array}$ & $\begin{array}{l}0 \\
0\end{array}$ \\
\hline $6-27$ & 2988 & 10 & 3 & 6 & 0 & 1 & 0 \\
\hline $0-29$ & $=$ & $=$ & - & $=$ & $=$ & $=$ & $=$ \\
\hline $7-03$ & -- & -- & -- & -- & -- & -- & -- \\
\hline $7-05$ & -- & -- & -- & - & -- & -- & -- \\
\hline $7-07$ & -- & -- & -- & -- & -- & -- & -- \\
\hline
\end{tabular}


TABLE 18.- NUMBER OF GREEN TRACER PARTICLES(1), PER 100 GRAMS OF BEDLOAD SAMPLE, COMPOSITED FROM CROSS-CHANNEL SAMPLING POSITIONS SPACED AT I-METER INTERVALS ACROSS THE CHANNEL WIDTH, EAST FORK RIVER, WYOMING, 1980

SECTION 112.0

\begin{tabular}{|c|c|c|c|c|c|c|c|}
\hline \multirow{3}{*}{ DATE } & \multirow{3}{*}{$\begin{array}{c}\text { DRY } \\
\text { MASS } \\
\text { OF } \\
\text { SAMPLE (2) } \\
\text { (G) }\end{array}$} & \multicolumn{6}{|c|}{ NUMBER OF GREEN TRACER PARTICLES } \\
\hline & & \multirow[b]{2}{*}{ TOTAL } & \multicolumn{5}{|c|}{ BY SIZE CLASS (MM) } \\
\hline & & & $\begin{array}{c}0.25 \\
\text { TO } \\
0.50\end{array}$ & $\begin{array}{l}0.50 \\
\text { TO } \\
1.00\end{array}$ & $\begin{array}{l}1.00 \\
\text { TO } \\
2.00\end{array}$ & $\begin{array}{l}2.00 \\
\text { TO } \\
4.00\end{array}$ & $\begin{array}{l}4.00 \\
\text { TO } \\
8.00\end{array}$ \\
\hline $\begin{array}{l}5-16 \\
5-19 \\
5-21 \\
5-22 \\
5-24\end{array}$ & $\begin{array}{c}72+ \\
273+ \\
3540 \\
5580 \\
2052\end{array}$ & $\begin{array}{l}0 \\
0 \\
0 \\
0 \\
0\end{array}$ & $\begin{array}{l}0 \\
0 \\
0 \\
0 \\
0\end{array}$ & $\begin{array}{l}0 \\
0 \\
0 \\
0 \\
0\end{array}$ & $\begin{array}{l}0 \\
0 \\
0 \\
0 \\
0\end{array}$ & $\begin{array}{l}0 \\
0 \\
0 \\
0 \\
0\end{array}$ & $\begin{array}{l}0 \\
0 \\
0 \\
0 \\
0\end{array}$ \\
\hline $\begin{array}{l}5-26 \\
5-27 \\
5-28\end{array}$ & $\begin{array}{l}3 \overline{0} \\
27 \overline{3}\end{array}$ & $\begin{array}{r}-\overline{0} \\
0\end{array}$ & $\begin{array}{r}--\overline{0} \\
0\end{array}$ & $\begin{array}{r}-\overline{0} \\
0\end{array}$ & $\begin{array}{r}-\overline{0} \\
0\end{array}$ & $\begin{array}{r}--\overline{0} \\
0\end{array}$ & $\begin{array}{r}--\overline{0} \\
0\end{array}$ \\
\hline $5-30$ & $103+$ & 0 & 0 & 0 & 0 & 0 & 0 \\
\hline $\begin{array}{l}6-01 \\
6-03 \\
6-05 \\
6-07 \\
6-09\end{array}$ & $\begin{array}{r}77++ \\
84++ \\
90+ \\
1350 \\
6295\end{array}$ & $\begin{array}{l}0 \\
0 \\
0 \\
0 \\
0\end{array}$ & $\begin{array}{l}0 \\
0 \\
0 \\
0 \\
0\end{array}$ & $\begin{array}{l}0 \\
0 \\
0 \\
0 \\
0\end{array}$ & $\begin{array}{l}0 \\
0 \\
0 \\
0 \\
0\end{array}$ & $\begin{array}{l}0 \\
0 \\
0 \\
0 \\
0\end{array}$ & $\begin{array}{l}0 \\
0 \\
0 \\
0 \\
0\end{array}$ \\
\hline $\begin{array}{l}6-10 \\
6-11 \\
6-12 \\
6-13 \\
6-14\end{array}$ & $\begin{array}{l}5355 \\
2111 \\
1473 \\
537 \\
1581\end{array}$ & $\begin{array}{l}0 \\
3 \\
0 \\
0 \\
0\end{array}$ & $\begin{array}{l}0 \\
3 \\
0 \\
0 \\
0\end{array}$ & $\begin{array}{l}0 \\
0 \\
0 \\
0 \\
0\end{array}$ & $\begin{array}{l}0 \\
0 \\
0 \\
0 \\
0\end{array}$ & $\begin{array}{l}0 \\
0 \\
0 \\
0 \\
0\end{array}$ & $\begin{array}{l}0 \\
0 \\
0 \\
0 \\
0\end{array}$ \\
\hline $6-15$ & 2074 & 0 & 0 & 0 & 0 & 0 & 0 \\
\hline $\begin{array}{l}0-10 \\
6-17 \\
6-18 \\
6-19\end{array}$ & $\begin{array}{l}1501 \\
4293 \\
2789\end{array}$ & $\begin{array}{r}4 \\
12\end{array}$ & $\begin{array}{r}4 \\
10\end{array}$ & $\begin{array}{l}0 \\
3 \\
2\end{array}$ & $\begin{array}{l}0 \\
0 \\
0\end{array}$ & $\begin{array}{l}0 \\
0 \\
0\end{array}$ & $\begin{array}{l}0 \\
0 \\
0\end{array}$ \\
\hline $\begin{array}{l}6-20 \\
6-21\end{array}$ & $\begin{array}{l}3904 \\
2718\end{array}$ & $\begin{array}{l}20 \\
13\end{array}$ & $\begin{array}{l}9 \\
6\end{array}$ & $1 \frac{1}{7}$ & $\begin{array}{l}0 \\
0\end{array}$ & $\begin{array}{l}0 \\
0\end{array}$ & $\begin{array}{l}0 \\
0\end{array}$ \\
\hline $\begin{array}{l}6-23 \\
6-25\end{array}$ & $\begin{array}{l}2504 \\
2506\end{array}$ & $\begin{array}{r}10 \\
8\end{array}$ & $\begin{array}{l}4 \\
0\end{array}$ & $\begin{array}{l}5 \\
6\end{array}$ & $\frac{1}{2}$ & $\begin{array}{l}0 \\
0\end{array}$ & $\begin{array}{l}0 \\
0\end{array}$ \\
\hline $\begin{array}{l}6-27 \\
6-20\end{array}$ & 4237 & 3 & $\underline{0}$ & 1 & $\underline{2}$ & $\underline{0}$ & $\underline{0}$ \\
\hline $\begin{array}{l}6-29 \\
7-01\end{array}$ & $\overline{--}$ & $\overline{--}$ & $\overline{--}$ & - & $\overline{--}$ & $\overline{--}$ & -- \\
\hline $\begin{array}{l}7-03 \\
7-05\end{array}$ & $=$ & $=$ & $=$ & $\overline{--}$ & $=$ & $=$ & $=$ \\
\hline $7-07$ & -- & -- & -- & -- & -- & -- & -- \\
\hline
\end{tabular}


TABLE 18.- NUMBER OF GREEN TRACER PARTICLES(1), PER 100 GRAMS OF BEDLOAD SAMPLE, COMPOSITED FROM CROSS-CHANNEL SAMPLING POSITIONS SPACED AT 1-METER INTERVALS ACROSS THE CHANNEL WIDTH, EAST FORK RIVER, WYOMING, 1980

SECTION 1155

\begin{tabular}{|c|c|c|c|c|c|c|c|}
\hline \multirow{3}{*}{ DATE } & \multirow{3}{*}{$\begin{array}{c}\text { DRY } \\
\text { MASS } \\
\text { OF } \\
\text { SAMPLE (2) } \\
\text { (G) }\end{array}$} & \multicolumn{6}{|c|}{ NUMBER OF GREEN TRACER PARTICLES } \\
\hline & & \multirow[b]{2}{*}{ TOTAL } & \multicolumn{5}{|c|}{ BY SIZE CLASS (MM) } \\
\hline & & & $\begin{array}{l}0.25 \\
\text { TO } \\
0.50\end{array}$ & $\begin{array}{l}0.50 \\
\text { TO } \\
1.00\end{array}$ & $\begin{array}{l}1.00 \\
\text { TO } \\
2.00\end{array}$ & $\begin{array}{l}2.00 \\
\text { TO } \\
4.00\end{array}$ & $\begin{array}{l}4.00 \\
\text { TO } \\
8.00\end{array}$ \\
\hline $\begin{array}{l}5-16 \\
5-19 \\
5-21 \\
5-22 \\
5-24\end{array}$ & $\begin{array}{c}17+++ \\
73^{+++} \\
2437^{++} \\
2980 \\
2253\end{array}$ & $\begin{array}{l}0 \\
0 \\
0 \\
0 \\
0\end{array}$ & $\begin{array}{l}0 \\
0 \\
0 \\
0 \\
0\end{array}$ & $\begin{array}{l}0 \\
0 \\
0 \\
0 \\
0\end{array}$ & $\begin{array}{l}0 \\
0 \\
0 \\
0 \\
0\end{array}$ & $\begin{array}{l}0 \\
0 \\
0 \\
0 \\
0\end{array}$ & $\begin{array}{l}0 \\
0 \\
0 \\
0 \\
0\end{array}$ \\
\hline $\begin{array}{l}5-26 \\
5-27 \\
5-28 \\
5-29\end{array}$ & $\begin{array}{l}\overline{25++} \\
51+\end{array}$ & $\begin{array}{r}-- \\
0 \\
--\end{array}$ & $\begin{array}{r}- \\
0 \\
-\end{array}$ & $\begin{array}{r}-- \\
0 \\
-\end{array}$ & $\begin{array}{r}-- \\
0 \\
--\end{array}$ & $\begin{array}{r}-- \\
0 \\
-\end{array}$ & $\begin{array}{r}- \\
0 \\
0\end{array}$ \\
\hline $5-30$ & $52+$ & 0 & 0 & 0 & 0 & 0 & 0 \\
\hline $\begin{array}{l}6-01 \\
6-03 \\
6-05 \\
6-07 \\
6-09\end{array}$ & $\begin{array}{c}46++ \\
87^{++} \\
23+++ \\
341 \\
2849\end{array}$ & $\begin{array}{l}0 \\
0 \\
0 \\
0 \\
0\end{array}$ & $\begin{array}{l}0 \\
0 \\
0 \\
0 \\
0\end{array}$ & $\begin{array}{l}0 \\
0 \\
0 \\
0 \\
0\end{array}$ & $\begin{array}{l}0 \\
0 \\
0 \\
0 \\
0\end{array}$ & $\begin{array}{l}0 \\
0 \\
0 \\
0 \\
0\end{array}$ & $\begin{array}{l}0 \\
0 \\
0 \\
0 \\
0\end{array}$ \\
\hline $\begin{array}{l}6-10 \\
6-11 \\
6-12 \\
6-13 \\
6-14\end{array}$ & $\begin{array}{r}4178 \\
1008 \\
776 \\
526 \\
2043\end{array}$ & $\begin{array}{l}0 \\
4 \\
0 \\
0 \\
0\end{array}$ & $\begin{array}{l}0 \\
3 \\
0 \\
0 \\
0\end{array}$ & $\begin{array}{l}0 \\
1 \\
0 \\
0 \\
0\end{array}$ & $\begin{array}{l}0 \\
0 \\
0 \\
0 \\
0\end{array}$ & $\begin{array}{l}0 \\
0 \\
0 \\
0 \\
0\end{array}$ & $\begin{array}{l}0 \\
0 \\
0 \\
0 \\
0\end{array}$ \\
\hline $6-15$ & 957 & 0 & 0 & 0 & 0 & 0 & 0 \\
\hline $\begin{array}{l}6-17 \\
6-18 \\
6-19\end{array}$ & $\begin{array}{r}659 \\
2916 \\
3706\end{array}$ & $\begin{array}{r}5 \\
26 \\
5\end{array}$ & $\begin{array}{r}4 \\
13 \\
2\end{array}$ & $\begin{array}{r}\frac{1}{3} \\
3\end{array}$ & $\begin{array}{l}0 \\
0 \\
0\end{array}$ & $\begin{array}{l}0 \\
0 \\
0\end{array}$ & $\begin{array}{l}0 \\
0 \\
0\end{array}$ \\
\hline $\begin{array}{l}6-20 \\
6-21\end{array}$ & $\begin{array}{l}1301 \\
2023\end{array}$ & $\begin{array}{r}15 \\
9\end{array}$ & $\begin{array}{l}3 \\
1\end{array}$ & $\begin{array}{l}8 \\
7\end{array}$ & $\begin{array}{l}3 \\
1\end{array}$ & $\begin{array}{l}1 \\
0\end{array}$ & $\begin{array}{l}0 \\
0\end{array}$ \\
\hline $\begin{array}{l}6-23 \\
6-25\end{array}$ & $\begin{array}{r}1384 \\
629\end{array}$ & $\begin{array}{l}3 \\
9\end{array}$ & $\begin{array}{l}2 \\
0\end{array}$ & $\begin{array}{l}0 \\
8\end{array}$ & $\begin{array}{l}1 \\
0\end{array}$ & $\begin{array}{l}0 \\
1\end{array}$ & $\begin{array}{l}0 \\
0\end{array}$ \\
\hline $6-27$ & 3473 & 2 & 0 & 2 & 0 & 0 & 0 \\
\hline $\begin{array}{l}6-29 \\
7-01\end{array}$ & $\overline{--}$ & $\overline{--}$ & $\overline{--}$ & $\overline{--}$ & $\overline{--}$ & $\overline{-}$ & $\overline{-}$ \\
\hline $7-03$ & -- & -- & -- & -- & - & -- & -- \\
\hline $7-05$ & -- & -- & -- & -- & -- & -- & -- \\
\hline $7-07$ & -- & -- & -- & -- & -- & -- & -- \\
\hline
\end{tabular}


TABLE 18.- NUMBER OF GREEN TRACER PARTICLES(1), PER 100 GRAMS OF BEDLOAD SAMPLE, COMPOSITED FROM CROSS-CHANNEL SAMPLING POSITIONS SPACED AT 1-METER INTERVALS ACROSS THE CHANNEL WIDTH, EAST FORK RIVER, WYOMING, 1980

SECTION 1202

\begin{tabular}{|c|c|c|c|c|c|c|c|}
\hline \multirow{3}{*}{\multicolumn{2}{|c|}{$\begin{array}{cc} & \\
\text { DATE } & \text { DRY } \\
& \text { MASS } \\
& \text { OF } \\
& \text { SAMPLE (2) } \\
& (\text { G }) \\
& \end{array}$}} & \multicolumn{6}{|c|}{ NUMBER OF GREEN TRACER PARTICLES } \\
\hline & & \multirow[b]{2}{*}{ TOTAL } & \multicolumn{5}{|c|}{ BY SIZE CLASS (MM) } \\
\hline & & & $\begin{array}{l}0.25 \\
\text { TO } \\
0.50\end{array}$ & $\begin{array}{l}0.50 \\
\text { TO } \\
1.00\end{array}$ & $\begin{array}{l}1.00 \\
\text { TO } \\
2.00\end{array}$ & $\begin{array}{l}2.00 \\
\text { TO } \\
4.00\end{array}$ & $\begin{array}{l}4.00 \\
\text { TO } \\
8.00\end{array}$ \\
\hline $\begin{array}{l}5-16 \\
5-19 \\
5-21 \\
5-22 \\
5-24\end{array}$ & $\begin{array}{l}323 \\
251+ \\
2833 \\
6017 \\
1764\end{array}$ & $\begin{array}{l}0 \\
0 \\
0 \\
0 \\
0\end{array}$ & $\begin{array}{l}0 \\
0 \\
0 \\
0 \\
0\end{array}$ & $\begin{array}{l}0 \\
0 \\
0 \\
0 \\
0\end{array}$ & $\begin{array}{l}0 \\
0 \\
0 \\
0 \\
0\end{array}$ & $\begin{array}{l}0 \\
0 \\
0 \\
0 \\
0\end{array}$ & $\begin{array}{l}0 \\
0 \\
0 \\
0 \\
0\end{array}$ \\
\hline $\begin{array}{l}5-26 \\
5-27 \\
5-28 \\
5-29\end{array}$ & $\begin{array}{l}4 \overline{0} \\
113+ \\
113+\end{array}$ & $\begin{array}{r}-- \\
0 \\
-\end{array}$ & $\begin{array}{r}-- \\
0 \\
-\end{array}$ & $\begin{array}{r}-- \\
0 \\
-\end{array}$ & $\begin{array}{r}-- \\
0 \\
-\end{array}$ & $\begin{array}{r}-- \\
0 \\
0\end{array}$ & $\begin{array}{r}-- \\
0 \\
0\end{array}$ \\
\hline $5-30$ & $35++$ & 0 & 0 & 0 & 0 & 0 & 0 \\
\hline $\begin{array}{l}6-01 \\
6-03 \\
6-05 \\
6-07 \\
6-09\end{array}$ & $\begin{array}{l}102++ \\
419 \\
1372 \\
1767 \\
7316\end{array}$ & $\begin{array}{l}0 \\
0 \\
0 \\
0 \\
0\end{array}$ & $\begin{array}{l}0 \\
0 \\
0 \\
0 \\
0\end{array}$ & $\begin{array}{l}0 \\
0 \\
0 \\
0 \\
0\end{array}$ & $\begin{array}{l}0 \\
0 \\
0 \\
0 \\
0\end{array}$ & $\begin{array}{l}0 \\
0 \\
0 \\
0 \\
0\end{array}$ & $\begin{array}{l}0 \\
0 \\
0 \\
0 \\
0\end{array}$ \\
\hline $\begin{array}{l}6-10 \\
6-11 \\
6-12 \\
6-13 \\
6-14\end{array}$ & $\begin{array}{r}4322 \\
1660 \\
907 \\
729 \\
2204\end{array}$ & $\begin{array}{l}0 \\
3 \\
9 \\
2 \\
0\end{array}$ & $\begin{array}{l}0 \\
2 \\
8 \\
2 \\
0\end{array}$ & $\begin{array}{l}0 \\
1 \\
1 \\
0 \\
0\end{array}$ & $\begin{array}{l}0 \\
0 \\
0 \\
0 \\
0\end{array}$ & $\begin{array}{l}0 \\
0 \\
0 \\
0 \\
0\end{array}$ & $\begin{array}{l}0 \\
0 \\
0 \\
0 \\
0\end{array}$ \\
\hline $6-15$ & 917 & 2 & 2 & 0 & 0 & $\underline{0}$ & 0 \\
\hline $\begin{array}{l}6-17 \\
6-18 \\
6-19\end{array}$ & $\begin{array}{l}1171 \\
1103 \\
4168\end{array}$ & $\begin{array}{l}7 \\
5 \\
9\end{array}$ & $\begin{array}{l}4 \\
3 \\
3\end{array}$ & $\begin{array}{l}3 \\
2 \\
4\end{array}$ & $\begin{array}{l}0 \\
0 \\
0\end{array}$ & $\begin{array}{l}0 \\
0 \\
2\end{array}$ & $\begin{array}{l}0 \\
0 \\
0\end{array}$ \\
\hline $\begin{array}{l}6-20 \\
6-21\end{array}$ & $\begin{array}{l}2102 \\
4380\end{array}$ & $\begin{array}{r}15 \\
8\end{array}$ & $\begin{array}{l}2 \\
3 \\
\end{array}$ & $\begin{array}{r}10 \\
3\end{array}$ & $\begin{array}{l}3 \\
2\end{array}$ & $\begin{array}{l}0 \\
0\end{array}$ & $\begin{array}{l}0 \\
0\end{array}$ \\
\hline $\begin{array}{l}6-23 \\
6-25\end{array}$ & $\begin{array}{l}2561 \\
5151\end{array}$ & $\begin{array}{r}17 \\
4\end{array}$ & $\begin{array}{l}6 \\
1\end{array}$ & $\begin{array}{r}10 \\
3\end{array}$ & $\begin{array}{l}0 \\
0\end{array}$ & $\begin{array}{l}1 \\
0\end{array}$ & $\begin{array}{l}0 \\
0\end{array}$ \\
\hline $6-27$ & 2402 & 2 & 0 & 2 & 0 & 0 & 0 \\
\hline $\begin{array}{l}0-29 \\
7-01\end{array}$ & - & - & - & $\overline{--}$ & $\overline{-}$ & - & $\overline{-}$ \\
\hline $\begin{array}{l}7-03 \\
7-05\end{array}$ & $\overline{--}$ & $\overline{-}$ & $\overline{--}$ & $\overline{--}$ & $\overline{--}$ & $\overline{--}$ & $\overline{--}$ \\
\hline $7-07$ & -- & -- & -- & - & -- & -- & -- \\
\hline
\end{tabular}


TABLE 18.- NUMBER OF GREEN TRACER PARTICLES(1), PER 100 GRAMS OF BEDLOAD SAMPLE, COMPOSITED FROM CROSS-CHANNEL SAMPLING POSITIONS SPACED AT 1-METER INTERVALS ACROSS THE CHANNEL WIDTH, EAST FORK RIVER, WYOMING, 1980

SECTION 1241

\begin{tabular}{|c|c|c|c|c|c|c|c|}
\hline \multirow{3}{*}{ DATE } & \multirow{3}{*}{$\begin{array}{c}\text { DRY } \\
\text { MASS } \\
\text { OF } \\
\text { SAMPLE (2) } \\
\text { (G) }\end{array}$} & \multicolumn{6}{|c|}{ NUMBER OF GREEN TRACER PARTICLES } \\
\hline & & \multirow[b]{2}{*}{ TOTAL } & \multicolumn{5}{|c|}{ BY SIZE CLASS (MM) } \\
\hline & & & $\begin{array}{l}0.25 \\
\text { TO } \\
0.50\end{array}$ & $\begin{array}{l}0.50 \\
\text { TO } \\
1.00\end{array}$ & $\begin{array}{l}1.00 \\
\text { TO } \\
2.00\end{array}$ & $\begin{array}{l}2.00 \\
\text { TO } \\
4.00\end{array}$ & $\begin{array}{l}4.00 \\
\text { TO } \\
8.00\end{array}$ \\
\hline $\begin{array}{l}5-16 \\
5-19 \\
5-21 \\
5-22 \\
5-24\end{array}$ & $\begin{array}{r}909 \\
359 \\
1312 \\
3206 \\
510\end{array}$ & $\begin{array}{l}0 \\
0 \\
0 \\
0 \\
0\end{array}$ & $\begin{array}{l}0 \\
0 \\
0 \\
0 \\
0\end{array}$ & $\begin{array}{l}0 \\
0 \\
0 \\
0 \\
0\end{array}$ & $\begin{array}{l}0 \\
0 \\
0 \\
0 \\
0\end{array}$ & $\begin{array}{l}0 \\
0 \\
0 \\
0 \\
0\end{array}$ & $\begin{array}{l}0 \\
0 \\
0 \\
0 \\
0\end{array}$ \\
\hline $\begin{array}{l}5-26 \\
5-27 \\
5-28 \\
5-29 \\
5-30\end{array}$ & $\begin{array}{l}\overline{56++} \\
170 \\
660 \\
101+\end{array}$ & $\begin{array}{c}-- \\
0 \\
0 \\
0 \\
0\end{array}$ & $\begin{array}{c}-\overline{0} \\
0 \\
0 \\
0\end{array}$ & $\begin{array}{r}-- \\
0 \\
0 \\
0 \\
0\end{array}$ & $\begin{array}{l}-\overline{0} \\
0 \\
0 \\
0\end{array}$ & $\begin{array}{l}- \\
0 \\
0 \\
0 \\
0\end{array}$ & $\begin{array}{c}-\overline{0} \\
0 \\
0 \\
0\end{array}$ \\
\hline $\begin{array}{l}6-01 \\
6-03 \\
6-05 \\
6-07 \\
6-09\end{array}$ & $\begin{array}{r}1777 \\
452 \\
759 \\
1177 \\
1501\end{array}$ & $\begin{array}{l}0 \\
0 \\
0 \\
0 \\
0\end{array}$ & $\begin{array}{l}0 \\
0 \\
0 \\
0 \\
0\end{array}$ & $\begin{array}{l}0 \\
0 \\
0 \\
0 \\
0\end{array}$ & $\begin{array}{l}0 \\
0 \\
0 \\
0 \\
0\end{array}$ & $\begin{array}{l}0 \\
0 \\
0 \\
0 \\
0\end{array}$ & $\begin{array}{l}0 \\
0 \\
0 \\
0 \\
0\end{array}$ \\
\hline $\begin{array}{l}6-10 \\
6-11 \\
6-12 \\
6-13 \\
6-14\end{array}$ & $\begin{array}{r}690 \\
945 \\
324 \\
308 \\
1093\end{array}$ & $\begin{array}{r}8 \\
12 \\
4 \\
2 \\
6\end{array}$ & $\begin{array}{r}8 \\
12 \\
3 \\
0 \\
1\end{array}$ & $\begin{array}{l}0 \\
0 \\
1 \\
2 \\
3\end{array}$ & $\begin{array}{l}0 \\
0 \\
0 \\
0 \\
1\end{array}$ & $\begin{array}{l}0 \\
0 \\
0 \\
0 \\
1\end{array}$ & $\begin{array}{l}0 \\
0 \\
0 \\
0 \\
0\end{array}$ \\
\hline $\begin{array}{l}6-15 \\
6-16 \\
6-17 \\
6-18 \\
6-19\end{array}$ & $\begin{array}{r}1350 \\
301 \\
1431 \\
3603 \\
1291\end{array}$ & $\begin{array}{l}14 \\
17 \\
23 \\
12 \\
10\end{array}$ & $\begin{array}{r}4 \\
12 \\
7 \\
1 \\
6\end{array}$ & $\begin{array}{r}7 \\
5 \\
16 \\
11 \\
4\end{array}$ & $\begin{array}{l}2 \\
0 \\
0 \\
0 \\
0\end{array}$ & $\begin{array}{l}1 \\
0 \\
0 \\
0 \\
0\end{array}$ & $\begin{array}{l}0 \\
0 \\
0 \\
0 \\
0\end{array}$ \\
\hline $\begin{array}{l}6-20 \\
6-21\end{array}$ & $\begin{array}{l}2036 \\
6118\end{array}$ & $\begin{array}{l}10 \\
11\end{array}$ & $\begin{array}{l}2 \\
2\end{array}$ & $\begin{array}{l}7 \\
6\end{array}$ & $\frac{1}{3}$ & $\begin{array}{l}0 \\
0\end{array}$ & $\begin{array}{l}0 \\
0\end{array}$ \\
\hline $\begin{array}{l}6-22 \\
6-23 \\
6-25\end{array}$ & $\begin{array}{r}4 \overline{53} \\
5117\end{array}$ & $\frac{3}{2}$ & $\overline{1}$ & $\overline{2}$ & $\overline{0}$ & $\overline{0}$ & $\overline{0}$ \\
\hline $6-27$ & 1604 & 3 & 0 & 2 & 1 & 0 & 0 \\
\hline $\begin{array}{l}6-29 \\
7-01\end{array}$ & -- & $\overline{-}$ & $\overline{--}$ & $\overline{-}$ & $\overline{--}$ & $\overline{--}$ & $\overline{--}$ \\
\hline $\begin{array}{l}7-03 \\
7-05\end{array}$ & $=$ & $=$ & $=$ & $=$ & $\overline{-}$ & $=$ & $=$ \\
\hline $7-07$ & -- & -- & - & -- & -- & -- & -- \\
\hline
\end{tabular}


TABLE 18.- NUMBER OF GREEN TRACER PARTICLES(1), PER 100 GRAMS OF BEDLOAD SAMPLE, COMPOSITED FROM CROSS-CHANNEL SAMPLING POSITIONS SPACED AT 1-METER INTERVALS ACROSS THE CHANNEL WIDTH, EAST FORK RIVER, WYOMING, 1980

SECTION 1284

\begin{tabular}{|c|c|c|c|c|c|c|c|}
\hline \multirow{3}{*}{ DATE } & \multirow{3}{*}{$\begin{array}{c}\text { DRY } \\
\text { MASS } \\
\text { OF } \\
\text { SAMPLE (2) } \\
\text { (G) }\end{array}$} & \multicolumn{6}{|c|}{ NUMBER OF GREEN TRACER PARTICLES } \\
\hline & & \multirow[b]{2}{*}{ TOTAL } & \multicolumn{5}{|c|}{ BY SIZE CLASS (MM) } \\
\hline & & & $\begin{array}{c}0.25 \\
\text { TO } \\
0.50\end{array}$ & $\begin{array}{l}0.50 \\
\text { TO } \\
1.00\end{array}$ & $\begin{array}{l}1.00 \\
\text { TO } \\
2.00\end{array}$ & $\begin{array}{l}2.00 \\
\text { TO } \\
4.00\end{array}$ & $\begin{array}{l}4.00 \\
\text { TO } \\
8.00\end{array}$ \\
\hline $\begin{array}{l}5-16 \\
5-19 \\
5-21 \\
5-22 \\
5-24\end{array}$ & $\begin{array}{l}3816 \\
1486 \\
1193 \\
1704 \\
763\end{array}$ & $\begin{array}{l}0 \\
0 \\
0 \\
0 \\
0\end{array}$ & $\begin{array}{l}0 \\
0 \\
0 \\
0 \\
0\end{array}$ & $\begin{array}{l}0 \\
0 \\
0 \\
0 \\
0\end{array}$ & $\begin{array}{l}0 \\
0 \\
0 \\
0 \\
0\end{array}$ & $\begin{array}{l}0 \\
0 \\
0 \\
0 \\
0\end{array}$ & $\begin{array}{l}0 \\
0 \\
0 \\
0 \\
0\end{array}$ \\
\hline $\begin{array}{l}5-26 \\
5-27 \\
5-28 \\
5-20\end{array}$ & $\begin{array}{r}1459 \\
1247\end{array}$ & $\begin{array}{r}--\overline{0} \\
0 \\
-\end{array}$ & $\begin{array}{l}-- \\
0 \\
0\end{array}$ & $\begin{array}{r}--\overline{0} \\
0 \\
--\end{array}$ & $\begin{array}{r}-- \\
0 \\
0\end{array}$ & $\begin{array}{r}-\overline{0} \\
0\end{array}$ & $\begin{array}{c}-- \\
0 \\
0\end{array}$ \\
\hline $5-30$ & 1345 & 0 & 0 & 0 & 0 & 0 & 0 \\
\hline $\begin{array}{l}6-01 \\
6-03 \\
6-05 \\
6-07 \\
6-09\end{array}$ & $\begin{array}{r}1178 \\
741 \\
944 \\
969 \\
2293\end{array}$ & $\begin{array}{l}0 \\
0 \\
0 \\
0 \\
0\end{array}$ & $\begin{array}{l}0 \\
0 \\
0 \\
0 \\
0\end{array}$ & $\begin{array}{l}0 \\
0 \\
0 \\
0 \\
0\end{array}$ & $\begin{array}{l}0 \\
0 \\
0 \\
0 \\
0\end{array}$ & $\begin{array}{l}0 \\
0 \\
0 \\
0 \\
0\end{array}$ & $\begin{array}{l}0 \\
0 \\
0 \\
0 \\
0\end{array}$ \\
\hline $\begin{array}{l}6-10 \\
6-11 \\
6-12 \\
6-13 \\
6-14\end{array}$ & $\begin{array}{r}1907 \\
879 \\
374 \\
470 \\
897\end{array}$ & $\begin{array}{l}22 \\
50 \\
25 \\
47 \\
29\end{array}$ & $\begin{array}{l}16 \\
45 \\
23 \\
37 \\
22\end{array}$ & $\begin{array}{r}6 \\
4 \\
2 \\
10 \\
7\end{array}$ & $\begin{array}{l}0 \\
1 \\
0 \\
0 \\
0\end{array}$ & $\begin{array}{l}0 \\
0 \\
0 \\
0 \\
0\end{array}$ & $\begin{array}{l}0 \\
0 \\
0 \\
0 \\
0\end{array}$ \\
\hline $\begin{array}{l}6-15 \\
6-16 \\
6-17 \\
6-18 \\
6-19\end{array}$ & $\begin{array}{l}2970 \\
1347 \\
6911 \\
2817 \\
2731\end{array}$ & $\begin{array}{r}18 \\
21 \\
21 \\
4 \\
9\end{array}$ & $\begin{array}{r}10 \\
8 \\
8 \\
1 \\
6\end{array}$ & $\begin{array}{r}7 \\
1 \frac{1}{12} \\
3 \\
2\end{array}$ & $\begin{array}{l}0 \\
1 \\
1 \\
0 \\
1\end{array}$ & $\begin{array}{l}1 \\
0 \\
0 \\
0 \\
0\end{array}$ & $\begin{array}{l}0 \\
1 \\
0 \\
0 \\
0\end{array}$ \\
\hline $\begin{array}{l}6-20 \\
6-21\end{array}$ & $\begin{array}{l}1814 \\
1452\end{array}$ & $\begin{array}{r}16 \\
4\end{array}$ & 7 & $\begin{array}{l}9 \\
2\end{array}$ & $\begin{array}{l}0 \\
1\end{array}$ & $\begin{array}{l}0 \\
0\end{array}$ & $\begin{array}{l}0 \\
0\end{array}$ \\
\hline $\begin{array}{l}6-23 \\
6-25\end{array}$ & $\begin{array}{l}2430 \\
2306\end{array}$ & $\begin{array}{l}9 \\
3\end{array}$ & 4 & 4 & 1 & $\begin{array}{l}0 \\
0\end{array}$ & $\begin{array}{l}0 \\
0\end{array}$ \\
\hline $\begin{array}{l}6-27 \\
6-29\end{array}$ & 3246 & 2 & 1 & 1 & -1 & 0 & -0 \\
\hline $7-0 \overline{1}$ & -- & -- & -- & -- & -- & -- & -- \\
\hline $7-05$ & - & - & - & - & -- & - & -- \\
\hline $7-07$ & -- & -- & -- & -- & -- & -- & -- \\
\hline
\end{tabular}


TABLE 18.- NUMBER OF GREEN TRACER PARTICLES(1), PER 100 GRAMS OF BEDLOAD SAMPLE, COMPOSITED FROM CROSS-CHANNEL SAMPLING POSITIONS SPACED AT 1-METER INTERVALS ACROSS THE CHANNEL WIDTH, EAST FORK RIVER, WYOMING, 1980

SECTION 1315

\begin{tabular}{|c|c|c|c|c|c|c|c|}
\hline \multirow{3}{*}{ DATE } & \multirow{3}{*}{$\begin{array}{c}\text { DRY } \\
\text { MASS } \\
\text { OF } \\
\text { SAMPLE (2) } \\
\text { (G) }\end{array}$} & \multicolumn{6}{|c|}{ NUMBER OF GREEN TRACER PARTICLES } \\
\hline & & \multirow[b]{2}{*}{ TOTAL } & \multicolumn{5}{|c|}{ BY SIZE CLASS (MM) } \\
\hline & & & $\begin{array}{l}0.25 \\
\text { TO } \\
0.50\end{array}$ & $\begin{array}{l}0.50 \\
\text { TO } \\
1.00\end{array}$ & $\begin{array}{l}1.00 \\
\text { TO } \\
2.00\end{array}$ & $\begin{array}{l}2.00 \\
\text { TO } \\
4.00\end{array}$ & $\begin{array}{l}4.00 \\
\text { TO } \\
8.00\end{array}$ \\
\hline $\begin{array}{l}5-16 \\
5-19 \\
5-21 \\
5-22 \\
5-24\end{array}$ & $\begin{array}{r}803 \\
351 \\
374 \\
684 \\
1499\end{array}$ & $\begin{array}{l}0 \\
0 \\
0 \\
0 \\
0\end{array}$ & $\begin{array}{l}0 \\
0 \\
0 \\
0 \\
0\end{array}$ & $\begin{array}{l}0 \\
0 \\
0 \\
0 \\
0\end{array}$ & $\begin{array}{l}0 \\
0 \\
0 \\
0 \\
0\end{array}$ & $\begin{array}{l}0 \\
0 \\
0 \\
0 \\
0\end{array}$ & $\begin{array}{l}0 \\
0 \\
0 \\
0 \\
0\end{array}$ \\
\hline $\begin{array}{l}5-26 \\
5-27 \\
5-28 \\
5-20\end{array}$ & $\begin{array}{r}12 \overline{6} \\
99+\end{array}$ & $\begin{array}{r}--\overline{0} \\
0\end{array}$ & $\begin{array}{r}-\overline{0} \\
0\end{array}$ & $\begin{array}{r}-- \\
0 \\
0\end{array}$ & $\begin{array}{r}-- \\
0 \\
0\end{array}$ & $\begin{array}{r}-- \\
0 \\
0\end{array}$ & $\begin{array}{r}-- \\
0 \\
-\end{array}$ \\
\hline $5-30$ & 1205 & 0 & 0 & 0 & 0 & 0 & 0 \\
\hline $\begin{array}{l}6-01 \\
6-03 \\
6-05 \\
6-07 \\
6-09\end{array}$ & $\begin{array}{c}1241 \\
217 \\
98+ \\
249 \\
483\end{array}$ & $\begin{array}{r}0 \\
1 \\
0 \\
0 \\
12\end{array}$ & $\begin{array}{r}0 \\
1 \\
0 \\
0 \\
10\end{array}$ & $\begin{array}{l}0 \\
0 \\
0 \\
0 \\
2\end{array}$ & $\begin{array}{l}0 \\
0 \\
0 \\
0 \\
0\end{array}$ & $\begin{array}{l}0 \\
0 \\
0 \\
0 \\
0\end{array}$ & $\begin{array}{l}0 \\
0 \\
0 \\
0 \\
0\end{array}$ \\
\hline $\begin{array}{l}6-10 \\
6-11 \\
6-12 \\
6-13 \\
6-14\end{array}$ & $\begin{array}{r}2803 \\
4062 \\
842 \\
846 \\
2194\end{array}$ & $\begin{array}{l}21 \\
33 \\
18 \\
15 \\
20\end{array}$ & $\begin{array}{l}19 \\
28 \\
10 \\
11 \\
13\end{array}$ & $\begin{array}{l}2 \\
5 \\
8 \\
4 \\
5\end{array}$ & $\begin{array}{l}0 \\
0 \\
0 \\
0 \\
2\end{array}$ & $\begin{array}{l}0 \\
0 \\
0 \\
0 \\
0\end{array}$ & $\begin{array}{l}0 \\
0 \\
0 \\
0 \\
0\end{array}$ \\
\hline $\begin{array}{l}6-15 \\
6-16 \\
6-17 \\
6-18 \\
6-19\end{array}$ & $\begin{array}{l}2768 \\
3105 \\
5091 \\
2403 \\
2294\end{array}$ & $\begin{array}{l}21 \\
29 \\
16 \\
21 \\
16\end{array}$ & $\begin{array}{r}9 \\
15 \\
6 \\
7 \\
6\end{array}$ & $\begin{array}{r}12 \\
11 \\
9 \\
12 \\
9\end{array}$ & $\begin{array}{l}0 \\
3 \\
1 \\
2 \\
1\end{array}$ & $\begin{array}{l}0 \\
0 \\
0 \\
0 \\
0\end{array}$ & $\begin{array}{l}0 \\
0 \\
0 \\
0 \\
0\end{array}$ \\
\hline $\begin{array}{l}6-20 \\
6-21 \\
6-22\end{array}$ & $\begin{array}{l}2348 \\
2830 \\
\end{array}$ & $\begin{array}{r}5 \\
9 \\
-\end{array}$ & $\begin{array}{r}1 \\
4\end{array}$ & $\begin{array}{r}2 \\
3 \\
-\end{array}$ & $\begin{array}{l}2 \\
2 \\
\end{array}$ & $\begin{array}{l}0 \\
0\end{array}$ & $\begin{array}{r}0 \\
0 \\
-\end{array}$ \\
\hline $\begin{array}{l}6-23 \\
6-25\end{array}$ & $\begin{array}{l}3316 \\
6606\end{array}$ & $\begin{array}{l}8 \\
5\end{array}$ & $\begin{array}{l}3 \\
1\end{array}$ & $\begin{array}{l}5 \\
4\end{array}$ & $\begin{array}{l}0 \\
0\end{array}$ & $\begin{array}{l}0 \\
0\end{array}$ & $\begin{array}{l}0 \\
0\end{array}$ \\
\hline $\begin{array}{l}6-27 \\
6-29\end{array}$ & 2276 & -3 & $\underline{0}$ & -3 & -0 & $\underline{0}$ & -1 \\
\hline $7-0 \frac{1}{2}$ & -- & -- & - & -- & - & -- & -- \\
\hline $7-05$ & $\overline{-}$ & $\overline{-}$ & $\overline{--}$ & $\overline{-}$ & - & $\overline{-}$ & $\overline{-}$ \\
\hline $7-07$ & -- & -- & -- & -- & -- & -- & - \\
\hline
\end{tabular}


TABLE 18.- NUMBER OF GREEN TRACER PARTICLES(1), PER 100 GRAMS OF BEDLOAD SAMPLE, COMPOSITED FROM CROSS-CHANNEL SAMPLING POSITIONS SPACED AT 1-METER INTERVALS ACROSS THE CHANNEL WIDTH, EAST FORK RIVER, WYOMING, 1980

SECTION $1360(4)$

\begin{tabular}{|c|c|c|c|c|c|c|c|}
\hline \multirow{3}{*}{ DATE } & \multirow{3}{*}{$\begin{array}{c}\text { DRY } \\
\text { MASS } \\
\text { OF } \\
\text { SAMPLE (2) } \\
\text { (G) }\end{array}$} & \multicolumn{6}{|c|}{ NUMBER OF GREEN TRACER PARTICLES } \\
\hline & & \multirow[b]{2}{*}{ TOTAL } & \multicolumn{5}{|c|}{ BY SIZE CLASS (MM) } \\
\hline & & & $\begin{array}{l}0.25 \\
\text { TO } \\
0.50\end{array}$ & $\begin{array}{l}0.50 \\
\text { TO } \\
1.00\end{array}$ & $\begin{array}{l}1.00 \\
\text { TO } \\
2.00\end{array}$ & $\begin{array}{l}2.00 \\
\text { TO } \\
4.00\end{array}$ & $\begin{array}{l}4.00 \\
\text { TO } \\
8.00\end{array}$ \\
\hline $\begin{array}{l}5-16 \\
5-19 \\
5-21 \\
5-22 \\
5-24\end{array}$ & $\begin{array}{l}114+ \\
255+ \\
197+ \\
1553 \\
1286\end{array}$ & $\begin{array}{l}0 \\
0 \\
0 \\
0 \\
0\end{array}$ & $\begin{array}{l}0 \\
0 \\
0 \\
0 \\
0\end{array}$ & $\begin{array}{l}0 \\
0 \\
0 \\
0 \\
0\end{array}$ & $\begin{array}{l}0 \\
0 \\
0 \\
0 \\
0\end{array}$ & $\begin{array}{l}0 \\
0 \\
0 \\
0 \\
0\end{array}$ & $\begin{array}{l}0 \\
0 \\
0 \\
0 \\
0\end{array}$ \\
\hline $\begin{array}{l}5-26 \\
5-27 \\
5-28\end{array}$ & $104 \overline{4}++$ & $\begin{array}{r}-\overline{0} \\
0\end{array}$ & $\begin{array}{r}-\overline{0} \\
0\end{array}$ & $\begin{array}{r}-\overline{0} \\
0\end{array}$ & $\begin{array}{r}-- \\
0 \\
0\end{array}$ & $\begin{array}{r}-\overline{0} \\
0\end{array}$ & $\begin{array}{r}-- \\
0 \\
0\end{array}$ \\
\hline $5-30$ & 724 & 0 & 0 & 0 & 0 & 0 & 0 \\
\hline $\begin{array}{l}6-01 \\
6-03 \\
6-05 \\
6-07 \\
6-09\end{array}$ & $\begin{aligned} & 31++ \\
& 235+ \\
& 147 \\
& 535 \\
& 1049\end{aligned}$ & $\begin{array}{r}0 \\
0 \\
0 \\
2 \\
48\end{array}$ & $\begin{array}{r}0 \\
0 \\
0 \\
1 \\
38\end{array}$ & $\begin{array}{r}0 \\
0 \\
0 \\
1 \\
10\end{array}$ & $\begin{array}{l}0 \\
0 \\
0 \\
0 \\
0\end{array}$ & $\begin{array}{l}0 \\
0 \\
0 \\
0 \\
0\end{array}$ & $\begin{array}{l}0 \\
0 \\
0 \\
0 \\
0\end{array}$ \\
\hline $\begin{array}{l}6-10 \\
6-11 \\
6-12 \\
6-13 \\
6-14\end{array}$ & $\begin{array}{r}1192 \\
1011 \\
419 \\
841 \\
612\end{array}$ & $\begin{array}{l}35 \\
25 \\
34 \\
22 \\
18\end{array}$ & $\begin{array}{r}30 \\
16 \\
19 \\
7 \\
4\end{array}$ & $\begin{array}{r}5 \\
9 \\
14 \\
13 \\
9\end{array}$ & $\begin{array}{l}0 \\
0 \\
1 \\
2 \\
5\end{array}$ & $\begin{array}{l}0 \\
0 \\
0 \\
0 \\
0\end{array}$ & $\begin{array}{l}0 \\
0 \\
0 \\
0 \\
0\end{array}$ \\
\hline $\begin{array}{l}6-15 \\
6-16 \\
6-17 \\
6-18 \\
6-19\end{array}$ & $\begin{array}{r}3832 \\
1042 \\
588 \\
520 \\
1603\end{array}$ & $\begin{array}{r}99 \\
11 \\
10 \\
4\end{array}$ & $\begin{array}{l}2 \\
6 \\
2 \\
5 \\
2\end{array}$ & $\begin{array}{l}5 \\
5 \\
0 \\
5 \\
1\end{array}$ & $\begin{array}{l}2 \\
0 \\
1 \\
0 \\
1\end{array}$ & $\begin{array}{l}0 \\
0 \\
0 \\
0 \\
0\end{array}$ & $\begin{array}{l}0 \\
0 \\
0 \\
0 \\
0\end{array}$ \\
\hline $\begin{array}{l}6-20 \\
6-21\end{array}$ & $\begin{array}{r}391 \\
1253\end{array}$ & $\frac{1}{4}$ & $\frac{1}{1}$ & $\begin{array}{l}0 \\
3\end{array}$ & $\begin{array}{l}0 \\
0\end{array}$ & $\begin{array}{l}0 \\
0\end{array}$ & $\begin{array}{l}0 \\
0\end{array}$ \\
\hline $\begin{array}{l}0-23 \\
6-23 \\
6-25\end{array}$ & $\begin{array}{l}265 \\
651\end{array}$ & 0 & 0 & 0 & $\begin{array}{l}0 \\
0\end{array}$ & $\begin{array}{l}0 \\
0\end{array}$ & 0 \\
\hline $6-27$ & 870 & 1 & 1 & 0 & 0 & 0 & 0 \\
\hline $\begin{array}{l}6-29 \\
7-01\end{array}$ & - & $\overline{--}$ & - & $=-$ & $\overline{-}$ & $\overline{-}$ & $\overline{--}$ \\
\hline $\begin{array}{l}7-03 \\
7-05\end{array}$ & $=$ & $=-$ & $=$ & $\overline{--}$ & $\overline{-}$ & $\overline{--}$ & $\overline{-}$ \\
\hline $7-07$ & -- & -- & -- & -- & -- & -- & -- \\
\hline
\end{tabular}


TABLE 18.- NUMBER OF GREEN TRACER PARTICLES(1), PER 100 GRAMS OF BEDLOAD SAMPLE, COMPOSITED FROM CROSS-CHANNEL SAMPLING POSITIONS SPACED AT 1-METER INTERVALS ACROSS THE CHANNEL WIDTH, EAST FORK RIVER, WYOMING, 1980

SECTION I396(4)

\begin{tabular}{|c|c|c|c|c|c|c|c|}
\hline \multirow{3}{*}{ DATE } & \multirow{3}{*}{$\begin{array}{c}\text { DRY } \\
\text { MASS } \\
\text { OF } \\
\text { SAMPLE (2) } \\
\text { (G) }\end{array}$} & \multicolumn{6}{|c|}{ NUMBER OF GREEN TRACER PARTICLES } \\
\hline & & \multirow[b]{2}{*}{ TOTAL } & \multicolumn{5}{|c|}{ BY SIZE CLASS (MM) } \\
\hline & & & $\begin{array}{l}0.25 \\
\text { To } \\
0.50\end{array}$ & $\begin{array}{l}0.50 \\
\text { TO } \\
1.00\end{array}$ & $\begin{array}{l}1.00 \\
\text { TO } \\
2.00\end{array}$ & $\begin{array}{l}2.00 \\
\text { TO } \\
4.00\end{array}$ & $\begin{array}{c}4.00 \\
\text { TO } \\
8.00\end{array}$ \\
\hline $\begin{array}{l}5-16 \\
5-19 \\
5-21 \\
5-22 \\
5-24\end{array}$ & $\begin{array}{r}331 \\
318 \\
1166 \\
589 \\
1626\end{array}$ & $\begin{array}{l}0 \\
0 \\
0 \\
0 \\
0\end{array}$ & $\begin{array}{l}0 \\
0 \\
0 \\
0 \\
0\end{array}$ & $\begin{array}{l}0 \\
0 \\
0 \\
0 \\
0\end{array}$ & $\begin{array}{l}0 \\
0 \\
0 \\
0 \\
0\end{array}$ & $\begin{array}{l}0 \\
0 \\
0 \\
0 \\
0\end{array}$ & $\begin{array}{l}0 \\
0 \\
0 \\
0 \\
0\end{array}$ \\
\hline $\begin{array}{l}5-26 \\
5-27 \\
5-28\end{array}$ & $\begin{array}{l}2 \overline{3} \\
439\end{array}$ & $\begin{array}{r}-- \\
0 \\
0\end{array}$ & $\begin{array}{r}-- \\
0 \\
0\end{array}$ & $\begin{array}{r}-- \\
0 \\
0\end{array}$ & $\begin{array}{r}-- \\
0 \\
0\end{array}$ & $\begin{array}{r}-\overline{0} \\
0\end{array}$ & $\begin{array}{r}--\overline{0} \\
0\end{array}$ \\
\hline $5-30$ & 398 & 0 & 0 & 0 & 0 & 0 & 0 \\
\hline $\begin{array}{l}6-01 \\
6-03 \\
6-05 \\
6-07 \\
6-09\end{array}$ & $\begin{array}{l}178 \\
472 \\
407 \\
532 \\
493\end{array}$ & $\begin{array}{r}0 \\
0 \\
1 \\
1 \\
34\end{array}$ & $\begin{array}{r}0 \\
0 \\
1 \\
0 \\
28\end{array}$ & $\begin{array}{l}0 \\
0 \\
0 \\
0 \\
6\end{array}$ & $\begin{array}{l}0 \\
0 \\
0 \\
1 \\
0\end{array}$ & $\begin{array}{l}0 \\
0 \\
0 \\
0 \\
0\end{array}$ & $\begin{array}{l}0 \\
0 \\
0 \\
0 \\
0\end{array}$ \\
\hline $\begin{array}{l}6-10 \\
6-11 \\
6-12 \\
6-13 \\
6-14\end{array}$ & $\begin{array}{r}2231 \\
1028 \\
467 \\
651 \\
2493\end{array}$ & $\begin{array}{r}32 \\
19 \\
20 \\
5 \\
5\end{array}$ & $\begin{array}{r}23 \\
8 \\
3 \\
0 \\
1\end{array}$ & $\begin{array}{r}8 \\
9 \\
13 \\
2 \\
1\end{array}$ & $\begin{array}{l}1 \\
1 \\
3 \\
2 \\
1\end{array}$ & $\begin{array}{l}0 \\
1 \\
1 \\
1 \\
\frac{1}{2}\end{array}$ & $\begin{array}{l}0 \\
0 \\
0 \\
0 \\
0\end{array}$ \\
\hline $\begin{array}{l}6-15 \\
6-16 \\
6-17 \\
6-18 \\
6-19\end{array}$ & $\begin{array}{l}1830 \\
1400 \\
751 \\
1513 \\
1126\end{array}$ & $\begin{array}{l}1 \\
7 \\
0 \\
9 \\
5\end{array}$ & $\begin{array}{l}0 \\
2 \\
0 \\
7 \\
1\end{array}$ & $\begin{array}{l}1 \\
3 \\
0 \\
2 \\
2\end{array}$ & $\begin{array}{l}0 \\
1 \\
0 \\
0 \\
1\end{array}$ & $\begin{array}{l}0 \\
1 \\
0 \\
0 \\
1\end{array}$ & $\begin{array}{l}0 \\
0 \\
0 \\
0 \\
0\end{array}$ \\
\hline $\begin{array}{l}6-20 \\
6-21\end{array}$ & $\begin{array}{l}432 \\
855\end{array}$ & $\begin{array}{l}2 \\
6\end{array}$ & $\begin{array}{l}0 \\
0\end{array}$ & $\begin{array}{l}2 \\
5\end{array}$ & $\begin{array}{l}0 \\
1\end{array}$ & $\begin{array}{l}0 \\
0\end{array}$ & $\begin{array}{l}0 \\
0\end{array}$ \\
\hline $\begin{array}{l}6-22 \\
6-23 \\
6-25\end{array}$ & $\begin{array}{l}754 \\
487\end{array}$ & $\overline{0}$ & 0 & 0 & 0 & 0 & $\begin{array}{l}0 \\
0\end{array}$ \\
\hline $6-27$ & 1773 & 0 & 0 & 0 & 0 & 0 & 0 \\
\hline $\begin{array}{l}6-29 \\
7-01\end{array}$ & $\overline{--}$ & $\overline{--}$ & $\overline{--}$ & $\overline{-}$ & $\overline{--}$ & $\overline{-}$ & $\overline{-}$ \\
\hline $7-03$ & -- & -- & -- & -- & -- & -- & -- \\
\hline $7-05$ & -- & -- & -- & -- & -- & -- & -- \\
\hline $7-07$ & -- & -- & -- & -- & -- & -- & -- \\
\hline
\end{tabular}


TABLE 18.- NUMBER OF GREEN TRACER PARTICLES(1), PER 100 GRAMS OF BEDLOAD SAMPLE, COMPOSITED FROM CROSS-CHANNEL SAMPLING POSITIONS SPACED AT I-METER INTERVALS ACROSS THE CHANNEL WIDTH, EAST FORK RIVER, WYOMING, 1980

SECTION $1400(5)$

\begin{tabular}{|c|c|c|c|c|c|c|c|}
\hline & & & BER OF & REEN ? & CER PL & ICLES & \\
\hline DATE & & & & $\mathrm{BY} \mathrm{SIZ}$ & CLASS & & \\
\hline & $\underset{\text { (G) }}{\text { OF }}$ & TOTAL & $\begin{array}{l}0.25 \\
\text { TO } \\
0.50\end{array}$ & $\begin{array}{l}0.50 \\
100 \\
1.00\end{array}$ & $\begin{array}{l}1.00 \\
2.00\end{array}$ & $\begin{array}{l}2.00 \\
\text { TO } \\
4.00\end{array}$ & $\begin{array}{l}4.00 \\
\text { TO } \\
8.00\end{array}$ \\
\hline $\begin{array}{l}5-16 \\
5-19 \\
5-21 \\
5-22 \\
5-24\end{array}$ & $\begin{array}{l}-- \\
\overline{37} \\
352 \\
1306\end{array}$ & $\begin{array}{c}-- \\
-\overline{0} \\
0 \\
0\end{array}$ & $\begin{array}{c}-- \\
-\overline{0} \\
0 \\
0\end{array}$ & $\begin{array}{c}-- \\
-\overline{0} \\
0 \\
0\end{array}$ & $\begin{array}{c}-- \\
-\overline{0} \\
0 \\
0\end{array}$ & $\begin{array}{l}-- \\
-\overline{0} \\
0 \\
0\end{array}$ & $\begin{array}{c}-- \\
-\overline{0} \\
0 \\
0\end{array}$ \\
\hline $\begin{array}{l}5-26 \\
5-27 \\
5-28 \\
5-29 \\
5-30\end{array}$ & $\begin{array}{c}3 \overline{62} \\
87+ \\
-\overline{0+++}\end{array}$ & $\begin{array}{r}-- \\
0 \\
-0 \\
-0\end{array}$ & $\begin{array}{r}-- \\
0 \\
-0 \\
0\end{array}$ & $\begin{array}{r}-\overline{0} \\
0 \\
-0\end{array}$ & $\begin{array}{r}-- \\
0 \\
-0 \\
0\end{array}$ & $\begin{array}{r}-- \\
0 \\
-0 \\
-0\end{array}$ & $\begin{array}{r}-- \\
0 \\
-0 \\
0\end{array}$ \\
\hline $\begin{array}{l}6-01 \\
6-03 \\
6-05 \\
6-07 \\
6-09\end{array}$ & $\begin{array}{c}-- \\
-- \\
-\overline{0}+++ \\
1103^{++}\end{array}$ & $\begin{array}{r}-- \\
-- \\
-0 \\
23\end{array}$ & $\begin{array}{l}-- \\
-- \\
-\overline{0} \\
6\end{array}$ & $\begin{array}{l}-- \\
-- \\
0 \\
16\end{array}$ & $\begin{array}{l}-- \\
-\overline{0} \\
1\end{array}$ & $\begin{array}{l}-- \\
-\overline{-} \\
0 \\
0\end{array}$ & $\begin{array}{l}-- \\
-- \\
-0 \\
0\end{array}$ \\
\hline $\begin{array}{l}6-10 \\
6-11 \\
6-12 \\
6-13 \\
6-14\end{array}$ & $\begin{array}{r}877 \\
867 \\
371 \\
1218 \\
1182\end{array}$ & $\begin{array}{r}75 \\
89 \\
104 \\
60 \\
36\end{array}$ & $\begin{array}{l}58 \\
65 \\
65 \\
22 \\
10\end{array}$ & $\begin{array}{l}16 \\
23 \\
37 \\
31 \\
21\end{array}$ & $\begin{array}{l}1 \\
1 \\
2 \\
7 \\
5\end{array}$ & $\begin{array}{l}0 \\
0 \\
0 \\
0 \\
0\end{array}$ & $\begin{array}{l}0 \\
0 \\
0 \\
0 \\
0\end{array}$ \\
\hline $\begin{array}{l}6-15 \\
6-16 \\
6-17 \\
6-18 \\
6-19\end{array}$ & $\begin{array}{r}2624 \\
620 \\
1053 \\
799 \\
1020\end{array}$ & $\begin{array}{l}18 \\
22 \\
19 \\
12 \\
26\end{array}$ & $\begin{array}{r}7 \\
6 \\
7 \\
2 \\
15\end{array}$ & $\begin{array}{r}6 \\
15 \\
7 \\
10 \\
11\end{array}$ & $\begin{array}{l}5 \\
1 \\
5 \\
0 \\
0\end{array}$ & $\begin{array}{l}0 \\
0 \\
0 \\
0 \\
0\end{array}$ & $\begin{array}{l}0 \\
0 \\
0 \\
0 \\
0\end{array}$ \\
\hline $\begin{array}{l}6-20 \\
6-21 \\
6-22 \\
6-23 \\
6-25\end{array}$ & $\begin{array}{r}351 \\
2623 \\
-- \\
908 \\
265\end{array}$ & $\begin{array}{r}18 \\
12 \\
-6 \\
6 \\
4\end{array}$ & $\begin{array}{r}15 \\
-3 \\
-\frac{2}{3}\end{array}$ & $\begin{array}{r}3 \\
-7 \\
-2 \\
1\end{array}$ & $\begin{array}{r}0 \\
-\frac{2}{1} \\
0\end{array}$ & $\begin{array}{r}0 \\
-0 \\
-1 \\
0\end{array}$ & $\begin{array}{r}0 \\
-0 \\
-0 \\
0\end{array}$ \\
\hline $\begin{array}{l}6-27 \\
6-29 \\
7-01 \\
7-03 \\
7-05\end{array}$ & $\begin{array}{r}1030 \\
=- \\
=- \\
=-\end{array}$ & $\begin{array}{l}3 \\
-- \\
-- \\
--\end{array}$ & $\begin{array}{l}2 \\
-- \\
--\end{array}$ & $\begin{array}{l}1 \\
-- \\
--\end{array}$ & $\begin{array}{l}0 \\
-- \\
-- \\
--\end{array}$ & $\begin{array}{l}0 \\
-- \\
-- \\
--\end{array}$ & $\begin{array}{l}0 \\
-- \\
-- \\
--\end{array}$ \\
\hline $7-07$ & -- & -- & -- & -- & -- & -- & -- \\
\hline
\end{tabular}


TABLE 18.- NUMBER OF GREEN TRACER PARTICLES(1), PER 100 GRAMS OF BEDLOAD SAMPLE, COMPOSITED FROM CROSS-CHANNEL SAMPLING POSITIONS SPACED AT 1-METER INTERVALS ACROSS THE CHANNEL WIDTH, EAST FORK RIVER, WYOMING, 1980

SECTION $1425(4)$

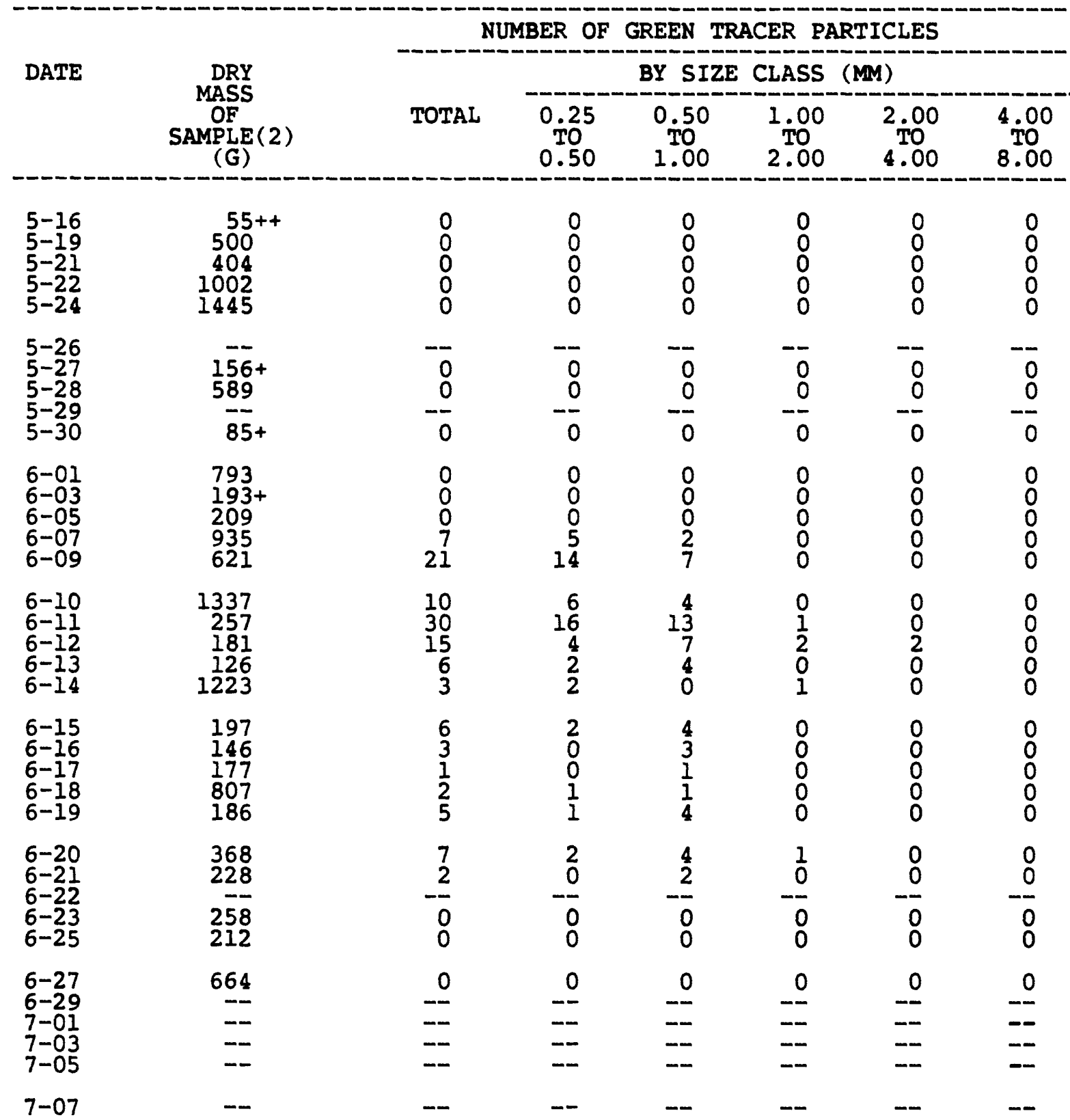


TABLE 18.- NUMBER OF GREEN TRACER PARTICLES(1), PER 100 GRAMS OF BEDLOAD SAMPLE, COMPOSITED FROM CROSS-CHANNEL SAMPLING POSITIONS SPACED AT 1-METER INTERVALS ACROSS THE CHANNEL WIDTH, EAST FORK RIVER, WYOMING, 1980

SECTION 1481

\begin{tabular}{|c|c|c|c|c|c|c|c|}
\hline \multirow{3}{*}{ DATE } & \multirow{3}{*}{$\begin{array}{c}\text { DRY } \\
\text { MASS } \\
\text { OF } \\
\text { SAMPLE (2) } \\
\text { (G) }\end{array}$} & \multicolumn{6}{|c|}{ NUMBER OF GREEN TRACER PARTICLES } \\
\hline & & \multirow[b]{2}{*}{ TOTAL } & \multicolumn{5}{|c|}{ BY SIZE CLASS (MM) } \\
\hline & & & $\begin{array}{c}0.25 \\
\text { TO } \\
0.50\end{array}$ & $\begin{array}{l}0.50 \\
\text { TO } \\
1.00\end{array}$ & $\begin{array}{l}1.00 \\
\text { TO } \\
2.00\end{array}$ & $\begin{array}{l}2.00 \\
\text { TO } \\
4.00\end{array}$ & $\begin{array}{l}4.00 \\
\text { TO } \\
8.00\end{array}$ \\
\hline $\begin{array}{l}5-16 \\
5-19 \\
5-21 \\
5-22 \\
5-24\end{array}$ & $\begin{array}{l}177^{++} \\
320 \\
329 \\
2322 \\
3171\end{array}$ & $\begin{array}{l}0 \\
0 \\
0 \\
0 \\
0\end{array}$ & $\begin{array}{l}0 \\
0 \\
0 \\
0 \\
0\end{array}$ & $\begin{array}{l}0 \\
0 \\
0 \\
0 \\
0\end{array}$ & $\begin{array}{l}0 \\
0 \\
0 \\
0 \\
0\end{array}$ & $\begin{array}{l}0 \\
0 \\
0 \\
0 \\
0\end{array}$ & $\begin{array}{l}0 \\
0 \\
0 \\
0 \\
0\end{array}$ \\
\hline $\begin{array}{l}5-26 \\
5-27 \\
5-28 \\
5-29 \\
5-30\end{array}$ & $\begin{array}{l}\frac{-\overline{3}}{295} \\
\frac{-5}{46}\end{array}$ & $\begin{array}{r}-\overline{0} \\
0 \\
-\overline{0}\end{array}$ & $\begin{array}{r}-- \\
0 \\
-0 \\
0\end{array}$ & $\begin{array}{r}-\overline{0} \\
-0 \\
-0\end{array}$ & $\begin{array}{r}--\overline{0} \\
-\overline{0} \\
-0\end{array}$ & $\begin{array}{r}-\overline{0} \\
-\frac{0}{0}\end{array}$ & $\begin{array}{r}-\overline{0} \\
0 \\
-\frac{1}{0}\end{array}$ \\
\hline $\begin{array}{l}6-01 \\
6-03 \\
6-05 \\
6-07 \\
6-09\end{array}$ & $\begin{array}{l}187+ \\
235 \\
197 \\
389 \\
2148\end{array}$ & $\begin{array}{r}0 \\
0 \\
0 \\
3 \\
16\end{array}$ & $\begin{array}{l}0 \\
0 \\
0 \\
3 \\
7\end{array}$ & $\begin{array}{l}0 \\
0 \\
0 \\
0 \\
9\end{array}$ & $\begin{array}{l}0 \\
0 \\
0 \\
0 \\
0\end{array}$ & $\begin{array}{l}0 \\
0 \\
0 \\
0 \\
0\end{array}$ & $\begin{array}{l}0 \\
0 \\
0 \\
0 \\
0\end{array}$ \\
\hline $\begin{array}{l}6-10 \\
6-11 \\
6-12 \\
6-13 \\
6-14\end{array}$ & $\begin{array}{r}2785 \\
1497 \\
685 \\
767 \\
1433\end{array}$ & $\begin{array}{r}37 \\
13 \\
20 \\
5 \\
6\end{array}$ & $\begin{array}{r}27 \\
6 \\
8 \\
1 \\
4\end{array}$ & $\begin{array}{r}10 \\
5 \\
9 \\
3 \\
1\end{array}$ & $\begin{array}{l}0 \\
1 \\
3 \\
1 \\
1\end{array}$ & $\begin{array}{l}0 \\
1 \\
0 \\
0 \\
0\end{array}$ & $\begin{array}{l}0 \\
0 \\
0 \\
0 \\
0\end{array}$ \\
\hline $\begin{array}{l}6-15 \\
6-16 \\
6-17 \\
6-18 \\
6-19\end{array}$ & $\begin{array}{r}2451 \\
824 \\
674 \\
2022 \\
1665\end{array}$ & $\begin{array}{l}2 \\
5 \\
1 \\
3 \\
2\end{array}$ & $\begin{array}{l}0 \\
4 \\
0 \\
2 \\
0\end{array}$ & $\begin{array}{l}\frac{1}{1} \\
\frac{1}{1} \\
\frac{1}{2}\end{array}$ & $\begin{array}{l}1 \\
0 \\
0 \\
0 \\
0\end{array}$ & $\begin{array}{l}0 \\
0 \\
0 \\
0 \\
0\end{array}$ & $\begin{array}{l}0 \\
0 \\
0 \\
0 \\
0\end{array}$ \\
\hline $\begin{array}{l}6-20 \\
6-21\end{array}$ & $\begin{array}{l}11466 \\
1233\end{array}$ & $\frac{4}{3}$ & $\begin{array}{l}0 \\
1\end{array}$ & $\frac{4}{2}$ & $\begin{array}{l}0 \\
0\end{array}$ & $\begin{array}{l}0 \\
0\end{array}$ & $\begin{array}{l}0 \\
0\end{array}$ \\
\hline $\begin{array}{l}6-22 \\
6-23 \\
6-25\end{array}$ & $\begin{array}{r}7 \overline{7 \overline{6}} \\
121 \overline{6}\end{array}$ & $\frac{1}{0}$ & $\overline{0}$ & $\frac{1}{0}$ & o & $\begin{array}{l}0 \\
0\end{array}$ & 0 \\
\hline $6-27$ & 1479 & 3 & 0 & 3 & 0 & 0 & 0 \\
\hline $\begin{array}{l}6-29 \\
7-01\end{array}$ & $\overline{-}$ & $\overline{-}$ & $\overline{-}$ & $\overline{-}$ & $\overline{-}$ & $\overline{--}$ & $\overline{--}$ \\
\hline $7-0 \overline{3}$ & - & -- & -- & - & -- & -- & -- \\
\hline $7-05$ & -- & -- & -- & -- & -- & -- & 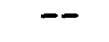 \\
\hline $7-07$ & -- & -- & -- & -- & -- & -- & -- \\
\hline
\end{tabular}


TABLE 18.- NUMBER OF GREEN TRACER PARTICLES(1), PER 100 GRAMS OF BEDLOAD SAMPLE, COMPOSITED FROM CROSS-CHANNEL SAMPLING POSITIONS SPACED AT I-METER INTERVALS ACROSS THE CHANNEL WIDTH, EAST FORK RIVER, WYOMING, 1980

SECTION 1481(3)

\begin{tabular}{|c|c|c|c|c|c|c|c|}
\hline \multirow{3}{*}{ DATE } & \multirow{3}{*}{$\begin{array}{c}\text { DRY } \\
\text { MASS } \\
\text { OF } \\
\text { SAMPLE (2) } \\
\text { (G) }\end{array}$} & \multicolumn{6}{|c|}{ NUMBER OF GREEN TRACER PARTICLES } \\
\hline & & \multirow[b]{2}{*}{ TOTAL } & \multicolumn{5}{|c|}{ BY SIZE CLASS (MM) } \\
\hline & & & $\begin{array}{r}0.25 \\
\text { TO } \\
0.50\end{array}$ & $\begin{array}{r}0.50 \\
\text { TO } \\
1.00\end{array}$ & $\begin{array}{l}1.00 \\
2.00\end{array}$ & $\begin{array}{l}2.00 \\
\text { TO } \\
4.00\end{array}$ & $\begin{array}{c}4.00 \\
\text { TO } \\
8.00\end{array}$ \\
\hline $\begin{array}{l}5-16 \\
5-19 \\
5-21 \\
5-22 \\
5-24\end{array}$ & $\begin{array}{l}-- \\
177+ \\
468 \\
--\end{array}$ & $\begin{array}{r}-- \\
-\overline{0} \\
0 \\
--\end{array}$ & $\begin{array}{r}-- \\
-\overline{0} \\
0 \\
--\end{array}$ & $\begin{array}{r}-- \\
-\overline{0} \\
--\end{array}$ & $\begin{array}{r}-- \\
-\overline{0} \\
0 \\
--\end{array}$ & $\begin{array}{r}-- \\
-\overline{0} \\
0 \\
--\end{array}$ & $\begin{array}{c}-- \\
-\overline{0} \\
0\end{array}$ \\
\hline $\begin{array}{l}5-26 \\
5-27 \\
5-28 \\
5-29 \\
5-30\end{array}$ & $\begin{array}{l}-\overline{0+++} \\
-\overline{-} \\
--\end{array}$ & $\begin{array}{l}-\overline{0} \\
-- \\
--\end{array}$ & $\begin{array}{l}-\overline{0} \\
-- \\
--\end{array}$ & $\begin{array}{l}-\overline{0} \\
-- \\
--\end{array}$ & $\begin{array}{l}-\overline{0} \\
-- \\
--\end{array}$ & $\begin{array}{l}-- \\
-- \\
--\end{array}$ & $\begin{array}{l}-- \\
-- \\
--\end{array}$ \\
\hline $\begin{array}{l}6-01 \\
6-03 \\
6-05 \\
6-07 \\
6-09\end{array}$ & 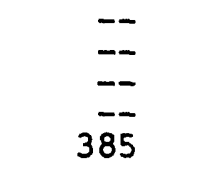 & $\begin{array}{l}\overline{-} \\
\overline{72}\end{array}$ & $\begin{array}{l}-1 \\
\overline{-1} \\
60\end{array}$ & 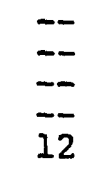 & $\begin{array}{l}-- \\
-\overline{0} \\
-\overline{0}\end{array}$ & $\overline{-}$ & $\overline{--}$ \\
\hline $\begin{array}{l}6-10 \\
6-11 \\
6-12 \\
6-13 \\
6-14\end{array}$ & $\begin{array}{r}3194 \\
1611 \\
345 \\
1365 \\
2335\end{array}$ & $\begin{array}{l}70 \\
54 \\
15 \\
14 \\
13\end{array}$ & $\begin{array}{r}46 \\
11 \\
\frac{1}{1} \\
\frac{1}{2}\end{array}$ & $\begin{array}{l}23 \\
32 \\
12 \\
10 \\
7\end{array}$ & $\begin{array}{r}1 \\
10 \\
2 \\
2 \\
2\end{array}$ & $\begin{array}{l}0 \\
1 \\
0 \\
1 \\
2\end{array}$ & $\begin{array}{l}0 \\
0 \\
0 \\
0 \\
0\end{array}$ \\
\hline $\begin{array}{l}6-15 \\
6-16 \\
6-17 \\
6-18 \\
6-19\end{array}$ & $\begin{array}{r}1054 \\
184 \\
360 \\
2209 \\
827\end{array}$ & $\begin{array}{r}3 \\
0 \\
2 \\
6 \\
11\end{array}$ & $\begin{array}{l}1 \\
0 \\
0 \\
3 \\
2\end{array}$ & $\begin{array}{l}2 \\
0 \\
1 \\
3 \\
8\end{array}$ & $\begin{array}{l}0 \\
0 \\
1 \\
0 \\
1\end{array}$ & $\begin{array}{l}0 \\
0 \\
0 \\
0 \\
0\end{array}$ & $\begin{array}{l}0 \\
0 \\
0 \\
0 \\
0\end{array}$ \\
\hline $\begin{array}{l}6-20 \\
6-21\end{array}$ & $\begin{array}{l}1600 \\
1029\end{array}$ & $\begin{array}{r}27 \\
0\end{array}$ & $\begin{array}{l}5 \\
0\end{array}$ & $\begin{array}{r}16 \\
0\end{array}$ & $\begin{array}{l}5 \\
0\end{array}$ & $\begin{array}{l}1 \\
0\end{array}$ & $\begin{array}{l}0 \\
0\end{array}$ \\
\hline $\begin{array}{l}6-23 \\
6-25\end{array}$ & $\begin{array}{l}465 \\
347\end{array}$ & $\begin{array}{l}3 \\
0\end{array}$ & $\begin{array}{l}0 \\
0\end{array}$ & $\begin{array}{l}3 \\
0\end{array}$ & $\begin{array}{l}0 \\
0\end{array}$ & $\begin{array}{l}0 \\
0\end{array}$ & $\begin{array}{l}0 \\
0\end{array}$ \\
\hline $\begin{array}{l}6-27 \\
6-29\end{array}$ & 1109 & 1 & $-\underline{0}$ & -1 & -0 & -0 & $-\underline{0}$ \\
\hline $7-01$ & - & - & -- & -- & - & - & - \\
\hline $7-05$ & - & -- & -- & - & $\overline{-}$ & 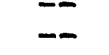 & - \\
\hline $7-07$ & -- & - & -- & -- & - & -- & - \\
\hline
\end{tabular}


TABLE 18.- NUMBER OF GREEN TRACER PARTICLES(1), PER 100 GRAMS OF BEDLOAD SAMPLE, COMPOSITED FROM CROSS-CHANNEL SAMPLING POSITIONS

SPACED AT 1-METER INTERVALS ACROSS THE CHANNEL WIDTH,

EAST FORK RIVER, WYOMING, 1980

SECTION 1533

\begin{tabular}{|c|c|c|c|c|c|c|c|}
\hline \multirow{3}{*}{\multicolumn{2}{|c|}{$\begin{array}{cc} & \\
\text { DATE } & \text { DRY } \\
& \text { MASS } \\
\text { OF } \\
\text { SAMPLE (2) } \\
& \text { (G) }\end{array}$}} & \multicolumn{6}{|c|}{ NUMBER OF GREEN TRACER PARTICLES } \\
\hline & & \multirow[b]{2}{*}{ TOTAL } & \multicolumn{5}{|c|}{ BY SIZE CLASS (MM) } \\
\hline & & & $\begin{array}{l}0.25 \\
\text { TO } \\
0.50\end{array}$ & $\begin{array}{l}0.50 \\
\text { TO } \\
1.00\end{array}$ & $\begin{array}{l}1.00 \\
2.00\end{array}$ & $\begin{array}{l}2.00 \\
\text { TO } \\
4.00\end{array}$ & $\begin{array}{l}4.00 \\
\text { TO } \\
8.00\end{array}$ \\
\hline $\begin{array}{l}5-16 \\
5-19 \\
5-21 \\
5-22 \\
5-24\end{array}$ & $\begin{array}{c}72++ \\
22+++ \\
256 \\
2210 \\
1455\end{array}$ & $\begin{array}{l}0 \\
0 \\
0 \\
0 \\
0\end{array}$ & $\begin{array}{l}0 \\
0 \\
0 \\
0 \\
0\end{array}$ & $\begin{array}{l}0 \\
0 \\
0 \\
0 \\
0\end{array}$ & $\begin{array}{l}0 \\
0 \\
0 \\
0 \\
0\end{array}$ & $\begin{array}{l}0 \\
0 \\
0 \\
0 \\
0\end{array}$ & $\begin{array}{l}0 \\
0 \\
0 \\
0 \\
0\end{array}$ \\
\hline $\begin{array}{l}5-26 \\
5-27 \\
5-28\end{array}$ & $\begin{array}{l}11 \overline{39} \\
1385\end{array}$ & $\begin{array}{c}-- \\
0\end{array}$ & $\begin{array}{r}-- \\
0\end{array}$ & $\begin{array}{c}-\overline{0} \\
0\end{array}$ & $\begin{array}{r}-- \\
0\end{array}$ & $\begin{array}{r}-- \\
0 \\
0\end{array}$ & $\begin{array}{c}-- \\
0\end{array}$ \\
\hline $\begin{array}{l}5-29 \\
5-30\end{array}$ & 249 & 0 & $\overline{0}$ & 0 & $\overline{0}$ & 0 & $\overline{0}$ \\
\hline $\begin{array}{l}6-01 \\
6-03 \\
6-05 \\
6-07 \\
6-09\end{array}$ & $\begin{array}{l}452 \\
169 \\
521 \\
342 \\
979\end{array}$ & $\begin{array}{r}0 \\
0 \\
7 \\
7 \\
128\end{array}$ & $\begin{array}{r}0 \\
0 \\
6 \\
1 \\
109\end{array}$ & $\begin{array}{r}0 \\
0 \\
1 \\
6 \\
19\end{array}$ & $\begin{array}{l}0 \\
0 \\
0 \\
0 \\
0\end{array}$ & $\begin{array}{l}0 \\
0 \\
0 \\
0 \\
0\end{array}$ & $\begin{array}{l}0 \\
0 \\
0 \\
0 \\
0\end{array}$ \\
\hline $\begin{array}{l}6-10 \\
6-11 \\
6-12 \\
6-13 \\
6-14\end{array}$ & $\begin{array}{l}4669 \\
1344 \\
1319 \\
3564 \\
3557\end{array}$ & $\begin{array}{r}33 \\
12 \\
7 \\
1 \\
0\end{array}$ & $\begin{array}{r}14 \\
5 \\
4 \\
0 \\
0\end{array}$ & $\begin{array}{r}13 \\
7 \\
2 \\
1 \\
0\end{array}$ & $\begin{array}{l}5 \\
0 \\
1 \\
0 \\
0\end{array}$ & $\begin{array}{l}1 \\
0 \\
0 \\
0 \\
0\end{array}$ & $\begin{array}{l}0 \\
0 \\
0 \\
0 \\
0\end{array}$ \\
\hline $6-15$ & 2663 & 1 & 0 & 1 & 0 & 0 & 0 \\
\hline $\begin{array}{l}6-17 \\
6-18 \\
6-19\end{array}$ & $\begin{array}{r}2076 \\
4222 \\
745\end{array}$ & $\begin{array}{r}1 \\
8 \\
12\end{array}$ & $\begin{array}{l}0 \\
4 \\
3\end{array}$ & $\begin{array}{l}\frac{1}{3} \\
7\end{array}$ & $\begin{array}{l}0 \\
1 \\
2\end{array}$ & $\begin{array}{l}0 \\
0 \\
0\end{array}$ & $\begin{array}{l}0 \\
0 \\
0\end{array}$ \\
\hline $\begin{array}{l}6-20 \\
6-21\end{array}$ & $\begin{array}{l}1383 \\
2034 \\
\end{array}$ & $\begin{array}{r}3 \\
2 \\
-\end{array}$ & $\begin{array}{r}2 \\
0 \\
-\end{array}$ & $\frac{1}{2}$ & $\begin{array}{l}0 \\
0\end{array}$ & $\begin{array}{l}0 \\
0\end{array}$ & $\begin{array}{r}0 \\
0 \\
-\end{array}$ \\
\hline $\begin{array}{l}6-23 \\
6-25\end{array}$ & $\begin{array}{l}1253 \\
1756\end{array}$ & 0 & 0 & $\begin{array}{l}0 \\
0\end{array}$ & $\begin{array}{l}0 \\
1\end{array}$ & $\begin{array}{l}0 \\
0\end{array}$ & 0 \\
\hline $\begin{array}{l}6-27 \\
6-20\end{array}$ & 3070 & 4 & 2 & 2 & $\underline{-0}$ & $\underline{-}$ & $-\underline{0}$ \\
\hline $7-01$ & -- & -- & -- & -- & -- & -- & -- \\
\hline $\begin{array}{l}7-0 \overline{3} \\
7-05\end{array}$ & $\begin{array}{l}1043 \\
1247\end{array}$ & $\begin{array}{l}2 \\
3\end{array}$ & $\begin{array}{l}0 \\
0\end{array}$ & $\begin{array}{l}2 \\
2\end{array}$ & $\begin{array}{l}0 \\
1\end{array}$ & $\begin{array}{l}0 \\
0\end{array}$ & $\begin{array}{l}0 \\
0\end{array}$ \\
\hline $7-07$ & $81+$ & 0 & 0 & 0 & 0 & 0 & 0 \\
\hline
\end{tabular}


TABLE 18.- NUMBER OF GREEN TRACER PARTICLES(1), PER 100 GRAMS OF BEDLOAD SAMPLE, COMPOSITED FROM CROSS-CHANNEL SAMPLING POSITIONS SPACED AT 1-METER INTERVALS ACROSS THE CHANNEL WIDTH, EAST FORK RIVER, WYOMING, 1980

SECTION 1573

\begin{tabular}{|c|c|c|c|c|c|c|c|}
\hline \multirow{3}{*}{ DATE } & \multirow{3}{*}{$\begin{array}{c}\text { DRY } \\
\text { MASS } \\
\text { OF } \\
\text { SAMPLE(2) } \\
\text { (G) }\end{array}$} & \multicolumn{6}{|c|}{ NUMBER OF GREEN TRACER PARTICLES } \\
\hline & & \multirow[b]{2}{*}{ TOTAL } & \multicolumn{5}{|c|}{ BY SIZE CLASS (MM) } \\
\hline & & & $\begin{array}{l}0.25 \\
\text { TO } \\
0.50\end{array}$ & $\begin{array}{l}0.50 \\
\text { TO } \\
1.00\end{array}$ & $\begin{array}{l}1.00 \\
\text { TO } \\
2.00\end{array}$ & $\begin{array}{l}2.00 \\
\text { TO } \\
4.00\end{array}$ & $\begin{array}{c}4.00 \\
\text { TO } \\
8.00\end{array}$ \\
\hline $\begin{array}{l}5-16 \\
5-19 \\
5-21 \\
5-22 \\
5-24\end{array}$ & $\begin{array}{l}0+++ \\
109+++ \\
223 \\
5886 \\
3327\end{array}$ & $\begin{array}{l}0 \\
0 \\
0 \\
0 \\
0\end{array}$ & $\begin{array}{l}0 \\
0 \\
0 \\
0 \\
0\end{array}$ & $\begin{array}{l}0 \\
0 \\
0 \\
0 \\
0\end{array}$ & $\begin{array}{l}0 \\
0 \\
0 \\
0 \\
0\end{array}$ & $\begin{array}{l}0 \\
0 \\
0 \\
0 \\
0\end{array}$ & $\begin{array}{l}0 \\
0 \\
0 \\
0 \\
0\end{array}$ \\
\hline $\begin{array}{l}5-26 \\
5-27 \\
5-28\end{array}$ & $\begin{array}{c}10 \overline{8} \\
102+\end{array}$ & $\begin{array}{r}-- \\
0 \\
0\end{array}$ & $\begin{array}{r}-\overline{0} \\
0\end{array}$ & $\begin{array}{r}-\overline{0} \\
0\end{array}$ & $\begin{array}{r}-\overline{0} \\
0\end{array}$ & $\begin{array}{r}-\overline{0} \\
0\end{array}$ & $\begin{array}{r}-\overline{0} \\
0\end{array}$ \\
\hline $5-30$ & 471 & 0 & 0 & 0 & 0 & 0 & 0 \\
\hline $\begin{array}{l}6-01 \\
6-03 \\
6-05 \\
6-07 \\
6-09\end{array}$ & $\begin{array}{c}93+ \\
110+ \\
45++ \\
59++ \\
2507\end{array}$ & $\begin{array}{r}0 \\
0 \\
0 \\
185 \\
77\end{array}$ & $\begin{array}{r}0 \\
0 \\
0 \\
92 \\
41\end{array}$ & $\begin{array}{r}0 \\
0 \\
0 \\
89 \\
32\end{array}$ & $\begin{array}{l}0 \\
0 \\
0 \\
4 \\
3\end{array}$ & $\begin{array}{l}0 \\
0 \\
0 \\
0 \\
0\end{array}$ & $\begin{array}{l}0 \\
0 \\
0 \\
0 \\
1\end{array}$ \\
\hline $\begin{array}{l}6-10 \\
6-11 \\
6-12 \\
6-13 \\
6-14\end{array}$ & $\begin{array}{l}2398 \\
1189 \\
4094 \\
3490 \\
5136\end{array}$ & $\begin{array}{r}108 \\
6 \\
2 \\
0 \\
1\end{array}$ & $\begin{array}{r}58 \\
3 \\
1 \\
0 \\
0\end{array}$ & $\begin{array}{r}42 \\
3 \\
0 \\
0 \\
1\end{array}$ & $\begin{array}{l}8 \\
0 \\
1 \\
0 \\
0\end{array}$ & $\begin{array}{l}0 \\
0 \\
0 \\
0 \\
0\end{array}$ & $\begin{array}{l}0 \\
0 \\
0 \\
0 \\
0\end{array}$ \\
\hline $6-15$ & 1507 & 1 & 0 & 1 & 0 & 0 & 0 \\
\hline $\begin{array}{l}6-17 \\
6-18 \\
6-19\end{array}$ & $\begin{array}{l}5589 \\
3202 \\
1359\end{array}$ & $\begin{array}{l}3 \\
8 \\
5\end{array}$ & $\frac{1}{1}$ & $\begin{array}{l}2 \\
6 \\
1\end{array}$ & $\begin{array}{l}0 \\
1 \\
1\end{array}$ & $\begin{array}{l}0 \\
0 \\
0\end{array}$ & $\begin{array}{l}0 \\
0 \\
0\end{array}$ \\
\hline $\begin{array}{l}6-20 \\
6-21\end{array}$ & $\begin{array}{l}4272 \\
3339\end{array}$ & $\frac{1}{1}$ & $\begin{array}{l}0 \\
1\end{array}$ & $\begin{array}{l}1 \\
0\end{array}$ & $\begin{array}{l}0 \\
0\end{array}$ & $\begin{array}{l}0 \\
0\end{array}$ & $\begin{array}{l}0 \\
0\end{array}$ \\
\hline $\begin{array}{l}6-23 \\
6-25\end{array}$ & $\begin{array}{l}1849 \\
2192\end{array}$ & $\begin{array}{l}0 \\
2\end{array}$ & $\begin{array}{l}0 \\
1\end{array}$ & $\begin{array}{l}0 \\
1\end{array}$ & $\begin{array}{l}0 \\
0\end{array}$ & $\begin{array}{l}0 \\
0\end{array}$ & $\begin{array}{l}0 \\
0\end{array}$ \\
\hline $6-27$ & 2560 & 10 & 3 & 5 & 2 & 0 & 0 \\
\hline $7-01$ & - & - & - & $=$ & -- & $=$ & $=$ \\
\hline $7-03$ & $=-$ & -- & -- & -- & -- & -- & -- \\
\hline $7-05$ & 269 & 2 & 0 & 1 & 1 & 0 & 0 \\
\hline $7-07$ & 393 & 0 & 0 & 0 & 0 & 0 & 0 \\
\hline
\end{tabular}


TABLE 18.- NUMBER OF GREEN TRACER PARTICLES(1), PER 100 GRAMS OF BEDLOAD SAMPLE, COMPOSITED FROM CROSS-CHANNEL SAMPLING POSITIONS SPACED AT 1-METER INTERVALS ACROSS THE CHANNEL WIDTH, EAST FORK RIVER, WYOMING, 1980

SECTION 1610

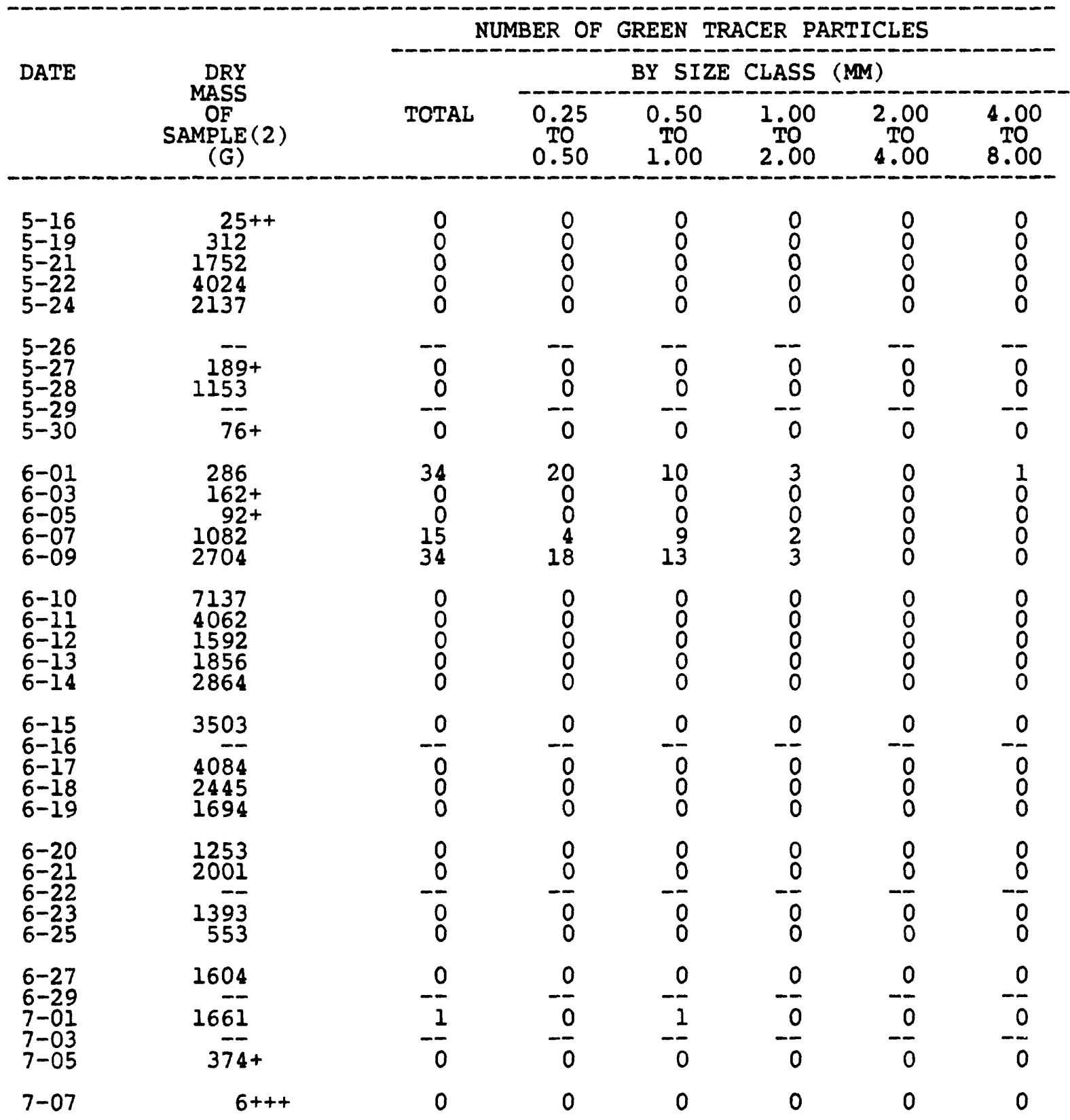


TABLE 18.- NUMBER OF GREEN TRACER PARTICLES(1), PER 100 GRAMS OF BEDLOAD SAMPLE, COMPOSITED FROM CROSS-CHANNEL SAMPLING POSITIONS SPACED AT 1-METER INTERVALS ACROSS THE CHANNEL WIDTH, EAST FORK RIVER, WYOMING, 1980--CONTINUED

(1) GREEN TRACER PARTICLES PLACED AS BED MATERIAL 10 METERS UPSTREAM

OF SECTION 1610 ON MAY 31, 1980.

(2) REPRESENTS TOTAL MASS, IN' GRAMS, RETAINED IN BEDLOAD SAMPLER AS MEASURED IN THE FIELD. FOR SUBSEQUENT ANALYSIS OF NUMBER AND SIZE OF TRACER, PARTICLES SMALLER THAN $0.25 \mathrm{MM}$ AND LARGER THAN $8.0 \mathrm{MM}$ WERE DISCÁRDED. REMAINING SAMPLES WEIGHING MORE THAN IOO GRAMS WERE CUT TO A STANDARD 100 GRAM-PORTION; SAMPLES WEIGHING LESS THAN 100 GRAMS WERE ANALYZED INTACT.

(3) SMALL OVERFLOW CHANNEL ADJACENT TO SECTION.

(4) DOES NOT INCLUDE TRACER PARTICLES MEASURED IN TRANSPORT AT SECTION 1400 , A BYPASS CHANNEL COMMON TO SECTIONS 1360, 1396, AND 1425.

(5) BYPASS CHANNELL. SEE FIGURE 3.

+ MASS OF SAMPLE ANALYZED WAS BETWEEN 50 AND 100 GRAMS; NUMBER OF TRACER PARTICLES ADJUSTED TO AN EOUIVALENT IOO-GRAM SAMPLE; EXTRAPOLATED NUMBERS ARE CONSIDERED REL IABLE.

+ MASS OF SAMPLE ANALYZED WAS BETWEEN 10 AND 50 GRAMS; NUMBER OF TRACER PARTICLES ADJUSTED TO AN EQUIVALENT 100-GRAM SAMPLE; CARE SHOULD BE TAKEN IN USING THE EXTRAPOLATED NUMBERS.

+++ MASS OF SAMPLE ANALYZED WAS BETWEEN 0 AND 10 GRAMS: NUMBER OF TRACER PARTICLES ADJUSTED TO AN EQUIVALENT 100-GRAM SAMPLE; EXTRAPOLATED NUMBERS ARE NOT CONSIDERED RELIABLE. 
TABLE 19.- NUMBER OF PINK TRACER PARTICLES(1), PER 100 GRAMS OF BEDLOAD SAMPLE, COLLECTED AT ONE OR MORE CROSS-CHANNEL SAMPLING POSITIONS SPACED AT I-METER INTERVALS ACROSS THE CHANNEL WIDTH, EAST FORK RIVER, WYOMING, 1980

SECTION 1241

\begin{tabular}{|c|c|c|c|c|c|c|c|c|c|}
\hline \multirow{3}{*}{ DATE } & \multirow{3}{*}{\multicolumn{2}{|c|}{$\begin{array}{l}\text { SAMPLING } \\
\text { POSITION } \\
\text { FROM TO }\end{array}$}} & \multirow{3}{*}{$\begin{array}{c}\text { DRY } \\
\text { MASS } \\
\text { OF } \\
\text { SAMPLE (2) } \\
\text { (G) }\end{array}$} & \multicolumn{6}{|c|}{ NUMBER OF PINK TRACER PARTICLES } \\
\hline & & & & \multirow[b]{2}{*}{ TOTAL } & \multicolumn{5}{|c|}{ BY SIZE CLASS (MM) } \\
\hline & & & & & $\begin{array}{l}0.25 \\
\text { TO } \\
0.50\end{array}$ & $\begin{array}{l}0.50 \\
\text { TO } \\
1.00\end{array}$ & $\begin{array}{l}1.00 \\
2.00\end{array}$ & $\begin{array}{l}2.00 \\
\text { TO } \\
4.00\end{array}$ & $\begin{array}{l}4.00 \\
\text { TO } \\
8.00\end{array}$ \\
\hline $6-01$ & 10 & 22 & 1777 & 5 & 3 & 2 & 0 & 0 & 0 \\
\hline $6-03$ & 10 & 22 & 452 & 6 & 2 & 4 & 0 & 0 & 0 \\
\hline $6-05$ & 10 & 22 & 759 & 0 & 0 & 0 & 0 & 0 & 0 \\
\hline $6-07$ & 10 & 21 & 1177 & 6 & 5 & 1 & 0 & 0 & 0 \\
\hline $6-09$ & 2 & 21 & 1501 & 0 & 0 & 0 & 0 & 0 & 0 \\
\hline $6-10$ & 2 & 21 & 690 & 0 & 0 & 0 & 0 & 0 & 0 \\
\hline $6-11$ & 3 & 21 & 945 & 4 & 3 & 1 & 0 & 0 & 0 \\
\hline $6-12$ & 2 & 21 & 324 & 4 & 3 & 1 & 0 & 0 & 0 \\
\hline $6-13$ & 7 & 20 & 308 & 0 & 0 & 0 & 0 & 0 & 0 \\
\hline $6-14$ & 2 & 21 & 1093 & 2 & 2 & 0 & 0 & 0 & 0 \\
\hline $6-15$ & 2 & 21 & 1350 & 4 & 1 & 2 & 1 & 0 & 0 \\
\hline $6-16$ & 10 & 22 & 301 & 11 & 3 & 7 & 1 & 0 & 0 \\
\hline $6-17$ & 7 & 22 & 1431 & 9 & 2 & 7 & 0 & 0 & 0 \\
\hline $6-18$ & 2 & 20 & 3603 & 1 & 0 & 0 & 1 & 0 & 0 \\
\hline $6-19$ & 2 & 22 & 1291 & 3 & 3 & 0 & 0 & 0 & 0 \\
\hline $6-20$ & 3 & 21 & 2036 & 7 & 1 & 4 & 1 & 1 & 0 \\
\hline $6-21$ & 14 & $\begin{array}{l}13 \\
25\end{array}$ & $\begin{array}{l}4824 \\
1293\end{array}$ & $\begin{array}{l}8 \\
5\end{array}$ & $\begin{array}{l}4 \\
0\end{array}$ & $\begin{array}{l}4 \\
5\end{array}$ & $\begin{array}{l}0 \\
0\end{array}$ & $\begin{array}{l}0 \\
0\end{array}$ & $\begin{array}{l}0 \\
0\end{array}$ \\
\hline $6-23$ & $\begin{array}{r}7 \\
12\end{array}$ & $\frac{11}{19}$ & $\begin{array}{l}187 \\
266\end{array}$ & $\begin{array}{r}13 \\
3\end{array}$ & $\begin{array}{l}4 \\
0\end{array}$ & $\begin{array}{l}8 \\
1\end{array}$ & $\frac{1}{2}$ & $\begin{array}{l}0 \\
0\end{array}$ & $\begin{array}{l}0 \\
0\end{array}$ \\
\hline $6-25$ & 9 & 21 & 5117 & 15 & 1 & 11 & 3 & 0 & 0 \\
\hline $6-27$ & 11 & $\begin{array}{l}10 \\
20\end{array}$ & $\begin{array}{l}667 \\
937\end{array}$ & $\begin{array}{l}21 \\
16\end{array}$ & $\begin{array}{l}5 \\
2\end{array}$ & 11 & $\begin{array}{l}4 \\
4\end{array}$ & $\frac{1}{1}$ & $\begin{array}{l}0 \\
0\end{array}$ \\
\hline
\end{tabular}


TABLE 19.- NUMBER OF PINK TRACER PARTICLES(1), PER 100 GRAMS OF BEDLOAD SAMPLE, COLLECTED AT ONE OR MORE CROSS-CHANNEL SAMPLING POSITIONS SPACED AT 1-METER INTERVALS ACROSS THE CHANNEL WIDTH, EAST FORK RIVER, WYOMING, 1980

\section{SECTION 1284}

\begin{tabular}{|c|c|c|c|c|c|c|c|c|c|}
\hline \multirow{3}{*}{ DATE } & \multirow{3}{*}{\multicolumn{2}{|c|}{$\begin{array}{l}\text { SAMPLING } \\
\text { POSITION } \\
\text { FROM TO }\end{array}$}} & \multirow{3}{*}{$\begin{array}{c}\text { DRY } \\
\text { MASS } \\
\text { OF } \\
\text { SAMPLE (2) } \\
\text { (G) }\end{array}$} & \multicolumn{2}{|c|}{ NUMBER OF } & \multicolumn{3}{|c|}{ PINK TRACER PARTICLES } & \multirow{2}{*}{$-\cdots$} \\
\hline & & & & \multirow[b]{2}{*}{ TOTAL } & \multicolumn{3}{|c|}{ BY SIZE CLASS } & $(\mathbf{M})$ & \\
\hline & & & & & $\begin{array}{l}0.25 \\
\text { To } \\
0.50\end{array}$ & $\begin{array}{l}0.50 \\
\text { TO } \\
1.00\end{array}$ & $\begin{array}{l}1.00 \\
2.00\end{array}$ & $\begin{array}{l}2.00 \\
400 \\
4.00\end{array}$ & $\begin{array}{l}4.00 \\
8.00\end{array}$ \\
\hline $6-01$ & 3 & 17 & 1178 & 3 & 0 & 3 & 0 & 0 & 0 \\
\hline $6-03$ & 1 & 18 & 741 & 1 & 0 & 1 & 0 & 0 & 0 \\
\hline $6-05$ & 3 & 16 & 944 & 0 & 0 & 0 & 0 & 0 & 0 \\
\hline $6-07$ & 2 & 17 & 969 & 5 & 3 & 2 & 0 & 0 & 0 \\
\hline $6-09$ & 2 & 17 & 2293 & 0 & 0 & 0 & 0 & 0 & 0 \\
\hline $6-10$ & $\begin{array}{r}3 \\
11\end{array}$ & $\begin{array}{l}10 \\
17\end{array}$ & $\begin{array}{r}867 \\
1039\end{array}$ & 4 & $\frac{1}{6}$ & $\begin{array}{l}2 \\
1\end{array}$ & $\begin{array}{l}0 \\
0\end{array}$ & $\frac{1}{0}$ & $\begin{array}{l}0 \\
0\end{array}$ \\
\hline $6-11$ & $\begin{array}{r}3 \\
12 \\
15\end{array}$ & $\begin{array}{l}11 \\
14 \\
18\end{array}$ & $\begin{array}{l}164 \\
475 \\
239\end{array}$ & $\begin{array}{l}4 \\
13 \\
10\end{array}$ & $\begin{array}{r}3 \\
10 \\
6\end{array}$ & $\begin{array}{l}1 \\
3 \\
4\end{array}$ & $\begin{array}{l}0 \\
0 \\
0\end{array}$ & $\begin{array}{l}0 \\
0 \\
0\end{array}$ & $\begin{array}{l}0 \\
0 \\
0\end{array}$ \\
\hline $6-12$ & 3 & 18 & 374 & 3 & 1 & 2 & 0 & 0 & 0 \\
\hline $6-13$ & 13 & $\begin{array}{l}10 \\
17\end{array}$ & $\begin{array}{l}141 \\
328\end{array}$ & $\begin{array}{l}0 \\
9\end{array}$ & $\begin{array}{l}0 \\
6\end{array}$ & $\begin{array}{l}0 \\
3\end{array}$ & $\begin{array}{l}0 \\
0\end{array}$ & $\begin{array}{l}0 \\
0\end{array}$ & $\begin{array}{l}0 \\
0\end{array}$ \\
\hline $6-14$ & 2 & 16 & 897 & 11 & 8 & 3 & 0 & 0 & 0 \\
\hline $6-15$ & $\begin{array}{r}2 \\
9 \\
14\end{array}$ & $\begin{array}{r}8 \\
13 \\
18\end{array}$ & $\begin{array}{l}1065 \\
725 \\
1178\end{array}$ & $\begin{array}{r}1 \\
5 \\
14\end{array}$ & $\begin{array}{l}0 \\
3 \\
3\end{array}$ & $\begin{array}{r}0 \\
2 \\
11\end{array}$ & $\begin{array}{l}0 \\
0 \\
0\end{array}$ & $\begin{array}{l}1 \\
0 \\
0\end{array}$ & $\begin{array}{l}0 \\
0 \\
0\end{array}$ \\
\hline $6-16$ & $\begin{array}{r}4 \\
7 \\
13\end{array}$ & $\begin{array}{r}6 \\
12 \\
16\end{array}$ & $\begin{array}{l}361 \\
697 \\
289\end{array}$ & $\begin{array}{l}1 \\
18 \\
17\end{array}$ & $\begin{array}{l}0 \\
8 \\
9\end{array}$ & $\begin{array}{r}0 \\
10 \\
8\end{array}$ & $\begin{array}{l}1 \\
0 \\
0\end{array}$ & $\begin{array}{l}0 \\
0 \\
0\end{array}$ & $\begin{array}{l}0 \\
0 \\
0\end{array}$ \\
\hline $6-17$ & $\begin{array}{r}2 \\
5 \\
6 \\
7 \\
8 \\
11 \\
12\end{array}$ & $\begin{array}{r}4 \\
5 \\
6 \\
7 \\
10 \\
11 \\
18\end{array}$ & $\begin{array}{c}43++ \\
45++ \\
47++ \\
783 \\
2725 \\
1767 \\
1497\end{array}$ & $\begin{array}{r}0 \\
0 \\
2 \\
13 \\
11 \\
19 \\
17\end{array}$ & $\begin{array}{r}0 \\
0 \\
0 \\
1 \\
1 \\
1 \\
10\end{array}$ & $\begin{array}{r}0 \\
0 \\
0 \\
11 \\
8 \\
15 \\
6\end{array}$ & $\begin{array}{l}0 \\
0 \\
0 \\
1 \\
2 \\
3 \\
1\end{array}$ & $\begin{array}{l}0 \\
0 \\
2 \\
0 \\
0 \\
0 \\
0\end{array}$ & $\begin{array}{l}0 \\
0 \\
0 \\
0 \\
0 \\
0 \\
0\end{array}$ \\
\hline $6-18$ & $\begin{array}{l}3 \\
8\end{array}$ & $\begin{array}{r}7 \\
18\end{array}$ & $\begin{array}{r}1887 \\
929\end{array}$ & $\frac{1}{3}$ & $\begin{array}{l}0 \\
2\end{array}$ & $\frac{1}{1}$ & $\begin{array}{l}0 \\
0\end{array}$ & $\begin{array}{l}0 \\
0\end{array}$ & $\begin{array}{l}0 \\
0\end{array}$ \\
\hline
\end{tabular}


TABLE 19.- NUMBER OF PINK TRACER PARTICLES(1), PER 100 GRAMS OF BEDLOAD SAMPLE, COLLECTED AT ONE OR MORE CROSS-CHANNEL SAMPLING POSITIONS SPACED AT 1-METER INTERVALS ACROSS THE CHANNEL WIDTH, EAST FORK RIVER, WYOMING, 1980--CONTINUED

\section{SECTION 1284}

\begin{tabular}{|c|c|c|c|c|c|c|c|c|c|}
\hline \multirow{3}{*}{ DATE } & & & & \multicolumn{6}{|c|}{ NUMBER OF PINK TRACER PARTICLES } \\
\hline & \multirow{2}{*}{\multicolumn{2}{|c|}{$\begin{array}{l}\text { SAMPLING } \\
\text { POSITION } \\
\text { FROM TO TO }\end{array}$}} & \multirow{2}{*}{$\begin{array}{c}\text { DRY } \\
\text { MASS } \\
\text { OF } \\
\text { SAMPLE (2) } \\
\text { (G) }\end{array}$} & \multirow[b]{2}{*}{ TOTAL } & \multirow[b]{2}{*}{$\begin{array}{l}0.25 \\
\text { TO } \\
0.50\end{array}$} & \multirow{2}{*}{$\begin{array}{c}\text { BY SIZE } \\
0.50 \\
\text { TO } \\
1.00\end{array}$} & \multirow{2}{*}{$\begin{array}{l}\text { CLASS } \\
1.00 \\
2.00\end{array}$} & \multirow{2}{*}{ 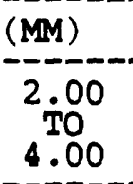 } & \multirow[b]{2}{*}{$\begin{array}{l}4.00 \\
\text { To } \\
8.00\end{array}$} \\
\hline & & & & & & & & & \\
\hline $6-19$ & $\begin{array}{r}3 \\
6 \\
9 \\
12 \\
15\end{array}$ & $\begin{array}{r}5 \\
8 \\
11 \\
14 \\
18\end{array}$ & $\begin{array}{l}40++ \\
721 \\
548 \\
986 \\
435\end{array}$ & $\begin{array}{l}0 \\
0 \\
6 \\
5 \\
7\end{array}$ & $\begin{array}{l}0 \\
0 \\
0 \\
3 \\
4\end{array}$ & $\begin{array}{l}0 \\
0 \\
0 \\
1 \\
1\end{array}$ & $\begin{array}{l}0 \\
0 \\
6 \\
1 \\
2\end{array}$ & $\begin{array}{l}0 \\
0 \\
0 \\
0 \\
0\end{array}$ & $\begin{array}{l}0 \\
0 \\
0 \\
0 \\
0\end{array}$ \\
\hline $6-20$ & $\begin{array}{r}3 \\
6 \\
9 \\
12 \\
15\end{array}$ & $\begin{array}{r}5 \\
8 \\
11 \\
14 \\
18\end{array}$ & $\begin{array}{c}53+ \\
46++ \\
262 \\
1010 \\
441\end{array}$ & $\begin{array}{r}0 \\
0 \\
11 \\
18 \\
34\end{array}$ & $\begin{array}{r}0 \\
0 \\
2 \\
4 \\
15\end{array}$ & $\begin{array}{r}0 \\
0 \\
5 \\
12 \\
18\end{array}$ & $\begin{array}{l}0 \\
0 \\
4 \\
2 \\
1\end{array}$ & $\begin{array}{l}0 \\
0 \\
0 \\
0 \\
0\end{array}$ & $\begin{array}{l}0 \\
0 \\
0 \\
0 \\
0\end{array}$ \\
\hline $6-21$ & $\begin{array}{r}3 \\
6 \\
9 \\
12 \\
15\end{array}$ & $\begin{array}{r}5 \\
8 \\
11 \\
14 \\
18\end{array}$ & $\begin{array}{l}43++ \\
266 \\
611 \\
401 \\
130\end{array}$ & $\begin{array}{r}0 \\
1 \\
7 \\
12 \\
14\end{array}$ & $\begin{array}{l}0 \\
0 \\
3 \\
2 \\
2\end{array}$ & $\begin{array}{r}0 \\
0 \\
2 \\
10 \\
6\end{array}$ & $\begin{array}{l}0 \\
1 \\
1 \\
0 \\
5\end{array}$ & $\begin{array}{l}0 \\
0 \\
0 \\
0 \\
1\end{array}$ & $\begin{array}{l}0 \\
0 \\
1 \\
0 \\
0\end{array}$ \\
\hline $6-23$ & $\begin{array}{r}3 \\
6 \\
9 \\
12 \\
15\end{array}$ & $\begin{array}{r}5 \\
8 \\
11 \\
14 \\
18\end{array}$ & $\begin{array}{l}1350^{4+++} \\
224 \\
360 \\
490\end{array}$ & $\begin{array}{r}0 \\
3 \\
8 \\
16 \\
25\end{array}$ & $\begin{array}{l}0 \\
1 \\
3 \\
7 \\
5\end{array}$ & $\begin{array}{r}0 \\
2 \\
5 \\
8 \\
18\end{array}$ & $\begin{array}{l}0 \\
0 \\
0 \\
1 \\
2\end{array}$ & $\begin{array}{l}0 \\
0 \\
0 \\
0 \\
0\end{array}$ & $\begin{array}{l}0 \\
0 \\
0 \\
0 \\
0\end{array}$ \\
\hline $6-25$ & $\begin{array}{r}3 \\
6 \\
9 \\
12 \\
15\end{array}$ & $\begin{array}{r}5 \\
8 \\
11 \\
14 \\
18\end{array}$ & $\begin{array}{c}54+ \\
155^{+} \\
9+++ \\
1529 \\
557\end{array}$ & $\begin{array}{r}0 \\
5 \\
0 \\
31 \\
0\end{array}$ & $\begin{array}{l}0 \\
0 \\
0 \\
2 \\
0\end{array}$ & $\begin{array}{r}0 \\
1 \\
0 \\
27 \\
0\end{array}$ & $\begin{array}{l}0 \\
1 \\
0 \\
2 \\
0\end{array}$ & $\begin{array}{l}0 \\
3 \\
0 \\
0 \\
0\end{array}$ & $\begin{array}{l}0 \\
0 \\
0 \\
0 \\
0\end{array}$ \\
\hline $6-27$ & $\begin{array}{r}3 \\
6 \\
9 \\
12 \\
15\end{array}$ & $\begin{array}{r}5 \\
8 \\
11 \\
14 \\
18\end{array}$ & $\begin{array}{r}19 \\
695 \\
10+++ \\
1583 \\
937\end{array}$ & $\begin{array}{r}0 \\
15 \\
0 \\
27 \\
29\end{array}$ & $\begin{array}{l}0 \\
1 \\
0 \\
4 \\
6\end{array}$ & $\begin{array}{r}0 \\
8 \\
0 \\
18 \\
20\end{array}$ & $\begin{array}{l}0 \\
5 \\
0 \\
4 \\
2\end{array}$ & $\begin{array}{l}0 \\
1 \\
0 \\
1 \\
1\end{array}$ & $\begin{array}{l}0 \\
0 \\
0 \\
0 \\
0\end{array}$ \\
\hline
\end{tabular}


TABLE 19.- NUMBER OF PINK TRACER PARTICLES(1), PER 100 GRAMS OF BEDLOAD SAMPLE, COLLECTED AT ONE OR MORE CROSS-CHANNEL SAMPLING POSITIONS SPACED AT 1-METER INTERVALS ACROSS THE CHANNEL WIDTH, EAST FORK RIVER, WYOMING, 1980--CONTINUED

SECTION 1315

\begin{tabular}{|c|c|c|c|c|c|c|c|c|c|}
\hline \multirow{3}{*}{ DATE } & \multirow{3}{*}{\multicolumn{2}{|c|}{$\begin{array}{r}\text { SAMPLING } \\
\text { POSITION } \\
\text { FROM TO }\end{array}$}} & \multirow{3}{*}{$\begin{array}{c}\text { DRY } \\
\text { MASS } \\
\text { OF } \\
\text { SAMPLE (2) } \\
(G)\end{array}$} & \multicolumn{6}{|c|}{ NUMBER OF PINK TRACER PARTICLES } \\
\hline & & & & & & BY SIZE & CLASS & $(\mathrm{MM})$ & \\
\hline & & & & TOTAL & $\begin{array}{l}0.25 \\
\text { TO } \\
0.50\end{array}$ & $\begin{array}{l}0.50 \\
\text { TO } \\
1.00\end{array}$ & $\begin{array}{l}1.00 \\
\text { TO } \\
2.00\end{array}$ & $\begin{array}{l}2.00 \\
\text { To } \\
4.00\end{array}$ & $\begin{array}{l}4.00 \\
\text { TO } \\
8.00\end{array}$ \\
\hline $6-01$ & 3 & 25 & 1241 & 2 & 2 & 0 & 0 & 0 & 0 \\
\hline $6-03$ & 3 & 22 & 217 & 2 & 0 & 0 & 2 & 0 & 0 \\
\hline $6-05$ & 3 & 24 & $98+$ & 2 & 2 & 0 & 0 & 0 & 0 \\
\hline $6-07$ & 3 & 25 & 249 & 10 & 3 & 7 & 0 & 0 & 0 \\
\hline $6-09$ & 3 & 25 & 483 & 0 & 0 & 0 & 0 & 0 & 0 \\
\hline $6-10$ & $\begin{array}{l}33 \\
12 \\
18 \\
21\end{array}$ & $\begin{array}{l}11 \\
17 \\
20 \\
25\end{array}$ & $\begin{array}{l}110+ \\
238 \\
508 \\
1945\end{array}$ & $\begin{array}{l}0 \\
5 \\
2 \\
5\end{array}$ & $\begin{array}{l}0 \\
5 \\
1 \\
5\end{array}$ & $\begin{array}{l}0 \\
0 \\
1 \\
0\end{array}$ & $\begin{array}{l}0 \\
0 \\
0 \\
0\end{array}$ & $\begin{array}{l}0 \\
0 \\
0 \\
0\end{array}$ & $\begin{array}{l}0 \\
0 \\
0 \\
0\end{array}$ \\
\hline $6-11$ & $\begin{array}{r}2 \\
13 \\
15\end{array}$ & $\begin{array}{l}12 \\
14 \\
17\end{array}$ & $\begin{array}{r}1882 \\
1720 \\
459\end{array}$ & $\begin{array}{l}3 \\
4 \\
3\end{array}$ & $\begin{array}{l}2 \\
4 \\
2\end{array}$ & $\begin{array}{l}1 \\
0 \\
1\end{array}$ & $\begin{array}{l}0 \\
0 \\
0\end{array}$ & $\begin{array}{l}0 \\
0 \\
0\end{array}$ & $\begin{array}{l}0 \\
0 \\
0\end{array}$ \\
\hline $6-12$ & 3 & 25 & 842 & 3 & 1 & 2 & 0 & 0 & 0 \\
\hline $6-13$ & $\begin{array}{r}3 \\
18\end{array}$ & $\begin{array}{l}17 \\
25\end{array}$ & $\begin{array}{l}223 \\
623\end{array}$ & $\begin{array}{l}6 \\
3\end{array}$ & $\begin{array}{l}2 \\
0\end{array}$ & $\begin{array}{l}4 \\
3\end{array}$ & $\begin{array}{l}0 \\
0\end{array}$ & $\begin{array}{l}0 \\
0\end{array}$ & $\begin{array}{l}0 \\
0\end{array}$ \\
\hline $6-14$ & $\begin{array}{r}3 \\
13\end{array}$ & $\frac{12}{25}$ & $\begin{array}{l}1095 \\
1099\end{array}$ & $\begin{array}{l}3 \\
5\end{array}$ & $\begin{array}{l}0 \\
4\end{array}$ & $\frac{1}{1}$ & $\begin{array}{l}2 \\
0\end{array}$ & $\begin{array}{l}0 \\
0\end{array}$ & $\begin{array}{l}0 \\
0\end{array}$ \\
\hline $6-15$ & $\begin{array}{l}3 \\
12 \\
17 \\
19\end{array}$ & $\begin{array}{l}11 \\
16 \\
18 \\
25\end{array}$ & $\begin{array}{l}804 \\
633 \\
484 \\
846\end{array}$ & $\begin{array}{r}10 \\
14 \\
7 \\
16\end{array}$ & $\begin{array}{l}1 \\
7 \\
2 \\
6\end{array}$ & $\begin{array}{r}7 \\
7 \\
5 \\
10\end{array}$ & $\begin{array}{l}2 \\
0 \\
0 \\
0\end{array}$ & $\begin{array}{l}0 \\
0 \\
0 \\
0\end{array}$ & $\begin{array}{l}0 \\
0 \\
0 \\
0\end{array}$ \\
\hline $6-16$ & $\begin{array}{r}6 \\
7 \\
9 \\
13 \\
18 \\
20\end{array}$ & $\begin{array}{r}6 \\
8 \\
12 \\
17 \\
19 \\
24\end{array}$ & $\begin{array}{c}56+ \\
46++ \\
641 \\
459 \\
838 \\
1062\end{array}$ & $\begin{array}{r}0 \\
0 \\
20 \\
23 \\
15 \\
16\end{array}$ & $\begin{array}{r}0 \\
0 \\
7 \\
17 \\
6 \\
8\end{array}$ & $\begin{array}{r}0 \\
0 \\
12 \\
6 \\
9 \\
8\end{array}$ & $\begin{array}{l}0 \\
0 \\
1 \\
0 \\
0 \\
0\end{array}$ & $\begin{array}{l}0 \\
0 \\
0 \\
0 \\
0 \\
0\end{array}$ & $\begin{array}{l}0 \\
0 \\
0 \\
0 \\
0 \\
0\end{array}$ \\
\hline
\end{tabular}


TABLE 19.- NUMBER OF PINK TRACER PARTICLES(1), PER 100 GRAMS OF BEDLOAD SAMPLE, COLLECTED AT ONE OR MORE CROSS-CHANNEL SAMPLING POSITIONS SPACED AT 1-METER INTERVALS ACROSS THE CHANNEL WIDTH, EAST FORK RIVER, WYOMING, 1980--CONTINUED

SECTION 1315

\begin{tabular}{|c|c|c|c|c|c|c|c|c|c|}
\hline \multirow{3}{*}{ DATE } & \multirow{3}{*}{\multicolumn{2}{|c|}{$\begin{array}{l}\text { SAMPLING } \\
\text { POSITION } \\
\text { FROM TO }\end{array}$}} & \multirow{3}{*}{$\begin{array}{c}\text { DRY } \\
\text { MASS } \\
\text { OF } \\
\text { SAMPLE (2) } \\
\text { (G) }\end{array}$} & \multicolumn{6}{|c|}{ NUMBER OF PINK TRACER PARTICLES } \\
\hline & & & & \multirow[b]{2}{*}{ TOTAL } & \multicolumn{5}{|c|}{ BY SIZE CLASS (MM) } \\
\hline & & & & & $\begin{array}{l}0.25 \\
\text { To } \\
0.50\end{array}$ & $\begin{array}{l}0.50 \\
\text { TO } \\
1.00\end{array}$ & $\begin{array}{l}1.00 \\
2.00\end{array}$ & $\begin{array}{l}2.00 \\
\text { TO } \\
4.00\end{array}$ & $\begin{array}{l}4.00 \\
\text { TO } \\
8.00\end{array}$ \\
\hline $6-17$ & $\begin{array}{r}3 \\
7 \\
8 \\
10 \\
13 \\
16 \\
19\end{array}$ & $\begin{array}{r}6 \\
7 \\
9 \\
12 \\
15 \\
18 \\
25\end{array}$ & $\begin{array}{c}17++ \\
20++ \\
378 \\
1811 \\
731 \\
867 \\
1265\end{array}$ & $\begin{array}{r}0 \\
0 \\
3 \\
10 \\
11 \\
10 \\
8\end{array}$ & $\begin{array}{l}0 \\
0 \\
1 \\
2 \\
2 \\
2 \\
2\end{array}$ & $\begin{array}{l}0 \\
0 \\
1 \\
6 \\
8 \\
4 \\
6\end{array}$ & $\begin{array}{l}0 \\
0 \\
1 \\
2 \\
1 \\
4 \\
0\end{array}$ & $\begin{array}{l}0 \\
0 \\
0 \\
0 \\
0 \\
0 \\
0\end{array}$ & $\begin{array}{l}0 \\
0 \\
0 \\
0 \\
0 \\
0 \\
0\end{array}$ \\
\hline $6-18$ & $\begin{array}{r}3 \\
5 \\
7 \\
9 \\
10 \\
14 \\
19\end{array}$ & $\begin{array}{r}4 \\
6 \\
8 \\
9 \\
13 \\
18 \\
25\end{array}$ & $\begin{array}{c}22++ \\
17++ \\
5+++ \\
38++ \\
519 \\
973 \\
828\end{array}$ & $\begin{array}{r}0 \\
0 \\
0 \\
0 \\
2 \\
15 \\
15\end{array}$ & $\begin{array}{l}0 \\
0 \\
0 \\
0 \\
0 \\
1 \\
4\end{array}$ & $\begin{array}{r}0 \\
0 \\
0 \\
0 \\
0 \\
12 \\
8\end{array}$ & $\begin{array}{l}0 \\
0 \\
0 \\
0 \\
2 \\
2 \\
3\end{array}$ & $\begin{array}{l}0 \\
0 \\
0 \\
0 \\
0 \\
0 \\
0\end{array}$ & $\begin{array}{l}0 \\
0 \\
0 \\
0 \\
0 \\
0 \\
0\end{array}$ \\
\hline $6-19$ & $\begin{array}{l}3 \\
14 \\
18\end{array}$ & $\begin{array}{l}13 \\
17 \\
25\end{array}$ & $\begin{array}{l}847 \\
705 \\
742\end{array}$ & $\begin{array}{r}6 \\
13 \\
13\end{array}$ & $\begin{array}{l}\frac{1}{3} \\
2\end{array}$ & $\begin{array}{l}3 \\
8 \\
8\end{array}$ & $\begin{array}{l}2 \\
2 \\
3\end{array}$ & $\begin{array}{l}0 . \\
0 \\
0\end{array}$ & $\begin{array}{l}0 \\
0 \\
0\end{array}$ \\
\hline $6-20$ & $\begin{array}{l}3 \\
11 \\
17\end{array}$ & $\begin{array}{l}10 \\
16 \\
25\end{array}$ & $\begin{array}{c}29+++ \\
1376 \\
942\end{array}$ & $\begin{array}{l}0 \\
9 \\
9\end{array}$ & $\begin{array}{l}0 \\
3 \\
0\end{array}$ & $\begin{array}{l}0 \\
4 \\
5\end{array}$ & $\begin{array}{l}0 \\
2 \\
3\end{array}$ & $\begin{array}{l}0 \\
0 \\
1\end{array}$ & $\begin{array}{l}0 \\
0 \\
0\end{array}$ \\
\hline $6-21$ & $\frac{4}{17}$ & $\begin{array}{l}10 \\
16 \\
25\end{array}$ & $\begin{array}{r}69+ \\
985 \\
1775\end{array}$ & $\begin{array}{r}0 \\
16 \\
17\end{array}$ & $\begin{array}{l}0 \\
7 \\
5\end{array}$ & $\begin{array}{r}0 \\
6 \\
10\end{array}$ & $\begin{array}{l}0 \\
3 \\
2\end{array}$ & $\begin{array}{l}0 \\
0 \\
0\end{array}$ & $\begin{array}{l}0 \\
0 \\
0\end{array}$ \\
\hline $6-23$ & $\begin{array}{r}3 \\
9 \\
13 \\
17\end{array}$ & $\begin{array}{r}8 \\
12 \\
16 \\
25\end{array}$ & $\begin{array}{r}130 \\
349 \\
246 \\
2589\end{array}$ & $\begin{array}{r}0 \\
2 \\
12 \\
10\end{array}$ & $\begin{array}{l}0 \\
0 \\
5 \\
1\end{array}$ & $\begin{array}{l}0 \\
0 \\
7 \\
7\end{array}$ & $\begin{array}{l}0 \\
2 \\
0 \\
1\end{array}$ & $\begin{array}{l}0 \\
0 \\
0 \\
1\end{array}$ & $\begin{array}{l}0 \\
0 \\
0 \\
0\end{array}$ \\
\hline $6-25$ & $\begin{array}{r}3 \\
10 \\
17\end{array}$ & $\begin{array}{r}9 \\
16 \\
25\end{array}$ & $\begin{array}{l}24++ \\
4383 \\
2198\end{array}$ & $\begin{array}{r}0 \\
26 \\
25\end{array}$ & $\begin{array}{l}0 \\
2 \\
2\end{array}$ & $\begin{array}{r}0 \\
16 \\
22\end{array}$ & $\begin{array}{l}0 \\
4 \\
1\end{array}$ & $\begin{array}{l}0 \\
4 \\
0\end{array}$ & $\begin{array}{l}0 \\
0 \\
0\end{array}$ \\
\hline $6-27$ & $\begin{array}{l}3 \\
11 \\
17\end{array}$ & $\begin{array}{l}10 \\
16 \\
25\end{array}$ & $\begin{array}{r}72+ \\
861 \\
1343\end{array}$ & $\begin{array}{r}0 \\
23 \\
31\end{array}$ & $\begin{array}{l}0 \\
6 \\
2\end{array}$ & $\begin{array}{r}0 \\
17 \\
26\end{array}$ & $\begin{array}{l}0 \\
0 \\
3\end{array}$ & $\begin{array}{l}0 \\
0 \\
0\end{array}$ & $\begin{array}{l}0 \\
0 \\
0\end{array}$ \\
\hline
\end{tabular}


TABLE 19.- NUMBER OF PINK TRACER PARTICLES(1), PER 100 GRAMS OF BEDLOAD SAMPLE, COLLECTED AT ONE OR MORE CROSS-CHANNEL SAMPLING POSITIONS SPACED AT I-METER INTERVALS ACROSS THE CHANNEL WIDTH, EAST FORK RIVER, WYOMING, 1980--CONTINUED

SECTION $1360(3)$

\begin{tabular}{|c|c|c|c|c|c|c|c|c|c|}
\hline \multirow{3}{*}{ DATE } & & & \multirow{3}{*}{$\begin{array}{c}\text { DRY } \\
\text { MASS } \\
\text { OF } \\
\text { SAMPLE (2) } \\
\text { (G) }\end{array}$} & \multicolumn{6}{|c|}{ NUMBER OF PINK TRACER PARTICLES } \\
\hline & \multirow{2}{*}{\multicolumn{2}{|c|}{$\begin{array}{l}\text { SAMPLING } \\
\text { POSITION } \\
\text { FROM TO }\end{array}$}} & & \multirow[b]{2}{*}{ TOTAL } & \multicolumn{5}{|c|}{ BY SIZE CLASS (MM) } \\
\hline & & & & & $\begin{array}{l}0.25 \\
\text { TO } \\
0.50\end{array}$ & $\begin{array}{l}0.50 \\
\text { TO } \\
1.00\end{array}$ & $\begin{array}{l}1.00 \\
2.00\end{array}$ & $\begin{array}{l}2.00 \\
40 \\
4.00\end{array}$ & $\begin{array}{l}4.00 \\
\text { TO } \\
8.00\end{array}$ \\
\hline $6-01$ & 8 & 23 & $31++$ & 0 & 0 & 0 & 0 & 0 & 0 \\
\hline $6-03$ & 8 & 23 & $235+$ & 5 & 2 & 2 & 1 & 0 & 0 \\
\hline $6-05$ & 8 & 22 & 147 & 5 & 0 & 5 & 0 & 0 & 0 \\
\hline $6-07$ & 9 & 22 & 535 & 8 & 5 & 3 & 0 & 0 & 0 \\
\hline $6-09$ & 2 & 22 & 1049 & 6 & 1 & 5 & 0 & 0 & 0 \\
\hline $6-10$ & 9 & 22 & 1192 & 7 & 2 & 5 & 0 & 0 & 0 \\
\hline $6-11$ & $\begin{array}{r}9 \\
17 \\
20\end{array}$ & $\begin{array}{l}16 \\
19 \\
22\end{array}$ & $\begin{array}{l}437 \\
573 \\
0+++\end{array}$ & $\begin{array}{l}7 \\
9 \\
0\end{array}$ & $\begin{array}{l}5 \\
1 \\
0\end{array}$ & $\begin{array}{l}0 \\
7 \\
0\end{array}$ & $\begin{array}{l}0 \\
1 \\
0\end{array}$ & $\begin{array}{l}2 \\
0 \\
0\end{array}$ & $\begin{array}{l}0 \\
0 \\
0\end{array}$ \\
\hline $6-12$ & $\begin{array}{r}9 \\
17\end{array}$ & $\begin{array}{l}16 \\
22\end{array}$ & $\begin{array}{l}156 \\
262\end{array}$ & 14 & $\begin{array}{l}7 \\
4\end{array}$ & $\begin{array}{l}7 \\
5\end{array}$ & $\begin{array}{l}0 \\
0\end{array}$ & $\begin{array}{l}0 \\
0\end{array}$ & $\begin{array}{l}0 \\
0\end{array}$ \\
\hline $6-13$ & 9 & 22 & 841 & 2 & 0 & 2 & 0 & 0 & 0 \\
\hline $6-14$ & $\begin{array}{r}7 \\
16\end{array}$ & $\frac{14}{21}$ & $\begin{array}{l}111 \\
501\end{array}$ & $\begin{array}{r}0 \\
14\end{array}$ & $\begin{array}{l}0 \\
5\end{array}$ & $\begin{array}{l}0 \\
8\end{array}$ & $\begin{array}{l}0 \\
1\end{array}$ & $\begin{array}{l}0 \\
0\end{array}$ & $\begin{array}{l}0 \\
0\end{array}$ \\
\hline $6-15$ & $\begin{array}{r}99 \\
17 \\
20\end{array}$ & $\begin{array}{l}16 \\
19 \\
23\end{array}$ & $\begin{array}{r}519 \\
1518 \\
1795\end{array}$ & $\begin{array}{r}0 \\
7 \\
17\end{array}$ & $\begin{array}{l}0 \\
2 \\
5\end{array}$ & $\begin{array}{r}0 \\
4 \\
10\end{array}$ & $\begin{array}{l}0 \\
1 \\
2\end{array}$ & $\begin{array}{l}0 \\
0 \\
0\end{array}$ & $\begin{array}{l}0 \\
0 \\
0\end{array}$ \\
\hline $6-16$ & $\begin{array}{l}14 \\
18 \\
20\end{array}$ & $\begin{array}{l}17 \\
19 \\
23\end{array}$ & $\begin{array}{l}170 \\
464 \\
408\end{array}$ & $\begin{array}{r}6 \\
12 \\
14\end{array}$ & $\begin{array}{l}1 \\
\frac{1}{4}\end{array}$ & $\begin{array}{l}4 \\
5 \\
9\end{array}$ & $\begin{array}{l}1 \\
4 \\
1\end{array}$ & $\begin{array}{l}0 \\
2 \\
0\end{array}$ & $\begin{array}{l}0 \\
0 \\
0\end{array}$ \\
\hline $6-17$ & $\begin{array}{r}9 \\
18\end{array}$ & $\begin{array}{l}17 \\
23\end{array}$ & $\begin{array}{c}85+ \\
502\end{array}$ & $\begin{array}{r}2 \\
15\end{array}$ & $\begin{array}{l}0 \\
3\end{array}$ & 12 & $\begin{array}{l}0 \\
1\end{array}$ & $\begin{array}{l}0 \\
0\end{array}$ & $\begin{array}{l}0 \\
0\end{array}$ \\
\hline $6-18$ & 19 & $\begin{array}{l}18 \\
23\end{array}$ & $\begin{array}{l}1444 \\
375\end{array}$ & $\begin{array}{l}8 \\
8\end{array}$ & $\begin{array}{l}0 \\
5\end{array}$ & $\begin{array}{l}7 \\
3\end{array}$ & $\begin{array}{l}0 \\
0\end{array}$ & $\frac{1}{0}$ & $\begin{array}{l}0 \\
0\end{array}$ \\
\hline $6-19$ & $\begin{array}{r}7 \\
18 \\
21\end{array}$ & $\begin{array}{l}17 \\
20 \\
23\end{array}$ & $\begin{array}{l}588 \\
786 \\
229\end{array}$ & $\begin{array}{r}8 \\
16 \\
16\end{array}$ & $\begin{array}{l}0 \\
1 \\
1\end{array}$ & $\begin{array}{r}6 \\
12 \\
13\end{array}$ & $\begin{array}{l}2 \\
3 \\
2\end{array}$ & $\begin{array}{l}0 \\
0 \\
0\end{array}$ & $\begin{array}{l}0 \\
0 \\
0\end{array}$ \\
\hline $6-20$ & 2 & 23 & 391 & 6 & 0 & 1 & 5 & 0 & 0 \\
\hline
\end{tabular}


TABLE 19.- NUMBER OF PINK TRACER PARTICLES(1), PER 100 GRAMS OF BEDLOAD SAMPLE, COLLECTED AT ONE OR MORE CROSS-CHANNEL SAMPL ING

POSITIONS SPACED AT 1-METER INTERVALS ACROSS THE CHANNEL WIDTH, EAST FORK RIVER, WYOMING, 1980--CONTINUED

SECTION 1360(3)

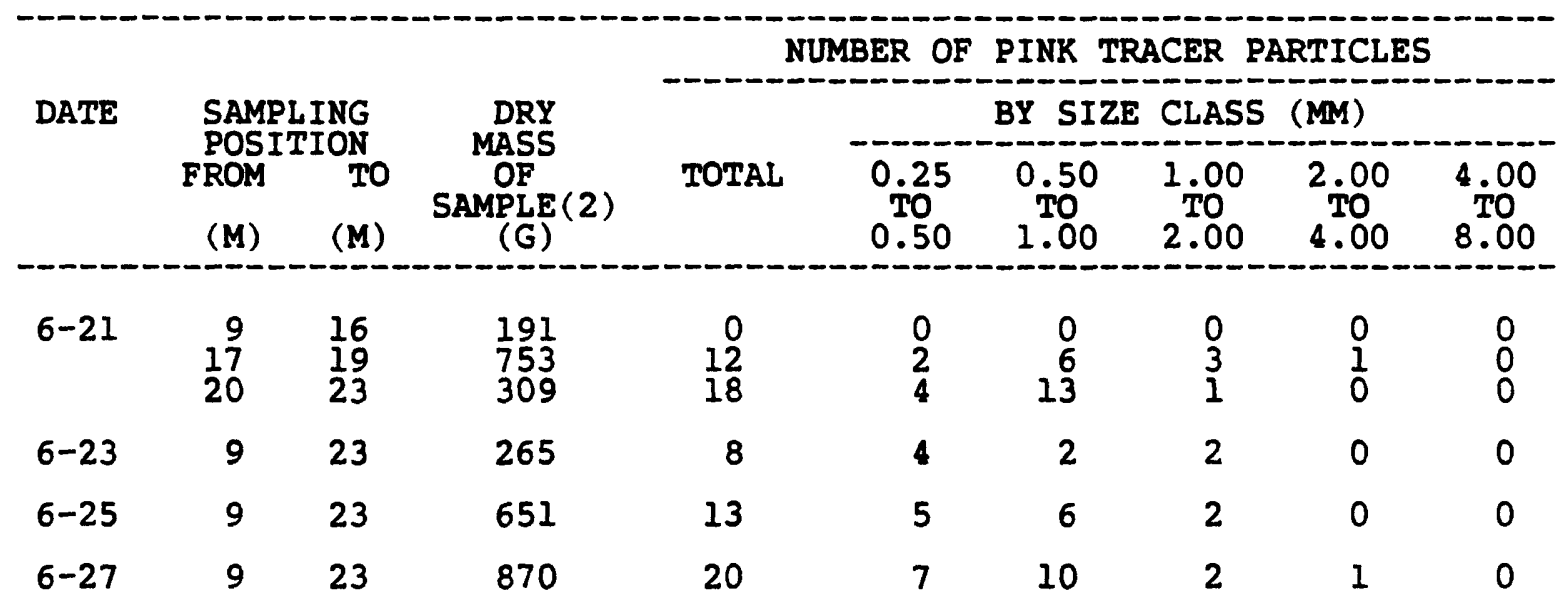


TABLE 19.- NUMBER OF PINK TRACER PARTICLES(1), PER 100 GRAMS OF BEDLOAD SAMPLE, COLLECTED AT ONE OR MORE CROSS-CHANNEL SAMPL ING POSITIONS SPACED AT 1-METER INTERVALS ACROSS THE CHANNEL WIDTH, EAST FORK RIVER, WYOMING, 1980--CONTINUED

SECTION 1396(3)

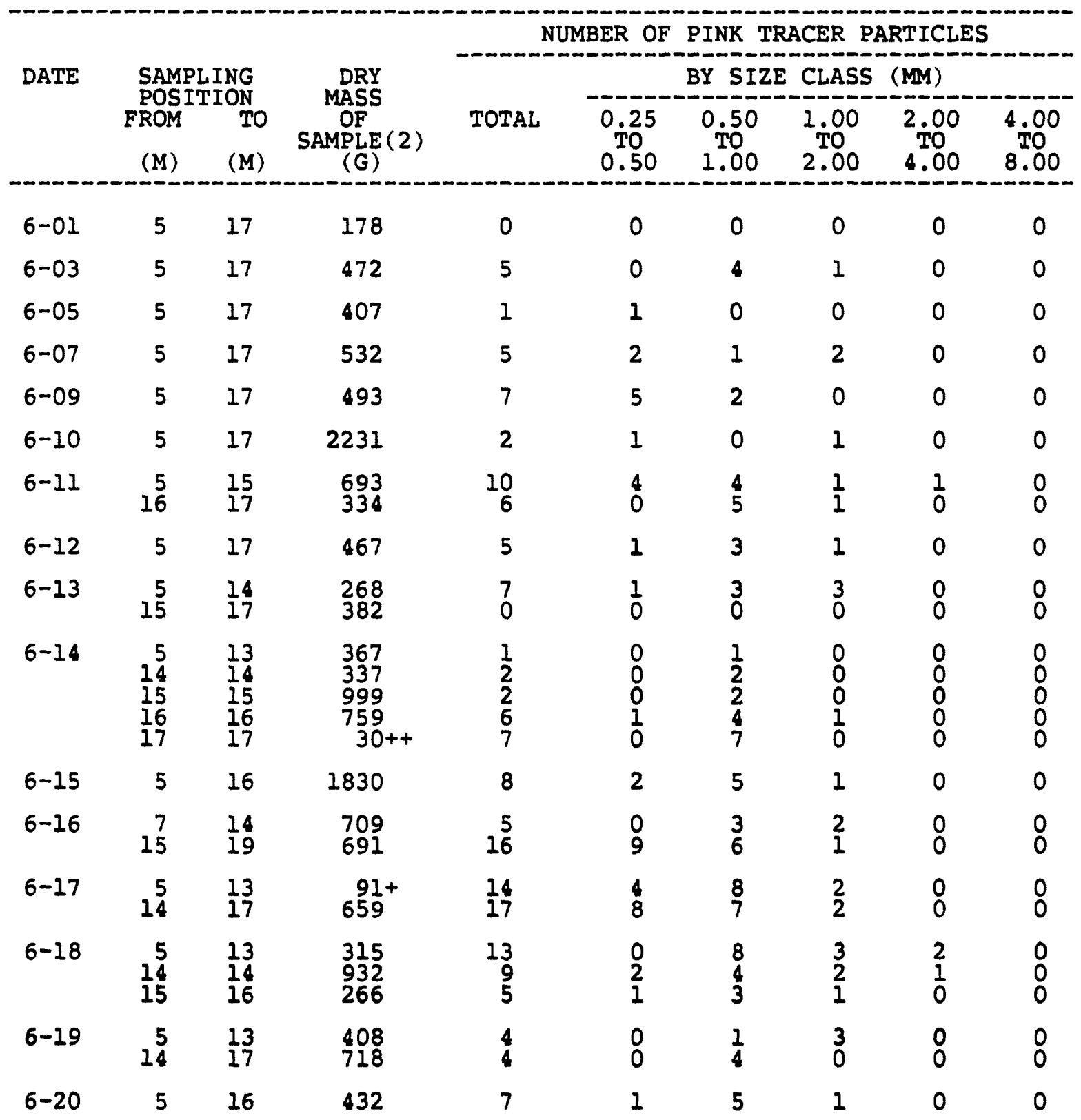


TABLE 19.- NUMBER OF PINK TRACER PARTICLES(1), PER 100 GRAMS OF BEDLOAD SAMPLE COLLECTED AT ONE OR MORE CROSS-CHANNEL SAMPLING POSITIONS SPACED AT 1-METER INTERVALS ACROSS THE CHANNEL WIDTH, EAST FORK RIVER, WYOMING, 1980--CONTINUED

SECTION $1396(3)$

\begin{tabular}{|c|c|c|c|c|c|c|c|c|c|}
\hline \multirow{3}{*}{ DATE } & \multirow{3}{*}{\multicolumn{2}{|c|}{$\begin{array}{l}\text { SAMPLING } \\
\text { POSITION } \\
\text { FROM TO }\end{array}$}} & \multirow{3}{*}{$\begin{array}{c}\text { DRY } \\
\text { MASS } \\
\text { OF } \\
\text { SAMPLE (2) } \\
\text { (G) }\end{array}$} & \multicolumn{6}{|c|}{ NUMBER OF PINK TRACER PARTICLES } \\
\hline & & & & \multirow[b]{2}{*}{ TOTAL } & \multicolumn{5}{|c|}{ BY SIZE CLASS (MM) } \\
\hline & & & & & $\begin{array}{l}0.25 \\
\text { To } \\
0.50\end{array}$ & $\begin{array}{l}0.50 \\
\text { To } \\
1.00\end{array}$ & $\begin{array}{l}1.00 \\
2.00\end{array}$ & $\begin{array}{l}2.00 \\
\text { TO } \\
4.00\end{array}$ & $\begin{array}{l}4.00 \\
\text { To } \\
8.00\end{array}$ \\
\hline $6-21$ & 5 & 16 & 855 & 9 & 0 & 6 & 1 & 2 & 0 \\
\hline $6-23$ & 5 & 16 & 754 & 6 & 0 & 4 & 1 & I & 0 \\
\hline $6-25$ & 5 & 16 & 487 & 8 & 2 & 5 & 0 & 1 & 0 \\
\hline $6-27$ & 5 & 16 & 1773 & 15 & 2 & 8 & 3 & 2 & 0 \\
\hline
\end{tabular}


TABLE 19.- NUMBER OF PINK TRACER PARTICLES(1), PER 100 GRAMS OF BEDLOAD SAMPLE, COLLECTED AT ONE OR MORE CROSS-CHANNEL SAMPLING POSITIONS SPACED AT 1-METER INTERVALS ACROSS THE CHANNEL WIDTH, EAST FORK RIVER, WYOMING, 1980--CONTINUED

SECTION $1425(3)$

\begin{tabular}{|c|c|c|c|c|c|c|c|c|c|}
\hline \multirow{3}{*}{ DATE } & \multirow{3}{*}{\multicolumn{2}{|c|}{$\begin{array}{l}\text { SAMPLING } \\
\text { POSITION } \\
\text { FROM TO }\end{array}$}} & \multirow{3}{*}{$\begin{array}{c}\text { DRY } \\
\text { MASS } \\
\text { OF } \\
\text { SAMPLE (2) } \\
\text { (G) }\end{array}$} & \multicolumn{6}{|c|}{ NUMBER OF PINK TRACER PARTICLES } \\
\hline & & & & & & BY SIZ & CLASS & $(\mathbf{M})$ & \\
\hline & & & & TOTAL & $\begin{array}{l}0.25 \\
\text { TO } \\
0.50\end{array}$ & $\begin{array}{l}0.50 \\
\text { TO } \\
1.00\end{array}$ & $\begin{array}{l}1.00 \\
2.00 \\
2.00\end{array}$ & $\begin{array}{l}2.00 \\
\text { TO } \\
4.00\end{array}$ & $\begin{array}{l}4.00 \\
\text { TO } \\
8.00\end{array}$ \\
\hline $6-01$ & 2 & 17 & 793 & 1 & 0 & 1 & 0 & 0 & 0 \\
\hline $6-03$ & 1 & 17 & $193+$ & 2 & 0 & 2 & 0 & 0 & 0 \\
\hline $6-05$ & 1 & 17 & 209 & 1 & 0 & 1 & 0 & 0 & 0 \\
\hline $6-07$ & 2 & 17 & 935 & 5 & 0 & 5 & 0 & 0 & 0 \\
\hline $6-09$ & 2 & 17 & 621 & 2 & 2 & 0 & 0 & 0 & 0 \\
\hline $6-10$ & $\begin{array}{r}2 \\
8 \\
11\end{array}$ & $\begin{array}{r}7 \\
10 \\
17\end{array}$ & $\begin{array}{l}119+ \\
833 \\
384\end{array}$ & $\begin{array}{r}7 \\
7 \\
12\end{array}$ & $\begin{array}{l}4 \\
2 \\
4\end{array}$ & $\begin{array}{l}3 \\
5 \\
8\end{array}$ & $\begin{array}{l}0 \\
0 \\
0\end{array}$ & $\begin{array}{l}0 \\
0 \\
0\end{array}$ & $\begin{array}{l}0 \\
0 \\
0\end{array}$ \\
\hline $6-11$ & $1 \frac{2}{13}$ & $\begin{array}{l}12 \\
17\end{array}$ & $\begin{array}{r}257 \\
0\end{array}$ & $\begin{array}{l}6 \\
0\end{array}$ & $\begin{array}{l}4 \\
0\end{array}$ & $\begin{array}{l}2 \\
0\end{array}$ & $\begin{array}{l}0 \\
0\end{array}$ & $\begin{array}{l}0 \\
0\end{array}$ & $\begin{array}{l}0 \\
0\end{array}$ \\
\hline $6-12$ & $\frac{1}{8}$ & $\begin{array}{r}7 \\
17\end{array}$ & $\begin{array}{l}153 \\
28++\end{array}$ & 11 & $\begin{array}{l}0 \\
4\end{array}$ & $\begin{array}{l}2 \\
7\end{array}$ & $\begin{array}{l}1 \\
0\end{array}$ & $\begin{array}{l}1 \\
0\end{array}$ & $\begin{array}{l}0 \\
0\end{array}$ \\
\hline $6-13$ & $\begin{array}{r}4 \\
9 \\
14\end{array}$ & $\begin{array}{r}8 \\
13 \\
17\end{array}$ & $\begin{array}{c}48++ \\
69+ \\
8+++\end{array}$ & $\begin{array}{l}0 \\
3 \\
0\end{array}$ & $\begin{array}{l}0 \\
0 \\
0\end{array}$ & $\begin{array}{l}0 \\
3 \\
0\end{array}$ & $\begin{array}{l}0 \\
0 \\
0\end{array}$ & $\begin{array}{l}0 \\
0 \\
0\end{array}$ & $\begin{array}{l}0 \\
0 \\
0\end{array}$ \\
\hline $6-14$ & $\begin{array}{l}7 \\
8 \\
9\end{array}$ & $\begin{array}{r}7 \\
8 \\
16\end{array}$ & $\begin{array}{l}654 \\
438 \\
130+\end{array}$ & $\begin{array}{r}9 \\
3 \\
12\end{array}$ & $\begin{array}{l}5 \\
0 \\
4\end{array}$ & $\begin{array}{l}3 \\
2 \\
6\end{array}$ & $\begin{array}{l}\frac{1}{1} \\
\frac{1}{2}\end{array}$ & $\begin{array}{l}0 \\
0 \\
0\end{array}$ & $\begin{array}{l}0 \\
0 \\
0\end{array}$ \\
\hline $6-15$ & $\begin{array}{r}3 \\
8 \\
13\end{array}$ & $\begin{array}{r}7 \\
12 \\
16\end{array}$ & $\begin{array}{c}51++ \\
120+ \\
24++\end{array}$ & $\begin{array}{r}8 \\
25 \\
0\end{array}$ & $\begin{array}{l}2 \\
8 \\
0\end{array}$ & $\begin{array}{r}6 \\
15 \\
0\end{array}$ & $\begin{array}{l}0 \\
2 \\
0\end{array}$ & $\begin{array}{l}0 \\
0 \\
0\end{array}$ & $\begin{array}{l}0 \\
0 \\
0\end{array}$ \\
\hline $6-16$ & $\begin{array}{r}4 \\
8 \\
12 \\
15\end{array}$ & $\begin{array}{l}7 \\
11 \\
14 \\
16\end{array}$ & $\begin{array}{c}21++ \\
82+ \\
42++ \\
0+++\end{array}$ & $\begin{array}{r}9 \\
10 \\
2 \\
0\end{array}$ & $\begin{array}{l}0 \\
2 \\
0 \\
0\end{array}$ & $\begin{array}{l}9 \\
4 \\
0 \\
0\end{array}$ & $\begin{array}{l}0 \\
2 \\
2 \\
0\end{array}$ & $\begin{array}{l}0 \\
2 \\
0 \\
0\end{array}$ & $\begin{array}{l}0 \\
0 \\
0 \\
0\end{array}$ \\
\hline $6-17$ & $\begin{array}{l}4 \\
7 \\
9\end{array}$ & $\begin{array}{r}6 \\
8 \\
14\end{array}$ & $\begin{array}{l}41+++ \\
56++ \\
79++\end{array}$ & $\begin{array}{l}0 \\
0 \\
9\end{array}$ & $\begin{array}{l}0 \\
0 \\
5\end{array}$ & $\begin{array}{l}0 \\
0 \\
3\end{array}$ & $\begin{array}{l}0 \\
0 \\
1\end{array}$ & $\begin{array}{l}0 \\
0 \\
0\end{array}$ & $\begin{array}{l}0 \\
0 \\
0\end{array}$ \\
\hline $6-18$ & $\begin{array}{l}2 \\
8\end{array}$ & $\begin{array}{r}7 \\
16\end{array}$ & $\begin{array}{l}565 \\
242\end{array}$ & $2 \frac{1}{1}$ & $\begin{array}{l}0 \\
8\end{array}$ & $\begin{array}{r}0 \\
12\end{array}$ & $\begin{array}{l}0 \\
1\end{array}$ & $\begin{array}{l}1 \\
0\end{array}$ & $\begin{array}{l}0 \\
0\end{array}$ \\
\hline
\end{tabular}


TABLE 19.- NUMBER OF PINK TRACER PARTICLES(1), PER 100 GRAMS OF BEDLOAD SAMPLE, COLLECTED AT ONE OR MORE CROSS-CHANNEL SAMPLING POSITIONS SPACED AT I-METER INTERVALS ACROSS THE CHANNEL WIDTH, EAST FORK RIVER, WYOMING, 1980--CONTINUED

SECTION $1425(3)$

\begin{tabular}{|c|c|c|c|c|c|c|c|c|c|}
\hline \multirow{3}{*}{ DATE } & & & & & ER $\quad$ O & PINK & ACER & RTICL & \\
\hline & \multicolumn{2}{|c|}{$\begin{array}{l}\text { SAMPLING } \\
\text { POSITION } \\
\text { FROM TO }\end{array}$} & \multirow{2}{*}{$\begin{array}{c}\text { DRY } \\
\text { MASS } \\
\text { OF } \\
\text { SAMPLE (2) } \\
\text { (G) }\end{array}$} & \multirow[b]{2}{*}{ TOTAL } & \multicolumn{5}{|c|}{ BY SIZE CLASS (MM) } \\
\hline & FROM & TO & & & $\begin{array}{l}0.25 \\
\text { TO } \\
0.50\end{array}$ & $\begin{array}{l}0.50 \\
\text { TO } \\
1.00\end{array}$ & $\begin{array}{l}1.00 \\
2.00\end{array}$ & $\begin{array}{l}2.00 \\
\text { TO } \\
4.00\end{array}$ & $\begin{array}{l}4.00 \\
\text { TO } \\
8.00\end{array}$ \\
\hline $6-19$ & $\begin{array}{r}3 \\
11\end{array}$ & $\begin{array}{l}10 \\
16\end{array}$ & $\begin{array}{l}55++ \\
130^{++}\end{array}$ & $\begin{array}{r}13 \\
7\end{array}$ & $\begin{array}{l}2 \\
3\end{array}$ & $\begin{array}{l}9 \\
4\end{array}$ & $\begin{array}{l}2 \\
0\end{array}$ & $\begin{array}{l}0 \\
0\end{array}$ & $\begin{array}{l}0 \\
0\end{array}$ \\
\hline $6-20$ & 12 & $\begin{array}{l}10 \\
16\end{array}$ & $\begin{array}{l}178 \\
190\end{array}$ & $\begin{array}{l}3 \\
8\end{array}$ & $\begin{array}{l}1 \\
0\end{array}$ & $\begin{array}{l}0 \\
6\end{array}$ & $\begin{array}{l}0 \\
2\end{array}$ & $\begin{array}{l}2 \\
0\end{array}$ & $\begin{array}{l}0 \\
0\end{array}$ \\
\hline $6-21$ & 2 & 15 & $228+$ & 18 & 3 & 13 & 2 & 0 & 0 \\
\hline $6-23$ & 1 & 17 & 258 & 19 & 11 & 8 & 0 & 0 & 0 \\
\hline $6-25$ & 2 & 16 & 212 & 14 & 3 & 10 & 1 & 0 & 0 \\
\hline $6-27$ & 8 & 16 & 664 & 14 & 3 & 7 & 3 & 1 & 0 \\
\hline
\end{tabular}


TABLE 19.- NUMBER OF PINK TRACER PARTICLES(1), PER 100 GRAMS OF BEDLOAD SAMPLE, COLLECTED AT ONE OR MORE CROSS-CHANNEL SAMPLING POSITIONS SPACED AT I-METER INTERVALS ACROSS THE CHANNEL WIDTH, EAST FORK RIVER, WYOMING, 1980--CONTINUED

SECTION 1481

\begin{tabular}{|c|c|c|c|c|c|c|c|c|c|}
\hline \multirow{3}{*}{ DATE } & & & & \multicolumn{6}{|c|}{ NUMBER OF PINK TRACER PARTICLES } \\
\hline & \multirow{2}{*}{\multicolumn{2}{|c|}{$\begin{array}{l}\text { SAMPLING } \\
\text { POSITION } \\
\text { FROM TO }\end{array}$}} & \multirow{2}{*}{$\begin{array}{c}\text { DRY } \\
\text { MASS } \\
\text { OF } \\
\text { SAMPLE (2) } \\
(G)\end{array}$} & \multirow[b]{2}{*}{ TOTAL } & \multicolumn{5}{|c|}{ BY SIZE CLASS (MM) } \\
\hline & & TO & & & $\begin{array}{l}0.25 \\
\text { TO } \\
0.50\end{array}$ & $\begin{array}{l}0.50 \\
\text { TO } \\
1.00\end{array}$ & $\begin{array}{l}1.00 \\
\text { To } \\
2.00\end{array}$ & $\begin{array}{l}2.00 \\
\text { TO } \\
4.00\end{array}$ & $\begin{array}{l}4.00 \\
\text { TO } \\
8.00\end{array}$ \\
\hline $6-01$ & 4 & 16 & $187+$ & 8 & 3 & 5 & 0 & 0 & 0 \\
\hline $6-03$ & 4 & 17 & 235 & 6 & 2 & 3 & 1 & 0 & 0 \\
\hline $6-05$ & 5 & 16 & 197 & 5 & 0 & 2 & 3 & 0 & 0 \\
\hline $6-07$ & 5 & 15 & 389 & 5 & 1 & 4 & 0 & 0 & 0 \\
\hline $6-09$ & $\begin{array}{r}14 \\
22\end{array}$ & $\begin{array}{r}9 \\
14 \\
26\end{array}$ & $\begin{array}{r}858 \\
1290 \\
385\end{array}$ & $\begin{array}{l}0 \\
7 \\
6\end{array}$ & $\begin{array}{l}0 \\
1 \\
3\end{array}$ & $\begin{array}{l}0 \\
5 \\
3\end{array}$ & $\begin{array}{l}0 \\
1 \\
0\end{array}$ & $\begin{array}{l}0 \\
0 \\
0\end{array}$ & $\begin{array}{l}0 \\
0 \\
0\end{array}$ \\
\hline $6-10$ & $\begin{array}{r}4 \\
9 \\
11 \\
12 \\
22 \\
25\end{array}$ & $\begin{array}{r}8 \\
10 \\
11 \\
15 \\
24 \\
25\end{array}$ & $\begin{array}{c}769 \\
765 \\
1148 \\
102+ \\
1420 \\
1773\end{array}$ & $\begin{array}{r}6 \\
9 \\
15 \\
9 \\
9 \\
23\end{array}$ & $\begin{array}{r}2 \\
5 \\
3 \\
2 \\
4 \\
11\end{array}$ & $\begin{array}{r}3 \\
2 \\
12 \\
5 \\
4 \\
11\end{array}$ & $\begin{array}{l}0 \\
2 \\
0 \\
2 \\
1 \\
1\end{array}$ & $\begin{array}{l}1 \\
0 \\
0 \\
0 \\
0 \\
0\end{array}$ & $\begin{array}{l}0 \\
0 \\
0 \\
0 \\
0 \\
0\end{array}$ \\
\hline $6-11$ & $\begin{array}{r}5 \\
9 \\
10 \\
11 \\
22 \\
24 \\
25\end{array}$ & $\begin{array}{r}8 \\
9 \\
10 \\
15 \\
23 \\
24 \\
26\end{array}$ & $\begin{array}{l}504 \\
368 \\
459 \\
164 \\
732 \\
623 \\
255\end{array}$ & $\begin{array}{r}0 \\
5 \\
13 \\
9 \\
9 \\
7 \\
18\end{array}$ & $\begin{array}{l}0 \\
1 \\
2 \\
2 \\
0 \\
0 \\
6\end{array}$ & $\begin{array}{r}0 \\
2 \\
8 \\
5 \\
6 \\
4 \\
10\end{array}$ & $\begin{array}{l}0 \\
2 \\
3 \\
2 \\
3 \\
3 \\
2\end{array}$ & $\begin{array}{l}0 \\
0 \\
0 \\
0 \\
0 \\
0 \\
0\end{array}$ & $\begin{array}{l}0 \\
0 \\
0 \\
0 \\
0 \\
0 \\
0\end{array}$ \\
\hline $6-12$ & $\begin{array}{l}4 \\
7 \\
8 \\
9 \\
10 \\
11 \\
12 \\
13 \\
14 \\
15 \\
16 \\
22 \\
24 \\
25 \\
26\end{array}$ & $\begin{array}{r}6 \\
7 \\
8 \\
9 \\
10 \\
11 \\
12 \\
13 \\
14 \\
15 \\
16 \\
23 \\
24 \\
25 \\
26\end{array}$ & $\begin{array}{c}1+++ \\
9+++ \\
7+++ \\
10+++ \\
73+ \\
83+ \\
16+++ \\
218 \\
216+ \\
0+++ \\
47++ \\
8+++ \\
14++ \\
308 \\
13+++\end{array}$ & $\begin{array}{r}0 \\
0 \\
13 \\
0 \\
3 \\
10 \\
6 \\
26 \\
22 \\
0 \\
13 \\
0 \\
0 \\
12 \\
15\end{array}$ & $\begin{array}{r}0 \\
0 \\
13 \\
0 \\
1 \\
1 \\
0 \\
12 \\
6 \\
0 \\
11 \\
0 \\
0 \\
0 \\
7\end{array}$ & $\begin{array}{r}0 \\
0 \\
0 \\
0 \\
0 \\
6 \\
6 \\
12 \\
15 \\
0 \\
2 \\
0 \\
0 \\
6 \\
8\end{array}$ & $\begin{array}{l}0 \\
0 \\
0 \\
0 \\
0 \\
1 \\
0 \\
2 \\
1 \\
0 \\
0 \\
0 \\
0 \\
6 \\
0\end{array}$ & $\begin{array}{l}0 \\
0 \\
0 \\
0 \\
2 \\
2 \\
0 \\
0 \\
0 \\
0 \\
0 \\
0 \\
0 \\
0 \\
0\end{array}$ & $\begin{array}{l}0 \\
0 \\
0 \\
0 \\
0 \\
0 \\
0 \\
0 \\
0 \\
0 \\
0 \\
0 \\
0 \\
0 \\
0\end{array}$ \\
\hline
\end{tabular}


TABLE 19.- NUMBER OF PINK TRACER PARTICLES(1), PER 100 GRAMS OF BEDLOAD SAMPLE, COLLECTED AT ONE OR MORE CROSS-CHANNEL SAMPLING POSITIONS SPACED AT I-METER INTERVALS ACROSS THE CHANNEL WIDTH, EAST FORK RIVER, WYOMING, 1980--CONTINUED

\section{SECTION 1481}

\begin{tabular}{|c|c|c|c|c|c|c|c|c|c|}
\hline \multirow{3}{*}{ DATE } & \multirow{3}{*}{\multicolumn{2}{|c|}{$\begin{array}{l}\text { SAMPLING } \\
\text { POSITION } \\
\text { FROM TO TO }\end{array}$}} & \multirow{3}{*}{$\begin{array}{c}\text { DRY } \\
\text { MASS } \\
\text { OF } \\
\text { SAMPLE (2) } \\
\text { (G) }\end{array}$} & \multicolumn{2}{|c|}{ NUMBER OF } & \multicolumn{3}{|c|}{ PINK TRACER PARTICLES } & \\
\hline & & & & \multirow[b]{2}{*}{ TOTAL } & \multicolumn{5}{|c|}{ BY SIZE CLASS (MM) } \\
\hline & & TO & & & $\begin{array}{l}0.25 \\
\text { TO } \\
0.50\end{array}$ & $\begin{array}{l}0.50 \\
\text { TO } \\
1.00\end{array}$ & $\begin{array}{l}1.00 \\
\text { TO } \\
2.00\end{array}$ & $\begin{array}{l}2.00 \\
\text { TO } \\
4.00\end{array}$ & $\begin{array}{l}4.00 \\
\text { TO } \\
8.00\end{array}$ \\
\hline $6-13$ & $\begin{array}{r}5 \\
6 \\
7 \\
8 \\
9 \\
10 \\
11 \\
12 \\
13 \\
22 \\
23 \\
24 \\
25 \\
26\end{array}$ & $\begin{array}{r}5 \\
6 \\
7 \\
8 \\
9 \\
10 \\
11 \\
12 \\
15 \\
22 \\
23 \\
24 \\
25 \\
26\end{array}$ & $\begin{array}{c}0+++ \\
7+++ \\
6+++ \\
17++ \\
80+ \\
240 \\
82+ \\
198 \\
133+ \\
340 \\
136 \\
146 \\
642 \\
100+\end{array}$ & $\begin{array}{r}0 \\
0 \\
0 \\
0 \\
0 \\
5 \\
12 \\
19 \\
16 \\
15 \\
8 \\
11 \\
0 \\
25\end{array}$ & $\begin{array}{l}0 \\
0 \\
0 \\
0 \\
0 \\
2 \\
1 \\
5 \\
4 \\
4 \\
1 \\
5 \\
0 \\
8\end{array}$ & $\begin{array}{r}0 \\
0 \\
0 \\
0 \\
0 \\
3 \\
9 \\
12 \\
12 \\
9 \\
6 \\
4 \\
0 \\
15\end{array}$ & $\begin{array}{l}0 \\
0 \\
0 \\
0 \\
0 \\
0 \\
1 \\
2 \\
0 \\
2 \\
1 \\
2 \\
0 \\
2\end{array}$ & $\begin{array}{l}0 \\
0 \\
0 \\
0 \\
0 \\
0 \\
1 \\
0 \\
0 \\
0 \\
0 \\
0 \\
0 \\
0\end{array}$ & $\begin{array}{l}0 \\
0 \\
0 \\
0 \\
0 \\
0 \\
0 \\
0 \\
0 \\
0 \\
0 \\
0 \\
0 \\
0\end{array}$ \\
\hline $6-14$ & $\begin{array}{r}7 \\
8 \\
9 \\
10 \\
11 \\
12 \\
13 \\
15 \\
23 \\
24 \\
25 \\
26 \\
27\end{array}$ & $\begin{array}{r}7 \\
8 \\
9 \\
10 \\
11 \\
12 \\
13 \\
15 \\
23 \\
24 \\
25 \\
26 \\
27\end{array}$ & $\begin{array}{c}37++ \\
34++ \\
630 \\
72+ \\
206 \\
69+ \\
306 \\
76+ \\
903 \\
138 \\
1203 \\
51++ \\
39++\end{array}$ & $\begin{array}{r}0 \\
0 \\
0 \\
4 \\
14 \\
9 \\
11 \\
0 \\
8 \\
8 \\
6 \\
6 \\
10\end{array}$ & $\begin{array}{l}0 \\
0 \\
0 \\
0 \\
5 \\
0 \\
9 \\
0 \\
1 \\
1 \\
0 \\
2 \\
0\end{array}$ & $\begin{array}{l}0 \\
0 \\
0 \\
4 \\
6 \\
9 \\
2 \\
0 \\
6 \\
5 \\
2 \\
4 \\
8\end{array}$ & $\begin{array}{l}0 \\
0 \\
0 \\
0 \\
3 \\
0 \\
0 \\
0 \\
1 \\
1 \\
4 \\
0 \\
2\end{array}$ & $\begin{array}{l}0 \\
0 \\
0 \\
0 \\
0 \\
0 \\
0 \\
0 \\
0 \\
1 \\
0 \\
0 \\
0\end{array}$ & $\begin{array}{l}0 \\
0 \\
0 \\
0 \\
0 \\
0 \\
0 \\
0 \\
0 \\
0 \\
0 \\
0 \\
0\end{array}$ \\
\hline $6-15$ & $\begin{array}{r}4 \\
7 \\
8 \\
9 \\
10 \\
11 \\
12 \\
22 \\
24\end{array}$ & $\begin{array}{r}6 \\
7 \\
8 \\
9 \\
10 \\
11 \\
14 \\
23 \\
27\end{array}$ & $\begin{array}{c}8+++ \\
60+ \\
90+ \\
412 \\
549 \\
473 \\
856 \\
457 \\
597\end{array}$ & $\begin{array}{r}0 \\
3 \\
2 \\
8 \\
8 \\
16 \\
18 \\
24 \\
35\end{array}$ & $\begin{array}{l}0 \\
0 \\
0 \\
0 \\
0 \\
1 \\
3 \\
5 \\
5\end{array}$ & $\begin{array}{r}0 \\
0 \\
2 \\
4 \\
3 \\
13 \\
12 \\
15 \\
30\end{array}$ & $\begin{array}{l}0 \\
2 \\
0 \\
4 \\
5 \\
2 \\
3 \\
4 \\
0\end{array}$ & $\begin{array}{l}0 \\
1 \\
0 \\
0 \\
0 \\
0 \\
0 \\
0 \\
0\end{array}$ & $\begin{array}{l}0 \\
0 \\
0 \\
0 \\
0 \\
0 \\
0 \\
0 \\
0\end{array}$ \\
\hline
\end{tabular}


TABLE 19.- NUMBER OF PINK TRACER PARTICLES(1), PER 100 GRAMS OF BEDLOAD SAMPLE, COLLECTED AT ONE OR MORE CROSS-CHANNEL SAMPLING POSITIONS SPACED AT 1-METER INTERVALS ACROSS THE CHANNEL WIDTH, EAST FORK RIVER, WYOMING, 1980--CONTINUED

SECTION 1481

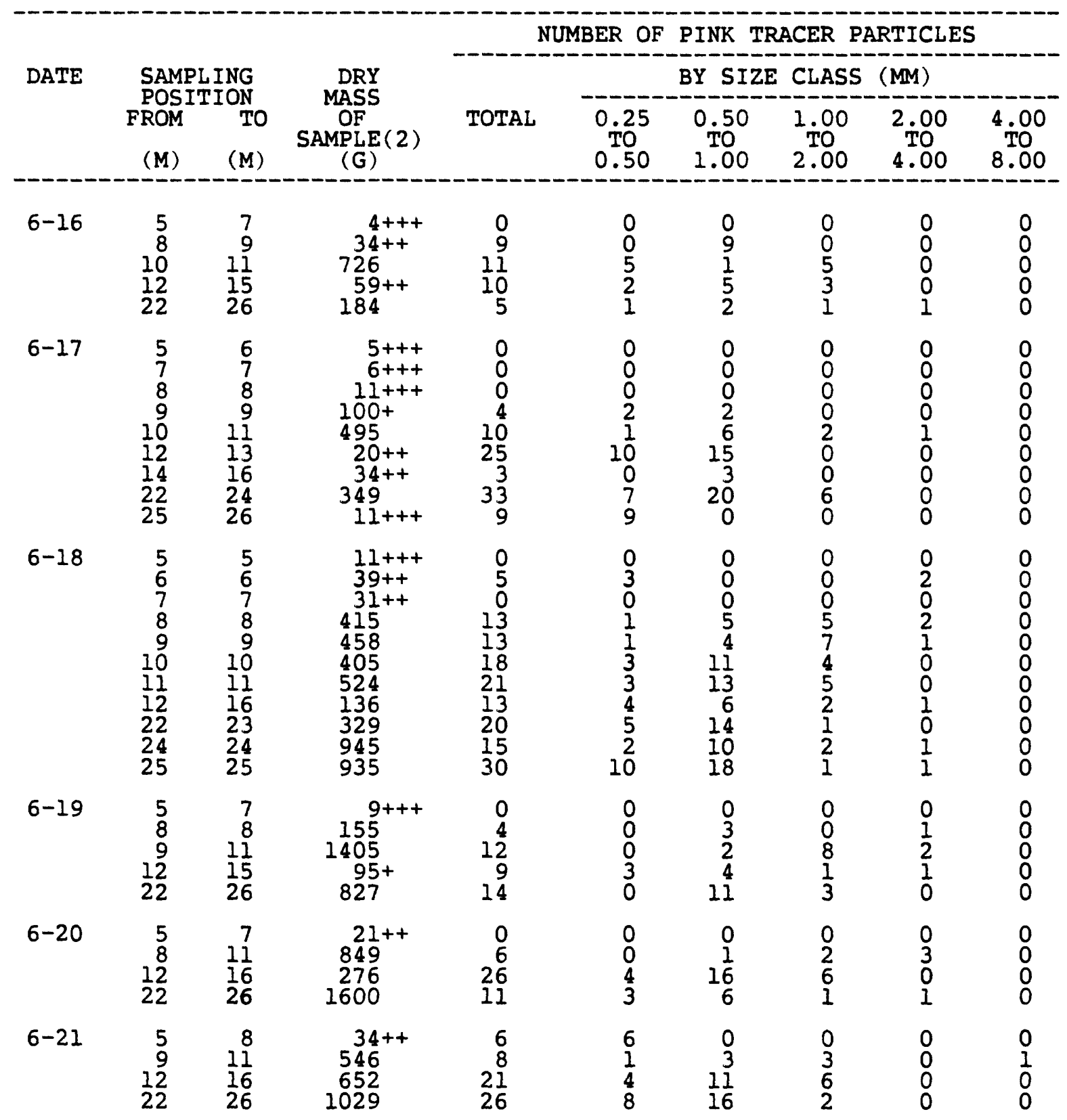


TABLE 19.- NUMBER OF PINK TRACER PARTICLES(1), PER 100 GRAMS OF BEDLOAD SAMPLE, COLLECTED AT ONE OR MORE CROSS-CHANNEL SAMPLING POSITIONS SPACED AT 1-METER INTERVALS ACROSS THE CHANNEL WIDTH, EAST FORK RIVER, WYOMING, 1980--CONTINUED

SECTION 1481

\begin{tabular}{|c|c|c|c|c|c|c|c|c|c|}
\hline \multirow{3}{*}{ DATE } & & & & \multicolumn{6}{|c|}{ NUMBER OF PINK TRACER PARTICLES } \\
\hline & \multicolumn{2}{|c|}{$\begin{array}{l}\text { SAMPLING } \\
\text { POSITION }\end{array}$} & \multirow{2}{*}{$\begin{array}{c}\text { DRY } \\
\text { MASS } \\
\text { OF } \\
\text { SAMPLE (2) } \\
\text { (G) }\end{array}$} & \multirow[b]{2}{*}{ TOTAL } & \multicolumn{5}{|c|}{ BY SIZE CLASS (MM) } \\
\hline & $\begin{array}{r}\text { FROM } \\
(M)\end{array}$ & TO & & & $\begin{array}{l}0.25 \\
\text { TO } \\
0.50\end{array}$ & $\begin{array}{l}0.50 \\
\text { TO } \\
1.00\end{array}$ & $\begin{array}{l}1.00 \\
\text { TO } \\
2.00\end{array}$ & $\begin{array}{l}2.00 \\
\text { TO } \\
4.00\end{array}$ & $\begin{array}{l}4.00 \\
\text { TO } \\
8.00\end{array}$ \\
\hline $6-23$ & $\begin{array}{r}5 \\
9 \\
22\end{array}$ & $\begin{array}{r}8 \\
16 \\
26\end{array}$ & $\begin{array}{l}111^{+++} \\
765 \\
465\end{array}$ & $\begin{array}{r}0 \\
14 \\
25\end{array}$ & $\begin{array}{l}0 \\
1 \\
5\end{array}$ & $\begin{array}{r}0 \\
8 \\
13\end{array}$ & $\begin{array}{l}0 \\
4 \\
5\end{array}$ & $\begin{array}{l}0 \\
1 \\
2\end{array}$ & $\begin{array}{l}0 \\
0 \\
0\end{array}$ \\
\hline $6-25$ & $\begin{array}{r}5 \\
22\end{array}$ & $\begin{array}{l}16 \\
26\end{array}$ & $\begin{array}{r}1216 \\
347\end{array}$ & 21 & $\begin{array}{l}2 \\
4\end{array}$ & $\begin{array}{l}11 \\
13\end{array}$ & $\begin{array}{l}7 \\
3\end{array}$ & $\frac{1}{1}$ & $\begin{array}{l}0 \\
0\end{array}$ \\
\hline $6-27$ & 25 & $\begin{array}{l}16 \\
26\end{array}$ & $\begin{array}{l}1479 \\
1109\end{array}$ & $\begin{array}{l}29 \\
27\end{array}$ & $\begin{array}{l}5 \\
7\end{array}$ & $\begin{array}{l}23 \\
16\end{array}$ & $\frac{1}{2}$ & $\begin{array}{l}0 \\
2\end{array}$ & $\begin{array}{l}0 \\
0\end{array}$ \\
\hline
\end{tabular}


TABLE 19.- NUMBER OF PINK TRACER PARTICLES(1), PER 100 GRAMS OF BEDLOAD SAMPLE, COLLECTED AT ONE OR MORE CROSS-CHANNEL SAMPLING POSITIONS SPACED AT 1-METER INTERVALS ACROSS THE CHANNEL WIDTH, EAST FORK RIVER, WYOMING, 1980--CONTINUED

SECTION 1533

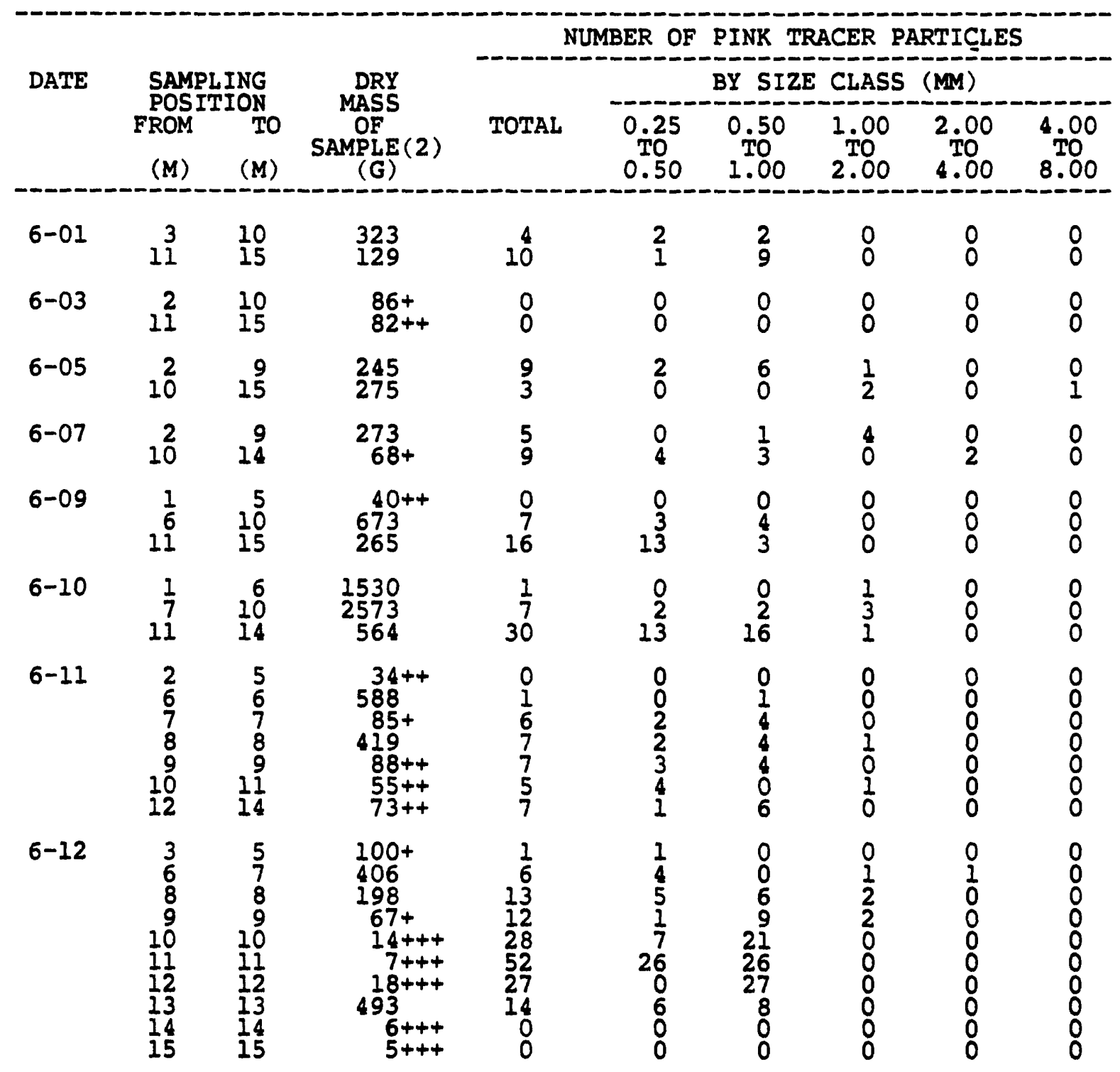


TABLE 19.- NUMBER OF PINK TRACER PARTICLES(1), PER 100 GRAMS OF BEDLOAD SAMPLE, COLLECTED AT ONE OR MORE CROSS-CHANNEL SAMPL ING POSITIONS SPACED AT I-METER INTERVALS ACROSS THE CHANNEL WIDTH, EAST FORK RIVER, WYOMING, 1980--CONTINUED

SECTION 1533

\begin{tabular}{|c|c|c|c|c|c|c|c|c|c|}
\hline \multirow{3}{*}{ DATE } & \multirow{3}{*}{\multicolumn{2}{|c|}{$\begin{array}{l}\text { SAMPLING } \\
\text { POSITION } \\
\text { FROM TO }\end{array}$}} & \multirow{3}{*}{$\begin{array}{c}\text { DRY } \\
\text { MASS } \\
\text { OF } \\
\text { SAMPLE (2) } \\
\text { (G) }\end{array}$} & \multicolumn{6}{|c|}{ NUMBER OF PINK TRACER PARTICLES } \\
\hline & & & & \multirow[b]{2}{*}{ TOTAL } & \multicolumn{5}{|c|}{ BY SIZE CLASS (MM) } \\
\hline & & & & & $\begin{array}{l}0.25 \\
\mathrm{TO} \\
0.50\end{array}$ & $\begin{array}{l}0.50 \\
\text { TO } \\
1.00\end{array}$ & $\begin{array}{l}1.00 \\
\text { To } \\
2.00\end{array}$ & $\begin{array}{r}2.00 \\
\text { TO } \\
4.00\end{array}$ & $\begin{array}{l}4.00 \\
\text { T.00 } \\
8.00\end{array}$ \\
\hline $6-13$ & $\begin{array}{r}2 \\
5 \\
6 \\
7 \\
8 \\
9 \\
10 \\
11 \\
12 \\
13\end{array}$ & $\begin{array}{r}4 \\
5 \\
6 \\
7 \\
8 \\
9 \\
10 \\
11 \\
12 \\
15\end{array}$ & $\begin{array}{c}11+++ \\
6+++ \\
130 \\
262 \\
503 \\
734 \\
881 \\
59++ \\
924 \\
49++\end{array}$ & $\begin{array}{r}0 \\
0 \\
0 \\
13 \\
15 \\
14 \\
13 \\
10 \\
29 \\
10\end{array}$ & $\begin{array}{l}0 \\
0 \\
0 \\
2 \\
2 \\
2 \\
1 \\
2 \\
9 \\
0\end{array}$ & $\begin{array}{r}0 \\
0 \\
0 \\
8 \\
10 \\
7 \\
10 \\
5 \\
17 \\
10\end{array}$ & $\begin{array}{l}0 \\
0 \\
0 \\
3 \\
3 \\
5 \\
2 \\
3 \\
3 \\
0\end{array}$ & $\begin{array}{l}0 \\
0 \\
0 \\
0 \\
0 \\
0 \\
0 \\
0 \\
0 \\
0\end{array}$ & $\begin{array}{l}0 \\
0 \\
0 \\
0 \\
0 \\
0 \\
0 \\
0 \\
0 \\
0\end{array}$ \\
\hline $6-14$ & $\begin{array}{r}6 \\
7 \\
8 \\
9 \\
10 \\
11 \\
12 \\
13 \\
14 \\
15\end{array}$ & $\begin{array}{r}6 \\
7 \\
8 \\
9 \\
10 \\
11 \\
12 \\
13 \\
14 \\
15\end{array}$ & $\begin{array}{c}862 \\
994 \\
403 \\
712 \\
459 \\
29++ \\
34+++ \\
37++ \\
15+++ \\
8+++\end{array}$ & $\begin{array}{r}7 \\
5 \\
11 \\
6 \\
11 \\
7 \\
6 \\
5 \\
13 \\
0\end{array}$ & $\begin{array}{l}0 \\
1 \\
3 \\
0 \\
0 \\
0 \\
3 \\
0 \\
0 \\
0\end{array}$ & $\begin{array}{r}5 \\
3 \\
5 \\
3 \\
10 \\
7 \\
3 \\
5 \\
13 \\
0\end{array}$ & $\begin{array}{l}0 \\
1 \\
3 \\
3 \\
0 \\
0 \\
0 \\
0 \\
0 \\
0\end{array}$ & $\begin{array}{l}2 \\
0 \\
0 \\
0 \\
1 \\
0 \\
0 \\
0 \\
0 \\
0\end{array}$ & $\begin{array}{l}0 \\
0 \\
0 \\
0 \\
0 \\
0 \\
0 \\
0 \\
0 \\
0\end{array}$ \\
\hline $6-15$ & $\begin{array}{r}2 \\
5 \\
6 \\
7 \\
8 \\
9 \\
10 \\
11 \\
12\end{array}$ & $\begin{array}{r}4 \\
5 \\
6 \\
7 \\
8 \\
9 \\
10 \\
11 \\
15\end{array}$ & $\begin{array}{c}32++ \\
20++ \\
171 \\
244 \\
124 \\
255 \\
245 \\
1365 \\
202\end{array}$ & $\begin{array}{r}0 \\
0 \\
1 \\
5 \\
17 \\
17 \\
15 \\
7 \\
24\end{array}$ & $\begin{array}{r}0 \\
0 \\
1 \\
1 \\
2 \\
0 \\
2 \\
0 \\
10\end{array}$ & $\begin{array}{r}0 \\
0 \\
0 \\
1 \\
9 \\
9 \\
12 \\
3 \\
14\end{array}$ & $\begin{array}{l}0 \\
0 \\
0 \\
2 \\
6 \\
7 \\
1 \\
\frac{1}{2} \\
0\end{array}$ & $\begin{array}{l}0 \\
0 \\
0 \\
1 \\
0 \\
1 \\
0 \\
2 \\
0\end{array}$ & $\begin{array}{l}0 \\
0 \\
0 \\
0 \\
0 \\
0 \\
0 \\
0 \\
0\end{array}$ \\
\hline $6-17$ & $\begin{array}{r}3 \\
7 \\
8 \\
9 \\
10 \\
12\end{array}$ & $\begin{array}{r}6 \\
7 \\
8 \\
9 \\
11 \\
15\end{array}$ & $\begin{array}{l}12+++ \\
107+ \\
294 \\
244 \\
1260 \\
155\end{array}$ & $\begin{array}{l}0 \\
10 \\
11 \\
12 \\
15 \\
31\end{array}$ & $\begin{array}{r}0 \\
0 \\
1 \\
3 \\
6 \\
11\end{array}$ & $\begin{array}{r}0 \\
7 \\
8 \\
5 \\
7 \\
18\end{array}$ & $\begin{array}{l}0 \\
1 \\
1 \\
\frac{1}{3} \\
2 \\
1\end{array}$ & $\begin{array}{l}0 \\
2 \\
1 \\
1 \\
0 \\
1\end{array}$ & $\begin{array}{l}0 \\
0 \\
0 \\
0 \\
0 \\
0\end{array}$ \\
\hline
\end{tabular}


TABLE 19.- NUMBER OF PINK TRACER PARTICLES(1), PER 100 GRAMS OF BEDLOAD SAMPLE, COLLECTED AT ONE OR MORE CROSS-CHANNEL SAMPLING POSITIONS SPACED AT 1-METER INTERVALS ACROSS THE CHANNEL WIDTH, EAST FORK RIVER, WYOMING, 1980--CONTINUED

SECTION 1533

\begin{tabular}{|c|c|c|c|c|c|c|c|c|c|}
\hline \multirow{3}{*}{ DATE } & & & \multirow{3}{*}{$\begin{array}{c}\text { DRY } \\
\text { MASS } \\
\text { OF } \\
\text { SAMPLE (2) } \\
\text { (G) }\end{array}$} & \multicolumn{2}{|c|}{ NUMBER OF } & \multicolumn{3}{|c|}{ PINK TRACER PARTICLES } & \\
\hline & \multirow{2}{*}{\multicolumn{2}{|c|}{$\begin{array}{l}\text { SAMPLING } \\
\text { POSITION } \\
\text { FROM TO }\end{array}$}} & & \multirow[b]{2}{*}{ TOTAL } & \multicolumn{5}{|c|}{ BY SIZE CLASS (MM) } \\
\hline & & & & & $\begin{array}{l}0.25 \\
\text { TO } \\
0.50\end{array}$ & $\begin{array}{l}0.50 \\
\text { TO } \\
1.00\end{array}$ & $\begin{array}{l}1.00 \\
2.00\end{array}$ & $\begin{array}{l}2.00 \\
40 \\
4.00\end{array}$ & $\begin{array}{l}4.00 \\
\text { TO } \\
8.00\end{array}$ \\
\hline $6-18$ & $\begin{array}{r}3 \\
5 \\
6 \\
7 \\
8 \\
9 \\
10 \\
12 \\
13\end{array}$ & $\begin{array}{r}4 \\
5 \\
6 \\
7 \\
8 \\
9 \\
11 \\
12 \\
15\end{array}$ & $\begin{array}{c}9+++ \\
6+++ \\
203 \\
1095 \\
1037 \\
192 \\
939 \\
523 \\
214\end{array}$ & $\begin{array}{r}0 \\
0 \\
14 \\
4 \\
6 \\
15 \\
27 \\
33 \\
21\end{array}$ & $\begin{array}{r}0 \\
0 \\
3 \\
0 \\
1 \\
1 \\
1 \\
18 \\
1\end{array}$ & $\begin{array}{r}0 \\
0 \\
6 \\
1 \\
3 \\
6 \\
23 \\
15 \\
18\end{array}$ & $\begin{array}{l}0 \\
0 \\
5 \\
2 \\
2 \\
7 \\
3 \\
0 \\
2\end{array}$ & $\begin{array}{l}0 \\
0 \\
0 \\
1 \\
0 \\
1 \\
0 \\
0 \\
0\end{array}$ & $\begin{array}{l}0 \\
0 \\
0 \\
0 \\
0 \\
0 \\
0 \\
0 \\
0\end{array}$ \\
\hline $6-19$ & $\begin{array}{r}3 \\
6 \\
10\end{array}$ & $\begin{array}{r}5 \\
9 \\
15\end{array}$ & $\begin{array}{l}111++ \\
481 \\
251\end{array}$ & $\begin{array}{r}0 \\
12 \\
20\end{array}$ & $\begin{array}{l}0 \\
0 \\
8\end{array}$ & $\begin{array}{r}0 \\
7 \\
12\end{array}$ & $\begin{array}{l}0 \\
2 \\
0\end{array}$ & $\begin{array}{l}0 \\
3 \\
0\end{array}$ & $\begin{array}{l}0 \\
0 \\
0\end{array}$ \\
\hline $6-20$ & $\begin{array}{r}3 \\
6 \\
8 \\
11\end{array}$ & $\begin{array}{r}5 \\
7 \\
10 \\
15\end{array}$ & $\begin{array}{l}10+++ \\
549 \\
385 \\
439\end{array}$ & $\begin{array}{r}0 \\
14 \\
30 \\
30\end{array}$ & $\begin{array}{r}0 \\
2 \\
10 \\
11\end{array}$ & $\begin{array}{r}0 \\
9 \\
16 \\
19\end{array}$ & $\begin{array}{l}0 \\
3 \\
4 \\
0\end{array}$ & $\begin{array}{l}0 \\
0 \\
0 \\
0\end{array}$ & $\begin{array}{l}0 \\
0 \\
0 \\
0\end{array}$ \\
\hline $6-21$ & $\begin{array}{r}3 \\
6 \\
7 \\
10 \\
12 \\
14\end{array}$ & $\begin{array}{r}5 \\
6 \\
9 \\
11 \\
13 \\
15\end{array}$ & $\begin{array}{l}102 \\
501 \\
328 \\
398 \\
658 \\
44++\end{array}$ & $\begin{array}{r}1 \\
4 \\
20 \\
20 \\
25 \\
27\end{array}$ & $\begin{array}{l}0 \\
0 \\
3 \\
5 \\
6 \\
9\end{array}$ & $\begin{array}{l}0 \\
3 \\
12 \\
11 \\
17 \\
11\end{array}$ & $\begin{array}{l}0 \\
0 \\
4 \\
2 \\
1 \\
7\end{array}$ & $\begin{array}{l}1 \\
1 \\
1 \\
2 \\
1 \\
0\end{array}$ & $\begin{array}{l}0 \\
0 \\
0 \\
0 \\
0 \\
0\end{array}$ \\
\hline $6-23$ & $\begin{array}{l}5 \\
9\end{array}$ & $\begin{array}{r}8 \\
15\end{array}$ & $\begin{array}{r}1045 \\
208\end{array}$ & $\begin{array}{r}8 \\
16\end{array}$ & $\begin{array}{l}0 \\
6\end{array}$ & $\begin{array}{l}4 \\
9\end{array}$ & $\begin{array}{l}3 \\
1\end{array}$ & $\begin{array}{l}1 \\
0\end{array}$ & $\begin{array}{l}0 \\
0\end{array}$ \\
\hline $6-25$ & $\begin{array}{r}3 \\
7 \\
8 \\
9 \\
11\end{array}$ & $\begin{array}{r}6 \\
7 \\
8 \\
10 \\
14\end{array}$ & $\begin{array}{l}34++ \\
366 \\
520 \\
761 \\
74++\end{array}$ & $\begin{array}{r}9 \\
14 \\
30 \\
9 \\
9\end{array}$ & $\begin{array}{l}0 \\
0 \\
0 \\
0 \\
0\end{array}$ & $\begin{array}{r}3 \\
8 \\
18 \\
6 \\
9\end{array}$ & $\begin{array}{r}3 \\
6 \\
10 \\
2 \\
0\end{array}$ & $\begin{array}{l}3 \\
0 \\
2 \\
1 \\
0\end{array}$ & $\begin{array}{l}0 \\
0 \\
0 \\
0 \\
0\end{array}$ \\
\hline $6-27$ & $\begin{array}{r}5 \\
7 \\
8 \\
9 \\
10 \\
11 \\
12\end{array}$ & $\begin{array}{r}6 \\
7 \\
8 \\
9 \\
10 \\
11 \\
14\end{array}$ & $\begin{array}{l}22++ \\
295 \\
704 \\
421 \\
770 \\
663 \\
191\end{array}$ & $\begin{array}{l}99 \\
20 \\
10 \\
21 \\
34 \\
28 \\
30\end{array}$ & $\begin{array}{r}0 \\
3 \\
0 \\
2 \\
5 \\
3 \\
12\end{array}$ & $\begin{array}{r}99 \\
11 \\
6 \\
16 \\
24 \\
15 \\
18\end{array}$ & $\begin{array}{r}0 \\
4 \\
2 \\
3 \\
5 \\
10 \\
0\end{array}$ & $\begin{array}{l}0 \\
2 \\
2 \\
0 \\
0 \\
0 \\
0\end{array}$ & $\begin{array}{l}0 \\
0 \\
0 \\
0 \\
0 \\
0 \\
0\end{array}$ \\
\hline
\end{tabular}


TABLE 19.- NUMBER OF PINK TRACER PARTICLES(1), PER 100 GRAMS OF BEDLOAD SAMPLE, COLLECTED AT ONE OR MORE CROSS-CHANNEL SAMPLING POSITIONS SPACED AT I-METER INTERVALS ACROSS THE CHANNEL WIDTH, EAST FORK RIVER, WYOMING, 1980--CONTINUED

SECTION 1533

\begin{tabular}{|c|c|c|c|c|c|c|c|c|c|}
\hline \multirow{3}{*}{ DATE } & \multirow{3}{*}{\multicolumn{2}{|c|}{$\begin{array}{l}\text { SAMPLING } \\
\text { POSITION } \\
\text { FROM TO }\end{array}$}} & \multirow{3}{*}{$\begin{array}{c}\text { DRY } \\
\text { MASS } \\
\text { OF } \\
\text { SAMPLE (2) } \\
(G)\end{array}$} & \multicolumn{6}{|c|}{ NUMBER OF PINK TRACER PARTICLES } \\
\hline & & & & \multirow[b]{2}{*}{ TOTAL } & \multicolumn{5}{|c|}{ BY SIZE CLASS (MM) } \\
\hline & & & & & $\begin{array}{l}0.25 \\
\text { TO } \\
0.50\end{array}$ & $\begin{array}{l}0.50 \\
\text { TO } \\
1.00\end{array}$ & $\begin{array}{l}1.00 \\
2.00\end{array}$ & $\begin{array}{l}2.00 \\
\text { TO } \\
4.00\end{array}$ & $\begin{array}{l}4.00 \\
\text { TO } \\
8.00\end{array}$ \\
\hline $7-03$ & $\begin{array}{r}5 \\
8 \\
10\end{array}$ & $\begin{array}{r}7 \\
9 \\
15\end{array}$ & $\begin{array}{l}442+ \\
480+ \\
121++\end{array}$ & $\begin{array}{r}7 \\
23 \\
4\end{array}$ & $\begin{array}{l}\frac{1}{3} \\
0\end{array}$ & $\begin{array}{r}0 \\
12 \\
4\end{array}$ & $\begin{array}{l}4 \\
5 \\
0\end{array}$ & $\begin{array}{l}2 \\
3 \\
0\end{array}$ & $\begin{array}{l}0 \\
0 \\
0\end{array}$ \\
\hline $7-05$ & $\begin{array}{r}6 \\
9 \\
11 \\
12\end{array}$ & $\begin{array}{r}8 \\
10 \\
11 \\
13\end{array}$ & $\begin{array}{l}540 \\
256++ \\
408+ \\
42+++\end{array}$ & $\begin{array}{r}13 \\
15 \\
13 \\
0\end{array}$ & $\begin{array}{l}\frac{1}{2} \\
3 \\
0\end{array}$ & $\begin{array}{l}3 \\
7 \\
5 \\
0\end{array}$ & $\begin{array}{l}8 \\
6 \\
5 \\
0\end{array}$ & $\begin{array}{l}1 \\
0 \\
0 \\
0\end{array}$ & $\begin{array}{l}0 \\
0 \\
0 \\
0\end{array}$ \\
\hline $7-07$ & $\begin{array}{r}6 \\
10\end{array}$ & $\begin{array}{r}9 \\
12\end{array}$ & $\begin{array}{l}62 \\
18++\end{array}$ & $\begin{array}{r}0 \\
21\end{array}$ & $\begin{array}{l}0 \\
0\end{array}$ & $\begin{array}{r}0 \\
16\end{array}$ & $\begin{array}{l}0 \\
5\end{array}$ & $\begin{array}{l}0 \\
0\end{array}$ & $\begin{array}{l}0 \\
0\end{array}$ \\
\hline
\end{tabular}


TABLE 19.- NUMBER OF PINK TRACER PARTICLES(1), PER 100 GRAMS OF BEDLOAD SAMPLE, COLLECTED AT ONE OR MORE CROSS-CHANNEL SAMPLING POSITIONS SPACED AT 1-METER INTERVALS ACROSS THE CHANNEL WIDTH, EAST FORK RIVER, WYOMING, 1980--CONTINUED

SECTION 1573

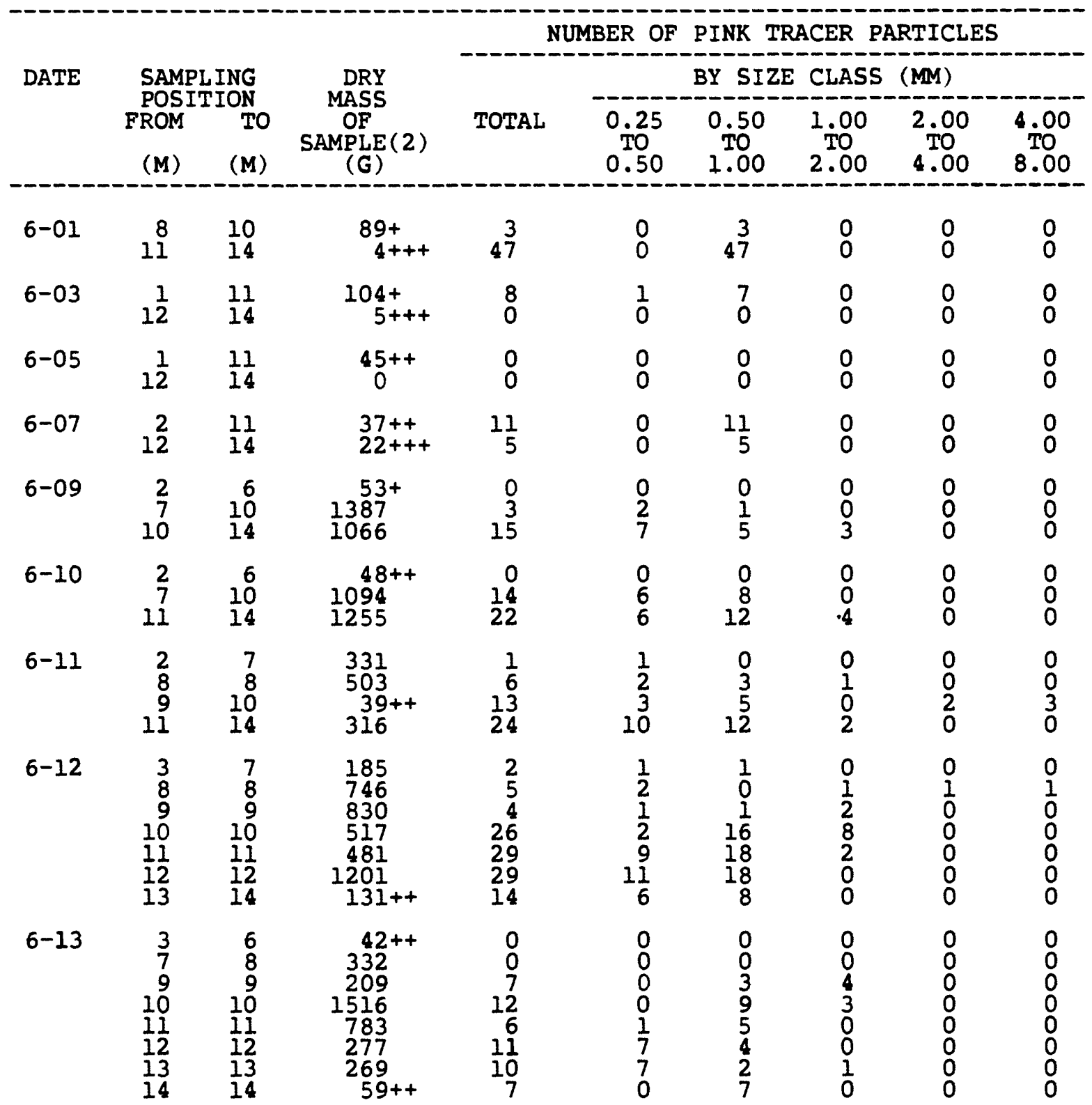


TABLE 19.- NUMBER OF PINK TRACER PARTICLES(1), PER 100 GRAMS OF BEDLOAD SAMPLE, COLLECTED AT ONE OR MORE CROSS-CHANNEL SAMPLING POSITIONS SPACED AT I-METER INTERVALS ACROSS THE CHANNEL WIDTH, EAST FORK RIVER, WYOMING, 1980--CONTINUED

SECTION 1573

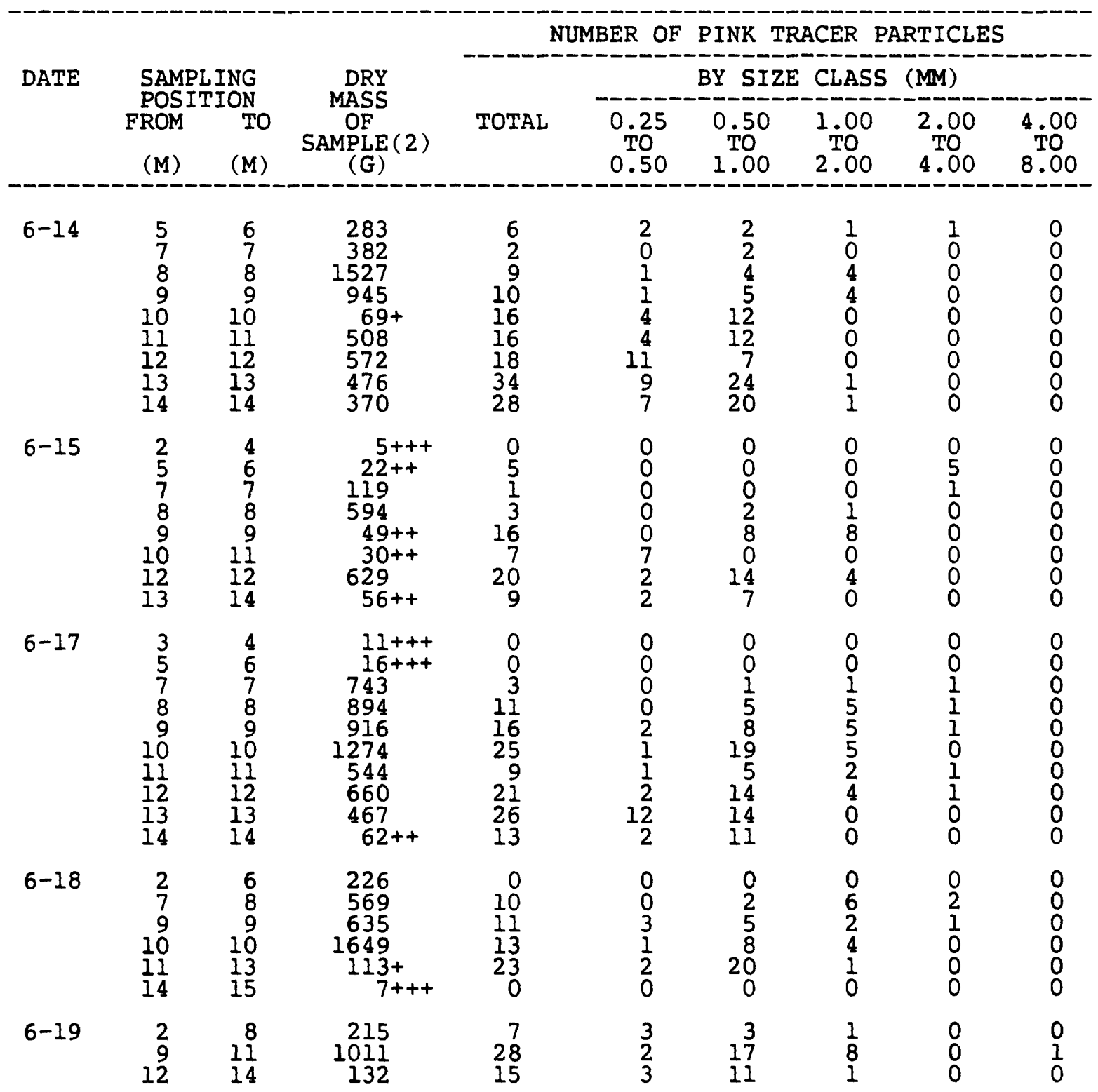


TABLE 19.- NUMBER OF PINK TRACER PARTICLES(1), PER 100 GRAMS OF BEDLOAD SAMPLE, COLLECTED AT ONE OR MORE CROSS-CHANNEL SAMPLING POSITIONS SPACED AT 1-METER INTERVALS ACROSS THE CHANNEL WIDTH, EAST FORK RIVER, WYOMING, 1980--CONTINUED

SECTION 1573

\begin{tabular}{|c|c|c|c|c|c|c|c|c|c|}
\hline \multirow{3}{*}{ DATE } & & & & \multicolumn{6}{|c|}{ NUMBER OF PINK TRACER PARTICLES } \\
\hline & \multicolumn{2}{|c|}{$\begin{array}{l}\text { SAMPLING } \\
\text { POSITION } \\
\text { FROM TO }\end{array}$} & \multirow{2}{*}{$\begin{array}{c}\text { DRY } \\
\text { MASS } \\
\text { OF } \\
\text { SAMPLE (2) } \\
\text { (G) }\end{array}$} & \multirow[b]{2}{*}{ TOTAL } & \multicolumn{5}{|c|}{ BY SIZE CLASS (MM) } \\
\hline & FROM & TO & & & $\begin{array}{l}0.25 \\
\text { TO } \\
0.50\end{array}$ & $\begin{array}{l}0.50 \\
\text { TO } \\
1.00\end{array}$ & $\begin{array}{l}1.00 \\
2.00 \\
\text { TO }\end{array}$ & $\begin{array}{l}2.00 \\
\text { TOO } \\
4.00\end{array}$ & $\begin{array}{l}4.00 \\
8.00 \\
\text { To }\end{array}$ \\
\hline $6-20$ & $\begin{array}{r}1 \\
8 \\
9 \\
12\end{array}$ & $\begin{array}{r}7 \\
8 \\
11 \\
14\end{array}$ & $\begin{array}{r}799 \\
1300 \\
1626 \\
544\end{array}$ & $\begin{array}{r}8 \\
9 \\
0 \\
27\end{array}$ & $\begin{array}{l}0 \\
1 \\
0 \\
9\end{array}$ & $\begin{array}{r}6 \\
4 \\
0 \\
17\end{array}$ & $\begin{array}{l}1 \\
2 \\
0 \\
1\end{array}$ & $\begin{array}{l}1 \\
1 \\
0 \\
0\end{array}$ & $\begin{array}{l}0 \\
1 \\
0 \\
0\end{array}$ \\
\hline $6-21$ & $\begin{array}{r}3 \\
8 \\
9 \\
10 \\
11 \\
12\end{array}$ & $\begin{array}{r}7 \\
8 \\
9 \\
10 \\
11 \\
14\end{array}$ & $\begin{array}{l}17++ \\
617 \\
936 \\
735 \\
878 \\
155+\end{array}$ & $\begin{array}{r}0 \\
14 \\
7 \\
15 \\
36 \\
31\end{array}$ & $\begin{array}{r}0 \\
0 \\
1 \\
2 \\
14 \\
12\end{array}$ & $\begin{array}{r}0 \\
7 \\
4 \\
8 \\
20 \\
17\end{array}$ & $\begin{array}{l}0 \\
6 \\
1 \\
5 \\
2 \\
2\end{array}$ & $\begin{array}{l}0 \\
1 \\
1 \\
0 \\
0 \\
0\end{array}$ & $\begin{array}{l}0 \\
0 \\
0 \\
0 \\
0 \\
0\end{array}$ \\
\hline $6-23$ & $\begin{array}{r}1 \\
8 \\
10 \\
13\end{array}$ & $\begin{array}{r}7 \\
99 \\
12 \\
14\end{array}$ & $\begin{array}{c}46++ \\
1261 \\
458 \\
83++\end{array}$ & $\begin{array}{r}0 \\
8 \\
26 \\
17\end{array}$ & $\begin{array}{l}0 \\
0 \\
6 \\
6\end{array}$ & $\begin{array}{r}0 \\
0 \\
19 \\
7\end{array}$ & $\begin{array}{l}0 \\
4 \\
1 \\
4\end{array}$ & $\begin{array}{l}0 \\
3 \\
0 \\
0\end{array}$ & $\begin{array}{l}0 \\
1 \\
0 \\
0\end{array}$ \\
\hline $6-25$ & $\begin{array}{l}2 \\
11 \\
12\end{array}$ & $\begin{array}{l}10 \\
11 \\
14\end{array}$ & $\begin{array}{r}1295 \\
500 \\
396\end{array}$ & $\begin{array}{l}28 \\
30 \\
28\end{array}$ & $\begin{array}{r}3 \\
9 \\
14\end{array}$ & $\begin{array}{l}23 \\
19 \\
13\end{array}$ & $\begin{array}{l}2 \\
2 \\
1\end{array}$ & $\begin{array}{l}0 \\
0 \\
0\end{array}$ & $\begin{array}{l}0 \\
0 \\
0\end{array}$ \\
\hline $6-27$ & $\begin{array}{r}5 \\
8 \\
9 \\
10 \\
11 \\
12 \\
13\end{array}$ & $\begin{array}{r}7 \\
8 \\
9 \\
10 \\
11 \\
12 \\
14\end{array}$ & $\begin{array}{l}5^{+++} \\
345 \\
317 \\
507 \\
646 \\
323 \\
413\end{array}$ & $\begin{array}{r}17 \\
7 \\
29 \\
17 \\
39 \\
0 \\
25\end{array}$ & $\begin{array}{l}0 \\
0 \\
4 \\
2 \\
4 \\
0 \\
8\end{array}$ & $\begin{array}{r}0 \\
2 \\
13 \\
9 \\
26 \\
0 \\
17\end{array}$ & $\begin{array}{r}0 \\
2 \\
10 \\
5 \\
9 \\
0 \\
0\end{array}$ & $\begin{array}{r}17 \\
3 \\
2 \\
1 \\
0 \\
0 \\
0\end{array}$ & $\begin{array}{l}0 \\
0 \\
0 \\
0 \\
0 \\
0 \\
0\end{array}$ \\
\hline $7-05$ & $\begin{array}{r}8 \\
12\end{array}$ & $\frac{11}{14}$ & $\begin{array}{l}139++ \\
130+\end{array}$ & $\begin{array}{r}5 \\
29\end{array}$ & $\frac{1}{7}$ & $\begin{array}{r}1 \\
14\end{array}$ & $\frac{1}{7}$ & $\begin{array}{l}2 \\
1\end{array}$ & $\begin{array}{l}0 \\
0\end{array}$ \\
\hline $7-07$ & $\begin{array}{r}8 \\
11\end{array}$ & $\begin{array}{l}10 \\
14\end{array}$ & $\begin{array}{c}341+ \\
51++\end{array}$ & $\begin{array}{l}15 \\
33\end{array}$ & $\begin{array}{r}3 \\
10\end{array}$ & 199 & $\begin{array}{l}2 \\
6\end{array}$ & $\frac{1}{6}$ & $\begin{array}{l}0 \\
0\end{array}$ \\
\hline
\end{tabular}


TABLE 19.- NUMBER OF PINK TRACER PARTICLES(1), PER 100 GRAMS OF BEDLOAD SAMPLE, COLLECTED AT ONE OR MORE CROSS-CHANNEL SAMPLING POSITIONS SPACED AT I-METER INTERVALS ACROSS THE CHANNEL WIDTH, EAST FORK RIVER, WYOMING, 1980--CONTINUED

SECTION 1610

\begin{tabular}{|c|c|c|c|c|c|c|c|c|c|}
\hline \multirow{3}{*}{ DATE } & \multirow{3}{*}{\multicolumn{2}{|c|}{$\begin{array}{l}\text { SAMPLING } \\
\text { POSITION } \\
\text { FROM TO }\end{array}$}} & \multirow{3}{*}{$\begin{array}{c}\text { DRY } \\
\text { MASS } \\
\text { OF } \\
\text { SAMPLE (2) } \\
\text { (G) }\end{array}$} & \multicolumn{2}{|c|}{ NUMBER OF } & \multicolumn{4}{|c|}{ PINK TRACER PARTICLES } \\
\hline & & & & \multirow[b]{2}{*}{ TOTAL } & \multicolumn{5}{|c|}{ BY SIZE CLASS (MM) } \\
\hline & & & & & $\begin{array}{l}0.25 \\
\text { To } \\
0.50\end{array}$ & $\begin{array}{l}0.50 \\
\text { To } \\
1.00\end{array}$ & $\begin{array}{l}1.00 \\
2.00\end{array}$ & $\begin{array}{l}2.00 \\
\text { To } \\
4.00\end{array}$ & $\begin{array}{r}4.00 \\
\text { To } \\
8.00\end{array}$ \\
\hline $6-01$ & 2 & 18 & 286 & 3 & 0 & 3 & 0 & 0 & 0 \\
\hline $6-03$ & $\begin{array}{r}2 \\
16\end{array}$ & $\begin{array}{l}15 \\
17\end{array}$ & $\underset{0+++}{161+}$ & $\begin{array}{l}0 \\
0\end{array}$ & $\begin{array}{l}0 \\
0\end{array}$ & $\begin{array}{l}0 \\
0\end{array}$ & $\begin{array}{l}0 \\
0\end{array}$ & $\begin{array}{l}0 \\
0\end{array}$ & $\begin{array}{l}0 \\
0\end{array}$ \\
\hline $6-05$ & $\begin{array}{r}2 \\
16\end{array}$ & $\begin{array}{l}15 \\
17\end{array}$ & $\begin{array}{c}91+ \\
0+++\end{array}$ & $\begin{array}{l}3 \\
0\end{array}$ & $\begin{array}{l}0 \\
0\end{array}$ & $\begin{array}{l}0 \\
0\end{array}$ & $\frac{1}{0}$ & $\begin{array}{l}2 \\
0\end{array}$ & $\begin{array}{l}0 \\
0\end{array}$ \\
\hline $6-07$ & $\begin{array}{r}2 \\
16\end{array}$ & $\begin{array}{l}15 \\
18\end{array}$ & $\begin{array}{l}1026 \\
55++\end{array}$ & $\begin{array}{r}10 \\
0\end{array}$ & $\begin{array}{l}0 \\
0\end{array}$ & $\begin{array}{l}8 \\
0\end{array}$ & $\begin{array}{l}2 \\
0\end{array}$ & $\begin{array}{l}0 \\
0\end{array}$ & $\begin{array}{l}0 \\
0\end{array}$ \\
\hline $6-09$ & 12 & $\begin{array}{l}10 \\
18\end{array}$ & $\begin{array}{c}677^{+} \\
2637^{-}\end{array}$ & $\begin{array}{l}0 \\
7\end{array}$ & $\begin{array}{l}0 \\
1\end{array}$ & $\begin{array}{l}0 \\
5\end{array}$ & $\begin{array}{l}0 \\
1\end{array}$ & $\begin{array}{l}0 \\
0\end{array}$ & $\begin{array}{l}0 \\
0\end{array}$ \\
\hline $6-10$ & $\begin{array}{r}3 \\
12 \\
13 \\
14 \\
16 \\
17\end{array}$ & $\begin{array}{l}12 \\
12 \\
13 \\
15 \\
16 \\
18\end{array}$ & $\begin{array}{r}1796 \\
1649 \\
1379 \\
560 \\
1282 \\
468\end{array}$ & $\begin{array}{l}13 \\
19 \\
13 \\
12 \\
15 \\
13\end{array}$ & $\begin{array}{l}3 \\
3 \\
0 \\
1 \\
2 \\
6\end{array}$ & $\begin{array}{r}9 \\
14 \\
10 \\
8 \\
11 \\
7\end{array}$ & $\begin{array}{l}1 \\
2 \\
2 \\
3 \\
2 \\
0\end{array}$ & $\begin{array}{l}0 \\
0 \\
1 \\
0 \\
0 \\
0\end{array}$ & $\begin{array}{l}0 \\
0 \\
0 \\
0 \\
0 \\
0\end{array}$ \\
\hline $6-11$ & $\begin{array}{r}2 \\
13 \\
15\end{array}$ & $\begin{array}{l}12 \\
14 \\
17\end{array}$ & $\begin{array}{r}1882 \\
1720 \\
459\end{array}$ & $\begin{array}{l}15 \\
15 \\
15\end{array}$ & $\begin{array}{l}4 \\
5 \\
4\end{array}$ & $\begin{array}{l}7 \\
8 \\
8\end{array}$ & $\begin{array}{l}4 \\
2 \\
2\end{array}$ & $\begin{array}{l}0 \\
0 \\
1\end{array}$ & $\begin{array}{l}0 \\
0 \\
0\end{array}$ \\
\hline $6-12$ & $\begin{array}{r}3 \\
14\end{array}$ & $\begin{array}{l}13 \\
18\end{array}$ & $\begin{array}{r}1067 \\
524\end{array}$ & $\begin{array}{l}10 \\
22\end{array}$ & $\begin{array}{l}2 \\
8\end{array}$ & 11 & $\begin{array}{l}3 \\
2\end{array}$ & $\frac{1}{1}$ & $\begin{array}{l}0 \\
0\end{array}$ \\
\hline $6-13$ & $\begin{array}{r}4 \\
16\end{array}$ & $\begin{array}{l}15 \\
18\end{array}$ & $\begin{array}{r}1659 \\
197\end{array}$ & $\begin{array}{r}3 \\
14\end{array}$ & $\begin{array}{l}0 \\
6\end{array}$ & $\frac{1}{4}$ & $\begin{array}{l}2 \\
4\end{array}$ & $\begin{array}{l}0 \\
0\end{array}$ & $\begin{array}{l}0 \\
0\end{array}$ \\
\hline $6-14$ & $\begin{array}{l}3 \\
11 \\
12 \\
13 \\
14 \\
15 \\
16 \\
17 \\
18\end{array}$ & $\begin{array}{l}10 \\
11 \\
12 \\
13 \\
14 \\
15 \\
16 \\
17 \\
18\end{array}$ & $\begin{array}{l}83+ \\
64+ \\
56++ \\
986 \\
516 \\
857 \\
11+++ \\
204 \\
84++\end{array}$ & $\begin{array}{r}0 \\
5 \\
2 \\
9 \\
9 \\
20 \\
0 \\
28 \\
14\end{array}$ & $\begin{array}{l}0 \\
0 \\
0 \\
0 \\
1 \\
7 \\
0 \\
8 \\
6\end{array}$ & $\begin{array}{r}0 \\
2 \\
2 \\
6 \\
6 \\
12 \\
0 \\
18 \\
8\end{array}$ & $\begin{array}{l}0 \\
3 \\
0 \\
3 \\
2 \\
1 \\
0 \\
2 \\
0\end{array}$ & $\begin{array}{l}0 \\
0 \\
0 \\
0 \\
0 \\
0 \\
0 \\
0 \\
0\end{array}$ & $\begin{array}{l}0 \\
0 \\
0 \\
0 \\
0 \\
0 \\
0 \\
0 \\
0\end{array}$ \\
\hline $6-15$ & $\begin{array}{r}2 \\
16\end{array}$ & $\begin{array}{l}15 \\
18\end{array}$ & $\begin{array}{l}1984 \\
1518\end{array}$ & $\begin{array}{l}13 \\
19\end{array}$ & $\frac{1}{2}$ & $\begin{array}{r}8 \\
13\end{array}$ & $\begin{array}{l}3 \\
4\end{array}$ & $\begin{array}{l}1 \\
0\end{array}$ & $\begin{array}{l}0 \\
0\end{array}$ \\
\hline
\end{tabular}


TABLE 19.- NUMBER OF PINK TRACER PARTICLES(1), PER 100 GRAMS OF BEDLOAD SAMPLE, COLLECTED AT ONE OR MORE CROSS-CHANNEL SAMPLING POSITIONS SPACED AT I-METER INTERVALS ACROSS THE CHANNEL WIDTH, EAST FORK RIVER, WYOMING, 1980--CONTINUED

SECTION 1610

\begin{tabular}{|c|c|c|c|c|c|c|c|c|c|}
\hline \multirow{3}{*}{ DATE } & \multirow{3}{*}{\multicolumn{2}{|c|}{$\begin{array}{l}\text { SAMPLING } \\
\text { POSITION } \\
\text { FROM TO }\end{array}$}} & \multirow{3}{*}{$\begin{array}{c}\text { DRY } \\
\text { MASS } \\
\text { OF } \\
\text { SAMPLE (2) } \\
\text { (G) }\end{array}$} & \multicolumn{6}{|c|}{ NUMBER OF PINK TRACER PARTICLES } \\
\hline & & & & \multirow[b]{2}{*}{ TOTAL } & \multicolumn{5}{|c|}{ BY SIZE CLASS (MM) } \\
\hline & & & & & $\begin{array}{c}0.25 \\
\mathrm{TO} \\
0.50\end{array}$ & $\begin{array}{l}0.50 \\
\text { TO } \\
1.00\end{array}$ & $\begin{array}{l}1.00 \\
\text { TO } \\
2.00\end{array}$ & $\begin{array}{l}2.00 \\
\text { TO } \\
4.00\end{array}$ & $\begin{array}{l}4.00 \\
\text { TO } \\
8.00\end{array}$ \\
\hline $6-17$ & 16 & $\begin{array}{l}15 \\
18\end{array}$ & $\begin{array}{r}3876 \\
208\end{array}$ & $\begin{array}{l}19 \\
26\end{array}$ & $\begin{array}{r}2 \\
16\end{array}$ & $\begin{array}{r}10 \\
9\end{array}$ & $\begin{array}{l}6 \\
1\end{array}$ & $\frac{1}{0}$ & $\begin{array}{l}0 \\
0\end{array}$ \\
\hline $6-18$ & $\begin{array}{r}2 \\
9 \\
12 \\
13 \\
14 \\
15 \\
16 \\
17\end{array}$ & $\begin{array}{l}8 \\
11 \\
12 \\
13 \\
14 \\
15 \\
16 \\
18\end{array}$ & $\begin{array}{l}17+++ \\
466 \\
176 \\
401 \\
430 \\
539 \\
396 \\
17++\end{array}$ & $\begin{array}{l}0 \\
13 \\
14 \\
24 \\
13 \\
17 \\
25 \\
12\end{array}$ & $\begin{array}{l}0 \\
0 \\
1 \\
2 \\
0 \\
3 \\
3 \\
0\end{array}$ & $\begin{array}{r}0 \\
3 \\
7 \\
17 \\
8 \\
10 \\
17 \\
12\end{array}$ & $\begin{array}{l}0 \\
9 \\
5 \\
5 \\
5 \\
4 \\
5 \\
0\end{array}$ & $\begin{array}{l}0 \\
1 \\
1 \\
0 \\
0 \\
0 \\
0 \\
0 \\
0\end{array}$ & $\begin{array}{l}0 \\
0 \\
0 \\
0 \\
0 \\
0 \\
0 \\
0\end{array}$ \\
\hline $6-19$ & $\begin{array}{r}36 \\
16\end{array}$ & $\begin{array}{l}15 \\
18\end{array}$ & $\begin{array}{r}1072 \\
622\end{array}$ & $\begin{array}{l}10 \\
25\end{array}$ & $\frac{1}{3}$ & $\begin{array}{r}6 \\
19\end{array}$ & $\begin{array}{l}2 \\
2\end{array}$ & $\frac{1}{1}$ & $\begin{array}{l}0 \\
0\end{array}$ \\
\hline $6-20$ & $\begin{array}{r}3 \\
16\end{array}$ & $\begin{array}{l}15 \\
18\end{array}$ & $\begin{array}{l}1134 \\
119+\end{array}$ & $\begin{array}{l}28 \\
17\end{array}$ & $\begin{array}{l}6 \\
7\end{array}$ & $\begin{array}{l}15 \\
10\end{array}$ & 6 & $\begin{array}{l}1 \\
0\end{array}$ & $\begin{array}{l}0 \\
0\end{array}$ \\
\hline $6-21$ & $1 \frac{3}{16}$ & $\begin{array}{l}15 \\
18\end{array}$ & $\begin{array}{r}388 \\
1613\end{array}$ & $\begin{array}{l}15 \\
28\end{array}$ & $\begin{array}{l}0 \\
4\end{array}$ & $2{ }^{6}$ & $\begin{array}{l}9 \\
2\end{array}$ & $\begin{array}{l}0 \\
0\end{array}$ & $\begin{array}{l}0 \\
0\end{array}$ \\
\hline $6-23$ & $\begin{array}{r}2 \\
16\end{array}$ & $\begin{array}{l}15 \\
18\end{array}$ & $\begin{array}{r}1327 \\
66++\end{array}$ & $\begin{array}{r}22 \\
9\end{array}$ & $\begin{array}{l}2 \\
0\end{array}$ & $\begin{array}{r}15 \\
9\end{array}$ & $\begin{array}{l}5 \\
0\end{array}$ & $\begin{array}{l}0 \\
0\end{array}$ & $\begin{array}{l}0 \\
0\end{array}$ \\
\hline $6-25$ & 2 & 18 & 553 & 28 & 7 & 16 & 2 & 3 & 0 \\
\hline $6-27$ & $\begin{array}{l}6 \\
11 \\
14 \\
15 \\
16\end{array}$ & $\begin{array}{l}10 \\
13 \\
14 \\
15 \\
17\end{array}$ & $\begin{array}{l}22++ \\
247 \\
761 \\
493 \\
79+\end{array}$ & $\begin{array}{r}9 \\
19 \\
25 \\
43 \\
20\end{array}$ & $\begin{array}{l}0 \\
2 \\
4 \\
5 \\
8\end{array}$ & $\begin{array}{r}9 \\
9 \\
16 \\
31 \\
11\end{array}$ & $\begin{array}{l}0 \\
6 \\
5 \\
7 \\
1\end{array}$ & $\begin{array}{l}0 \\
2 \\
0 \\
0 \\
0\end{array}$ & $\begin{array}{l}0 \\
0 \\
0 \\
0 \\
0\end{array}$ \\
\hline $7-01$ & $\begin{array}{l}11 \\
12 \\
13\end{array}$ & $\begin{array}{l}11 \\
12 \\
17\end{array}$ & $\begin{array}{l}476 \\
807 \\
377+\end{array}$ & $\begin{array}{l}14 \\
24 \\
20\end{array}$ & $\begin{array}{l}0 \\
1 \\
5\end{array}$ & $\begin{array}{r}6 \\
14 \\
13\end{array}$ & $\begin{array}{l}4 \\
8 \\
2\end{array}$ & $\begin{array}{l}4 \\
1 \\
0\end{array}$ & $\begin{array}{l}0 \\
0 \\
0\end{array}$ \\
\hline $7-05$ & 9 & 17 & $374+$ & 19 & 3 & 14 & 1 & 1 & 0 \\
\hline $7-07$ & 12 & 17 & $6+++$ & 0 & 0 & 0 & 0 & 0 & 0 \\
\hline
\end{tabular}


TABLE 19.- NUMBER OF PINK TRACER PARTICLES(1), PER 100 GRAMS OF BEDLOAD SAMPLE, COLLECTED AT ONE OR MORE CROSS-CHANNEL SAMPLING POSITIONS SPACED AT I-METER INTERVALS ACROSS THE CHANNEL WIDTH, EAST FORK RIVER, WYOMING, 1980--CONTINUED

(1) PINK TRACER PARTICLES PLACED AS BED MATERIAL 10 METERS DOWNSTREAM

(2) REPECSTION 3047 ON MAY 18 , 1979 . MEASURED IN THE FIELD' FOR SUBSEQUENT ANALYSIS OF NUMBER AND SIZE OF TRACER, PARTICLES SMALLER THAN 0.25 MM AND LARGER THAN $8.0 \mathrm{MM}$ WERE DISCARDED. REMAINING SAMPLES WEIGHING MORE THAN 100 GRAMS WERE CUT TO A STANDARD 100 GRAM-PORTION; SAMPLES WEIGHING LESS THAN 100 GRAMS WERE ANALYZED INTACT.

(3) DOES NOT INCLUDE TRACER PARTICLES MEASURED IN TRANSPORT AT SECTION 1400, A BYPASS CHANNEL COMMON TO SECTIONS 1360, 1396, AND 1425. SEE FIGURE 3.

$+\quad$ MASS OF SAMPLE ANALYZED WAS BETWEEN 50 AND 100 GRAMS; NUMBER OF TRACER PARTICLES ADJUSTED TO AN EOUIVALENT 100-GRAM SAMPLE; EXTRAPOLATED NUMBERS ARE CONSIDERED RELIABLE.

+ MASS OF SAMPLE ANALYZED WAS BETWEEN 10 AND 50 GRAMS; NUMBER OF TRACER PARTICLES ADJUSTED TO AN EQUIVALENT 100-GRAM SAMPLE; CARE SHOULD BE TAKEN IN USING THE EXTRAPOLATED NUMBERS.

++ MASS OF SAMPLE ANALYZED WAS BETWEEN 0 AND 10 GRAMS : NUMBER OF TRACER PARTICLES ADJUSTED TO AN EQUIVALENT 100-GRAM SAMPLE; EXTRAPOLATED NUMBERS ARE CONSIDERED RELIABLE. 
TABLE 20.- NUMBER OF BLUE TRACER PARTICLES(1), PER 100 GRAMS OF BEDLOAD SAMPLE, COLLECTED AT ONE OR MORE CROSS-CHANNEL SAMPL ING POSITIONS SPACED AT I-METER INTERVALS ACROSS THE CHANNEL WIDTH, EAST FORK RIVER, WYOMING, 1980

SECTION 1241

\begin{tabular}{|c|c|c|c|c|c|c|c|c|c|}
\hline \multirow{3}{*}{ DATE } & \multirow{3}{*}{\multicolumn{2}{|c|}{$\begin{array}{l}\text { SAMPLING } \\
\text { POSITION } \\
\text { FROM TO }\end{array}$}} & \multirow{3}{*}{$\begin{array}{c}\text { DRY } \\
\text { MASS } \\
\text { OF } \\
\text { SAMPLE(2) } \\
\text { (G) }\end{array}$} & \multicolumn{6}{|c|}{ NUMBER OF BLUE TRACER PARTICLES } \\
\hline & & & & \multirow[b]{2}{*}{ TOTAL } & \multicolumn{5}{|c|}{ BY SIZE CLASS (MM) } \\
\hline & & & & & $\begin{array}{l}0.25 \\
\text { TO } \\
0.50\end{array}$ & $\begin{array}{l}0.50 \\
\text { TO } \\
1.00\end{array}$ & $\begin{array}{l}1.00 \\
2.00\end{array}$ & $\begin{array}{l}2.00 \\
\text { TO } \\
4.00\end{array}$ & $\begin{array}{l}4.00 \\
\text { TO } \\
8.00\end{array}$ \\
\hline $6-01$ & 10 & 22 & 1777 & 11 & 0 & 6 & 4 & 1 & 0 \\
\hline $6-03$ & 10 & 22 & 452 & 12 & 2 & 7 & 3 & 0 & 0 \\
\hline $6-05$ & 10 & 22 & 759 & 7 & 0 & 2 & 4 & 1 & 0 \\
\hline $6-07$ & 10 & 21 & 1177 & 9 & 0 & 5 & 4 & 0 & 0 \\
\hline $6-09$ & 2 & 21 & 1501 & 5 & 2 & 1 & 2 & 0 & 0 \\
\hline $6-10$ & 2 & 21 & 690 & 3 & 2 & 0 & 1 & 0 & 0 \\
\hline $6-11$ & 3 & 21 & 945 & 12 & 5 & 6 & 1 & 0 & 0 \\
\hline $6-12$ & 2 & 21 & 324 & 12 & 1 & 6 & 4 & 1 & 0 \\
\hline $6-13$ & 7 & 20 & 308 & 18 & 4 & 11 & 1 & 1 & 1 \\
\hline $6-14$ & 2 & 21 & 1093 & 11 & 5 & 5 & 1 & 0 & 0 \\
\hline $6-15$ & 2 & 21 & 1350 & 12 & 0 & 7 & 4 & 1 & 0 \\
\hline $6-16$ & 10 & 22 & 301 & 15 & 4 & 9 & 2 & 0 & 0 \\
\hline $6-17$ & 7 & 22 & 1431 & 5 & 0 & 2 & 1 & 2 & 0 \\
\hline $6-18$ & 2 & 20 & 3603 & 6 & 1 & 1 & 3 & 1 & 0 \\
\hline $6-19$ & 2 & 22 & 1291 & 0 & 0 & 0 & 0 & 0 & 0 \\
\hline $6-20$ & 3 & 21 & 2036 & 3 & 0 & 2 & 1 & 0 & 0 \\
\hline $6-21$ & $1 \frac{1}{14}$ & $\begin{array}{l}13 \\
25\end{array}$ & $\begin{array}{l}4824 \\
1293\end{array}$ & $\begin{array}{l}2 \\
5\end{array}$ & $\begin{array}{l}0 \\
0\end{array}$ & $\begin{array}{l}2 \\
4\end{array}$ & $\begin{array}{l}0 \\
1\end{array}$ & $\begin{array}{l}0 \\
0\end{array}$ & $\begin{array}{l}0 \\
0\end{array}$ \\
\hline $6-23$ & $\begin{array}{r}7 \\
12\end{array}$ & $\begin{array}{l}11 \\
19\end{array}$ & $\begin{array}{l}187 \\
266\end{array}$ & 4 & $\begin{array}{l}1 \\
0\end{array}$ & $\begin{array}{l}3 \\
0\end{array}$ & $\begin{array}{l}0 \\
0\end{array}$ & $\begin{array}{l}0 \\
0\end{array}$ & $\begin{array}{l}0 \\
0\end{array}$ \\
\hline $6-25$ & 9 & 21 & 5117 & 0 & 0 & 0 & 0 & 0 & 0 \\
\hline $6-27$ & 11 & $\begin{array}{l}10 \\
20\end{array}$ & $\begin{array}{l}667 \\
937\end{array}$ & $\begin{array}{l}2 \\
2\end{array}$ & $\begin{array}{l}0 \\
0\end{array}$ & $\begin{array}{l}2 \\
2\end{array}$ & $\begin{array}{l}0 \\
0\end{array}$ & $\begin{array}{l}0 \\
0\end{array}$ & $\begin{array}{l}0 \\
0\end{array}$ \\
\hline
\end{tabular}


TABLE 20.- NUMBER OF BLUE TRACER PARTICLES(1), PER 100 GRAMS OF BEDLOAD SAMPLE, COLLECTED AT ONE OR MORE CROSS-CHANNEL SAMPLING POSITIONS SPACED AT I-METER INTERVALS ACROSS THE CHANNEL WIDTH, EAST FORK RIVER, WYOMING, 1980

SECTION 1284

\begin{tabular}{|c|c|c|c|c|c|c|c|c|c|}
\hline \multirow{3}{*}{ DATE } & \multirow{3}{*}{\multicolumn{2}{|c|}{$\begin{array}{l}\text { SAMPLING } \\
\text { POSITION } \\
\text { FROM TO }\end{array}$}} & \multirow{3}{*}{$\begin{array}{c}\text { DRY } \\
\text { MASS } \\
\text { OF } \\
\text { SAMPLE (2) } \\
\text { (G) }\end{array}$} & \multicolumn{6}{|c|}{ NUMBER OF BLUE TRACER PARTICLES } \\
\hline & & & & \multirow[b]{2}{*}{ TOTAL } & \multicolumn{5}{|c|}{ BY SIZE CLASS (MM) } \\
\hline & & & & & $\begin{array}{l}0.25 \\
\text { TO } \\
0.50\end{array}$ & $\begin{array}{l}0.50 \\
\text { TO } \\
1.00\end{array}$ & $\begin{array}{l}1.00 \\
2.00\end{array}$ & $\begin{array}{l}2.00 \\
\text { TO } \\
4.00\end{array}$ & $\begin{array}{l}4.00 \\
\text { TO } \\
8.00\end{array}$ \\
\hline $6-01$ & 3 & 17 & 1178 & 12 & 2 & 7 & 3 & 0 & 0 \\
\hline $6-03$ & 1 & 18 & 741 & 2 & 0 & 1 & 1 & 0 & 0 \\
\hline $6-05$ & 3 & 16 & 944 & 5 & 1 & 4 & 0 & 0 & 0 \\
\hline $6-07$ & 2 & 17 & 969 & 2 & 0 & 0 & 2 & 0 & 0 \\
\hline $6-09$ & 2 & 17 & 2293 & 0 & 0 & 0 & 0 & 0 & 0 \\
\hline $6-10$ & $\begin{array}{r}3 \\
11\end{array}$ & $\begin{array}{l}10 \\
17\end{array}$ & $\begin{array}{r}867 \\
1039\end{array}$ & $1 \frac{1}{16}$ & 12 & $\begin{array}{l}0 \\
2\end{array}$ & $\begin{array}{l}0 \\
2\end{array}$ & $\begin{array}{l}0 \\
0\end{array}$ & $\begin{array}{l}0 \\
0\end{array}$ \\
\hline $6-11$ & $\begin{array}{r}3 \\
12 \\
15\end{array}$ & $\begin{array}{l}11 \\
14 \\
18\end{array}$ & $\begin{array}{l}164 \\
475 \\
239\end{array}$ & $\begin{array}{l}2 \\
8 \\
5\end{array}$ & $\begin{array}{l}0 \\
2 \\
5\end{array}$ & $\begin{array}{l}1 \\
4 \\
0\end{array}$ & $\begin{array}{l}1 \\
2 \\
0\end{array}$ & $\begin{array}{l}0 \\
0 \\
0\end{array}$ & $\begin{array}{l}0 \\
0 \\
0\end{array}$ \\
\hline $6-12$ & 3 & 18 & 374 & 2 & 0 & 2 & 0 & 0 & 0 \\
\hline $6-13$ & $\begin{array}{r}3 \\
11\end{array}$ & $\begin{array}{l}10 \\
17\end{array}$ & $\begin{array}{l}141 \\
328\end{array}$ & 4 & $\begin{array}{l}2 \\
0\end{array}$ & $\begin{array}{l}0 \\
0\end{array}$ & $\begin{array}{l}1 \\
0\end{array}$ & $\begin{array}{l}1 \\
0\end{array}$ & $\begin{array}{l}0 \\
0\end{array}$ \\
\hline $6-14$ & 2 & 16 & 897 & 11 & 3 & 8 & 0 & 0 & 0 \\
\hline $6-15$ & $\begin{array}{r}2 \\
9 \\
14\end{array}$ & $\begin{array}{r}8 \\
13 \\
18\end{array}$ & $\begin{array}{r}1065 \\
725 \\
1178\end{array}$ & $\begin{array}{r}0 \\
1 \\
12\end{array}$ & $\begin{array}{l}0 \\
0 \\
5\end{array}$ & $\begin{array}{l}0 \\
0 \\
3\end{array}$ & $\begin{array}{l}0 \\
1 \\
3\end{array}$ & $\begin{array}{l}0 \\
0 \\
1\end{array}$ & $\begin{array}{l}0 \\
0 \\
0\end{array}$ \\
\hline $6-16$ & $\begin{array}{r}4 \\
13\end{array}$ & $\begin{array}{l}6 \\
12 \\
16\end{array}$ & $\begin{array}{l}361 \\
697 \\
289\end{array}$ & $\begin{array}{r}0 \\
10 \\
3\end{array}$ & $\begin{array}{l}0 \\
2 \\
2\end{array}$ & $\begin{array}{l}0 \\
7 \\
1\end{array}$ & $\begin{array}{l}0 \\
1 \\
0\end{array}$ & $\begin{array}{l}0 \\
0 \\
0\end{array}$ & $\begin{array}{l}0 \\
0 \\
0\end{array}$ \\
\hline $6-17$ & $\begin{array}{r}2 \\
5 \\
6 \\
7 \\
8 \\
11 \\
12\end{array}$ & $\begin{array}{r}4 \\
5 \\
6 \\
7 \\
10 \\
11 \\
18\end{array}$ & $\begin{array}{c}43++ \\
45++ \\
47++ \\
783 \\
2725 \\
1767 \\
1497\end{array}$ & $\begin{array}{l}0 \\
0 \\
0 \\
2 \\
2 \\
9 \\
6\end{array}$ & $\begin{array}{l}0 \\
0 \\
0 \\
1 \\
0 \\
1 \\
0\end{array}$ & $\begin{array}{l}0 \\
0 \\
0 \\
1 \\
0 \\
5 \\
2\end{array}$ & $\begin{array}{l}0 \\
0 \\
0 \\
0 \\
2 \\
3 \\
3\end{array}$ & $\begin{array}{l}0 \\
0 \\
0 \\
0 \\
0 \\
0 \\
1\end{array}$ & $\begin{array}{l}0 \\
0 \\
0 \\
0 \\
0 \\
0 \\
0\end{array}$ \\
\hline $6-18$ & $\begin{array}{l}3 \\
8\end{array}$ & $\begin{array}{r}7 \\
18\end{array}$ & $\begin{array}{r}1887 \\
929\end{array}$ & $\begin{array}{l}2 \\
8\end{array}$ & $\begin{array}{l}0 \\
2\end{array}$ & $\begin{array}{l}0 \\
5\end{array}$ & $\frac{1}{1}$ & $\begin{array}{l}1 \\
0\end{array}$ & $\begin{array}{l}0 \\
0\end{array}$ \\
\hline
\end{tabular}


TABLE 20.- NUMBER OF BLUE TRACER PARTICLES(1), PER 100 GRAMS OF BEDLOAD SAMPLE, COLLECTED AT ONE OR MORE CROSS-CHANNEL SAMPLING POSITIONS SPACED AT 1-METER INTERVALS ACROSS THE CHANNEL WIDTH, EAST FORK RIVER, WYOMING, 1980--CONTINUED

SECTION 1284

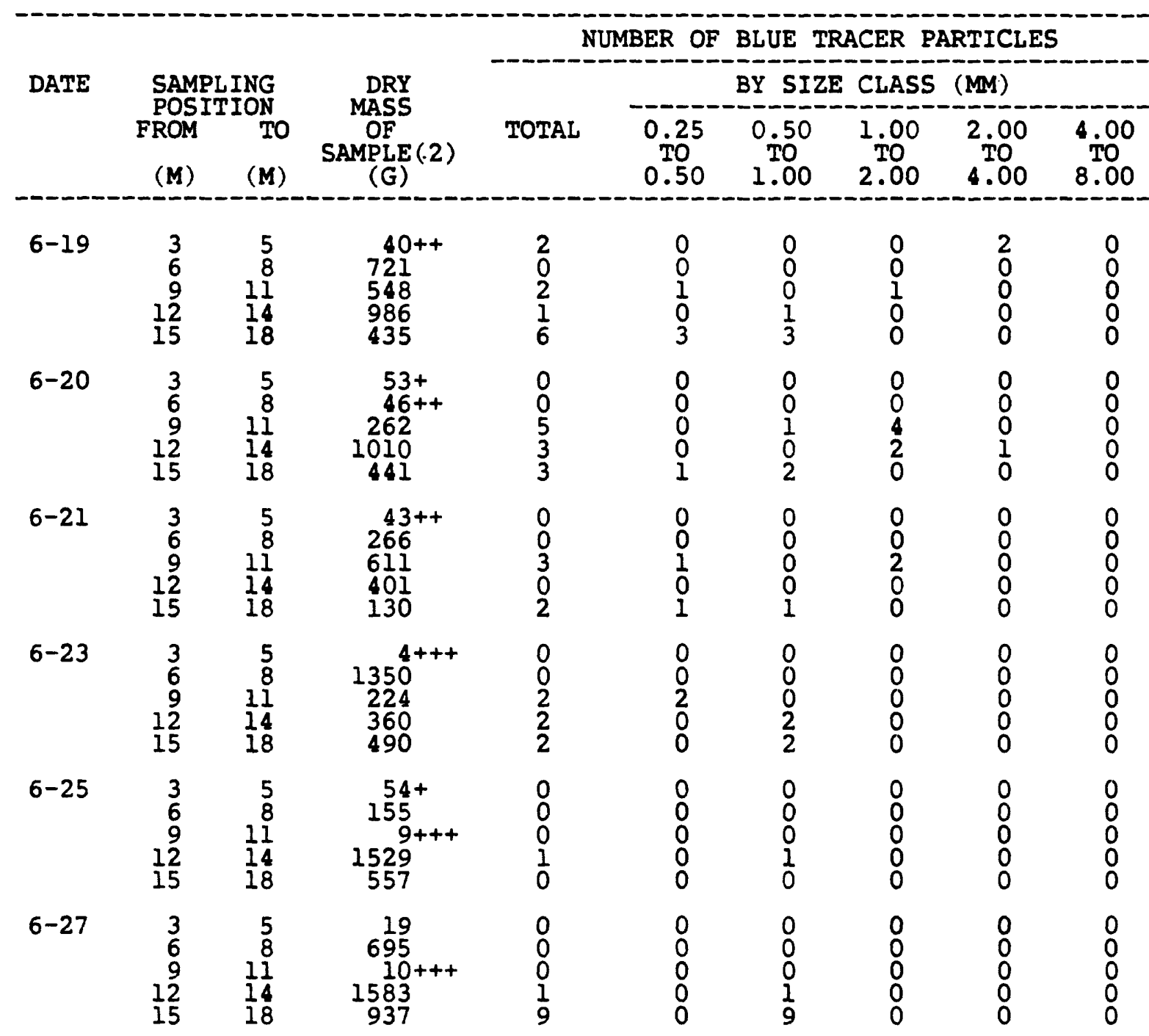


TABLE 20.- NUMBER OF BLUE TRACER PARTICLES(1), PER 100 GRAMS OF BEDLOAD SAMPLE, COLLECTED AT ONE OR MORE CROSS-CHANNEL SAMPLING POSITIONS SPACED AT I-METER INTERVALS ACROSS THE CHANNEL WIDTH, EAST FORK RIVER, WYOMING, 1980--CONTINUED

SECTION 1315

\begin{tabular}{|c|c|c|c|c|c|c|c|c|c|}
\hline \multirow{3}{*}{ DATE } & & & & \multicolumn{6}{|c|}{ NUMBER OF BLUE TRACER PARTICLES } \\
\hline & \multirow{2}{*}{\multicolumn{2}{|c|}{$\begin{array}{l}\text { SAMPLING } \\
\text { POSITION } \\
\text { FROM TO }\end{array}$}} & \multirow{2}{*}{$\begin{array}{c}\text { DRY } \\
\text { MASS } \\
\text { OF } \\
\text { SAMPLE (2) } \\
\text { (G) }\end{array}$} & \multirow[b]{2}{*}{ TOTAL } & \multicolumn{5}{|c|}{ BY SIZE CLASS (MM) } \\
\hline & & & & & $\begin{array}{l}0.25 \\
\text { TO } \\
0.50\end{array}$ & $\begin{array}{l}0.50 \\
\text { TO } \\
1.00\end{array}$ & $\begin{array}{l}1.00 \\
2.00\end{array}$ & $\begin{array}{l}2.00 \\
4.00\end{array}$ & $\begin{array}{l}4.00 \\
\mathrm{TO} \\
8.00\end{array}$ \\
\hline $6-01$ & 3 & 25 & 1241 & 4 & 0 & 1 & 3 & 0 . & 0 \\
\hline $6-03$ & 3 & 22 & 217 & 9 & 3 & 5 & 1 & 0 & 0 \\
\hline $6-05$ & 3 & 24 & $98+$ & 3 & 0 & 2 & 1 & 0 & 0 \\
\hline $6-07$ & 3 & 25 & 249 & 11 & 3 & 7 & 1 & 0 & 0 \\
\hline $6-09$ & 3 & 25 & 483 & 5 & 3 & 2 & 0 & 0 & 0 \\
\hline $6-10$ & $\begin{array}{l}3 \\
12 \\
18 \\
21\end{array}$ & $\begin{array}{l}11 \\
17 \\
20 \\
25\end{array}$ & $\begin{array}{l}110+ \\
238 \\
508 \\
1945\end{array}$ & $\begin{array}{r}1 \\
4 \\
20 \\
9\end{array}$ & $\begin{array}{l}0 \\
3 \\
7 \\
2\end{array}$ & $\begin{array}{r}1 \\
0 \\
13 \\
7\end{array}$ & $\begin{array}{l}0 \\
1 \\
0 \\
0\end{array}$ & $\begin{array}{l}0 \\
0 \\
0 \\
0\end{array}$ & $\begin{array}{l}0 \\
0 \\
0 \\
0\end{array}$ \\
\hline $6-11$ & $\begin{array}{l}2 \\
13 \\
15\end{array}$ & $\begin{array}{l}12 \\
14 \\
17\end{array}$ & $\begin{array}{r}1882 \\
1720 \\
459\end{array}$ & $\begin{array}{r}4 \\
6 \\
16\end{array}$ & $\begin{array}{l}0 \\
0 \\
4\end{array}$ & $\begin{array}{r}3 \\
12\end{array}$ & $\begin{array}{l}\frac{1}{3} \\
0\end{array}$ & $\begin{array}{l}0 \\
2 \\
0\end{array}$ & $\begin{array}{l}0 \\
0 \\
0\end{array}$ \\
\hline $6-12$ & 3 & 25 & 842 & 6 & 0 & 3 & 3 & 0 & 0 \\
\hline $6-13$ & $\begin{array}{r}3 \\
18\end{array}$ & $\begin{array}{l}17 \\
25\end{array}$ & $\begin{array}{l}223 \\
623\end{array}$ & $\frac{1}{5}$ & $\frac{1}{1}$ & $\begin{array}{l}0 \\
3\end{array}$ & $\begin{array}{l}0 \\
1\end{array}$ & $\begin{array}{l}0 \\
0\end{array}$ & $\begin{array}{l}0 \\
0\end{array}$ \\
\hline $6-14$ & $\begin{array}{r}3 \\
13\end{array}$ & $\begin{array}{l}12 \\
25\end{array}$ & $\begin{array}{l}1095 \\
1099\end{array}$ & $\begin{array}{l}4 \\
0\end{array}$ & $\frac{1}{0}$ & $\frac{1}{0}$ & $\begin{array}{l}2 \\
0\end{array}$ & $\begin{array}{l}0 \\
0\end{array}$ & $\begin{array}{l}0 \\
0\end{array}$ \\
\hline $6-15$ & $\begin{array}{r}3 \\
12 \\
17 \\
19\end{array}$ & $\begin{array}{l}11 \\
16 \\
18 \\
25\end{array}$ & $\begin{array}{l}804 \\
633 \\
484 \\
846\end{array}$ & $\begin{array}{l}1 \\
2 \\
5 \\
3\end{array}$ & $\begin{array}{l}0 \\
0 \\
0 \\
0\end{array}$ & $\begin{array}{l}0 \\
1 \\
5 \\
3\end{array}$ & $\begin{array}{l}0 \\
1 \\
0 \\
0\end{array}$ & $\begin{array}{l}0 \\
0 \\
0 \\
0\end{array}$ & $\begin{array}{l}1 \\
0 \\
0 \\
0\end{array}$ \\
\hline $6-16$ & $\begin{array}{r}6 \\
7 \\
9 \\
13 \\
18 \\
20\end{array}$ & $\begin{array}{r}6 \\
8 \\
12 \\
17 \\
19 \\
24\end{array}$ & $\begin{array}{c}56+ \\
46++ \\
641 \\
459 \\
838 \\
1062\end{array}$ & $\begin{array}{l}2 \\
0 \\
7 \\
5 \\
3 \\
1\end{array}$ & $\begin{array}{l}0 \\
0 \\
4 \\
1 \\
1 \\
0\end{array}$ & $\begin{array}{l}0 \\
0 \\
3 \\
3 \\
1 \\
1\end{array}$ & $\begin{array}{l}0 \\
0 \\
0 \\
1 \\
1 \\
0\end{array}$ & $\begin{array}{l}2 \\
0 \\
0 \\
0 \\
0 \\
0\end{array}$ & $\begin{array}{l}0 \\
0 \\
0 \\
0 \\
0 \\
0\end{array}$ \\
\hline
\end{tabular}


TABLE 20.- NUMBER OF BLUE TRACER PARTICLES(1), PER 100 GRAMS OF BEDLOAD SAMPLE, COLLECTED AT ONE OR MORE CROSS-CHANNEL SAMPLING POSITIONS SPACED AT I-METER INTERVALS ACROSS THE CHANNEL WIDTH, EAST FORK RIVER, WYOMING, 1980--CONTINUED

SECTION 1315

\begin{tabular}{|c|c|c|c|c|c|c|c|c|c|}
\hline \multirow{3}{*}{ DATE } & & & & \multicolumn{6}{|c|}{ NUMBER OF BLUE TRACER PARTICLES } \\
\hline & \multirow{2}{*}{\multicolumn{2}{|c|}{$\begin{array}{c}\text { SAMPLING } \\
\text { POSITION } \\
\text { FROM TO }\end{array}$}} & \multirow{2}{*}{$\begin{array}{c}\text { DRY } \\
\text { MASS } \\
\text { OF } \\
\text { SAMPLE (2) } \\
(G)\end{array}$} & \multirow[b]{2}{*}{ TOTAL } & \multicolumn{5}{|c|}{ BY SIZE CLASS (MM) } \\
\hline & & & & & $\begin{array}{l}0.25 \\
0.50\end{array}$ & $\begin{array}{l}0.50 \\
\text { TO } \\
1.00\end{array}$ & $\begin{array}{l}1.00 \\
2.00\end{array}$ & $\begin{array}{l}2.00 \\
\text { TO } \\
4.00\end{array}$ & $\begin{array}{l}4.00 \\
\text { TO } \\
8.00\end{array}$ \\
\hline $6-17$ & $\begin{array}{r}3 \\
7 \\
8 \\
10 \\
13 \\
16 \\
19\end{array}$ & $\begin{array}{r}6 \\
7 \\
9 \\
12 \\
15 \\
18 \\
25\end{array}$ & $\begin{array}{r}17++ \\
20++ \\
378 \\
1811 \\
731 \\
867 \\
1265\end{array}$ & $\begin{array}{l}0 \\
0 \\
0 \\
4 \\
1 \\
6 \\
5\end{array}$ & $\begin{array}{l}0 \\
0 \\
0 \\
1 \\
1 \\
1 \\
0\end{array}$ & $\begin{array}{l}0 \\
0 \\
0 \\
1 \\
0 \\
4 \\
1\end{array}$ & $\begin{array}{l}0 \\
0 \\
0 \\
2 \\
0 \\
0 \\
2\end{array}$ & $\begin{array}{l}0 \\
0 \\
0 \\
0 \\
0 \\
1 \\
2\end{array}$ & $\begin{array}{l}0 \\
0 \\
0 \\
0 \\
0 \\
0 \\
0\end{array}$ \\
\hline $6-18$ & $\begin{array}{r}3 \\
5 \\
7 \\
9 \\
10 \\
14 \\
19\end{array}$ & $\begin{array}{r}4 \\
6 \\
8 \\
9 \\
13 \\
18 \\
25\end{array}$ & $\begin{array}{c}22++ \\
17++ \\
5+++ \\
38++ \\
519 \\
973 \\
828\end{array}$ & $\begin{array}{l}0 \\
0 \\
0 \\
0 \\
0 \\
0 \\
3\end{array}$ & $\begin{array}{l}0 \\
0 \\
0 \\
0 \\
0 \\
0 \\
0\end{array}$ & $\begin{array}{l}0 \\
0 \\
0 \\
0 \\
0 \\
0 \\
3\end{array}$ & $\begin{array}{l}0 \\
0 \\
0 \\
0 \\
0 \\
0 \\
0\end{array}$ & $\begin{array}{l}0 \\
0 \\
0 \\
0 \\
0 \\
0 \\
0\end{array}$ & $\begin{array}{l}0 \\
0 \\
0 \\
0 \\
0 \\
0 \\
0\end{array}$ \\
\hline $6-19$ & $\begin{array}{l}3 \\
14 \\
18\end{array}$ & $\begin{array}{l}13 \\
17 \\
25\end{array}$ & $\begin{array}{l}847 \\
705 \\
742\end{array}$ & $\begin{array}{l}0 \\
0 \\
3\end{array}$ & $\begin{array}{l}0 \\
0 \\
0\end{array}$ & $\begin{array}{l}0 \\
0 \\
3\end{array}$ & $\begin{array}{l}.0 \\
0 \\
0\end{array}$ & $\begin{array}{l}0 \\
0 \\
0\end{array}$ & $\begin{array}{l}0 \\
0 \\
0\end{array}$ \\
\hline $6-20$ & $\frac{13}{17}$ & $\begin{array}{l}10 \\
16 \\
25\end{array}$ & $\begin{array}{c}29+++ \\
1376^{6} \\
942\end{array}$ & $\begin{array}{l}0 \\
0 \\
2\end{array}$ & $\begin{array}{l}0 \\
0 \\
1\end{array}$ & $\begin{array}{l}0 \\
0 \\
1\end{array}$ & $\begin{array}{l}0 \\
0 \\
0\end{array}$ & $\begin{array}{l}0 \\
0 \\
0\end{array}$ & $\begin{array}{l}0 \\
0 \\
0\end{array}$ \\
\hline $6-21$ & $\frac{4}{17}$ & $\begin{array}{l}10 \\
16 \\
25\end{array}$ & $\begin{array}{r}69+ \\
985 \\
1775\end{array}$ & $\begin{array}{l}0 \\
0 \\
2\end{array}$ & $\begin{array}{l}0 \\
0 \\
0\end{array}$ & $\begin{array}{l}0 \\
0 \\
2\end{array}$ & $\begin{array}{l}0 \\
0 \\
0\end{array}$ & $\begin{array}{l}0 \\
0 \\
0\end{array}$ & $\begin{array}{l}0 \\
0 \\
0\end{array}$ \\
\hline $6-23$ & $\begin{array}{r}3 \\
9 \\
13 \\
17\end{array}$ & $\begin{array}{l}8 \\
12 \\
16 \\
25\end{array}$ & $\begin{array}{r}130 \\
349 \\
246 \\
2589\end{array}$ & $\begin{array}{l}1 \\
0 \\
0 \\
2\end{array}$ & $\begin{array}{l}0 \\
0 \\
0 \\
0\end{array}$ & $\begin{array}{l}0 \\
0 \\
0 \\
0\end{array}$ & $\begin{array}{l}0 \\
0 \\
0 \\
0\end{array}$ & $\begin{array}{l}0 \\
0 \\
0 \\
2\end{array}$ & $\begin{array}{l}1 \\
0 \\
0 \\
0\end{array}$ \\
\hline $6-25$ & $\begin{array}{r}3 \\
10 \\
17\end{array}$ & $\begin{array}{r}99 \\
16 \\
25\end{array}$ & $\begin{array}{l}244^{++} \\
4383 \\
2198\end{array}$ & $\begin{array}{l}0 \\
0 \\
2\end{array}$ & $\begin{array}{l}0 \\
0 \\
0\end{array}$ & $\begin{array}{l}0 \\
0 \\
2\end{array}$ & $\begin{array}{l}0 \\
0 \\
0\end{array}$ & $\begin{array}{l}0 \\
0 \\
0\end{array}$ & $\begin{array}{l}0 \\
0 \\
0\end{array}$ \\
\hline $6-27$ & $\begin{array}{r}3 \\
11 \\
17\end{array}$ & $\begin{array}{l}10 \\
16 \\
25\end{array}$ & $\begin{array}{c}72+ \\
861 \\
1343\end{array}$ & $\begin{array}{r}0 \\
10 \\
3\end{array}$ & $\begin{array}{l}0 \\
2 \\
2\end{array}$ & $\begin{array}{l}0 \\
8 \\
1\end{array}$ & $\begin{array}{l}0 \\
0 \\
0\end{array}$ & $\begin{array}{l}0 \\
0 \\
0\end{array}$ & $\begin{array}{l}0 \\
0 \\
0\end{array}$ \\
\hline
\end{tabular}


TABLE 20.- NUMBER OF BLUE TRACER PARTICLES(1), PER 100 GRAMS OF BEDLOAD SAMPLE, COLLECTED AT ONE OR MORE CROSS-CHANNEL SAMPLING POSITIONS SPACED AT 1-METER INTERVALS ACROSS THE CHANNEL WIDTH, EAST FORK RIVER, WYOMING, 1980--CONTINUED

SECTION $1360(3)$

\begin{tabular}{|c|c|c|c|c|c|c|c|c|c|}
\hline \multirow{3}{*}{ DATE } & \multirow{3}{*}{\multicolumn{2}{|c|}{$\begin{array}{r}\text { SAMPLING } \\
\text { POSITION } \\
\text { FROM TO }\end{array}$}} & \multirow{3}{*}{$\begin{array}{c}\text { DRY } \\
\text { MASS } \\
\text { OF } \\
\text { SAMPLE (2) } \\
\text { (G) }\end{array}$} & \multicolumn{6}{|c|}{ NUMBER OF BLUE TRACER PARTICLES } \\
\hline & & & & \multirow[b]{2}{*}{ TOTAL } & \multicolumn{5}{|c|}{ BY SIZE CLASS (MM) } \\
\hline & & & & & $\begin{array}{l}0.25 \\
\text { TO } \\
0.50\end{array}$ & $\begin{array}{l}0.50 \\
\text { TO } \\
1.00\end{array}$ & $\begin{array}{l}1.00 \\
\text { TO } \\
2.00\end{array}$ & $\begin{array}{l}2.00 \\
\text { TO } \\
4.00\end{array}$ & $\begin{array}{l}4.00 \\
\text { TO } \\
8.00\end{array}$ \\
\hline $6-01$ & 8 & 23 & $31++$ & 0 & 0 & 0 & 0 & 0 & 0 \\
\hline $6-03$ & 8 & 23 & $235+$ & 2 & 0 & 1 & 1 & 0 & 0 \\
\hline $6-05$ & 8 & 22 & 147 & 3 & 0 & 3 & 0 & 0 & 0 \\
\hline $6-07$ & 9 & 22 & 535 & 3 & 0 & 0 & 2 & 1 & 0 \\
\hline $6-09$ & 2 & 22 & 1049 & 0 & 0 & 0 & 0 & 0 & 0 \\
\hline $6-10$ & 9 & 22 & 1192 & 3 & 2 & 1 & 0 & 0 & 0 \\
\hline $6-11$ & $\begin{array}{r}99 \\
17 \\
20\end{array}$ & $\begin{array}{l}16 \\
19 \\
22\end{array}$ & $\begin{array}{l}437 \\
573 \\
0+++\end{array}$ & $\begin{array}{l}3 \\
3 \\
0\end{array}$ & $\begin{array}{l}1 \\
0 \\
0\end{array}$ & $\begin{array}{l}0 \\
1 \\
0\end{array}$ & $\begin{array}{l}1 \\
1 \\
0\end{array}$ & $\begin{array}{l}1 \\
1 \\
0\end{array}$ & $\begin{array}{l}0 \\
0 \\
0\end{array}$ \\
\hline $6-12$ & $\begin{array}{r}9 \\
1.7\end{array}$ & $\begin{array}{l}16 \\
22\end{array}$ & $\begin{array}{l}156 \\
262\end{array}$ & $\frac{1}{1}$ & $\begin{array}{l}0 \\
1\end{array}$ & $\frac{1}{0}$ & $\begin{array}{l}0 \\
0\end{array}$ & $\begin{array}{l}0 \\
0\end{array}$ & $\begin{array}{l}0 \\
0\end{array}$ \\
\hline $6-13$ & 9 & 22 & 841 & 0 & 0 & 0 & 0 & 0 & 0 \\
\hline $6-14$ & $\begin{array}{r}7 \\
16\end{array}$ & $\frac{14}{21}$ & $\begin{array}{l}111 \\
501\end{array}$ & $\begin{array}{l}0 \\
2\end{array}$ & $\begin{array}{l}0 \\
0\end{array}$ & $\begin{array}{l}0 \\
2\end{array}$ & $\begin{array}{l}0 \\
0\end{array}$ & $\begin{array}{l}0 \\
0\end{array}$ & $\begin{array}{l}0 \\
0\end{array}$ \\
\hline $6-15$ & $\begin{array}{r}99 \\
17 \\
20\end{array}$ & $\begin{array}{l}16 \\
199 \\
23\end{array}$ & $\begin{array}{r}519 \\
1518 \\
1795\end{array}$ & $\begin{array}{l}1 \\
0 \\
0\end{array}$ & $\begin{array}{l}0 \\
0 \\
0\end{array}$ & $\begin{array}{l}0 \\
0 \\
0\end{array}$ & $\begin{array}{l}0 \\
0 \\
0\end{array}$ & $\begin{array}{l}0 \\
0 \\
0\end{array}$ & $\begin{array}{l}1 \\
0 \\
0\end{array}$ \\
\hline $6-16$ & $\begin{array}{l}14 \\
18 \\
20\end{array}$ & $\begin{array}{l}17 \\
19 \\
23\end{array}$ & $\begin{array}{l}170 \\
464 \\
408\end{array}$ & $\begin{array}{l}0 \\
3 \\
5\end{array}$ & $\begin{array}{l}0 \\
0 \\
1\end{array}$ & $\begin{array}{l}0 \\
1 \\
3\end{array}$ & $\begin{array}{l}0 \\
1 \\
1\end{array}$ & $\begin{array}{l}0 \\
1 \\
0\end{array}$ & $\begin{array}{l}0 \\
0 \\
0\end{array}$ \\
\hline $6-17$ & $\begin{array}{r}9 \\
18\end{array}$ & $\begin{array}{l}17 \\
23\end{array}$ & $\begin{array}{l}85+ \\
502^{+}\end{array}$ & $\begin{array}{l}0 \\
2\end{array}$ & $\begin{array}{l}0 \\
1\end{array}$ & $\begin{array}{l}0 \\
1\end{array}$ & $\begin{array}{l}0 \\
0\end{array}$ & $\begin{array}{l}0 \\
0\end{array}$ & $\begin{array}{l}0 \\
0\end{array}$ \\
\hline $6-18$ & $\begin{array}{r}99 \\
19\end{array}$ & $\begin{array}{l}18 \\
23\end{array}$ & $\begin{array}{l}144 \\
375\end{array}$ & $\begin{array}{l}1 \\
0\end{array}$ & $\begin{array}{l}1 \\
0\end{array}$ & $\begin{array}{l}0 \\
0\end{array}$ & $\begin{array}{l}0 \\
0\end{array}$ & $\begin{array}{l}0 \\
0\end{array}$ & $\begin{array}{l}0 \\
0\end{array}$ \\
\hline $6-19$ & $\begin{array}{r}7 \\
18 \\
21\end{array}$ & $\begin{array}{l}17 \\
20 \\
23\end{array}$ & $\begin{array}{l}588 \\
786 \\
229\end{array}$ & $\begin{array}{l}0 \\
0 \\
0\end{array}$ & $\begin{array}{l}0 \\
0 \\
0\end{array}$ & $\begin{array}{l}0 \\
0 \\
0\end{array}$ & $\begin{array}{l}0 \\
0 \\
0\end{array}$ & $\begin{array}{l}0 \\
0 \\
0\end{array}$ & $\begin{array}{l}0 \\
0 \\
0\end{array}$ \\
\hline $6-20$ & 2 & 23 & 391 & 0 & 0 & 0 & 0 & 0 & 0 \\
\hline
\end{tabular}


TABLE 20.- NUMBER OF BLUE TRACER PARTICLES(1), PER 100 GRAMS OF BEDLOAD SAMPLE, COLLECTED AT ONE OR MORE CROSS-CHANNEL SAMPLING POSITIONS SPACED AT I-METER INTERVALS ACROSS THE CHANNEL WIDTH, EAST FORK RIVER, WYOMING, 1980-CONTINUED

SECTION $1360(3)$

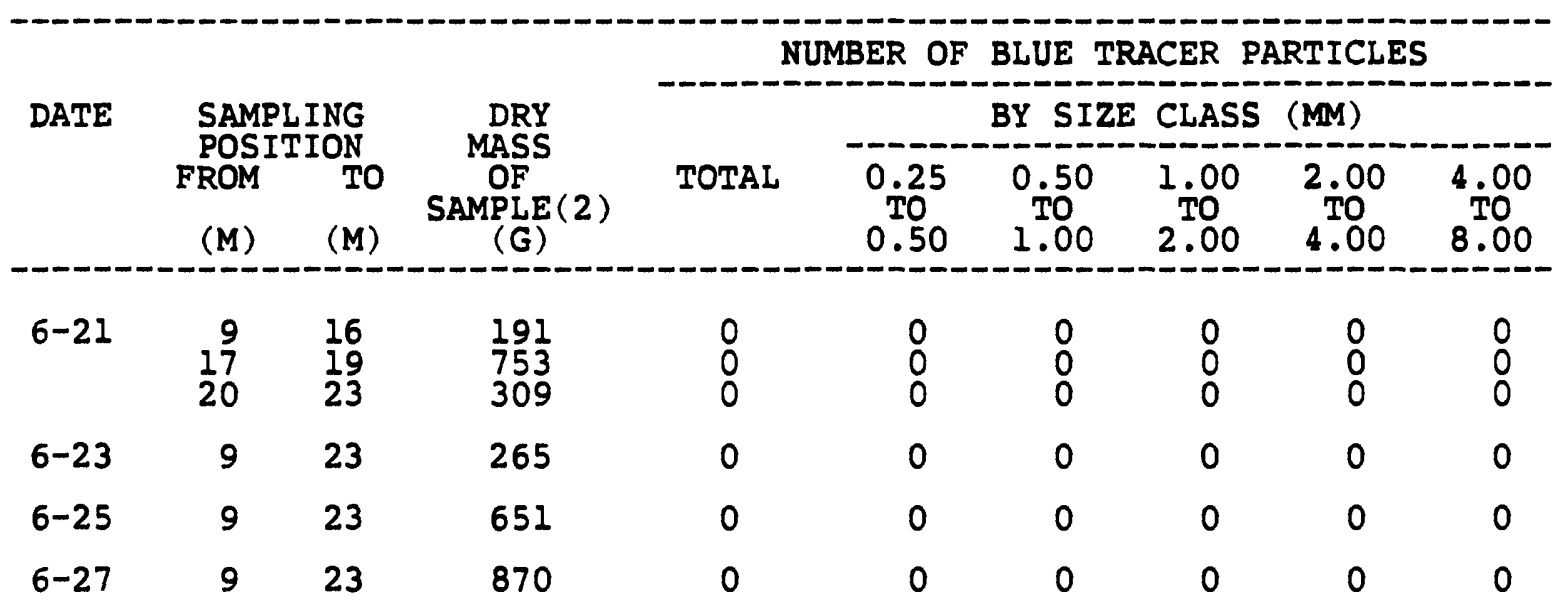


TABLE 20.- NUMBER OF BLUE TRACER PARTICLES(1), PER 100 GRAMS OF BEDLOAD SAMPLE, COLLECTED AT ONE OR MORE CROSS-CHANNEL SAMPLING POSITIONS SPACED AT 1-METER INTERVALS ACROSS THE CHANNEL WIDTH, EAST FORK RIVER, WYOMING, 1980--CONTINUED

SECTION 1396(3)

\begin{tabular}{|c|c|c|c|c|c|c|c|c|c|}
\hline \multirow{3}{*}{ DATE } & \multirow{2}{*}{\multicolumn{2}{|c|}{$\begin{array}{l}\text { SAMPLING } \\
\text { POSITION } \\
\text { FROM TO }\end{array}$}} & \multirow{3}{*}{$\begin{array}{c}\text { DRY } \\
\text { MASS } \\
\text { OF } \\
\text { SAMPLE (2) } \\
\text { (G) }\end{array}$} & \multicolumn{6}{|c|}{ NUMBER OF BLUE TRACER PARTICLES } \\
\hline & & & & \multirow[b]{2}{*}{ TOTAL } & \multicolumn{5}{|c|}{ BY SIZE CLASS (MM) } \\
\hline & FROM & (M) & & & $\begin{array}{l}0.25 \\
\text { TO } \\
0.50\end{array}$ & $\begin{array}{l}0.50 \\
\text { TO } \\
1.00\end{array}$ & $\begin{array}{l}1.00 \\
2.00\end{array}$ & $\begin{array}{l}2.00 \\
\text { TO } \\
4.00\end{array}$ & $\begin{array}{l}4.00 \\
\text { TO } \\
8.00\end{array}$ \\
\hline $6-01$ & 5 & 17 & 178 & 0 & 0 & 0 & 0 & 0 & 0 \\
\hline $6-03$ & 5 & 17 & 472 & 2 & 0 & 2 & 0 & 0 & 0 \\
\hline $6-05$ & 5 & 17 & 407 & 1 & 1 & 0 & 0 & 0 & 0 \\
\hline $6-07$ & 5 & 17 & 532 & 1 & 0 & 1 & 0 & 0 & 0 \\
\hline $6-09$ & 5 & 17 & 493 & 0 & 0 & 0 & 0 & 0 & 0 \\
\hline $6-10$ & 5 & 17 & 2231 & 1 & 0 & 0 & 1 & 0 & 0 \\
\hline $6-11$ & $\begin{array}{r}5 \\
16\end{array}$ & $\begin{array}{l}15 \\
17\end{array}$ & $\begin{array}{l}693 \\
334\end{array}$ & $\begin{array}{l}0 \\
2\end{array}$ & $\begin{array}{l}0 \\
1\end{array}$ & $\begin{array}{l}0 \\
1\end{array}$ & $\begin{array}{l}0 \\
0\end{array}$ & $\begin{array}{l}0 \\
0\end{array}$ & $\begin{array}{l}0 \\
0\end{array}$ \\
\hline $6-12$ & 5 & 17 & 467 & 2 & 0 & 0 & 2 & 0 & 0 \\
\hline $6-13$ & $\begin{array}{r}5 \\
15\end{array}$ & $\begin{array}{l}14 \\
17\end{array}$ & $\begin{array}{l}268 \\
382\end{array}$ & $\begin{array}{l}2 \\
0\end{array}$ & $\begin{array}{l}0 \\
0\end{array}$ & $\begin{array}{l}1 \\
0\end{array}$ & $\begin{array}{l}0 \\
0\end{array}$ & $\frac{1}{0}$ & $\begin{array}{l}0 \\
0\end{array}$ \\
\hline $6-14$ & $\begin{array}{l}5 \\
14 \\
15 \\
16 \\
17\end{array}$ & $\begin{array}{l}13 \\
14 \\
15 \\
16 \\
17\end{array}$ & $\begin{array}{l}367 \\
337 \\
999 \\
759 \\
30++\end{array}$ & $\begin{array}{l}0 \\
0 \\
1 \\
3 \\
0\end{array}$ & $\begin{array}{l}0 \\
0 \\
0 \\
1 \\
0\end{array}$ & $\begin{array}{l}0 \\
0 \\
1 \\
2 \\
0\end{array}$ & $\begin{array}{l}0 \\
0 \\
0 \\
0 \\
0\end{array}$ & $\begin{array}{l}0 \\
0 \\
0 \\
0 \\
0\end{array}$ & $\begin{array}{l}0 \\
0 \\
0 \\
0 \\
0\end{array}$ \\
\hline $6-15$ & 5 & 16 & 1830 & 2 & 0 & 0 & 1 & 1 & 0 \\
\hline $6-16$ & $\begin{array}{r}7 \\
15\end{array}$ & $\begin{array}{l}14 \\
19\end{array}$ & $\begin{array}{l}709 \\
691\end{array}$ & $\begin{array}{l}0 \\
0\end{array}$ & $\begin{array}{l}0 \\
0\end{array}$ & $\begin{array}{l}0 \\
0\end{array}$ & $\begin{array}{l}0 \\
0\end{array}$ & $\begin{array}{l}0 \\
0\end{array}$ & $\begin{array}{l}0 \\
0\end{array}$ \\
\hline $6-17$ & $\begin{array}{r}5 \\
14\end{array}$ & $\begin{array}{l}13 \\
17\end{array}$ & $\begin{array}{c}91+ \\
659\end{array}$ & $\begin{array}{l}0 \\
3\end{array}$ & $\begin{array}{l}0 \\
0\end{array}$ & $\begin{array}{l}0 \\
1\end{array}$ & $\begin{array}{l}0 \\
1\end{array}$ & $\begin{array}{l}0 \\
1\end{array}$ & $\begin{array}{l}0 \\
0\end{array}$ \\
\hline $6-18$ & $\begin{array}{r}5 \\
14 \\
15\end{array}$ & $\begin{array}{l}13 \\
14 \\
16\end{array}$ & $\begin{array}{l}315 \\
932 \\
266\end{array}$ & $\begin{array}{l}0 \\
0 \\
0\end{array}$ & $\begin{array}{l}0 \\
0 \\
0\end{array}$ & $\begin{array}{l}0 \\
0 \\
0\end{array}$ & $\begin{array}{l}0 \\
0 \\
0\end{array}$ & $\begin{array}{l}0 \\
0 \\
0\end{array}$ & $\begin{array}{l}0 \\
0 \\
0\end{array}$ \\
\hline $6-19$ & $\begin{array}{r}5 \\
14\end{array}$ & $\begin{array}{l}13 \\
17\end{array}$ & $\begin{array}{l}408 \\
718\end{array}$ & $\begin{array}{l}0 \\
0\end{array}$ & $\begin{array}{l}0 \\
0\end{array}$ & $\begin{array}{l}0 \\
0\end{array}$ & $\begin{array}{l}0 \\
0\end{array}$ & $\begin{array}{l}0 \\
0\end{array}$ & $\begin{array}{l}0 \\
0\end{array}$ \\
\hline $6-20$ & 5 & 16 & 432 & 0 & 0 & 0 & 0 & 0 & 0 \\
\hline
\end{tabular}


TABLE 20.- NUMBER OF BLUE TRACER PARTICLES(1), PER 100 GRAMS OF BEDLOAD SAMPLE, COLLECTED AT ONE OR MORE CROSS-CHANNEL SAMPLING

POSITIONS SPACED AT 1-METER INTERVALS ACROSS THE CHANNEL WIDTH, EAST FORK RIVER, WYOMING, 1980--CONTINUED

SECTION $1396(3)$

\begin{tabular}{|c|c|c|c|c|c|c|c|c|c|}
\hline \multirow{3}{*}{ DATE } & & & \multirow{3}{*}{$\begin{array}{c}\text { DRY } \\
\text { MASS } \\
\text { OF } \\
\text { SAMPLE (2) } \\
(\mathrm{G})\end{array}$} & 1 & BER 0 & BLUE & ACER & RTICLE & \\
\hline & \multicolumn{2}{|c|}{$\begin{array}{l}\text { SAMPLING } \\
\text { POSITION } \\
\text { FROM TO TO }\end{array}$} & & \multirow[b]{2}{*}{ TOTAL } & \multicolumn{5}{|c|}{ BY SIZE CLASS (MM) } \\
\hline & $\begin{array}{r}\text { FROM } \\
(M)\end{array}$ & TO & & & $\begin{array}{l}0.25 \\
\text { TO } \\
0.50\end{array}$ & $\begin{array}{l}0.50 \\
\text { TO } \\
1.00\end{array}$ & $\begin{array}{l}1.00 \\
\text { TO } \\
2.00\end{array}$ & $\begin{array}{l}2.00 \\
\text { TO } \\
4.00\end{array}$ & $\begin{array}{l}4.00 \\
\text { TO } \\
8.00\end{array}$ \\
\hline $6-21$ & 5 & 16 & 855 & 0 & 0 & 0 & 0 & 0 & 0 \\
\hline $6-23$ & 5 & 16 & 754 & 0 & 0 & 0 & 0 & 0 & 0 \\
\hline $6-25$ & 5 & 16 & 487 & 0 & 0 & 0 & 0 & 0 & 0 \\
\hline $6-27$ & 5 & 16 & 1773 & 1 & 0 & 0 & 1 & 0 & 0 \\
\hline
\end{tabular}


TABLE 20.- NUMBER OF BLUE TRACER PARTICLES(1), PER 100 GRAMS OF BEDLOAD SAMPLE COLLECTED AT ONE OR MORE CROSS-CHANNEL SAMPLING POSITIONS SPACED AT 1-METER INTERVALS ACROSS THE CHANNEL WIDTH, EAST FORK RIVER, WYOMING, 1980--CONTINUED

SECTION $1425(3)$

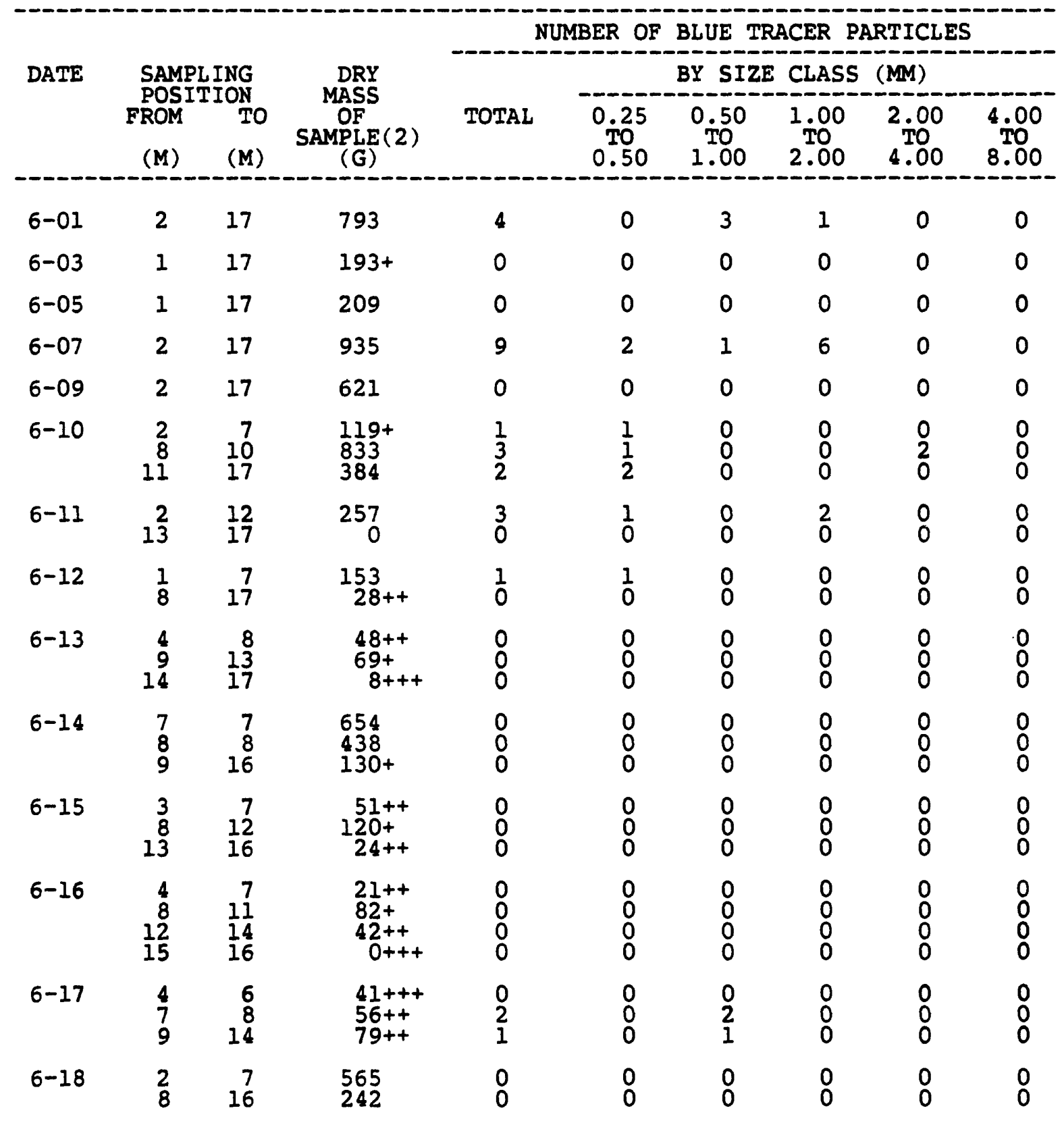


TABLE 20.- NUMBER OF BLUE TRACER PARTICLES(1), PER 100 GRAMS OF BEDLOAD SAMPLE COLLECTED AT ONE OR MORE CROSS-CHANNEL SAMPLING

POSITIONS SPACED AT 1-METER INTERVALS ACROSS THE CHANNEL WIDTH, EAST FORK RIVER, WYOMING, 1980--CONTINUED

SECTION $1425(3)$

\begin{tabular}{|c|c|c|c|c|c|c|c|c|c|}
\hline \multirow{3}{*}{ DATE } & \multirow{2}{*}{\multicolumn{2}{|c|}{$\begin{array}{l}\text { SAMPLING } \\
\text { POSITION }\end{array}$}} & \multirow{3}{*}{$\begin{array}{c}\text { DRY } \\
\text { MASS } \\
\text { OF } \\
\text { SAMPLE (2) } \\
\text { (G) }\end{array}$} & \multicolumn{6}{|c|}{ NUMBER OF BLUE TRACER PARTICLES } \\
\hline & & & & \multirow[b]{2}{*}{ TOTAL } & \multicolumn{5}{|c|}{ BY SIZE CLASS (MM) } \\
\hline & $\begin{array}{r}\text { FROM } \\
(\mathrm{M})\end{array}$ & $\begin{array}{l}\text { TO } \\
\text { (M) }\end{array}$ & & & $\begin{array}{c}0.25 \\
\text { TO } \\
0.50\end{array}$ & $\begin{array}{c}0.50 \\
\text { TO } \\
1.00\end{array}$ & $\begin{array}{c}1.00 \\
\text { TO } \\
2.00\end{array}$ & $\begin{array}{r}2.00 \\
\text { TO } \\
4.00\end{array}$ & $\begin{array}{l}4.00 \\
\text { TO } \\
8.00\end{array}$ \\
\hline $6-19$ & $\begin{array}{r}3 \\
11\end{array}$ & $\begin{array}{l}10 \\
16\end{array}$ & $\begin{array}{c}55++ \\
130^{++}\end{array}$ & $\begin{array}{l}0 \\
0\end{array}$ & $\begin{array}{l}0 \\
0\end{array}$ & $\begin{array}{l}0 \\
0\end{array}$ & $\begin{array}{l}0 \\
0\end{array}$ & $\begin{array}{l}0 \\
0\end{array}$ & $\begin{array}{l}0 \\
0\end{array}$ \\
\hline $6-20$ & 2 & $\begin{array}{l}10 \\
16\end{array}$ & $\begin{array}{l}178 \\
190\end{array}$ & $\begin{array}{l}0 \\
0\end{array}$ & $\begin{array}{l}0 \\
0\end{array}$ & $\begin{array}{l}0 \\
0\end{array}$ & $\begin{array}{l}0 \\
0\end{array}$ & $\begin{array}{l}0 \\
0\end{array}$ & $\begin{array}{l}0 \\
0\end{array}$ \\
\hline $6-21$ & 2 & 15 & $228+$ & 0 & 0 & 0 & 0 & 0 & 0 \\
\hline $6-23$ & 1 & 17 & 258 & 0 & 0 & 0 & 0 & 0 & 0 \\
\hline $6-25$ & 2 & 16 & 212 & 0 & 0 & 0 & 0 & 0 & 0 \\
\hline $6-27$ & 8 & 16 & 664 & 1 & 0 & 0 & 0 & 0 & 1 \\
\hline
\end{tabular}


TABLE 20.- NUMBER OF BLUE TRACER PARTICLES(1), PER 100 GRAMS OF BEDLOAD SAMPLE, COLLECTED AT ONE OR MORE CROSS-CHANNEL SAMPLING POSITIONS SPACED AT I-METER INTERVALS ACROSS THE CHANNEL WIDTH, EAST FORK RIVER, WYOMING, 1980--CONTINUED

\section{SECTION 1481}

\begin{tabular}{|c|c|c|c|c|c|c|c|c|c|}
\hline \multirow{3}{*}{ DATE } & \multirow{3}{*}{\multicolumn{2}{|c|}{$\begin{array}{l}\text { SAMPLING } \\
\text { POSITION } \\
\text { FROM TO }\end{array}$}} & \multirow{3}{*}{$\begin{array}{c}\text { DRY } \\
\text { MASS } \\
\text { OF } \\
\text { SAMPLE (2) } \\
\text { (G) }\end{array}$} & \multicolumn{6}{|c|}{ NUMBER OF BLUE TRACER PARTICLES } \\
\hline & & & & \multirow[b]{2}{*}{ TOTAL } & \multicolumn{5}{|c|}{ BY SIZE CLASS } \\
\hline & & & & & $\begin{array}{l}0.25 \\
\text { TO } \\
0.50\end{array}$ & $\begin{array}{l}0.50 \\
\text { TO } \\
1.00\end{array}$ & $\begin{array}{l}1.00 \\
\text { TO } \\
2.00\end{array}$ & $\begin{array}{l}2.00 \\
\text { TO } \\
4.00\end{array}$ & $\begin{array}{l}4.00 \\
\text { TO } \\
8.00\end{array}$ \\
\hline $6-01$ & 4 & 16 & $187+$ & 5 & 0 & 3 & 2 & 0 & 0 \\
\hline $6-03$ & 4 & 17 & 235 & 3 & 0 & 0 & 3 & 0 & 0 \\
\hline $6-05$ & 5 & 16 & 197 & 2 & 0 & 0 & 2 & 0 & 0 \\
\hline $6-07$ & 5 & 15 & 389 & 1 & 0 & 0 & 1 & 0 & 0 \\
\hline $6-09$ & $\begin{array}{r}4 \\
10 \\
22\end{array}$ & $\begin{array}{r}9 \\
14 \\
26\end{array}$ & $\begin{array}{r}858 \\
1290 \\
385\end{array}$ & $\begin{array}{l}0 \\
0 \\
0\end{array}$ & $\begin{array}{l}0 \\
0 \\
0\end{array}$ & $\begin{array}{l}0 \\
0 \\
0\end{array}$ & $\begin{array}{l}0 \\
0 \\
0\end{array}$ & $\begin{array}{l}0 \\
0 \\
0\end{array}$ & $\begin{array}{l}0 \\
0 \\
0\end{array}$ \\
\hline $6-10$ & $\begin{array}{r}4 \\
9 \\
11 \\
12 \\
22 \\
25\end{array}$ & $\begin{array}{r}8 \\
10 \\
11 \\
15 \\
24 \\
25\end{array}$ & $\begin{array}{r}769 \\
765 \\
1148 \\
102+ \\
1420 \\
1773\end{array}$ & $\begin{array}{l}1 \\
0 \\
1 \\
2 \\
3 \\
0\end{array}$ & $\begin{array}{l}1 \\
0 \\
0 \\
0 \\
1 \\
0\end{array}$ & $\begin{array}{l}0 \\
0 \\
1 \\
0 \\
1 \\
0\end{array}$ & $\begin{array}{l}0 \\
0 \\
0 \\
2 \\
0 \\
0\end{array}$ & $\begin{array}{l}0 \\
0 \\
0 \\
0 \\
1 \\
0\end{array}$ & $\begin{array}{l}0 \\
0 \\
0 \\
0 \\
0 \\
0\end{array}$ \\
\hline $6-11$ & $\begin{array}{r}5 \\
9 \\
10 \\
11 \\
22 \\
24 \\
25\end{array}$ & $\begin{array}{r}8 \\
9 \\
10 \\
15 \\
23 \\
24 \\
26\end{array}$ & $\begin{array}{l}504 \\
368 \\
459 \\
164 \\
732 \\
623 \\
255\end{array}$ & $\begin{array}{l}\frac{1}{1} \\
\frac{1}{3} \\
5 \\
2 \\
9 \\
2\end{array}$ & $\begin{array}{l}1 \\
0 \\
1 \\
1 \\
1 \\
3 \\
0\end{array}$ & $\begin{array}{l}0 \\
0 \\
2 \\
4 \\
1 \\
3 \\
2\end{array}$ & $\begin{array}{l}0 \\
0 \\
0 \\
0 \\
0 \\
3 \\
0\end{array}$ & $\begin{array}{l}0 \\
0 \\
0 \\
0 \\
0 \\
0 \\
0\end{array}$ & $\begin{array}{l}0 \\
1 \\
0 \\
0 \\
0 \\
0 \\
0\end{array}$ \\
\hline $6-12$ & $\begin{array}{l}4 \\
7 \\
8 \\
9 \\
10 \\
11 \\
12 \\
13 \\
14 \\
15 \\
16 \\
22 \\
24 \\
25 \\
26\end{array}$ & $\begin{array}{r}6 \\
7 \\
8 \\
9 \\
10 \\
11 \\
12 \\
13 \\
14 \\
15 \\
16 \\
23 \\
24 \\
25 \\
26\end{array}$ & $\begin{array}{c}1+++ \\
9+++ \\
7+++ \\
10+++ \\
73+ \\
83+ \\
16+++ \\
218 \\
216+ \\
0+++ \\
47++ \\
8+++ \\
14++ \\
308 \\
13+++\end{array}$ & $\begin{array}{l}0 \\
0 \\
0 \\
0 \\
0 \\
1 \\
0 \\
4 \\
2 \\
0 \\
0 \\
0 \\
0 \\
2 \\
0\end{array}$ & $\begin{array}{l}0 \\
0 \\
0 \\
0 \\
0 \\
1 \\
0 \\
1 \\
0 \\
0 \\
0 \\
0 \\
0 \\
1 \\
0\end{array}$ & $\begin{array}{l}0 \\
0 \\
0 \\
0 \\
0 \\
0 \\
0 \\
3 \\
2 \\
0 \\
0 \\
0 \\
0 \\
1 \\
0\end{array}$ & $\begin{array}{l}0 \\
0 \\
0 \\
0 \\
0 \\
0 \\
0 \\
0 \\
0 \\
0 \\
0 \\
0 \\
0 \\
0 \\
0\end{array}$ & $\begin{array}{l}0 \\
0 \\
0 \\
0 \\
0 \\
0 \\
0 \\
0 \\
0 \\
0 \\
0 \\
0 \\
0 \\
0 \\
0\end{array}$ & $\begin{array}{l}0 \\
0 \\
0 \\
0 \\
0 \\
0 \\
0 \\
0 \\
0 \\
0 \\
0 \\
0 \\
0 \\
0 \\
0\end{array}$ \\
\hline
\end{tabular}


TABLE 20.- NUMBER OF BLUE TRACER PARTICLES(1), PER 100 GRAMS OF BEDLOAD SAMPLE, COLLECTED AT ONE OR MORE CROSS-CHANNEL SAMPLING POSITIONS SPACED AT 1-METER INTERVALS ACROSS THE CHANNEL WIDTH, EAST FORK RIVER, WYOMING, 1980--CONTINUED

SECTION 1481

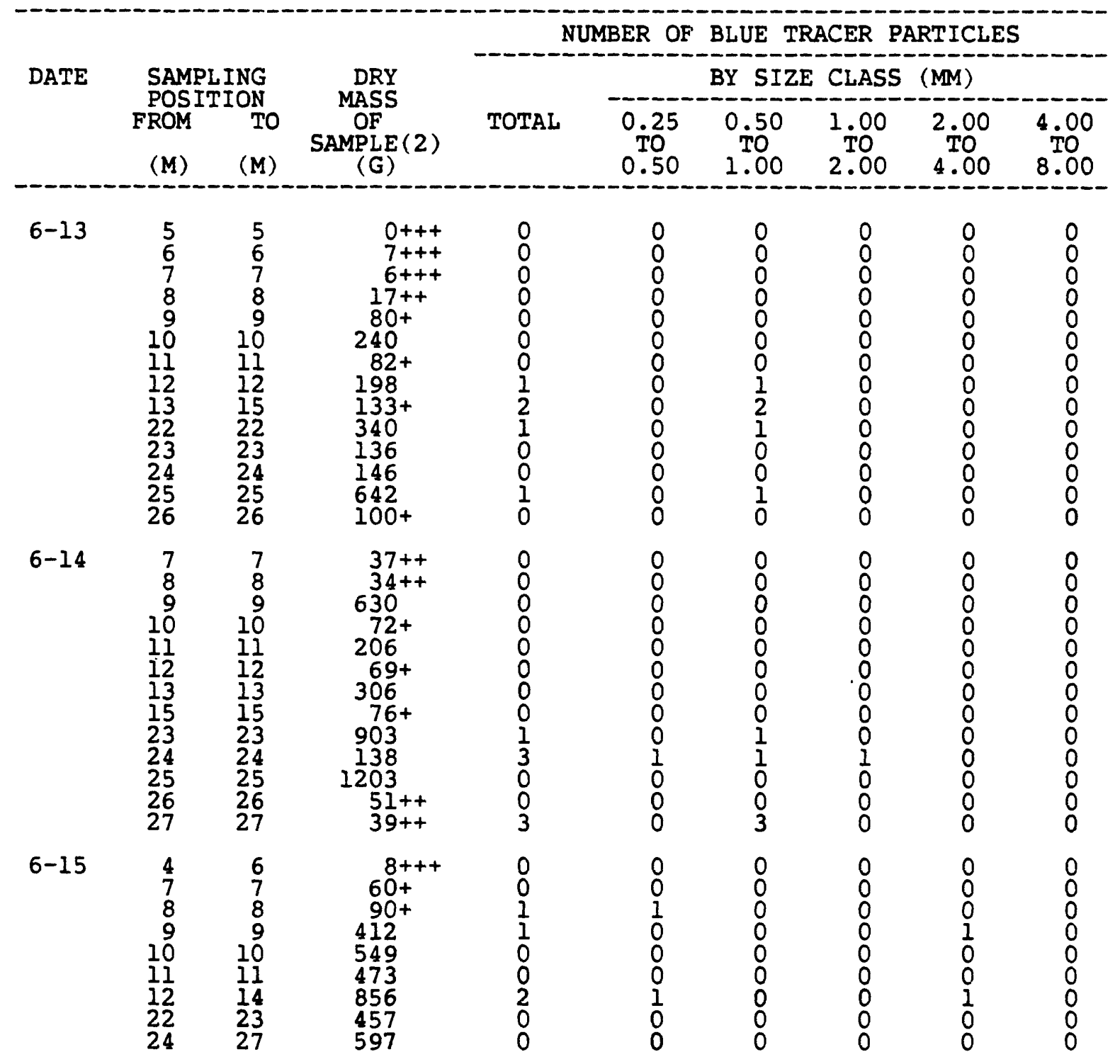


TABLE 20.- NUMBER OF BLUE TRACER PARTICLES(1), PER 100 GRAMS OF BEDLOAD SAMPLE, COLLECTED AT ONE OR MORE CROSS-CHANNEL SAMPLING POSITIONS SPACED AT 1-METER INTERVALS ACROSS THE CHANNEL WIDTH, EAST FORK RIVER, WYOMING, 1980--CONTINUED

SECTION 1481

\begin{tabular}{|c|c|c|c|c|c|c|c|c|c|}
\hline \multirow{3}{*}{ DATE } & & & & \multicolumn{6}{|c|}{ NUMBER OF BLUE TRACER PARTICLES } \\
\hline & \multicolumn{2}{|c|}{$\begin{array}{l}\text { SAMPLING } \\
\text { POSITION } \\
\text { FROM TO }\end{array}$} & \multirow{2}{*}{$\begin{array}{c}\text { DRY } \\
\text { MASS } \\
\text { OF } \\
\text { SAMPLE (2) } \\
\text { (G) }\end{array}$} & \multirow[b]{2}{*}{ TOTAL } & \multicolumn{5}{|c|}{ BY SIZE CLASS (MM) } \\
\hline & FROM & (M) & & & $\begin{array}{l}0.25 \\
\text { TO } \\
0.50\end{array}$ & $\begin{array}{l}0.50 \\
\text { TO } \\
1.00\end{array}$ & $\begin{array}{l}1.00 \\
\text { TO } \\
2.00\end{array}$ & $\begin{array}{l}2.00 \\
\text { TO } \\
4.00\end{array}$ & $\begin{array}{l}4.00 \\
\text { TO } \\
8.00\end{array}$ \\
\hline $6-16$ & $\begin{array}{r}5 \\
8 \\
10 \\
12 \\
22\end{array}$ & $\begin{array}{r}7 \\
9 \\
11 \\
15 \\
26\end{array}$ & $\begin{array}{c}4+++ \\
34++ \\
726 \\
59++ \\
184\end{array}$ & $\begin{array}{l}0 \\
3 \\
0 \\
0 \\
0\end{array}$ & $\begin{array}{l}0 \\
0 \\
0 \\
0 \\
0\end{array}$ & $\begin{array}{l}0 \\
3 \\
0 \\
0 \\
0\end{array}$ & $\begin{array}{l}0 \\
0 \\
0 \\
0 \\
0\end{array}$ & $\begin{array}{l}0 \\
0 \\
0 \\
0 \\
0\end{array}$ & $\begin{array}{l}0 \\
0 \\
0 \\
0 \\
0\end{array}$ \\
\hline $6-17$ & $\begin{array}{r}5 \\
7 \\
8 \\
9 \\
10 \\
12 \\
14 \\
22 \\
25\end{array}$ & $\begin{array}{r}6 \\
7 \\
8 \\
9 \\
11 \\
13 \\
16 \\
24 \\
26\end{array}$ & $\begin{array}{r}5+++ \\
6+++ \\
11+++ \\
100+ \\
495 \\
20++ \\
34++ \\
349 \\
11+++\end{array}$ & $\begin{array}{l}0 \\
0 \\
0 \\
0 \\
0 \\
0 \\
0 \\
0 \\
0\end{array}$ & $\begin{array}{l}0 \\
0 \\
0 \\
0 \\
0 \\
0 \\
0 \\
0 \\
0\end{array}$ & $\begin{array}{l}0 \\
0 \\
0 \\
0 \\
0 \\
0 \\
0 \\
0 \\
0\end{array}$ & $\begin{array}{l}0 \\
0 \\
0 \\
0 \\
0 \\
0 \\
0 \\
0 \\
0\end{array}$ & $\begin{array}{l}0 \\
0 \\
0 \\
0 \\
0 \\
0 \\
0 \\
0 \\
0\end{array}$ & $\begin{array}{l}0 \\
0 \\
0 \\
0 \\
0 \\
0 \\
0 \\
0 \\
0\end{array}$ \\
\hline $6-18$ & $\begin{array}{r}5 \\
6 \\
7 \\
8 \\
9 \\
10 \\
11 \\
12 \\
22 \\
24 \\
25\end{array}$ & $\begin{array}{r}5 \\
6 \\
7 \\
8 \\
9 \\
10 \\
11 \\
16 \\
23 \\
24 \\
25\end{array}$ & $\begin{array}{l}11+++ \\
39++ \\
31++ \\
415 \\
458 \\
405 \\
524 \\
136 \\
329 \\
945 \\
935\end{array}$ & $\begin{array}{l}0 \\
0 \\
0 \\
0 \\
0 \\
0 \\
0 \\
1 \\
1 \\
0 \\
0\end{array}$ & $\begin{array}{l}0 \\
0 \\
0 \\
0 \\
0 \\
0 \\
0 \\
0 \\
0 \\
0 \\
0\end{array}$ & $\begin{array}{l}0 \\
0 \\
0 \\
0 \\
0 \\
0 \\
0 \\
0 \\
1 \\
0 \\
0\end{array}$ & $\begin{array}{l}0 \\
0 \\
0 \\
0 \\
0 \\
0 \\
0 \\
0 \\
0 \\
0 \\
0\end{array}$ & $\begin{array}{l}0 \\
0 \\
0 \\
0 \\
0 \\
0 \\
0 \\
1 \\
0 \\
0 \\
0\end{array}$ & $\begin{array}{l}0 \\
0 \\
0 \\
0 \\
0 \\
0 \\
0 \\
0 \\
0 \\
0 \\
0\end{array}$ \\
\hline $6-19$ & $\begin{array}{r}5 \\
8 \\
9 \\
12 \\
22\end{array}$ & $\begin{array}{r}7 \\
8 \\
11 \\
15 \\
26\end{array}$ & $\begin{array}{c}99^{+++} \\
155^{++} \\
1405 \\
95+ \\
827\end{array}$ & $\begin{array}{l}0 \\
0 \\
0 \\
2 \\
0\end{array}$ & $\begin{array}{l}0 \\
0 \\
0 \\
0 \\
0\end{array}$ & $\begin{array}{l}0 \\
0 \\
0 \\
2 \\
0\end{array}$ & $\begin{array}{l}0 \\
0 \\
0 \\
0 \\
0\end{array}$ & $\begin{array}{l}0 \\
0 \\
0 \\
0 \\
0\end{array}$ & $\begin{array}{l}0 \\
0 \\
0 \\
0 \\
0\end{array}$ \\
\hline $6-20$ & $\begin{array}{r}5 \\
8 \\
12 \\
22\end{array}$ & $\begin{array}{l}7 \\
11 \\
16 \\
26\end{array}$ & $\begin{array}{l}21^{++} \\
849 \\
276 \\
1600\end{array}$ & $\begin{array}{l}0 \\
0 \\
0 \\
0\end{array}$ & $\begin{array}{l}0 \\
0 \\
0 \\
0\end{array}$ & $\begin{array}{l}0 \\
0 \\
0 \\
0\end{array}$ & $\begin{array}{l}0 \\
0 \\
0 \\
0\end{array}$ & $\begin{array}{l}0 \\
0 \\
0 \\
0\end{array}$ & $\begin{array}{l}0 \\
0 \\
0 \\
0\end{array}$ \\
\hline $6-21$ & $\begin{array}{r}5 \\
9 \\
12 \\
22\end{array}$ & $\begin{array}{r}8 \\
11 \\
16 \\
26\end{array}$ & $\begin{array}{l}34++ \\
546 \\
652 \\
1029\end{array}$ & $\begin{array}{l}0 \\
0 \\
0 \\
0\end{array}$ & $\begin{array}{l}0 \\
0 \\
0 \\
0\end{array}$ & $\begin{array}{l}0 \\
0 \\
0 \\
0\end{array}$ & $\begin{array}{l}0 \\
0 \\
0 \\
0\end{array}$ & $\begin{array}{l}0 \\
0 \\
0 \\
0\end{array}$ & $\begin{array}{l}0 \\
0 \\
0 \\
0\end{array}$ \\
\hline
\end{tabular}


TABLE 20.- NUMBER OF BLUE TRACER PARTICLES(1), PER 100 GRAMS OF BEDLOAD SAMPLE, COLLECTED AT ONE OR MORE CROSS-CHANNEL SAMPLING

POSITIONS SPACED AT 1-METER INTERVALS ACROSS THE CHANNEL WIDTH, EAST FORK RIVER, WYOMING, 1980--CONTINUED

SECTION 1481

\begin{tabular}{|c|c|c|c|c|c|c|c|c|c|}
\hline \multirow{3}{*}{ DATE } & \multirow{2}{*}{\multicolumn{2}{|c|}{$\begin{array}{l}\text { SAMPLING } \\
\text { POSITION }\end{array}$}} & \multirow{3}{*}{$\begin{array}{c}\text { DRY } \\
\text { MASS } \\
\text { OF } \\
\text { SAMPLE (2) } \\
\text { (G) }\end{array}$} & \multicolumn{2}{|c|}{ NUMBER OF } & \multicolumn{3}{|c|}{ BLUE TRACER PARTICLES } & \\
\hline & & & & & & BY SIZ & CLASS & $(\mathbf{M M})$ & \\
\hline & $\begin{array}{l}\text { FROM } \\
(M)\end{array}$ & TO & & TOTAL & $\begin{array}{l}0.25 \\
\text { TO } \\
0.50\end{array}$ & $\begin{array}{l}0.50 \\
\text { TO } \\
1.00\end{array}$ & $\begin{array}{l}1.00 \\
2.00\end{array}$ & $\begin{array}{l}2.00 \\
\text { TO } \\
4.00\end{array}$ & $\begin{array}{l}4.00 \\
\text { TO } \\
8.00\end{array}$ \\
\hline $6-23$ & $\begin{array}{r}5 \\
9 \\
22\end{array}$ & $\begin{array}{r}8 \\
16 \\
26\end{array}$ & $\begin{array}{l}111+++ \\
765 \\
465\end{array}$ & $\begin{array}{l}0 \\
0 \\
0\end{array}$ & $\begin{array}{l}0 \\
0 \\
0\end{array}$ & $\begin{array}{l}0 \\
0 \\
0\end{array}$ & $\begin{array}{l}0 \\
0 \\
0\end{array}$ & $\begin{array}{l}0 \\
0 \\
0\end{array}$ & $\begin{array}{l}0 \\
0 \\
0\end{array}$ \\
\hline $6-25$ & $\begin{array}{r}5 \\
22\end{array}$ & $\begin{array}{l}16 \\
26\end{array}$ & $\begin{array}{r}1216 \\
347\end{array}$ & $\begin{array}{l}0 \\
0\end{array}$ & $\begin{array}{l}0 \\
0\end{array}$ & $\begin{array}{l}0 \\
0\end{array}$ & $\begin{array}{l}0 \\
0\end{array}$ & $\begin{array}{l}0 \\
0\end{array}$ & $\begin{array}{l}0 \\
0\end{array}$ \\
\hline $6-27$ & $\begin{array}{r}5 \\
22\end{array}$ & $\begin{array}{l}16 \\
26\end{array}$ & $\begin{array}{l}1479 \\
1109\end{array}$ & $\begin{array}{l}0 \\
0\end{array}$ & $\begin{array}{l}0 \\
0\end{array}$ & $\begin{array}{l}0 \\
0\end{array}$ & $\begin{array}{l}0 \\
0\end{array}$ & $\begin{array}{l}0 \\
0\end{array}$ & $\begin{array}{l}0 \\
0\end{array}$ \\
\hline
\end{tabular}


TABLE 20.- NUMBER OF BLUE TRACER PARTICLES(1), PER 100 GRAMS OF BEDLOAD SAMPLE, COLLECTED AT ONE OR MORE CROSS-CHANNEL SAMPLING

POSITIONS SPACED AT 1-METER INTERVALS ACROSS THE CHANNEL WIDTH, EAST FORK RIVER, WYOMING, 1980--CONTINUED

SECTION 1533

\begin{tabular}{|c|c|c|c|c|c|c|c|c|c|}
\hline \multirow{3}{*}{ DATE } & & & & \multicolumn{6}{|c|}{ NUMBER OF BLUE TRACER PARTICLES } \\
\hline & \multirow{2}{*}{\multicolumn{2}{|c|}{$\begin{array}{l}\text { SAMPLING } \\
\text { POSITION } \\
\text { FROM TO }\end{array}$}} & \multirow{2}{*}{$\begin{array}{c}\text { DRY } \\
\text { MASS } \\
\text { OF } \\
\text { SAMPLE (2) } \\
(G)\end{array}$} & \multirow[b]{2}{*}{ TOTAL } & \multicolumn{5}{|c|}{ BY SIZE CLASS (MM) } \\
\hline & & (M) & & & $\begin{array}{l}0.25 \\
\text { TO } \\
0.50\end{array}$ & $\begin{array}{l}0.50 \\
\text { TO } \\
1.00\end{array}$ & $\begin{array}{l}1.00 \\
2.00\end{array}$ & $\begin{array}{l}2.00 \\
\text { T0 } \\
4.00\end{array}$ & $\begin{array}{l}4.00 \\
\text { TO } \\
8.00\end{array}$ \\
\hline $6-01$ & $\begin{array}{r}3 \\
11\end{array}$ & $\begin{array}{l}10 \\
15\end{array}$ & $\begin{array}{l}323 \\
129\end{array}$ & $\begin{array}{l}3 \\
2\end{array}$ & $\begin{array}{l}0 \\
0\end{array}$ & $\begin{array}{l}3 \\
2\end{array}$ & $\begin{array}{l}0 \\
0\end{array}$ & $\begin{array}{l}0 \\
0\end{array}$ & $\begin{array}{l}0 \\
0\end{array}$ \\
\hline $6-03$ & 12 & $\begin{array}{l}10 \\
15\end{array}$ & $\begin{array}{l}86+ \\
82++\end{array}$ & $\begin{array}{l}0 \\
1\end{array}$ & $\begin{array}{l}0 \\
0\end{array}$ & $\begin{array}{l}0 \\
1\end{array}$ & $\begin{array}{l}0 \\
0\end{array}$ & $\begin{array}{l}0 \\
0\end{array}$ & $\begin{array}{l}0 \\
0\end{array}$ \\
\hline $6-05$ & $\begin{array}{r}2 \\
10\end{array}$ & $\begin{array}{r}9 \\
15\end{array}$ & $\begin{array}{l}245 \\
275\end{array}$ & $\begin{array}{l}0 \\
1\end{array}$ & $\begin{array}{l}0 \\
0\end{array}$ & $\begin{array}{l}0 \\
0\end{array}$ & $\begin{array}{l}0 \\
0\end{array}$ & $\begin{array}{l}0 \\
1\end{array}$ & $\begin{array}{l}0 \\
0\end{array}$ \\
\hline $6-07$ & $\begin{array}{r}2 \\
10\end{array}$ & $\begin{array}{r}9 \\
14\end{array}$ & $\begin{array}{c}273 \\
68+\end{array}$ & $\begin{array}{l}4 \\
3\end{array}$ & $\frac{1}{3}$ & $\begin{array}{l}3 \\
0\end{array}$ & $\begin{array}{l}0 \\
0\end{array}$ & $\begin{array}{l}0 \\
0\end{array}$ & $\begin{array}{l}0 \\
0\end{array}$ \\
\hline $6-09$ & $\begin{array}{r}1 \\
6 \\
11\end{array}$ & $\begin{array}{r}5 \\
10 \\
15\end{array}$ & $\begin{array}{l}40++ \\
673 \\
265\end{array}$ & $\begin{array}{l}0 \\
0 \\
0\end{array}$ & $\begin{array}{l}0 \\
0 \\
0\end{array}$ & $\begin{array}{l}0 \\
0 \\
0\end{array}$ & $\begin{array}{l}0 \\
0 \\
0\end{array}$ & $\begin{array}{l}0 \\
0 \\
0\end{array}$ & $\begin{array}{l}0 \\
0 \\
0\end{array}$ \\
\hline $6-10$ & $\begin{array}{r}1 \\
11\end{array}$ & $\begin{array}{r}6 \\
10 \\
14\end{array}$ & $\begin{array}{r}1530 \\
2573 \\
564\end{array}$ & $\begin{array}{l}3 \\
0 \\
0\end{array}$ & $\begin{array}{l}0 \\
0 \\
0\end{array}$ & $\begin{array}{l}2 \\
0 \\
0\end{array}$ & $\begin{array}{l}0 \\
0 \\
0\end{array}$ & $\begin{array}{l}1 \\
0 \\
0\end{array}$ & $\begin{array}{l}0 \\
0 \\
0\end{array}$ \\
\hline $6-11$ & $\begin{array}{r}2 \\
6 \\
7 \\
8 \\
9 \\
10 \\
12\end{array}$ & $\begin{array}{r}5 \\
6 \\
7 \\
8 \\
9 \\
11 \\
14\end{array}$ & $\begin{array}{c}34++ \\
588 \\
85+ \\
419 \\
88++ \\
55++ \\
73++\end{array}$ & $\begin{array}{l}0 \\
0 \\
0 \\
0 \\
0 \\
2 \\
0\end{array}$ & $\begin{array}{l}0 \\
0 \\
0 \\
0 \\
0 \\
0 \\
0\end{array}$ & $\begin{array}{l}0 \\
0 \\
0 \\
0 \\
0 \\
2 \\
0\end{array}$ & $\begin{array}{l}0 \\
0 \\
0 \\
0 \\
0 \\
0 \\
0\end{array}$ & $\begin{array}{l}0 \\
0 \\
0 \\
0 \\
0 \\
0 \\
0\end{array}$ & $\begin{array}{l}0 \\
0 \\
0 \\
0 \\
0 \\
0 \\
0\end{array}$ \\
\hline $6-12$ & $\begin{array}{r}3 \\
6 \\
8 \\
9 \\
10 \\
11 \\
12 \\
13 \\
14 \\
15\end{array}$ & $\begin{array}{r}5 \\
7 \\
8 \\
9 \\
10 \\
11 \\
12 \\
13 \\
14 \\
15\end{array}$ & $\begin{array}{c}100+ \\
406 \\
198 \\
67+ \\
14+++ \\
7+++ \\
18+++ \\
493 \\
6+++ \\
5+++\end{array}$ & $\begin{array}{l}0 \\
0 \\
0 \\
0 \\
0 \\
0 \\
0 \\
0 \\
0 \\
0\end{array}$ & $\begin{array}{l}0 \\
0 \\
0 \\
0 \\
0 \\
0 \\
0 \\
0 \\
0 \\
0\end{array}$ & $\begin{array}{l}0 \\
0 \\
0 \\
0 \\
0 \\
0 \\
0 \\
0 \\
0 \\
0\end{array}$ & $\begin{array}{l}0 \\
0 \\
0 \\
0 \\
0 \\
0 \\
0 \\
0 \\
0 \\
0\end{array}$ & $\begin{array}{l}0 \\
0 \\
0 \\
0 \\
0 \\
0 \\
0 \\
0 \\
0 \\
0\end{array}$ & $\begin{array}{l}0 \\
0 \\
0 \\
0 \\
0 \\
0 \\
0 \\
0 \\
0 \\
0\end{array}$ \\
\hline
\end{tabular}


TABLE 20.- NUMBER OF BLUE TRACER PARTICLES(1), PER 100 GRAMS OF BEDLOAD SAMPLE, COLLECTED AT ONE OR MORE CROSS-CHANNEL SAMPLING

POSITIONS SPACED AT 1-METER INTERVALS ACROSS THE CHANNEL WIDTH, EAST FORK RIVER, WYOMING, 1980--CONTINUED

SECTION 1533

\begin{tabular}{|c|c|c|c|c|c|c|c|c|c|}
\hline \multirow{3}{*}{ DATE } & & & & \multicolumn{2}{|c|}{ NUMBER OF } & \multicolumn{3}{|c|}{ BLUE TRACER PARTICLES } & \\
\hline & \multicolumn{2}{|c|}{$\begin{array}{l}\text { SAMPLING } \\
\text { POSITION } \\
\text { FROM TO }\end{array}$} & \multirow{2}{*}{$\begin{array}{c}\text { DRY } \\
\text { MASS } \\
\text { OF } \\
\text { SAMPLE (2) } \\
(\mathrm{G})\end{array}$} & \multirow[b]{2}{*}{ TOTAL } & \multicolumn{5}{|c|}{ BY SIZE CLASS (MM) } \\
\hline & $\begin{array}{r}\text { FROM } \\
(M)\end{array}$ & (M) & & & $\begin{array}{l}0.25 \\
\text { TO } \\
0.50\end{array}$ & $\begin{array}{l}0.50 \\
\text { TO } \\
1.00\end{array}$ & $\begin{array}{l}1.00 \\
2.00\end{array}$ & $\begin{array}{l}2.00 \\
\text { TO } \\
4.00\end{array}$ & $\begin{array}{l}4.00 \\
\text { TO } \\
8.00\end{array}$ \\
\hline $6-13$ & $\begin{array}{r}2 \\
5 \\
6 \\
7 \\
8 \\
9 \\
10 \\
11 \\
12 \\
13\end{array}$ & $\begin{array}{r}4 \\
5 \\
6 \\
7 \\
8 \\
9 \\
10 \\
11 \\
12 \\
15\end{array}$ & $\begin{array}{c}11+++ \\
6+++ \\
130 \\
262 \\
503 \\
734 \\
881 \\
59++ \\
924 \\
49++\end{array}$ & $\begin{array}{l}0 \\
0 \\
0 \\
0 \\
0 \\
2 \\
0 \\
5 \\
1 \\
0\end{array}$ & $\begin{array}{l}0 \\
0 \\
0 \\
0 \\
0 \\
0 \\
0 \\
0 \\
0 \\
0\end{array}$ & $\begin{array}{l}0 \\
0 \\
0 \\
0 \\
0 \\
1 \\
0 \\
2 \\
1 \\
0\end{array}$ & $\begin{array}{l}0 \\
0 \\
0 \\
0 \\
0 \\
1 \\
0 \\
3 \\
0 \\
0\end{array}$ & $\begin{array}{l}0 \\
0 \\
0 \\
0 \\
0 \\
0 \\
0 \\
0 \\
0 \\
0\end{array}$ & $\begin{array}{l}0 \\
0 \\
0 \\
0 \\
0 \\
0 \\
0 \\
0 \\
0 \\
0\end{array}$ \\
\hline $6-14$ & $\begin{array}{r}6 \\
7 \\
8 \\
9 \\
10 \\
11 \\
12 \\
13 \\
14 \\
15\end{array}$ & $\begin{array}{r}6 \\
7 \\
8 \\
9 \\
10 \\
11 \\
12 \\
13 \\
14 \\
15\end{array}$ & $\begin{array}{c}862 \\
994 \\
403 \\
712 \\
459 \\
29++ \\
34+++ \\
37++ \\
15+++ \\
8+++\end{array}$ & $\begin{array}{l}0 \\
1 \\
0 \\
0 \\
0 \\
0 \\
0 \\
0 \\
0 \\
0\end{array}$ & $\begin{array}{l}0 \\
0 \\
0 \\
0 \\
0 \\
0 \\
0 \\
0 \\
0 \\
0\end{array}$ & $\begin{array}{l}0 \\
0 \\
0 \\
0 \\
0 \\
0 \\
0 \\
0 \\
0 \\
0\end{array}$ & $\begin{array}{l}0 \\
1 \\
0 \\
0 \\
0 \\
0 \\
0 \\
0 \\
0 \\
0\end{array}$ & $\begin{array}{l}0 \\
0 \\
0 \\
0 \\
0 \\
0 \\
0 \\
0 \\
0 \\
0\end{array}$ & $\begin{array}{l}0 \\
0 \\
0 \\
0 \\
0 \\
0 \\
0 \\
0 \\
0 \\
0\end{array}$ \\
\hline $6-15$ & $\begin{array}{r}2 \\
5 \\
6 \\
7 \\
8 \\
9 \\
10 \\
11 \\
12\end{array}$ & $\begin{array}{r}4 \\
5 \\
6 \\
7 \\
8 \\
9 \\
10 \\
11 \\
15\end{array}$ & $\begin{array}{c}32++ \\
20++ \\
171 \\
244 \\
124 \\
255 \\
245 \\
1365 \\
202\end{array}$ & $\begin{array}{l}0 \\
0 \\
0 \\
0 \\
0 \\
0 \\
2 \\
1 \\
0\end{array}$ & $\begin{array}{l}0 \\
0 \\
0 \\
0 \\
0 \\
0 \\
0 \\
0 \\
0\end{array}$ & $\begin{array}{l}0 \\
0 \\
0 \\
0 \\
0 \\
0 \\
2 \\
0 \\
0\end{array}$ & $\begin{array}{l}0 \\
0 \\
0 \\
0 \\
0 \\
0 \\
0 \\
0 \\
0\end{array}$ & $\begin{array}{l}0 \\
0 \\
0 \\
0 \\
0 \\
0 \\
0 \\
1 \\
0\end{array}$ & $\begin{array}{l}0 \\
0 \\
0 \\
0 \\
0 \\
0 \\
0 \\
0 \\
0\end{array}$ \\
\hline $6-17$ & $\begin{array}{r}3 \\
7 \\
8 \\
9 \\
10 \\
12\end{array}$ & $\begin{array}{r}6 \\
7 \\
8 \\
9 \\
11 \\
15\end{array}$ & $\begin{array}{l}12+++ \\
107+ \\
294 \\
244 \\
1260 \\
155\end{array}$ & $\begin{array}{l}0 \\
0 \\
0 \\
0 \\
0 \\
0\end{array}$ & $\begin{array}{l}0 \\
0 \\
0 \\
0 \\
0 \\
0\end{array}$ & $\begin{array}{l}0 \\
0 \\
0 \\
0 \\
0 \\
0\end{array}$ & $\begin{array}{l}0 \\
0 \\
0 \\
0 \\
0 \\
0\end{array}$ & $\begin{array}{l}0 \\
0 \\
0 \\
0 \\
0 \\
0\end{array}$ & $\begin{array}{l}0 \\
0 \\
0 \\
0 \\
0 \\
0\end{array}$ \\
\hline
\end{tabular}


TABLE 20.- NUMBER OF BLUE TRACER PARTICLES(1), PER 100 GRAMS OF BEDLOAD SAMPLE, COLLECTED AT ONE OR MORE CROSS-CHANNEL SAMPLING POSITIONS SPACED AT 1-METER INTERVALS ACROSS THE CHANNEL WIDTH, EAST FORK RIVER, WYOMING, 1980--CONTINUED

SECTION 1533

\begin{tabular}{|c|c|c|c|c|c|c|c|c|c|}
\hline \multirow{3}{*}{ DATE } & \multirow{3}{*}{\multicolumn{2}{|c|}{$\begin{array}{l}\text { SAMPLING } \\
\text { POSITION } \\
\text { FROM TO }\end{array}$}} & \multirow{3}{*}{$\begin{array}{c}\text { DRY } \\
\text { MASS } \\
\text { OF } \\
\text { SAMPLE (2) } \\
\text { (G) }\end{array}$} & \multicolumn{2}{|c|}{ NUMBER OF } & \multicolumn{3}{|c|}{ BLUE TRACER PARTICLE } & \\
\hline & & & & & & BY SIZ & CLASS & $(\mathbf{M})$ & \\
\hline & & & & TOTAL & $\begin{array}{l}0.25 \\
\text { TO } \\
0.50\end{array}$ & $\begin{array}{l}0.50 \\
\text { To } \\
1.00\end{array}$ & $\begin{array}{l}1.00 \\
2.00\end{array}$ & $\begin{array}{l}2.00 \\
\text { TO } \\
4.00\end{array}$ & $\begin{array}{l}4.00 \\
\text { TO } \\
8.00\end{array}$ \\
\hline $6-18$ & $\begin{array}{r}3 \\
5 \\
6 \\
7 \\
8 \\
9 \\
10 \\
12 \\
13\end{array}$ & $\begin{array}{r}4 \\
5 \\
6 \\
7 \\
8 \\
9 \\
11 \\
12 \\
15\end{array}$ & $\begin{array}{l}9+++ \\
6+++ \\
203 \\
1095 \\
1037 \\
192 \\
939 \\
523 \\
214\end{array}$ & $\begin{array}{l}0 \\
0 \\
1 \\
0 \\
0 \\
0 \\
1 \\
0 \\
0\end{array}$ & $\begin{array}{l}0 \\
0 \\
1 \\
0 \\
0 \\
0 \\
0 \\
0 \\
0\end{array}$ & $\begin{array}{l}0 \\
0 \\
0 \\
0 \\
0 \\
0 \\
0 \\
0 \\
0\end{array}$ & $\begin{array}{l}0 \\
0 \\
0 \\
0 \\
0 \\
0 \\
1 \\
0 \\
0\end{array}$ & $\begin{array}{l}0 \\
0 \\
0 \\
0 \\
0 \\
0 \\
0 \\
0 \\
0\end{array}$ & $\begin{array}{l}0 \\
0 \\
0 \\
0 \\
0 \\
0 \\
0 \\
0 \\
0\end{array}$ \\
\hline $6-19$ & $\begin{array}{r}3 \\
6 \\
10\end{array}$ & $\begin{array}{r}5 \\
9 \\
15\end{array}$ & $\begin{array}{l}11++ \\
481 \\
251\end{array}$ & $\begin{array}{l}0 \\
2 \\
0\end{array}$ & $\begin{array}{l}0 \\
0 \\
0\end{array}$ & $\begin{array}{l}0 \\
1 \\
0\end{array}$ & $\begin{array}{l}0 \\
1 \\
0\end{array}$ & $\begin{array}{l}0 \\
0 \\
0\end{array}$ & $\begin{array}{l}0 \\
0 \\
0\end{array}$ \\
\hline $6-20$ & $\begin{array}{r}3 \\
6 \\
8 \\
11\end{array}$ & $\begin{array}{r}5 \\
7 \\
10 \\
15\end{array}$ & $\begin{array}{l}10+++ \\
549 \\
385 \\
439\end{array}$ & $\begin{array}{l}0 \\
0 \\
0 \\
0\end{array}$ & $\begin{array}{l}0 \\
0 \\
0 \\
0\end{array}$ & $\begin{array}{l}0 \\
0 \\
0 \\
0\end{array}$ & $\begin{array}{l}0 \\
0 \\
0 \\
0\end{array}$ & $\begin{array}{l}0 \\
0 \\
0 \\
0\end{array}$ & $\begin{array}{l}0 \\
0 \\
0 \\
0\end{array}$ \\
\hline $6-21$ & $\begin{array}{r}3 \\
6 \\
7 \\
10 \\
12 \\
14\end{array}$ & $\begin{array}{r}5 \\
6 \\
9 \\
11 \\
13 \\
15\end{array}$ & $\begin{array}{l}102 \\
501 \\
328 \\
398 \\
658 \\
44++\end{array}$ & $\begin{array}{l}0 \\
0 \\
0 \\
0 \\
0 \\
0\end{array}$ & $\begin{array}{l}0 \\
0 \\
0 \\
0 \\
0 \\
0\end{array}$ & $\begin{array}{l}0 \\
0 \\
0 \\
0 \\
0 \\
0\end{array}$ & $\begin{array}{l}0 \\
0 \\
0 \\
0 \\
0 \\
0\end{array}$ & $\begin{array}{l}0 \\
0 \\
0 \\
0 \\
0 \\
0\end{array}$ & $\begin{array}{l}0 \\
0 \\
0 \\
0 \\
0 \\
0\end{array}$ \\
\hline $6-23$ & $\begin{array}{l}5 \\
9\end{array}$ & $\begin{array}{r}8 \\
15\end{array}$ & $\begin{array}{r}1045 \\
208\end{array}$ & $\begin{array}{l}0 \\
0\end{array}$ & $\begin{array}{l}0 \\
0\end{array}$ & $\begin{array}{l}0 \\
0\end{array}$ & $\begin{array}{l}0 \\
0\end{array}$ & $\begin{array}{l}0 \\
0\end{array}$ & $\begin{array}{l}0 \\
0\end{array}$ \\
\hline $6-25$ & $\begin{array}{r}3 \\
7 \\
8 \\
9 \\
11\end{array}$ & $\begin{array}{r}6 \\
7 \\
8 \\
10 \\
14\end{array}$ & $\begin{array}{l}34++ \\
366 \\
520 \\
761 \\
74++\end{array}$ & $\begin{array}{r}0 \\
1 \\
0 \\
12 \\
0\end{array}$ & $\begin{array}{l}0 \\
1 \\
0 \\
1 \\
0\end{array}$ & $\begin{array}{l}0 \\
0 \\
0 \\
9 \\
0\end{array}$ & $\begin{array}{l}0 \\
0 \\
0 \\
2 \\
0\end{array}$ & $\begin{array}{l}0 \\
0 \\
0 \\
0 \\
0\end{array}$ & $\begin{array}{l}0 \\
0 \\
0 \\
0 \\
0\end{array}$ \\
\hline $6-27$ & $\begin{array}{r}5 \\
7 \\
8 \\
9 \\
10 \\
11 \\
12\end{array}$ & $\begin{array}{r}6 \\
7 \\
8 \\
9 \\
10 \\
11 \\
14\end{array}$ & $\begin{array}{l}22++ \\
295 \\
704 \\
421 \\
770 \\
663 \\
191\end{array}$ & $\begin{array}{l}0 \\
0 \\
0 \\
0 \\
0 \\
0 \\
0\end{array}$ & $\begin{array}{l}0 \\
0 \\
0 \\
0 \\
0 \\
0 \\
0\end{array}$ & $\begin{array}{l}0 \\
0 \\
0 \\
0 \\
0 \\
0 \\
0\end{array}$ & $\begin{array}{l}0 \\
0 \\
0 \\
0 \\
0 \\
0 \\
0\end{array}$ & $\begin{array}{l}0 \\
0 \\
0 \\
0 \\
0 \\
0 \\
0\end{array}$ & $\begin{array}{l}0 \\
0 \\
0 \\
0 \\
0 \\
0 \\
0\end{array}$ \\
\hline
\end{tabular}


TABLE 20.- NUMBER OF BLUE TRACER PARTICLES(1), PER 100 GRAMS OF BEDLOAD SAMPLE, COLLECTED AT ONE OR MORE CROSS-CHANNEL SAMPLING POSITIONS SPACED AT I-METER INTERVALS ACROSS THE CHANNEL WIDTH, EAST FORK RIVER, WYOMING, 1980--CONTINUED

SECTION 1533

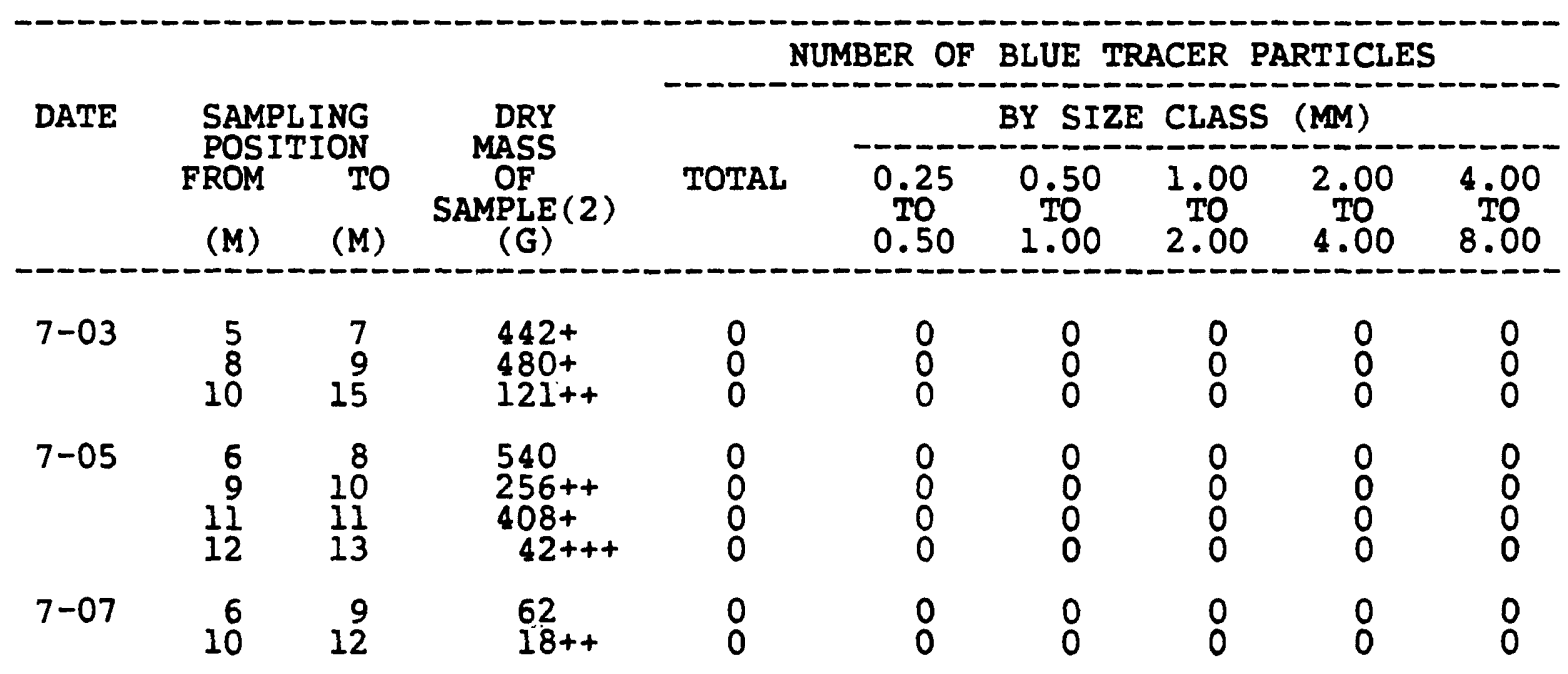


TABLE 20.- NUMBER OF BLUE TRACER PARTICLES(1), PER 100 GRAMS OF BEDLOAD SAMPLE, COLLECTED AT ONE OR MORE CROSS-CHANNEL SAMPLING POSITIONS SPACED AT I-METER INTERVALS ACROSS THE CHANNEL WIDTH, EAST FORK RIVER, WYOMING, 1980--CONTINUED

SECTION 1573

\begin{tabular}{|c|c|c|c|c|c|c|c|c|c|}
\hline \multirow{3}{*}{ DATE } & \multirow{3}{*}{\multicolumn{2}{|c|}{$\begin{array}{l}\text { SAMPLING } \\
\text { POSITION } \\
\text { FROM TO }\end{array}$}} & \multirow{3}{*}{$\begin{array}{c}\text { DRY } \\
\text { MASS } \\
\text { OF } \\
\text { SAMPLE (2) } \\
\text { (G) }\end{array}$} & \multicolumn{6}{|c|}{ NUMBER OF BLUE TRACER PARTICLES } \\
\hline & & & & \multirow[b]{2}{*}{ TOTAL } & & \multirow{2}{*}{$\begin{array}{c}\text { BY SIZ } \\
0.50 \\
\text { TO } \\
1.00\end{array}$} & \multirow{2}{*}{$\begin{array}{l}\text { CLASS } \\
1.00 \\
2.00 \\
2.00\end{array}$} & \multirow{2}{*}{$\begin{array}{l}\text { (MM) } \\
2.00 \\
40 \\
4.00\end{array}$} & \multirow[b]{2}{*}{$\begin{array}{l}4.00 \\
\text { TO } \\
8.00\end{array}$} \\
\hline & & & & & $\begin{array}{l}0.25 \\
\text { TO } \\
0.50\end{array}$ & & & & \\
\hline $6-01$ & $\begin{array}{r}8 \\
11\end{array}$ & $\begin{array}{l}10 \\
14\end{array}$ & $\underset{4+++}{89+}$ & $\begin{array}{l}3 \\
0\end{array}$ & $\frac{1}{0}$ & $\begin{array}{l}2 \\
0\end{array}$ & $\begin{array}{l}0 \\
0\end{array}$ & $\begin{array}{l}0 \\
0\end{array}$ & $\begin{array}{l}0 \\
0\end{array}$ \\
\hline $6-03$ & $1 \frac{1}{2}$ & $\frac{11}{14}$ & $\underset{5+++}{104+}$ & $\begin{array}{l}4 \\
0\end{array}$ & $\frac{1}{0}$ & $\frac{1}{0}$ & $\begin{array}{l}2 \\
0\end{array}$ & $\begin{array}{l}0 \\
0\end{array}$ & $\begin{array}{l}0 \\
0\end{array}$ \\
\hline $6-05$ & $1 \frac{1}{2}$ & $\begin{array}{l}11 \\
14\end{array}$ & $\begin{array}{c}45++ \\
0\end{array}$ & $\begin{array}{r}15 \\
0\end{array}$ & $\begin{array}{l}2 \\
0\end{array}$ & $\begin{array}{l}7 \\
0\end{array}$ & $\begin{array}{l}6 \\
0\end{array}$ & $\begin{array}{l}0 \\
0\end{array}$ & $\begin{array}{l}0 \\
0\end{array}$ \\
\hline $6-07$ & $\begin{array}{r}2 \\
12\end{array}$ & $\begin{array}{l}11 \\
14\end{array}$ & $\begin{array}{l}37++ \\
22+++\end{array}$ & $\begin{array}{l}0 \\
0\end{array}$ & $\begin{array}{l}0 \\
0\end{array}$ & $\begin{array}{l}0 \\
0\end{array}$ & $\begin{array}{l}0 \\
0\end{array}$ & $\begin{array}{l}0 \\
0\end{array}$ & $\begin{array}{l}0 \\
0\end{array}$ \\
\hline $6-09$ & $\begin{array}{r}2 \\
7 \\
10\end{array}$ & $\begin{array}{r}6 \\
10 \\
14\end{array}$ & $\begin{array}{c}53+ \\
1387^{+} \\
1066\end{array}$ & $\begin{array}{l}0 \\
0 \\
0\end{array}$ & $\begin{array}{l}0 \\
0 \\
0\end{array}$ & $\begin{array}{l}0 \\
0 \\
0\end{array}$ & $\begin{array}{l}0 \\
0 \\
0\end{array}$ & $\begin{array}{l}0 \\
0 \\
0\end{array}$ & $\begin{array}{l}0 \\
0 \\
0\end{array}$ \\
\hline $6-10$ & $\begin{array}{r}2 \\
71\end{array}$ & $\begin{array}{r}6 \\
10 \\
14\end{array}$ & $\begin{array}{l}108^{++} \\
1255 \\
1255\end{array}$ & $\begin{array}{l}0 \\
0 \\
1\end{array}$ & $\begin{array}{l}0 \\
0 \\
0\end{array}$ & $\begin{array}{l}0 \\
0 \\
1\end{array}$ & $\begin{array}{l}0 \\
0 \\
0\end{array}$ & $\begin{array}{l}0 \\
0 \\
0\end{array}$ & $\begin{array}{l}0 \\
0 \\
0\end{array}$ \\
\hline $6-11$ & $\begin{array}{r}2 \\
8 \\
9 \\
11\end{array}$ & $\begin{array}{r}7 \\
8 \\
10 \\
14\end{array}$ & $\begin{array}{l}331 \\
503 \\
39++ \\
316\end{array}$ & $\begin{array}{l}1 \\
1 \\
0 \\
0\end{array}$ & $\begin{array}{l}1 \\
0 \\
0 \\
0\end{array}$ & $\begin{array}{l}0 \\
0 \\
0 \\
0\end{array}$ & $\begin{array}{l}0 \\
0 \\
0 \\
0\end{array}$ & $\begin{array}{l}0 \\
1 \\
0 \\
0\end{array}$ & $\begin{array}{l}0 \\
0 \\
0 \\
0\end{array}$ \\
\hline $6-12$ & $\begin{array}{r}3 \\
8 \\
9 \\
10 \\
11 \\
12 \\
13\end{array}$ & $\begin{array}{r}7 \\
8 \\
9 \\
10 \\
11 \\
12 \\
14\end{array}$ & $\begin{array}{r}185 \\
746 \\
830 \\
517 \\
481 \\
1201 \\
131++\end{array}$ & $\begin{array}{l}0 \\
2 \\
0 \\
0 \\
0 \\
2 \\
0\end{array}$ & $\begin{array}{l}0 \\
0 \\
0 \\
0 \\
0 \\
0 \\
0\end{array}$ & $\begin{array}{l}0 \\
0 \\
0 \\
0 \\
0 \\
2 \\
0\end{array}$ & $\begin{array}{l}0 \\
2 \\
0 \\
0 \\
0 \\
0 \\
0\end{array}$ & $\begin{array}{l}0 \\
0 \\
0 \\
0 \\
0 \\
0 \\
0\end{array}$ & $\begin{array}{l}0 \\
0 \\
0 \\
0 \\
0 \\
0 \\
0\end{array}$ \\
\hline $6-13$ & $\begin{array}{r}3 \\
7 \\
9 \\
10 \\
11 \\
12 \\
13 \\
14\end{array}$ & $\begin{array}{r}6 \\
8 \\
9 \\
10 \\
11 \\
12 \\
13 \\
14\end{array}$ & $\begin{array}{c}42++ \\
332 \\
209 \\
1516 \\
783 \\
277 \\
269 \\
59++\end{array}$ & $\begin{array}{l}0 \\
0 \\
2 \\
0 \\
1 \\
0 \\
0 \\
3\end{array}$ & $\begin{array}{l}0 \\
0 \\
0 \\
0 \\
0 \\
0 \\
0 \\
2\end{array}$ & $\begin{array}{l}0 \\
0 \\
2 \\
0 \\
0 \\
0 \\
0 \\
1\end{array}$ & $\begin{array}{l}0 \\
0 \\
0 \\
0 \\
1 \\
0 \\
0 \\
0\end{array}$ & $\begin{array}{l}0 \\
0 \\
0 \\
0 \\
0 \\
0 \\
0 \\
0\end{array}$ & $\begin{array}{l}0 \\
0 \\
0 \\
0 \\
0 \\
0 \\
0 \\
0\end{array}$ \\
\hline
\end{tabular}


TABLE 20.- NUMBER OF BLUE TRACER PARTICLES(1), PER 100 GRAMS OF BEDLOAD SAMPLE, COLLECTED AT ONE OR MORE CROSS-CHANNEL SAMPLING POSITIONS SPACED AT 1-METER INTERVALS ACROSS THE CHANNEL WIDTH, EAST FORK RIVER, WYOMING, 1980--CONTINUED

SECTION 1573

\begin{tabular}{|c|c|c|c|c|c|c|c|c|c|}
\hline \multirow{3}{*}{ DATE } & & & & \multicolumn{6}{|c|}{ NUMBER OF BLUE TRACER PARTICLES } \\
\hline & \multirow{2}{*}{\multicolumn{2}{|c|}{$\begin{array}{l}\text { SAMPLING } \\
\text { POSITION } \\
\text { FROM TO }\end{array}$}} & \multirow{2}{*}{$\begin{array}{c}\text { DRY } \\
\text { MASS } \\
\text { OF } \\
\text { SAMPLE (2) } \\
\text { (G) }\end{array}$} & \multirow[b]{2}{*}{ TOTAL } & \multicolumn{5}{|c|}{ BY SIZE CLASS (MM) } \\
\hline & & & & & $\begin{array}{l}0.25 \\
\text { TO } \\
0.50\end{array}$ & $\begin{array}{l}0.50 \\
\text { TO } \\
1.00\end{array}$ & $\begin{array}{l}1.00 \\
\text { TO } \\
2.00\end{array}$ & $\begin{array}{l}2.00 \\
\text { TO } \\
4.00\end{array}$ & $\begin{array}{l}4.00 \\
\text { TO } \\
8.00\end{array}$ \\
\hline $6-14$ & $\begin{array}{r}5 \\
7 \\
8 \\
9 \\
10 \\
11 \\
12 \\
13 \\
14\end{array}$ & $\begin{array}{r}6 \\
7 \\
8 \\
9 \\
10 \\
11 \\
12 \\
13 \\
14\end{array}$ & $\begin{array}{c}283 \\
382 \\
1527 \\
945 \\
69+ \\
508 \\
572 \\
476 \\
370\end{array}$ & $\begin{array}{l}0 \\
0 \\
1 \\
0 \\
0 \\
1 \\
0 \\
1 \\
1\end{array}$ & $\begin{array}{l}0 \\
0 \\
0 \\
0 \\
0 \\
0 \\
0 \\
0 \\
0\end{array}$ & $\begin{array}{l}0 \\
0 \\
1 \\
0 \\
0 \\
1 \\
0 \\
1 \\
1\end{array}$ & $\begin{array}{l}0 \\
0 \\
0 \\
0 \\
0 \\
0 \\
0 \\
0 \\
0\end{array}$ & $\begin{array}{l}0 \\
0 \\
0 \\
0 \\
0 \\
0 \\
0 \\
0 \\
0\end{array}$ & $\begin{array}{l}0 \\
0 \\
0 \\
0 \\
0 \\
0 \\
0 \\
0 \\
0\end{array}$ \\
\hline $6-15$ & $\begin{array}{r}2 \\
5 \\
7 \\
8 \\
9 \\
10 \\
12 \\
13\end{array}$ & $\begin{array}{r}4 \\
6 \\
7 \\
8 \\
9 \\
11 \\
12 \\
14\end{array}$ & $\begin{array}{c}5+++ \\
22++ \\
119 \\
594 \\
49++ \\
30++ \\
629 \\
56++\end{array}$ & $\begin{array}{l}0 \\
0 \\
0 \\
0 \\
0 \\
0 \\
1 \\
0\end{array}$ & $\begin{array}{l}0 \\
0 \\
0 \\
0 \\
0 \\
0 \\
0 \\
0\end{array}$ & $\begin{array}{l}0 \\
0 \\
0 \\
0 \\
0 \\
0 \\
1 \\
0\end{array}$ & $\begin{array}{l}0 \\
0 \\
0 \\
0 \\
0 \\
0 \\
0 \\
0\end{array}$ & $\begin{array}{l}0 \\
0 \\
0 \\
0 \\
0 \\
0 \\
0 \\
0\end{array}$ & $\begin{array}{l}0 \\
0 \\
0 \\
0 \\
0 \\
0 \\
0 \\
0\end{array}$ \\
\hline $6-17$ & $\begin{array}{r}3 \\
5 \\
7 \\
8 \\
9 \\
10 \\
11 \\
12 \\
13 \\
14\end{array}$ & $\begin{array}{r}4 \\
6 \\
7 \\
8 \\
9 \\
10 \\
11 \\
12 \\
13 \\
14\end{array}$ & $\begin{array}{r}11+++ \\
16+++ \\
743 \\
894 \\
916 \\
1274 \\
544 \\
660 \\
467 \\
62++\end{array}$ & $\begin{array}{l}0 \\
0 \\
0 \\
1 \\
1 \\
0 \\
1 \\
0 \\
0 \\
2\end{array}$ & $\begin{array}{l}0 \\
0 \\
0 \\
0 \\
0 \\
0 \\
0 \\
0 \\
0 \\
0\end{array}$ & $\begin{array}{l}0 \\
0 \\
0 \\
0 \\
0 \\
0 \\
1 \\
0 \\
0 \\
0\end{array}$ & $\begin{array}{l}0 \\
0 \\
0 \\
1 \\
1 \\
0 \\
0 \\
0 \\
0 \\
2\end{array}$ & $\begin{array}{l}0 \\
0 \\
0 \\
0 \\
0 \\
0 \\
0 \\
0 \\
0 \\
0\end{array}$ & $\begin{array}{l}0 \\
0 \\
0 \\
0 \\
0 \\
0 \\
0 \\
0 \\
0 \\
0\end{array}$ \\
\hline $6-18$ & $\begin{array}{r}2 \\
7 \\
9 \\
10 \\
11 \\
14\end{array}$ & $\begin{array}{r}6 \\
8 \\
9 \\
10 \\
13 \\
15\end{array}$ & $\begin{array}{c}226 \\
569 \\
635 \\
1649 \\
113+ \\
7+++\end{array}$ & $\begin{array}{l}0 \\
0 \\
0 \\
0 \\
0 \\
0\end{array}$ & $\begin{array}{l}0 \\
0 \\
0 \\
0 \\
0 \\
0\end{array}$ & $\begin{array}{l}0 \\
0 \\
0 \\
0 \\
0 \\
0\end{array}$ & $\begin{array}{l}0 \\
0 \\
0 \\
0 \\
0 \\
0\end{array}$ & $\begin{array}{l}0 \\
0 \\
0 \\
0 \\
0 \\
0\end{array}$ & $\begin{array}{l}0 \\
0 \\
0 \\
0 \\
0 \\
0\end{array}$ \\
\hline $6-19$ & $\begin{array}{r}2 \\
9 \\
12\end{array}$ & $\begin{array}{r}8 \\
11 \\
14\end{array}$ & $\begin{array}{r}215 \\
1011 \\
132\end{array}$ & $\begin{array}{l}0 \\
0 \\
0\end{array}$ & $\begin{array}{l}0 \\
0 \\
0\end{array}$ & $\begin{array}{l}0 \\
0 \\
0\end{array}$ & $\begin{array}{l}0 \\
0 \\
0\end{array}$ & $\begin{array}{l}0 \\
0 \\
0\end{array}$ & $\begin{array}{l}0 \\
0 \\
0\end{array}$ \\
\hline
\end{tabular}


TABLE 20.- NUMBER OF BLUE TRACER PARTICLES(1), PER 100 GRAMS OF BEDLOAD SAMPLE, COLLECTED AT ONE OR MORE CROSS-CHANNEL SAMPLING POSITIONS SPACED AT 1-METER INTERVALS ACROSS THE CHANNEL WIDTH, EAST FORK RIVER, WYOMING, 1980--CONTINUED

SECTION 1573

\begin{tabular}{|c|c|c|c|c|c|c|c|c|c|}
\hline \multirow{3}{*}{ DATE } & \multirow{3}{*}{\multicolumn{2}{|c|}{$\begin{array}{l}\text { SAMPLING } \\
\text { POSITION } \\
\text { FROM TO }\end{array}$}} & \multirow{3}{*}{$\begin{array}{c}\text { DRY } \\
\text { MASS } \\
\text { OF } \\
\text { SAMPLE(2) } \\
\text { (G) }\end{array}$} & \multicolumn{6}{|c|}{ NUMBER OF BLUE TRACER PARTICLES } \\
\hline & & & & \multirow[b]{2}{*}{ TOTAL } & \multicolumn{5}{|c|}{ BY SIZE CLASS } \\
\hline & & & & & $\begin{array}{l}0.25 \\
\text { TO } \\
0.50\end{array}$ & $\begin{array}{l}0.50 \\
\text { TO } \\
1.00\end{array}$ & $\begin{array}{l}1.00 \\
2.00\end{array}$ & $\begin{array}{l}2.00 \\
\text { TO } \\
4.00\end{array}$ & $\begin{array}{l}4.00 \\
\text { TO } \\
8.00\end{array}$ \\
\hline $6-20$ & $\begin{array}{r}1 \\
8 \\
9 \\
12\end{array}$ & $\begin{array}{r}7 \\
8 \\
11 \\
14\end{array}$ & $\begin{array}{r}799 \\
1300 \\
1626 \\
544\end{array}$ & $\begin{array}{l}0 \\
0 \\
0 \\
0\end{array}$ & $\begin{array}{l}0 \\
0 \\
0 \\
0\end{array}$ & $\begin{array}{l}0 \\
0 \\
0 \\
0\end{array}$ & $\begin{array}{l}0 \\
0 \\
0 \\
0\end{array}$ & $\begin{array}{l}0 \\
0 \\
0 \\
0\end{array}$ & $\begin{array}{l}0 \\
0 \\
0 \\
0\end{array}$ \\
\hline $6-21$ & $\begin{array}{r}3 \\
8 \\
9 \\
10 \\
11 \\
12\end{array}$ & $\begin{array}{r}7 \\
8 \\
9 \\
10 \\
11 \\
14\end{array}$ & $\begin{array}{l}17++ \\
617 \\
936 \\
735 \\
878 \\
155+\end{array}$ & $\begin{array}{l}0 \\
0 \\
0 \\
0 \\
0 \\
0\end{array}$ & $\begin{array}{l}0 \\
0 \\
0 \\
0 \\
0 \\
0\end{array}$ & $\begin{array}{l}0 \\
0 \\
0 \\
0 \\
0 \\
0\end{array}$ & $\begin{array}{l}0 \\
0 \\
0 \\
0 \\
0 \\
0\end{array}$ & $\begin{array}{l}0 \\
0 \\
0 \\
0 \\
0 \\
0\end{array}$ & $\begin{array}{l}0 \\
0 \\
0 \\
0 \\
0 \\
0\end{array}$ \\
\hline $6-23$ & $\begin{array}{r}1 \\
8 \\
10 \\
13\end{array}$ & $\begin{array}{r}7 \\
9 \\
12 \\
14\end{array}$ & $\begin{array}{c}46++ \\
1261 \\
458 \\
83++\end{array}$ & $\begin{array}{l}0 \\
0 \\
0 \\
0\end{array}$ & $\begin{array}{l}0 \\
0 \\
0 \\
0\end{array}$ & $\begin{array}{l}0 \\
0 \\
0 \\
0\end{array}$ & $\begin{array}{l}0 \\
0 \\
0 \\
0\end{array}$ & $\begin{array}{l}0 \\
0 \\
0 \\
0\end{array}$ & $\begin{array}{l}0 \\
0 \\
0 \\
0\end{array}$ \\
\hline $6-25$ & $\begin{array}{r}2 \\
11 \\
12\end{array}$ & $\begin{array}{l}10 \\
11 \\
14\end{array}$ & $\begin{array}{r}1295 \\
500 \\
396\end{array}$ & $\begin{array}{l}0 \\
0 \\
1\end{array}$ & $\begin{array}{l}0 \\
0 \\
0\end{array}$ & $\begin{array}{l}0 \\
0 \\
1\end{array}$ & $\begin{array}{l}0 \\
0 \\
0\end{array}$ & $\begin{array}{l}0 \\
0 \\
0\end{array}$ & $\begin{array}{l}0 \\
0 \\
0\end{array}$ \\
\hline $6-27$ & $\begin{array}{r}5 \\
8 \\
9 \\
10 \\
11 \\
12 \\
13\end{array}$ & $\begin{array}{r}7 \\
8 \\
9 \\
10 \\
11 \\
12 \\
14\end{array}$ & $\begin{array}{l}5^{+++} \\
345 \\
317 \\
507 \\
646 \\
323 \\
413\end{array}$ & $\begin{array}{l}0 \\
0 \\
0 \\
0 \\
0 \\
0 \\
0\end{array}$ & $\begin{array}{l}0 \\
0 \\
0 \\
0 \\
0 \\
0 \\
0\end{array}$ & $\begin{array}{l}0 \\
0 \\
0 \\
0 \\
0 \\
0 \\
0\end{array}$ & $\begin{array}{l}0 \\
0 \\
0 \\
0 \\
0 \\
0 \\
0\end{array}$ & $\begin{array}{l}0 \\
0 \\
0 \\
0 \\
0 \\
0 \\
0\end{array}$ & $\begin{array}{l}0 \\
0 \\
0 \\
0 \\
0 \\
0 \\
0\end{array}$ \\
\hline $7-05$ & $\begin{array}{r}8 \\
12\end{array}$ & $\frac{11}{14}$ & $\begin{array}{l}139++ \\
130+\end{array}$ & $\begin{array}{l}0 \\
0\end{array}$ & $\begin{array}{l}0 \\
0\end{array}$ & $\begin{array}{l}0 \\
0\end{array}$ & $\begin{array}{l}0 \\
0\end{array}$ & $\begin{array}{l}0 \\
0\end{array}$ & $\begin{array}{l}0 \\
0\end{array}$ \\
\hline $7-07$ & $\begin{array}{r}8 \\
11\end{array}$ & $\begin{array}{l}10 \\
14\end{array}$ & $\begin{array}{c}341+ \\
51++\end{array}$ & $\begin{array}{l}0 \\
0\end{array}$ & $\begin{array}{l}0 \\
0\end{array}$ & $\begin{array}{l}0 \\
0\end{array}$ & $\begin{array}{l}0 \\
0\end{array}$ & $\begin{array}{l}0 \\
0\end{array}$ & $\begin{array}{l}0 \\
0\end{array}$ \\
\hline
\end{tabular}


TABLE 20.- NUMBER OF BLUE TRACER PARTICLES(1), PER 100 GRAMS OF BEDLOAD SAMPLE, COLLECTED AT ONE OR MORE CROSS-CHANNEL SAMPLING POSITIONS SPACED AT 1-METER INTERVALS ACROSS THE CHANNEL WIDTH, EAST FORK RIVER, WYOMING, 1980--CONTINUED

SECTION 1610

\begin{tabular}{|c|c|c|c|c|c|c|c|c|c|}
\hline \multirow{3}{*}{ DATE } & \multirow{3}{*}{\multicolumn{2}{|c|}{$\begin{array}{l}\text { SAMPLING } \\
\text { POSITION } \\
\text { FROM TO }\end{array}$}} & \multirow{3}{*}{$\begin{array}{c}\text { DRY } \\
\text { MASS } \\
\text { OF } \\
\text { SAMPLE (2) } \\
\text { (G) }\end{array}$} & \multicolumn{6}{|c|}{ NUMBER OF BLUE TRACER PARTICLES } \\
\hline & & & & \multirow[b]{2}{*}{ TOTAL } & \multicolumn{5}{|c|}{ BY SIZE CLASS (MM) } \\
\hline & & & & & $\begin{array}{l}0.25 \\
\text { TO } \\
0.50\end{array}$ & $\begin{array}{l}0.50 \\
\text { TO } \\
1.00\end{array}$ & $\begin{array}{l}1.00 \\
2.00\end{array}$ & $\begin{array}{l}2.00 \\
\text { TO } \\
4.00\end{array}$ & $\begin{array}{l}4.00 \\
\text { TO } \\
8.00\end{array}$ \\
\hline $6-01$ & 2 & 18 & 286 & 0 & 0 & 0 & 0 & 0 & 0 \\
\hline $6-03$ & $\begin{array}{r}2 \\
16\end{array}$ & $\begin{array}{l}15 \\
17\end{array}$ & $\begin{array}{c}161+ \\
0+++\end{array}$ & $\begin{array}{l}0 \\
0\end{array}$ & $\begin{array}{l}0 \\
0\end{array}$ & $\begin{array}{l}0 \\
0\end{array}$ & $\begin{array}{l}0 \\
0\end{array}$ & $\begin{array}{l}0 \\
0\end{array}$ & $\begin{array}{l}0 \\
0\end{array}$ \\
\hline $6-05$ & $\begin{array}{r}2 \\
16\end{array}$ & $\begin{array}{l}15 \\
17\end{array}$ & 91+ & $\begin{array}{l}3 \\
0\end{array}$ & $\begin{array}{l}0 \\
0\end{array}$ & $\begin{array}{l}2 \\
0\end{array}$ & $\begin{array}{l}1 \\
0\end{array}$ & $\begin{array}{l}0 \\
0\end{array}$ & $\begin{array}{l}0 \\
0\end{array}$ \\
\hline $6-07$ & $\begin{array}{r}2 \\
16\end{array}$ & $\begin{array}{l}15 \\
18\end{array}$ & $\begin{array}{c}1026 \\
55++\end{array}$ & $\begin{array}{l}0 \\
0\end{array}$ & $\begin{array}{l}0 \\
0\end{array}$ & $\begin{array}{l}0 \\
0\end{array}$ & $\begin{array}{l}0 \\
0\end{array}$ & $\begin{array}{l}0 \\
0\end{array}$ & $\begin{array}{l}0 \\
0\end{array}$ \\
\hline $6-09$ & $\begin{array}{l}2 \\
11\end{array}$ & $\begin{array}{l}10 \\
18\end{array}$ & $\begin{array}{c}67+ \\
2637^{+}\end{array}$ & $\begin{array}{l}0 \\
0\end{array}$ & $\begin{array}{l}0 \\
0\end{array}$ & $\begin{array}{l}0 \\
0\end{array}$ & $\begin{array}{l}0 \\
0\end{array}$ & $\begin{array}{l}0 \\
0\end{array}$ & $\begin{array}{l}0 \\
0\end{array}$ \\
\hline $6-10$ & $\begin{array}{l}3 \\
12 \\
13 \\
14 \\
16 \\
17\end{array}$ & $\begin{array}{l}12 \\
12 \\
13 \\
15 \\
16 \\
18\end{array}$ & $\begin{array}{r}1796 \\
1649 \\
1379 \\
560 \\
1282 \\
468\end{array}$ & $\begin{array}{l}0 \\
0 \\
1 \\
0 \\
2 \\
0\end{array}$ & $\begin{array}{l}0 \\
0 \\
1 \\
0 \\
1 \\
0\end{array}$ & $\begin{array}{l}0 \\
0 \\
0 \\
0 \\
1 \\
0\end{array}$ & $\begin{array}{l}0 \\
0 \\
0 \\
0 \\
0 \\
0\end{array}$ & $\begin{array}{l}0 \\
0 \\
0 \\
0 \\
0 \\
0\end{array}$ & $\begin{array}{l}0 \\
0 \\
0 \\
0 \\
0 \\
0\end{array}$ \\
\hline $6-11$ & $\begin{array}{r}2 \\
13 \\
15\end{array}$ & $\begin{array}{l}12 \\
14 \\
17\end{array}$ & $\begin{array}{r}1882 \\
1720 \\
459\end{array}$ & $\begin{array}{l}4 \\
1\end{array}$ & $\frac{1}{1}$ & $\begin{array}{l}2 \\
0 \\
0\end{array}$ & $\begin{array}{l}1 \\
0 \\
0\end{array}$ & $\begin{array}{l}0 \\
0 \\
0\end{array}$ & $\begin{array}{l}0 \\
0 \\
0\end{array}$ \\
\hline $6-12$ & $\begin{array}{r}3 \\
14\end{array}$ & $\begin{array}{l}13 \\
18\end{array}$ & $\begin{array}{r}1067 \\
524\end{array}$ & $\frac{1}{2}$ & $\begin{array}{l}0 \\
0\end{array}$ & $\begin{array}{l}0 \\
2\end{array}$ & $\frac{1}{0}$ & $\begin{array}{l}0 \\
0\end{array}$ & $\begin{array}{l}0 \\
0\end{array}$ \\
\hline $6-13$ & $\begin{array}{r}4 \\
16\end{array}$ & $\begin{array}{l}15 \\
18\end{array}$ & $\begin{array}{r}1659 \\
197\end{array}$ & $\frac{1}{2}$ & $\begin{array}{l}0 \\
0\end{array}$ & $\frac{1}{0}$ & $\begin{array}{l}0 \\
2\end{array}$ & $\begin{array}{l}0 \\
0\end{array}$ & $\begin{array}{l}0 \\
0\end{array}$ \\
\hline $6-14$ & $\begin{array}{l}3 \\
11 \\
12 \\
13 \\
14 \\
15 \\
16 \\
17 \\
18\end{array}$ & $\begin{array}{l}10 \\
11 \\
12 \\
13 \\
14 \\
15 \\
16 \\
17 \\
18\end{array}$ & $\begin{array}{c}83+ \\
64+ \\
56++ \\
986 \\
516 \\
857 \\
11+++ \\
204 \\
84++\end{array}$ & $\begin{array}{l}0 \\
0 \\
0 \\
0 \\
1 \\
0 \\
0 \\
1 \\
0\end{array}$ & $\begin{array}{l}0 \\
0 \\
0 \\
0 \\
0 \\
0 \\
0 \\
0 \\
0\end{array}$ & $\begin{array}{l}0 \\
0 \\
0 \\
0 \\
1 \\
0 \\
0 \\
0 \\
0\end{array}$ & $\begin{array}{l}0 \\
0 \\
0 \\
0 \\
0 \\
0 \\
0 \\
1 \\
0\end{array}$ & $\begin{array}{l}0 \\
0 \\
0 \\
0 \\
0 \\
0 \\
0 \\
0 \\
0\end{array}$ & $\begin{array}{l}0 \\
0 \\
0 \\
0 \\
0 \\
0 \\
0 \\
0 \\
0\end{array}$ \\
\hline $6-15$ & $\begin{array}{r}2 \\
16\end{array}$ & $\begin{array}{l}15 \\
18\end{array}$ & $\begin{array}{l}1984 \\
1518\end{array}$ & $\begin{array}{l}0 \\
0\end{array}$ & $\begin{array}{l}0 \\
0\end{array}$ & $\begin{array}{l}0 \\
0\end{array}$ & $\begin{array}{l}0 \\
0\end{array}$ & $\begin{array}{l}0 \\
0\end{array}$ & $\begin{array}{l}0 \\
0\end{array}$ \\
\hline
\end{tabular}


TABLE 20.- NUMBER OF BLUE TRACER PARTICLES(1), PER 100 GRAMS OF BEDLOAD SAMPLE, COLLECTED AT ONE OR MORE CROSS-CHANNEL SAMPLING POSITIONS SPACED AT I-METER INTERVALS ACROSS THE CHANNEL WIDTH, EAST FORK RIVER, WYOMING, 1980--CONTINUED

SECIION 1610

\begin{tabular}{|c|c|c|c|c|c|c|c|c|c|}
\hline \multirow{3}{*}{ DATE } & & & & \multicolumn{2}{|c|}{ NUMBER OF } & \multicolumn{3}{|c|}{ BLUE TRACER PARTICLES } & \\
\hline & \multicolumn{2}{|c|}{$\begin{array}{l}\text { SAMPLING } \\
\text { POSITION }\end{array}$} & \multirow{2}{*}{$\begin{array}{c}\text { DRY } \\
\text { MASS } \\
\text { OF } \\
\text { SAMPLE (2) } \\
\text { (G) }\end{array}$} & \multirow[b]{2}{*}{ TOTAL } & \multicolumn{3}{|c|}{ BY SIZE CLASS } & \multicolumn{2}{|l|}{ (MM) } \\
\hline & $\begin{array}{l}\text { FROM } \\
(\mathrm{M})\end{array}$ & (M) & & & $\begin{array}{l}0.25 \\
\text { TO } \\
0.50\end{array}$ & $\begin{array}{l}0.50 \\
\text { TO } \\
1.00\end{array}$ & $\begin{array}{l}1.00 \\
\text { TO } \\
2.00\end{array}$ & $\begin{array}{c}2.00 \\
\text { TO } \\
4.00\end{array}$ & $\begin{array}{l}4.00 \\
\text { TO } \\
8.00\end{array}$ \\
\hline $6-17$ & 16 & $\begin{array}{l}15 \\
18\end{array}$ & $\begin{array}{r}3876 \\
208\end{array}$ & $\begin{array}{l}0 \\
0\end{array}$ & $\begin{array}{l}0 \\
0\end{array}$ & $\begin{array}{l}0 \\
0\end{array}$ & $\begin{array}{l}0 \\
0\end{array}$ & $\begin{array}{l}0 \\
0\end{array}$ & $\begin{array}{l}0 \\
0\end{array}$ \\
\hline $6-18$ & $\begin{array}{r}2 \\
9 \\
12 \\
13 \\
14 \\
15 \\
16 \\
17\end{array}$ & $\begin{array}{l}8 \\
11 \\
12 \\
13 \\
14 \\
15 \\
16 \\
18\end{array}$ & $\begin{array}{l}17+++ \\
466 \\
176 \\
401 \\
430 \\
539 \\
396 \\
17++\end{array}$ & $\begin{array}{l}0 \\
0 \\
0 \\
0 \\
1 \\
0 \\
0 \\
0\end{array}$ & $\begin{array}{l}0 \\
0 \\
0 \\
0 \\
0 \\
0 \\
0 \\
0\end{array}$ & $\begin{array}{l}0 \\
0 \\
0 \\
0 \\
0 \\
0 \\
0 \\
0\end{array}$ & $\begin{array}{l}0 \\
0 \\
0 \\
0 \\
1 \\
0 \\
0 \\
0\end{array}$ & $\begin{array}{l}0 \\
0 \\
0 \\
0 \\
0 \\
0 \\
0 \\
0\end{array}$ & $\begin{array}{l}0 \\
0 \\
0 \\
0 \\
0 \\
0 \\
0 \\
0\end{array}$ \\
\hline $6-19$ & $\begin{array}{r}3 \\
16\end{array}$ & $\begin{array}{l}15 \\
18\end{array}$ & $\begin{array}{r}1072 \\
622\end{array}$ & $\begin{array}{l}0 \\
0\end{array}$ & $\begin{array}{l}0 \\
0\end{array}$ & $\begin{array}{l}0 \\
0\end{array}$ & $\begin{array}{l}0 \\
0\end{array}$ & $\begin{array}{l}0 \\
0\end{array}$ & $\begin{array}{l}0 \\
0\end{array}$ \\
\hline $6-20$ & $\begin{array}{r}3 \\
16\end{array}$ & $\begin{array}{l}15 \\
18\end{array}$ & $\begin{array}{l}1134 \\
119+\end{array}$ & $\begin{array}{l}0 \\
0\end{array}$ & $\begin{array}{l}0 \\
0\end{array}$ & $\begin{array}{l}0 \\
0\end{array}$ & $\begin{array}{l}0 \\
0\end{array}$ & $\begin{array}{l}0 \\
0\end{array}$ & $\begin{array}{l}0 \\
0\end{array}$ \\
\hline $6-21$ & $1 \frac{3}{16}$ & $\begin{array}{l}15 \\
18\end{array}$ & $\begin{array}{r}388 \\
1613\end{array}$ & $\begin{array}{l}0 \\
0\end{array}$ & $\begin{array}{l}0 \\
0\end{array}$ & $\begin{array}{l}0 \\
0\end{array}$ & $\begin{array}{l}0 \\
0\end{array}$ & $\begin{array}{l}0 \\
0\end{array}$ & $\begin{array}{l}0 \\
0\end{array}$ \\
\hline $6-23$ & $\begin{array}{r}2 \\
16\end{array}$ & $\begin{array}{l}15 \\
18\end{array}$ & $\begin{array}{l}1327 \\
66++\end{array}$ & $\begin{array}{l}0 \\
0\end{array}$ & $\begin{array}{l}0 \\
0\end{array}$ & $\begin{array}{l}0 \\
0\end{array}$ & $\begin{array}{l}0 \\
0\end{array}$ & $\begin{array}{l}0 \\
0\end{array}$ & $\begin{array}{l}0 \\
0\end{array}$ \\
\hline $6-25$ & 2 & 18 & 553 & 0 & 0 & 0 & 0 & 0 & 0 \\
\hline $6-27$ & $\begin{array}{l}6 \\
11 \\
14 \\
15 \\
16\end{array}$ & $\begin{array}{l}10 \\
13 \\
14 \\
15 \\
17\end{array}$ & $\begin{array}{l}22++ \\
247 \\
761 \\
493 \\
79+\end{array}$ & $\begin{array}{l}0 \\
0 \\
0 \\
0 \\
0\end{array}$ & $\begin{array}{l}0 \\
0 \\
0 \\
0 \\
0\end{array}$ & $\begin{array}{l}0 \\
0 \\
0 \\
0 \\
0\end{array}$ & $\begin{array}{l}0 \\
0 \\
0 \\
0 \\
0\end{array}$ & $\begin{array}{l}0 \\
0 \\
0 \\
0 \\
0\end{array}$ & $\begin{array}{l}0 \\
0 \\
0 \\
0 \\
0\end{array}$ \\
\hline $7-01$ & $\begin{array}{l}11 \\
12 \\
13\end{array}$ & $\begin{array}{l}11 \\
12 \\
17\end{array}$ & $\begin{array}{l}476 \\
807 \\
377+\end{array}$ & $\begin{array}{l}0 \\
0 \\
0\end{array}$ & $\begin{array}{l}0 \\
0 \\
0\end{array}$ & $\begin{array}{l}0 \\
0 \\
0\end{array}$ & $\begin{array}{l}0 \\
0 \\
0\end{array}$ & $\begin{array}{l}0 \\
0 \\
0\end{array}$ & $\begin{array}{l}0 \\
0 \\
0\end{array}$ \\
\hline $7-05$ & 9 & 17 & $374+$ & 0 & 0 & 0 & 0 & 0 & 0 \\
\hline $7-07$ & 12 & 17 & $6+++$ & 0 & 0 & 0 & 0 & 0 & 0 \\
\hline
\end{tabular}


TABLE 20.- NUMBER OF BLUE TRACER PARTICLES(1), PER 100 GRAMS OF BEDLOAD SAMPLE, COLLECTED AT ONE OR MORE CROSS-CHANNEL SAMPLING POSITIONS SPACED AT I-METER INTERVALS ACROSS THE CHANNEL WIDTH, EAST FORK RIVER, WYOMING, 1980--CONTINUED

(1) BLUE TRACER PARTICLES PLACED AS BED MATERIAL 10 METERS DOWNSTREAM OF SECTION 1695 ON MAY 19, 1979.

(2) REPRESENTS TOTAL MASS, IN GRAMS, RETAINED IN BEDLOAD SAMPLER AS MEASURED IN THE FIELD. FOR SUBSEQUENT ANALYSIS OF NUMBER AND SIZE OF TRACER, PARTICLES SMALLER THAN $0.25 \mathrm{MM}$ AND LARGER THAN $8.0 \mathrm{MM}$ WERE DISCARDED. REMAINING SAMPLES WEIGHING MORE THAN 100 GRAMS WERE CUT TO A STANDARD 100 GRAM-PORTION; SAMPLES WEIGHING LESS THAN 100 GRAMS WERE ANALYZED INTACT.

(3) DOES NOT INCLUDE TRACER PARTICLES MEASURED IN TRANSPORT AT SECTION 1400 , A BYPASS CHANNEL COMMON TO SECTIONS 1360, 1396, AND 1425. SEE FIGURE 3 .

$+\quad$ MASS OF SAMPLE ANALYZED WAS BETWEEN 50 AND 100 GRAMS; NUMBER OF TRACER PARTICLES ADJUSTED TO AN EQUIVALENT 100-GRAM SAMPLE; EXTRAPOLATED NUMBERS ARE CONSIDERED RELIABLE.

+ MASS OF SAMPLE ANALYZED WAS BETWEEN 10 AND 50 GRAMS; NUMBER OF TRACER PARTICLES ADJUSTED TO AN EQUIVALENT 100-GRAM SAMPLE; CARE SHOULD BE TAKEN IN USING THE EXTRAPOLATED NUMBERS.

+++ MASS OF SAMPLE ANALYZED WAS BETWEEN 0 AND 10 GRAMS: NUMBER OF TRACER PARTICLES ADJUSTED TO AN EQUIVALENT 100-GRAM SAMPLE; EXTRAPOLATED NUMBERS ARE CONSIDERED RELIABLE. 
TABLE 21.- NUMBER OF GREEN TRACER PARTICLES(1), PER 100 GRAMS OF BEDLOAD SAMPLE, COLLECTED AT ONE OR MORE CROSS-CHANNEL SAMPLING POSITIONS SPACED AT I-METER INTERVALS ACROSS THE CHANNEL WIDTH, EAST FORK RIVER, WYOMING, 1980

SECTION 1241

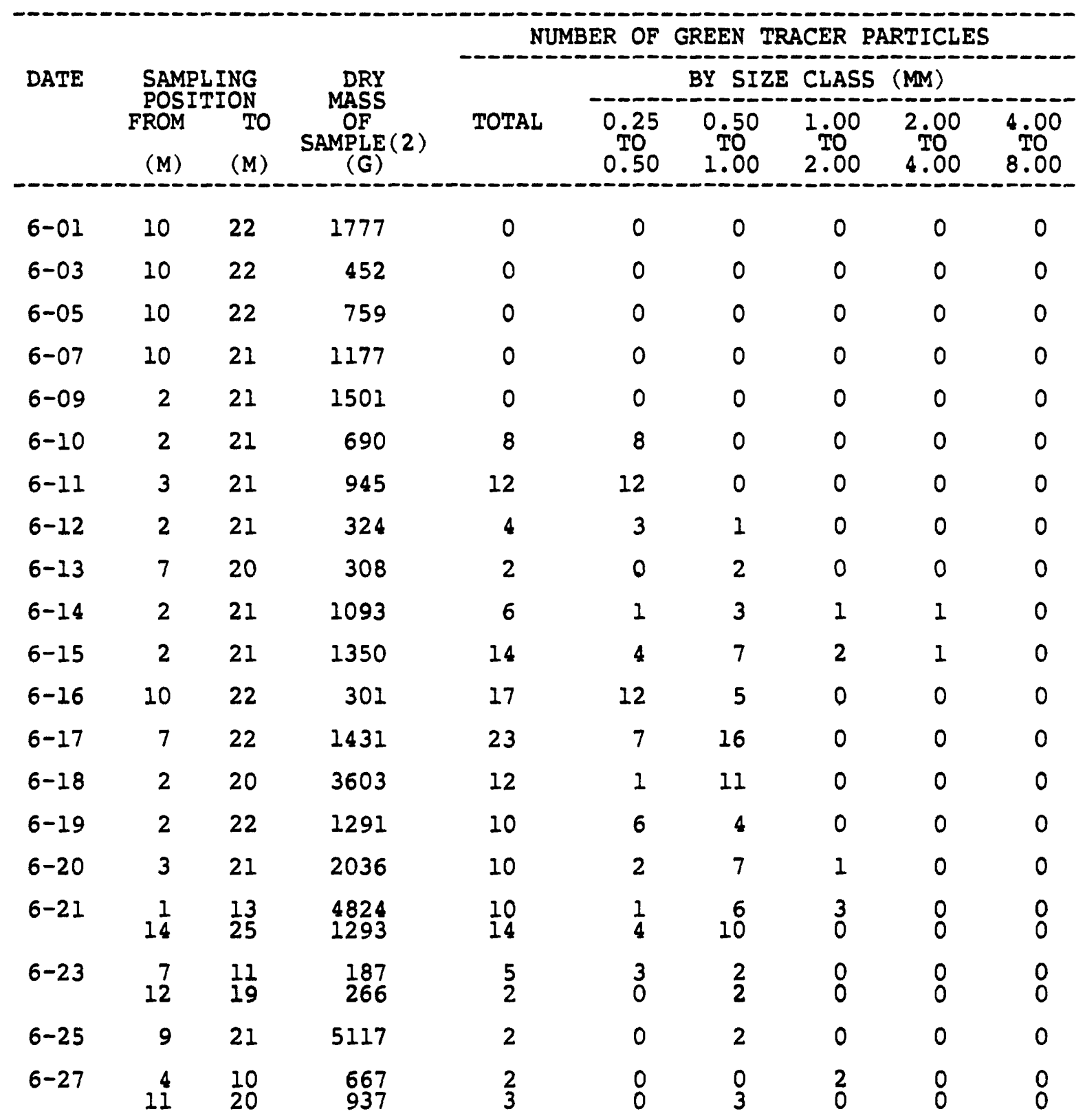


TABLE 21.- NUMBER OF GREEN TRACER PARTICLES(1), PER 100 GRAMS OF BEDLOAD SAMPLE, COLLECTED AT ONE OR MORE CROSS-CHANNEL SAMPLING POSITIONS SPACED AT 1-METER INTERVALS ACROSS THE CHANNEL WIDTH, EAST FORK RIVER, WYOMING, 1980

SECTION 1284

\begin{tabular}{|c|c|c|c|c|c|c|c|c|c|}
\hline \multicolumn{10}{|c|}{ NUMBER OF GREEN TRACER PARTICLES } \\
\hline \multirow[t]{2}{*}{ DATE } & \multicolumn{2}{|c|}{$\begin{array}{l}\text { SAMPLING } \\
\text { POSITION } \\
\text { FROM TO }\end{array}$} & \multirow{2}{*}{$\begin{array}{c}\text { DRY } \\
\text { MASS } \\
\text { OF } \\
\text { SAMPLE (2) } \\
\text { (G) }\end{array}$} & \multirow[b]{2}{*}{ TOTAL } & \multicolumn{5}{|c|}{ BY SIZE CLASS (MM) } \\
\hline & FROM & TO & & & $\begin{array}{l}0.25 \\
\text { TO } \\
0.50\end{array}$ & $\begin{array}{l}0.50 \\
100 \\
1.00\end{array}$ & $\begin{array}{l}1.00 \\
2.00\end{array}$ & $\begin{array}{l}2.00 \\
40 \\
4.00\end{array}$ & $\begin{array}{l}4.00 \\
\text { TO } \\
8.00\end{array}$ \\
\hline $6-01$ & 3 & 17 & 1178 & 0 & 0 & 0 & 0 & 0 & 0 \\
\hline $6-03$ & 1 & 18 & 741 & 0 & 0 & 0 & 0 & 0 & 0 \\
\hline $6-05$ & 3 & 16 & 944 & 0 & 0 & 0 & 0 & 0 & 0 \\
\hline $6-07$ & 2 & 17 & 969 & 0 & 0 & 0 & 0 & 0 & 0 \\
\hline $6-09$ & 2 & 17 & 2293 & 0 & 0 & 0 & 0 & 0 & 0 \\
\hline $6-10$ & $\begin{array}{r}3 \\
11\end{array}$ & $\begin{array}{l}10 \\
17\end{array}$ & $\begin{array}{r}867 \\
1039\end{array}$ & $\begin{array}{r}7 \\
34\end{array}$ & $\begin{array}{r}7 \\
24\end{array}$ & $\begin{array}{r}0 \\
10\end{array}$ & $\begin{array}{l}0 \\
0\end{array}$ & $\begin{array}{l}0 \\
0\end{array}$ & $\begin{array}{l}0 \\
0\end{array}$ \\
\hline $6-11$ & $\begin{array}{r}32 \\
12 \\
15\end{array}$ & $\begin{array}{l}11 \\
14 \\
18\end{array}$ & $\begin{array}{l}164 \\
475 \\
239\end{array}$ & $\begin{array}{l}14 \\
56 \\
63\end{array}$ & $\begin{array}{l}14 \\
49 \\
58\end{array}$ & $\begin{array}{l}0 \\
5 \\
5\end{array}$ & $\begin{array}{l}0 \\
2 \\
0\end{array}$ & $\begin{array}{l}0 \\
0 \\
0\end{array}$ & $\begin{array}{l}0 \\
0 \\
0\end{array}$ \\
\hline $6-12$ & 3 & 18 & 374 & 25 & 23 & 2 & 0 & 0 & 0 \\
\hline $6-13$ & 13 & $\begin{array}{l}10 \\
17\end{array}$ & $\begin{array}{l}141 \\
328\end{array}$ & $\begin{array}{r}0 \\
67\end{array}$ & $\begin{array}{r}0 \\
53\end{array}$ & $\begin{array}{r}0 \\
14\end{array}$ & $\begin{array}{l}0 \\
0\end{array}$ & $\begin{array}{l}0 \\
0\end{array}$ & $\begin{array}{l}0 \\
0\end{array}$ \\
\hline $6-14$ & 2 & 16 & 897 & 29 & 22 & 7 & 0 & 0 & 0 \\
\hline $6-15$ & $\begin{array}{r}2 \\
9 \\
14\end{array}$ & $\begin{array}{r}8 \\
13 \\
18\end{array}$ & $\begin{array}{r}1065 \\
725 \\
1178\end{array}$ & $\begin{array}{r}2 \\
17 \\
32\end{array}$ & $\begin{array}{r}0 \\
7 \\
22\end{array}$ & $\begin{array}{r}1 \\
8 \\
10\end{array}$ & $\begin{array}{l}0 \\
2 \\
0\end{array}$ & $\begin{array}{l}1 \\
0 \\
0\end{array}$ & $\begin{array}{l}0 \\
0 \\
0\end{array}$ \\
\hline $6-16$ & $\begin{array}{r}4 \\
7 \\
13\end{array}$ & $\begin{array}{l}6 \\
12 \\
16\end{array}$ & $\begin{array}{l}361 \\
697 \\
289\end{array}$ & $\begin{array}{r}2 \\
23 \\
38\end{array}$ & $\begin{array}{l}0 \\
10 \\
14\end{array}$ & $\begin{array}{r}0 \\
10 \\
24\end{array}$ & $\begin{array}{l}0 \\
2 \\
0\end{array}$ & $\begin{array}{l}1 \\
1 \\
0\end{array}$ & $\begin{array}{l}1 \\
0 \\
0\end{array}$ \\
\hline $6-17$ & $\begin{array}{r}2 \\
5 \\
6 \\
7 \\
8 \\
11 \\
12\end{array}$ & $\begin{array}{r}4 \\
5 \\
6 \\
7 \\
10 \\
11 \\
18\end{array}$ & $\begin{array}{c}43++ \\
45++ \\
47^{++} \\
783 \\
2725 \\
1767 \\
1497\end{array}$ & $\begin{array}{r}0 \\
0 \\
6 \\
7 \\
12 \\
28 \\
38\end{array}$ & $\begin{array}{r}0 \\
0 \\
0 \\
1 \\
3 \\
11 \\
20\end{array}$ & $\begin{array}{r}0 \\
0 \\
4 \\
5 \\
8 \\
15 \\
18\end{array}$ & $\begin{array}{l}0 \\
0 \\
2 \\
0 \\
1 \\
2 \\
0\end{array}$ & $\begin{array}{l}0 \\
0 \\
0 \\
1 \\
0 \\
0 \\
0\end{array}$ & $\begin{array}{l}0 \\
0 \\
0 \\
0 \\
0 \\
0 \\
0\end{array}$ \\
\hline $6-18$ & $\begin{array}{l}3 \\
8\end{array}$ & $\begin{array}{r}7 \\
18\end{array}$ & $\begin{array}{r}1887 \\
929\end{array}$ & $\begin{array}{r}0 \\
13\end{array}$ & $\begin{array}{l}0 \\
3\end{array}$ & $\begin{array}{l}0 \\
9\end{array}$ & $\begin{array}{l}0 \\
1\end{array}$ & $\begin{array}{l}0 \\
0\end{array}$ & $\begin{array}{l}0 \\
0\end{array}$ \\
\hline
\end{tabular}


TABLE 21.- NUMBER OF GREEN TRACER PARTICLES(1), PER 100 GRAMS OF BEDLOAD SAMPLE, COLLECTED AT ONE OR MORE CROSS-CHANNEL SAMPLING POSITIONS SPACED AT I-METER INTERVALS ACROSS THE CHANNEL WIDTH, EAST FORK RIVER, WYOMING, 1980--CONTINUED

SECTION 1284

\begin{tabular}{|c|c|c|c|c|c|c|c|c|c|}
\hline \multirow{3}{*}{ DATE } & & & \multirow{3}{*}{$\begin{array}{c}\text { DRY } \\
\text { MASS } \\
\text { OF } \\
\text { SAMPLE (2) } \\
(G)\end{array}$} & \multicolumn{6}{|c|}{ NUMBER OF GREEN TRACER PARTICLES } \\
\hline & \multicolumn{2}{|c|}{$\begin{array}{l}\text { SAMPLING } \\
\text { POSITION } \\
\text { FROM TO }\end{array}$} & & \multirow[b]{2}{*}{ TOTAL } & \multicolumn{5}{|c|}{ BY SIZE CLASS (MM) } \\
\hline & FROM & TO & & & $\begin{array}{l}0.25 \\
\text { TO } \\
0.50\end{array}$ & $\begin{array}{l}0.50 \\
\text { TO } \\
1.00\end{array}$ & $\begin{array}{l}1.00 \\
2.00\end{array}$ & $\begin{array}{l}2.00 \\
\text { TO } \\
4.00\end{array}$ & $\begin{array}{l}4.00 \\
\text { TO } \\
8.00\end{array}$ \\
\hline $6-19$ & $\begin{array}{r}3 \\
6 \\
9 \\
12 \\
15\end{array}$ & $\begin{array}{r}5 \\
8 \\
11 \\
14 \\
18\end{array}$ & $\begin{array}{l}40++ \\
721 \\
548 \\
986 \\
435\end{array}$ & $\begin{array}{r}0 \\
0 \\
12 \\
8 \\
21\end{array}$ & $\begin{array}{r}0 \\
0 \\
5 \\
7 \\
18\end{array}$ & $\begin{array}{l}0 \\
0 \\
3 \\
1 \\
3\end{array}$ & $\begin{array}{l}0 \\
0 \\
4 \\
0 \\
0\end{array}$ & $\begin{array}{l}0 \\
0 \\
0 \\
0 \\
0\end{array}$ & $\begin{array}{l}0 \\
0 \\
0 \\
0 \\
0\end{array}$ \\
\hline $6-20$ & $\begin{array}{r}3 \\
6 \\
9 \\
12 \\
15\end{array}$ & $\begin{array}{r}5 \\
8 \\
11 \\
14 \\
18\end{array}$ & $\begin{array}{c}53+ \\
46++ \\
262 \\
1010 \\
441\end{array}$ & $\begin{array}{r}0 \\
0 \\
11 \\
16 \\
23\end{array}$ & $\begin{array}{r}0 \\
0 \\
1 \\
8 \\
12\end{array}$ & $\begin{array}{r}0 \\
0 \\
10 \\
8 \\
11\end{array}$ & $\begin{array}{l}0 \\
0 \\
0 \\
0 \\
0\end{array}$ & $\begin{array}{l}0 \\
0 \\
0 \\
0 \\
0\end{array}$ & $\begin{array}{l}0 \\
0 \\
0 \\
0 \\
0\end{array}$ \\
\hline $6-21$ & $\begin{array}{r}3 \\
6 \\
9 \\
12 \\
15\end{array}$ & $\begin{array}{r}5 \\
8 \\
11 \\
14 \\
18\end{array}$ & $\begin{array}{l}43++ \\
266 \\
611 \\
401 \\
130\end{array}$ & $\begin{array}{r}0 \\
1 \\
0 \\
9 \\
13\end{array}$ & $\begin{array}{l}0 \\
0 \\
0 \\
2 \\
1\end{array}$ & $\begin{array}{l}0 \\
0 \\
0 \\
6 \\
9\end{array}$ & $\begin{array}{l}0 \\
0 \\
0 \\
1 \\
3\end{array}$ & $\begin{array}{l}0 \\
1 \\
0 \\
0 \\
0\end{array}$ & $\begin{array}{l}0 \\
0 \\
0 \\
0 \\
0\end{array}$ \\
\hline $6-23$ & $\begin{array}{r}3 \\
6 \\
9 \\
12 \\
15\end{array}$ & $\begin{array}{r}5 \\
8 \\
11 \\
14 \\
18\end{array}$ & $\begin{array}{l}1.4+++ \\
1350 \\
224 \\
360 \\
490\end{array}$ & $\begin{array}{r}0 \\
2 \\
9 \\
17 \\
20\end{array}$ & $\begin{array}{r}0 \\
0 \\
6 \\
8 \\
12\end{array}$ & $\begin{array}{l}0 \\
2 \\
3 \\
8 \\
8\end{array}$ & $\begin{array}{l}0 \\
0 \\
0 \\
1 \\
0\end{array}$ & $\begin{array}{l}0 \\
0 \\
0 \\
0 \\
0\end{array}$ & $\begin{array}{l}0 \\
0 \\
0 \\
0 \\
0\end{array}$ \\
\hline $6-25$ & $\begin{array}{r}3 \\
6 \\
9 \\
12 \\
15\end{array}$ & $\begin{array}{l}5 \\
8 \\
11 \\
14 \\
18\end{array}$ & $\begin{array}{c}54+ \\
155 \\
9+++ \\
1529 \\
557\end{array}$ & $\begin{array}{r}0 \\
1 \\
32 \\
4 \\
0\end{array}$ & $\begin{array}{r}0 \\
0 \\
21 \\
1 \\
0\end{array}$ & $\begin{array}{r}0 \\
0 \\
11 \\
3 \\
0\end{array}$ & $\begin{array}{l}0 \\
0 \\
0 \\
0 \\
0\end{array}$ & $\begin{array}{l}0 \\
1 \\
0 \\
0 \\
0\end{array}$ & $\begin{array}{l}0 \\
0 \\
0 \\
0 \\
0\end{array}$ \\
\hline $6-27$ & $\begin{array}{r}3 \\
6 \\
9 \\
12 \\
15\end{array}$ & $\begin{array}{r}5 \\
8 \\
11 \\
14 \\
18\end{array}$ & $\begin{array}{c}19 \\
695 \\
10+++ \\
1583 \\
937\end{array}$ & $\begin{array}{l}0 \\
3 \\
0 \\
2 \\
3\end{array}$ & $\begin{array}{l}0 \\
0 \\
0 \\
0 \\
2\end{array}$ & $\begin{array}{l}0 \\
0 \\
0 \\
2 \\
1\end{array}$ & $\begin{array}{l}0 \\
3 \\
0 \\
0 \\
0\end{array}$ & $\begin{array}{l}0 \\
0 \\
0 \\
0 \\
0\end{array}$ & $\begin{array}{l}0 \\
0 \\
0 \\
0 \\
0\end{array}$ \\
\hline
\end{tabular}


TABLE 21.- NUMBER OF GREEN TRACER PARTICLES(1), PER 100 GRAMS OF BEDLOAD SAMPLE, COLLECTED AT ONE OR MORE CROSS-CHANNEL SAMPLING POSITIONS SPACED AT I-METER INTERVALS ACROSS THE CHANNEL WIDTH, EAST FORK RIVER, WYOMING, 1980--CONTINUED

SECTION 1315

\begin{tabular}{|c|c|c|c|c|c|c|c|c|c|}
\hline \multirow{3}{*}{ DATE } & \multirow{2}{*}{\multicolumn{2}{|c|}{$\begin{array}{l}\text { SAMPLING } \\
\text { POSITION } \\
\text { FROM TO }\end{array}$}} & \multirow{3}{*}{$\begin{array}{c}\text { DRY } \\
\text { MASS } \\
\text { OF } \\
\text { SAMPLE (2) } \\
\text { (G) }\end{array}$} & \multicolumn{6}{|c|}{ NUMBER OF GREEN TRACER PARTICLES } \\
\hline & & & & & & BY SIZ & CLASS & $(\mathbf{M})$ & \\
\hline & FROM & TO & & TOTAL & $\begin{array}{l}0.25 \\
\text { TO } \\
0.50\end{array}$ & $\begin{array}{l}0.50 \\
.00 \\
1.00\end{array}$ & $\begin{array}{l}1.00 \\
2.00\end{array}$ & $\begin{array}{l}2.00 \\
\text { To } \\
4.00\end{array}$ & $\begin{array}{l}4.00 \\
\text { TO } \\
8.00\end{array}$ \\
\hline $6-01$ & 3 & 25 & 1241 & 0 & 0 & 0 & 0 & 0 & 0 \\
\hline $6-03$ & 3 & 22 & 217 & 1 & 1 & 0 & 0 & 0 & 0 \\
\hline $6-05$ & 3 & 24 & $98+$ & 0 & 0 & 0 & 0 & 0 & 0 \\
\hline $6-07$ & 3 & 25 & 249 & 0 & 0 & 0 & 0 & 0 & 0 \\
\hline $6-09$ & 3 & 25 & 483 & 12 & 10 & 2 & 0 & 0 & 0 \\
\hline $6-10$ & $\begin{array}{l}33 \\
12 \\
18 \\
21\end{array}$ & $\begin{array}{l}11 \\
17 \\
20 \\
25\end{array}$ & $\begin{array}{l}110+ \\
238 \\
508 \\
1945\end{array}$ & $\begin{array}{l}17 \\
42 \\
18 \\
19\end{array}$ & $\begin{array}{l}16 \\
30 \\
17 \\
19\end{array}$ & $\begin{array}{r}1 \\
12 \\
1 \\
0\end{array}$ & $\begin{array}{l}0 \\
0 \\
0 \\
0\end{array}$ & $\begin{array}{l}0 \\
0 \\
0 \\
0\end{array}$ & $\begin{array}{l}0 \\
0 \\
0 \\
0\end{array}$ \\
\hline $6-11$ & $\begin{array}{r}2 \\
13 \\
15\end{array}$ & $\begin{array}{l}12 \\
14 \\
17\end{array}$ & $\begin{array}{r}1882 \\
1720 \\
459\end{array}$ & $\begin{array}{l}37 \\
29 \\
29\end{array}$ & $\begin{array}{l}33 \\
24 \\
22\end{array}$ & $\begin{array}{l}4 \\
5 \\
7\end{array}$ & $\begin{array}{l}0 \\
0 \\
0\end{array}$ & $\begin{array}{l}0 \\
0 \\
0\end{array}$ & $\begin{array}{l}0 \\
0 \\
0\end{array}$ \\
\hline $6-12$ & 3 & 25 & 842 & 18 & 10 & 8 & 0 & 0 & 0 \\
\hline $6-13$ & $\begin{array}{r}3 \\
18\end{array}$ & $\frac{17}{25}$ & $\begin{array}{l}223 \\
623\end{array}$ & $\begin{array}{l}13 \\
16\end{array}$ & $\begin{array}{l}10 \\
12\end{array}$ & $\begin{array}{l}3 \\
4\end{array}$ & $\begin{array}{l}0 \\
0\end{array}$ & $\begin{array}{l}0 \\
0\end{array}$ & $\begin{array}{l}0 \\
0\end{array}$ \\
\hline $6-14$ & $\begin{array}{r}3 \\
13\end{array}$ & $\begin{array}{l}12 \\
25\end{array}$ & $\begin{array}{l}1095 \\
1099\end{array}$ & $\begin{array}{l}13 \\
26\end{array}$ & 25 & $\begin{array}{l}5 \\
5\end{array}$ & $\begin{array}{l}3 \\
0\end{array}$ & $\begin{array}{l}0 \\
0\end{array}$ & $\begin{array}{l}0 \\
0\end{array}$ \\
\hline $6-15$ & $\begin{array}{l}3 \\
12 \\
17 \\
19\end{array}$ & $\begin{array}{l}11 \\
16 \\
18 \\
25\end{array}$ & $\begin{array}{l}804 \\
633 \\
484 \\
846\end{array}$ & $\begin{array}{l}24 \\
21 \\
38\end{array}$ & $\begin{array}{r}11 \\
11 \\
17\end{array}$ & $\begin{array}{l}2 \\
10 \\
17 \\
20\end{array}$ & $\begin{array}{l}1 \\
0 \\
0 \\
1\end{array}$ & $\begin{array}{l}0 \\
0 \\
0 \\
0\end{array}$ & $\begin{array}{l}0 \\
0 \\
0 \\
0\end{array}$ \\
\hline $6-16$ & $\begin{array}{r}6 \\
7 \\
9 \\
13 \\
18 \\
20\end{array}$ & $\begin{array}{r}6 \\
8 \\
12 \\
17 \\
19 \\
24\end{array}$ & $\begin{array}{c}56+ \\
46++ \\
641 \\
459 \\
838 \\
1062\end{array}$ & $\begin{array}{r}0 \\
2 \\
18 \\
32 \\
21 \\
42\end{array}$ & $\begin{array}{r}0 \\
2 \\
9 \\
17 \\
99 \\
23\end{array}$ & $\begin{array}{r}0 \\
0 \\
9 \\
11 \\
8 \\
16\end{array}$ & $\begin{array}{l}0 \\
0 \\
0 \\
4 \\
4 \\
3\end{array}$ & $\begin{array}{l}0 \\
0 \\
0 \\
0 \\
0 \\
0\end{array}$ & $\begin{array}{l}0 \\
0 \\
0 \\
0 \\
0 \\
0\end{array}$ \\
\hline
\end{tabular}


TABLE 21.- NUMBER OF GREEN TRACER PARTICLES(1), PER 100 GRAMS OF BEDLOAD SAMPLE, COLLECTED AT ONE OR MORE CROSS-CHANNEL SAMPLING POSITIONS SPACED AT I-METER INTERVALS ACROSS THE CHANNEL WIDTH, EAST FORK RIVER, WYOMING, 1980--CONTINUED

SECTION 1315

\begin{tabular}{|c|c|c|c|c|c|c|c|c|c|}
\hline \multirow{3}{*}{ DATE } & \multirow{3}{*}{\multicolumn{2}{|c|}{$\begin{array}{l}\text { SAMPLING } \\
\text { POSITION } \\
\text { FROM TO }\end{array}$}} & \multirow{3}{*}{$\begin{array}{c}\text { DRY } \\
\text { MASS } \\
\text { OF } \\
\text { SAMPLE (2) } \\
\text { (G) }\end{array}$} & \multicolumn{6}{|c|}{ NUMBER OF GREEN TRACER PARTICLES } \\
\hline & & & & \multirow[b]{2}{*}{ TOTAL } & \multicolumn{5}{|c|}{ BY SIZE CLASS (MM) } \\
\hline & & (M) & & & $\begin{array}{l}0.25 \\
\text { TO } \\
0.50\end{array}$ & $\begin{array}{l}0.50 \\
\text { TO } \\
1.00\end{array}$ & $\begin{array}{l}1.00 \\
2.00\end{array}$ & $\begin{array}{l}2.00 \\
\text { TO } \\
4.00\end{array}$ & $\begin{array}{l}4.00 \\
\text { TO } \\
8.00\end{array}$ \\
\hline $6-17$ & $\begin{array}{r}3 \\
7 \\
8 \\
10 \\
13 \\
16 \\
19\end{array}$ & $\begin{array}{r}6 \\
7 \\
9 \\
12 \\
15 \\
18 \\
25\end{array}$ & $\begin{array}{c}17++ \\
20++ \\
378 \\
1811 \\
731 \\
867 \\
1265\end{array}$ & $\begin{array}{r}0 \\
5 \\
0 \\
19 \\
99 \\
21 \\
16\end{array}$ & $\begin{array}{l}0 \\
0 \\
0 \\
7 \\
2 \\
8 \\
7\end{array}$ & $\begin{array}{r}0 \\
0 \\
0 \\
11 \\
7 \\
13 \\
8\end{array}$ & $\begin{array}{l}0 \\
0 \\
0 \\
1 \\
0 \\
0 \\
1\end{array}$ & $\begin{array}{l}0 \\
0 \\
0 \\
0 \\
0 \\
0 \\
0\end{array}$ & $\begin{array}{l}0 \\
5 \\
0 \\
0 \\
0 \\
0 \\
0\end{array}$ \\
\hline $6-18$ & $\begin{array}{r}3 \\
5 \\
7 \\
9 \\
10 \\
14 \\
19\end{array}$ & $\begin{array}{r}4 \\
6 \\
8 \\
9 \\
13 \\
18 \\
25\end{array}$ & $\begin{array}{c}22++ \\
17++ \\
5+++ \\
38++ \\
519 \\
973 \\
828\end{array}$ & $\begin{array}{r}0 \\
0 \\
0 \\
C \\
0 \\
21 \\
35\end{array}$ & $\begin{array}{r}0 \\
0 \\
0 \\
0 \\
0 \\
7 \\
11\end{array}$ & $\begin{array}{r}0 \\
0 \\
0 \\
0 \\
0 \\
13 \\
22\end{array}$ & $\begin{array}{l}0 \\
0 \\
0 \\
0 \\
0 \\
1 \\
2\end{array}$ & $\begin{array}{l}0 \\
0 \\
0 \\
0 \\
0 \\
0 \\
0\end{array}$ & $\begin{array}{l}0 \\
0 \\
0 \\
0 \\
0 \\
0 \\
0\end{array}$ \\
\hline $6-19$ & $\begin{array}{r}3 \\
14 \\
18\end{array}$ & $\begin{array}{l}13 \\
17 \\
25\end{array}$ & $\begin{array}{l}847 \\
705 \\
742\end{array}$ & $\begin{array}{r}5 \\
27 \\
17\end{array}$ & $\begin{array}{r}1 \\
14 \\
5\end{array}$ & $\begin{array}{r}3 \\
13 \\
10\end{array}$ & $\begin{array}{l}1 \\
0 \\
2\end{array}$ & $\begin{array}{l}0 \\
0 \\
0\end{array}$ & $\begin{array}{l}0 \\
0 \\
0\end{array}$ \\
\hline $6-20$ & $\begin{array}{r}3 \\
11 \\
17\end{array}$ & $\begin{array}{l}10 \\
16 \\
25\end{array}$ & $\begin{array}{c}29+++ \\
1376 \\
942\end{array}$ & $\begin{array}{r}54 \\
0 \\
13\end{array}$ & $\begin{array}{r}13 \\
0 \\
3\end{array}$ & $\begin{array}{r}34 \\
0 \\
5\end{array}$ & $\begin{array}{l}3 \\
0 \\
5\end{array}$ & $\begin{array}{l}4 \\
0 \\
0\end{array}$ & $\begin{array}{l}0 \\
0 \\
0\end{array}$ \\
\hline $6-21$ & $1 \frac{4}{17}$ & $\begin{array}{l}10 \\
16 \\
25\end{array}$ & $\begin{array}{r}69+ \\
985 \\
1775\end{array}$ & $\begin{array}{r}0 \\
7 \\
10\end{array}$ & $\begin{array}{l}0 \\
3 \\
5\end{array}$ & $\begin{array}{l}0 \\
4 \\
3\end{array}$ & $\begin{array}{l}0 \\
0 \\
2\end{array}$ & $\begin{array}{l}0 \\
0 \\
0\end{array}$ & $\begin{array}{l}0 \\
0 \\
0\end{array}$ \\
\hline $6-23$ & $\begin{array}{r}3 \\
9 \\
13 \\
17\end{array}$ & $\begin{array}{r}8 \\
12 \\
16 \\
25\end{array}$ & $\begin{array}{r}130 \\
349 \\
246 \\
2589\end{array}$ & $\begin{array}{r}0 \\
2 \\
14 \\
9\end{array}$ & $\begin{array}{l}0 \\
0 \\
8 \\
3\end{array}$ & $\begin{array}{l}0 \\
0 \\
6 \\
6\end{array}$ & $\begin{array}{l}0 \\
0 \\
0 \\
0\end{array}$ & $\begin{array}{l}0 \\
2 \\
0 \\
0\end{array}$ & $\begin{array}{l}0 \\
0 \\
0 \\
0\end{array}$ \\
\hline $6-25$ & $\begin{array}{r}3 \\
10 \\
17\end{array}$ & $\begin{array}{r}99 \\
16 \\
25\end{array}$ & $\begin{array}{l}24++ \\
4383 \\
2198\end{array}$ & $\begin{array}{r}0 \\
2 \\
10\end{array}$ & $\begin{array}{l}0 \\
0 \\
4\end{array}$ & $\begin{array}{l}0 \\
2 \\
6\end{array}$ & $\begin{array}{l}0 \\
0 \\
0\end{array}$ & $\begin{array}{l}0 \\
0 \\
0\end{array}$ & $\begin{array}{l}0 \\
0 \\
0\end{array}$ \\
\hline $6-27$ & $\frac{3}{17}$ & $\begin{array}{l}10 \\
16 \\
25\end{array}$ & $\begin{array}{r}72+ \\
861 \\
1343\end{array}$ & $\begin{array}{l}0 \\
\frac{1}{5}\end{array}$ & $\begin{array}{l}0 \\
0 \\
0\end{array}$ & $\begin{array}{l}0 \\
1 \\
4\end{array}$ & $\begin{array}{l}0 \\
0 \\
1\end{array}$ & $\begin{array}{l}0 \\
0 \\
0\end{array}$ & $\begin{array}{l}0 \\
0 \\
0\end{array}$ \\
\hline
\end{tabular}


TABLE 21.- NUMBER OF GREEN TRACER PARTICLES(1), PER 100 GRAMS OF BEDLOAD SAMPLE, COLLECTED AT ONE OR MORE CROSS-CHANNEL SAMPLING

POSITIONS SPACED AT I-METER INTERVALS ACROSS THE CHANNEL

WIDTH, EAST FORK RIVER, WYOMING, 1980--CONTINUED

\section{SECTION $1360(3)$}

\begin{tabular}{|c|c|c|c|c|c|c|c|c|c|}
\hline \multirow{3}{*}{ DATE } & \multirow{3}{*}{\multicolumn{2}{|c|}{$\begin{array}{l}\text { SAMPLING } \\
\text { POSITION } \\
\text { FROM TO }\end{array}$}} & \multirow{3}{*}{$\begin{array}{c}\text { DRY } \\
\text { MASS } \\
\text { OF } \\
\text { SAMPLE (2) } \\
(G)\end{array}$} & \multicolumn{6}{|c|}{ NUMBER OF GREEN TRACER PARTICLES } \\
\hline & & & & & & BY SI2 & CLASS & (MM) & \\
\hline & & (M) & & TOTAL & $\begin{array}{l}0.25 \\
\text { TO } \\
0.50\end{array}$ & $\begin{array}{l}0.50 \\
\text { TO } \\
1.00\end{array}$ & $\begin{array}{l}1.00 \\
2.00\end{array}$ & $\begin{array}{l}2.00 \\
\text { TO } \\
4.00\end{array}$ & $\begin{array}{l}4.00 \\
\text { TO } \\
8.00\end{array}$ \\
\hline $6-01$ & 8 & 23 & $31++$ & 0 & 0 & 0 & 0 & 0 & 0 \\
\hline $6-03$ & 8 & 23 & $235+$ & 0 & 0 & 0 & 0 & 0 & 0 \\
\hline $6-05$ & 8 & 22 & 147 & 0 & 0 & 0 & 0 & 0 & 0 \\
\hline $6-07$ & 9 & 22 & 535 & 2 & 1 & 1 & 0 & 0 & 0 \\
\hline $6-09$ & 2 & 22 & 1049 & 48 & 38 & 10 & 0 & 0 & 0 \\
\hline $6-10$ & 9 & 22 & 1192 & 35 & 30 & 5 & 0 & 0 & 0 \\
\hline $6-11$ & $\begin{array}{r}9 \\
17 \\
20\end{array}$ & $\begin{array}{l}16 \\
19 \\
22\end{array}$ & $\begin{array}{l}437 \\
573 \\
0+++\end{array}$ & $\begin{array}{r}11 \\
36 \\
0\end{array}$ & $\begin{array}{r}6 \\
23 \\
0\end{array}$ & $\begin{array}{r}4 \\
13 \\
0\end{array}$ & $\begin{array}{l}1 \\
0 \\
0\end{array}$ & $\begin{array}{l}0 \\
0 \\
0\end{array}$ & $\begin{array}{l}0 \\
0 \\
0\end{array}$ \\
\hline $6-12$ & $\begin{array}{r}99 \\
17\end{array}$ & $\begin{array}{l}16 \\
22\end{array}$ & $\begin{array}{l}156 \\
262\end{array}$ & $\begin{array}{l}47 \\
26\end{array}$ & $\begin{array}{l}28 \\
13\end{array}$ & $\begin{array}{l}19 \\
12\end{array}$ & $\begin{array}{l}0 \\
1\end{array}$ & $\begin{array}{l}0 \\
0\end{array}$ & $\begin{array}{l}0 \\
0\end{array}$ \\
\hline $6-13$ & 9 & 22 & 841 & 22 & 7 & 13 & 2 & 0 & 0 \\
\hline $6-14$ & $\begin{array}{r}7 \\
16\end{array}$ & $\frac{14}{21}$ & $\begin{array}{l}111 \\
501\end{array}$ & $23^{3}$ & $\begin{array}{l}0 \\
5\end{array}$ & $\begin{array}{r}0 \\
11\end{array}$ & $\begin{array}{l}2 \\
5\end{array}$ & $\frac{1}{0}$ & $\begin{array}{l}0 \\
0\end{array}$ \\
\hline $6-15$ & $\begin{array}{r}99 \\
17 \\
20\end{array}$ & $\begin{array}{l}16 \\
19 \\
23\end{array}$ & $\begin{array}{r}519 \\
1518 \\
1795\end{array}$ & $\begin{array}{l}0 \\
11 \\
10\end{array}$ & $\begin{array}{l}0 \\
4 \\
1\end{array}$ & $\begin{array}{l}0 \\
6 \\
6\end{array}$ & $\begin{array}{l}0 \\
\frac{1}{3}\end{array}$ & $\begin{array}{l}0 \\
0 \\
0\end{array}$ & $\begin{array}{l}0 \\
0 \\
0\end{array}$ \\
\hline $6-16$ & $\begin{array}{l}14 \\
18 \\
20\end{array}$ & $\begin{array}{l}17 \\
19 \\
23\end{array}$ & $\begin{array}{l}170 \\
464 \\
408\end{array}$ & $\begin{array}{r}\frac{1}{7} \\
20\end{array}$ & $\begin{array}{l}0 \\
5 \\
9\end{array}$ & $\begin{array}{r}1 \\
11\end{array}$ & $\begin{array}{l}0 \\
0 \\
0\end{array}$ & $\begin{array}{l}0 \\
0 \\
0\end{array}$ & $\begin{array}{l}0 \\
0 \\
0\end{array}$ \\
\hline $6-17$ & $\begin{array}{r}9 \\
18\end{array}$ & $\begin{array}{l}17 \\
23\end{array}$ & $502^{85+}$ & $\begin{array}{l}0 \\
4\end{array}$ & $\begin{array}{l}0 \\
2\end{array}$ & $\begin{array}{l}0 \\
0\end{array}$ & $\begin{array}{l}0 \\
2\end{array}$ & $\begin{array}{l}0 \\
0\end{array}$ & $\begin{array}{l}0 \\
0\end{array}$ \\
\hline $6-18$ & $\begin{array}{r}9 \\
19\end{array}$ & $\begin{array}{l}18 \\
23\end{array}$ & $\begin{array}{l}144 \\
375\end{array}$ & $1 \frac{1}{3}$ & $\frac{1}{7}$ & $\begin{array}{l}0 \\
6\end{array}$ & $\begin{array}{l}0 \\
0\end{array}$ & $\begin{array}{l}0 \\
0\end{array}$ & $\begin{array}{l}0 \\
0\end{array}$ \\
\hline $6-19$ & $\begin{array}{r}7 \\
18 \\
21\end{array}$ & $\begin{array}{l}17 \\
20 \\
23\end{array}$ & $\begin{array}{l}588 \\
786 \\
229\end{array}$ & $\begin{array}{l}0 \\
6 \\
4\end{array}$ & $\begin{array}{l}0 \\
4 \\
1\end{array}$ & $\begin{array}{l}0 \\
2 \\
1\end{array}$ & $\begin{array}{l}0 \\
0 \\
2\end{array}$ & $\begin{array}{l}0 \\
0 \\
0\end{array}$ & $\begin{array}{l}0 \\
0 \\
0\end{array}$ \\
\hline $6-20$ & 2 & 23 & 391 & 1 & 1 & 0 & 0 & 0 & 0 \\
\hline
\end{tabular}


TABLE 21.- NUMBER OF GREEN TRACER PARTICLES(1), PER 100 GRAMS OF BEDLOAD SAMPLE, COLLECTED AT ONE OR MORE CROSS-CHANNEL SAMPLING POSITIONS SPACED AT I-METER INTERVALS ACROSS THE CHANNEL WIDTH, EAST FORK RIVER, WYOMING, 1980--CONTINUED

SECTION $1360(3)$

\begin{tabular}{|c|c|c|c|c|c|c|c|c|c|}
\hline \multirow{3}{*}{ DATE } & & & \multirow{3}{*}{$\begin{array}{c}\text { DRY } \\
\text { MASS } \\
\text { OF } \\
\text { SAMPLE (2) } \\
\text { (G) }\end{array}$} & \multicolumn{6}{|c|}{ NUMBER OF GREEN TRACER PARTICLES } \\
\hline & \multicolumn{2}{|c|}{$\begin{array}{l}\text { SAMPLING } \\
\text { POSITION } \\
\text { FROM TO }\end{array}$} & & \multirow[b]{2}{*}{ TOTAL } & \multicolumn{5}{|c|}{ BY SIZE CLASS (MM) } \\
\hline & $\begin{array}{r}\text { FROM } \\
\text { (M) }\end{array}$ & & & & $\begin{array}{l}0.25 \\
\text { TO } \\
0.50\end{array}$ & $\begin{array}{l}0.50 \\
\text { TO } \\
1.00\end{array}$ & $\begin{array}{l}1.00 \\
\text { TO } \\
2.00\end{array}$ & $\begin{array}{l}2.00 \\
\text { TO } \\
4.00\end{array}$ & $\begin{array}{l}4.00 \\
\text { TO } \\
8.00\end{array}$ \\
\hline $6-21$ & $\begin{array}{r}9 \\
17 \\
20\end{array}$ & $\begin{array}{l}16 \\
19 \\
23\end{array}$ & $\begin{array}{l}191 \\
753 \\
309\end{array}$ & $\begin{array}{r}0 \\
3 \\
10\end{array}$ & $\begin{array}{l}0 \\
1 \\
3\end{array}$ & $\begin{array}{l}0 \\
2 \\
7\end{array}$ & $\begin{array}{l}0 \\
0 \\
0\end{array}$ & $\begin{array}{l}0 \\
0 \\
0\end{array}$ & $\begin{array}{l}0 \\
0 \\
0\end{array}$ \\
\hline $6-23$ & 9 & 23 & 265 & 0 & 0 & 0 & 0 & 0 & 0 \\
\hline $6-25$ & 9 & 23 & 651 & 1 & 1 & 0 & 0 & 0 & 0 \\
\hline $6-27$ & 9 & 23 & 870 & 0 & 0 & 0 & 0 & 0 & 0 \\
\hline
\end{tabular}


TABLE 21.- NUMBER OF GREEN TRACER PARTICLES(1), PER 100 GRAMS OF BEDLOAD SAMPLE, COLLECTED AT ONE OR MORE CROSSS-CHANNEL SAMPLING POSITIONS SPACED AT I-METER INTERVALS ACROSS THE CHANNEL WIDTH, EAST FORK RIVER, WYOMING, 1980--CONTINUED

SECTION 1396(3)

\begin{tabular}{|c|c|c|c|c|c|c|c|c|c|}
\hline \multirow{3}{*}{ DATE } & & & & \multicolumn{6}{|c|}{ NUMBER OF GREEN TRACER PARTICLES } \\
\hline & \multirow{2}{*}{\multicolumn{2}{|c|}{$\begin{array}{l}\text { SAMPLING } \\
\text { POSITION } \\
\text { FROM TO }\end{array}$}} & \multirow{2}{*}{$\begin{array}{c}\text { DRY } \\
\text { MASS } \\
\text { OF } \\
\text { SAMPLE (2) } \\
\text { (G) }\end{array}$} & \multirow[b]{2}{*}{ TOTAL } & \multicolumn{5}{|c|}{ BY SIZE CLASS (MM) } \\
\hline & & & & & $\begin{array}{l}0.25 \\
\text { TO } \\
0.50\end{array}$ & $\begin{array}{l}0.50 \\
\text { TO } \\
1.00\end{array}$ & $\begin{array}{l}1.00 \\
2.00\end{array}$ & $\begin{array}{l}2.00 \\
\text { TO } \\
4.00\end{array}$ & $\begin{array}{l}4.00 \\
\text { TO } \\
8.00\end{array}$ \\
\hline $6-01$ & 5 & 17 & 178 & 0 & 0 & 0 & 0 & 0 & 0 \\
\hline $6-03$ & 5 & 17 & 472 & 0 & 0 & 0 & 0 & 0 & 0 \\
\hline $6-05$ & 5 & 17 & 407 & 1 & 1 & 0 & 0 & 0 & 0 \\
\hline $6-07$ & 5 & 17 & 532 & 1 & 0 & 0 & 1 & 0 & 0 \\
\hline $6-09$ & 5 & 17 & 493 & 34 & 28 & 6 & 0 & 0 & 0 \\
\hline $6-10$ & 5 & 17 & 2231 & 32 & 23 & 8 & 1 & 0 & 0 \\
\hline $6-11$ & $\begin{array}{r}5 \\
16\end{array}$ & $\begin{array}{l}15 \\
17\end{array}$ & $\begin{array}{l}693 \\
334\end{array}$ & $\begin{array}{l}21 \\
14\end{array}$ & $\begin{array}{l}9 \\
6\end{array}$ & 11 & $\frac{1}{1}$ & $\begin{array}{l}0 \\
1\end{array}$ & $\begin{array}{l}0 \\
0\end{array}$ \\
\hline $6-12$ & 5 & 17 & 467 & 20 & 3 & 13 & 3 & 1 & 0 \\
\hline $6-13$ & $\begin{array}{r}5 \\
15\end{array}$ & $\begin{array}{l}14 \\
17\end{array}$ & $\begin{array}{l}268 \\
382\end{array}$ & $\begin{array}{r}12 \\
0\end{array}$ & $\begin{array}{l}0 \\
0\end{array}$ & $\begin{array}{l}5 \\
0\end{array}$ & $\begin{array}{l}5 \\
0\end{array}$ & $\begin{array}{l}2 \\
0\end{array}$ & $\begin{array}{l}0 \\
0\end{array}$ \\
\hline $6-14$ & $\begin{array}{l}5 \\
14 \\
15 \\
16 \\
17\end{array}$ & $\begin{array}{l}13 \\
14 \\
15 \\
16 \\
17\end{array}$ & $\begin{array}{l}367 \\
337 \\
999 \\
759 \\
30++\end{array}$ & $\begin{array}{r}0 \\
0 \\
4 \\
10 \\
7\end{array}$ & $\begin{array}{l}0 \\
0 \\
2 \\
1 \\
0\end{array}$ & $\begin{array}{l}0 \\
0 \\
0 \\
2 \\
7\end{array}$ & $\begin{array}{l}0 \\
0 \\
2 \\
2 \\
0\end{array}$ & $\begin{array}{l}0 \\
0 \\
0 \\
5 \\
0\end{array}$ & $\begin{array}{l}0 \\
0 \\
0 \\
0 \\
0\end{array}$ \\
\hline $6-15$ & 5 & 16 & 1830 & 1 & 0 & 1 & 0 & 0 & 0 \\
\hline $6-16$ & $\begin{array}{r}7 \\
15\end{array}$ & $\begin{array}{l}14 \\
19\end{array}$ & $\begin{array}{l}709 \\
691\end{array}$ & $\begin{array}{r}2 \\
12\end{array}$ & $\begin{array}{l}0 \\
4\end{array}$ & $\frac{1}{5}$ & $\begin{array}{l}0 \\
2\end{array}$ & $\frac{1}{1}$ & $\begin{array}{l}0 \\
0\end{array}$ \\
\hline $6-17$ & $\begin{array}{r}5 \\
14\end{array}$ & $\begin{array}{l}13 \\
17\end{array}$ & $\begin{array}{c}91+ \\
659\end{array}$ & $\begin{array}{l}2 \\
0\end{array}$ & $\frac{1}{0}$ & $\frac{1}{0}$ & $\begin{array}{l}0 \\
0\end{array}$ & $\begin{array}{l}0 \\
0\end{array}$ & $\begin{array}{l}0 \\
0\end{array}$ \\
\hline $6-18$ & $\begin{array}{r}5 \\
14 \\
15\end{array}$ & $\begin{array}{l}13 \\
14 \\
16\end{array}$ & $\begin{array}{l}315 \\
932 \\
266\end{array}$ & $\begin{array}{r}4 \\
13 \\
0\end{array}$ & $\begin{array}{r}0 \\
11 \\
0\end{array}$ & $\begin{array}{l}4 \\
2 \\
0\end{array}$ & $\begin{array}{l}0 \\
0 \\
0\end{array}$ & $\begin{array}{l}0 \\
0 \\
0\end{array}$ & $\begin{array}{l}0 \\
0 \\
0\end{array}$ \\
\hline $6-19$ & $\begin{array}{r}5 \\
14\end{array}$ & $\begin{array}{l}13 \\
17\end{array}$ & $\begin{array}{l}408 \\
718\end{array}$ & $\frac{1}{7}$ & $\frac{1}{1}$ & $\begin{array}{l}0 \\
3\end{array}$ & $\begin{array}{l}0 \\
2\end{array}$ & $\begin{array}{l}0 \\
1\end{array}$ & $\begin{array}{l}0 \\
0\end{array}$ \\
\hline $6-20$ & 5 & 16 & 432 & 2 & 0 & 2 & 0 & 0 & 0 \\
\hline
\end{tabular}


TABLE 21.- NUMBER OF GREEN TRACER PARTICLES(1), PER 100 GRAMS OF BEDLOAD SAMPLE, COLLECTED AT ONE OR MORE CROSS-CHANNEL SAMPLING POSITIONS SPACED AT I-METER INTERVALS ACROSS THE CHANNEL WIDTH, EAST FORK RIVER, WYOMING, 1980--CONTINUED

SECTION $1396(3)$

\begin{tabular}{|c|c|c|c|c|c|c|c|c|c|}
\hline \multirow{3}{*}{ DATE } & & & & \multicolumn{6}{|c|}{ NUMBER OF GREEN TRACER PARTICLES } \\
\hline & \multicolumn{2}{|c|}{$\begin{array}{l}\text { SAMPLING } \\
\text { POSITION } \\
\text { FROM TO }\end{array}$} & \multirow{2}{*}{$\begin{array}{c}\text { DRY } \\
\text { MASS } \\
\text { OF } \\
\text { SAMPLE (2) } \\
\text { (G) } \\
\end{array}$} & \multirow[b]{2}{*}{ TOTAL } & \multicolumn{3}{|c|}{ BY SIZE CLASS } & \multicolumn{2}{|l|}{ (MM) } \\
\hline & $\begin{array}{r}\text { FROM } \\
(\mathrm{M})\end{array}$ & (M) & & & $\begin{array}{l}0.25 \\
\text { TO } \\
0.50\end{array}$ & $\begin{array}{l}0.50 \\
\text { TO } \\
1.00\end{array}$ & $\begin{array}{l}1.00 \\
\text { TO } \\
2.00\end{array}$ & $\begin{array}{l}2.00 \\
\text { TO } \\
4.00\end{array}$ & $\begin{array}{l}4.00 \\
\text { TO } \\
8.00\end{array}$ \\
\hline $6-21$ & 5 & 16 & 855 & 6 & 0 & 5 & 1 & 0 & 0 \\
\hline $6-23$ & 5 & 16 & 754 & 0 & 0 & 0 & 0 & 0 & 0 \\
\hline $6-25$ & 5 & 16 & 487 & 0 & 0 & 0 & 0 & 0 & 0 \\
\hline $6-27$ & 5 & 16 & 1773 & 0 & 0 & 0 & 0 & 0 & 0 \\
\hline
\end{tabular}


TABLE 21.- NUMBER OF GREEN TRACER PARTICLES(1), PER 100 GRAMS OF BEDLOAD SAMPLE, COLLECTED AT ONE OR MORE CROSS-CHANNEL SAMPLING

POSITIONS SPACED AT I-METER INTERVALS ACROSS THE CHANNEL

WIDTH, EAST FORK RIVER, WYOMING, 1980--CONTINUED

SECTION $1425(3)$

\begin{tabular}{|c|c|c|c|c|c|c|c|c|c|}
\hline \multirow{3}{*}{ DATE } & \multirow{3}{*}{\multicolumn{2}{|c|}{$\begin{array}{c}\text { SAMPLING } \\
\text { POSITION } \\
\text { FROM TO }\end{array}$}} & \multirow{3}{*}{$\begin{array}{c}\text { DRY } \\
\text { MASS } \\
\text { OF } \\
\text { SAMPLE (2) } \\
(G)\end{array}$} & \multicolumn{6}{|c|}{ NUMBER OF GREEN TRACER PARTICLES } \\
\hline & & & & \multirow[b]{2}{*}{ TOTAL } & \multicolumn{5}{|c|}{ BY SIZE CLASS (MM) } \\
\hline & & & & & $\begin{array}{l}0.25 \\
\text { TO } \\
0.50\end{array}$ & $\begin{array}{l}0.50 \\
\text { TO } \\
1.00\end{array}$ & $\begin{array}{l}1.00 \\
2.00\end{array}$ & $\begin{array}{l}2.00 \\
\text { TO } \\
4.00\end{array}$ & $\begin{array}{l}4.00 \\
\text { TO } \\
8.00\end{array}$ \\
\hline $6-01$ & 2 & 17 & 793 & 0 & 0 & 0 & 0 & 0 & 0 \\
\hline $6-03$ & 1 & 17 & $193+$ & 0 & 0 & 0 & 0 & 0 & 0 \\
\hline $6-05$ & 1 & 17 & 209 & 0 & 0 & 0 & 0 & 0 & 0 \\
\hline $6-07$ & 2 & 17 & 935 & 7 & 5 & 2 & 0 & 0 & 0 \\
\hline $6-09$ & 2 & 17 & 621 & 21 & 14 & 7 & 0 & 0 & 0 \\
\hline $6-10$ & $\begin{array}{r}2 \\
8 \\
11\end{array}$ & $\begin{array}{r}7 \\
10 \\
17\end{array}$ & $\begin{array}{l}119+ \\
833 \\
384\end{array}$ & $\begin{array}{r}8 \\
12 \\
5\end{array}$ & $\begin{array}{l}6 \\
8 \\
3\end{array}$ & $\begin{array}{l}2 \\
4 \\
2\end{array}$ & $\begin{array}{l}0 \\
0 \\
0\end{array}$ & $\begin{array}{l}0 \\
0 \\
0\end{array}$ & $\begin{array}{l}0 \\
0 \\
0\end{array}$ \\
\hline $6-11$ & $\begin{array}{r}2 \\
13\end{array}$ & $\begin{array}{l}12 \\
17\end{array}$ & $\begin{array}{r}257 \\
0\end{array}$ & $\begin{array}{r}30 \\
0\end{array}$ & $\begin{array}{r}16 \\
0\end{array}$ & $\begin{array}{r}13 \\
0\end{array}$ & $\begin{array}{l}1 \\
0\end{array}$ & $\begin{array}{l}0 \\
0\end{array}$ & $\begin{array}{l}0 \\
0\end{array}$ \\
\hline $6-12$ & $\frac{1}{8}$ & $\begin{array}{r}7 \\
17\end{array}$ & $\begin{array}{l}153 \\
28++\end{array}$ & $\begin{array}{l}17 \\
18\end{array}$ & $\begin{array}{l}5 \\
0\end{array}$ & $\begin{array}{r}7 \\
18\end{array}$ & $\begin{array}{l}3 \\
0\end{array}$ & $\begin{array}{l}2 \\
0\end{array}$ & $\begin{array}{l}0 \\
0\end{array}$ \\
\hline $6-13$ & $\begin{array}{r}4 \\
9 \\
14\end{array}$ & $\begin{array}{r}8 \\
13 \\
17\end{array}$ & $\begin{array}{c}48++ \\
69+ \\
8+++\end{array}$ & $\begin{array}{r}17 \\
9 \\
0\end{array}$ & $\begin{array}{r}10 \\
1 \\
0\end{array}$ & $\begin{array}{l}7 \\
8 \\
0\end{array}$ & $\begin{array}{l}0 \\
0 \\
0\end{array}$ & $\begin{array}{l}0 \\
0 \\
0\end{array}$ & $\begin{array}{l}0 \\
0 \\
0\end{array}$ \\
\hline $6-14$ & $\begin{array}{l}7 \\
8 \\
9\end{array}$ & $\begin{array}{r}7 \\
8 \\
16\end{array}$ & $\begin{array}{l}654 \\
438 \\
130+\end{array}$ & $\begin{array}{l}4 \\
2 \\
1\end{array}$ & $\begin{array}{l}2 \\
2 \\
0\end{array}$ & $\begin{array}{l}1 \\
0 \\
0\end{array}$ & $\begin{array}{l}1 \\
0 \\
0\end{array}$ & $\begin{array}{l}0 \\
0 \\
1\end{array}$ & $\begin{array}{l}0 \\
0 \\
0\end{array}$ \\
\hline $6-15$ & $\begin{array}{r}3 \\
8 \\
13\end{array}$ & $\begin{array}{r}7 \\
12 \\
16\end{array}$ & $\begin{array}{c}51++ \\
120+ \\
24++\end{array}$ & $\begin{array}{l}0 \\
9 \\
4\end{array}$ & $\begin{array}{l}0 \\
3 \\
0\end{array}$ & $\begin{array}{l}0 \\
6 \\
4\end{array}$ & $\begin{array}{l}0 \\
0 \\
0\end{array}$ & $\begin{array}{l}0 \\
0 \\
0\end{array}$ & $\begin{array}{l}0 \\
0 \\
0\end{array}$ \\
\hline $6-16$ & $\begin{array}{r}4 \\
8 \\
12 \\
15\end{array}$ & $\begin{array}{l}7 \\
11 \\
14 \\
16\end{array}$ & $\begin{array}{l}21++ \\
82+ \\
42++ \\
0+++\end{array}$ & $\begin{array}{l}5 \\
6 \\
2 \\
0\end{array}$ & $\begin{array}{l}0 \\
1 \\
0 \\
0\end{array}$ & $\begin{array}{l}5 \\
5 \\
2 \\
0\end{array}$ & $\begin{array}{l}0 \\
0 \\
0 \\
0\end{array}$ & $\begin{array}{l}0 \\
0 \\
0 \\
0\end{array}$ & $\begin{array}{l}0 \\
0 \\
0 \\
0\end{array}$ \\
\hline $6-17$ & $\begin{array}{l}4 \\
7 \\
9\end{array}$ & $\begin{array}{r}6 \\
8 \\
14\end{array}$ & $\begin{array}{l}41+++ \\
56++ \\
79++\end{array}$ & $\begin{array}{l}0 \\
0 \\
3\end{array}$ & $\begin{array}{l}0 \\
0 \\
1\end{array}$ & $\begin{array}{l}0 \\
0 \\
2\end{array}$ & $\begin{array}{l}0 \\
0 \\
0\end{array}$ & $\begin{array}{l}0 \\
0 \\
0\end{array}$ & $\begin{array}{l}0 \\
0 \\
0\end{array}$ \\
\hline $6-18$ & $\begin{array}{l}2 \\
8\end{array}$ & $\begin{array}{r}7 \\
16\end{array}$ & $\begin{array}{l}565 \\
242\end{array}$ & $\begin{array}{l}0 \\
8\end{array}$ & $\begin{array}{l}0 \\
3\end{array}$ & $\begin{array}{l}0 \\
5\end{array}$ & $\begin{array}{l}0 \\
0\end{array}$ & $\begin{array}{l}0 \\
0\end{array}$ & $\begin{array}{l}0 \\
0\end{array}$ \\
\hline
\end{tabular}


TABLE 21.- NUMBER OF GREEN TRACER PARTICLES(1), PER 100 GRAMS OF BEDLOAD SAMPLE, COLLECTED AT ONE OR MORE CRÓSS-CHANNEL SAMPLING POSITIONS SPACED AT I-METER INTERVALS ACROSS THE CHANNEL WIDTH, EAST FORK RIVER, WYOMING, 1980--CONTINUED

SECTION 1425(3)

\begin{tabular}{|c|c|c|c|c|c|c|c|c|c|}
\hline \multirow{3}{*}{ DATE } & & & \multirow{3}{*}{$\begin{array}{c}\text { DRY } \\
\text { MASS } \\
\text { OF } \\
\text { SAMPLE (2) } \\
\text { (G) }\end{array}$} & \multicolumn{6}{|c|}{ NUMBER OF GREEN TRACER PARTICLES } \\
\hline & \multicolumn{2}{|c|}{$\begin{array}{l}\text { SAMPLING } \\
\text { POSITION } \\
\text { FROM TO }\end{array}$} & & \multirow[b]{2}{*}{ TOTAL } & \multicolumn{5}{|c|}{ BY SIZE CLASS (MM) } \\
\hline & $\begin{array}{l}\text { FROM } \\
(\mathrm{M})\end{array}$ & (M) & & & $\begin{array}{l}0.25 \\
\text { TO } \\
0.50\end{array}$ & $\begin{array}{l}0.50 \\
\text { TO } \\
1.00\end{array}$ & $\begin{array}{l}1.00 \\
2.00\end{array}$ & $\begin{array}{l}2.00 \\
\text { TO } \\
4.00\end{array}$ & $\begin{array}{l}4.00 \\
\text { TO } \\
8.00\end{array}$ \\
\hline $6-19$ & $\begin{array}{r}3 \\
11\end{array}$ & $\begin{array}{l}10 \\
16\end{array}$ & $\begin{array}{l}55++ \\
130^{++}\end{array}$ & $\begin{array}{l}2 \\
6\end{array}$ & 2 & $\begin{array}{l}0 \\
5\end{array}$ & $\begin{array}{l}0 \\
0\end{array}$ & $\begin{array}{l}0 \\
0\end{array}$ & $\begin{array}{l}0 \\
0\end{array}$ \\
\hline $6-20$ & II & $\begin{array}{l}10 \\
16\end{array}$ & $\begin{array}{l}178 \\
190\end{array}$ & $10^{4}$ & $\frac{1}{2}$ & $\frac{1}{8}$ & $\begin{array}{l}2 \\
0\end{array}$ & $\begin{array}{l}0 \\
0\end{array}$ & $\begin{array}{l}0 \\
0\end{array}$ \\
\hline $6-21$ & 2 & 15 & $228+$ & 2 & 0 & 2 & 0 & 0 & 0 \\
\hline $6-23$ & 1 & 17 & 258 & 0 & 0 & 0 & 0 & 0 & 0 \\
\hline $6-25$ & 2 & 16 & 212 & 0 & 0 & 0 & 0 & 0 & 0 \\
\hline $6-27$ & 8 & 16 & 664 & 0 & 0 & 0 & 0 & 0 & 0 \\
\hline
\end{tabular}


TABLE 21. - NUMBER OF GREEN TRACER PARTICLES(1), PER 100 GRAMS OF BEDLOAD SAMPLE, COLLECTED AT ONE OR MORE CROSS-CHANNEL SAMPLING POSITIONS SPACED AT I-METER INTERVALS ACROSS THE CHANNEL WIDTH, EAST FORK RIVER, WYOMING, 1980--CONTINUED

SECTION 1481

\begin{tabular}{|c|c|c|c|c|c|c|c|c|c|}
\hline \multirow{3}{*}{ DATE } & & & & \multicolumn{6}{|c|}{ NUMBER OF GREEN TRACER PARTICLES } \\
\hline & \multirow{2}{*}{\multicolumn{2}{|c|}{$\begin{array}{l}\text { SAMPLING } \\
\text { POSITION } \\
\text { FROM TO }\end{array}$}} & \multirow{2}{*}{$\begin{array}{c}\text { DRY } \\
\text { MASS } \\
\text { OF } \\
\text { SAMPLE (2) } \\
\text { (G) }\end{array}$} & \multirow[b]{2}{*}{ TOTAL } & \multicolumn{5}{|c|}{ BY SIZE CLASS (MM) } \\
\hline & & & & & $\begin{array}{l}0.25 \\
\text { TO } \\
0.50\end{array}$ & $\begin{array}{l}0.50 \\
\text { TO } \\
1.00\end{array}$ & $\begin{array}{l}1.00 \\
2.00\end{array}$ & $\begin{array}{l}2.00 \\
\text { TO } \\
4.00\end{array}$ & $\begin{array}{l}4.00 \\
\text { TO } \\
8.00\end{array}$ \\
\hline $6-01$ & 4 & 16 & $187+$ & 0 & 0 & 0 & 0 & 0 & 0 \\
\hline $6-03$ & 4 & 17 & 235 & 0 & 0 & 0 & 0 & 0 & 0 \\
\hline $6-05$ & 5 & 16 & 197 & 0 & 0 & 0 & 0 & 0 & 0 \\
\hline $6-07$ & 5 & 15 & 389 & 3 & 3 & 0 & 0 & 0 & 0 \\
\hline $6-09$ & $\begin{array}{r}4 \\
10 \\
22\end{array}$ & $\begin{array}{r}9 \\
14 \\
26\end{array}$ & $\begin{array}{r}858 \\
1290 \\
385\end{array}$ & $\begin{array}{r}5 \\
23 \\
72\end{array}$ & $\begin{array}{l}2 \\
11 \\
60\end{array}$ & $\begin{array}{r}3 \\
12 \\
12\end{array}$ & $\begin{array}{l}0 \\
0 \\
0\end{array}$ & $\begin{array}{l}0 \\
0 \\
0\end{array}$ & $\begin{array}{l}0 \\
0 \\
0\end{array}$ \\
\hline $6-10$ & $\begin{array}{r}4 \\
9 \\
11 \\
12 \\
22 \\
25\end{array}$ & $\begin{array}{r}8 \\
10 \\
11 \\
15 \\
24 \\
25\end{array}$ & $\begin{array}{r}769 \\
765 \\
1148 \\
102+ \\
1420 \\
1773\end{array}$ & $\begin{array}{l}13 \\
31 \\
58 \\
18 \\
37 \\
97\end{array}$ & $\begin{array}{l}7 \\
25 \\
44 \\
7 \\
20 \\
67\end{array}$ & $\begin{array}{r}5 \\
5 \\
14 \\
10 \\
15 \\
29\end{array}$ & $\begin{array}{l}0 \\
1 \\
0 \\
0 \\
2 \\
1\end{array}$ & $\begin{array}{l}0 \\
0 \\
0 \\
1 \\
0 \\
0\end{array}$ & $\begin{array}{l}1 \\
0 \\
0 \\
0 \\
0 \\
0\end{array}$ \\
\hline $6-11$ & $\begin{array}{r}5 \\
9 \\
10 \\
11 \\
22 \\
24 \\
25\end{array}$ & $\begin{array}{r}8 \\
9 \\
10 \\
15 \\
23 \\
24 \\
26\end{array}$ & $\begin{array}{l}504 \\
368 \\
459 \\
164 \\
732 \\
623 \\
255\end{array}$ & $\begin{array}{r}3 \\
17 \\
15 \\
31 \\
54 \\
51 \\
61\end{array}$ & $\begin{array}{r}1 \\
10 \\
8 \\
8 \\
11 \\
8 \\
20\end{array}$ & $\begin{array}{r}0 \\
6 \\
4 \\
16 \\
33 \\
30 \\
33\end{array}$ & $\begin{array}{r}1 \\
0 \\
2 \\
6 \\
9 \\
12 \\
6\end{array}$ & $\begin{array}{l}1 \\
1 \\
1 \\
1 \\
1 \\
1 \\
2\end{array}$ & $\begin{array}{l}0 \\
0 \\
0 \\
0 \\
0 \\
0 \\
0\end{array}$ \\
\hline $6-12$ & $\begin{array}{l}4 \\
7 \\
8 \\
9 \\
10 \\
11 \\
12 \\
13 \\
14 \\
15 \\
16 \\
22 \\
24 \\
25 \\
26\end{array}$ & $\begin{array}{r}6 \\
7 \\
8 \\
9 \\
10 \\
11 \\
12 \\
13 \\
14 \\
15 \\
16 \\
23 \\
24 \\
25 \\
26\end{array}$ & $\begin{array}{c}1+++ \\
9+++ \\
7+++ \\
10+++ \\
73+ \\
83+ \\
16+++ \\
218 \\
216+ \\
0+++ \\
47++ \\
8+++ \\
14++ \\
308 \\
13+++\end{array}$ & $\begin{array}{r}0 \\
0 \\
0 \\
0 \\
1 \\
8 \\
12 \\
25 \\
34 \\
0 \\
8 \\
0 \\
28 \\
17 \\
15\end{array}$ & $\begin{array}{r}0 \\
0 \\
0 \\
0 \\
0 \\
6 \\
12 \\
9 \\
13 \\
0 \\
6 \\
0 \\
0 \\
1 \\
0\end{array}$ & $\begin{array}{r}0 \\
0 \\
0 \\
0 \\
0 \\
1 \\
0 \\
11 \\
18 \\
0 \\
2 \\
0 \\
7 \\
13 \\
15\end{array}$ & $\begin{array}{l}0 \\
0 \\
0 \\
0 \\
0 \\
1 \\
0 \\
4 \\
3 \\
0 \\
0 \\
0 \\
21 \\
2 \\
0\end{array}$ & $\begin{array}{l}0 \\
0 \\
0 \\
0 \\
1 \\
0 \\
0 \\
1 \\
0 \\
0 \\
0 \\
0 \\
0 \\
1 \\
0\end{array}$ & $\begin{array}{l}0 \\
0 \\
0 \\
0 \\
0 \\
0 \\
0 \\
0 \\
0 \\
0 \\
0 \\
0 \\
0 \\
0 \\
0\end{array}$ \\
\hline
\end{tabular}


TABLE 21.- NUMBER OF GREEN TRACER PARTICLES(1), PER 100 GRAMS OF BEDLOAD SAMPLE, COLLECTED AT ONE OR MORE CROSS-CHANNEL SAMPLING POSITIONS SPACED AT 1-METER INTERVALS ACROSS THE CHANNEL WIDTH, EAST FORK RIVER, WYOMING, 1980--CONTINUED

SECTICN 1481

\begin{tabular}{|c|c|c|c|c|c|c|c|c|c|}
\hline \multirow{3}{*}{ DATE } & & & & \multicolumn{6}{|c|}{ NUMBER OF GREEN TRACER PARTICLES } \\
\hline & \multicolumn{2}{|c|}{$\begin{array}{l}\text { SAMPLING } \\
\text { POSITION } \\
\text { FROM TO }\end{array}$} & \multirow{2}{*}{$\begin{array}{c}\text { DRY } \\
\text { MASS } \\
\text { OF } \\
\text { SAMPLE (2) } \\
\text { (G) }\end{array}$} & \multirow[b]{2}{*}{ TOTAL } & \multicolumn{5}{|c|}{ BY SIZE CLASS (MM) } \\
\hline & FROM & (M) & & & $\begin{array}{l}0.25 \\
\text { TO } \\
0.50\end{array}$ & $\begin{array}{l}0.50 \\
\text { TO } \\
1.00\end{array}$ & $\begin{array}{l}1.00 \\
\text { TO } \\
2.00\end{array}$ & $\begin{array}{l}2.00 \\
\text { TO } \\
4.00\end{array}$ & $\begin{array}{l}4.00 \\
\text { TO } \\
8.00\end{array}$ \\
\hline $6-13$ & $\begin{array}{r}5 \\
6 \\
7 \\
8 \\
9 \\
10 \\
11 \\
12 \\
13 \\
22 \\
23 \\
24 \\
25 \\
26\end{array}$ & $\begin{array}{r}5 \\
6 \\
7 \\
8 \\
9 \\
10 \\
11 \\
12 \\
15 \\
22 \\
23 \\
24 \\
25 \\
26\end{array}$ & $\begin{array}{c}0+++ \\
7+++ \\
6+++ \\
17++ \\
80+ \\
240 \\
82+ \\
198 \\
133+ \\
340 \\
136 \\
146 \\
642 \\
100+\end{array}$ & $\begin{array}{l}0 \\
0 \\
0 \\
0 \\
0 \\
4 \\
1 \\
9 \\
8 \\
10 \\
17 \\
11 \\
16 \\
13\end{array}$ & $\begin{array}{l}0 \\
0 \\
0 \\
0 \\
0 \\
1 \\
0 \\
5 \\
0 \\
4 \\
1 \\
0 \\
0 \\
2\end{array}$ & $\begin{array}{r}0 \\
0 \\
0 \\
0 \\
0 \\
2 \\
1 \\
3 \\
6 \\
3 \\
11 \\
5 \\
15 \\
10\end{array}$ & $\begin{array}{l}0 \\
0 \\
0 \\
0 \\
0 \\
1 \\
0 \\
1 \\
2 \\
3 \\
2 \\
5 \\
1 \\
1\end{array}$ & $\begin{array}{l}0 \\
0 \\
0 \\
0 \\
0 \\
0 \\
0 \\
0 \\
0 \\
0 \\
3 \\
1 \\
0 \\
0\end{array}$ & $\begin{array}{l}0 \\
0 \\
0 \\
0 \\
0 \\
0 \\
0 \\
0 \\
0 \\
0 \\
0 \\
0 \\
0 \\
0\end{array}$ \\
\hline $6-14$ & $\begin{array}{r}7 \\
8 \\
9 \\
10 \\
11 \\
12 \\
13 \\
15 \\
23 \\
24 \\
25 \\
26 \\
27\end{array}$ & $\begin{array}{r}7 \\
8 \\
9 \\
10 \\
11 \\
12 \\
13 \\
15 \\
23 \\
24 \\
25 \\
26 \\
27\end{array}$ & $\begin{array}{c}37++ \\
34++ \\
630 \\
72+ \\
206 \\
69+ \\
306 \\
76+ \\
903 \\
138 \\
1203 \\
51++ \\
39++\end{array}$ & $\begin{array}{r}0 \\
0 \\
0 \\
4 \\
2 \\
7 \\
23 \\
9 \\
12 \\
10 \\
15 \\
27 \\
8\end{array}$ & $\begin{array}{r}0 \\
0 \\
0 \\
3 \\
1 \\
1 \\
19 \\
19 \\
1 \\
0 \\
6 \\
2 \\
12 \\
5\end{array}$ & $\begin{array}{r}0 \\
0 \\
0 \\
1 \\
1 \\
3 \\
4 \\
2 \\
11 \\
4 \\
6 \\
13 \\
3\end{array}$ & $\begin{array}{l}0 \\
0 \\
0 \\
0 \\
0 \\
3 \\
0 \\
6 \\
1 \\
0 \\
2 \\
2 \\
0\end{array}$ & $\begin{array}{l}0 \\
0 \\
0 \\
0 \\
0 \\
0 \\
0 \\
0 \\
0 \\
0 \\
4 \\
0 \\
0\end{array}$ & $\begin{array}{l}0 \\
0 \\
0 \\
0 \\
0 \\
0 \\
0 \\
0 \\
0 \\
0 \\
1 \\
0 \\
0\end{array}$ \\
\hline $6-15$ & $\begin{array}{r}4 \\
7 \\
8 \\
9 \\
10 \\
11 \\
12 \\
22 \\
24\end{array}$ & $\begin{array}{r}6 \\
7 \\
8 \\
9 \\
10 \\
11 \\
14 \\
23 \\
27\end{array}$ & $\begin{array}{c}8+++ \\
60+ \\
90+ \\
412 \\
549 \\
473 \\
856 \\
457 \\
597\end{array}$ & $\begin{array}{l}0 \\
0 \\
1 \\
0 \\
1 \\
0 \\
5 \\
1 \\
4\end{array}$ & $\begin{array}{l}0 \\
0 \\
0 \\
0 \\
0 \\
0 \\
0 \\
0 \\
2\end{array}$ & $\begin{array}{l}0 \\
0 \\
0 \\
0 \\
1 \\
0 \\
3 \\
1 \\
2\end{array}$ & $\begin{array}{l}0 \\
0 \\
0 \\
0 \\
0 \\
0 \\
2 \\
0 \\
0\end{array}$ & $\begin{array}{l}0 \\
0 \\
1 \\
0 \\
0 \\
0 \\
0 \\
0 \\
0\end{array}$ & $\begin{array}{l}0 \\
0 \\
0 \\
0 \\
0 \\
0 \\
0 \\
0 \\
0\end{array}$ \\
\hline
\end{tabular}


TABLE 21.- NUMBER OF GREEN TRACER PARTICLES(1), PER 100 GRAMS OF BEDLOAD SAMPLE, COLLECTED AT ONE OR MORE CROSS-CHANNEL SAMPLING POSITIONS SPACED AT I-METER INTERVALS ACROSS THE CHANNEL WIDTH, EAST FORK RIVER, WYOMING, 1980--CONTINUED

\section{SECTION 1481}

\begin{tabular}{|c|c|c|c|c|c|c|c|c|c|}
\hline \multirow{3}{*}{ DATE } & & & & \multicolumn{6}{|c|}{ NUMBER OF GREEN TRACER PARTICLES } \\
\hline & \multicolumn{2}{|c|}{$\begin{array}{l}\text { SAMPLING } \\
\text { POSITION } \\
\text { FROM TO }\end{array}$} & \multirow{2}{*}{$\begin{array}{c}\text { DRY } \\
\text { MASS } \\
\text { OF } \\
\text { SAMPLE(2) } \\
(G)\end{array}$} & & \multicolumn{5}{|c|}{ BY SIZE CLASS } \\
\hline & FROM & TO & & TOTAL & $\begin{array}{l}0.25 \\
\text { TO } \\
0.50\end{array}$ & $\begin{array}{l}0.50 \\
\text { TO } \\
1.00\end{array}$ & $\begin{array}{l}1.00 \\
2.00\end{array}$ & $\begin{array}{l}2.00 \\
\text { To } \\
4.00\end{array}$ & $\begin{array}{l}4.00 \\
\text { TO } \\
8.00\end{array}$ \\
\hline $6-16$ & $\begin{array}{r}5 \\
8 \\
10 \\
12 \\
22\end{array}$ & $\begin{array}{r}7 \\
9 \\
11 \\
15 \\
26\end{array}$ & $\begin{array}{c}4+++ \\
34++ \\
726 \\
59++ \\
184\end{array}$ & $\begin{array}{l}0 \\
0 \\
6 \\
0 \\
0\end{array}$ & $\begin{array}{l}0 \\
0 \\
5 \\
0 \\
0\end{array}$ & $\begin{array}{l}0 \\
0 \\
1 \\
0 \\
0\end{array}$ & $\begin{array}{l}0 \\
0 \\
0 \\
0 \\
0\end{array}$ & $\begin{array}{l}0 \\
0 \\
0 \\
0 \\
0\end{array}$ & $\begin{array}{l}0 \\
0 \\
0 \\
0 \\
0\end{array}$ \\
\hline $6-17$ & $\begin{array}{r}5 \\
7 \\
8 \\
9 \\
10 \\
12 \\
14 \\
22 \\
25\end{array}$ & $\begin{array}{r}6 \\
7 \\
8 \\
9 \\
11 \\
13 \\
16 \\
24 \\
26\end{array}$ & $\begin{array}{r}5+++ \\
6+++ \\
11+++ \\
100+ \\
495 \\
20++ \\
34++ \\
349 \\
11+++\end{array}$ & $\begin{array}{l}0 \\
0 \\
0 \\
1 \\
1 \\
5 \\
3 \\
2 \\
0\end{array}$ & $\begin{array}{l}0 \\
0 \\
0 \\
0 \\
0 \\
0 \\
0 \\
0 \\
0\end{array}$ & $\begin{array}{l}0 \\
0 \\
0 \\
1 \\
1 \\
5 \\
0 \\
1 \\
0\end{array}$ & $\begin{array}{l}0 \\
0 \\
0 \\
0 \\
0 \\
0 \\
0 \\
1 \\
0\end{array}$ & $\begin{array}{l}0 \\
0 \\
0 \\
0 \\
0 \\
0 \\
3 \\
0 \\
0\end{array}$ & $\begin{array}{l}0 \\
0 \\
0 \\
0 \\
0 \\
0 \\
0 \\
0 \\
0\end{array}$ \\
\hline $6-18$ & $\begin{array}{r}5 \\
6 \\
7 \\
8 \\
9 \\
10 \\
11 \\
12 \\
22 \\
24 \\
25\end{array}$ & $\begin{array}{r}5 \\
6 \\
7 \\
8 \\
9 \\
10 \\
11 \\
16 \\
23 \\
24 \\
25\end{array}$ & $\begin{array}{c}11+++ \\
39++ \\
31++ \\
415 \\
458 \\
405 \\
524 \\
136 \\
329 \\
945 \\
935\end{array}$ & $\begin{array}{r}0 \\
0 \\
0 \\
2 \\
1 \\
6 \\
5 \\
5 \\
9 \\
2 \\
10\end{array}$ & $\begin{array}{l}0 \\
0 \\
0 \\
1 \\
0 \\
4 \\
2 \\
3 \\
8 \\
0 \\
4\end{array}$ & $\begin{array}{l}0 \\
0 \\
0 \\
1 \\
1 \\
2 \\
3 \\
1 \\
1 \\
1 \\
6\end{array}$ & $\begin{array}{l}0 \\
0 \\
0 \\
0 \\
0 \\
0 \\
0 \\
1 \\
0 \\
1 \\
0\end{array}$ & $\begin{array}{l}0 \\
0 \\
0 \\
0 \\
0 \\
0 \\
0 \\
0 \\
0 \\
0 \\
0\end{array}$ & $\begin{array}{l}0 \\
0 \\
0 \\
0 \\
0 \\
0 \\
0 \\
0 \\
0 \\
0 \\
0\end{array}$ \\
\hline $6-19$ & $\begin{array}{r}5 \\
8 \\
9 \\
12 \\
22\end{array}$ & $\begin{array}{r}7 \\
8 \\
11 \\
15 \\
26\end{array}$ & $\begin{array}{c}159^{+++} \\
1405 \\
95+ \\
827\end{array}$ & $\begin{array}{r}0 \\
0 \\
2 \\
6 \\
11\end{array}$ & $\begin{array}{l}0 \\
0 \\
0 \\
3 \\
2\end{array}$ & $\begin{array}{l}0 \\
0 \\
2 \\
3 \\
8\end{array}$ & $\begin{array}{l}0 \\
0 \\
0 \\
0 \\
1\end{array}$ & $\begin{array}{l}0 \\
0 \\
0 \\
0 \\
0\end{array}$ & $\begin{array}{l}0 \\
0 \\
0 \\
0 \\
0\end{array}$ \\
\hline $6-20$ & $\begin{array}{r}5 \\
8 \\
12 \\
22\end{array}$ & $\begin{array}{r}7 \\
11 \\
16 \\
26\end{array}$ & $\begin{array}{l}21++ \\
849 \\
276 \\
1600\end{array}$ & $\begin{array}{r}0 \\
2 \\
10 \\
27\end{array}$ & $\begin{array}{l}0 \\
0 \\
2 \\
5\end{array}$ & $\begin{array}{r}0 \\
2 \\
8 \\
16\end{array}$ & $\begin{array}{l}0 \\
0 \\
0 \\
5\end{array}$ & $\begin{array}{l}0 \\
0 \\
0 \\
1\end{array}$ & $\begin{array}{l}0 \\
0 \\
0 \\
0\end{array}$ \\
\hline $6-21$ & $\begin{array}{r}5 \\
9 \\
12 \\
22\end{array}$ & $\begin{array}{l}8 \\
11 \\
16 \\
26\end{array}$ & $\begin{array}{c}34++ \\
546 \\
652 \\
1029\end{array}$ & $\begin{array}{l}0 \\
0 \\
5 \\
0\end{array}$ & $\begin{array}{l}0 \\
0 \\
2 \\
0\end{array}$ & $\begin{array}{l}0 \\
0 \\
3 \\
0\end{array}$ & $\begin{array}{l}0 \\
0 \\
0 \\
0\end{array}$ & $\begin{array}{l}0 \\
0 \\
0 \\
0\end{array}$ & $\begin{array}{l}0 \\
0 \\
0 \\
0\end{array}$ \\
\hline
\end{tabular}


TABLE 21.- NUMBER OF GREEN TRACER PARTICLES(1), PER 100 GRAMS OF BEDLOAD SAMPLE, COLLECTED AT ONE OR MORE CROSS-CHANNEL SAMPLING POSITIONS SPACED AT 1-METER INTERVALS ACROSS THE CHANNEL WIDTH, EAST FORK RIVER, WYOMING, 1980--CONTINUED

SECTION 1481

\begin{tabular}{|c|c|c|c|c|c|c|c|c|c|}
\hline \multirow{3}{*}{ DATE } & & & & \multicolumn{6}{|c|}{ NUMBER OF GREEN TRACER PARTICLES } \\
\hline & \multicolumn{2}{|c|}{$\begin{array}{l}\text { SAMPLING } \\
\text { POSITION } \\
\text { FROM TO }\end{array}$} & \multirow{2}{*}{$\begin{array}{c}\text { DRY } \\
\text { MASS } \\
\text { OF } \\
\text { SAMPLE (2) } \\
\text { (G) }\end{array}$} & \multirow[b]{2}{*}{ TOTAL } & \multicolumn{3}{|c|}{ BY SIZE CLASS } & \multicolumn{2}{|l|}{$(\mathrm{MM})$} \\
\hline & $\begin{array}{l}\text { FROM } \\
\qquad(M)\end{array}$ & $\begin{array}{l}\text { TO } \\
\text { (M) }\end{array}$ & & & $\begin{array}{l}0.25 \\
\text { TO } \\
0.50\end{array}$ & $\begin{array}{l}0.50 \\
\text { TO } \\
1.00\end{array}$ & $\begin{array}{l}1.00 \\
\text { TO } \\
2.00\end{array}$ & $\begin{array}{l}2.00 \\
\text { TOO } \\
4.00\end{array}$ & $\begin{array}{l}4.00 \\
\text { TO } \\
8.00\end{array}$ \\
\hline $6-23$ & $\begin{array}{r}5 \\
9 \\
22\end{array}$ & $\begin{array}{r}8 \\
16 \\
26\end{array}$ & $\begin{array}{l}111+++ \\
765 \\
465\end{array}$ & $\begin{array}{l}0 \\
0 \\
3\end{array}$ & $\begin{array}{l}0 \\
0 \\
0\end{array}$ & $\begin{array}{l}0 \\
0 \\
3\end{array}$ & $\begin{array}{l}0 \\
0 \\
0\end{array}$ & $\begin{array}{l}0 \\
0 \\
0\end{array}$ & $\begin{array}{l}0 \\
0 \\
0\end{array}$ \\
\hline $6-25$ & $\begin{array}{r}5 \\
22\end{array}$ & $\begin{array}{l}16 \\
26\end{array}$ & $\begin{array}{r}1216 \\
347\end{array}$ & $\begin{array}{l}0 \\
1\end{array}$ & $\begin{array}{l}0 \\
0\end{array}$ & $\begin{array}{l}0 \\
1\end{array}$ & $\begin{array}{l}0 \\
0\end{array}$ & $\begin{array}{l}0 \\
0\end{array}$ & $\begin{array}{l}0 \\
0\end{array}$ \\
\hline $6-27$ & 22 & $\begin{array}{l}16 \\
26\end{array}$ & $\begin{array}{l}1479 \\
1109\end{array}$ & $\begin{array}{l}3 \\
3\end{array}$ & $\begin{array}{l}0 \\
2\end{array}$ & $\begin{array}{l}3 \\
1\end{array}$ & $\begin{array}{l}0 \\
0\end{array}$ & $\begin{array}{l}0 \\
0\end{array}$ & $\begin{array}{l}0 \\
0\end{array}$ \\
\hline
\end{tabular}


TABLE 21.- NUMBER OF GREEN TRACER PARTICLES(1), PER 100 GRAMS OF BEDLOAD SAMPLE, COLLECTED AT ONE OR MORE CRÓSS-CHANNEL SAMPLING POSITIONS SPACED AT 1-METER INTERVALS ACROSS THE CHANNEL WIDTH, EAST FORK RIVER, WYOMING, 1980--CONTINUED

SECTION 1533

\begin{tabular}{|c|c|c|c|c|c|c|c|c|c|}
\hline \multirow{3}{*}{ DATE } & & & \multirow{3}{*}{$\begin{array}{c}\text { DRY } \\
\text { MASS } \\
\text { OF } \\
\text { SAMPLE (2) } \\
\text { (G) }\end{array}$} & \multicolumn{6}{|c|}{ NUMBER OF GREEN TRACER PARTICLES } \\
\hline & \multirow{2}{*}{\multicolumn{2}{|c|}{$\begin{array}{l}\text { SAMPLING } \\
\text { POSITION } \\
\text { FROM TO }\end{array}$}} & & \multirow[b]{2}{*}{ TOTAL } & \multicolumn{5}{|c|}{ BY SIZE CLASS (MM) } \\
\hline & & & & & $\begin{array}{l}0.25 \\
\text { TO } \\
0.50\end{array}$ & $\begin{array}{l}0.50 \\
\text { To } \\
1.00\end{array}$ & $\begin{array}{l}1.00 \\
2.00\end{array}$ & $\begin{array}{l}2.00 \\
40 \\
4.00\end{array}$ & $\begin{array}{l}4.00 \\
\text { TO } \\
8.00\end{array}$ \\
\hline $6-01$ & 13 & $\begin{array}{l}10 \\
15\end{array}$ & $\begin{array}{l}323 \\
129\end{array}$ & $\begin{array}{l}0 \\
0\end{array}$ & $\begin{array}{l}0 \\
0\end{array}$ & $\begin{array}{l}0 \\
0\end{array}$ & $\begin{array}{l}0 \\
0\end{array}$ & $\begin{array}{l}0 \\
0\end{array}$ & $\begin{array}{l}0 \\
0\end{array}$ \\
\hline $6-03$ & $\begin{array}{r}2 \\
11\end{array}$ & $\begin{array}{l}10 \\
15\end{array}$ & $\begin{array}{l}86+ \\
82++\end{array}$ & $\begin{array}{l}0 \\
0\end{array}$ & $\begin{array}{l}0 \\
0\end{array}$ & $\begin{array}{l}0 \\
0\end{array}$ & $\begin{array}{l}0 \\
0\end{array}$ & $\begin{array}{l}0 \\
0\end{array}$ & $\begin{array}{l}0 \\
0\end{array}$ \\
\hline $6-05$ & $\begin{array}{r}2 \\
10\end{array}$ & $\begin{array}{r}9 \\
15\end{array}$ & $\begin{array}{l}245 \\
275\end{array}$ & $\begin{array}{l}8 \\
6\end{array}$ & $\begin{array}{l}8 \\
5\end{array}$ & $\begin{array}{l}0 \\
1\end{array}$ & $\begin{array}{l}0 \\
0\end{array}$ & $\begin{array}{l}0 \\
0\end{array}$ & $\begin{array}{l}0 \\
0\end{array}$ \\
\hline $6-07$ & $\begin{array}{r}2 \\
10\end{array}$ & $\begin{array}{r}9 \\
14\end{array}$ & $\begin{array}{c}273 \\
68+\end{array}$ & $\begin{array}{r}5 \\
19\end{array}$ & $\begin{array}{l}0 \\
6\end{array}$ & $\begin{array}{r}5 \\
13\end{array}$ & $\begin{array}{l}0 \\
0\end{array}$ & $\begin{array}{l}0 \\
0\end{array}$ & $\begin{array}{l}0 \\
0\end{array}$ \\
\hline $6-09$ & $\begin{array}{r}1 \\
11 \\
11\end{array}$ & $\begin{array}{r}5 \\
10 \\
15\end{array}$ & $\begin{array}{l}40++ \\
673 \\
265\end{array}$ & $\begin{array}{r}0 \\
45 \\
357\end{array}$ & $\begin{array}{r}0 \\
42 \\
294\end{array}$ & $\begin{array}{r}0 \\
3 \\
62\end{array}$ & $\begin{array}{l}0 \\
0 \\
1\end{array}$ & $\begin{array}{l}0 \\
0 \\
0\end{array}$ & $\begin{array}{l}0 \\
0 \\
0\end{array}$ \\
\hline $6-10$ & $\begin{array}{r}\frac{1}{7} \\
11\end{array}$ & $\begin{array}{r}6 \\
10 \\
14\end{array}$ & $\begin{array}{r}1530 \\
2573 \\
564\end{array}$ & $\begin{array}{r}7 \\
17 \\
175\end{array}$ & $\begin{array}{r}0 \\
3 \\
102\end{array}$ & $\begin{array}{r}3 \\
6 \\
68\end{array}$ & $\begin{array}{l}3 \\
7 \\
5\end{array}$ & $\begin{array}{l}1 \\
1 \\
0\end{array}$ & $\begin{array}{l}0 \\
0 \\
0\end{array}$ \\
\hline $6-11$ & $\begin{array}{r}2 \\
6 \\
7 \\
8 \\
9 \\
10 \\
12\end{array}$ & $\begin{array}{r}5 \\
6 \\
7 \\
8 \\
9 \\
11 \\
14\end{array}$ & $\begin{array}{c}34++ \\
588 \\
85+ \\
419 \\
88++ \\
55++ \\
73++\end{array}$ & $\begin{array}{r}0 \\
2 \\
3 \\
29 \\
29 \\
22 \\
3\end{array}$ & $\begin{array}{r}0 \\
1 \\
1 \\
11 \\
11 \\
9 \\
1\end{array}$ & $\begin{array}{r}0 \\
1 \\
2 \\
16 \\
17 \\
13 \\
2\end{array}$ & $\begin{array}{l}0 \\
0 \\
0 \\
2 \\
1 \\
0 \\
0\end{array}$ & $\begin{array}{l}0 \\
0 \\
0 \\
0 \\
0 \\
0 \\
0\end{array}$ & $\begin{array}{l}0 \\
0 \\
0 \\
0 \\
0 \\
0 \\
0\end{array}$ \\
\hline $6-12$ & $\begin{array}{r}3 \\
6 \\
8 \\
9 \\
10 \\
11 \\
12 \\
13 \\
14 \\
15\end{array}$ & $\begin{array}{r}5 \\
7 \\
8 \\
9 \\
10 \\
11 \\
12 \\
13 \\
14 \\
15\end{array}$ & $\begin{array}{c}100+ \\
406 \\
198 \\
67+ \\
14+++ \\
7+++ \\
18+++ \\
493 \\
6+++ \\
5+++\end{array}$ & $\begin{array}{r}1 \\
1 \\
0 \\
3 \\
7 \\
0 \\
11 \\
17 \\
15 \\
0\end{array}$ & $\begin{array}{l}1 \\
1 \\
0 \\
0 \\
0 \\
0 \\
5 \\
9 \\
0 \\
0\end{array}$ & $\begin{array}{r}0 \\
0 \\
0 \\
3 \\
7 \\
0 \\
0 \\
5 \\
15 \\
0\end{array}$ & $\begin{array}{l}0 \\
0 \\
0 \\
0 \\
0 \\
0 \\
6 \\
3 \\
0 \\
0\end{array}$ & $\begin{array}{l}0 \\
0 \\
0 \\
0 \\
0 \\
0 \\
0 \\
0 \\
0 \\
0\end{array}$ & $\begin{array}{l}0 \\
0 \\
0 \\
0 \\
0 \\
0 \\
0 \\
0 \\
0 \\
0\end{array}$ \\
\hline
\end{tabular}


TABLE 21.- NUMBER OF GREEN TRACER PARTICLES(1), PER 100 GRAMS OF BEDLOAD SAMPLE, COLLECTED AT ONE OR MORE CROSS-CHANNEL SAMPLING POSITIONS SPACED AT 1-METER INTERVALS ACROSS THE CHANNEL WIDTH, EAST FORK RIVER, WYOMING, 1980--CONTINUED

SECTION 1533

\begin{tabular}{|c|c|c|c|c|c|c|c|c|c|}
\hline \multirow{3}{*}{ DATE } & \multirow{3}{*}{\multicolumn{2}{|c|}{$\begin{array}{l}\text { SAMPLING } \\
\text { POSITION } \\
\text { FROM TO }\end{array}$}} & \multirow{3}{*}{$\begin{array}{c}\text { DRY } \\
\text { MASS } \\
\text { OF } \\
\text { SAMPLE (2) } \\
(G)\end{array}$} & \multicolumn{6}{|c|}{ NUMBER OF GREEN TRACER PARTICLES } \\
\hline & & & & \multirow[b]{2}{*}{ TOTAL } & \multicolumn{4}{|c|}{ BY SIZE CLASS (MM) } & \multirow[b]{2}{*}{$\begin{array}{l}4.00 \\
8.00\end{array}$} \\
\hline & & (M) & & & $\begin{array}{l}0.25 \\
\text { TO } \\
0.50\end{array}$ & $\begin{array}{l}0.50 \\
\text { TO } \\
1.00\end{array}$ & $\begin{array}{l}1.00 \\
2.00\end{array}$ & $\begin{array}{l}2.00 \\
4.00\end{array}$ & \\
\hline $6-13$ & $\begin{array}{r}2 \\
5 \\
6 \\
7 \\
8 \\
9 \\
10 \\
11 \\
12 \\
13\end{array}$ & $\begin{array}{r}4 \\
5 \\
6 \\
7 \\
8 \\
9 \\
10 \\
11 \\
12 \\
15\end{array}$ & $\begin{array}{c}11+++ \\
6+++ \\
130 \\
262 \\
503 \\
734 \\
881 \\
59++ \\
924 \\
49++\end{array}$ & $\begin{array}{l}0 \\
0 \\
0 \\
0 \\
1 \\
1 \\
0 \\
0 \\
2 \\
4\end{array}$ & $\begin{array}{l}0 \\
0 \\
0 \\
0 \\
0 \\
0 \\
0 \\
0 \\
0 \\
0\end{array}$ & $\begin{array}{l}0 \\
0 \\
0 \\
0 \\
0 \\
0 \\
0 \\
0 \\
2 \\
4\end{array}$ & $\begin{array}{l}0 \\
0 \\
0 \\
0 \\
1 \\
1 \\
0 \\
0 \\
0 \\
0\end{array}$ & $\begin{array}{l}0 \\
0 \\
0 \\
0 \\
0 \\
0 \\
0 \\
0 \\
0 \\
0\end{array}$ & $\begin{array}{l}0 \\
0 \\
0 \\
0 \\
0 \\
0 \\
0 \\
0 \\
0 \\
0\end{array}$ \\
\hline $6-14$ & $\begin{array}{r}6 \\
7 \\
8 \\
9 \\
10 \\
11 \\
12 \\
13 \\
14 \\
15\end{array}$ & $\begin{array}{r}6 \\
7 \\
8 \\
9 \\
10 \\
11 \\
12 \\
13 \\
14 \\
15\end{array}$ & $\begin{array}{c}862 \\
994 \\
403 \\
712 \\
459 \\
29++ \\
34+++ \\
37++ \\
15+++ \\
8+++\end{array}$ & $\begin{array}{l}0 \\
0 \\
1 \\
1 \\
0 \\
3 \\
0 \\
0 \\
0 \\
0\end{array}$ & $\begin{array}{l}0 \\
0 \\
0 \\
0 \\
0 \\
0 \\
0 \\
0 \\
0 \\
0\end{array}$ & $\begin{array}{l}0 \\
0 \\
1 \\
1 \\
0 \\
3 \\
0 \\
0 \\
0 \\
0\end{array}$ & $\begin{array}{l}0 \\
0 \\
0 \\
0 \\
0 \\
0 \\
0 \\
0 \\
0 \\
0\end{array}$ & $\begin{array}{l}0 \\
0 \\
0 \\
0 \\
0 \\
0 \\
0 \\
0 \\
0 \\
0\end{array}$ & $\begin{array}{l}0 \\
0 \\
0 \\
0 \\
0 \\
0 \\
0 \\
0 \\
0 \\
0\end{array}$ \\
\hline $6-15$ & $\begin{array}{r}2 \\
5 \\
6 \\
7 \\
8 \\
9 \\
10 \\
11 \\
12\end{array}$ & $\begin{array}{r}4 \\
5 \\
6 \\
7 \\
8 \\
9 \\
10 \\
11 \\
15\end{array}$ & $\begin{array}{r}32++ \\
20++ \\
171 \\
244 \\
124 \\
255 \\
245 \\
1365 \\
202\end{array}$ & $\begin{array}{l}0 \\
0 \\
0 \\
1 \\
0 \\
1 \\
1 \\
0 \\
5\end{array}$ & $\begin{array}{l}0 \\
0 \\
0 \\
0 \\
0 \\
0 \\
0 \\
0 \\
4\end{array}$ & $\begin{array}{l}0 \\
0 \\
0 \\
0 \\
0 \\
1 \\
1 \\
0 \\
0\end{array}$ & $\begin{array}{l}0 \\
0 \\
0 \\
1 \\
0 \\
0 \\
0 \\
0 \\
0\end{array}$ & $\begin{array}{l}0 \\
0 \\
0 \\
0 \\
0 \\
0 \\
0 \\
0 \\
1\end{array}$ & $\begin{array}{l}0 \\
0 \\
0 \\
0 \\
0 \\
0 \\
0 \\
0 \\
0\end{array}$ \\
\hline $6-17$ & $\begin{array}{r}3 \\
7 \\
8 \\
9 \\
10 \\
12\end{array}$ & $\begin{array}{r}6 \\
7 \\
8 \\
9 \\
11 \\
15\end{array}$ & $\begin{array}{l}12+++ \\
107+ \\
294 \\
244 \\
1260 \\
155\end{array}$ & $\begin{array}{l}0 \\
0 \\
0 \\
0 \\
0 \\
9\end{array}$ & $\begin{array}{l}0 \\
0 \\
0 \\
0 \\
0 \\
5\end{array}$ & $\begin{array}{l}0 \\
0 \\
0 \\
0 \\
0 \\
4\end{array}$ & $\begin{array}{l}0 \\
0 \\
0 \\
0 \\
0 \\
0\end{array}$ & $\begin{array}{l}0 \\
0 \\
0 \\
0 \\
0 \\
0\end{array}$ & $\begin{array}{l}0 \\
0 \\
0 \\
0 \\
0 \\
0\end{array}$ \\
\hline
\end{tabular}


TABLE 21.- NUMBER OF GREEN TRACER PARTICLES(1), PER 100 GRAMS OF BEDLOAD SAMPLE, COLLECTED AT ONE OR MORE CROSS-CHANNEL SAMPLING POSITIONS SPACED AT 1-METER INTERVALS ACROSS THE CHANNEL WIDTH, EAST FORK RIVER, WYOMING, 1980--CONTINUED

SECTION 1533

\begin{tabular}{|c|c|c|c|c|c|c|c|c|c|}
\hline \multirow{3}{*}{ DATE } & & & \multirow{3}{*}{$\begin{array}{c}\text { DRY } \\
\text { MASS } \\
\text { OF } \\
\text { SAMPLE (2) } \\
(G)\end{array}$} & \multicolumn{6}{|c|}{ NUMBER OF GREEN TRACER PARTICLES } \\
\hline & \multirow{2}{*}{\multicolumn{2}{|c|}{$\begin{array}{l}\text { SAMPLING } \\
\text { POSITION } \\
\text { FROM TO }\end{array}$}} & & & \multicolumn{5}{|c|}{ BY SIZE CLASS (MM) } \\
\hline & & & & TOTAL & $\begin{array}{l}0.25 \\
\text { TO } \\
0.50\end{array}$ & $\begin{array}{l}0.50 \\
\text { TO } \\
1.00\end{array}$ & $\begin{array}{l}1.00 \\
2.00\end{array}$ & $\begin{array}{l}2.00 \\
\text { TO } \\
4.00\end{array}$ & $\begin{array}{l}4.00 \\
\text { TO } \\
8.00\end{array}$ \\
\hline $6-18$ & $\begin{array}{r}3 \\
5 \\
6 \\
7 \\
8 \\
9 \\
10 \\
12 \\
13\end{array}$ & $\begin{array}{r}4 \\
5 \\
6 \\
7 \\
8 \\
9 \\
11 \\
12 \\
15\end{array}$ & $\begin{array}{c}9+++ \\
6+++ \\
203 \\
1095 \\
1037 \\
192 \\
939 \\
523 \\
214\end{array}$ & $\begin{array}{r}0 \\
0 \\
11 \\
0 \\
1 \\
2 \\
9 \\
28 \\
26\end{array}$ & $\begin{array}{r}0 \\
0 \\
8 \\
0 \\
0 \\
0 \\
2 \\
22 \\
14\end{array}$ & $\begin{array}{r}0 \\
0 \\
2 \\
0 \\
0 \\
0 \\
6 \\
6 \\
10\end{array}$ & $\begin{array}{l}0 \\
0 \\
1 \\
0 \\
1 \\
1 \\
1 \\
0 \\
2\end{array}$ & $\begin{array}{l}0 \\
0 \\
0 \\
0 \\
0 \\
1 \\
0 \\
0 \\
0\end{array}$ & $\begin{array}{l}0 \\
0 \\
0 \\
0 \\
0 \\
0 \\
0 \\
0 \\
0\end{array}$ \\
\hline $6-19$ & $\begin{array}{r}3 \\
6 \\
10\end{array}$ & $\begin{array}{r}5 \\
9 \\
15\end{array}$ & $\begin{array}{l}111++ \\
481 \\
251\end{array}$ & $\begin{array}{r}0 \\
5 \\
27\end{array}$ & $\begin{array}{l}0 \\
0 \\
9\end{array}$ & $\begin{array}{r}0 \\
3 \\
15\end{array}$ & $\begin{array}{l}0 \\
2 \\
3\end{array}$ & $\begin{array}{l}0 \\
0 \\
0\end{array}$ & $\begin{array}{l}0 \\
0 \\
0\end{array}$ \\
\hline $6-20$ & $\begin{array}{r}3 \\
6 \\
8 \\
11\end{array}$ & $\begin{array}{r}5 \\
7 \\
10 \\
15\end{array}$ & $\begin{array}{l}10+++ \\
549 \\
385 \\
439\end{array}$ & $\begin{array}{l}0 \\
1 \\
2 \\
7\end{array}$ & $\begin{array}{l}0 \\
1 \\
1 \\
4\end{array}$ & $\begin{array}{l}0 \\
0 \\
1 \\
2\end{array}$ & $\begin{array}{l}0 \\
0 \\
0 \\
0\end{array}$ & $\begin{array}{l}0 \\
0 \\
0 \\
1\end{array}$ & $\begin{array}{l}0 \\
0 \\
0 \\
0\end{array}$ \\
\hline $6-21$ & $\begin{array}{r}3 \\
6 \\
7 \\
10 \\
12 \\
14\end{array}$ & $\begin{array}{r}5 \\
6 \\
9 \\
11 \\
13 \\
15\end{array}$ & $\begin{array}{l}102 \\
501 \\
328 \\
398 \\
658 \\
44++\end{array}$ & $\begin{array}{l}0 \\
0 \\
4 \\
1 \\
3 \\
0\end{array}$ & $\begin{array}{l}0 \\
0 \\
0 \\
0 \\
1 \\
0\end{array}$ & $\begin{array}{l}0 \\
0 \\
3 \\
1 \\
2 \\
0\end{array}$ & $\begin{array}{l}0 \\
0 \\
1 \\
0 \\
0 \\
0\end{array}$ & $\begin{array}{l}0 \\
0 \\
0 \\
0 \\
0 \\
0\end{array}$ & $\begin{array}{l}0 \\
0 \\
0 \\
0 \\
0 \\
0\end{array}$ \\
\hline $6-23$ & $\begin{array}{l}5 \\
9\end{array}$ & $\begin{array}{r}8 \\
15\end{array}$ & $\begin{array}{r}1045 \\
208\end{array}$ & $\begin{array}{l}0 \\
0\end{array}$ & $\begin{array}{l}0 \\
0\end{array}$ & $\begin{array}{l}0 \\
0\end{array}$ & $\begin{array}{l}0 \\
0\end{array}$ & $\begin{array}{l}0 \\
0\end{array}$ & $\begin{array}{l}0 \\
0\end{array}$ \\
\hline $6-25$ & $\begin{array}{r}3 \\
7 \\
8 \\
9 \\
11\end{array}$ & $\begin{array}{r}6 \\
7 \\
8 \\
10 \\
14\end{array}$ & $\begin{array}{l}34++ \\
366 \\
520 \\
761 \\
74++\end{array}$ & $\begin{array}{l}0 \\
0 \\
1 \\
1 \\
1\end{array}$ & $\begin{array}{l}0 \\
0 \\
0 \\
0 \\
1\end{array}$ & $\begin{array}{l}0 \\
0 \\
0 \\
0 \\
0\end{array}$ & $\begin{array}{l}0 \\
0 \\
1 \\
1 \\
0\end{array}$ & $\begin{array}{l}0 \\
0 \\
0 \\
0 \\
0\end{array}$ & $\begin{array}{l}0 \\
0 \\
0 \\
0 \\
0\end{array}$ \\
\hline $6-27$ & $\begin{array}{r}5 \\
7 \\
8 \\
9 \\
10 \\
11 \\
12\end{array}$ & $\begin{array}{r}6 \\
7 \\
8 \\
9 \\
10 \\
11 \\
14\end{array}$ & $\begin{array}{l}22++ \\
295 \\
704 \\
421 \\
770 \\
663 \\
191\end{array}$ & $\begin{array}{r}0 \\
1 \\
0 \\
1 \\
5 \\
2 \\
32\end{array}$ & $\begin{array}{r}0 \\
0 \\
0 \\
0 \\
2 \\
1 \\
27\end{array}$ & $\begin{array}{l}0 \\
0 \\
0 \\
1 \\
3 \\
1 \\
5\end{array}$ & $\begin{array}{l}0 \\
1 \\
0 \\
0 \\
0 \\
0 \\
0\end{array}$ & $\begin{array}{l}0 \\
0 \\
0 \\
0 \\
0 \\
0 \\
0\end{array}$ & $\begin{array}{l}0 \\
0 \\
0 \\
0 \\
0 \\
0 \\
0\end{array}$ \\
\hline
\end{tabular}


TABLE 21.- NUMBER OF GREEN TRACER PARTICLES(1), PER 100 GRAMS OF BEDLOAD SAMPLE, COLLECTED AT ONE OR MORE CROSS-CHANNEL SAMPLING POSITIONS SPACED AT I-METER INTERVALS ACROSS THE CHANNEL WIDTH, EAST FORK RIVER, WYOMING, 1980--CONTINUED

SECTION 1533

\begin{tabular}{|c|c|c|c|c|c|c|c|c|c|}
\hline \multirow{3}{*}{ DATE } & & & \multirow{3}{*}{$\begin{array}{c}\text { DRY } \\
\text { MASS } \\
\text { OF } \\
\text { SAMPLE (2) } \\
\text { (G) }\end{array}$} & \multicolumn{6}{|c|}{ NUMBER OF GREEN TRACER PARTICLES } \\
\hline & \multirow{2}{*}{\multicolumn{2}{|c|}{$\begin{array}{l}\text { SAMPLING } \\
\text { POSITION } \\
\text { FROM TO }\end{array}$}} & & \multirow[b]{2}{*}{ TOTAL } & \multicolumn{5}{|c|}{ BY SIZE CLASS (MM) } \\
\hline & & & & & $\begin{array}{l}0.25 \\
\text { TO } \\
0.50\end{array}$ & $\begin{array}{l}0.50 \\
\text { TO } \\
1.00\end{array}$ & $\begin{array}{l}1.00 \\
2.00\end{array}$ & $\begin{array}{l}2.00 \\
\text { TO } \\
4.00\end{array}$ & $\begin{array}{l}4.00 \\
\text { TO } \\
8.00\end{array}$ \\
\hline $7-03$ & $\begin{array}{r}5 \\
8 \\
10\end{array}$ & $\begin{array}{r}7 \\
9 \\
15\end{array}$ & $\begin{array}{l}442+ \\
480+ \\
121++\end{array}$ & $\begin{array}{l}0 \\
3 \\
4\end{array}$ & $\begin{array}{l}0 \\
0 \\
0\end{array}$ & $\begin{array}{l}0 \\
3 \\
4\end{array}$ & $\begin{array}{l}0 \\
0 \\
0\end{array}$ & $\begin{array}{l}0 \\
0 \\
0\end{array}$ & $\begin{array}{l}0 \\
0 \\
0\end{array}$ \\
\hline $7-05$ & $\begin{array}{r}6 \\
9 \\
11 \\
12\end{array}$ & $\begin{array}{r}8 \\
10 \\
11 \\
13\end{array}$ & $\begin{array}{l}540 \\
256++ \\
408+ \\
42+++\end{array}$ & $\begin{array}{l}1 \\
3 \\
5 \\
0\end{array}$ & $\begin{array}{l}0 \\
0 \\
0 \\
0\end{array}$ & $\begin{array}{l}0 \\
3 \\
4 \\
0\end{array}$ & $\begin{array}{l}1 \\
0 \\
1 \\
0\end{array}$ & $\begin{array}{l}0 \\
0 \\
0 \\
0\end{array}$ & $\begin{array}{l}0 \\
0 \\
0 \\
0\end{array}$ \\
\hline $7-07$ & $\begin{array}{r}6 \\
10\end{array}$ & $\begin{array}{r}99 \\
12\end{array}$ & $\begin{array}{l}62 \\
18++\end{array}$ & $\begin{array}{l}0 \\
0\end{array}$ & $\begin{array}{l}0 \\
0\end{array}$ & $\begin{array}{l}0 \\
0\end{array}$ & $\begin{array}{l}0 \\
0\end{array}$ & $\begin{array}{l}0 \\
0\end{array}$ & $\begin{array}{l}0 \\
0\end{array}$ \\
\hline
\end{tabular}


TABLE 21.- NUMBER OF GREEN TRACER PARTICLES(1), PER 100 GRAMS OF BEDLOAD SAMPLE, COLLECTED AT ONE OR MORE CROSS-CHANNEL SAMPLING POSITIONS SPACED AT I-METER INTERVALS ACROSS THE CHANNEL WIDTH, EAST FORK RIVER, WYOMING, 1980--CONTINUED

SECTION 1573

\begin{tabular}{|c|c|c|c|c|c|c|c|c|c|}
\hline \multicolumn{10}{|c|}{ NUMBER OF GREEN TRACER PARTICLES } \\
\hline \multirow[t]{2}{*}{ DATE } & \multicolumn{2}{|c|}{$\begin{array}{l}\text { SAMPLING } \\
\text { POSITION } \\
\text { FROM TO }\end{array}$} & \multirow{2}{*}{$\begin{array}{c}\text { DRY } \\
\text { MASS } \\
\text { OF } \\
\text { SAMPLE (2) } \\
\text { (G) }\end{array}$} & \multirow[b]{2}{*}{ TOTAL } & \multicolumn{5}{|c|}{ BY SIZE CLASS (MM) } \\
\hline & $\begin{array}{r}\text { FROM } \\
\text { (M) }\end{array}$ & (M) & & & $\begin{array}{l}0.25 \\
\text { TO } \\
0.50\end{array}$ & $\begin{array}{l}0.50 \\
\text { TO } \\
1.00\end{array}$ & $\begin{array}{l}1.00 \\
2.00\end{array}$ & $\begin{array}{l}2.00 \\
\text { TO } \\
4.00\end{array}$ & $\begin{array}{l}4.00 \\
\text { TO } \\
8.00\end{array}$ \\
\hline $6-01$ & $\begin{array}{r}8 \\
11\end{array}$ & $\begin{array}{l}10 \\
14\end{array}$ & $\underset{4+++}{89+}$ & $\begin{array}{l}0 \\
0\end{array}$ & $\begin{array}{l}0 \\
0\end{array}$ & $\begin{array}{l}0 \\
0\end{array}$ & $\begin{array}{l}0 \\
0\end{array}$ & $\begin{array}{l}0 \\
0\end{array}$ & $\begin{array}{l}0 \\
0\end{array}$ \\
\hline $6-03$ & $1 \frac{1}{2}$ & $\begin{array}{l}11 \\
14\end{array}$ & $\underset{5+++}{104+}$ & $\begin{array}{l}0 \\
0\end{array}$ & $\begin{array}{l}0 \\
0\end{array}$ & $\begin{array}{l}0 \\
0\end{array}$ & $\begin{array}{l}0 \\
0\end{array}$ & $\begin{array}{l}0 \\
0\end{array}$ & $\begin{array}{l}0 \\
0\end{array}$ \\
\hline $6-05$ & $1 \frac{1}{2}$ & $\begin{array}{l}11 \\
14\end{array}$ & $\begin{array}{l}45++ \\
0\end{array}$ & $\begin{array}{l}0 \\
0\end{array}$ & $\begin{array}{l}0 \\
0\end{array}$ & $\begin{array}{l}0 \\
0\end{array}$ & $\begin{array}{l}0 \\
0\end{array}$ & $\begin{array}{l}0 \\
0\end{array}$ & $\begin{array}{l}0 \\
0\end{array}$ \\
\hline $6-07$ & $\begin{array}{r}2 \\
12\end{array}$ & $\frac{11}{14}$ & $\begin{array}{l}37++ \\
22+++\end{array}$ & $\begin{array}{l}422 \\
113\end{array}$ & $\begin{array}{r}214 \\
50\end{array}$ & $\begin{array}{r}200 \\
63\end{array}$ & $\begin{array}{l}8 \\
0\end{array}$ & $\begin{array}{l}0 \\
0\end{array}$ & $\begin{array}{l}0 \\
0\end{array}$ \\
\hline $6-09$ & $\begin{array}{r}2 \\
7 \\
10\end{array}$ & $\begin{array}{r}6 \\
10 \\
14\end{array}$ & $\begin{array}{l}53+ \\
1387 \\
1066\end{array}$ & $\begin{array}{r}0 \\
6 \\
173\end{array}$ & $\begin{array}{r}0 \\
1 \\
95\end{array}$ & $\begin{array}{r}0 \\
4 \\
71\end{array}$ & $\begin{array}{l}0 \\
0 \\
7\end{array}$ & $\begin{array}{l}0 \\
0 \\
0\end{array}$ & $\begin{array}{l}0 \\
1 \\
0\end{array}$ \\
\hline $6-10$ & $\begin{array}{r}2 \\
7 \\
11\end{array}$ & $\begin{array}{r}6 \\
10 \\
14\end{array}$ & $\begin{array}{l}48++ \\
1094 \\
1255\end{array}$ & $\begin{array}{r}20 \\
20 \\
189\end{array}$ & $\begin{array}{r}0 \\
10 \\
102\end{array}$ & $\begin{array}{r}0 \\
7 \\
74\end{array}$ & $\begin{array}{r}0 \\
2 \\
13\end{array}$ & $\begin{array}{l}0 \\
1 \\
0\end{array}$ & $\begin{array}{l}0 \\
0 \\
0\end{array}$ \\
\hline $6-11$ & $\begin{array}{r}2 \\
8 \\
9 \\
11\end{array}$ & $\begin{array}{r}7 \\
8 \\
10 \\
14\end{array}$ & $\begin{array}{l}331 \\
503 \\
39++ \\
316\end{array}$ & $\begin{array}{r}0 \\
0 \\
3 \\
22\end{array}$ & $\begin{array}{r}0 \\
0 \\
0 \\
10\end{array}$ & $\begin{array}{r}0 \\
0 \\
3 \\
11\end{array}$ & $\begin{array}{l}0 \\
0 \\
0 \\
1\end{array}$ & $\begin{array}{l}0 \\
0 \\
0 \\
0\end{array}$ & $\begin{array}{l}0 \\
0 \\
0 \\
0\end{array}$ \\
\hline $6-12$ & $\begin{array}{r}3 \\
8 \\
9 \\
10 \\
11 \\
12 \\
13\end{array}$ & $\begin{array}{r}7 \\
8 \\
9 \\
10 \\
11 \\
12 \\
14\end{array}$ & $\begin{array}{c}185 \\
746 \\
830 \\
517 \\
481 \\
1201 \\
131++\end{array}$ & $\begin{array}{l}0 \\
0 \\
0 \\
0 \\
8 \\
2 \\
2\end{array}$ & $\begin{array}{l}0 \\
0 \\
0 \\
0 \\
3 \\
1 \\
0\end{array}$ & $\begin{array}{l}0 \\
0 \\
0 \\
0 \\
5 \\
0 \\
2\end{array}$ & $\begin{array}{l}0 \\
0 \\
0 \\
0 \\
0 \\
1 \\
0\end{array}$ & $\begin{array}{l}0 \\
0 \\
0 \\
0 \\
0 \\
0 \\
0\end{array}$ & $\begin{array}{l}0 \\
0 \\
0 \\
0 \\
0 \\
0 \\
0\end{array}$ \\
\hline $6-13$ & $\begin{array}{r}3 \\
7 \\
9 \\
10 \\
11 \\
12 \\
13 \\
14\end{array}$ & $\begin{array}{r}6 \\
8 \\
9 \\
10 \\
11 \\
12 \\
13 \\
14\end{array}$ & $\begin{array}{c}42++ \\
332 \\
209 \\
1516 \\
783 \\
277 \\
269 \\
59++\end{array}$ & $\begin{array}{l}0 \\
0 \\
0 \\
0 \\
0 \\
0 \\
0 \\
0\end{array}$ & $\begin{array}{l}0 \\
0 \\
0 \\
0 \\
0 \\
0 \\
0 \\
0\end{array}$ & $\begin{array}{l}0 \\
0 \\
0 \\
0 \\
0 \\
0 \\
0 \\
0\end{array}$ & $\begin{array}{l}0 \\
0 \\
0 \\
0 \\
0 \\
0 \\
0 \\
0\end{array}$ & $\begin{array}{l}0 \\
0 \\
0 \\
0 \\
0 \\
0 \\
0 \\
0\end{array}$ & $\begin{array}{l}0 \\
0 \\
0 \\
0 \\
0 \\
0 \\
0 \\
0\end{array}$ \\
\hline
\end{tabular}


TABLE 21. - NUMBER OF GREEN TRACER PARTICLES(1), PER 100 GRAMS OF BEDLOAD SAMPLE, COLLECTED AT ONE OR MORE CROSS-CHANNEL SAMPLING POSITIONS SPACED AT 1-METER INTERVALS ACROSS THE CHANNEL WIDTH, EAST FORK RIVER, WYOMING, 1980--CONTINUED

SECTION 1573

\begin{tabular}{|c|c|c|c|c|c|c|c|c|c|}
\hline \multirow{3}{*}{ DATE } & \multirow{3}{*}{\multicolumn{2}{|c|}{$\begin{array}{l}\text { SAMPLING } \\
\text { POSITION } \\
\text { FROM TO }\end{array}$}} & \multirow{3}{*}{$\begin{array}{c}\text { DRY } \\
\text { MASS } \\
\text { OF } \\
\text { SAMPLE (2) } \\
(G)\end{array}$} & \multicolumn{6}{|c|}{ NUMBER OF GREEN TRACER PARTICLES } \\
\hline & & & & \multirow[b]{2}{*}{ TOTAL } & \multicolumn{5}{|c|}{ BY SIZE CLASS (MM) } \\
\hline & & & & & $\begin{array}{l}0.25 \\
\text { TO } \\
0.50\end{array}$ & $\begin{array}{l}0.50 \\
\text { TO } \\
1.00\end{array}$ & $\begin{array}{l}1.00 \\
2.00\end{array}$ & $\begin{array}{l}2.00 \\
\text { TO } \\
4.00\end{array}$ & $\begin{array}{l}4.00 \\
\text { TO } \\
8.00\end{array}$ \\
\hline $6-14$ & $\begin{array}{r}5 \\
7 \\
8 \\
9 \\
10 \\
11 \\
12 \\
13 \\
14\end{array}$ & $\begin{array}{r}6 \\
7 \\
8 \\
9 \\
10 \\
11 \\
12 \\
13 \\
14\end{array}$ & $\begin{array}{c}283 \\
382 \\
1527 \\
945 \\
69+ \\
508 \\
572 \\
476 \\
370\end{array}$ & $\begin{array}{l}0 \\
0 \\
0 \\
1 \\
0 \\
2 \\
0 \\
1 \\
2\end{array}$ & $\begin{array}{l}0 \\
0 \\
0 \\
1 \\
0 \\
2 \\
0 \\
0 \\
1\end{array}$ & $\begin{array}{l}0 \\
0 \\
0 \\
0 \\
0 \\
0 \\
0 \\
1 \\
1\end{array}$ & $\begin{array}{l}0 \\
0 \\
0 \\
0 \\
0 \\
0 \\
0 \\
0 \\
0\end{array}$ & $\begin{array}{l}0 \\
0 \\
0 \\
0 \\
0 \\
0 \\
0 \\
0 \\
0\end{array}$ & $\begin{array}{l}0 \\
0 \\
0 \\
0 \\
0 \\
0 \\
0 \\
0 \\
0\end{array}$ \\
\hline $6-15$ & $\begin{array}{r}2 \\
5 \\
7 \\
8 \\
9 \\
10 \\
12 \\
13\end{array}$ & $\begin{array}{r}4 \\
6 \\
7 \\
8 \\
9 \\
11 \\
12 \\
14\end{array}$ & $\begin{array}{c}5+++ \\
22++ \\
119 \\
594 \\
49++ \\
30++ \\
629 \\
56++\end{array}$ & $\begin{array}{l}0 \\
0 \\
0 \\
0 \\
0 \\
0 \\
2 \\
0\end{array}$ & $\begin{array}{l}0 \\
0 \\
0 \\
0 \\
0 \\
0 \\
1 \\
0\end{array}$ & $\begin{array}{l}0 \\
0 \\
0 \\
0 \\
0 \\
0 \\
1 \\
0\end{array}$ & $\begin{array}{l}0 \\
0 \\
0 \\
0 \\
0 \\
0 \\
0 \\
0\end{array}$ & $\begin{array}{l}0 \\
0 \\
0 \\
0 \\
0 \\
0 \\
0 \\
0\end{array}$ & $\begin{array}{l}0 \\
0 \\
0 \\
0 \\
0 \\
0 \\
0 \\
0\end{array}$ \\
\hline $6-17$ & $\begin{array}{r}3 \\
5 \\
7 \\
8 \\
9 \\
10 \\
11 \\
12 \\
13 \\
14\end{array}$ & $\begin{array}{r}4 \\
6 \\
7 \\
8 \\
9 \\
10 \\
11 \\
12 \\
13 \\
14\end{array}$ & $\begin{array}{c}11+++ \\
16+++ \\
743 \\
894 \\
916 \\
1274 \\
544 \\
660 \\
467 \\
62++\end{array}$ & $\begin{array}{r}0 \\
0 \\
0 \\
0 \\
1 \\
2 \\
4 \\
7 \\
11 \\
8\end{array}$ & $\begin{array}{l}0 \\
0 \\
0 \\
0 \\
0 \\
0 \\
3 \\
1 \\
7 \\
2\end{array}$ & $\begin{array}{l}0 \\
0 \\
0 \\
0 \\
1 \\
2 \\
1 \\
6 \\
4 \\
6\end{array}$ & $\begin{array}{l}0 \\
0 \\
0 \\
0 \\
0 \\
0 \\
0 \\
0 \\
0 \\
0\end{array}$ & $\begin{array}{l}0 \\
0 \\
0 \\
0 \\
0 \\
0 \\
0 \\
0 \\
0 \\
0\end{array}$ & $\begin{array}{l}0 \\
0 \\
0 \\
0 \\
0 \\
0 \\
0 \\
0 \\
0 \\
0\end{array}$ \\
\hline $6-18$ & $\begin{array}{r}2 \\
7 \\
9 \\
10 \\
11 \\
14\end{array}$ & $\begin{array}{r}6 \\
8 \\
9 \\
10 \\
13 \\
15\end{array}$ & $\begin{array}{c}226 \\
569 \\
635 \\
1649 \\
113+ \\
7+++\end{array}$ & $\begin{array}{r}0 \\
3 \\
6 \\
9 \\
48 \\
0\end{array}$ & $\begin{array}{r}0 \\
0 \\
1 \\
1 \\
15 \\
0\end{array}$ & $\begin{array}{r}0 \\
2 \\
5 \\
7 \\
32 \\
0\end{array}$ & $\begin{array}{l}0 \\
0 \\
0 \\
1 \\
0 \\
0\end{array}$ & $\begin{array}{l}0 \\
1 \\
0 \\
0 \\
0 \\
0\end{array}$ & $\begin{array}{l}0 \\
0 \\
0 \\
0 \\
1 \\
0\end{array}$ \\
\hline $6-19$ & $\begin{array}{r}2 \\
9 \\
12\end{array}$ & $\begin{array}{r}8 \\
11 \\
14\end{array}$ & $\begin{array}{r}215 \\
1011 \\
132\end{array}$ & $\begin{array}{r}0 \\
5 \\
14\end{array}$ & $\begin{array}{l}0 \\
3 \\
9\end{array}$ & $\begin{array}{l}0 \\
1 \\
4\end{array}$ & $\begin{array}{l}0 \\
1 \\
1\end{array}$ & $\begin{array}{l}0 \\
0 \\
0\end{array}$ & $\begin{array}{l}0 \\
0 \\
0\end{array}$ \\
\hline
\end{tabular}


TABLE 21.- NUMBER OF GREEN TRACER PARTICLES(1), PER 100 GRAMS OF BEDLOAD SAMPLE, COLLECTED AT ONE OR MORE CROSS-CHANNEL SAMPLING POSITIONS SPACED AT 1-METER INTERVALS ACROSS THE CHANNEL WIDTH, EAST FORK RIVER, WYOMING, 1980--CONTINUED

SECTION 1573

\begin{tabular}{|c|c|c|c|c|c|c|c|c|c|}
\hline \multirow{3}{*}{ DATE } & \multirow{3}{*}{\multicolumn{2}{|c|}{$\begin{array}{l}\text { SAMPLING } \\
\text { POSITION } \\
\text { FROM TO }\end{array}$}} & \multirow{3}{*}{$\begin{array}{c}\text { DRY } \\
\text { MASS } \\
\text { OF } \\
\text { SAMPLE (2) } \\
\text { (G) }\end{array}$} & \multicolumn{6}{|c|}{ NUMBER OF GREEN TRACER PARTICLES } \\
\hline & & & & \multirow[b]{2}{*}{ TOTAL } & \multicolumn{5}{|c|}{ BY SIZE CLASS (MM) } \\
\hline & & & & & $\begin{array}{l}0.25 \\
\text { TO } \\
0.50\end{array}$ & $\begin{array}{l}0.50 \\
\text { TO } \\
1.00\end{array}$ & $\begin{array}{l}1.00 \\
\text { TO } \\
2.00\end{array}$ & $\begin{array}{l}2.00 \\
\text { TO } \\
4.00\end{array}$ & $\begin{array}{l}4.00 \\
\text { TO } \\
8.00\end{array}$ \\
\hline $6-20$ & $\begin{array}{r}1 \\
8 \\
9 \\
12\end{array}$ & $\begin{array}{r}7 \\
8 \\
11 \\
14\end{array}$ & $\begin{array}{r}799 \\
1300 \\
1626 \\
544\end{array}$ & $\begin{array}{l}0 \\
0 \\
1 \\
3\end{array}$ & $\begin{array}{l}0 \\
0 \\
0 \\
1\end{array}$ & $\begin{array}{l}0 \\
0 \\
1 \\
2\end{array}$ & $\begin{array}{l}0 \\
0 \\
0 \\
0\end{array}$ & $\begin{array}{l}0 \\
0 \\
0 \\
0\end{array}$ & $\begin{array}{l}0 \\
0 \\
0 \\
0\end{array}$ \\
\hline $6-21$ & $\begin{array}{r}3 \\
8 \\
9 \\
10 \\
11 \\
12\end{array}$ & $\begin{array}{r}7 \\
8 \\
9 \\
10 \\
11 \\
14\end{array}$ & $\begin{array}{l}17++ \\
617 \\
936 \\
735 \\
878 \\
155+\end{array}$ & $\begin{array}{l}0 \\
0 \\
2 \\
1 \\
0 \\
0\end{array}$ & $\begin{array}{l}0 \\
0 \\
2 \\
0 \\
0 \\
0\end{array}$ & $\begin{array}{l}0 \\
0 \\
0 \\
1 \\
0 \\
0\end{array}$ & $\begin{array}{l}0 \\
0 \\
0 \\
0 \\
0 \\
0\end{array}$ & $\begin{array}{l}0 \\
0 \\
0 \\
0 \\
0 \\
0\end{array}$ & $\begin{array}{l}0 \\
0 \\
0 \\
0 \\
0 \\
0\end{array}$ \\
\hline $6-23$ & $\begin{array}{r}1 \\
8 \\
10 \\
13\end{array}$ & $\begin{array}{r}7 \\
9 \\
12 \\
14\end{array}$ & $\begin{array}{c}46++ \\
1261 \\
458 \\
83++\end{array}$ & $\begin{array}{l}0 \\
0 \\
0 \\
0\end{array}$ & $\begin{array}{l}0 \\
0 \\
0 \\
0\end{array}$ & $\begin{array}{l}0 \\
0 \\
0 \\
0\end{array}$ & $\begin{array}{l}0 \\
0 \\
0 \\
0\end{array}$ & $\begin{array}{l}0 \\
0 \\
0 \\
0\end{array}$ & $\begin{array}{l}0 \\
0 \\
0 \\
0\end{array}$ \\
\hline $6-25$ & $\begin{array}{l}2 \\
12\end{array}$ & $\begin{array}{l}10 \\
11 \\
14\end{array}$ & $\begin{array}{r}1295 \\
500 \\
396\end{array}$ & $\begin{array}{l}0 \\
3 \\
5\end{array}$ & $\begin{array}{l}0 \\
0 \\
4\end{array}$ & $\begin{array}{l}0 \\
3 \\
1\end{array}$ & $\begin{array}{l}0 \\
0 \\
0\end{array}$ & $\begin{array}{l}0 \\
0 \\
0\end{array}$ & $\begin{array}{l}0 \\
0 \\
0\end{array}$ \\
\hline $6-27$ & $\begin{array}{r}5 \\
8 \\
9 \\
10 \\
11 \\
12 \\
13\end{array}$ & $\begin{array}{r}7 \\
8 \\
9 \\
10 \\
11 \\
12 \\
14\end{array}$ & $\begin{array}{l}5^{+++} \\
345 \\
317 \\
507 \\
646 \\
323 \\
413\end{array}$ & $\begin{array}{r}0 \\
0 \\
2 \\
3 \\
17 \\
0 \\
27\end{array}$ & $\begin{array}{r}0 \\
0 \\
0 \\
0 \\
3 \\
0 \\
11\end{array}$ & $\begin{array}{r}0 \\
0 \\
2 \\
2 \\
12 \\
0 \\
13\end{array}$ & $\begin{array}{l}0 \\
0 \\
0 \\
1 \\
2 \\
0 \\
3\end{array}$ & $\begin{array}{l}0 \\
0 \\
0 \\
0 \\
0 \\
0 \\
0\end{array}$ & $\begin{array}{l}0 \\
0 \\
0 \\
0 \\
0 \\
0 \\
0\end{array}$ \\
\hline $7-05$ & $\begin{array}{r}8 \\
12\end{array}$ & $\begin{array}{l}11 \\
14\end{array}$ & $\begin{array}{l}139++ \\
130+\end{array}$ & $\begin{array}{l}0 \\
4\end{array}$ & $\begin{array}{l}0 \\
0\end{array}$ & $\begin{array}{l}0 \\
3\end{array}$ & $\begin{array}{l}0 \\
1\end{array}$ & $\begin{array}{l}0 \\
0\end{array}$ & $\begin{array}{l}0 \\
0\end{array}$ \\
\hline $7-07$ & $\begin{array}{r}8 \\
11\end{array}$ & $\begin{array}{l}10 \\
14\end{array}$ & $\begin{array}{c}341+ \\
51++\end{array}$ & $\begin{array}{l}0 \\
8\end{array}$ & $\begin{array}{l}0 \\
4\end{array}$ & $\begin{array}{l}0 \\
4\end{array}$ & $\begin{array}{l}0 \\
0\end{array}$ & $\begin{array}{l}0 \\
0\end{array}$ & $\begin{array}{l}0 \\
0\end{array}$ \\
\hline
\end{tabular}


TABLE 21.- NUMBER OF GREEN TRACER PARTICLES(1), PER 100 GRAMS OF BEDLOAD SAMPLE, COLLECTED AT ONE OR MORE CROSS-CHANNEL SAMPLING POSITIONS SPACED AT I-METER INTERVALS ACROSS THE CHANNEL WIDTH, EAST FORK RIVER, WYOMING, 1980--CONTINUED

SECTION 1610

\begin{tabular}{|c|c|c|c|c|c|c|c|c|c|}
\hline \multirow{3}{*}{ DATE } & \multirow{3}{*}{\multicolumn{2}{|c|}{$\begin{array}{l}\text { SAMPLING } \\
\text { POSITION } \\
\text { FROM TO TO }\end{array}$}} & \multirow{3}{*}{$\begin{array}{c}\text { DRY } \\
\text { MASS } \\
\text { OF } \\
\text { SAMPLE (2) } \\
\text { (G) }\end{array}$} & \multicolumn{6}{|c|}{ NUMBER OF GREEN TRACER PARTICLES } \\
\hline & & & & \multirow[b]{2}{*}{ TOTAL } & \multicolumn{5}{|c|}{ BY SIZE CLASS (MM) } \\
\hline & & & & & $\begin{array}{l}0.25 \\
\text { TO } \\
0.50\end{array}$ & $\begin{array}{l}0.50 \\
\text { TO } \\
1.00\end{array}$ & $\begin{array}{l}1.00 \\
2.00\end{array}$ & $\begin{array}{l}2.00 \\
\text { TO } \\
4.00\end{array}$ & $\begin{array}{l}4.00 \\
\text { TO } \\
8.00\end{array}$ \\
\hline $6-01$ & 2 & 18 & 286 & 34 & 20 & 10 & 3 & 0 & 1 \\
\hline $6-03$ & $\begin{array}{r}2 \\
16\end{array}$ & $\begin{array}{l}15 \\
17\end{array}$ & $\begin{array}{c}161+ \\
0+++\end{array}$ & $\begin{array}{l}0 \\
0\end{array}$ & $\begin{array}{l}0 \\
0\end{array}$ & $\begin{array}{l}0 \\
0\end{array}$ & $\begin{array}{l}0 \\
0\end{array}$ & $\begin{array}{l}0 \\
0\end{array}$ & $\begin{array}{l}0 \\
0\end{array}$ \\
\hline $6-05$ & $\begin{array}{r}2 \\
16\end{array}$ & $\begin{array}{l}15 \\
17\end{array}$ & $\begin{array}{c}91+ \\
0+++\end{array}$ & $\begin{array}{l}0 \\
0\end{array}$ & $\begin{array}{l}0 \\
0\end{array}$ & $\begin{array}{l}0 \\
0\end{array}$ & $\begin{array}{l}0 \\
0\end{array}$ & $\begin{array}{l}0 \\
0\end{array}$ & $\begin{array}{l}0 \\
0\end{array}$ \\
\hline $6-07$ & $\begin{array}{r}2 \\
16\end{array}$ & $\begin{array}{l}15 \\
18\end{array}$ & $\begin{array}{l}1026 \\
55++\end{array}$ & 509 & $\begin{array}{r}0 \\
127\end{array}$ & 311 & $\begin{array}{r}0 \\
69\end{array}$ & $\begin{array}{l}0 \\
2\end{array}$ & $\begin{array}{l}0 \\
0\end{array}$ \\
\hline $6-09$ & $\begin{array}{r}2 \\
11\end{array}$ & $\begin{array}{l}10 \\
18\end{array}$ & $\begin{array}{r}67+ \\
2637^{+}\end{array}$ & $\begin{array}{r}0 \\
35\end{array}$ & $\begin{array}{r}0 \\
19\end{array}$ & $\begin{array}{r}0 \\
13\end{array}$ & $\begin{array}{l}0 \\
3\end{array}$ & $\begin{array}{l}0 \\
0\end{array}$ & $\begin{array}{l}0 \\
0\end{array}$ \\
\hline $6-10$ & $\begin{array}{r}3 \\
12 \\
13 \\
14 \\
16 \\
17\end{array}$ & $\begin{array}{l}12 \\
12 \\
13 \\
15 \\
16 \\
18\end{array}$ & $\begin{array}{r}1796 \\
1649 \\
1379 \\
560 \\
1282 \\
468\end{array}$ & $\begin{array}{l}1 \\
0 \\
0 \\
0 \\
0 \\
0\end{array}$ & $\begin{array}{l}1 \\
0 \\
0 \\
0 \\
0 \\
0\end{array}$ & $\begin{array}{l}0 \\
0 \\
0 \\
0 \\
0 \\
0\end{array}$ & $\begin{array}{l}0 \\
0 \\
0 \\
0 \\
0 \\
0\end{array}$ & $\begin{array}{l}0 \\
0 \\
0 \\
0 \\
0 \\
0\end{array}$ & $\begin{array}{l}0 \\
0 \\
0 \\
0 \\
0 \\
0\end{array}$ \\
\hline $6-11$ & $\begin{array}{r}2 \\
13 \\
15\end{array}$ & $\begin{array}{l}12 \\
14 \\
17\end{array}$ & $\begin{array}{r}1882 \\
1720 \\
459\end{array}$ & $\begin{array}{l}1 \\
0 \\
0\end{array}$ & $\begin{array}{l}1 \\
0 \\
0\end{array}$ & $\begin{array}{l}0 \\
0 \\
0\end{array}$ & $\begin{array}{l}0 \\
0 \\
0\end{array}$ & $\begin{array}{l}0 \\
0 \\
0\end{array}$ & $\begin{array}{l}0 \\
0 \\
0\end{array}$ \\
\hline $6-12$ & $\begin{array}{r}3 \\
14\end{array}$ & $\begin{array}{l}13 \\
18\end{array}$ & $\begin{array}{r}1067 \\
524\end{array}$ & $\begin{array}{l}0 \\
0\end{array}$ & $\begin{array}{l}0 \\
0\end{array}$ & $\begin{array}{l}0 \\
0\end{array}$ & $\begin{array}{l}0 \\
0\end{array}$ & $\begin{array}{l}0 \\
0\end{array}$ & $\begin{array}{l}0 \\
0\end{array}$ \\
\hline $6-13$ & $\begin{array}{r}4 \\
16\end{array}$ & $\begin{array}{l}15 \\
18\end{array}$ & $\begin{array}{r}1659 \\
197\end{array}$ & $\begin{array}{l}0 \\
0\end{array}$ & $\begin{array}{l}0 \\
0\end{array}$ & $\begin{array}{l}0 \\
0\end{array}$ & $\begin{array}{l}0 \\
0\end{array}$ & $\begin{array}{l}0 \\
0\end{array}$ & $\begin{array}{l}0 \\
0\end{array}$ \\
\hline $6-14$ & $\begin{array}{l}3 \\
11 \\
12 \\
13 \\
14 \\
15 \\
16 \\
17 \\
18\end{array}$ & $\begin{array}{l}10 \\
11 \\
12 \\
13 \\
14 \\
15 \\
16 \\
17 \\
18\end{array}$ & $\begin{array}{c}83+ \\
64+ \\
56++ \\
986 \\
516 \\
857 \\
11+++ \\
204 \\
84++\end{array}$ & $\begin{array}{l}0 \\
0 \\
0 \\
0 \\
0 \\
0 \\
0 \\
0 \\
0\end{array}$ & $\begin{array}{l}0 \\
0 \\
0 \\
0 \\
0 \\
0 \\
0 \\
0 \\
0\end{array}$ & $\begin{array}{l}0 \\
0 \\
0 \\
0 \\
0 \\
0 \\
0 \\
0 \\
0\end{array}$ & $\begin{array}{l}0 \\
0 \\
0 \\
0 \\
0 \\
0 \\
0 \\
0 \\
0\end{array}$ & $\begin{array}{l}0 \\
0 \\
0 \\
0 \\
0 \\
0 \\
0 \\
0 \\
0\end{array}$ & $\begin{array}{l}0 \\
0 \\
0 \\
0 \\
0 \\
0 \\
0 \\
0 \\
0\end{array}$ \\
\hline $6-15$ & $\begin{array}{r}2 \\
16\end{array}$ & $\begin{array}{l}15 \\
18\end{array}$ & $\begin{array}{l}1984 \\
1518\end{array}$ & $\begin{array}{l}0 \\
0\end{array}$ & $\begin{array}{l}0 \\
0\end{array}$ & $\begin{array}{l}0 \\
0\end{array}$ & $\begin{array}{l}0 \\
0\end{array}$ & $\begin{array}{l}0 \\
0\end{array}$ & $\begin{array}{l}0 \\
0\end{array}$ \\
\hline
\end{tabular}


TABLE 21.- NUMBER OF GREEN TRACER PARTICLES(1), PER 100 GRAMS OF BEDLOAD SAMPLE, COLLECTED AT ONE OR MORE CRÓSS-CHANNEL SAMPLING POSITIONS SPACED AT 1-METER INTERVALS ACROSS THE CHANNEL WIDTH, EAST FORK RIVER, WYOMING, 1980--CONTINUED

SECTION 1610

\begin{tabular}{|c|c|c|c|c|c|c|c|c|c|}
\hline \multirow{3}{*}{ DATE } & \multirow{3}{*}{\multicolumn{2}{|c|}{$\begin{array}{l}\text { SAMPLING } \\
\text { POSITION } \\
\text { FROM TO }\end{array}$}} & \multirow{3}{*}{$\begin{array}{c}\text { DRY } \\
\text { MASS } \\
\text { OF } \\
\text { SAMPLE (2) } \\
\text { (G) }\end{array}$} & \multicolumn{6}{|c|}{ NUMBER OF GREEN TRACER PARTICLES } \\
\hline & & & & \multirow[b]{2}{*}{ TOTAL } & \multicolumn{5}{|c|}{ BY SIZE CLASS (MM) } \\
\hline & & & & & $\begin{array}{l}0.25 \\
\text { TO } \\
0.50\end{array}$ & $\begin{array}{l}0.50 \\
\text { TO } \\
1.00\end{array}$ & $\begin{array}{l}1.00 \\
2.00\end{array}$ & $\begin{array}{l}2.00 \\
\text { TO } \\
4.00\end{array}$ & $\begin{array}{l}4.00 \\
\text { TO } \\
8.00\end{array}$ \\
\hline $6-17$ & $\begin{array}{r}4 \\
16\end{array}$ & $\begin{array}{l}15 \\
18\end{array}$ & $\begin{array}{r}3876 \\
208\end{array}$ & $\begin{array}{l}0 \\
2\end{array}$ & $\begin{array}{l}0 \\
1\end{array}$ & $\begin{array}{l}0 \\
1\end{array}$ & $\begin{array}{l}0 \\
0\end{array}$ & $\begin{array}{l}0 \\
0\end{array}$ & $\begin{array}{l}0 \\
0\end{array}$ \\
\hline $6-18$ & $\begin{array}{r}2 \\
9 \\
12 \\
13 \\
14 \\
15 \\
16 \\
17\end{array}$ & $\begin{array}{l}8 \\
11 \\
12 \\
13 \\
14 \\
15 \\
16 \\
18\end{array}$ & $\begin{array}{l}17+++ \\
466 \\
176 \\
401 \\
430 \\
539 \\
396 \\
17++\end{array}$ & $\begin{array}{l}0 \\
0 \\
0 \\
0 \\
0 \\
0 \\
0 \\
0\end{array}$ & $\begin{array}{l}0 \\
0 \\
0 \\
0 \\
0 \\
0 \\
0 \\
0\end{array}$ & $\begin{array}{l}0 \\
0 \\
0 \\
0 \\
0 \\
0 \\
0 \\
0\end{array}$ & $\begin{array}{l}0 \\
0 \\
0 \\
0 \\
0 \\
0 \\
0 \\
0\end{array}$ & $\begin{array}{l}0 \\
0 \\
0 \\
0 \\
0 \\
0 \\
0 \\
0\end{array}$ & $\begin{array}{l}0 \\
0 \\
0 \\
0 \\
0 \\
0 \\
0 \\
0\end{array}$ \\
\hline $6-19$ & $\begin{array}{r}3 \\
16\end{array}$ & $\begin{array}{l}15 \\
18\end{array}$ & $\begin{array}{r}1072 \\
622\end{array}$ & $\begin{array}{l}0 \\
0\end{array}$ & $\begin{array}{l}0 \\
0\end{array}$ & $\begin{array}{l}0 \\
0\end{array}$ & $\begin{array}{l}0 \\
0\end{array}$ & $\begin{array}{l}0 \\
0\end{array}$ & $\begin{array}{l}0 \\
0\end{array}$ \\
\hline $6-20$ & $\begin{array}{r}3 \\
16\end{array}$ & $\begin{array}{l}15 \\
18\end{array}$ & $\begin{array}{l}1134 \\
119+\end{array}$ & $\begin{array}{l}0 \\
0\end{array}$ & $\begin{array}{l}0 \\
0\end{array}$ & $\begin{array}{l}0 \\
0\end{array}$ & $\begin{array}{l}0 \\
0\end{array}$ & $\begin{array}{l}0 \\
0\end{array}$ & $\begin{array}{l}0 \\
0\end{array}$ \\
\hline $6-21$ & $\begin{array}{r}3 \\
16\end{array}$ & $\begin{array}{l}15 \\
18\end{array}$ & $\begin{array}{r}388 \\
1613\end{array}$ & $\begin{array}{l}0 \\
0\end{array}$ & $\begin{array}{l}0 \\
0\end{array}$ & $\begin{array}{l}0 \\
0\end{array}$ & $\begin{array}{l}0 \\
0\end{array}$ & $\begin{array}{l}0 \\
0\end{array}$ & $\begin{array}{l}0 \\
0\end{array}$ \\
\hline $6-23$ & $\begin{array}{r}2 \\
16\end{array}$ & $\begin{array}{l}15 \\
18\end{array}$ & $\begin{array}{r}1327 \\
66++\end{array}$ & $\begin{array}{l}0 \\
0\end{array}$ & $\begin{array}{l}0 \\
0\end{array}$ & $\begin{array}{l}0 \\
0\end{array}$ & $\begin{array}{l}0 \\
0\end{array}$ & $\begin{array}{l}0 \\
0\end{array}$ & $\begin{array}{l}0 \\
0\end{array}$ \\
\hline $6-25$ & 2 & 18 & 553 & 0 & 0 & 0 & 0 & 0 & 0 \\
\hline $6-27$ & $\begin{array}{l}6 \\
11 \\
14 \\
15 \\
16\end{array}$ & $\begin{array}{l}10 \\
13 \\
14 \\
15 \\
17\end{array}$ & $\begin{array}{l}22++ \\
247 \\
761 \\
493 \\
79+\end{array}$ & $\begin{array}{l}0 \\
1 \\
0 \\
0 \\
0\end{array}$ & $\begin{array}{l}0 \\
0 \\
0 \\
0 \\
0\end{array}$ & $\begin{array}{l}0 \\
1 \\
0 \\
0 \\
0\end{array}$ & $\begin{array}{l}0 \\
0 \\
0 \\
0 \\
0\end{array}$ & $\begin{array}{l}0 \\
0 \\
0 \\
0 \\
0\end{array}$ & $\begin{array}{l}0 \\
0 \\
0 \\
0 \\
0\end{array}$ \\
\hline $7-01$ & $\begin{array}{l}11 \\
12 \\
13\end{array}$ & $\begin{array}{l}11 \\
12 \\
17\end{array}$ & $\begin{array}{l}476 \\
807 \\
377+\end{array}$ & $\begin{array}{l}0 \\
0 \\
3\end{array}$ & $\begin{array}{l}0 \\
0 \\
2\end{array}$ & $\begin{array}{l}0 \\
0 \\
1\end{array}$ & $\begin{array}{l}0 \\
0 \\
0\end{array}$ & $\begin{array}{l}0 \\
0 \\
0\end{array}$ & $\begin{array}{l}0 \\
0 \\
0\end{array}$ \\
\hline $7-05$ & 9 & 17 & $374+$ & 0 & 0 & 0 & 0 & 0 & 0 \\
\hline $7-07$ & 12 & 17 & $6+++$ & 0 & 0 & 0 & 0 & 0 & 0 \\
\hline
\end{tabular}


TABLE 21.- NUMBER OF GREEN TRACER PARTICLES(1), PER 100 GRAMS OF BEDLOAD SAMPLE, COLLECTED AT ONE OR MORE CROSS-CHANNEL SAMPLING POSITIONS SPACED AT I-METER INTERVALS ACROSS THE CHANNEL WIDTH, EAST FORK RIVER, WYOMING, 1980--CONTINUED

(1) GREEN TRACER PARTICLES PLACED AS BED MATERIAL 42 METERS DOWNSTREAM OF SECTION 1662 ON MAY 31, 1980.

(2) REPRESENTS TOTAL MASS, IN' GRAMS, RETAINED IN BEDLOAD SAMPLER AS MEASURED IN THE FIELD: FOR SUBSEQUENT ANALYSIS OF NUMBER AND SIZE OF TRACER, PARTICLES SMALLER THAN 0.25 MM AND LARGER THAN $8.0 \mathrm{MM}$ WERE DISCARDED. REMAINING SAMPLES WEIGHING MORE THAN 100 GRAMS WERE CUT TO A STANDARD 100 GRAM-PORTION; SAMPLES WEIGHING LESS THAN 100 GRAMS WERE ANALYZED INTACT.

(3) DOES NOT INCLUDE TRACER PARTICLES MEASURED IN TRANSPORT AT SECTION 1400, A BYPASS CHANNEL COMMON TO SECTIONS 1360, 1396, AND 1425. SEE FIGURE 3.

$+\quad$ MASS OF SAMPLE ANALYZED WAS BETWEEN 50 AND 100 GRAMS; NUMBER OF TRACER PARTICLES ADJUSTED TO AN EQUIVALENT 100-GRAM SAMPLE; EXTRAPOLATED NUMBERS ARE CONSIDERED RELIABLE.

+ MASS OF SAMPLE ANALYZED WAS BETWEEN 10 AND 50 GRAMS; NUMBER OF TRACER PARTICLES ADJUSTED TO AN EQUIVALENT 100-GRAM SAMPLE; CARE SHOULD BE TAKEN IN USING THE EXTRAPOLATED NUMBERS.

+++ MASS OF SAMPLE ANALYZED WAS BETWEEN 0 AND 10 GRAMS: NUMBER OF TRACER PARTICLES ADJUSTED TO AN EQUIVALENT 100-GRAM SAMPLE; EXTRAPOLATED NUMBERS ARE CONSIDERED RELIABLE. 
TABLE 22.- NUMBER OF PINK TRACER PARTICLES(1), PER 100 GRAMS OF BED MATERIAL SAMPLE, EAST FORK RIVER, WYOMING, 1982

\begin{tabular}{|c|c|c|c|c|c|c|c|}
\hline \multirow{3}{*}{ SECTION } & \multirow{3}{*}{ DATE } & & MBER C & PINK ? & ER P & ICLES & \\
\hline & & \multirow[b]{2}{*}{ TOTAL } & \multicolumn{5}{|c|}{ BY SIZE CLASS (MM) } \\
\hline & & & $\begin{array}{l}0.25 \\
\text { TO } \\
0.50\end{array}$ & $\begin{array}{l}0.50 \\
\text { TO } \\
1.00\end{array}$ & $\begin{array}{l}1.00 \\
\text { TO } \\
2.00\end{array}$ & $\begin{array}{l}2.00 \\
\text { TO } \\
4.00\end{array}$ & $\begin{array}{l}4.00 \\
\text { TO } \\
8.00\end{array}$ \\
\hline \multirow{4}{*}{$\begin{array}{l}0043 \\
0075 \\
0137 \\
0178 \\
0220\end{array}$} & \multirow{4}{*}{$\begin{array}{l}8-20 \\
8-20 \\
8-20\end{array}$} & -- & -- & - & -- & - & - \\
\hline & & -- & $-\overline{-}$ & $-\bar{c}$ & $-\overline{0}$ & $\overline{0}$ & $-\overline{-}$ \\
\hline & & $\frac{1}{9}$ & $\frac{1}{0}$ & 0 & $\begin{array}{l}0 \\
0\end{array}$ & $\begin{array}{l}0 \\
0\end{array}$ & $\begin{array}{l}0 \\
0\end{array}$ \\
\hline & & $\frac{1}{0}$ & 0 & 0 & 0 & 0 & 0 \\
\hline \multirow{4}{*}{$\begin{array}{l}0257 \\
0257(2) \\
0301 \\
0301(2) \\
0348\end{array}$} & \multirow{4}{*}{$\begin{array}{l}8-20 \\
8-20 \\
8-20 \\
8-20 \\
8-21\end{array}$} & 4 & 0 & 2 & 2 & 0 & 0 \\
\hline & & 15 & 2 & 8 & 5 & 0 & $\begin{array}{l}0 \\
0\end{array}$ \\
\hline & & 8 & $\frac{1}{0}$ & 5 & $\frac{1}{3}$ & 0 & 0 \\
\hline & & 0 & 0 & 0 & 0 & 0 & 0 \\
\hline \multirow{4}{*}{$\begin{array}{l}0421 \\
0460 \\
0516 \\
0556 \\
0602\end{array}$} & \multirow{4}{*}{$\begin{array}{l}8-21 \\
8-21 \\
8-21 \\
8-23 \\
8-23\end{array}$} & 0 & 0 & 0 & 0 & 0 & 0 \\
\hline & & 0 & 0 & $\underset{\sigma}{0}$ & 0 & $\stackrel{0}{0}$ & 0 \\
\hline & & 6 & $\frac{1}{0}$ & $\begin{array}{l}6 \\
2\end{array}$ & 1 & $\begin{array}{l}0 \\
3\end{array}$ & 0 \\
\hline & & 6 & 0 & 2 & 4 & 0 & 0 \\
\hline \multirow{4}{*}{$\begin{array}{l}0653 \\
0653(2) \\
0708 \\
0757 \\
0808\end{array}$} & \multirow{4}{*}{$\begin{array}{l}8-23 \\
8-23 \\
8-23 \\
8-23\end{array}$} & 0 & 0 & 0 & 0 & 0 & 0 \\
\hline & & 3 & 2 & $\frac{1}{1}$ & 0 & 0 & $\begin{array}{l}0 \\
0\end{array}$ \\
\hline & & 18 & 10 & $\frac{1}{8}$ & 0 & 0 & 0 \\
\hline & & $=$ & $=-$ & - & -- & - & - \\
\hline \multirow{4}{*}{$\begin{array}{l}0808(2) \\
0853 \\
0898 \\
0940 \\
0985\end{array}$} & $8-23$ & 13 & 8 & 5 & 0 & 0 & 0 \\
\hline & $8-23$ & 0 & 0 & 0 & 0 & 0 & 0 \\
\hline & $8-23$ & 3 & 1 & 2 & 0 & 0 & 0 \\
\hline & $8-23$ & 3 & $\overline{0}$ & $\overline{3}$ & 0 & 0 & 0 \\
\hline \multirow{4}{*}{$\begin{array}{l}1038 \\
1077 \\
1120 \\
1155 \\
1202\end{array}$} & \multirow{4}{*}{$\begin{array}{l}8-23 \\
8-24 \\
8-24 \\
8-24\end{array}$} & 3 & 0 & 1 & 2 & 0 & 0 \\
\hline & & 2 & 0 & 2 & 0 & 0 & 0 \\
\hline & & 8 & 0 & 1 & $\frac{1}{5}$ & 2 & 0 \\
\hline & & & & & . & & \\
\hline \multirow{4}{*}{$\begin{array}{l}1241 \\
1284 \\
1315 \\
1360 \\
1396\end{array}$} & $8-24$ & 17 & 6 & 9 & 1 & 0 & 1 \\
\hline & & & 1 & 2 & 2 & 0 & 0 \\
\hline & $\begin{array}{l}8-24 \\
8-24\end{array}$ & $\begin{array}{l}0 \\
3\end{array}$ & $\overline{0}$ & $\overline{0}$ & $\overline{0}$ & 0 & 0 \\
\hline & & 3 & 1 & 0 & 0 & 2 & 0 \\
\hline
\end{tabular}


TABLE 22.- NUMBER OF PINK TRACER PARTICLES(1), PER 100 GRAMS OF BED MATERIAL SAMPLE, EAST FORK RIVER, WYOMING, 1982--CONTINUED

\begin{tabular}{|c|c|c|c|c|c|c|c|}
\hline \multirow{3}{*}{ SECTION } & \multirow{3}{*}{ DATE } & \multicolumn{6}{|c|}{ NUMBER OF PINK TRACER PARTICLES } \\
\hline & & \multirow[b]{2}{*}{ TOTAL } & \multicolumn{5}{|c|}{ BY SIZE CLASS (MM) } \\
\hline & & & $\begin{array}{l}0.25 \\
\text { To } \\
0.50\end{array}$ & $\begin{array}{l}0.50 \\
\text { To } \\
1.00\end{array}$ & $\begin{array}{l}1.00 \\
2.00 \\
2.00\end{array}$ & $\begin{array}{r}2.00 \\
\text { To } \\
4.00\end{array}$ & $\begin{array}{r}4.00 \\
\text { TO } \\
8.00\end{array}$ \\
\hline $\begin{array}{l}1400(3) \\
1425 \\
1481 \\
1481(2) \\
1533\end{array}$ & $\begin{array}{l}8-24 \\
8-24 \\
8-24 \\
8-24 \\
8-24\end{array}$ & $\begin{array}{l}3 \\
0 \\
0 \\
0 \\
3\end{array}$ & $\begin{array}{l}1 \\
0 \\
0 \\
0 \\
0\end{array}$ & $\begin{array}{l}0 \\
0 \\
0 \\
0 \\
0\end{array}$ & $\begin{array}{l}2 \\
0 \\
0 \\
0 \\
3\end{array}$ & $\begin{array}{l}0 \\
0 \\
0 \\
0 \\
0\end{array}$ & $\begin{array}{l}0 \\
0 \\
0 \\
0 \\
0\end{array}$ \\
\hline $\begin{array}{l}1573 \\
1610 \\
1662 \\
1695 \\
1730\end{array}$ & $\begin{array}{l}8-24 \\
8-22 \\
8-22 \\
8-22 \\
8-22\end{array}$ & $\begin{array}{l}2 \\
7 \\
0 \\
1 \\
0\end{array}$ & $\begin{array}{l}0 \\
2 \\
0 \\
0 \\
0\end{array}$ & $\begin{array}{l}2 \\
4 \\
0 \\
0 \\
0\end{array}$ & $\begin{array}{l}0 \\
1 \\
0 \\
1 \\
0\end{array}$ & $\begin{array}{l}0 \\
0 \\
0 \\
0 \\
0\end{array}$ & $\begin{array}{l}0 \\
0 \\
0 \\
0 \\
0\end{array}$ \\
\hline $\begin{array}{l}1766 \\
1800 \\
1830\end{array}$ & $\begin{array}{l}8-22 \\
8-22 \\
8-22\end{array}$ & $\begin{array}{l}2 \\
1 \\
0\end{array}$ & $\begin{array}{l}0 \\
0 \\
0\end{array}$ & $\begin{array}{l}2 \\
0 \\
0\end{array}$ & $\begin{array}{l}0 \\
1 \\
0\end{array}$ & $\begin{array}{l}0 \\
0 \\
0\end{array}$ & $\begin{array}{l}0 \\
0 \\
0\end{array}$ \\
\hline
\end{tabular}

(1) PINK TRACER PARTICLES PLACED AS BED MATERIAL 10 METERS DOWNSTREAM

(2) SM SECTION 3047 ON MAY 19 ' 1979.

(3) BYPASS CHANNEL. SEE FIGURE 3. 
TABLE 23.- NUMBER OF BLUE TRACER PARTICLES(1), PER 100 GRAMS OF BED MATERIAL SAMPLE, EAST FORK RIVER, WYOMING, 1982

\begin{tabular}{|c|c|c|c|c|c|c|c|}
\hline \multirow{3}{*}{ SECTION } & \multirow{3}{*}{ DATE } & \multicolumn{6}{|c|}{ NUMBER OF BLUE TRACER PARTICLES } \\
\hline & & \multirow[b]{2}{*}{ TOTAL } & \multicolumn{5}{|c|}{ BY SIZE CLASS (MM) } \\
\hline & & & $\begin{array}{l}0.25 \\
\text { TO } \\
0.50\end{array}$ & $\begin{array}{l}0.50 \\
\text { TO } \\
1.00\end{array}$ & $\begin{array}{l}1.00 \\
\text { TO } \\
2.00\end{array}$ & $\begin{array}{l}2.00 \\
\text { TO } \\
4.00\end{array}$ & $\begin{array}{l}4.00 \\
\text { TO } \\
8.00\end{array}$ \\
\hline $\begin{array}{l}0043 \\
0075 \\
0137 \\
0178 \\
0220\end{array}$ & $\begin{array}{l}8-20 \\
8-20 \\
8-20\end{array}$ & $\begin{array}{c}-- \\
-\frac{1}{1} \\
1 \\
0\end{array}$ & $\begin{array}{c}-- \\
-\overline{0} \\
0 \\
0\end{array}$ & $\begin{array}{c}-- \\
-\frac{1}{1} \\
0\end{array}$ & $\begin{array}{c}-- \\
-\overline{0} \\
0 \\
0\end{array}$ & $\begin{array}{c}-- \\
-\overline{0} \\
0 \\
0\end{array}$ & $\begin{array}{c}-- \\
-\overline{0} \\
0 \\
0\end{array}$ \\
\hline $\begin{array}{l}0257 \\
0257(2) \\
0301 \\
0301(2) \\
0348\end{array}$ & $\begin{array}{l}8-20 \\
8-20 \\
8-20 \\
8-20 \\
8-21\end{array}$ & $\begin{array}{l}2 \\
8 \\
3 \\
9 \\
1\end{array}$ & $\begin{array}{l}2 \\
1 \\
2 \\
5 \\
1\end{array}$ & $\begin{array}{l}0 \\
6 \\
0 \\
4 \\
0\end{array}$ & $\begin{array}{l}0 \\
1 \\
0 \\
0 \\
0\end{array}$ & $\begin{array}{l}0 \\
0 \\
1 \\
0 \\
0\end{array}$ & $\begin{array}{l}0 \\
0 \\
0 \\
0 \\
0\end{array}$ \\
\hline $\begin{array}{l}0421 \\
0460 \\
0516 \\
0556 \\
0602\end{array}$ & $\begin{array}{l}8-21 \\
8-21 \\
8-21 \\
8-23 \\
8-23\end{array}$ & $\begin{array}{l}0 \\
0 \\
2 \\
0 \\
1\end{array}$ & $\begin{array}{l}0 \\
0 \\
2 \\
0 \\
0\end{array}$ & $\begin{array}{l}0 \\
0 \\
0 \\
0 \\
0\end{array}$ & $\begin{array}{l}0 \\
0 \\
0 \\
0 \\
1\end{array}$ & $\begin{array}{l}0 \\
0 \\
0 \\
0 \\
0\end{array}$ & $\begin{array}{l}0 \\
0 \\
0 \\
0 \\
0\end{array}$ \\
\hline $\begin{array}{l}0653 \\
0653(2) \\
0708 \\
0757 \\
0808\end{array}$ & $\begin{array}{l}8-23 \\
8-23 \\
8-23 \\
8-23\end{array}$ & $\begin{array}{r}0 \\
2 \\
4 \\
14 \\
--\end{array}$ & $\begin{array}{r}0 \\
0 \\
3 \\
8 \\
--\end{array}$ & $\begin{array}{r}0 \\
2 \\
1 \\
6 \\
--\end{array}$ & $\begin{array}{r}0 \\
0 \\
0 \\
0 \\
--\end{array}$ & $\begin{array}{r}0 \\
0 \\
0 \\
0 \\
--\end{array}$ & $\begin{array}{r}0 \\
0 \\
0 \\
0 \\
--\end{array}$ \\
\hline $\begin{array}{l}0808(2) \\
0853\end{array}$ & $\begin{array}{l}8-23 \\
8-23\end{array}$ & $\begin{array}{l}8 \\
8\end{array}$ & $\begin{array}{l}7 \\
5\end{array}$ & $\begin{array}{l}0 \\
3\end{array}$ & $\begin{array}{l}1 \\
0\end{array}$ & $\begin{array}{l}0 \\
0\end{array}$ & $\begin{array}{l}0 \\
0\end{array}$ \\
\hline $\begin{array}{l}0940 \\
0985\end{array}$ & $\begin{array}{l}8-23 \\
8-23\end{array}$ & $\begin{array}{l}1 \\
0\end{array}$ & $\begin{array}{l}0 \\
0\end{array}$ & $\begin{array}{l}0 \\
0\end{array}$ & $\begin{array}{l}1 \\
0\end{array}$ & $\begin{array}{l}0 \\
0\end{array}$ & $\begin{array}{l}0 \\
0\end{array}$ \\
\hline $\begin{array}{l}1038 \\
1077 \\
1120 \\
1155 \\
1202\end{array}$ & $\begin{array}{l}8-23 \\
8-24 \\
8-24 \\
8-24\end{array}$ & $\begin{array}{r}0 \\
1 \\
0 \\
1 \\
--\end{array}$ & $\begin{array}{r}0 \\
0 \\
0 \\
0 \\
--\end{array}$ & $\begin{array}{r}0 \\
1 \\
0 \\
1 \\
--\end{array}$ & $\begin{array}{r}0 \\
0 \\
0 \\
0 \\
--\end{array}$ & $\begin{array}{r}0 \\
0 \\
0 \\
0 \\
--\end{array}$ & $\begin{array}{l}0 \\
0 \\
0 \\
0 \\
--\end{array}$ \\
\hline & $8-24$ & 1 & $\underline{0}$ & 1 & $\underline{0}$ & 0 & $\underline{0}$ \\
\hline $\begin{array}{l}1315 \\
1360 \\
1396\end{array}$ & $\begin{array}{l}8-24 \\
8-24 \\
8-24\end{array}$ & $\begin{array}{l}2 \\
0 \\
0\end{array}$ & $\begin{array}{l}0 \\
0 \\
0\end{array}$ & $\begin{array}{l}2 \\
0 \\
0\end{array}$ & $\begin{array}{l}0 \\
0 \\
0\end{array}$ & $\begin{array}{l}0 \\
0 \\
0\end{array}$ & $\begin{array}{l}0 \\
0 \\
0\end{array}$ \\
\hline
\end{tabular}


TABLE 23.- NUMBER OF BLUE TRACER PARTICLES(1), PER 100 GRAMS OF BED MATERIAL SAMPLE, EAST FORK RIVER, WYOMING, 1982--CONTINUED

\begin{tabular}{|c|c|c|c|c|c|c|c|}
\hline \multirow{3}{*}{ SECTION } & \multirow{3}{*}{ DATE } & \multicolumn{6}{|c|}{ NUMBER OF BLUE TRACER PARTICLES } \\
\hline & & \multirow[b]{2}{*}{ TOTAL } & \multicolumn{5}{|c|}{ BY SIZE CLASS (MM) } \\
\hline & & & $\begin{array}{l}0.25 \\
\text { TO } \\
0.50\end{array}$ & $\begin{array}{l}0.50 \\
\text { To } \\
1.00\end{array}$ & $\begin{array}{l}1.00 \\
\text { TO } \\
2.00\end{array}$ & $\begin{array}{l}2.00 \\
\text { TO } \\
4.00\end{array}$ & $\begin{array}{l}4.00 \\
\text { TO } \\
8.00\end{array}$ \\
\hline $\begin{array}{l}1400(3) \\
1425 \\
1481 \\
1481(2) \\
1533\end{array}$ & $\begin{array}{l}8-24 \\
8-24 \\
8-24 \\
8-24 \\
8-24\end{array}$ & $\begin{array}{l}0 \\
0 \\
2 \\
2 \\
0\end{array}$ & $\begin{array}{l}0 \\
0 \\
0 \\
0 \\
0\end{array}$ & $\begin{array}{l}0 \\
0 \\
2 \\
0 \\
0\end{array}$ & $\begin{array}{l}0 \\
0 \\
0 \\
2 \\
0\end{array}$ & $\begin{array}{l}0 \\
0 \\
0 \\
0 \\
0\end{array}$ & $\begin{array}{l}0 \\
0 \\
0 \\
0 \\
0\end{array}$ \\
\hline $\begin{array}{l}1573 \\
1610 \\
1662\end{array}$ & $\begin{array}{l}8-24 \\
8-22 \\
8-22\end{array}$ & $\begin{array}{l}0 \\
0 \\
0\end{array}$ & $\begin{array}{l}0 \\
0 \\
0\end{array}$ & $\begin{array}{l}0 \\
0 \\
0\end{array}$ & $\begin{array}{l}0 \\
0 \\
0\end{array}$ & $\begin{array}{l}0 \\
0 \\
0\end{array}$ & $\begin{array}{l}0 \\
0 \\
0\end{array}$ \\
\hline
\end{tabular}

(1) BLUE TRACER PARTICLES PLACED AS BED MATERIAL 10 METERS DOWNSTREAM

(2) SMALL OVERFLOW CHANNEL ADJACENT TO SECTION.

(3) BYPASS CHANNEL. SEE FIGURE 3 . 
TABLE 24.- NUMBER OF ORANGE TRACER PARTICLES(1), PER 100 GRAMS OF BED MATERIAL SAMPLE, EAST FORK RIVER, WYOMING, 1982

\begin{tabular}{|c|c|c|c|c|c|c|c|}
\hline \multirow{3}{*}{ SECTION } & \multirow{3}{*}{ DATE } & \multicolumn{6}{|c|}{ NUMBER OF ORANGE TRACER PARTICLES } \\
\hline & & \multirow[b]{2}{*}{ TOTAL } & \multicolumn{5}{|c|}{ BY SIZE CLASS (MM) } \\
\hline & & & $\begin{array}{l}0.25 \\
\text { TO } \\
0.50\end{array}$ & $\begin{array}{l}0.50 \\
\text { TO } \\
1.00\end{array}$ & $\begin{array}{l}1.00 \\
\text { TO } \\
2.00\end{array}$ & $\begin{array}{l}2.00 \\
\text { TO } \\
4.00\end{array}$ & $\begin{array}{l}4.00 \\
\text { TO } \\
8.00\end{array}$ \\
\hline $\begin{array}{l}0043 \\
0075 \\
0137 \\
0178 \\
0220\end{array}$ & $\begin{array}{l}8-20 \\
8-20 \\
8-20\end{array}$ & $\begin{array}{l}=- \\
\overline{16} \\
14 \\
16\end{array}$ & $\begin{array}{l}-- \\
\overline{16} \\
13 \\
15\end{array}$ & $\begin{array}{c}-- \\
-\overline{0} \\
1 \\
1\end{array}$ & $\begin{array}{c}-- \\
-\overline{0} \\
0 \\
0\end{array}$ & $\begin{array}{c}-- \\
-\overline{0} \\
0 \\
0\end{array}$ & $\begin{array}{c}-- \\
-\overline{0} \\
0 \\
0\end{array}$ \\
\hline $\begin{array}{l}0257 \\
0257(2) \\
0301 \\
0301(2) \\
0348\end{array}$ & $\begin{array}{l}8-20 \\
8-20 \\
8-20 \\
8-20 \\
8-21\end{array}$ & $\begin{array}{r}9 \\
0 \\
11 \\
2 \\
0\end{array}$ & $\begin{array}{l}8 \\
0 \\
7 \\
0 \\
0\end{array}$ & $\begin{array}{l}1 \\
0 \\
4 \\
2 \\
0\end{array}$ & $\begin{array}{l}0 \\
0 \\
0 \\
0 \\
0\end{array}$ & $\begin{array}{l}0 \\
0 \\
0 \\
0 \\
0\end{array}$ & $\begin{array}{l}0 \\
0 \\
0 \\
0 \\
0\end{array}$ \\
\hline $\begin{array}{l}0421 \\
0460\end{array}$ & $\begin{array}{l}8-21 \\
8-21\end{array}$ & $\begin{array}{l}0 \\
0\end{array}$ & $\begin{array}{l}0 \\
0\end{array}$ & $\begin{array}{l}0 \\
0\end{array}$ & $\begin{array}{l}0 \\
0\end{array}$ & $\begin{array}{l}0 \\
0\end{array}$ & $\begin{array}{l}0 \\
0\end{array}$ \\
\hline
\end{tabular}

(1) ORANGE TRACER PARTICLES PLACED AS BED MATERIAL 10 METERS DOWNSTREAM (2) SMALL OVERFLOW CHANNEL ADJACENT TO SECTION. 
TABLE 25.- NUMBER OF GREEN TRACER PARTICLES(1), PER 100 GRAMS OF BED MATERIAL SAMPLE, EAST FORK RIVER, WYOMING, 1982

\begin{tabular}{|c|c|c|c|c|c|c|c|}
\hline \multirow{3}{*}{ SECTION } & \multirow{3}{*}{ DATE } & \multicolumn{6}{|c|}{ NUMBER OF GREEN TRACER PARTICLES } \\
\hline & & \multirow[b]{2}{*}{ TOTAL } & \multicolumn{4}{|c|}{ BY SIZE CLASS (MM) } & \multirow[b]{2}{*}{$\begin{array}{c}4.00 \\
\text { TO } \\
8.00\end{array}$} \\
\hline & & & $\begin{array}{l}0.25 \\
\text { TO } \\
0.50\end{array}$ & $\begin{array}{l}0.50 \\
\text { TO } \\
1.00\end{array}$ & $\begin{array}{l}1.00 \\
2.00\end{array}$ & $\begin{array}{l}2.00 \\
\text { TO } \\
4.00\end{array}$ & \\
\hline $\begin{array}{l}0043 \\
0075 \\
0137 \\
0178 \\
0220\end{array}$ & $\begin{array}{l}8-20 \\
8-20 \\
8-20\end{array}$ & $\begin{array}{c}-- \\
-\overline{0} \\
0 \\
0\end{array}$ & $\begin{array}{c}-- \\
-\overline{0} \\
0 \\
0\end{array}$ & $\begin{array}{c}-- \\
-\overline{0} \\
0 \\
0\end{array}$ & $\begin{array}{c}-- \\
-\overline{0} \\
0 \\
0\end{array}$ & $\begin{array}{l}-- \\
-\overline{0} \\
0 \\
0\end{array}$ & $\begin{array}{c}-- \\
-\overline{0} \\
0 \\
0\end{array}$ \\
\hline $\begin{array}{l}0257 \\
0257(2) \\
0301 \\
0301(2) \\
0348\end{array}$ & $\begin{array}{l}8-20 \\
8-20 \\
8-20 \\
8-20 \\
8-21\end{array}$ & $\begin{array}{l}0 \\
0 \\
0 \\
0 \\
0\end{array}$ & $\begin{array}{l}0 \\
0 \\
0 \\
0 \\
0\end{array}$ & $\begin{array}{l}0 \\
0 \\
0 \\
0 \\
0\end{array}$ & $\begin{array}{l}0 \\
0 \\
0 \\
0 \\
0\end{array}$ & $\begin{array}{l}0 \\
0 \\
0 \\
0 \\
0\end{array}$ & $\begin{array}{l}0 \\
0 \\
0 \\
0 \\
0\end{array}$ \\
\hline $\begin{array}{l}0421 \\
0460 \\
0516 \\
0556 \\
0602\end{array}$ & $\begin{array}{l}8-21 \\
8-21 \\
8-21 \\
8-23 \\
8-23\end{array}$ & $\begin{array}{l}0 \\
0 \\
0 \\
0 \\
0\end{array}$ & $\begin{array}{l}0 \\
0 \\
0 \\
0 \\
0\end{array}$ & $\begin{array}{l}0 \\
0 \\
0 \\
0 \\
0\end{array}$ & $\begin{array}{l}0 \\
0 \\
0 \\
0 \\
0\end{array}$ & $\begin{array}{l}0 \\
0 \\
0 \\
0 \\
0\end{array}$ & $\begin{array}{l}0 \\
0 \\
0 \\
0 \\
0\end{array}$ \\
\hline $\begin{array}{l}0653 \\
0653(2) \\
0708 \\
0757 \\
0808\end{array}$ & $\begin{array}{l}8-23 \\
8-23 \\
8-23 \\
8-23\end{array}$ & $\begin{array}{r}0 \\
0 \\
0 \\
0 \\
--\end{array}$ & $\begin{array}{r}0 \\
0 \\
0 \\
0 \\
--\end{array}$ & $\begin{array}{r}0 \\
0 \\
0 \\
0 \\
--\end{array}$ & $\begin{array}{r}0 \\
0 \\
0 \\
0 \\
--\end{array}$ & $\begin{array}{r}0 \\
0 \\
0 \\
0 \\
--\end{array}$ & $\begin{array}{r}0 \\
0 \\
0 \\
0 \\
--\end{array}$ \\
\hline $\begin{array}{l}0808(2) \\
0853\end{array}$ & $\begin{array}{l}8-23 \\
8-23\end{array}$ & $\begin{array}{l}0 \\
0\end{array}$ & $\begin{array}{r}0 \\
0 \\
-\end{array}$ & $\begin{array}{l}0 \\
0\end{array}$ & $\begin{array}{l}0 \\
0\end{array}$ & $\begin{array}{l}0 \\
0\end{array}$ & $\begin{array}{l}0 \\
0\end{array}$ \\
\hline $\begin{array}{l}0940 \\
0985\end{array}$ & $\begin{array}{l}8-23 \\
8-23\end{array}$ & $\begin{array}{l}0 \\
0\end{array}$ & $\begin{array}{l}0 \\
0\end{array}$ & $\begin{array}{l}0 \\
0\end{array}$ & $\begin{array}{l}0 \\
0\end{array}$ & $\begin{array}{l}0 \\
0\end{array}$ & $\begin{array}{l}0 \\
0\end{array}$ \\
\hline $\begin{array}{l}1038 \\
1077 \\
1120 \\
1155 \\
1202\end{array}$ & $\begin{array}{l}8-23 \\
8-24 \\
8-24 \\
8-24\end{array}$ & $\begin{array}{r}0 \\
0 \\
0 \\
0 \\
--\end{array}$ & $\begin{array}{r}0 \\
0 \\
0 \\
0 \\
--\end{array}$ & $\begin{array}{r}0 \\
0 \\
0 \\
0 \\
--\end{array}$ & $\begin{array}{r}0 \\
0 \\
0 \\
0 \\
--\end{array}$ & $\begin{array}{r}0 \\
0 \\
0 \\
0 \\
--\end{array}$ & $\begin{array}{r}0 \\
0 \\
0 \\
0 \\
--\end{array}$ \\
\hline 1241 & $8-24$ & $-\underline{0}$ & -0 & -0 & $\underline{0}$ & $-\underline{0}$ & $\underline{0}$ \\
\hline $\begin{array}{l}1315 \\
1360 \\
1396\end{array}$ & $\begin{array}{l}8-24 \\
8-24 \\
8-24\end{array}$ & $\begin{array}{l}0 \\
0 \\
0\end{array}$ & $\begin{array}{l}0 \\
0 \\
0\end{array}$ & $\begin{array}{l}0 \\
0 \\
0\end{array}$ & $\begin{array}{l}0 \\
0 \\
0\end{array}$ & $\begin{array}{l}0 \\
0 \\
0\end{array}$ & $\begin{array}{l}0 \\
0 \\
0\end{array}$ \\
\hline
\end{tabular}


TABLE 25.- NUMBER OF GREEN TRACER PARTICLES(1), PER 100 GRAMS OF BED MATERIAL SAMPLE, EAST FORK RIVER, WYOMING, 1982--CONTINUED

\begin{tabular}{|c|c|c|c|c|c|c|c|}
\hline \multirow{3}{*}{ SECTION } & \multirow{3}{*}{ DATE } & \multicolumn{6}{|c|}{ NUMBER OF GREEN TRFCER PARTICLES } \\
\hline & & \multirow[b]{2}{*}{ TOTAL } & \multicolumn{5}{|c|}{ BY SIZE CLASS (MM) } \\
\hline & & & $\begin{array}{l}0.25 \\
\text { TO } \\
0.50\end{array}$ & $\begin{array}{l}0.50 \\
\text { TO } \\
1.00\end{array}$ & $\begin{array}{l}1.00 \\
\text { TO } \\
2.00\end{array}$ & $\begin{array}{l}2.00 \\
\text { TO } \\
4.00\end{array}$ & $\begin{array}{l}4.00 \\
\text { TO } \\
8.00\end{array}$ \\
\hline $\begin{array}{l}1400(3) \\
1425 \\
1481 \\
1481(2) \\
1533\end{array}$ & $\begin{array}{l}8-24 \\
8-24 \\
8-24 \\
8-24 \\
8-24\end{array}$ & $\begin{array}{l}0 \\
0 \\
0 \\
0 \\
0\end{array}$ & $\begin{array}{l}0 \\
0 \\
0 \\
0 \\
0\end{array}$ & $\begin{array}{l}0 \\
0 \\
0 \\
0 \\
0\end{array}$ & $\begin{array}{l}0 \\
0 \\
0 \\
0 \\
0\end{array}$ & $\begin{array}{l}0 \\
0 \\
0 \\
0 \\
0\end{array}$ & $\begin{array}{l}0 \\
0 \\
0 \\
0 \\
0\end{array}$ \\
\hline $\begin{array}{l}1573 \\
1610\end{array}$ & $\begin{array}{l}8-24 \\
8-22\end{array}$ & $\begin{array}{l}0 \\
0\end{array}$ & $\begin{array}{l}0 \\
0\end{array}$ & $\begin{array}{l}0 \\
0\end{array}$ & $\begin{array}{l}0 \\
0\end{array}$ & $\begin{array}{l}0 \\
0\end{array}$ & $\begin{array}{l}0 \\
0\end{array}$ \\
\hline
\end{tabular}

(1) GREEN TRACER PARTICLES PLACED AS BED MATERIAL 42 METERS DOWNSTREAM

(2) SMALL OVERFLOW CHANNEL ADJACENT TO SECTION.

(3) BYPASS CHANNEL. SEE FIGURE 3 . 